\title{
Frit Development for High Level Waste Sludge Batch 5: Compositional Trends for Varying Aluminum Concentrations
}

K. M. Fox

T. B. Edwards

D. R. Best

I. A. Reamer

R. J. Workman

August 2008

Environmental \& Chemical Process Technology Savannah River National Laboratory Aiken, SC 29808

This document was prepared in conjunction with work accomplished under Contract No. DE-AC09-08SR22470 with the U.S. Department of Energy.

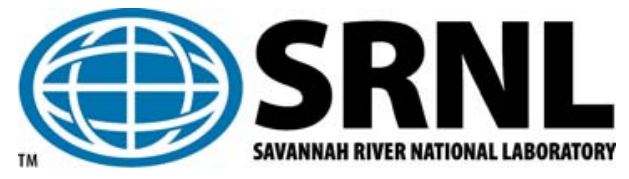


SRNS-STI-2008-00060

Revision 0

\section{DISCLAIMER}

This work was prepared under an agreement with and funded by the U.S. Government. Neither the U.S. Government or its employees, nor any of its contractors, subcontractors or their employees, makes any express or implied: 1 . warranty or assumes any legal liability for the accuracy, completeness, or for the use or results of such use of any information, product, or process disclosed; or 2. representation that such use or results of such use would not infringe privately owned rights; or 3. endorsement or recommendation of any specifically identified commercial product, process, or service. Any views and opinions of authors expressed in this work do not necessarily state or reflect those of the United States Government, or its contractors, or subcontractors.

This document was prepared in conjunction with work accomplished under Contract No. DE-AC09-08SR22470 with the U.S. Department of Energy. 
Keywords: $D W P F$, frit, glass formulation, sludge batch 5

Retention: permanent

\section{Frit Development for High Level Waste Sludge Batch 5: Compositional Trends for Varying Aluminum Concentrations}

K. M. Fox

T. B. Edwards

D. R. Best

I. A. Reamer

R. J. Workman

August 2008

Environmental \& Chemical Process Technology Savannah River National Laboratory Aiken, SC 29808

This document was prepared in conjunction with work accomplished under Contract No. DE-AC09-08SR22470 with the U.S. Department of Energy. 


\section{REVIEWS AND APPROVALS}

\section{AUTHORS:}

\begin{tabular}{lc}
\hline K.M. Fox, Process Engineering Technology & Date
\end{tabular}

\begin{tabular}{ll}
\hline T.B. Edwards, Statistical Consulting Section & Date
\end{tabular}

\begin{tabular}{ll}
\hline D.R. Best, Process Engineering Technology & Date
\end{tabular}

\begin{tabular}{lc}
\hline I.A. Reamer, Process Engineering Technology Date & Den
\end{tabular}

\begin{tabular}{ll}
\hline R.J. Workman, Process Engineering Technology & Date
\end{tabular}

\section{TECHNICAL REVIEWER:}

D.K. Peeler, Process Engineering Technology

Date

APPROVERS:

C.C. Herman, Manager, Process Engineering Technology Group

Date

J.C. Griffin, Manager,

Date

Environmental \& Chemical Process Technology Research Programs

J.E. Occhipinti, Manager,

Date

Waste Solidification Engineering 


\section{EXECUTIVE SUMMARY}

The objective of this study was to experimentally measure the properties and performance of a series of glasses with compositions that could represent Sludge Batch 5 (SB5) as processed at the Defense Waste Processing Facility (DWPF). The data was used to provide recommendations to the Liquid Waste Organization (LWO) regarding blending and washing strategies in preparing SB5 based on acceptability of the glass compositions. These data were also used to guide frit optimization efforts as the SB5 composition was finalized.

Glass compositions for this study were developed by combining a series of SB5 composition projections with a group of frits. Three composition projections for SB5 were developed using a model-based approach at Savannah River National Laboratory (SRNL). These compositions, referred to as SB5 Cases B, C and D, projected removal of 25, 50 and 75\% (respectively) of the aluminum in Tank 51 through the low temperature aluminum dissolution process. The frits for this study (Frits 530 through 537) were selected based on their predicted operating windows (i.e., ranges of waste loadings over which the predicted properties of the glasses were acceptable) and their potential (based on historical trends) to provide acceptable melt rates for SB5.

Six additional glasses were designed to evaluate alternatives for uranium in DWPF-type glasses used for variability studies and some scoping studies. Since special measures are necessary when working with uranium-containing glasses in the laboratory, it is desirable as a cost and time saving measure to find an alternative for uranium to support frit optimization efforts. Hafnium and neodymium were investigated as potential surrogates for uranium, and other glasses were made by simply excluding the radioactive components and renormalizing the glass composition.

The study glasses were fabricated and characterized at SRNL. Chemical composition analyses suggested only minor difficulties in meeting the targeted compositions for some of the oxides for some of the glasses. Although minor differences were observed, they did not have a significant impact on the conclusions made in this study. Several of the study compositions showed retention of more than $0.5 \mathrm{wt} \% \mathrm{SO}_{4}{ }^{2-}$ in glass.

Trevorite (a spinel) was the only crystalline phase that was positively identified in a few of the study glasses after the canister centerline cooled (CCC) heat treatment. Spinels are not of concern as they have been shown to have little impact on the durability of high level waste glasses. The crystallization behavior of the surrogate glasses was generally the same as that of their $\mathrm{U}_{3} \mathrm{O}_{8}$-containing counterparts. There are two pairs that were exceptions: SB5-04 (amorphous) and SB5-24 (possible trevorite), along with SB5-07 (amorphous) and SB5-25 (trevorite). In these cases, the surrogate glasses (SB5-24 and SB5-25) appear to be more conservative (more prone to crystallization) than their $\mathrm{U}_{3} \mathrm{O}_{8}$-containing counterparts.

Chemical durability was quantified using the Product Consistency Test (PCT). The normalized leachate (NL) values for $\mathrm{B}, \mathrm{Li}, \mathrm{Na}$ and $\mathrm{Si}$ for all of the study glasses were well below those of the Environmental Assessment (EA) benchmark glass, regardless of heat treatment or compositional view. This indicates that all of the glasses had very acceptable durability performance. The highest NL [B] for the study glasses was $0.914 \mathrm{~g} / \mathrm{L}$ (the quenched version of glass SB5-13), normalized using the measured, bias-correct composition. There was little practical impact of the $\mathrm{CCC}$ heat treatment on the PCT responses of the study glasses. The measured PCT responses were predictable by the current $\Delta \mathrm{G}_{\mathrm{p}}$ models. 
In general, the PCT responses for the surrogate glasses or the glasses without $\mathrm{U}_{3} \mathrm{O}_{8}$ were quite similar to their $\mathrm{U}_{3} \mathrm{O}_{8}$-containing counterparts. The average percent error in NL [B] normalized by the measured, bias-corrected compositions for the surrogate glasses compared with their radioactive counterparts was $8.8 \%$. The largest difference in NL [B] was $0.152 \mathrm{~g} / \mathrm{L}$.

While similarities existed in crystallization behavior and PCT response for both the surrogates and the glasses renormalized without $\mathrm{U}_{3} \mathrm{O}_{8}$, additional data must be collected before the best solution to fabricating test glasses without radioactive components can be identified. Further surrogate studies are currently underway at SRNL.

The results of this study indicate that a frit composition can be identified that will provide a processable and durable glass when combined with SB5 at the DWPF. Additional studies are underway to recommend a frit that continues to meet process and performance requirements as well as to provide an enhanced melt rate for improved waste throughput. 


\section{TABLE OF CONTENTS}

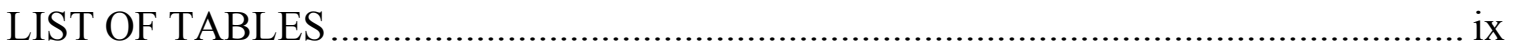

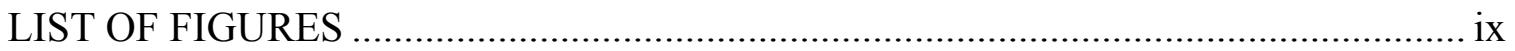

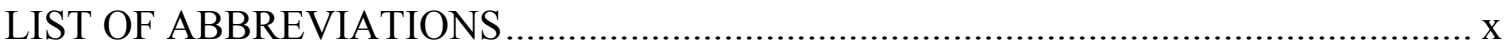

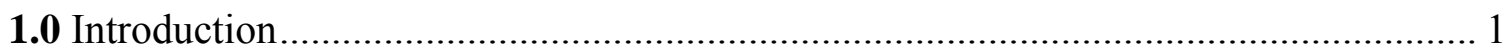

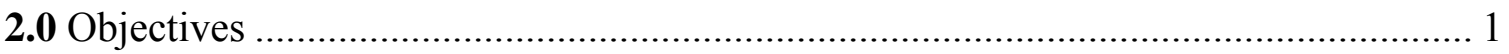

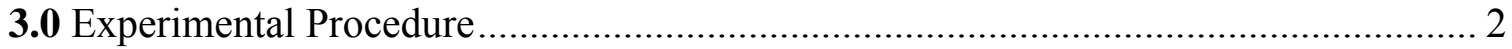

3.1 Selection of Glass Compositions .......................................................................... 2

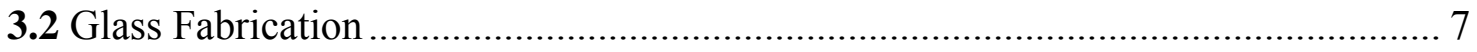

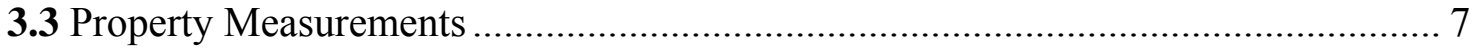

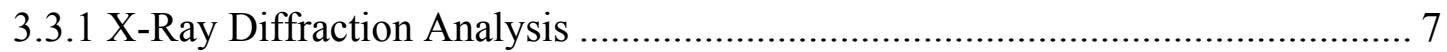

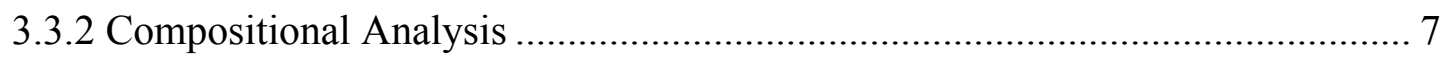

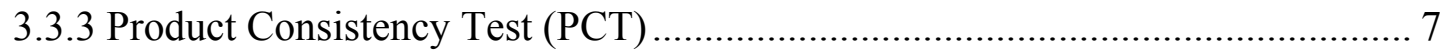

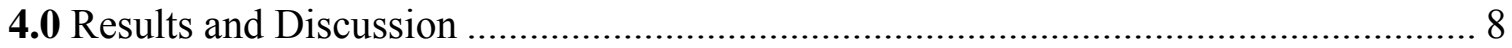

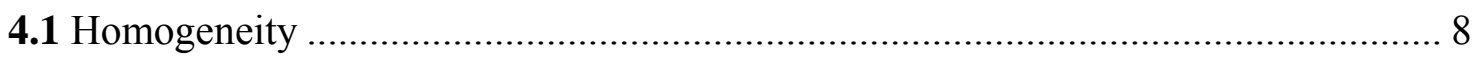

4.2 A Statistical Review of the Chemical Composition Measurements....................... 11

4.2.1 Measurements in Analytical Sequence ......................................................... 12

4.2.2 Composition Measurements by Glass Identifier .............................................. 12

4.2.3 Batch 1 and Uranium Standard Results..................................................... 12

4.2.4 Composition Measurements by Glass Identifier with Targeted Compositions. 13

4.2.5 Measured versus Targeted Compositions ........................................................ 14

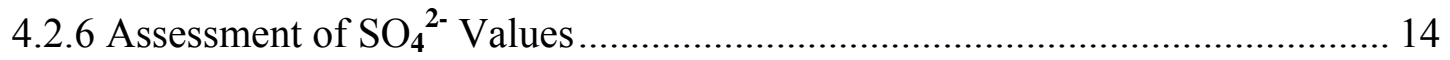

4.2.7 MAR Assessment of the SB4/Second Decant VS Glasses ............................. 15

4.3 A Statistical Review of the PCT Results for These Initial SB5 Glasses ................ 17

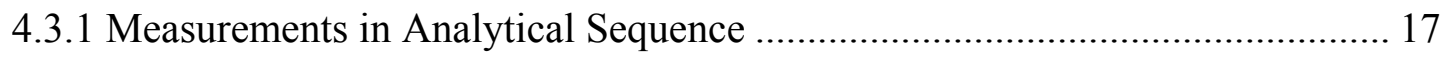

4.3.2 Results for the Samples of the Multi-Element Solution Standard ................... 17

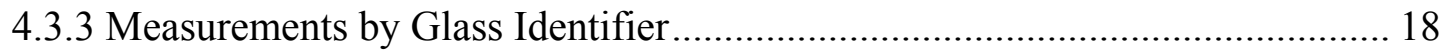

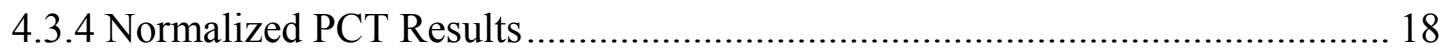

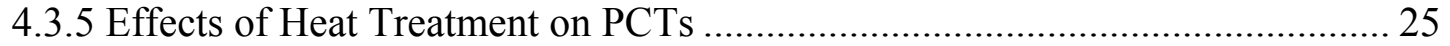

4.3.6 Initial Comparisons of Surrogate Pairs .......................................................... 25

4.3.7 Predicted versus Measured Durability ....................................................... 25 
SRNS-STI-2008-00060

Revision 0

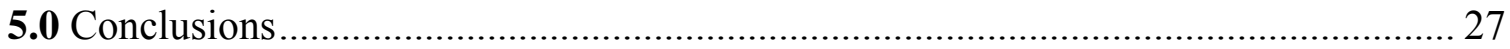

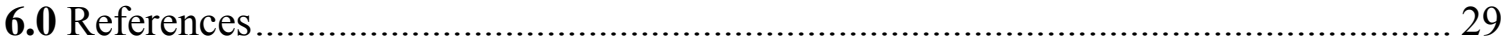

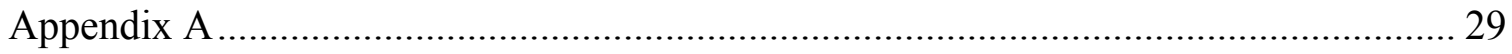

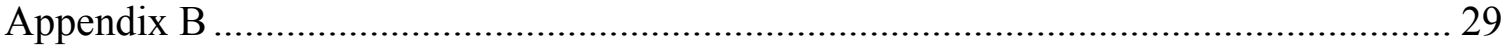




\section{LIST OF TABLES}

Table 3-1. SB5 composition projections used in this study. ..................................................... 2

Table 3-2. Compositions of the candidate frits used in this study................................................ 3

Table 3-3. Target compositions of the study glasses................................................................ 4

Table 3-4. Non-radioactive glass compositions with and without surrogates for uranium........... 6

Table 4-1. Visual observations and XRD results for each of the study glasses. .......................... 9

Table 4-2. Results of MAR Assessment of SB4 VS Glasses for Measured, Bias-Corrected, and

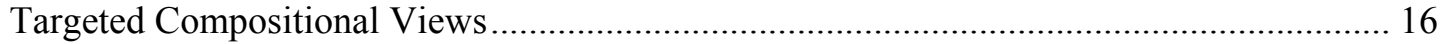

Table 4-3. Results from Samples of the Multi-Element Solution Standard ................................. 18

Table 4-4. Normalized PCTs by Glass ID and Compositional View for Initial SB5 Study Glasses.

\section{LIST OF FIGURES}

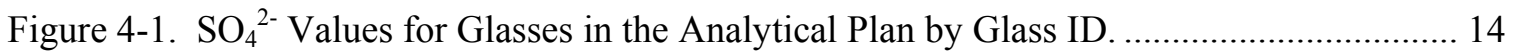

Figure 4-2. Normalized Leachate for Boron versus $\Delta \mathrm{G}_{\mathrm{p}}$ Model Prediction with $95 \%$ Confidence Interval for Individual PCTs. 


\section{LIST OF ABBREVIATIONS}

$\begin{array}{ll}\text { ANOVA } & \text { Analysis of Variance } \\ \text { ARM } & \text { Approved Reference Material } \\ \text { bc } & \text { bias-corrected } \\ \text { CCC } & \text { Canister Centerline Cooled } \\ \text { CPC } & \text { Chemical Process Cell } \\ \text { DWPF } & \text { Defense Waste Processing Facility } \\ \text { EA } & \text { Environmental Assessment } \\ \text { ICP-ES } & \text { Inductively Coupled Plasma - Emission Spectroscopy } \\ \text { LM } & \text { Lithium Metaborate } \\ \text { LWO } & \text { Liquid Waste Organization } \\ \text { MAR } & \text { Measurement Acceptability Region } \\ \text { NL } & \text { Normalized Leachate } \\ \text { PCCS } & \text { Product Composition Control System } \\ \text { PCT } & \text { Product Consistency Test } \\ \text { PF } & \text { Peroxide Fusion } \\ \text { PSAL } & \text { Process Science Analytical Laboratory } \\ \text { SB5 } & \text { Sludge Batch 5 } \\ \text { SRNL } & \text { Savannah River National Laboratory } \\ \text { SRS } & \text { Savannah River Site } \\ \mathrm{T}_{\mathrm{L}} & \text { Liquidus Temperature } \\ \text { WL } & \text { Waste Loading } \\ \text { XRD } & \text { X-ray Diffraction }\end{array}$




\subsection{Introduction}

The contents of Tank 51 have been blended with Purex sludge from Tank 7 to constitute Sludge Batch 5 (SB5). The Savannah River Site (SRS) Liquid Waste Organization (LWO) performed low-temperature Al-dissolution in Tank 51 to reduce the total mass of sludge solids and Al being fed to the Defense Waste Processing Facility (DWPF). A radioactive demonstration using a $3 \mathrm{~L}$ Tank 51 sludge slurry sample was performed to verify the Tank Farm processing parameters. ${ }^{1}$ The aluminum dissolved sludge was used to determine potential downstream impacts so that technical issues could be identified before the start of SB5 processing. The potential downstream impacts assessed included the Tank Farm washing and concentration process and the DWPF Chemical Process Cell (CPC) and melter processing envelopes. The chemical composition of this 3 L Tank 51 sample was used to project potential compositions of SB5 as it will be processed by the DWPF. These projections were used by the Savannah River National Laboratory (SRNL) to develop frit compositions for SB5 that will produce glasses that will be acceptable for disposition to the federal repository.

\subsection{Objectives}

The objective of this study is to experimentally measure the properties and performance of a series of glasses with compositions that are anticipated to represent SB5 as processed at the DWPF. The data will be used to provide recommendations to the LWO regarding blending and washing strategies in preparing SB5 based on acceptability of the glass compositions. These data will also be used to guide frit optimization efforts as the SB5 composition is finalized. 


\subsection{Experimental Procedure}

\subsection{Selection of Glass Compositions}

Glass compositions for this study were developed by combining a series of SB5 composition projections with a group of frits. Three composition projections for SB5 were developed using a model-based approach at SRNL. These compositions, referred to as SB5 Cases B, C and D, project removal of 25,50 and $75 \%$ (respectively) of the aluminum in Tank 51 through the low temperature aluminum dissolution process. The development of these SB5 composition projections is described in further detail in a previous report. ${ }^{2}$ A fourth SB5 composition was provided by LWO in 2007. 'This composition, which will be referred to as 'LWO Al-Diss', represents 50\% removal of aluminum from Tank 51 and a blend with the heel of SB4 occurring on August 31, 2008. The compositions of these four SB5 projections are given in Table 3-1.

Table 3-1. SB5 composition projections used in this study.

\begin{tabular}{|c|c|c|c|c|}
\hline Oxide & SB5 Case B & SB5 Case C & SB5 Case D & LWO Al-Diss \\
\hline $\mathrm{Ag}_{2} \mathrm{O}$ & 0.010 & 0.010 & 0.012 & 0.000 \\
\hline $\mathrm{Al}_{2} \mathrm{O}_{3}$ & 28.972 & 24.894 & 20.914 & 18.993 \\
\hline $\mathrm{BaO}$ & 0.102 & 0.113 & 0.121 & 0.165 \\
\hline $\mathrm{CaO}$ & 1.781 & 1.966 & 2.108 & 2.252 \\
\hline $\mathrm{CdO}$ & 0.061 & 0.066 & 0.072 & 0.000 \\
\hline $\mathrm{Ce}_{2} \mathrm{O}_{3}$ & 0.371 & 0.409 & 0.439 & 0.266 \\
\hline $\mathrm{CoO}$ & 0.024 & 0.026 & 0.028 & 0.000 \\
\hline $\mathrm{Cr}_{2} \mathrm{O}_{3}$ & 0.373 & 0.411 & 0.440 & 0.245 \\
\hline $\mathrm{CuO}$ & 0.012 & 0.013 & 0.013 & 0.087 \\
\hline $\mathrm{Fe}_{2} \mathrm{O}_{3}$ & 23.246 & 25.644 & 27.491 & 27.641 \\
\hline $\mathrm{K}_{2} \mathrm{O}$ & 0.067 & 0.072 & 0.080 & 0.202 \\
\hline $\mathrm{La}_{2} \mathrm{O}_{3}$ & 0.163 & 0.180 & 0.193 & 0.046 \\
\hline $\mathrm{MgO}$ & 1.178 & 1.299 & 1.393 & 1.228 \\
\hline $\mathrm{MnO}$ & 4.861 & 5.362 & 5.748 & 6.094 \\
\hline $\mathrm{Na}_{2} \mathrm{O}$ & 24.832 & 24.194 & 24.500 & 27.206 \\
\hline $\mathrm{NiO}$ & 2.730 & 3.011 & 3.228 & 3.154 \\
\hline $\mathrm{P}_{2} \mathrm{O}_{5}$ & 0.528 & 0.581 & 0.622 & 0.000 \\
\hline $\mathrm{PbO}$ & 0.022 & 0.023 & 0.026 & 0.096 \\
\hline $\mathrm{SO}_{4}{ }^{2-}$ & 0.729 & 0.761 & 0.815 & 1.439 \\
\hline $\mathrm{SiO}_{2}$ & 1.886 & 2.081 & 2.234 & 1.838 \\
\hline $\mathrm{SrO}$ & 0.319 & 0.351 & 0.377 & 0.000 \\
\hline $\mathrm{ThO}_{2}$ & 0.000 & 0.000 & 0.000 & 0.011 \\
\hline $\mathrm{TiO}_{2}$ & 0.026 & 0.029 & 0.031 & 0.922 \\
\hline $\mathrm{U}_{3} \mathrm{O}_{8}$ & 7.436 & 8.203 & 8.794 & 7.696 \\
\hline $\mathrm{ZnO}$ & 0.016 & 0.017 & 0.018 & 0.111 \\
\hline $\mathrm{ZrO}_{2}$ & 0.258 & 0.285 & 0.306 & 0.307 \\
\hline
\end{tabular}

The frits for this study were selected based on their predicted operating windows (i.e., ranges of waste loadings over which the predicted properties of the glasses were acceptable) and potential to provide acceptable melt rates for SB5. The selection process for the frits used in this study is also described in a previous report. ${ }^{2}$ The compositions of each frit are given in Table 3-2.

\footnotetext{
${ }^{a}$ Data provided by H. B. Shah, Washington Savannah River Company, via email on July 31, 2007.
} 
Table 3-2. Compositions of the candidate frits used in this study.

\begin{tabular}{||c|c|c|c|c|c||}
\hline Frit ID & $\mathbf{B}_{\mathbf{2}} \mathbf{O}_{3}$ & $\mathbf{C a O}$ & $\mathbf{L i}_{\mathbf{2}} \mathbf{O}$ & $\mathbf{N a}_{\mathbf{2}} \mathbf{O}$ & $\mathbf{S i O}_{\mathbf{2}}$ \\
\hline 530 & 10 & 4 & 7 & 7 & 72 \\
\hline 531 & 11 & 2 & 7 & 7 & 73 \\
\hline 532 & 14 & 2 & 6 & 7 & 71 \\
\hline 533 & 16 & 0 & 5 & 8 & 71 \\
\hline 534 & 15 & 0 & 9 & 4 & 72 \\
\hline 535 & 14 & 0 & 7 & 8 & 71 \\
\hline 536 & 15 & 0 & 5 & 10 & 70 \\
\hline 537 & 16 & 0 & 4 & 7 & 73 \\
\hline
\end{tabular}

Frits 530, 531, 532 and 533 were each combined with the four SB5 composition projections to form the first 16 glass compositions (glasses SB5-01 through SB5-16). All of the study glasses targeted 38\% waste loading (WL) in anticipation of higher WL targets for DWPF processing of SB5. Frits 534, 535, 536 and 537 were combined with SB5 Case B, SB5 Case C, SB5 Case D and LWO Al-diss, respectively, as these frits provided good operating windows with these specific SB5 composition projections. That is, while the earlier frits provide good operating windows with all four of the SB5 composition projections, these frits are tailored specifically for the individual composition projections that they are combined with. These combinations form glasses SB5-17 through SB5-20. The compositions of the first 20 study glasses are given in Table 3-3. 
Table 3-3. Target compositions of the study glasses.

\begin{tabular}{|c|c|c|c|c|c|c|c|c|c|c|c|c|}
\hline "Glass ID & SB5-01 & SB5-02 & SB5-03 & SB5-04 & SB5-05 & SB5-06 & SB5-07 & SB5-08 & SB5-09 & SB5-10 & SB5-11 & "SB5-12 \\
\hline Frit ID & 530 & 531 & 532 & 533 & 530 & 531 & 532 & 533 & 530 & 531 & 532 & 533 \\
\hline Sludge Type & Case B & Case B & Case B & Case B & Case C & Case C & Case C & Case C & Case D & Case D & Case D & Case D \\
\hline $\mathrm{Ag}_{2} \mathrm{O}$ & 0.004 & 0.004 & 0.004 & 0.004 & 0.004 & 0.004 & 0.004 & 0.004 & 0.004 & 0.004 & 0.004 & 0.004 \\
\hline $\mathrm{Al}_{2} \mathrm{O}_{3}$ & 11.009 & 11.009 & 11.009 & 11.009 & 9.460 & 9.460 & 9.460 & 9.460 & 7.947 & 7.947 & 7.947 & 7.947 \\
\hline $\mathrm{B}_{2} \mathrm{O}_{3}$ & 6.200 & 6.820 & 8.680 & 9.920 & 6.200 & 6.820 & 8.680 & 9.920 & 6.200 & 6.820 & 8.680 & 9.920 \\
\hline $\mathrm{BaO}$ & 0.039 & 0.039 & 0.039 & 0.039 & 0.043 & 0.043 & 0.043 & 0.043 & 0.046 & 0.046 & 0.046 & 0.046 \\
\hline $\mathrm{CaO}$ & 3.157 & 1.917 & 1.917 & 0.677 & 3.227 & 1.987 & 1.987 & 0.747 & 3.281 & 2.041 & 2.041 & 0.801 \\
\hline $\mathrm{CdO}$ & 0.023 & 0.023 & 0.023 & 0.023 & 0.025 & 0.025 & 0.025 & 0.025 & 0.027 & 0.027 & 0.027 & 0.027 \\
\hline $\mathrm{Ce}_{2} \mathrm{O}_{3}$ & 0.141 & 0.141 & 0.141 & 0.141 & 0.155 & 0.155 & 0.155 & 0.155 & 0.167 & 0.167 & 0.167 & 0.167 \\
\hline $\mathrm{CoO}$ & 0.009 & 0.009 & 0.009 & 0.009 & 0.010 & 0.010 & 0.010 & 0.010 & 0.011 & 0.011 & 0.011 & 0.011 \\
\hline $\mathrm{Cr}_{2} \mathrm{O}_{3}$ & 0.142 & 0.142 & 0.142 & 0.142 & 0.156 & 0.156 & 0.156 & 0.156 & 0.167 & 0.167 & 0.167 & 0.167 \\
\hline $\mathrm{CuO}$ & 0.005 & 0.005 & 0.005 & 0.005 & 0.005 & 0.005 & 0.005 & 0.005 & 0.005 & 0.005 & 0.005 & 0.005 \\
\hline $\mathrm{Fe}_{2} \mathrm{O}_{3}$ & 8.834 & 8.834 & 8.834 & 8.834 & 9.745 & 9.745 & 9.745 & 9.745 & 10.447 & 10.447 & 10.447 & 10.447 \\
\hline $\mathrm{HfO}_{2}$ & 0.000 & 0.000 & 0.000 & 0.000 & 0.000 & 0.000 & 0.000 & 0.000 & 0.000 & 0.000 & 0.000 & 0.000 \\
\hline $\mathrm{K}_{2} \mathrm{O}$ & 0.025 & 0.025 & 0.025 & 0.025 & 0.027 & 0.027 & 0.027 & 0.027 & 0.030 & 0.030 & 0.030 & 0.030 \\
\hline $\mathrm{La}_{2} \mathrm{O}_{3}$ & 0.062 & 0.062 & 0.062 & 0.062 & 0.068 & 0.068 & 0.068 & 0.068 & 0.073 & 0.073 & 0.073 & 0.073 \\
\hline $\mathrm{Li}_{2} \mathrm{O}$ & 4.340 & 4.340 & 3.720 & 3.100 & 4.340 & 4.340 & 3.720 & 3.100 & 4.340 & 4.340 & 3.720 & 3.100 \\
\hline $\mathrm{MgO}$ & 0.447 & 0.447 & 0.447 & 0.447 & 0.494 & 0.494 & 0.494 & 0.494 & 0.529 & 0.529 & 0.529 & 0.529 \\
\hline $\mathrm{MnO}$ & 1.847 & 1.847 & 1.847 & 1.847 & 2.038 & 2.038 & 2.038 & 2.038 & 2.184 & 2.184 & 2.184 & 2.184 \\
\hline $\mathrm{Na}_{2} \mathrm{O}$ & 13.776 & 13.776 & 13.776 & 14.396 & 13.534 & 13.534 & 13.534 & 14.154 & 13.650 & 13.650 & 13.650 & 14.270 \\
\hline $\mathrm{Nd}_{2} \mathrm{O}_{3}$ & 0.000 & 0.000 & 0.000 & 0.000 & 0.000 & 0.000 & 0.000 & 0.000 & 0.000 & 0.000 & 0.000 & 0.000 \\
\hline $\mathrm{NiO}$ & 1.037 & 1.037 & 1.037 & 1.037 & 1.144 & 1.144 & 1.144 & 1.144 & 1.227 & 1.227 & 1.227 & 1.227 \\
\hline $\mathrm{P}_{2} \mathrm{O}_{5}$ & 0.201 & 0.201 & 0.201 & 0.201 & 0.221 & 0.221 & 0.221 & 0.221 & 0.236 & 0.236 & 0.236 & 0.236 \\
\hline $\mathrm{PbO}$ & 0.008 & 0.008 & 0.008 & 0.008 & 0.009 & 0.009 & 0.009 & 0.009 & 0.010 & 0.010 & 0.010 & 0.010 \\
\hline $\mathrm{SiO}_{2}$ & 45.357 & 45.977 & 44.737 & 44.737 & 45.431 & 46.051 & 44.811 & 44.811 & 45.489 & 46.109 & 44.869 & 44.869 \\
\hline $\mathrm{SO}_{4}$ & 0.277 & 0.277 & 0.277 & 0.277 & 0.289 & 0.289 & 0.289 & 0.289 & 0.310 & 0.310 & 0.310 & 0.310 \\
\hline $\mathrm{SrO}$ & 0.121 & 0.121 & 0.121 & 0.121 & 0.134 & 0.134 & 0.134 & 0.134 & 0.143 & 0.143 & 0.143 & 0.143 \\
\hline $\mathrm{ThO}_{2}$ & 0.000 & 0.000 & 0.000 & 0.000 & 0.000 & 0.000 & 0.000 & 0.000 & 0.000 & 0.000 & 0.000 & 0.000 \\
\hline $\mathrm{TiO}_{2}$ & 0.010 & 0.010 & 0.010 & 0.010 & 0.011 & 0.011 & 0.011 & 0.011 & 0.012 & 0.012 & 0.012 & 0.012 \\
\hline $\mathrm{U}_{3} \mathrm{O}_{8}$ & 2.826 & 2.826 & 2.826 & 2.826 & 3.117 & 3.117 & 3.117 & 3.117 & 3.342 & 3.342 & 3.342 & 3.342 \\
\hline $\mathrm{ZnO}$ & 0.006 & 0.006 & 0.006 & 0.006 & 0.006 & 0.006 & 0.006 & 0.006 & 0.007 & 0.007 & 0.007 & 0.007 \\
\hline $\mathrm{ZrO}_{2}$ & 0.098 & 0.098 & 0.098 & 0.098 & 0.108 & 0.108 & 0.108 & 0.108 & 0.116 & 0.116 & 0.116 & 0.116 \\
\hline
\end{tabular}


Table 3-3. Target compositions of the study glasses. (continued)

\begin{tabular}{|c|c|c|c|c|c|c|c|c|}
\hline Glass ID & SB5-13 & SB5-14 & SB5-15 & SB5-16 & SB5-17 & SB5-18 & SB5-19 & SB5-20 \\
\hline Frit ID & 530 & 531 & 532 & 533 & 534 & 535 & 536 & 537 \\
\hline Sludge Type & LWO Al-Diss & LWO Al-Diss & LWO Al-Diss & LWO Al-Diss & Case B & Case C & Case D & LWO Al-Diss \\
\hline $\mathrm{Ag}_{2} \mathrm{O}$ & 0.000 & 0.000 & 0.000 & 0.000 & 0.004 & 0.004 & 0.004 & 0.000 \\
\hline $\mathrm{Al}_{2} \mathrm{O}_{3}$ & 7.217 & 7.217 & 7.217 & 7.217 & 11.009 & 9.460 & 7.947 & 7.217 \\
\hline $\mathrm{B}_{2} \mathrm{O}_{3}$ & 6.200 & 6.820 & 8.680 & 9.920 & 9.300 & 8.680 & 9.300 & 9.920 \\
\hline $\mathrm{BaO}$ & 0.063 & 0.063 & 0.063 & 0.063 & 0.039 & 0.043 & 0.046 & 0.063 \\
\hline $\mathrm{CaO}$ & 3.336 & 2.096 & 2.096 & 0.856 & 0.677 & 0.747 & 0.801 & 0.856 \\
\hline $\mathrm{CdO}$ & 0.000 & 0.000 & 0.000 & 0.000 & 0.023 & 0.025 & 0.027 & 0.000 \\
\hline $\mathrm{Ce}_{2} \mathrm{O}_{3}$ & 0.101 & 0.101 & 0.101 & 0.101 & 0.141 & 0.155 & 0.167 & 0.101 \\
\hline $\mathrm{CoO}$ & 0.000 & 0.000 & 0.000 & 0.000 & 0.009 & 0.010 & 0.011 & 0.000 \\
\hline $\mathrm{Cr}_{2} \mathrm{O}_{3}$ & 0.093 & 0.093 & 0.093 & 0.093 & 0.142 & 0.156 & 0.167 & 0.093 \\
\hline $\mathrm{CuO}$ & 0.033 & 0.033 & 0.033 & 0.033 & 0.005 & 0.005 & 0.005 & 0.033 \\
\hline $\mathrm{Fe}_{2} \mathrm{O}_{3}$ & 10.504 & 10.504 & 10.504 & 10.504 & 8.834 & 9.745 & 10.447 & 10.504 \\
\hline $\mathrm{HfO}_{2}$ & 0.000 & 0.000 & 0.000 & 0.000 & 0.000 & 0.000 & 0.000 & 0.000 \\
\hline $\mathrm{K}_{2} \mathrm{O}$ & 0.077 & 0.077 & 0.077 & 0.077 & 0.025 & 0.027 & 0.030 & 0.077 \\
\hline $\mathrm{La}_{2} \mathrm{O}_{3}$ & 0.018 & 0.018 & 0.018 & 0.018 & 0.062 & 0.068 & 0.073 & 0.018 \\
\hline $\mathrm{Li}_{2} \mathrm{O}$ & 4.340 & 4.340 & 3.720 & 3.100 & 5.580 & 4.340 & 3.100 & 2.480 \\
\hline $\mathrm{MgO}$ & 0.467 & 0.467 & 0.467 & 0.467 & 0.447 & 0.494 & 0.529 & 0.467 \\
\hline $\mathrm{MnO}$ & 2.316 & 2.316 & 2.316 & 2.316 & 1.847 & 2.038 & 2.184 & 2.316 \\
\hline $\mathrm{Na}_{2} \mathrm{O}$ & 14.678 & 14.678 & 14.678 & 15.298 & 11.916 & 14.154 & 15.510 & 14.678 \\
\hline $\mathrm{Nd}_{2} \mathrm{O}_{3}$ & 0.000 & 0.000 & 0.000 & 0.000 & 0.000 & 0.000 & 0.000 & 0.000 \\
\hline $\mathrm{NiO}$ & 1.198 & 1.198 & 1.198 & 1.198 & 1.037 & 1.144 & 1.227 & 1.198 \\
\hline $\mathrm{P}_{2} \mathrm{O}_{5}$ & 0.000 & 0.000 & 0.000 & 0.000 & 0.201 & 0.221 & 0.236 & 0.000 \\
\hline $\mathrm{PbO}$ & 0.036 & 0.036 & 0.036 & 0.036 & 0.008 & 0.009 & 0.010 & 0.036 \\
\hline $\mathrm{SiO}_{2}$ & 45.338 & 45.958 & 44.718 & 44.718 & 45.357 & 44.811 & 44.249 & 45.958 \\
\hline $\mathrm{SO}_{4}$ & 0.547 & 0.547 & 0.547 & 0.547 & 0.277 & 0.289 & 0.310 & 0.547 \\
\hline $\mathrm{SrO}$ & 0.000 & 0.000 & 0.000 & 0.000 & 0.121 & 0.134 & 0.143 & 0.000 \\
\hline $\mathrm{ThO}_{2}$ & 0.004 & 0.004 & 0.004 & 0.004 & 0.000 & 0.000 & 0.000 & 0.004 \\
\hline $\mathrm{TiO}_{2}$ & 0.350 & 0.350 & 0.350 & 0.350 & 0.010 & 0.011 & 0.012 & 0.350 \\
\hline $\mathrm{U}_{3} \mathrm{O}_{8}$ & 2.925 & 2.925 & 2.925 & 2.925 & 2.826 & 3.117 & 3.342 & 2.925 \\
\hline $\mathrm{ZnO}$ & 0.042 & 0.042 & 0.042 & 0.042 & 0.006 & 0.006 & 0.007 & 0.042 \\
\hline $\mathrm{ZrO}_{2}$ & 0.117 & 0.117 & 0.117 & 0.117 & 0.098 & 0.108 & 0.116 & 0.117 \\
\hline
\end{tabular}


Six additional glasses were designed to evaluate possible alternatives for uranium in DWPF-type glasses used for variability studies and some scoping studies. Since special measures are necessary when working with uranium-containing glasses in the laboratory, it is desirable as a cost and time saving measure to find an alternative for uranium to support frit development efforts. Frits 532 and 533 were combined with SB5 Cases B, C and D at 38\% WL to form these six glasses. Previous studies have suggested neodymium and hafnium as possible surrogates for uranium in nuclear waste glasses. ${ }^{3,4}$ For the first two glass compositions, neodymium was substituted for uranium and thorium on an oxide mass basis. For the next two glass compositions, hafnium was substituted for uranium and thorium on an oxide mass basis. Uranium and thorium were removed from the last two glass compositions and the concentrations of the remaining components were renormalized to $100 \%$. The compositions of the six non-radioactive glasses are given in Table 3-4.

Table 3-4. Non-radioactive glass compositions with and without surrogates for uranium.

\begin{tabular}{|c|c|c|c|c|c|c|}
\hline Glass ID & SB5-21 & SB5-22 & SB5-23 & SB5-24 & SB5-25 & SB5-26 \\
\hline Frit ID & 532 & 533 & 532 & 533 & 532 & 533 \\
\hline $\begin{array}{c}\text { Sludge } \\
\text { Type } \\
\end{array}$ & Case B w/Nd & Case C w/Nd & Case D w/Hf & Case B w/Hf & Case C w/o U & Case D w/o U \\
\hline $\mathrm{Ag}_{2} \mathrm{O}$ & 0.004 & 0.004 & 0.004 & 0.004 & 0.004 & 0.005 \\
\hline $\mathrm{Al}_{2} \mathrm{O}_{3}$ & 11.009 & 9.460 & 7.947 & 11.009 & 10.305 & 8.714 \\
\hline $\mathrm{B}_{2} \mathrm{O}_{3}$ & 8.680 & 9.920 & 8.680 & 9.920 & 8.680 & 9.920 \\
\hline $\mathrm{BaO}$ & 0.039 & 0.043 & 0.046 & 0.039 & 0.047 & 0.050 \\
\hline $\mathrm{CaO}$ & 1.917 & 0.747 & 2.041 & 0.677 & 2.054 & 0.878 \\
\hline $\mathrm{CdO}$ & 0.023 & 0.025 & 0.027 & 0.023 & 0.027 & 0.030 \\
\hline $\mathrm{Ce}_{2} \mathrm{O}_{3}$ & 0.141 & 0.155 & 0.167 & 0.141 & 0.169 & 0.183 \\
\hline $\mathrm{CoO}$ & 0.009 & 0.010 & 0.011 & 0.009 & 0.011 & 0.012 \\
\hline $\mathrm{Cr}_{2} \mathrm{O}_{3}$ & 0.142 & 0.156 & 0.167 & 0.142 & 0.170 & 0.183 \\
\hline $\mathrm{CuO}$ & 0.005 & 0.005 & 0.005 & 0.005 & 0.005 & 0.005 \\
\hline $\mathrm{Fe}_{2} \mathrm{O}_{3}$ & 8.834 & 9.745 & 10.447 & 8.834 & 10.615 & 11.454 \\
\hline $\mathrm{HfO}_{2}$ & 0.000 & 0.000 & 3.342 & 2.826 & 0.000 & 0.000 \\
\hline $\mathrm{K}_{2} \mathrm{O}$ & 0.025 & 0.027 & 0.030 & 0.025 & 0.030 & 0.033 \\
\hline $\mathrm{La}_{2} \mathrm{O}_{3}$ & 0.062 & 0.068 & 0.073 & 0.062 & 0.074 & 0.080 \\
\hline $\mathrm{Li}_{2} \mathrm{O}$ & 3.720 & 3.100 & 3.720 & 3.100 & 3.720 & 3.100 \\
\hline $\mathrm{MgO}$ & 0.447 & 0.494 & 0.529 & 0.447 & 0.538 & 0.580 \\
\hline $\mathrm{MnO}$ & 1.847 & 2.038 & 2.184 & 1.847 & 2.220 & 2.395 \\
\hline $\mathrm{Na}_{2} \mathrm{O}$ & 13.776 & 14.154 & 13.650 & 14.396 & 14.355 & 15.168 \\
\hline $\mathrm{Nd}_{2} \mathrm{O}_{3}$ & 2.826 & 3.117 & 0.000 & 0.000 & 0.000 & 0.000 \\
\hline $\mathrm{NiO}$ & 1.037 & 1.144 & 1.227 & 1.037 & 1.247 & 1.345 \\
\hline $\mathrm{P}_{2} \mathrm{O}_{5}$ & 0.201 & 0.221 & 0.236 & 0.201 & 0.240 & 0.259 \\
\hline $\mathrm{PbO}$ & 0.008 & 0.009 & 0.010 & 0.008 & 0.010 & 0.011 \\
\hline $\mathrm{SiO}_{2}$ & 44.737 & 44.811 & 44.869 & 44.737 & 44.882 & 44.951 \\
\hline $\mathrm{SO}_{4}$ & 0.277 & 0.289 & 0.310 & 0.277 & 0.315 & 0.340 \\
\hline $\mathrm{SrO}$ & 0.121 & 0.134 & 0.143 & 0.121 & 0.145 & 0.157 \\
\hline $\mathrm{ThO}_{2}$ & 0.000 & 0.000 & 0.000 & 0.000 & 0.000 & 0.000 \\
\hline $\mathrm{TiO}_{2}$ & 0.010 & 0.011 & 0.012 & 0.010 & 0.012 & 0.013 \\
\hline $\mathrm{U}_{3} \mathrm{O}_{8}$ & 0.000 & 0.000 & 0.000 & 0.000 & 0.000 & 0.000 \\
\hline $\mathrm{ZnO}$ & 0.006 & 0.006 & 0.007 & 0.006 & 0.007 & 0.008 \\
\hline $\mathrm{ZrO}_{2}$ & 0.098 & 0.108 & 0.116 & 0.098 & 0.118 & 0.127 \\
\hline
\end{tabular}




\subsection{Glass Fabrication}

Each of the study glasses was prepared from the proper proportions of reagent-grade metal oxides, carbonates, boric acid and salts in $150 \mathrm{~g}$ batches. ${ }^{5}$ The raw materials were thoroughly mixed and placed into platinum/rhodium, $250 \mathrm{ml}$ crucibles. The batch was placed into a high-temperature furnace at the target melt temperature of $1150{ }^{\circ} \mathrm{C} .{ }^{6}$ The crucible was removed from the furnace after a one hour isothermal hold. The glass was poured onto a clean, stainless steel plate and allowed to air cool (quench). The glass pour patty was used as a sampling stock for the various property measurements, including chemical composition and durability testing.

Approximately $25 \mathrm{~g}$ of each glass was heat-treated to simulate cooling along the centerline of a DWPF-type canister ${ }^{7}$ to gauge the effects of thermal history on the product performance. This cooling schedule is referred to as the centerline canister cooling (CCC) curve. Visual observations on both quenched and CCC glasses were documented.

\subsection{Property Measurements}

This section provides a general discussion of the methodology used to perform X-ray diffraction (XRD) analyses, chemical composition analyses, and the Product Consistency Tests (PCT) on the study glasses.

\subsubsection{X-Ray Diffraction Analysis}

Representative samples of all the CCC glasses were submitted to SRNL Analytical Development for XRD analysis. Samples were run under conditions providing a detection limit of approximately $0.5 \mathrm{vol} \%$. That is, if crystals (or undissolved batch material) were present at $0.5 \mathrm{vol} \%$ or greater, the diffractometer would not only be capable of detecting the crystals but would also allow for a qualitative determination of the type of crystal(s) present. Otherwise, a characteristically high background signal (amorphous hump) devoid of crystalline spectral peaks indicates that the glass product is free of crystallization, suggesting either a completely amorphous product or that the degree of crystallization is below the detection limit.

\subsubsection{Compositional Analysis}

To confirm that the as-fabricated glasses met the target compositions, a representative sample from each quenched glass was submitted to the SRNL Process Science Analytical Laboratory (PSAL) for chemical analysis under the auspices of two analytical plans, with the first plan ${ }^{8}$ covering the twenty uranium-containing glasses and the second plan ${ }^{9}$ covering the six nonradioactive glasses. The analytical plans were developed in such a way as to provide the opportunity to evaluate potential sources of bias and error. Glass standards were also intermittently measured to assess the performance of the analytical instrumentation over the course of these analyses.

\subsubsection{Product Consistency Test (PCT)}

The PCT Method- $\mathrm{A}^{10}$ was performed in triplicate on each quenched and $\mathrm{CCC}$ glass to assess chemical durability. Also included in the experimental test matrix was the Environmental Assessment (EA) benchmark glass, ${ }^{11}$ the Approved Reference Material (ARM) glass, and blanks from the sample cleaning batch. Samples were ground, washed, and prepared following the standard procedure. ${ }^{10}$ Fifteen milliliters of Type-I ASTM water were added to $1.5 \mathrm{~g}$ of glass in stainless steel vessels. The vessels were closed, sealed, and placed in an oven at $90 \pm 2{ }^{\circ} \mathrm{C}$ where the samples were maintained at temperature for 7 days. Once cooled, the resulting solutions were sampled (filtered and acidified), then labeled and analyzed by PSAL under the auspices of two analytical plans, one for the twenty uranium-containing glasses ${ }^{12}$ and one for the six nonradioactive glasses. ${ }^{13}$ The aim of the plans was to provide an opportunity to assess the 
consistency (repeatability) of the PCT and analytical procedures in evaluating the chemical durability of the glasses. Samples of a multi-element, standard solution were also included in the analytical plans as a check on the accuracy of the analytical instrumentation used for these measurements. Normalized release rates were calculated based on target, measured, and biascorrected (bc) compositions using the average of the common logarithms of the leachate concentrations.

\subsection{Results and Discussion}

\subsection{Homogeneity}

Visual observations were recorded for each of the study glasses both after quenching and after the CCC heat treatment, as shown in Table 4-1. Descriptions such as clean or homogeneous indicate that no crystallization was apparent to the unaided eye. Descriptions such as streaks, film, sheen, haze, and dull or matte indicate that crystallization was visible on the surface of the glass.

XRD results are also summarized for each glass in Table 4-1. Only the CCC version of each glass was submitted for XRD analysis as the visual observations of the quenched glasses (and later the PCT performance) indicated that crystallization in the quenched glasses was unlikely. Trevorite (a spinel) was the only crystalline phase that was positively identified in a few of the study glasses. Spinels are not of concern as they have been shown to have little impact on the durability of high level waste glasses. ${ }^{14}$ In some cases, crystallization was visible on the surface of a CCC glass but not detected by XRD. It is likely that the volume fraction of crystallization in these glasses was below the XRD detection limit of about $0.5 \mathrm{vol} \%$.

The crystallization behavior of the surrogate glasses (SB5-21 through SB5-26) was generally the same as that of their $\mathrm{U}_{3} \mathrm{O}_{8}$-containing counterparts. There are two pairs that are exceptions: SB5-04 (amorphous by XRD) and SB5-24 (possible trevorite identified by XRD), along with SB5-07 (amorphous) and SB5-25 (trevorite). In these cases, the surrogate glasses (SB5-24 and SB5-25) appear to be more conservative (more prone to crystallization) than their $\mathrm{U}_{3} \mathrm{O}_{8^{-}}$ containing counterparts. Again, additional data are required in order to determine the best method of removing the radioactive components from study glass compositions without influencing performance. 
Table 4-1. Visual observations and XRD results for each of the study glasses.

\begin{tabular}{|c|c|c|c|}
\hline $\begin{array}{c}\text { Glass } \\
\text { ID }\end{array}$ & $\begin{array}{c}\text { Heat } \\
\text { Treatment }\end{array}$ & Visual Observations & XRD Results \\
\hline \multirow{2}{*}{ SB5-01 } & Quenched & Patty: black and shiny, homogeneous; Crucible: clean & - \\
\hline & $\mathrm{CCC}$ & Surface: Dull film with crystals; Bulk: clean & Amorphous \\
\hline \multirow{2}{*}{ SB5-02 } & Quenched & Patty: black and shiny with one tiny silver streak on surface, bulk clean; Crucible: clean & - \\
\hline & $\mathrm{CCC}$ & Surface: Mirror-like film; Bulk: clean & Amorphous \\
\hline \multirow{2}{*}{ SB5-03 } & Quenched & Patty: Several silver spots on surface, bulk clean; Crucible: clean & - \\
\hline & $\mathrm{CCC}$ & Surface: Mirror-like film; Bulk: clean & Amorphous \\
\hline SB5-04 & Quenched & Patty: Tiny silvery specks on surface, possible crystals in bulk; Crucible: silvery specks on glass surface & - \\
\hline \multirow{2}{*}{ SB5-05 } & Quenched & Patty: Three tiny silver streaks on surface, bulk clean; Crucible: clean & 1 \\
\hline & $\mathrm{CCC}$ & Surface: Dull, silvery film with crystals; Bulk: clean & Possible Bunsenite (NiO) \\
\hline \multirow{2}{*}{ SB5-06 } & Quenched & Patty: Two tiny silvery streaks on surface, bulk clean; Crucible: clean & - \\
\hline & $\mathrm{CCC}$ & Surface: Shiny, mirror-like film with crystals; Bulk: clean & Amorphous \\
\hline \multirow{2}{*}{ SB5-07 } & Quenched & Patty: Two tiny silvery streaks on surface, bulk clean; Crucible: clean & $T_{1}-T_{0}$ \\
\hline & $\mathrm{CCC}$ & Surface: Mirror-like sheen; Bulk: clean & Amorphous \\
\hline \multirow{2}{*}{ SB5-08 } & Quenched & Patty: Lots of silvery specks on surface, possible crystals in bulk; Crucible: clean & - \\
\hline & $\mathrm{CCC}$ & Surface: Shiny film with crystals; Bulk: clean & Amorphous \\
\hline SB5-09 & $\mathrm{CCC}$ & Surface: Shiny film with crystals; Bulk: clean & Amorphous \\
\hline \multirow{2}{*}{ SB5-10 } & Quenched & Patty: A few tiny specks and streaks on surface, bulk clean; Crucible: clean & $x_{1}$ \\
\hline & $\mathrm{CCC}$ & Surface: Mirror-like shine; Bulk: clean & Amorphous \\
\hline \multirow{2}{*}{ SB5-11 } & Quenched & Patty: A few tiny streaks on surface, bulk clean; Crucible: clean & 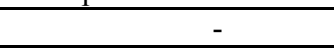 \\
\hline & $\mathrm{CCC}$ & Surface: Shiny, mirror-like sheen; Bulk: clean & Amorphous \\
\hline \multirow{2}{*}{ SB5-12 } & Quenched & Patty: A few tiny, silvery specs and streaks on surface, bulk clean; Crucible: clean & 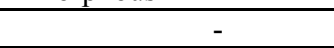 \\
\hline & $\mathrm{CCC}$ & Surface: Mirror-like sheen; Bulk: clean & Amorphous \\
\hline \multirow{2}{*}{ SB5-13 } & Quenched & Patty: A few tiny specks on surface, bulk clean; Crucible: clean & - \\
\hline & $\mathrm{CCC}$ & Surface: Dull, matte with crystals; Bulk: clean & Amorphous \\
\hline \multirow{2}{*}{ SB5-14 } & Quenched & Patty: Black, shiny and clean; Crucible: clean & - \\
\hline & $\mathrm{CCC}$ & Surface: Shiny metallic haze; Bulk: clean & Amorphous \\
\hline \multirow{2}{*}{ SB5-15 } & Quenched & Patty: Black, shiny and clean; Crucible: clean & - \\
\hline & $\mathrm{CCC}$ & Surface: Shiny metallic haze; Bulk: clean & Trevorite $\left(\mathrm{NiFe}_{2} \mathrm{O}_{4}\right)$ \\
\hline \multirow{2}{*}{ SB5-16 } & Quenched & Patty: Black, shiny and clean; Crucible: clean & - \\
\hline & $\mathrm{CCC}$ & Surface: Silver, mirror-like metallic haze; Bulk: clean & Trevorite $\left(\mathrm{NiFe}_{2} \mathrm{O}_{4}\right)$ \\
\hline
\end{tabular}


Table 4-1. Visual observations and XRD results for each of the study glasses. (continued)

\begin{tabular}{|c|c|c|c|}
\hline $\begin{array}{c}\text { Glass } \\
\text { ID } \\
\end{array}$ & $\begin{array}{c}\text { Heat } \\
\text { Treatment } \\
\end{array}$ & Visual Observations & XRD Results \\
\hline \multirow{2}{*}{ SB5-17 } & Quenched & Patty: Several small silver spots on surface; Crucible: clean & - \\
\hline & $\mathrm{CCC}$ & Surface: Silver, metallic haze with crystals; Bulk: clean & Trevorite $\left(\mathrm{NiFe}_{2} \mathrm{O}_{4}\right)$ \\
\hline \multirow{2}{*}{ SB5-18 } & Quenched & Patty: Black, shiny and clean; Crucible: clean & -4 \\
\hline & $\mathrm{CCC}$ & Surface: Metallic, mirror-like shine; Bulk: clean & Trevorite $\left(\mathrm{NiFe}_{2} \mathrm{O}_{4}\right)$ \\
\hline \multirow{2}{*}{ SB5-19 } & Quenched & Patty: Four tiny spots of silver and a few metallic swirls; Crucible: clean & 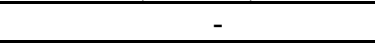 \\
\hline & $\mathrm{CCC}$ & Surface, Silvery, mirror-like shine with crystals; Bulk: clean & Amorphous \\
\hline SB5-20 & Quenched & Patty: Black, shiny and clean; Crucible: clean & - \\
\hline \multirow{2}{*}{ SB5-21 } & Quenched & Patty: Black, shiny and homogeneous; Crucible: clean & 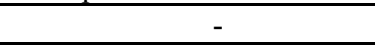 \\
\hline & $\mathrm{CCC}$ & Surface: Mirror-like shine; Bulk: clean & Amorphous \\
\hline \multirow{2}{*}{ SB5-22 } & Quenched & Patty: Black, shiny and homogeneous; Crucible: clean & $x_{2}$ \\
\hline & $\mathrm{CCC}$ & Surface: Mirror-like shine; Bulk: clean & Amorphous \\
\hline \multirow{2}{*}{ SB5-23 } & Quenched & Patty: Black, shiny and homogeneous; Crucible: clean & $T_{1}$ \\
\hline & $\mathrm{CCC}$ & Surface: Mirror-like shine; Bulk: clean & Amorphous \\
\hline \multirow{2}{*}{ SB5-24 } & Quenched & Patty: Black and shiny with four tiny metallic spots on surface; Crucible: clean & - \\
\hline & $\mathrm{CCC}$ & Surface: Mirror-like shine; Bulk: clean & Possible Trevorite $\left(\mathrm{NiFe}_{2} \mathrm{O}_{4}\right)$ \\
\hline \multirow{2}{*}{ SB5-26 } & Quenched & Patty: Black and shiny with three very tiny silver spots on surface; Crucible: clean & - \\
\hline & $\mathrm{CCC}$ & Surface: Mirror-like shine; Bulk: clean & Amorphous \\
\hline
\end{tabular}




\subsection{A Statistical Review of the Chemical Composition Measurements}

In this section, the measured versus targeted compositions of the study glasses are presented and compared. Glasses with identifiers SB5-1 through SB5-20 contained depleted uranium while the glasses with identifiers SB5-21 through SB5-26 were batched with the uranium normalized out of the compositions or with a surrogate such as neodymium or hafnium substituted for uranium. The identifiers and targeted compositions for these glasses are provided in Table A1 of Appendix A.

Two dissolution methods were utilized by PSAL in measuring the chemical compositions of the glasses for this study: lithium metaborate (LM) and sodium peroxide fusion (PF). For the plans in SRNL-SCS-2008-00003, the LM dissolution method was used to measure elemental concentrations of aluminum $(\mathrm{Al})$, barium $(\mathrm{Ba})$, calcium $(\mathrm{Ca})$, cadmium $(\mathrm{Cd})$, cerium $(\mathrm{Ce})$, cobalt $(\mathrm{Co})$, chromium $(\mathrm{Cr})$, copper $(\mathrm{Cu})$, iron $(\mathrm{Fe})$, potassium $(\mathrm{K})$, lanthanum $(\mathrm{La})$, magnesium $(\mathrm{Mg})$, manganese $(\mathrm{Mn})$, sodium $(\mathrm{Na})$, niobium $(\mathrm{Nb})$, nickel $(\mathrm{Ni})$, phosphorus $(\mathrm{P})$, lead $(\mathrm{Pb})$, sulfur $(\mathrm{S})$, strontium (Sr), thorium (Th), titanium (Ti), uranium (U), zinc $(\mathrm{Zn})$, and zirconium $(\mathrm{Zr})$ concentrations. For the plans in SRNL-SCS-2008-00003, samples prepared by PF were measured for boron (B), lithium (Li), and silicon ( $\mathrm{Si}$ ) concentrations.

For the glasses of the plan in SRNL-SCS-2008-00043, the LM dissolution method was used to measure elemental concentrations of silver $(\mathrm{Ag})$, aluminum $(\mathrm{Al})$, barium $(\mathrm{Ba})$, calcium $(\mathrm{Ca})$, cerium $(\mathrm{Ce})$, chromium $(\mathrm{Cr})$, iron $(\mathrm{Fe})$, hafnium $(\mathrm{Hf})$, potassium $(\mathrm{K})$, lanthanum $(\mathrm{La})$, magnesium $(\mathrm{Mg})$, manganese $(\mathrm{Mn})$, sodium $(\mathrm{Na})$, neodymium $(\mathrm{Nd})$, nickel $(\mathrm{Ni})$, lead $(\mathrm{Pb})$, sulfur $(\mathrm{S})$, silicon (Si), titanium (Ti), zinc ( $\mathrm{Zn})$, and zirconium ( $\mathrm{Zr}$ ), while samples from glasses prepared by $\mathrm{PF}$ dissolution were used to measure elemental concentrations of boron (B) and lithium (Li).

It should be noted that several of the elements in this list are not present or are present at very small concentrations. For each study glass, measurements were obtained from samples prepared in duplicate by each of these dissolution methods. All of the prepared samples were analyzed (twice for each element of interest) by Inductively Coupled Plasma - Atomic Emission Spectroscopy (ICP-AES) (with the instrumentation being re-calibrated between the duplicate analyses).

Table A2 in Appendix A provides the elemental concentration measurements derived from the samples prepared using LM in SRNL-SCS-2008-00003, and Table A3 in Appendix A provides the measurements derived from the samples prepared using PF in SRNL-SCS-2008-00003. The analytical plan and the analytical set are included as part of the information in these tables. Measurements for standards (Batch 1 and a uranium standard, $\mathrm{U}_{\text {std }}$ ) that were included in the analytical plans along with the study glasses are also provided in these two tables.

Table A4 in Appendix A provides the elemental concentration measurements derived from the samples prepared using LM in SRNL-SCS-2008-00043, and Table A5 in Appendix A provides the measurements derived from the samples prepared using PF in SRNL-SCS-2008-00043. The analytical plan and the analytical set are included as part of the information in these tables. Measurements for standards (Batch 1 and $U_{\text {std }}$ ) that were included in the analytical plan along with the study glasses are also provided in these two tables.

The elemental concentrations were converted to oxide concentrations by multiplying the values for each element by the gravimetric factor for the corresponding oxide. During this process, an elemental concentration that was determined to be below the detection limit of the analytical 
procedures used by the PSAL was reduced to half of that detection limit as the oxide concentration was determined.

In the sections that follow, the analytical sequences of the measurements are explored, the measurements of the standards are investigated and used for bias correction, the measurements for each glass are reviewed, the average chemical compositions (measured and bias-corrected) for each glass are determined, and comparisons are made between the measurements and the targeted compositions for the glasses.

\subsubsection{Measurements in Analytical Sequence}

Exhibit A1 in Appendix A provides plots of the measurements generated by the PSAL for samples by preparation method, by analytical plan, and by oxide. The plots are in analytical sequence with different symbols and colors being used to represent each of the study and standard glasses. These plots include all of the measurement data from Tables A2 through A5. Additional opportunities for review of these measurements are provided in the discussions that follow.

\subsubsection{Composition Measurements by Glass Identifier}

Exhibit A2 in Appendix A provides plots of the oxide concentration measurements by analytical plan, by preparation method by Glass ID (including Batch 1 and $\mathrm{U}_{\text {std }}$ ). Different symbols and colors are used to represent the different glasses. These plots show the individual measurements across the duplicates of each preparation method and the two ICP-AES calibrations within each analytical set. A review of the plots presented in these exhibits reveals the repeatability of the four individual values for each oxide for each glass, although care should be taken in interpreting these results due to the varying scale of the y-axis depending on the concentration of each oxide. There is some scatter in the $\mathrm{Fe}_{2} \mathrm{O}_{3}$ and $\mathrm{SiO}_{2}$ results, particularly for the glass standards. The low $\mathrm{NiO}$ value noted above was measured for one the $\mathrm{U}_{\text {std }}$ samples in SRNL-SCS-2008-00003. These issues will not impact the outcome of the study.

\subsubsection{Batch 1 and Uranium Standard Results}

In this section, the PSAL measurements of the chemical compositions of the Batch 1 and $\mathrm{U}_{\text {std }}$ glasses are reviewed. The measurements are investigated across the ICP-AES analytical blocks for each of the analytical sets for both SRNL-SCS-2008-00003 and SRNL-SCS-2008-00043.

Exhibit A3 in Appendix A provides statistical analyses of the Batch 1 and $\mathrm{U}_{\text {std }}$ (where included) results generated by both preparation methods by calibration block for each oxide of interest for each of the analytical plans. The results include analysis of variance (ANOVA) investigations looking for statistically significant differences between the means of these groups for each of the oxides for each of the standards. The results from the statistical tests for the Batch 1 standard for SRNL-SCS-2008-00003, Set 2 indicate that $\mathrm{Al}_{2} \mathrm{O}_{3}, \mathrm{CaO}, \mathrm{Ce}_{2} \mathrm{O}_{3}, \mathrm{CuO}, \mathrm{K} 2 \mathrm{O}, \mathrm{Na}_{2} \mathrm{O}, \mathrm{Nb}_{2} \mathrm{O}_{5}, \mathrm{TiO}_{2}$, and $\mathrm{ZrO}_{2}$ have measurements with a significant ICP-AES calibration effect on the block averages at the 5\% significance level. The results for $\mathrm{U}_{\text {std }}$ for SRNL-SCS-2008-00003, Set 2 indicate that $\mathrm{Al}_{2} \mathrm{O}_{3}, \mathrm{CaO}, \mathrm{K}_{2} \mathrm{O}_{3}, \mathrm{Li}_{2} \mathrm{O}, \mathrm{Na}_{2} \mathrm{O}, \mathrm{Nb}_{2} \mathrm{O}_{5}, \mathrm{SiO}_{2}, \mathrm{TiO}_{2}$, and $\mathrm{U}_{3} \mathrm{O}_{8}$ have measurements with a significant ICP-AES calibration effect on the block averages at the 5\% significance level. The results from the statistical tests for the Batch 1 standard for SRNL-SCS-2008-00003, Set 3 indicate that $\mathrm{Al}_{2} \mathrm{O}_{3}, \mathrm{Ce}_{2} \mathrm{O}_{3}, \mathrm{~K}_{2} \mathrm{O}$, and $\mathrm{Nb}_{2} \mathrm{O}_{5}$ have measurements with a significant ICP-AES calibration effect on the block averages at the 5\% significance level. The results for $\mathrm{U}_{\text {std }}$ for SRNL-SCS-2008-00003, Set 3 indicate that $\mathrm{Al}_{2} \mathrm{O}_{3}, \mathrm{~K}_{2} \mathrm{O}_{3}, \mathrm{Na}_{2} \mathrm{O}, \mathrm{Nb}_{2} \mathrm{O}_{5}$, and $\mathrm{U}_{3} \mathrm{O}_{8}$ have measurements with a significant ICP-AES calibration effect on the block averages at the 5\% significance level. The results from the statistical tests for the Batch 1 standard for SRNL-SCS2008-00043 indicate that $\mathrm{BaO}, \mathrm{Cr}_{2} \mathrm{O}_{3}, \mathrm{Li}_{2} \mathrm{O}, \mathrm{MgO}, \mathrm{TiO}_{2}$ and $\mathrm{ZrO}_{2}$ have measurements with a 
significant ICP-AES calibration effect on the block averages at the 5\% significance level. The reference values for the oxide concentrations of the standard are given in the header for each set of measurements in the exhibit.

Some of the results from these statistical analyses provide incentive for adjusting the measurements by the effects of the ICP-AES calibration. Therefore, the oxide measurements of the study glasses are to be bias corrected for the effect of the ICP-AES calibration on each of the analytical blocks and sub-blocks. The basis for this bias correction is presented as part of Exhibit A3 - the average measurement for Batch 1 for each ICP-AES block/sub-block for $\mathrm{Al}_{2} \mathrm{O}_{3}, \mathrm{~B}_{2} \mathrm{O}_{3}$, $\mathrm{BaO}, \mathrm{CaO}, \mathrm{Cr}_{2} \mathrm{O}_{3}, \mathrm{CuO}, \mathrm{Fe}_{2} \mathrm{O}_{3}, \mathrm{Li}_{2} \mathrm{O}, \mathrm{MgO}, \mathrm{MnO}, \mathrm{Na}_{2} \mathrm{O}, \mathrm{NiO}, \mathrm{SiO}_{2}$, and $\mathrm{TiO}_{2}$ and the average measurement for $\mathrm{U}_{\text {std }}$ for each ICP-AES set/block for $\mathrm{U}_{3} \mathrm{O}_{8}$. The Batch 1 results served as the basis for bias correcting all of the oxides (that were bias corrected) except uranium. The $\mathrm{U}_{\text {std }}$ results were used to bias correct for uranium. For the other oxides, the Batch 1 results were used to conduct the bias correction as long as the reference value for the oxide concentration in the Batch 1 glass was greater than or equal to $0.1 \mathrm{wt} \%$. Thus, applying this approach and based upon the information in the exhibits, the Batch 1 results were used to bias correct the $\mathrm{Al}_{2} \mathrm{O}_{3}, \mathrm{~B}_{2} \mathrm{O}_{3}, \mathrm{BaO}$, $\mathrm{CaO}, \mathrm{Cr}_{2} \mathrm{O}_{3}, \mathrm{CuO}, \mathrm{Fe}_{2} \mathrm{O}_{3}, \mathrm{~K}_{2} \mathrm{O}, \mathrm{Li}_{2} \mathrm{O}, \mathrm{MgO}, \mathrm{MnO}, \mathrm{Na}_{2} \mathrm{O}, \mathrm{Nd}_{2} \mathrm{O}_{3}, \mathrm{NiO}, \mathrm{SiO}_{2}$, and $\mathrm{TiO}_{2}$ measurements, and the $\mathrm{U}_{\text {std }}$ values were used to bias correct the $\mathrm{U}_{3} \mathrm{O}_{8}$. No bias correction was conducted for $\mathrm{Ce}_{2} \mathrm{O}_{3}, \mathrm{La}_{2} \mathrm{O}_{3}, \mathrm{PbO}, \mathrm{SO}_{4}{ }^{2-}$, or $\mathrm{ZrO}_{2}$.

The bias correction was conducted as follows: For each oxide, let $\bar{a}_{i j}$ be the average measurement for the $i^{\text {th }}$ oxide at analytical block $\mathrm{j}$ for Batch 1 (or $\mathrm{U}_{\text {std }}$ for uranium), and let $t_{i}$ be the reference value for the $i^{\text {th }}$ oxide for Batch 1 (or for $\mathrm{U}_{\text {std }}$ if uranium). (The averages and reference values are provided in Exhibits A3 and A4 in Appendix A.) Let $\bar{c}_{i j k}$ be the average measurement for the $i^{\text {th }}$ oxide at analytical block $\mathrm{j}$ for the $k^{\text {th }}$ glass. The bias adjustment was conducted as follows:

$$
\bar{c}_{i j k} \bullet\left(1-\frac{\bar{a}_{i j}-t_{i}}{\bar{a}_{i j}}\right)=\bar{c}_{i j k} \bullet \frac{t_{i}}{\bar{a}_{i j}}
$$

Bias-corrected measurements are indicated by a "bc" suffix, and such adjustments were performed for all of the oxides of this study except for $\mathrm{Ce}_{2} \mathrm{O}_{3}, \mathrm{La}_{2} \mathrm{O}_{3}, \mathrm{PbO}, \mathrm{SO}_{4}{ }^{2-}$, and $\mathrm{ZrO}_{2}$. Both measured and measured-bc values are included in the discussion that follows. In these discussions, the original values for $\mathrm{Ce}_{2} \mathrm{O}_{3}, \mathrm{La}_{2} \mathrm{O}_{3}, \mathrm{PbO}, \mathrm{SO}_{4}{ }^{2-}$, and $\mathrm{ZrO}_{2}$ are included for completeness (e.g., to allow a sum of oxides to be computed for the bias-corrected results). These bias-corrected values are the same as the original $\mathrm{Ce}_{2} \mathrm{O}_{3}, \mathrm{La}_{2} \mathrm{O}_{3}, \mathrm{PbO}, \mathrm{SO}_{4}{ }^{2-}$, and $\mathrm{ZrO}_{2}$ values.

\subsubsection{Composition Measurements by Glass Identifier with Targeted Compositions}

To finalize the measurements that were used to characterize the compositions of the study glasses, Exhibit A4 in Appendix A was prepared. This exhibit provides plots of the oxide concentration measurements by Glass ID (including Batch 1 and $\mathrm{U}_{\text {std }}$ ) by Lab ID for the LM and PF preparation methods for each set of analyses. The plots are grouped by analytical plan and preparation method, and they show the individual measurements across the duplicates of each preparation method and the replicate ICP-AES calibrations. A review of the plots presented in these exhibits reveals the repeatability of the individual oxide values for each glass. The targeted concentration for each of the oxides for each of the study glasses and the reference values for the standards are also provided as part of these plots. 


\subsubsection{Measured versus Targeted Compositions}

The measurements for each oxide for each glass were averaged to determine a representative chemical composition for each glass. These determinations were conducted both for the measured and for the bias-corrected data. A sum of oxides was also computed for each glass based upon both the measured and bias-corrected values. Exhibit A5 in Appendix A provides plots showing results for each glass for each oxide to help highlight the comparisons among the measured, bias-corrected, and targeted values. These plots are grouped by analytical plan and, where appropriate, by analytical set.

Some observations from the plots of Exhibit A5 are offered: For the SRNL-SCS-2008-00003 results, the measured $\mathrm{Fe}_{2} \mathrm{O}_{3}$ (set 2), $\mathrm{NiO}$ (sets 2 and 3), and $\mathrm{SiO}_{2}$ (sets 2 and 3) values for most of the study glasses fall below their targeted values. For the SRNL-SCS-2008-00043 results, the $\mathrm{SiO}_{2}$ values for most of the study glasses fall above their targeted values. These issues are not expected to impact the outcome of the study.

Table A6 in Appendix A provides a summary of the average compositions as well as the targeted compositions and some associated differences and relative differences. Notice that the targeted sums of oxides for the standard glasses do not sum to $100 \%$ due to an incomplete coverage of the oxides in the Batch 1 and $\mathrm{U}_{\text {std }}$ glasses. All of the sums of oxides (both measured and biascorrected) for the study glasses fall within the interval of 95 to $105 \mathrm{wt} \%$. Entries in Table A5 show the relative differences between the measured or bias-corrected values and the targeted values. These differences are shaded when they are greater than or equal to $5 \%$. Overall, these comparisons between the measured and targeted compositions suggest only minor difficulties in meeting the targeted compositions for some of the oxides for some of the glasses.

\subsubsection{Assessment of $\mathrm{SO}_{4}{ }^{2-}$ Values}

The targeted $\mathrm{SO}_{4}{ }^{2-}$ concentrations for the study glasses fell within the interval of 0.277 to $0.547 \mathrm{wt} \%$. Figure 4-1 provides a plot of the measured versus targeted $\mathrm{SO}_{4}{ }^{2-}$ values for each of the glasses over all the analytical plans (this plot is a compilation of three of the plots in Exhibit A5).

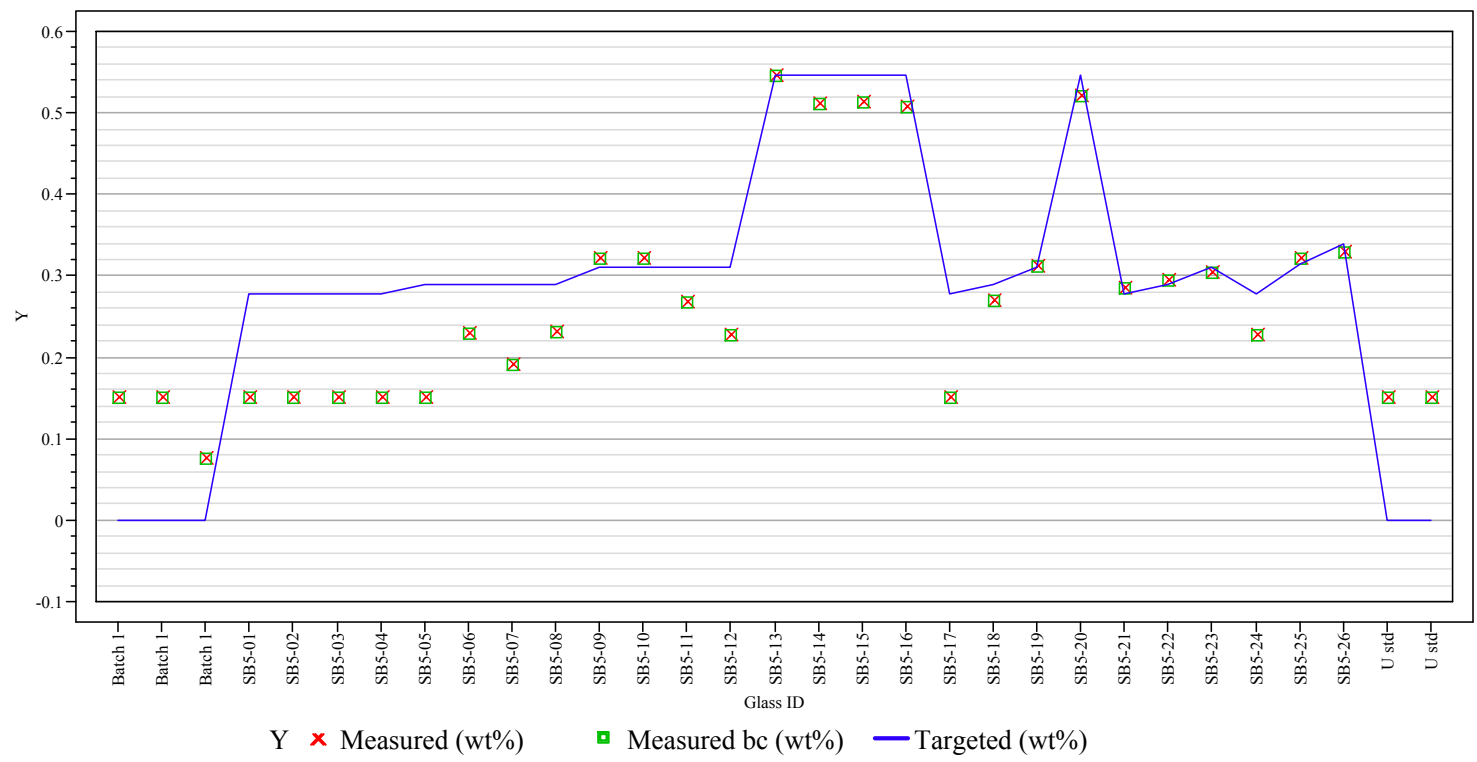

Figure 4-1. $\mathrm{SO}_{4}{ }^{2-}$ Values for Glasses in the Analytical Plan by Glass ID. 
Since Batch 1 and the $\mathrm{U}_{\text {std }}$ standard contain no $\mathrm{SO}_{4}{ }^{2-}$, their measurements are below the detection limit for sulfur (see Tables A2 and A4 in Appendix A). For many of the study glasses (even those with the highest targeted $\mathrm{SO}_{4}{ }^{2-}$ concentrations), the average, measured values fall near the targeted concentrations. Some volatilization of $\mathrm{SO}_{4}{ }^{2-}$ may have occurred during melting of the glasses where the measured $\mathrm{SO}_{4}{ }^{2-}$ concentration falls below the target value. No issues with sulfur retention are apparent in the frit/sludge combinations studied here.

\subsubsection{MAR Assessment of the SB4/Second Decant VS Glasses}

Another assessment that can be made for these initial SB5 study glasses is how well they satisfy the Measurement Acceptability Region (MAR) criteria of the DWPF Product Composition Control System (PCCS). Glasses SB5-21 through SB5-26 are not incorporated in this evaluation since $\mathrm{Nd}_{2} \mathrm{O}_{3}$ and $\mathrm{HfO}_{2}$ are not included in the current calculations conducted by PCCS.

Table 4-2 provides the outcome of the MAR assessment. The columns in the table give the glass identifier, compositional view, the free energy of hydration value for boron $\left(B \Delta G_{p}\right.$ Value), the predicted normalized leachate for boron (NL $[B])$, the predicted liquidus temperature $\left(\mathrm{T}_{\mathrm{L}}\right.$ Pred), the viscosity prediction (Visc Pred), the nepheline discriminator value (Neph), and the overall MAR assessment outcome excluding any limitation on $\mathrm{Na}_{2} \mathrm{SO}_{4}$ concentration. All but one of the study glasses would pass the PCCS MAR and be deemed processable by and acceptable for the DWPF. Glass SB5-04, based on the measured bc composition, fails the nepheline discriminator criterion. This is not considered to be a critical issue since, as will be shown in the following section, the PCT response for this glass is very acceptable. 
Table 4-2. Results of MAR Assessment of SB4 VS Glasses for Measured, Bias-Corrected, and Targeted Compositional Views

\begin{tabular}{|c|c|c|c|c|c|c|c|}
\hline Glass ID & $\begin{array}{c}\text { Compositional } \\
\text { View }\end{array}$ & $\begin{array}{c}\text { B } \Delta G_{p} \\
\text { (kcal / } 100 \text { g glass) }\end{array}$ & $\begin{array}{c}\text { NL [B] } \\
(\mathrm{g} / \mathrm{L})\end{array}$ & $\begin{array}{c}\text { TL Pred } \\
\left({ }^{\circ} \mathrm{C}\right) \\
\end{array}$ & $\begin{array}{c}\text { Visc Pred } \\
\text { (P) }\end{array}$ & Neph & MAR Status \\
\hline SB5-01 & Measured & -9.640 & 0.70 & 939.7 & 61.4 & 0.649 & Acceptable \\
\hline SB5-02 & Measured & -9.389 & 0.63 & 939.6 & 56.4 & 0.653 & Acceptable \\
\hline SB5-03 & Measured & -9.317 & 0.61 & 952.0 & 61.9 & 0.644 & Acceptable \\
\hline SB5-04 & Measured & -9.591 & 0.69 & 944.5 & 50.2 & 0.633 & Acceptable \\
\hline SB5-05 & Measured & -10.380 & 0.95 & 952.8 & 44.1 & 0.659 & Acceptable \\
\hline SB5-06 & Measured & -10.101 & 0.85 & 951.1 & 46.8 & 0.662 & Acceptable \\
\hline SB5-07 & Measured & -9.719 & 0.72 & 971.5 & 48.0 & 0.663 & Acceptable \\
\hline SB5-08 & Measured & -9.677 & 0.71 & 974.1 & 49.3 & 0.657 & Acceptable \\
\hline SB5-09 & Measured & -10.894 & 1.18 & 961.9 & 37.5 & 0.675 & Acceptable \\
\hline SB5-10 & Measured & -10.515 & 1.01 & 954.6 & 43.1 & 0.684 & Acceptable \\
\hline SB5-11 & Measured & -10.231 & 0.90 & 973.5 & 40.1 & 0.678 & Acceptable \\
\hline SB5-12 & Measured & -10.150 & 0.87 & 981.3 & 41.5 & 0.675 & Acceptable \\
\hline SB5-13 & Measured & -11.994 & 1.87 & 930.7 & 31.6 & 0.679 & Acceptable \\
\hline SB5-14 & Measured & -11.638 & 1.61 & 916.8 & 31.6 & 0.681 & Acceptable \\
\hline SB5-15 & Measured & -11.583 & 1.58 & 926.1 & 29.3 & 0.670 & Acceptable \\
\hline SB5-16 & Measured & -11.215 & 1.35 & 939.0 & 30.5 & 0.667 & Acceptable \\
\hline SB5-17 & Measured & -9.296 & 0.61 & 942.0 & 38.4 & 0.662 & Acceptable \\
\hline SB5-18 & Measured & -10.497 & 1.00 & 953.7 & 37.2 & 0.657 & Acceptable \\
\hline SB5-19 & Measured & -11.149 & 1.31 & 958.3 & 32.1 & 0.656 & Acceptable \\
\hline SB5-20 & Measured & -10.213 & 0.89 & 962.9 & 51.3 & 0.685 & Acceptable \\
\hline SB5-01 & Measured bc & -9.897 & 0.78 & 939.0 & 56.6 & 0.640 & Acceptable \\
\hline SB5-02 & Measured bc & -9.631 & 0.70 & 939.4 & 51.9 & 0.644 & Acceptable \\
\hline SB5-03 & Measured bc & -9.323 & 0.61 & 956.9 & 56.3 & 0.641 & Acceptable \\
\hline SB5-04 & Measured bc & -9.867 & 0.77 & 943.3 & 45.3 & 0.624 & Neph \\
\hline SB5-05 & Measured bc & -10.661 & 1.07 & 952.4 & 40.2 & 0.650 & Acceptable \\
\hline SB5-06 & Measured bc & -10.067 & 0.84 & 956.7 & 43.3 & 0.660 & Acceptable \\
\hline SB5-07 & Measured bc & -9.960 & 0.80 & 970.9 & 44.3 & 0.654 & Acceptable \\
\hline SB5-08 & Measured bc & -9.661 & 0.71 & 980.3 & 45.0 & 0.655 & Acceptable \\
\hline SB5-09 & Measured bc & -10.941 & 1.21 & 966.1 & 33.3 & 0.672 & Acceptable \\
\hline SB5-10 & Measured bc & -10.486 & 1.00 & 960.4 & 39.7 & 0.682 & Acceptable \\
\hline SB5-11 & Measured bc & -10.565 & 1.03 & 972.5 & 35.5 & 0.669 & Acceptable \\
\hline SB5-12 & Measured bc & -10.459 & 0.99 & 980.5 & 37.1 & 0.666 & Acceptable \\
\hline SB5-13 & Measured bc & -11.974 & 1.85 & 930.5 & 28.4 & 0.674 & Acceptable \\
\hline SB5-14 & Measured bc & -11.688 & 1.65 & 915.3 & 27.4 & 0.676 & Acceptable \\
\hline SB5-15 & Measured bc & -11.733 & 1.68 & 931.0 & 27.2 & 0.666 & Acceptable \\
\hline SB5-16 & Measured bc & -11.340 & 1.42 & 943.7 & 28.4 & 0.663 & Acceptable \\
\hline SB5-17 & Measured bc & -9.337 & 0.62 & 946.3 & 37.1 & 0.657 & Acceptable \\
\hline SB5-18 & Measured bc & -10.448 & 0.98 & 953.5 & 33.7 & 0.651 & Acceptable \\
\hline SB5-19 & Measured bc & -11.184 & 1.33 & 963.5 & 31.3 & 0.652 & Acceptable \\
\hline SB5-20 & Measured bc & -10.209 & 0.89 & 963.3 & 46.1 & 0.679 & Acceptable \\
\hline SB5-01 & Targeted & -9.877 & 0.77 & 956.0 & 55.3 & 0.647 & Acceptable \\
\hline SB5-02 & Targeted & -9.623 & 0.70 & 954.9 & 55.5 & 0.650 & Acceptable \\
\hline SB5-03 & Targeted & -9.487 & 0.66 & 966.8 & 54.2 & 0.643 & Acceptable \\
\hline SB5-04 & Targeted & -9.400 & 0.63 & 965.1 & 55.2 & 0.638 & Acceptable \\
\hline SB5-05 & Targeted & -10.255 & 0.91 & 976.9 & 46.7 & 0.664 & Acceptable \\
\hline SB5-06 & Targeted & -10.001 & 0.81 & 975.9 & 47.0 & 0.667 & Acceptable \\
\hline SB5-07 & Targeted & -9.865 & 0.77 & 989.4 & 45.6 & 0.661 & Acceptable \\
\hline SB5-08 & Targeted & -9.778 & 0.74 & 988.2 & 46.5 & 0.655 & Acceptable \\
\hline SB5-09 & Targeted & -10.924 & 1.20 & 980.9 & 37.8 & 0.678 & Acceptable \\
\hline SB5-10 & Targeted & -10.670 & 1.08 & 980.2 & 38.1 & 0.681 & Acceptable \\
\hline SB5-11 & Targeted & -10.533 & 1.02 & 994.9 & 36.8 & 0.675 & Acceptable \\
\hline SB5-12 & Targeted & -10.446 & 0.98 & 994.3 & 37.5 & 0.669 & Acceptable \\
\hline SB5-13 & Targeted & -12.076 & 1.94 & 924.6 & 28.9 & 0.674 & Acceptable \\
\hline SB5-14 & Targeted & -11.822 & 1.74 & 924.0 & 29.3 & 0.677 & Acceptable \\
\hline SB5-15 & Targeted & -11.685 & 1.64 & 937.7 & 28.0 & 0.671 & Acceptable \\
\hline SB5-16 & Targeted & -11.598 & 1.59 & 937.6 & 28.6 & 0.665 & Acceptable \\
\hline SB5-17 & Targeted & -9.136 & 0.57 & 969.8 & 39.0 & 0.664 & Acceptable \\
\hline SB5-18 & Targeted & -10.590 & 1.04 & 966.3 & 33.1 & 0.655 & Acceptable \\
\hline SB5-19 & Targeted & -11.457 & 1.50 & 969.6 & 30.0 & 0.654 & Acceptable \\
\hline SB5-20 & Targeted & -10.485 & 1.00 & 959.9 & 44.0 & 0.677 & Acceptable \\
\hline
\end{tabular}




\subsection{A Statistical Review of the PCT Results for These Initial SB5 Glasses}

Table B1 in Appendix B provides the elemental leachate concentration measurements (column headings include "ar," for as received) determined by the PSAL for the solution samples generated by the PCTs for each for analytical plan (2 sets for SRNL-SCS-2008-00005 and 1 set for SRNL-SCS-2008-00042). One of the quality control checkpoints for the PCT procedure is solution-weight loss over the course of the 7-day test. None of these PCT results indicated a solution-weight loss problem. Any measurement in Table B1 below the detection limit of the analytical procedure (indicated by a " $<$ ") was replaced by one half of the detection limit in subsequent analyses. In addition to adjustments for detection limits, the values were adjusted for the dilution factors: the values for the study glasses, the blanks, and the ARM glass in Table B1 were multiplied by 1.6667 to determine the values in parts per million (ppm) and the values for EA were multiplied by 16.6667 . The last four columns of Table B1 provide the resulting measurements.

In the following sections,

- the analytical sequence of the measurements is explored,

- the measurements of the standards are investigated and used to assess the overall accuracy of the measurement process,

- the measurements for each glass are reviewed,

- plots are provided that explore the effects of heat treatment on the PCTs for these glasses,

- the PCTs are normalized using the compositions (targeted, measured, and bias-corrected) presented in Table A5, and

- the normalized PCTs of glasses SB5-01 through SB4-20 are compared to durability predictions for these compositions generated from the current DWPF models. ${ }^{15}$

\subsubsection{Measurements in Analytical Sequence}

Exhibit B1 in Appendix B provides plots of the leachate concentrations (as common logarithms of the ppm values) in analytical sequence as generated by the PSAL for all of the measurements from Table B1 by analytical set within each analytical plan. An " $x$ " is used to represent a value for a standard (EA, ARM, blank, or the solution standard). A red square ( $\square$ ) is used to represent a CCC result for a study glass, and a "+" is used to represent a quenched result for a study glass.

\subsubsection{Results for the Samples of the Multi-Element Solution Standard}

Exhibit B2 in Appendix B provides analyses of the PSAL measurements of the samples of the multi-element solution standard by ICP-AES calibration block within analytical plan and set. An ANOVA investigating for statistically significant differences among the block averages for these samples for each element of interest is included in these exhibits. There was no indication of a statistically significant (at a 5\% level) difference among the averages of these measurements for any of the elements of interest except for B and Si in SRNL-SCS-2008-00042 (set u). However, averaging the ppm values for each set of triplicates helps to minimize the impact of any potential ICP-AES effects.

Table 4-3 summarizes the average measurements and the reference values for the four primary elements of interest. The results indicate consistent and accurate measurements from the PSAL processes used to conduct these analyses. 
Table 4-3. Results from Samples of the Multi-Element Solution Standard

\begin{tabular}{|c|c|c|c|c|c|c|}
\hline Analytical Plan & $\begin{array}{c}\text { Analytical } \\
\text { Set } \\
\end{array}$ & $\begin{array}{c}\text { Analytical } \\
\text { Block }\end{array}$ & Avg B & $\operatorname{Avg} \mathbf{L i}$ & Avg $\mathrm{Na}$ & Avg Si \\
\hline SRNL-SCS-2008-00005 & $q$ & 1 & 20.20 & 9.95 & 81.20 & 49.37 \\
\hline SRNL-SCS-2008-00005 & $\mathrm{q}$ & 2 & 20.27 & 9.85 & 80.13 & 50.63 \\
\hline SRNL-SCS-2008-00005 & $q$ & 3 & 19.40 & 9.88 & 80.43 & 50.40 \\
\hline SRNL-SCS-2008-00005 & $\mathrm{r}$ & 1 & 20.13 & 9.80 & 79.27 & 50.00 \\
\hline SRNL-SCS-2008-00005 & $\mathrm{r}$ & 2 & 20.40 & 9.79 & 80.10 & 52.00 \\
\hline SRNL-SCS-2008-00005 & $\mathrm{r}$ & 3 & 20.83 & 10.00 & 80.73 & 51.50 \\
\hline SRNL-SCS-2008-00005 & $\mathrm{s}$ & 1 & 19.70 & 9.78 & 79.87 & 50.93 \\
\hline SRNL-SCS-2008-00005 & $\mathrm{s}$ & 2 & 19.03 & 9.57 & 80.17 & 48.70 \\
\hline SRNL-SCS-2008-00005 & $\mathrm{s}$ & 3 & 19.33 & 9.67 & 81.00 & 49.90 \\
\hline SRNL-SCS-2008-00042 & $\mathrm{u}$ & 1 & 19.37 & 9.99 & 83.27 & 50.73 \\
\hline SRNL-SCS-2008-00042 & $\mathrm{u}$ & 2 & 21.33 & 10.15 & 82.87 & 53.70 \\
\hline \multirow[t]{4}{*}{ SRNL-SCS-2008-00042 } & $\mathrm{u}$ & 3 & 20.00 & 10.03 & 83.43 & 51.60 \\
\hline & & Grand Average & 20.00 & 9.87 & 81.04 & 50.79 \\
\hline & & $\begin{array}{c}\text { Reference } \\
\text { Value } \\
\end{array}$ & 20 & 10 & 81 & 50 \\
\hline & & \% difference & $0.00 \%$ & $-1.29 \%$ & $0.05 \%$ & $1.58 \%$ \\
\hline
\end{tabular}

\subsubsection{Measurements by Glass Identifier}

Exhibit B3 in Appendix B provide plots of the leachate concentrations for each type of submitted sample by analytical plan and set: the study glasses by heat treatment and the standards (EA, ARM, the multi-element solution standard, and blanks). Exhibit B4 in Appendix B provide plots of the leachate concentrations for the PCT results of just the study glasses by heat treatment grouped by analytical plan and set. These plots allow for the assessment of the repeatability of the measurements, which suggests some scatter in the triplicate values for some analytes for some of the glasses. Also, note the small differences between the values for the two heat treatments for the study glasses. More will be said regarding comparisons between the heat treatments in the discussions that follow.

\subsubsection{Normalized PCT Results}

PCT leachate concentrations are typically normalized using the cation composition (expressed as a weight percent) in the glass to obtain a grams-per-liter $(\mathrm{g} / \mathrm{L})$ leachate concentration. The normalization of the PCT results is usually conducted using the measured compositions of the glasses. This is the preferred normalization process for the PCTs. For completeness, the targeted cation and the bias-corrected cation compositions were also used to conduct this normalization.

As is the usual convention, the common logarithm of the normalized PCT (normalized leachate, $\mathrm{NL}$ ) for each element of interest was determined and used for comparison. To accomplish this computation, one must:

1. Determine the common logarithm of the elemental parts per million (ppm) leachate concentration for each of the triplicates and each of the elements of interest (these values are provided in Table B1 of Appendix B), 
2. Average the common logarithms over the triplicates for each element of interest, and then,

Normalizing Using Measured Composition (preferred method),

3. Subtract a quantity equal to 1 plus the common logarithm of the average cation measured concentration (expressed as a weight percent of the glass) from the average computed in step 2 .

Or Normalizing Using Target Composition,

3. Subtract a quantity equal to 1 plus the common logarithm of the target cation concentration (expressed as a weight percent of the glass) from the average computed in step 2 .

Or Normalizing Using Measured Bias-Corrected Composition,

3 . Subtract a quantity equal to 1 plus the common logarithm of the measured biascorrected cation concentration (expressed as a weight percent of the glass) from the average computed in step 2 .

Exhibit B5 in Appendix B provides scatter plots for these results by analytical plan and set and offers an opportunity to investigate the consistency in the leaching across the elements for the glasses of this study. All combinations of the normalizations of the PCTs (i.e., those generated using the targeted, measured, and bias-corrected compositional views) and both heat treatments are represented in the series of scatter plots. Consistency in the leaching across the elements is typically demonstrated by a high degree of linear correlation among the values for pairs of these elements. The smallest correlation in these plot is that for B and Li (SRNL-SCS-2008-00005, Set s), with a value of $\sim 95 \%$, indicating a high degree of correlation for all of the measurements.

Table 4-4 summarizes the normalized PCT results for the glasses of this study. The glasses are listed by glass identifier. Also, note that the nepheline discriminator value is shown for each glass (based upon the compositional view indicated) and that a pairing value is indicated where a surrogate was developed for the study glass. The normalized leachate values for $\mathrm{B}, \mathrm{Li}, \mathrm{Na}$ and $\mathrm{Si}$ for all of the study glasses are well below those of the EA benchmark glass, regardless of heat treatment or compositional view. This indicates that all of the glasses have very acceptable durability performance. 
Table 4-4. Normalized PCTs by Glass ID and Compositional View for Initial SB5 Study Glasses.

\begin{tabular}{|c|c|c|c|c|c|c|c|c|c|c|c|c|c|}
\hline Glass ID & Pair & $\begin{array}{c}\text { Heat } \\
\text { Treatment }\end{array}$ & $\begin{array}{c}\text { Composition } \\
\text { View }\end{array}$ & $\begin{array}{c}\text { Nepheline } \\
\text { Discriminator }\end{array}$ & $\Delta \mathbf{G}_{\mathrm{p}}$ & $\begin{array}{c}\log N L \\
{[B(g / L)]}\end{array}$ & $\begin{array}{c}\log N L \\
{[\mathrm{Li}(\mathrm{g} / \mathrm{L})]}\end{array}$ & $\begin{array}{c}\log \mathrm{NL} \\
{[\mathrm{Na}(\mathrm{g} / \mathrm{L})]}\end{array}$ & $\begin{array}{c}\log \mathrm{NL} \\
{[\mathrm{Si}(\mathrm{g} / \mathrm{L})]}\end{array}$ & $\begin{array}{c}\text { NL } \\
\text { B (g/L) }\end{array}$ & $\begin{array}{c}\mathrm{NL} \\
\mathrm{Li}(\mathrm{g} / \mathrm{L}) \\
\end{array}$ & $\begin{array}{c}\mathrm{NL} \\
\mathrm{Na}(\mathrm{g} / \mathrm{L})\end{array}$ & $\begin{array}{c}\mathrm{NL} \\
\mathrm{Si}(\mathrm{g} / \mathrm{L}) \\
\end{array}$ \\
\hline ARM & 0 & ref & reference & 0.753 & -8.905 & -0.3244 & -0.2494 & -0.3000 & -0.5647 & 0.474 & 0.563 & 0.501 & 0.272 \\
\hline ARM & 0 & ref & reference & 0.753 & -8.905 & $\begin{array}{l}-0.3363 \\
\end{array}$ & -0.2554 & $\begin{array}{l}-0.3003 \\
\end{array}$ & -0.5645 & 0.461 & 0.555 & 0.501 & 0.273 \\
\hline ARM & 0 & ref & reference & 0.753 & -8.905 & -0.2241 & -0.1742 & -0.2291 & -0.4928 & 0.597 & 0.670 & 0.590 & 0.322 \\
\hline EA & 0 & ref & reference & 0.704 & -14.941 & 1.2409 & 0.9589 & 1.1084 & 0.5969 & 17.413 & 9.096 & 12.835 & 3.953 \\
\hline EA & 0 & ref & reference & 0.704 & -14.941 & 1.2285 & 0.9668 & 1.1265 & 0.5869 & 16.922 & 9.264 & 13.381 & 3.863 \\
\hline EA & 0 & ref & reference & 0.704 & -14.941 & 1.2686 & 0.9967 & 1.1474 & 0.6196 & 18.562 & 9.925 & 14.040 & 4.165 \\
\hline SB5-01 & 0 & $\mathrm{CCC}$ & Measured & 0.649 & $\begin{array}{l}-9.640 \\
\end{array}$ & $\begin{array}{l}-0.2900 \\
\end{array}$ & -0.2003 & $\begin{array}{l}-0.1761 \\
\end{array}$ & -0.4738 & 0.513 & 0.630 & 0.667 & 0.336 \\
\hline SB5-02 & 0 & $\mathrm{CCC}$ & Measured & 0.653 & -9.389 & $\begin{array}{l}-0.2729 \\
\end{array}$ & -0.2157 & -0.1805 & -0.4121 & 0.533 & 0.609 & 0.660 & 0.387 \\
\hline SB5-03 & 1 & $\mathrm{CCC}$ & Measured & 0.644 & -9.318 & -0.2451 & -0.0518 & -0.1280 & -0.3543 & 0.569 & 0.888 & 0.745 & 0.442 \\
\hline SB5-04 & 4 & $\mathrm{CCC}$ & Measured & 0.633 & -9.592 & -0.2600 & -0.2256 & -0.2450 & -0.3972 & 0.550 & 0.595 & 0.569 & 0.401 \\
\hline SB5-05 & 0 & $\mathrm{CCC}$ & Measured & 0.659 & -10.381 & -0.2090 & -0.1386 & -0.1015 & -0.4001 & 0.618 & 0.727 & 0.792 & 0.398 \\
\hline SB5-06 & 0 & $\mathrm{CCC}$ & Measured & 0.662 & -10.102 & -0.1859 & -0.2895 & -0.2149 & -0.4534 & 0.652 & 0.513 & 0.610 & 0.352 \\
\hline SB5-07 & 5 & $\mathrm{CCC}$ & Measured & 0.663 & -9.719 & -0.2380 & $\begin{array}{l}-0.1953 \\
\end{array}$ & $\begin{array}{l}-0.1756 \\
\end{array}$ & -0.4272 & 0.578 & 0.638 & 0.667 & 0.374 \\
\hline SB5-08 & 2 & $\mathrm{CCC}$ & Measured & 0.657 & -9.677 & -0.2371 & -0.2161 & -0.2241 & -0.4185 & 0.579 & 0.608 & 0.597 & 0.382 \\
\hline SB5-09 & 0 & $\mathrm{CCC}$ & Measured & 0.675 & $\begin{array}{l}-10.894 \\
\end{array}$ & -0.1286 & -0.0740 & -0.0358 & -0.3381 & 0.744 & 0.843 & 0.921 & 0.459 \\
\hline SB5-10 & 0 & $\mathrm{CCC}$ & Measured & 0.684 & -10.515 & -0.1330 & -0.1128 & -0.0802 & -0.3364 & 0.736 & 0.771 & 0.831 & 0.461 \\
\hline SB5-11 & 3 & $\mathrm{CCC}$ & Measured & 0.678 & -10.230 & -0.1438 & -0.1264 & -0.1084 & -0.3544 & 0.718 & 0.747 & 0.779 & 0.442 \\
\hline SB5-12 & 6 & $\mathrm{CCC}$ & Measured & 0.675 & -10.150 & -0.1970 & $\begin{array}{l}-0.1774 \\
\end{array}$ & -0.1350 & -0.3739 & 0.635 & 0.665 & 0.733 & 0.423 \\
\hline SB5-13 & 0 & $\mathrm{CCC}$ & Measured & 0.679 & -11.994 & $\begin{array}{l}-0.0699 \\
\end{array}$ & -0.0285 & 0.0342 & -0.2914 & 0.851 & 0.937 & 1.082 & 0.511 \\
\hline SB5-14 & 0 & $\mathrm{CCC}$ & Measured & 0.681 & -11.636 & -0.0846 & -0.0732 & -0.0226 & $\begin{array}{l}-0.2992 \\
\end{array}$ & 0.823 & 0.845 & 0.949 & 0.502 \\
\hline SB5-15 & 0 & $\mathrm{CCC}$ & Measured & 0.670 & -11.582 & -0.1203 & -0.1197 & -0.0875 & -0.3579 & 0.758 & 0.759 & 0.818 & 0.439 \\
\hline SB5-16 & 0 & $\mathrm{CCC}$ & Measured & 0.667 & -11.215 & -0.1298 & -0.1572 & -0.1208 & -0.3671 & 0.742 & 0.696 & 0.757 & 0.429 \\
\hline SB5-17 & 0 & $\mathrm{CCC}$ & Measured & 0.662 & $\begin{array}{l}-9.296 \\
\end{array}$ & -0.1868 & -0.1585 & -0.2217 & -0.3220 & 0.650 & 0.694 & 0.600 & 0.476 \\
\hline SB5-18 & 0 & $\mathrm{CCC}$ & Measured & 0.657 & -10.498 & -0.1586 & -0.1553 & -0.1335 & -0.3543 & 0.694 & 0.699 & 0.735 & 0.442 \\
\hline SB5-19 & 0 & $\mathrm{CCC}$ & Measured & 0.656 & -11.150 & -0.1625 & $\begin{array}{l}-0.1917 \\
\end{array}$ & -0.1392 & -0.3695 & 0.688 & 0.643 & 0.726 & 0.427 \\
\hline SB5-20 & 0 & $\mathrm{CCC}$ & Measured & 0.685 & -10.212 & $\begin{array}{l}-0.1851 \\
\end{array}$ & -0.1976 & -0.2101 & -0.4249 & 0.653 & 0.635 & 0.616 & 0.376 \\
\hline SB5-21 & 1 & $\mathrm{CCC}$ & Measured & 0.642 & -9.708 & -0.2493 & -0.1962 & -0.1879 & -0.4696 & 0.563 & 0.636 & 0.649 & 0.339 \\
\hline SB5-22 & 2 & $\mathrm{CCC}$ & Measured & 0.662 & -9.932 & -0.2363 & -0.1978 & -0.2073 & -0.4581 & 0.580 & 0.634 & 0.620 & 0.348 \\
\hline SB5-23 & 3 & $\mathrm{CCC}$ & Measured & 0.677 & -10.373 & -0.2569 & -0.1955 & -0.1905 & -0.4696 & 0.553 & 0.638 & 0.645 & 0.339 \\
\hline SB5-24 & 4 & $\mathrm{CCC}$ & Measured & 0.639 & -9.306 & -0.3889 & $\begin{array}{l}-0.3031 \\
\end{array}$ & -0.3382 & -0.5268 & 0.408 & 0.498 & 0.459 & 0.297 \\
\hline SB5-25 & 5 & $\mathrm{CCC}$ & Measured & 0.651 & -9.850 & -0.2787 & -0.2055 & -0.1812 & -0.4430 & 0.526 & 0.623 & 0.659 & 0.361 \\
\hline SB5-26 & 6 & $\mathrm{CCC}$ & Measured & 0.657 & -10.757 & -0.2250 & -0.2094 & -0.1811 & -0.3981 & 0.596 & 0.617 & 0.659 & 0.400 \\
\hline SB5-01 & 0 & quenched & Measured & 0.649 & -9.640 & -0.2846 & -0.2039 & -0.1474 & -0.4689 & 0.519 & 0.625 & 0.712 & 0.340 \\
\hline SB5-02 & 0 & quenched & Measured & 0.653 & -9.389 & -0.2757 & -0.2265 & -0.1608 & -0.4173 & 0.530 & 0.594 & 0.691 & 0.383 \\
\hline SB5-03 & 1 & quenched & Measured & 0.644 & -9.318 & -0.2597 & -0.2048 & -0.1994 & -0.4456 & 0.550 & 0.624 & 0.632 & 0.358 \\
\hline SB5-04 & 4 & quenched & Measured & 0.633 & -9.592 & -0.2461 & -0.2281 & -0.2029 & $\begin{array}{l}-0.4090 \\
\end{array}$ & 0.567 & 0.591 & 0.627 & 0.390 \\
\hline SB5-05 & 0 & quenched & Measured & 0.659 & -10.381 & -0.2205 & -0.1510 & -0.0845 & $\begin{array}{l}-0.4079 \\
\end{array}$ & 0.602 & 0.706 & 0.823 & 0.391 \\
\hline
\end{tabular}


Table 4-4. Normalized PCTs by Glass ID and Compositional View for Initial SB5 Study Glasses. (continued)

\begin{tabular}{|c|c|c|c|c|c|c|c|c|c|c|c|c|c|}
\hline Glass ID & Pair & $\begin{array}{c}\text { Heat } \\
\text { Treatment }\end{array}$ & $\begin{array}{c}\text { Composition } \\
\text { View }\end{array}$ & $\begin{array}{c}\text { Nepheline } \\
\text { Discriminator }\end{array}$ & $\Delta \mathbf{G}_{\mathrm{p}}$ & $\begin{array}{c}\log \mathrm{NL} \\
{[\mathrm{B}(\mathrm{g} / \mathrm{L})]}\end{array}$ & $\begin{array}{c}\log N L \\
{[\mathrm{Li}(\mathrm{g} / \mathrm{L})]}\end{array}$ & $\begin{array}{c}\log \mathrm{NL} \\
{[\mathrm{Na}(\mathrm{g} / \mathrm{L})]}\end{array}$ & $\begin{array}{c}\log N L \\
{[\mathrm{Si}(\mathrm{g} / \mathrm{L})]}\end{array}$ & $\begin{array}{c}\mathrm{NL} \\
\mathrm{B}(\mathrm{g} / \mathrm{L})\end{array}$ & $\begin{array}{c}\mathrm{NL} \\
\mathrm{Li}(\mathrm{g} / \mathrm{L})\end{array}$ & $\begin{array}{c}\mathrm{NL} \\
\mathrm{Na}(\mathrm{g} / \mathrm{L})\end{array}$ & $\begin{array}{c}\mathrm{NL} \\
\mathrm{Si}(\mathrm{g} / \mathrm{L})\end{array}$ \\
\hline SB5-06 & 0 & quenched & Measured & 0.662 & -10.102 & -0.1989 & -0.1602 & -0.1004 & -0.3787 & 0.633 & 0.692 & 0.794 & 0.418 \\
\hline SB5-07 & 5 & quenched & Measured & 0.663 & -9.719 & -0.2042 & -0.1841 & -0.1465 & -0.4092 & 0.625 & 0.654 & 0.714 & 0.390 \\
\hline SB5-08 & 2 & quenched & Measured & 0.657 & -9.677 & -0.2413 & -0.2142 & -0.2035 & -0.4161 & 0.574 & 0.611 & 0.626 & 0.384 \\
\hline $\begin{array}{l}\text { SB5-09 } \\
\end{array}$ & 0 & quenched & Measured & 0.675 & -10.894 & -0.1081 & $\begin{array}{l}-0.0660 \\
\end{array}$ & 0.0129 & -0.3250 & 0.780 & 0.859 & 1.030 & 0.473 \\
\hline SB5-10 & 0 & quenched & Measured & 0.684 & -10.515 & $\begin{array}{l}-0.0945 \\
\end{array}$ & $\begin{array}{l}-0.1143 \\
\end{array}$ & $\begin{array}{l}-0.0389 \\
\end{array}$ & -0.3380 & 0.805 & 0.769 & 0.914 & 0.459 \\
\hline SB5-11 & 3 & quenched & Measured & 0.678 & -10.230 & -0.1711 & -0.1452 & $\begin{array}{l}-0.1017 \\
\end{array}$ & $\begin{array}{l}-0.3775 \\
\end{array}$ & 0.674 & 0.716 & 0.791 & 0.419 \\
\hline SB5-12 & 6 & quenched & Measured & 0.675 & -10.150 & -0.1860 & $\begin{array}{l}-0.1759 \\
\end{array}$ & $\begin{array}{l}-0.1302 \\
\end{array}$ & $\begin{array}{l}-0.3858 \\
\end{array}$ & 0.652 & 0.667 & 0.741 & 0.411 \\
\hline SB5-13 & 0 & quenched & Measured & 0.679 & -11.994 & -0.0507 & $\begin{array}{l}-0.0198 \\
\end{array}$ & 0.0616 & $\begin{array}{l}-0.2793 \\
\end{array}$ & 0.890 & 0.956 & 1.152 & 0.526 \\
\hline SB5-14 & 0 & quenched & Measured & 0.681 & -11.636 & $\begin{array}{l}-0.0677 \\
\end{array}$ & -0.0887 & -0.0089 & -0.2854 & 0.856 & 0.815 & 0.980 & 0.518 \\
\hline SB5-15 & 0 & quenched & Measured & 0.670 & -11.582 & -0.0699 & -0.1028 & -0.0486 & -0.3220 & 0.851 & 0.789 & 0.894 & 0.476 \\
\hline SB5-16 & 0 & quenched & Measured & 0.667 & -11.215 & -0.0910 & -0.1343 & -0.0732 & -0.3500 & 0.811 & 0.734 & 0.845 & 0.447 \\
\hline SB5-17 & 0 & quenched & Measured & 0.662 & -9.296 & -0.1937 & -0.1547 & -0.2055 & -0.3373 & 0.640 & 0.700 & 0.623 & 0.460 \\
\hline SB5-18 & 0 & quenched & Measured & 0.657 & -10.498 & -0.1718 & -0.1757 & -0.1319 & -0.3711 & 0.673 & 0.667 & 0.738 & 0.425 \\
\hline SB5-19 & 0 & quenched & Measured & 0.656 & -11.150 & -0.1289 & -0.1730 & -0.1016 & -0.3561 & 0.743 & 0.671 & 0.791 & 0.440 \\
\hline SB5-20 & 0 & quenched & Measured & 0.685 & -10.212 & -0.1920 & -0.2058 & $\begin{array}{l}-0.1934 \\
\end{array}$ & -0.4392 & 0.643 & 0.623 & 0.641 & 0.364 \\
\hline SB5-21 & 1 & quenched & Measured & 0.642 & -9.708 & $\begin{array}{l}-0.2469 \\
\end{array}$ & $\begin{array}{l}-0.1971 \\
\end{array}$ & $\begin{array}{l}-0.1503 \\
\end{array}$ & -0.4616 & 0.566 & 0.635 & 0.707 & 0.345 \\
\hline SB5-22 & 2 & quenched & Measured & 0.662 & -9.932 & $\begin{array}{l}-0.2447 \\
\end{array}$ & $\begin{array}{c}-0.2080 \\
\end{array}$ & $\begin{array}{l}-0.1900 \\
\end{array}$ & -0.4635 & 0.569 & 0.620 & 0.646 & 0.344 \\
\hline SB5-23 & 3 & quenched & Measured & 0.677 & -10.373 & $\begin{array}{l}-0.2658 \\
\end{array}$ & -0.2165 & $\begin{array}{l}-0.1856 \\
\end{array}$ & $\begin{array}{l}-0.4851 \\
\end{array}$ & 0.542 & 0.607 & 0.652 & 0.327 \\
\hline SB5-24 & 4 & quenched & Measured & 0.639 & $\begin{array}{l}-9.306 \\
\end{array}$ & -0.3720 & $\begin{array}{l}-0.2911 \\
\end{array}$ & -0.2992 & -0.5202 & 0.425 & 0.512 & 0.502 & 0.302 \\
\hline SB5-25 & 5 & quenched & Measured & 0.651 & -9.850 & $\begin{array}{l}-0.2449 \\
\end{array}$ & $\begin{array}{l}-0.1984 \\
\end{array}$ & -0.1508 & -0.4263 & 0.569 & 0.633 & 0.707 & 0.375 \\
\hline SB5-26 & 6 & quenched & Measured & 0.657 & -10.757 & -0.2324 & -0.2346 & -0.1645 & -0.4148 & 0.586 & 0.583 & 0.685 & 0.385 \\
\hline SB5-01 & 0 & $\mathrm{CCC}$ & Measured bc & 0.640 & -9.897 & -0.2847 & -0.2155 & -0.1847 & -0.4687 & 0.519 & 0.609 & 0.654 & 0.340 \\
\hline SB5-02 & 0 & $\mathrm{CCC}$ & Measured bc & 0.644 & -9.631 & -0.2676 & -0.2309 & -0.1890 & -0.4071 & 0.540 & 0.588 & 0.647 & 0.392 \\
\hline SB5-03 & 1 & $\mathrm{CCC}$ & Measured bc & 0.641 & $\begin{array}{l}-9.323 \\
\end{array}$ & $\begin{array}{l}-0.2473 \\
\end{array}$ & $\begin{array}{l}-0.0743 \\
\end{array}$ & -0.1201 & $\begin{array}{l}-0.3453 \\
\end{array}$ & 0.566 & 0.843 & 0.758 & 0.452 \\
\hline SB5-04 & 4 & $\mathrm{CCC}$ & Measured bc & 0.624 & $\begin{array}{l}-9.867 \\
\end{array}$ & -0.2623 & -0.2481 & -0.2535 & $\begin{array}{l}-0.3921 \\
\end{array}$ & 0.547 & 0.565 & 0.558 & 0.405 \\
\hline SB5-05 & 0 & $\mathrm{CCC}$ & Measured bc & 0.650 & -10.661 & -0.2037 & -0.1537 & -0.1101 & -0.3951 & 0.626 & 0.702 & 0.776 & 0.403 \\
\hline SB5-06 & 0 & $\mathrm{CCC}$ & Measured bc & 0.660 & -10.067 & $\begin{array}{l}-0.1806 \\
\end{array}$ & $\begin{array}{l}-0.3047 \\
\end{array}$ & -0.2072 & $\begin{array}{l}-0.4444 \\
\end{array}$ & 0.660 & 0.496 & 0.621 & 0.359 \\
\hline SB5-07 & 5 & $\mathrm{CCC}$ & Measured bc & 0.654 & -9.961 & $\begin{array}{l}-0.2327 \\
\end{array}$ & $\begin{array}{l}-0.2104 \\
\end{array}$ & -0.1842 & -0.4222 & 0.585 & 0.616 & 0.654 & 0.378 \\
\hline $\begin{array}{l}\text { SB5-08 } \\
\end{array}$ & 2 & $\mathrm{CCC}$ & Measured bc & 0.655 & -9.661 & -0.2393 & $\begin{array}{l}-0.2386 \\
\end{array}$ & $\begin{array}{l}-0.2163 \\
\end{array}$ & $\begin{array}{l}-0.4094 \\
\end{array}$ & 0.576 & 0.577 & 0.608 & 0.390 \\
\hline $\begin{array}{l}\text { SB5-09 } \\
\text { SB }\end{array}$ & 0 & $\mathrm{CCC}$ & Measured bc & 0.672 & -10.941 & -0.1309 & -0.0965 & $\begin{array}{l}-0.0279 \\
\end{array}$ & $\begin{array}{l}-0.3291 \\
\end{array}$ & 0.740 & 0.801 & 0.938 & 0.469 \\
\hline SB5-10 & 0 & $\mathrm{CCC}$ & Measured bc & 0.682 & -10.486 & $\begin{array}{l}-0.1277 \\
\end{array}$ & -0.1280 & $\begin{array}{l}-0.0723 \\
\end{array}$ & -0.3273 & 0.745 & 0.745 & 0.847 & 0.471 \\
\hline SB5-11 & 3 & $\mathrm{CCC}$ & Measured bc & 0.669 & -10.565 & $\begin{array}{l}-0.1460 \\
\end{array}$ & -0.1490 & -0.1169 & -0.3494 & 0.715 & 0.710 & 0.764 & 0.447 \\
\hline SB5-12 & 6 & $\mathrm{CCC}$ & Measured bc & 0.666 & -10.460 & $\begin{array}{l}-0.1992 \\
\end{array}$ & -0.1999 & -0.1436 & -0.3689 & 0.632 & 0.631 & 0.718 & 0.428 \\
\hline SB5-13 & 0 & $\mathrm{CCC}$ & Measured bc & 0.674 & -11.974 & -0.0582 & -0.0393 & 0.0422 & -0.2755 & 0.875 & 0.914 & 1.102 & 0.530 \\
\hline SB5-14 & 0 & $\mathrm{CCC}$ & Measured bc & 0.676 & -11.688 & -0.0928 & -0.0876 & -0.0147 & -0.2833 & 0.808 & 0.817 & 0.967 & 0.521 \\
\hline SB5-15 & 0 & $\mathrm{CCC}$ & Measured bc & 0.666 & -11.732 & -0.1286 & -0.1342 & -0.0896 & -0.3551 & 0.744 & 0.734 & 0.814 & 0.441 \\
\hline SB5-16 & 0 & $\mathrm{CCC}$ & Measured bc & 0.663 & -11.340 & -0.1380 & -0.1716 & -0.1229 & -0.3642 & 0.728 & 0.674 & 0.754 & 0.432 \\
\hline SB5-17 & 0 & $\mathrm{CCC}$ & Measured bc & 0.657 & -9.337 & $\begin{array}{l}-0.1751 \\
\end{array}$ & -0.1693 & -0.2238 & -0.3193 & 0.668 & 0.677 & 0.597 & 0.479 \\
\hline
\end{tabular}


Table 4-4. Normalized PCTs by Glass ID and Compositional View for Initial SB5 Study Glasses. (continued)

\begin{tabular}{|c|c|c|c|c|c|c|c|c|c|c|c|c|c|}
\hline Glass ID & Pair & $\begin{array}{c}\text { Heat } \\
\text { Treatment }\end{array}$ & $\begin{array}{c}\text { Composition } \\
\text { View }\end{array}$ & $\begin{array}{c}\text { Nepheline } \\
\text { Discriminator }\end{array}$ & $\Delta \mathbf{G}_{\mathrm{p}}$ & $\begin{array}{c}\log \mathrm{NL} \\
{[\mathrm{B}(\mathrm{g} / \mathrm{L})]}\end{array}$ & $\begin{array}{c}\log N L \\
{[\mathrm{Li}(\mathrm{g} / \mathrm{L})]}\end{array}$ & $\begin{array}{c}\log \mathrm{NL} \\
{[\mathrm{Na}(\mathrm{g} / \mathrm{L})]}\end{array}$ & $\begin{array}{c}\log \mathrm{NL} \\
{[\mathrm{Si}(\mathrm{g} / \mathrm{L})]}\end{array}$ & $\begin{array}{c}\mathrm{NL} \\
\mathrm{B}(\mathrm{g} / \mathrm{L})\end{array}$ & $\begin{array}{c}\mathrm{NL} \\
\mathrm{Li}(\mathrm{g} / \mathrm{L})\end{array}$ & $\begin{array}{c}\mathrm{NL} \\
\mathrm{Na}(\mathrm{g} / \mathrm{L})\end{array}$ & $\begin{array}{c}\mathrm{NL} \\
\mathrm{Si}(\mathrm{g} / \mathrm{L})\end{array}$ \\
\hline SB5-18 & 0 & $\mathrm{CCC}$ & Measured bc & 0.651 & -10.448 & -0.1471 & -0.1661 & -0.1254 & -0.3384 & 0.713 & 0.682 & 0.749 & 0.459 \\
\hline SB5-19 & 0 & $\mathrm{CCC}$ & Measured bc & 0.652 & -11.183 & $\begin{array}{l}-0.1509 \\
\end{array}$ & -0.2025 & -0.1413 & -0.3666 & 0.706 & 0.627 & 0.722 & 0.430 \\
\hline SB5-20 & 0 & $\mathrm{CCC}$ & Measured bc & 0.679 & -10.209 & $\begin{array}{l}-0.1933 \\
\end{array}$ & -0.2120 & -0.2022 & $\begin{array}{l}-0.4091 \\
\end{array}$ & 0.641 & 0.614 & 0.628 & 0.390 \\
\hline SB5-21 & 1 & $\mathrm{CCC}$ & Measured bc & 0.644 & -9.370 & -0.2416 & $\begin{array}{l}-0.1974 \\
\end{array}$ & -0.1780 & $\begin{array}{l}-0.4706 \\
\end{array}$ & 0.573 & 0.635 & 0.664 & 0.338 \\
\hline SB5-22 & 2 & $\mathrm{CCC}$ & Measured bc & 0.664 & -9.602 & -0.2286 & $\begin{array}{l}-0.1990 \\
\end{array}$ & $\begin{array}{l}-0.1973 \\
\end{array}$ & $\begin{array}{l}-0.4591 \\
\end{array}$ & 0.591 & 0.632 & 0.635 & 0.347 \\
\hline SB5-23 & 3 & $\mathrm{CCC}$ & Measured bc & 0.679 & -10.025 & $\begin{array}{l}-0.2492 \\
\end{array}$ & $\begin{array}{l}-0.1967 \\
\end{array}$ & $\begin{array}{l}-0.1805 \\
\end{array}$ & $\begin{array}{l}-0.4706 \\
\end{array}$ & 0.563 & 0.636 & 0.660 & 0.338 \\
\hline SB5-24 & 4 & $\mathrm{CCC}$ & Measured bc & 0.641 & -8.922 & $\begin{array}{l}-0.3813 \\
\end{array}$ & $\begin{array}{l}-0.3043 \\
\end{array}$ & $\begin{array}{l}-0.3282 \\
\end{array}$ & $\begin{array}{l}-0.5278 \\
\end{array}$ & 0.416 & 0.496 & 0.470 & 0.297 \\
\hline SB5-25 & 5 & $\mathrm{CCC}$ & Measured bc & 0.652 & $\begin{array}{l}-9.478 \\
\end{array}$ & -0.2710 & -0.2067 & -0.1712 & -0.4440 & 0.536 & 0.621 & 0.674 & 0.360 \\
\hline SB5-26 & 6 & $\mathrm{CCC}$ & Measured bc & 0.659 & $\begin{array}{l}-10.373 \\
\end{array}$ & -0.2173 & -0.2106 & -0.1710 & -0.3991 & 0.606 & 0.616 & 0.675 & 0.399 \\
\hline SB5-01 & 0 & quenched & Measured bc & 0.640 & -9.897 & -0.2793 & -0.2191 & -0.1560 & -0.4638 & 0.526 & 0.604 & 0.698 & 0.344 \\
\hline SB5-02 & 0 & quenched & Measured bc & 0.644 & -9.631 & -0.2703 & -0.2417 & -0.1694 & -0.4122 & 0.537 & 0.573 & 0.677 & 0.387 \\
\hline SB5-03 & 1 & quenched & Measured bc & 0.641 & -9.323 & -0.2619 & -0.2273 & -0.1915 & -0.4366 & 0.547 & 0.593 & 0.643 & 0.366 \\
\hline SB5-04 & 4 & quenched & Measured bc & 0.624 & -9.867 & -0.2484 & -0.2506 & -0.2115 & -0.4040 & 0.564 & 0.562 & 0.615 & 0.394 \\
\hline SB5-05 & 0 & quenched & Measured bc & 0.650 & $\begin{array}{l}-10.661 \\
\end{array}$ & -0.2152 & -0.1662 & $\begin{array}{l}-0.0931 \\
\end{array}$ & -0.4028 & 0.609 & 0.682 & 0.807 & 0.396 \\
\hline SB5-06 & 0 & quenched & Measured bc & 0.660 & -10.067 & $\begin{array}{l}-0.1936 \\
\end{array}$ & -0.1753 & $\begin{array}{l}-0.0926 \\
\end{array}$ & -0.3697 & 0.640 & 0.668 & 0.808 & 0.427 \\
\hline SB5-07 & 5 & quenched & Measured bc & 0.654 & -9.961 & $\begin{array}{l}-0.1989 \\
\end{array}$ & $\begin{array}{l}-0.1993 \\
\end{array}$ & -0.1551 & -0.4042 & 0.633 & 0.632 & 0.700 & 0.394 \\
\hline $\begin{array}{l}\text { SB5-08 } \\
\text { SB }\end{array}$ & 2 & quenched & Measured bc & 0.655 & -9.661 & -0.2435 & $\begin{array}{c}-0.2367 \\
\end{array}$ & $\begin{array}{l}-0.1956 \\
\end{array}$ & $\begin{array}{l}-0.4070 \\
\end{array}$ & 0.571 & 0.580 & 0.637 & 0.392 \\
\hline SB5-09 & 0 & quenched & Measured bc & 0.672 & -10.941 & -0.1103 & $\begin{array}{l}-0.0885 \\
\end{array}$ & 0.0208 & $\begin{array}{l}-0.3160 \\
\end{array}$ & 0.776 & 0.816 & 1.049 & 0.483 \\
\hline SB5-10 & 0 & quenched & Measured bc & 0.682 & -10.486 & -0.0892 & -0.1295 & -0.0311 & -0.3290 & 0.814 & 0.742 & 0.931 & 0.469 \\
\hline SB5-11 & 3 & quenched & Measured bc & 0.669 & -10.565 & $\begin{array}{l}-0.1732 \\
\end{array}$ & -0.1677 & -0.1103 & -0.3725 & 0.671 & 0.680 & 0.776 & 0.424 \\
\hline SB5-12 & 6 & quenched & Measured bc & 0.666 & $\begin{array}{l}-10.460 \\
\end{array}$ & $\begin{array}{l}-0.1883 \\
\end{array}$ & -0.1984 & -0.1388 & -0.3807 & 0.648 & 0.633 & 0.727 & 0.416 \\
\hline SB5-13 & 0 & quenched & Measured bc & 0.674 & -11.974 & -0.0389 & -0.0306 & 0.0696 & $\begin{array}{l}-0.2634 \\
\end{array}$ & 0.914 & 0.932 & 1.174 & 0.545 \\
\hline SB5-14 & 0 & quenched & Measured bc & 0.676 & -11.688 & -0.0759 & -0.1031 & -0.0010 & -0.2695 & 0.840 & 0.789 & 0.998 & 0.538 \\
\hline SB5-15 & 0 & quenched & Measured bc & 0.666 & -11.732 & -0.0782 & -0.1173 & -0.0507 & -0.3192 & 0.835 & 0.763 & 0.890 & 0.480 \\
\hline SB5-16 & 0 & quenched & Measured bc & 0.663 & -11.340 & -0.0992 & $\begin{array}{l}-0.1487 \\
\end{array}$ & $\begin{array}{l}-0.0753 \\
\end{array}$ & $\begin{array}{l}-0.3471 \\
\end{array}$ & 0.796 & 0.710 & 0.841 & 0.450 \\
\hline SB5-17 & 0 & quenched & Measured bc & 0.657 & -9.337 & -0.1820 & -0.1655 & -0.2076 & -0.3346 & 0.658 & 0.683 & 0.620 & 0.463 \\
\hline SB5-18 & 0 & quenched & Measured bc & 0.651 & -10.448 & -0.1604 & -0.1865 & $\begin{array}{l}-0.1239 \\
\end{array}$ & -0.3552 & 0.691 & 0.651 & 0.752 & 0.441 \\
\hline SB5-19 & 0 & quenched & Measured bc & 0.652 & -11.183 & $\begin{array}{l}-0.1173 \\
\end{array}$ & $\begin{array}{l}-0.1838 \\
\end{array}$ & $\begin{array}{l}-0.1036 \\
\end{array}$ & -0.3533 & 0.763 & 0.655 & 0.788 & 0.443 \\
\hline SB5-20 & 0 & quenched & Measured bc & 0.679 & -10.209 & -0.2002 & -0.2202 & $\begin{array}{l}-0.1855 \\
\end{array}$ & $\begin{array}{l}-0.4234 \\
\end{array}$ & 0.631 & 0.602 & 0.652 & 0.377 \\
\hline SB5-21 & 1 & quenched & Measured bc & 0.644 & -9.370 & -0.2392 & -0.1982 & -0.1404 & -0.4626 & 0.576 & 0.634 & 0.724 & 0.345 \\
\hline SB5-22 & 2 & quenched & Measured bc & 0.664 & $\begin{array}{l}-9.602 \\
\end{array}$ & -0.2370 & -0.2091 & -0.1800 & -0.4645 & 0.579 & 0.618 & 0.661 & 0.343 \\
\hline SB5-23 & 3 & quenched & Measured bc & 0.679 & -10.025 & $\begin{array}{l}-0.2581 \\
\end{array}$ & -0.2177 & -0.1756 & $\begin{array}{l}-0.4861 \\
\end{array}$ & 0.552 & 0.606 & 0.667 & 0.327 \\
\hline SB5-24 & 4 & quenched & Measured bc & 0.641 & -8.922 & $\begin{array}{l}-0.3643 \\
\end{array}$ & -0.2922 & -0.2893 & $\begin{array}{l}-0.5212 \\
\end{array}$ & 0.432 & 0.510 & 0.514 & 0.301 \\
\hline SB5-25 & 5 & quenched & Measured bc & 0.652 & -9.478 & -0.2373 & -0.1996 & -0.1408 & -0.4273 & 0.579 & 0.632 & 0.723 & 0.374 \\
\hline SB5-26 & 6 & quenched & Measured bc & 0.659 & -10.373 & -0.2248 & -0.2358 & -0.1545 & -0.4158 & 0.596 & 0.581 & 0.701 & 0.384 \\
\hline SB5-01 & 0 & $\mathrm{CCC}$ & Targeted & 0.647 & -9.877 & -0.2896 & -0.2120 & -0.1832 & -0.4723 & 0.513 & 0.614 & 0.656 & 0.337 \\
\hline SB5-02 & 0 & $\mathrm{CCC}$ & Targeted & 0.650 & -9.623 & $\begin{array}{l}-0.2609 \\
\end{array}$ & -0.2138 & $\begin{array}{l}-0.1969 \\
\end{array}$ & $\begin{array}{l}-0.4191 \\
\end{array}$ & 0.548 & 0.611 & 0.635 & 0.381 \\
\hline SB5-03 & 1 & $\mathrm{CCC}$ & Targeted & 0.643 & -9.487 & $\begin{array}{l}-0.2538 \\
\end{array}$ & -0.0706 & -0.1275 & $\begin{array}{l}-0.3504 \\
\end{array}$ & 0.557 & 0.850 & 0.746 & 0.446 \\
\hline
\end{tabular}


Table 4-4. Normalized PCTs by Glass ID and Compositional View for Initial SB5 Study Glasses. (continued)

\begin{tabular}{|c|c|c|c|c|c|c|c|c|c|c|c|c|c|}
\hline Glass ID & Pair & $\begin{array}{c}\text { Heat } \\
\text { Treatment }\end{array}$ & $\begin{array}{c}\text { Composition } \\
\text { View }\end{array}$ & $\begin{array}{c}\text { Nepheline } \\
\text { Discriminator }\end{array}$ & $\Delta \mathbf{G}_{\mathrm{p}}$ & $\begin{array}{c}\log \mathrm{NL} \\
{[\mathrm{B}(\mathrm{g} / \mathrm{L})]}\end{array}$ & $\begin{array}{c}\log N L \\
{[\mathrm{Li}(\mathrm{g} / \mathrm{L})]}\end{array}$ & $\begin{array}{c}\log \mathrm{NL} \\
{[\mathrm{Na}(\mathrm{g} / \mathrm{L})]}\end{array}$ & $\begin{array}{c}\log N L \\
{[\mathrm{Si}(\mathrm{g} / \mathrm{L})]}\end{array}$ & $\begin{array}{c}\mathrm{NL} \\
\mathrm{B}(\mathrm{g} / \mathrm{L})\end{array}$ & $\begin{array}{c}\mathrm{NL} \\
\mathrm{Li}(\mathrm{g} / \mathrm{L})\end{array}$ & $\begin{array}{c}\mathrm{NL} \\
\mathrm{Na}(\mathrm{g} / \mathrm{L})\end{array}$ & $\begin{array}{c}\mathrm{NL} \\
\mathrm{Si}(\mathrm{g} / \mathrm{L})\end{array}$ \\
\hline SB5-04 & 4 & $\mathrm{CCC}$ & Targeted & 0.638 & -9.400 & -0.2435 & -0.2233 & -0.2421 & -0.4037 & 0.571 & 0.598 & 0.573 & 0.395 \\
\hline SB5-05 & 0 & $\mathrm{CCC}$ & Targeted & 0.664 & -10.255 & -0.1887 & -0.1382 & -0.1014 & -0.4076 & 0.648 & 0.727 & 0.792 & 0.391 \\
\hline SB5-06 & 0 & $\mathrm{CCC}$ & Targeted & 0.667 & -10.001 & $\begin{array}{l}-0.1694 \\
\end{array}$ & -0.2935 & -0.2090 & $\begin{array}{l}-0.4539 \\
\end{array}$ & 0.677 & 0.509 & 0.618 & 0.352 \\
\hline SB5-07 & 5 & $\mathrm{CCC}$ & Targeted & 0.661 & -9.864 & -0.2401 & $\begin{array}{l}-0.1985 \\
\end{array}$ & $\begin{array}{l}-0.1819 \\
\end{array}$ & $\begin{array}{l}-0.4277 \\
\end{array}$ & 0.575 & 0.633 & 0.658 & 0.374 \\
\hline SB5-08 & 2 & $\mathrm{CCC}$ & Targeted & 0.655 & -9.778 & -0.2271 & $\begin{array}{l}-0.2184 \\
\end{array}$ & -0.2277 & $\begin{array}{l}-0.4148 \\
\end{array}$ & 0.593 & 0.605 & 0.592 & 0.385 \\
\hline $\begin{array}{l}\text { SB5-09 } \\
\text { SB }\end{array}$ & 0 & $\mathrm{CCC}$ & Targeted & 0.678 & -10.924 & $\begin{array}{l}-0.1127 \\
\end{array}$ & $\begin{array}{l}-0.0796 \\
\end{array}$ & $\begin{array}{l}-0.0378 \\
\end{array}$ & -0.3435 & 0.772 & 0.833 & 0.917 & 0.453 \\
\hline SB5-10 & 0 & $\mathrm{CCC}$ & Targeted & 0.681 & -10.670 & -0.1290 & $\begin{array}{l}-0.1135 \\
\end{array}$ & $\begin{array}{l}-0.0838 \\
\end{array}$ & -0.3284 & 0.743 & 0.770 & 0.825 & 0.469 \\
\hline SB5-11 & 3 & $\mathrm{CCC}$ & Targeted & 0.675 & -10.533 & -0.1448 & -0.1354 & -0.1235 & $\begin{array}{l}-0.3611 \\
\end{array}$ & 0.717 & 0.732 & 0.753 & 0.435 \\
\hline SB5-12 & 6 & $\mathrm{CCC}$ & Targeted & 0.669 & -10.446 & $\begin{array}{l}-0.1915 \\
\end{array}$ & -0.1759 & $\begin{array}{l}-0.1495 \\
\end{array}$ & -0.3734 & 0.643 & 0.667 & 0.709 & 0.423 \\
\hline SB5-13 & 0 & $\mathrm{CCC}$ & Targeted & 0.674 & -12.076 & $\begin{array}{l}-0.0486 \\
\end{array}$ & -0.0271 & 0.0322 & -0.2791 & 0.894 & 0.940 & 1.077 & 0.526 \\
\hline SB5-14 & 0 & $\mathrm{CCC}$ & Targeted & 0.677 & -11.822 & -0.0842 & -0.0712 & -0.0287 & -0.2939 & 0.824 & 0.849 & 0.936 & 0.508 \\
\hline SB5-15 & 0 & $\mathrm{CCC}$ & Targeted & 0.671 & -11.685 & -0.1352 & -0.1307 & -0.0896 & -0.3622 & 0.733 & 0.740 & 0.814 & 0.434 \\
\hline SB5-16 & 0 & $\mathrm{CCC}$ & Targeted & 0.665 & -11.598 & -0.1435 & -0.1648 & -0.1316 & -0.3709 & 0.719 & 0.684 & 0.739 & 0.426 \\
\hline SB5-17 & 0 & $\mathrm{CCC}$ & Targeted & 0.664 & -9.136 & -0.1765 & -0.1626 & -0.2173 & -0.3251 & 0.666 & 0.688 & 0.606 & 0.473 \\
\hline SB5-18 & 0 & $\mathrm{CCC}$ & Targeted & 0.655 & -10.590 & -0.1547 & -0.1582 & -0.1292 & -0.3410 & 0.700 & 0.695 & 0.743 & 0.456 \\
\hline SB5-19 & 0 & $\mathrm{CCC}$ & Targeted & 0.654 & -11.457 & $\begin{array}{l}-0.1649 \\
\end{array}$ & -0.1962 & $\begin{array}{l}-0.1489 \\
\end{array}$ & $\begin{array}{l}-0.3707 \\
\end{array}$ & 0.684 & 0.636 & 0.710 & 0.426 \\
\hline SB5-20 & 0 & $\mathrm{CCC}$ & Targeted & 0.677 & -10.485 & $\begin{array}{l}-0.1955 \\
\end{array}$ & $\begin{array}{l}-0.1936 \\
\end{array}$ & -0.2139 & $\begin{array}{l}-0.4087 \\
\end{array}$ & 0.638 & 0.640 & 0.611 & 0.390 \\
\hline SB5-21 & 1 & $\mathrm{CCC}$ & Targeted & 0.643 & -9.724 & -0.2343 & $\begin{array}{l}-0.1963 \\
\end{array}$ & $\begin{array}{l}-0.1877 \\
\end{array}$ & $\begin{array}{l}-0.4657 \\
\end{array}$ & 0.583 & 0.636 & 0.649 & 0.342 \\
\hline SB5-22 & 2 & $\mathrm{CCC}$ & Targeted & 0.655 & -10.039 & -0.2322 & -0.2016 & $\begin{array}{l}-0.2094 \\
\end{array}$ & $\begin{array}{l}-0.4443 \\
\end{array}$ & 0.586 & 0.629 & 0.618 & 0.360 \\
\hline SB5-23 & 3 & $\mathrm{CCC}$ & Targeted & 0.675 & -10.439 & -0.2526 & -0.1956 & $\begin{array}{l}-0.1949 \\
\end{array}$ & -0.4670 & 0.559 & 0.637 & 0.638 & 0.341 \\
\hline SB5-24 & 4 & $\mathrm{CCC}$ & Targeted & 0.638 & -9.320 & $\begin{array}{l}-0.3793 \\
\end{array}$ & -0.3023 & $\begin{array}{l}-0.3374 \\
\end{array}$ & -0.5178 & 0.418 & 0.498 & 0.460 & 0.304 \\
\hline SB5-25 & 5 & $\mathrm{CCC}$ & Targeted & 0.645 & -10.172 & $\begin{array}{l}-0.2688 \\
\end{array}$ & -0.2165 & -0.1925 & -0.4385 & 0.538 & 0.608 & 0.642 & 0.364 \\
\hline SB5-26 & 6 & $\mathrm{CCC}$ & Targeted & 0.653 & -10.843 & -0.2119 & -0.2049 & -0.1880 & -0.3932 & 0.614 & 0.624 & 0.649 & 0.404 \\
\hline SB5-01 & 0 & quenched & Targeted & 0.647 & -9.877 & -0.2842 & -0.2156 & -0.1545 & -0.4674 & 0.520 & 0.609 & 0.701 & 0.341 \\
\hline SB5-02 & 0 & quenched & Targeted & 0.650 & $\begin{array}{l}-9.623 \\
\end{array}$ & -0.2637 & -0.2245 & -0.1772 & $\begin{array}{l}-0.4243 \\
\end{array}$ & 0.545 & 0.596 & 0.665 & 0.376 \\
\hline $\begin{array}{l}\text { SB5-03 } \\
\end{array}$ & 1 & quenched & Targeted & 0.643 & $\begin{array}{l}-9.487 \\
\end{array}$ & -0.2684 & -0.2235 & $\begin{array}{l}-0.1989 \\
\end{array}$ & -0.4417 & 0.539 & 0.598 & 0.633 & 0.362 \\
\hline SB5-04 & 4 & quenched & Targeted & 0.638 & -9.400 & $\begin{array}{l}-0.2296 \\
\end{array}$ & $\begin{array}{l}-0.2258 \\
\end{array}$ & $\begin{array}{l}-0.2000 \\
\end{array}$ & -0.4156 & 0.589 & 0.595 & 0.631 & 0.384 \\
\hline SB5-05 & 0 & quenched & Targeted & 0.664 & -10.255 & $\begin{array}{l}-0.2002 \\
\end{array}$ & -0.1507 & $\begin{array}{l}-0.0844 \\
\end{array}$ & -0.4154 & 0.631 & 0.707 & 0.823 & 0.384 \\
\hline SB5-06 & 0 & quenched & Targeted & 0.667 & -10.001 & -0.1824 & -0.1642 & $\begin{array}{l}-0.0945 \\
\end{array}$ & $\begin{array}{l}-0.3792 \\
\end{array}$ & 0.657 & 0.685 & 0.805 & 0.418 \\
\hline SB5-07 & 5 & quenched & Targeted & 0.661 & -9.864 & -0.2063 & $\begin{array}{l}-0.1874 \\
\end{array}$ & -0.1529 & $\begin{array}{l}-0.4097 \\
\end{array}$ & 0.622 & 0.650 & 0.703 & 0.389 \\
\hline SB5-08 & 2 & quenched & Targeted & 0.655 & $\begin{array}{l}-9.778 \\
\end{array}$ & -0.2313 & -0.2164 & -0.2071 & -0.4124 & 0.587 & 0.608 & 0.621 & 0.387 \\
\hline $\begin{array}{l}\text { SB5-09 } \\
\end{array}$ & 0 & quenched & Targeted & 0.678 & -10.924 & $\begin{array}{l}-0.0921 \\
\end{array}$ & -0.0716 & 0.0109 & -0.3304 & 0.809 & 0.848 & 1.025 & 0.467 \\
\hline SB5-10 & 0 & quenched & Targeted & 0.681 & -10.670 & -0.0905 & -0.1150 & -0.0425 & -0.3301 & 0.812 & 0.767 & 0.907 & 0.468 \\
\hline SB5-11 & 3 & quenched & Targeted & 0.675 & -10.533 & -0.1720 & -0.1542 & -0.1168 & -0.3843 & 0.673 & 0.701 & 0.764 & 0.413 \\
\hline SB5-12 & 6 & quenched & Targeted & 0.669 & -10.446 & -0.1805 & -0.1743 & -0.1446 & -0.3852 & 0.660 & 0.669 & 0.717 & 0.412 \\
\hline SB5-13 & 0 & quenched & Targeted & 0.674 & -12.076 & -0.0293 & -0.0183 & 0.0596 & -0.2671 & 0.935 & 0.959 & 1.147 & 0.541 \\
\hline SB5-14 & 0 & quenched & Targeted & 0.677 & -11.822 & -0.0673 & -0.0867 & -0.0150 & $\begin{array}{l}-0.2801 \\
\end{array}$ & 0.856 & 0.819 & 0.966 & 0.525 \\
\hline SB5-15 & 0 & quenched & Targeted & 0.671 & -11.685 & $\begin{array}{l}-0.0848 \\
\end{array}$ & -0.1137 & -0.0508 & -0.3263 & 0.823 & 0.770 & 0.890 & 0.472 \\
\hline
\end{tabular}


Table 4-4. Normalized PCTs by Glass ID and Compositional View for Initial SB5 Study Glasses. (continued)

\begin{tabular}{|c|c|c|c|c|c|c|c|c|c|c|c|c|c|}
\hline Glass ID & Pair & $\begin{array}{c}\text { Heat } \\
\text { Treatment }\end{array}$ & $\begin{array}{c}\text { Composition } \\
\text { View }\end{array}$ & $\begin{array}{c}\text { Nepheline } \\
\text { Discriminator }\end{array}$ & $\Delta \mathbf{G}_{\mathbf{p}}$ & $\begin{array}{c}\log \mathrm{NL} \\
{[\mathrm{B}(\mathrm{g} / \mathrm{L})]}\end{array}$ & $\begin{array}{c}\log \mathrm{NL} \\
{[\mathrm{Li}(\mathrm{g} / \mathrm{L})]}\end{array}$ & $\begin{array}{c}\log \mathrm{NL} \\
{[\mathrm{Na}(\mathrm{g} / \mathrm{L})]}\end{array}$ & $\begin{array}{c}\log \mathrm{NL} \\
{[\mathrm{Si}(\mathrm{g} / \mathrm{L})]}\end{array}$ & $\begin{array}{c}\mathrm{NL} \\
\mathbf{B}(\mathbf{g} / \mathbf{L}) \\
\end{array}$ & $\begin{array}{c}\mathrm{NL} \\
\mathrm{Li}(\mathrm{g} / \mathrm{L}) \\
\end{array}$ & $\begin{array}{c}\mathrm{NL} \\
\mathrm{Na}(\mathrm{g} / \mathrm{L})\end{array}$ & $\begin{array}{c}\mathrm{NL} \\
\mathrm{Si}(\mathrm{g} / \mathrm{L}) \\
\end{array}$ \\
\hline SB5-16 & 0 & quenched & Targeted & 0.665 & -11.598 & -0.1047 & -0.1419 & -0.0840 & -0.3537 & 0.786 & 0.721 & 0.824 & 0.443 \\
\hline SB5-17 & 0 & quenched & Targeted & 0.664 & -9.136 & $\begin{array}{l}-0.1834 \\
\end{array}$ & $\begin{array}{l}-0.1588 \\
\end{array}$ & -0.2011 & -0.3405 & 0.656 & 0.694 & 0.629 & 0.457 \\
\hline SB5-18 & 0 & quenched & Targeted & 0.655 & $\begin{array}{l}-10.590 \\
\end{array}$ & $\begin{array}{l}-0.1679 \\
\end{array}$ & -0.1785 & -0.1277 & $\begin{array}{l}-0.3578 \\
\end{array}$ & 0.679 & 0.663 & 0.745 & 0.439 \\
\hline SB5-19 & 0 & quenched & Targeted & 0.654 & -11.457 & $\begin{array}{l}-0.1313 \\
\end{array}$ & -0.1775 & -0.1113 & -0.3574 & 0.739 & 0.664 & 0.774 & 0.439 \\
\hline SB5-20 & 0 & quenched & Targeted & 0.677 & -10.485 & $\begin{array}{l}-0.2024 \\
\end{array}$ & $\begin{array}{l}-0.2018 \\
\end{array}$ & $\begin{array}{l}-0.1971 \\
\end{array}$ & -0.4230 & 0.627 & 0.628 & 0.635 & 0.378 \\
\hline SB5-21 & 1 & quenched & Targeted & 0.643 & $\begin{array}{l}-9.724 \\
\end{array}$ & -0.2320 & $\begin{array}{l}-0.1972 \\
\end{array}$ & $\begin{array}{l}-0.1501 \\
\end{array}$ & $\begin{array}{l}-0.4577 \\
\end{array}$ & 0.586 & 0.635 & 0.708 & 0.349 \\
\hline SB5-22 & 2 & quenched & Targeted & 0.655 & -10.039 & -0.2406 & -0.2117 & -0.1921 & -0.4496 & 0.575 & 0.614 & 0.643 & 0.355 \\
\hline SB5-23 & 3 & quenched & Targeted & 0.675 & -10.439 & -0.2615 & -0.2166 & -0.1900 & -0.4825 & 0.548 & 0.607 & 0.646 & 0.329 \\
\hline SB5-24 & 4 & quenched & Targeted & 0.638 & -9.320 & -0.3623 & -0.2903 & -0.2984 & -0.5112 & 0.434 & 0.513 & 0.503 & 0.308 \\
\hline SB5-25 & 5 & quenched & Targeted & 0.645 & -10.172 & -0.2351 & -0.2094 & -0.1621 & -0.4218 & 0.582 & 0.617 & 0.689 & 0.379 \\
\hline SB5-26 & 6 & quenched & Targeted & 0.653 & -10.843 & -0.2194 & -0.2301 & -0.1714 & -0.4099 & 0.603 & 0.589 & 0.674 & 0.389 \\
\hline
\end{tabular}




\subsubsection{Effects of Heat Treatment on PCTs}

Exhibit B6 in Appendix B provides a series of plots and statistical comparisons that show the effects of heat treatment on the common logarithm ppm responses of interest of the triplicate PCTs for each element for each study glass. The quenched version of a given glass yielded measurements indicating a significantly (at the 5\% significance level) different mean $\log (\mathrm{ppm})$ response as compared to the CCC version of the glass for a given element if the Prob $>|\mathbf{t}|$ value in the exhibit is 0.05 or smaller. There was a statistically significant difference in the mean response for $\mathrm{Na}$ (the quenched version was higher) for SB5-01, SB5-04, SB5-05, SB5-06, SB5-13, SB5-15, SB5-19, SB5-21, SB5-25, and SB5-26. There was a statistically significant difference in the mean response for $\mathrm{Na}$ (the $\mathrm{CCC}$ version was higher) for SB5-03. There was a statistically significant difference in the mean response for $\mathrm{Si}$ - the CCC version was higher for SB5-03 and the quenched version was higher for SB5-06. There were statistically significant differences in the mean response for $\mathrm{Li}$ - the CCC versions of SB5-03, SB5-11 and SB5-26 were higher than their CCC counterparts and the quenched version was higher for SB5-06. Exhibit B7 in Appendix B provides a series of plots that show the effects of heat treatment on the normalized PCT response based on the three different compositional views: measured, measured biascorrected, and targeted.

While some statistically significant differences in PCT responses relative to heat treatment were identified, from a practical perspective, these differences are insiginificant since the normalized release values remain well below those of the EA glass regardless of thermal history.

\subsubsection{Initial Comparisons of Surrogate Pairs}

Exhibit B8 in Appendix B provides a series of plots to facilitate comparisons between pairs of study glasses, one with $\mathrm{U}_{3} \mathrm{O}_{8}$ and one either without $\mathrm{U}_{3} \mathrm{O}_{8}$ or with a surrogate for $\mathrm{U}_{3} \mathrm{O}_{8}$. The plots show the leachate concentrations in ppm and in common logarithms of the ppm values for each of the triplicate PCTs for the quenched and CCC versions of each of the glasses. The mean values of each set of three measurements for each pair are connected in these plots. This exhibit provides an opportunity to gauge the differences in the mean responses for the pairs of glasses relative to the repeatability of each set of triplicate PCTs.

Additional plots of the normalized PCT responses also are provided in this exhibit. These plots provide an opportunity to compare the PCT responses over the three compositional views for the glasses. In general, the PCT responses for the surrogate glasses or the glasses without $\mathrm{U}_{3} \mathrm{O}_{8}$ were quite similar to their $\mathrm{U}_{3} \mathrm{O}_{8}$-containing counterparts. The average percent error in NL [B] normalized by the measured bc compositions for the surrogate glasses compared with their radioactive counterparts is $8.8 \%$. The largest difference in NL [B] is $0.152 \mathrm{~g} / \mathrm{L}$ (see Table 4-4). While this is a promising result for both the surrogates and the glasses renormalized without $\mathrm{U}_{3} \mathrm{O}_{8}$, additional data must be collected before the best solution to fabricating test glasses without radioactive components can be identified.

\subsubsection{Predicted versus Measured Durability}

As seen in Table 4-4, the durabilities of the study glasses are all very acceptable when compared to the durability of the EA benchmark glass. The ability of the DWPF models to correctly predict the durability of glasses SB5-01 through SB5-20 is investigated in this section. Exhibit B9 in Appendix B provides plots of the DWPF models that relate the logarithm of the normalized PCT (for each element of interest) to a linear function of a free energy of hydration term $\left(\Delta \mathrm{G}_{\mathrm{p}}\right.$, $\mathrm{kcal} / 100 \mathrm{~g}$ glass) derived from all of the glass compositional views and heat treatments for these glasses. ${ }^{15}$ Prediction limits (at a 95\% confidence) for an individual PCT result are also plotted 
along with the linear fit. The EA and ARM results are indicated on these plots as well.

Exhibit B10 in Appendix B provides a version of these plots for the quenched glasses only while Exhibit B11 in Appendix B provides a version for CCC glasses only. All of these plots show good predictability by the DWPF durability models of PCT responses for the SB5-01 through SB5-20 glasses.

Figure 4-2 provides a closer look at the PCT response for boron, which is used as an indicator for the leaching rate of radioactive species. These plots illustrate good predictability of the study glasses SB5-01 through SB5-20 by the current $\Delta \mathrm{G}_{\mathrm{p}}$ models.
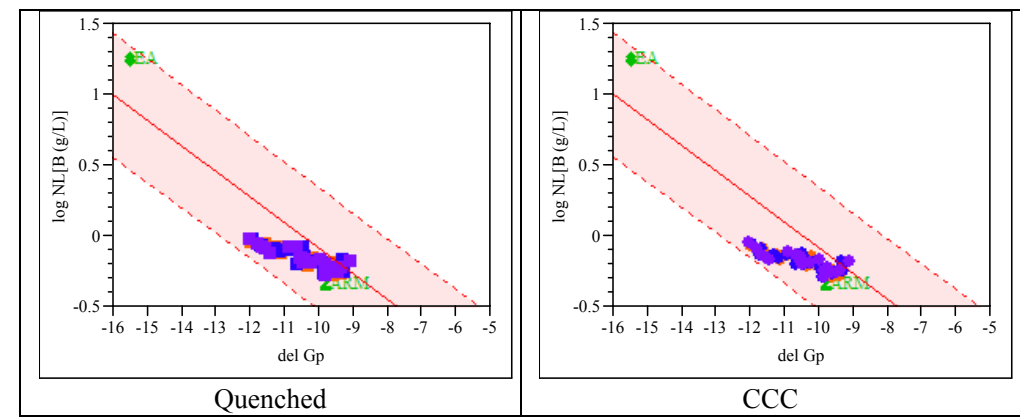

Figure 4-2. Normalized Leachate for Boron versus $\Delta G_{p}$ Model Prediction with $\mathbf{9 5 \%}$ Confidence Interval for Individual PCTs. 


\subsection{Conclusions}

The objective of this study was to experimentally measure the properties and performance of a series of glasses with compositions that could represent SB5 as processed at the DWPF. The data were used to provide recommendations to LWO regarding blending and washing strategies in preparing SB5 based on acceptability of the glass compositions. These data were also used to guide frit optimization efforts as the SB5 composition was finalized.

Glass compositions for this study were developed by combining a series of SB5 composition projections with a group of frits. Three composition projections for SB5 were developed using a model-based approach at SRNL. These compositions, referred to as SB5 Cases B, C and D, projected removal of 25,50 and $75 \%$ (respectively) of the aluminum in Tank 51 through the low temperature aluminum dissolution process. The frits for this study were selected based on their predicted operating windows (i.e., ranges of waste loadings over which the predicted properties of the glasses were acceptable) and their potential (based on historical trends) to provide acceptable melt rates for SB5.

Six additional glasses were designed to evaluate alternatives for uranium in DWPF-type glasses used for variability studies and some scoping studies. Since special measures are necessary when working with uranium-containing glasses in the laboratory, it is desirable as a cost and time saving measure to find an alternative for uranium to support frit optimization efforts. Hafnium and neodymium were investigated as potential surrogates for uranium, and other glasses were made by simply excluding the radioactive components and renormalizing the glass composition.

The study glasses were fabricated and characterized at SRNL. Chemical composition analyses suggested only minor difficulties in meeting the targeted compositions for some of the oxides for some of the glasses. Although minor differences were observed, they did not have a significant impact on the conclusions made in this study. Several of the study compositions showed retention of more than $0.5 \mathrm{wt} \% \mathrm{SO}_{4}{ }^{2-}$ in glass.

Trevorite (a spinel) was the only crystalline phase that was positively identified in a few of the study glasses after the CCC heat treatment. Spinels are not of concern as they have been shown to have little impact on the durability of high level waste glasses. ${ }^{14}$ The crystallization behavior of the surrogate glasses was generally the same as that of their $\mathrm{U}_{3} \mathrm{O}_{8}$-containing counterparts. There are two pairs that were exceptions: SB5-04 (amorphous) and SB5-24 (possible trevorite), along with SB5-07 (amorphous) and SB5-25 (trevorite). In these cases, the surrogate glasses (SB5-24 and SB5-25) appear to be more conservative (more prone to crystallization) than their $\mathrm{U}_{3} \mathrm{O}_{8}$-containing counterparts.

Chemical durability was quantified using the PCT. The normalized leachate values for B, Li, Na and Si for all of the study glasses were well below those of the EA benchmark glass, regardless of heat treatment or compositional view. This indicates that all of the glasses had very acceptable durability performance. The highest NL [B] for the study glasses was $0.914 \mathrm{~g} / \mathrm{L}$ (the quenched version of glass SB5-13), normalized using the measured, bias-correct composition. There was little practical impact of the CCC heat treatment on the PCT responses of the study glasses. The measured PCT responses were predictable by the current $\Delta \mathrm{G}_{\mathrm{p}}$ models.

In general, the PCT responses for the surrogate glasses or the glasses without $\mathrm{U}_{3} \mathrm{O}_{8}$ were quite similar to their $\mathrm{U}_{3} \mathrm{O}_{8}$-containing counterparts. The average percent error in $\mathrm{NL}$ [B] normalized by 
the measured bc compositions for the surrogate glasses compared with their radioactive counterparts was $8.8 \%$. The largest difference in NL [B] was $0.152 \mathrm{~g} / \mathrm{L}$.

While similarities existed in crystallization behavior and PCT response for both the surrogates and the glasses renormalized without $\mathrm{U}_{3} \mathrm{O}_{8}$, additional data must be collected before the best solution to fabricating test glasses without radioactive components can be identified. Further surrogate studies are currently underway at SRNL.

The results of this study indicate that a frit composition can be identified that will provide a processable and durable glass when combined with SB5 at the DWPF. Additional studies are underway to recommend a frit that continues to meet process and performance requirements as well as to provide an enhanced melt rate for improved waste throughput. 


\subsection{References}

1. Hay, M. S., J. M. Pareizs, C. J. Bannochie, M. E. Stone, D. R. Click and D. J. McCabe, "Characterization and Aluminum Dissolution Demonstration with a 3 Liter Tank 51H Sample," U.S. Department of Energy Report WSRC-STI-2007-00697, Revision 0, Washington Savannah River Company, Aiken, SC (2008).

2. Fox, K. M. and T. B. Edwards, "SB5 with the Estimated Impact of Low-Temperature Aluminum Dissolution: Preliminary Frits for Melt Rate Testing," U.S. Department of Energy Report WSRC-STI-2008-00006, Revision 0, Washington Savannah River Company, Aiken, SC (2008).

3. Lopez, C., X. Deschanels, J. M. Bart, J. M. Boubals, C. D. Auwer and E. Simoni, "Solubility of Actinide Surrogates in Nuclear Glasses," Journal of Nuclear Materials, 312 76-80 (2003).

4. Ramsey, W. G., N. E. Bibler and T. F. Meaker, "Compositions and Durabilities of Glasses for Immobilization of Plutonium and Uranium," U.S. Department of Energy Report WSRC-MS-940550, Westinghouse Savannah River Company, Aiken, SC (1994).

5. SRNL, "Glass Batching," U.S. Department of Energy Report SRTC Procedure Manual, L29, ITS-0001, Westinghouse Savannah River Company, Aiken, SC (2002).

6. SRNL, "Glass Melting," U.S. Department of Energy Report SRTC Procedure Manual, L29, ITS-0003, Westinghouse Savannah River Company, Aiken, SC (2002).

7. Marra, S. L. and C. M. Jantzen, "Characterization of Projected DWPF Glass Heat Treated to Simulate Canister Centerline Cooling," U.S. Department of Energy Report WSRC-TR-92-142, Revision 1, Westinghouse Savannah River Company, Aiken, SC (1993).

8. Edwards, T. B., "Analytical Plans for Measuring the Chemical Compositions of Glasses from an EM-20 Study and Glasses from Two DWPF Studies," U.S. Department of Energy Report SRNL-SCS-2008-00003, Washington Savannah River Company, Aiken, SC (2008).

9. Edwards, T. B., "An Analytical Plan for Measuring the Chemical Compositions of Some SB5 Glasses Without Uranium," U.S. Department of Energy Report SRNL-SCS-2008-00043, Washington Savannah River Company, Aiken, SC (2008).

10. ASTM, "Standard Test Methods for Determining Chemical Durability of Nuclear Waste Glasses: The Product Consistency Test (PCT)," ASTM C-1285, (2002).

11. Jantzen, C. M., N. E. Bibler, D. C. Beam, C. L. Crawford and M. A. Pickett, "Characterization of the Defense Waste Processing Facility (DWPF) Environmental Assessment (EA) Glass Standard Reference Material," U.S. Department of Energy Report WSRC-TR-92-346, Revision 1, Westinghouse Savannah River Company, Aiken, SC (1993).

12. Edwards, T. B., "Analytical Plans for Measuring the PCT Solutions of Glasses from an EM20 Study and Glasses from Two DWPF Studies," U.S. Department of Energy Report SRNL-SCS2008-00005, Washington Savannah River Company, Aiken, SC (2008). 
13. Edwards, T. B., "An Analytical Plan for Measuring the PCT Solutions for Some SB5 Glasses without Uranium," U.S. Department of Energy Report SRNL-SCS-2008-00042, Washington Savannah River Company, Aiken, SC (2008).

14. Bickford, D. F. and C. M. Jantzen, "Devitrification of SRL Defense Waste Glass," Sci. Basis for Nuclear Waste Management VII, edited by G. L. McVay. Elsevier, New York, pp. 557-565 (1984).

15. Jantzen, C. M., J. B. Picket, K. G. Brown, T. B. Edwards and D. C. Beam, "Process/Product Models for the Defense Waste Processing Facility (DWPF): Part I. Predicting Glass Durability from Composition Using a Thermodynamic Hydration Energy Reaction Model (THERMO)," U.S. Department of Energy Report WSRC-TR-93-672, Revision 1, Westinghouse Savannah River Company, Aiken, SC (1995). 
SRNS-STI-2008-00060

Revision 0

\section{Appendix A}

Tables and Exhibits Supporting the Analysis of the Chemical Composition Measurements 
Table A1. Targeted Oxide Concentrations (in wt\%) for the Initial SB5 Glass Study (Part 1).

\begin{tabular}{|c|c|c|c|c|c|c|c|c|c|c|c|c|c|c|c|}
\hline Glass ID & $\mathrm{Ag}_{2} \mathrm{O}$ & $\mathbf{A l}_{2} \mathbf{O}_{3}$ & $\mathbf{B}_{2} \mathbf{O}_{3}$ & $\mathrm{BaO}$ & $\mathrm{CaO}$ & CdO & $\mathrm{Ce}_{2} \mathrm{O}_{3}$ & $\mathrm{CoO}$ & $\mathrm{Cr}_{2} \mathrm{O}_{3}$ & $\mathrm{CuO}$ & $\mathrm{Fe}_{2} \mathrm{O}_{3}$ & $\mathbf{H f O}_{2}$ & $\mathbf{K}_{2} \mathbf{O}$ & $\mathbf{L a}_{2} \mathbf{O}_{3}$ & $\mathbf{L i}_{2} \mathbf{O}$ \\
\hline SB5-01 & 0.004 & 11.009 & 6.200 & 0.039 & 3.157 & 0.023 & 0.141 & 0.009 & 0.142 & 0.005 & 8.834 & 0.000 & 0.025 & 0.062 & 4.340 \\
\hline SB5-02 & 0.004 & 11.009 & 6.820 & 0.039 & 1.917 & 0.023 & 0.141 & 0.009 & 0.142 & 0.005 & 8.834 & 0.000 & 0.025 & 0.062 & 4.340 \\
\hline SB5-03 & 0.004 & 11.009 & 8.680 & 0.039 & 1.917 & 0.023 & 0.141 & 0.009 & 0.142 & 0.005 & 8.834 & 0.000 & 0.025 & 0.062 & 3.720 \\
\hline SB5-04 & 0.004 & 11.009 & 9.920 & 0.039 & 0.677 & 0.023 & 0.141 & 0.009 & 0.142 & 0.005 & 8.834 & 0.000 & 0.025 & 0.062 & 3.100 \\
\hline SB5-05 & 0.004 & 9.460 & 6.200 & 0.043 & 3.227 & 0.025 & 0.155 & 0.010 & 0.156 & 0.005 & 9.745 & 0.000 & 0.027 & 0.068 & 4.340 \\
\hline SB5-06 & 0.004 & 9.460 & 6.820 & 0.043 & 1.987 & 0.025 & 0.155 & 0.010 & 0.156 & 0.005 & 9.745 & 0.000 & 0.027 & 0.068 & 4.340 \\
\hline SB5-07 & 0.004 & 9.460 & 8.680 & 0.043 & 1.987 & 0.025 & 0.155 & 0.010 & 0.156 & 0.005 & 9.745 & 0.000 & 0.027 & 0.068 & 3.720 \\
\hline SB5-08 & 0.004 & 9.460 & 9.920 & 0.043 & 0.747 & 0.025 & 0.155 & 0.010 & 0.156 & 0.005 & 9.745 & 0.000 & 0.027 & 0.068 & 3.100 \\
\hline SB5-09 & 0.004 & 7.947 & 6.200 & 0.046 & 3.281 & 0.027 & 0.167 & 0.011 & 0.167 & 0.005 & 10.447 & 0.000 & 0.030 & 0.073 & 4.340 \\
\hline SB5-10 & 0.004 & 7.947 & 6.820 & 0.046 & 2.041 & 0.027 & 0.167 & 0.011 & 0.167 & 0.005 & 10.447 & 0.000 & 0.030 & 0.073 & 4.340 \\
\hline SB5-11 & 0.004 & 7.947 & 8.680 & 0.046 & 2.041 & 0.027 & 0.167 & 0.011 & 0.167 & 0.005 & 10.447 & 0.000 & 0.030 & 0.073 & 3.720 \\
\hline SB5-12 & 0.004 & 7.947 & 9.920 & 0.046 & 0.801 & 0.027 & 0.167 & 0.011 & 0.167 & 0.005 & 10.447 & 0.000 & 0.030 & 0.073 & 3.100 \\
\hline SB5-13 & 0.000 & 7.217 & 6.200 & 0.063 & 3.336 & 0.000 & 0.101 & 0.000 & 0.093 & 0.033 & 10.504 & 0.000 & 0.077 & 0.018 & 4.340 \\
\hline SB5-14 & 0.000 & 7.217 & 6.820 & 0.063 & 2.096 & 0.000 & 0.101 & 0.000 & 0.093 & 0.033 & 10.504 & 0.000 & 0.077 & 0.018 & 4.340 \\
\hline SB5-15 & 0.000 & 7.217 & 8.680 & 0.063 & 2.096 & 0.000 & 0.101 & 0.000 & 0.093 & 0.033 & 10.504 & 0.000 & 0.077 & 0.018 & 3.720 \\
\hline SB5-16 & 0.000 & 7.217 & 9.920 & 0.063 & 0.856 & 0.000 & 0.101 & 0.000 & 0.093 & 0.033 & 10.504 & 0.000 & 0.077 & 0.018 & 3.100 \\
\hline SB5-17 & 0.004 & 11.009 & 9.300 & 0.039 & 0.677 & 0.023 & 0.141 & 0.009 & 0.142 & 0.005 & 8.834 & 0.000 & 0.025 & 0.062 & 5.580 \\
\hline SB5-18 & 0.004 & 9.460 & 8.680 & 0.043 & 0.747 & 0.025 & 0.155 & 0.010 & 0.156 & 0.005 & 9.745 & 0.000 & 0.027 & 0.068 & 4.340 \\
\hline SB5-19 & 0.004 & 7.947 & 9.300 & 0.046 & 0.801 & 0.027 & 0.167 & 0.011 & 0.167 & 0.005 & 10.447 & 0.000 & 0.030 & 0.073 & 3.100 \\
\hline SB5-20 & 0.000 & 7.217 & 9.920 & 0.063 & 0.856 & 0.000 & 0.101 & 0.000 & 0.093 & 0.033 & 10.504 & 0.000 & 0.077 & 0.018 & 2.480 \\
\hline SB5-21 & 0.004 & 11.009 & 8.680 & 0.039 & 1.917 & 0.023 & 0.141 & 0.009 & 0.142 & 0.005 & 8.834 & 0.000 & 0.025 & 0.062 & 3.720 \\
\hline SB5-22 & 0.004 & 9.460 & 9.920 & 0.043 & 0.747 & 0.025 & 0.155 & 0.010 & 0.156 & 0.005 & 9.745 & 0.000 & 0.027 & 0.068 & 3.100 \\
\hline SB5-23 & 0.004 & 7.947 & 8.680 & 0.046 & 2.041 & 0.027 & 0.167 & 0.011 & 0.167 & 0.005 & 10.447 & 3.342 & 0.030 & 0.073 & 3.720 \\
\hline SB5-24 & 0.004 & 11.009 & 9.920 & 0.039 & 0.677 & 0.023 & 0.141 & 0.009 & 0.142 & 0.005 & 8.834 & 2.826 & 0.025 & 0.062 & 3.100 \\
\hline SB5-25 & 0.004 & 10.305 & 8.680 & 0.047 & 2.054 & 0.027 & 0.169 & 0.011 & 0.170 & 0.005 & 10.615 & 0.000 & 0.030 & 0.074 & 3.720 \\
\hline SB5-26 & 0.005 & 8.714 & 9.920 & 0.050 & 0.878 & 0.030 & 0.183 & 0.012 & 0.183 & 0.005 & 11.454 & 0.000 & 0.033 & 0.080 & 3.100 \\
\hline
\end{tabular}


Table A1. Targeted Oxide Concentrations (in wt\%) for the Initial SB5 Glass Study (Part 2).

\begin{tabular}{|c|c|c|c|c|c|c|c|c|c|c|c|c|c|c|c|}
\hline Glass ID & MgO & MnO & $\mathrm{Na}_{2} \mathrm{O}$ & $\mathbf{N d}_{2} \mathbf{O}_{3}$ & $\mathrm{NiO}$ & $\mathbf{P}_{2} \mathbf{O}_{5}$ & PbO & $\mathrm{SiO}_{2}$ & $\mathrm{SO}_{4}{ }^{2-}$ & SrO & $\mathrm{ThO}_{2}$ & $\mathrm{TiO}_{2}$ & $\mathbf{U}_{3} \mathbf{O}_{8}$ & ZnO & $\mathrm{ZrO}_{2}$ \\
\hline SB5-01 & 0.447 & 1.847 & 13.776 & 0.000 & 1.037 & 0.201 & 0.008 & 45.357 & 0.277 & 0.121 & 0.000 & 0.010 & 2.826 & 0.006 & 0.098 \\
\hline SB5-02 & 0.447 & 1.847 & 13.776 & 0.000 & 1.037 & 0.201 & 0.008 & 45.977 & 0.277 & 0.121 & 0.000 & 0.010 & 2.826 & 0.006 & 0.098 \\
\hline SB5-03 & 0.447 & 1.847 & 13.776 & 0.000 & 1.037 & 0.201 & 0.008 & 44.737 & 0.277 & 0.121 & 0.000 & 0.010 & 2.826 & 0.006 & 0.098 \\
\hline SB5-04 & 0.447 & 1.847 & 14.396 & 0.000 & 1.037 & 0.201 & 0.008 & 44.737 & 0.277 & 0.121 & 0.000 & 0.010 & 2.826 & 0.006 & 0.098 \\
\hline SB5-05 & 0.494 & 2.038 & 13.534 & 0.000 & 1.144 & 0.221 & 0.009 & 45.431 & 0.289 & 0.134 & 0.000 & 0.011 & 3.117 & 0.006 & 0.108 \\
\hline SB5-06 & 0.494 & 2.038 & 13.534 & 0.000 & 1.144 & 0.221 & 0.009 & 46.051 & 0.289 & 0.134 & 0.000 & 0.011 & 3.117 & 0.006 & 0.108 \\
\hline SB5-07 & 0.494 & 2.038 & 13.534 & 0.000 & 1.144 & 0.221 & 0.009 & 44.811 & 0.289 & 0.134 & 0.000 & 0.011 & 3.117 & 0.006 & 0.108 \\
\hline SB5-08 & 0.494 & 2.038 & 14.154 & 0.000 & 1.144 & 0.221 & 0.009 & 44.811 & 0.289 & 0.134 & 0.000 & 0.011 & 3.117 & 0.006 & 0.108 \\
\hline SB5-09 & 0.529 & 2.184 & 13.650 & 0.000 & 1.227 & 0.236 & 0.010 & 45.489 & 0.310 & 0.143 & 0.000 & 0.012 & 3.342 & 0.007 & 0.116 \\
\hline SB5-10 & 0.529 & 2.184 & 13.650 & 0.000 & 1.227 & 0.236 & 0.010 & 46.109 & 0.310 & 0.143 & 0.000 & 0.012 & 3.342 & 0.007 & 0.116 \\
\hline SB5-11 & 0.529 & 2.184 & 13.650 & 0.000 & 1.227 & 0.236 & 0.010 & 44.869 & 0.310 & 0.143 & 0.000 & 0.012 & 3.342 & 0.007 & 0.116 \\
\hline SB5-12 & 0.529 & 2.184 & 14.270 & 0.000 & 1.227 & 0.236 & 0.010 & 44.869 & 0.310 & 0.143 & 0.000 & 0.012 & 3.342 & 0.007 & 0.116 \\
\hline SB5-13 & 0.467 & 2.316 & 14.678 & 0.000 & 1.198 & 0.000 & 0.036 & 45.338 & 0.547 & 0.000 & 0.004 & 0.350 & 2.925 & 0.042 & 0.117 \\
\hline SB5-14 & 0.467 & 2.316 & 14.678 & 0.000 & 1.198 & 0.000 & 0.036 & 45.958 & 0.547 & 0.000 & 0.004 & 0.350 & 2.925 & 0.042 & 0.117 \\
\hline SB5-15 & 0.467 & 2.316 & 14.678 & 0.000 & 1.198 & 0.000 & 0.036 & 44.718 & 0.547 & 0.000 & 0.004 & 0.350 & 2.925 & 0.042 & 0.117 \\
\hline SB5-16 & 0.467 & 2.316 & 15.298 & 0.000 & 1.198 & 0.000 & 0.036 & 44.718 & 0.547 & 0.000 & 0.004 & 0.350 & 2.925 & 0.042 & 0.117 \\
\hline SB5-17 & 0.447 & 1.847 & 11.916 & 0.000 & 1.037 & 0.201 & 0.008 & 45.357 & 0.277 & 0.121 & 0.000 & 0.010 & 2.826 & 0.006 & 0.098 \\
\hline SB5-18 & 0.494 & 2.038 & 14.154 & 0.000 & 1.144 & 0.221 & 0.009 & 44.811 & 0.289 & 0.134 & 0.000 & 0.011 & 3.117 & 0.006 & 0.108 \\
\hline SB5-19 & 0.529 & 2.184 & 15.510 & 0.000 & 1.227 & 0.236 & 0.010 & 44.249 & 0.310 & 0.143 & 0.000 & 0.012 & 3.342 & 0.007 & 0.116 \\
\hline SB5-20 & 0.467 & 2.316 & 14.678 & 0.000 & 1.198 & 0.000 & 0.036 & 45.958 & 0.547 & 0.000 & 0.004 & 0.350 & 2.925 & 0.042 & 0.117 \\
\hline SB5-21 & 0.447 & 1.847 & 13.776 & 2.826 & 1.037 & 0.201 & 0.008 & 44.737 & 0.277 & 0.121 & 0.000 & 0.010 & 0.000 & 0.006 & 0.098 \\
\hline SB5-22 & 0.494 & 2.038 & 14.154 & 3.117 & 1.144 & 0.221 & 0.009 & 44.811 & 0.289 & 0.134 & 0.000 & 0.011 & 0.000 & 0.006 & 0.108 \\
\hline SB5-23 & 0.529 & 2.184 & 13.650 & 0.000 & 1.227 & 0.236 & 0.010 & 44.869 & 0.310 & 0.143 & 0.000 & 0.012 & 0.000 & 0.007 & 0.116 \\
\hline SB5-24 & 0.447 & 1.847 & 14.396 & 0.000 & 1.037 & 0.201 & 0.008 & 44.737 & 0.277 & 0.121 & 0.000 & 0.010 & 0.000 & 0.006 & 0.098 \\
\hline SB5-25 & 0.538 & 2.220 & 14.355 & 0.000 & 1.247 & 0.240 & 0.010 & 44.882 & 0.315 & 0.145 & 0.000 & 0.012 & 0.000 & 0.007 & 0.118 \\
\hline SB5-26 & 0.580 & 2.395 & 15.168 & 0.000 & 1.345 & 0.259 & 0.011 & 44.951 & 0.340 & 0.157 & 0.000 & 0.013 & 0.000 & 0.008 & 0.127 \\
\hline
\end{tabular}


Table A2. Measured Elemental Concentrations (wt\%) for Samples Prepared Using Lithium Metaborate (Part 1).

\begin{tabular}{|c|c|c|c|c|c|c|c|c|c|c|c|c|c|c|c|c|}
\hline Analytical Plan & Set & Type & Glass ID & Block & $\begin{array}{l}\text { Sub- } \\
\text { Block }\end{array}$ & Sequence & Lab ID & Al & Ba & Ca & Cd & Ce & Co & $\mathrm{Cr}$ & $\mathbf{C u}$ & $\mathbf{F e}$ \\
\hline SRNL-SCS-2008-00003 & 2 & Batch 1 & Batch 1 & 1 & 1 & 1 & BCHLM2-111 & 2.49 & 0.126 & 0.834 & $<0.010$ & 0.011 & $<0.010$ & 0.073 & 0.295 & 9.33 \\
\hline SRNL-SCS-2008-00003 & 2 & Ustd & U std & 1 & 1 & 2 & UstdLM2-111 & 2.03 & $<0.010$ & 0.892 & $<0.010$ & 0.001 & $<0.010$ & 0.162 & $<0.010$ & 9.5 \\
\hline SRNL-SCS-2008-00003 & 2 & SB5 & SB5-01 & 1 & 1 & 3 & N02LM11 & 5.73 & 0.031 & 2.41 & 0.014 & 0.084 & $<0.010$ & 0.077 & $<0.010$ & 6.26 \\
\hline $\begin{array}{l}\text { SRNL-SCS-2008-00003 } \\
\end{array}$ & 2 & SB5 & SB5-05 & 1 & 1 & 4 & N07LM11 & 5.12 & 0.035 & 2.5 & 0.013 & 0.052 & $<0.010$ & 0.087 & $<0.010$ & 6.87 \\
\hline SRNL-SCS-2008-00003 & 2 & SB5 & SB5-04 & 1 & 1 & 5 & N03LM21 & 5.89 & 0.033 & 0.463 & 0.016 & 0.057 & $<0.010$ & 0.077 & 0.01 & 6.15 \\
\hline $\begin{array}{l}\text { SRNL-SCS-2008-00003 } \\
\end{array}$ & 2 & SB5 & SB5-04 & 1 & 1 & 6 & N03LM11 & 5.9 & 0.033 & 0.47 & 0.016 & 0.058 & $<0.010$ & 0.078 & 0.011 & 6.09 \\
\hline SRNL-SCS-2008-00003 & 2 & SB5 & SB5-12 & 1 & 1 & 7 & N13LM21 & 4.21 & 0.038 & 0.568 & 0.017 & 0.126 & $<0.010$ & 0.109 & 0.011 & 6.85 \\
\hline $\begin{array}{l}\text { SRNL-SCS-2008-00003 } \\
\end{array}$ & 2 & SB5 & $\begin{array}{l}\text { SB5-02 } \\
\end{array}$ & 1 & 1 & 8 & N12LM21 & 5.76 & 0.032 & 1.47 & 0.014 & 0.083 & $<0.010$ & 0.08 & $<0.010$ & 5.79 \\
\hline SRNL-SCS-2008-00003 & 2 & SB5 & SB5-11 & 1 & 1 & 9 & N14LM11 & 4.18 & 0.037 & 1.57 & 0.018 & 0.126 & $<0.010$ & 0.098 & 0.011 & 6.54 \\
\hline SRNL-SCS-2008-00003 & 2 & SB5 & SB5-07 & 1 & 1 & 10 & N15LM21 & 5.07 & 0.035 & 1.5 & 0.015 & 0.083 & $<0.010$ & 0.086 & 0.01 & 6.51 \\
\hline SRNL-SCS-2008-00003 & 2 & Batch 1 & Batch 1 & 1 & 1 & 11 & BCHLM2-112 & 2.44 & 0.126 & 0.839 & $<0.010$ & 0.011 & $<0.010$ & 0.074 & 0.298 & 9.18 \\
\hline SRNL-SCS-2008-00003 & 2 & Ustd & U std & 1 & 1 & 12 & UstdLM1-112 & 2.04 & $<0.010$ & 0.89 & $<0.010$ & 0.066 & $<0.010$ & 0.162 & $<0.010$ & 9.47 \\
\hline $\begin{array}{l}\text { SRNL-SCS-2008-00003 } \\
\end{array}$ & 2 & SB5 & SB5-02 & 1 & 1 & 13 & N12LM11 & 5.75 & 0.032 & 1.43 & 0.014 & 0.084 & $<0.010$ & 0.083 & $<0.010$ & 6 \\
\hline SRNL-SCS-2008-00003 & 2 & SB5 & SB5-11 & 1 & 1 & 14 & N14LM21 & 4.13 & 0.038 & 1.56 & 0.018 & 0.126 & $<0.010$ & 0.096 & 0.011 & 6.96 \\
\hline $\begin{array}{l}\text { SRNL-SCS-2008-00003 } \\
\end{array}$ & 2 & SB4 & SB4VAR33 & 1 & 1 & 15 & N05LM21 & 5.18 & 0.023 & 0.747 & $<0.010$ & 0.019 & $<0.010$ & 0.038 & 0.024 & 7.44 \\
\hline SRNL-SCS-2008-00003 & 2 & SB5 & SB5-05 & 1 & 1 & 16 & N07LM21 & 5.11 & 0.034 & 2.55 & 0.012 & 0.051 & $<0.010$ & 0.085 & $<0.010$ & 6.42 \\
\hline $\begin{array}{l}\text { SRNL-SCS-2008-00003 } \\
\end{array}$ & 2 & SB5 & SB5-12 & 1 & 1 & 17 & N13LM11 & 4.18 & 0.038 & 0.576 & 0.017 & 0.125 & $<0.010$ & 0.109 & 0.01 & 6.82 \\
\hline SRNL-SCS-2008-00003 & 2 & SB4 & SB4VAR33 & 1 & 1 & 18 & N05LM11 & 5.17 & 0.023 & 0.745 & $<0.010$ & 0.02 & $<0.010$ & 0.038 & 0.023 & 7.58 \\
\hline SRNL-SCS-2008-00003 & 2 & SB5 & $\begin{array}{l}\text { SB5-01 } \\
\end{array}$ & 1 & 1 & 19 & N02LM21 & 5.96 & 0.032 & 2.56 & 0.014 & 0.087 & $<0.010$ & 0.078 & $<0.010$ & 6.32 \\
\hline SRNL-SCS-2008-00003 & 2 & SB5 & SB5-07 & 1 & 1 & 20 & N15LM11 & 5.03 & 0.035 & 1.54 & 0.016 & 0.085 & $<0.010$ & 0.089 & 0.014 & 6.75 \\
\hline SRNL-SCS-2008-00003 & 2 & Batch 1 & Batch 1 & 1 & 1 & 21 & BCHLM2-113 & 2.53 & 0.126 & 0.85 & $<0.010$ & 0.011 & $<0.010$ & 0.073 & 0.3 & 9.28 \\
\hline SRNL-SCS-2008-00003 & 2 & Ustd & U std & 1 & 1 & 22 & UstdLM2-113 & 2.04 & $<0.010$ & 0.896 & $<0.010$ & 0.073 & $<0.010$ & 0.161 & $<0.010$ & 9.5 \\
\hline SRNL-SCS-2008-00003 & 2 & Batch 1 & Batch 1 & 1 & 2 & 1 & BCHLM2-121 & 2.49 & 0.126 & 0.84 & $<0.010$ & 0.01 & $<0.010$ & 0.073 & 0.3 & 8.63 \\
\hline $\begin{array}{l}\text { SRNL-SCS-2008-00003 } \\
\end{array}$ & 2 & Ustd & U std & 1 & 2 & 2 & UstdLM2-121 & 2.03 & $<0.010$ & 0.892 & $<0.010$ & 0 & $<0.010$ & 0.161 & $<0.010$ & 8.72 \\
\hline SRNL-SCS-2008-00003 & 2 & SB5 & SB5-12 & 1 & 2 & 3 & N13LM22 & 4.13 & 0.037 & 0.568 & 0.015 & 0.125 & $<0.010$ & 0.108 & 0.011 & 6.48 \\
\hline SRNL-SCS-2008-00003 & 2 & SB5 & SB5-05 & 1 & 2 & 4 & N07LM22 & 5.02 & 0.032 & 2.46 & 0.009 & 0.05 & $<0.010$ & 0.083 & $<0.010$ & 6.47 \\
\hline SRNL-SCS-2008-00003 & 2 & SB4 & SB4VAR33 & 1 & 2 & 5 & N05LM22 & 5.19 & 0.021 & 0.747 & $<0.010$ & 0.019 & $<0.010$ & 0.037 & 0.023 & 7.43 \\
\hline $\begin{array}{l}\text { SRNL-SCS-2008-00003 } \\
\end{array}$ & 2 & SB4 & SB4VAR33 & 1 & 2 & 6 & N05LM12 & 5.17 & 0.022 & 0.734 & $<0.010$ & 0.019 & $<0.010$ & 0.038 & 0.022 & 7.57 \\
\hline SRNL-SCS-2008-00003 & 2 & SB5 & SB5-01 & 1 & 2 & 7 & N02LM12 & 5.83 & 0.029 & 2.33 & 0.011 & 0.082 & $<0.010$ & 0.074 & $<0.010$ & 5.8 \\
\hline SRNL-SCS-2008-00003 & 2 & SB5 & SB5-04 & 1 & 2 & 8 & N03LM12 & 5.83 & 0.032 & 0.464 & 0.014 & 0.057 & $<0.010$ & 0.077 & 0.011 & 5.95 \\
\hline SRNL-SCS-2008-00003 & 2 & SB5 & SB5-01 & 1 & 2 & 9 & N02LM22 & 5.87 & 0.03 & 2.42 & 0.012 & 0.085 & $<0.010$ & 0.077 & $<0.010$ & 5.87 \\
\hline SRNL-SCS-2008-00003 & 2 & SB5 & SB5-07 & 1 & 2 & 10 & N15LM12 & 4.9 & 0.033 & 1.48 & 0.013 & 0.084 & $<0.010$ & 0.086 & 0.014 & 6.5 \\
\hline SRNL-SCS-2008-00003 & 2 & Batch 1 & Batch 1 & 1 & 2 & 11 & BCHLM2-122 & 2.44 & 0.123 & 0.832 & $<0.010$ & 0.01 & $<0.010$ & 0.071 & 0.298 & 8.95 \\
\hline SRNL-SCS-2008-00003 & 2 & Ustd & U std & 1 & 2 & 12 & UstdLM2-122 & 2.07 & $<0.010$ & 0.882 & $<0.010$ & 0.028 & $<0.010$ & 0.157 & $<0.010$ & 9.5 \\
\hline $\begin{array}{l}\text { SRNL-SCS-2008-00003 } \\
\end{array}$ & 2 & SB5 & $\begin{array}{l}\text { SB5-04 } \\
\end{array}$ & 1 & 2 & 13 & N03LM22 & 5.76 & 0.031 & 0.469 & 0.013 & 0.056 & $<0.010$ & 0.075 & 0.01 & 6.19 \\
\hline SRNL-SCS-2008-00003 & 2 & SB5 & SB5-11 & 1 & 2 & 14 & N14LM22 & 4.17 & 0.037 & 1.52 & 0.016 & 0.125 & $<0.010$ & 0.094 & 0.011 & 6.83 \\
\hline $\begin{array}{l}\text { SRNL-SCS-2008-00003 } \\
\end{array}$ & 2 & SB5 & SB5-05 & 1 & 2 & 15 & N07LM12 & 5.03 & 0.034 & 2.43 & 0.01 & 0.052 & $<0.010$ & 0.087 & $<0.010$ & 6.64 \\
\hline SRNL-SCS-2008-00003 & 2 & SB5 & SB5-02 & 1 & 2 & 16 & N12LM12 & 5.7 & 0.031 & 1.38 & 0.012 & 0.082 & $<0.010$ & 0.083 & $<0.010$ & 5.89 \\
\hline SRNL-SCS-2008-00003 & 2 & SB5 & SB5-11 & 1 & 2 & 17 & N14LM12 & 4.09 & 0.037 & 1.47 & 0.016 & 0.126 & $<0.010$ & 0.099 & 0.011 & 6.65 \\
\hline SRNL-SCS-2008-00003 & 2 & SB5 & $\begin{array}{l}\text { SB5-02 } \\
\end{array}$ & 1 & 2 & 18 & N12LM22 & 5.66 & 0.031 & 1.37 & 0.012 & 0.083 & $<0.010$ & 0.081 & $<0.010$ & 5.73 \\
\hline SRNL-SCS-2008-00003 & 2 & SB5 & SB5-12 & 1 & 2 & 19 & N13LM12 & 4.11 & 0.037 & 0.569 & 0.015 & 0.125 & $<0.010$ & 0.109 & 0.01 & 7.43 \\
\hline $\begin{array}{l}\text { SRNL-SCS-2008-00003 } \\
\end{array}$ & 2 & SB5 & $\begin{array}{l}\text { SB5-07 } \\
\end{array}$ & 1 & 2 & 20 & N15LM22 & 5.01 & 0.034 & 1.46 & 0.013 & 0.082 & $<0.010$ & 0.086 & 0.01 & 7.13 \\
\hline
\end{tabular}


Table A2. Measured Elemental Concentrations (wt\%) for Samples Prepared Using Lithium Metaborate (Part 1, continued).

\begin{tabular}{|c|c|c|c|c|c|c|c|c|c|c|c|c|c|c|c|c|}
\hline Analytical Plan & Set & Type & Glass ID & Block & $\begin{array}{l}\text { Sub- } \\
\text { Block }\end{array}$ & Sequence & Lab ID & Al & Ba & Ca & Cd & $\mathrm{Ce}$ & Co & $\mathrm{Cr}$ & $\mathbf{C u}$ & $\mathbf{F e}$ \\
\hline SRNL-SCS-2008-00003 & 2 & Batch 1 & Batch 1 & 1 & 2 & 21 & BCHLM2-123 & 2.53 & 0.126 & 0.832 & $<0.010$ & 0.01 & $<0.010$ & 0.073 & 0.295 & 9.61 \\
\hline SRNL-SCS-2008-00003 & 2 & Ustd & U std & 1 & 2 & 22 & UstdLM2-123 & 2.04 & $<0.010$ & 0.877 & $<0.010$ & 0 & $<0.010$ & 0.164 & $<0.010$ & 9.81 \\
\hline SRNL-SCS-2008-00003 & 2 & Batch 1 & Batch 1 & 2 & 1 & 1 & BCHLM2-211 & 2.49 & 0.129 & 0.873 & $<0.010$ & 0.013 & $<0.010$ & 0.075 & 0.311 & 8.99 \\
\hline SRNL-SCS-2008-00003 & 2 & Ustd & U std & 2 & 1 & 2 & UstdLM2-211 & 2.04 & $<0.010$ & 0.927 & $<0.010$ & 0.002 & $<0.010$ & 0.163 & $<0.010$ & 9.32 \\
\hline SRNL-SCS-2008-00003 & 2 & SB4 & SB4VAR32 & 2 & 1 & 3 & N11LM21 & 4.66 & 0.022 & 0.722 & $<0.010$ & 0.018 & $<0.010$ & 0.041 & 0.023 & 6.82 \\
\hline SRNL-SCS-2008-00003 & 2 & SB4 & SB4VAR31 & 2 & 1 & 4 & N06LM11 & 4.14 & 0.021 & 0.628 & $<0.010$ & 0.018 & $<0.010$ & 0.038 & 0.02 & 6.01 \\
\hline SRNL-SCS-2008-00003 & 2 & SB5 & SB5-03 & 2 & 1 & 5 & N09LM11 & 5.72 & 0.033 & 1.38 & 0.014 & 0.089 & $<0.010$ & 0.08 & 0.012 & 6.05 \\
\hline SRNL-SCS-2008-00003 & 2 & SB5 & SB5-09 & 2 & 1 & 6 & N04LM11 & 4.17 & 0.036 & 2.43 & 0.015 & 0.084 & $<0.010$ & 0.09 & 0.011 & 7.38 \\
\hline SRNL-SCS-2008-00003 & 2 & SB5 & SB5-03 & 2 & 1 & 7 & N09LM21 & 5.79 & 0.034 & 1.39 & 0.014 & 0.091 & $<0.010$ & 0.082 & 0.013 & 6.37 \\
\hline SRNL-SCS-2008-00003 & 2 & SB4 & SB4VAR32 & 2 & 1 & 8 & N11LM11 & 4.75 & 0.022 & 0.711 & $<0.010$ & 0.018 & $<0.010$ & 0.047 & 0.022 & 7.6 \\
\hline SRNL-SCS-2008-00003 & 2 & SB5 & SB5-10 & 2 & 1 & 9 & N081LM11 & 4.27 & 0.038 & 1.57 & 0.018 & 0.126 & $<0.010$ & 0.084 & 0.011 & 7.55 \\
\hline SRNL-SCS-2008-00003 & 2 & Batch 1 & Batch 1 & 2 & 1 & 10 & BCHLM2-212 & 2.52 & 0.127 & 0.867 & $<0.010$ & 0.013 & $<0.010$ & 0.074 & 0.305 & 9.48 \\
\hline SRNL-SCS-2008-00003 & 2 & Ustd & U std & 2 & 1 & 11 & UstdLM2-212 & 2.05 & $<0.010$ & 0.944 & $<0.010$ & 0.002 & $<0.010$ & 0.166 & $<0.010$ & 9.23 \\
\hline SRNL-SCS-2008-00003 & 2 & SB5 & SB5-08 & 2 & 1 & 12 & N10LM21 & 4.83 & 0.037 & 0.533 & 0.014 & 0.056 & $<0.010$ & 0.088 & 0.01 & 6.6 \\
\hline SRNL-SCS-2008-00003 & 2 & SB5 & SB5-06 & 2 & 1 & 13 & N01LM11 & 4.99 & 0.037 & 1.42 & 0.015 & 0.094 & $<0.010$ & 0.083 & 0.011 & 6.88 \\
\hline SRNL-SCS-2008-00003 & 2 & SB4 & SB4VAR31 & 2 & 1 & 14 & N06LM21 & 4.22 & 0.021 & 0.636 & $<0.010$ & 0.018 & $<0.010$ & 0.038 & 0.02 & 6.42 \\
\hline SRNL-SCS-2008-00003 & 2 & SB5 & SB5-10 & 2 & 1 & 15 & N08LM21 & 4.17 & 0.039 & 1.46 & 0.018 & 0.128 & $<0.010$ & 0.085 & 0.011 & 7 \\
\hline SRNL-SCS-2008-00003 & 2 & SB5 & SB5-08 & 2 & 1 & 16 & N10LM11 & 5.03 & 0.038 & 0.564 & 0.016 & 0.056 & $<0.010$ & 0.093 & 0.012 & 6.72 \\
\hline SRNL-SCS-2008-00003 & 2 & SB5 & SB5-09 & 2 & 1 & 17 & N04LM21 & 4.16 & 0.038 & 2.3 & 0.016 & 0.088 & $<0.010$ & 0.094 & 0.011 & 7.16 \\
\hline SRNL-SCS-2008-00003 & 2 & SB5 & SB5-06 & 2 & 1 & 18 & N01LM21 & 5.21 & 0.033 & 1.54 & 0.013 & 0.088 & $<0.010$ & 0.075 & 0.01 & 6.75 \\
\hline SRNL-SCS-2008-00003 & 2 & Batch 1 & Batch 1 & 2 & 1 & 19 & BCHLM2-213 & 2.54 & 0.129 & 0.881 & $<0.010$ & 0.013 & $<0.010$ & 0.075 & 0.313 & 9.36 \\
\hline SRNL-SCS-2008-00003 & 2 & Ustd & U std & 2 & 1 & 20 & UstdLM2-213 & 2.05 & $<0.010$ & 0.933 & $<0.010$ & 0.002 & $<0.010$ & 0.165 & $<0.010$ & 9.92 \\
\hline SRNL-SCS-2008-00003 & 2 & Batch 1 & Batch 1 & 2 & 2 & 1 & BCHLM2-221 & 2.62 & 0.127 & 0.853 & $<0.010$ & 0.01 & $<0.010$ & 0.073 & 0.302 & 8.77 \\
\hline SRNL-SCS-2008-00003 & 2 & Ustd & U std & 2 & 2 & 2 & UstdLM2-221 & 2.21 & $<0.010$ & 0.906 & $<0.010$ & $<0.010$ & $<0.010$ & 0.163 & $<0.010$ & 9.38 \\
\hline SRNL-SCS-2008-00003 & 2 & SB4 & SB4VAR31 & 2 & 2 & 3 & N06LM22 & 4.4 & 0.019 & 0.622 & $<0.010$ & 0.015 & $<0.010$ & 0.035 & 0.016 & 6.11 \\
\hline SRNL-SCS-2008-00003 & 2 & SB4 & SB4VAR32 & 2 & 2 & 4 & N11LM22 & 4.91 & 0.02 & 0.692 & $<0.010$ & 0.016 & $<0.010$ & 0.039 & 0.018 & 7.18 \\
\hline SRNL-SCS-2008-00003 & 2 & SB5 & SB5-10 & 2 & 2 & 5 & N08LM12 & 4.39 & 0.036 & 1.6 & 0.015 & 0.121 & $<0.010$ & 0.081 & $<0.010$ & 7.16 \\
\hline SRNL-SCS-2008-00003 & 2 & SB5 & SB5-06 & 2 & 2 & 6 & N01LM22 & 5.21 & 0.033 & 1.56 & 0.011 & 0.089 & $<0.010$ & 0.078 & $<0.010$ & 6.74 \\
\hline SRNL-SCS-2008-00003 & 2 & SB4 & SB4VAR31 & 2 & 2 & 7 & N06LM12 & 4.37 & 0.019 & 0.609 & $<0.010$ & 0.016 & $<0.010$ & 0.036 & 0.016 & 6.15 \\
\hline SRNL-SCS-2008-00003 & 2 & SB5 & SB5-09 & 2 & 2 & 8 & N04LM12 & 4.43 & 0.034 & 2.59 & 0.012 & 0.081 & $<0.010$ & 0.089 & $<0.010$ & 7.28 \\
\hline SRNL-SCS-2008-00003 & 2 & SB5 & SB5-08 & 2 & 2 & 9 & N10LM12 & 5.27 & 0.037 & 0.541 & 0.014 & 0.052 & $<0.010$ & 0.092 & $<0.010$ & 6.73 \\
\hline SRNL-SCS-2008-00003 & 2 & Batch 1 & Batch 1 & 2 & 2 & 10 & BCHLM2-222 & 2.66 & 0.128 & 0.859 & $<0.010$ & 0.01 & $<0.010$ & 0.073 & 0.304 & 8.91 \\
\hline SRNL-SCS-2008-00003 & 2 & Ustd & U std & 2 & 2 & 11 & UstdLM2-222 & 2.2 & $<0.010$ & 0.928 & $<0.010$ & $<0.010$ & $<0.010$ & 0.164 & $<0.010$ & 9.39 \\
\hline SRNL-SCS-2008-00003 & 2 & SB5 & SB5-03 & 2 & 2 & 12 & N09LM12 & 6.14 & 0.032 & 1.55 & 0.012 & 0.086 & $<0.010$ & 0.081 & $<0.010$ & 5.85 \\
\hline SRNL-SCS-2008-00003 & 2 & SB5 & SB5-10 & 2 & 2 & 13 & N08LM22 & 4.41 & 0.037 & 1.62 & 0.016 & 0.122 & $<0.010$ & 0.085 & $<0.010$ & 6.99 \\
\hline SRNL-SCS-2008-00003 & 2 & SB5 & SB5-09 & 2 & 2 & 14 & N04LM22 & 4.34 & 0.038 & 2.58 & 0.014 & 0.085 & $<0.010$ & 0.096 & $<0.010$ & 6.63 \\
\hline SRNL-SCS-2008-00003 & 2 & SB5 & SB5-03 & 2 & 2 & 15 & N09LM22 & 5.96 & 0.034 & 1.51 & 0.013 & 0.089 & $<0.010$ & 0.085 & $<0.010$ & 5.59 \\
\hline SRNL-SCS-2008-00003 & 2 & SB5 & SB5-08 & 2 & 2 & 16 & N10LM22 & 5.01 & 0.037 & 0.513 & 0.012 & 0.054 & $<0.010$ & 0.089 & $<0.010$ & 6.59 \\
\hline SRNL-SCS-2008-00003 & 2 & SB5 & SB5-06 & 2 & 2 & 17 & N01LM12 & 5.19 & 0.036 & 1.56 & 0.012 & 0.092 & $<0.010$ & 0.083 & $<0.010$ & 6.51 \\
\hline SRNL-SCS-2008-00003 & 2 & SB4 & SB4VAR32 & 2 & 2 & 18 & N11LM12 & 4.92 & 0.021 & 0.677 & $<0.010$ & 0.016 & $<0.010$ & 0.046 & 0.018 & 6.72 \\
\hline SRNL-SCS-2008-00003 & 2 & Batch 1 & Batch 1 & 2 & 2 & 19 & BCHLM2-223 & 2.62 & 0.134 & 0.833 & $<0.010$ & 0.011 & $<0.010$ & 0.077 & 0.304 & 8.91 \\
\hline SRNL-SCS-2008-00003 & 2 & Ustd & U std & 2 & 2 & 20 & UstdLM2-223 & 2.2 & $<0.010$ & 0.881 & $<<0.010$ & $<0.010$ & $<0.010$ & 0.173 & $<0.010$ & 9.16 \\
\hline SRNL-SCS-2008-00003 & 3 & Batch 1 & Batch 1 & 1 & 1 & 1 & BCHLM3-111 & 2.58 & 0.125 & 0.841 & $<0.010$ & 0.013 & $<0.010$ & 0.072 & 0.305 & 9.37 \\
\hline
\end{tabular}


Table A2. Measured Elemental Concentrations (wt\%) for Samples Prepared Using Lithium Metaborate (Part 1, continued).

\begin{tabular}{|c|c|c|c|c|c|c|c|c|c|c|c|c|c|c|c|c|}
\hline Analytical Plan & Set & Type & Glass ID & Block & $\begin{array}{l}\text { Sub- } \\
\text { Block }\end{array}$ & Sequence & Lab ID & Al & Ba & Ca & Cd & $\mathrm{Ce}$ & Co & $\mathrm{Cr}$ & $\mathbf{C u}$ & $\mathrm{Fe}$ \\
\hline SRNL-SCS-2008-00003 & 3 & Ustd & U std & 1 & 1 & 2 & UstdLM3-111 & 2.14 & $<0.010$ & 0.904 & $<0.010$ & $<0.010$ & $<0.010$ & 0.156 & $<0.010$ & 9.7 \\
\hline SRNL-SCS-2008-00003 & 3 & HWL & HWL-28 & 1 & 1 & 3 & P11LM11 & 2.38 & 0.066 & 0.702 & $<0.010$ & 0.188 & $<0.010$ & 0.067 & 0.041 & 8.93 \\
\hline SRNL-SCS-2008-00003 & 3 & HWL & HWL-25 & 1 & 1 & 4 & $\begin{array}{l}\text { P05LM21 } \\
\end{array}$ & 2.16 & 0.058 & 0.606 & $<0.010$ & 0.213 & $<0.010$ & 0.057 & 0.034 & 7.68 \\
\hline SRNL-SCS-2008-00003 & 3 & SB5 & SB5-14 & 1 & 1 & 5 & $\begin{array}{l}\text { P08LM21 } \\
\end{array}$ & 3.93 & 0.049 & 1.55 & $<0.010$ & 0.069 & $<0.010$ & 0.061 & 0.031 & 7.62 \\
\hline SRNL-SCS-2008-00003 & 3 & SB5 & SB5-20 & 1 & 1 & 6 & P01LM11 & 3.94 & 0.052 & 0.584 & $<0.010$ & 0.075 & $<0.010$ & 0.06 & 0.029 & 7.72 \\
\hline SRNL-SCS-2008-00003 & 3 & SB5 & SB5-14 & 1 & 1 & 7 & P08LM11 & 3.88 & 0.051 & 1.52 & $<0.010$ & 0.073 & $<0.010$ & 0.064 & 0.032 & 7.89 \\
\hline SRNL-SCS-2008-00003 & 3 & SB5 & SB5-20 & 1 & 1 & 8 & $\begin{array}{l}\text { P01LM21 } \\
\end{array}$ & 3.96 & 0.052 & 0.591 & $<0.010$ & 0.14 & $<0.010$ & 0.06 & 0.029 & 7.82 \\
\hline SRNL-SCS-2008-00003 & 3 & HWL & HWL-25 & 1 & 1 & 9 & $\begin{array}{l}\text { P05LM11 } \\
\end{array}$ & 2.17 & 0.059 & 0.613 & $<0.010$ & 0.145 & $<0.010$ & 0.056 & 0.033 & 7.83 \\
\hline SRNL-SCS-2008-00003 & 3 & Batch 1 & Batch 1 & 1 & 1 & 10 & BCHLM3-112 & 2.6 & 0.13 & 0.817 & $<0.010$ & 0.013 & $<0.010$ & 0.075 & 0.303 & 9.82 \\
\hline SRNL-SCS-2008-00003 & 3 & Ustd & U std & 1 & 1 & 11 & UstdLM3-112 & 2.16 & $<0.010$ & 0.874 & $<0.010$ & $<0.010$ & $<0.010$ & 0.163 & $<0.010$ & 10.07 \\
\hline SRNL-SCS-2008-00003 & 3 & SB5 & SB5-18 & 1 & 1 & 12 & P09LM21 & 5.31 & 0.034 & 0.494 & 0.015 & 0.11 & $<0.010$ & 0.086 & $<0.010$ & 7.09 \\
\hline SRNL-SCS-2008-00003 & 3 & SB5 & SB5-18 & 1 & 1 & 13 & P09LM11 & 5.29 & 0.036 & 0.498 & 0.016 & 0.114 & $<0.010$ & 0.089 & 0.01 & 7.12 \\
\hline SRNL-SCS-2008-00003 & 3 & HWL & HWL-23 & 1 & 1 & 14 & P10LM11 & 2.49 & 0.063 & 0.577 & $<0.010$ & 0.12 & $<0.010$ & 0.07 & 0.042 & 7.32 \\
\hline SRNL-SCS-2008-00003 & 3 & HWL & HWL-23 & 1 & 1 & 15 & P10LM21 & 2.49 & 0.06 & 0.572 & $<0.010$ & 0.115 & $<0.010$ & 0.066 & 0.042 & 7.14 \\
\hline SRNL-SCS-2008-00003 & 3 & SB5 & SB5-13 & 1 & 1 & 16 & P14LM11 & 4.01 & 0.058 & 2.57 & $<0.010$ & 0.075 & $<0.010$ & 0.066 & 0.029 & 7.94 \\
\hline SRNL-SCS-2008-00003 & 3 & HWL & HWL-28 & 1 & 1 & 17 & P11LM21 & 2.43 & 0.068 & 0.673 & $<0.010$ & 0.172 & $<0.010$ & 0.067 & 0.041 & 9.26 \\
\hline SRNL-SCS-2008-00003 & 3 & SB5 & SB5-13 & 1 & 1 & 18 & P14LM21 & 4.03 & 0.057 & 2.59 & $<0.010$ & 0.085 & $<0.010$ & 0.065 & 0.029 & 7.85 \\
\hline SRNL-SCS-2008-00003 & 3 & Batch 1 & Batch 1 & 1 & 1 & 19 & BCHLM3-113 & 2.65 & 0.13 & 0.807 & $<0.010$ & 0.012 & $<0.010$ & 0.075 & 0.3 & 9.48 \\
\hline SRNL-SCS-2008-00003 & 3 & Ustd & U std & 1 & 1 & 20 & UstdLM3-113 & 2.16 & $<0.010$ & 0.862 & $<0.010$ & $<0.010$ & $<0.010$ & 0.163 & $<0.010$ & 9.78 \\
\hline SRNL-SCS-2008-00003 & 3 & Batch 1 & Batch 1 & 1 & 2 & 1 & BCHLM3-121 & 2.54 & 0.128 & 0.849 & $<0.010$ & 0.012 & $<0.010$ & 0.074 & 0.307 & 9.1 \\
\hline SRNL-SCS-2008-00003 & 3 & Ustd & U std & 1 & 2 & 2 & UstdLM3-121 & 2.1 & $<0.010$ & 0.887 & $<0.010$ & $<0.010$ & $<0.010$ & 0.158 & $<0.010$ & 9.17 \\
\hline SRNL-SCS-2008-00003 & 3 & HWL & HWL-23 & 1 & 2 & 3 & P10LM12 & 2.41 & 0.061 & 0.596 & $<0.010$ & 0.121 & $<0.010$ & 0.069 & 0.041 & 6.74 \\
\hline SRNL-SCS-2008-00003 & 3 & SB5 & SB5-14 & 1 & 2 & 4 & P08LM12 & 3.82 & 0.049 & 1.52 & $<0.010$ & 0.071 & $<0.010$ & 0.062 & 0.029 & 7.37 \\
\hline SRNL-SCS-2008-00003 & 3 & HWL & HWL-23 & 1 & 2 & 5 & P10LM22 & 2.39 & 0.059 & 0.607 & $<0.010$ & 0.118 & $<0.010$ & 0.066 & 0.042 & 7.02 \\
\hline SRNL-SCS-2008-00003 & 3 & HWL & HWL-28 & 1 & 2 & 6 & P11LM22 & 2.32 & 0.068 & 0.729 & $<0.010$ & 0.179 & $<0.010$ & 0.067 & 0.04 & 9.25 \\
\hline SRNL-SCS-2008-00003 & 3 & SB5 & SB5-13 & 1 & 2 & 7 & P14LM12 & 3.85 & 0.058 & 2.42 & $<0.010$ & 0.077 & $<0.010$ & 0.065 & 0.029 & 8 \\
\hline SRNL-SCS-2008-00003 & 3 & SB5 & SB5-20 & 1 & 2 & 8 & $\begin{array}{l}\text { P01LM12 } \\
\end{array}$ & 3.84 & 0.053 & 0.626 & $<0.010$ & 0.077 & $<0.010$ & 0.061 & 0.028 & 8.18 \\
\hline SRNL-SCS-2008-00003 & 3 & SB5 & SB5-18 & 1 & 2 & 9 & P09LM22 & 5.06 & 0.034 & 0.541 & 0.016 & 0.114 & $<0.010$ & 0.086 & $<0.010$ & 7.64 \\
\hline SRNL-SCS-2008-00003 & 3 & Batch 1 & Batch 1 & 1 & 2 & 10 & BCHLM3-122 & 2.56 & 0.129 & 0.889 & $<0.010$ & 0.012 & $<0.010$ & 0.075 & 0.319 & 9.92 \\
\hline SRNL-SCS-2008-00003 & 3 & Ustd & U std & 1 & 2 & 11 & UstdLM3-122 & 2.16 & $<0.010$ & 0.914 & $<0.010$ & $<0.010$ & $<0.010$ & 0.16 & $<0.010$ & 10.55 \\
\hline SRNL-SCS-2008-00003 & 3 & HWL & HWL-25 & 1 & 2 & 12 & P05LM12 & 2.17 & 0.058 & 0.636 & $<0.010$ & 0.147 & $<0.010$ & 0.055 & 0.031 & 7.41 \\
\hline SRNL-SCS-2008-00003 & 3 & SB5 & SB5-20 & 1 & 2 & 13 & P01LM22 & 3.97 & 0.051 & 0.608 & $<0.010$ & 0.141 & $<0.010$ & 0.059 & 0.027 & 7.44 \\
\hline SRNL-SCS-2008-00003 & 3 & HWL & HWL-25 & 1 & 2 & 14 & P05LM22 & 2.13 & 0.059 & 0.629 & $<0.010$ & 0.219 & $<0.010$ & 0.058 & 0.033 & 7.53 \\
\hline SRNL-SCS-2008-00003 & 3 & HWL & HWL-28 & 1 & 2 & 15 & P11LM12 & 2.35 & 0.066 & 0.74 & $<0.010$ & 0.195 & $<0.010$ & 0.068 & 0.041 & 8.39 \\
\hline SRNL-SCS-2008-00003 & 3 & SB5 & SB5-13 & 1 & 2 & 16 & P14LM22 & 3.85 & 0.056 & 2.51 & $<0.010$ & 0.087 & $<0.010$ & 0.064 & 0.028 & 7.29 \\
\hline SRNL-SCS-2008-00003 & 3 & SB5 & SB5-14 & 1 & 2 & 17 & $\begin{array}{l}\text { P08LM22 } \\
\end{array}$ & 3.86 & 0.048 & 1.55 & $<0.010$ & 0.069 & $<0.010$ & 0.061 & 0.029 & 7.04 \\
\hline SRNL-SCS-2008-00003 & 3 & SB5 & SB5-18 & 1 & 2 & 18 & P09LM12 & 5.1 & 0.036 & 0.534 & 0.016 & 0.116 & $<0.010$ & 0.089 & $<0.010$ & 6.69 \\
\hline SRNL-SCS-2008-00003 & 3 & Batch 1 & Batch 1 & 1 & 2 & 19 & BCHLM3-123 & 2.56 & 0.128 & 0.858 & $<0.010$ & 0.011 & $<0.010$ & 0.075 & 0.302 & 8.96 \\
\hline SRNL-SCS-2008-00003 & 3 & Ustd & U std & 1 & 2 & 20 & UstdLM3-123 & 2.13 & $<0.010$ & 0.923 & $<0.010$ & $<0.010$ & $<0.010$ & 0.162 & $<0.010$ & 9.29 \\
\hline SRNL-SCS-2008-00003 & 3 & Batch 1 & Batch 1 & 2 & 1 & 1 & BCHLM3-211 & 2.5 & 0.126 & 0.826 & $<0.010$ & 0.01 & $<0.010$ & 0.072 & 0.3 & 9.48 \\
\hline SRNL-SCS-2008-00003 & 3 & Ustd & U std & 2 & 1 & 2 & UstdLM3-211 & 2.06 & $<0.010$ & 0.877 & $<0.010$ & $<0.010$ & $<0.010$ & 0.158 & $<0.010$ & 9.77 \\
\hline SRNL-SCS-2008-00003 & 3 & HWL & HWL-27 & 2 & 1 & 3 & P13LM11 & 2.12 & 0.057 & 0.631 & $<0.010$ & 0.16 & $<0.010$ & 0.055 & 0.033 & 7.66 \\
\hline SRNL-SCS-2008-00003 & 3 & HWL & HWL-27 & 2 & 1 & 4 & P13LM21 & 2.15 & 0.055 & 0.644 & $<0.010$ & 0.164 & $<0.010$ & 0.053 & 0.032 & 7.56 \\
\hline
\end{tabular}


Table A2. Measured Elemental Concentrations (wt\%) for Samples Prepared Using Lithium Metaborate (Part 1, continued).

\begin{tabular}{|c|c|c|c|c|c|c|c|c|c|c|c|c|c|c|c|c|}
\hline Analytical Plan & Set & Type & Glass ID & Block & $\begin{array}{l}\text { Sub- } \\
\text { Block }\end{array}$ & Sequence & Lab ID & Al & Ba & Ca & Cd & $\mathrm{Ce}$ & Co & $\mathrm{Cr}$ & $\mathbf{C u}$ & $\mathbf{F e}$ \\
\hline SRNL-SCS-2008-00003 & 3 & $\mathrm{HWL}$ & HWL-26 & 2 & 1 & 5 & P03LM11 & 2.4 & 0.065 & 0.729 & $<0.010$ & 0.167 & $<0.010$ & 0.059 & 0.038 & 8.31 \\
\hline SRNL-SCS-2008-00003 & 3 & HWL & HWL-24 & 2 & 1 & 6 & P07LM11 & 2.39 & 0.065 & 0.606 & $<0.010$ & 0.089 & $<0.010$ & 0.06 & 0.042 & 7.36 \\
\hline SRNL-SCS-2008-00003 & 3 & SB5 & SB5-16 & 2 & 1 & 7 & $\begin{array}{l}\text { P06LM11 } \\
\end{array}$ & 3.87 & 0.051 & 0.591 & $<0.010$ & 0.077 & $<0.010$ & 0.059 & 0.027 & 7.55 \\
\hline $\begin{array}{l}\text { SRNL-SCS-2008-00003 } \\
\end{array}$ & 3 & HWL & HWL-24 & 2 & 1 & 8 & P07LM21 & 2.4 & 0.065 & 0.612 & $<0.010$ & 0.089 & $<0.010$ & 0.062 & 0.045 & 7.77 \\
\hline SRNL-SCS-2008-00003 & 3 & SB5 & SB5-19 & 2 & 1 & 9 & P04LM11 & 4.23 & 0.031 & 0.555 & 0.018 & 0.123 & $<0.010$ & 0.097 & $<0.010$ & 7.77 \\
\hline SRNL-SCS-2008-00003 & 3 & Batch 1 & Batch 1 & 2 & 1 & 10 & BCHLM3-212 & 2.53 & 0.127 & 0.838 & $<0.010$ & 0.01 & $<0.010$ & 0.073 & 0.303 & 10.21 \\
\hline SRNL-SCS-2008-00003 & 3 & Ustd & U std & 2 & 1 & 11 & UstdLM3-212 & 2.11 & $<0.010$ & 0.91 & $<0.010$ & $<0.010$ & $<0.010$ & 0.159 & $<0.010$ & 10.29 \\
\hline SRNL-SCS-2008-00003 & 3 & SB5 & SB5-15 & 2 & 1 & 12 & P12LM21 & 3.8 & 0.05 & 1.53 & $<0.010$ & 0.075 & $<0.010$ & 0.063 & 0.026 & 7.3 \\
\hline SRNL-SCS-2008-00003 & 3 & SB5 & SB5-19 & 2 & 1 & 13 & P04LM21 & 4.23 & 0.031 & 0.569 & 0.018 & 0.19 & $<0.010$ & 0.096 & $<0.010$ & 7.04 \\
\hline SRNL-SCS-2008-00003 & 3 & SB5 & SB5-16 & 2 & 1 & 14 & P06LM21 & 3.84 & 0.05 & 0.601 & $<0.010$ & 0.147 & $<0.010$ & 0.058 & 0.027 & 7.21 \\
\hline SRNL-SCS-2008-00003 & 3 & HWL & HWL-26 & 2 & 1 & 15 & P03LM21 & 2.36 & 0.066 & 0.731 & $<0.010$ & 0.252 & $<0.010$ & 0.059 & 0.038 & 7.62 \\
\hline SRNL-SCS-2008-00003 & 3 & SB5 & SB5-15 & 2 & 1 & 16 & P12LM11 & 3.83 & 0.052 & 1.6 & $<0.010$ & 0.076 & $<0.010$ & 0.064 & 0.026 & 6.73 \\
\hline SRNL-SCS-2008-00003 & 3 & SB5 & SB5-17 & 2 & 1 & 17 & P02LM11 & 5.85 & 0.031 & 0.477 & 0.012 & 0.104 & $<0.010$ & 0.075 & $<0.010$ & 5.64 \\
\hline SRNL-SCS-2008-00003 & 3 & SB5 & SB5-17 & 2 & 1 & 18 & P02LM21 & 5.74 & 0.032 & 0.479 & 0.013 & 0.179 & $<0.010$ & 0.078 & $<0.010$ & 5.69 \\
\hline SRNL-SCS-2008-00003 & 3 & Batch 1 & Batch 1 & 2 & 1 & 19 & BCHLM3-213 & 2.51 & 0.127 & 0.865 & $<0.010$ & 0.01 & $<0.010$ & 0.073 & 0.309 & 8.62 \\
\hline SRNL-SCS-2008-00003 & 3 & Ustd & U std & 2 & 1 & 20 & UstdLM3-213 & 2.08 & $<0.010$ & 0.905 & $<0.010$ & $<0.010$ & $<0.010$ & 0.157 & $<0.010$ & 8.24 \\
\hline SRNL-SCS-2008-00003 & 3 & Batch 1 & Batch 1 & 2 & 2 & 1 & BCHLM3-221 & 2.56 & 0.126 & 0.858 & $<0.010$ & 0.011 & $<0.010$ & 0.073 & 0.309 & 8.79 \\
\hline SRNL-SCS-2008-00003 & 3 & Ustd & U std & 2 & 2 & 2 & UstdLM3-221 & 2.08 & $<0.010$ & 0.877 & $<0.010$ & $<0.010$ & $<0.010$ & 0.157 & $<0.010$ & 9.47 \\
\hline SRNL-SCS-2008-00003 & 3 & $\mathrm{HWL}$ & HWL-24 & 2 & 2 & 3 & P07LM12 & 2.36 & 0.066 & 0.587 & $<0.010$ & 0.088 & $<0.010$ & 0.06 & 0.043 & 7.09 \\
\hline SRNL-SCS-2008-00003 & 3 & SB5 & SB5-19 & 2 & 2 & 4 & P04LM12 & 4.2 & 0.032 & 0.546 & 0.019 & 0.122 & $<0.010$ & 0.096 & $<0.010$ & 7.27 \\
\hline SRNL-SCS-2008-00003 & 3 & HWL & HWL-24 & 2 & 2 & 5 & P07LM22 & 2.39 & 0.065 & 0.587 & $<0.010$ & 0.087 & $<0.010$ & 0.061 & 0.045 & 7.25 \\
\hline SRNL-SCS-2008-00003 & 3 & SB5 & SB5-16 & 2 & 2 & 6 & P06LM22 & 3.83 & 0.052 & 0.566 & $<0.010$ & 0.142 & $<0.010$ & 0.058 & 0.027 & 7.29 \\
\hline SRNL-SCS-2008-00003 & 3 & SB5 & SB5-17 & 2 & 2 & 7 & P02LM12 & 5.86 & 0.032 & 0.455 & 0.013 & 0.101 & $<0.010$ & 0.074 & $<0.010$ & 6.09 \\
\hline SRNL-SCS-2008-00003 & 3 & SB5 & SB5-19 & 2 & 2 & 8 & P04LM22 & 4.21 & 0.033 & 0.538 & 0.019 & 0.186 & $<0.010$ & 0.097 & $<0.010$ & 7.08 \\
\hline SRNL-SCS-2008-00003 & 3 & SB5 & SB5-15 & 2 & 2 & 9 & P12LM12 & 3.82 & 0.053 & 1.56 & $<0.010$ & 0.075 & $<0.010$ & 0.064 & 0.026 & 6.97 \\
\hline SRNL-SCS-2008-00003 & 3 & Batch 1 & Batch 1 & 2 & 2 & 10 & BCHLM3-222 & 2.52 & 0.125 & 0.804 & $<0.010$ & 0.01 & $<0.010$ & 0.072 & 0.293 & 8.88 \\
\hline SRNL-SCS-2008-00003 & 3 & Ustd & U std & 2 & 2 & 11 & UstdLM3-222 & 2.05 & $<0.010$ & 0.874 & $<0.010$ & $<0.010$ & $<0.010$ & 0.157 & $<0.010$ & 9.32 \\
\hline SRNL-SCS-2008-00003 & 3 & HWL & HWL-26 & 2 & 2 & 12 & P03LM12 & 2.38 & 0.067 & 0.723 & $<0.010$ & 0.168 & $<0.010$ & 0.06 & 0.039 & 7.84 \\
\hline SRNL-SCS-2008-00003 & 3 & SB5 & SB5-17 & 2 & 2 & 13 & P02LM22 & 5.82 & 0.034 & 0.471 & 0.014 & 0.178 & $<0.010$ & 0.079 & $<0.010$ & 5.95 \\
\hline SRNL-SCS-2008-00003 & 3 & SB5 & SB5-15 & 2 & 2 & 14 & P12LM22 & 3.77 & 0.052 & 1.51 & $<0.010$ & 0.074 & $<0.010$ & 0.063 & 0.027 & 7.4 \\
\hline SRNL-SCS-2008-00003 & 3 & HWL & HWL-26 & 2 & 2 & 15 & P03LM22 & 2.32 & 0.066 & 0.709 & $<0.010$ & 0.248 & $<0.010$ & 0.058 & 0.039 & 7.83 \\
\hline SRNL-SCS-2008-00003 & 3 & HWL & HWL-27 & 2 & 2 & 16 & P13LM12 & 2.06 & 0.057 & 0.62 & $<0.010$ & 0.159 & $<0.010$ & 0.055 & 0.033 & 7.1 \\
\hline $\begin{array}{l}\text { SRNL-SCS-2008-00003 } \\
\end{array}$ & 3 & SB5 & SB5-16 & 2 & 2 & 17 & P06LM12 & 3.74 & 0.053 & 0.588 & $<0.010$ & 0.078 & $<0.010$ & 0.059 & 0.028 & 7.58 \\
\hline SRNL-SCS-2008-00003 & 3 & HWL & HWL-27 & 2 & 2 & 18 & P13LM22 & 2.09 & 0.056 & 0.631 & $<0.010$ & 0.165 & $<0.010$ & 0.054 & 0.033 & 7.56 \\
\hline SRNL-SCS-2008-00003 & 3 & Batch 1 & Batch 1 & 2 & 2 & 19 & BCHLM3-223 & 2.47 & 0.128 & 0.839 & $<0.010$ & 0.011 & $<0.010$ & 0.073 & 0.3 & 8.24 \\
\hline SRNL-SCS-2008-00003 & 3 & Ustd & U std & 2 & 2 & 20 & UstdLM3-223 & 2.05 & $<0.010$ & 0.891 & $<0.010$ & $<0.010$ & $<0.010$ & 0.158 & $<0.010$ & 8.13 \\
\hline
\end{tabular}


Table A2. Measured Elemental Concentrations (wt\%) for Samples Prepared Using Lithium Metaborate (Part 2).

\begin{tabular}{|c|c|c|c|c|c|c|c|c|c|c|c|c|c|c|c|c|}
\hline Analytical Plan & Set & Type & Glass ID & Block & $\begin{array}{c}\text { Sub- } \\
\text { Block }\end{array}$ & Sequence & Lab ID & $\mathbf{K}$ & La & Mg & Mn & $\mathrm{Na}$ & $\mathbf{N b}$ & $\mathrm{Ni}$ & $\mathbf{P}$ & $\mathbf{P b}$ \\
\hline SRNL-SCS-2008-00003 & 2 & Batch 1 & Batch 1 & 1 & 1 & 1 & BCHLM2-111 & 2.49 & $<0.100$ & 0.819 & 1.42 & 6.59 & 0.03 & 0.551 & $<0.100$ & $<0.020$ \\
\hline SRNL-SCS-2008-00003 & 2 & Ustd & U std & 1 & 1 & 2 & UstdLM2-111 & 2.22 & $<0.100$ & 0.71 & 2.27 & 8.36 & 0.048 & 0.793 & $<0.100$ & $<0.020$ \\
\hline SRNL-SCS-2008-00003 & 2 & SB5 & SB5-01 & 1 & 1 & 3 & N02LM11 & 0.014 & $<0.100$ & 0.263 & 1.51 & 9.83 & $<0.010$ & 0.705 & $<0.100$ & $<0.020$ \\
\hline SRNL-SCS-2008-00003 & 2 & SB5 & SB5-05 & 1 & 1 & 4 & N07LM11 & 0.013 & $<0.100$ & 0.296 & 1.66 & 10.1 & $<0.010$ & 0.802 & $<0.100$ & $<0.020$ \\
\hline SRNL-SCS-2008-00003 & 2 & SB5 & SB5-04 & 1 & 1 & 5 & N03LM21 & 0.012 & $<0.100$ & 0.273 & 1.5 & 10.8 & $<0.010$ & 0.724 & $<0.100$ & $<0.020$ \\
\hline SRNL-SCS-2008-00003 & 2 & SB5 & SB5-04 & 1 & 1 & 6 & N03LM11 & 0.012 & $<0.100$ & 0.276 & 1.5 & 10.8 & $<0.010$ & 0.729 & $<0.100$ & $<0.020$ \\
\hline SRNL-SCS-2008-00003 & 2 & SB5 & SB5-12 & 1 & 1 & 7 & N13LM21 & 0.016 & $<0.100$ & 0.326 & 1.71 & 10.5 & $<0.010$ & 0.882 & $<0.100$ & $<0.020$ \\
\hline SRNL-SCS-2008-00003 & 2 & SB5 & SB5-02 & 1 & 1 & 8 & N12LM21 & 0.012 & $<0.100$ & 0.271 & 1.43 & 9.96 & $<0.010$ & 0.72 & $<0.100$ & $<0.020$ \\
\hline SRNL-SCS-2008-00003 & 2 & SB5 & SB5-11 & 1 & 1 & 9 & N14LM11 & 0.016 & $<0.100$ & 0.316 & 1.63 & 9.95 & $<0.010$ & 0.864 & $<0.100$ & $<0.020$ \\
\hline SRNL-SCS-2008-00003 & 2 & SB5 & SB5-07 & 1 & 1 & 10 & N15LM21 & 0.018 & $<0.100$ & 0.295 & 1.6 & 10.1 & $<0.010$ & 0.778 & $<0.100$ & $<0.020$ \\
\hline SRNL-SCS-2008-00003 & 2 & Batch 1 & Batch 1 & 1 & 1 & 11 & BCHLM2-112 & 2.51 & $<0.100$ & 0.824 & 1.4 & 6.37 & 0.029 & 0.551 & $<0.100$ & $<0.020$ \\
\hline SRNL-SCS-2008-00003 & 2 & Ustd & U std & 1 & 1 & 12 & UstdLM1-112 & 2.22 & $<0.100$ & 0.705 & 2.28 & 8.42 & 0.047 & 0.795 & $<0.100$ & $<0.020$ \\
\hline SRNL-SCS-2008-00003 & 2 & SB5 & SB5-02 & 1 & 1 & 13 & N12LM11 & 0.011 & $<0.100$ & 0.271 & 1.47 & 9.83 & $<0.010$ & 0.732 & $<0.100$ & $<0.020$ \\
\hline SRNL-SCS-2008-00003 & 2 & SB5 & SB5-11 & 1 & 1 & 14 & N14LM21 & 0.017 & $<0.100$ & 0.322 & 1.75 & 9.67 & $<0.010$ & 0.884 & $<0.100$ & $<0.020$ \\
\hline SRNL-SCS-2008-00003 & 2 & SB4 & SB4VAR33 & 1 & 1 & 15 & N05LM21 & 0.126 & $<0.100$ & 0.619 & 1.76 & 9.26 & $<0.010$ & 0.432 & $<0.100$ & 0.026 \\
\hline SRNL-SCS-2008-00003 & 2 & SB5 & SB5-05 & 1 & 1 & 16 & N07LM21 & 0.017 & $<0.100$ & 0.289 & 1.56 & 10.1 & $<0.010$ & 0.777 & $<0.100$ & $<0.020$ \\
\hline SRNL-SCS-2008-00003 & 2 & SB5 & SB5-12 & 1 & 1 & 17 & N13LM11 & 0.016 & $<0.100$ & 0.325 & 1.71 & 10.2 & $<0.010$ & 0.879 & $<0.100$ & $<0.020$ \\
\hline SRNL-SCS-2008-00003 & 2 & SB4 & SB4VAR33 & 1 & 1 & 18 & N05LM11 & 0.117 & $<0.100$ & 0.635 & 1.79 & 9.26 & $<0.010$ & 0.447 & $<0.100$ & 0.026 \\
\hline SRNL-SCS-2008-00003 & 2 & SB5 & SB5-01 & 1 & 1 & 19 & N02LM21 & 0.013 & $<0.100$ & 0.271 & 1.53 & 10.19 & $<0.010$ & 0.739 & $<0.100$ & $<0.020$ \\
\hline SRNL-SCS-2008-00003 & 2 & SB5 & SB5-07 & 1 & 1 & 20 & N15LM11 & 0.014 & $<0.100$ & 0.305 & 1.65 & $\begin{aligned} 9.9 \\
\end{aligned}$ & $<0.010$ & 0.816 & $<0.100$ & $<0.020$ \\
\hline SRNL-SCS-2008-00003 & 2 & Batch 1 & Batch 1 & 1 & 1 & 21 & BCHLM2-113 & 2.51 & $<0.100$ & 0.827 & 1.41 & 6.69 & 0.029 & 0.553 & $<0.100$ & $<0.020$ \\
\hline SRNL-SCS-2008-00003 & 2 & Ustd & U std & 1 & 1 & 22 & UstdLM2-113 & 2.22 & $<0.100$ & 0.702 & 2.28 & 8.37 & 0.047 & 0.794 & $<0.100$ & $<0.020$ \\
\hline SRNL-SCS-2008-00003 & 2 & Batch 1 & Batch 1 & 1 & 2 & 1 & BCHLM2-121 & 2.49 & $<0.100$ & 0.828 & 1.3 & 6.6 & 0.029 & 0.551 & $<0.100$ & $<0.020$ \\
\hline SRNL-SCS-2008-00003 & 2 & Ustd & U std & 1 & 2 & 2 & UstdLM2-121 & 2.21 & $<0.100$ & 0.707 & 2.08 & 8.56 & 0.047 & 0.79 & $<0.100$ & $<0.020$ \\
\hline SRNL-SCS-2008-00003 & 2 & SB5 & SB5-12 & 1 & 2 & 3 & N13LM22 & 0.016 & $<0.100$ & 0.326 & 1.61 & 10.3 & $<0.010$ & 0.878 & $<0.100$ & $<0.020$ \\
\hline SRNL-SCS-2008-00003 & 2 & SB5 & SB5-05 & 1 & 2 & 4 & N07LM22 & 0.017 & $<0.100$ & 0.286 & 1.55 & 10 & $<0.010$ & 0.772 & $<0.100$ & $<0.020$ \\
\hline SRNL-SCS-2008-00003 & 2 & SB4 & SB4VAR33 & 1 & 2 & 5 & N05LM22 & 0.127 & $<0.100$ & 0.62 & 1.74 & 9.29 & $<0.010$ & 0.429 & $<0.100$ & 0.024 \\
\hline SRNL-SCS-2008-00003 & 2 & SB4 & SB4VAR33 & 1 & 2 & 6 & N05LM12 & 0.114 & $<0.100$ & 0.629 & 1.76 & 9.26 & $<0.010$ & 0.444 & $<0.100$ & 0.025 \\
\hline SRNL-SCS-2008-00003 & 2 & SB5 & SB5-01 & 1 & 2 & 7 & N02LM12 & 0.013 & $<0.100$ & 0.257 & 1.39 & 10.1 & $<0.010$ & 0.69 & $<0.100$ & $<0.020$ \\
\hline SRNL-SCS-2008-00003 & 2 & SB5 & SB5-04 & 1 & 2 & 8 & N03LM12 & 0.012 & $<0.100$ & 0.273 & 1.44 & 10.8 & $<0.010$ & 0.721 & $<0.100$ & $<0.020$ \\
\hline SRNL-SCS-2008-00003 & 2 & SB5 & SB5-01 & 1 & 2 & 9 & N02LM22 & 0.012 & $<0.100$ & 0.267 & 1.41 & 10.1 & $<0.010$ & 0.727 & $<0.100$ & $<0.020$ \\
\hline SRNL-SCS-2008-00003 & 2 & SB5 & SB5-07 & 1 & 2 & 10 & N15LM12 & 0.014 & $<0.100$ & 0.299 & 1.57 & 9.75 & $<0.010$ & 0.8 & $<0.100$ & $<0.020$ \\
\hline SRNL-SCS-2008-00003 & 2 & Batch 1 & Batch 1 & 1 & 2 & 11 & BCHLM2-122 & 2.47 & $<0.100$ & 0.817 & 1.34 & 6.42 & 0.027 & 0.545 & $<0.100$ & $<0.020$ \\
\hline SRNL-SCS-2008-00003 & 2 & Ustd & U std & 1 & 2 & 12 & UstdLM2-122 & 2.18 & $<0.100$ & 0.688 & 2.26 & 8.56 & 0.045 & 0.078 & $<0.100$ & $<0.020$ \\
\hline SRNL-SCS-2008-00003 & 2 & SB5 & SB5-04 & 1 & 2 & 13 & N03LM22 & 0.012 & $<0.100$ & 0.264 & 1.5 & 10.6 & $<0.010$ & 0.705 & $<0.100$ & $<0.020$ \\
\hline SRNL-SCS-2008-00003 & 2 & SB5 & SB5-11 & 1 & 2 & 14 & N14LM22 & 0.017 & $<0.100$ & 0.317 & 1.68 & 9.81 & $<0.010$ & 0.871 & $<0.100$ & $<0.020$ \\
\hline SRNL-SCS-2008-00003 & 2 & SB5 & SB5-05 & 1 & 2 & 15 & N07LM12 & 0.013 & $<0.100$ & 0.299 & 1.59 & 9.97 & $<0.010$ & 0.811 & $<0.100$ & $<0.020$ \\
\hline SRNL-SCS-2008-00003 & 2 & SB5 & SB5-02 & 1 & 2 & 16 & N12LM12 & 0.011 & $<0.100$ & 0.274 & 1.42 & 9.8 & $<0.010$ & 0.738 & $<0.100$ & $<0.020$ \\
\hline SRNL-SCS-2008-00003 & 2 & SB5 & SB5-11 & 1 & 2 & 17 & N14LM12 & 0.016 & $<0.100$ & 0.325 & 1.63 & 9.69 & $<0.010$ & 0.886 & $<0.100$ & $<0.020$ \\
\hline SRNL-SCS-2008-00003 & 2 & SB5 & SB5-02 & 1 & 2 & 18 & N12LM22 & 0.012 & $<0.100$ & 0.276 & 1.38 & 9.77 & $<0.010$ & 0.735 & $<0.100$ & $<0.020$ \\
\hline SRNL-SCS-2008-00003 & 2 & SB5 & SB5-12 & 1 & 2 & 19 & N13LM12 & 0.016 & $<0.100$ & 0.329 & 1.83 & 9.96 & $<0.010$ & 0.89 & $<0.100$ & $<0.020$ \\
\hline SRNL-SCS-2008-00003 & 2 & SB5 & SB5-07 & 1 & 2 & 20 & N15LM22 & 0.017 & $<0.100$ & 0.297 & 1.72 & 9.83 & $<0.010$ & 0.782 & $<0.100$ & $<0.020$ \\
\hline SRNL-SCS-2008-00003 & 2 & Batch 1 & Batch 1 & 1 & 2 & 21 & BCHLM2-123 & 2.45 & $<0.100$ & 0.832 & 1.44 & 6.62 & 0.028 & 0.557 & $<0.100$ & $<0.020$ \\
\hline
\end{tabular}


Table A2. Measured Elemental Concentrations (wt\%) for Samples Prepared Using Lithium Metaborate (Part 2, continued).

\begin{tabular}{|c|c|c|c|c|c|c|c|c|c|c|c|c|c|c|c|c|}
\hline Analytical Plan & Set & Type & Glass ID & Block & $\begin{array}{c}\text { Sub- } \\
\text { Block }\end{array}$ & Sequence & Lab ID & $\mathbf{K}$ & La & Mg & Mn & $\mathrm{Na}$ & $\mathbf{N b}$ & $\mathrm{Ni}$ & $\mathbf{P}$ & $\mathbf{P b}$ \\
\hline SRNL-SCS-2008-00003 & 2 & Ustd & U std & 1 & 2 & 22 & UstdLM2-123 & 2.16 & $<0.100$ & 0.724 & 2.3 & 8.42 & 0.047 & 0.804 & $<0.100$ & $<0.020$ \\
\hline SRNL-SCS-2008-00003 & 22 & Batch 1 & Batch 1 & 2 & 1 & 1 & BCHLM2-211 & 2.6 & $<0.100$ & 0.826 & 1.35 & 6.62 & 0.032 & 0.557 & $<0.100$ & $<0.020$ \\
\hline SRNL-SCS-2008-00003 & 2 & Ustd & U std & 2 & 1 & 2 & UstdLM2-211 & 2.31 & $<0.100$ & 0.704 & 2.21 & 8.55 & 0.051 & 0.799 & $<0.100$ & $<0.020$ \\
\hline SRNL-SCS-2008-00003 & 2 & SB4 & SB4VAR32 & 2 & 1 & 3 & N11LM21 & 0.119 & $<0.100$ & 0.563 & 1.58 & 8.97 & $<0.010$ & 0.399 & $<0.100$ & $<0.020$ \\
\hline SRNL-SCS-2008-00003 & 2 & SB4 & SB4VAR31 & 2 & 1 & 4 & N06LM11 & 0.091 & $<0.100$ & 0.53 & 1.39 & 8.56 & $<0.010$ & 0.38 & $<0.100$ & $<0.020$ \\
\hline SRNL-SCS-2008-00003 & 2 & SB5 & SB5-03 & 2 & 1 & 5 & N09LM11 & 0.01 & $<0.100$ & 0.271 & 1.47 & 9.9 & $<0.010$ & 0.726 & $<0.100$ & $<0.020$ \\
\hline SRNL-SCS-2008-00003 & 2 & SB5 & SB5-09 & 2 & 1 & 6 & N04LM11 & 0.021 & $<0.100$ & 0.315 & 1.75 & 9.82 & $<0.010$ & 0.836 & $<0.100$ & $<0.020$ \\
\hline SRNL-SCS-2008-00003 & 2 & SB5 & SB5-03 & 2 & 1 & 7 & $\begin{array}{l}\text { N09LM21 } \\
\end{array}$ & 0.008 & $<0.100$ & 0.278 & 1.55 & 9.93 & $<0.010$ & 0.753 & $<0.100$ & $<0.020$ \\
\hline SRNL-SCS-2008-00003 & 22 & SB4 & SB4VAR32 & 2 & 1 & 8 & N11LM11 & 0.116 & $<0.100$ & 0.569 & 1.74 & 8.93 & $<0.010$ & 0.408 & $<0.100$ & $<0.020$ \\
\hline SRNL-SCS-2008-00003 & 2 & SB5 & SB5-10 & 2 & 1 & 9 & N08ILM11 & 0.019 & $<0.100$ & 0.316 & 1.85 & 9.87 & $<0.010$ & 0.849 & $<0.100$ & $<0.020$ \\
\hline SRNL-SCS-2008-00003 & 22 & Batch 1 & Batch 1 & 2 & 1 & 10 & BCHLM2-212 & 2.6 & $<0.100$ & 0.818 & 1.42 & 6.59 & 0.031 & 0.553 & $<0.100$ & $<0.020$ \\
\hline SRNL-SCS-2008-00003 & 2 & Ustd & U std & 2 & 1 & 11 & UstdLM2-212 & 2.36 & $<0.100$ & 0.719 & 2.19 & 8.58 & 0.051 & 0.812 & $<0.100$ & $<0.020$ \\
\hline SRNL-SCS-2008-00003 & 2 & SB5 & SB5-08 & 2 & 1 & 12 & N10LM21 & 0.012 & $<0.100$ & 0.299 & 1.58 & 9.96 & $<0.010$ & 0.808 & $<0.100$ & $<0.020$ \\
\hline SRNL-SCS-2008-00003 & 2 & SB5 & SB5-06 & 2 & 1 & 13 & N01LM11 & 0.013 & $<0.100$ & 0.305 & 1.65 & 9.91 & $<0.010$ & 0.819 & $<0.100$ & $<0.020$ \\
\hline SRNL-SCS-2008-00003 & 22 & SB4 & SB4VAR31 & 2 & 1 & 14 & N06LM21 & 0.106 & $<0.100$ & 0.514 & 1.49 & 8.69 & $<0.010$ & 0.362 & $<0.100$ & $<0.020$ \\
\hline SRNL-SCS-2008-00003 & 2 & SB5 & SB5-10 & 2 & 1 & 15 & N08LM21 & 0.018 & $<0.100$ & 0.32 & 1.72 & 9.8 & $<0.010$ & 0.86 & $<0.100$ & $<0.020$ \\
\hline SRNL-SCS-2008-00003 & 22 & SB5 & SB5-08 & 2 & 1 & 16 & N10LM11 & 0.078 & $<0.100$ & 0.31 & 1.61 & 10.4 & $<0.010$ & 0.877 & $<0.100$ & $<0.020$ \\
\hline SRNL-SCS-2008-00003 & 2 & SB5 & SB5-09 & 2 & 1 & 17 & N04LM21 & 0.016 & $<0.100$ & 0.329 & 1.68 & 9.8 & $<0.010$ & 0.89 & $<0.100$ & $<0.020$ \\
\hline SRNL-SCS-2008-00003 & 2 & SB5 & SB5-06 & 2 & 1 & 18 & N01LM21 & 0.013 & $<0.100$ & 0.278 & 1.52 & 10.2 & $<0.010$ & 0.74 & $<0.100$ & $<0.020$ \\
\hline SRNL-SCS-2008-00003 & 22 & Batch 1 & Batch 1 & 2 & 1 & 19 & BCHLM2-213 & 2.63 & $<0.100$ & 0.824 & 1.41 & 6.66 & 0.031 & 0.558 & $<0.100$ & $<0.020$ \\
\hline SRNL-SCS-2008-00003 & 2 & Ustd & U std & 2 & 1 & 20 & UstdLM2-213 & 2.33 & $<0.100$ & 0.71 & 2.35 & 8.49 & 0.051 & 0.81 & $<0.100$ & $<0.020$ \\
\hline SRNL-SCS-2008-00003 & 2 & Batch 1 & Batch 1 & 2 & 2 & 1 & BCHLM2-221 & 2.53 & $<0.100$ & 0.836 & 1.31 & 6.91 & 0.028 & 0.557 & $<0.100$ & $<0.020$ \\
\hline SRNL-SCS-2008-00003 & 2 & Ustd & U std & 2 & 2 & 2 & UstdLM2-221 & 2.23 & $<0.100$ & 0.721 & 2.22 & 9.13 & 0.047 & 0.805 & $<0.100$ & $<0.020$ \\
\hline SRNL-SCS-2008-00003 & 2 & SB4 & SB4VAR31 & 2 & 2 & 3 & N06LM22 & 0.104 & $<0.100$ & 0.517 & 1.41 & 9.02 & $<0.010$ & 0.357 & $<0.100$ & $<0.020$ \\
\hline SRNL-SCS-2008-00003 & 2 & SB4 & SB4VAR32 & 2 & 2 & 4 & N11LM22 & 0.11 & $<0.100$ & 0.578 & 1.64 & 9.24 & $<0.010$ & 0.405 & $<0.100$ & $<0.020$ \\
\hline SRNL-SCS-2008-00003 & 2 & SB5 & $\begin{array}{l}\text { SB5-10 } \\
\end{array}$ & 2 & 2 & 5 & N08LM12 & 0.018 & $<0.100$ & 0.314 & 1.74 & 10.1 & $<0.010$ & 0.839 & $<0.100$ & $<0.020$ \\
\hline SRNL-SCS-2008-00003 & 2 & SB5 & SB5-06 & 2 & 2 & 6 & N01LM22 & 0.012 & $<0.100$ & 0.297 & 1.6 & 10.2 & $<0.010$ & 0.789 & $<0.100$ & $<0.020$ \\
\hline SRNL-SCS-2008-00003 & 22 & SB4 & SB4VAR31 & 2 & 2 & 7 & N06LM12 & 0.087 & $<0.100$ & 0.538 & 1.41 & 8.97 & $<0.010$ & 0.38 & $<0.100$ & $<0.020$ \\
\hline SRNL-SCS-2008-00003 & 22 & SB5 & SB5-09 & 2 & 2 & 8 & N04LM12 & 0.021 & $<0.100$ & 0.32 & 1.71 & 10.4 & $<0.010$ & 0.843 & $<0.100$ & $<0.020$ \\
\hline SRNL-SCS-2008-00003 & 2 & SB5 & SB5-08 & 2 & 2 & 9 & N10LM12 & 0.073 & $<0.100$ & 0.314 & 1.59 & 10.9 & $<0.010$ & 0.881 & $<0.100$ & $<0.020$ \\
\hline SRNL-SCS-2008-00003 & 22 & Batch 1 & Batch 1 & 2 & 2 & 10 & BCHLM2-222 & 2.53 & $<0.100$ & 0.843 & 1.31 & 7.1 & 0.027 & 0.562 & $<0.100$ & $<0.020$ \\
\hline SRNL-SCS-2008-00003 & 2 & Ustd & U std & 2 & 2 & 11 & UstdLM2-222 & 2.26 & $<0.100$ & 0.731 & 2.2 & 9.17 & 0.047 & 0.812 & $<0.100$ & $<0.020$ \\
\hline SRNL-SCS-2008-00003 & 2 & SB5 & SB5-03 & 2 & 2 & 12 & N09LM12 & 0.008 & $<0.100$ & 0.282 & 1.41 & 10.7 & $<0.010$ & 0.747 & $<0.100$ & $<0.020$ \\
\hline SRNL-SCS-2008-00003 & 2 & SB5 & SB5-10 & 2 & 2 & 13 & N08LM22 & 0.015 & $<0.100$ & 0.33 & 1.71 & 10.4 & $<0.010$ & 0.877 & $<0.100$ & $<0.020$ \\
\hline SRNL-SCS-2008-00003 & 22 & SB5 & SB5-09 & 2 & 2 & 14 & N04LM22 & 0.014 & $<0.100$ & 0.348 & 1.57 & 10.3 & $<0.010$ & 0.926 & $<0.100$ & $<0.020$ \\
\hline SRNL-SCS-2008-00003 & 2 & SB5 & SB5-03 & 2 & 2 & 15 & N09LM22 & 0.007 & $<0.100$ & 0.298 & 1.36 & 10.4 & $<0.010$ & 0.796 & $<0.100$ & $<0.020$ \\
\hline SRNL-SCS-2008-00003 & 22 & SB5 & SB5-08 & 2 & 2 & 16 & N10LM22 & 0.01 & $<0.100$ & 0.314 & 1.48 & 10.4 & $<0.010$ & 0.838 & $<0.100$ & $<0.020$ \\
\hline SRNL-SCS-2008-00003 & 2 & SB5 & SB5-06 & 2 & 2 & 17 & N01LM12 & 0.012 & $<0.100$ & 0.315 & 1.56 & 10.4 & $<0.010$ & 0.84 & $<0.100$ & $<0.020$ \\
\hline SRNL-SCS-2008-00003 & 2 & SB4 & SB4VAR32 & 2 & 2 & 18 & N11LM12 & 0.106 & $<0.100$ & 0.6 & 1.55 & 9.61 & $<0.010$ & 0.419 & $<0.100$ & $<0.020$ \\
\hline SRNL-SCS-2008-00003 & 2 & Batch 1 & Batch 1 & 2 & 2 & 19 & BCHLM2-223 & 2.48 & $<0.100$ & 0.888 & 1.34 & 6.93 & 0.028 & 0.586 & $<0.100$ & $<0.020$ \\
\hline SRNL-SCS-2008-00003 & 22 & Ustd & U std & 2 & 2 & 20 & UstdLM2-223 & 2.17 & $<0.100$ & 0.767 & 2.18 & 9.21 & 0.048 & 0.851 & $<0.100$ & $<0.020$ \\
\hline SRNL-SCS-2008-00003 & 3 & Batch 1 & Batch 1 & 1 & 1 & 1 & BCHLM3-111 & 2.54 & $<0.100$ & 0.817 & 1.4 & 6.79 & 0.033 & 0.549 & $<0.100$ & $<0.020$ \\
\hline SRNL-SCS-2008-00003 & 3 & Ustd & U std & 1 & 1 & 2 & UstdLM3-111 & 2.38 & $<0.100$ & 0.681 & 2.31 & 8.98 & 0.049 & 0.763 & $<0.100$ & $<0.020$ \\
\hline $\begin{array}{l}\text { SRNL-SCS-2008-00003 } \\
\end{array}$ & 3 & $\mathrm{HWL}$ & HWL-28 & 1 & 1 & 3 & P11LM11 & 0.05 & $<0.100$ & 0.091 & 2.26 & 9.35 & 0.512 & 0.251 & $<0.100$ & 0.109 \\
\hline
\end{tabular}


Table A2. Measured Elemental Concentrations (wt\%) for Samples Prepared Using Lithium Metaborate (Part 2, continued).

\begin{tabular}{|c|c|c|c|c|c|c|c|c|c|c|c|c|c|c|c|c|}
\hline Analytical Plan & Set & Type & Glass ID & Block & $\begin{array}{c}\text { Sub- } \\
\text { Block }\end{array}$ & Sequence & Lab ID & $\mathbf{K}$ & La & Mg & Mn & $\mathrm{Na}$ & $\mathbf{N b}$ & $\mathrm{Ni}$ & $\mathbf{P}$ & $\mathbf{P b}$ \\
\hline SRNL-SCS-2008-00003 & 3 & HWL & HWL-25 & 1 & 1 & 4 & P05LM21 & 0.048 & $<0.100$ & 0.089 & 1.94 & 5.56 & 0.419 & 0.29 & $<0.100$ & 0.095 \\
\hline SRNL-SCS-2008-00003 & 3 & SB5 & SB5-14 & 1 & 1 & 5 & P08LM21 & 0.077 & $<0.100$ & 0.268 & 1.93 & 10.85 & 0.022 & 0.782 & $<0.100$ & 0.034 \\
\hline SRNL-SCS-2008-00003 & 3 & SB5 & SB5-20 & 1 & 1 & 6 & $\begin{array}{l}\text { P01LM11 } \\
\end{array}$ & 0.063 & $<0.100$ & 0.29 & 1.98 & 10.83 & 0.021 & 0.856 & $<0.100$ & 0.034 \\
\hline SRNL-SCS-2008-00003 & 3 & SB5 & $\begin{array}{l}\text { SB5-14 } \\
\end{array}$ & 1 & 1 & 7 & P08LM11 & 0.066 & $<0.100$ & 0.282 & 2 & 10.77 & 0.023 & 0.837 & $<0.100$ & 0.037 \\
\hline SRNL-SCS-2008-00003 & 3 & SB5 & SB5-20 & 1 & 1 & 8 & P01LM21 & 0.067 & $<0.100$ & 0.289 & 2 & 10.91 & 0.021 & 0.851 & $<0.100$ & 0.034 \\
\hline SRNL-SCS-2008-00003 & 3 & HWL & HWL-25 & 1 & 1 & 9 & P05LM11 & 0.043 & $<0.100$ & 0.092 & 2.05 & 5.78 & 0.455 & 0.23 & $<0.100$ & 0.097 \\
\hline SRNL-SCS-2008-00003 & 3 & Batch 1 & Batch 1 & 1 & 1 & 10 & BCHLM3-112 & 2.45 & $<0.100$ & 0.853 & 1.48 & 6.8 & 0.034 & 0.57 & $<0.100$ & $<0.020$ \\
\hline SRNL-SCS-2008-00003 & 3 & Ustd & U std & 1 & 1 & 11 & UstdLM3-112 & 2.28 & $<0.100$ & 0.716 & 2.42 & 9.07 & 0.05 & 0.797 & $<0.100$ & $<0.020$ \\
\hline SRNL-SCS-2008-00003 & 3 & SB5 & SB5-18 & 1 & 1 & 12 & P09LM21 & 0.018 & $<0.100$ & 0.288 & 1.75 & 10.93 & $<0.020$ & 0.764 & $<0.100$ & $<0.020$ \\
\hline SRNL-SCS-2008-00003 & 3 & SB5 & SB5-18 & 1 & 1 & 13 & P09LM11 & 0.016 & $<0.100$ & 0.301 & 1.77 & 10.89 & $<0.020$ & 0.806 & $<0.100$ & $<0.020$ \\
\hline SRNL-SCS-2008-00003 & 3 & HWL & HWL-23 & 1 & 1 & 14 & P10LM11 & 0.047 & $<0.100$ & 0.088 & 2.84 & 6.14 & 0.585 & 0.486 & $<0.100$ & 0.088 \\
\hline SRNL-SCS-2008-00003 & 3 & HWL & HWL-23 & 1 & 1 & 15 & P10LM21 & 0.052 & $<0.100$ & 0.084 & 2.79 & 6.04 & 0.554 & 0.458 & $<0.100$ & 0.083 \\
\hline SRNL-SCS-2008-00003 & 3 & SB5 & SB5-13 & 1 & 1 & 16 & P14LM11 & 0.06 & $<0.100$ & 0.291 & 2.05 & 11.15 & 0.026 & 0.875 & $<0.100$ & 0.038 \\
\hline SRNL-SCS-2008-00003 & 3 & HWL & HWL-28 & 1 & 1 & 17 & P11LM21 & 0.045 & $<0.100$ & 0.095 & 2.34 & 9.75 & 0.521 & 0.259 & $<0.100$ & 0.112 \\
\hline SRNL-SCS-2008-00003 & 3 & SB5 & SB5-13 & 1 & 1 & 18 & P14LM21 & 0.061 & $<0.100$ & 0.288 & 2.02 & 11.18 & 0.026 & 0.866 & $<0.100$ & 0.038 \\
\hline SRNL-SCS-2008-00003 & 3 & Batch 1 & Batch 1 & 1 & 1 & 19 & BCHLM3-113 & 2.401 & $<0.100$ & 0.855 & 1.43 & 7.13 & 0.033 & 0.57 & $<0.100$ & $<0.020$ \\
\hline SRNL-SCS-2008-00003 & 3 & Ustd & U std & 1 & 1 & 20 & UstdLM3-113 & 2.248 & $<0.100$ & 0.715 & 2.33 & 8.99 & 0.05 & 0.798 & $<0.100$ & $<0.020$ \\
\hline SRNL-SCS-2008-00003 & 3 & Batch 1 & Batch 1 & 1 & 2 & 1 & BCHLM3-121 & 2.61 & $<0.100$ & 0.833 & 1.37 & 6.74 & 0.031 & 0.561 & $<0.100$ & $<0.020$ \\
\hline SRNL-SCS-2008-00003 & 3 & Ustd & U std & 1 & 2 & 2 & UstdLM3-121 & 2.41 & $<0.100$ & 0.691 & 2.18 & 8.7 & 0.048 & 0.78 & $<0.100$ & $<0.020$ \\
\hline SRNL-SCS-2008-00003 & 3 & HWL & HWL-23 & 1 & 2 & 3 & P10LM12 & 0.048 & $<0.100$ & 0.086 & 2.61 & 5.9 & 0.599 & 0.475 & $<0.100$ & 0.086 \\
\hline SRNL-SCS-2008-00003 & 3 & SB5 & SB5-14 & 1 & 2 & 4 & P08LM12 & 0.068 & $<0.100$ & 0.272 & 1.85 & 10.49 & 0.02 & 0.816 & $<0.100$ & 0.036 \\
\hline SRNL-SCS-2008-00003 & 3 & HWL & HWL-23 & 1 & 2 & 5 & P10LM22 & 0.057 & $<0.100$ & 0.082 & 2.72 & 5.79 & 0.577 & 0.455 & $<0.100$ & 0.082 \\
\hline SRNL-SCS-2008-00003 & 3 & HWL & HWL-28 & 1 & 2 & 6 & P11LM22 & 0.051 & $<0.100$ & 0.095 & 2.31 & 9 & 0.548 & 0.261 & $<0.100$ & 0.112 \\
\hline SRNL-SCS-2008-00003 & 3 & SB5 & SB5-13 & 1 & 2 & 7 & P14LM12 & 0.067 & $<0.100$ & 0.289 & 2.04 & 10.31 & 0.024 & 0.876 & $<0.100$ & 0.039 \\
\hline SRNL-SCS-2008-00003 & 3 & SB5 & SB5-20 & 1 & 2 & 8 & P01LM12 & 0.07 & $<0.100$ & 0.291 & 2.08 & 10.32 & 0.019 & 0.868 & $<0.100$ & 0.035 \\
\hline SRNL-SCS-2008-00003 & 3 & SB5 & SB5-18 & 1 & 2 & 9 & P09LM22 & 0.02 & $<0.100$ & 0.287 & 1.88 & 10.09 & $<0.020$ & 0.767 & $<0.100$ & $<0.020$ \\
\hline SRNL-SCS-2008-00003 & 3 & Batch 1 & Batch 1 & 1 & 2 & 10 & BCHLM3-122 & 2.73 & $<0.100$ & 0.844 & 1.48 & 6.63 & 0.031 & 0.569 & $<0.100$ & $<0.020$ \\
\hline SRNL-SCS-2008-00003 & 3 & Ustd & U std & 1 & 2 & 11 & UstdLM3-122 & 2.48 & $<0.100$ & 0.697 & 2.49 & 8.44 & 0.048 & 0.782 & $<0.100$ & $<0.020$ \\
\hline SRNL-SCS-2008-00003 & 3 & HWL & HWL-25 & 1 & 2 & 12 & P05LM12 & 0.045 & $<0.100$ & 0.09 & 1.93 & 5.76 & 0.468 & 0.227 & $<0.100$ & 0.097 \\
\hline SRNL-SCS-2008-00003 & 3 & SB5 & SB5-20 & 1 & 2 & 13 & P01LM22 & 0.069 & $<0.100$ & 0.285 & 1.93 & 11.12 & 0.019 & 0.844 & $<0.100$ & 0.033 \\
\hline SRNL-SCS-2008-00003 & 3 & HWL & HWL-25 & 1 & 2 & 14 & P05LM22 & 0.05 & $<0.100$ & 0.09 & 1.9 & 5.49 & 0.44 & 0.294 & $<0.100$ & 0.097 \\
\hline SRNL-SCS-2008-00003 & 3 & HWL & HWL-28 & 1 & 2 & 15 & P11LM12 & 0.054 & $<0.100$ & 0.092 & 2.12 & 9.29 & 0.539 & 0.254 & $<0.100$ & 0.109 \\
\hline SRNL-SCS-2008-00003 & 3 & SB5 & SB5-13 & 1 & 2 & 16 & P14LM22 & 0.068 & $<0.100$ & 0.28 & 1.88 & 10.72 & 0.024 & 0.846 & $<0.100$ & 0.037 \\
\hline SRNL-SCS-2008-00003 & 3 & SB5 & SB5-14 & 1 & 2 & 17 & P08LM22 & 0.08 & $<0.100$ & 0.265 & 1.79 & 10.84 & 0.019 & 0.784 & $<0.100$ & 0.036 \\
\hline SRNL-SCS-2008-00003 & 3 & SB5 & $\begin{array}{l}\text { SB5-18 } \\
\end{array}$ & 1 & 2 & 18 & P09LM12 & 0.017 & $<0.100$ & 0.296 & 1.65 & 10.5 & $<0.020$ & 0.797 & $<0.100$ & $<0.020$ \\
\hline SRNL-SCS-2008-00003 & 3 & Batch 1 & Batch 1 & 1 & 2 & 19 & BCHLM3-123 & 2.65 & $<0.100$ & 0.838 & 1.35 & 6.72 & 0.031 & 0.567 & $<0.100$ & $<0.020$ \\
\hline SRNL-SCS-2008-00003 & 3 & Ustd & U std & 1 & 2 & 20 & UstdLM3-123 & 2.5 & $<0.100$ & 0.7 & 2.22 & 8.85 & 0.049 & 0.788 & $<0.100$ & $<0.020$ \\
\hline SRNL-SCS-2008-00003 & 3 & Batch 1 & Batch 1 & 2 & 1 & 1 & BCHLM3-211 & 2.54 & $<0.100$ & 0.829 & 1.43 & 6.47 & 0.036 & 0.557 & $<0.100$ & $<0.020$ \\
\hline SRNL-SCS-2008-00003 & 3 & Ustd & U std & 2 & 1 & 2 & UstdLM3-211 & 2.36 & $<0.100$ & 0.691 & 2.31 & 8.51 & 0.052 & 0.775 & $<0.100$ & $<0.020$ \\
\hline SRNL-SCS-2008-00003 & 3 & HWL & HWL-27 & 2 & 1 & 3 & P13LM11 & 0.037 & $<0.100$ & 0.081 & 1.94 & 9.18 & 0.461 & 0.225 & $<0.100$ & 0.1 \\
\hline SRNL-SCS-2008-00003 & 3 & HWL & HWL-27 & 2 & 1 & 4 & P13LM21 & 0.042 & $<0.100$ & 0.079 & 1.92 & 9.22 & 0.447 & 0.218 & $<0.100$ & 0.096 \\
\hline SRNL-SCS-2008-00003 & 3 & HWL & HWL-26 & 2 & 1 & 5 & P03LM11 & 0.045 & $<0.100$ & 0.093 & 2.2 & 6.35 & 0.521 & 0.253 & $<0.100$ & 0.109 \\
\hline SRNL-SCS-2008-00003 & 3 & HWL & HWL-24 & 2 & 1 & 6 & P07LM11 & 0.041 & $<0.100$ & 0.082 & 2.74 & 9.04 & 0.598 & 0.488 & $<0.100$ & 0.086 \\
\hline $\begin{array}{l}\text { SRNL-SCS-2008-00003 } \\
\end{array}$ & 3 & SB5 & SB5-16 & 2 & 1 & 7 & $\begin{array}{l}\text { P06LM11 } \\
\end{array}$ & 0.055 & $<0.100$ & 0.291 & 1.91 & 11.16 & 0.026 & 0.881 & $<0.100$ & 0.036 \\
\hline
\end{tabular}


Table A2. Measured Elemental Concentrations (wt\%) for Samples Prepared Using Lithium Metaborate (Part 2, continued).

\begin{tabular}{|c|c|c|c|c|c|c|c|c|c|c|c|c|c|c|c|c|}
\hline Analytical Plan & Set & Type & Glass ID & Block & $\begin{array}{c}\text { Sub- } \\
\text { Block }\end{array}$ & Sequence & Lab ID & $\mathbf{K}$ & La & Mg & Mn & $\mathrm{Na}$ & $\mathbf{N b}$ & $\mathrm{Ni}$ & $\mathbf{P}$ & $\mathbf{P b}$ \\
\hline SRNL-SCS-2008-00003 & 3 & HWL & HWL-24 & 2 & 1 & 8 & $\begin{array}{l}\text { P07LM21 } \\
\end{array}$ & 0.045 & $<0.100$ & 0.082 & 2.89 & 9.05 & 0.602 & 0.483 & $<0.100$ & 0.087 \\
\hline SRNL-SCS-2008-00003 & 3 & SB5 & SB5-19 & 2 & 1 & 9 & P04LM11 & 0.014 & $<0.100$ & 0.317 & 1.89 & 11.17 & 0.009 & 0.867 & $<0.100$ & $<0.020$ \\
\hline SRNL-SCS-2008-00003 & 3 & Batch 1 & Batch 1 & 2 & 1 & 10 & BCHLM3-212 & 2.58 & $<0.100$ & 0.836 & 1.52 & 6.48 & 0.035 & 0.559 & $<0.100$ & $<0.020$ \\
\hline SRNL-SCS-2008-00003 & 3 & Ustd & U std & 2 & 1 & 11 & UstdLM3-212 & 2.43 & $<0.100$ & 0.698 & 2.44 & 8.65 & 0.053 & 0.78 & $<0.100$ & $<0.020$ \\
\hline SRNL-SCS-2008-00003 & 3 & SB5 & SB5-15 & 2 & 1 & 12 & P12LM21 & 0.06 & $<0.100$ & 0.28 & 1.8 & 10.7 & 0.028 & 0.845 & $<0.100$ & 0.034 \\
\hline SRNL-SCS-2008-00003 & 3 & SB5 & SB5-19 & 2 & 1 & 13 & P04LM21 & 0.013 & $<0.100$ & 0.316 & 1.7 & 11.27 & 0.01 & 0.865 & $<0.100$ & $<0.020$ \\
\hline SRNL-SCS-2008-00003 & 3 & SB5 & SB5-16 & 2 & 1 & 14 & P06LM21 & 0.059 & $<0.100$ & 0.287 & 1.84 & 11.03 & 0.026 & 0.856 & $<0.100$ & 0.035 \\
\hline SRNL-SCS-2008-00003 & 3 & HWL & HWL-26 & 2 & 1 & 15 & P03LM21 & 0.046 & $<0.100$ & 0.094 & 2.02 & 6.45 & 0.529 & 0.255 & $<0.100$ & 0.108 \\
\hline SRNL-SCS-2008-00003 & 3 & SB5 & SB5-15 & 2 & 1 & 16 & P12LM11 & 0.058 & $<0.100$ & 0.289 & 1.71 & 10.99 & 0.029 & 0.868 & $<0.100$ & 0.035 \\
\hline SRNL-SCS-2008-00003 & 3 & SB5 & SB5-17 & 2 & 1 & 17 & $\begin{array}{l}\text { P02LM11 } \\
\end{array}$ & 0.013 & $<0.100$ & 0.266 & 1.39 & 9.17 & $<0.020$ & 0.705 & $<0.100$ & $<0.020$ \\
\hline SRNL-SCS-2008-00003 & 3 & SB5 & SB5-17 & 2 & 1 & 18 & P02LM21 & 0.009 & $<0.100$ & 0.279 & 1.4 & 8.8 & $<0.020$ & 0.753 & $<0.100$ & $<0.020$ \\
\hline SRNL-SCS-2008-00003 & 3 & Batch 1 & Batch 1 & 2 & 1 & 19 & BCHLM3-213 & 2.63 & $<0.100$ & 0.826 & 1.3 & 6.7 & 0.036 & 0.558 & $<0.100$ & $<0.020$ \\
\hline SRNL-SCS-2008-00003 & 3 & Ustd & U std & 2 & 1 & 20 & UstdLM3-213 & 2.42 & $<0.100$ & 0.686 & 1.97 & 8.81 & 0.052 & 0.769 & $<0.100$ & $<0.020$ \\
\hline SRNL-SCS-2008-00003 & 3 & Batch 1 & Batch 1 & 2 & 2 & 1 & BCHLM3-221 & 2.63 & $<0.100$ & 0.817 & 1.33 & 6.92 & 0.038 & 0.554 & $<0.100$ & $<0.020$ \\
\hline SRNL-SCS-2008-00003 & 3 & Ustd & U std & 2 & 2 & 2 & UstdLM3-221 & 2.36 & $<0.100$ & 0.684 & 2.27 & 8.61 & 0.053 & 0.767 & $<0.100$ & $<0.020$ \\
\hline SRNL-SCS-2008-00003 & 3 & HWL & HWL-24 & 2 & 2 & 3 & P07LM12 & 0.043 & $<0.100$ & 0.081 & 2.67 & 8.93 & 0.587 & 0.482 & $<0.100$ & 0.086 \\
\hline SRNL-SCS-2008-00003 & 3 & SB5 & SB5-19 & 2 & 2 & 4 & P04LM12 & 0.016 & $<0.100$ & 0.313 & 1.78 & 11.17 & $<0.020$ & 0.856 & $<0.100$ & $<0.020$ \\
\hline SRNL-SCS-2008-00003 & 3 & HWL & HWL-24 & 2 & 2 & 5 & P07LM22 & 0.045 & $<0.100$ & 0.081 & 2.71 & 9.03 & 0.588 & 0.479 & $<0.100$ & 0.085 \\
\hline SRNL-SCS-2008-00003 & 3 & SB5 & SB5-16 & 2 & 2 & 6 & P06LM22 & 0.056 & $<0.100$ & 0.284 & 1.87 & 11.11 & 0.028 & 0.851 & $<0.100$ & 0.037 \\
\hline SRNL-SCS-2008-00003 & 3 & SB5 & SB5-17 & 2 & 2 & 7 & P02LM12 & 0.014 & $<0.100$ & 0.262 & 1.5 & 8.86 & $<0.020$ & 0.696 & $<0.100$ & $<0.020$ \\
\hline SRNL-SCS-2008-00003 & 3 & SB5 & SB5-19 & 2 & 2 & 8 & P04LM22 & 0.015 & $<0.100$ & 0.314 & 1.74 & 11.4 & $<0.020$ & 0.857 & $<0.100$ & $<0.020$ \\
\hline SRNL-SCS-2008-00003 & 3 & SB5 & SB5-15 & 2 & 2 & 9 & P12LM12 & 0.057 & $<0.100$ & 0.283 & 1.78 & 11.01 & 0.03 & 0.858 & $<0.100$ & 0.036 \\
\hline SRNL-SCS-2008-00003 & 3 & Batch 1 & Batch 1 & 2 & 2 & 10 & BCHLM3-222 & 2.5 & $<0.100$ & 0.824 & 1.35 & 6.68 & 0.037 & 0.551 & $<0.100$ & $<0.020$ \\
\hline SRNL-SCS-2008-00003 & 3 & Ustd & U std & 2 & 2 & 11 & UstdLM3-222 & 2.35 & $<0.100$ & 0.692 & 2.24 & 8.51 & 0.053 & 0.773 & $<0.100$ & $<0.020$ \\
\hline SRNL-SCS-2008-00003 & 3 & HWL & HWL-26 & 2 & 2 & 12 & P03LM12 & 0.048 & $<0.100$ & 0.093 & 2.1 & 6.34 & 0.524 & 0.257 & $<0.100$ & 0.11 \\
\hline SRNL-SCS-2008-00003 & 3 & SB5 & SB5-17 & 2 & 2 & 13 & P02LM22 & 0.012 & $<0.100$ & 0.279 & 1.47 & 8.89 & $<0.020$ & 0.755 & $<0.100$ & $<0.020$ \\
\hline SRNL-SCS-2008-00003 & 3 & SB5 & SB5-15 & 2 & 2 & 14 & P12LM22 & 0.059 & $<0.100$ & 0.279 & 1.86 & 10.64 & 0.03 & 0.844 & $<0.100$ & 0.036 \\
\hline SRNL-SCS-2008-00003 & 3 & HWL & HWL-26 & 2 & 2 & 15 & P03LM22 & 0.047 & $<0.100$ & 0.092 & 2.09 & 6.19 & 0.519 & 0.253 & $<0.100$ & 0.109 \\
\hline SRNL-SCS-2008-00003 & 3 & HWL & HWL-27 & 2 & 2 & 16 & P13LM12 & 0.04 & $<0.100$ & 0.08 & 1.81 & 8.96 & 0.454 & 0.223 & $<0.100$ & 0.098 \\
\hline SRNL-SCS-2008-00003 & 3 & SB5 & SB5-16 & 2 & 2 & 17 & P06LM12 & 0.059 & $<0.100$ & 0.291 & 1.73 & 10.98 & 0.029 & 0.882 & $<0.100$ & 0.037 \\
\hline SRNL-SCS-2008-00003 & 3 & HWL & HWL-27 & 2 & 2 & 18 & P13LM22 & 0.044 & $<0.100$ & 0.078 & 1.7 & 9.13 & 0.444 & 0.217 & $<0.100$ & 0.097 \\
\hline SRNL-SCS-2008-00003 & 3 & Batch 1 & Batch 1 & 2 & 2 & 19 & BCHLM3-223 & 2.58 & $<0.100$ & 0.832 & 1.24 & 6.64 & 0.038 & 0.561 & $<0.100$ & $<0.020$ \\
\hline SRNL-SCS-2008-00003 & 3 & Ustd & U std & 2 & 2 & 20 & UstdLM3-223 & 2.42 & $<0.100$ & 0.697 & 1.95 & 8.71 & 0.054 & 0.777 & $<0.100$ & $<0.020$ \\
\hline
\end{tabular}


Table A2. Measured Elemental Concentrations (wt\%) for Samples Prepared Using Lithium Metaborate (Part 3).

\begin{tabular}{|c|c|c|c|c|c|c|c|c|c|c|c|c|c|c|c|c|}
\hline Analytical Plan & Set & Type & Glass ID & Block & Sub-Block & Sequence & Lab ID & $\mathbf{P b}$ & $\mathbf{S}$ & Si & $\mathrm{Sr}$ & Th & Ti & $\mathbf{U}$ & Zn & $\overline{\mathrm{Zr}}$ \\
\hline $\begin{array}{l}\text { SRNL-SCS-2008-00003 } \\
\end{array}$ & 2 & Batch 1 & Batch 1 & 1 & 1 & 1 & BCHLM2-111 & $<0.020$ & $<0.100$ & 23.9 & $<0.010$ & $<0.100$ & 0.387 & $<0.100$ & $<0.010$ & 0.062 \\
\hline SRNL-SCS-2008-00003 & 2 & Ustd & U std & 1 & 1 & 2 & UstdLM2-111 & $<0.020$ & $<0.100$ & 21.2 & $<0.010$ & $<0.100$ & 0.563 & 1.93 & $<0.010$ & $<0.010$ \\
\hline $\begin{array}{l}\text { SRNL-SCS-2008-00003 } \\
\end{array}$ & 2 & SB5 & SB5-01 & 1 & 1 & 3 & N02LM11 & $<0.020$ & $<0.100$ & 21.3 & 0.096 & $<0.100$ & $<0.010$ & 2.26 & $<0.010$ & 0.065 \\
\hline SRNL-SCS-2008-00003 & 2 & SB5 & SB5-05 & 1 & 1 & 4 & $\begin{array}{l}\text { N07LM11 } \\
\end{array}$ & $<0.020$ & $<0.100$ & 21.3 & 0.108 & $<0.100$ & 0.01 & 2.59 & $<0.010$ & 0.073 \\
\hline SRNL-SCS-2008-00003 & 2 & SB5 & SB5-04 & 1 & 1 & 5 & N03LM21 & $<0.020$ & $<0.100$ & 20.7 & 0.089 & $<0.100$ & $<0.010$ & 2.34 & $<0.010$ & 0.068 \\
\hline SRNL-SCS-2008-00003 & 2 & SB5 & SB5-04 & 1 & 1 & 6 & N03LM11 & $<0.020$ & $<0.100$ & 20.8 & 0.09 & $<0.100$ & 0.01 & 2.34 & $<0.010$ & 0.068 \\
\hline SRNL-SCS-2008-00003 & 2 & SB5 & SB5-12 & 1 & 1 & 7 & N13LM21 & $<0.020$ & 0.102 & 20.9 & 0.109 & $<0.100$ & 0.011 & 2.74 & $<0.010$ & 0.08 \\
\hline SRNL-SCS-2008-00003 & 2 & SB5 & SB5-02 & 1 & 1 & 8 & N12LM21 & $<0.020$ & $<0.100$ & 21.2 & 0.09 & $<0.100$ & 0.011 & 2.26 & $<0.010$ & 0.067 \\
\hline SRNL-SCS-2008-00003 & 2 & SB5 & SB5-11 & 1 & 1 & 9 & N14LM11 & $<<0.020$ & 0.102 & 20.3 & 0.112 & $<0.100$ & 0.011 & 2.67 & $<0.010$ & 0.082 \\
\hline $\begin{array}{l}\text { SRNL-SCS-2008-00003 } \\
\end{array}$ & 2 & SB5 & SB5-07 & 1 & 1 & 10 & N15LM21 & $<0.020$ & $<0.100$ & 20.9 & 0.108 & $<0.100$ & 0.01 & 2.58 & $<0.010$ & 0.071 \\
\hline SRNL-SCS-2008-00003 & 2 & Batch 1 & Batch 1 & 1 & 1 & 11 & BCHLM2-112 & $<0.020$ & $<0.100$ & 23.6 & $<0.010$ & $<0.100$ & 0.386 & $<0.100$ & $<0.010$ & 0.063 \\
\hline $\begin{array}{l}\text { SRNL-SCS-2008-00003 } \\
\end{array}$ & 2 & Ustd & U std & 1 & 1 & 12 & UstdLM1-112 & $<0.020$ & $<0.100$ & 21.4 & $<0.010$ & $<0.100$ & 0.564 & 1.93 & $<0.010$ & $<0.010$ \\
\hline SRNL-SCS-2008-00003 & 2 & SB5 & SB5-02 & 1 & 1 & 13 & N12LM11 & $<0.020$ & $<0.100$ & 21.4 & 0.091 & $<0.100$ & 0.011 & 2.29 & $<0.010$ & 0.065 \\
\hline SRNL-SCS-2008-00003 & 2 & SB5 & SB5-11 & 1 & 1 & 14 & N14LM21 & $<0.020$ & 0.104 & 20.8 & 0.113 & $<0.100$ & 0.011 & 2.69 & $<0.010$ & 0.082 \\
\hline SRNL-SCS-2008-00003 & 2 & SB4 & SB4VAR33 & 1 & 1 & 15 & N05LM21 & 0.026 & 0.127 & 19.8 & $<0.010$ & $<0.100$ & 0.013 & 2.79 & $<0.010$ & 0.02 \\
\hline SRNL-SCS-2008-00003 & 2 & SB5 & SB5-05 & 1 & 1 & 16 & N07LM21 & $<0.020$ & $<0.100$ & 20.8 & 0.11 & $<0.100$ & 0.011 & 2.55 & $<0.010$ & 0.071 \\
\hline SRNL-SCS-2008-00003 & 2 & SB5 & SB5-12 & 1 & 1 & 17 & N13LM11 & $<0.020$ & 0.1 & 20.8 & 0.111 & $<0.100$ & 0.011 & 2.71 & $<0.010$ & 0.079 \\
\hline SRNL-SCS-2008-00003 & 2 & SB4 & SB4VAR33 & 1 & 1 & 18 & N05LM11 & 0.026 & 0.124 & 19.8 & $<0.010$ & $<0.100$ & 0.014 & 2.81 & $<0.010$ & 0.021 \\
\hline SRNL-SCS-2008-00003 & 2 & SB5 & SB5-01 & 1 & 1 & 19 & N02LM21 & $<0.020$ & $<0.100$ & 21.9 & 0.099 & $<0.100$ & $<0.010$ & 2.38 & $<0.010$ & 0.062 \\
\hline SRNL-SCS-2008-00003 & 2 & SB5 & SB5-07 & 1 & 1 & 20 & N15LM11 & $<0.020$ & 0.102 & 21 & 0.107 & $<0.100$ & 0.01 & 2.56 & $<0.010$ & 0.072 \\
\hline $\begin{array}{l}\text { SRNL-SCS-2008-00003 } \\
\end{array}$ & 2 & Batch 1 & Batch 1 & 1 & 1 & 21 & BCHLM2-113 & $<0.020$ & $<0.100$ & 24.1 & $<0.010$ & $<0.100$ & 0.388 & $<0.100$ & $<0.010$ & 0.064 \\
\hline SRNL-SCS-2008-00003 & 2 & Ustd & U std & 1 & 1 & 22 & UstdLM2-113 & $<0.020$ & $<0.100$ & 21.3 & $<0.010$ & $<0.100$ & 0.558 & 1.94 & $<0.010$ & $<0.010$ \\
\hline SRNL-SCS-2008-00003 & 2 & Batch 1 & Batch 1 & 1 & 2 & 1 & BCHLM2-121 & $<0.020$ & $<0.100$ & 23.2 & $<0.010$ & $<0.100$ & 0.389 & $<0.100$ & $<0.010$ & 0.062 \\
\hline SRNL-SCS-2008-00003 & 2 & Ustd & U std & 1 & 2 & 2 & UstdLM2-121 & $<0.020$ & $<0.100$ & 21.5 & $<0.010$ & $<0.100$ & 0.564 & 1.94 & $<0.010$ & $<0.010$ \\
\hline SRNL-SCS-2008-00003 & 2 & SB5 & SB5-12 & 1 & 2 & 3 & N13LM22 & $<0.020$ & $<0.100$ & 21.2 & 0.108 & $<0.100$ & $<0.010$ & 2.69 & $<0.010$ & 0.078 \\
\hline SRNL-SCS-2008-00003 & 2 & SB5 & SB5-05 & 1 & 2 & 4 & N07LM22 & $<0.020$ & $<0.100$ & 20.6 & 0.109 & $<0.100$ & $<0.010$ & 2.54 & $<0.010$ & 0.07 \\
\hline SRNL-SCS-2008-00003 & 2 & SB4 & SB4VAR33 & 1 & 2 & 5 & N05LM22 & 0.024 & 0.122 & 19.7 & $<0.010$ & $<0.100$ & 0.012 & 2.8 & $<0.010$ & 0.019 \\
\hline SRNL-SCS-2008-00003 & 2 & SB4 & SB4VAR33 & 1 & 2 & 6 & N05LM12 & 0.025 & 0.123 & 19.7 & $<0.010$ & $<0.100$ & 0.013 & 2.8 & $<0.010$ & 0.02 \\
\hline SRNL-SCS-2008-00003 & 2 & SB5 & SB5-01 & 1 & 2 & 7 & N02LM12 & $<0.020$ & $<0.100$ & 20.9 & 0.095 & $<0.100$ & $<0.010$ & 2.29 & $<0.010$ & 0.063 \\
\hline $\begin{array}{l}\text { SRNL-SCS-2008-00003 } \\
\end{array}$ & 2 & SB5 & SB5-04 & 1 & 2 & 8 & N03LM12 & $<0.020$ & $<0.100$ & 20.4 & 0.088 & $<0.100$ & $<0.010$ & 2.33 & $<0.010$ & 0.067 \\
\hline SRNL-SCS-2008-00003 & 2 & SB5 & SB5-01 & 1 & 2 & 9 & N02LM22 & $<<0.020$ & $<0.100$ & 21 & 0.096 & $<0.100$ & $<0.010$ & 2.3 & $<0.010$ & 0.061 \\
\hline SRNL-SCS-2008-00003 & 2 & SB5 & SB5-07 & 1 & 2 & 10 & N15LM12 & $<0.020$ & $<0.100$ & 20.4 & 0.105 & $<0.100$ & $<0.010$ & 2.52 & $<0.010$ & 0.07 \\
\hline SRNL-SCS-2008-00003 & 2 & Batch 1 & Batch 1 & 1 & 2 & 11 & BCHLM2-122 & $<<0.020$ & $<0.100$ & 23.2 & $<0.010$ & $<0.100$ & 0.384 & $<0.100$ & $<0.010$ & 0.062 \\
\hline SRNL-SCS-2008-00003 & 2 & Ustd & U std & 1 & 2 & 12 & UstdLM2-122 & $<0.020$ & $<0.100$ & 21.5 & $<0.010$ & $<0.100$ & 0.552 & 1.98 & $<0.010$ & $<0.010$ \\
\hline SRNL-SCS-2008-00003 & 2 & SB5 & SB5-04 & 1 & 2 & 13 & N03LM22 & $<0.020$ & $<0.100$ & 20.5 & 0.089 & $<0.100$ & $<0.010$ & 2.33 & $<0.010$ & 0.065 \\
\hline SRNL-SCS-2008-00003 & 2 & SB5 & SB5-11 & 1 & 2 & 14 & N14LM22 & $<0.020$ & $<0.100$ & 20.7 & 0.112 & $<0.100$ & 0.01 & 2.73 & $<0.010$ & 0.081 \\
\hline SRNL-SCS-2008-00003 & 2 & SB5 & SB5-05 & 1 & 2 & 15 & N07LM12 & $<0.020$ & $<0.100$ & 20.8 & 0.106 & $<0.100$ & $<0.010$ & 2.56 & $<0.010$ & 0.073 \\
\hline SRNL-SCS-2008-00003 & 2 & SB5 & SB5-02 & 1 & 2 & 16 & N12LM12 & $<0.020$ & $<0.100$ & 21.2 & 0.089 & $<0.100$ & $<0.010$ & 2.28 & $<0.010$ & 0.066 \\
\hline SRNL-SCS-2008-00003 & 2 & SB5 & SB5-11 & 1 & 2 & 17 & N14LM12 & $<0.020$ & 0.1 & 20.8 & 0.111 & $<0.100$ & $<0.010$ & 2.66 & $<0.010$ & 0.082 \\
\hline SRNL-SCS-2008-00003 & 2 & SB5 & SB5-02 & 1 & 2 & 18 & N12LM22 & $<0.020$ & $<0.100$ & 20.8 & 0.09 & $<0.100$ & $<0.010$ & 2.24 & $<0.010$ & 0.067 \\
\hline SRNL-SCS-2008-00003 & 2 & SB5 & SB5-12 & 1 & 2 & 19 & N13LM12 & $<0.020$ & $<0.100$ & 21.1 & 0.109 & $<0.100$ & $<0.010$ & 2.72 & $<0.010$ & 0.079 \\
\hline SRNL-SCS-2008-00003 & 2 & SB5 & SB5-07 & 1 & 2 & 20 & N15LM22 & $<<0.020$ & $<0.100$ & 21.4 & 0.106 & $<0.100$ & $<0.010$ & 2.57 & $<0.010$ & 0.071 \\
\hline SRNL-SCS-2008-00003 & 2 & Batch 1 & Batch 1 & 1 & 2 & 21 & BCHLM2-123 & $<0.020$ & $<0.100$ & 24.5 & $<0.010$ & $<0.100$ & 0.387 & $<0.100$ & $<0.010$ & 0.063 \\
\hline SRNL-SCS-2008-00003 & 2 & Ustd & U std & 1 & 2 & 22 & UstdLM2-123 & $<0.020$ & $<0.100$ & 21.5 & $<0.010$ & $<0.100$ & 0.562 & 1.95 & $<0.010$ & $<0.010$ \\
\hline SRNL-SCS-2008-00003 & 2 & Batch 1 & Batch 1 & 2 & 1 & 1 & BCHLM2-211 & $<0.020$ & $<0.100$ & 23.5 & $<0.010$ & $<0.100$ & 0.397 & $<0.100$ & $<0.010$ & 0.065 \\
\hline
\end{tabular}


Table A2. Measured Elemental Concentrations (wt\%) for Samples Prepared Using Lithium Metaborate (Part 3, continued).

\begin{tabular}{|c|c|c|c|c|c|c|c|c|c|c|c|c|c|c|c|c|}
\hline Analytical Plan & Set & Type & Glass ID & Block & Sub-Block & Sequence & Lab ID & $\mathbf{P b}$ & $\mathrm{S}$ & Si & Sr & Th & Ti & $\mathbf{U}$ & $\mathbf{Z n}$ & $\mathbf{Z r}$ \\
\hline $\begin{array}{l}\text { SRNL-SCS-2008-00003 } \\
\end{array}$ & 2 & Ustd & U std & 2 & 1 & 2 & UstdLM2-211 & $<0.020$ & $<0.100$ & 21.2 & $<<0.010$ & $<0.100$ & 0.575 & 1.94 & $<0.010$ & $<0.010$ \\
\hline SRNL-SCS-2008-00003 & 2 & SB4 & SB4VAR32 & 2 & 1 & 3 & N11LM21 & $<0.020$ & 0.116 & 21 & $<<0.010$ & $<0.100$ & 0.013 & 2.51 & $<0.010$ & 0.019 \\
\hline $\begin{array}{l}\text { SRNL-SCS-2008-00003 } \\
\end{array}$ & 2 & SB4 & SB4VAR31 & 2 & 1 & 4 & N06LM11 & $<0.020$ & 0.104 & 22 & $<<0.010$ & $<0.100$ & 0.015 & 2.21 & $<0.010$ & 0.018 \\
\hline SRNL-SCS-2008-00003 & 2 & SB5 & SB5-03 & 2 & 1 & 5 & N09LM11 & $<0.020$ & $<0.100$ & 20.8 & 0.099 & $<0.100$ & 0.01 & 2.29 & $<0.010$ & 0.069 \\
\hline SRNL-SCS-2008-00003 & 2 & SB5 & SB5-09 & 2 & 1 & 6 & N04LM11 & $<0.020$ & 0.104 & 21 & 0.123 & $<0.100$ & 0.012 & 2.69 & $<0.010$ & 0.085 \\
\hline SRNL-SCS-2008-00003 & 2 & SB5 & SB5-03 & 2 & 1 & 7 & N09LM21 & $<0.020$ & $<0.100$ & 21.3 & 0.097 & $<0.100$ & 0.011 & 2.34 & $<0.010$ & 0.071 \\
\hline SRNL-SCS-2008-00003 & 2 & SB4 & SB4VAR32 & 2 & 1 & 8 & N11LM11 & $<0.020$ & 0.117 & 21.9 & $<0.010$ & $<0.100$ & 0.013 & 2.57 & $<0.010$ & 0.019 \\
\hline SRNL-SCS-2008-00003 & 2 & SB5 & SB5-10 & 2 & 1 & 9 & N081LM11 & $<0.020$ & 0.11 & 22.2 & 0.122 & $<0.100$ & 0.013 & 2.75 & $<0.010$ & 0.08 \\
\hline SRNL-SCS-2008-00003 & 2 & Batch 1 & Batch 1 & 2 & 1 & 10 & BCHLM2-212 & $<0.020$ & $<0.100$ & 24.1 & $<0.010$ & $<0.100$ & 0.393 & $<0.100$ & $<0.010$ & 0.065 \\
\hline $\begin{array}{l}\text { SRNL-SCS-2008-00003 } \\
\end{array}$ & 2 & Ustd & U std & 2 & 1 & 11 & UstdLM2-212 & $<0.020$ & $<0.100$ & 21.1 & $<0.010$ & $<0.100$ & 0.585 & 1.94 & $<0.010$ & $<0.010$ \\
\hline SRNL-SCS-2008-00003 & 2 & SB5 & SB5-08 & 2 & 1 & 12 & N10LM21 & $<0.020$ & $<0.100$ & 21.2 & 0.104 & $<0.100$ & 0.012 & 2.49 & $<0.010$ & 0.072 \\
\hline $\begin{array}{l}\text { SRNL-SCS-2008-00003 } \\
\end{array}$ & 2 & SB5 & SB5-06 & 2 & 1 & 13 & N01LM11 & $<0.020$ & 0.102 & 21.2 & 0.111 & $<0.100$ & 0.011 & 2.55 & $<0.010$ & 0.076 \\
\hline SRNL-SCS-2008-00003 & 2 & SB4 & SB4VAR31 & 2 & 1 & 14 & N06LM21 & $<0.020$ & 0.103 & 22.8 & $<<0.010$ & $<0.100$ & 0.015 & 2.26 & $<0.010$ & 0.017 \\
\hline SRNL-SCS-2008-00003 & 2 & SB5 & SB5-10 & 2 & 1 & 15 & N08LM21 & $<0.020$ & 0.107 & 21.4 & 0.121 & $<0.100$ & 0.012 & 2.69 & $<0.010$ & 0.081 \\
\hline SRNL-SCS-2008-00003 & 2 & SB5 & SB5-08 & 2 & 1 & 16 & N10LM11 & $<0.020$ & 0.105 & 20.7 & 0.11 & $<0.100$ & 0.012 & 2.56 & $<0.010$ & 0.075 \\
\hline SRNL-SCS-2008-00003 & 22 & SB5 & SB5-09 & 2 & 1 & 17 & N04LM21 & $<0.020$ & 0.107 & 20.7 & 0.118 & $<0.100$ & 0.012 & 2.68 & $<0.010$ & 0.089 \\
\hline SRNL-SCS-2008-00003 & 2 & SB5 & SB5-06 & 2 & 1 & 18 & N01LM21 & $<0.020$ & $<0.100$ & 21.7 & 0.106 & $<0.100$ & 0.01 & 2.65 & $<0.010$ & 0.071 \\
\hline SRNL-SCS-2008-00003 & 2 & Batch 1 & Batch 1 & 2 & 1 & 19 & BCHLM2-213 & $<0.020$ & $<0.100$ & 24.2 & $<0.010$ & $<0.100$ & 0.399 & $<0.100$ & $<0.010$ & 0.066 \\
\hline SRNL-SCS-2008-00003 & 2 & Ustd & U std & 2 & 1 & 20 & UstdLM2-213 & $<0.020$ & $<0.100$ & 21.7 & $<0.010$ & $<0.100$ & 0.578 & 1.96 & $<0.010$ & $<0.010$ \\
\hline SRNL-SCS-2008-00003 & 2 & Batch 1 & Batch 1 & 2 & 2 & 1 & BCHLM2-221 & $<0.020$ & $<0.100$ & 23.7 & $<0.010$ & $<0.100$ & 0.394 & $<0.100$ & $<0.010$ & 0.062 \\
\hline $\begin{array}{l}\text { SRNL-SCS-2008-00003 } \\
\end{array}$ & 2 & Ustd & U std & 2 & 2 & 2 & UstdLM2-221 & $<0.020$ & $<0.100$ & 22 & $<0.010$ & $<0.100$ & 0.575 & 2.11 & $<0.010$ & $<0.010$ \\
\hline SRNL-SCS-2008-00003 & 2 & SB4 & SB4VAR31 & 2 & 2 & 3 & N06LM22 & $<0.020$ & 0.104 & 22.9 & $<<0.010$ & $<0.100$ & 0.012 & 2.41 & $<0.010$ & 0.014 \\
\hline SRNL-SCS-2008-00003 & 2 & SB4 & SB4VAR32 & 2 & 2 & 4 & N11LM22 & $<0.020$ & 0.115 & 21.9 & $<0.010$ & $<0.100$ & 0.01 & 2.71 & $<0.010$ & 0.016 \\
\hline SRNL-SCS-2008-00003 & 2 & SB5 & SB5-10 & 2 & 2 & 5 & N08LM12 & $<0.020$ & 0.107 & 22.1 & 0.118 & $<0.100$ & $<0.010$ & 2.88 & $<0.010$ & 0.077 \\
\hline SRNL-SCS-2008-00003 & 2 & SB5 & SB5-06 & 2 & 2 & 6 & N01LM22 & $<0.020$ & $<0.100$ & 21.7 & 0.107 & $<0.100$ & $<0.010$ & 2.69 & $<0.010$ & 0.072 \\
\hline SRNL-SCS-2008-00003 & 2 & SB4 & SB4VAR31 & 2 & 2 & 7 & N06LM12 & $<0.020$ & 0.104 & 22.9 & $<0.010$ & $<0.100$ & 0.012 & 2.38 & $<0.010$ & 0.016 \\
\hline SRNL-SCS-2008-00003 & 2 & SB5 & SB5-09 & 2 & 2 & 8 & N04LM12 & $<0.020$ & 0.107 & 21.6 & 0.12 & $<0.100$ & $<0.010$ & 2.87 & $<0.010$ & 0.083 \\
\hline SRNL-SCS-2008-00003 & 2 & SB5 & SB5-08 & 2 & 2 & 9 & N10LM12 & $<0.020$ & 0.101 & 21.3 & 0.105 & $<0.100$ & $<0.010$ & 2.71 & $<0.010$ & 0.073 \\
\hline SRNL-SCS-2008-00003 & 2 & Batch 1 & Batch 1 & 2 & 2 & 10 & BCHLM2-222 & $<0.020$ & $<0.100$ & 24.2 & $<0.010$ & $<0.100$ & 0.396 & $<0.100$ & $<0.010$ & 0.063 \\
\hline $\begin{array}{l}\text { SRNL-SCS-2008-00003 } \\
\end{array}$ & 2 & Ustd & U std & 2 & 2 & 11 & UstdLM2-222 & $<0.020$ & $<0.100$ & 22.1 & $<<0.010$ & $<0.100$ & 0.579 & 2.08 & $<0.010$ & $<0.010$ \\
\hline SRNL-SCS-2008-00003 & 2 & SB5 & SB5-03 & 2 & 2 & 12 & N09LM12 & $<0.020$ & $<0.100$ & 21.5 & 0.093 & $<0.100$ & $<0.010$ & 2.46 & $<0.010$ & 0.068 \\
\hline SRNL-SCS-2008-00003 & 2 & SB5 & SB5-10 & 2 & 2 & 13 & N08LM22 & $<0.020$ & 0.104 & 22.1 & 0.111 & $<0.100$ & $<0.010$ & 2.86 & $<0.010$ & 0.078 \\
\hline SRNL-SCS-2008-00003 & 2 & SB5 & SB5-09 & 2 & 2 & 14 & N04LM22 & $<0.020$ & 0.108 & 20.7 & 0.112 & $<0.100$ & $<0.010$ & 2.82 & $<0.010$ & 0.088 \\
\hline SRNL-SCS-2008-00003 & 2 & SB5 & SB5-03 & 2 & 2 & 15 & N09LM22 & $<0.020$ & $<0.100$ & 20.8 & 0.092 & $<0.100$ & $<0.010$ & 2.42 & $<0.010$ & 0.071 \\
\hline SRNL-SCS-2008-00003 & 2 & SB5 & SB5-08 & 2 & 2 & 16 & N10LM22 & $<0.020$ & $<0.100$ & 21.3 & 0.098 & $<0.100$ & $<0.010$ & 2.57 & $<0.010$ & 0.071 \\
\hline SRNL-SCS-2008-00003 & 2 & SB5 & SB5-06 & 2 & 2 & 17 & N01LM12 & $<0.020$ & 0.103 & 21.4 & 0.106 & $<0.100$ & $<0.010$ & 2.65 & $<0.010$ & 0.075 \\
\hline SRNL-SCS-2008-00003 & 2 & SB4 & SB4VAR32 & 2 & 2 & 18 & N11LM12 & $<0.020$ & 0.118 & 21.4 & $<0.010$ & $<0.100$ & 0.01 & 2.66 & $<0.010$ & 0.017 \\
\hline SRNL-SCS-2008-00003 & 22 & Batch 1 & Batch 1 & 2 & 2 & 19 & BCHLM2-223 & $<0.020$ & $<0.100$ & 24.1 & $<0.010$ & $<0.100$ & 0.407 & $<0.100$ & $<0.010$ & 0.064 \\
\hline SRNL-SCS-2008-00003 & 2 & Ustd & U std & 2 & 2 & 20 & UstdLM2-223 & $<0.020$ & $<0.100$ & 22 & $<<0.010$ & $<0.100$ & 0.592 & 2.09 & $<0.010$ & $<0.010$ \\
\hline SRNL-SCS-2008-00003 & 3 & Batch 1 & Batch 1 & 1 & 1 & 1 & BCHLM3-111 & $<0.020$ & $<0.100$ & 24.4 & $<0.010$ & $<0.100$ & 0.388 & $<0.100$ & $<0.010$ & 0.063 \\
\hline SRNL-SCS-2008-00003 & 3 & Ustd & U std & 1 & 1 & 2 & UstdLM3-111 & $<0.020$ & $<0.100$ & 21.9 & $<0.010$ & $<0.100$ & 0.55 & 2.07 & $<0.010$ & $<0.010$ \\
\hline SRNL-SCS-2008-00003 & 3 & HWL & HWL-28 & 1 & 1 & 3 & P11LM11 & 0.109 & $<0.100$ & 23.2 & $<0.010$ & 0.806 & 1.71 & 0.417 & 0.017 & 0.441 \\
\hline SRNL-SCS-2008-00003 & 3 & HWL & HWL-25 & 1 & 1 & 4 & $\begin{array}{l}\text { P05LM21 } \\
\end{array}$ & 0.095 & $<0.100$ & 25.2 & $<0.010$ & 0.713 & 1.51 & 0.375 & 0.016 & 0.369 \\
\hline SRNL-SCS-2008-00003 & 3 & SB5 & SB5-14 & 1 & 1 & 5 & P08LM21 & 0.034 & 0.165 & 22.2 & $<<0.010$ & $<0.100$ & 0.194 & 2.49 & 0.033 & 0.08 \\
\hline SRNL-SCS-2008-00003 & 3 & SB5 & SB5-20 & 1 & 1 & 6 & P01LM11 & 0.034 & 0.169 & 22.2 & $<0.010$ & $<0.100$ & 0.199 & 2.53 & 0.033 & 0.078 \\
\hline
\end{tabular}


Table A2. Measured Elemental Concentrations (wt\%) for Samples Prepared Using Lithium Metaborate (Part 3, continued).

\begin{tabular}{|c|c|c|c|c|c|c|c|c|c|c|c|c|c|c|c|c|}
\hline Analytical Plan & Set & Type & Glass ID & Block & Sub-Block & Sequence & Lab ID & $\mathbf{P b}$ & $\mathrm{S}$ & Si & Sr & Th & Ti & $\mathbf{U}$ & $\mathbf{Z n}$ & $\mathrm{Zr}$ \\
\hline $\begin{array}{l}\text { SRNL-SCS-2008-00003 } \\
\end{array}$ & 3 & SB5 & SB5-14 & 1 & 1 & 7 & $\begin{array}{l}\text { P08LM11 } \\
\end{array}$ & 0.037 & 0.172 & 22.2 & $<<0.010$ & $<0.100$ & 0.203 & 2.49 & 0.033 & 0.081 \\
\hline SRNL-SCS-2008-00003 & 3 & SB5 & SB5-20 & 1 & 1 & 8 & P01LM21 & 0.034 & 0.17 & 22.5 & $<<0.010$ & $<0.100$ & 0.199 & 2.52 & 0.034 & 0.078 \\
\hline $\begin{array}{l}\text { SRNL-SCS-2008-00003 } \\
\end{array}$ & 3 & HWL & HWL-25 & 1 & 1 & 9 & $\begin{array}{l}\text { P05LM11 } \\
\end{array}$ & 0.097 & $<0.100$ & 25.4 & $<0.010$ & 0.716 & 1.51 & 0.374 & 0.017 & 0.397 \\
\hline SRNL-SCS-2008-00003 & 3 & Batch 1 & Batch 1 & 1 & 1 & 10 & BCHLM3-112 & $<0.020$ & $<0.100$ & 24.9 & $<0.010$ & $<0.100$ & 0.395 & $<0.100$ & $<0.010$ & 0.066 \\
\hline SRNL-SCS-2008-00003 & 3 & Ustd & U std & 1 & 1 & 11 & UstdLM3-112 & $<0.020$ & $<0.100$ & 22.2 & $<0.010$ & $<0.100$ & 0.559 & 2.11 & $<0.010$ & $<0.010$ \\
\hline SRNL-SCS-2008-00003 & 3 & SB5 & SB5-18 & 1 & 1 & 12 & P09LM21 & $<0.020$ & $<0.100$ & 21.8 & 0.101 & $<0.100$ & 0.013 & 2.76 & $<0.010$ & 0.071 \\
\hline SRNL-SCS-2008-00003 & 3 & SB5 & SB5-18 & 1 & 1 & 13 & P09LM11 & $<0.020$ & 0.102 & 21.9 & 0.1 & $<0.100$ & 0.013 & 2.77 & $<0.010$ & 0.073 \\
\hline SRNL-SCS-2008-00003 & 3 & HWL & HWL-23 & 1 & 1 & 14 & $\begin{array}{l}\text { P10LM11 } \\
\end{array}$ & 0.088 & $<0.100$ & 23.8 & $<0.010$ & 0.791 & 1.27 & 0.752 & 0.028 & 0.513 \\
\hline SRNL-SCS-2008-00003 & 3 & HWL & HWL-23 & 1 & 1 & 15 & P10LM21 & 0.083 & $<0.100$ & 23.7 & $<0.010$ & 0.755 & 1.21 & 0.715 & 0.027 & 0.492 \\
\hline $\begin{array}{l}\text { SRNL-SCS-2008-00003 } \\
\end{array}$ & 3 & SB5 & SB5-13 & 1 & 1 & 16 & $\begin{array}{l}\text { P14LM11 } \\
\end{array}$ & 0.038 & 0.181 & 22.3 & $<0.010$ & $<0.100$ & 0.217 & 2.6 & 0.036 & 0.085 \\
\hline SRNL-SCS-2008-00003 & 3 & HWL & HWL-28 & 1 & 1 & 17 & P11LM21 & 0.112 & $<0.100$ & 24 & $<0.010$ & 0.819 & 1.73 & 0.413 & 0.017 & 0.446 \\
\hline $\begin{array}{l}\text { SRNL-SCS-2008-00003 } \\
\end{array}$ & 3 & SB5 & SB5-13 & 1 & 1 & 18 & P14LM21 & 0.038 & 0.179 & 22.2 & $<<0.010$ & $<0.100$ & 0.215 & 2.59 & 0.036 & 0.084 \\
\hline SRNL-SCS-2008-00003 & 3 & Batch 1 & Batch 1 & 1 & 1 & 19 & BCHLM3-113 & $<0.020$ & $<0.100$ & 24.8 & $<<0.010$ & $<0.100$ & 0.393 & $<0.100$ & $<0.010$ & 0.065 \\
\hline SRNL-SCS-2008-00003 & 3 & Ustd & U std & 1 & 1 & 20 & UstdLM3-113 & $<0.020$ & $<0.100$ & 22 & $<0.010$ & $<0.100$ & 0.556 & 2.1 & $<0.010$ & $<0.010$ \\
\hline SRNL-SCS-2008-00003 & 3 & Batch 1 & Batch 1 & 1 & 2 & 1 & BCHLM3-121 & $<0.020$ & $<0.100$ & 23.8 & $<0.010$ & $<0.100$ & 0.393 & $<0.100$ & $<0.010$ & 0.064 \\
\hline SRNL-SCS-2008-00003 & 3 & Ustd & U std & 1 & 2 & 2 & UstdLM3-121 & $<0.020$ & $<0.100$ & 21.2 & $<0.010$ & $<0.100$ & 0.551 & 2.02 & $<0.010$ & $<0.010$ \\
\hline SRNL-SCS-2008-00003 & 3 & HWL & HWL-23 & 1 & 2 & 3 & P10LM12 & 0.086 & $<0.100$ & 22.5 & $<0.010$ & 0.792 & 1.28 & 0.776 & 0.027 & 0.513 \\
\hline SRNL-SCS-2008-00003 & 3 & SB5 & SB5-14 & 1 & 2 & 4 & P08LM12 & 0.036 & 0.17 & 21.3 & $<0.010$ & $<0.100$ & 0.201 & 2.38 & 0.032 & 0.08 \\
\hline SRNL-SCS-2008-00003 & 3 & HWL & HWL-23 & 1 & 2 & 5 & P10LM22 & 0.082 & $<0.100$ & 22.8 & $<0.010$ & 0.765 & 1.24 & 0.751 & 0.027 & 0.5 \\
\hline SRNL-SCS-2008-00003 & 3 & HWL & HWL-28 & 1 & 2 & 6 & P11LM22 & 0.112 & $<0.100$ & 23.2 & $<0.010$ & 0.839 & 1.73 & 0.437 & 0.017 & 0.46 \\
\hline $\begin{array}{l}\text { SRNL-SCS-2008-00003 } \\
\end{array}$ & 3 & SB5 & SB5-13 & 1 & 2 & 7 & $\begin{array}{l}\text { P14LM12 } \\
\end{array}$ & 0.039 & 0.183 & 21.6 & $<<0.010$ & $<0.100$ & 0.222 & 2.45 & 0.035 & 0.087 \\
\hline SRNL-SCS-2008-00003 & 3 & SB5 & SB5-20 & 1 & 2 & 8 & P01LM12 & 0.035 & 0.179 & 22.3 & $<<0.010$ & $<0.100$ & 0.205 & 2.46 & 0.033 & 0.08 \\
\hline SRNL-SCS-2008-00003 & 3 & SB5 & SB5-18 & 1 & 2 & 9 & $\begin{array}{l}\text { P09LM22 } \\
\end{array}$ & $<0.020$ & 0.101 & 21.7 & 0.109 & $<0.100$ & 0.013 & 2.65 & $<0.010$ & 0.074 \\
\hline SRNL-SCS-2008-00003 & 3 & Batch 1 & Batch 1 & 1 & 2 & 10 & BCHLM3-122 & $<0.020$ & $<0.100$ & 24.5 & $<<0.010$ & $<0.100$ & 0.401 & $<0.100$ & $<0.010$ & 0.066 \\
\hline SRNL-SCS-2008-00003 & 3 & Ustd & U std & 1 & 2 & 11 & UstdLM3-122 & $<0.020$ & $<0.100$ & 22.2 & $<0.010$ & $<0.100$ & 0.561 & 2.05 & $<0.010$ & $<0.010$ \\
\hline SRNL-SCS-2008-00003 & 3 & HWL & HWL-25 & 1 & 2 & 12 & P05LM12 & 0.097 & $<0.100$ & 24.8 & $<0.010$ & 0.723 & 1.53 & 0.383 & 0.017 & 0.4 \\
\hline SRNL-SCS-2008-00003 & 3 & SB5 & SB5-20 & 1 & 2 & 13 & P01LM22 & 0.033 & 0.175 & 22.2 & $<0.010$ & $<0.100$ & 0.2 & 2.52 & 0.033 & 0.079 \\
\hline SRNL-SCS-2008-00003 & 3 & HWL & HWL-25 & 1 & 2 & 14 & P05LM22 & 0.097 & $<0.100$ & 25 & $<0.010$ & 0.729 & 1.54 & 0.388 & 0.016 & 0.377 \\
\hline SRNL-SCS-2008-00003 & 3 & HWL & HWL-28 & 1 & 2 & 15 & P11LM12 & 0.109 & $<0.100$ & 22.7 & $<0.010$ & 0.828 & 1.78 & 0.436 & 0.017 & 0.457 \\
\hline $\begin{array}{l}\text { SRNL-SCS-2008-00003 } \\
\end{array}$ & 3 & SB5 & SB5-13 & 1 & 2 & 16 & P14LM22 & 0.037 & 0.183 & 21.1 & $<0.010$ & $<0.100$ & 0.216 & 2.47 & 0.034 & 0.085 \\
\hline SRNL-SCS-2008-00003 & 3 & SB5 & SB5-14 & 1 & 2 & 17 & P08LM22 & 0.036 & 0.173 & 21.3 & $<<0.010$ & $<0.100$ & 0.196 & 2.42 & 0.032 & 0.08 \\
\hline SRNL-SCS-2008-00003 & 3 & SB5 & SB5-18 & 1 & 2 & 18 & $\begin{array}{l}\text { P09LM12 } \\
\end{array}$ & $<0.020$ & 0.104 & 21 & 0.105 & $<0.100$ & 0.013 & 2.64 & $<0.010$ & 0.074 \\
\hline SRNL-SCS-2008-00003 & 3 & Batch 1 & Batch 1 & 1 & 2 & 19 & BCHLM3-123 & $<0.020$ & $<0.100$ & 23.7 & $<<0.010$ & $<0.100$ & 0.398 & $<0.100$ & $<0.010$ & 0.065 \\
\hline SRNL-SCS-2008-00003 & 3 & Ustd & U std & 1 & 2 & 20 & UstdLM3-123 & $<0.020$ & $<0.100$ & 21.4 & $<0.010$ & $<0.100$ & 0.562 & 2.05 & $<0.010$ & $<0.010$ \\
\hline SRNL-SCS-2008-00003 & 3 & Batch 1 & Batch 1 & 2 & 1 & 1 & BCHLM3-211 & $<0.020$ & $<0.100$ & 23.9 & $<0.010$ & $<0.100$ & 0.388 & $<0.100$ & $<0.010$ & 0.062 \\
\hline SRNL-SCS-2008-00003 & 3 & Ustd & U std & 2 & 1 & 2 & UstdLM3-211 & $<0.020$ & $<0.100$ & 21.4 & $<0.010$ & $<0.100$ & 0.547 & 1.98 & $<0.010$ & $<0.010$ \\
\hline SRNL-SCS-2008-00003 & 3 & HWL & HWL-27 & 2 & 1 & 3 & $\begin{array}{l}\text { P13LM11 } \\
\end{array}$ & 0.1 & $<0.100$ & 24.8 & $<0.010$ & 0.714 & 1.54 & 0.375 & 0.017 & 0.39 \\
\hline SRNL-SCS-2008-00003 & 3 & HWL & HWL-27 & 2 & 1 & 4 & P13LM21 & 0.096 & $<0.100$ & 24.9 & $<0.010$ & 0.696 & 1.51 & 0.369 & 0.015 & 0.382 \\
\hline SRNL-SCS-2008-00003 & 3 & HWL & HWL-26 & 2 & 1 & 5 & $\begin{array}{l}\text { P03LM11 } \\
\end{array}$ & 0.109 & $<0.100$ & 23.1 & $<0.010$ & 0.818 & 1.72 & 0.432 & 0.016 & 0.443 \\
\hline SRNL-SCS-2008-00003 & 3 & HWL & HWL-24 & 2 & 1 & 6 & P07LM11 & 0.086 & $<0.100$ & 23.4 & $<0.010$ & 0.809 & 1.33 & 0.754 & 0.028 & 0.505 \\
\hline SRNL-SCS-2008-00003 & 3 & SB5 & SB5-16 & 2 & 1 & 7 & $\begin{array}{l}\text { P06LM11 } \\
\end{array}$ & 0.036 & 0.172 & 21.2 & $<0.010$ & $<0.100$ & 0.212 & 2.48 & 0.034 & 0.085 \\
\hline SRNL-SCS-2008-00003 & 3 & HWL & HWL-24 & 2 & 1 & 8 & P07LM21 & 0.087 & $<0.100$ & 24.1 & $<<0.010$ & 0.811 & 1.34 & 0.756 & 0.028 & 0.507 \\
\hline SRNL-SCS-2008-00003 & 3 & SB5 & SB5-19 & 2 & 1 & 9 & P04LM11 & $<0.020$ & 0.105 & 21 & 0.108 & $<0.100$ & 0.011 & 2.82 & $<0.010$ & 0.078 \\
\hline SRNL-SCS-2008-00003 & 3 & Batch 1 & Batch 1 & 2 & 1 & 10 & BCHLM3-212 & $<0.020$ & $<0.100$ & 24.8 & $<<0.010$ & $<0.100$ & 0.391 & $<0.100$ & $<0.010$ & 0.063 \\
\hline SRNL-SCS-2008-00003 & 3 & Ustd & U std & 2 & 1 & 11 & UstdLM3-212 & $<0.020$ & $<0.100$ & 22.1 & $<0.010$ & $<0.100$ & 0.557 & 2.05 & $<0.010$ & $<0.010$ \\
\hline
\end{tabular}


Table A2. Measured Elemental Concentrations (wt\%) for Samples Prepared Using Lithium Metaborate (Part 3, continued).

\begin{tabular}{|c|c|c|c|c|c|c|c|c|c|c|c|c|c|c|c|c|}
\hline Analytical Plan & Set & Type & Glass ID & Block & Sub-Block & Sequence & \begin{tabular}{|l|l} 
Lab ID \\
\end{tabular} & $\overline{\mathbf{P b}}$ & $\overline{\mathrm{S}}$ & $\overline{\mathrm{Si}}$ & $\overline{\mathrm{Sr}}$ & Th & 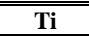 & $\overline{\mathrm{U}}$ & $\overline{Z \mathbf{Z n}}$ & $\overline{\mathbf{Z r}}$ \\
\hline SRNL-SCS-2008-00003 & 3 & SB5 & SB5-15 & 2 & 1 & 12 & P12LM21 & 0.034 & 0.174 & 21 & $<0.010$ & $<0.100$ & 0.217 & 2.41 & 0.032 & 0.081 \\
\hline SRNL-SCS-2008-00003 & 3 & SB5 & SB5-19 & 2 & 1 & 13 & $\begin{array}{l}\text { P04LM21 } \\
\end{array}$ & $<0.020$ & 0.104 & 20.3 & 0.11 & $<0.100$ & 0.013 & 2.81 & $<0.010$ & 0.077 \\
\hline SRNL-SCS-2008-00003 & 3 & SB5 & SB5-16 & 2 & 1 & 14 & P06LM21 & 0.035 & 0.165 & 20.7 & $<0.010$ & $<0.100$ & 0.211 & 2.44 & 0.033 & 0.083 \\
\hline SRNL-SCS-2008-00003 & 3 & HWL & HWL-26 & 2 & 1 & 15 & $\begin{array}{l}\text { P03LM21 } \\
\end{array}$ & 0.108 & $<0.100$ & 22.1 & $<0.010$ & 0.829 & 1.73 & 0.438 & 0.017 & 0.448 \\
\hline SRNL-SCS-2008-00003 & 3 & SB5 & SB5-15 & 2 & 1 & 16 & P12LM11 & 0.035 & 0.173 & 20.3 & $<0.010$ & $<0.100$ & 0.223 & 2.45 & 0.033 & 0.087 \\
\hline SRNL-SCS-2008-00003 & 3 & SB5 & SB5-17 & 2 & 1 & 17 & P02LM11 & $<0.020$ & $<0.100$ & 20.8 & 0.092 & $<0.100$ & $<0.010$ & 2.37 & $<0.010$ & 0.067 \\
\hline SRNL-SCS-2008-00003 & 3 & SB5 & SB5-17 & 2 & 1 & 18 & P02LM21 & $<0.020$ & $<0.100$ & 20.6 & 0.09 & $<0.100$ & $<0.010$ & 2.33 & $<0.010$ & 0.07 \\
\hline SRNL-SCS-2008-00003 & 3 & Batch 1 & Batch 1 & 2 & 1 & 19 & BCHLM3-213 & $<0.020$ & $<0.100$ & 23.2 & $<0.010$ & $<0.100$ & 0.396 & $<0.100$ & $<0.010$ & 0.064 \\
\hline SRNL-SCS-2008-00003 & 3 & Ustd & U std & 2 & 1 & 20 & UstdLM3-213 & $<0.020$ & $<0.100$ & 20.2 & $<0.010$ & $<0.100$ & 0.552 & 1.98 & $<0.010$ & $<0.010$ \\
\hline SRNL-SCS-2008-00003 & 3 & Batch 1 & Batch 1 & 2 & 2 & 1 & BCHLM3-221 & $<0.020$ & $<0.100$ & 23.8 & $<0.010$ & $<0.100$ & 0.391 & $<0.100$ & $<0.010$ & 0.063 \\
\hline SRNL-SCS-2008-00003 & 3 & Ustd & U std & 2 & 2 & 2 & UstdLM3-221 & $<0.020$ & $<0.100$ & 21.5 & $<0.010$ & $<0.100$ & 0.546 & 2.04 & $<0.010$ & $<0.010$ \\
\hline SRNL-SCS-2008-00003 & 3 & HWL & HWL-24 & 2 & 2 & 3 & P07LM12 & 0.086 & $<0.100$ & 23.2 & $<0.010$ & 0.795 & 1.32 & 0.737 & 0.029 & 0.49 \\
\hline SRNL-SCS-2008-00003 & 3 & SB5 & SB5-19 & $\frac{2}{2}$ & $\frac{2}{2}$ & 4 & P04LM12 & $<0.020$ & 0.102 & 20.7 & 0.107 & $<0.100$ & 0.011 & 2.79 & $<0.010$ & 0.077 \\
\hline SRNL-SCS-2008-00003 & 3 & HWL & HWL-24 & 2 & 2 & 5 & P07LM22 & 0.085 & $<0.100$ & 23.5 & $<0.010$ & 0.79 & 1.31 & 0.731 & 0.029 & 0.49 \\
\hline SRNL-SCS-2008-00003 & 3 & SB5 & SB5-16 & 2 & 2 & 6 & P06LM22 & 0.037 & 0.165 & 20.9 & $<0.010$ & $<0.100$ & 0.204 & 2.42 & 0.034 & 0.083 \\
\hline SRNL-SCS-2008-00003 & 3 & SB5 & SB5-17 & 2 & 2 & 7 & P02LM12 & $<0.020$ & $<0.100$ & 21.5 & 0.088 & $<0.100$ & $<0.010$ & 2.37 & $<0.010$ & 0.066 \\
\hline SRNL-SCS-2008-00003 & 3 & SB5 & SB5-19 & 2 & 2 & 8 & P04LM22 & $<0.020$ & 0.104 & 20.5 & 0.105 & $<0.100$ & 0.013 & 2.78 & $<0.010$ & 0.076 \\
\hline SRNL-SCS-2008-00003 & 3 & SB5 & SB5-15 & 2 & 2 & 9 & P12LM12 & 0.036 & 0.169 & 20.7 & $<0.010$ & $<0.100$ & 0.219 & 2.45 & 0.034 & 0.085 \\
\hline SRNL-SCS-2008-00003 & 3 & Batch 1 & Batch 1 & 2 & 2 & 10 & BCHLM3-222 & $<0.020$ & $<0.100$ & 23.5 & $<0.010$ & $<0.100$ & 0.384 & $<0.100$ & $<0.010$ & 0.062 \\
\hline SRNL-SCS-2008-00003 & 3 & Ustd & U std & 2 & 2 & 11 & UstdLM3-222 & $<0.020$ & $<0.100$ & 21.1 & $<0.010$ & $<0.100$ & 0.552 & 1.99 & $<0.010$ & $<0.010$ \\
\hline SRNL-SCS-2008-00003 & 3 & HWL & HWL-26 & 2 & 2 & 12 & P03LM12 & 0.11 & $<0.100$ & 22.7 & $<0.010$ & 0.822 & 1.72 & 0.432 & 0.018 & 0.44 \\
\hline SRNL-SCS-2008-00003 & 3 & SB5 & SB5-17 & 2 & 2 & 13 & P02LM22 & $<0.020$ & $<0.100$ & 21.3 & 0.09 & $<0.100$ & $<0.010$ & 2.38 & $<0.010$ & 0.072 \\
\hline SRNL-SCS-2008-00003 & 3 & SB5 & SB5-15 & 2 & 2 & 14 & P12LM22 & 0.036 & 0.168 & 20.8 & $<0.010$ & $<0.100$ & 0.217 & 2.41 & 0.033 & 0.082 \\
\hline SRNL-SCS-2008-00003 & 3 & HWL & HWL-26 & 2 & 2 & 15 & P03LM22 & 0.109 & $<0.100$ & 22.2 & $<0.010$ & 0.811 & 1.71 & 0.428 & 0.017 & 0.438 \\
\hline SRNL-SCS-2008-00003 & 3 & HWL & HWL-27 & 2 & 2 & 16 & P13LM12 & 0.098 & $<0.100$ & 24 & $<0.010$ & 0.704 & 1.53 & 0.369 & 0.018 & 0.387 \\
\hline SRNL-SCS-2008-00003 & 3 & SB5 & SB5-16 & 2 & 2 & 17 & P06LM12 & 0.037 & 0.174 & 20.1 & $<0.010$ & $<0.100$ & 0.211 & 2.39 & 0.035 & 0.085 \\
\hline SRNL-SCS-2008-00003 & 3 & HWL & HWL-27 & 2 & 2 & 18 & P13LM22 & 0.097 & $<0.100$ & 24.6 & $<0.010$ & 0.692 & 1.5 & 0.365 & 0.016 & 0.377 \\
\hline SRNL-SCS-2008-00003 & 3 & Batch 1 & Batch 1 & 2 & 2 & 19 & BCHLM3-223 & $<0.020$ & $<0.100$ & 22.6 & $<0.010$ & $<0.100$ & 0.392 & $<0.100$ & $<0.010$ & 0.065 \\
\hline SRNL-SCS-2008-00003 & 3 & Ustd & U std & 2 & 2 & 20 & UstdLM3-223 & $<0.020$ & $<0.100$ & 21 & $<0.010$ & $<0.100$ & 0.553 & 1.96 & $<0.010$ & $<0.010$ \\
\hline
\end{tabular}


Table A3. Measured Elemental Concentrations (wt\%) for Samples in SRNL-SCS-2008-00003 Prepared Using Peroxide Fusion.

\begin{tabular}{|c|c|c|c|c|c|c|c|c|c|}
\hline Analytical Plan & Set & Type & Glass ID & Block & Sub-Block & Sequence & Lab ID & B & $\mathbf{L i}$ \\
\hline SRNL-SCS-2008-00003 & 2 & Batch 1 & Batch 1 & 1 & 1 & 1 & BCHPF2-111 & 2.45 & 1.97 \\
\hline SRNL-SCS-2008-00003 & 2 & Ustd & U std & 1 & 1 & 2 & UstdPF2-111 & 2.8 & 1.35 \\
\hline SRNL-SCS-2008-00003 & 2 & SB5 & SB5-04 & 1 & 1 & 3 & N03PF11 & 3.13 & 1.46 \\
\hline SRNL-SCS-2008-00003 & 2 & SB5 & SB5-09 & 1 & 1 & 4 & N04PF21 & 2.02 & 1.98 \\
\hline SRNL-SCS-2008-00003 & 2 & SB5 & SB5-08 & 1 & 1 & 5 & N10PF21 & 3.12 & 1.42 \\
\hline SRNL-SCS-2008-00003 & 2 & SB5 & SB5-09 & 1 & 1 & 6 & N04PF11 & 1.99 & 1.98 \\
\hline SRNL-SCS-2008-00003 & 2 & SB5 & SB5-04 & 1 & 1 & 7 & N03PF21 & 3.26 & 1.43 \\
\hline SRNL-SCS-2008-00003 & 2 & SB5 & SB5-08 & 1 & 1 & 8 & N10PF11 & 3.14 & 1.42 \\
\hline SRNL-SCS-2008-00003 & 2 & SB5 & SB5-03 & 1 & 1 & 9 & N09PF11 & 2.56 & 1.6 \\
\hline SRNL-SCS-2008-00003 & 2 & Batch 1 & Batch 1 & 1 & 1 & 10 & BCHPF2-112 & 2.3 & 1.91 \\
\hline SRNL-SCS-2008-00003 & 2 & Ustd & U std & 1 & 1 & 11 & UstdPF2-112 & 2.78 & 1.35 \\
\hline SRNL-SCS-2008-00003 & 2 & SB4 & SB4VAR32 & 1 & 1 & 12 & N11PF11 & 2.86 & 2.32 \\
\hline SRNL-SCS-2008-00003 & 2 & SB5 & SB5-12 & 1 & 1 & 13 & N13PF21 & 3.15 & 1.45 \\
\hline SRNL-SCS-2008-00003 & 2 & SB5 & SB5-11 & 1 & 1 & 14 & N14PF11 & 2.68 & 1.68 \\
\hline SRNL-SCS-2008-00003 & 2 & SB5 & SB5-12 & 1 & 1 & 15 & N13PF11 & 3.11 & 1.43 \\
\hline SRNL-SCS-2008-00003 & 2 & SB5 & SB5-03 & 1 & 1 & 16 & N09PF21 & 2.65 & 1.68 \\
\hline SRNL-SCS-2008-00003 & 2 & SB4 & SB4VAR32 & 1 & 1 & 17 & N11PF21 & 2.7 & 2.32 \\
\hline SRNL-SCS-2008-00003 & 2 & SB5 & SB5-11 & 1 & 1 & 18 & N14PF21 & 2.62 & 1.69 \\
\hline SRNL-SCS-2008-00003 & 2 & Batch 1 & Batch 1 & 1 & 1 & 19 & BCHPF2-113 & 2.36 & 1.95 \\
\hline SRNL-SCS-2008-00003 & 2 & Ustd & U std & 1 & 1 & 20 & UstdPF2-113 & 2.71 & 1.34 \\
\hline SRNL-SCS-2008-00003 & 2 & Batch 1 & Batch 1 & 1 & 2 & 1 & BCHPF1-121 & 2.5 & 1.98 \\
\hline SRNL-SCS-2008-00003 & 2 & Ustd & U std & 1 & 2 & 2 & UstdPF1-121 & 2.85 & 1.36 \\
\hline SRNL-SCS-2008-00003 & 2 & SB5 & SB5-12 & 1 & 2 & 3 & N13PF22 & 3.16 & 1.45 \\
\hline SRNL-SCS-2008-00003 & 2 & SB5 & SB5-11 & 1 & 2 & 4 & N14PF12 & 2.72 & 1.7 \\
\hline SRNL-SCS-2008-00003 & 2 & SB5 & SB5-03 & 1 & 2 & 5 & N09PF22 & 2.76 & 1.72 \\
\hline SRNL-SCS-2008-00003 & 2 & SB5 & SB5-11 & 1 & 2 & 6 & N14PF22 & 2.74 & 1.7 \\
\hline SRNL-SCS-2008-00003 & 2 & SB5 & SB5-03 & 1 & 2 & 7 & N09PF12 & 2.6 & 1.62 \\
\hline SRNL-SCS-2008-00003 & 2 & SB5 & SB5-08 & 1 & 2 & 8 & N10PF12 & 3.18 & 1.45 \\
\hline SRNL-SCS-2008-00003 & 2 & SB4 & SB4VAR32 & 1 & 2 & 9 & N11PF12 & 2.84 & 2.32 \\
\hline SRNL-SCS-2008-00003 & 2 & Batch 1 & Batch 1 & 1 & 2 & 10 & BCHPF1-122 & 2.44 & 1.97 \\
\hline SRNL-SCS-2008-00003 & 2 & Ustd & U std & 1 & 2 & 11 & UstdPF1-122 & 2.83 & 1.36 \\
\hline SRNL-SCS-2008-00003 & 2 & SB5 & SB5-08 & 1 & 2 & 12 & N10PF22 & 3.17 & 1.44 \\
\hline SRNL-SCS-2008-00003 & 2 & SB4 & SB4VAR32 & 1 & 2 & 13 & N11PF22 & 2.79 & 2.36 \\
\hline SRNL-SCS-2008-00003 & 2 & SB5 & SB5-04 & 1 & 2 & 14 & N03PF12 & 3.19 & 1.45 \\
\hline SRNL-SCS-2008-00003 & 2 & SB5 & SB5-04 & 1 & 2 & 15 & N03PF22 & 3.22 & 1.45 \\
\hline SRNL-SCS-2008-00003 & 2 & SB5 & SB5-09 & 1 & 2 & 16 & N04PF22 & 1.98 & 2 \\
\hline SRNL-SCS-2008-00003 & 2 & SB5 & SB5-12 & 1 & 2 & 17 & N13PF12 & 3.06 & 1.45 \\
\hline SRNL-SCS-2008-00003 & 2 & SB5 & SB5-09 & 1 & 2 & 18 & N04PF12 & 2 & 2 \\
\hline SRNL-SCS-2008-00003 & 2 & Batch 1 & Batch 1 & 1 & 2 & 19 & BCHPF2-123 & 2.37 & 1.94 \\
\hline SRNL-SCS-2008-00003 & 2 & Ustd & U std & 1 & 2 & 20 & UstdPF1-123 & 2.79 & 1.36 \\
\hline SRNL-SCS-2008-00003 & 2 & Batch 1 & Batch 1 & 2 & 1 & 1 & BCHPF2-211 & 2.5 & 1.98 \\
\hline SRNL-SCS-2008-00003 & 2 & Ustd & U std & 2 & 1 & 2 & UstdPF2-211 & 2.84 & 1.37 \\
\hline SRNL-SCS-2008-00003 & 2 & SB5 & SB5-06 & 2 & 1 & 3 & N01PF21 & 2.19 & 2 \\
\hline SRNL-SCS-2008-00003 & 2 & SB5 & SB5-05 & 2 & 1 & 4 & N07PF11 & 2.01 & 2.02 \\
\hline SRNL-SCS-2008-00003 & 2 & SB4 & SB4VAR33 & 2 & 1 & 5 & N05PF11 & 2.67 & 2.23 \\
\hline SRNL-SCS-2008-00003 & 2 & SB5 & SB5-10 & 2 & 1 & 6 & N08PF21 & 2.17 & 2 \\
\hline SRNL-SCS-2008-00003 & 2 & SB5 & SB5-02 & 2 & 1 & 7 & N12PF11 & 2.15 & 2.01 \\
\hline SRNL-SCS-2008-00003 & 2 & SB5 & SB5-07 & 2 & 1 & 8 & N15PF21 & 2.62 & 1.7 \\
\hline SRNL-SCS-2008-00003 & 2 & SB5 & SB5-01 & 2 & 1 & 9 & N02PF11 & 1.89 & 1.96 \\
\hline SRNL-SCS-2008-00003 & 2 & SB5 & SB5-02 & 2 & 1 & 10 & N12PF21 & 2.12 & 2 \\
\hline SRNL-SCS-2008-00003 & 2 & Batch 1 & Batch 1 & 2 & 1 & 11 & BCHPF2-212 & 2.35 & 1.97 \\
\hline SRNL-SCS-2008-00003 & 2 & Ustd & U std & 2 & 1 & 12 & UstdPF2-212 & 2.77 & 1.37 \\
\hline SRNL-SCS-2008-00003 & 2 & SB4 & SB4VAR31 & 2 & 1 & 13 & N06PF21 & 2.96 & 2.48 \\
\hline SRNL-SCS-2008-00003 & 2 & SB5 & SB5-05 & 2 & 1 & 14 & N07PF21 & 1.97 & 1.99 \\
\hline SRNL-SCS-2008-00003 & 2 & SB4 & SB4VAR33 & 2 & 1 & 15 & N05PF21 & 2.57 & 2.2 \\
\hline SRNL-SCS-2008-00003 & 2 & SB5 & SB5-01 & 2 & 1 & 16 & N02PF21 & 1.9 & 1.96 \\
\hline SRNL-SCS-2008-00003 & 2 & SB5 & SB5-10 & 2 & 1 & 17 & N08PF11 & 2.05 & 2 \\
\hline SRNL-SCS-2008-00003 & 2 & SB4 & SB4VAR31 & 2 & 1 & 18 & N06PF11 & 2.91 & 2.5 \\
\hline SRNL-SCS-2008-00003 & 2 & SB5 & SB5-06 & 2 & 1 & 19 & N01PF11 & 2.16 & 1.97 \\
\hline SRNL-SCS-2008-00003 & 2 & SB5 & SB5-07 & 2 & 1 & 20 & N15PF11 & 2.68 & 1.71 \\
\hline SRNL-SCS-2008-00003 & 2 & Batch 1 & Batch 1 & 2 & 1 & 21 & BCHPF2-213 & 2.37 & 1.99 \\
\hline SRNL-SCS-2008-00003 & 2 & Ustd & U std & 2 & 1 & 22 & UstdPF2-213 & 2.72 & 1.36 \\
\hline SRNL-SCS-2008-00003 & 2 & Batch 1 & Batch 1 & 2 & 2 & 1 & BCHPF2-221 & 2.5 & 1.98 \\
\hline SRNL-SCS-2008-00003 & 2 & Ustd & U std & 2 & 2 & 2 & UstdPF2-221 & 2.83 & 1.38 \\
\hline SRNL-SCS-2008-00003 & 2 & SB5 & SB5-06 & 2 & 2 & 3 & N01PF12 & 2.27 & 2.01 \\
\hline SRNL-SCS-2008-00003 & 2 & SB4 & SB4VAR33 & 2 & 2 & 4 & N05PF22 & 2.66 & 2.23 \\
\hline SRNL-SCS-2008-00003 & 2 & SB5 & SB5-01 & 2 & 2 & 5 & N02PF22 & 1.93 & 1.95 \\
\hline
\end{tabular}


Table A3. Measured Elemental Concentrations (wt\%) for Samples in SRNL-SCS-2008-00003 Prepared Using Peroxide Fusion. (continued)

\begin{tabular}{|c|c|c|c|c|c|c|c|c|c|}
\hline Analytical Plan & Set & Type & Glass ID & Block & Sub-Block & Sequence & Lab ID & B & $\mathbf{L i}$ \\
\hline SRNL-SCS-2008-00003 & 2 & SB5 & SB5-10 & 2 & 2 & 6 & N08PF22 & 2.19 & 2.02 \\
\hline SRNL-SCS-2008-00003 & 2 & SB5 & SB5-05 & 2 & 2 & 7 & N07PF22 & 2 & 2.02 \\
\hline SRNL-SCS-2008-00003 & 2 & SB4 & SB4VAR31 & 2 & 2 & 8 & N06PF22 & 3.03 & 2.48 \\
\hline SRNL-SCS-2008-00003 & 2 & SB5 & SB5-07 & 2 & 2 & 9 & N15PF12 & 2.78 & 1.73 \\
\hline SRNL-SCS-2008-00003 & 2 & SB4 & SB4VAR33 & 2 & 2 & 10 & N05PF12 & 2.64 & 2.23 \\
\hline SRNL-SCS-2008-00003 & 2 & Batch 1 & Batch 1 & 2 & 2 & 11 & BCHPF2-222 & 2.43 & 1.98 \\
\hline SRNL-SCS-2008-00003 & 2 & Ustd & $\mathrm{U}$ std & 2 & 2 & 12 & UstdPF2-222 & 2.79 & 1.38 \\
\hline SRNL-SCS-2008-00003 & 2 & SB5 & SB5-05 & 2 & 2 & 13 & N07PF12 & 2.09 & 2.04 \\
\hline SRNL-SCS-2008-00003 & 2 & SB5 & SB5-01 & 2 & 2 & 14 & N02PF12 & 1.99 & 1.98 \\
\hline SRNL-SCS-2008-00003 & 2 & SB5 & SB5-06 & 2 & 2 & 15 & N01PF22 & 2.18 & 2.01 \\
\hline SRNL-SCS-2008-00003 & 2 & SB5 & SB5-10 & 2 & 2 & 16 & N08PF12 & 2.14 & 2.03 \\
\hline SRNL-SCS-2008-00003 & 2 & SB5 & SB5-02 & 2 & 2 & 17 & N12PF22 & 2.18 & 2.03 \\
\hline SRNL-SCS-2008-00003 & 2 & SB5 & SB5-07 & 2 & 2 & 18 & N15PF22 & 2.65 & 1.72 \\
\hline SRNL-SCS-2008-00003 & 2 & SB5 & SB5-02 & 2 & 2 & 19 & N12PF12 & 2.26 & 2.06 \\
\hline SRNL-SCS-2008-00003 & 2 & SB4 & SB4VAR31 & 2 & 2 & 20 & N06PF12 & 3.08 & 2.58 \\
\hline SRNL-SCS-2008-00003 & 2 & Batch 1 & Batch 1 & 2 & 2 & 21 & BCHPF2-223 & 2.52 & 2.02 \\
\hline SRNL-SCS-2008-00003 & 2 & Ustd & U std & 2 & 2 & 22 & UstdPF2-223 & 2.88 & 1.4 \\
\hline SRNL-SCS-2008-00003 & 3 & Batch 1 & Batch 1 & 1 & 1 & 1 & BCHPF3-111 & 2.57 & 2.01 \\
\hline SRNL-SCS-2008-00003 & 3 & Ustd & U std & 1 & 1 & 2 & UstdPF3-111 & 2.8 & 1.37 \\
\hline SRNL-SCS-2008-00003 & 3 & SB5 & SB5-19 & 1 & 1 & 3 & P04PF21 & 2.88 & 1.39 \\
\hline SRNL-SCS-2008-00003 & 3 & SB5 & SB5-17 & 1 & 1 & 4 & P02PF21 & 2.98 & 2.58 \\
\hline SRNL-SCS-2008-00003 & 3 & HWL & HWL-23 & 1 & 1 & 5 & P10PF21 & 3.8 & 2.39 \\
\hline SRNL-SCS-2008-00003 & 3 & SB5 & SB5-13 & 1 & 1 & 6 & P14PF11 & 2.08 & 2.03 \\
\hline SRNL-SCS-2008-00003 & 3 & SB5 & SB5-13 & 1 & 1 & 7 & P14PF21 & 2.08 & 2.03 \\
\hline SRNL-SCS-2008-00003 & 3 & SB5 & SB5-19 & 1 & 1 & 8 & P04PF11 & 2.96 & 1.45 \\
\hline SRNL-SCS-2008-00003 & 3 & SB5 & SB5-18 & 1 & 1 & 9 & P09PF21 & 2.73 & 1.99 \\
\hline SRNL-SCS-2008-00003 & 3 & Batch 1 & Batch 1 & 1 & 1 & 10 & BCHPF3-112 & 2.46 & 1.99 \\
\hline SRNL-SCS-2008-00003 & 3 & Ustd & U std & 1 & 1 & 11 & UstdPF3-112 & 2.88 & 1.38 \\
\hline SRNL-SCS-2008-00003 & 3 & SB5 & SB5-17 & 1 & 1 & 12 & P02PF11 & 3.04 & 2.56 \\
\hline SRNL-SCS-2008-00003 & 3 & HWL & HWL-27 & 1 & 1 & 13 & P13PF11 & 1.84 & 2.54 \\
\hline SRNL-SCS-2008-00003 & 3 & HWL & HWL-25 & 1 & 1 & 14 & P05PF21 & 3.66 & 1.89 \\
\hline SRNL-SCS-2008-00003 & 3 & SB5 & SB5-18 & 1 & 1 & 15 & P09PF11 & 2.74 & 1.99 \\
\hline SRNL-SCS-2008-00003 & 3 & HWL & HWL-27 & 1 & 1 & 16 & P13PF21 & 1.67 & 2.45 \\
\hline SRNL-SCS-2008-00003 & 3 & HWL & HWL-23 & 1 & 1 & 17 & P10PF11 & 3.71 & 2.39 \\
\hline SRNL-SCS-2008-00003 & 3 & HWL & HWL-25 & 1 & 1 & 18 & P05PF11 & 4.06 & 1.93 \\
\hline SRNL-SCS-2008-00003 & 3 & Batch 1 & Batch 1 & 1 & 1 & 19 & BCHPF3-113 & 2.63 & 2.02 \\
\hline SRNL-SCS-2008-00003 & 3 & Ustd & U std & 1 & 1 & 20 & UstdPF3-113 & 3 & 1.42 \\
\hline SRNL-SCS-2008-00003 & 3 & Batch 1 & Batch 1 & 1 & 2 & 1 & BCHPF3-121 & 2.52 & 2.01 \\
\hline SRNL-SCS-2008-00003 & 3 & Ustd & U std & 1 & 2 & 2 & UstdPF3-121 & 2.87 & 1.41 \\
\hline SRNL-SCS-2008-00003 & 3 & SB5 & SB5-18 & 1 & 2 & 3 & P09PF12 & 2.79 & 2.04 \\
\hline SRNL-SCS-2008-00003 & 3 & HWL & HWL-23 & 1 & 2 & 4 & P10PF12 & 3.56 & 2.39 \\
\hline SRNL-SCS-2008-00003 & 3 & SB5 & SB5-17 & 1 & 2 & 5 & P02PF12 & 2.86 & 2.53 \\
\hline SRNL-SCS-2008-00003 & 3 & SB5 & SB5-19 & 1 & 2 & 6 & P04PF12 & 2.85 & 1.45 \\
\hline SRNL-SCS-2008-00003 & 3 & HWL & HWL-25 & 1 & 2 & 7 & P05PF22 & 3.62 & 1.89 \\
\hline SRNL-SCS-2008-00003 & 3 & HWL & HWL-27 & 1 & 2 & 8 & P13PF12 & 1.71 & 2.51 \\
\hline SRNL-SCS-2008-00003 & 3 & SB5 & SB5-17 & 1 & 2 & 9 & P02PF22 & 2.95 & 2.6 \\
\hline SRNL-SCS-2008-00003 & 3 & Batch 1 & Batch 1 & 1 & 2 & 10 & BCHPF3-122 & 2.43 & 2.03 \\
\hline SRNL-SCS-2008-00003 & 3 & Ustd & U std & 1 & 2 & 11 & UstdPF3-122 & 2.75 & 1.39 \\
\hline SRNL-SCS-2008-00003 & 3 & HWL & HWL-25 & 1 & 2 & 12 & P05PF12 & 3.77 & 1.88 \\
\hline SRNL-SCS-2008-00003 & 3 & SB5 & SB5-13 & 1 & 2 & 13 & P14PF22 & 1.95 & 2 \\
\hline SRNL-SCS-2008-00003 & 3 & SB5 & SB5-13 & 1 & 2 & 14 & P14PF12 & 1.98 & 2.03 \\
\hline SRNL-SCS-2008-00003 & 3 & SB5 & SB5-19 & 1 & 2 & 15 & P04PF22 & 2.8 & 1.41 \\
\hline SRNL-SCS-2008-00003 & 3 & HWL & HWL-27 & 1 & 2 & 16 & P13PF22 & 1.67 & 2.51 \\
\hline SRNL-SCS-2008-00003 & 3 & HWL & HWL-23 & 1 & 2 & 17 & P10PF22 & 3.5 & 2.36 \\
\hline SRNL-SCS-2008-00003 & 3 & SB5 & SB5-18 & 1 & 2 & 18 & P09PF22 & 2.62 & 1.99 \\
\hline SRNL-SCS-2008-00003 & 3 & Batch 1 & Batch 1 & 1 & 2 & 19 & BCHPF3-123 & 2.28 & 1.98 \\
\hline SRNL-SCS-2008-00003 & 3 & Ustd & U std & 1 & 2 & 20 & UstdPF3-123 & 2.7 & 1.38 \\
\hline SRNL-SCS-2008-00003 & 3 & Batch 1 & Batch 1 & 2 & 1 & 1 & BCHPF3-211 & 2.36 & 1.95 \\
\hline SRNL-SCS-2008-00003 & 3 & Ustd & U std & 2 & 1 & 2 & UstdPF3-211 & 2.81 & 1.38 \\
\hline SRNL-SCS-2008-00003 & 3 & HWL & HWL-24 & 2 & 1 & 3 & P07PF21 & 1.72 & 2.46 \\
\hline SRNL-SCS-2008-00003 & 3 & SB5 & SB5-14 & 2 & 1 & 4 & P08PF11 & 2.14 & 2.05 \\
\hline SRNL-SCS-2008-00003 & 3 & HWL & HWL-26 & 2 & 1 & 5 & P03PF21 & 3.58 & 1.82 \\
\hline SRNL-SCS-2008-00003 & 3 & HWL & HWL-24 & 2 & 1 & 6 & P07PF11 & 1.68 & 2.4 \\
\hline SRNL-SCS-2008-00003 & 3 & SB5 & SB5-20 & 2 & 1 & 7 & P01PF11 & 2.95 & 1.12 \\
\hline SRNL-SCS-2008-00003 & 3 & SB5 & SB5-20 & 2 & 1 & 8 & P01PF21 & 3.1 & 1.18 \\
\hline SRNL-SCS-2008-00003 & 3 & SB5 & SB5-16 & 2 & 1 & 9 & P06PF11 & 2.95 & 1.39 \\
\hline SRNL-SCS-2008-00003 & 3 & Batch 1 & Batch 1 & 2 & 1 & 10 & BCHPF3-212 & 2.4 & 2.01 \\
\hline
\end{tabular}


Table A3. Measured Elemental Concentrations (wt\%) for Samples in SRNL-SCS-2008-00003 Prepared Using Peroxide Fusion. (continued)

\begin{tabular}{|c|c|c|c|c|c|c|c|c|c|}
\hline Analytical Plan & Set & Type & Glass ID & Block & Sub-Block & Sequence & Lab ID & B & $\mathbf{L i}$ \\
\hline SRNL-SCS-2008-00003 & 3 & Ustd & U std & 2 & 1 & 11 & UstdPF3-212 & 2.86 & 1.39 \\
\hline SRNL-SCS-2008-00003 & 3 & SB5 & SB5-14 & 2 & 1 & 12 & P08PF21 & 2.13 & 2 \\
\hline SRNL-SCS-2008-00003 & 3 & SB5 & SB5-15 & 2 & 1 & 13 & P12PF21 & 2.59 & 1.68 \\
\hline SRNL-SCS-2008-00003 & 3 & HWL & HWL-28 & 2 & 1 & 14 & P11PF11 & 1.68 & 2.38 \\
\hline SRNL-SCS-2008-00003 & 3 & SB5 & SB5-15 & 2 & 1 & 15 & P12PF11 & 2.6 & 1.69 \\
\hline SRNL-SCS-2008-00003 & 3 & SB5 & SB5-16 & 2 & 1 & 16 & P06PF21 & 3.11 & 1.44 \\
\hline SRNL-SCS-2008-00003 & 3 & HWL & HWL-28 & 2 & 1 & 17 & P11PF21 & 1.66 & 2.38 \\
\hline SRNL-SCS-2008-00003 & 3 & HWL & HWL-26 & 2 & 1 & 18 & P03PF11 & 3.64 & 1.82 \\
\hline SRNL-SCS-2008-00003 & 3 & Batch 1 & Batch 1 & 2 & 1 & 19 & BCHPF3-213 & 2.43 & 2.02 \\
\hline SRNL-SCS-2008-00003 & 3 & Ustd & U std & 2 & 1 & 20 & UstdPF3-213 & 2.82 & 1.4 \\
\hline SRNL-SCS-2008-00003 & 3 & Batch 1 & Batch 1 & 2 & 2 & 1 & BCHPF3-221 & 2.46 & 2.02 \\
\hline SRNL-SCS-2008-00003 & 3 & Ustd & U std & 2 & 2 & 2 & UstdPF3-221 & 2.76 & 1.38 \\
\hline SRNL-SCS-2008-00003 & 3 & HWL & HWL-26 & 2 & 2 & 3 & P03PF22 & 3.6 & 1.83 \\
\hline SRNL-SCS-2008-00003 & 3 & SB5 & SB5-16 & 2 & 2 & 4 & P06PF12 & 2.96 & 1.4 \\
\hline SRNL-SCS-2008-00003 & 3 & SB5 & SB5-15 & 2 & 2 & 5 & P12PF22 & 2.58 & 1.67 \\
\hline SRNL-SCS-2008-00003 & 3 & SB5 & SB5-14 & 2 & 2 & 6 & P08PF12 & 2.1 & 2.04 \\
\hline SRNL-SCS-2008-00003 & 3 & SB5 & SB5-20 & 2 & 2 & 7 & P01PF12 & 3.06 & 1.18 \\
\hline SRNL-SCS-2008-00003 & 3 & HWL & HWL-28 & 2 & 2 & 8 & P11PF22 & 1.67 & 2.4 \\
\hline SRNL-SCS-2008-00003 & 3 & SB5 & SB5-14 & 2 & 2 & 9 & P08PF22 & 2.11 & 2.01 \\
\hline SRNL-SCS-2008-00003 & 3 & Batch 1 & Batch 1 & 2 & 2 & 10 & BCHPF3-222 & 2.34 & 1.99 \\
\hline SRNL-SCS-2008-00003 & 3 & Ustd & U std & 2 & 2 & 11 & UstdPF3-222 & 2.65 & 1.38 \\
\hline SRNL-SCS-2008-00003 & 3 & HWL & HWL-28 & 2 & 2 & 12 & P11PF12 & 1.67 & 2.41 \\
\hline SRNL-SCS-2008-00003 & 3 & HWL & HWL-26 & 2 & 2 & 13 & P03PF12 & 3.44 & 1.76 \\
\hline SRNL-SCS-2008-00003 & 3 & HWL & HWL-24 & 2 & 2 & 14 & P07PF12 & 1.61 & 2.4 \\
\hline SRNL-SCS-2008-00003 & 3 & SB5 & SB5-16 & 2 & 2 & 15 & P06PF22 & 2.92 & 1.43 \\
\hline SRNL-SCS-2008-00003 & 3 & HWL & HWL-24 & 2 & 2 & 16 & P07PF22 & 1.57 & 2.42 \\
\hline SRNL-SCS-2008-00003 & 3 & SB5 & SB5-15 & 2 & 2 & 17 & P12PF12 & 2.65 & 1.7 \\
\hline SRNL-SCS-2008-00003 & 3 & SB5 & SB5-20 & 2 & 2 & 18 & P01PF22 & 2.92 & 1.17 \\
\hline SRNL-SCS-2008-00003 & 3 & Batch 1 & Batch 1 & 2 & 2 & 19 & BCHPF3-223 & 2.23 & 1.95 \\
\hline SRNL-SCS-2008-00003 & 3 & Ustd & U std & 2 & 2 & 20 & UstdPF3-223 & 2.67 & 1.38 \\
\hline
\end{tabular}


Table A4. Measured Elemental Concentrations (wt\%) for Samples in

SRNL-SCS-2008-00043 Prepared Using Lithium Metaborate. (Part 1)

\begin{tabular}{|c|c|c|c|c|c|c|c|c|c|c|c|c|c|c|c|}
\hline PAnalytical Plan & $\begin{array}{l}\text { Glass ID } \\
\end{array}$ & Block & Sequence & $\begin{array}{l}\text { Lab ID } \\
\end{array}$ & $\overline{\mathrm{Ag}}$ & $\overline{\mathrm{Al}}$ & $\overline{\mathbf{B a}}$ & $\overline{\mathrm{Ca}}$ & 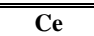 & $\overline{\mathrm{Cr}}$ & $\overline{F e}$ & $\overline{\mathrm{Hff}}$ & $\overline{\bar{K}}$ & 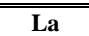 & $\overline{\mathrm{Mg}}$ \\
\hline SRNL-SCS-2008-00043 & Batch 1 & 1 & 1 & BCHLM11 & $<0.100$ & 2.48 & 0.12 & 0.902 & $<0.010$ & 0.075 & 8.76 & $<0.010$ & 2.62 & $<0.010$ & 0.84 \\
\hline SRNL-SCS-2008-00043 & SB5-21 & 1 & 2 & V06LM21 & $<0.100$ & 6.1 & 0.027 & 1.45 & 0.142 & 0.087 & 6.05 & $<0.010$ & $<0.100$ & 0.053 & 0.286 \\
\hline SRNL-SCS-2008-00043 & SB5-26 & 1 & 3 & V05LM11 & $<0.100$ & 4.67 & 0.037 & 0.627 & 0.158 & 0.103 & 7.77 & $<0.010$ & $<0.100$ & 0.068 & 0.386 \\
\hline SRNL-SCS-2008-00043 & SB5-21 & 1 & 4 & V06LM11 & $<0.100$ & 5.99 & 0.029 & 1.4 & 0.15 & 0.094 & 6.47 & $<0.010$ & $<0.100$ & 0.056 & 0.297 \\
\hline SRNL-SCS-2008-00043 & SB5-25 & 1 & 5 & V03LM11 & $<0.100$ & 5.53 & 0.034 & 1.46 & 0.135 & 0.096 & 7.35 & $<0.010$ & $<0.100$ & 0.061 & 0.335 \\
\hline SRNL-SCS-2008-00043 & SB5-24 & 1 & 6 & V01LM11 & $<0.100$ & 6.02 & 0.032 & 0.494 & 0.12 & 0.088 & 6.03 & 2.08 & $<0.100$ & 0.054 & 0.298 \\
\hline SRNL-SCS-2008-00043 & SB5-26 & 1 & 7 & V05LM21 & $<0.100$ & 4.69 & 0.039 & 0.629 & 0.151 & 0.103 & 7.78 & $<0.010$ & $<0.100$ & 0.07 & 0.382 \\
\hline SRNL-SCS-2008-00043 & Batch 1 & 1 & 8 & BCHLM12 & $<0.100$ & 2.53 & 0.127 & 0.901 & $<0.010$ & 0.078 & 9.22 & $<0.010$ & 2.6 & $<0.010$ & 0.837 \\
\hline SRNL-SCS-2008-00043 & SB5-25 & 1 & 9 & V03LM21 & $<0.100$ & 5.5 & 0.037 & 1.49 & 0.138 & 0.105 & 7.42 & $<0.010$ & $<0.100$ & 0.063 & 0.354 \\
\hline SRNL-SCS-2008-00043 & SB5-22 & 1 & 10 & V02LM11 & $<0.100$ & 5.14 & 0.032 & 0.573 & 0.157 & 0.092 & 6.85 & $<0.010$ & $<0.100$ & 0.057 & 0.31 \\
\hline SRNL-SCS-2008-00043 & SB5-23 & 1 & 11 & V04LM11 & $<0.100$ & 4.34 & 0.036 & 1.48 & 0.138 & 0.096 & 7.47 & 2.58 & $<0.100$ & 0.062 & 0.34 \\
\hline SRNL-SCS-2008-00043 & SB5-24 & 1 & 12 & V01LM21 & $<0.100$ & 6.11 & 0.03 & 0.507 & 0.118 & 0.083 & 6.11 & 2.13 & $<0.100$ & 0.051 & 0.279 \\
\hline SRNL-SCS-2008-00043 & SB5-22 & 1 & 13 & V02LM21 & $<0.100$ & 5.03 & 0.029 & 0.574 & 0.15 & 0.09 & 6.8 & $<0.010$ & $<0.100$ & 0.058 & 0.311 \\
\hline SRNL-SCS-2008-00043 & SB5-23 & 1 & 14 & V04LM21 & $<0.100$ & 4.22 & 0.036 & 1.44 & 0.134 & 0.094 & 7.24 & 2.51 & $<0.100$ & 0.06 & 0.338 \\
\hline SRNL-SCS-2008-00043 & Batch 1 & 1 & 15 & BCHLM13 & $<0.100$ & 2.53 & 0.126 & 0.895 & $<0.010$ & 0.077 & 9.28 & $<0.010$ & 2.56 & $<0.010$ & 0.827 \\
\hline SRNL-SCS-2008-00043 & Batch 1 & 2 & 1 & BCHLM21 & $<0.100$ & 2.52 & 0.118 & 0.896 & $<0.010$ & 0.072 & 8.73 & $<0.010$ & 2.61 & $<0.010$ & 0.8 \\
\hline SRNL-SCS-2008-00043 & SB5-24 & 2 & 2 & V01LM12 & $<0.100$ & 5.96 & 0.03 & 0.495 & 0.118 & 0.082 & 5.7 & 2 & $<0.100$ & 0.05 & 0.286 \\
\hline SRNL-SCS-2008-00043 & SB5-23 & 2 & 3 & V04LM22 & $<0.100$ & 4.18 & 0.033 & 1.46 & 0.13 & 0.085 & 6.72 & 2.4 & $<0.100$ & 0.056 & 0.314 \\
\hline SRNL-SCS-2008-00043 & SB5-25 & 2 & 4 & V03LM22 & $<0.100$ & 5.53 & 0.033 & 1.49 & 0.133 & 0.095 & 6.96 & $<0.010$ & $<0.100$ & 0.058 & 0.328 \\
\hline SRNL-SCS-2008-00043 & SB5-21 & 2 & 5 & V06LM12 & $<0.100$ & 5.94 & 0.027 & 1.42 & 0.147 & 0.088 & 6.05 & $<0.010$ & $<0.100$ & 0.054 & 0.291 \\
\hline SRNL-SCS-2008-00043 & SB5-22 & 2 & 6 & V02LM12 & $<0.100$ & 5.04 & 0.029 & 0.582 & 0.15 & 0.083 & 6.4 & $<0.010$ & $<0.100$ & 0.052 & 0.281 \\
\hline SRNL-SCS-2008-00043 & SB5-25 & 2 & 7 & V03LM12 & $<0.100$ & 5.4 & 0.032 & 1.48 & 0.132 & 0.09 & 6.92 & $<0.010$ & $<0.100$ & 0.057 & 0.32 \\
\hline SRNL-SCS-2008-00043 & Batch 1 & 2 & 8 & BCHLM22 & $<0.100$ & 2.48 & 0.118 & 0.902 & $<0.010$ & 0.073 & 8.61 & $<0.010$ & 2.63 & $<0.010$ & 0.809 \\
\hline SRNL-SCS-2008-00043 & SB5-24 & 2 & 9 & V01LM22 & $<0.100$ & 5.98 & 0.029 & 0.508 & 0.116 & 0.078 & 5.62 & 1.99 & $<0.100$ & 0.049 & 0.275 \\
\hline SRNL-SCS-2008-00043 & SB5-21 & 2 & 10 & V06LM22 & $<0.100$ & 5.97 & 0.026 & 1.43 & 0.139 & 0.082 & 5.89 & $<0.010$ & $<0.100$ & 0.05 & 0.27 \\
\hline SRNL-SCS-2008-00043 & SB5-22 & 2 & 11 & V02LM22 & $<0.100$ & 5.05 & 0.032 & 0.581 & 0.151 & 0.084 & 6.45 & $<0.010$ & $<0.100$ & 0.057 & 0.289 \\
\hline SRNL-SCS-2008-00043 & SB5-26 & 2 & 12 & V05LM12 & $<0.100$ & 4.67 & 0.035 & 0.629 & 0.153 & 0.099 & 7.27 & $<0.010$ & $<0.100$ & 0.065 & 0.363 \\
\hline SRNL-SCS-2008-00043 & SB5-23 & 2 & 13 & V04LM12 & $<0.100$ & 4.28 & 0.033 & 1.52 & 0.133 & 0.09 & 6.85 & 2.45 & $<0.100$ & 0.057 & 0.32 \\
\hline SRNL-SCS-2008-00043 & SB5-26 & 2 & 14 & V05LM22 & $<0.100$ & 4.68 & 0.036 & 0.626 & 0.147 & 0.094 & 7.23 & $<0.010$ & $<0.100$ & 0.064 & 0.362 \\
\hline SRNL-SCS-2008-00043 & Batch 1 & 2 & 15 & BCHLM23 & $<0.100$ & 2.5 & 0.116 & 0.909 & $<0.010$ & 0.071 & 8.5 & $<0.010$ & 2.65 & $<0.010$ & 0.775 \\
\hline
\end{tabular}


Table A4. Measured Elemental Concentrations (wt\%) for Samples in

SRNL-SCS-2008-00043 Prepared Using Lithium Metaborate. (Part 2)

\begin{tabular}{|c|c|c|c|c|c|c|c|c|c|c|c|c|c|c|}
\hline Analytical Plan & Glass ID & Block & Sequence & Lab ID & Mn (wt\%) & $\mathrm{Na}(\mathrm{wt} \%)$ & $\mathrm{Nd}(\mathrm{wt} \%)$ & Ni (wt\%) & $\mathrm{Pb}(\mathrm{wt} \%)$ & $\mathrm{S}(\mathrm{wt} \%)$ & Si (wt\%) & Ti (wt \%) & Zn (wt \%) & $\mathrm{Zr}(\mathrm{wt} \%)$ \\
\hline SRNL-SCS-2008-00043 & Batch 1 & 1 & 1 & BCHLM11 & 1.33 & 6.54 & 0.104 & 0.45 & $<0.020$ & $<0.050$ & 23.6 & 0.374 & $<0.010$ & 0.062 \\
\hline SRNL-SCS-2008-00043 & SB5-21 & 1 & 2 & V06LM21 & 1.47 & 10.3 & 2.41 & 0.72 & $<0.020$ & 0.085 & 21.2 & $<0.010$ & $<0.010$ & 0.068 \\
\hline SRNL-SCS-2008-00043 & SB5-26 & 1 & 3 & V05LM11 & 1.97 & 11 & $<0.100$ & 0.96 & $<0.020$ & 0.112 & 21.6 & $<0.010$ & $<0.010$ & 0.09 \\
\hline SRNL-SCS-2008-00043 & SB5-21 & 1 & 4 & V06LM11 & 1.54 & 10.2 & 2.43 & 0.804 & $<0.020$ & 0.099 & 21.6 & $<0.010$ & $<0.010$ & 0.072 \\
\hline $\begin{array}{l}\text { SRNL-SCS-2008-00043 } \\
\end{array}$ & SB5-25 & 1 & 5 & V03LM11 & 1.8 & 10.2 & $<0.100$ & 0.836 & $<0.020$ & 0.106 & 21.3 & $<0.010$ & $<0.010$ & 0.077 \\
\hline SRNL-SCS-2008-00043 & SB5-24 & 1 & 6 & V01LM11 & 1.54 & 10.6 & $<0.100$ & 0.743 & $<0.020$ & 0.068 & 21.6 & $<0.010$ & $<0.010$ & 0.063 \\
\hline SRNL-SCS-2008-00043 & SB5-26 & 1 & 7 & V05LM21 & 1.97 & 11 & $<0.100$ & 0.944 & $<0.020$ & 0.108 & 21.7 & $<0.010$ & $<0.010$ & 0.089 \\
\hline $\begin{array}{l}\text { SRNL-SCS-2008-00043 } \\
\end{array}$ & Batch 1 & 1 & 8 & BCHLM12 & 1.39 & 6.67 & 0.105 & 0.463 & $<0.020$ & $<0.050$ & 24.2 & 0.381 & $<0.010$ & 0.064 \\
\hline SRNL-SCS-2008-00043 & SB5-25 & 1 & 9 & V03LM21 & 1.84 & 10.6 & $<0.100$ & 0.852 & $<0.020$ & 0.108 & 21.8 & $<0.010$ & $<0.010$ & 0.086 \\
\hline SRNL-SCS-2008-00043 & SB5-22 & 1 & 10 & V02LM11 & 1.68 & 10.5 & 2.66 & 0.743 & $<0.020$ & 0.091 & 21.8 & $<0.010$ & $<0.010$ & 0.069 \\
\hline SRNL-SCS-2008-00043 & SB5-23 & 1 & 11 & V04LM11 & 1.83 & 10.3 & $<0.100$ & 0.842 & $<0.020$ & 0.105 & 21.9 & $<0.010$ & $<0.010$ & 0.092 \\
\hline SRNL-SCS-2008-00043 & SB5-24 & 1 & 12 & V01LM21 & 1.56 & 10.8 & $<0.100$ & 0.701 & $<0.020$ & 0.073 & 22 & $<0.010$ & $<0.010$ & 0.062 \\
\hline SRNL-SCS-2008-00043 & SB5-22 & 1 & 13 & \begin{tabular}{|l|} 
V02LM21 \\
\end{tabular} & 1.69 & 10.4 & 2.67 & 0.745 & $<0.020$ & 0.1 & 21.9 & $<0.010$ & $<0.010$ & 0.064 \\
\hline SRNL-SCS-2008-00043 & SB5-23 & 1 & 14 & \begin{tabular}{|l} 
V04LM21 \\
\end{tabular} & 1.77 & 9.73 & $<0.100$ & 0.816 & $<0.020$ & 0.105 & 21.2 & $<0.010$ & $<0.010$ & 0.082 \\
\hline SRNL-SCS-2008-00043 & Batch 1 & 1 & 15 & BCHLM13 & 1.4 & 6.58 & 0.105 & 0.465 & $<0.020$ & $<0.050$ & 24.4 & 0.373 & $<0.010$ & 0.064 \\
\hline SRNL-SCS-2008-00043 & Batch 1 & 2 & 1 & BCHLM21 & 1.33 & 7.7 & 0.104 & 0.449 & $<0.020$ & $<0.050$ & 23.6 & 0.363 & $<0.010$ & 0.06 \\
\hline SRNL-SCS-2008-00043 & SB5-24 & 2 & 2 & V01LM12 & 1.46 & 10.7 & $<0.100$ & 0.722 & $<0.020$ & 0.079 & 20.9 & $<0.010$ & $<0.010$ & 0.06 \\
\hline SRNL-SCS-2008-00043 & SB5-23 & 2 & 3 & V04LM22 & 1.66 & 9.87 & $<0.100$ & 0.785 & $<0.020$ & 0.095 & 20.4 & $<0.010$ & $<0.010$ & 0.078 \\
\hline $\begin{array}{l}\text { SRNL-SCS-2008-00043 } \\
\end{array}$ & SB5-25 & 2 & 4 & V03LM22 & 1.73 & 10.4 & $<0.100$ & 0.821 & $<0.020$ & 0.115 & 21 & $<0.010$ & $<0.010$ & 0.082 \\
\hline $\begin{array}{l}\text { SRNL-SCS-2008-00043 } \\
\end{array}$ & SB5-21 & 2 & 5 & V06LM12 & 1.44 & 10.2 & 2.38 & 0.792 & $<0.020$ & 0.107 & 20.8 & $<0.010$ & $<0.010$ & 0.069 \\
\hline SRNL-SCS-2008-00043 & SB5-22 & 2 & 6 & V02LM12 & 1.58 & 10.4 & 2.63 & 0.701 & $<0.020$ & 0.1 & 21 & $<0.010$ & $<0.010$ & 0.064 \\
\hline SRNL-SCS-2008-00043 & SB5-25 & 2 & 7 & V03LM12 & 1.71 & 10.3 & $<0.100$ & 0.821 & $<0.020$ & 0.098 & 20.7 & $<0.010$ & $<0.010$ & 0.074 \\
\hline SRNL-SCS-2008-00043 & Batch 1 & 2 & 8 & BCHLM22 & 1.31 & 6.62 & 0.102 & 0.446 & $<0.020$ & $<0.050$ & 23.3 & 0.369 & $<0.010$ & 0.06 \\
\hline SRNL-SCS-2008-00043 & SB5-24 & 2 & 9 & V01LM22 & 1.45 & 10.7 & $<0.100$ & 0.692 & $<0.020$ & 0.081 & 20.9 & $<0.010$ & $<0.010$ & 0.059 \\
\hline SRNL-SCS-2008-00043 & SB5-21 & 2 & 10 & V06LM22 & 1.44 & 10.2 & 2.39 & 0.707 & $<0.020$ & 0.088 & 20.8 & $<0.010$ & $<0.010$ & 0.065 \\
\hline SRNL-SCS-2008-00043 & SB5-22 & 2 & 11 & V02LM22 & 1.59 & 10.5 & 2.64 & 0.702 & $<0.020$ & 0.101 & 21.8 & $<0.010$ & $<0.010$ & 0.069 \\
\hline SRNL-SCS-2008-00043 & SB5-26 & 2 & 12 & V05LM12 & 1.85 & 11.2 & $<0.100$ & 0.939 & $<0.020$ & 0.107 & 20.9 & $<0.010$ & $<0.010$ & 0.085 \\
\hline SRNL-SCS-2008-00043 & SB5-23 & 2 & 13 & V04LM12 & 1.69 & 10.2 & $<0.100$ & 0.81 & $<0.020$ & 0.098 & 20.9 & $<0.010$ & $<0.010$ & 0.088 \\
\hline SRNL-SCS-2008-00043 & SB5-26 & 2 & 14 & V05LM22 & 1.84 & 11.1 & $<0.100$ & 0.912 & $<0.020$ & 0.11 & 20.8 & $<0.010$ & $<0.010$ & 0.085 \\
\hline $\begin{array}{l}\text { SRNL-SCS-2008-00043 } \\
\end{array}$ & Batch 1 & 2 & 15 & BCHLM23 & 1.3 & 6.67 & 0.103 & 0.442 & $<0.020$ & $<0.050$ & 23.2 & 0.361 & $<0.010$ & 0.06 \\
\hline
\end{tabular}


SRNS-STI-2008-00060

Revision 0

Appendix A

Table A5. Measured Elemental Concentrations (wt\%) for Samples in SRNL-SCS-2008-00043 Prepared Using Peroxide Fusion.

\begin{tabular}{|c|c|c|c|c|c|c|}
\hline Analytical Plan & Glass ID & Block & Sequence & Lab ID & B & $\mathbf{L i}$ \\
\hline SRNL-SCS-2008-00043 & Batch 1 & 1 & 1 & BCHPF11 & 2.54 & 2.04 \\
\hline SRNL-SCS-2008-00043 & SB5-24 & 1 & 2 & V01PF11 & 3.14 & 1.43 \\
\hline SRNL-SCS-2008-00043 & SB5-26 & 1 & 3 & V05PF21 & 3.17 & 1.44 \\
\hline SRNL-SCS-2008-00043 & SB5-22 & 1 & 4 & V02PF21 & 3.12 & 1.43 \\
\hline SRNL-SCS-2008-00043 & SB5-26 & 1 & 5 & V05PF11 & 3.15 & 1.44 \\
\hline SRNL-SCS-2008-00043 & SB5-21 & 1 & 6 & V06PF11 & 2.79 & 1.73 \\
\hline SRNL-SCS-2008-00043 & SB5-22 & 1 & 7 & V02PF11 & 3.11 & 1.41 \\
\hline SRNL-SCS-2008-00043 & Batch 1 & 1 & 8 & BCHPF12 & 2.44 & 2.04 \\
\hline SRNL-SCS-2008-00043 & SB5-24 & 1 & 9 & V01PF21 & 3.17 & 1.43 \\
\hline SRNL-SCS-2008-00043 & SB5-25 & 1 & 10 & V03PF11 & 2.74 & 1.66 \\
\hline SRNL-SCS-2008-00043 & SB5-23 & 1 & 11 & V04PF11 & 2.7 & 1.72 \\
\hline SRNL-SCS-2008-00043 & SB5-23 & 1 & 12 & V04PF21 & 2.77 & 1.74 \\
\hline SRNL-SCS-2008-00043 & SB5-25 & 1 & 13 & V03PF21 & 2.78 & 1.69 \\
\hline SRNL-SCS-2008-00043 & SB5-21 & 1 & 14 & V06PF21 & 2.76 & 1.7 \\
\hline SRNL-SCS-2008-00043 & Batch 1 & 1 & 15 & BCHPF13 & 2.4 & 2.03 \\
\hline SRNL-SCS-2008-00043 & Batch 1 & 2 & 1 & BCHPF21 & 2.46 & 2.05 \\
\hline SRNL-SCS-2008-00043 & SB5-25 & 2 & 2 & V03PF22 & 2.73 & 1.69 \\
\hline SRNL-SCS-2008-00043 & SB5-23 & 2 & 3 & V04PF22 & 2.72 & 1.74 \\
\hline SRNL-SCS-2008-00043 & SB5-24 & 2 & 4 & V01PF12 & 3.02 & 1.43 \\
\hline SRNL-SCS-2008-00043 & SB5-21 & 2 & 5 & V06PF22 & 2.76 & 1.71 \\
\hline SRNL-SCS-2008-00043 & SB5-22 & 2 & 6 & V02PF12 & 3.14 & 1.43 \\
\hline SRNL-SCS-2008-00043 & SB5-26 & 2 & 7 & V05PF22 & 3.21 & 1.48 \\
\hline SRNL-SCS-2008-00043 & Batch 1 & 2 & 8 & BCHPF22 & 2.46 & 2.07 \\
\hline SRNL-SCS-2008-00043 & SB5-26 & 2 & 9 & V05PF12 & 3.17 & 1.46 \\
\hline SRNL-SCS-2008-00043 & SB5-22 & 2 & 10 & V02PF22 & 3.07 & 1.44 \\
\hline SRNL-SCS-2008-00043 & SB5-23 & 2 & 11 & V04PF12 & 2.7 & 1.71 \\
\hline SRNL-SCS-2008-00043 & SB5-25 & 2 & 12 & V03PF12 & 2.78 & 1.7 \\
\hline SRNL-SCS-2008-00043 & SB5-24 & 2 & 13 & V01PF22 & 3.27 & 1.48 \\
\hline SRNL-SCS-2008-00043 & SB5-21 & 2 & 14 & V06PF12 & 2.85 & 1.77 \\
\hline SRNL-SCS-2008-00043 & Batch 1 & 2 & 15 & BCHPF23 & 2.45 & 2.08 \\
\hline
\end{tabular}


SRNS-STI-2008-00060

Revision 0

Appendix A

Table A6. Average Measured and Bias-Corrected Chemical Compositions Versus

Targeted Compositions by Oxide by Study Glass.

\begin{tabular}{|c|c|c|c|c|c|c|c|c|c|}
\hline Analytical Plan/Set & $\begin{array}{l}\text { Glass } \\
\text { ID }\end{array}$ & Oxide & $\begin{array}{c}\text { Measured } \\
\text { (wt \%) }\end{array}$ & $\begin{array}{c}\text { Measured BC } \\
\text { (wt\%) }\end{array}$ & $\begin{array}{c}\text { Targeted } \\
\text { (wt \%) }\end{array}$ & $\begin{array}{c}\text { Diff of } \\
\text { Measured }\end{array}$ & $\begin{array}{c}\text { Diff of } \\
\text { Meas BC }\end{array}$ & $\begin{array}{l}\text { \% Diff of } \\
\text { Measured }\end{array}$ & $\begin{array}{l}\text { \% Diff of } \\
\text { Meas BC }\end{array}$ \\
\hline SRNL-SCS-2008-00003/Set 2 & Batch 1 & $\mathrm{~A} 12 \mathrm{O} 3(\mathrm{wt} \%)$ & 4.7820 & 4.8770 & 4.8770 & -0.0950 & 0.0000 & $-1.9 \%$ & $0.0 \%$ \\
\hline SRNL-SCS-2008-00003/Set 2 & Batch 1 & $\mathrm{~B} 2 \mathrm{O} 3(\mathrm{wt} \%)$ & 7.8056 & 7.7770 & 7.7770 & 0.0286 & 0.0000 & $0.4 \%$ & $0.0 \%$ \\
\hline SRNL-SCS-2008-00003/Set 2 & Batch 1 & $\mathrm{BaO}(\mathrm{wt} \%)$ & 0.1421 & 0.1510 & 0.1510 & -0.0089 & 0.0000 & $-5.9 \%$ & $0.0 \%$ \\
\hline SRNL-SCS-2008-00003/Set 2 & Batch 1 & $\mathrm{CaO}(\mathrm{wt} \%)$ & 1.1885 & 1.2200 & 1.2200 & -0.0315 & 0.0000 & $-2.6 \%$ & $0.0 \%$ \\
\hline SRNL-SCS-2008-00003/Set 2 & Batch 1 & $\mathrm{CdO}(\mathrm{wt} \%)$ & 0.0057 & 0.0057 & 0.0000 & 0.0057 & 0.0057 & & \\
\hline SRNL-SCS-2008-00003/Set 2 & Batch 1 & $\mathrm{Ce} 2 \mathrm{O} 3(\mathrm{wt} \%)$ & 0.0130 & 0.0130 & 0.0000 & 0.0130 & 0.0130 & & \\
\hline SRNL-SCS-2008-00003/Set 2 & Batch 1 & $\mathrm{Cr} 2 \mathrm{O} 3(\mathrm{wt} \%)$ & 0.1077 & 0.1070 & 0.1070 & 0.0007 & 0.0000 & $0.6 \%$ & $0.0 \%$ \\
\hline SRNL-SCS-2008-00003/Set 2 & Batch 1 & $\mathrm{CuO}(\mathrm{wt} \%)$ & 0.3781 & 0.3990 & 0.3990 & -0.0209 & 0.0000 & $-5.2 \%$ & $0.0 \%$ \\
\hline SRNL-SCS-2008-00003/Set 2 & Batch 1 & $\mathrm{Fe} 2 \mathrm{O} 3(\mathrm{wt} \%)$ & 13.0341 & 12.8390 & 12.8390 & 0.1951 & 0.0000 & $1.5 \%$ & $0.0 \%$ \\
\hline SRNL-SCS-2008-00003/Set 2 & Batch 1 & $\mathrm{~K} 2 \mathrm{O}(\mathrm{wt} \%)$ & 3.0406 & 3.3270 & 3.3270 & -0.2864 & 0.0000 & $-8.6 \%$ & $0.0 \%$ \\
\hline SRNL-SCS-2008-00003/Set 2 & Batch 1 & $\mathrm{La} 2 \mathrm{O} 3(\mathrm{wt} \%)$ & 0.0586 & 0.0586 & 0.0000 & 0.0586 & 0.0586 & & \\
\hline SRNL-SCS-2008-00003/Set 2 & Batch 1 & $\mathrm{Li} 2 \mathrm{O}(\mathrm{wt} \%)$ & 4.2412 & 4.4290 & 4.4290 & -0.1878 & 0.0000 & $-4.2 \%$ & $0.0 \%$ \\
\hline SRNL-SCS-2008-00003/Set 2 & Batch 1 & $\mathrm{MgO}\left(\mathrm{wt}^{\circ} \%\right)$ & 1.3794 & 1.4190 & 1.4190 & -0.0396 & 0.0000 & $-2.8 \%$ & $0.0 \%$ \\
\hline SRNL-SCS-2008-00003/Set 2 & Batch 1 & $\mathrm{MnO}\left(\mathrm{wt}^{\%} \%\right)$ & 1.7700 & 1.7260 & 1.7260 & 0.0440 & 0.0000 & $2.6 \%$ & $0.0 \%$ \\
\hline SRNL-SCS-2008-00003/Set 2 & Batch 1 & $\mathrm{Na} 2 \mathrm{O}(\mathrm{wt} \%)$ & 8.9979 & 9.0030 & 9.0030 & -0.0051 & 0.0000 & $-0.1 \%$ & $0.0 \%$ \\
\hline SRNL-SCS-2008-00003/Set 2 & Batch 1 & $\mathrm{Nb} 2 \mathrm{O} 5(\mathrm{wt} \%)$ & 0.0416 & 0.0416 & 0.0000 & 0.0416 & 0.0416 & & \\
\hline SRNL-SCS-2008-00003/Set 2 & Batch 1 & $\mathrm{NiO}(\mathrm{wt} \%)$ & 0.7085 & 0.7510 & 0.7510 & -0.0425 & 0.0000 & $-5.7 \%$ & $0.0 \%$ \\
\hline SRNL-SCS-2008-00003/Set 2 & Batch 1 & $\mathrm{PbO}(\mathrm{wt} \%)$ & 0.0108 & 0.0108 & 0.0000 & 0.0108 & 0.0108 & & \\
\hline SRNL-SCS-2008-00003/Set 2 & Batch 1 & $\mathrm{SiO} 2\left(\mathrm{wt}^{\circ} \%\right)$ & 51.0401 & 50.2200 & 50.2200 & 0.8201 & 0.0000 & $1.6 \%$ & $0.0 \%$ \\
\hline SRNL-SCS-2008-00003/Set 2 & Batch 1 & SO4 (wt \%) & 0.1498 & 0.1498 & 0.0000 & 0.1498 & 0.1498 & & \\
\hline SRNL-SCS-2008-00003/Set 2 & Batch 1 & ThO2 (wt $\%)$ & 0.0569 & 0.0569 & 0.0000 & 0.0569 & 0.0569 & & \\
\hline SRNL-SCS-2008-00003/Set 2 & Batch 1 & $\mathrm{TiO} 2(\mathrm{wt} \%)$ & 0.6543 & 0.6770 & 0.6770 & -0.0227 & 0.0000 & $-3.4 \%$ & $0.0 \%$ \\
\hline SRNL-SCS-2008-00003/Set 2 & Batch 1 & $\mathrm{U} 3 \mathrm{O} 8(\mathrm{wt} \%)$ & 0.0590 & 0.0607 & 0.0000 & 0.0590 & 0.0607 & & \\
\hline SRNL-SCS-2008-00003/Set 2 & Batch 1 & $\mathrm{ZnO}(\mathrm{wt} \%)$ & 0.0062 & 0.0062 & 0.0000 & 0.0062 & 0.0062 & & \\
\hline SRNL-SCS-2008-00003/Set 2 & Batch 1 & $\mathrm{ZrO} 2(\mathrm{wt} \%)$ & 0.0857 & 0.0857 & 0.0980 & -0.0123 & -0.0123 & $-12.6 \%$ & $-12.6 \%$ \\
\hline SRNL-SCS-2008-00003/Set 2 & Batch 1 & Sum & 99.7574 & 99.4110 & 99.0200 & 0.7374 & 0.3910 & $0.7 \%$ & $0.4 \%$ \\
\hline SRNL-SCS-2008-00003/Set 2 & SB5-01 & $\mathrm{A} 12 \mathrm{O} 3(\mathrm{wt} \%)$ & 11.0489 & 11.4685 & 11.0090 & 0.0399 & 0.4595 & $0.4 \%$ & $4.2 \%$ \\
\hline SRNL-SCS-2008-00003/Set 2 & SB5-01 & $\mathrm{B} 2 \mathrm{O} 3(\mathrm{wt} \%)$ & 6.2064 & 6.1308 & 6.2000 & 0.0064 & -0.0692 & $0.1 \%$ & $-1.1 \%$ \\
\hline SRNL-SCS-2008-00003/Set 2 & SB5-01 & $\mathrm{BaO}(\mathrm{wt} \%)$ & 0.0341 & 0.0367 & 0.0390 & -0.0049 & -0.0023 & $-12.7 \%$ & $-5.9 \%$ \\
\hline SRNL-SCS-2008-00003/Set 2 & SB5-01 & $\mathrm{CaO}(\mathrm{wt} \%)$ & 3.4001 & 3.5382 & 3.1570 & 0.2431 & 0.3812 & $7.7 \%$ & $12.1 \%$ \\
\hline SRNL-SCS-2008-00003/Set 2 & SB5-01 & $\mathrm{CdO}(\mathrm{wt} \%)$ & 0.0146 & 0.0146 & 0.0230 & -0.0084 & -0.0084 & $-36.7 \%$ & $-36.7 \%$ \\
\hline SRNL-SCS-2008-00003/Set 2 & SB5-01 & $\mathrm{Ce} 2 \mathrm{O} 3(\mathrm{wt} \%)$ & 0.0990 & 0.0990 & 0.1410 & -0.0420 & -0.0420 & $-29.8 \%$ & $-29.8 \%$ \\
\hline SRNL-SCS-2008-00003/Set 2 & SB5-01 & $\mathrm{Cr} 2 \mathrm{O} 3(\mathrm{wt} \%)$ & 0.1118 & 0.1124 & 0.1420 & -0.0302 & -0.0296 & $-21.3 \%$ & $-20.9 \%$ \\
\hline SRNL-SCS-2008-00003/Set 2 & SB5-01 & $\mathrm{CuO}(\mathrm{wt} \%)$ & 0.0063 & 0.0067 & 0.0050 & 0.0013 & 0.0017 & $25.2 \%$ & $34.0 \%$ \\
\hline SRNL-SCS-2008-00003/Set 2 & SB5-01 & $\mathrm{Fe} 2 \mathrm{O} 3(\mathrm{wt} \%)$ & 8.6676 & 8.4919 & 8.8340 & -0.1664 & -0.3421 & $-1.9 \%$ & $-3.9 \%$ \\
\hline SRNL-SCS-2008-00003/Set 2 & SB5-01 & $\mathrm{K} 2 \mathrm{O}(\mathrm{wt} \%)$ & 0.0157 & 0.0174 & 0.0250 & -0.0093 & -0.0076 & $-37.4 \%$ & $-30.4 \%$ \\
\hline SRNL-SCS-2008-00003/Set 2 & SB5-01 & $\mathrm{La2O} 3(\mathrm{wt} \%)$ & 0.0586 & 0.0586 & 0.0620 & -0.0034 & -0.0034 & $-5.4 \%$ & $-5.4 \%$ \\
\hline SRNL-SCS-2008-00003/Set 2 & SB5-01 & $\mathrm{Li} 2 \mathrm{O}(\mathrm{wt} \%)$ & 4.2251 & 4.3752 & 4.3400 & -0.1149 & 0.0352 & $-2.6 \%$ & $0.8 \%$ \\
\hline SRNL-SCS-2008-00003/Set 2 & SB5-01 & $\mathrm{MgO}(\mathrm{wt} \%)$ & 0.4386 & 0.4552 & 0.4470 & -0.0084 & 0.0082 & $-1.9 \%$ & $1.8 \%$ \\
\hline SRNL-SCS-2008-00003/Set 2 & SB5-01 & $\mathrm{MnO}(\mathrm{wt} \%)$ & 1.8852 & 1.8187 & 1.8470 & 0.0382 & -0.0283 & $2.1 \%$ & $-1.5 \%$ \\
\hline SRNL-SCS-2008-00003/Set 2 & SB5-01 & $\mathrm{Na} 2 \mathrm{O}(\mathrm{wt} \%)$ & 13.5541 & 13.8242 & 13.7760 & -0.2219 & 0.0482 & $-1.6 \%$ & $0.3 \%$ \\
\hline SRNL-SCS-2008-00003/Set 2 & SB5-01 & $\mathrm{Nb} 2 \mathrm{O} 5(\mathrm{wt} \%)$ & 0.0072 & 0.0072 & 0.0000 & 0.0072 & 0.0072 & & \\
\hline SRNL-SCS-2008-00003/Set 2 & SB5-01 & $\mathrm{NiO}(w \mathrm{t} \%)$ & 0.9102 & 0.9743 & 1.0370 & -0.1268 & -0.0627 & $-12.2 \%$ & $-6.0 \%$ \\
\hline SRNL-SCS-2008-00003/Set 2 & SB5-01 & $\mathrm{PbO}(\mathrm{wt} \%)$ & 0.0108 & 0.0108 & 0.0080 & 0.0028 & 0.0028 & $34.7 \%$ & $34.7 \%$ \\
\hline SRNL-SCS-2008-00003/Set 2 & SB5-01 & $\mathrm{SiO} 2(\mathrm{wt} \%)$ & 45.5136 & 44.9843 & 45.3570 & 0.1566 & -0.3727 & $0.3 \%$ & $-0.8 \%$ \\
\hline SRNL-SCS-2008-00003/Set 2 & SB5-01 & SO4 (wt \%) & 0.1498 & 0.1498 & 0.2770 & -0.1272 & -0.1272 & $-45.9 \%$ & $-45.9 \%$ \\
\hline SRNL-SCS-2008-00003/Set 2 & SB5-01 & ThO2 (wt $\%)$ & 0.0569 & 0.0569 & 0.0000 & 0.0569 & 0.0569 & & \\
\hline SRNL-SCS-2008-00003/Set 2 & SB5-01 & $\mathrm{TiO} 2\left(\mathrm{wt}^{\circ} \%\right)$ & 0.0083 & 0.0088 & 0.0100 & -0.0017 & -0.0012 & $-16.6 \%$ & $-12.5 \%$ \\
\hline SRNL-SCS-2008-00003/Set 2 & SB5-01 & $\mathrm{U} 3 \mathrm{O} 8(\mathrm{wt} \%)$ & 2.7210 & 2.8546 & 2.8260 & -0.1050 & 0.0286 & $-3.7 \%$ & $1.0 \%$ \\
\hline SRNL-SCS-2008-00003/Set 2 & SB5-01 & $\mathrm{ZnO}(\mathrm{wt} \%)$ & 0.0062 & 0.0062 & 0.0060 & 0.0002 & 0.0002 & $3.7 \%$ & $3.7 \%$ \\
\hline SRNL-SCS-2008-00003/Set 2 & SB5-01 & $\mathrm{ZrO} 2(\mathrm{wt} \%)$ & 0.0848 & 0.0848 & 0.0980 & -0.0132 & -0.0132 & $-13.5 \%$ & $-13.5 \%$ \\
\hline SRNL-SCS-2008-00003/Set 2 & SB5-01 & Sum & 99.2345 & 99.5854 & 99.6660 & -0.4315 & -0.0806 & $-0.4 \%$ & $-0.1 \%$ \\
\hline SRNL-SCS-2008-00003/Set 2 & SB5-02 & $\mathrm{A} 12 \mathrm{O} 3(\mathrm{wt} \%)$ & 10.8032 & 11.2135 & 11.0090 & -0.2058 & 0.2045 & $-1.9 \%$ & $1.9 \%$ \\
\hline SRNL-SCS-2008-00003/Set 2 & SB5-02 & $\mathrm{B} 2 \mathrm{O} 3(\mathrm{wt} \%)$ & 7.0113 & 6.9257 & 6.8200 & 0.1913 & 0.1057 & $2.8 \%$ & $1.6 \%$ \\
\hline SRNL-SCS-2008-00003/Set 2 & SB5-02 & $\mathrm{BaO}(\mathrm{wt} \%)$ & 0.0352 & 0.0379 & 0.0390 & -0.0038 & -0.0011 & $-9.8 \%$ & $-2.8 \%$ \\
\hline SRNL-SCS-2008-00003/Set 2 & SB5-02 & $\mathrm{CaO}(\mathrm{wt} \%)$ & 1.9764 & 2.0566 & 1.9170 & 0.0594 & 0.1396 & $3.1 \%$ & $7.3 \%$ \\
\hline SRNL-SCS-2008-00003/Set 2 & SB5-02 & $\mathrm{CdO}(\mathrm{wt} \%)$ & 0.0148 & 0.0148 & 0.0230 & -0.0082 & -0.0082 & $-35.4 \%$ & $-35.4 \%$ \\
\hline SRNL-SCS-2008-00003/Set 2 & SB5-02 & $\mathrm{Ce} 2 \mathrm{O} 3(\mathrm{wt} \%)$ & 0.0972 & 0.0972 & 0.1410 & -0.0438 & -0.0438 & $-31.1 \%$ & $-31.1 \%$ \\
\hline SRNL-SCS-2008-00003/Set 2 & SB5-02 & $\mathrm{Cr} 2 \mathrm{O} 3(\mathrm{wt} \%)$ & 0.1195 & 0.1201 & 0.1420 & -0.0225 & -0.0219 & $-15.9 \%$ & $-15.4 \%$ \\
\hline SRNL-SCS-2008-00003/Set 2 & SB5-02 & $\mathrm{CuO}(\mathrm{wt} \%)$ & 0.0063 & 0.0067 & 0.0050 & 0.0013 & 0.0017 & $25.2 \%$ & $34.0 \%$ \\
\hline SRNL-SCS-2008-00003/Set 2 & SB5-02 & $\mathrm{Fe} 2 \mathrm{O} 3(\mathrm{wt} \%)$ & 8.3673 & 8.2004 & 8.8340 & -0.4667 & -0.6336 & $-5.3 \%$ & $-7.2 \%$ \\
\hline SRNL-SCS-2008-00003/Set 2 & SB5-02 & $\mathrm{K} 2 \mathrm{O}(\mathrm{wt} \%)$ & 0.0139 & 0.0154 & 0.0250 & -0.0111 & -0.0096 & $-44.6 \%$ & $-38.5 \%$ \\
\hline SRNL-SCS-2008-00003/Set 2 & SB5-02 & $\mathrm{La} 2 \mathrm{O} 3(\mathrm{wt} \%)$ & 0.0586 & 0.0586 & 0.0620 & -0.0034 & -0.0034 & $-5.4 \%$ & $-5.4 \%$ \\
\hline SRNL-SCS-2008-00003/Set 2 & SB5-02 & $\mathrm{Li} 2 \mathrm{O}(\mathrm{wt} \%)$ & 4.3596 & 4.5144 & 4.3400 & 0.0196 & 0.1744 & $0.5 \%$ & $4.0 \%$ \\
\hline SRNL-SCS-2008-00003/Set 2 & SB5-02 & $\mathrm{MgO}(\mathrm{wt} \%)$ & 0.4527 & 0.4698 & 0.4470 & 0.0057 & 0.0228 & $1.3 \%$ & $5.1 \%$ \\
\hline SRNL-SCS-2008-00003/Set 2 & SB5-02 & $\mathrm{MnO}(\mathrm{wt} \%)$ & 1.8400 & 1.7759 & 1.8470 & -0.0070 & -0.0711 & $-0.4 \%$ & $-3.9 \%$ \\
\hline SRNL-SCS-2008-00003/Set 2 & SB5-02 & $\mathrm{Na} 2 \mathrm{O}(\mathrm{wt} \%)$ & 13.2643 & 13.5285 & 13.7760 & -0.5117 & -0.2475 & $-3.7 \%$ & $-1.8 \%$ \\
\hline
\end{tabular}


SRNS-STI-2008-00060

Revision 0

Appendix A

Table A6. Average Measured and Bias-Corrected Chemical Compositions Versus Targeted Compositions by Oxide by Study Glass. (continued)

\begin{tabular}{|c|c|c|c|c|c|c|c|c|c|}
\hline Analytical Plan/Set & $\begin{array}{l}\text { Glass } \\
\text { ID }\end{array}$ & Oxide & $\begin{array}{c}\text { Measured } \\
\text { (wt\%) }\end{array}$ & $\begin{array}{c}\text { Measured BC } \\
\text { (wt\%) }\end{array}$ & $\begin{array}{c}\text { Targeted } \\
\text { (wt \%) }\end{array}$ & $\begin{array}{c}\text { Diff of } \\
\text { Measured }\end{array}$ & $\begin{array}{c}\text { Diff of } \\
\text { Meas BC }\end{array}$ & $\begin{array}{l}\text { \% Diff of } \\
\text { Measured }\end{array}$ & $\begin{array}{l}\text { \% Diff of } \\
\text { Meas BC }\end{array}$ \\
\hline SRNL-SCS-2008-00003/Set 2 & SB5-02 & $\mathrm{Nb} 2 \mathrm{O} 5(\mathrm{wt} \%)$ & 0.0072 & 0.0072 & 0.0000 & 0.0072 & 0.0072 & & \\
\hline SRNL-SCS-2008-00003/Set 2 & SB5-02 & $\mathrm{NiO}(\mathrm{wt} \%)$ & 0.9305 & 0.9961 & 1.0370 & -0.1065 & -0.0409 & $-10.3 \%$ & $-3.9 \%$ \\
\hline SRNL-SCS-2008-00003/Set 2 & SB5-02 & $\mathrm{PbO}(\mathrm{wt} \%)$ & 0.0108 & 0.0108 & 0.0080 & 0.0028 & 0.0028 & $34.7 \%$ & $34.7 \%$ \\
\hline SRNL-SCS-2008-00003/Set 2 & SB5-02 & $\mathrm{SiO} 2\left(\mathrm{wt}^{\circ} \%\right)$ & 45.2462 & 44.7218 & 45.9770 & -0.7308 & -1.2552 & $-1.6 \%$ & $-2.7 \%$ \\
\hline SRNL-SCS-2008-00003/Set 2 & SB5-02 & SO4 $(w t \%)$ & 0.1498 & 0.1498 & 0.2770 & -0.1272 & -0.1272 & $-45.9 \%$ & $-45.9 \%$ \\
\hline SRNL-SCS-2008-00003/Set 2 & SB5-02 & ThO2 (wt $\%)$ & 0.0569 & 0.0569 & 0.0000 & 0.0569 & 0.0569 & & \\
\hline SRNL-SCS-2008-00003/Set 2 & SB5-02 & $\mathrm{TiO} 2\left(\mathrm{wt}^{\%} \%\right)$ & 0.0133 & 0.0140 & 0.0100 & 0.0033 & 0.0040 & $33.4 \%$ & $40.0 \%$ \\
\hline SRNL-SCS-2008-00003/Set 2 & SB5-02 & $\mathrm{U} 3 \mathrm{O} 8(\mathrm{wt} \%)$ & 2.6738 & 2.8050 & 2.8260 & -0.1522 & -0.0210 & $-5.4 \%$ & $-0.7 \%$ \\
\hline SRNL-SCS-2008-00003/Set 2 & SB5-02 & $\mathrm{ZnO}(\mathrm{wt} \%)$ & 0.0062 & 0.0062 & 0.0060 & 0.0002 & 0.0002 & $3.7 \%$ & $3.7 \%$ \\
\hline SRNL-SCS-2008-00003/Set 2 & SB5-02 & $\mathrm{ZrO} 2(\mathrm{wt} \%)$ & 0.0895 & 0.0895 & 0.0980 & -0.0085 & -0.0085 & $-8.7 \%$ & $-8.7 \%$ \\
\hline SRNL-SCS-2008-00003/Set 2 & SB5-02 & Sum & 97.6046 & 97.8929 & 99.6660 & -2.0614 & -1.7731 & $-2.1 \%$ & $-1.8 \%$ \\
\hline SRNL-SCS-2008-00003/Set 2 & SB5-03 & $\mathrm{Al} 2 \mathrm{O} 3(\mathrm{wt} \%)$ & 11.1528 & 11.1786 & 11.0090 & 0.1438 & 0.1696 & $1.3 \%$ & $1.5 \%$ \\
\hline SRNL-SCS-2008-00003/Set 2 & SB5-03 & $\mathrm{B} 2 \mathrm{O} 3(\mathrm{wt} \%)$ & 8.5086 & 8.5509 & 8.6800 & -0.1714 & -0.1291 & $-2.0 \%$ & $-1.5 \%$ \\
\hline SRNL-SCS-2008-00003/Set 2 & SB5-03 & $\mathrm{BaO}\left(\mathrm{wt}^{\%} \%\right)$ & 0.0371 & 0.0389 & 0.0390 & -0.0019 & -0.0001 & $-4.8 \%$ & $-0.2 \%$ \\
\hline SRNL-SCS-2008-00003/Set 2 & SB5-03 & $\mathrm{CaO}(\mathrm{wt} \%)$ & 2.0393 & 2.0672 & 1.9170 & 0.1223 & 0.1502 & $6.4 \%$ & $7.8 \%$ \\
\hline SRNL-SCS-2008-00003/Set 2 & SB5-03 & $\mathrm{CdO}(\mathrm{wt} \%)$ & 0.0151 & 0.0151 & 0.0230 & -0.0079 & -0.0079 & $-34.2 \%$ & $-34.2 \%$ \\
\hline SRNL-SCS-2008-00003/Set 2 & SB5-03 & $\mathrm{Ce} 2 \mathrm{O} 3(\mathrm{wt} \%)$ & 0.1040 & 0.1040 & 0.1410 & -0.0370 & -0.0370 & $-26.3 \%$ & $-26.3 \%$ \\
\hline SRNL-SCS-2008-00003/Set 2 & SB5-03 & $\mathrm{Cr} 2 \mathrm{O} 3(\mathrm{wt} \%)$ & 0.1199 & 0.1178 & 0.1420 & -0.0221 & -0.0242 & $-15.6 \%$ & $-17.1 \%$ \\
\hline SRNL-SCS-2008-00003/Set 2 & SB5-03 & $\mathrm{CuO}(\mathrm{wt} \%)$ & 0.0110 & 0.0113 & 0.0050 & 0.0060 & 0.0063 & $119.1 \%$ & $126.8 \%$ \\
\hline SRNL-SCS-2008-00003/Set 2 & SB5-03 & $\mathrm{Fe} 2 \mathrm{O} 3(\mathrm{wt} \%)$ & 8.5282 & 8.4402 & 8.8340 & -0.3058 & -0.3938 & $-3.5 \%$ & $-4.5 \%$ \\
\hline SRNL-SCS-2008-00003/Set 2 & SB5-03 & $\mathrm{K} 2 \mathrm{O}(\mathrm{wt} \%)$ & 0.0099 & 0.0107 & 0.0250 & -0.0151 & -0.0143 & $-60.2 \%$ & $-57.2 \%$ \\
\hline SRNL-SCS-2008-00003/Set 2 & SB5-03 & $\mathrm{La} 2 \mathrm{O} 3(\mathrm{wt} \%)$ & 0.0586 & 0.0586 & 0.0620 & -0.0034 & -0.0034 & $-5.4 \%$ & $-5.4 \%$ \\
\hline SRNL-SCS-2008-00003/Set 2 & SB5-03 & $\mathrm{Li} 2 \mathrm{O}(\mathrm{wt} \%)$ & 3.5630 & 3.7525 & 3.7200 & -0.1570 & 0.0325 & $-4.2 \%$ & $0.9 \%$ \\
\hline SRNL-SCS-2008-00003/Set 2 & SB5-03 & $\mathrm{MgO}(\mathrm{wt} \%)$ & 0.4681 & 0.4772 & 0.4470 & 0.0211 & 0.0302 & $4.7 \%$ & $6.8 \%$ \\
\hline SRNL-SCS-2008-00003/Set 2 & SB5-03 & $\mathrm{MnO}\left(\mathrm{wt}^{\circ} \%\right)$ & 1.8690 & 1.8408 & 1.8470 & 0.0220 & -0.0062 & $1.2 \%$ & $-0.3 \%$ \\
\hline SRNL-SCS-2008-00003/Set 2 & SB5-03 & $\mathrm{Na} 2 \mathrm{O}(\mathrm{wt} \%)$ & 13.7934 & 13.5425 & 13.7760 & 0.0174 & -0.2335 & $0.1 \%$ & $-1.7 \%$ \\
\hline SRNL-SCS-2008-00003/Set 2 & SB5-03 & $\mathrm{Nb} 2 \mathrm{O} 5(\mathrm{wt} \%)$ & 0.0072 & 0.0072 & 0.0000 & 0.0072 & 0.0072 & & \\
\hline SRNL-SCS-2008-00003/Set 2 & SB5-03 & $\mathrm{NiO}(\mathrm{wt} \%)$ & 0.9614 & 1.0092 & 1.0370 & -0.0756 & -0.0278 & $-7.3 \%$ & $-2.7 \%$ \\
\hline SRNL-SCS-2008-00003/Set 2 & SB5-03 & $\mathrm{PbO}(\mathrm{wt} \%)$ & 0.0108 & 0.0108 & 0.0080 & 0.0028 & 0.0028 & $34.7 \%$ & $34.7 \%$ \\
\hline SRNL-SCS-2008-00003/Set 2 & SB5-03 & $\mathrm{SiO} 2(\mathrm{wt} \%)$ & 45.1392 & 44.2131 & 44.7370 & 0.4022 & -0.5239 & $0.9 \%$ & $-1.2 \%$ \\
\hline SRNL-SCS-2008-00003/Set 2 & SB5-03 & $\mathrm{SO} 4(\mathrm{wt} \%)$ & 0.1498 & 0.1498 & 0.2770 & -0.1272 & -0.1272 & $-45.9 \%$ & $-45.9 \%$ \\
\hline SRNL-SCS-2008-00003/Set 2 & SB5-03 & ThO2 (wt $\%)$ & 0.0569 & 0.0569 & 0.0000 & 0.0569 & 0.0569 & & \\
\hline SRNL-SCS-2008-00003/Set 2 & SB5-03 & $\mathrm{TiO} 2(\mathrm{wt} \%)$ & 0.0129 & 0.0132 & 0.0100 & 0.0029 & 0.0032 & $29.3 \%$ & $32.1 \%$ \\
\hline SRNL-SCS-2008-00003/Set 2 & SB5-03 & $\mathrm{U} 3 \mathrm{O} 8(\mathrm{wt} \%)$ & 2.8035 & 2.8328 & 2.8260 & -0.0225 & 0.0068 & $-0.8 \%$ & $0.2 \%$ \\
\hline SRNL-SCS-2008-00003/Set 2 & SB5-03 & $\mathrm{ZnO}(\mathrm{wt} \%)$ & 0.0062 & 0.0062 & 0.0060 & 0.0002 & 0.0002 & $3.7 \%$ & $3.7 \%$ \\
\hline SRNL-SCS-2008-00003/Set 2 & SB5-03 & $\mathrm{ZrO} 2(\mathrm{wt} \%)$ & 0.0942 & 0.0942 & 0.0980 & -0.0038 & -0.0038 & $-3.9 \%$ & $-3.9 \%$ \\
\hline SRNL-SCS-2008-00003/Set 2 & SB5-03 & Sum & 99.5201 & 98.5997 & 99.6660 & -0.1459 & -1.0663 & $-0.1 \%$ & $-1.1 \%$ \\
\hline SRNL-SCS-2008-00003/Set 2 & SB5-04 & $\mathrm{A} 12 \mathrm{O} 3(\mathrm{wt} \%)$ & 11.0441 & 11.4636 & 11.0090 & 0.0351 & 0.4546 & $0.3 \%$ & $4.1 \%$ \\
\hline SRNL-SCS-2008-00003/Set 2 & SB5-04 & $\mathrm{B} 2 \mathrm{O} 3(\mathrm{wt} \%)$ & 10.3037 & 10.3567 & 9.9200 & 0.3837 & 0.4367 & $3.9 \%$ & $4.4 \%$ \\
\hline SRNL-SCS-2008-00003/Set 2 & SB5-04 & $\mathrm{BaO}(\mathrm{wt} \%)$ & 0.0360 & 0.0388 & 0.0390 & -0.0030 & -0.0002 & $-7.7 \%$ & $-0.5 \%$ \\
\hline SRNL-SCS-2008-00003/Set 2 & SB5-04 & $\mathrm{CaO}(\mathrm{wt} \%)$ & 0.6527 & 0.6793 & 0.6770 & -0.0243 & 0.0023 & $-3.6 \%$ & $0.3 \%$ \\
\hline SRNL-SCS-2008-00003/Set 2 & SB5-04 & $\mathrm{CdO}(\mathrm{wt} \%)$ & 0.0168 & 0.0168 & 0.0230 & -0.0062 & -0.0062 & $-26.7 \%$ & $-26.7 \%$ \\
\hline SRNL-SCS-2008-00003/Set 2 & SB5-04 & $\mathrm{Ce} 2 \mathrm{O} 3(\mathrm{wt} \%)$ & 0.0668 & 0.0668 & 0.1410 & -0.0742 & -0.0742 & $-52.6 \%$ & $-52.6 \%$ \\
\hline SRNL-SCS-2008-00003/Set 2 & SB5-04 & $\mathrm{Cr} 2 \mathrm{O} 3(\mathrm{wt} \%)$ & 0.1122 & 0.1128 & 0.1420 & -0.0298 & -0.0292 & $-21.0 \%$ & $-20.6 \%$ \\
\hline SRNL-SCS-2008-00003/Set 2 & SB5-04 & $\mathrm{CuO}(\mathrm{wt} \%)$ & 0.0131 & 0.0141 & 0.0050 & 0.0081 & 0.0091 & $162.9 \%$ & $181.5 \%$ \\
\hline SRNL-SCS-2008-00003/Set 2 & SB5-04 & $\mathrm{Fe} 2 \mathrm{O} 3(\mathrm{wt} \%)$ & 8.7140 & 8.5405 & 8.8340 & -0.1200 & -0.2935 & $-1.4 \%$ & $-3.3 \%$ \\
\hline SRNL-SCS-2008-00003/Set 2 & SB5-04 & $\mathrm{K} 2 \mathrm{O}(\mathrm{wt} \%)$ & 0.0145 & 0.0161 & 0.0250 & -0.0105 & -0.0089 & $-42.2 \%$ & $-35.8 \%$ \\
\hline SRNL-SCS-2008-00003/Set 2 & SB5-04 & $\mathrm{La} 2 \mathrm{O} 3(\mathrm{wt} \%)$ & 0.0586 & 0.0586 & 0.0620 & -0.0034 & -0.0034 & $-5.4 \%$ & $-5.4 \%$ \\
\hline SRNL-SCS-2008-00003/Set 2 & SB5-04 & $\mathrm{Li} 2 \mathrm{O}(\mathrm{wt} \%)$ & 3.1163 & 3.2821 & 3.1000 & 0.0163 & 0.1821 & $0.5 \%$ & $5.9 \%$ \\
\hline SRNL-SCS-2008-00003/Set 2 & SB5-04 & $\mathrm{MgO}(\mathrm{wt} \%)$ & 0.4502 & 0.4673 & 0.4470 & 0.0032 & 0.0203 & $0.7 \%$ & $4.5 \%$ \\
\hline SRNL-SCS-2008-00003/Set 2 & SB5-04 & $\mathrm{MnO}(\mathrm{wt} \%)$ & 1.9174 & 1.8509 & 1.8470 & 0.0704 & 0.0039 & $3.8 \%$ & $0.2 \%$ \\
\hline SRNL-SCS-2008-00003/Set 2 & SB5-04 & $\mathrm{Na} 2 \mathrm{O}(\mathrm{wt} \%)$ & 14.4910 & 14.7797 & 14.3960 & 0.0950 & 0.3837 & $0.7 \%$ & $2.7 \%$ \\
\hline SRNL-SCS-2008-00003/Set 2 & SB5-04 & $\mathrm{Nb} 2 \mathrm{O} 5(\mathrm{wt} \%)$ & 0.0072 & 0.0072 & 0.0000 & 0.0072 & 0.0072 & & \\
\hline SRNL-SCS-2008-00003/Set 2 & SB5-04 & $\mathrm{NiO}(\mathrm{wt} \%)$ & 0.9159 & 0.9804 & 1.0370 & -0.1211 & -0.0566 & $-11.7 \%$ & $-5.5 \%$ \\
\hline SRNL-SCS-2008-00003/Set 2 & SB5-04 & $\mathrm{PbO}(\mathrm{wt} \%)$ & 0.0108 & 0.0108 & 0.0080 & 0.0028 & 0.0028 & $34.7 \%$ & $34.7 \%$ \\
\hline SRNL-SCS-2008-00003/Set 2 & SB5-04 & $\mathrm{SiO} 2\left(w^{\circ} \%\right)$ & 44.0696 & 43.5587 & 44.7370 & -0.6674 & -1.1783 & $-1.5 \%$ & $-2.6 \%$ \\
\hline SRNL-SCS-2008-00003/Set 2 & SB5-04 & SO4 $(\mathrm{wt} \%)$ & 0.1498 & 0.1498 & 0.2770 & -0.1272 & -0.1272 & $-45.9 \%$ & $-45.9 \%$ \\
\hline SRNL-SCS-2008-00003/Set 2 & SB5-04 & ThO2 (wt $\%)$ & 0.0569 & 0.0569 & 0.0000 & 0.0569 & 0.0569 & & \\
\hline SRNL-SCS-2008-00003/Set 2 & SB5-04 & $\mathrm{TiO} 2(\mathrm{wt} \%)$ & 0.0104 & 0.0109 & 0.0100 & 0.0004 & 0.0009 & $4.3 \%$ & $9.4 \%$ \\
\hline SRNL-SCS-2008-00003/Set 2 & SB5-04 & $\mathrm{U} 3 \mathrm{O} 8(\mathrm{wt} \%)$ & 2.7534 & 2.8885 & 2.8260 & -0.0726 & 0.0625 & $-2.6 \%$ & $2.2 \%$ \\
\hline SRNL-SCS-2008-00003/Set 2 & SB5-04 & $\mathrm{ZnO}(\mathrm{wt} \%)$ & 0.0062 & 0.0062 & 0.0060 & 0.0002 & 0.0002 & $3.7 \%$ & $3.7 \%$ \\
\hline SRNL-SCS-2008-00003/Set 2 & SB5-04 & $\mathrm{ZrO} 2(\mathrm{wt} \%)$ & 0.0905 & 0.0905 & 0.0980 & -0.0075 & -0.0075 & $-7.6 \%$ & $-7.6 \%$ \\
\hline SRNL-SCS-2008-00003/Set 2 & SB5-04 & Sum & 99.0782 & 99.5039 & 99.6660 & -0.5878 & -0.1621 & $-0.6 \%$ & $-0.2 \%$ \\
\hline SRNL-SCS-2008-00003/Set 2 & SB5-05 & $\mathrm{A} 12 \mathrm{O} 3(\mathrm{wt} \%)$ & 9.5798 & 9.9436 & 9.4600 & 0.1198 & 0.4836 & $1.3 \%$ & $5.1 \%$ \\
\hline SRNL-SCS-2008-00003/Set 2 & SB5-05 & $\mathrm{B} 2 \mathrm{O} 3(\mathrm{wt} \%)$ & 6.4961 & 6.4174 & 6.2000 & 0.2961 & 0.2174 & $4.8 \%$ & $3.5 \%$ \\
\hline SRNL-SCS-2008-00003/Set 2 & SB5-05 & $\mathrm{BaO}(\mathrm{wt} \%)$ & 0.0377 & 0.0406 & 0.0430 & -0.0053 & -0.0024 & $-12.4 \%$ & $-5.6 \%$ \\
\hline SRNL-SCS-2008-00003/Set 2 & SB5-05 & $\mathrm{CaO}(\mathrm{wt} \%)$ & 3.4770 & 3.6183 & 3.2270 & 0.2500 & 0.3913 & $7.7 \%$ & $12.1 \%$ \\
\hline SRNL-SCS-2008-00003/Set 2 & SB5-05 & $\mathrm{CdO}(\mathrm{wt} \%)$ & 0.0126 & 0.0126 & 0.0250 & -0.0124 & -0.0124 & $-49.7 \%$ & $-49.7 \%$ \\
\hline
\end{tabular}


SRNS-STI-2008-00060

Revision 0

Appendix A

Table A6. Average Measured and Bias-Corrected Chemical Compositions Versus Targeted Compositions by Oxide by Study Glass. (continued)

\begin{tabular}{|c|c|c|c|c|c|c|c|c|c|}
\hline Analytical Plan/Set & $\begin{array}{c}\text { Glass } \\
\text { ID }\end{array}$ & Oxide & $\begin{array}{c}\text { Measured } \\
\text { (wt\%) }\end{array}$ & $\begin{array}{c}\text { Measured BC } \\
\text { (wt \%) }\end{array}$ & $\begin{array}{c}\text { Targeted } \\
\text { (wt \%) }\end{array}$ & $\begin{array}{c}\text { Diff of } \\
\text { Measured }\end{array}$ & $\begin{array}{c}\text { Diff of } \\
\text { Meas BC }\end{array}$ & $\begin{array}{l}\text { \% Diff of } \\
\text { Measured }\end{array}$ & $\begin{array}{l}\text { \% Diff of } \\
\text { Meas BC }\end{array}$ \\
\hline SRNL-SCS-2008-00003/Set 2 & SB5-05 & $\mathrm{Ce} 2 \mathrm{O} 3(\mathrm{wt} \%)$ & 0.0600 & 0.0600 & 0.1550 & -0.0950 & -0.0950 & $-61.3 \%$ & $-61.3 \%$ \\
\hline SRNL-SCS-2008-00003/Set 2 & SB5-05 & $\mathrm{Cr} 2 \mathrm{O} 3(\mathrm{wt} \%)$ & 0.1250 & 0.1256 & 0.1560 & -0.0310 & -0.0304 & $-19.9 \%$ & $-19.5 \%$ \\
\hline SRNL-SCS-2008-00003/Set 2 & SB5-05 & $\mathrm{CuO}(\mathrm{wt} \%)$ & 0.0063 & 0.0067 & 0.0050 & 0.0013 & 0.0017 & $25.2 \%$ & $34.0 \%$ \\
\hline SRNL-SCS-2008-00003/Set 2 & SB5-05 & $\mathrm{Fe} 2 \mathrm{O} 3(\mathrm{wt} \%)$ & 9.4360 & 9.2479 & 9.7450 & -0.3090 & -0.4971 & $-3.2 \%$ & $-5.1 \%$ \\
\hline SRNL-SCS-2008-00003/Set 2 & SB5-05 & $\mathrm{K} 2 \mathrm{O}(\mathrm{wt} \%)$ & 0.0181 & 0.0201 & 0.0270 & -0.0089 & -0.0069 & $-33.1 \%$ & $-25.7 \%$ \\
\hline SRNL-SCS-2008-00003/Set 2 & SB5-05 & $\mathrm{La} 2 \mathrm{O} 3(\mathrm{wt} \%)$ & 0.0586 & 0.0586 & 0.0680 & -0.0094 & -0.0094 & $-13.8 \%$ & $-13.8 \%$ \\
\hline SRNL-SCS-2008-00003/Set 2 & SB5-05 & $\mathrm{Li} 2 \mathrm{O}(\mathrm{wt} \%)$ & 4.3435 & 4.4977 & 4.3400 & 0.0035 & 0.1577 & $0.1 \%$ & $3.6 \%$ \\
\hline SRNL-SCS-2008-00003/Set 2 & SB5-05 & $\mathrm{MgO}\left(\mathrm{wt}^{\%} \%\right)$ & 0.4851 & 0.5034 & 0.4940 & -0.0089 & 0.0094 & $-1.8 \%$ & $1.9 \%$ \\
\hline SRNL-SCS-2008-00003/Set 2 & SB5-05 & $\mathrm{MnO}(\mathrm{wt} \%)$ & 2.0530 & 1.9817 & 2.0380 & 0.0150 & -0.0563 & $0.7 \%$ & $-2.8 \%$ \\
\hline SRNL-SCS-2008-00003/Set 2 & SB5-05 & $\mathrm{Na} 2 \mathrm{O}(\mathrm{wt} \%)$ & 13.5373 & 13.8069 & 13.5340 & 0.0033 & 0.2729 & $0.0 \%$ & $2.0 \%$ \\
\hline SRNL-SCS-2008-00003/Set 2 & SB5-05 & $\mathrm{Nb} 2 \mathrm{O} 5(\mathrm{wt} \%)$ & 0.0072 & 0.0072 & 0.0000 & 0.0072 & 0.0072 & & \\
\hline SRNL-SCS-2008-00003/Set 2 & SB5-05 & $\mathrm{NiO}(\mathrm{wt} \%)$ & 1.0059 & 1.0768 & 1.1440 & -0.1381 & -0.0672 & $-12.1 \%$ & $-5.9 \%$ \\
\hline SRNL-SCS-2008-00003/Set 2 & SB5-05 & $\mathrm{PbO}(\mathrm{wt} \%)$ & 0.0108 & 0.0108 & 0.0090 & 0.0018 & 0.0018 & $19.7 \%$ & $19.7 \%$ \\
\hline SRNL-SCS-2008-00003/Set 2 & SB5-05 & $\mathrm{SiO} 2(\mathrm{wt} \%)$ & 44.6579 & 44.1400 & 45.4310 & -0.7731 & -1.2910 & $-1.7 \%$ & $-2.8 \%$ \\
\hline SRNL-SCS-2008-00003/Set 2 & SB5-05 & $\mathrm{SO} 4(\mathrm{wt} \%)$ & 0.1498 & 0.1498 & 0.2890 & -0.1392 & -0.1392 & $-48.2 \%$ & $-48.2 \%$ \\
\hline SRNL-SCS-2008-00003/Set 2 & SB5-05 & ThO2 (wt $\%)$ & 0.0569 & 0.0569 & 0.0000 & 0.0569 & 0.0569 & & \\
\hline SRNL-SCS-2008-00003/Set 2 & SB5-05 & $\mathrm{TiO} 2(\mathrm{wt} \%)$ & 0.0129 & 0.0136 & 0.0110 & 0.0019 & 0.0026 & $17.5 \%$ & $23.3 \%$ \\
\hline SRNL-SCS-2008-00003/Set 2 & SB5-05 & $\mathrm{U} 3 \mathrm{O} 8(\mathrm{wt} \%)$ & 3.0188 & 3.1669 & 3.1170 & -0.0982 & 0.0499 & $-3.2 \%$ & $1.6 \%$ \\
\hline SRNL-SCS-2008-00003/Set 2 & SB5-05 & $\mathrm{ZnO}\left(\mathrm{wt}^{\%} \%\right)$ & 0.0062 & 0.0062 & 0.0060 & 0.0002 & 0.0002 & $3.7 \%$ & $3.7 \%$ \\
\hline SRNL-SCS-2008-00003/Set 2 & SB5-05 & $\mathrm{ZrO} 2\left(\mathrm{wt}^{\circ} \%\right)$ & 0.0969 & 0.0969 & 0.1080 & -0.0111 & -0.0111 & $-10.3 \%$ & $-10.3 \%$ \\
\hline SRNL-SCS-2008-00003/Set 2 & SB5-05 & Sum & 98.7492 & 99.0661 & 99.6320 & -0.8828 & -0.5659 & $-0.9 \%$ & $-0.6 \%$ \\
\hline SRNL-SCS-2008-00003/Set 2 & SB5-06 & $\mathrm{Al} 2 \mathrm{O} 3(\mathrm{wt} \%)$ & 9.7309 & 9.7569 & 9.4600 & 0.2709 & 0.2969 & $2.9 \%$ & $3.1 \%$ \\
\hline SRNL-SCS-2008-00003/Set 2 & SB5-06 & $\mathrm{B} 2 \mathrm{O} 3(\mathrm{wt} \%)$ & 7.0838 & 6.9982 & 6.8200 & 0.2638 & 0.1782 & $3.9 \%$ & $2.6 \%$ \\
\hline SRNL-SCS-2008-00003/Set 2 & SB5-06 & $\mathrm{BaO}(\mathrm{wt} \%)$ & 0.0388 & 0.0407 & 0.0430 & -0.0042 & -0.0023 & $-9.8 \%$ & $-5.4 \%$ \\
\hline SRNL-SCS-2008-00003/Set 2 & SB5-06 & $\mathrm{CaO}(\mathrm{wt} \%)$ & 2.1268 & 2.1551 & 1.9870 & 0.1398 & 0.1681 & $7.0 \%$ & $8.5 \%$ \\
\hline SRNL-SCS-2008-00003/Set 2 & SB5-06 & $\mathrm{CdO}(\mathrm{wt} \%)$ & 0.0146 & 0.0146 & 0.0250 & -0.0104 & -0.0104 & $-41.7 \%$ & $-41.7 \%$ \\
\hline SRNL-SCS-2008-00003/Set 2 & SB5-06 & $\mathrm{Ce} 2 \mathrm{O} 3(\mathrm{wt} \%)$ & 0.1063 & 0.1063 & 0.1550 & -0.0487 & -0.0487 & $-31.4 \%$ & $-31.4 \%$ \\
\hline SRNL-SCS-2008-00003/Set 2 & SB5-06 & $\mathrm{Cr} 2 \mathrm{O} 3(\mathrm{wt} \%)$ & 0.1166 & 0.1145 & 0.1560 & -0.0394 & -0.0415 & $-25.3 \%$ & $-26.6 \%$ \\
\hline SRNL-SCS-2008-00003/Set 2 & SB5-06 & $\mathrm{CuO}(\mathrm{wt} \%)$ & 0.0097 & 0.0101 & 0.0050 & 0.0047 & 0.0051 & $94.0 \%$ & $101.1 \%$ \\
\hline SRNL-SCS-2008-00003/Set 2 & SB5-06 & $\mathrm{Fe} 2 \mathrm{O} 3(\mathrm{wt} \%)$ & 9.6076 & 9.5143 & 9.7450 & -0.1374 & -0.2307 & $-1.4 \%$ & $-2.4 \%$ \\
\hline SRNL-SCS-2008-00003/Set 2 & SB5-06 & $\mathrm{K} 2 \mathrm{O}(\mathrm{wt} \%)$ & 0.0151 & 0.0162 & 0.0270 & -0.0119 & -0.0108 & $-44.2 \%$ & $-39.9 \%$ \\
\hline SRNL-SCS-2008-00003/Set 2 & SB5-06 & $\mathrm{La} 2 \mathrm{O} 3(\mathrm{wt} \%)$ & 0.0586 & 0.0586 & 0.0680 & -0.0094 & -0.0094 & $-13.8 \%$ & $-13.8 \%$ \\
\hline SRNL-SCS-2008-00003/Set 2 & SB5-06 & $\mathrm{Li} 2 \mathrm{O}(\mathrm{wt} \%)$ & 4.3004 & 4.4531 & 4.3400 & -0.0396 & 0.1131 & $-0.9 \%$ & $2.6 \%$ \\
\hline SRNL-SCS-2008-00003/Set 2 & SB5-06 & $\mathrm{MgO}(\mathrm{wt} \%)$ & 0.4954 & 0.5051 & 0.4940 & 0.0014 & 0.0111 & $0.3 \%$ & $2.3 \%$ \\
\hline SRNL-SCS-2008-00003/Set 2 & SB5-06 & $\mathrm{MnO}(\mathrm{wt} \%)$ & 2.0433 & 2.0147 & 2.0380 & 0.0053 & -0.0233 & $0.3 \%$ & $-1.1 \%$ \\
\hline SRNL-SCS-2008-00003/Set 2 & SB5-06 & $\mathrm{Na} 2 \mathrm{O}(\mathrm{wt} \%)$ & 13.7193 & 13.4764 & 13.5340 & 0.1853 & -0.0576 & $1.4 \%$ & $-0.4 \%$ \\
\hline SRNL-SCS-2008-00003/Set 2 & SB5-06 & $\mathrm{Nb} 2 \mathrm{O} 5(\mathrm{wt} \%)$ & 0.0072 & 0.0072 & 0.0000 & 0.0072 & 0.0072 & & \\
\hline SRNL-SCS-2008-00003/Set 2 & SB5-06 & $\mathrm{NiO}(\mathrm{wt} \%)$ & 1.0142 & 1.0646 & 1.1440 & -0.1298 & -0.0794 & $-11.3 \%$ & $-6.9 \%$ \\
\hline SRNL-SCS-2008-00003/Set 2 & SB5-06 & $\mathrm{PbO}(\mathrm{wt} \%)$ & 0.0108 & 0.0108 & 0.0090 & 0.0018 & 0.0018 & $19.7 \%$ & $19.7 \%$ \\
\hline SRNL-SCS-2008-00003/Set 2 & SB5-06 & $\mathrm{SiO} 2(\mathrm{wt} \%)$ & 45.9950 & 45.0513 & 46.0510 & -0.0560 & -0.9997 & $-0.1 \%$ & $-2.2 \%$ \\
\hline SRNL-SCS-2008-00003/Set 2 & SB5-06 & SO4 $(w t \%)$ & 0.2284 & 0.2284 & 0.2890 & -0.0606 & -0.0606 & $-21.0 \%$ & $-21.0 \%$ \\
\hline SRNL-SCS-2008-00003/Set 2 & SB5-06 & ThO2 (wt $\%)$ & 0.0569 & 0.0569 & 0.0000 & 0.0569 & 0.0569 & & \\
\hline SRNL-SCS-2008-00003/Set 2 & SB5-06 & $\mathrm{TiO} 2\left(\mathrm{wt}^{\%} \%\right)$ & 0.0129 & 0.0132 & 0.0110 & 0.0019 & 0.0022 & $17.5 \%$ & $20.1 \%$ \\
\hline SRNL-SCS-2008-00003/Set 2 & SB5-06 & $\mathrm{U} 3 \mathrm{O} 8(\mathrm{wt} \%)$ & 3.1072 & 3.1411 & 3.1170 & -0.0098 & 0.0241 & $-0.3 \%$ & $0.8 \%$ \\
\hline SRNL-SCS-2008-00003/Set 2 & SB5-06 & $\mathrm{ZnO}(\mathrm{wt} \%)$ & 0.0062 & 0.0062 & 0.0060 & 0.0002 & 0.0002 & $3.7 \%$ & $3.7 \%$ \\
\hline SRNL-SCS-2008-00003/Set 2 & SB5-06 & $\mathrm{ZrO} 2(\mathrm{wt} \%)$ & 0.0993 & 0.0993 & 0.1080 & -0.0087 & -0.0087 & $-8.1 \%$ & $-8.1 \%$ \\
\hline SRNL-SCS-2008-00003/Set 2 & SB5-06 & Sum & 100.0051 & 98.9138 & 99.6320 & 0.3731 & -0.7182 & $0.4 \%$ & $-0.7 \%$ \\
\hline SRNL-SCS-2008-00003/Set 2 & SB5-07 & $\mathrm{Al} 2 \mathrm{O} 3(\mathrm{wt} \%)$ & 9.4522 & 9.8112 & 9.4600 & -0.0078 & 0.3512 & $-0.1 \%$ & $3.7 \%$ \\
\hline SRNL-SCS-2008-00003/Set 2 & SB5-07 & $\mathrm{B} 2 \mathrm{O} 3(\mathrm{wt} \%)$ & 8.6374 & 8.5329 & 8.6800 & -0.0426 & -0.1471 & $-0.5 \%$ & $-1.7 \%$ \\
\hline SRNL-SCS-2008-00003/Set 2 & SB5-07 & $\mathrm{BaO}(\mathrm{wt} \%)$ & 0.0382 & 0.0412 & 0.0430 & -0.0048 & -0.0018 & $-11.1 \%$ & $-4.2 \%$ \\
\hline SRNL-SCS-2008-00003/Set 2 & SB5-07 & $\mathrm{CaO}(\mathrm{wt} \%)$ & 2.0918 & 2.1768 & 1.9870 & 0.1048 & 0.1898 & $5.3 \%$ & $9.6 \%$ \\
\hline SRNL-SCS-2008-00003/Set 2 & SB5-07 & $\mathrm{CdO}(\mathrm{wt} \%)$ & 0.0163 & 0.0163 & 0.0250 & -0.0087 & -0.0087 & $-34.9 \%$ & $-34.9 \%$ \\
\hline SRNL-SCS-2008-00003/Set 2 & SB5-07 & $\mathrm{Ce} 2 \mathrm{O} 3(\mathrm{wt} \%)$ & 0.0978 & 0.0978 & 0.1550 & -0.0572 & -0.0572 & $-36.9 \%$ & $-36.9 \%$ \\
\hline SRNL-SCS-2008-00003/Set 2 & SB5-07 & $\mathrm{Cr} 2 \mathrm{O} 3(\mathrm{wt} \%)$ & 0.1268 & 0.1274 & 0.1560 & -0.0292 & -0.0286 & $-18.7 \%$ & $-18.3 \%$ \\
\hline SRNL-SCS-2008-00003/Set 2 & SB5-07 & $\mathrm{CuO}\left(\mathrm{wt}^{\%} \%\right)$ & 0.0150 & 0.0161 & 0.0050 & 0.0100 & 0.0111 & $200.4 \%$ & $221.7 \%$ \\
\hline SRNL-SCS-2008-00003/Set 2 & SB5-07 & $\mathrm{Fe} 2 \mathrm{O} 3(\mathrm{wt} \%)$ & 9.6112 & 9.4216 & 9.7450 & -0.1338 & -0.3234 & $-1.4 \%$ & $-3.3 \%$ \\
\hline SRNL-SCS-2008-00003/Set 2 & SB5-07 & $\mathrm{K} 2 \mathrm{O}(\mathrm{wt} \%)$ & 0.0190 & 0.0211 & 0.0270 & -0.0080 & -0.0059 & $-29.7 \%$ & $-22.0 \%$ \\
\hline SRNL-SCS-2008-00003/Set 2 & SB5-07 & $\mathrm{La} 2 \mathrm{O} 3(\mathrm{wt} \%)$ & 0.0586 & 0.0586 & 0.0680 & -0.0094 & -0.0094 & $-13.8 \%$ & $-13.8 \%$ \\
\hline SRNL-SCS-2008-00003/Set 2 & SB5-07 & $\mathrm{Li} 2 \mathrm{O}(\mathrm{wt} \%)$ & 3.6922 & 3.8233 & 3.7200 & -0.0278 & 0.1033 & $-0.7 \%$ & $2.8 \%$ \\
\hline SRNL-SCS-2008-00003/Set 2 & SB5-07 & $\mathrm{MgO}(\mathrm{wt} \%)$ & 0.4958 & 0.5146 & 0.4940 & 0.0018 & 0.0206 & $0.4 \%$ & $4.2 \%$ \\
\hline SRNL-SCS-2008-00003/Set 2 & SB5-07 & $\mathrm{MnO}(\mathrm{wt} \%)$ & 2.1111 & 2.0384 & 2.0380 & 0.0731 & 0.0004 & $3.6 \%$ & $0.0 \%$ \\
\hline SRNL-SCS-2008-00003/Set 2 & SB5-07 & $\mathrm{Na} 2 \mathrm{O}(\mathrm{wt} \%)$ & 13.3385 & 13.6041 & 13.5340 & -0.1955 & 0.0701 & $-1.4 \%$ & $0.5 \%$ \\
\hline SRNL-SCS-2008-00003/Set 2 & SB5-07 & $\mathrm{Nb} 2 \mathrm{O} 5(\mathrm{wt} \%)$ & 0.0072 & 0.0072 & 0.0000 & 0.0072 & 0.0072 & & \\
\hline SRNL-SCS-2008-00003/Set 2 & SB5-07 & $\mathrm{NiO}(\mathrm{wt} \%)$ & 1.0104 & 1.0815 & 1.1440 & -0.1336 & -0.0625 & $-11.7 \%$ & $-5.5 \%$ \\
\hline SRNL-SCS-2008-00003/Set 2 & SB5-07 & $\mathrm{PbO}(\mathrm{wt} \%)$ & 0.0108 & 0.0108 & 0.0090 & 0.0018 & 0.0018 & $19.7 \%$ & $19.7 \%$ \\
\hline SRNL-SCS-2008-00003/Set 2 & SB5-07 & $\mathrm{SiO} 2(\mathrm{wt} \%)$ & 44.7649 & 44.2473 & 44.8110 & -0.0461 & -0.5637 & $-0.1 \%$ & $-1.3 \%$ \\
\hline SRNL-SCS-2008-00003/Set 2 & SB5-07 & SO4 $(w t \%)$ & 0.1887 & 0.1887 & 0.2890 & -0.1003 & -0.1003 & $-34.7 \%$ & $-34.7 \%$ \\
\hline SRNL-SCS-2008-00003/Set 2 & SB5-07 & ThO2 (wt\%) & 0.0569 & 0.0569 & 0.0000 & 0.0569 & 0.0569 & & \\
\hline
\end{tabular}


SRNS-STI-2008-00060

Revision 0

Appendix A

Table A6. Average Measured and Bias-Corrected Chemical Compositions Versus Targeted Compositions by Oxide by Study Glass. (continued)

\begin{tabular}{|c|c|c|c|c|c|c|c|c|c|}
\hline Analytical Plan/Set & $\begin{array}{c}\text { Glass } \\
\text { ID }\end{array}$ & Oxide & $\begin{array}{c}\text { Measured } \\
\text { (wt\%) }\end{array}$ & $\begin{array}{c}\text { Measured BC } \\
\text { (wt \%) }\end{array}$ & $\begin{array}{c}\text { Targeted } \\
\text { (wt \%) }\end{array}$ & $\begin{array}{c}\text { Diff of } \\
\text { Measured }\end{array}$ & $\begin{array}{c}\text { Diff of } \\
\text { Meas BC }\end{array}$ & $\begin{array}{l}\text { \% Diff of } \\
\text { Measured }\end{array}$ & $\begin{array}{l}\text { \% Diff of } \\
\text { Meas BC }\end{array}$ \\
\hline SRNL-SCS-2008-00003/Set 2 & SB5-07 & $\mathrm{TiO} 2(\mathrm{wt} \%)$ & 0.0125 & 0.0131 & 0.0110 & 0.0015 & 0.0021 & $13.7 \%$ & $19.3 \%$ \\
\hline SRNL-SCS-2008-00003/Set 2 & SB5-07 & $\mathrm{U} 3 \mathrm{O} 8(\mathrm{wt} \%)$ & 3.0158 & 3.1638 & 3.1170 & -0.1012 & 0.0468 & $-3.2 \%$ & $1.5 \%$ \\
\hline SRNL-SCS-2008-00003/Set 2 & SB5-07 & $\mathrm{ZnO}(\mathrm{wt} \%)$ & 0.0062 & 0.0062 & 0.0060 & 0.0002 & 0.0002 & $3.7 \%$ & $3.7 \%$ \\
\hline SRNL-SCS-2008-00003/Set 2 & SB5-07 & $\mathrm{ZrO} 2(\mathrm{wt} \%)$ & 0.0959 & 0.0959 & 0.1080 & -0.0121 & -0.0121 & $-11.2 \%$ & $-11.2 \%$ \\
\hline SRNL-SCS-2008-00003/Set 2 & SB5-07 & Sum & 98.9712 & 99.1730 & 99.6320 & -0.6608 & -0.4590 & $-0.7 \%$ & $-0.5 \%$ \\
\hline SRNL-SCS-2008-00003/Set 2 & SB5-08 & $\mathrm{Al} 2 \mathrm{O} 3(\mathrm{wt} \%)$ & 9.5136 & 9.5366 & 9.4600 & 0.0536 & 0.0766 & $0.6 \%$ & $0.8 \%$ \\
\hline SRNL-SCS-2008-00003/Set 2 & SB5-08 & $\mathrm{B} 2 \mathrm{O} 3(\mathrm{wt} \%)$ & 10.1507 & 10.2022 & 9.9200 & 0.2307 & 0.2822 & $2.3 \%$ & $2.8 \%$ \\
\hline SRNL-SCS-2008-00003/Set 2 & SB5-08 & $\mathrm{BaO}(\mathrm{wt} \%)$ & 0.0416 & 0.0436 & 0.0430 & -0.0014 & 0.0006 & $-3.3 \%$ & $1.4 \%$ \\
\hline SRNL-SCS-2008-00003/Set 2 & SB5-08 & $\mathrm{CaO}(\mathrm{wt} \%)$ & 0.7524 & 0.7619 & 0.7470 & 0.0054 & 0.0149 & $0.7 \%$ & $2.0 \%$ \\
\hline SRNL-SCS-2008-00003/Set 2 & SB5-08 & $\mathrm{CdO}(\mathrm{wt} \%)$ & 0.0160 & 0.0160 & 0.0250 & -0.0090 & -0.0090 & $-36.0 \%$ & $-36.0 \%$ \\
\hline SRNL-SCS-2008-00003/Set 2 & SB5-08 & $\mathrm{Ce} 2 \mathrm{O} 3(\mathrm{wt} \%)$ & 0.0638 & 0.0638 & 0.1550 & -0.0912 & -0.0912 & $-58.8 \%$ & $-58.8 \%$ \\
\hline SRNL-SCS-2008-00003/Set 2 & SB5-08 & $\mathrm{Cr} 2 \mathrm{O} 3(\mathrm{wt} \%)$ & 0.1323 & 0.1300 & 0.1560 & -0.0237 & -0.0260 & $-15.2 \%$ & $-16.7 \%$ \\
\hline SRNL-SCS-2008-00003/Set 2 & SB5-08 & $\mathrm{CuO}(\mathrm{wt} \%)$ & 0.0100 & 0.0104 & 0.0050 & 0.0050 & 0.0054 & $100.3 \%$ & $107.5 \%$ \\
\hline SRNL-SCS-2008-00003/Set 2 & SB5-08 & $\mathrm{Fe} 2 \mathrm{O} 3(\mathrm{wt} \%)$ & 9.5218 & 9.4324 & 9.7450 & -0.2232 & -0.3126 & $-2.3 \%$ & $-3.2 \%$ \\
\hline SRNL-SCS-2008-00003/Set 2 & SB5-08 & $\mathrm{K} 2 \mathrm{O}(\mathrm{wt} \%)$ & 0.0521 & 0.0561 & 0.0270 & 0.0251 & 0.0291 & $93.0 \%$ & $108.0 \%$ \\
\hline SRNL-SCS-2008-00003/Set 2 & SB5-08 & $\mathrm{La} 2 \mathrm{O} 3(\mathrm{wt} \%)$ & 0.0586 & 0.0586 & 0.0680 & -0.0094 & -0.0094 & $-13.8 \%$ & $-13.8 \%$ \\
\hline SRNL-SCS-2008-00003/Set 2 & SB5-08 & $\mathrm{Li} 2 \mathrm{O}(\mathrm{wt} \%)$ & 3.0840 & 3.2480 & 3.1000 & -0.0160 & 0.1480 & $-0.5 \%$ & $4.8 \%$ \\
\hline SRNL-SCS-2008-00003/Set 2 & SB5-08 & $\mathrm{MgO}\left(\mathrm{wt}^{\%} \%\right)$ & 0.5128 & 0.5230 & 0.4940 & 0.0188 & 0.0290 & $3.8 \%$ & $5.9 \%$ \\
\hline SRNL-SCS-2008-00003/Set 2 & SB5-08 & $\mathrm{MnO}(\mathrm{wt} \%)$ & 2.0207 & 1.9915 & 2.0380 & -0.0173 & -0.0465 & $-0.8 \%$ & $-2.3 \%$ \\
\hline SRNL-SCS-2008-00003/Set 2 & SB5-08 & $\mathrm{Na} 2 \mathrm{O}(\mathrm{wt} \%)$ & 14.0394 & 13.7871 & 14.1540 & -0.1146 & -0.3669 & $-0.8 \%$ & $-2.6 \%$ \\
\hline SRNL-SCS-2008-00003/Set 2 & SB5-08 & $\mathrm{Nb} 2 \mathrm{O} 5(\mathrm{wt} \%)$ & 0.0072 & 0.0072 & 0.0000 & 0.0072 & 0.0072 & & \\
\hline SRNL-SCS-2008-00003/Set 2 & SB5-08 & $\mathrm{NiO}(\mathrm{wt} \%)$ & 1.0829 & 1.1369 & 1.1440 & -0.0611 & -0.0071 & $-5.3 \%$ & $-0.6 \%$ \\
\hline SRNL-SCS-2008-00003/Set 2 & SB5-08 & $\mathrm{PbO}(\mathrm{wt} \%)$ & 0.0108 & 0.0108 & 0.0090 & 0.0018 & 0.0018 & $19.7 \%$ & $19.7 \%$ \\
\hline SRNL-SCS-2008-00003/Set 2 & SB5-08 & $\mathrm{SiO} 2(\mathrm{wt} \%)$ & 45.1927 & 44.2651 & 44.8110 & 0.3817 & -0.5459 & $0.9 \%$ & $-1.2 \%$ \\
\hline SRNL-SCS-2008-00003/Set 2 & SB5-08 & $\mathrm{SO} 4(\mathrm{wt} \%)$ & 0.2292 & 0.2292 & 0.2890 & -0.0598 & -0.0598 & $-20.7 \%$ & $-20.7 \%$ \\
\hline SRNL-SCS-2008-00003/Set 2 & SB5-08 & ThO2 (wt $\%)$ & 0.0569 & 0.0569 & 0.0000 & 0.0569 & 0.0569 & & \\
\hline SRNL-SCS-2008-00003/Set 2 & SB5-08 & $\mathrm{TiO} 2(\mathrm{wt} \%)$ & 0.0142 & 0.0145 & 0.0110 & 0.0032 & 0.0035 & $28.9 \%$ & $31.7 \%$ \\
\hline SRNL-SCS-2008-00003/Set 2 & SB5-08 & $\mathrm{U} 3 \mathrm{O} 8(\mathrm{wt} \%)$ & 3.0453 & 3.0776 & 3.1170 & -0.0717 & -0.0394 & $-2.3 \%$ & $-1.3 \%$ \\
\hline SRNL-SCS-2008-00003/Set 2 & SB5-08 & $\mathrm{ZnO}(\mathrm{wt} \%)$ & 0.0062 & 0.0062 & 0.0060 & 0.0002 & 0.0002 & $3.7 \%$ & $3.7 \%$ \\
\hline SRNL-SCS-2008-00003/Set 2 & SB5-08 & $\mathrm{ZrO} 2(\mathrm{wt} \%)$ & 0.0983 & 0.0983 & 0.1080 & -0.0097 & -0.0097 & $-9.0 \%$ & $-9.0 \%$ \\
\hline SRNL-SCS-2008-00003/Set 2 & SB5-08 & Sum & 99.7136 & 98.7638 & 99.6320 & 0.0816 & -0.8682 & $0.1 \%$ & $-0.9 \%$ \\
\hline SRNL-SCS-2008-00003/Set 2 & SB5-09 & $\mathrm{A} 12 \mathrm{O} 3(\mathrm{wt} \%)$ & 8.0776 & 8.0962 & 7.9470 & 0.1306 & 0.1492 & $1.6 \%$ & $1.9 \%$ \\
\hline SRNL-SCS-2008-00003/Set 2 & SB5-09 & $\mathrm{B} 2 \mathrm{O} 3(\mathrm{wt} \%)$ & 6.4318 & 6.4653 & 6.2000 & 0.2318 & 0.2653 & $3.7 \%$ & $4.3 \%$ \\
\hline SRNL-SCS-2008-00003/Set 2 & SB5-09 & $\mathrm{BaO}(\mathrm{wt} \%)$ & 0.0408 & 0.0427 & 0.0460 & -0.0052 & -0.0033 & $-11.4 \%$ & $-7.1 \%$ \\
\hline SRNL-SCS-2008-00003/Set 2 & SB5-09 & $\mathrm{CaO}(\mathrm{wt} \%)$ & 3.4630 & 3.5100 & 3.2810 & 0.1820 & 0.2290 & $5.5 \%$ & $7.0 \%$ \\
\hline SRNL-SCS-2008-00003/Set 2 & SB5-09 & $\mathrm{CdO}(\mathrm{wt} \%)$ & 0.0163 & 0.0163 & 0.0270 & -0.0107 & -0.0107 & $-39.7 \%$ & $-39.7 \%$ \\
\hline SRNL-SCS-2008-00003/Set 2 & SB5-09 & $\mathrm{Ce} 2 \mathrm{O} 3(\mathrm{wt} \%)$ & 0.0990 & 0.0990 & 0.1670 & -0.0680 & -0.0680 & $-40.7 \%$ & $-40.7 \%$ \\
\hline SRNL-SCS-2008-00003/Set 2 & SB5-09 & $\mathrm{Cr} 2 \mathrm{O} 3(\mathrm{wt} \%)$ & 0.1348 & 0.1325 & 0.1670 & -0.0322 & -0.0345 & $-19.3 \%$ & $-20.7 \%$ \\
\hline SRNL-SCS-2008-00003/Set 2 & SB5-09 & $\mathrm{CuO}(\mathrm{wt} \%)$ & 0.0100 & 0.0104 & 0.0050 & 0.0050 & 0.0054 & $100.3 \%$ & $107.5 \%$ \\
\hline SRNL-SCS-2008-00003/Set 2 & SB5-09 & $\mathrm{Fe} 2 \mathrm{O} 3(\mathrm{wt} \%)$ & 10.1687 & 10.0682 & 10.4470 & -0.2783 & -0.3788 & $-2.7 \%$ & $-3.6 \%$ \\
\hline SRNL-SCS-2008-00003/Set 2 & SB5-09 & $\mathrm{K} 2 \mathrm{O}(\mathrm{wt} \%)$ & 0.0217 & 0.0234 & 0.0300 & -0.0083 & -0.0066 & $-27.7 \%$ & $-22.1 \%$ \\
\hline SRNL-SCS-2008-00003/Set 2 & SB5-09 & $\mathrm{La} 2 \mathrm{O} 3(\mathrm{wt} \%)$ & 0.0586 & 0.0586 & 0.0730 & -0.0144 & -0.0144 & $-19.7 \%$ & $-19.7 \%$ \\
\hline SRNL-SCS-2008-00003/Set 2 & SB5-09 & $\mathrm{Li} 2 \mathrm{O}(\mathrm{wt} \%)$ & 4.2843 & 4.5121 & 4.3400 & -0.0557 & 0.1721 & $-1.3 \%$ & $4.0 \%$ \\
\hline SRNL-SCS-2008-00003/Set 2 & SB5-09 & $\mathrm{MgO}(\mathrm{wt} \%)$ & 0.5439 & 0.5547 & 0.5290 & 0.0149 & 0.0257 & $2.8 \%$ & $4.8 \%$ \\
\hline SRNL-SCS-2008-00003/Set 2 & SB5-09 & $\mathrm{MnO}(\mathrm{wt} \%)$ & 2.1660 & 2.1344 & 2.1840 & -0.0180 & -0.0496 & $-0.8 \%$ & $-2.3 \%$ \\
\hline SRNL-SCS-2008-00003/Set 2 & SB5-09 & $\mathrm{Na} 2 \mathrm{O}(\mathrm{wt} \%)$ & 13.5878 & 13.3422 & 13.6500 & -0.0622 & -0.3078 & $-0.5 \%$ & $-2.3 \%$ \\
\hline SRNL-SCS-2008-00003/Set 2 & SB5-09 & $\mathrm{Nb} 2 \mathrm{O} 5(\mathrm{wt} \%)$ & 0.0072 & 0.0072 & 0.0000 & 0.0072 & 0.0072 & & \\
\hline SRNL-SCS-2008-00003/Set 2 & SB5-09 & $\mathrm{NiO}\left(\mathrm{wt}^{0} \%\right)$ & 1.1118 & 1.1672 & 1.2270 & -0.1152 & -0.0598 & $-9.4 \%$ & $-4.9 \%$ \\
\hline SRNL-SCS-2008-00003/Set 2 & SB5-09 & $\mathrm{PbO}(\mathrm{wt} \%)$ & 0.0108 & 0.0108 & 0.0100 & 0.0008 & 0.0008 & $7.7 \%$ & $7.7 \%$ \\
\hline SRNL-SCS-2008-00003/Set 2 & SB5-09 & $\mathrm{SiO} 2(\mathrm{wt} \%)$ & 44.9253 & 44.0033 & 45.4890 & -0.5637 & -1.4857 & $-1.2 \%$ & $-3.3 \%$ \\
\hline SRNL-SCS-2008-00003/Set 2 & SB5-09 & SO4 (wt $\%)$ & 0.3191 & 0.3191 & 0.3100 & 0.0091 & 0.0091 & $2.9 \%$ & $2.9 \%$ \\
\hline SRNL-SCS-2008-00003/Set 2 & SB5-09 & ThO2 (wt $\%)$ & 0.0569 & 0.0569 & 0.0000 & 0.0569 & 0.0569 & & \\
\hline SRNL-SCS-2008-00003/Set 2 & SB5-09 & $\mathrm{TiO} 2(\mathrm{wt} \%)$ & 0.0142 & 0.0145 & 0.0120 & 0.0022 & 0.0025 & $18.2 \%$ & $20.8 \%$ \\
\hline SRNL-SCS-2008-00003/Set 2 & SB5-09 & $\mathrm{U} 3 \mathrm{O} 8(\mathrm{wt} \%)$ & 3.2605 & 3.2942 & 3.3420 & -0.0815 & -0.0478 & $-2.4 \%$ & $-1.4 \%$ \\
\hline SRNL-SCS-2008-00003/Set 2 & SB5-09 & $\mathrm{ZnO}(\mathrm{wt} \%)$ & 0.0062 & 0.0062 & 0.0070 & -0.0008 & -0.0008 & $-11.1 \%$ & $-11.1 \%$ \\
\hline SRNL-SCS-2008-00003/Set 2 & SB5-09 & $\mathrm{ZrO} 2(\mathrm{wt} \%)$ & 0.1165 & 0.1165 & 0.1160 & 0.0005 & 0.0005 & $0.4 \%$ & $0.4 \%$ \\
\hline SRNL-SCS-2008-00003/Set 2 & SB5-09 & Sum & 98.9327 & 98.0619 & 99.6060 & -0.6733 & -1.5441 & $-0.7 \%$ & $-1.6 \%$ \\
\hline SRNL-SCS-2008-00003/Set 2 & SB5-10 & $\mathrm{A} 12 \mathrm{O} 3(\mathrm{wt} \%)$ & 8.1437 & 8.1634 & 7.9470 & 0.1967 & 0.2164 & $2.5 \%$ & $2.7 \%$ \\
\hline SRNL-SCS-2008-00003/Set 2 & SB5-10 & $\mathrm{B} 2 \mathrm{O} 3(\mathrm{wt} \%)$ & 6.8825 & 6.7992 & 6.8200 & 0.0625 & -0.0208 & $0.9 \%$ & $-0.3 \%$ \\
\hline SRNL-SCS-2008-00003/Set 2 & SB5-10 & $\mathrm{BaO}(\mathrm{wt} \%)$ & 0.0419 & 0.0439 & 0.0460 & -0.0041 & -0.0021 & $-9.0 \%$ & $-4.6 \%$ \\
\hline SRNL-SCS-2008-00003/Set 2 & SB5-10 & $\mathrm{CaO}(\mathrm{wt} \%)$ & 2.1863 & 2.2155 & 2.0410 & 0.1453 & 0.1745 & $7.1 \%$ & $8.5 \%$ \\
\hline SRNL-SCS-2008-00003/Set 2 & SB5-10 & $\mathrm{CdO}(\mathrm{wt} \%)$ & 0.0191 & 0.0191 & 0.0270 & -0.0079 & -0.0079 & $-29.1 \%$ & $-29.1 \%$ \\
\hline SRNL-SCS-2008-00003/Set 2 & SB5-10 & $\mathrm{Ce} 2 \mathrm{O} 3(\mathrm{wt} \%)$ & 0.1455 & 0.1455 & 0.1670 & -0.0215 & -0.0215 & $-12.9 \%$ & $-12.9 \%$ \\
\hline SRNL-SCS-2008-00003/Set 2 & SB5-10 & $\mathrm{Cr} 2 \mathrm{O} 3(\mathrm{wt} \%)$ & 0.1224 & 0.1203 & 0.1670 & -0.0446 & -0.0467 & $-26.7 \%$ & $-28.0 \%$ \\
\hline SRNL-SCS-2008-00003/Set 2 & SB5-10 & $\mathrm{CuO}(\mathrm{wt} \%)$ & 0.0100 & 0.0104 & 0.0050 & 0.0050 & 0.0054 & $100.3 \%$ & $107.5 \%$ \\
\hline SRNL-SCS-2008-00003/Set 2 & SB5-10 & $\mathrm{Fe} 2 \mathrm{O} 3(\mathrm{wt} \%)$ & 10.2581 & 10.1586 & 10.4470 & -0.1889 & -0.2884 & $-1.8 \%$ & $-2.8 \%$ \\
\hline SRNL-SCS-2008-00003/Set 2 & SB5-10 & $\mathrm{K} 2 \mathrm{O}(\mathrm{wt} \%)$ & 0.0211 & 0.0227 & 0.0300 & -0.0089 & -0.0073 & $-29.7 \%$ & $-24.3 \%$ \\
\hline SRNL-SCS-2008-00003/Set 2 & SB5-10 & $\mathrm{La} 2 \mathrm{O} 3(\mathrm{wt} \%)$ & 0.0586 & 0.0586 & 0.0730 & -0.0144 & -0.0144 & $-19.7 \%$ & $-19.7 \%$ \\
\hline
\end{tabular}


SRNS-STI-2008-00060

Revision 0

Appendix A

Table A6. Average Measured and Bias-Corrected Chemical Compositions Versus Targeted Compositions by Oxide by Study Glass. (continued)

\begin{tabular}{|c|c|c|c|c|c|c|c|c|c|}
\hline Analytical Plan/Set & $\begin{array}{c}\text { Glass } \\
\text { ID }\end{array}$ & Oxide & $\begin{array}{c}\text { Measured } \\
\text { (wt\%) }\end{array}$ & $\begin{array}{c}\text { Measured BC } \\
\text { (wt\%) }\end{array}$ & $\begin{array}{c}\text { Targeted } \\
\text { (wt \%) }\end{array}$ & $\begin{array}{c}\text { Diff of } \\
\text { Measured }\end{array}$ & $\begin{array}{c}\text { Diff of } \\
\text { Meas BC }\end{array}$ & $\begin{array}{l}\text { \% Diff of } \\
\text { Measured }\end{array}$ & $\begin{array}{l}\text { \% Diff of } \\
\text { Meas BC }\end{array}$ \\
\hline SRNL-SCS-2008-00003/Set 2 & SB5-10 & $\mathrm{Li} 2 \mathrm{O}(\mathrm{wt} \%)$ & 4.3327 & 4.4865 & 4.3400 & -0.0073 & 0.1465 & $-0.2 \%$ & $3.4 \%$ \\
\hline SRNL-SCS-2008-00003/Set 2 & SB5-10 & $\mathrm{MgO}\left(\mathrm{wt}^{\circ} \%\right)$ & 0.5307 & 0.5413 & 0.5290 & 0.0017 & 0.0123 & $0.3 \%$ & $2.3 \%$ \\
\hline SRNL-SCS-2008-00003/Set 2 & SB5-10 & $\mathrm{MnO}\left(\mathrm{wt}^{0} \%\right)$ & 2.2661 & 2.2334 & 2.1840 & 0.0821 & 0.0494 & $3.8 \%$ & $2.3 \%$ \\
\hline SRNL-SCS-2008-00003/Set 2 & SB5-10 & $\mathrm{Na} 2 \mathrm{O}(\mathrm{wt} \%)$ & 13.5373 & 13.2947 & 13.6500 & -0.1127 & -0.3553 & $-0.8 \%$ & $-2.6 \%$ \\
\hline SRNL-SCS-2008-00003/Set 2 & SB5-10 & $\mathrm{Nb} 2 \mathrm{O} 5(\mathrm{wt} \%)$ & 0.0072 & 0.0072 & 0.0000 & 0.0072 & 0.0072 & & \\
\hline SRNL-SCS-2008-00003/Set 2 & SB5-10 & $\mathrm{NiO}(\mathrm{wt} \%)$ & 1.0896 & 1.1440 & 1.2270 & -0.1374 & -0.0830 & $-11.2 \%$ & $-6.8 \%$ \\
\hline SRNL-SCS-2008-00003/Set 2 & SB5-10 & $\mathrm{PbO}(\mathrm{wt} \%)$ & 0.0108 & 0.0108 & 0.0100 & 0.0008 & 0.0008 & $7.7 \%$ & $7.7 \%$ \\
\hline SRNL-SCS-2008-00003/Set 2 & SB5-10 & $\mathrm{SiO} 2(\mathrm{wt} \%)$ & 46.9576 & 45.9939 & 46.1090 & 0.8486 & -0.1151 & $1.8 \%$ & $-0.2 \%$ \\
\hline SRNL-SCS-2008-00003/Set 2 & SB5-10 & SO4 $(\mathrm{wt} \%)$ & 0.3206 & 0.3206 & 0.3100 & 0.0106 & 0.0106 & $3.4 \%$ & $3.4 \%$ \\
\hline SRNL-SCS-2008-00003/Set 2 & SB5-10 & $\mathrm{ThO} 2(\mathrm{wt} \%)$ & 0.0569 & 0.0569 & 0.0000 & 0.0569 & 0.0569 & & \\
\hline SRNL-SCS-2008-00003/Set 2 & SB5-10 & $\mathrm{TiO} 2(\mathrm{wt} \%)$ & 0.0146 & 0.0149 & 0.0120 & 0.0026 & 0.0029 & $21.6 \%$ & $24.3 \%$ \\
\hline SRNL-SCS-2008-00003/Set 2 & SB5-10 & $\mathrm{U} 3 \mathrm{O} 8(\mathrm{wt} \%)$ & 3.2959 & 3.3302 & 3.3420 & -0.0461 & -0.0118 & $-1.4 \%$ & $-0.4 \%$ \\
\hline SRNL-SCS-2008-00003/Set 2 & SB5-10 & $\mathrm{ZnO}(\mathrm{wt} \%)$ & 0.0062 & 0.0062 & 0.0070 & -0.0008 & -0.0008 & $-11.1 \%$ & $-11.1 \%$ \\
\hline SRNL-SCS-2008-00003/Set 2 & SB5-10 & $\mathrm{ZrO} 2(\mathrm{wt} \%)$ & 0.1067 & 0.1067 & 0.1160 & -0.0093 & -0.0093 & $-8.0 \%$ & $-8.0 \%$ \\
\hline SRNL-SCS-2008-00003/Set 2 & SB5-10 & Sum & 100.4220 & 99.3044 & 99.6060 & 0.8160 & -0.3016 & $0.8 \%$ & $-0.3 \%$ \\
\hline SRNL-SCS-2008-00003/Set 2 & SB5-11 & $\mathrm{A} 12 \mathrm{O} 3(\mathrm{wt} \%)$ & 7.8273 & 8.1245 & 7.9470 & -0.1197 & 0.1775 & $-1.5 \%$ & $2.2 \%$ \\
\hline SRNL-SCS-2008-00003/Set 2 & SB5-11 & $\mathrm{B} 2 \mathrm{O} 3(\mathrm{wt} \%)$ & 8.6615 & 8.7045 & 8.6800 & -0.0185 & 0.0245 & $-0.2 \%$ & $0.3 \%$ \\
\hline SRNL-SCS-2008-00003/Set 2 & SB5-11 & $\mathrm{BaO}(\mathrm{wt} \%)$ & 0.0416 & 0.0448 & 0.0460 & -0.0044 & -0.0012 & $-9.6 \%$ & $-2.6 \%$ \\
\hline SRNL-SCS-2008-00003/Set 2 & SB5-11 & $\mathrm{CaO}(\mathrm{wt} \%)$ & 2.1408 & 2.2277 & 2.0410 & 0.0998 & 0.1867 & $4.9 \%$ & $9.1 \%$ \\
\hline SRNL-SCS-2008-00003/Set 2 & SB5-11 & $\mathrm{CdO}(\mathrm{wt} \%)$ & 0.0194 & 0.0194 & 0.0270 & -0.0076 & -0.0076 & $-28.1 \%$ & $-28.1 \%$ \\
\hline SRNL-SCS-2008-00003/Set 2 & SB5-11 & $\mathrm{Ce} 2 \mathrm{O} 3(\mathrm{wt} \%)$ & 0.1473 & 0.1473 & 0.1670 & -0.0197 & -0.0197 & $-11.8 \%$ & $-11.8 \%$ \\
\hline SRNL-SCS-2008-00003/Set 2 & SB5-11 & $\mathrm{Cr} 2 \mathrm{O} 3(\mathrm{wt} \%)$ & 0.1414 & 0.1421 & 0.1670 & -0.0256 & -0.0249 & $-15.3 \%$ & $-14.9 \%$ \\
\hline SRNL-SCS-2008-00003/Set 2 & SB5-11 & $\mathrm{CuO}(\mathrm{wt} \%)$ & 0.0138 & 0.0147 & 0.0050 & 0.0088 & 0.0097 & $175.4 \%$ & $194.9 \%$ \\
\hline SRNL-SCS-2008-00003/Set 2 & SB5-11 & $\mathrm{Fe} 2 \mathrm{O} 3(\mathrm{wt} \%)$ & 9.6433 & 9.4517 & 10.4470 & -0.8037 & -0.9953 & $-7.7 \%$ & $-9.5 \%$ \\
\hline SRNL-SCS-2008-00003/Set 2 & SB5-11 & $\mathrm{K} 2 \mathrm{O}(\mathrm{wt} \%)$ & 0.0199 & 0.0221 & 0.0300 & -0.0101 & -0.0079 & $-33.7 \%$ & $-26.4 \%$ \\
\hline SRNL-SCS-2008-00003/Set 2 & SB5-11 & $\mathrm{La} 2 \mathrm{O} 3(\mathrm{wt} \%)$ & 0.0586 & 0.0586 & 0.0730 & -0.0144 & -0.0144 & $-19.7 \%$ & $-19.7 \%$ \\
\hline SRNL-SCS-2008-00003/Set 2 & SB5-11 & $\mathrm{Li} 2 \mathrm{O}(\mathrm{wt} \%)$ & 3.6438 & 3.8376 & 3.7200 & -0.0762 & 0.1176 & $-2.0 \%$ & $3.2 \%$ \\
\hline SRNL-SCS-2008-00003/Set 2 & SB5-11 & $\mathrm{MgO}(\mathrm{wt} \%)$ & 0.5307 & 0.5507 & 0.5290 & 0.0017 & 0.0217 & $0.3 \%$ & $4.1 \%$ \\
\hline SRNL-SCS-2008-00003/Set 2 & SB5-11 & $\mathrm{MnO}(\mathrm{wt} \%)$ & 2.1595 & 2.0846 & 2.1840 & -0.0245 & -0.0994 & $-1.1 \%$ & $-4.6 \%$ \\
\hline SRNL-SCS-2008-00003/Set 2 & SB5-11 & $\mathrm{Na} 2 \mathrm{O}(\mathrm{wt} \%)$ & 13.1834 & 13.4461 & 13.6500 & -0.4666 & -0.2039 & $-3.4 \%$ & $-1.5 \%$ \\
\hline SRNL-SCS-2008-00003/Set 2 & SB5-11 & $\mathrm{Nb} 2 \mathrm{O} 5(\mathrm{wt} \%)$ & 0.0072 & 0.0072 & 0.0000 & 0.0072 & 0.0072 & & \\
\hline SRNL-SCS-2008-00003/Set 2 & SB5-11 & $\mathrm{NiO}(\mathrm{wt} \%)$ & 1.1150 & 1.1936 & 1.2270 & -0.1120 & -0.0334 & $-9.1 \%$ & $-2.7 \%$ \\
\hline SRNL-SCS-2008-00003/Set 2 & SB5-11 & $\mathrm{PbO}(\mathrm{wt} \%)$ & 0.0108 & 0.0108 & 0.0100 & 0.0008 & 0.0008 & $7.7 \%$ & $7.7 \%$ \\
\hline SRNL-SCS-2008-00003/Set 2 & SB5-11 & $\mathrm{SiO} 2(\mathrm{wt} \%)$ & 44.1765 & 43.6671 & 44.8690 & -0.6925 & -1.2019 & $-1.5 \%$ & $-2.7 \%$ \\
\hline SRNL-SCS-2008-00003/Set 2 & SB5-11 & SO4 $(\mathrm{wt} \%)$ & 0.2666 & 0.2666 & 0.3100 & -0.0434 & -0.0434 & $-14.0 \%$ & $-14.0 \%$ \\
\hline SRNL-SCS-2008-00003/Set 2 & SB5-11 & $\mathrm{ThO} 2(\mathrm{wt} \%)$ & 0.0569 & 0.0569 & 0.0000 & 0.0569 & 0.0569 & & \\
\hline SRNL-SCS-2008-00003/Set 2 & SB5-11 & $\mathrm{TiO} 2(\mathrm{wt} \%)$ & 0.0154 & 0.0162 & 0.0120 & 0.0034 & 0.0042 & $28.6 \%$ & $34.9 \%$ \\
\hline SRNL-SCS-2008-00003/Set 2 & SB5-11 & $\mathrm{U} 3 \mathrm{O} 8(\mathrm{wt} \%)$ & 3.1691 & 3.3245 & 3.3420 & -0.1729 & -0.0175 & $-5.2 \%$ & $-0.5 \%$ \\
\hline SRNL-SCS-2008-00003/Set 2 & SB5-11 & $\mathrm{ZnO}(\mathrm{wt} \%)$ & 0.0062 & 0.0062 & 0.0070 & -0.0008 & -0.0008 & $-11.1 \%$ & $-11.1 \%$ \\
\hline SRNL-SCS-2008-00003/Set 2 & SB5-11 & $\mathrm{ZrO} 2(\mathrm{wt} \%)$ & 0.1104 & 0.1104 & 0.1160 & -0.0056 & -0.0056 & $-4.8 \%$ & $-4.8 \%$ \\
\hline SRNL-SCS-2008-00003/Set 2 & SB5-11 & Sum & 97.1665 & 97.5359 & 99.6060 & -2.4395 & -2.0701 & $-2.4 \%$ & $-2.1 \%$ \\
\hline SRNL-SCS-2008-00003/Set 2 & SB5-12 & $\mathrm{A} 12 \mathrm{O} 3(\mathrm{wt} \%)$ & 7.8556 & 8.1539 & 7.9470 & -0.0914 & 0.2069 & $-1.2 \%$ & $2.6 \%$ \\
\hline SRNL-SCS-2008-00003/Set 2 & SB5-12 & $\mathrm{B} 2 \mathrm{O} 3(\mathrm{wt} \%)$ & 10.0461 & 10.0985 & 9.9200 & 0.1261 & 0.1785 & $1.3 \%$ & $1.8 \%$ \\
\hline SRNL-SCS-2008-00003/Set 2 & SB5-12 & $\mathrm{BaO}(\mathrm{wt} \%)$ & 0.0419 & 0.0451 & 0.0460 & -0.0041 & -0.0009 & $-9.0 \%$ & $-1.9 \%$ \\
\hline SRNL-SCS-2008-00003/Set 2 & SB5-12 & $\mathrm{CaO}(\mathrm{wt} \%)$ & 0.7979 & 0.8304 & 0.8010 & -0.0031 & 0.0294 & $-0.4 \%$ & $3.7 \%$ \\
\hline SRNL-SCS-2008-00003/Set 2 & SB5-12 & $\mathrm{CdO}(\mathrm{wt} \%)$ & 0.0183 & 0.0183 & 0.0270 & -0.0087 & -0.0087 & $-32.3 \%$ & $-32.3 \%$ \\
\hline SRNL-SCS-2008-00003/Set 2 & SB5-12 & $\mathrm{Ce} 2 \mathrm{O} 3(\mathrm{wt} \%)$ & 0.1467 & 0.1467 & 0.1670 & -0.0203 & -0.0203 & $-12.2 \%$ & $-12.2 \%$ \\
\hline SRNL-SCS-2008-00003/Set 2 & SB5-12 & $\mathrm{Cr} 2 \mathrm{O} 3(\mathrm{wt} \%)$ & 0.1589 & 0.1598 & 0.1670 & -0.0081 & -0.0072 & $-4.8 \%$ & $-4.3 \%$ \\
\hline SRNL-SCS-2008-00003/Set 2 & SB5-12 & $\mathrm{CuO}(\mathrm{wt} \%)$ & 0.0131 & 0.0141 & 0.0050 & 0.0081 & 0.0091 & $162.9 \%$ & $181.5 \%$ \\
\hline SRNL-SCS-2008-00003/Set 2 & SB5-12 & $\mathrm{Fe} 2 \mathrm{O} 3(\mathrm{wt} \%)$ & 9.8578 & 9.6628 & 10.4470 & -0.5892 & -0.7842 & $-5.6 \%$ & $-7.5 \%$ \\
\hline SRNL-SCS-2008-00003/Set 2 & SB5-12 & $\mathrm{K} 2 \mathrm{O}(\mathrm{wt} \%)$ & 0.0193 & 0.0214 & 0.0300 & -0.0107 & -0.0086 & $-35.8 \%$ & $-28.6 \%$ \\
\hline SRNL-SCS-2008-00003/Set 2 & SB5-12 & La2O3 (wt $\%)$ & 0.0586 & 0.0586 & 0.0730 & -0.0144 & -0.0144 & $-19.7 \%$ & $-19.7 \%$ \\
\hline SRNL-SCS-2008-00003/Set 2 & SB5-12 & $\mathrm{Li} 2 \mathrm{O}(\mathrm{wt} \%)$ & 3.1109 & 3.2764 & 3.1000 & 0.0109 & 0.1764 & $0.4 \%$ & $5.7 \%$ \\
\hline SRNL-SCS-2008-00003/Set 2 & SB5-12 & $\mathrm{MgO}\left(\mathrm{wt}^{\mathrm{O}} \%\right)$ & 0.5414 & 0.5619 & 0.5290 & 0.0124 & 0.0329 & $2.4 \%$ & $6.2 \%$ \\
\hline SRNL-SCS-2008-00003/Set 2 & SB5-12 & $\mathrm{MnO}\left(\mathrm{wt}^{\circ} \%\right)$ & 2.2144 & 2.1381 & 2.1840 & 0.0304 & -0.0459 & $1.4 \%$ & $-2.1 \%$ \\
\hline SRNL-SCS-2008-00003/Set 2 & SB5-12 & $\mathrm{Na} 2 \mathrm{O}(\mathrm{wt} \%)$ & 13.8035 & 14.0785 & 14.2700 & -0.4665 & -0.1915 & $-3.3 \%$ & $-1.3 \%$ \\
\hline SRNL-SCS-2008-00003/Set 2 & SB5-12 & $\mathrm{Nb} 2 \mathrm{O} 5(\mathrm{wt} \%)$ & 0.0072 & 0.0072 & 0.0000 & 0.0072 & 0.0072 & & \\
\hline SRNL-SCS-2008-00003/Set 2 & SB5-12 & $\mathrm{NiO}(\mathrm{wt} \%)$ & 1.1227 & 1.2018 & 1.2270 & -0.1043 & -0.0252 & $-8.5 \%$ & $-2.1 \%$ \\
\hline SRNL-SCS-2008-00003/Set 2 & SB5-12 & $\mathrm{PbO}(\mathrm{wt} \%)$ & 0.0108 & 0.0108 & 0.0100 & 0.0008 & 0.0008 & $7.7 \%$ & $7.7 \%$ \\
\hline SRNL-SCS-2008-00003/Set 2 & SB5-12 & $\mathrm{SiO} 2\left(\mathrm{wt}^{\%} \%\right)$ & 44.9253 & 44.4077 & 44.8690 & 0.0563 & -0.4613 & $0.1 \%$ & $-1.0 \%$ \\
\hline SRNL-SCS-2008-00003/Set 2 & SB5-12 & SO4 (wt \%) & 0.2262 & 0.2262 & 0.3100 & -0.0838 & -0.0838 & $-27.0 \%$ & $-27.0 \%$ \\
\hline SRNL-SCS-2008-00003/Set 2 & SB5-12 & ThO2 (wt $\%)$ & 0.0569 & 0.0569 & 0.0000 & 0.0569 & 0.0569 & & \\
\hline SRNL-SCS-2008-00003/Set 2 & SB5-12 & $\mathrm{TiO} 2(\mathrm{wt} \%)$ & 0.0133 & 0.0140 & 0.0120 & 0.0013 & 0.0020 & $11.2 \%$ & $16.7 \%$ \\
\hline SRNL-SCS-2008-00003/Set 2 & SB5-12 & $\mathrm{U} 3 \mathrm{O} 8(\mathrm{wt} \%)$ & 3.2015 & 3.3586 & 3.3420 & -0.1405 & 0.0166 & $-4.2 \%$ & $0.5 \%$ \\
\hline SRNL-SCS-2008-00003/Set 2 & SB5-12 & $\mathrm{ZnO}(\mathrm{wt} \%)$ & 0.0062 & 0.0062 & 0.0070 & -0.0008 & -0.0008 & $-11.1 \%$ & $-11.1 \%$ \\
\hline SRNL-SCS-2008-00003/Set 2 & SB5-12 & $\mathrm{ZrO} 2(\mathrm{wt} \%)$ & 0.1067 & 0.1067 & 0.1160 & -0.0093 & -0.0093 & $-8.0 \%$ & $-8.0 \%$ \\
\hline SRNL-SCS-2008-00003/Set 2 & SB5-12 & Sum & 98.3613 & 98.6605 & 99.6060 & -1.2447 & -0.9455 & $-1.2 \%$ & $-0.9 \%$ \\
\hline SRNL-SCS-2008-00003/Set 2 & U std & $\mathrm{A} 12 \mathrm{O} 3(\mathrm{wt} \%)$ & 3.9365 & 4.0138 & 4.1000 & -0.1635 & -0.0862 & $-4.0 \%$ & $-2.1 \%$ \\
\hline
\end{tabular}


SRNS-STI-2008-00060

Revision 0

Appendix A

Table A6. Average Measured and Bias-Corrected Chemical Compositions Versus Targeted Compositions by Oxide by Study Glass. (continued)

\begin{tabular}{|c|c|c|c|c|c|c|c|c|c|}
\hline Analytical Plan/Set & $\begin{array}{l}\text { Glass } \\
\text { ID }\end{array}$ & Oxide & $\begin{array}{c}\text { Measured } \\
\text { (wt\%) }\end{array}$ & $\begin{array}{c}\text { Measured BC } \\
\text { (wt\%) }\end{array}$ & $\begin{array}{c}\text { Targeted } \\
\text { (wt \%) }\end{array}$ & $\begin{array}{c}\text { Diff of } \\
\text { Measured }\end{array}$ & $\begin{array}{c}\text { Diff of } \\
\text { Meas BC }\end{array}$ & $\begin{array}{l}\% \text { Diff of } \\
\text { Measured }\end{array}$ & $\begin{array}{l}\text { \% Diff of } \\
\text { Meas BC }\end{array}$ \\
\hline SRNL-SCS-2008-00003/Set 2 & U std & $\mathrm{B} 2 \mathrm{O} 3(\mathrm{wt} \%)$ & 9.0130 & 8.9811 & 9.2090 & -0.1960 & -0.2279 & $-2.1 \%$ & $-2.5 \%$ \\
\hline SRNL-SCS-2008-00003/Set 2 & U std & $\mathrm{BaO}(\mathrm{wt} \%)$ & 0.0056 & 0.0059 & 0.0000 & 0.0056 & 0.0059 & & \\
\hline SRNL-SCS-2008-00003/Set 2 & U std & $\mathrm{CaO}(\mathrm{wt} \%)$ & 1.2649 & 1.2983 & 1.3010 & -0.0361 & -0.0027 & $-2.8 \%$ & $-0.2 \%$ \\
\hline SRNL-SCS-2008-00003/Set 2 & U std & $\mathrm{CdO}(\mathrm{wt} \%)$ & 0.0057 & 0.0057 & 0.0000 & 0.0057 & 0.0057 & & \\
\hline SRNL-SCS-2008-00003/Set 2 & U std & $\mathrm{Ce} 2 \mathrm{O} 3(\mathrm{wt} \%)$ & 0.0184 & 0.0184 & 0.0000 & 0.0184 & 0.0184 & & \\
\hline SRNL-SCS-2008-00003/Set 2 & U std & $\mathrm{Cr} 2 \mathrm{O} 3(\mathrm{wt} \%)$ & 0.2388 & 0.2374 & 0.0000 & 0.2388 & 0.2374 & & \\
\hline SRNL-SCS-2008-00003/Set 2 & U std & $\mathrm{CuO}(\mathrm{wt} \%)$ & 0.0063 & 0.0066 & 0.0000 & 0.0063 & 0.0066 & & \\
\hline SRNL-SCS-2008-00003/Set 2 & U std & $\mathrm{Fe} 2 \mathrm{O} 3(\mathrm{wt} \%)$ & 13.4511 & 13.2523 & 13.1960 & 0.2551 & 0.0563 & $1.9 \%$ & $0.4 \%$ \\
\hline SRNL-SCS-2008-00003/Set 2 & U std & $\mathrm{K} 2 \mathrm{O}(\mathrm{wt} \%)$ & 2.6973 & 2.9511 & 2.9990 & -0.3017 & -0.0479 & $-10.1 \%$ & $-1.6 \%$ \\
\hline SRNL-SCS-2008-00003/Set 2 & U std & La2O3 (wt $\%)$ & 0.0586 & 0.0586 & 0.0000 & 0.0586 & 0.0586 & & \\
\hline SRNL-SCS-2008-00003/Set 2 & U std & $\mathrm{Li} 2 \mathrm{O}(\mathrm{wt} \%)$ & 2.9387 & 3.0688 & 3.0570 & -0.1183 & 0.0118 & $-3.9 \%$ & $0.4 \%$ \\
\hline SRNL-SCS-2008-00003/Set 2 & U std & $\mathrm{MgO}(\mathrm{wt} \%)$ & 1.1868 & 1.2208 & 1.2100 & -0.0232 & 0.0108 & $-1.9 \%$ & $0.9 \%$ \\
\hline SRNL-SCS-2008-00003/Set 2 & U std & $\mathrm{MnO}\left(\mathrm{wt}^{\circ} \%\right)$ & 2.8858 & 2.8149 & 2.8920 & -0.0062 & -0.0771 & $-0.2 \%$ & $-2.7 \%$ \\
\hline SRNL-SCS-2008-00003/Set 2 & U std & $\mathrm{Na} 2 \mathrm{O}(\mathrm{wt} \%)$ & 11.6624 & 11.6666 & 11.7950 & -0.1326 & -0.1284 & $-1.1 \%$ & $-1.1 \%$ \\
\hline SRNL-SCS-2008-00003/Set 2 & U std & $\mathrm{Nb} 2 \mathrm{O} 5(\mathrm{wt} \%)$ & 0.0687 & 0.0687 & 0.0000 & 0.0687 & 0.0687 & & \\
\hline SRNL-SCS-2008-00003/Set 2 & U std & $\mathrm{NiO}(\mathrm{wt} \%)$ & 0.9483 & 1.0044 & 1.1200 & -0.1717 & -0.1156 & $-15.3 \%$ & $-10.3 \%$ \\
\hline SRNL-SCS-2008-00003/Set 2 & U std & $\mathrm{PbO}(\mathrm{wt} \%)$ & 0.0108 & 0.0108 & 0.0000 & 0.0108 & 0.0108 & & \\
\hline SRNL-SCS-2008-00003/Set 2 & U std & $\mathrm{SiO} 2(\mathrm{wt} \%)$ & 46.0841 & 45.3438 & 45.3530 & 0.7311 & -0.0092 & $1.6 \%$ & $0.0 \%$ \\
\hline SRNL-SCS-2008-00003/Set 2 & U std & SO4 (wt $\%)$ & 0.1498 & 0.1498 & 0.0000 & 0.1498 & 0.1498 & & \\
\hline SRNL-SCS-2008-00003/Set 2 & U std & ThO2 (wt $\%)$ & 0.0569 & 0.0569 & 0.0000 & 0.0569 & 0.0569 & & \\
\hline SRNL-SCS-2008-00003/Set 2 & U std & $\mathrm{TiO} 2(\mathrm{wt} \%)$ & 0.9517 & 0.9847 & 1.0490 & -0.0973 & -0.0643 & $-9.3 \%$ & $-6.1 \%$ \\
\hline SRNL-SCS-2008-00003/Set 2 & U std & $\mathrm{U} 3 \mathrm{O} 8(\mathrm{wt} \%)$ & 2.3378 & 2.4060 & 2.4060 & -0.0682 & 0.0000 & $-2.8 \%$ & $0.0 \%$ \\
\hline SRNL-SCS-2008-00003/Set 2 & U std & $\mathrm{ZnO}(\mathrm{wt} \%)$ & 0.0062 & 0.0062 & 0.0000 & 0.0062 & 0.0062 & & \\
\hline SRNL-SCS-2008-00003/Set 2 & U std & $\mathrm{ZrO} 2(\mathrm{wt} \%)$ & 0.0068 & 0.0068 & 0.0000 & 0.0068 & 0.0068 & & \\
\hline SRNL-SCS-2008-00003/Set 2 & U std & Sum & 99.9911 & 99.6385 & 99.6870 & 0.3041 & -0.0485 & $0.3 \%$ & $0.0 \%$ \\
\hline SRNL-SCS-2008-00003/Set 3 & Batch 1 & $\mathrm{~A} 12 \mathrm{O} 3(\mathrm{wt} \%)$ & 4.8151 & 4.8770 & 4.8770 & -0.0619 & 0.0000 & $-1.3 \%$ & $0.0 \%$ \\
\hline SRNL-SCS-2008-00003/Set 3 & Batch 1 & $\mathrm{~B} 2 \mathrm{O} 3(\mathrm{wt} \%)$ & 7.8109 & 7.7770 & 7.7770 & 0.0339 & 0.0000 & $0.4 \%$ & $0.0 \%$ \\
\hline SRNL-SCS-2008-00003/Set 3 & Batch 1 & $\mathrm{BaO}(\mathrm{wt} \%)$ & 0.1423 & 0.1510 & 0.1510 & -0.0087 & 0.0000 & $-5.8 \%$ & $0.0 \%$ \\
\hline SRNL-SCS-2008-00003/Set 3 & Batch 1 & $\mathrm{CaO}(\mathrm{wt} \%)$ & 1.1766 & 1.2200 & 1.2200 & -0.0434 & 0.0000 & $-3.6 \%$ & $0.0 \%$ \\
\hline SRNL-SCS-2008-00003/Set 3 & Batch 1 & $\mathrm{CdO}(\mathrm{wt} \%)$ & 0.0057 & 0.0057 & 0.0000 & 0.0057 & 0.0057 & & \\
\hline SRNL-SCS-2008-00003/Set 3 & Batch 1 & $\mathrm{Ce} 2 \mathrm{O} 3(\mathrm{wt} \%)$ & 0.0132 & 0.0132 & 0.0000 & 0.0132 & 0.0132 & & \\
\hline SRNL-SCS-2008-00003/Set 3 & Batch 1 & $\mathrm{Cr} 2 \mathrm{O} 3(\mathrm{wt} \%)$ & 0.1074 & 0.1070 & 0.1070 & 0.0004 & 0.0000 & $0.4 \%$ & $0.0 \%$ \\
\hline SRNL-SCS-2008-00003/Set 3 & Batch 1 & $\mathrm{CuO}(\mathrm{wt} \%)$ & 0.3808 & 0.3990 & 0.3990 & -0.0182 & 0.0000 & $-4.6 \%$ & $0.0 \%$ \\
\hline SRNL-SCS-2008-00003/Set 3 & Batch 1 & $\mathrm{Fe} 2 \mathrm{O} 3(\mathrm{wt} \%)$ & 13.2092 & 12.8390 & 12.8390 & 0.3702 & 0.0000 & $2.9 \%$ & $0.0 \%$ \\
\hline SRNL-SCS-2008-00003/Set 3 & Batch 1 & $\mathrm{~K} 2 \mathrm{O}(\mathrm{wt} \%)$ & 3.0959 & 3.3270 & 3.3270 & -0.2311 & 0.0000 & $-6.9 \%$ & $0.0 \%$ \\
\hline SRNL-SCS-2008-00003/Set 3 & Batch 1 & $\mathrm{La} 2 \mathrm{O} 3(\mathrm{wt} \%)$ & 0.0586 & 0.0586 & 0.0000 & 0.0586 & 0.0586 & & \\
\hline SRNL-SCS-2008-00003/Set 3 & Batch 1 & $\mathrm{Li} 2 \mathrm{O}(\mathrm{wt} \%)$ & 4.3022 & 4.4290 & 4.4290 & -0.1268 & 0.0000 & $-2.9 \%$ & $0.0 \%$ \\
\hline SRNL-SCS-2008-00003/Set 3 & Batch 1 & $\mathrm{MgO}(\mathrm{wt} \%)$ & 1.3825 & 1.4190 & 1.4190 & -0.0365 & 0.0000 & $-2.6 \%$ & $0.0 \%$ \\
\hline SRNL-SCS-2008-00003/Set 3 & Batch 1 & $\mathrm{MnO}\left(\mathrm{wt}^{0} \%\right)$ & 1.7948 & 1.7260 & 1.7260 & 0.0688 & 0.0000 & $4.0 \%$ & $0.0 \%$ \\
\hline SRNL-SCS-2008-00003/Set 3 & Batch 1 & $\mathrm{Na} 2 \mathrm{O}(\mathrm{wt} \%)$ & 9.0653 & 9.0030 & 9.0030 & 0.0623 & 0.0000 & $0.7 \%$ & $0.0 \%$ \\
\hline SRNL-SCS-2008-00003/Set 3 & Batch 1 & $\mathrm{Nb} 2 \mathrm{O} 5(\mathrm{wt} \%)$ & 0.0492 & 0.0492 & 0.0000 & 0.0492 & 0.0492 & & \\
\hline SRNL-SCS-2008-00003/Set 3 & Batch 1 & $\mathrm{NiO}(\mathrm{wt} \%)$ & 0.7132 & 0.7510 & 0.7510 & -0.0378 & 0.0000 & $-5.0 \%$ & $0.0 \%$ \\
\hline SRNL-SCS-2008-00003/Set 3 & Batch 1 & $\mathrm{PbO}(\mathrm{wt} \%)$ & 0.0108 & 0.0108 & 0.0000 & 0.0108 & 0.0108 & & \\
\hline SRNL-SCS-2008-00003/Set 3 & Batch 1 & $\mathrm{SiO} 2(\mathrm{wt} \%)$ & 51.3254 & 50.2200 & 50.2200 & 1.1054 & 0.0000 & $2.2 \%$ & $0.0 \%$ \\
\hline SRNL-SCS-2008-00003/Set 3 & Batch 1 & SO4 (wt $\%)$ & 0.1498 & 0.1498 & 0.0000 & 0.1498 & 0.1498 & & \\
\hline SRNL-SCS-2008-00003/Set 3 & Batch 1 & ThO2 (wt $\%)$ & 0.0569 & 0.0569 & 0.0000 & 0.0569 & 0.0569 & & \\
\hline SRNL-SCS-2008-00003/Set 3 & Batch 1 & $\mathrm{TiO} 2(\mathrm{wt} \%)$ & 0.6547 & 0.6770 & 0.6770 & -0.0223 & 0.0000 & $-3.3 \%$ & $0.0 \%$ \\
\hline SRNL-SCS-2008-00003/Set 3 & Batch 1 & $\mathrm{U} 3 \mathrm{O} 8(\mathrm{wt} \%)$ & 0.0590 & 0.0592 & 0.0000 & 0.0590 & 0.0592 & & \\
\hline SRNL-SCS-2008-00003/Set 3 & Batch 1 & $\mathrm{ZnO}(\mathrm{wt} \%)$ & 0.0062 & 0.0062 & 0.0000 & 0.0062 & 0.0062 & & \\
\hline SRNL-SCS-2008-00003/Set 3 & Batch 1 & $\mathrm{ZrO} 2(\mathrm{wt} \%)$ & 0.0865 & 0.0865 & 0.0980 & -0.0115 & -0.0115 & $-11.8 \%$ & $-11.8 \%$ \\
\hline SRNL-SCS-2008-00003/Set 3 & Batch 1 & Sum & 100.4721 & 99.4181 & 99.0200 & 1.4521 & 0.3981 & $1.5 \%$ & $0.4 \%$ \\
\hline SRNL-SCS-2008-00003/Set 3 & SB5-13 & $\mathrm{A} 12 \mathrm{O} 3(\mathrm{wt} \%)$ & 7.4352 & 7.4327 & 7.2170 & 0.2182 & 0.2157 & $3.0 \%$ & $3.0 \%$ \\
\hline SRNL-SCS-2008-00003/Set 3 & SB5-13 & $\mathrm{B} 2 \mathrm{O} 3(\mathrm{wt} \%)$ & 6.5122 & 6.3382 & 6.2000 & 0.3122 & 0.1382 & $5.0 \%$ & $2.2 \%$ \\
\hline SRNL-SCS-2008-00003/Set 3 & SB5-13 & $\mathrm{BaO}(\mathrm{wt} \%)$ & 0.0639 & 0.0674 & 0.0630 & 0.0009 & 0.0044 & $1.5 \%$ & $6.9 \%$ \\
\hline SRNL-SCS-2008-00003/Set 3 & SB5-13 & $\mathrm{CaO}(\mathrm{wt} \%)$ & 3.5295 & 3.6530 & 3.3360 & 0.1935 & 0.3170 & $5.8 \%$ & $9.5 \%$ \\
\hline SRNL-SCS-2008-00003/Set 3 & SB5-13 & $\mathrm{CdO}(\mathrm{wt} \%)$ & 0.0057 & 0.0057 & 0.0000 & 0.0057 & 0.0057 & & \\
\hline SRNL-SCS-2008-00003/Set 3 & SB5-13 & $\mathrm{Ce} 2 \mathrm{O} 3(\mathrm{wt} \%)$ & 0.0949 & 0.0949 & 0.1010 & -0.0061 & -0.0061 & $-6.1 \%$ & $-6.1 \%$ \\
\hline SRNL-SCS-2008-00003/Set 3 & SB5-13 & $\mathrm{Cr} 2 \mathrm{O} 3(\mathrm{wt} \%)$ & 0.0950 & 0.0936 & 0.0930 & 0.0020 & 0.0006 & $2.2 \%$ & $0.6 \%$ \\
\hline SRNL-SCS-2008-00003/Set 3 & SB5-13 & $\mathrm{CuO}(\mathrm{wt} \%)$ & 0.0360 & 0.0375 & 0.0330 & 0.0030 & 0.0045 & $9.1 \%$ & $13.6 \%$ \\
\hline SRNL-SCS-2008-00003/Set 3 & SB5-13 & $\mathrm{Fe} 2 \mathrm{O} 3(\mathrm{wt} \%)$ & 11.1088 & 10.5653 & 10.5040 & 0.6048 & 0.0613 & $5.8 \%$ & $0.6 \%$ \\
\hline SRNL-SCS-2008-00003/Set 3 & SB5-13 & $\mathrm{K} 2 \mathrm{O}(\mathrm{wt} \%)$ & 0.0771 & 0.0830 & 0.0770 & 0.0001 & 0.0060 & $0.1 \%$ & $7.8 \%$ \\
\hline SRNL-SCS-2008-00003/Set 3 & SB5-13 & $\mathrm{La} 2 \mathrm{O} 3(\mathrm{wt} \%)$ & 0.0586 & 0.0586 & 0.0180 & 0.0406 & 0.0406 & $225.8 \%$ & $225.8 \%$ \\
\hline SRNL-SCS-2008-00003/Set 3 & SB5-13 & $\mathrm{Li} 2 \mathrm{O}(\mathrm{wt} \%)$ & 4.3542 & 4.4639 & 4.3400 & 0.0142 & 0.1239 & $0.3 \%$ & $2.9 \%$ \\
\hline SRNL-SCS-2008-00003/Set 3 & SB5-13 & $\mathrm{MgO}\left(\mathrm{wt}^{\circ} \%\right)$ & 0.4759 & 0.4848 & 0.4670 & 0.0089 & 0.0178 & $1.9 \%$ & $3.8 \%$ \\
\hline SRNL-SCS-2008-00003/Set 3 & SB5-13 & $\mathrm{MnO}(\mathrm{wt} \%)$ & 2.5792 & 2.4306 & 2.3160 & 0.2632 & 0.1146 & $11.4 \%$ & $4.9 \%$ \\
\hline SRNL-SCS-2008-00003/Set 3 & SB5-13 & $\mathrm{Na} 2 \mathrm{O}(\mathrm{wt} \%)$ & 14.6123 & 14.3451 & 14.6780 & -0.0657 & -0.3329 & $-0.4 \%$ & $-2.3 \%$ \\
\hline SRNL-SCS-2008-00003/Set 3 & SB5-13 & $\mathrm{Nb} 2 \mathrm{O} 5(\mathrm{wt} \%)$ & 0.0358 & 0.0358 & 0.0000 & 0.0358 & 0.0358 & & \\
\hline SRNL-SCS-2008-00003/Set 3 & SB5-13 & $\mathrm{NiO}(\mathrm{wt} \%)$ & 1.1017 & 1.1521 & 1.1980 & -0.0963 & -0.0459 & $-8.0 \%$ & $-3.8 \%$ \\
\hline
\end{tabular}


SRNS-STI-2008-00060

Revision 0

Appendix A

Table A6. Average Measured and Bias-Corrected Chemical Compositions Versus Targeted Compositions by Oxide by Study Glass. (continued)

\begin{tabular}{|c|c|c|c|c|c|c|c|c|c|}
\hline Analytical Plan/Set & $\begin{array}{l}\text { Glass } \\
\text { ID }\end{array}$ & Oxide & $\begin{array}{c}\text { Measured } \\
\text { (wt\%) }\end{array}$ & $\begin{array}{c}\text { Measured BC } \\
\text { (wt \%) }\end{array}$ & $\begin{array}{c}\text { Targeted } \\
\text { (wt \%) }\end{array}$ & $\begin{array}{c}\text { Diff of } \\
\text { Measured }\end{array}$ & $\begin{array}{c}\text { Diff of } \\
\text { Meas BC }\end{array}$ & $\begin{array}{l}\text { \% Diff of } \\
\text { Measured }\end{array}$ & $\begin{array}{l}\text { \% Diff of } \\
\text { Meas BC }\end{array}$ \\
\hline SRNL-SCS-2008-00003/Set 3 & SB5-13 & $\mathrm{PbO}(\mathrm{wt} \%)$ & 0.0409 & 0.0409 & 0.0360 & 0.0049 & 0.0049 & $13.7 \%$ & $13.7 \%$ \\
\hline SRNL-SCS-2008-00003/Set 3 & SB5-13 & $\mathrm{SiO} 2\left(\mathrm{wt}^{\circ} \%\right)$ & 46.6367 & 44.9568 & 45.3380 & 1.2987 & -0.3812 & $2.9 \%$ & $-0.8 \%$ \\
\hline SRNL-SCS-2008-00003/Set 3 & SB5-13 & SO4 $\left(w t^{\%} \%\right)$ & 0.5438 & 0.5438 & 0.5470 & -0.0032 & -0.0032 & $-0.6 \%$ & $-0.6 \%$ \\
\hline SRNL-SCS-2008-00003/Set 3 & SB5-13 & $\mathrm{ThO} 2(\mathrm{wt} \%)$ & 0.0569 & 0.0569 & 0.0000 & 0.0569 & 0.0569 & & \\
\hline SRNL-SCS-2008-00003/Set 3 & SB5-13 & $\mathrm{TiO} 2(w t \%)$ & 0.3628 & 0.3731 & 0.3500 & 0.0128 & 0.0231 & $3.7 \%$ & $6.6 \%$ \\
\hline SRNL-SCS-2008-00003/Set 3 & SB5-13 & $\mathrm{U} 3 \mathrm{O} 8(\mathrm{wt} \%)$ & 2.9804 & 2.9420 & 2.9250 & 0.0554 & 0.0170 & $1.9 \%$ & $0.6 \%$ \\
\hline SRNL-SCS-2008-00003/Set 3 & SB5-13 & $\mathrm{ZnO}\left(\mathrm{wt}^{\%} \%\right)$ & 0.0439 & 0.0439 & 0.0420 & 0.0019 & 0.0019 & $4.5 \%$ & $4.5 \%$ \\
\hline SRNL-SCS-2008-00003/Set 3 & SB5-13 & $\mathrm{ZrO} 2(\mathrm{wt} \%)$ & 0.1152 & 0.1152 & 0.1170 & -0.0018 & -0.0018 & $-1.6 \%$ & $-1.6 \%$ \\
\hline SRNL-SCS-2008-00003/Set 3 & SB5-13 & Sum & 102.9566 & 100.4147 & 99.9960 & 2.9606 & 0.4187 & $3.0 \%$ & $0.4 \%$ \\
\hline SRNL-SCS-2008-00003/Set 3 & SB5-14 & $\mathrm{Al} 2 \mathrm{O} 3(\mathrm{wt} \%)$ & 7.3171 & 7.3157 & 7.2170 & 0.1001 & 0.0987 & $1.4 \%$ & $1.4 \%$ \\
\hline SRNL-SCS-2008-00003/Set 3 & SB5-14 & $\mathrm{B} 2 \mathrm{O} 3(\mathrm{wt} \%)$ & 6.8262 & 6.9570 & 6.8200 & 0.0062 & 0.1370 & $0.1 \%$ & $2.0 \%$ \\
\hline SRNL-SCS-2008-00003/Set 3 & SB5-14 & $\mathrm{BaO}(\mathrm{wt} \%)$ & 0.0550 & 0.0579 & 0.0630 & -0.0080 & -0.0051 & $-12.7 \%$ & $-8.0 \%$ \\
\hline SRNL-SCS-2008-00003/Set 3 & SB5-14 & $\mathrm{CaO}(\mathrm{wt} \%)$ & 2.1478 & 2.2216 & 2.0960 & 0.0518 & 0.1256 & $2.5 \%$ & $6.0 \%$ \\
\hline SRNL-SCS-2008-00003/Set 3 & SB5-14 & $\mathrm{CdO}\left(\mathrm{wt}^{\%} \%\right)$ & 0.0057 & 0.0057 & 0.0000 & 0.0057 & 0.0057 & & \\
\hline SRNL-SCS-2008-00003/Set 3 & SB5-14 & $\mathrm{Ce} 2 \mathrm{O} 3(\mathrm{wt} \%)$ & 0.0826 & 0.0826 & 0.1010 & -0.0184 & -0.0184 & $-18.2 \%$ & $-18.2 \%$ \\
\hline SRNL-SCS-2008-00003/Set 3 & SB5-14 & $\mathrm{Cr} 2 \mathrm{O} 3(\mathrm{wt} \%)$ & 0.0906 & 0.0893 & 0.0930 & -0.0024 & -0.0037 & $-2.6 \%$ & $-4.0 \%$ \\
\hline SRNL-SCS-2008-00003/Set 3 & SB5-14 & $\mathrm{CuO}(\mathrm{wt} \%)$ & 0.0379 & 0.0395 & 0.0330 & 0.0049 & 0.0065 & $14.7 \%$ & $19.6 \%$ \\
\hline SRNL-SCS-2008-00003/Set 3 & SB5-14 & $\mathrm{Fe} 2 \mathrm{O} 3(\mathrm{wt} \%)$ & 10.6942 & 10.1684 & 10.5040 & 0.1902 & -0.3356 & $1.8 \%$ & $-3.2 \%$ \\
\hline SRNL-SCS-2008-00003/Set 3 & SB5-14 & $\mathrm{K} 2 \mathrm{O}(\mathrm{wt} \%)$ & 0.0876 & 0.0945 & 0.0770 & 0.0106 & 0.0175 & $13.8 \%$ & $22.7 \%$ \\
\hline SRNL-SCS-2008-00003/Set 3 & SB5-14 & $\mathrm{La} 2 \mathrm{O} 3(\mathrm{wt} \%)$ & 0.0586 & 0.0586 & 0.0180 & 0.0406 & 0.0406 & $225.8 \%$ & $225.8 \%$ \\
\hline SRNL-SCS-2008-00003/Set 3 & SB5-14 & $\mathrm{Li2O}(\mathrm{wt} \%)$ & 4.3596 & 4.5069 & 4.3400 & 0.0196 & 0.1669 & $0.5 \%$ & $3.8 \%$ \\
\hline SRNL-SCS-2008-00003/Set 3 & SB5-14 & $\mathrm{MgO}(\mathrm{wt} \%)$ & 0.4506 & 0.4591 & 0.4670 & -0.0164 & -0.0079 & $-3.5 \%$ & $-1.7 \%$ \\
\hline SRNL-SCS-2008-00003/Set 3 & SB5-14 & $\mathrm{MnO}(\mathrm{wt} \%)$ & 2.4436 & 2.3023 & 2.3160 & 0.1276 & -0.0137 & $5.5 \%$ & $-0.6 \%$ \\
\hline SRNL-SCS-2008-00003/Set 3 & SB5-14 & $\mathrm{Na} 2 \mathrm{O}(\mathrm{wt} \%)$ & 14.4742 & 14.2146 & 14.6780 & -0.2039 & -0.4634 & $-1.4 \%$ & $-3.2 \%$ \\
\hline SRNL-SCS-2008-00003/Set 3 & SB5-14 & $\mathrm{Nb} 2 \mathrm{O} 5(\mathrm{wt} \%)$ & 0.0300 & 0.0300 & 0.0000 & 0.0300 & 0.0300 & & \\
\hline SRNL-SCS-2008-00003/Set 3 & SB5-14 & $\mathrm{NiO}(\mathrm{wt} \%)$ & 1.0240 & 1.0710 & 1.1980 & -0.1740 & -0.1270 & $-14.5 \%$ & $-10.6 \%$ \\
\hline SRNL-SCS-2008-00003/Set 3 & SB5-14 & $\mathrm{PbO}(\mathrm{wt} \%)$ & 0.0385 & 0.0385 & 0.0360 & 0.0025 & 0.0025 & $7.0 \%$ & $7.0 \%$ \\
\hline SRNL-SCS-2008-00003/Set 3 & SB5-14 & $\mathrm{SiO} 2(\mathrm{wt} \%)$ & 46.5298 & 44.8536 & 45.9580 & 0.5718 & -1.1044 & $1.2 \%$ & $-2.4 \%$ \\
\hline SRNL-SCS-2008-00003/Set 3 & SB5-14 & SO4 $(w t \%)$ & 0.5093 & 0.5093 & 0.5470 & -0.0377 & -0.0377 & $-6.9 \%$ & $-6.9 \%$ \\
\hline SRNL-SCS-2008-00003/Set 3 & SB5-14 & ThO2 (wt $\%)$ & 0.0569 & 0.0569 & 0.0000 & 0.0569 & 0.0569 & & \\
\hline SRNL-SCS-2008-00003/Set 3 & SB5-14 & $\mathrm{TiO} 2(\mathrm{wt} \%)$ & 0.3311 & 0.3405 & 0.3500 & -0.0189 & -0.0095 & $-5.4 \%$ & $-2.7 \%$ \\
\hline SRNL-SCS-2008-00003/Set 3 & SB5-14 & $\mathrm{U} 3 \mathrm{O} 8(\mathrm{wt} \%)$ & 2.8831 & 2.8463 & 2.9250 & -0.0419 & -0.0787 & $-1.4 \%$ & $-2.7 \%$ \\
\hline SRNL-SCS-2008-00003/Set 3 & SB5-14 & $\mathrm{ZnO}(\mathrm{wt} \%)$ & 0.0405 & 0.0405 & 0.0420 & -0.0015 & -0.0015 & $-3.7 \%$ & $-3.7 \%$ \\
\hline SRNL-SCS-2008-00003/Set 3 & SB5-14 & $\mathrm{ZrO} 2(\mathrm{wt} \%)$ & 0.1084 & 0.1084 & 0.1170 & -0.0086 & -0.0086 & $-7.3 \%$ & $-7.3 \%$ \\
\hline SRNL-SCS-2008-00003/Set 3 & SB5-14 & Sum & 100.6829 & 98.4686 & 99.9960 & 0.6869 & -1.5274 & $0.7 \%$ & $-1.5 \%$ \\
\hline SRNL-SCS-2008-00003/Set 3 & SB5-15 & $\mathrm{A} 12 \mathrm{O} 3(\mathrm{wt} \%)$ & 7.1895 & 7.3785 & 7.2170 & -0.0275 & 0.1615 & $-0.4 \%$ & $2.2 \%$ \\
\hline SRNL-SCS-2008-00003/Set 3 & SB5-15 & $\mathrm{B} 2 \mathrm{O} 3(\mathrm{wt} \%)$ & 8.3878 & 8.5496 & 8.6800 & -0.2922 & -0.1304 & $-3.4 \%$ & $-1.5 \%$ \\
\hline SRNL-SCS-2008-00003/Set 3 & SB5-15 & $\mathrm{BaO}(\mathrm{wt} \%)$ & 0.0578 & 0.0618 & 0.0630 & -0.0052 & -0.0012 & $-8.3 \%$ & $-1.9 \%$ \\
\hline SRNL-SCS-2008-00003/Set 3 & SB5-15 & $\mathrm{CaO}(\mathrm{wt} \%)$ & 2.1688 & 2.2556 & 2.0960 & 0.0728 & 0.1596 & $3.5 \%$ & $7.6 \%$ \\
\hline SRNL-SCS-2008-00003/Set 3 & SB5-15 & $\mathrm{CdO}(\mathrm{wt} \%)$ & 0.0057 & 0.0057 & 0.0000 & 0.0057 & 0.0057 & & \\
\hline SRNL-SCS-2008-00003/Set 3 & SB5-15 & $\mathrm{Ce} 2 \mathrm{O} 3(\mathrm{wt} \%)$ & 0.0878 & 0.0878 & 0.1010 & -0.0132 & -0.0132 & $-13.0 \%$ & $-13.0 \%$ \\
\hline SRNL-SCS-2008-00003/Set 3 & SB5-15 & $\mathrm{Cr} 2 \mathrm{O} 3(\mathrm{wt} \%)$ & 0.0928 & 0.0935 & 0.0930 & -0.0002 & 0.0005 & $-0.2 \%$ & $0.5 \%$ \\
\hline SRNL-SCS-2008-00003/Set 3 & SB5-15 & $\mathrm{CuO}(\mathrm{wt} \%)$ & 0.0329 & 0.0346 & 0.0330 & -0.0001 & 0.0016 & $-0.4 \%$ & $5.0 \%$ \\
\hline SRNL-SCS-2008-00003/Set 3 & SB5-15 & $\mathrm{Fe} 2 \mathrm{O} 3(\mathrm{wt} \%)$ & 10.1509 & 10.1126 & 10.5040 & -0.3531 & -0.3914 & $-3.4 \%$ & $-3.7 \%$ \\
\hline SRNL-SCS-2008-00003/Set 3 & SB5-15 & $\mathrm{K} 2 \mathrm{O}(\mathrm{wt} \%)$ & 0.0705 & 0.0755 & 0.0770 & -0.0065 & -0.0015 & $-8.5 \%$ & $-1.9 \%$ \\
\hline SRNL-SCS-2008-00003/Set 3 & SB5-15 & $\mathrm{La} 2 \mathrm{O} 3(\mathrm{wt} \%)$ & 0.0586 & 0.0586 & 0.0180 & 0.0406 & 0.0406 & $225.8 \%$ & $225.8 \%$ \\
\hline SRNL-SCS-2008-00003/Set 3 & SB5-15 & $\mathrm{Li} 2 \mathrm{O}(\mathrm{wt} \%)$ & 3.6276 & 3.7502 & 3.7200 & -0.0924 & 0.0302 & $-2.5 \%$ & $0.8 \%$ \\
\hline SRNL-SCS-2008-00003/Set 3 & SB5-15 & $\mathrm{MgO}(\mathrm{wt} \%)$ & 0.4689 & 0.4850 & 0.4670 & 0.0019 & 0.0180 & $0.4 \%$ & $3.8 \%$ \\
\hline SRNL-SCS-2008-00003/Set 3 & SB5-15 & $\mathrm{MnO}(\mathrm{wt} \%)$ & 2.3080 & 2.2711 & 2.3160 & -0.0080 & -0.0449 & $-0.3 \%$ & $-1.9 \%$ \\
\hline SRNL-SCS-2008-00003/Set 3 & SB5-15 & $\mathrm{Na} 2 \mathrm{O}(\mathrm{wt} \%)$ & 14.6056 & 14.6759 & 14.6780 & -0.0724 & -0.0021 & $-0.5 \%$ & $0.0 \%$ \\
\hline SRNL-SCS-2008-00003/Set 3 & SB5-15 & $\mathrm{Nb} 2 \mathrm{O} 5(\mathrm{wt} \%)$ & 0.0418 & 0.0418 & 0.0000 & 0.0418 & 0.0418 & & \\
\hline SRNL-SCS-2008-00003/Set 3 & SB5-15 & $\mathrm{NiO}(\mathrm{wt} \%)$ & 1.0864 & 1.1518 & 1.1980 & -0.1116 & -0.0462 & $-9.3 \%$ & $-3.9 \%$ \\
\hline SRNL-SCS-2008-00003/Set 3 & SB5-15 & $\mathrm{PbO}(\mathrm{wt} \%)$ & 0.0380 & 0.0380 & 0.0360 & 0.0020 & 0.0020 & $5.5 \%$ & $5.5 \%$ \\
\hline SRNL-SCS-2008-00003/Set 3 & SB5-15 & $\mathrm{SiO} 2(\mathrm{wt} \%)$ & 44.2835 & 43.9970 & 44.7180 & -0.4345 & -0.7210 & $-1.0 \%$ & $-1.6 \%$ \\
\hline SRNL-SCS-2008-00003/Set 3 & SB5-15 & $\mathrm{SO} 4(\mathrm{wt} \%)$ & 0.5123 & 0.5123 & 0.5470 & -0.0347 & -0.0347 & $-6.3 \%$ & $-6.3 \%$ \\
\hline SRNL-SCS-2008-00003/Set 3 & SB5-15 & ThO2 (wt $\%)$ & 0.0569 & 0.0569 & 0.0000 & 0.0569 & 0.0569 & & \\
\hline SRNL-SCS-2008-00003/Set 3 & SB5-15 & $\mathrm{TiO} 2(\mathrm{wt} \%)$ & 0.3653 & 0.3798 & 0.3500 & 0.0153 & 0.0298 & $4.4 \%$ & $8.5 \%$ \\
\hline SRNL-SCS-2008-00003/Set 3 & SB5-15 & $\mathrm{U} 3 \mathrm{O} 8(\mathrm{wt} \%)$ & 2.8655 & 2.9233 & 2.9250 & -0.0595 & -0.0017 & $-2.0 \%$ & $-0.1 \%$ \\
\hline SRNL-SCS-2008-00003/Set 3 & SB5-15 & $\mathrm{ZnO}(\mathrm{wt} \%)$ & 0.0411 & 0.0411 & 0.0420 & -0.0009 & -0.0009 & $-2.2 \%$ & $-2.2 \%$ \\
\hline SRNL-SCS-2008-00003/Set 3 & SB5-15 & $\mathrm{ZrO} 2(\mathrm{wt} \%)$ & 0.1131 & 0.1131 & 0.1170 & -0.0039 & -0.0039 & $-3.3 \%$ & $-3.3 \%$ \\
\hline SRNL-SCS-2008-00003/Set 3 & SB5-15 & Sum & 98.7171 & 99.1513 & 99.9960 & -1.2789 & -0.8447 & $-1.3 \%$ & $-0.8 \%$ \\
\hline SRNL-SCS-2008-00003/Set 3 & SB5-16 & $\mathrm{A} 12 \mathrm{O} 3(\mathrm{wt} \%)$ & 7.2179 & 7.4077 & 7.2170 & 0.0009 & 0.1907 & $0.0 \%$ & $2.6 \%$ \\
\hline SRNL-SCS-2008-00003/Set 3 & SB5-16 & $\mathrm{B} 2 \mathrm{O} 3(\mathrm{wt} \%)$ & 9.6114 & 9.7947 & 9.9200 & -0.3086 & -0.1253 & $-3.1 \%$ & $-1.3 \%$ \\
\hline SRNL-SCS-2008-00003/Set 3 & SB5-16 & $\mathrm{BaO}(\mathrm{wt} \%)$ & 0.0575 & 0.0615 & 0.0630 & -0.0055 & -0.0015 & $-8.7 \%$ & $-2.4 \%$ \\
\hline SRNL-SCS-2008-00003/Set 3 & SB5-16 & $\mathrm{CaO}(\mathrm{wt} \%)$ & 0.8206 & 0.8535 & 0.8560 & -0.0354 & -0.0025 & $-4.1 \%$ & $-0.3 \%$ \\
\hline SRNL-SCS-2008-00003/Set 3 & SB5-16 & $\mathrm{CdO}(\mathrm{wt} \%)$ & 0.0057 & 0.0057 & 0.0000 & 0.0057 & 0.0057 & & \\
\hline SRNL-SCS-2008-00003/Set 3 & SB5-16 & $\mathrm{Ce} 2 \mathrm{O} 3(\mathrm{wt} \%)$ & 0.1300 & 0.1300 & 0.1010 & 0.0290 & 0.0290 & $28.7 \%$ & $28.7 \%$ \\
\hline SRNL-SCS-2008-00003/Set 3 & SB5-16 & $\mathrm{Cr} 2 \mathrm{O} 3(\mathrm{wt} \%)$ & 0.0855 & 0.0861 & 0.0930 & -0.0075 & -0.0069 & $-8.1 \%$ & $-7.4 \%$ \\
\hline
\end{tabular}


SRNS-STI-2008-00060

Revision 0

Appendix A

Table A6. Average Measured and Bias-Corrected Chemical Compositions Versus Targeted Compositions by Oxide by Study Glass. (continued)

\begin{tabular}{|c|c|c|c|c|c|c|c|c|c|}
\hline Analytical Plan/Set & $\begin{array}{c}\text { Glass } \\
\text { ID }\end{array}$ & Oxide & $\begin{array}{c}\text { Measured } \\
\text { (wt\%) }\end{array}$ & $\begin{array}{c}\text { Measured BC } \\
\text { (wt\%) }\end{array}$ & $\begin{array}{c}\text { Targeted } \\
\text { (wt \%) }\end{array}$ & $\begin{array}{c}\text { Diff of } \\
\text { Measured }\end{array}$ & $\begin{array}{c}\text { Diff of } \\
\text { Meas BC }\end{array}$ & $\begin{array}{l}\text { \% Diff of } \\
\text { Measured }\end{array}$ & $\begin{array}{l}\text { \% Diff of } \\
\text { Meas BC }\end{array}$ \\
\hline SRNL-SCS-2008-00003/Set 3 & SB5-16 & $\mathrm{CuO}(\mathrm{wt} \%)$ & 0.0341 & 0.0360 & 0.0330 & 0.0011 & 0.0030 & $3.4 \%$ & $9.0 \%$ \\
\hline SRNL-SCS-2008-00003/Set 3 & SB5-16 & $\mathrm{Fe} 2 \mathrm{O} 3(\mathrm{wt} \%)$ & 10.5905 & 10.5467 & 10.5040 & 0.0865 & 0.0427 & $0.8 \%$ & $0.4 \%$ \\
\hline SRNL-SCS-2008-00003/Set 3 & SB5-16 & $\mathrm{K} 2 \mathrm{O}(\mathrm{wt} \%)$ & 0.0690 & 0.0739 & 0.0770 & -0.0080 & -0.0031 & $-10.4 \%$ & $-4.0 \%$ \\
\hline SRNL-SCS-2008-00003/Set 3 & SB5-16 & $\mathrm{La} 2 \mathrm{O} 3(\mathrm{wt} \%)$ & 0.0586 & 0.0586 & 0.0180 & 0.0406 & 0.0406 & $225.8 \%$ & $225.8 \%$ \\
\hline SRNL-SCS-2008-00003/Set 3 & SB5-16 & $\mathrm{Li} 2 \mathrm{O}(\mathrm{wt} \%)$ & 3.0464 & 3.1493 & 3.1000 & -0.0536 & 0.0493 & $-1.7 \%$ & $1.6 \%$ \\
\hline SRNL-SCS-2008-00003/Set 3 & SB5-16 & $\mathrm{MgO}\left(\mathrm{wt}^{0} \%\right)$ & 0.4780 & 0.4944 & 0.4670 & 0.0110 & 0.0274 & $2.4 \%$ & $5.9 \%$ \\
\hline SRNL-SCS-2008-00003/Set 3 & SB5-16 & $\mathrm{MnO}(\mathrm{wt} \%)$ & 2.3726 & 2.3310 & 2.3160 & 0.0566 & 0.0150 & $2.4 \%$ & $0.6 \%$ \\
\hline SRNL-SCS-2008-00003/Set 3 & SB5-16 & $\mathrm{Na} 2 \mathrm{O}(\mathrm{wt} \%)$ & 14.9224 & 14.9945 & 15.2980 & -0.3756 & -0.3035 & $-2.5 \%$ & $-2.0 \%$ \\
\hline SRNL-SCS-2008-00003/Set 3 & SB5-16 & $\mathrm{Nb} 2 \mathrm{O} 5(\mathrm{wt} \%)$ & 0.0390 & 0.0390 & 0.0000 & 0.0390 & 0.0390 & & \\
\hline SRNL-SCS-2008-00003/Set 3 & SB5-16 & $\mathrm{NiO}\left(\mathrm{wt}^{0} \%\right)$ & 1.1039 & 1.1703 & 1.1980 & -0.0941 & -0.0277 & $-7.9 \%$ & $-2.3 \%$ \\
\hline SRNL-SCS-2008-00003/Set 3 & SB5-16 & $\mathrm{PbO}(\mathrm{wt} \%)$ & 0.0390 & 0.0390 & 0.0360 & 0.0030 & 0.0030 & $8.5 \%$ & $8.5 \%$ \\
\hline SRNL-SCS-2008-00003/Set 3 & SB5-16 & $\mathrm{SiO} 2(\mathrm{wt} \%)$ & 44.3370 & 44.0419 & 44.7180 & -0.3810 & -0.6761 & $-0.9 \%$ & $-1.5 \%$ \\
\hline SRNL-SCS-2008-00003/Set 3 & SB5-16 & $\mathrm{SO} 4(\mathrm{wt} \%)$ & 0.5063 & 0.5063 & 0.5470 & -0.0407 & -0.0407 & $-7.4 \%$ & $-7.4 \%$ \\
\hline SRNL-SCS-2008-00003/Set 3 & SB5-16 & ThO2 (wt $\%)$ & 0.0569 & 0.0569 & 0.0000 & 0.0569 & 0.0569 & & \\
\hline SRNL-SCS-2008-00003/Set 3 & SB5-16 & $\mathrm{TiO} 2(\mathrm{wt} \%)$ & 0.3494 & 0.3634 & 0.3500 & -0.0006 & 0.0134 & $-0.2 \%$ & $3.8 \%$ \\
\hline SRNL-SCS-2008-00003/Set 3 & SB5-16 & $\mathrm{U} 3 \mathrm{O} 8(\mathrm{wt} \%)$ & 2.8684 & 2.9263 & 2.9250 & -0.0566 & 0.0013 & $-1.9 \%$ & $0.0 \%$ \\
\hline SRNL-SCS-2008-00003/Set 3 & SB5-16 & $\mathrm{ZnO}(\mathrm{wt} \%)$ & 0.0423 & 0.0423 & 0.0420 & 0.0003 & 0.0003 & $0.8 \%$ & $0.8 \%$ \\
\hline SRNL-SCS-2008-00003/Set 3 & SB5-16 & $\mathrm{ZrO} 2(\mathrm{wt} \%)$ & 0.1135 & 0.1135 & 0.1170 & -0.0035 & -0.0035 & $-3.0 \%$ & $-3.0 \%$ \\
\hline SRNL-SCS-2008-00003/Set 3 & SB5-16 & Sum & 98.9559 & 99.3225 & 99.9960 & -1.0401 & -0.6735 & $-1.0 \%$ & $-0.7 \%$ \\
\hline SRNL-SCS-2008-00003/Set 3 & SB5-17 & $\mathrm{Al} 2 \mathrm{O} 3(\mathrm{wt} \%)$ & 10.9922 & 11.2811 & 11.0090 & -0.0168 & 0.2721 & $-0.2 \%$ & $2.5 \%$ \\
\hline SRNL-SCS-2008-00003/Set 3 & SB5-17 & $\mathrm{B} 2 \mathrm{O} 3(\mathrm{wt} \%)$ & 9.5229 & 9.2711 & 9.3000 & 0.2229 & -0.0289 & $2.4 \%$ & $-0.3 \%$ \\
\hline SRNL-SCS-2008-00003/Set 3 & SB5-17 & $\mathrm{BaO}(\mathrm{wt} \%)$ & 0.0360 & 0.0385 & 0.0390 & -0.0030 & -0.0005 & $-7.7 \%$ & $-1.3 \%$ \\
\hline SRNL-SCS-2008-00003/Set 3 & SB5-17 & $\mathrm{CaO}(\mathrm{wt} \%)$ & 0.6583 & 0.6847 & 0.6770 & -0.0187 & 0.0077 & $-2.8 \%$ & $1.1 \%$ \\
\hline SRNL-SCS-2008-00003/Set 3 & SB5-17 & $\mathrm{CdO}(\mathrm{wt} \%)$ & 0.0148 & 0.0148 & 0.0230 & -0.0082 & -0.0082 & $-35.4 \%$ & $-35.4 \%$ \\
\hline SRNL-SCS-2008-00003/Set 3 & SB5-17 & $\mathrm{Ce} 2 \mathrm{O} 3(\mathrm{wt} \%)$ & 0.1646 & 0.1646 & 0.1410 & 0.0236 & 0.0236 & $16.7 \%$ & $16.7 \%$ \\
\hline SRNL-SCS-2008-00003/Set 3 & SB5-17 & $\mathrm{Cr} 2 \mathrm{O} 3(\mathrm{wt} \%)$ & 0.1118 & 0.1126 & 0.1420 & -0.0302 & -0.0294 & $-21.3 \%$ & $-20.7 \%$ \\
\hline SRNL-SCS-2008-00003/Set 3 & SB5-17 & $\mathrm{CuO}(\mathrm{wt} \%)$ & 0.0063 & 0.0066 & 0.0050 & 0.0013 & 0.0016 & $25.2 \%$ & $32.0 \%$ \\
\hline SRNL-SCS-2008-00003/Set 3 & SB5-17 & $\mathrm{Fe} 2 \mathrm{O} 3(\mathrm{wt} \%)$ & 8.3530 & 8.3283 & 8.8340 & -0.4810 & -0.5057 & $-5.4 \%$ & $-5.7 \%$ \\
\hline SRNL-SCS-2008-00003/Set 3 & SB5-17 & $\mathrm{K} 2 \mathrm{O}(\mathrm{wt} \%)$ & 0.0145 & 0.0155 & 0.0250 & -0.0105 & -0.0095 & $-42.2 \%$ & $-38.0 \%$ \\
\hline SRNL-SCS-2008-00003/Set 3 & SB5-17 & $\mathrm{La} 2 \mathrm{O} 3(\mathrm{wt} \%)$ & 0.0586 & 0.0586 & 0.0620 & -0.0034 & -0.0034 & $-5.4 \%$ & $-5.4 \%$ \\
\hline SRNL-SCS-2008-00003/Set 3 & SB5-17 & $\mathrm{Li} 2 \mathrm{O}(\mathrm{wt} \%)$ & 5.5276 & 5.6668 & 5.5800 & -0.0524 & 0.0868 & $-0.9 \%$ & $1.6 \%$ \\
\hline SRNL-SCS-2008-00003/Set 3 & SB5-17 & $\mathrm{MgO}(\mathrm{wt} \%)$ & 0.4502 & 0.4657 & 0.4470 & 0.0032 & 0.0187 & $0.7 \%$ & $4.2 \%$ \\
\hline SRNL-SCS-2008-00003/Set 3 & SB5-17 & $\mathrm{MnO}(\mathrm{wt} \%)$ & 1.8593 & 1.8306 & 1.8470 & 0.0123 & -0.0164 & $0.7 \%$ & $-0.9 \%$ \\
\hline SRNL-SCS-2008-00003/Set 3 & SB5-17 & $\mathrm{Na} 2 \mathrm{O}(\mathrm{wt} \%)$ & 12.0376 & 12.0965 & 11.9160 & 0.1216 & 0.1805 & $1.0 \%$ & $1.5 \%$ \\
\hline SRNL-SCS-2008-00003/Set 3 & SB5-17 & $\mathrm{Nb} 2 \mathrm{O} 5(\mathrm{wt} \%)$ & 0.0143 & 0.0143 & 0.0000 & 0.0143 & 0.0143 & & \\
\hline SRNL-SCS-2008-00003/Set 3 & SB5-17 & $\mathrm{NiO}(\mathrm{wt} \%)$ & 0.9254 & 0.9811 & 1.0370 & -0.1116 & -0.0559 & $-10.8 \%$ & $-5.4 \%$ \\
\hline SRNL-SCS-2008-00003/Set 3 & SB5-17 & $\mathrm{PbO}(\mathrm{wt} \%)$ & 0.0108 & 0.0108 & 0.0080 & 0.0028 & 0.0028 & $34.7 \%$ & $34.7 \%$ \\
\hline SRNL-SCS-2008-00003/Set 3 & SB5-17 & $\mathrm{SiO} 2(\mathrm{wt} \%)$ & 45.0323 & 44.7499 & 45.3570 & -0.3247 & -0.6071 & $-0.7 \%$ & $-1.3 \%$ \\
\hline SRNL-SCS-2008-00003/Set 3 & SB5-17 & SO4 (wt $\%)$ & 0.1498 & 0.1498 & 0.2770 & -0.1272 & -0.1272 & $-45.9 \%$ & $-45.9 \%$ \\
\hline SRNL-SCS-2008-00003/Set 3 & SB5-17 & ThO2 (wt $\%)$ & 0.0569 & 0.0569 & 0.0000 & 0.0569 & 0.0569 & & \\
\hline SRNL-SCS-2008-00003/Set 3 & SB5-17 & $\mathrm{TiO} 2(\mathrm{wt} \%)$ & 0.0083 & 0.0087 & 0.0100 & -0.0017 & -0.0013 & $-16.6 \%$ & $-13.3 \%$ \\
\hline SRNL-SCS-2008-00003/Set 3 & SB5-17 & $\mathrm{U} 3 \mathrm{O} 8(\mathrm{wt} \%)$ & 2.7859 & 2.8421 & 2.8260 & -0.0401 & 0.0161 & $-1.4 \%$ & $0.6 \%$ \\
\hline SRNL-SCS-2008-00003/Set 3 & SB5-17 & $\mathrm{ZnO}(\mathrm{wt} \%)$ & 0.0062 & 0.0062 & 0.0060 & 0.0002 & 0.0002 & $3.7 \%$ & $3.7 \%$ \\
\hline SRNL-SCS-2008-00003/Set 3 & SB5-17 & $\mathrm{ZrO} 2(\mathrm{wt} \%)$ & 0.0929 & 0.0929 & 0.0980 & -0.0051 & -0.0051 & $-5.2 \%$ & $-5.2 \%$ \\
\hline SRNL-SCS-2008-00003/Set 3 & SB5-17 & Sum & 98.8905 & 98.9488 & 99.6660 & -0.7755 & -0.7172 & $-0.8 \%$ & $-0.7 \%$ \\
\hline SRNL-SCS-2008-00003/Set 3 & SB5-18 & $\mathrm{A} 12 \mathrm{O} 3(\mathrm{wt} \%)$ & 9.8065 & 9.8033 & 9.4600 & 0.3465 & 0.3433 & $3.7 \%$ & $3.6 \%$ \\
\hline SRNL-SCS-2008-00003/Set 3 & SB5-18 & $\mathrm{B} 2 \mathrm{O} 3(\mathrm{wt} \%)$ & 8.7581 & 8.5296 & 8.6800 & 0.0781 & -0.1504 & $0.9 \%$ & $-1.7 \%$ \\
\hline SRNL-SCS-2008-00003/Set 3 & SB5-18 & $\mathrm{BaO}(\mathrm{wt} \%)$ & 0.0391 & 0.0412 & 0.0430 & -0.0039 & -0.0018 & $-9.1 \%$ & $-4.2 \%$ \\
\hline SRNL-SCS-2008-00003/Set 3 & SB5-18 & $\mathrm{CaO}(\mathrm{wt} \%)$ & 0.7230 & 0.7471 & 0.7470 & -0.0240 & 0.0001 & $-3.2 \%$ & $0.0 \%$ \\
\hline SRNL-SCS-2008-00003/Set 3 & SB5-18 & $\mathrm{CdO}(\mathrm{wt} \%)$ & 0.0180 & 0.0180 & 0.0250 & -0.0070 & -0.0070 & $-28.0 \%$ & $-28.0 \%$ \\
\hline SRNL-SCS-2008-00003/Set 3 & SB5-18 & $\mathrm{Ce} 2 \mathrm{O} 3(\mathrm{wt} \%)$ & 0.1329 & 0.1329 & 0.1550 & -0.0221 & -0.0221 & $-14.2 \%$ & $-14.2 \%$ \\
\hline SRNL-SCS-2008-00003/Set 3 & SB5-18 & $\mathrm{Cr} 2 \mathrm{O} 3(\mathrm{wt} \%)$ & 0.1279 & 0.1260 & 0.1560 & -0.0281 & -0.0300 & $-18.0 \%$ & $-19.3 \%$ \\
\hline SRNL-SCS-2008-00003/Set 3 & SB5-18 & $\mathrm{CuO}(\mathrm{wt} \%)$ & 0.0078 & 0.0082 & 0.0050 & 0.0028 & 0.0032 & $56.5 \%$ & $63.4 \%$ \\
\hline SRNL-SCS-2008-00003/Set 3 & SB5-18 & $\mathrm{Fe} 2 \mathrm{O} 3(\mathrm{wt} \%)$ & 10.2009 & 9.7043 & 9.7450 & 0.4559 & -0.0407 & $4.7 \%$ & $-0.4 \%$ \\
\hline SRNL-SCS-2008-00003/Set 3 & SB5-18 & $\mathrm{K} 2 \mathrm{O}(\mathrm{wt} \%)$ & 0.0214 & 0.0230 & 0.0270 & -0.0056 & -0.0040 & $-20.8 \%$ & $-14.7 \%$ \\
\hline SRNL-SCS-2008-00003/Set 3 & SB5-18 & $\mathrm{La} 2 \mathrm{O} 3(\mathrm{wt} \%)$ & 0.0586 & 0.0586 & 0.0680 & -0.0094 & -0.0094 & $-13.8 \%$ & $-13.8 \%$ \\
\hline SRNL-SCS-2008-00003/Set 3 & SB5-18 & $\mathrm{Li} 2 \mathrm{O}(\mathrm{wt} \%)$ & 4.3112 & 4.4198 & 4.3400 & -0.0288 & 0.0798 & $-0.7 \%$ & $1.8 \%$ \\
\hline SRNL-SCS-2008-00003/Set 3 & SB5-18 & $\mathrm{MgO}(\mathrm{wt} \%)$ & 0.4859 & 0.4950 & 0.4940 & -0.0081 & 0.0010 & $-1.6 \%$ & $0.2 \%$ \\
\hline SRNL-SCS-2008-00003/Set 3 & SB5-18 & $\mathrm{MnO}(\mathrm{wt} \%)$ & 2.2757 & 2.1452 & 2.0380 & 0.2377 & 0.1072 & $11.7 \%$ & $5.3 \%$ \\
\hline SRNL-SCS-2008-00003/Set 3 & SB5-18 & $\mathrm{Na} 2 \mathrm{O}(\mathrm{wt} \%)$ & 14.2922 & 14.0310 & 14.1540 & 0.1382 & -0.1230 & $1.0 \%$ & $-0.9 \%$ \\
\hline SRNL-SCS-2008-00003/Set 3 & SB5-18 & $\mathrm{Nb} 2 \mathrm{O} 5(\mathrm{wt} \%)$ & 0.0143 & 0.0143 & 0.0000 & 0.0143 & 0.0143 & & \\
\hline SRNL-SCS-2008-00003/Set 3 & SB5-18 & $\mathrm{NiO}\left(\mathrm{wt}^{\mathrm{O}} \%\right)$ & 0.9970 & 1.0427 & 1.1440 & -0.1470 & -0.1013 & $-12.8 \%$ & $-8.9 \%$ \\
\hline SRNL-SCS-2008-00003/Set 3 & SB5-18 & $\mathrm{PbO}(\mathrm{wt} \%)$ & 0.0108 & 0.0108 & 0.0090 & 0.0018 & 0.0018 & $19.7 \%$ & $19.7 \%$ \\
\hline SRNL-SCS-2008-00003/Set 3 & SB5-18 & $\mathrm{SiO} 2(\mathrm{wt} \%)$ & 46.2089 & 44.5501 & 44.8110 & 1.3979 & -0.2609 & $3.1 \%$ & $-0.6 \%$ \\
\hline SRNL-SCS-2008-00003/Set 3 & SB5-18 & SO4 (wt \%) & 0.2674 & 0.2674 & 0.2890 & -0.0216 & -0.0216 & $-7.5 \%$ & $-7.5 \%$ \\
\hline SRNL-SCS-2008-00003/Set 3 & SB5-18 & ThO2 (wt \%) & 0.0569 & 0.0569 & 0.0000 & 0.0569 & 0.0569 & & \\
\hline SRNL-SCS-2008-00003/Set 3 & SB5-18 & $\mathrm{TiO} 2(\mathrm{wt} \%)$ & 0.0217 & 0.0223 & 0.0110 & 0.0107 & 0.0113 & $97.1 \%$ & $102.7 \%$ \\
\hline SRNL-SCS-2008-00003/Set 3 & SB5-18 & $\mathrm{U} 3 \mathrm{O} 8(\mathrm{wt} \%)$ & 3.1897 & 3.1488 & 3.1170 & 0.0727 & 0.0318 & $2.3 \%$ & $1.0 \%$ \\
\hline
\end{tabular}


SRNS-STI-2008-00060

Revision 0

Appendix A

Table A6. Average Measured and Bias-Corrected Chemical Compositions Versus Targeted Compositions by Oxide by Study Glass. (continued)

\begin{tabular}{|c|c|c|c|c|c|c|c|c|c|}
\hline Analytical Plan/Set & $\begin{array}{l}\text { Glass } \\
\text { ID }\end{array}$ & Oxide & $\begin{array}{c}\text { Measured } \\
\text { (wt\%) }\end{array}$ & $\begin{array}{c}\text { Measured BC } \\
\text { (wt \%) }\end{array}$ & $\begin{array}{c}\text { Targeted } \\
\text { (wt \%) }\end{array}$ & $\begin{array}{c}\text { Diff of } \\
\text { Measured }\end{array}$ & $\begin{array}{c}\text { Diff of } \\
\text { Meas BC }\end{array}$ & $\begin{array}{l}\% \text { Diff of } \\
\text { Measured }\end{array}$ & $\begin{array}{l}\text { \% Diff of } \\
\text { Meas BC }\end{array}$ \\
\hline SRNL-SCS-2008-00003/Set 3 & SB5-18 & $\mathrm{ZnO}(\mathrm{wt} \%)$ & 0.0062 & 0.0062 & 0.0060 & 0.0002 & 0.0002 & $3.7 \%$ & $3.7 \%$ \\
\hline SRNL-SCS-2008-00003/Set 3 & SB5-18 & $\mathrm{ZrO} 2(\mathrm{wt} \%)$ & 0.0986 & 0.0986 & 0.1080 & -0.0094 & -0.0094 & $-8.7 \%$ & $-8.7 \%$ \\
\hline SRNL-SCS-2008-00003/Set 3 & SB5-18 & Sum & 102.1308 & 99.5013 & 99.6320 & 2.4988 & -0.1307 & $2.5 \%$ & $-0.1 \%$ \\
\hline SRNL-SCS-2008-00003/Set 3 & SB5-19 & $\mathrm{A} 12 \mathrm{O} 3(\mathrm{wt} \%)$ & 7.9690 & 8.1784 & 7.9470 & 0.0220 & 0.2314 & $0.3 \%$ & $2.9 \%$ \\
\hline SRNL-SCS-2008-00003/Set 3 & SB5-19 & $\mathrm{B} 2 \mathrm{O} 3(\mathrm{wt} \%)$ & 9.2492 & 9.0050 & 9.3000 & -0.0508 & -0.2950 & $-0.5 \%$ & $-3.2 \%$ \\
\hline SRNL-SCS-2008-00003/Set 3 & SB5-19 & $\mathrm{BaO}(\mathrm{wt} \%)$ & 0.0354 & 0.0379 & 0.0460 & -0.0106 & -0.0081 & $-22.9 \%$ & $-17.6 \%$ \\
\hline SRNL-SCS-2008-00003/Set 3 & SB5-19 & $\mathrm{CaO}(\mathrm{wt} \%)$ & 0.7724 & 0.8033 & 0.8010 & -0.0286 & 0.0023 & $-3.6 \%$ & $0.3 \%$ \\
\hline SRNL-SCS-2008-00003/Set 3 & SB5-19 & $\mathrm{CdO}(\mathrm{wt} \%)$ & 0.0211 & 0.0211 & 0.0270 & -0.0059 & -0.0059 & $-21.7 \%$ & $-21.7 \%$ \\
\hline SRNL-SCS-2008-00003/Set 3 & SB5-19 & $\mathrm{Ce} 2 \mathrm{O} 3(\mathrm{wt} \%)$ & 0.1818 & 0.1818 & 0.1670 & 0.0148 & 0.0148 & $8.9 \%$ & $8.9 \%$ \\
\hline SRNL-SCS-2008-00003/Set 3 & SB5-19 & $\mathrm{Cr} 2 \mathrm{O} 3(\mathrm{wt} \%)$ & 0.1410 & 0.1421 & 0.1670 & -0.0260 & -0.0249 & $-15.5 \%$ & $-14.9 \%$ \\
\hline SRNL-SCS-2008-00003/Set 3 & SB5-19 & $\mathrm{CuO}(\mathrm{wt} \%)$ & 0.0063 & 0.0066 & 0.0050 & 0.0013 & 0.0016 & $25.2 \%$ & $32.0 \%$ \\
\hline SRNL-SCS-2008-00003/Set 3 & SB5-19 & $\mathrm{Fe} 2 \mathrm{O} 3(\mathrm{wt} \%)$ & 10.4225 & 10.3705 & 10.4470 & -0.0245 & -0.0765 & $-0.2 \%$ & $-0.7 \%$ \\
\hline SRNL-SCS-2008-00003/Set 3 & SB5-19 & $\mathrm{K} 2 \mathrm{O}(\mathrm{wt} \%)$ & 0.0175 & 0.0187 & 0.0300 & -0.0125 & -0.0113 & $-41.8 \%$ & $-37.6 \%$ \\
\hline SRNL-SCS-2008-00003/Set 3 & SB5-19 & $\mathrm{La} 2 \mathrm{O} 3(\mathrm{wt} \%)$ & 0.0586 & 0.0586 & 0.0730 & -0.0144 & -0.0144 & $-19.7 \%$ & $-19.7 \%$ \\
\hline SRNL-SCS-2008-00003/Set 3 & SB5-19 & $\mathrm{Li} 2 \mathrm{O}(\mathrm{wt} \%)$ & 3.0679 & 3.1452 & 3.1000 & -0.0321 & 0.0452 & $-1.0 \%$ & $1.5 \%$ \\
\hline SRNL-SCS-2008-00003/Set 3 & SB5-19 & $\mathrm{MgO}\left(\mathrm{wt}^{\mathrm{O}} \%\right)$ & 0.5224 & 0.5403 & 0.5290 & -0.0066 & 0.0113 & $-1.3 \%$ & $2.1 \%$ \\
\hline SRNL-SCS-2008-00003/Set 3 & SB5-19 & $\mathrm{MnO}\left(\mathrm{wt}^{\%} \%\right)$ & 2.2951 & 2.2559 & 2.1840 & 0.1111 & 0.0719 & $5.1 \%$ & $3.3 \%$ \\
\hline SRNL-SCS-2008-00003/Set 3 & SB5-19 & $\mathrm{Na} 2 \mathrm{O}(\mathrm{wt} \%)$ & 15.1684 & 15.2405 & 15.5100 & -0.3416 & -0.2695 & $-2.2 \%$ & $-1.7 \%$ \\
\hline SRNL-SCS-2008-00003/Set 3 & SB5-19 & $\mathrm{Nb} 2 \mathrm{O} 5(\mathrm{wt} \%)$ & 0.0139 & 0.0139 & 0.0000 & 0.0139 & 0.0139 & & \\
\hline SRNL-SCS-2008-00003/Set 3 & SB5-19 & $\mathrm{NiO}(\mathrm{wt} \%)$ & 1.0959 & 1.1619 & 1.2270 & -0.1311 & -0.0651 & $-10.7 \%$ & $-5.3 \%$ \\
\hline SRNL-SCS-2008-00003/Set 3 & SB5-19 & $\mathrm{PbO}(\mathrm{wt} \%)$ & 0.0108 & 0.0108 & 0.0100 & 0.0008 & 0.0008 & $7.7 \%$ & $7.7 \%$ \\
\hline SRNL-SCS-2008-00003/Set 3 & SB5-19 & $\mathrm{SiO} 2(\mathrm{wt} \%)$ & 44.1231 & 43.8354 & 44.2490 & -0.1259 & -0.4136 & $-0.3 \%$ & $-0.9 \%$ \\
\hline SRNL-SCS-2008-00003/Set 3 & SB5-19 & SO4 (wt $\%)$ & 0.3108 & 0.3108 & 0.3100 & 0.0008 & 0.0008 & $0.3 \%$ & $0.3 \%$ \\
\hline SRNL-SCS-2008-00003/Set 3 & SB5-19 & ThO2 (wt $\%)$ & 0.0569 & 0.0569 & 0.0000 & 0.0569 & 0.0569 & & \\
\hline SRNL-SCS-2008-00003/Set 3 & SB5-19 & $\mathrm{TiO} 2\left(\mathrm{wt}^{\circ} \%\right)$ & 0.0200 & 0.0208 & 0.0120 & 0.0080 & 0.0088 & $66.8 \%$ & $73.4 \%$ \\
\hline SRNL-SCS-2008-00003/Set 3 & SB5-19 & $\mathrm{U} 3 \mathrm{O} 8(\mathrm{wt} \%)$ & 3.3018 & 3.3684 & 3.3420 & -0.0402 & 0.0264 & $-1.2 \%$ & $0.8 \%$ \\
\hline SRNL-SCS-2008-00003/Set 3 & SB5-19 & $\mathrm{ZnO}(\mathrm{wt} \%)$ & 0.0062 & 0.0062 & 0.0070 & -0.0008 & -0.0008 & $-11.1 \%$ & $-11.1 \%$ \\
\hline SRNL-SCS-2008-00003/Set 3 & SB5-19 & $\mathrm{ZrO} 2(\mathrm{wt} \%)$ & 0.1040 & 0.1040 & 0.1160 & -0.0120 & -0.0120 & $-10.3 \%$ & $-10.3 \%$ \\
\hline SRNL-SCS-2008-00003/Set 3 & SB5-19 & Sum & 98.9720 & 98.8951 & 99.6060 & -0.6340 & -0.7109 & $-0.6 \%$ & $-0.7 \%$ \\
\hline SRNL-SCS-2008-00003/Set 3 & SB5-20 & $\mathrm{A} 12 \mathrm{O} 3(\mathrm{wt} \%)$ & 7.4210 & 7.4198 & 7.2170 & 0.2040 & 0.2028 & $2.8 \%$ & $2.8 \%$ \\
\hline SRNL-SCS-2008-00003/Set 3 & SB5-20 & $\mathrm{B} 2 \mathrm{O} 3(\mathrm{wt} \%)$ & 9.6838 & 9.8695 & 9.9200 & -0.2362 & -0.0505 & $-2.4 \%$ & $-0.5 \%$ \\
\hline SRNL-SCS-2008-00003/Set 3 & SB5-20 & $\mathrm{BaO}(\mathrm{wt} \%)$ & 0.0581 & 0.0612 & 0.0630 & -0.0049 & -0.0018 & $-7.8 \%$ & $-2.9 \%$ \\
\hline SRNL-SCS-2008-00003/Set 3 & SB5-20 & $\mathrm{CaO}(\mathrm{wt} \%)$ & 0.8427 & 0.8711 & 0.8560 & -0.0133 & 0.0151 & $-1.6 \%$ & $1.8 \%$ \\
\hline SRNL-SCS-2008-00003/Set 3 & SB5-20 & $\mathrm{CdO}(\mathrm{wt} \%)$ & 0.0057 & 0.0057 & 0.0000 & 0.0057 & 0.0057 & & \\
\hline SRNL-SCS-2008-00003/Set 3 & SB5-20 & $\mathrm{Ce} 2 \mathrm{O} 3(\mathrm{wt} \%)$ & 0.1268 & 0.1268 & 0.1010 & 0.0258 & 0.0258 & $25.5 \%$ & $25.5 \%$ \\
\hline SRNL-SCS-2008-00003/Set 3 & SB5-20 & $\mathrm{Cr} 2 \mathrm{O} 3(\mathrm{wt} \%)$ & 0.0877 & 0.0864 & 0.0930 & -0.0053 & -0.0066 & $-5.7 \%$ & $-7.1 \%$ \\
\hline SRNL-SCS-2008-00003/Set 3 & SB5-20 & $\mathrm{CuO}(\mathrm{wt} \%)$ & 0.0354 & 0.0369 & 0.0330 & 0.0024 & 0.0039 & $7.2 \%$ & $11.7 \%$ \\
\hline SRNL-SCS-2008-00003/Set 3 & SB5-20 & $\mathrm{Fe} 2 \mathrm{O} 3(\mathrm{wt} \%)$ & 11.1374 & 10.5949 & 10.5040 & 0.6334 & 0.0909 & $6.0 \%$ & $0.9 \%$ \\
\hline SRNL-SCS-2008-00003/Set 3 & SB5-20 & $\mathrm{K} 2 \mathrm{O}(\mathrm{wt} \%)$ & 0.0810 & 0.0873 & 0.0770 & 0.0040 & 0.0103 & $5.2 \%$ & $13.4 \%$ \\
\hline SRNL-SCS-2008-00003/Set 3 & SB5-20 & $\mathrm{La} 2 \mathrm{O} 3(\mathrm{wt} \%)$ & 0.0586 & 0.0586 & 0.0180 & 0.0406 & 0.0406 & $225.8 \%$ & $225.8 \%$ \\
\hline SRNL-SCS-2008-00003/Set 3 & SB5-20 & $\mathrm{Li} 2 \mathrm{O}(\mathrm{wt} \%)$ & 2.5027 & 2.5873 & 2.4800 & 0.0227 & 0.1073 & $0.9 \%$ & $4.3 \%$ \\
\hline SRNL-SCS-2008-00003/Set 3 & SB5-20 & $\mathrm{MgO}\left(\mathrm{wt}^{\circ} \%\right)$ & 0.4788 & 0.4878 & 0.4670 & 0.0118 & 0.0208 & $2.5 \%$ & $4.4 \%$ \\
\hline SRNL-SCS-2008-00003/Set 3 & SB5-20 & $\mathrm{MnO}\left(\mathrm{wt}^{\%} \%\right)$ & 2.5792 & 2.4313 & 2.3160 & 0.2632 & 0.1153 & $11.4 \%$ & $5.0 \%$ \\
\hline SRNL-SCS-2008-00003/Set 3 & SB5-20 & $\mathrm{Na} 2 \mathrm{O}(\mathrm{wt} \%)$ & 14.5517 & 14.2906 & 14.6780 & -0.1263 & -0.3874 & $-0.9 \%$ & $-2.6 \%$ \\
\hline SRNL-SCS-2008-00003/Set 3 & SB5-20 & $\mathrm{Nb} 2 \mathrm{O} 5(\mathrm{wt} \%)$ & 0.0286 & 0.0286 & 0.0000 & 0.0286 & 0.0286 & & \\
\hline SRNL-SCS-2008-00003/Set 3 & SB5-20 & $\mathrm{NiO}(\mathrm{wt} \%)$ & 1.0877 & 1.1375 & 1.1980 & -0.1103 & -0.0605 & $-9.2 \%$ & $-5.1 \%$ \\
\hline SRNL-SCS-2008-00003/Set 3 & SB5-20 & $\mathrm{PbO}(\mathrm{wt} \%)$ & 0.0366 & 0.0366 & 0.0360 & 0.0006 & 0.0006 & $1.7 \%$ & $1.7 \%$ \\
\hline SRNL-SCS-2008-00003/Set 3 & SB5-20 & $\mathrm{SiO} 2(\mathrm{wt} \%)$ & 47.7064 & 46.0001 & 45.9580 & 1.7484 & 0.0421 & $3.8 \%$ & $0.1 \%$ \\
\hline SRNL-SCS-2008-00003/Set 3 & SB5-20 & SO4 (wt\%) & 0.5190 & 0.5190 & 0.5470 & -0.0280 & -0.0280 & $-5.1 \%$ & $-5.1 \%$ \\
\hline SRNL-SCS-2008-00003/Set 3 & SB5-20 & ThO2 (wt $\%)$ & 0.0569 & 0.0569 & 0.0000 & 0.0569 & 0.0569 & & \\
\hline SRNL-SCS-2008-00003/Set 3 & SB5-20 & $\mathrm{TiO} 2(\mathrm{wt} \%)$ & 0.3349 & 0.3444 & 0.3500 & -0.0151 & -0.0056 & $-4.3 \%$ & $-1.6 \%$ \\
\hline SRNL-SCS-2008-00003/Set 3 & SB5-20 & U3O8 (wt\%) & 2.9568 & 2.9194 & 2.9250 & 0.0318 & -0.0056 & $1.1 \%$ & $-0.2 \%$ \\
\hline SRNL-SCS-2008-00003/Set 3 & SB5-20 & $\mathrm{ZnO}(\mathrm{wt} \%)$ & 0.0414 & 0.0414 & 0.0420 & -0.0006 & -0.0006 & $-1.5 \%$ & $-1.5 \%$ \\
\hline SRNL-SCS-2008-00003/Set 3 & SB5-20 & $\mathrm{ZrO} 2\left(\mathrm{wt}^{\circ} \%\right)$ & 0.1064 & 0.1064 & 0.1170 & -0.0106 & -0.0106 & $-9.1 \%$ & $-9.1 \%$ \\
\hline SRNL-SCS-2008-00003/Set 3 & SB5-20 & Sum & 102.5253 & 100.2056 & 99.9960 & 2.5293 & 0.2096 & $2.5 \%$ & $0.2 \%$ \\
\hline SRNL-SCS-2008-00003/Set 3 & U std & $\mathrm{A} 12 \mathrm{O} 3(\mathrm{wt} \%)$ & 3.9805 & 4.0317 & 4.1000 & -0.1195 & -0.0683 & $-2.9 \%$ & $-1.7 \%$ \\
\hline SRNL-SCS-2008-00003/Set 3 & U std & $\mathrm{B} 2 \mathrm{O} 3(\mathrm{wt} \%)$ & 9.0077 & 8.9709 & 9.2090 & -0.2013 & -0.2381 & $-2.2 \%$ & $-2.6 \%$ \\
\hline SRNL-SCS-2008-00003/Set 3 & U std & $\mathrm{BaO}(\mathrm{wt} \%)$ & 0.0056 & 0.0059 & 0.0000 & 0.0056 & 0.0059 & & \\
\hline SRNL-SCS-2008-00003/Set 3 & U std & $\mathrm{CaO}(\mathrm{wt} \%)$ & 1.2474 & 1.2935 & 1.3010 & -0.0536 & -0.0075 & $-4.1 \%$ & $-0.6 \%$ \\
\hline SRNL-SCS-2008-00003/Set 3 & U std & $\mathrm{CdO}(\mathrm{wt} \%)$ & 0.0057 & 0.0057 & 0.0000 & 0.0057 & 0.0057 & & \\
\hline SRNL-SCS-2008-00003/Set 3 & U std & $\mathrm{Ce} 2 \mathrm{O} 3(\mathrm{wt} \%)$ & 0.0059 & 0.0059 & 0.0000 & 0.0059 & 0.0059 & & \\
\hline SRNL-SCS-2008-00003/Set 3 & U std & $\mathrm{Cr} 2 \mathrm{O} 3(\mathrm{wt} \%)$ & 0.2324 & 0.2315 & 0.0000 & 0.2324 & 0.2315 & & \\
\hline SRNL-SCS-2008-00003/Set 3 & U std & $\mathrm{CuO}(\mathrm{wt} \%)$ & 0.0063 & 0.0066 & 0.0000 & 0.0063 & 0.0066 & & \\
\hline SRNL-SCS-2008-00003/Set 3 & U std & $\mathrm{Fe} 2 \mathrm{O} 3(\mathrm{wt} \%)$ & 13.5559 & 13.1797 & 13.1960 & 0.3599 & -0.0163 & $2.7 \%$ & $-0.1 \%$ \\
\hline SRNL-SCS-2008-00003/Set 3 & U std & $\mathrm{K} 2 \mathrm{O}(\mathrm{wt} \%)$ & 2.8748 & 3.0897 & 2.9990 & -0.1242 & 0.0907 & $-4.1 \%$ & $3.0 \%$ \\
\hline SRNL-SCS-2008-00003/Set 3 & U std & $\mathrm{La} 2 \mathrm{O} 3(\mathrm{wt} \%)$ & 0.0586 & 0.0586 & 0.0000 & 0.0586 & 0.0586 & & \\
\hline SRNL-SCS-2008-00003/Set 3 & U std & $\mathrm{Li} 2 \mathrm{O}\left(\mathrm{wt}^{\mathrm{N}} \%\right)$ & 2.9889 & 3.0770 & 3.0570 & -0.0681 & 0.0200 & $-2.2 \%$ & $0.7 \%$ \\
\hline SRNL-SCS-2008-00003/Set 3 & U std & $\mathrm{MgO}(\mathrm{wt} \%)$ & 1.1536 & 1.1841 & 1.2100 & -0.0564 & -0.0259 & $-4.7 \%$ & $-2.1 \%$ \\
\hline
\end{tabular}


SRNS-STI-2008-00060

Revision 0

Appendix A

Table A6. Average Measured and Bias-Corrected Chemical Compositions Versus Targeted Compositions by Oxide by Study Glass. (continued)

\begin{tabular}{|c|c|c|c|c|c|c|c|c|c|}
\hline Analytical Plan/Set & $\begin{array}{l}\text { Glass } \\
\text { ID }\end{array}$ & Oxide & $\begin{array}{c}\text { Measured } \\
\text { (wt\%) }\end{array}$ & $\begin{array}{c}\text { Measured BC } \\
\text { (wt\%) }\end{array}$ & $\begin{array}{c}\text { Targeted } \\
\text { (wt \%) }\end{array}$ & $\begin{array}{c}\text { Diff of } \\
\text { Measured }\end{array}$ & $\begin{array}{c}\text { Diff of } \\
\text { Meas BC }\end{array}$ & $\begin{array}{l}\text { \% Diff of } \\
\text { Measured }\end{array}$ & $\begin{array}{l}\text { \% Diff of } \\
\text { Meas BC }\end{array}$ \\
\hline SRNL-SCS-2008-00003/Set 3 & U std & $\mathrm{MnO}(\mathrm{wt} \%)$ & 2.9192 & 2.8081 & 2.8920 & 0.0272 & -0.0839 & $0.9 \%$ & $-2.9 \%$ \\
\hline SRNL-SCS-2008-00003/Set 3 & U std & $\mathrm{Na} 2 \mathrm{O}(\mathrm{wt} \%)$ & 11.7759 & 11.6960 & 11.7950 & -0.0191 & -0.0990 & $-0.2 \%$ & $-0.8 \%$ \\
\hline SRNL-SCS-2008-00003/Set 3 & U std & $\mathrm{Nb} 2 \mathrm{O} 5(\mathrm{wt} \%)$ & 0.0728 & 0.0728 & 0.0000 & 0.0728 & 0.0728 & & \\
\hline SRNL-SCS-2008-00003/Set 3 & U std & $\mathrm{NiO}(\mathrm{wt} \%)$ & 0.9914 & 1.0439 & 1.1200 & -0.1286 & -0.0761 & $-11.5 \%$ & $-6.8 \%$ \\
\hline SRNL-SCS-2008-00003/Set 3 & U std & $\mathrm{PbO}(\mathrm{wt} \%)$ & 0.0108 & 0.0108 & 0.0000 & 0.0108 & 0.0108 & & \\
\hline SRNL-SCS-2008-00003/Set 3 & U std & $\mathrm{SiO} 2(\mathrm{wt} \%)$ & 46.0306 & 45.0456 & 45.3530 & 0.6776 & -0.3074 & $1.5 \%$ & $-0.7 \%$ \\
\hline SRNL-SCS-2008-00003/Set 3 & U std & SO4 (wt $\%)$ & 0.1498 & 0.1498 & 0.0000 & 0.1498 & 0.1498 & & \\
\hline SRNL-SCS-2008-00003/Set 3 & U std & $\mathrm{ThO} 2(\mathrm{wt} \%)$ & 0.0569 & 0.0569 & 0.0000 & 0.0569 & 0.0569 & & \\
\hline SRNL-SCS-2008-00003/Set 3 & U std & $\mathrm{TiO} 2(w t \%)$ & 0.9238 & 0.9553 & 1.0490 & -0.1252 & -0.0937 & $-11.9 \%$ & $-8.9 \%$ \\
\hline SRNL-SCS-2008-00003/Set 3 & U std & $\mathrm{U} 3 \mathrm{O} 8\left(\mathrm{wt}^{\%} \%\right)$ & 2.3977 & 2.4060 & 2.4060 & -0.0083 & 0.0000 & $-0.3 \%$ & $0.0 \%$ \\
\hline SRNL-SCS-2008-00003/Set 3 & U std & $\mathrm{ZnO}(\mathrm{wt} \%)$ & 0.0062 & 0.0062 & 0.0000 & 0.0062 & 0.0062 & & \\
\hline SRNL-SCS-2008-00003/Set 3 & U std & $\mathrm{ZrO} 2(\mathrm{wt} \%)$ & 0.0068 & 0.0068 & 0.0000 & 0.0068 & 0.0068 & & \\
\hline SRNL-SCS-2008-00003/Set 3 & U std & Sum & 100.4652 & 99.3990 & 99.6870 & 0.7782 & -0.2880 & $0.8 \%$ & $-0.3 \%$ \\
\hline SRNL-SCS-2008-00043/Set 1 & Batch 1 & $\mathrm{~A} 12 \mathrm{O} 3(\mathrm{wt} \%)$ & 4.7363 & 4.8130 & 4.8770 & -0.1407 & -0.0640 & $-2.9 \%$ & $-1.3 \%$ \\
\hline SRNL-SCS-2008-00043/Set 1 & Batch 1 & $\mathrm{~B} 2 \mathrm{O} 3(\mathrm{wt} \%)$ & 7.9156 & 7.7770 & 7.7770 & 0.1386 & 0.0000 & $1.8 \%$ & $0.0 \%$ \\
\hline SRNL-SCS-2008-00043/Set 1 & Batch 1 & $\mathrm{BaO}(\mathrm{wt} \%)$ & 0.1349 & 0.1449 & 0.1510 & -0.0161 & -0.0061 & $-10.7 \%$ & $-4.0 \%$ \\
\hline SRNL-SCS-2008-00043/Set 1 & Batch 1 & $\mathrm{CaO}(\mathrm{wt} \%)$ & 1.2604 & 1.2392 & 1.2200 & 0.0404 & 0.0192 & $3.3 \%$ & $1.6 \%$ \\
\hline SRNL-SCS-2008-00043/Set 1 & Batch 1 & $\mathrm{Ce} 2 \mathrm{O} 3(\mathrm{wt} \%)$ & 0.0059 & 0.0059 & 0.0000 & 0.0059 & 0.0059 & & \\
\hline SRNL-SCS-2008-00043/Set 1 & Batch 1 & $\mathrm{Cr} 2 \mathrm{O} 3(\mathrm{wt} \%)$ & 0.1086 & 0.1095 & 0.1070 & 0.0016 & 0.0025 & $1.5 \%$ & $2.4 \%$ \\
\hline SRNL-SCS-2008-00043/Set 1 & Batch 1 & $\mathrm{Fe} 2 \mathrm{O} 3(\mathrm{wt} \%)$ & 12.6528 & 12.9151 & 12.8390 & -0.1862 & 0.0761 & $-1.4 \%$ & $0.6 \%$ \\
\hline SRNL-SCS-2008-00043/Set 1 & Batch 1 & $\mathrm{HfO} 2(\mathrm{wt} \%)$ & 0.0059 & 0.0059 & 0.0000 & 0.0059 & 0.0059 & & \\
\hline SRNL-SCS-2008-00043/Set 1 & Batch 1 & $\mathrm{~K} 2 \mathrm{O}(\mathrm{wt} \%)$ & 3.1460 & 3.2255 & 3.3270 & -0.1810 & -0.1015 & $-5.4 \%$ & $-3.1 \%$ \\
\hline SRNL-SCS-2008-00043/Set 1 & Batch 1 & $\mathrm{La} 2 \mathrm{O} 3(\mathrm{wt} \%)$ & 0.0059 & 0.0059 & 0.0000 & 0.0059 & 0.0059 & & \\
\hline SRNL-SCS-2008-00043/Set 1 & Batch 1 & $\mathrm{Li} 2 \mathrm{O}(\mathrm{wt} \%)$ & 4.4170 & 4.4290 & 4.4290 & -0.0120 & 0.0000 & $-0.3 \%$ & $0.0 \%$ \\
\hline SRNL-SCS-2008-00043/Set 1 & Batch 1 & $\mathrm{MgO}\left(\mathrm{wt}^{\circ} \%\right)$ & 1.3510 & 1.4016 & 1.4190 & -0.0680 & -0.0174 & $-4.8 \%$ & $-1.2 \%$ \\
\hline SRNL-SCS-2008-00043/Set 1 & Batch 1 & $\mathrm{MnO}(\mathrm{wt} \%)$ & 1.7345 & 1.7496 & 1.7260 & 0.0085 & 0.0236 & $0.5 \%$ & $1.4 \%$ \\
\hline SRNL-SCS-2008-00043/Set 1 & Batch 1 & $\mathrm{Na} 2 \mathrm{O}(\mathrm{wt} \%)$ & 9.1619 & 8.9477 & 9.0030 & 0.1589 & -0.0553 & $1.8 \%$ & $-0.6 \%$ \\
\hline SRNL-SCS-2008-00043/Set 1 & Batch 1 & $\mathrm{Nd} 2 \mathrm{O} 3(\mathrm{wt} \%)$ & 0.1211 & 0.1345 & 0.1470 & -0.0259 & -0.0125 & $-17.6 \%$ & $-8.5 \%$ \\
\hline SRNL-SCS-2008-00043/Set 1 & Batch 1 & $\mathrm{NiO}(\mathrm{wt} \%)$ & 0.5758 & 0.6678 & 0.7510 & -0.1752 & -0.0832 & $-23.3 \%$ & $-11.1 \%$ \\
\hline SRNL-SCS-2008-00043/Set 1 & Batch 1 & $\mathrm{PbO}(\mathrm{wt} \%)$ & 0.0108 & 0.0108 & 0.0000 & 0.0108 & 0.0108 & & \\
\hline SRNL-SCS-2008-00043/Set 1 & Batch 1 & $\mathrm{SiO} 2(\mathrm{wt} \%)$ & 50.7371 & 50.8529 & 50.2200 & 0.5171 & 0.6329 & $1.0 \%$ & $1.3 \%$ \\
\hline SRNL-SCS-2008-00043/Set 1 & Batch 1 & SO4 (wt $\%)$ & 0.0749 & 0.0749 & 0.0000 & 0.0749 & 0.0749 & & \\
\hline SRNL-SCS-2008-00043/Set 1 & Batch 1 & $\mathrm{TiO} 2(\mathrm{wt} \%)$ & 0.6174 & 0.6521 & 0.6770 & -0.0596 & -0.0249 & $-8.8 \%$ & $-3.7 \%$ \\
\hline SRNL-SCS-2008-00043/Set 1 & Batch 1 & $\mathrm{ZnO}(\mathrm{wt} \%)$ & 0.0062 & 0.0062 & 0.0000 & 0.0062 & 0.0062 & & \\
\hline SRNL-SCS-2008-00043/Set 1 & Batch 1 & $\mathrm{ZrO} 2(\mathrm{wt} \%)$ & 0.0833 & 0.0833 & 0.0980 & -0.0147 & -0.0147 & $-15.0 \%$ & $-15.0 \%$ \\
\hline SRNL-SCS-2008-00043/Set 1 & Batch 1 & Sum & 98.8634 & 99.2521 & 98.7680 & 0.0954 & 0.4841 & $0.1 \%$ & $0.5 \%$ \\
\hline SRNL-SCS-2008-00043/Set 1 & SB5-21 & $\mathrm{A} 12 \mathrm{O} 3(\mathrm{wt} \%)$ & 11.3370 & 11.5195 & 11.0090 & 0.3280 & 0.5105 & $3.0 \%$ & $4.6 \%$ \\
\hline SRNL-SCS-2008-00043/Set 1 & SB5-21 & $\mathrm{B} 2 \mathrm{O} 3(\mathrm{wt} \%)$ & 8.9835 & 8.8263 & 8.6800 & 0.3035 & 0.1463 & $3.5 \%$ & $1.7 \%$ \\
\hline SRNL-SCS-2008-00043/Set 1 & SB5-21 & $\mathrm{BaO}(\mathrm{wt} \%)$ & 0.0304 & 0.0327 & 0.0390 & -0.0086 & -0.0063 & $-22.0 \%$ & $-16.2 \%$ \\
\hline SRNL-SCS-2008-00043/Set 1 & SB5-21 & $\mathrm{CaO}(\mathrm{wt} \%)$ & 1.9939 & 1.9603 & 1.9170 & 0.0769 & 0.0433 & $4.0 \%$ & $2.3 \%$ \\
\hline SRNL-SCS-2008-00043/Set 1 & SB5-21 & $\mathrm{Ce} 2 \mathrm{O} 3(\mathrm{wt} \%)$ & 0.1693 & 0.1693 & 0.1410 & 0.0283 & 0.0283 & $20.0 \%$ & $20.0 \%$ \\
\hline SRNL-SCS-2008-00043/Set 1 & SB5-21 & $\mathrm{Cr} 2 \mathrm{O} 3(\mathrm{wt} \%)$ & 0.1283 & 0.1293 & 0.1420 & -0.0137 & -0.0127 & $-9.7 \%$ & $-8.9 \%$ \\
\hline SRNL-SCS-2008-00043/Set 1 & SB5-21 & $\mathrm{Fe} 2 \mathrm{O} 3(\mathrm{wt} \%)$ & 8.7426 & 8.9244 & 8.8340 & -0.0914 & 0.0904 & $-1.0 \%$ & $1.0 \%$ \\
\hline SRNL-SCS-2008-00043/Set 1 & SB5-21 & $\mathrm{HfO} 2(\mathrm{wt} \%)$ & 0.0059 & 0.0059 & 0.0000 & 0.0059 & 0.0059 & & \\
\hline SRNL-SCS-2008-00043/Set 1 & SB5-21 & $\mathrm{K} 2 \mathrm{O}(\mathrm{wt} \%)$ & 0.0602 & 0.0617 & 0.0250 & 0.0352 & 0.0367 & $140.9 \%$ & $147.0 \%$ \\
\hline SRNL-SCS-2008-00043/Set 1 & SB5-21 & La2O3 (wt $\%)$ & 0.0625 & 0.0625 & 0.0620 & 0.0005 & 0.0005 & $0.7 \%$ & $0.7 \%$ \\
\hline SRNL-SCS-2008-00043/Set 1 & SB5-21 & $\mathrm{Li} 2 \mathrm{O}(\mathrm{wt} \%)$ & 3.7191 & 3.7292 & 3.7200 & -0.0009 & 0.0092 & $0.0 \%$ & $0.2 \%$ \\
\hline SRNL-SCS-2008-00043/Set 1 & SB5-21 & $\mathrm{MgO}\left(\mathrm{wt}^{\circ} \%\right)$ & 0.4743 & 0.4921 & 0.4470 & 0.0273 & 0.0451 & $6.1 \%$ & $10.1 \%$ \\
\hline SRNL-SCS-2008-00043/Set 1 & SB5-21 & $\mathrm{MnO}\left(\mathrm{wt}^{\circ} \%\right)$ & 1.9013 & 1.9179 & 1.8470 & 0.0543 & 0.0709 & $2.9 \%$ & $3.8 \%$ \\
\hline SRNL-SCS-2008-00043/Set 1 & SB5-21 & $\mathrm{Na} 2 \mathrm{O}(\mathrm{wt} \%)$ & 13.7833 & 13.4710 & 13.7760 & 0.0073 & -0.3050 & $0.1 \%$ & $-2.2 \%$ \\
\hline SRNL-SCS-2008-00043/Set 1 & SB5-21 & $\mathrm{Nd} 2 \mathrm{O} 3(\mathrm{wt} \%)$ & 2.8023 & 3.1133 & 2.8260 & -0.0237 & 0.2873 & $-0.8 \%$ & $10.2 \%$ \\
\hline SRNL-SCS-2008-00043/Set 1 & SB5-21 & $\mathrm{NiO}(\mathrm{wt} \%)$ & 0.9617 & 1.1163 & 1.0370 & -0.0753 & 0.0793 & $-7.3 \%$ & $7.6 \%$ \\
\hline SRNL-SCS-2008-00043/Set 1 & SB5-21 & $\mathrm{PbO}(\mathrm{wt} \%)$ & 0.0108 & 0.0108 & 0.0080 & 0.0028 & 0.0028 & $34.7 \%$ & $34.7 \%$ \\
\hline SRNL-SCS-2008-00043/Set 1 & SB5-21 & $\mathrm{SiO} 2(\mathrm{wt} \%)$ & 45.1392 & 45.2424 & 44.7370 & 0.4022 & 0.5054 & $0.9 \%$ & $1.1 \%$ \\
\hline SRNL-SCS-2008-00043/Set 1 & SB5-21 & SO4 (wt \%) & 0.2839 & 0.2839 & 0.2770 & 0.0069 & 0.0069 & $2.5 \%$ & $2.5 \%$ \\
\hline SRNL-SCS-2008-00043/Set 1 & SB5-21 & $\mathrm{TiO} 2(\mathrm{wt} \%)$ & 0.0083 & 0.0088 & 0.0100 & -0.0017 & -0.0012 & $-16.6 \%$ & $-11.8 \%$ \\
\hline SRNL-SCS-2008-00043/Set 1 & SB5-21 & $\mathrm{ZnO}(\mathrm{wt} \%)$ & 0.0062 & 0.0062 & 0.0060 & 0.0002 & 0.0002 & $3.7 \%$ & $3.7 \%$ \\
\hline SRNL-SCS-2008-00043/Set 1 & SB5-21 & $\mathrm{ZrO} 2(\mathrm{wt} \%)$ & 0.0925 & 0.0925 & 0.0980 & -0.0055 & -0.0055 & $-5.6 \%$ & $-5.6 \%$ \\
\hline SRNL-SCS-2008-00043/Set 1 & SB5-21 & Sum & 100.6964 & 101.1761 & 99.6380 & 1.0584 & 1.5381 & $1.1 \%$ & $1.5 \%$ \\
\hline SRNL-SCS-2008-00043/Set 1 & SB5-22 & $\mathrm{A} 12 \mathrm{O} 3(\mathrm{wt} \%)$ & 9.5703 & 9.7249 & 9.4600 & 0.1103 & 0.2649 & $1.2 \%$ & $2.8 \%$ \\
\hline SRNL-SCS-2008-00043/Set 1 & SB5-22 & $\mathrm{B} 2 \mathrm{O} 3(\mathrm{wt} \%)$ & 10.0139 & 9.8386 & 9.9200 & 0.0939 & -0.0814 & $0.9 \%$ & $-0.8 \%$ \\
\hline SRNL-SCS-2008-00043/Set 1 & SB5-22 & $\mathrm{BaO}(\mathrm{wt} \%)$ & 0.0341 & 0.0367 & 0.0430 & -0.0089 & -0.0063 & $-20.8 \%$ & $-14.8 \%$ \\
\hline SRNL-SCS-2008-00043/Set 1 & SB5-22 & $\mathrm{CaO}(\mathrm{wt} \%)$ & 0.8080 & 0.7943 & 0.7470 & 0.0610 & 0.0473 & $8.2 \%$ & $6.3 \%$ \\
\hline SRNL-SCS-2008-00043/Set 1 & SB5-22 & $\mathrm{Ce} 2 \mathrm{O} 3(\mathrm{wt} \%)$ & 0.1780 & 0.1780 & 0.1550 & 0.0230 & 0.0230 & $14.9 \%$ & $14.9 \%$ \\
\hline SRNL-SCS-2008-00043/Set 1 & SB5-22 & $\mathrm{Cr} 2 \mathrm{O} 3(\mathrm{wt} \%)$ & 0.1275 & 0.1285 & 0.1560 & -0.0285 & -0.0275 & $-18.3 \%$ & $-17.6 \%$ \\
\hline SRNL-SCS-2008-00043/Set 1 & SB5-22 & $\mathrm{Fe} 2 \mathrm{O} 3(\mathrm{wt} \%)$ & 9.4718 & 9.6674 & 9.7450 & -0.2732 & -0.0776 & $-2.8 \%$ & $-0.8 \%$ \\
\hline SRNL-SCS-2008-00043/Set 1 & SB5-22 & $\mathrm{HfO} 2(\mathrm{wt} \%)$ & 0.0059 & 0.0059 & 0.0000 & 0.0059 & 0.0059 & & \\
\hline SRNL-SCS-2008-00043/Set 1 & SB5-22 & $\mathrm{K} 2 \mathrm{O}(\mathrm{wt} \%)$ & 0.0602 & 0.0617 & 0.0270 & 0.0332 & 0.0347 & $123.1 \%$ & $128.7 \%$ \\
\hline
\end{tabular}


SRNS-STI-2008-00060

Revision 0

Appendix A

Table A6. Average Measured and Bias-Corrected Chemical Compositions Versus Targeted Compositions by Oxide by Study Glass. (continued)

\begin{tabular}{|c|c|c|c|c|c|c|c|c|c|}
\hline Analytical Plan/Set & $\begin{array}{c}\text { Glass } \\
\text { ID }\end{array}$ & Oxide & $\begin{array}{c}\text { Measured } \\
\text { (wt\%) }\end{array}$ & $\begin{array}{c}\text { Measured BC } \\
\text { (wt\%) }\end{array}$ & $\begin{array}{c}\text { Targeted } \\
\text { (wt \%) }\end{array}$ & $\begin{array}{c}\text { Diff of } \\
\text { Measured }\end{array}$ & $\begin{array}{c}\text { Diff of } \\
\text { Meas BC }\end{array}$ & $\begin{array}{l}\text { \% Diff of } \\
\text { Measured }\end{array}$ & $\begin{array}{l}\text { \% Diff of } \\
\text { Meas BC }\end{array}$ \\
\hline SRNL-SCS-2008-00043/Set 1 & SB5-22 & La2O3 (wt\%) & 0.0657 & 0.0657 & 0.0680 & -0.0023 & -0.0023 & $-3.4 \%$ & $-3.4 \%$ \\
\hline SRNL-SCS-2008-00043/Set 1 & SB5-22 & $\mathrm{Li} 2 \mathrm{O}(\mathrm{wt} \%)$ & 3.0733 & 3.0816 & 3.1000 & -0.0267 & -0.0184 & $-0.9 \%$ & $-0.6 \%$ \\
\hline SRNL-SCS-2008-00043/Set 1 & SB5-22 & $\mathrm{MgO}\left(\mathrm{wt}^{0} \%\right)$ & 0.4938 & 0.5119 & 0.4940 & -0.0002 & 0.0179 & $0.0 \%$ & $3.6 \%$ \\
\hline SRNL-SCS-2008-00043/Set 1 & SB5-22 & $\mathrm{MnO}\left(\mathrm{wt}^{0} \%\right)$ & 2.1111 & 2.1293 & 2.0380 & 0.0731 & 0.0913 & $3.6 \%$ & $4.5 \%$ \\
\hline SRNL-SCS-2008-00043/Set 1 & SB5-22 & $\mathrm{Na} 2 \mathrm{O}(\mathrm{wt} \%)$ & 14.0866 & 13.7666 & 14.1540 & -0.0674 & -0.3874 & $-0.5 \%$ & $-2.7 \%$ \\
\hline SRNL-SCS-2008-00043/Set 1 & SB5-22 & $\mathrm{Nd} 2 \mathrm{O} 3(\mathrm{wt} \%)$ & 3.0910 & 3.4345 & 3.1170 & -0.0260 & 0.3175 & $-0.8 \%$ & $10.2 \%$ \\
\hline SRNL-SCS-2008-00043/Set 1 & SB5-22 & $\mathrm{NiO}(\mathrm{wt} \%)$ & 0.9197 & 1.0644 & 1.1440 & -0.2243 & -0.0796 & $-19.6 \%$ & $-7.0 \%$ \\
\hline SRNL-SCS-2008-00043/Set 1 & SB5-22 & $\mathrm{PbO}(\mathrm{wt} \%)$ & 0.0108 & 0.0108 & 0.0090 & 0.0018 & 0.0018 & $19.7 \%$ & $19.7 \%$ \\
\hline SRNL-SCS-2008-00043/Set 1 & SB5-22 & $\mathrm{SiO} 2(\mathrm{wt} \%)$ & 46.2624 & 46.3685 & 44.8110 & 1.4514 & 1.5575 & $3.2 \%$ & $3.5 \%$ \\
\hline SRNL-SCS-2008-00043/Set 1 & SB5-22 & SO4 $(w t \%)$ & 0.2936 & 0.2936 & 0.2890 & 0.0046 & 0.0046 & $1.6 \%$ & $1.6 \%$ \\
\hline SRNL-SCS-2008-00043/Set 1 & SB5-22 & $\mathrm{TiO} 2\left(\mathrm{wt}^{0} \%\right)$ & 0.0083 & 0.0088 & 0.0110 & -0.0027 & -0.0022 & $-24.2 \%$ & $-19.9 \%$ \\
\hline SRNL-SCS-2008-00043/Set 1 & SB5-22 & $\mathrm{ZnO}(\mathrm{wt} \%)$ & 0.0062 & 0.0062 & 0.0060 & 0.0002 & 0.0002 & $3.7 \%$ & $3.7 \%$ \\
\hline SRNL-SCS-2008-00043/Set 1 & SB5-22 & $\mathrm{ZrO} 2(\mathrm{wt} \%)$ & 0.0898 & 0.0898 & 0.1080 & -0.0182 & -0.0182 & $-16.8 \%$ & $-16.8 \%$ \\
\hline SRNL-SCS-2008-00043/Set 1 & SB5-22 & Sum & 100.7819 & 101.2679 & 99.6020 & 1.1799 & 1.6659 & $1.2 \%$ & $1.7 \%$ \\
\hline SRNL-SCS-2008-00043/Set 1 & SB5-23 & $\mathrm{A} 12 \mathrm{O} 3(\mathrm{wt} \%)$ & 8.0398 & 8.1695 & 7.9470 & 0.0928 & 0.2225 & $1.2 \%$ & $2.8 \%$ \\
\hline SRNL-SCS-2008-00043/Set 1 & SB5-23 & $\mathrm{B} 2 \mathrm{O} 3(\mathrm{wt} \%)$ & 8.7662 & 8.6127 & 8.6800 & 0.0862 & -0.0673 & $1.0 \%$ & $-0.8 \%$ \\
\hline SRNL-SCS-2008-00043/Set 1 & SB5-23 & $\mathrm{BaO}(\mathrm{wt} \%)$ & 0.0385 & 0.0413 & 0.0460 & -0.0075 & -0.0047 & $-16.3 \%$ & $-10.1 \%$ \\
\hline SRNL-SCS-2008-00043/Set 1 & SB5-23 & $\mathrm{CaO}(\mathrm{wt} \%)$ & 2.0638 & 2.0287 & 2.0410 & 0.0228 & -0.0123 & $1.1 \%$ & $-0.6 \%$ \\
\hline SRNL-SCS-2008-00043/Set 1 & SB5-23 & $\mathrm{Ce} 2 \mathrm{O} 3(\mathrm{wt} \%)$ & 0.1567 & 0.1567 & 0.1670 & -0.0103 & -0.0103 & $-6.2 \%$ & $-6.2 \%$ \\
\hline SRNL-SCS-2008-00043/Set 1 & SB5-23 & $\mathrm{Cr} 2 \mathrm{O} 3(\mathrm{wt} \%)$ & 0.1334 & 0.1344 & 0.1670 & -0.0336 & -0.0326 & $-20.1 \%$ & $-19.5 \%$ \\
\hline SRNL-SCS-2008-00043/Set 1 & SB5-23 & $\mathrm{Fe} 2 \mathrm{O} 3(\mathrm{wt} \%)$ & 10.1080 & 10.3146 & 10.4470 & -0.3390 & -0.1324 & $-3.2 \%$ & $-1.3 \%$ \\
\hline SRNL-SCS-2008-00043/Set 1 & SB5-23 & $\mathrm{HfO} 2(\mathrm{wt} \%)$ & 2.9306 & 2.9306 & 3.3420 & -0.4114 & -0.4114 & $-12.3 \%$ & $-12.3 \%$ \\
\hline SRNL-SCS-2008-00043/Set 1 & SB5-23 & $\mathrm{K} 2 \mathrm{O}(\mathrm{wt} \%)$ & 0.0602 & 0.0617 & 0.0300 & 0.0302 & 0.0317 & $100.8 \%$ & $105.8 \%$ \\
\hline SRNL-SCS-2008-00043/Set 1 & SB5-23 & $\mathrm{La} 2 \mathrm{O} 3(\mathrm{wt} \%)$ & 0.0689 & 0.0689 & 0.0730 & -0.0041 & -0.0041 & $-5.6 \%$ & $-5.6 \%$ \\
\hline SRNL-SCS-2008-00043/Set 1 & SB5-23 & $\mathrm{Li} 2 \mathrm{O}(\mathrm{wt} \%)$ & 3.7191 & 3.7294 & 3.7200 & -0.0009 & 0.0094 & $0.0 \%$ & $0.3 \%$ \\
\hline SRNL-SCS-2008-00043/Set 1 & SB5-23 & $\mathrm{MgO}\left(\mathrm{wt}^{\%} \%\right)$ & 0.5439 & 0.5641 & 0.5290 & 0.0149 & 0.0351 & $2.8 \%$ & $6.6 \%$ \\
\hline SRNL-SCS-2008-00043/Set 1 & SB5-23 & $\mathrm{MnO}(\mathrm{wt} \%)$ & 2.2435 & 2.2627 & 2.1840 & 0.0595 & 0.0787 & $2.7 \%$ & $3.6 \%$ \\
\hline SRNL-SCS-2008-00043/Set 1 & SB5-23 & $\mathrm{Na} 2 \mathrm{O}(\mathrm{wt} \%)$ & 13.5137 & 13.2064 & 13.6500 & -0.1363 & -0.4436 & $-1.0 \%$ & $-3.2 \%$ \\
\hline SRNL-SCS-2008-00043/Set 1 & SB5-23 & $\mathrm{Nd} 2 \mathrm{O} 3(\mathrm{wt} \%)$ & 0.0583 & 0.0648 & 0.0000 & 0.0583 & 0.0648 & & \\
\hline SRNL-SCS-2008-00043/Set 1 & SB5-23 & $\mathrm{NiO}(\mathrm{wt} \%)$ & 1.0349 & 1.1994 & 1.2270 & -0.1921 & -0.0276 & $-15.7 \%$ & $-2.3 \%$ \\
\hline SRNL-SCS-2008-00043/Set 1 & SB5-23 & $\mathrm{PbO}(\mathrm{wt} \%)$ & 0.0108 & 0.0108 & 0.0100 & 0.0008 & 0.0008 & $7.7 \%$ & $7.7 \%$ \\
\hline SRNL-SCS-2008-00043/Set 1 & SB5-23 & $\mathrm{SiO} 2\left(\mathrm{wt}^{0} \%\right)$ & 45.1392 & 45.2416 & 44.8690 & 0.2702 & 0.3726 & $0.6 \%$ & $0.8 \%$ \\
\hline SRNL-SCS-2008-00043/Set 1 & SB5-23 & SO4 (wt \%) & 0.3018 & 0.3018 & 0.3100 & -0.0082 & -0.0082 & $-2.6 \%$ & $-2.6 \%$ \\
\hline SRNL-SCS-2008-00043/Set 1 & SB5-23 & $\mathrm{TiO} 2(\mathrm{wt} \%)$ & 0.0083 & 0.0088 & 0.0120 & -0.0037 & -0.0032 & $-30.5 \%$ & $-26.5 \%$ \\
\hline SRNL-SCS-2008-00043/Set 1 & SB5-23 & $\mathrm{ZnO}(\mathrm{wt} \%)$ & 0.0062 & 0.0062 & 0.0070 & -0.0008 & -0.0008 & $-11.1 \%$ & $-11.1 \%$ \\
\hline SRNL-SCS-2008-00043/Set 1 & SB5-23 & $\mathrm{ZrO} 2(\mathrm{wt} \%)$ & 0.1148 & 0.1148 & 0.1160 & -0.0012 & -0.0012 & $-1.0 \%$ & $-1.0 \%$ \\
\hline SRNL-SCS-2008-00043/Set 1 & SB5-23 & Sum & 99.0607 & 99.2300 & 99.5740 & -0.5133 & -0.3440 & $-0.5 \%$ & $-0.3 \%$ \\
\hline SRNL-SCS-2008-00043/Set 1 & SB5-24 & $\mathrm{A} 12 \mathrm{O} 3(\mathrm{wt} \%)$ & 11.3701 & 11.5530 & 11.0090 & 0.3611 & 0.5440 & $3.3 \%$ & $4.9 \%$ \\
\hline SRNL-SCS-2008-00043/Set 1 & SB5-24 & $\mathrm{B} 2 \mathrm{O} 3(\mathrm{wt} \%)$ & 10.1427 & 9.9651 & 9.9200 & 0.2227 & 0.0451 & $2.2 \%$ & $0.5 \%$ \\
\hline SRNL-SCS-2008-00043/Set 1 & SB5-24 & $\mathrm{BaO}(\mathrm{wt} \%)$ & 0.0338 & 0.0363 & 0.0390 & -0.0052 & -0.0027 & $-13.4 \%$ & $-7.0 \%$ \\
\hline SRNL-SCS-2008-00043/Set 1 & SB5-24 & $\mathrm{CaO}(\mathrm{wt} \%)$ & 0.7010 & 0.6892 & 0.6770 & 0.0240 & 0.0122 & $3.5 \%$ & $1.8 \%$ \\
\hline SRNL-SCS-2008-00043/Set 1 & SB5-24 & $\mathrm{Ce} 2 \mathrm{O} 3(\mathrm{wt} \%)$ & 0.1382 & 0.1382 & 0.1410 & -0.0028 & -0.0028 & $-2.0 \%$ & $-2.0 \%$ \\
\hline SRNL-SCS-2008-00043/Set 1 & SB5-24 & $\mathrm{Cr} 2 \mathrm{O} 3(\mathrm{wt} \%)$ & 0.1209 & 0.1219 & 0.1420 & -0.0211 & -0.0201 & $-14.8 \%$ & $-14.1 \%$ \\
\hline SRNL-SCS-2008-00043/Set 1 & SB5-24 & $\mathrm{Fe} 2 \mathrm{O} 3(\mathrm{wt} \%)$ & 8.3852 & 8.5575 & 8.8340 & -0.4488 & -0.2765 & $-5.1 \%$ & $-3.1 \%$ \\
\hline SRNL-SCS-2008-00043/Set 1 & SB5-24 & $\mathrm{HfO} 2(\mathrm{wt} \%)$ & 2.4176 & 2.4176 & 2.8260 & -0.4084 & -0.4084 & $-14.5 \%$ & $-14.5 \%$ \\
\hline SRNL-SCS-2008-00043/Set 1 & SB5-24 & $\mathrm{K} 2 \mathrm{O}(\mathrm{wt} \%)$ & 0.0602 & 0.0617 & 0.0250 & 0.0352 & 0.0367 & $140.9 \%$ & $147.0 \%$ \\
\hline SRNL-SCS-2008-00043/Set 1 & SB5-24 & $\mathrm{La} 2 \mathrm{O} 3(\mathrm{wt} \%)$ & 0.0598 & 0.0598 & 0.0620 & -0.0022 & -0.0022 & $-3.5 \%$ & $-3.5 \%$ \\
\hline SRNL-SCS-2008-00043/Set 1 & SB5-24 & $\mathrm{Li} 2 \mathrm{O}(\mathrm{wt} \%)$ & 3.1056 & 3.1139 & 3.1000 & 0.0056 & 0.0139 & $0.2 \%$ & $0.4 \%$ \\
\hline SRNL-SCS-2008-00043/Set 1 & SB5-24 & $\mathrm{MgO}(\mathrm{wt} \%)$ & 0.4718 & 0.4896 & 0.4470 & 0.0248 & 0.0426 & $5.5 \%$ & $9.5 \%$ \\
\hline SRNL-SCS-2008-00043/Set 1 & SB5-24 & $\mathrm{MnO}(\mathrm{wt} \%)$ & 1.9400 & 1.9568 & 1.8470 & 0.0930 & 0.1098 & $5.0 \%$ & $5.9 \%$ \\
\hline SRNL-SCS-2008-00043/Set 1 & SB5-24 & $\mathrm{Na} 2 \mathrm{O}(\mathrm{wt} \%)$ & 14.4236 & 14.0959 & 14.3960 & 0.0276 & -0.3001 & $0.2 \%$ & $-2.1 \%$ \\
\hline SRNL-SCS-2008-00043/Set 1 & SB5-24 & $\mathrm{Nd} 2 \mathrm{O} 3(\mathrm{wt} \%)$ & 0.0583 & 0.0648 & 0.0000 & 0.0583 & 0.0648 & & \\
\hline SRNL-SCS-2008-00043/Set 1 & SB5-24 & $\mathrm{NiO}(\mathrm{wt} \%)$ & 0.9092 & 1.0551 & 1.0370 & -0.1278 & 0.0181 & $-12.3 \%$ & $1.7 \%$ \\
\hline SRNL-SCS-2008-00043/Set 1 & SB5-24 & $\mathrm{PbO}(\mathrm{wt} \%)$ & 0.0108 & 0.0108 & 0.0080 & 0.0028 & 0.0028 & $34.7 \%$ & $34.7 \%$ \\
\hline SRNL-SCS-2008-00043/Set 1 & SB5-24 & $\mathrm{SiO} 2(\mathrm{wt} \%)$ & 45.6741 & 45.7777 & 44.7370 & 0.9371 & 1.0407 & $2.1 \%$ & $2.3 \%$ \\
\hline SRNL-SCS-2008-00043/Set 1 & SB5-24 & $\mathrm{SO} 4(\mathrm{wt} \%)$ & 0.2254 & 0.2254 & 0.2770 & -0.0516 & -0.0516 & $-18.6 \%$ & $-18.6 \%$ \\
\hline SRNL-SCS-2008-00043/Set 1 & SB5-24 & $\mathrm{TiO} 2(\mathrm{wt} \%)$ & 0.0083 & 0.0088 & 0.0100 & -0.0017 & -0.0012 & $-16.6 \%$ & $-11.8 \%$ \\
\hline SRNL-SCS-2008-00043/Set 1 & SB5-24 & $\mathrm{ZnO}(\mathrm{wt} \%)$ & 0.0062 & 0.0062 & 0.0060 & 0.0002 & 0.0002 & $3.7 \%$ & $3.7 \%$ \\
\hline SRNL-SCS-2008-00043/Set 1 & SB5-24 & $\mathrm{ZrO} 2(\mathrm{wt} \%)$ & 0.0824 & 0.0824 & 0.0980 & -0.0156 & -0.0156 & $-15.9 \%$ & $-15.9 \%$ \\
\hline SRNL-SCS-2008-00043/Set 1 & SB5-24 & Sum & 100.3452 & 100.4879 & 99.6380 & 0.7072 & 0.8499 & $0.7 \%$ & $0.9 \%$ \\
\hline SRNL-SCS-2008-00043/Set 1 & SB5-25 & $\mathrm{A} 12 \mathrm{O} 3(\mathrm{wt} \%)$ & 10.3734 & 10.5409 & 10.3050 & 0.0684 & 0.2359 & $0.7 \%$ & $2.3 \%$ \\
\hline SRNL-SCS-2008-00043/Set 1 & SB5-25 & $\mathrm{B} 2 \mathrm{O} 3(\mathrm{wt} \%)$ & 8.8789 & 8.7234 & 8.6800 & 0.1989 & 0.0434 & $2.3 \%$ & $0.5 \%$ \\
\hline SRNL-SCS-2008-00043/Set 1 & SB5-25 & $\mathrm{BaO}(\mathrm{wt} \%)$ & 0.0380 & 0.0407 & 0.0470 & -0.0090 & -0.0063 & $-19.2 \%$ & $-13.3 \%$ \\
\hline SRNL-SCS-2008-00043/Set 1 & SB5-25 & $\mathrm{CaO}(\mathrm{wt} \%)$ & 2.0708 & 2.0358 & 2.0540 & 0.0168 & -0.0182 & $0.8 \%$ & $-0.9 \%$ \\
\hline SRNL-SCS-2008-00043/Set 1 & SB5-25 & $\mathrm{Ce} 2 \mathrm{O} 3(\mathrm{wt} \%)$ & 0.1575 & 0.1575 & 0.1690 & -0.0115 & -0.0115 & $-6.8 \%$ & $-6.8 \%$ \\
\hline SRNL-SCS-2008-00043/Set 1 & SB5-25 & $\mathrm{Cr} 2 \mathrm{O} 3(\mathrm{wt} \%)$ & 0.1410 & 0.1422 & 0.1700 & -0.0290 & -0.0278 & $-17.0 \%$ & $-16.4 \%$ \\
\hline SRNL-SCS-2008-00043/Set 1 & SB5-25 & $\mathrm{Fe} 2 \mathrm{O} 3(\mathrm{wt} \%)$ & 10.2402 & 10.4515 & 10.6150 & -0.3748 & -0.1635 & $-3.5 \%$ & $-1.5 \%$ \\
\hline SRNL-SCS-2008-00043/Set 1 & SB5-25 & $\mathrm{HfO} 2(\mathrm{wt} \%)$ & 0.0059 & 0.0059 & 0.0000 & 0.0059 & 0.0059 & & \\
\hline
\end{tabular}


SRNS-STI-2008-00060

Revision 0

Appendix A

Table A6. Average Measured and Bias-Corrected Chemical Compositions Versus Targeted Compositions by Oxide by Study Glass. (continued)

\begin{tabular}{|c|c|c|c|c|c|c|c|c|c|}
\hline Analytical Plan/Set & $\begin{array}{l}\text { Glass } \\
\text { ID } \\
\end{array}$ & Oxide & $\begin{array}{c}\text { Measured } \\
\text { (wt\%) }\end{array}$ & $\begin{array}{c}\text { Measured BC } \\
\text { (wt\%) }\end{array}$ & $\begin{array}{c}\text { Targeted } \\
\text { (wt \%) }\end{array}$ & $\begin{array}{c}\text { Diff of } \\
\text { Measured }\end{array}$ & $\begin{array}{c}\text { Diff of } \\
\text { Meas BC }\end{array}$ & $\begin{array}{r}\text { \% Diff of } \\
\text { Measured }\end{array}$ & $\begin{array}{l}\text { \% Diff of } \\
\text { Meas BC }\end{array}$ \\
\hline SRNL-SCS-2008-00043/Set 1 & SB5-25 & $\mathrm{K} 2 \mathrm{O}(\mathrm{wt} \%)$ & 0.0602 & 0.0617 & 0.0300 & 0.0302 & 0.0317 & $100.8 \%$ & $105.8 \%$ \\
\hline SRNL-SCS-2008-00043/Set 1 & SB5-25 & $\mathrm{La} 2 \mathrm{O} 3(\mathrm{wt} \%)$ & 0.0701 & 0.0701 & 0.0740 & -0.0039 & -0.0039 & $-5.3 \%$ & $-5.3 \%$ \\
\hline SRNL-SCS-2008-00043/Set 1 & SB5-25 & $\mathrm{Li} 2 \mathrm{O}(\mathrm{wt} \%)$ & 3.6276 & 3.6375 & 3.7200 & -0.0924 & -0.0825 & $-2.5 \%$ & $-2.2 \%$ \\
\hline SRNL-SCS-2008-00043/Set 1 & SB5-25 & $\mathrm{MgO}(\mathrm{wt} \%)$ & 0.5543 & 0.5749 & 0.5380 & 0.0163 & 0.0369 & $3.0 \%$ & $6.9 \%$ \\
\hline SRNL-SCS-2008-00043/Set 1 & SB5-25 & $\mathrm{MnO}(\mathrm{wt} \%)$ & 2.2854 & 2.3052 & 2.2200 & 0.0654 & 0.0852 & $2.9 \%$ & $3.8 \%$ \\
\hline SRNL-SCS-2008-00043/Set 1 & SB5-25 & $\mathrm{Na} 2 \mathrm{O}(\mathrm{wt} \%)$ & 13.9855 & 13.6686 & 14.3550 & -0.3695 & -0.6864 & $-2.6 \%$ & $-4.8 \%$ \\
\hline SRNL-SCS-2008-00043/Set 1 & SB5-25 & $\mathrm{Nd} 2 \mathrm{O} 3(\mathrm{wt} \%)$ & 0.0583 & 0.0648 & 0.0000 & 0.0583 & 0.0648 & & \\
\hline SRNL-SCS-2008-00043/Set 1 & SB5-25 & $\mathrm{NiO}\left(\mathrm{wt}^{\circ} \%\right)$ & 1.0594 & 1.2287 & 1.2470 & -0.1876 & -0.0183 & $-15.0 \%$ & $-1.5 \%$ \\
\hline SRNL-SCS-2008-00043/Set 1 & SB5-25 & $\mathrm{PbO}(\mathrm{wt} \%)$ & 0.0108 & 0.0108 & 0.0100 & 0.0008 & 0.0008 & $7.7 \%$ & $7.7 \%$ \\
\hline SRNL-SCS-2008-00043/Set 1 & SB5-25 & $\mathrm{SiO} 2(\mathrm{wt} \%)$ & 45.3532 & 45.4565 & 44.8820 & 0.4712 & 0.5745 & $1.0 \%$ & $1.3 \%$ \\
\hline SRNL-SCS-2008-00043/Set 1 & SB5-25 & SO4 $(\mathrm{wt} \%)$ & 0.3198 & 0.3198 & 0.3150 & 0.0048 & 0.0048 & $1.5 \%$ & $1.5 \%$ \\
\hline SRNL-SCS-2008-00043/Set 1 & SB5-25 & $\mathrm{TiO} 2\left(\mathrm{wt}^{\mathrm{O}} \%\right)$ & 0.0083 & 0.0088 & 0.0120 & -0.0037 & -0.0032 & $-30.5 \%$ & $-26.5 \%$ \\
\hline SRNL-SCS-2008-00043/Set 1 & SB5-25 & $\mathrm{ZnO}(\mathrm{wt} \%)$ & 0.0062 & 0.0062 & 0.0070 & -0.0008 & -0.0008 & $-11.1 \%$ & $-11.1 \%$ \\
\hline SRNL-SCS-2008-00043/Set 1 & SB5-25 & $\mathrm{ZrO} 2(\mathrm{wt} \%)$ & 0.1077 & 0.1077 & 0.1180 & -0.0103 & -0.0103 & $-8.7 \%$ & $-8.7 \%$ \\
\hline SRNL-SCS-2008-00043/Set 1 & SB5-25 & Sum & 99.4126 & 99.6194 & 99.5680 & -0.1554 & 0.0514 & $-0.2 \%$ & $0.1 \%$ \\
\hline SRNL-SCS-2008-00043/Set 1 & SB5-26 & $\mathrm{A} 12 \mathrm{O} 3(\mathrm{wt} \%)$ & 8.8381 & 8.9814 & 8.7140 & 0.1241 & 0.2674 & $1.4 \%$ & $3.1 \%$ \\
\hline SRNL-SCS-2008-00043/Set 1 & SB5-26 & $\mathrm{B} 2 \mathrm{O} 3(\mathrm{wt} \%)$ & 10.2232 & 10.0442 & 9.9200 & 0.3032 & 0.1242 & $3.1 \%$ & $1.3 \%$ \\
\hline SRNL-SCS-2008-00043/Set 1 & SB5-26 & $\mathrm{BaO}(\mathrm{wt} \%)$ & 0.0410 & 0.0441 & 0.0500 & -0.0090 & -0.0059 & $-17.9 \%$ & $-11.9 \%$ \\
\hline SRNL-SCS-2008-00043/Set 1 & SB5-26 & $\mathrm{CaO}(\mathrm{wt} \%)$ & 0.8783 & 0.8636 & 0.8780 & 0.0003 & -0.0144 & $0.0 \%$ & $-1.6 \%$ \\
\hline SRNL-SCS-2008-00043/Set 1 & SB5-26 & $\mathrm{Ce} 2 \mathrm{O} 3(\mathrm{wt} \%)$ & 0.1783 & 0.1783 & 0.1830 & -0.0047 & -0.0047 & $-2.6 \%$ & $-2.6 \%$ \\
\hline SRNL-SCS-2008-00043/Set 1 & SB5-26 & $\mathrm{Cr} 2 \mathrm{O} 3(\mathrm{wt} \%)$ & 0.1458 & 0.1470 & 0.1830 & -0.0372 & -0.0360 & $-20.3 \%$ & $-19.7 \%$ \\
\hline SRNL-SCS-2008-00043/Set 1 & SB5-26 & $\mathrm{Fe} 2 \mathrm{O} 3(\mathrm{wt} \%)$ & 10.7406 & 10.9614 & 11.4540 & -0.7134 & -0.4926 & $-6.2 \%$ & $-4.3 \%$ \\
\hline SRNL-SCS-2008-00043/Set 1 & SB5-26 & $\mathrm{HfO} 2(\mathrm{wt} \%)$ & 0.0059 & 0.0059 & 0.0000 & 0.0059 & 0.0059 & & \\
\hline SRNL-SCS-2008-00043/Set 1 & SB5-26 & $\mathrm{K} 2 \mathrm{O}(\mathrm{wt} \%)$ & 0.0602 & 0.0617 & 0.0330 & 0.0272 & 0.0287 & $82.5 \%$ & $87.1 \%$ \\
\hline SRNL-SCS-2008-00043/Set 1 & SB5-26 & $\mathrm{La} 2 \mathrm{O} 3(\mathrm{wt} \%)$ & 0.0783 & 0.0783 & 0.0800 & -0.0017 & -0.0017 & $-2.1 \%$ & $-2.1 \%$ \\
\hline SRNL-SCS-2008-00043/Set 1 & SB5-26 & $\mathrm{Li} 2 \mathrm{O}(\mathrm{wt} \%)$ & 3.1325 & 3.1409 & 3.1000 & 0.0325 & 0.0409 & $1.0 \%$ & $1.3 \%$ \\
\hline SRNL-SCS-2008-00043/Set 1 & SB5-26 & $\mathrm{MgO}(\mathrm{wt} \%)$ & 0.6190 & 0.6420 & 0.5800 & 0.0390 & 0.0620 & $6.7 \%$ & $10.7 \%$ \\
\hline SRNL-SCS-2008-00043/Set 1 & SB5-26 & $\mathrm{MnO}(\mathrm{wt} \%)$ & 2.4630 & 2.4842 & 2.3950 & 0.0680 & 0.0892 & $2.8 \%$ & $3.7 \%$ \\
\hline SRNL-SCS-2008-00043/Set 1 & SB5-26 & $\mathrm{Na} 2 \mathrm{O}(\mathrm{wt} \%)$ & 14.9291 & 14.5877 & 15.1680 & -0.2389 & -0.5803 & $-1.6 \%$ & $-3.8 \%$ \\
\hline SRNL-SCS-2008-00043/Set 1 & SB5-26 & $\mathrm{Nd} 2 \mathrm{O} 3(\mathrm{wt} \%)$ & 0.0583 & 0.0648 & 0.0000 & 0.0583 & 0.0648 & & \\
\hline SRNL-SCS-2008-00043/Set 1 & SB5-26 & $\mathrm{NiO}(\mathrm{wt} \%)$ & 1.1946 & 1.3855 & 1.3450 & -0.1504 & 0.0405 & $-11.2 \%$ & $3.0 \%$ \\
\hline SRNL-SCS-2008-00043/Set 1 & SB5-26 & $\mathrm{PbO}(\mathrm{wt} \%)$ & 0.0108 & 0.0108 & 0.0110 & -0.0002 & -0.0002 & $-2.1 \%$ & $-2.1 \%$ \\
\hline SRNL-SCS-2008-00043/Set 1 & SB5-26 & $\mathrm{SiO} 2(\mathrm{wt} \%)$ & 45.4601 & 45.5635 & 44.9510 & 0.5091 & 0.6125 & $1.1 \%$ & $1.4 \%$ \\
\hline SRNL-SCS-2008-00043/Set 1 & SB5-26 & $\mathrm{SO} 4(\mathrm{wt} \%)$ & 0.3273 & 0.3273 & 0.3400 & -0.0127 & -0.0127 & $-3.7 \%$ & $-3.7 \%$ \\
\hline SRNL-SCS-2008-00043/Set 1 & SB5-26 & $\mathrm{TiO} 2(\mathrm{wt} \%)$ & 0.0083 & 0.0088 & 0.0130 & -0.0047 & -0.0042 & $-35.8 \%$ & $-32.2 \%$ \\
\hline SRNL-SCS-2008-00043/Set 1 & SB5-26 & $\mathrm{ZnO}(\mathrm{wt} \%)$ & 0.0062 & 0.0062 & 0.0080 & -0.0018 & -0.0018 & $-22.2 \%$ & $-22.2 \%$ \\
\hline SRNL-SCS-2008-00043/Set 1 & SB5-26 & $\mathrm{ZrO} 2(\mathrm{wt} \%)$ & 0.1179 & 0.1179 & 0.1270 & -0.0091 & -0.0091 & $-7.2 \%$ & $-7.2 \%$ \\
\hline SRNL-SCS-2008-00043/Set 1 & SB5-26 & Sum & 99.5168 & 99.7055 & 99.5330 & -0.0162 & 0.1725 & $0.0 \%$ & $0.2 \%$ \\
\hline
\end{tabular}




\section{Exhibit A1. Oxide Measurements in Analytical Sequence for Samples by Prep Method and Analytical Plan Memo.}

Measured By Analytical Sequence Prep=LM,

Analytical Plan=SRNL-SCS-2008-00003, Oxide=Al2O3 (wt \%)

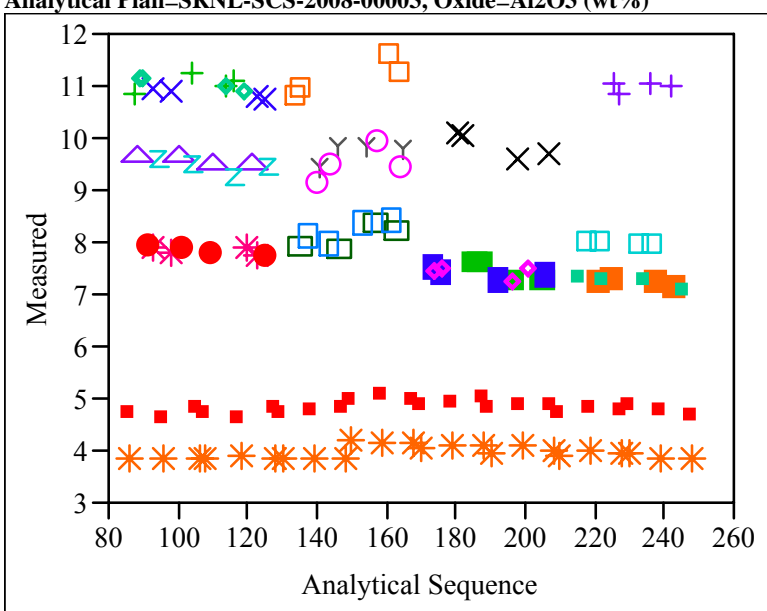

Measured By Analytical Sequence Prep=LM,

Analytical Plan $=$ SRNL-SCS-2008-00003, Oxide $=\mathrm{BaO}(\mathrm{wt} \%)$

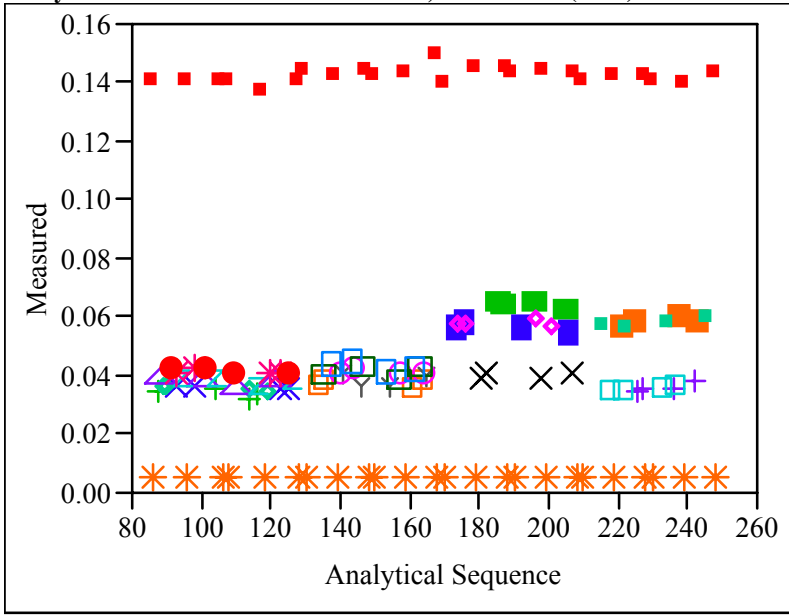

Measured By Analytical Sequence Prep=LM,

Analytical Plan =SRNL-SCS-2008-00003, Oxide $=\mathrm{CaO}$ (wt \%)

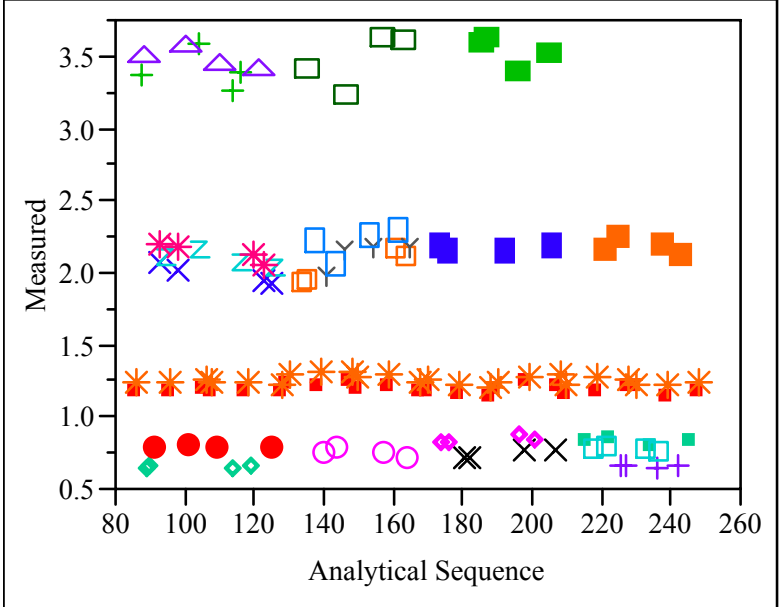

Measured By Analytical Sequence Prep=LM,

Analytical Plan $=$ SRNL-SCS-2008-00003, Oxide $=$ CdO (wt $\%)$

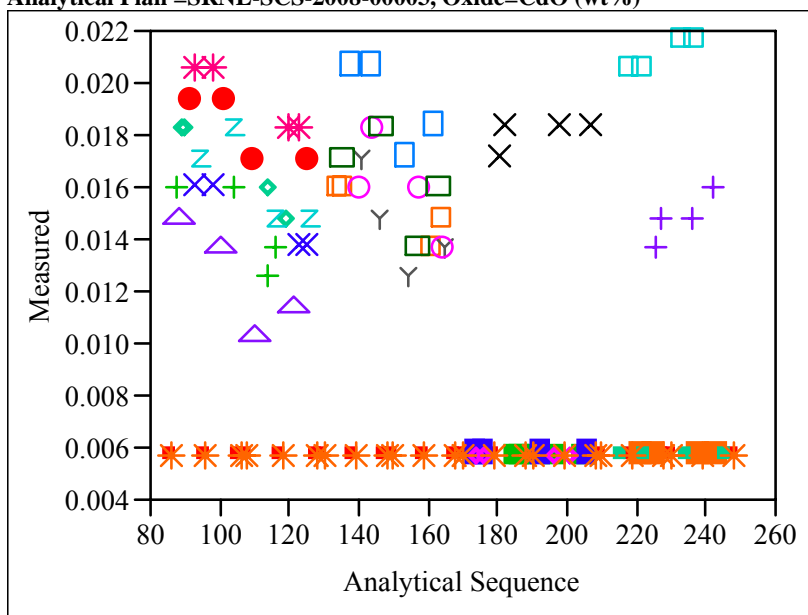

Measured By Analytical Sequence Prep=LM,

Analytical Plan =SRNL-SCS-2008-00003, Oxide $=$ Ce2O3 (wt \%)

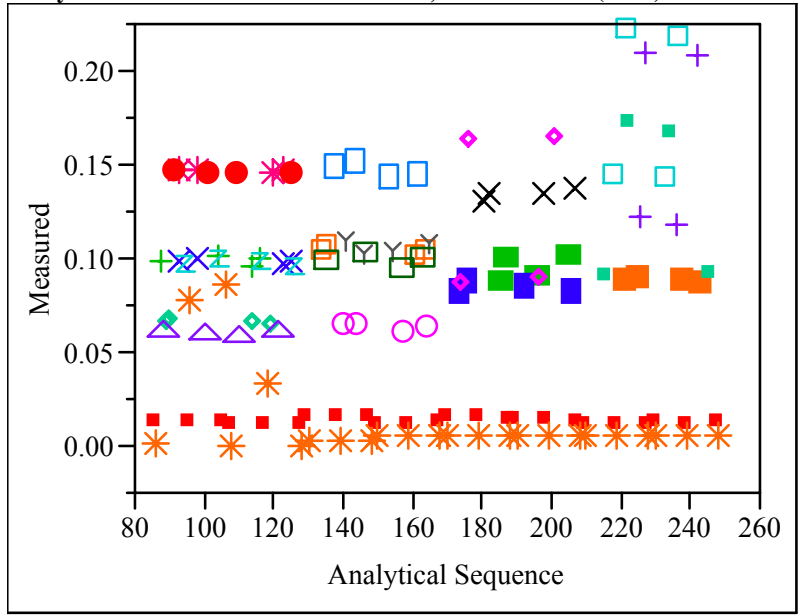

Measured By Analytical Sequence Prep=LM,

Analytical Plan =SRNL-SCS-2008-00003, Oxide $=$ Cr2O3 (wt \%)

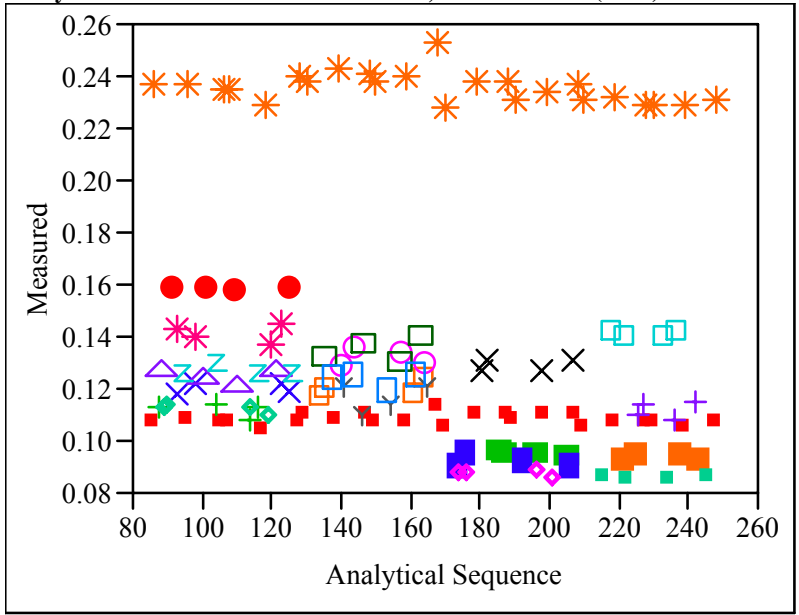


Exhibit A1. Oxide Measurements in Analytical Sequence for Samples by Prep Method and Analytical Plan Memo. (continued)

Measured By Analytical Sequence Prep $=$ LM, Analytical Plan $=$ SRNL-SCS-2008-00003, Oxide $=\mathrm{CuO}(\mathrm{wt} \%)$

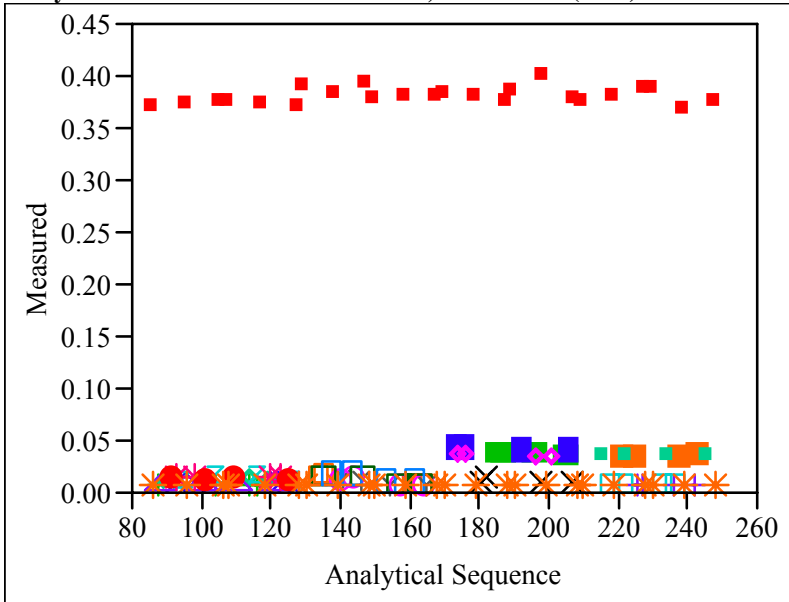

Measured By Analytical Sequence Prep=LM, Analytical Plan =SRNL-SCS-2008-00003, Oxide=Fe2O3 (wt \%)

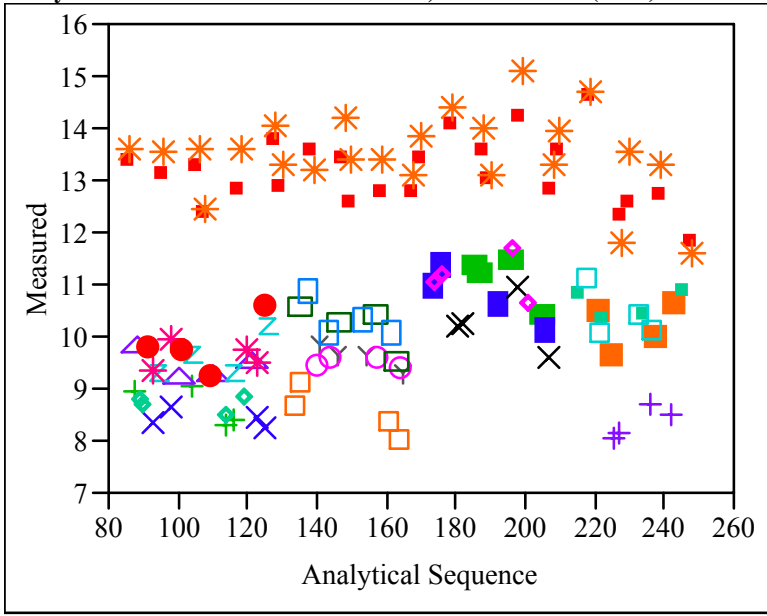

Measured By Analytical Sequence Prep=LM,

Analytical Plan =SRNL-SCS-2008-00003, Oxide=K2O (wt \%)

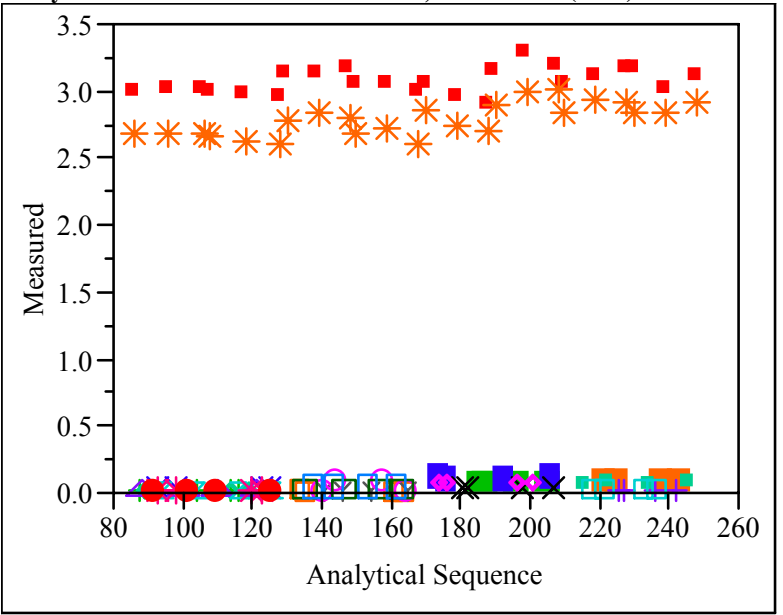

Measured By Analytical Sequence Prep=LM,

Analytical Plan =SRNL-SCS-2008-00003, Oxide=La2O3 (wt \%)

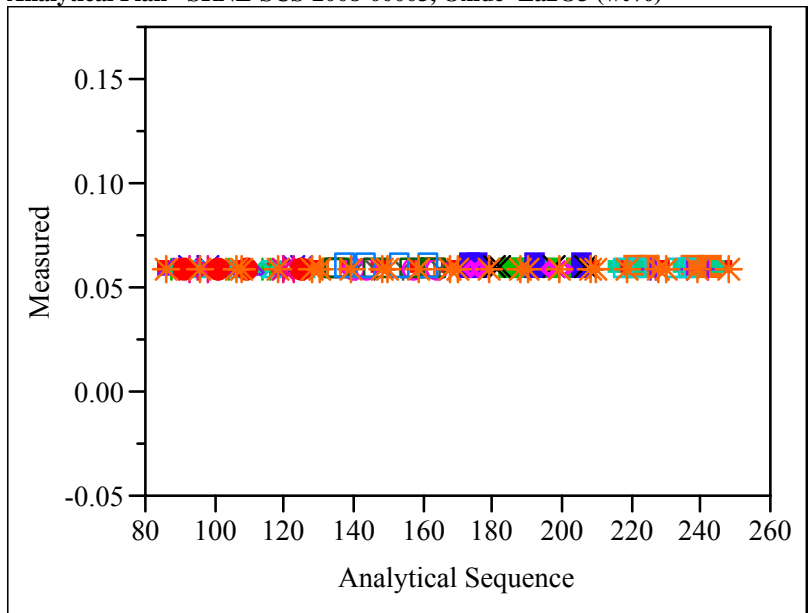

Measured By Analytical Sequence Prep=LM,

Analytical Plan =SRNL-SCS-2008-00003, Oxide=MgO (wt \%)

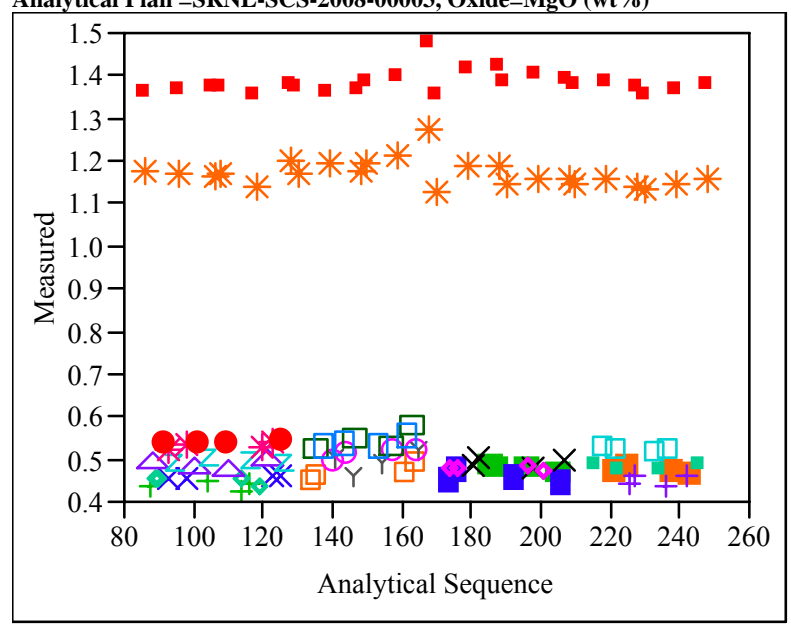

Measured By Analytical Sequence Prep=LM,

Analytical Plan =SRNL-SCS-2008-00003, Oxide=MnO (wt \%)

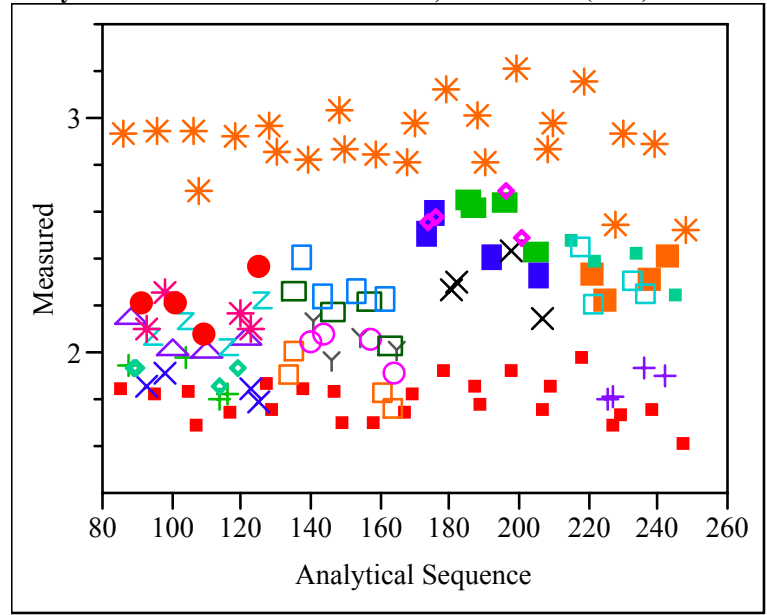


Exhibit A1. Oxide Measurements in Analytical Sequence for

Samples by Prep Method and Analytical Plan Memo. (continued)

Measured By Analytical Sequence Prep=LM,

Analytical Plan =SRNL-SCS-2008-00003, Oxide=Na2O (wt \%)

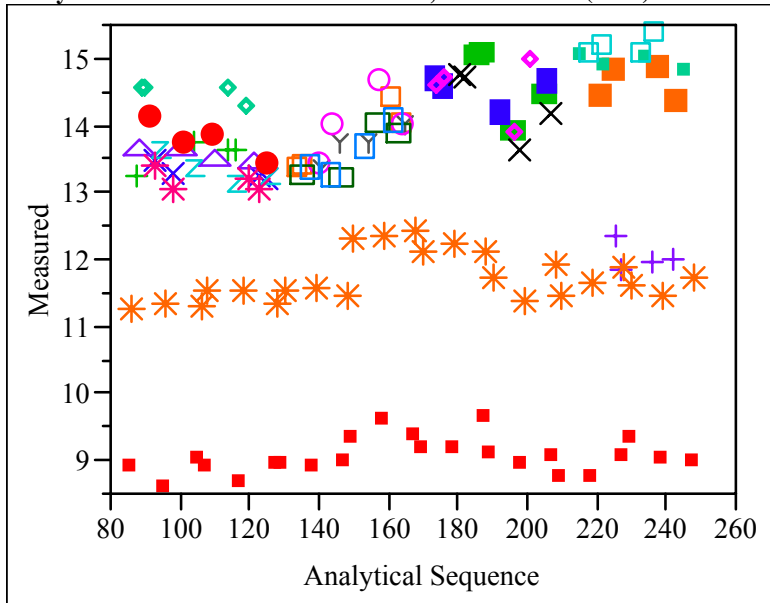

Measured By Analytical Sequence Prep=LM,

Analytical Plan =SRNL-SCS-2008-00003, Oxide=Nb2O5 (wt \%)

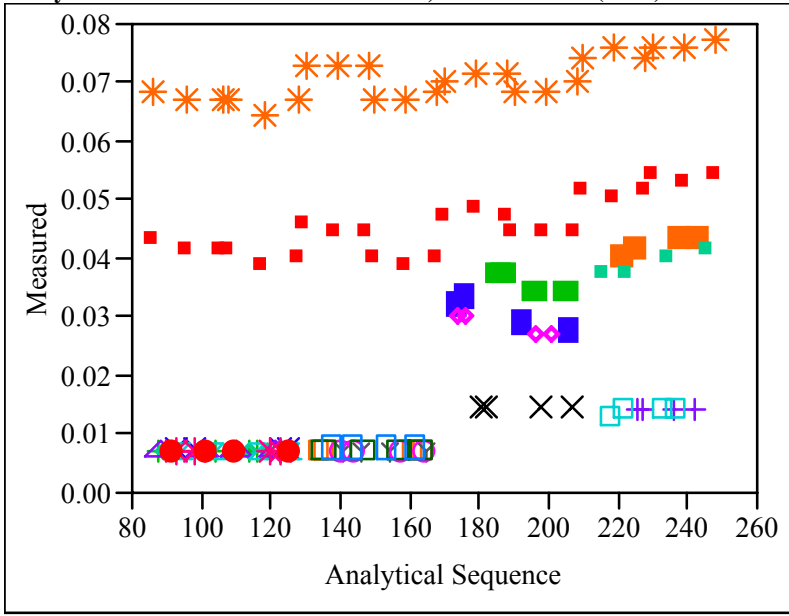

Measured By Analytical Sequence Prep=LM,

Analytical Plan =SRNL-SCS-2008-00003, Oxide=NiO (wt \%)

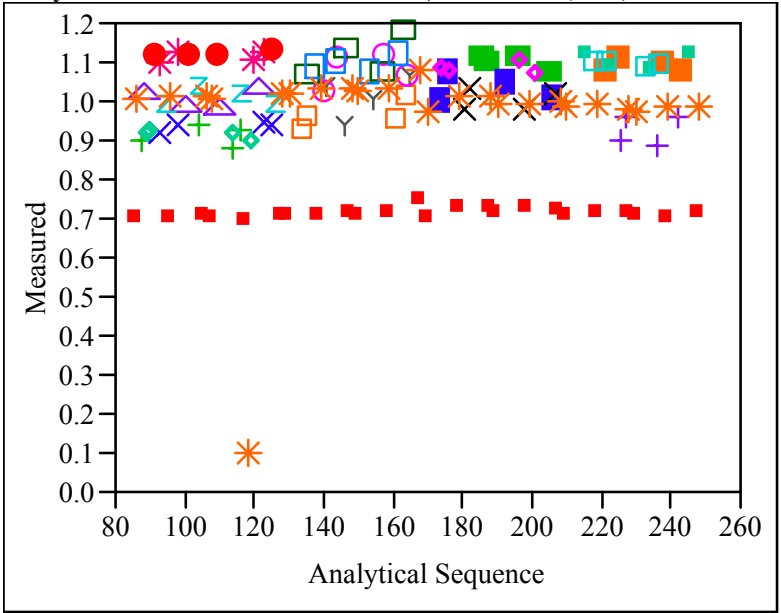

Measured By Analytical Sequence Prep=LM,

Analytical Plan $=$ SRNL-SCS-2008-00003, Oxide $=$ PbO (wt $\%)$

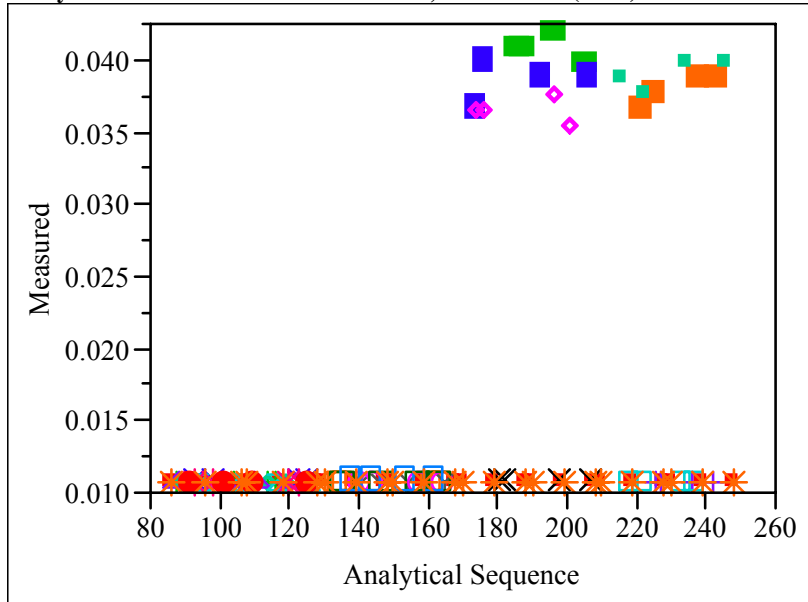

Measured By Analytical Sequence Prep=LM,

Analytical Plan =SRNL-SCS-2008-00003, Oxide=SiO2 (wt \%)

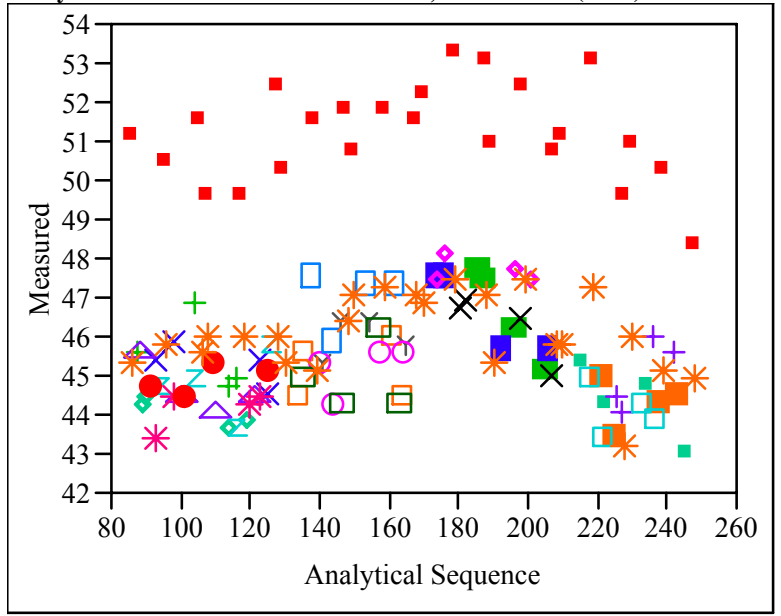

Measured By Analytical Sequence Prep=LM,

Analytical Plan =SRNL-SCS-2008-00003, Oxide=SO4 (wt \%)

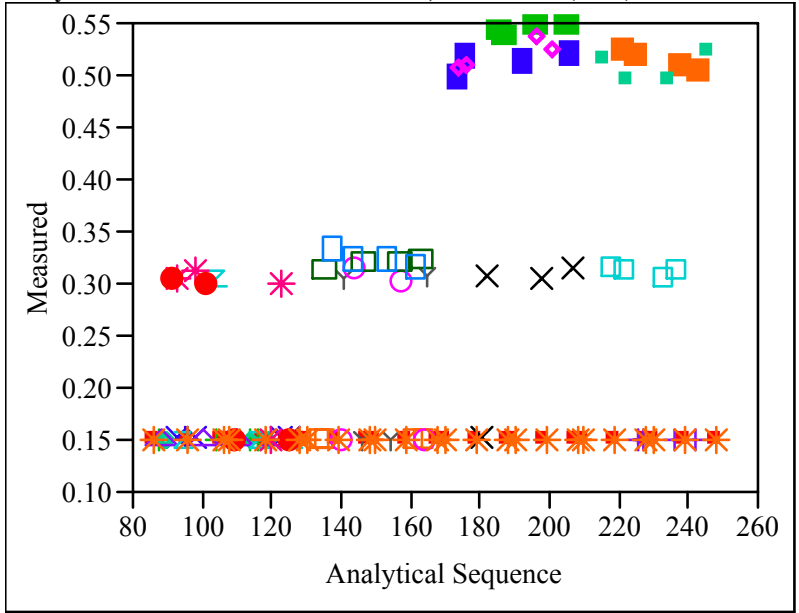


Exhibit A1. Oxide Measurements in Analytical Sequence for Samples by Prep Method and Analytical Plan Memo. (continued)

Measured By Analytical Sequence Prep $=$ LM, Analytical Plan =SRNL-SCS-2008-00003, Oxide=ThO2 (wt \%)

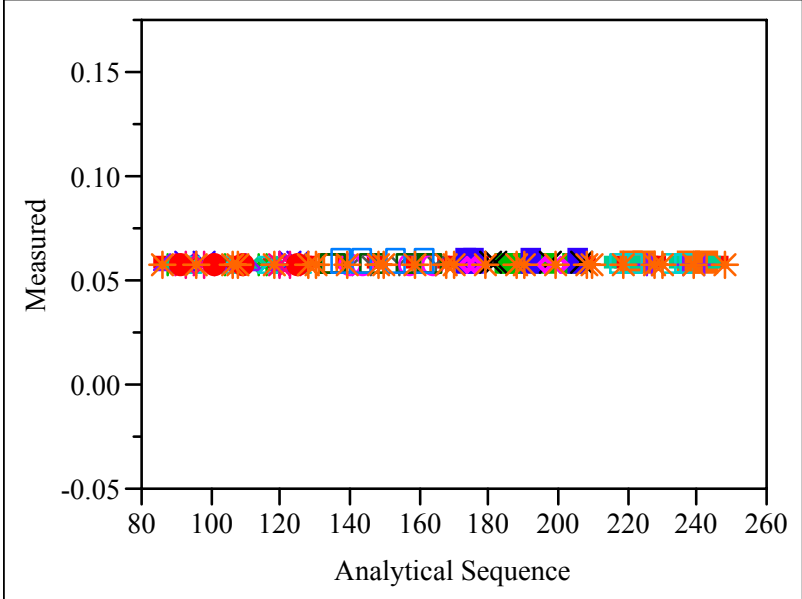

Measured By Analytical Sequence Prep=LM, Analytical Plan $=$ SRNL-SCS-2008-00003, Oxide=TiO2 (wt\%)

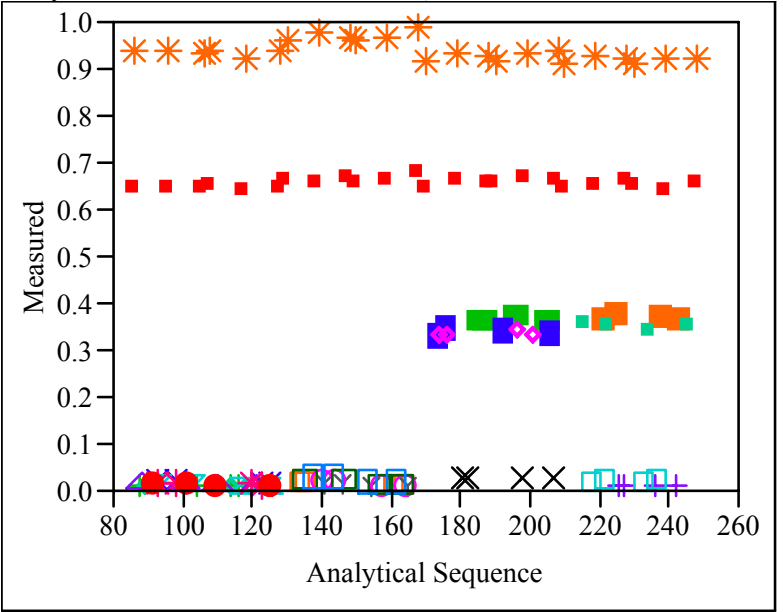

Measured By Analytical Sequence Prep=LM,

Analytical Plan $=$ SRNL-SCS-2008-00003, Oxide $=\mathrm{U} 308$ (wt \%)

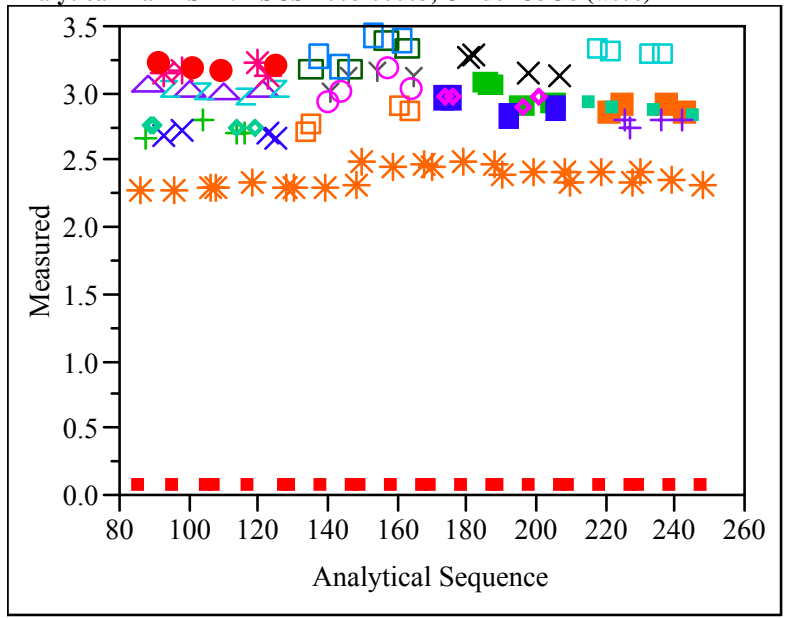

Measured By Analytical Sequence Prep=LM, Analytical Plan $=$ SRNL-SCS-2008-00003, Oxide $=\mathrm{ZnO}$ (wt \%)

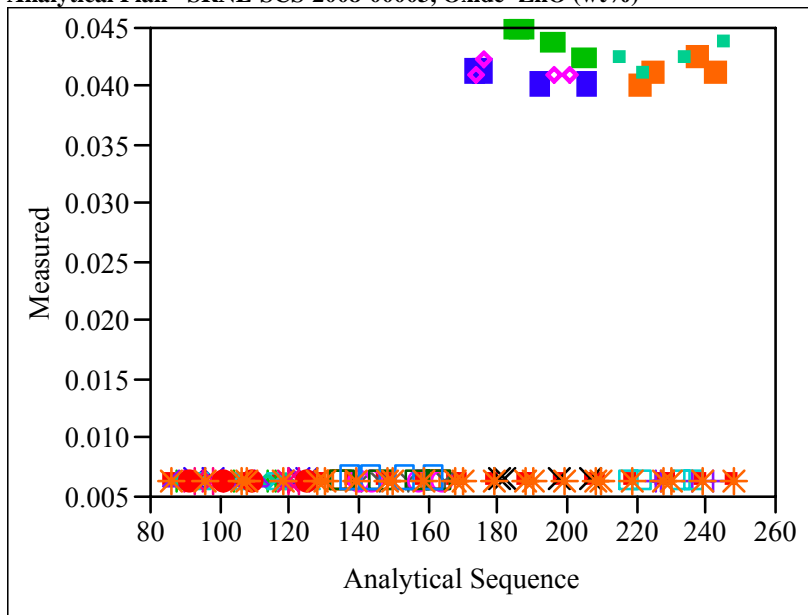

Measured By Analytical Sequence Prep=LM,

Analytical Plan =SRNL-SCS-2008-00003, Oxide=ZrO2 (wt \%)

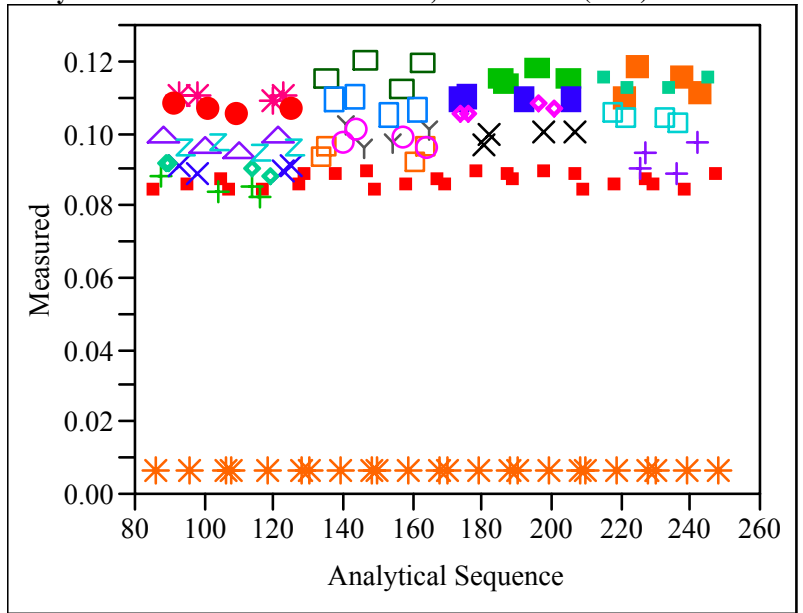

Measured By Analytical Sequence Prep=LM,

Analytical Plan =SRNL-SCS-2008-00043, Oxide=Al2O3 (wt \%)

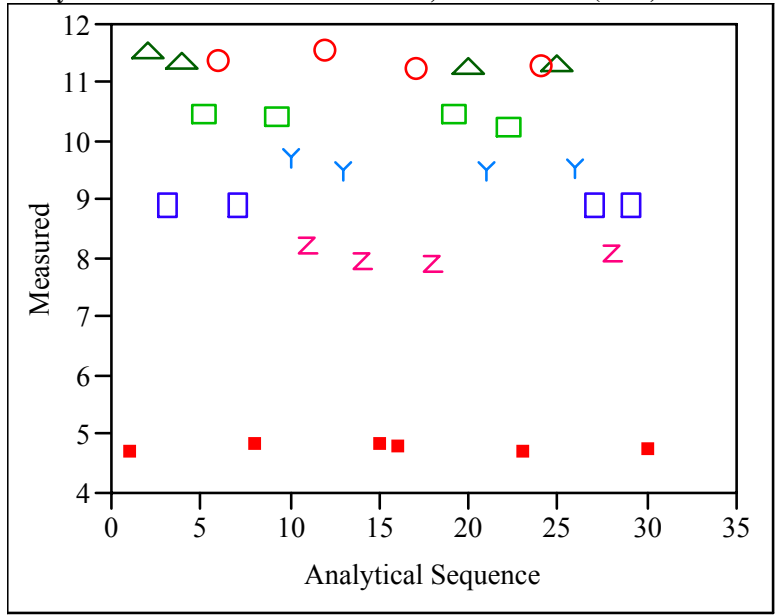


Exhibit A1. Oxide Measurements in Analytical Sequence for Samples by Prep Method and Analytical Plan Memo. (continued)

Measured By Analytical Sequence Prep $=$ LM, Analytical Plan $=$ SRNL-SCS-2008-00043, Oxide $=$ BaO $(w t \%)$

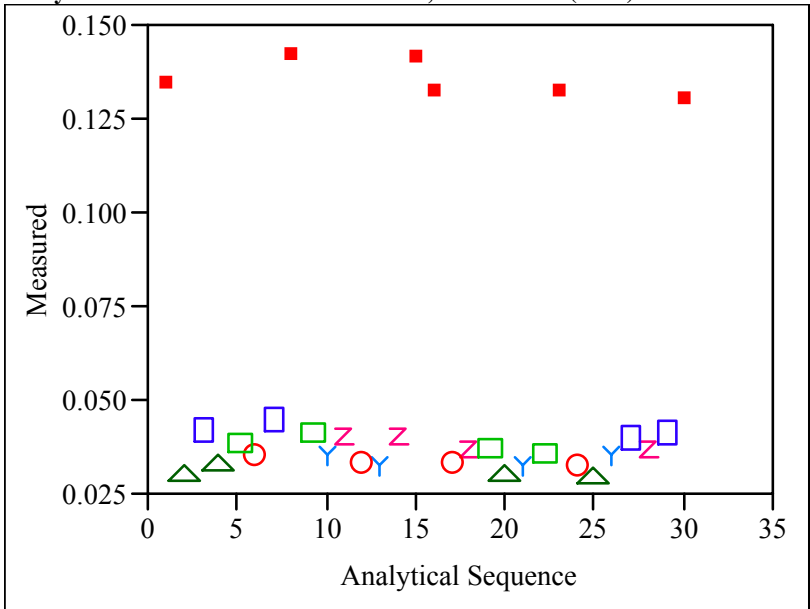

Measured By Analytical Sequence Prep=LM, Analytical Plan =SRNL-SCS-2008-00043, Oxide $=\mathrm{CaO}(\mathrm{wt} \%)$

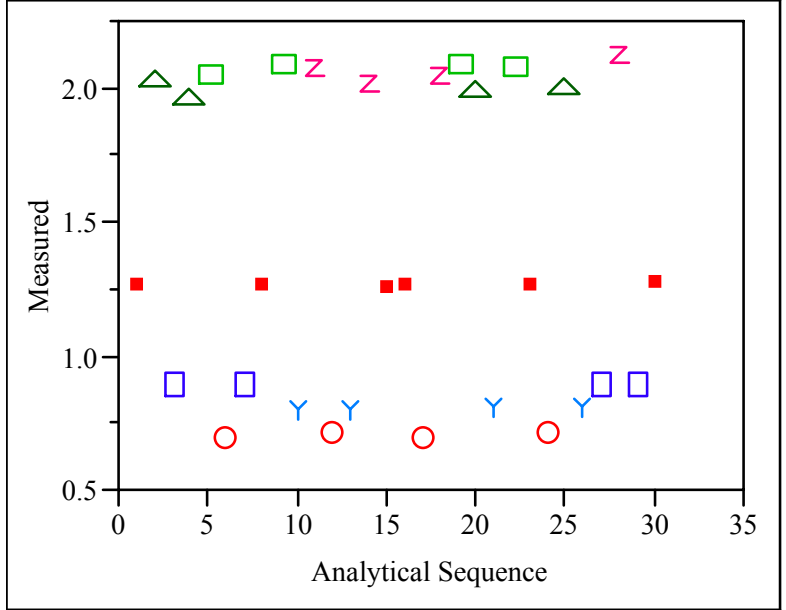

Measured By Analytical Sequence Prep=LM,

Analytical Plan $=$ SRNL-SCS-2008-00043, Oxide $=\mathrm{Ce} 2 \mathrm{O} 3$ (wt \%)

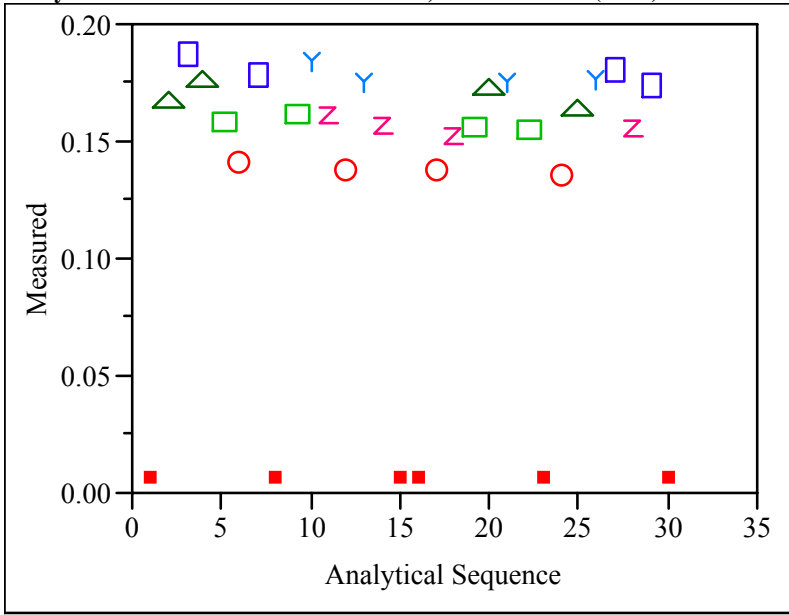

Measured By Analytical Sequence Prep=LM, Analytical Plan $=$ SRNL-SCS-2008-00043, Oxide $=$ Cr2O3 (wt \%)

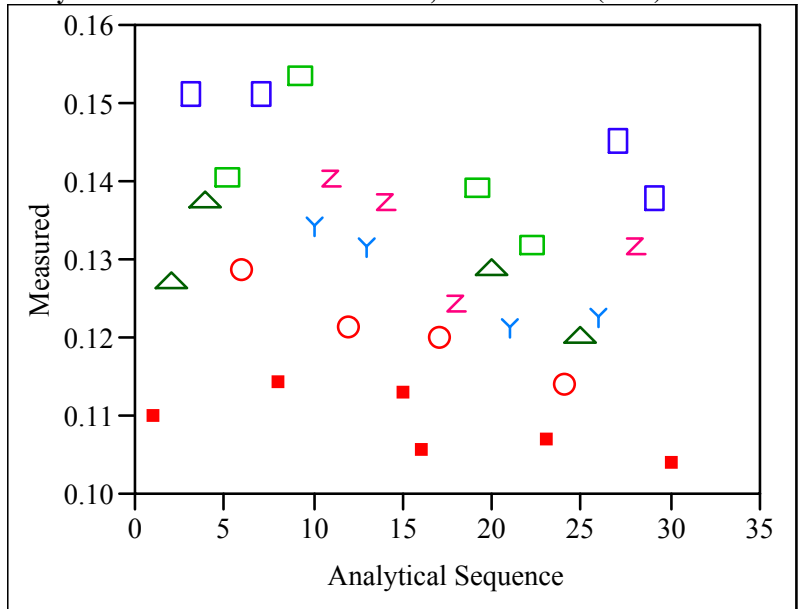

Measured By Analytical Sequence Prep=LM,

Analytical Plan =SRNL-SCS-2008-00043, Oxide=Fe2O3 (wt \%)

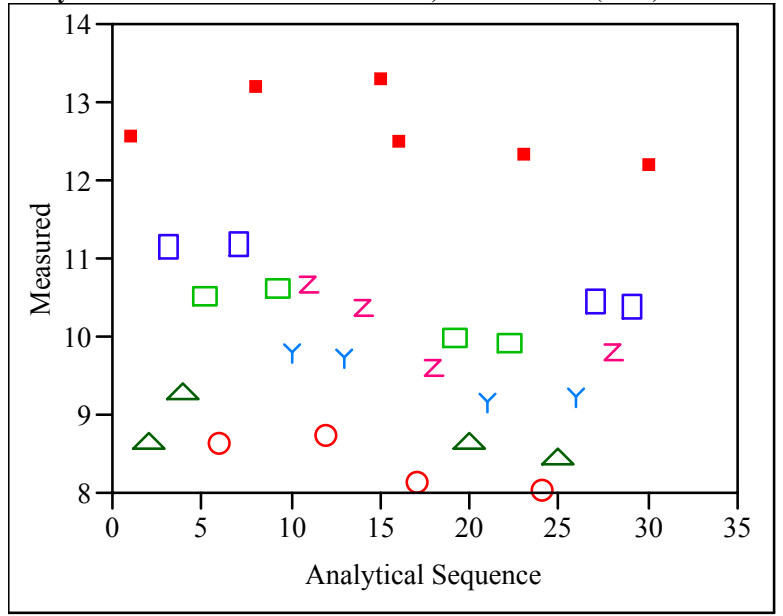

Measured By Analytical Sequence Prep=LM, Analytical Plan =SRNL-SCS-2008-00043, Oxide=HfO2 (wt \%)

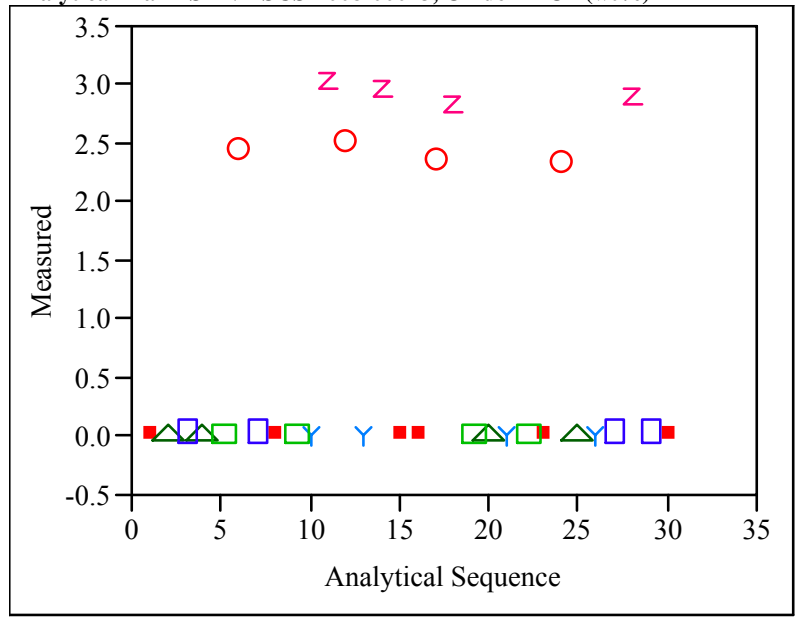


Exhibit A1. Oxide Measurements in Analytical Sequence for Samples by Prep Method and Analytical Plan Memo. (continued)

Measured By Analytical Sequence Prep $=$ LM, Analytical Plan =SRNL-SCS-2008-00043, Oxide=K2O (wt \%)

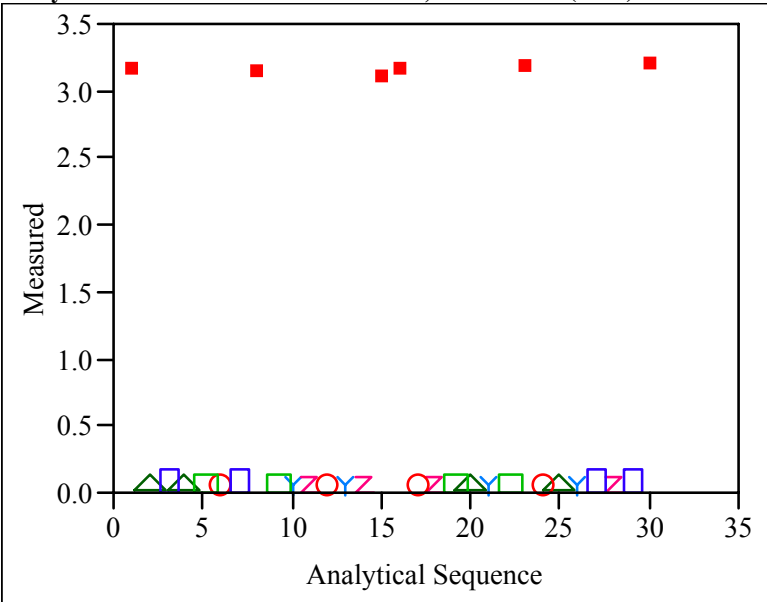

Measured By Analytical Sequence Prep=LM, Analytical Plan =SRNL-SCS-2008-00043, Oxide $=$ La2O3 (wt \%)

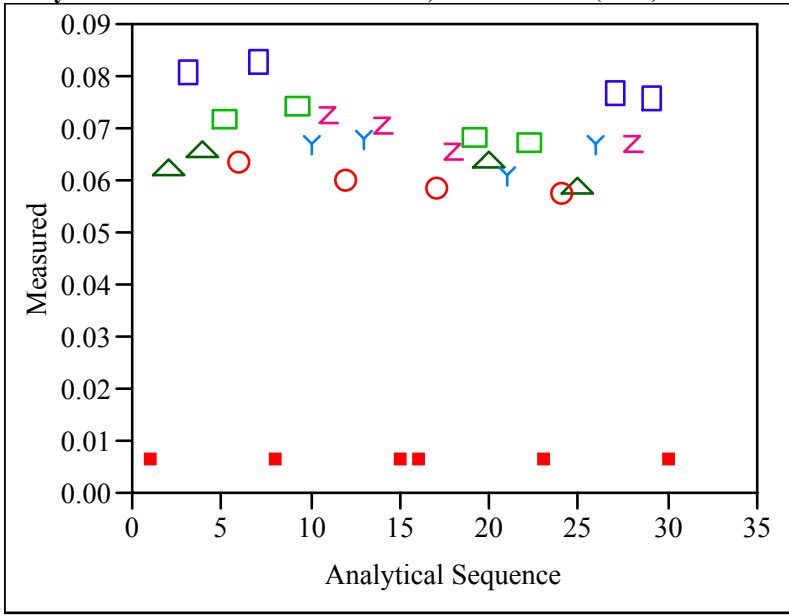

Measured By Analytical Sequence Prep=LM, Analytical Plan $=$ SRNL-SCS-2008-00043, Oxide $=$ MgO (wt $\%$ )

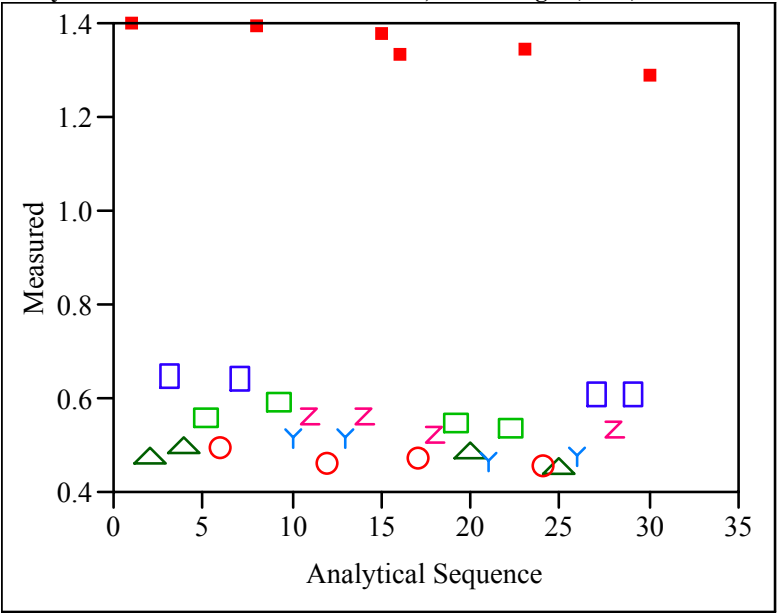

Measured By Analytical Sequence Prep=LM, Analytical Plan =SRNL-SCS-2008-00043, Oxide=MnO (wt \%)

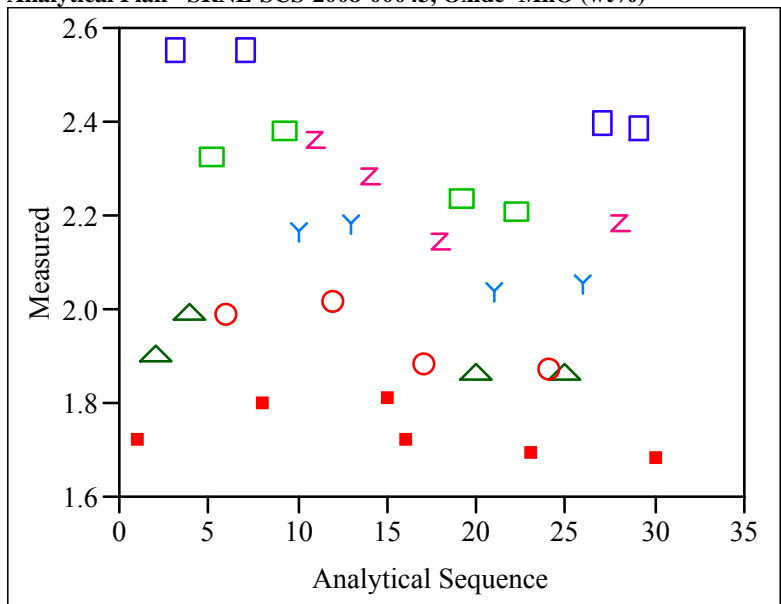

Measured By Analytical Sequence Prep=LM,

Analytical Plan =SRNL-SCS-2008-00043, Oxide=Na2O (wt \%)

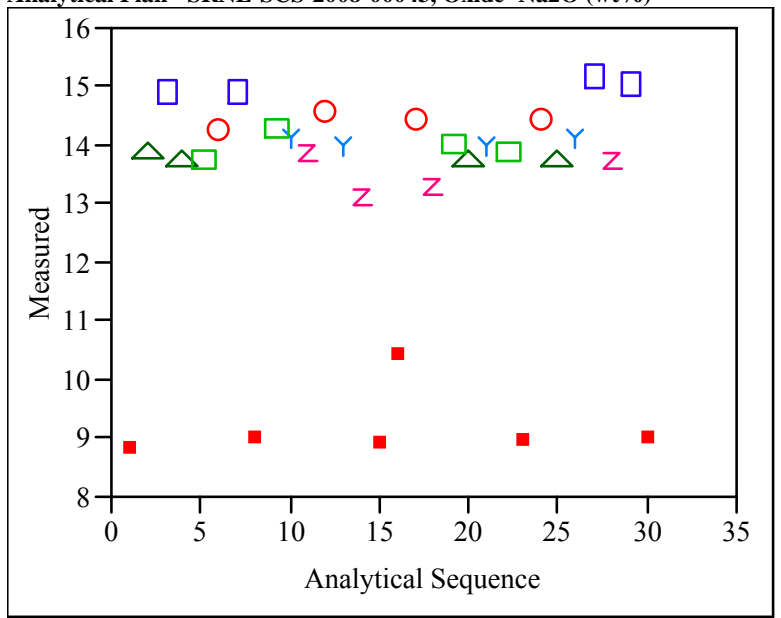

Measured By Analytical Sequence Prep=LM, Analytical Plan =SRNL-SCS-2008-00043, Oxide=Nd2O3 (wt \%)

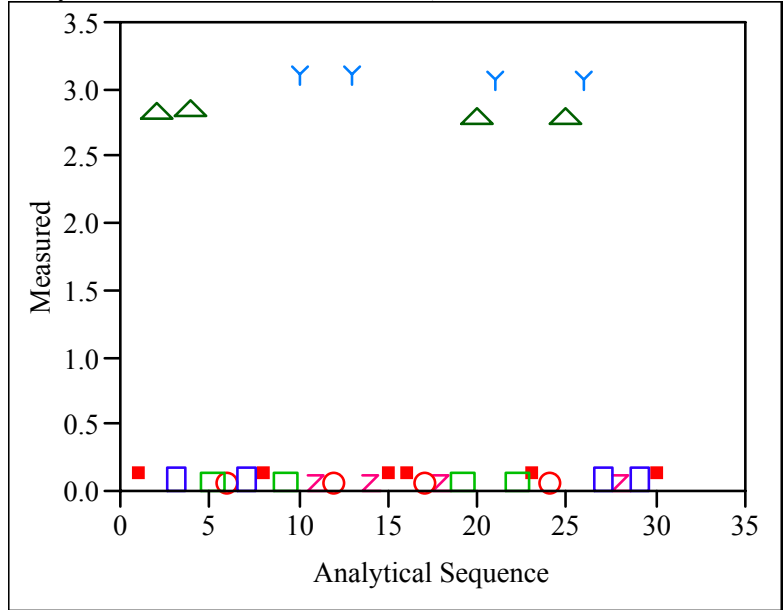


Exhibit A1. Oxide Measurements in Analytical Sequence for Samples by Prep Method and Analytical Plan Memo. (continued)

Measured By Analytical Sequence Prep=LM, Analytical Plan $=$ SRNL-SCS-2008-00043, Oxide $=\mathrm{NiO}(\mathrm{wt} \%)$

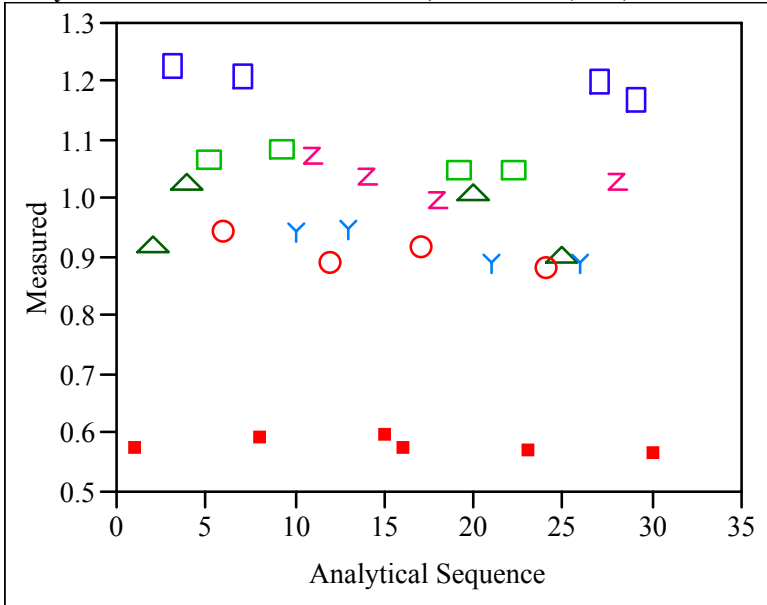

Measured By Analytical Sequence Prep=LM, Analytical Plan =SRNL-SCS-2008-00043, Oxide=PbO (wt \%)

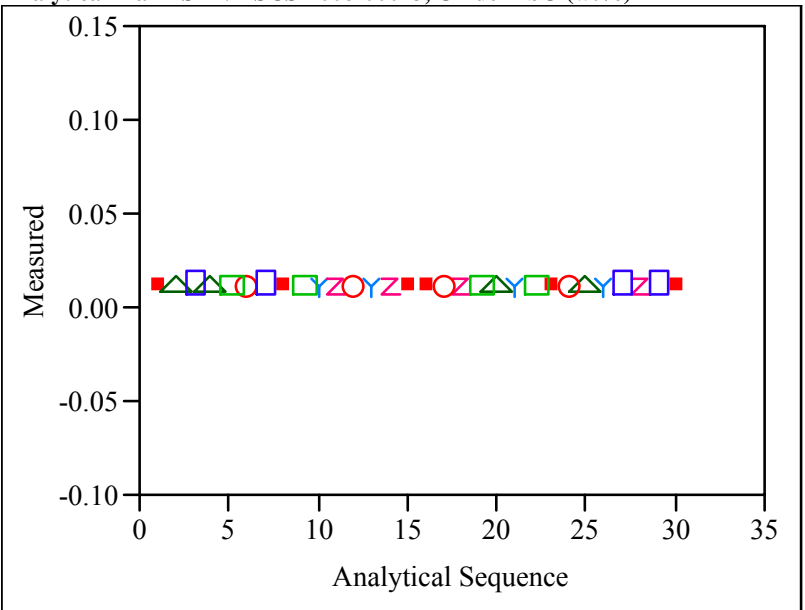

Measured By Analytical Sequence Prep=LM,

Analytical Plan =SRNL-SCS-2008-00043, Oxide=SiO2 (wt \%)

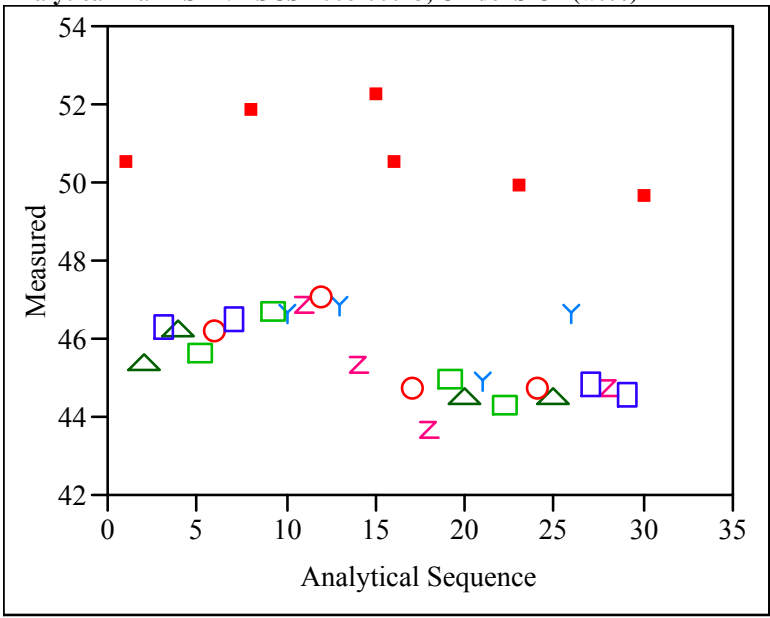

Measured By Analytical Sequence Prep=LM, Analytical Plan =SRNL-SCS-2008-00043, Oxide=SO4 (wt \%)

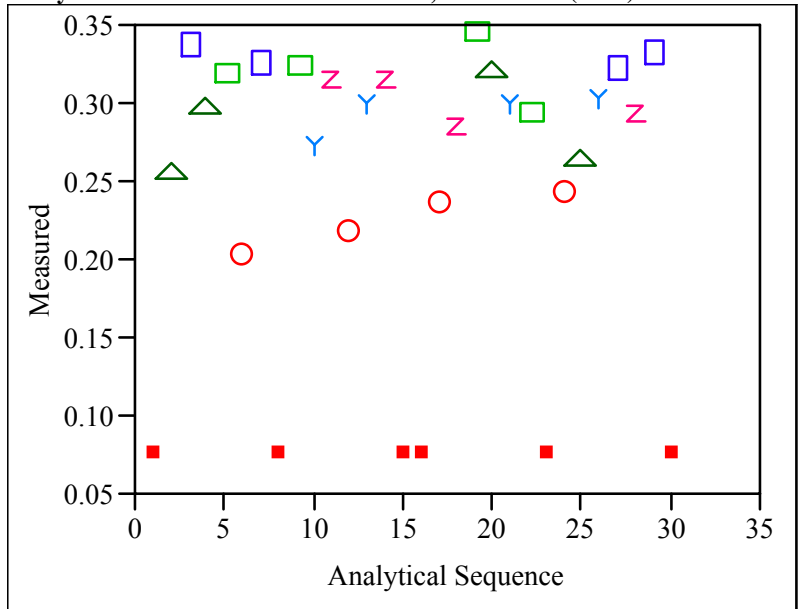

Measured By Analytical Sequence Prep=LM, Analytical Plan =SRNL-SCS-2008-00043, Oxide=TiO2 (wt \%)

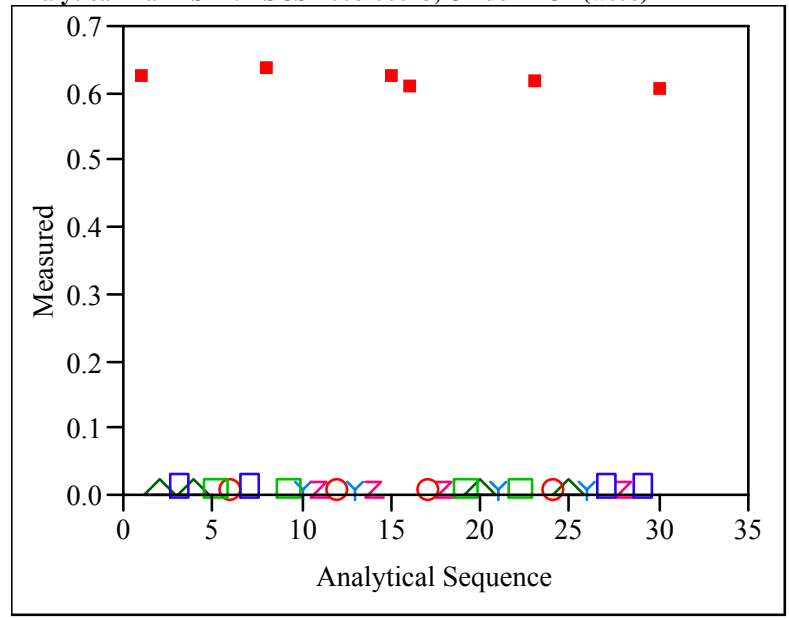

Measured By Analytical Sequence Prep=LM, Analytical Plan =SRNL-SCS-2008-00043, Oxide=ZnO (wt \%)

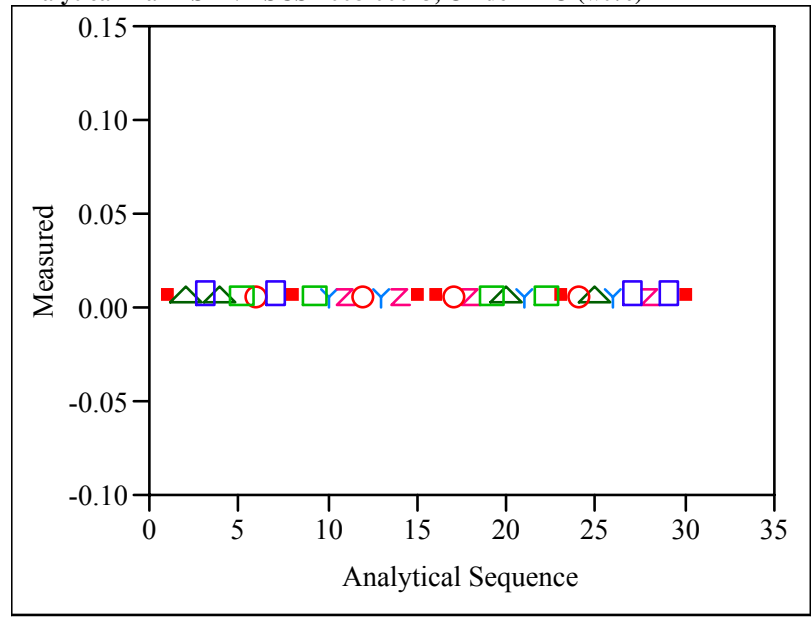


Exhibit A1. Oxide Measurements in Analytical Sequence for Samples by Prep Method and Analytical Plan Memo. (continued)

Measured By Analytical Sequence Prep=LM, Analytical Plan =SRNL-SCS-2008-00043, Oxide=ZrO2 (wt\%)

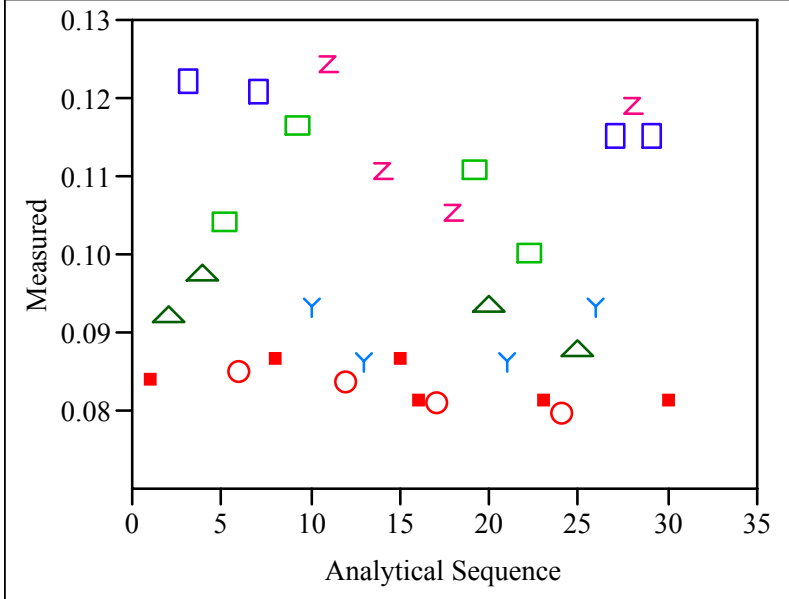

Measured By Analytical Sequence Prep=PF, Analytical Plan =SRNL-SCS-2008-00003, Oxide=B2O3 (wt \%)

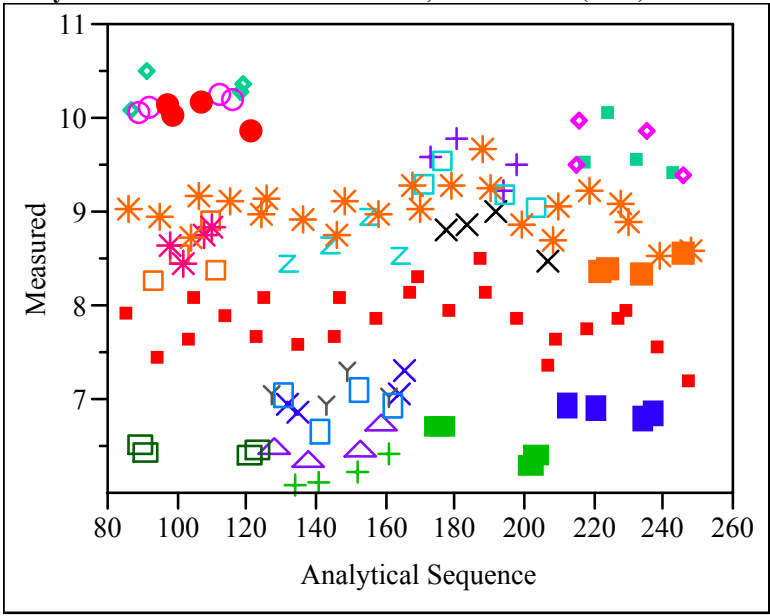

Measured By Analytical Sequence Prep=PF,

Analytical Plan $=$ SRNL-SCS-2008-00003, Oxide $=$ Li2O (wt \%)

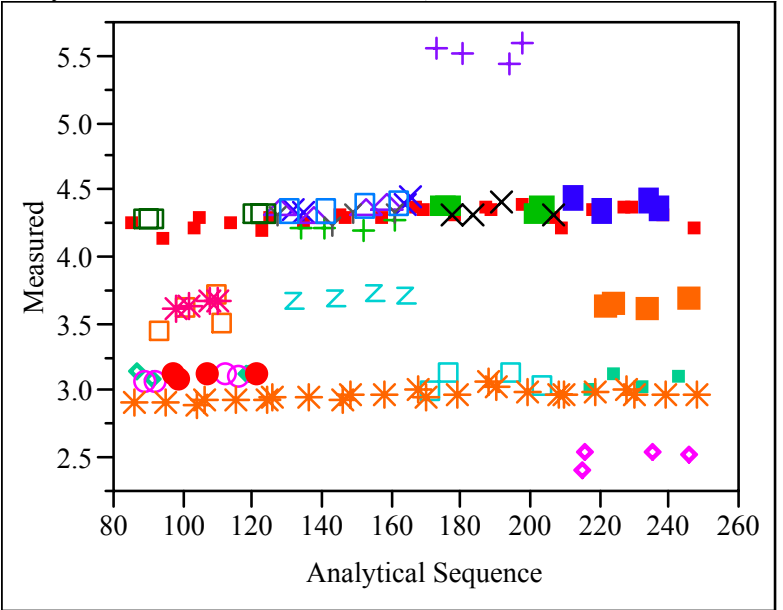

Measured By Analytical Sequence Prep=PF, Analytical Plan =SRNL-SCS-2008-00043, Oxide $=$ B2O3 (wt \%)

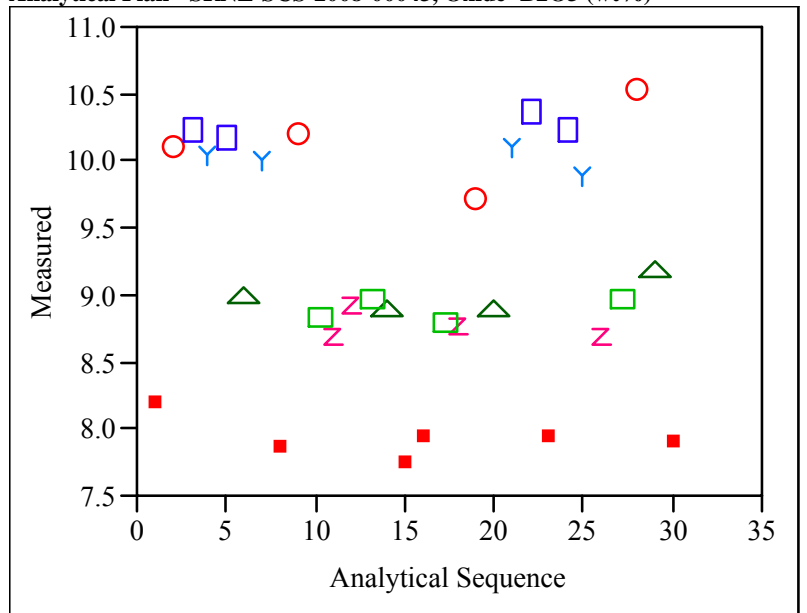

Measured By Analytical Sequence Prep=PF, Analytical Plan =SRNL-SCS-2008-00043, Oxide $=$ Li2O (wt \%)

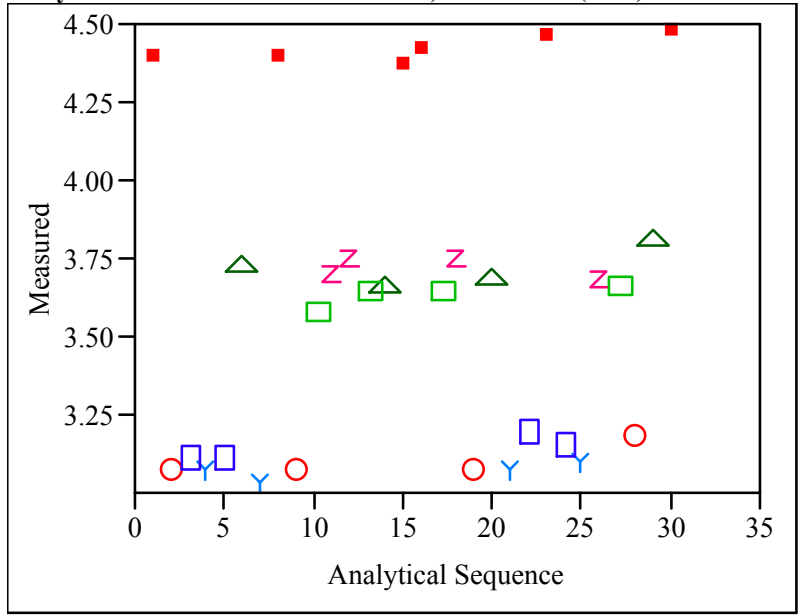


Exhibit A2. Oxide Measurements by Lab ID within Glass ID for Samples by Prep Method and Analytical Plan Memo.

Variability Plot for Analytical Plan =SRNL-SCS-2008-00003, Prep=LM, Oxide=Al2O3 (wt \%)

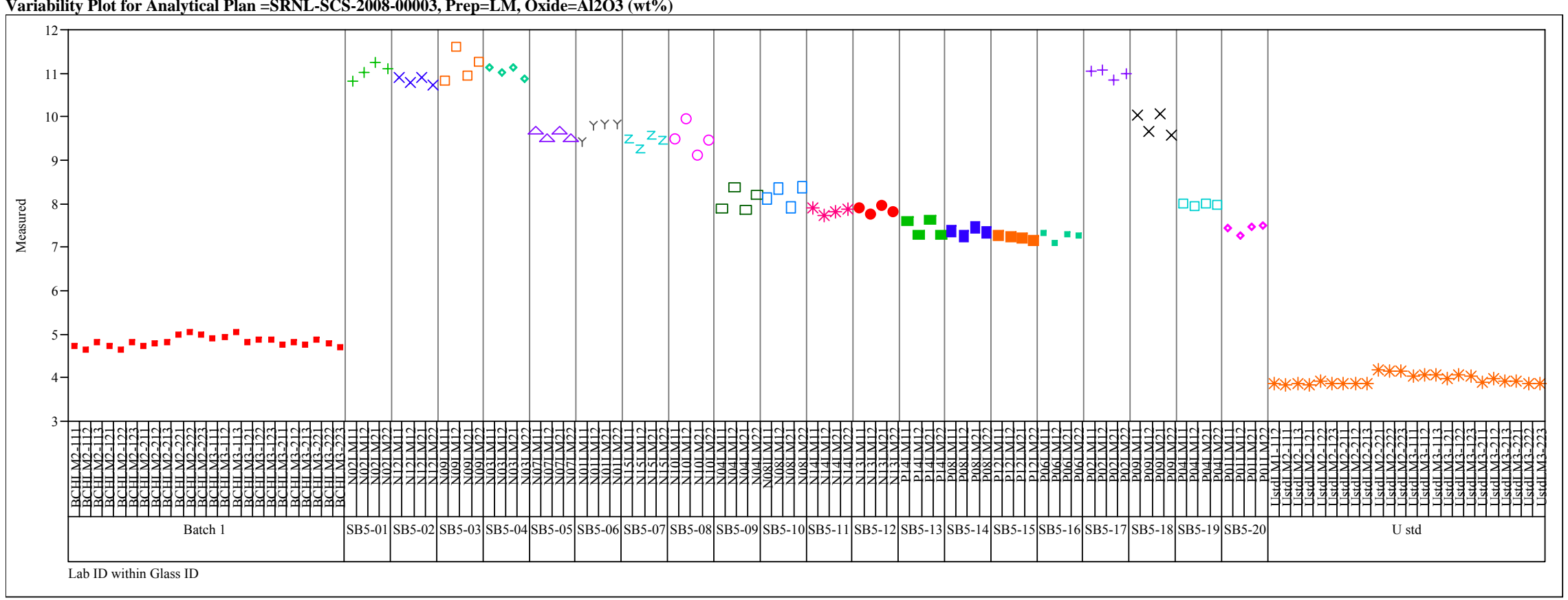


Exhibit A2. Oxide Measurements by Lab ID within Glass ID for Samples by Prep Method and Analytical Plan Memo. (continued) Variability Plot for Analytical Plan =SRNL-SCS-2008-00003, Prep=LM, Oxide=BaO (wt \%)

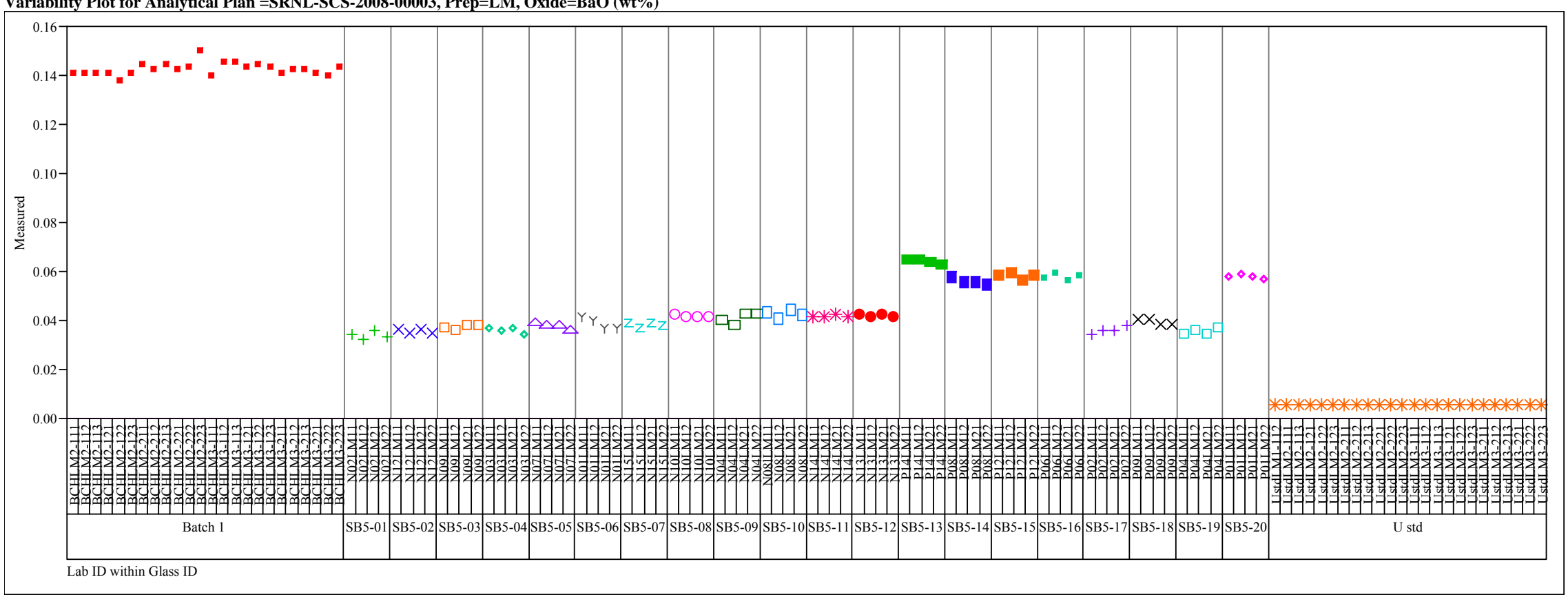


Exhibit A2. Oxide Measurements by Lab ID within Glass ID for Samples by Prep Method and Analytical Plan Memo. (continued) Variability Plot for Analytical Plan =SRNL-SCS-2008-00003, Prep=LM, Oxide=CaO (wt\%)

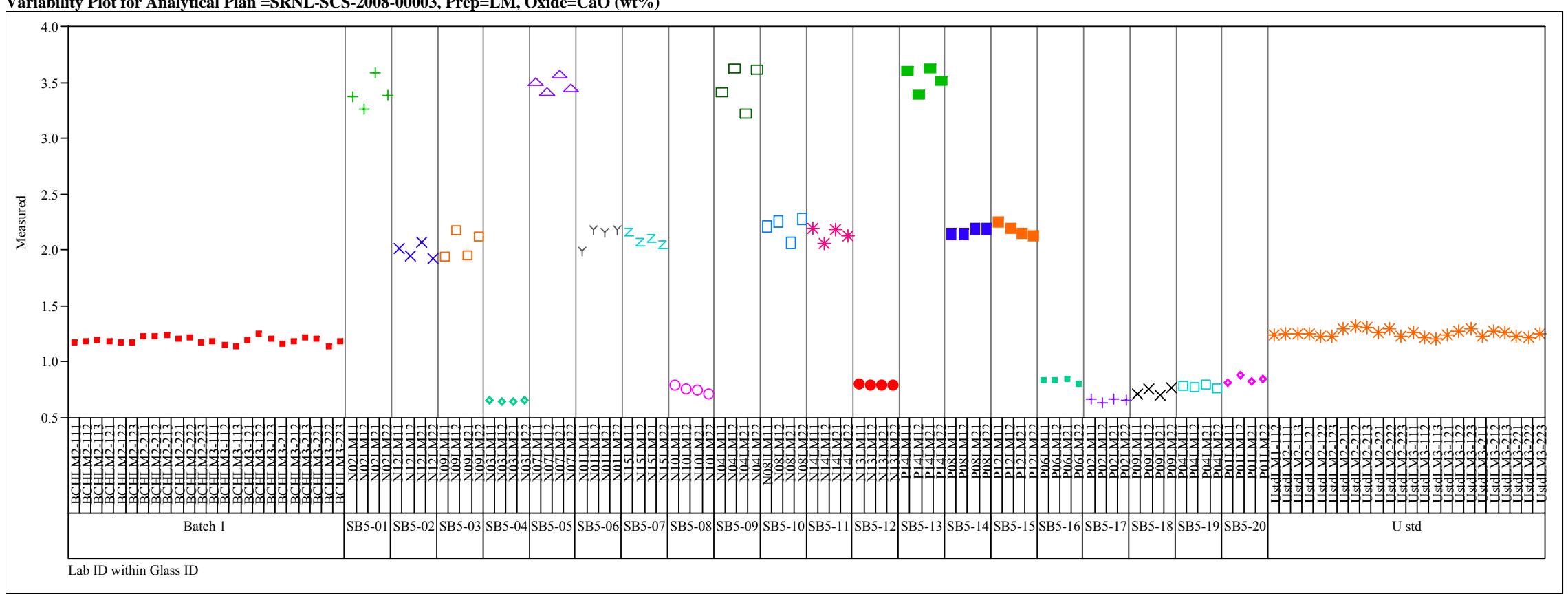


Exhibit A2. Oxide Measurements by Lab ID within Glass ID for Samples by Prep Method and Analytical Plan Memo. (continued) Variability Plot for Analytical Plan =SRNL-SCS-2008-00003, Prep=LM, Oxide=CdO (wt \%)

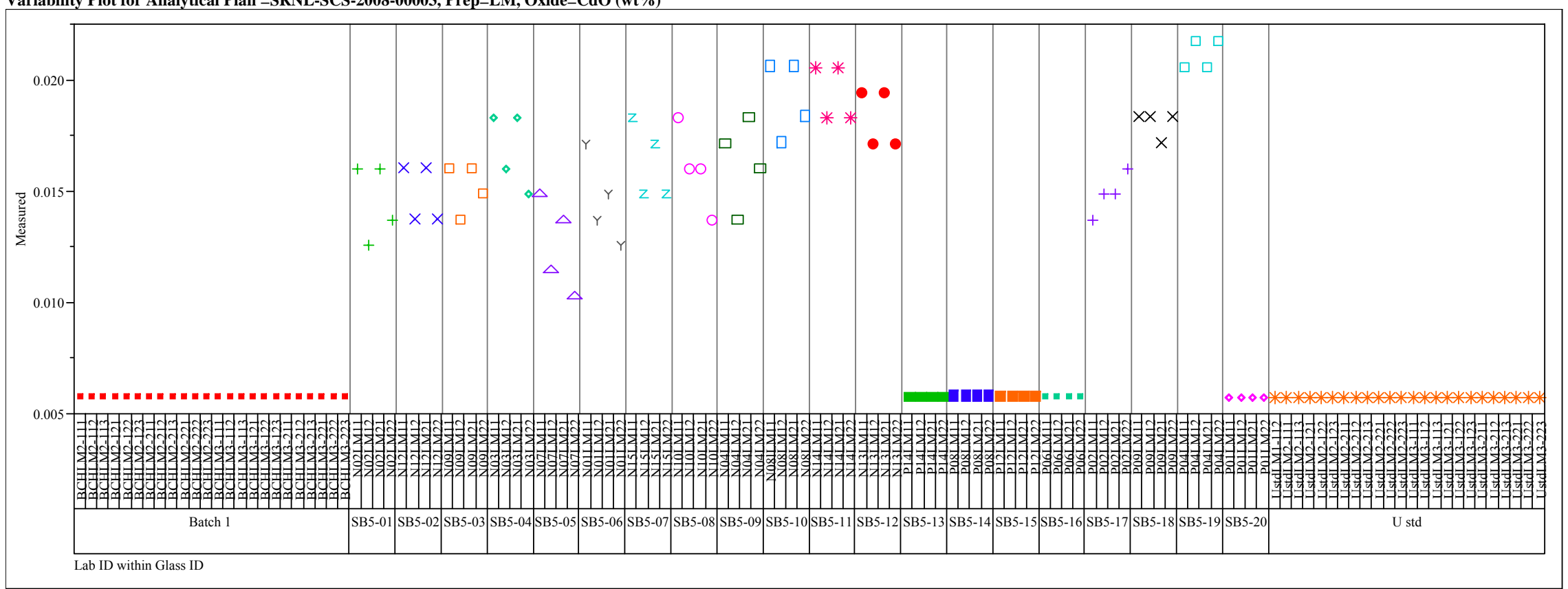


Exhibit A2. Oxide Measurements by Lab ID within Glass ID for Samples by Prep Method and Analytical Plan Memo. (continued) Variability Plot for Analytical Plan =SRNL-SCS-2008-00003, Prep=LM, Oxide=Ce2O3 (wt\%)




Exhibit A2. Oxide Measurements by Lab ID within Glass ID for Samples by Prep Method and Analytical Plan Memo. (continued) Variability Plot for Analytical Plan =SRNL-SCS-2008-00003, Prep=LM, Oxide=Cr2O3 (wt\%)

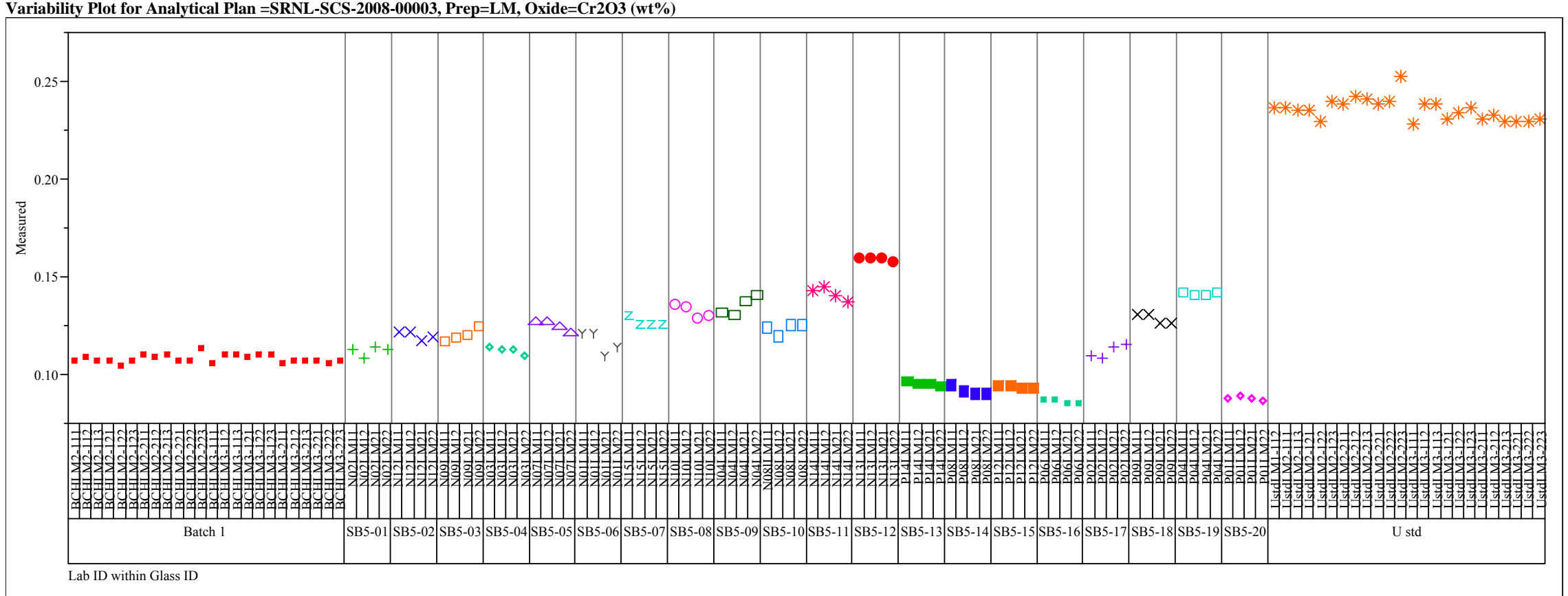


Exhibit A2. Oxide Measurements by Lab ID within Glass ID for Samples by Prep Method and Analytical Plan Memo. (continued) Variability Plot for Analytical Plan =SRNL-SCS-2008-00003, Prep=LM, Oxide=CuO (wt \%)

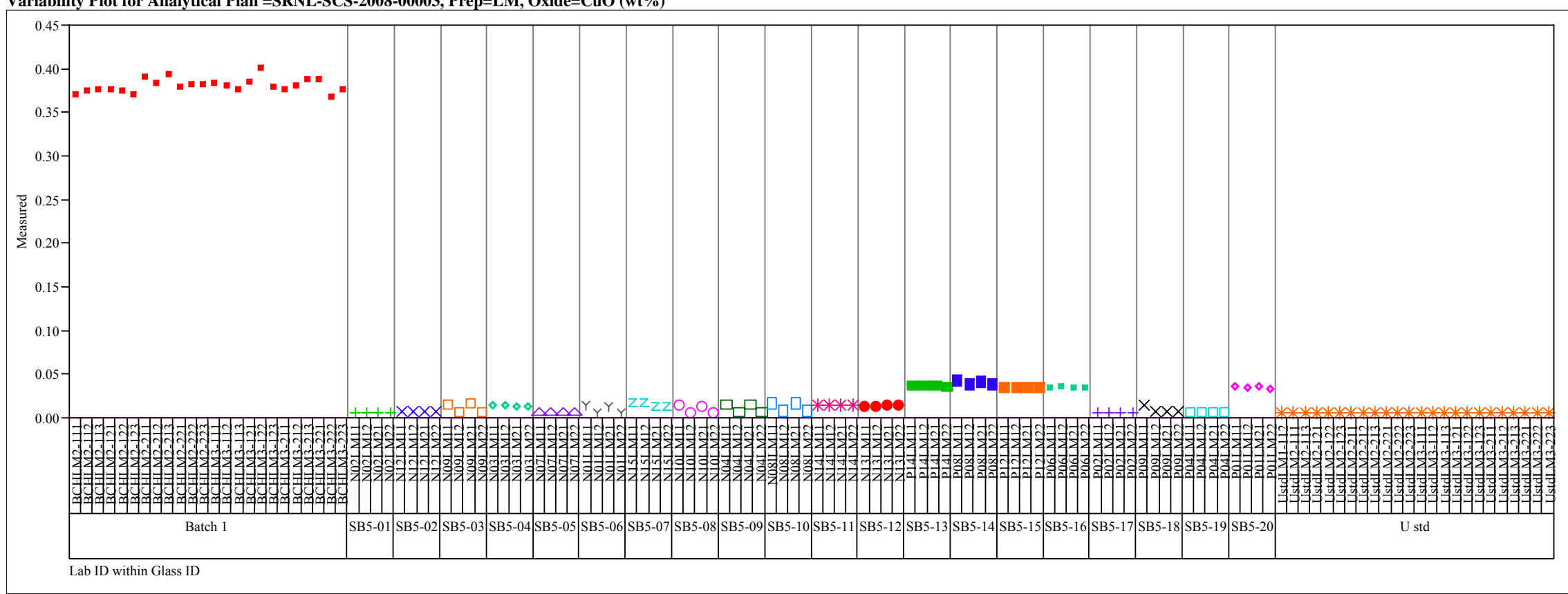


Exhibit A2. Oxide Measurements by Lab ID within Glass ID for Samples by Prep Method and Analytical Plan Memo. (continued) Variability Plot for Analytical Plan =SRNL-SCS-2008-00003, Prep=LM, Oxide=Fe2O3 (wt\%)

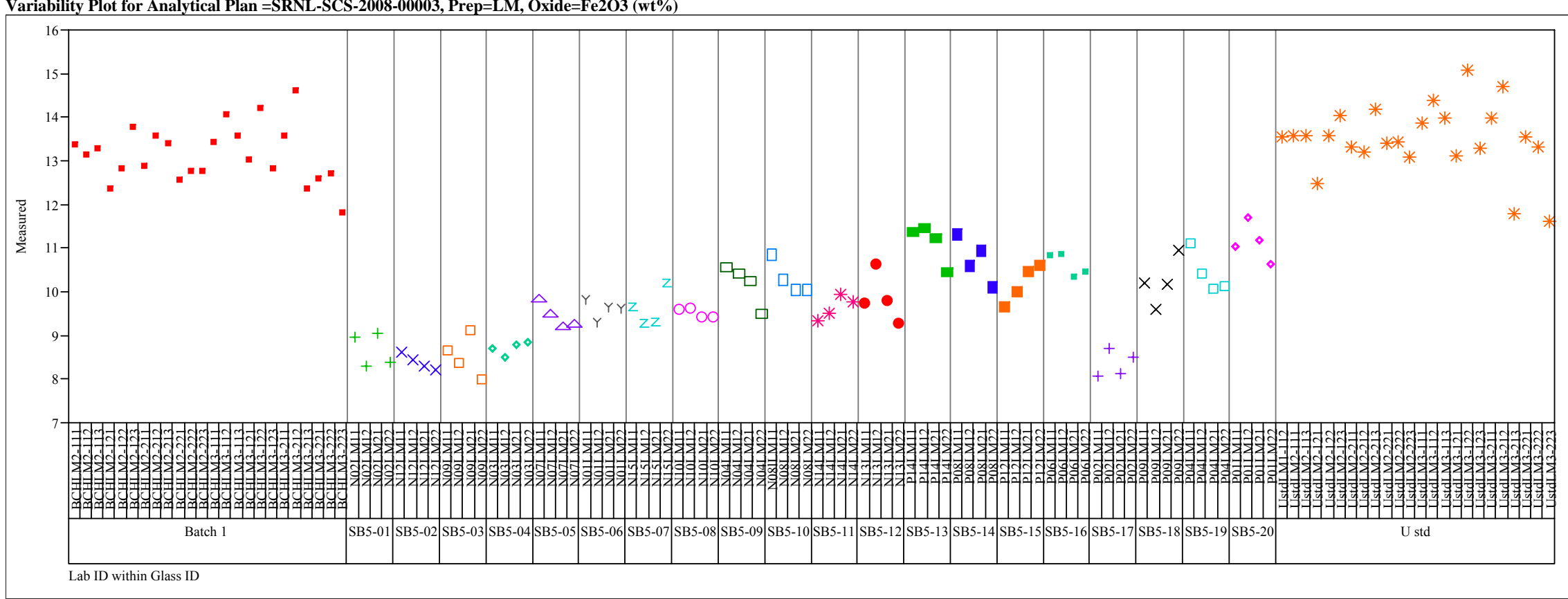


Exhibit A2. Oxide Measurements by Lab ID within Glass ID for Samples by Prep Method and Analytical Plan Memo. (continued) Variability Plot for Analytical Plan =SRNL-SCS-2008-00003, Prep=LM, Oxide=K2O (wt \%)

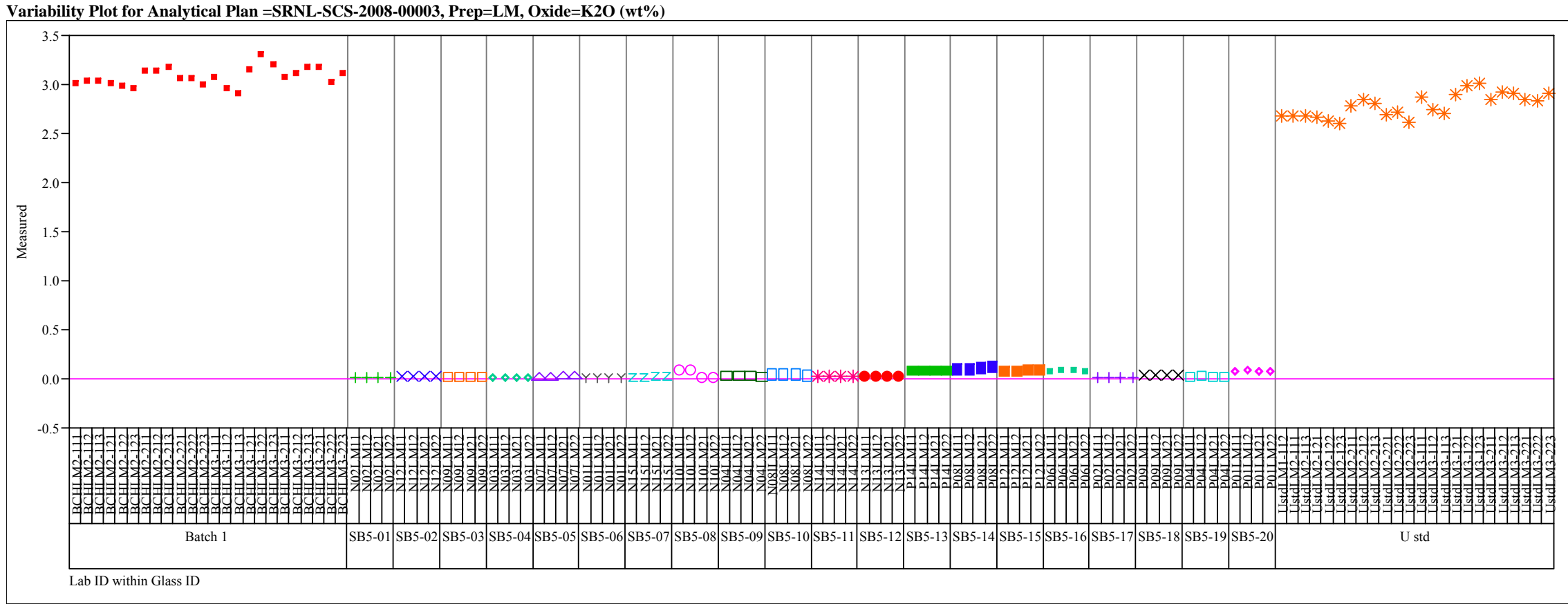


Exhibit A2. Oxide Measurements by Lab ID within Glass ID for Samples by Prep Method and Analytical Plan Memo. (continued) Variability Plot for Analytical Plan =SRNL-SCS-2008-00003, Prep=LM, Oxide=La2O3 (wt\%)

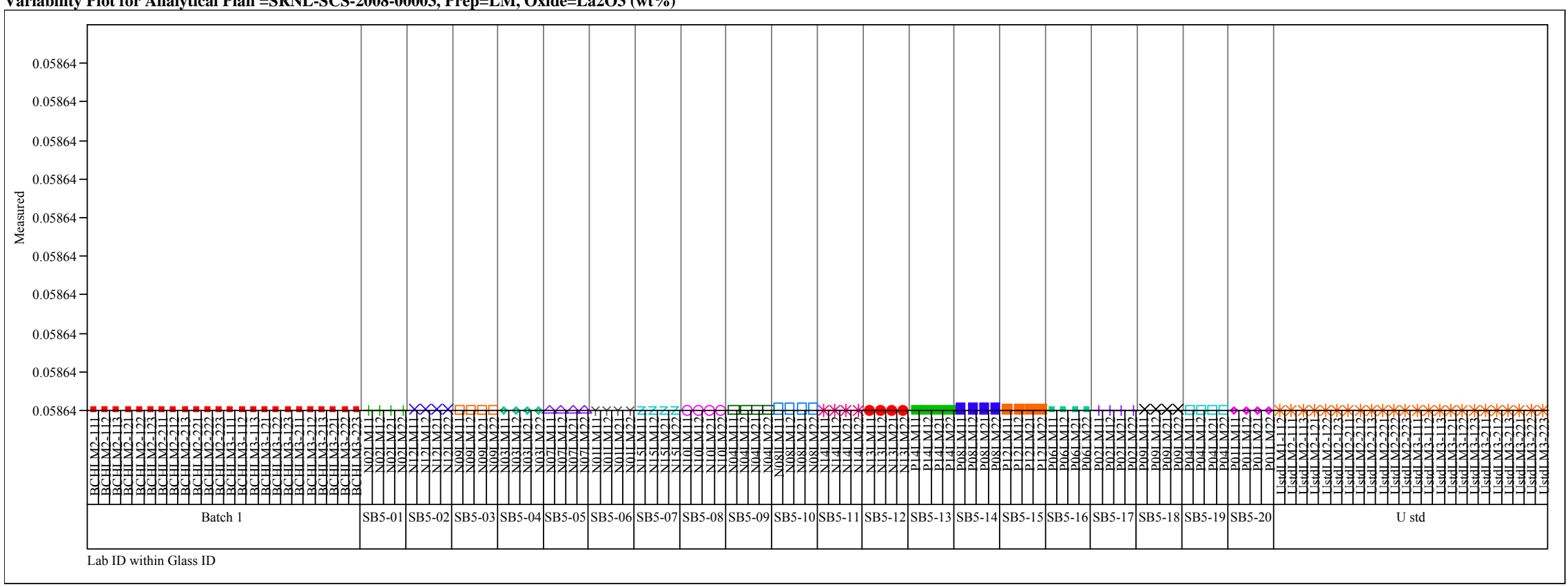


Exhibit A2. Oxide Measurements by Lab ID within Glass ID for Samples by Prep Method and Analytical Plan Memo. (continued) Variability Plot for Analytical Plan =SRNL-SCS-2008-00003, Prep=LM, Oxide=MgO (wt \%)

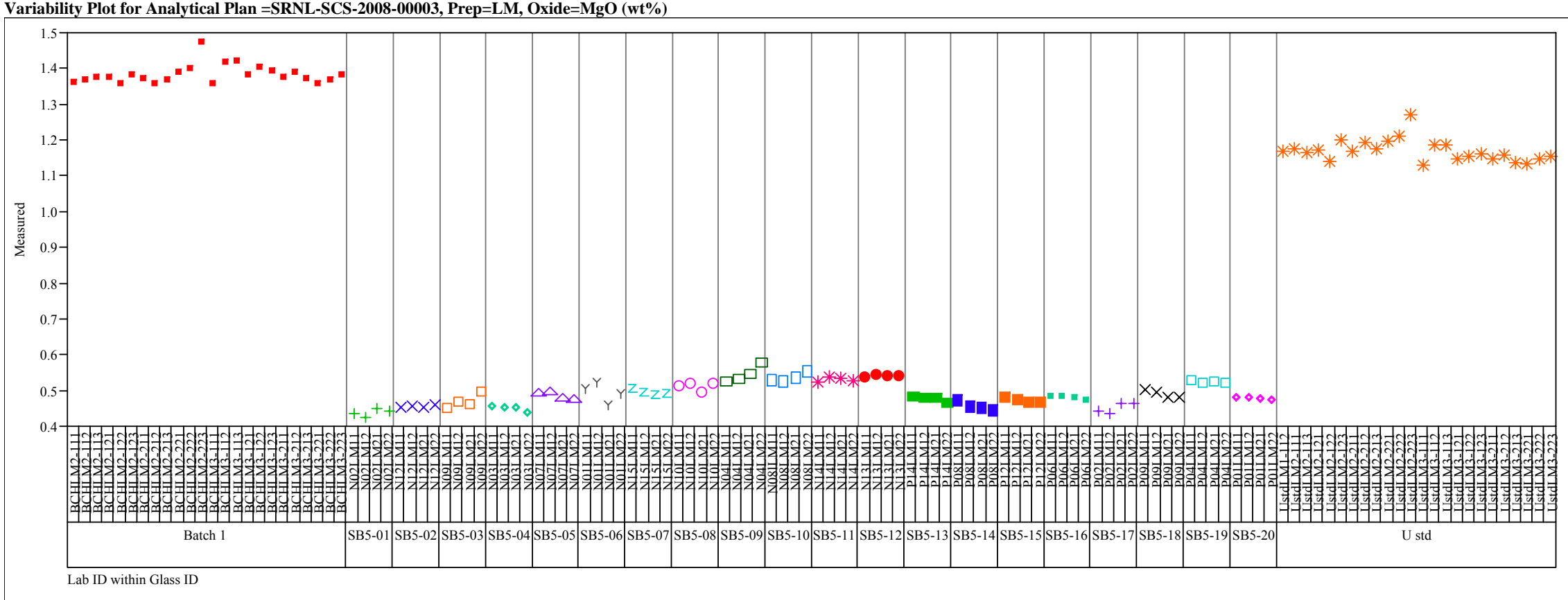


Exhibit A2. Oxide Measurements by Lab ID within Glass ID for Samples by Prep Method and Analytical Plan Memo. (continued) Variability Plot for Analytical Plan =SRNL-SCS-2008-00003, Prep=LM, Oxide=MnO (wt\%)

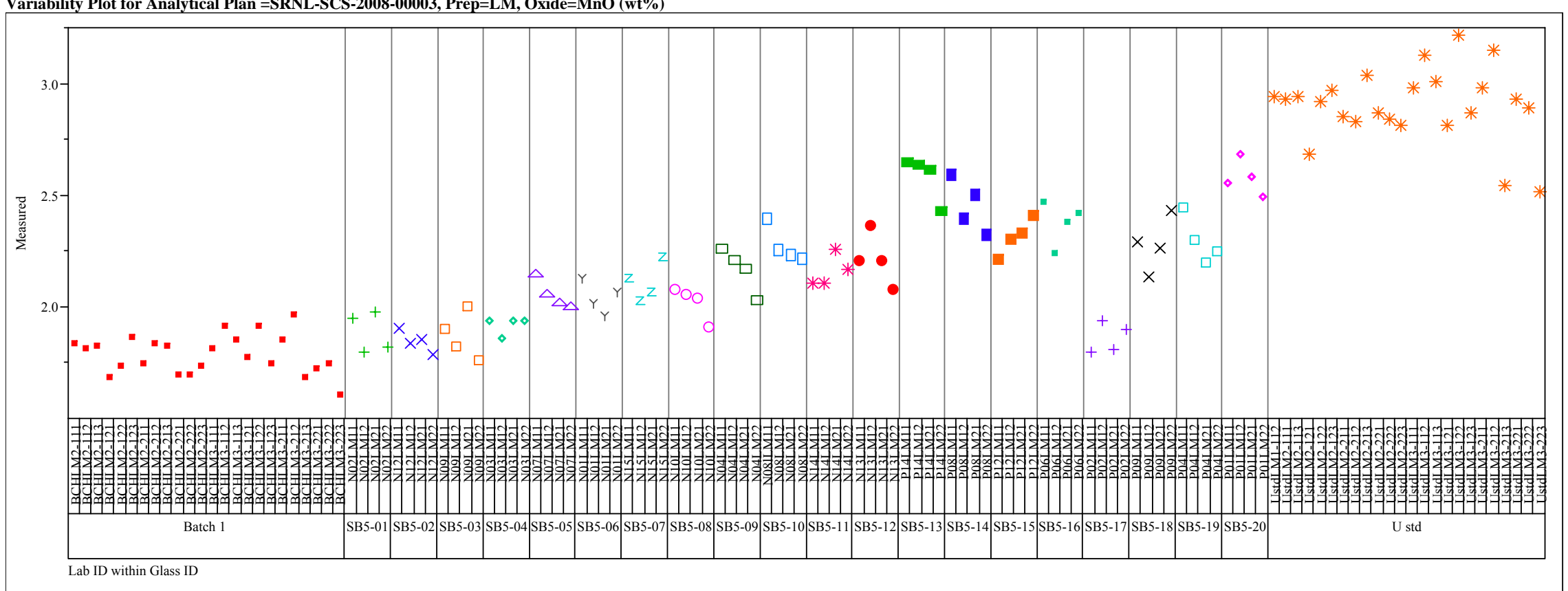


Exhibit A2. Oxide Measurements by Lab ID within Glass ID for Samples by Prep Method and Analytical Plan Memo. (continued) Variability Plot for Analytical Plan =SRNL-SCS-2008-00003, Prep=LM, Oxide=Na2O (wt\%)

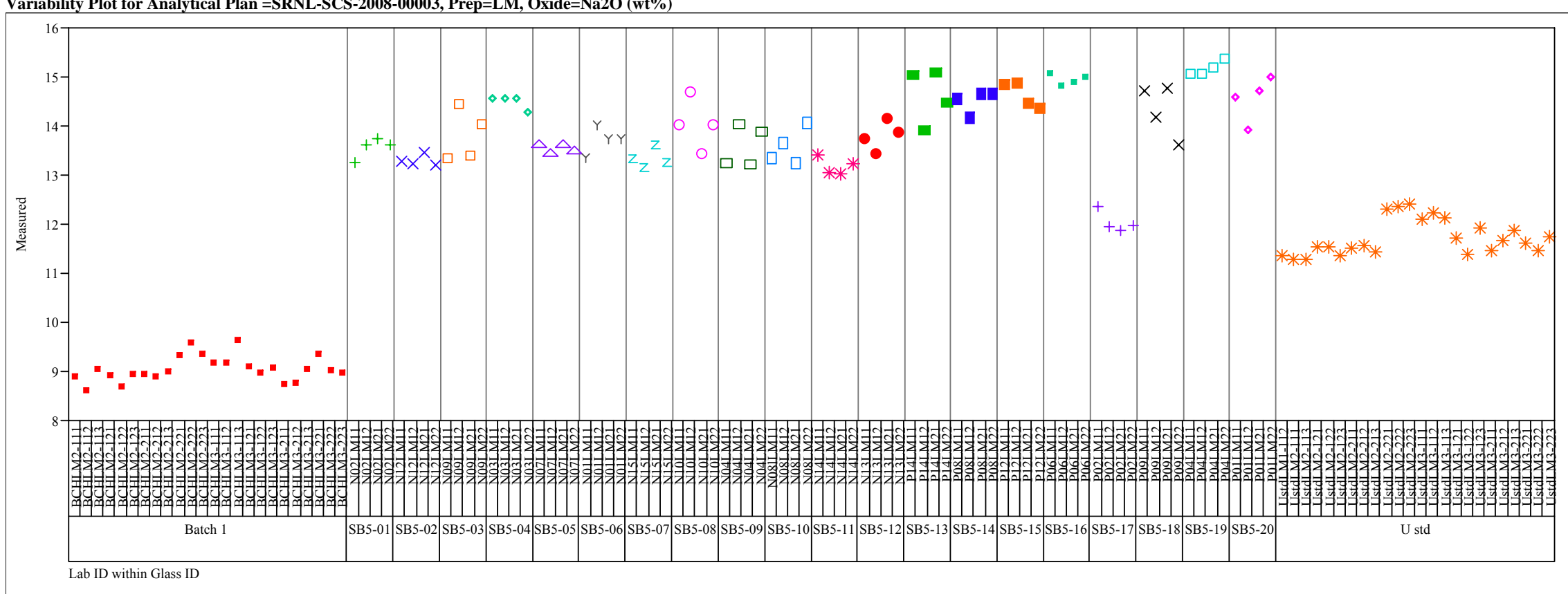


Exhibit A2. Oxide Measurements by Lab ID within Glass ID for Samples by Prep Method and Analytical Plan Memo. (continued) Variability Plot for Analytical Plan =SRNL-SCS-2008-00003, Prep=LM, Oxide=Nb2O5 (wt \%)

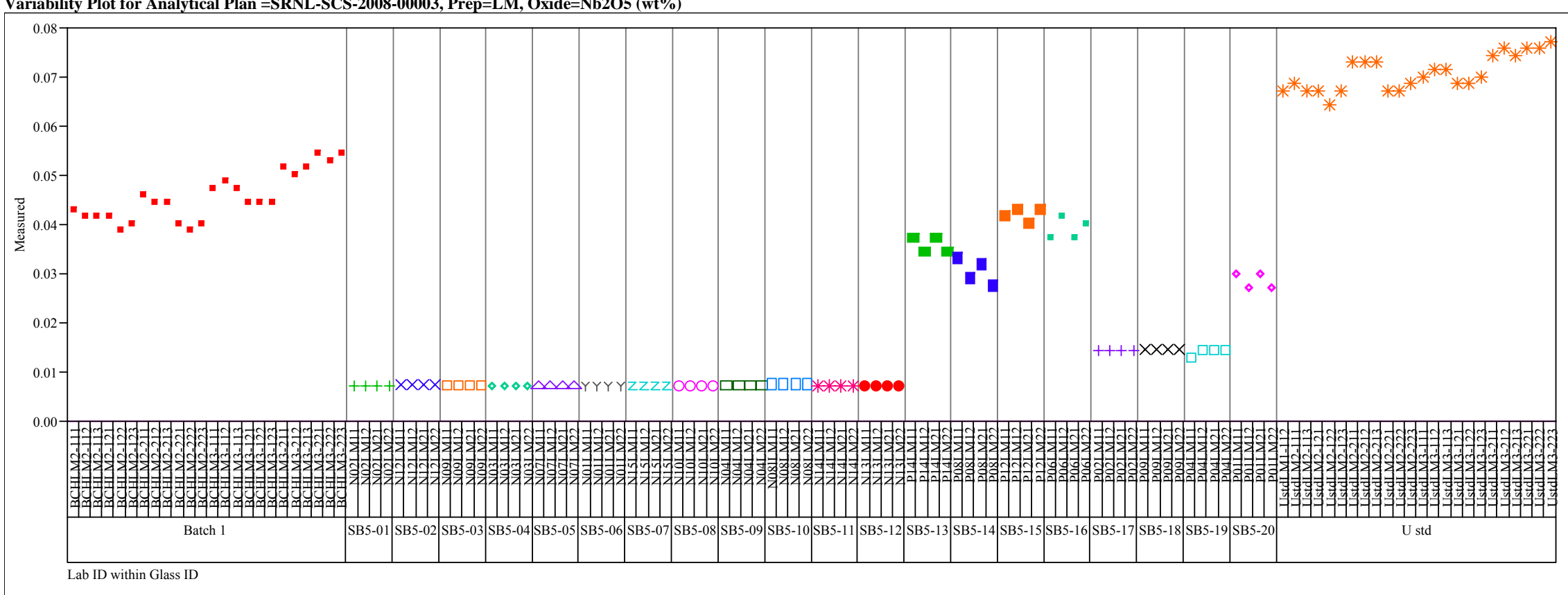


Exhibit A2. Oxide Measurements by Lab ID within Glass ID for Samples by Prep Method and Analytical Plan Memo. (continued) Variability Plot for Analytical Plan =SRNL-SCS-2008-00003, Prep=LM, Oxide=NiO (wt\%)

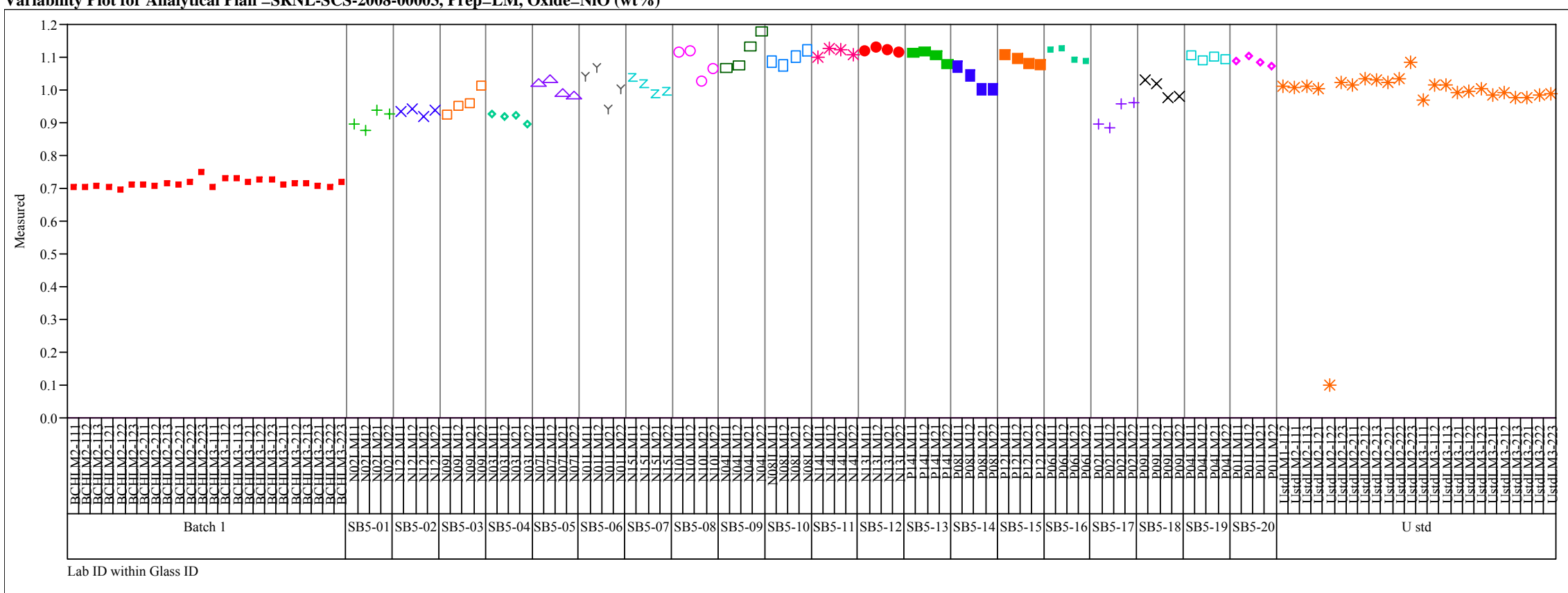


Exhibit A2. Oxide Measurements by Lab ID within Glass ID for Samples by Prep Method and Analytical Plan Memo. (continued) Variability Plot for Analytical Plan =SRNL-SCS-2008-00003, Prep=LM, Oxide=PbO (wt\%)

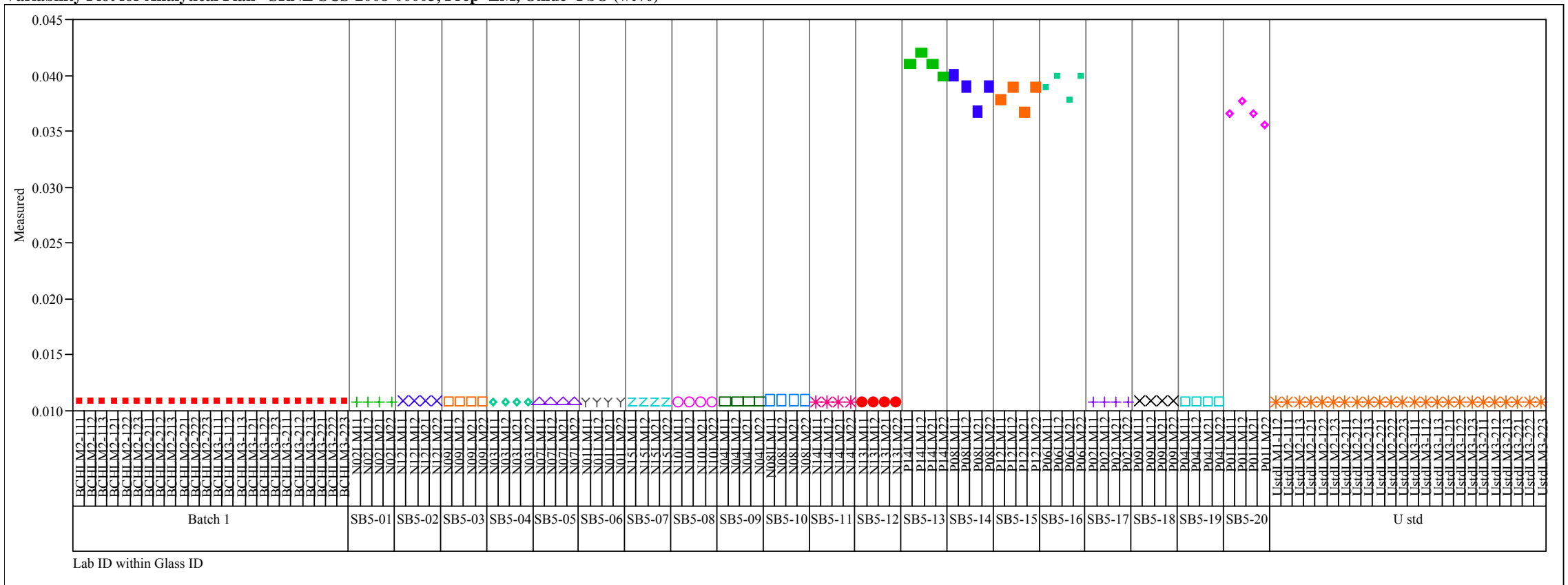


Exhibit A2. Oxide Measurements by Lab ID within Glass ID for Samples by Prep Method and Analytical Plan Memo. (continued) Variability Plot for Analytical Plan =SRNL-SCS-2008-00003, Prep=LM, Oxide=SiO2 (wt \%)

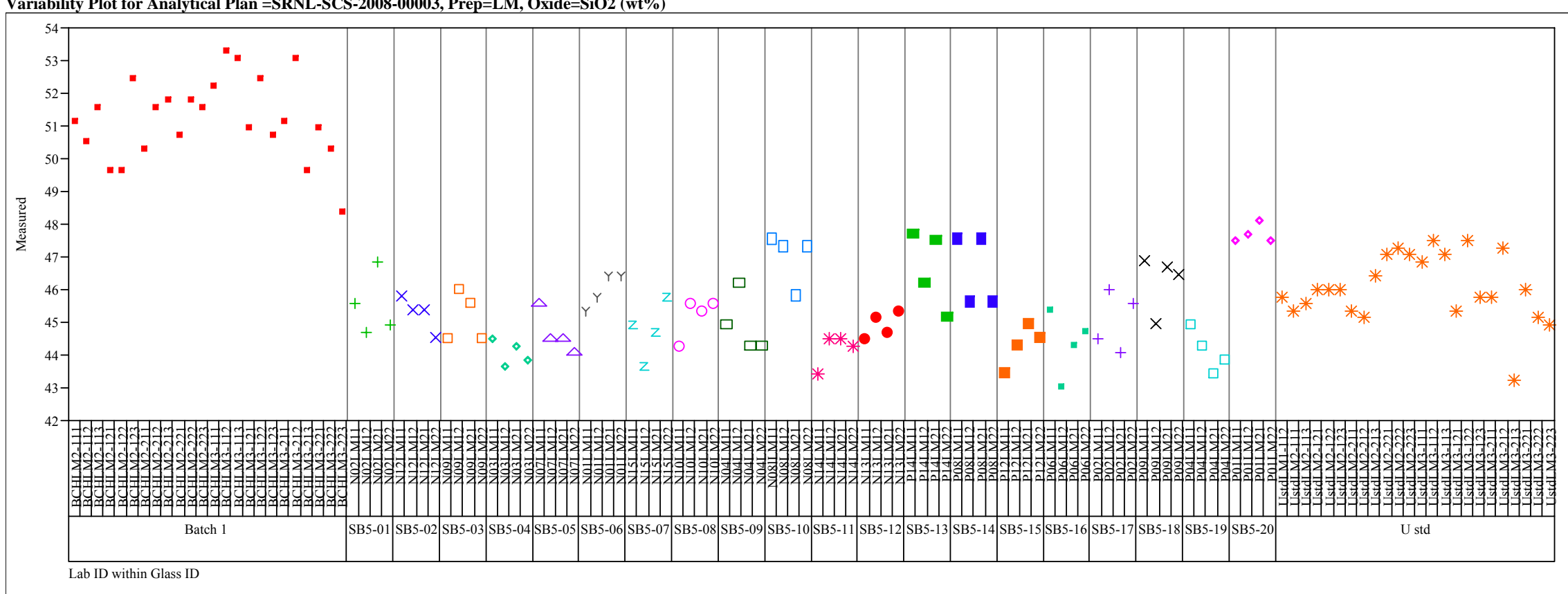


Exhibit A2. Oxide Measurements by Lab ID within Glass ID for Samples by Prep Method and Analytical Plan Memo. (continued) Variability Plot for Analytical Plan =SRNL-SCS-2008-00003, Prep=LM, Oxide=SO4 (wt \%)

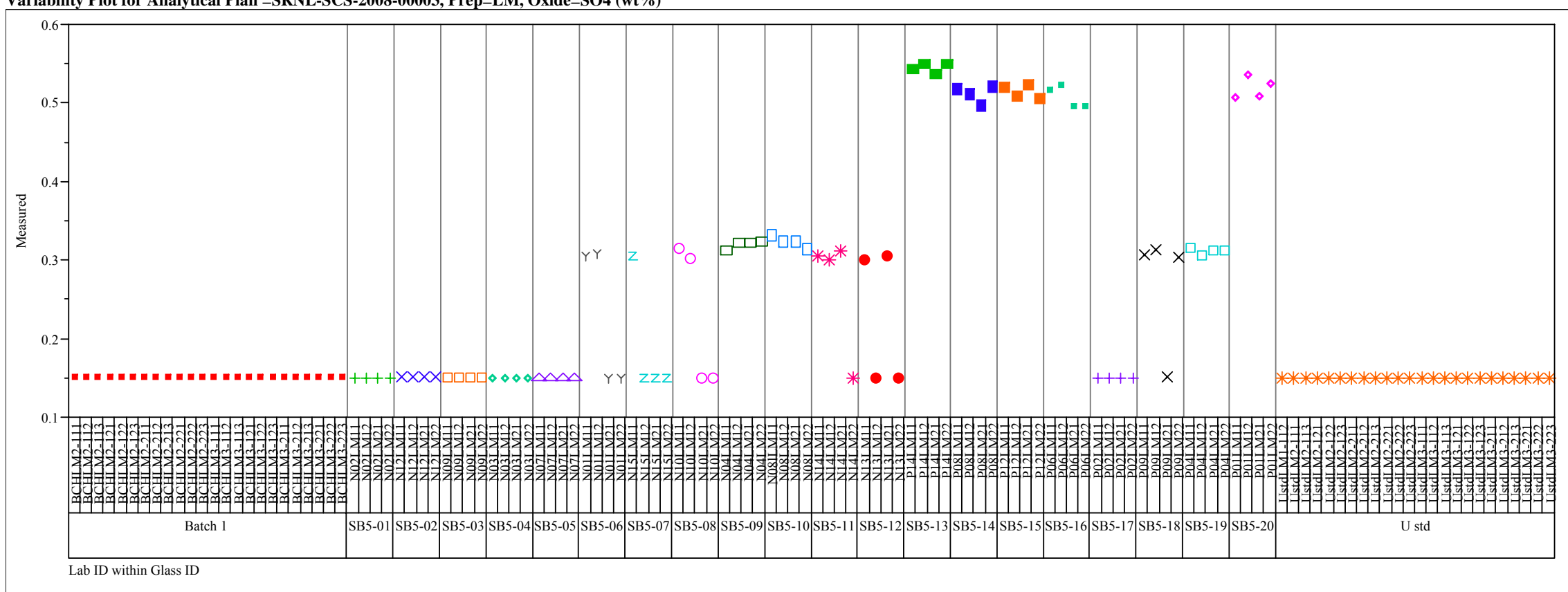


Exhibit A2. Oxide Measurements by Lab ID within Glass ID for Samples by Prep Method and Analytical Plan Memo. (continued) Variability Plot for Analytical Plan =SRNL-SCS-2008-00003, Prep=LM, Oxide=ThO2 (wt\%)

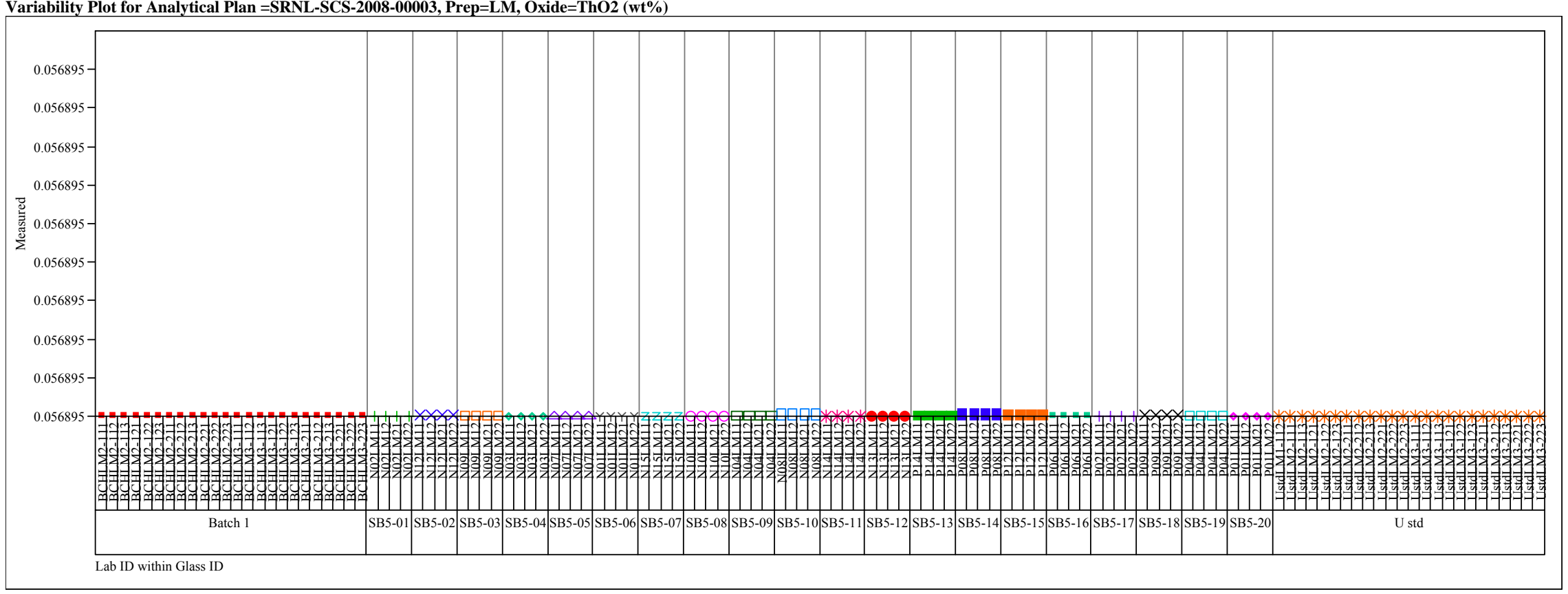


Exhibit A2. Oxide Measurements by Lab ID within Glass ID for Samples by Prep Method and Analytical Plan Memo. (continued) Variability Plot for Analytical Plan =SRNL-SCS-2008-00003, Prep=LM, Oxide=TiO2 (wt\%)

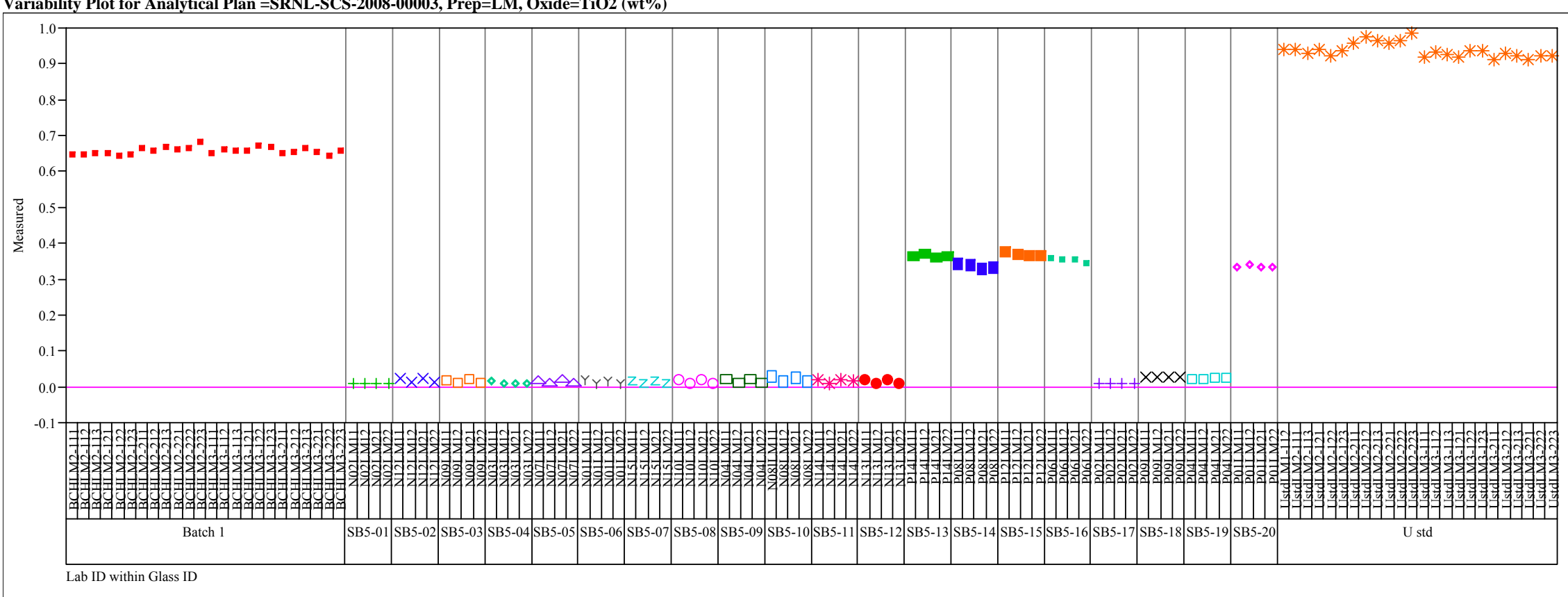


Exhibit A2. Oxide Measurements by Lab ID within Glass ID for Samples by Prep Method and Analytical Plan Memo. (continued) Variability Plot for Analytical Plan =SRNL-SCS-2008-00003, Prep=LM, Oxide=U3O8 (wt\%)

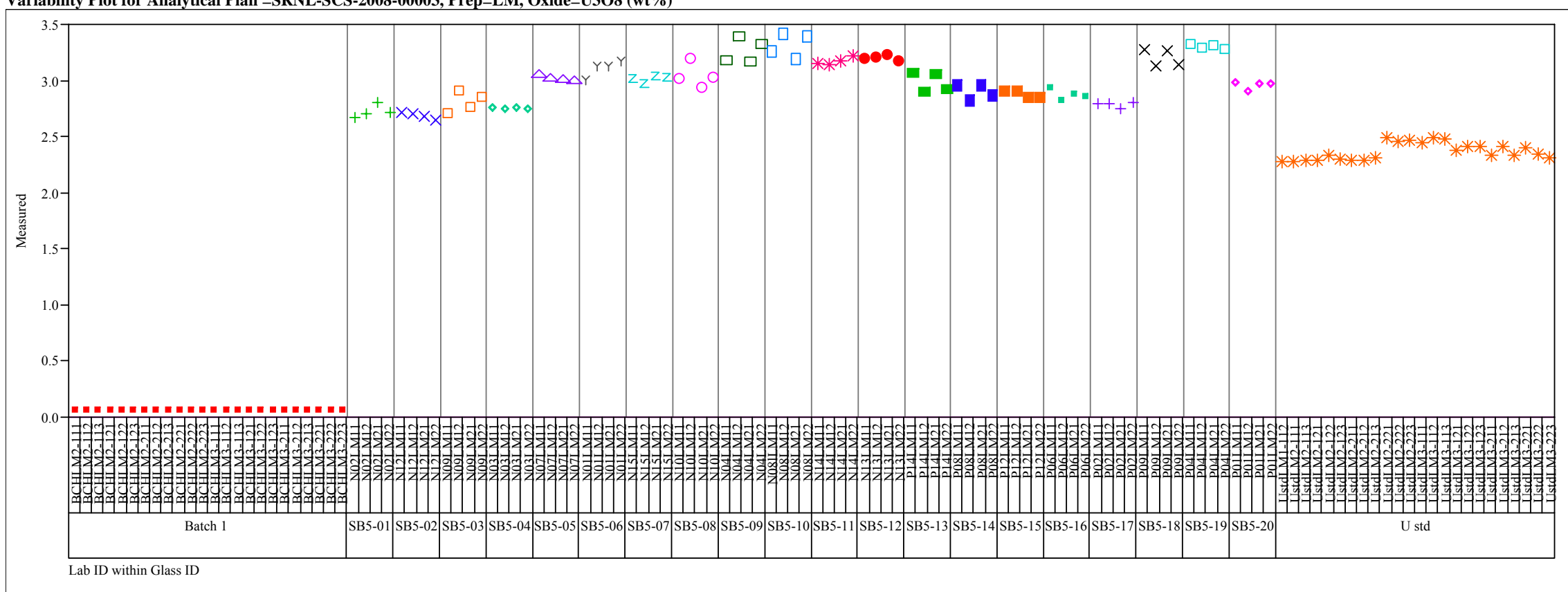


Exhibit A2. Oxide Measurements by Lab ID within Glass ID for Samples by Prep Method and Analytical Plan Memo. (continued) Variability Plot for Analytical Plan =SRNL-SCS-2008-00003, Prep=LM, Oxide=ZnO (wt\%)

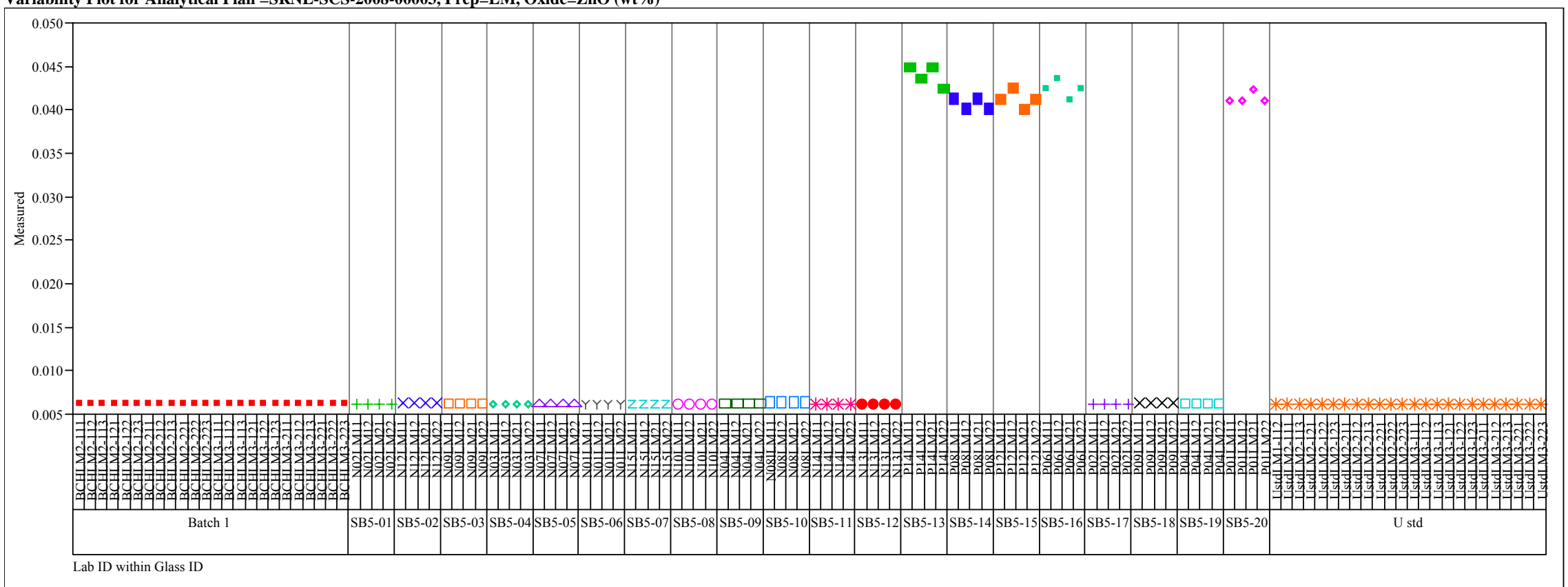


Exhibit A2. Oxide Measurements by Lab ID within Glass ID for Samples by Prep Method and Analytical Plan Memo. (continued) Variability Plot for Analytical Plan =SRNL-SCS-2008-00003, Prep=LM, Oxide=ZrO2 (wt\%)

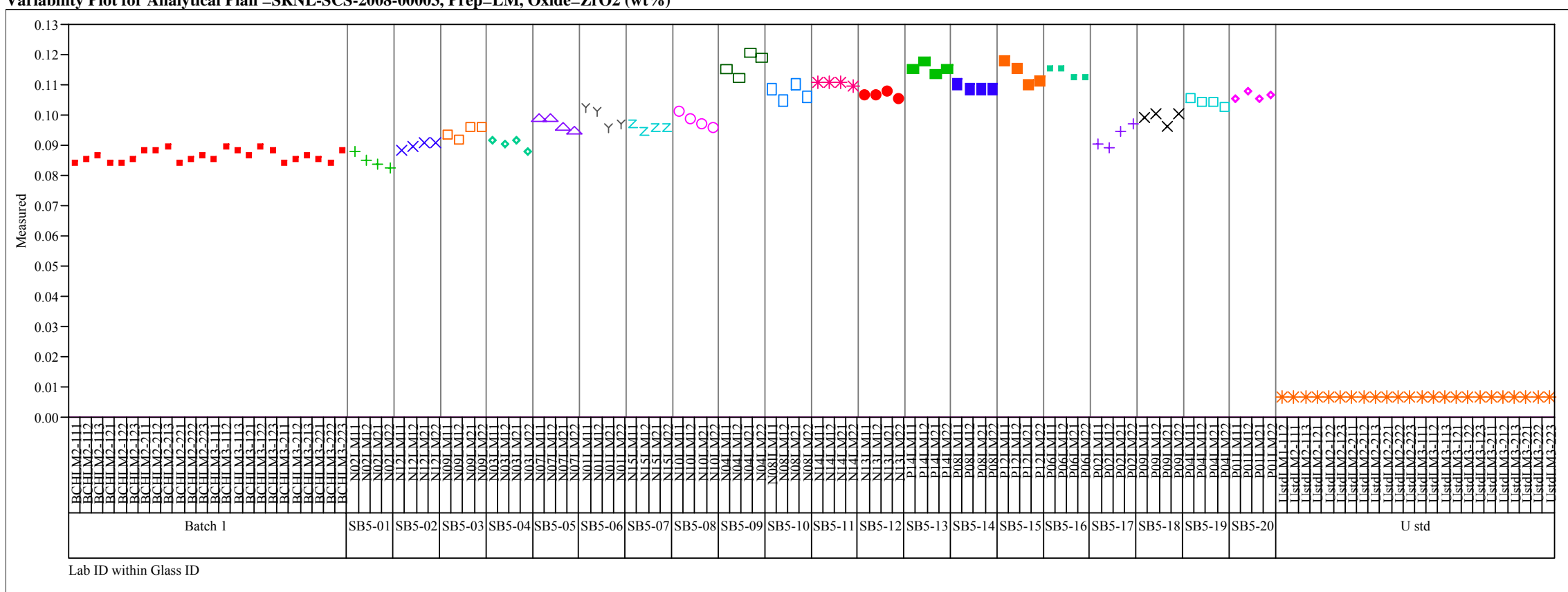


Exhibit A2. Oxide Measurements by Lab ID within Glass ID for Samples by Prep Method and Analytical Plan Memo. (continued) Variability Plot for Analytical Plan =SRNL-SCS-2008-00003, Prep=PF, Oxide=B2O3 (wt \%)

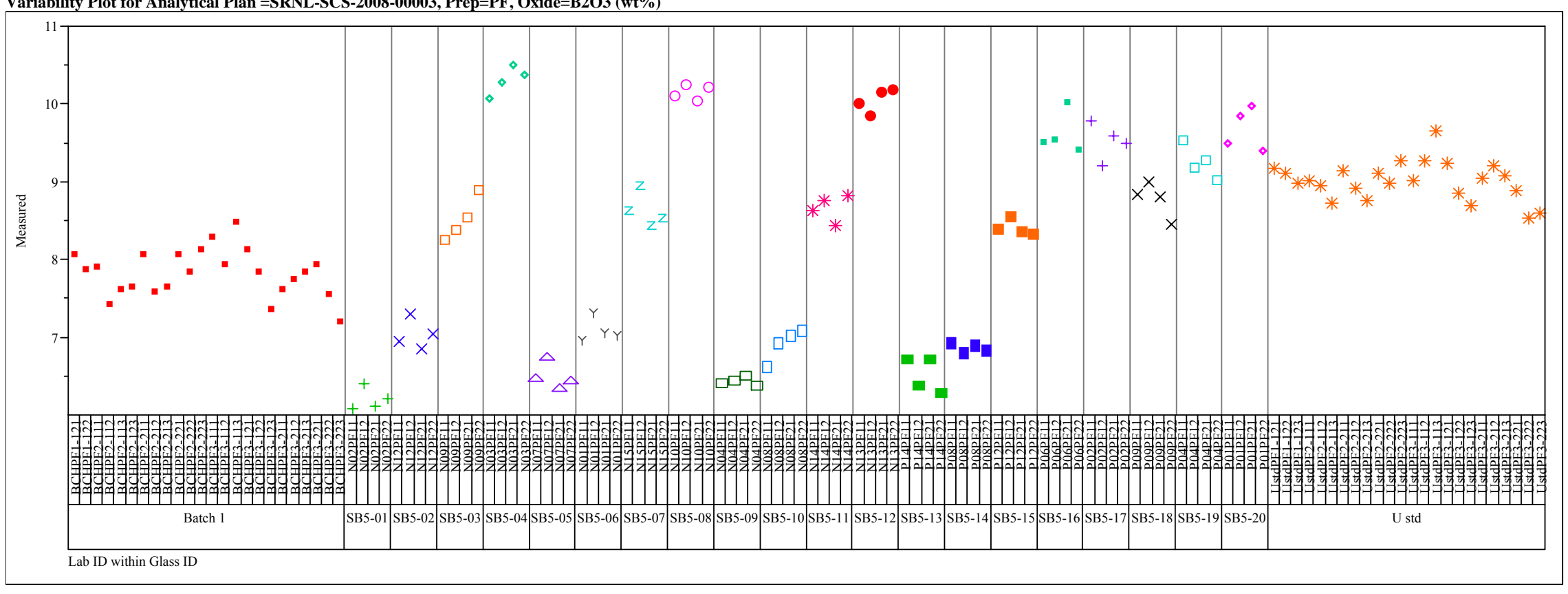


Exhibit A2. Oxide Measurements by Lab ID within Glass ID for Samples by Prep Method and Analytical Plan Memo. (continued) Variability Plot for Analytical Plan =SRNL-SCS-2008-00003, Prep=PF, Oxide=Li2O (wt \%)

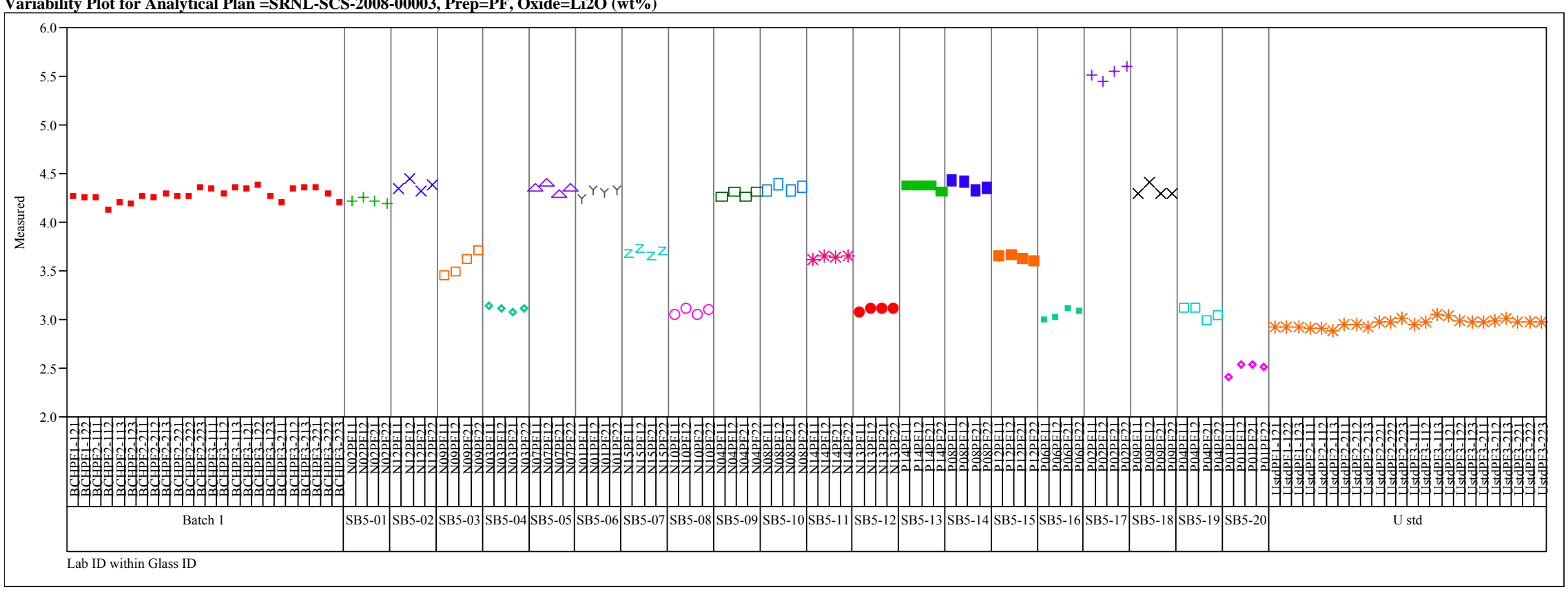


Exhibit A2. Oxide Measurements by Lab ID within Glass ID for Samples by Prep Method and Analytical Plan Memo. (continued) Variability Plot for Analytical Plan =SRNL-SCS-2008-00043, Prep=LM, Oxide=Al2O3 (wt\%)

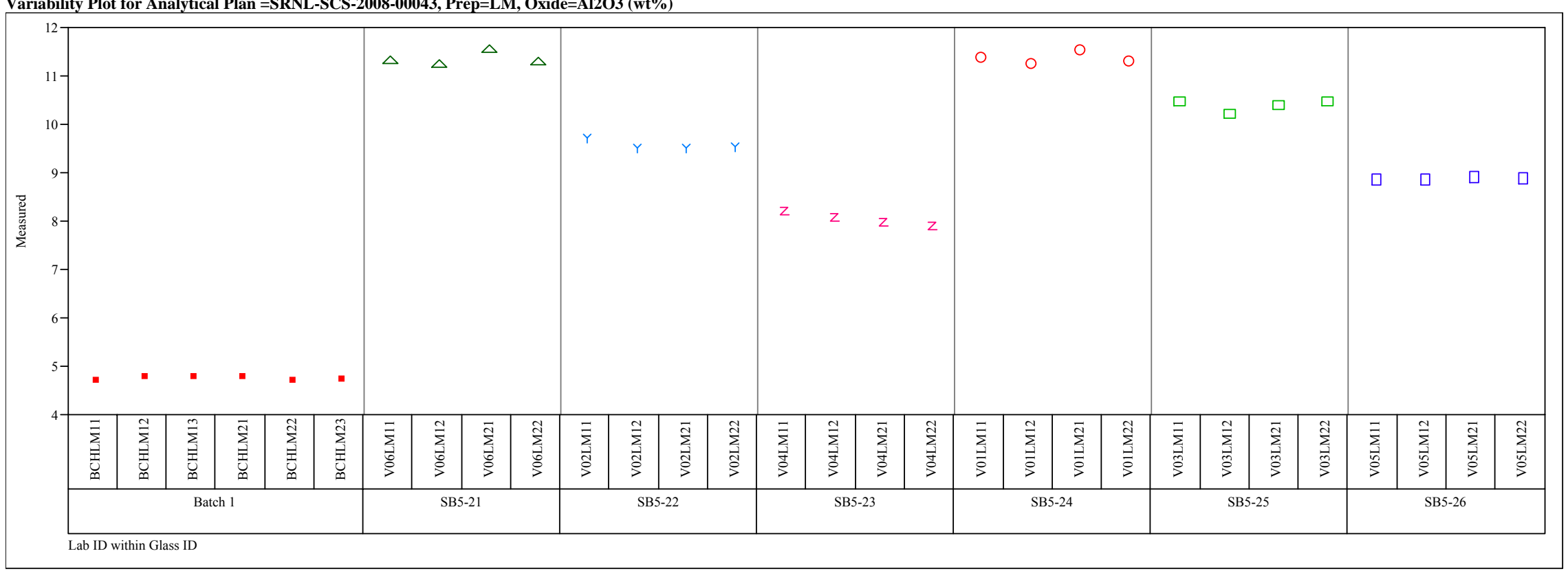


Exhibit A2. Oxide Measurements by Lab ID within Glass ID for Samples by Prep Method and Analytical Plan Memo. (continued)

Variability Plot for Analytical Plan =SRNL-SCS-2008-00043, Prep=LM, Oxide=BaO (wt\%)

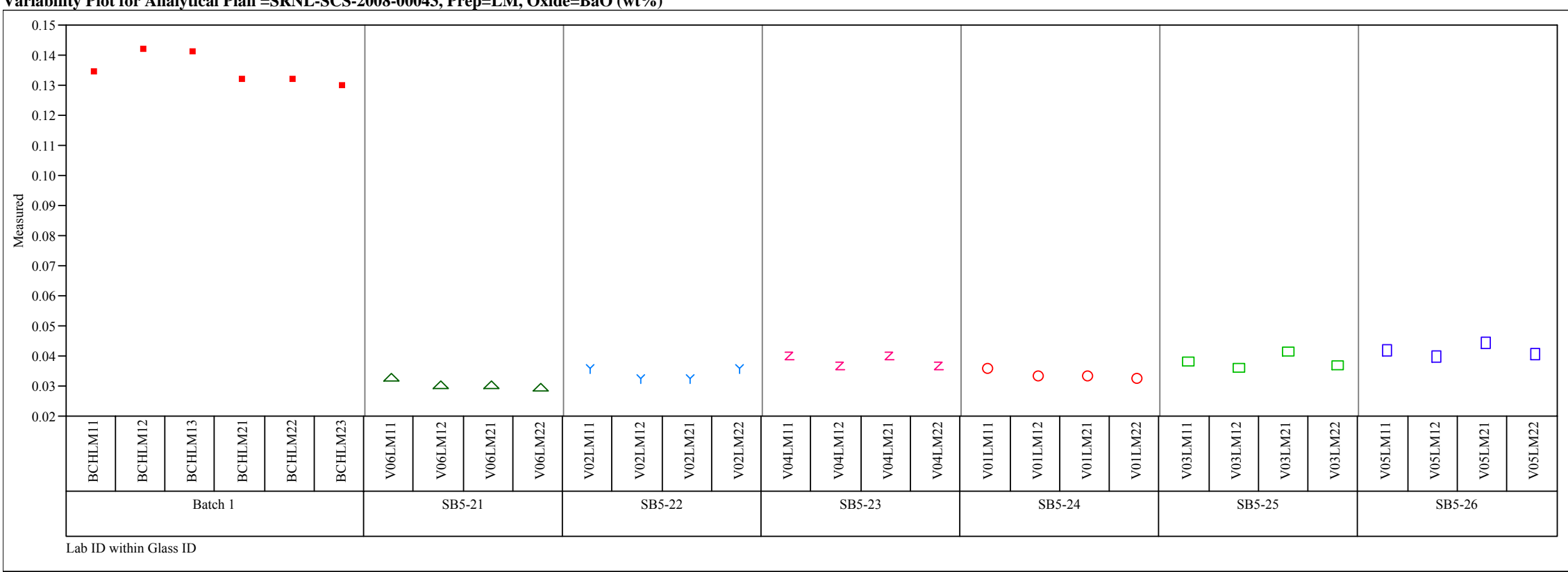


Exhibit A2. Oxide Measurements by Lab ID within Glass ID for Samples by Prep Method and Analytical Plan Memo. (continued) Variability Plot for Analytical Plan =SRNL-SCS-2008-00043, Prep=LM, Oxide=CaO (wt\%)

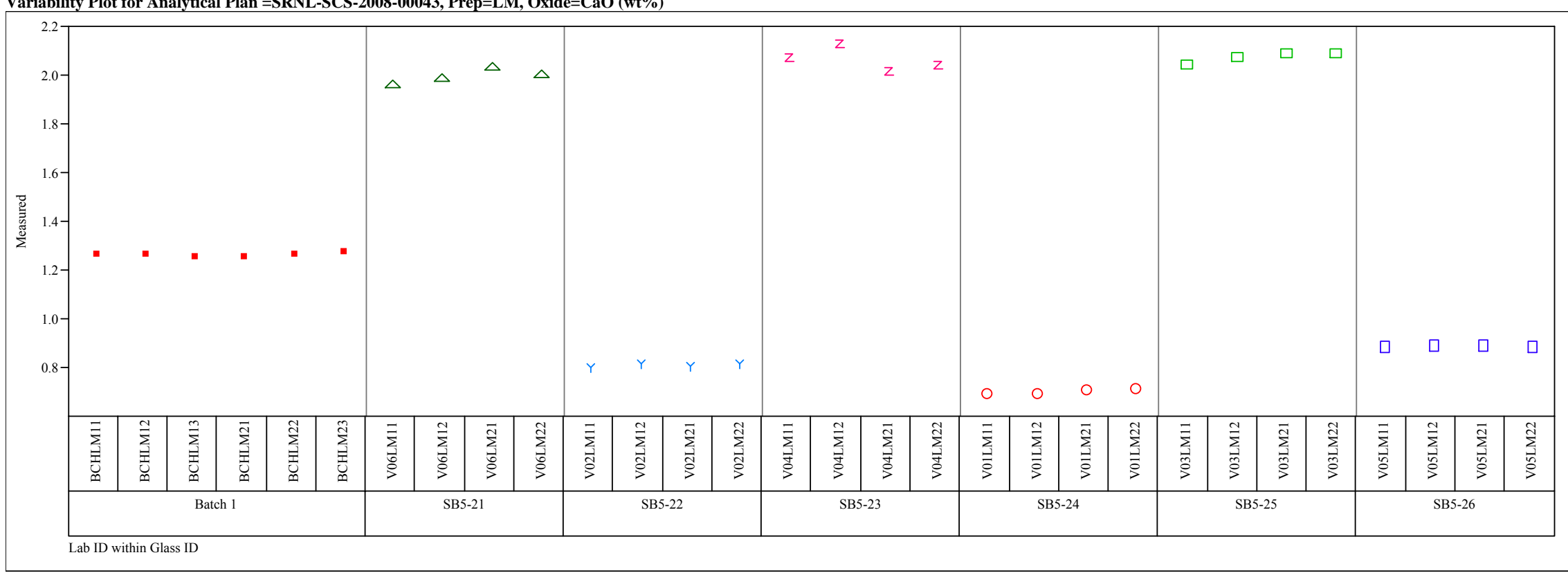


Exhibit A2. Oxide Measurements by Lab ID within Glass ID for Samples by Prep Method and Analytical Plan Memo. (continued) Variability Plot for Analytical Plan =SRNL-SCS-2008-00043, Prep=LM, Oxide=Ce2O3 (wt \%)

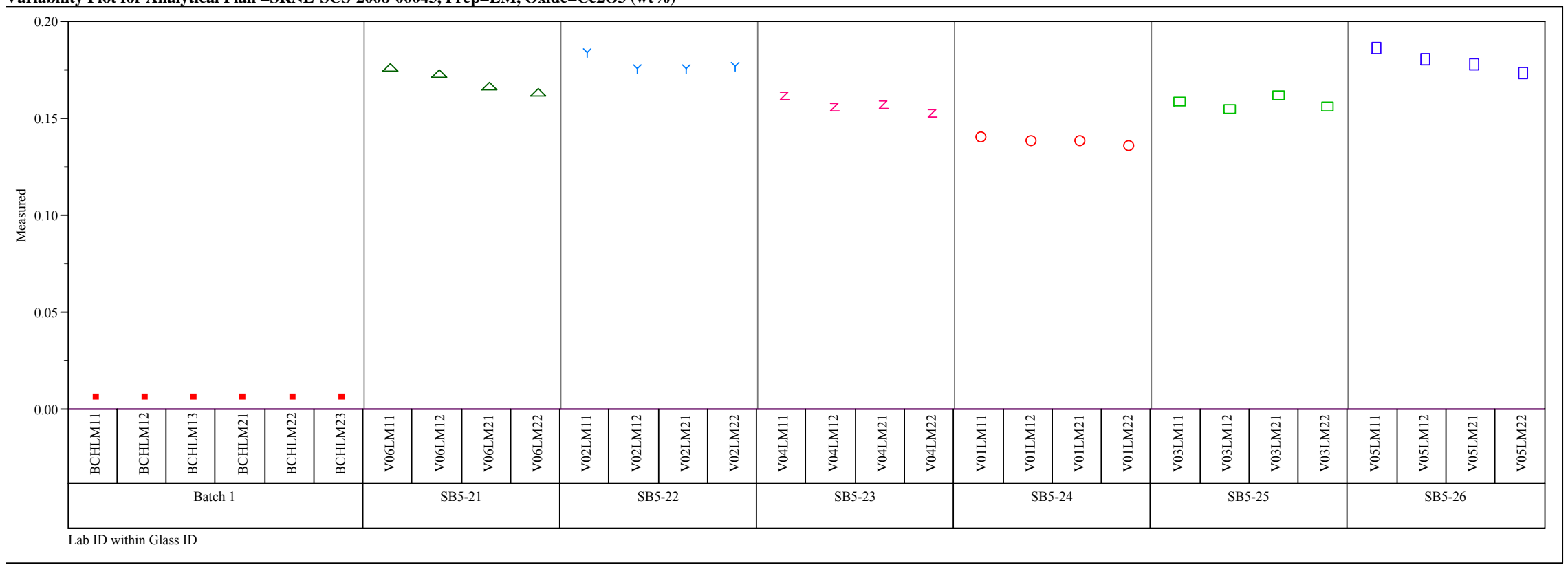


Exhibit A2. Oxide Measurements by Lab ID within Glass ID for Samples by Prep Method and Analytical Plan Memo. (continued) Variability Plot for Analytical Plan $=$ SRNL-SCS-2008-00043, Prep $=$ LM, Oxide=Cr2O3 (wt\%)

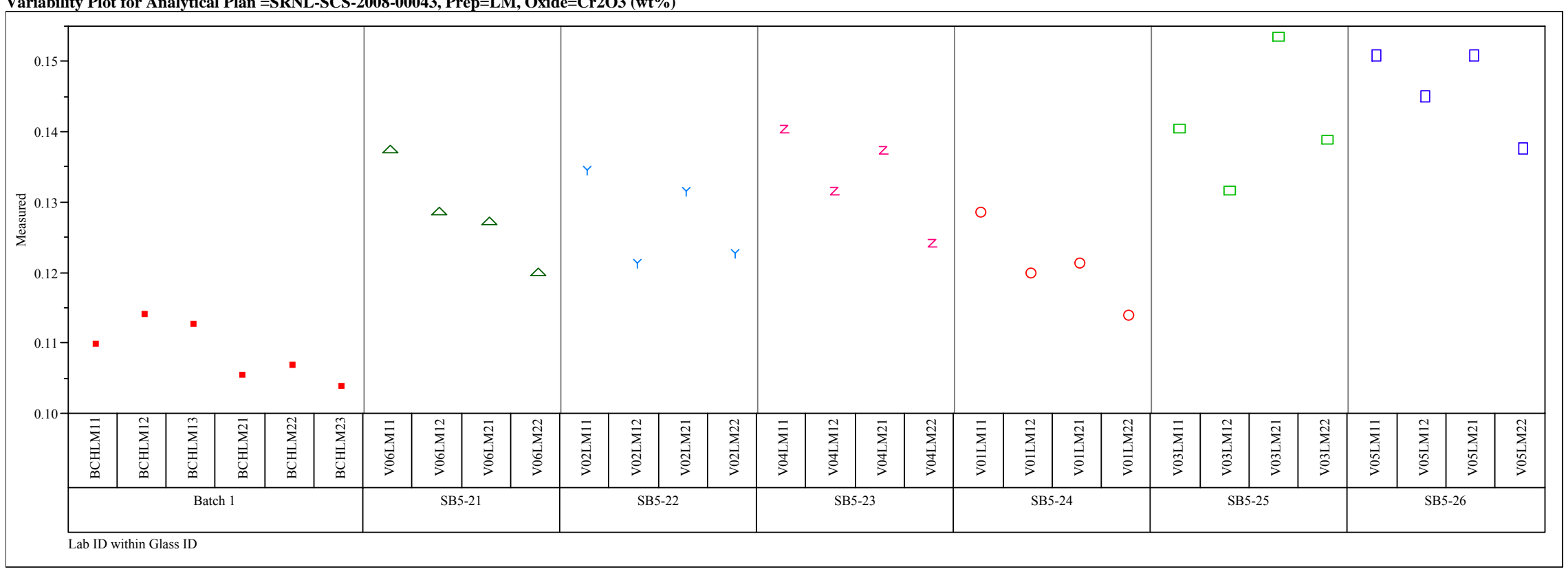


Exhibit A2. Oxide Measurements by Lab ID within Glass ID for Samples by Prep Method and Analytical Plan Memo. (continued) Variability Plot for Analytical Plan $=$ SRNL-SCS-2008-00043, Prep $=$ LM, Oxide=Fe2O3 (wt\%)

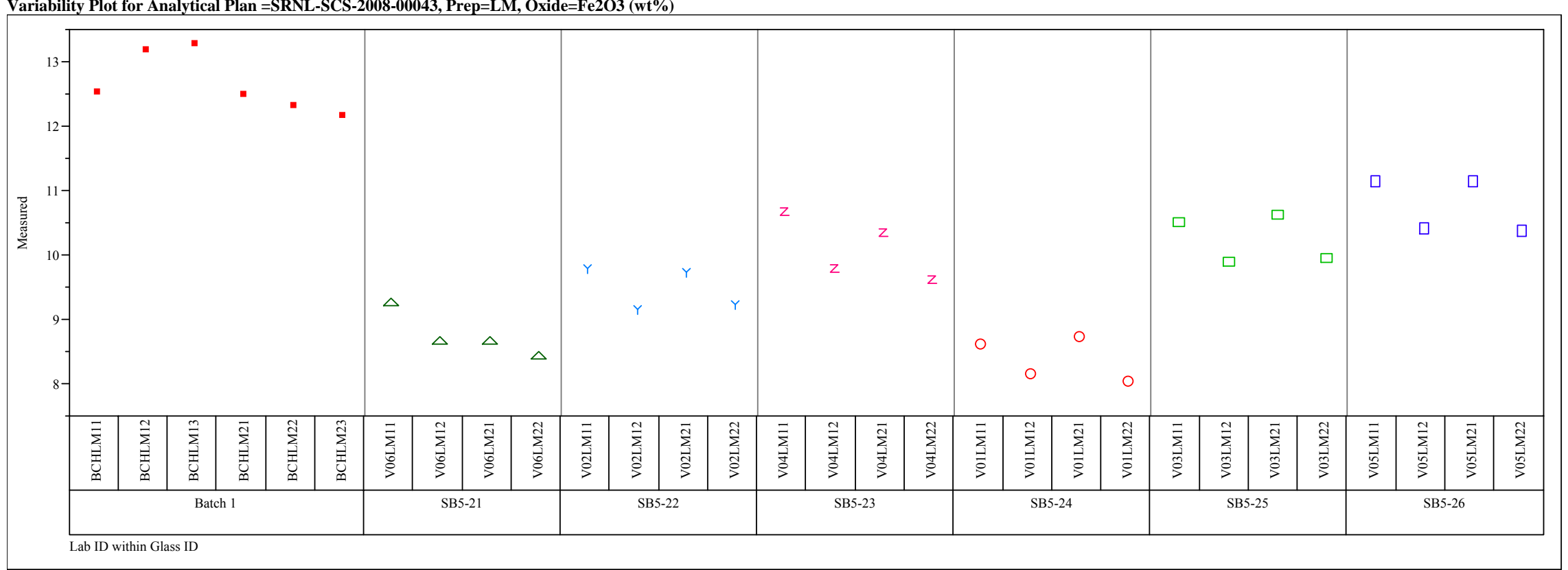


Exhibit A2. Oxide Measurements by Lab ID within Glass ID for Samples by Prep Method and Analytical Plan Memo. (continued) Variability Plot for Analytical Plan =SRNL-SCS-2008-00043, Prep=LM, Oxide=HfO2 (wt\%)

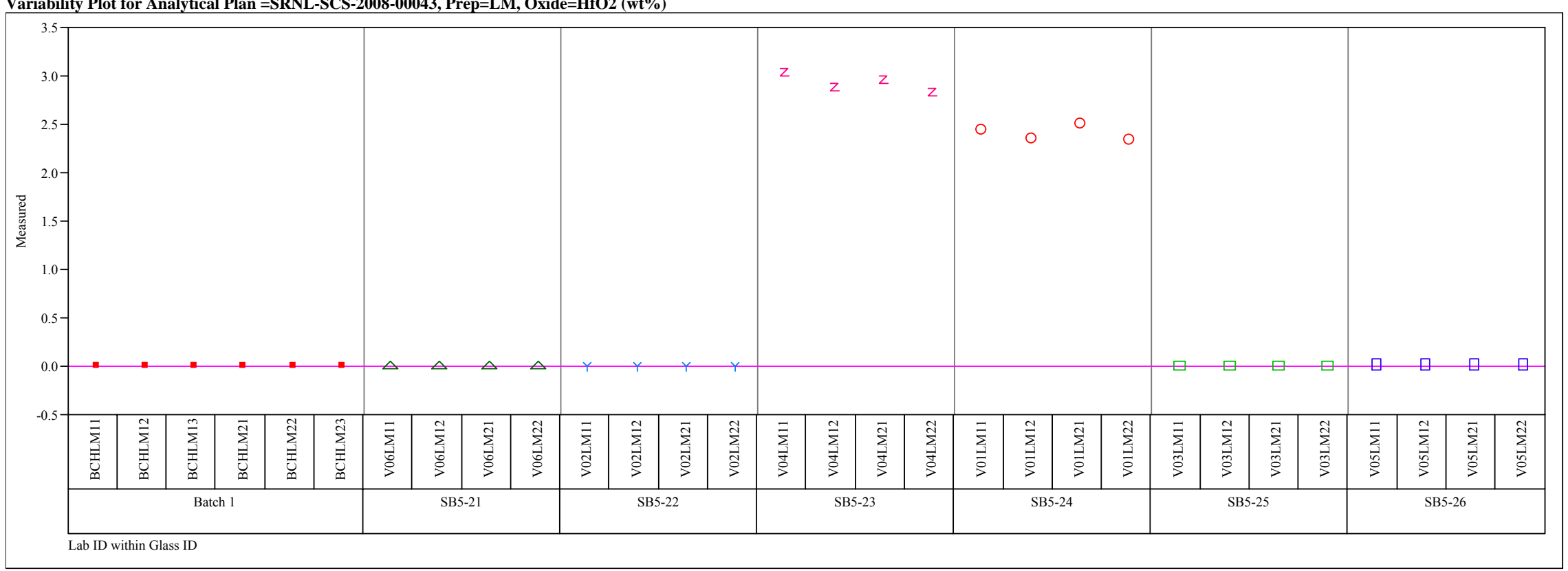


Exhibit A2. Oxide Measurements by Lab ID within Glass ID for Samples by Prep Method and Analytical Plan Memo. (continued) Variability Plot for Analytical Plan =SRNL-SCS-2008-00043, Prep=LM, Oxide=K2O (wt\%)

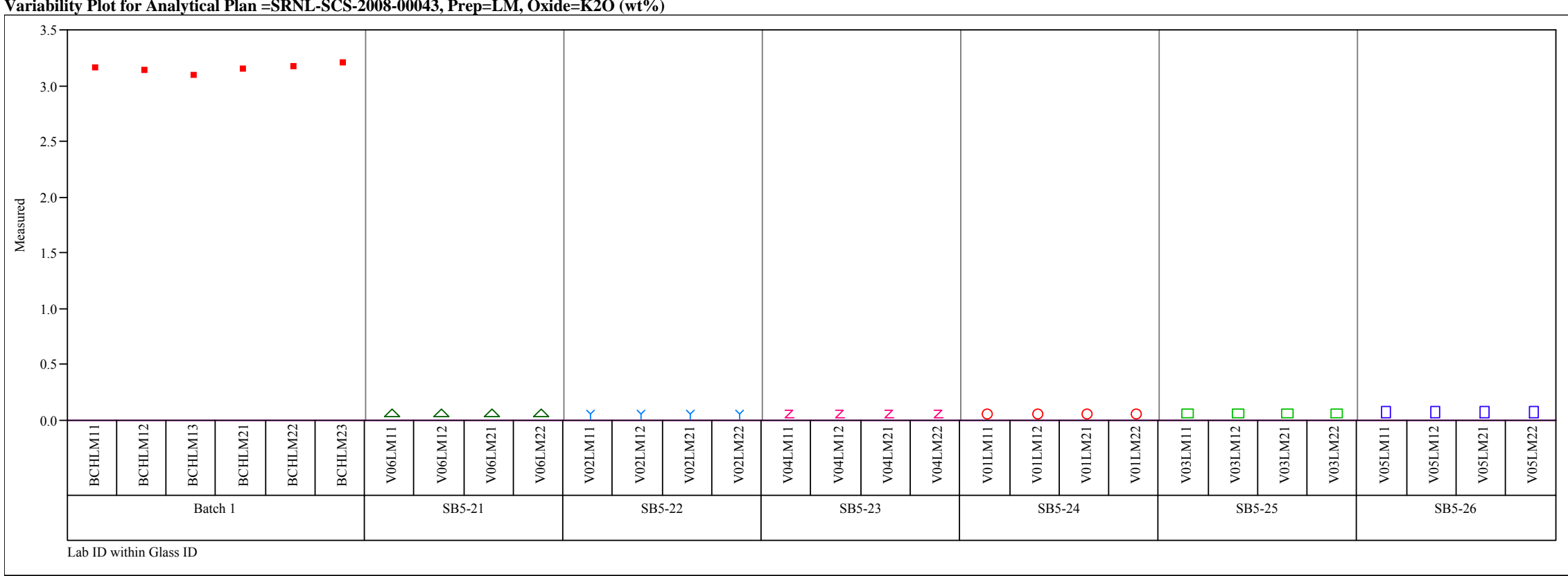


Exhibit A2. Oxide Measurements by Lab ID within Glass ID for Samples by Prep Method and Analytical Plan Memo. (continued) Variability Plot for Analytical Plan =SRNL-SCS-2008-00043, Prep=LM, Oxide=La2O3 (wt \%)

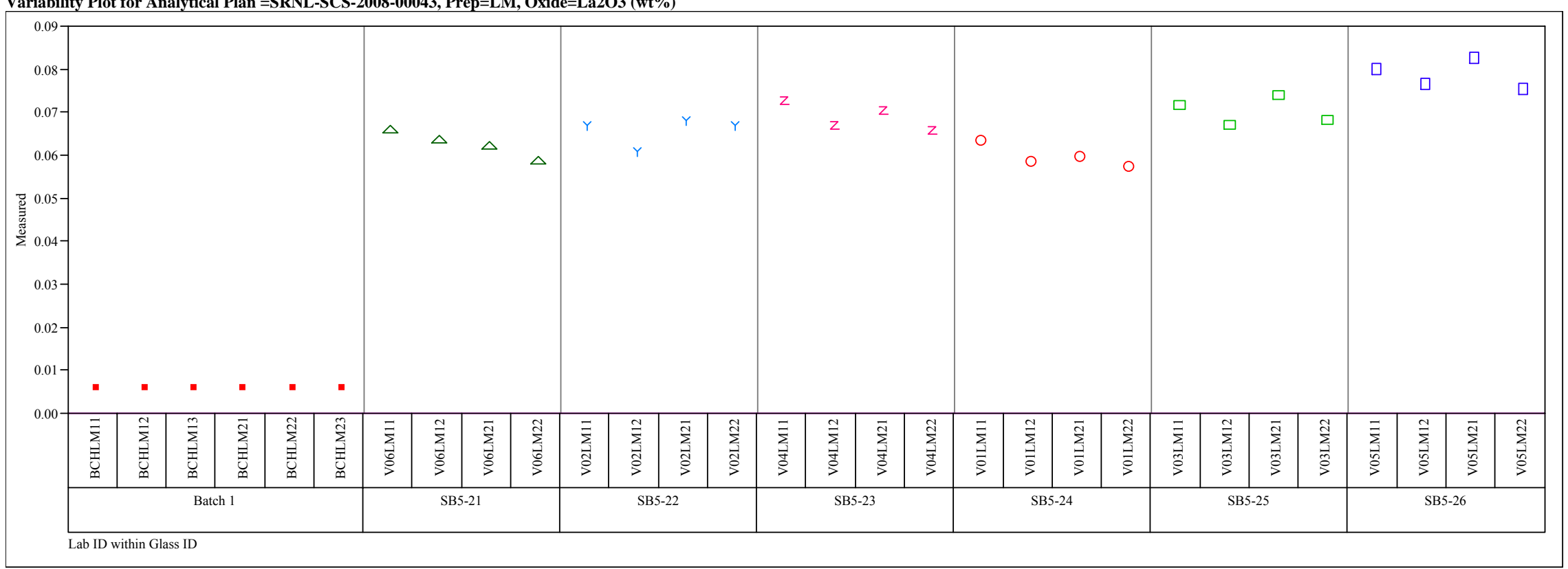


Exhibit A2. Oxide Measurements by Lab ID within Glass ID for Samples by Prep Method and Analytical Plan Memo. (continued) Variability Plot for Analytical Plan =SRNL-SCS-2008-00043, Prep=LM, Oxide=MgO (wt\%)

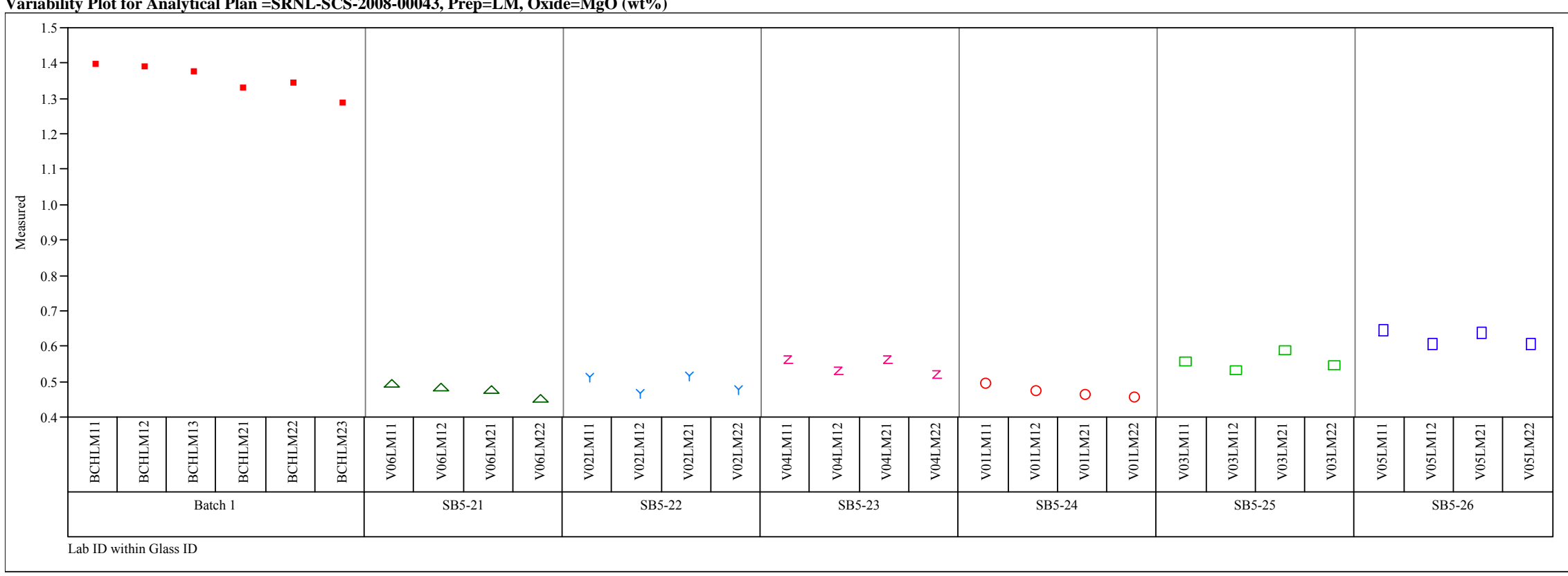


Exhibit A2. Oxide Measurements by Lab ID within Glass ID for Samples by Prep Method and Analytical Plan Memo. (continued) Variability Plot for Analytical Plan =SRNL-SCS-2008-00043, Prep=LM, Oxide=MnO (wt \%)

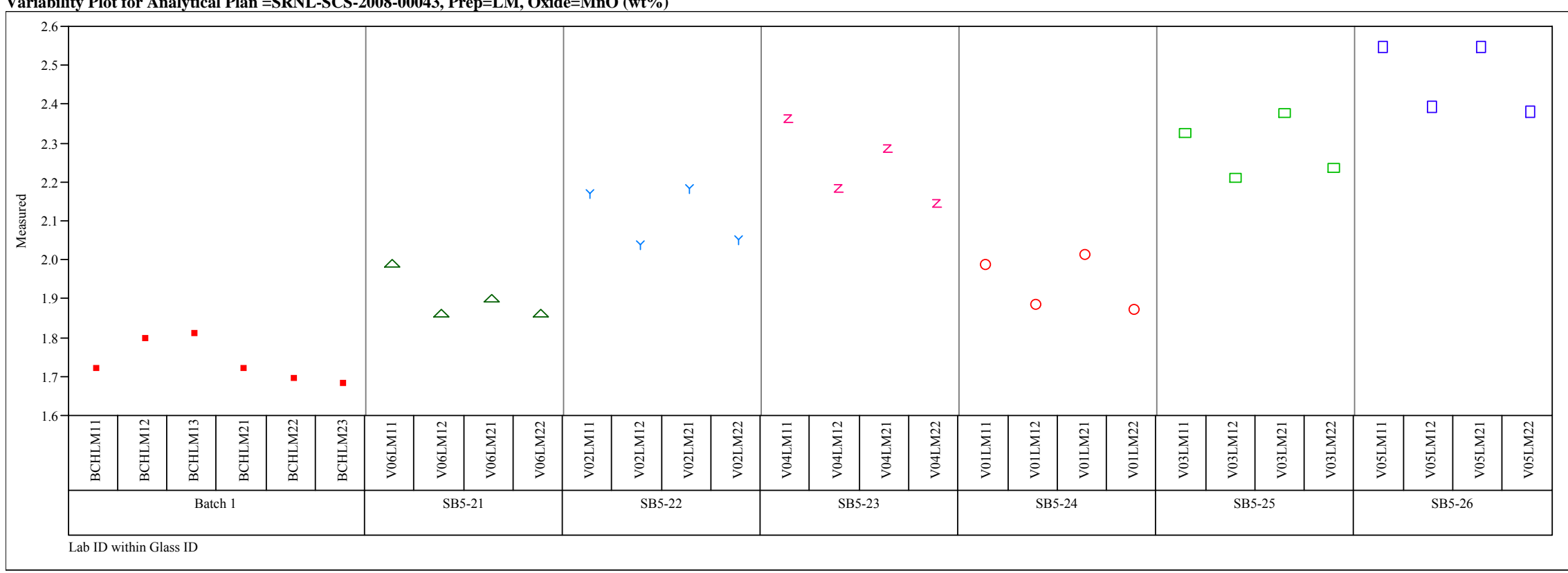


Exhibit A2. Oxide Measurements by Lab ID within Glass ID for Samples by Prep Method and Analytical Plan Memo. (continued) Variability Plot for Analytical Plan =SRNL-SCS-2008-00043, Prep=LM, Oxide=Na2O (wt\%)

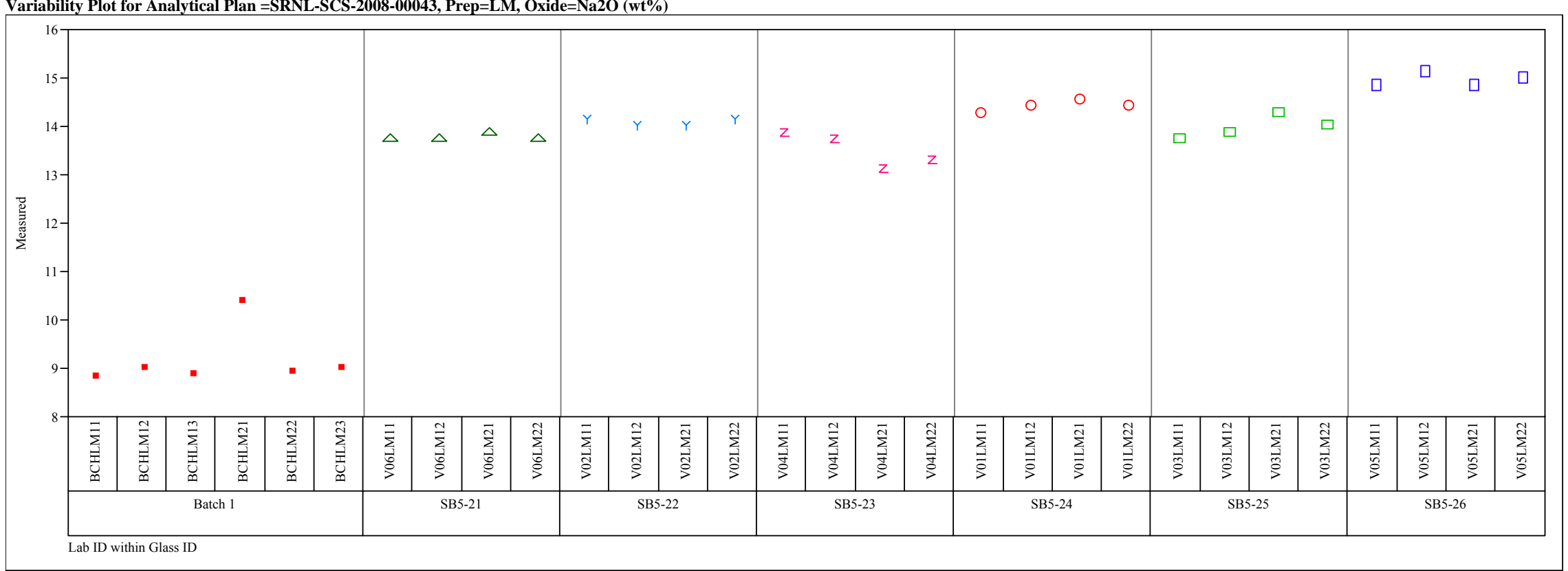


Exhibit A2. Oxide Measurements by Lab ID within Glass ID for Samples by Prep Method and Analytical Plan Memo. (continued) Variability Plot for Analytical Plan =SRNL-SCS-2008-00043, Prep=LM, Oxide=Nd2O3 (wt \%)

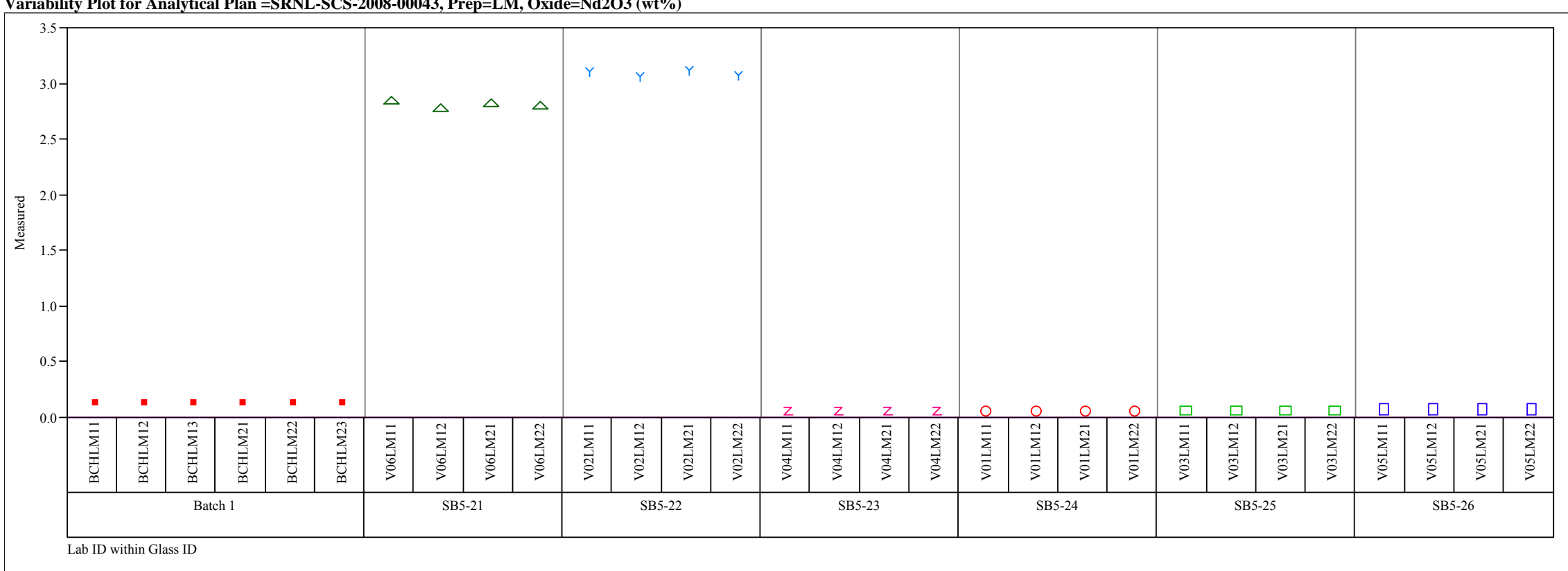


Exhibit A2. Oxide Measurements by Lab ID within Glass ID for Samples by Prep Method and Analytical Plan Memo. (continued) Variability Plot for Analytical Plan =SRNL-SCS-2008-00043, Prep=LM, Oxide=NiO (wt \%)

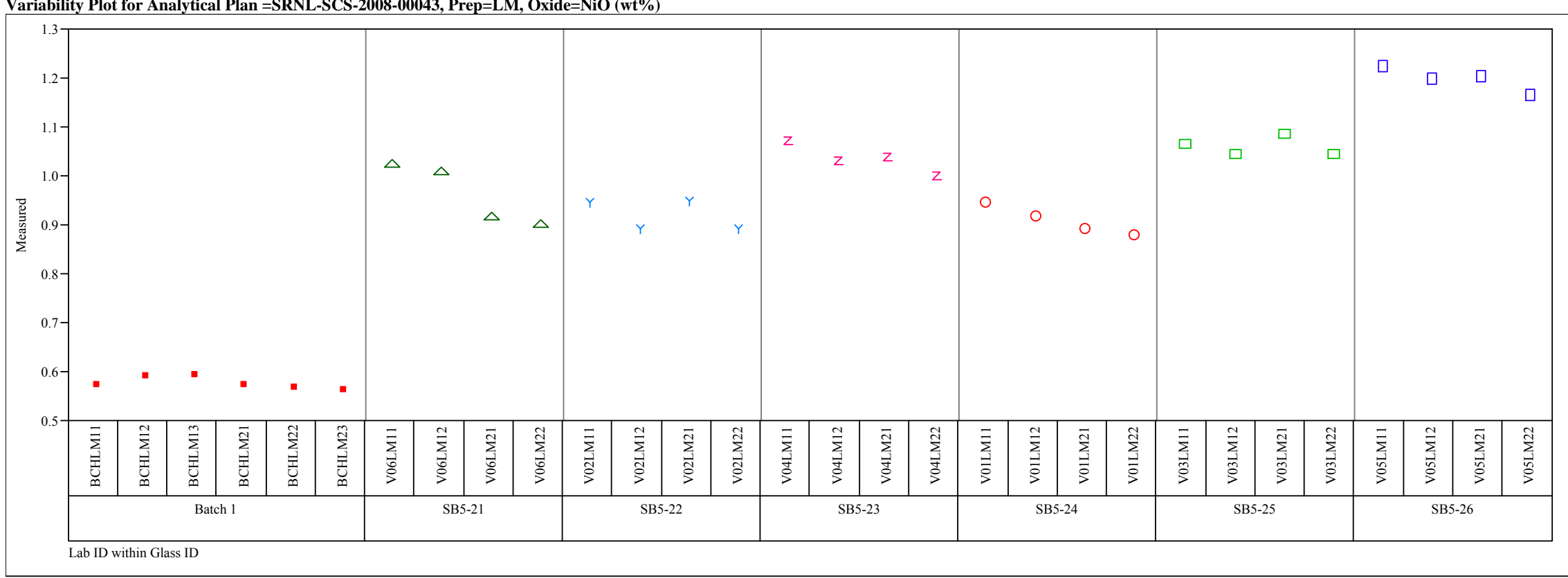


Exhibit A2. Oxide Measurements by Lab ID within Glass ID for Samples by Prep Method and Analytical Plan Memo. (continued)

Variability Plot for Analytical Plan =SRNL-SCS-2008-00043, Prep=LM, Oxide=PbO (wt \%)

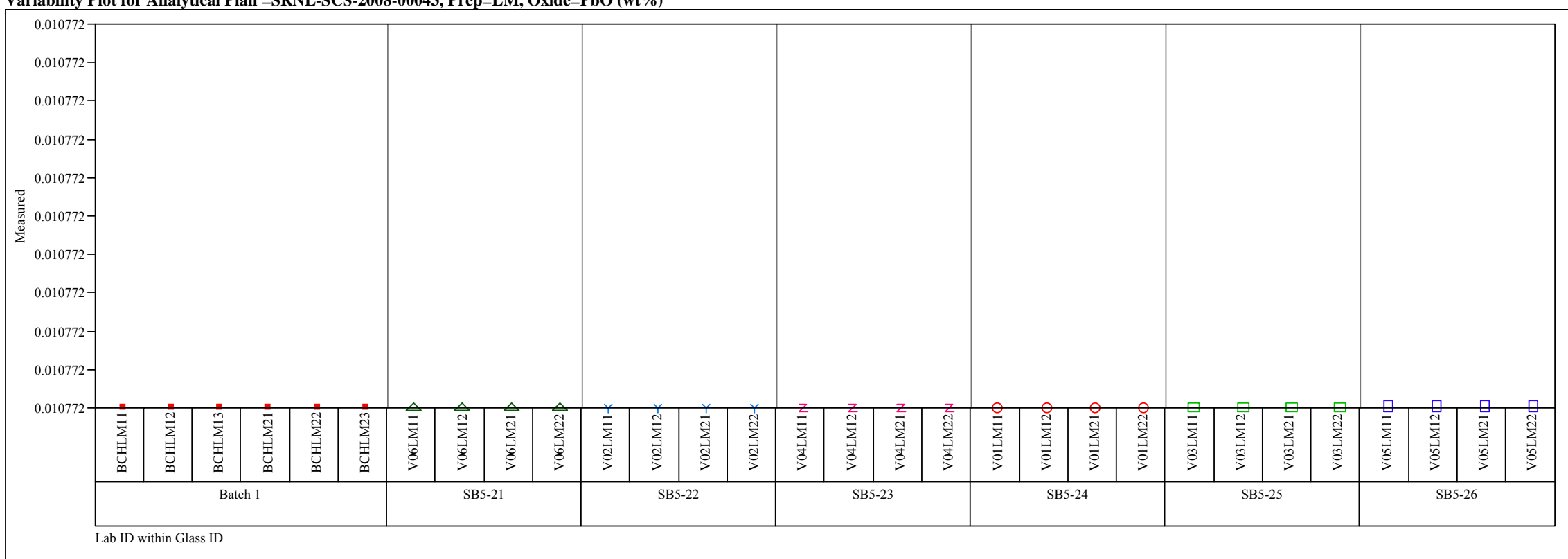


Exhibit A2. Oxide Measurements by Lab ID within Glass ID for Samples by Prep Method and Analytical Plan Memo. (continued) Variability Plot for Analytical Plan =SRNL-SCS-2008-00043, Prep=LM, Oxide=SiO2 (wt \%)

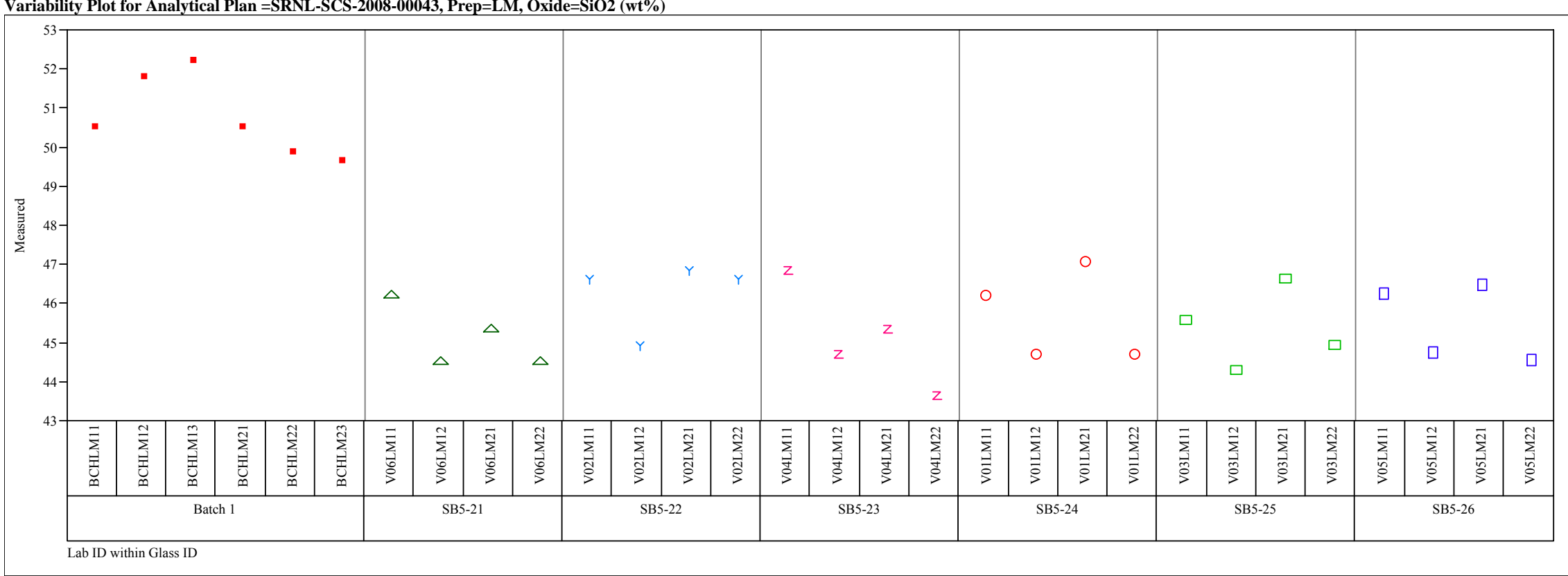


Exhibit A2. Oxide Measurements by Lab ID within Glass ID for Samples by Prep Method and Analytical Plan Memo. (continued) Variability Plot for Analytical Plan =SRNL-SCS-2008-00043, Prep=LM, Oxide=SO4 (wt\%)

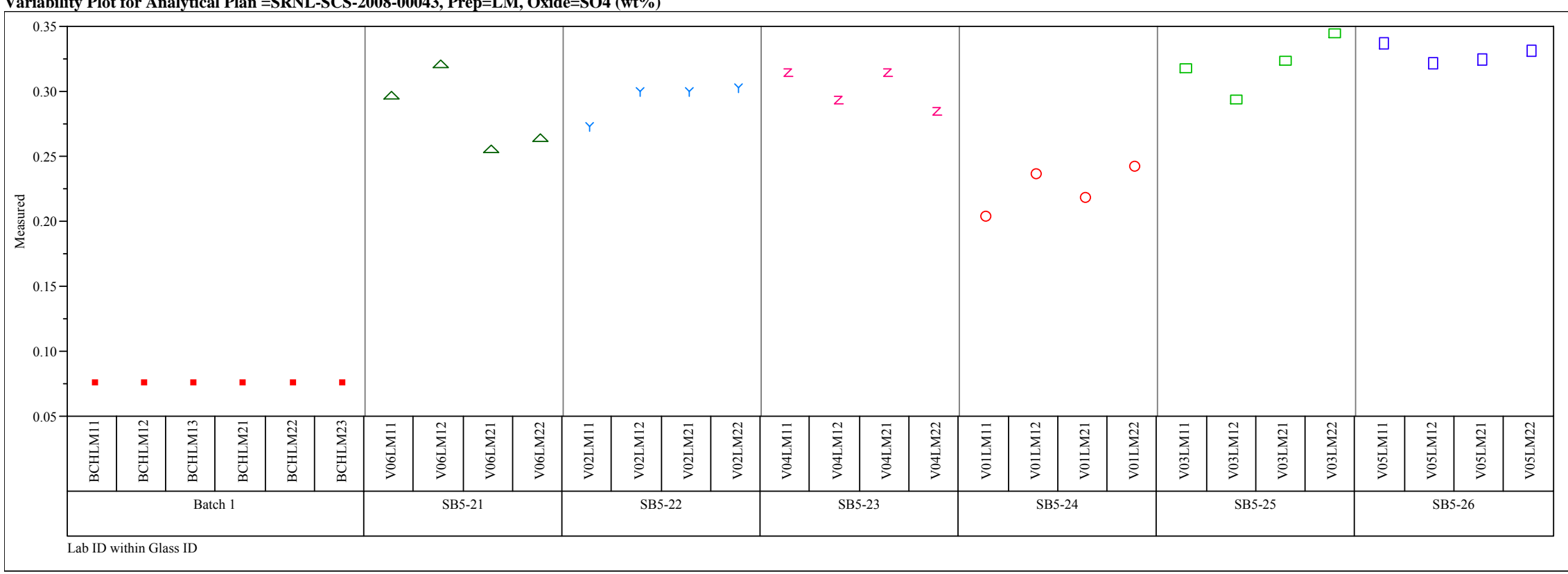


Exhibit A2. Oxide Measurements by Lab ID within Glass ID for Samples by Prep Method and Analytical Plan Memo. (continued) Variability Plot for Analytical Plan =SRNL-SCS-2008-00043, Prep=LM, Oxide=TiO2 (wt\%)

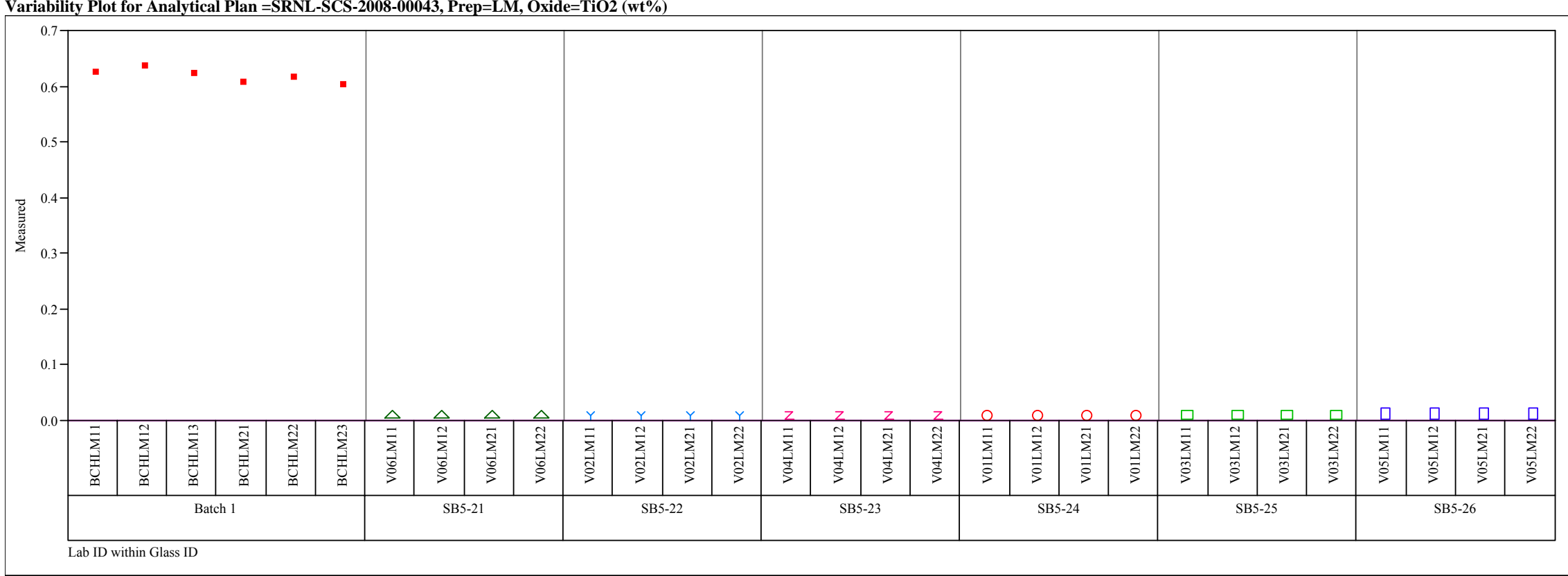


Exhibit A2. Oxide Measurements by Lab ID within Glass ID for Samples by Prep Method and Analytical Plan Memo. (continued)

Variability Plot for Analytical Plan =SRNL-SCS-2008-00043, Prep=LM, Oxide=ZnO (wt\%)

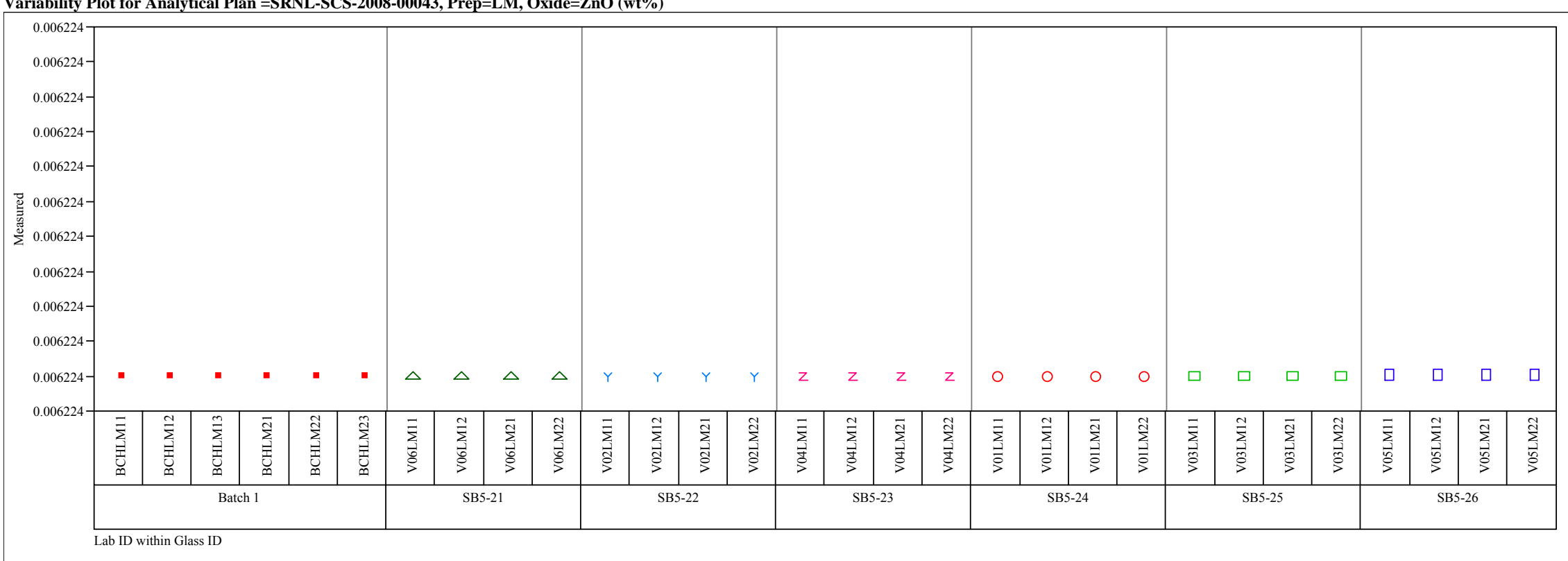


Exhibit A2. Oxide Measurements by Lab ID within Glass ID for Samples by Prep Method and Analytical Plan Memo. (continued) Variability Plot for Analytical Plan =SRNL-SCS-2008-00043, Prep=LM, Oxide=ZrO2 (wt\%)

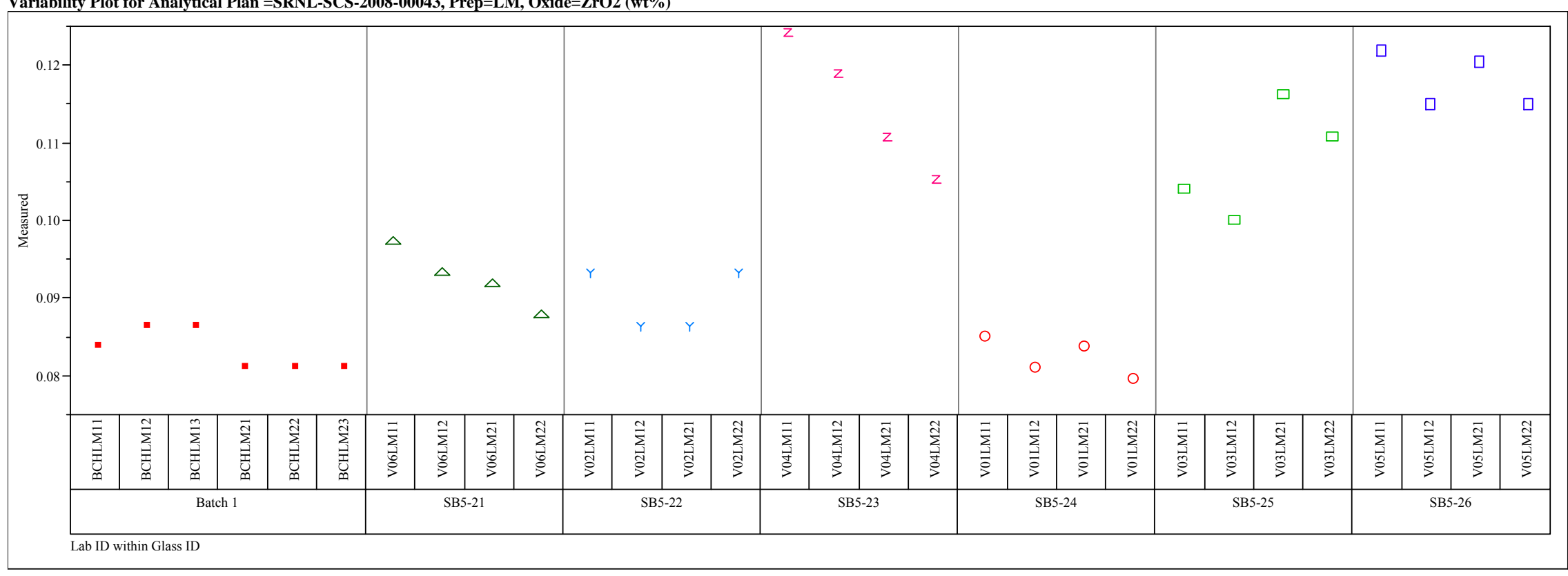


Exhibit A2. Oxide Measurements by Lab ID within Glass ID for Samples by Prep Method and Analytical Plan Memo. (continued) Variability Plot for Analytical Plan =SRNL-SCS-2008-00043, Prep=PF, Oxide=B2O3 (wt\%)

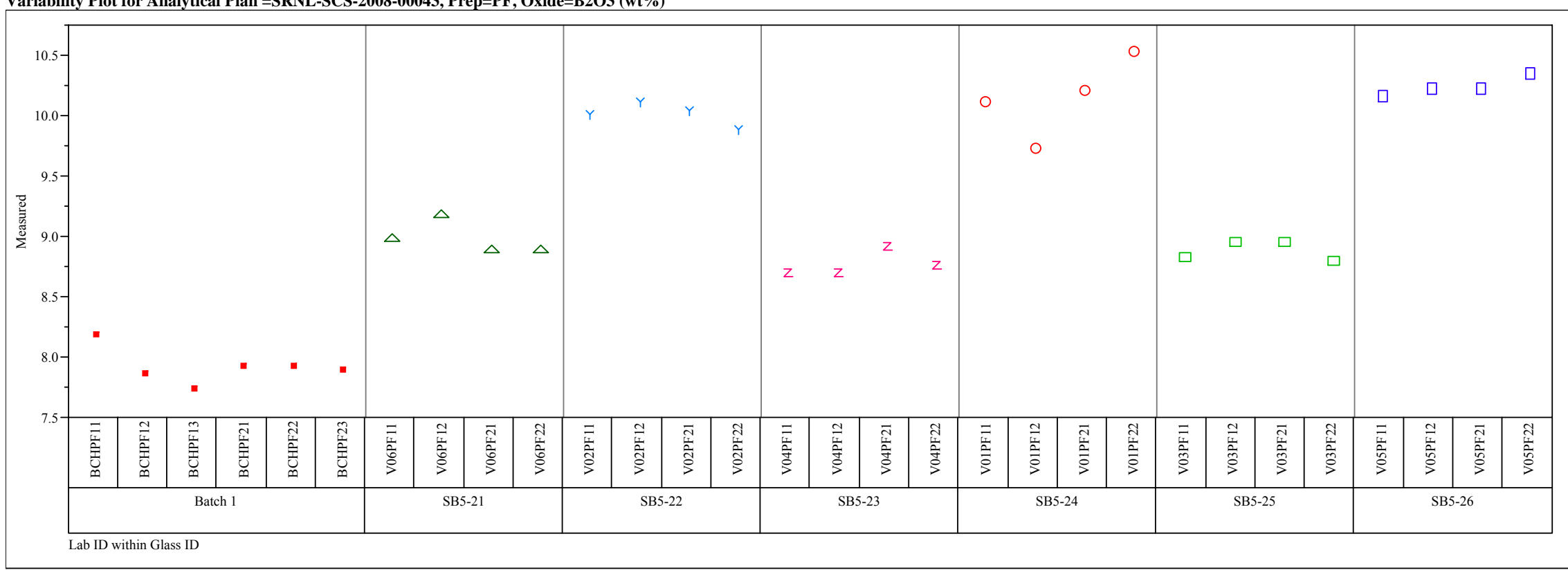


Exhibit A2. Oxide Measurements by Lab ID within Glass ID for Samples by Prep Method and Analytical Plan Memo. (continued) Variability Plot for Analytical Plan =SRNL-SCS-2008-00043, Prep=PF, Oxide=Li2O (wt\%)

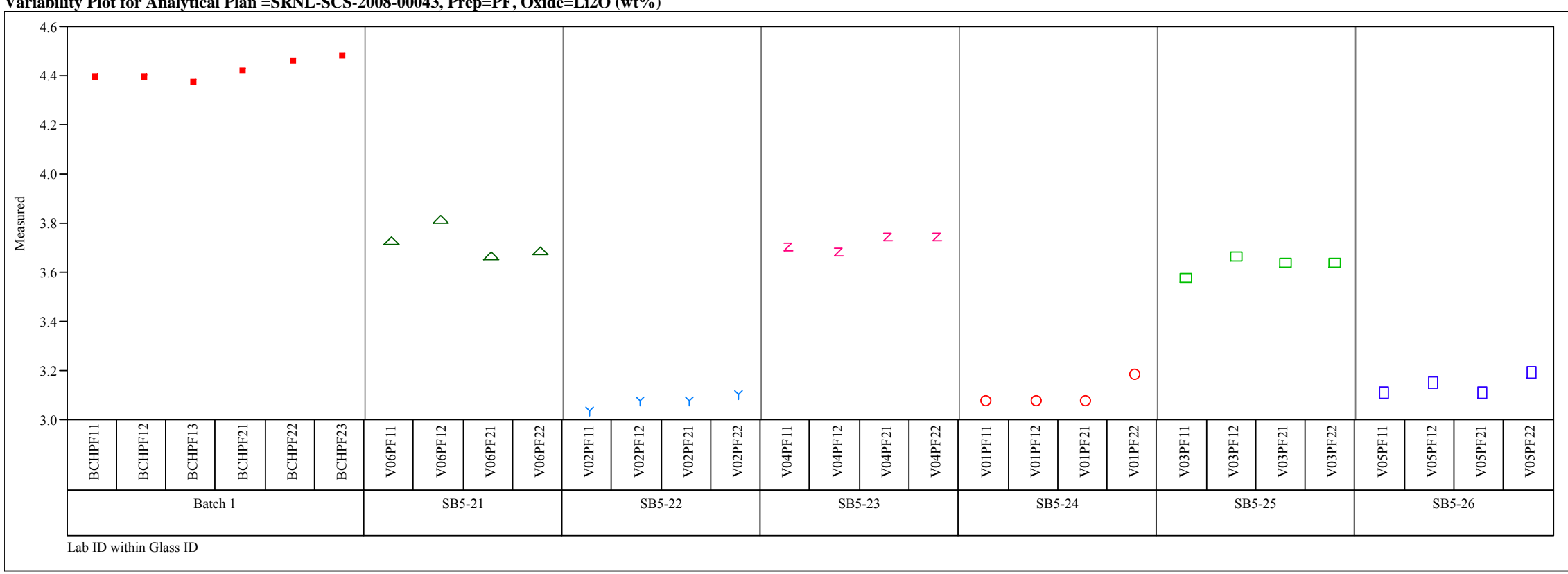


Exhibit A3. PSAL Measurements by ICP Calibration Block for Samples of the

Standard Glasses by Preparation Method by Set by Analytical Plan Memo.

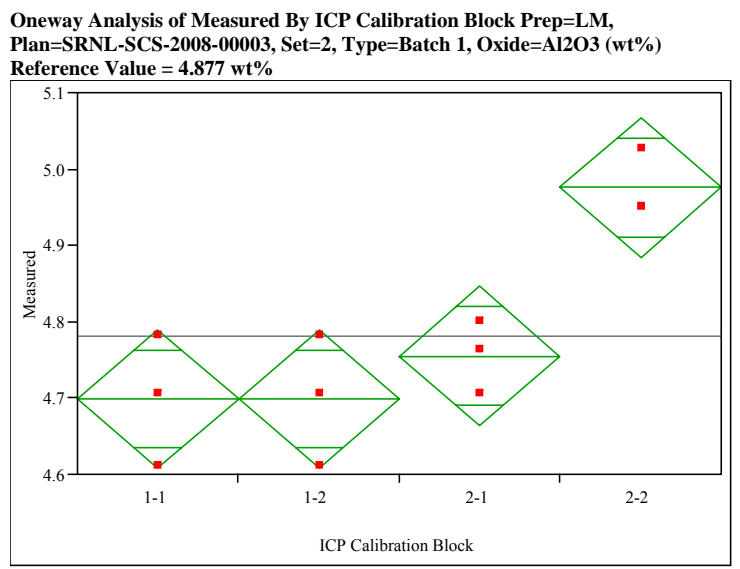

\section{Oneway Anova
Summary of Fit}

Oneway Anova
Summary of Fit

$$
\begin{array}{lr}
\text { Rsquare } & 0.807214 \\
\text { Adj Rsquare } & 0.734919 \\
\text { Root Mean Square Error } & 0.068345 \\
\text { Mean of Response } & 4.78201 \\
\text { Observations (or Sum Wgts) } & 12
\end{array}
$$

Analysis of Variance

$\begin{array}{lrrrrr}\text { Source } & \text { DF } & \text { Sum of Squares } & \text { Mean Square } & \text { F Ratio } & \text { Prob }>\text { F } \\ \text { ICP Calibration Block } & 3 & 0.15646446 & 0.052155 & 11.1656 & 0.0031\end{array}$

Error

$\begin{array}{lllll}3 & 0.15646446 & 0.052155 & 11.1656 & 0.0031 \\ 8 & 0.03736820 & 0.004671 & & \end{array}$

C. Tota

$11 \quad 0.19383266$

\begin{tabular}{lrrrrr}
\multicolumn{2}{c}{ Means for Oneway Anova } & & \\
Level & Number & Mean & Std Error & Lower $95 \%$ & Upper $95 \%$ \\
$1-1$ & 3 & 4.69856 & 0.03946 & 4.6076 & 4.7895 \\
$1-2$ & 3 & 4.69856 & 0.03946 & 4.6076 & 4.7895 \\
$2-1$ & 3 & 4.75524 & 0.03946 & 4.6642 & 4.8462 \\
$2-2$ & 3 & 4.97568 & 0.03946 & 4.8847 & 5.0667
\end{tabular}

Std Error uses a pooled estimate of error variance

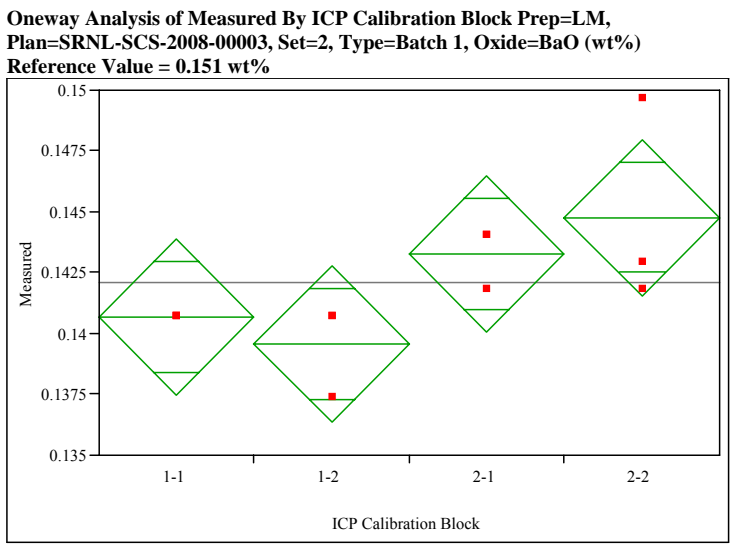

Oneway Analysis of Measured By ICP Calibration Block Prep=LM, Plan=SRNL-SCS-2008-00003, Set=2, Type=Batch 1, Oxide=CaO (wto) Reference Value $=1.220 \mathrm{wt}^{\%}$

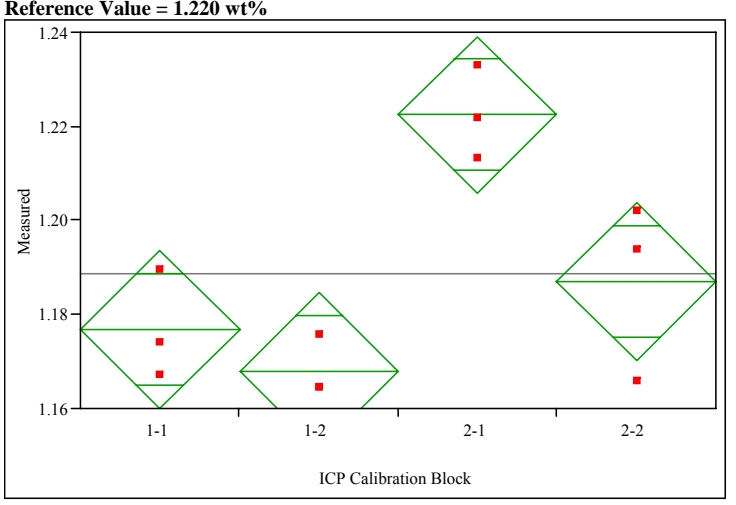

Oneway Anova

Summary of Fit

Rsquare

$\begin{array}{ll}\text { Rsquare } & 0.522897 \\ \text { Adj Rsquare } & 0.343983\end{array}$

Root Mean Square Error $\quad 0.002412$

$\begin{array}{ll}\text { Mean of Response } & 0.142075 \\ \text { Observations (or Sum W } & \end{array}$

Analysis of Variance

Source DF Sum of Squares Mean Square F Ratio Prob $>F$

$\begin{array}{lrrrrr}\text { ICP Calibration Block } & 3 & 0.00005101 & 0.000017 & 2.9226 & 0.1001 \\ \text { Error } & 8 & 0.00004654 & 5.817 \mathrm{e}-6 & & \end{array}$

Error

nova

Level Number Mean Std Error Lower 95\% Upper 95\%

$\begin{array}{lrrrrrr}1-1 & 3 & 0.140679 & 0.00139 & 0.13747 & 0.14389 \\ 1-2 & 3 & 0.13563 & 0.0139 & 0.13635 & 0.1279\end{array}$

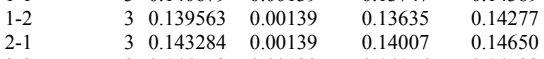

Std Enor ues a poted estimete of er vainec 0.14798

Std Error uses a pooled estimate of error variance Mean of Response $\quad 1.188504$ Observations (or Sum Wgts) $\quad 12$

Analysis of Variance $\begin{array}{lrrrrr}\text { Source } & \text { DF } & \text { Sum of Squares } & \text { Mean Square } & \text { F Ratio } & \text { Prob }>\text { F } \\ \text { ICP Calibration Block } & 3 & 0.00515462 & 0.001718 & 10.8686 & 0.0034\end{array}$ $\begin{array}{lrrr}\text { ICP Calibration Block } & 3 & 0.00515462 & 0.001718 \\ \text { Error } & 8 & 0.00126471 & 0.000158 \\ \text { C. Total } & 11 & 0.00641933 & \end{array}$

Means for Oneway Anova

Level Number Mean Std Error Lower 95\% Upper 95\%

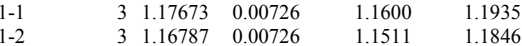

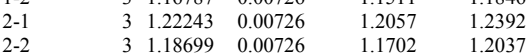

Std Error uses a pooled estimate of error variance $\begin{array}{ll}\text { Adj Rsquare } & 0.802984 \\ \text { Root Mean Square Error } & 0.729103 \\ 0.012573\end{array}$ 
Exhibit A3. PSAL Measurements by ICP Calibration Block for Samples of the

Standard Glasses by Preparation Method by Set by Analytical Plan Memo. (continued)

Oneway Analysis of Measured By ICP Calibration Block Prep $=$ LM, Plan =SRNL-SCS-2008-00003, Set=2, Type=Batch 1, Oxide=CdO (wt $\%)$

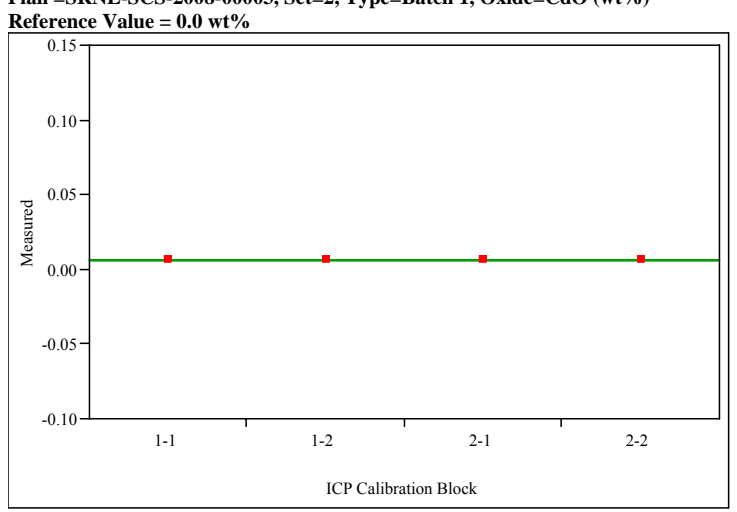

Oneway Anova

Rsquare

Root Mean Square Error

$\begin{array}{lr}\text { Mean of Response } & 0.005712 \\ \text { Observations (or Sum Wgts) } & 12\end{array}$

Analysis of Variance $\quad$ DF Sum of Squares Mean Square F Ratio Prob $>$ F

$\begin{array}{lrrr}\text { Error } & 3 & 0 & 0 \\ \text { C. Total } & 8 & 0 & 0 \\ & 11 & 0 & \end{array}$

Means for Oneway Anova

$\begin{array}{crrr}\text { Level Number } & \text { Mean Std Error } & \text { Lower 95\% Upper 95\% } \\ 1-1 & 0.005712 & 0 & 0.00571\end{array}$

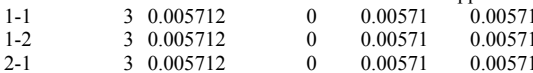

$\begin{array}{llllll}2-2 & 3 & 0.005712 & 0 & 0.000571 & 0.00571 \\ & & & 0.00571\end{array}$

Std Error uses a pooled estimate of error variance

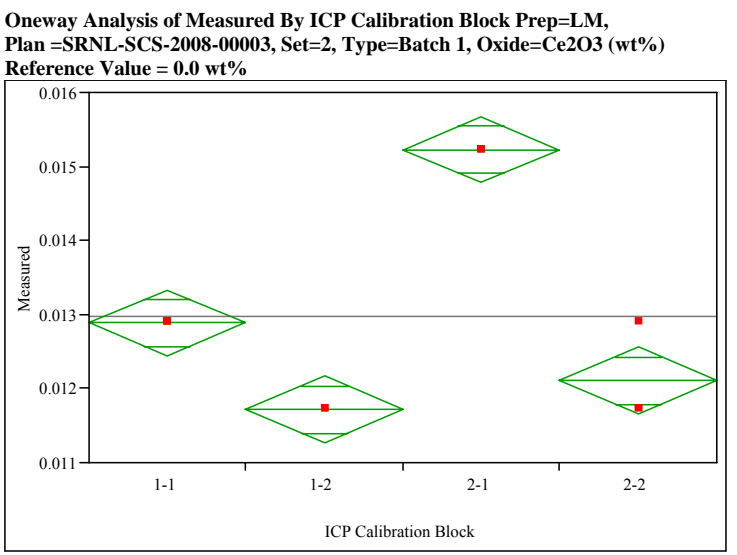

Oneway Anova

$\begin{array}{lr}\text { Rsquare } & 0.960591 \\ \text { Adj Rsquare } & 0.945813 \\ \text { Root Mean Square Error } & 0.000338 \\ \text { Mean of Response } & 0.012982 \\ \text { Observations (or Sum Wgts) } & 12\end{array}$

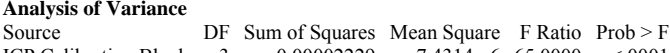

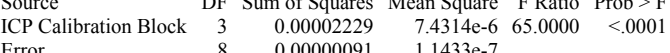

$\begin{array}{lrrr}\text { Error } & 8 & 0.00000091 & 1.1433 \mathrm{e}-7 \\ \text { C. Total } & 11 & 0.00002321 & \end{array}$

Means for Oneway Anova

Level Number Mean Std Error Lower 95\% Upper 95\%

$\begin{array}{lllllll}1-1 & 3 & 0.012884 & 0.00020 & 0.01243 & 0.0133 \\ 1-2 & 3 & 0.011713 & 0.00020 & 0.0126 & 0.01216\end{array}$

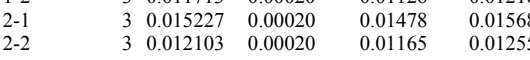

Std Error uses a pooled estimate of error variance
Oneway Analysis of Measured By ICP Calibration Block Prep=LM, Plan =SRNL-SCS-2008-00003, Set=2, Type=Batch 1, Oxide $=\mathrm{Cr} 203(\mathrm{wt} \%)$ Reference Value $=0.107 \mathrm{wt} \%$

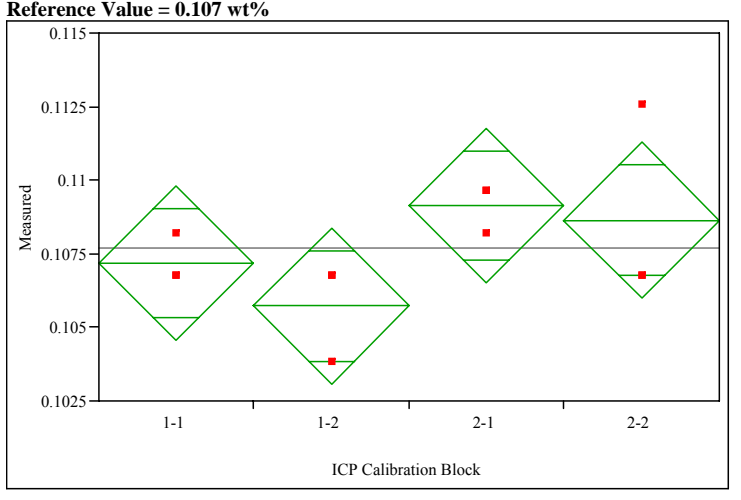

Oneway Anova

Rsquare

0.405405

Root Mean Square Error $\quad 0.001979$

$\begin{array}{lr}\text { Mean of Response } & 0.107671 \\ \text { Observations (or Sum Wgts) } & 12\end{array}$

Analysis of Variance

$\begin{array}{lllr}\text { Source } & \text { DF Sum of Squares Mean Square F Ratio Prob }>\text { F } \\ \text { ICP Calibration Block } & \text { S } & \end{array}$

Calibration Block 3 S 0.00002136

Error $\quad 8,0.00003133$

$3.9165 \mathrm{e}-6$

Means for Oneway Anov

Level Number Mean Std Error Lower 95\% Upper 95\%

$\begin{array}{lllllll}1-1 & 3 & 0.107184 & 0.00114 & 0.10455 & 0.10982\end{array}$

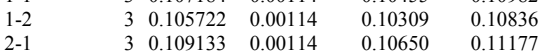

$\begin{array}{llllll}2-2 & 3 & 0.108646 & 0.00114 & 0.10601 & 0.11128\end{array}$

Std Error uses a pooled estimate of error variance 
Exhibit A3. PSAL Measurements by ICP Calibration Block for Samples of the

Standard Glasses by Preparation Method by Set by Analytical Plan Memo. (continued)

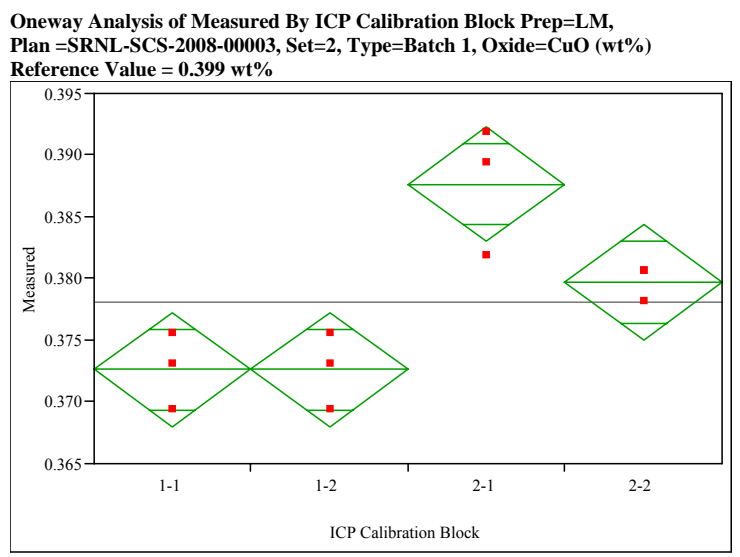

Oneway Anova

Rsquare
Adj Rsquare

$$
\begin{array}{r}
0.824422 \\
0.75858
\end{array}
$$

0.75858
Root Mean Square Error $\quad 0.003504$

$\begin{array}{lr}\text { Mean of Response } & 0.378148 \\ \text { Observations (or Sum Wgts) } & 12\end{array}$

$\begin{array}{lrrrrr}\text { Analysis of Variance } & & & & & \\ \text { Source } & \text { DF } & \text { Sum of Squares } & \text { Mean Square } & \text { F Ratio } & \text { Prob }>\text { F } \\ \text { ICP Calibration Block } & 3 & 0.00046109 & 0.000154 & 12.5213 & 0.0022 \\ \text { Error } & 8 & 0.00000820 & 0.000012 & & \\ \text { C. Total } & 11 & 0.00055929 & & & \end{array}$

Means for Oneway Anova

$\begin{array}{lrrrrr}\text { Level } & \text { Number } & \text { Mean } & \text { Std Error } & \text { Lower 95\% } & \text { Upper 95\% } \\ 1-1 & 3 & 0.372619 & 0.00202 & 0.36795 & 0.37728\end{array}$

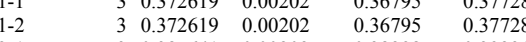

$\begin{array}{lllllll}2-1 & & 3 & 0.387641 & 0.00202 & 0.38298 & 0.39231 \\ 2-2 & & 3 & 0.379713 & 0.00202 & 0.37505 & 0.38438\end{array}$

Std Error uses a pooled estimate of error variance

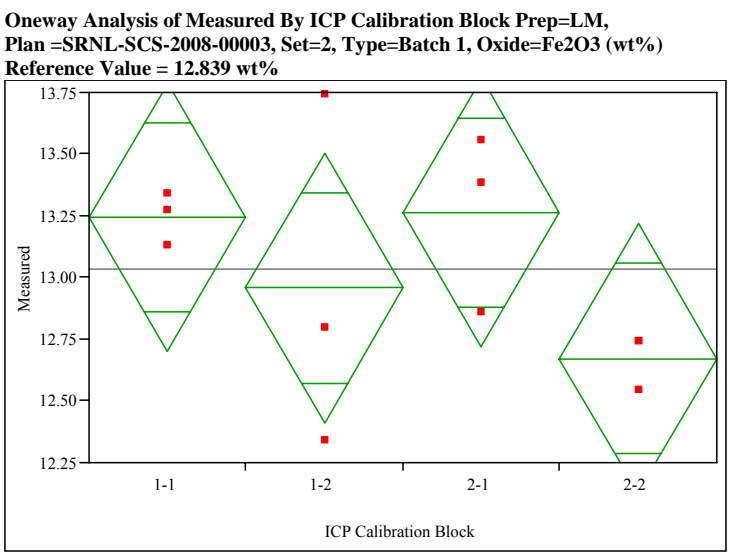

Oneway Anova

ummary of Fit

$\begin{array}{lr}\text { Rsquare } & 0.343407 \\ \text { Adj Rsquare } & 0.097185 \\ \text { Root Mean Square Error } & 0.408988 \\ \text { Mean of Response } & 13.0341 \\ \text { Observations (or Sum Wgts) } & 12\end{array}$

Analysis of Variance
Source DF Sum of Squares Mean Square F Ratio Prob $>$ F

$\begin{array}{lrrrrr} & & & & \\ \text { Source } & & & & & \\ \text { ICP Calibration Block } & 3 & 0.6998800 & 0.233293 & 1.3947 & 0.3133 \\ \text { Error } & 8 & 1.3381662 & 0.167271 & & \end{array}$ 0.167271

Means for Oneway Anova

Level Number Mean Std Error Lower 95\% Upper 95\%

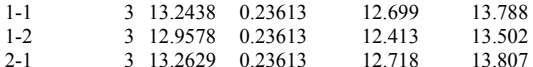

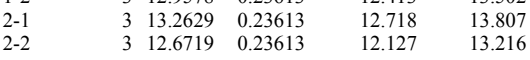

Std Error uses a pooled estimate of error variance
Oneway Analysis of Measured By ICP Calibration Block Prep=LM, Plan =SRNL-SCS-2008-00003, Set=2, Type=Batch 1, Oxide=K20 (wto) Reference Value $=3.327 \mathrm{wt} \%$

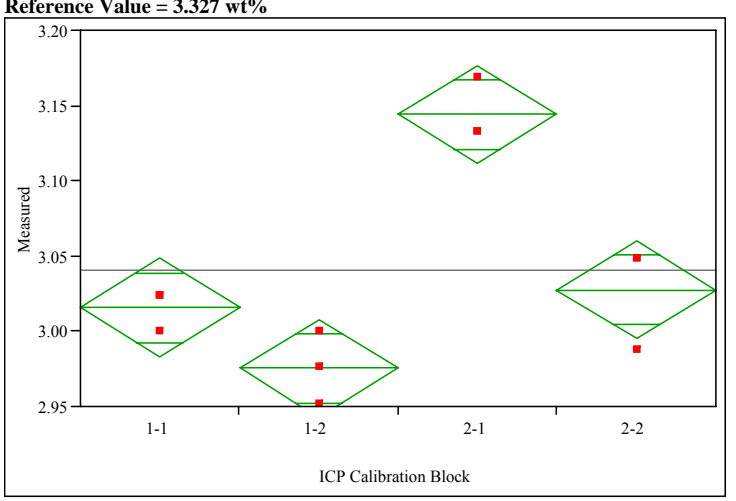

Oneway Anova

Summary of Fi

Rsquare

$-0.907128$

re Error $\quad 0.024589$ $\begin{array}{lr}\text { Mean of Response } & 3.040611 \\ \text { Observations (or Sum Wgts) } & 12\end{array}$

Analysis of Variance

Source DF Sum of Squares Mean Square F Ratio Prob $>$ F $\begin{array}{lrrrrr}\text { Source } & \text { DF } & \text { Sum of Squares } & \text { Mean Square } & \text { F Ratio } & \text { Prob }>\text { F } \\ \text { ICP Calibration Block } & 3 & 0.04724413 & 0.015748 & 26.0467 & 0.0002 \\ \text { Error } & 8 & 0.00483687 & 0.000605 & & \end{array}$ Crror 0.000605

Means for Oneway Anova

Level Number Mean Std Error Lower 95\% Upper 95\%

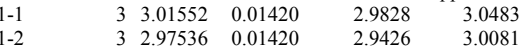

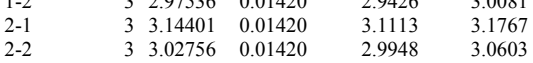

Std Error uses a pooled estimate of error variance 
Exhibit A3. PSAL Measurements by ICP Calibration Block for Samples of the

Standard Glasses by Preparation Method by Set by Analytical Plan Memo. (continued)

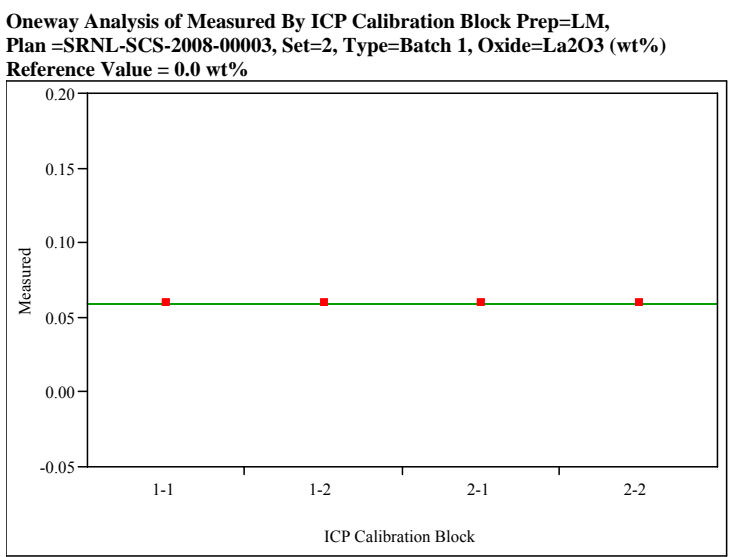

Oneway Anova

Rsquare

Adj Rsquare Root Mean Square Error

$\begin{array}{lr}\text { Mean of Response } & 0.05864 \\ \text { Observations (or Sum Wgts) } & 12\end{array}$

Analysis of Variance
Source

$\begin{array}{lrrr}\text { Source } & \text { DF } & \text { Sum of Squares } & \text { Mean Square } \\ \text { ICP Calibration Block } & 3 & 0 & 0 \\ \text { Error } & 8 & 0 & 0\end{array}$

C. Total

8
11

Oneway Analysis of Measured By ICP Calibration Block Prep=LM,

Plan $=$ SRNL-SCS-2008-00003, Set $=2$, Type=Batch 1, Oxide $=\mathrm{MgO}(\mathrm{wt} \%)$

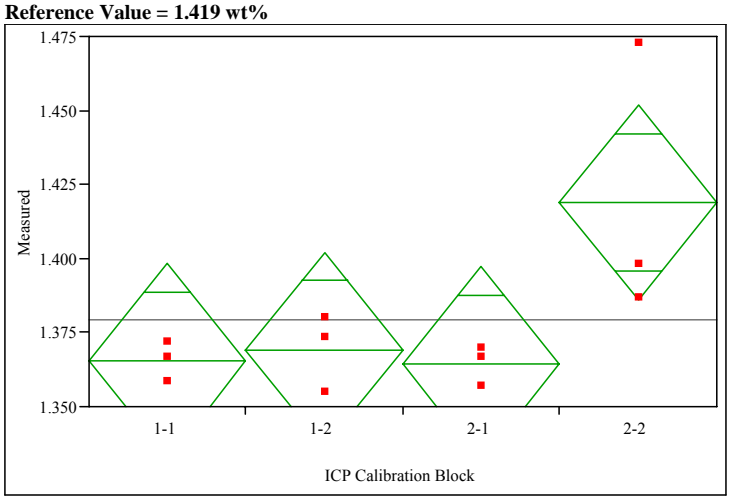

Oneway Anova

mmary of $F$

Rsquare
Adj Rsquare

\subsection{9}

0.398078
Root Mean Square Error $\quad 0.02474$

Mean of Response $\quad 1.37942$

Observations (or Sum Wgts) 12

Analysis of Variance
Source

$\begin{array}{lrrrrr}\text { Source } & & & & & \\ \text { ICP Calibration Block } & 3 & 0.00628916 & 0.002096 & 3.4249 & 0.0727\end{array}$

$\begin{array}{lrrr}\text { Error } & 8 & 0.00489676 & 0.000612 \\ \text { C. Total } & 11 & 0.01118592 & \end{array}$

Means for Oneway Anova

Level Number Mean Std Error Lower 95\% Upper 95\%

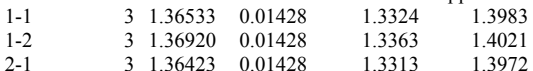

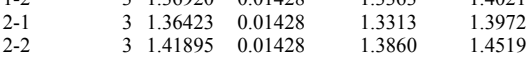

Std Error uses a pooled estimate of error variance
Oneway Analysis of Measured By ICP Calibration Block Prep=LM, Plan =SRNL-SCS-2008-00003, Set=2, Type=Batch 1, Oxide=MnO (wt $\%)$ Reference Value $=1.726 \mathrm{wt} \%$

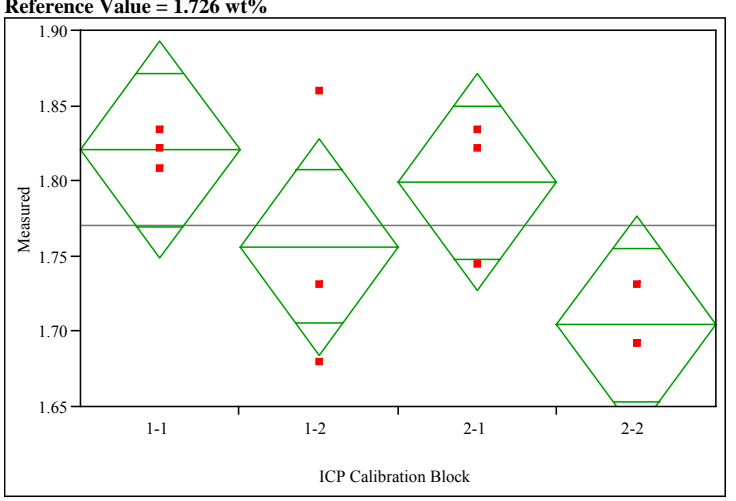

Oneway Anova

Summary of Fi

Rsquare

0.502798

re Error $\quad 0.054143$ $\begin{array}{lr}\text { Mean of Response } & 1.77002 \\ \text { Observations (or Sum Wgts) } & 12\end{array}$

Analysis of Variance

Source DF Sum of Squares Mean Square F Ratio Prob $>$ F $\begin{array}{lrrrrr}\text { ICP Calibration Block } & 3 & 0.02271588 & 0.007905 & 2.6967 & 0.1165 \\ \text { Error } & 8 & 0.02345191 & 0.002931 & & \end{array}$ C. Total 0.002931

Means for Oneway Anova

Level Number Mean Std Error Lower 95\% Upper 95\% $\begin{array}{lllllll}1-1 & & 3 & 1.82059 & 0.03126 & 1.7485 & 1.8927 \\ 1-2 & & 3 & 1.75603 & 0.03126 & 1.6839 & 1.8281\end{array}$

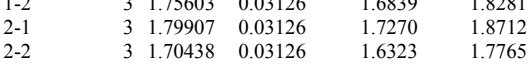

Std Error uses a pooled estimate of error variance 
Exhibit A3. PSAL Measurements by ICP Calibration Block for Samples of the

Standard Glasses by Preparation Method by Set by Analytical Plan Memo. (continued)

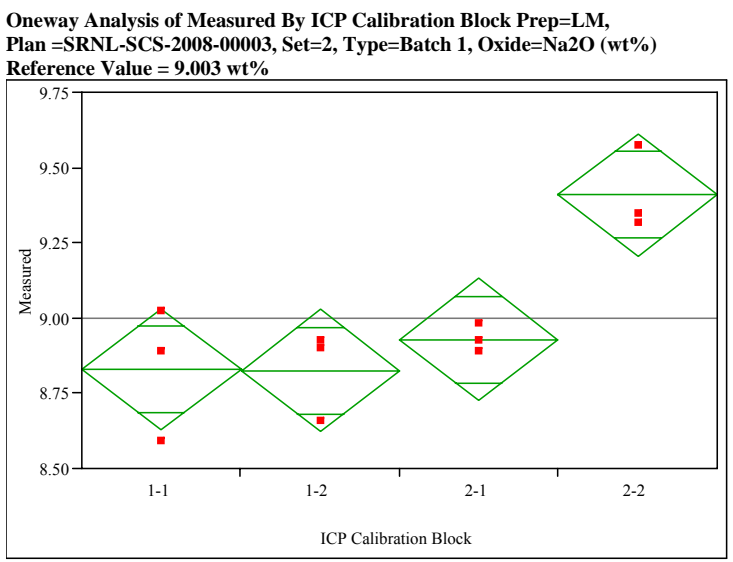

Oneway Anova
Summary of Fit

$\begin{array}{lr}\text { Rsquare } & 0.789633 \\ \text { Adj Rsquare } & 0.710745 \\ \text { Root Mean Square Error } & 0.15231 \\ \text { Mean of Response } & 8.9979 \\ \text { Observations (or Sum Wgts) } & 12\end{array}$

Analysis of Variance
Source

$\begin{array}{lrrrrr}\text { Source } & \text { DF } & \text { Sum of Squares } & \text { Mean Square } & \text { F Ratio } & \text { Prob }>\text { F } \\ \text { ICP Calibration Block } & 3 & 0.69661710 & 0.232206 & 10.0096 & 0.0044\end{array}$

$\begin{array}{lrrr}\text { ICP Calibration Block } & 3 & 0.69661710 & 0.232206 \\ \text { Error } & 8 & 0.18558689 & 0.023198 \\ \text { C. Total } & 11 & 0.88220399 & \end{array}$

Means for Oneway Anova

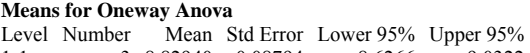

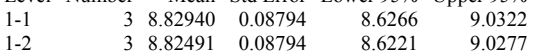

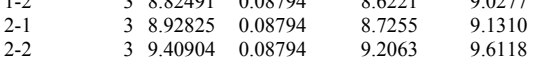

Std Error uses a pooled estimate of error variance
Oneway Analysis of Measured By ICP Calibration Block Prep=LM, Plan =SRNL-SCS-2008-00003, Set=2, Type=Batch 1, Oxide=Nb2O5 (wt \%) Reference Value $=0.0 \mathrm{wt} \%$

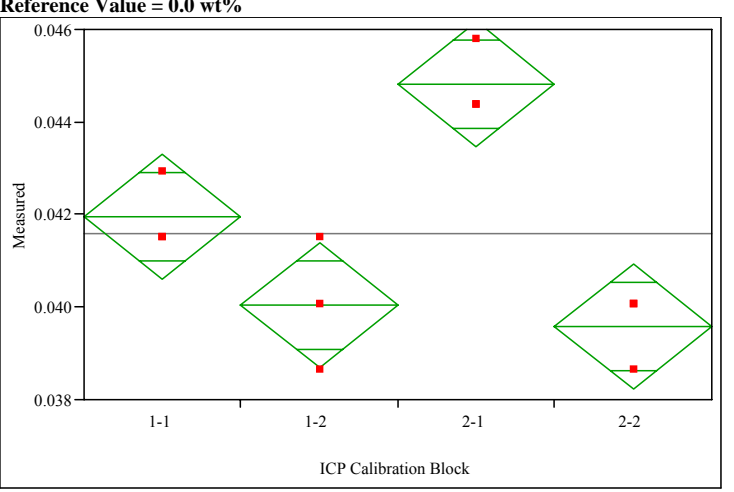

Oneway Anova

ummary of Fit

$\begin{array}{lr}\text { Rsquare } & 0.861671 \\ \text { Adj Rsquare } & 0.809798 \\ \text { Root Mean Square Error } & 0.001012 \\ \text { Mean of Response } & 0.041604 \\ \text { Observations (or Sum Wgts) } & 12\end{array}$

$\begin{array}{lllll}\text { Analysis of Variance } & & & \\ \text { Source } & \text { DF Sum of Squares Mean Square F Ratio Prob }>\text { F }\end{array}$

$\begin{array}{lllllll} & & 0.00005099 & 0.000017 & 16.6111 & 0.0008\end{array}$

Error
C. Total

$\begin{array}{rr}8 & 0.00000819 \\ 11 & 0.00005917\end{array}$

$1.023 \mathrm{e}-6$

Means for Oneway Anova

Level Number Mean Std Error Lower 95\% Upper 95\%

$\begin{array}{lllllll}1-1 & 3 & 0.041961 & 0.00058 & 0.04061 & 0.0433 \\ -2 & & 3 & 0.040054 & 0.00058 & 0.03871 & 0.04140\end{array}$

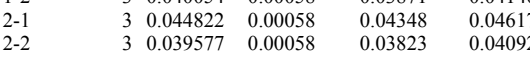

Std Error uses a pooled estimate of error variance
Oneway Analysis of Measured By ICP Calibration Block Prep=LM, Plan =SRNL-SCS-2008-00003, Set=2, Type=Batch 1 , Oxide=NiO (wt\%) Reference Value $=0.751 \mathrm{wt} \%$

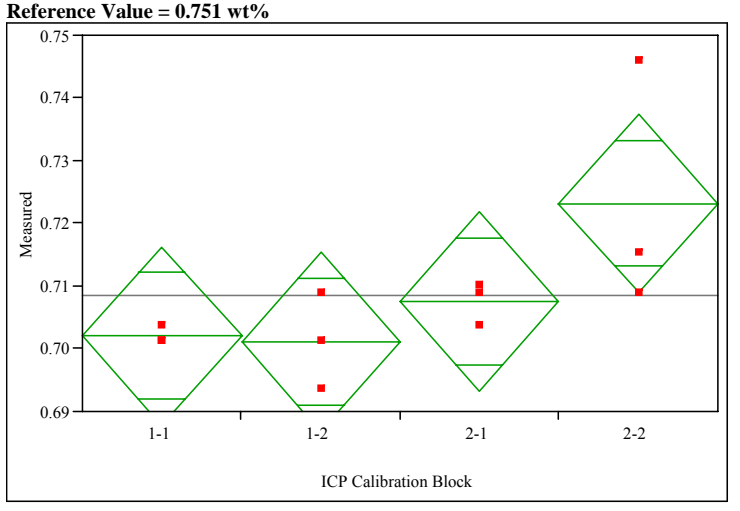

Oneway Anova

Summary of Fit

Rsquare $\begin{array}{lr}\text { Mean of Response } & 0.708464 \\ \text { Observations (or Sum Wgts) } & 12\end{array}$

Analysis of Variance

Source DF Sum of Squares Mean Square F Ratio Prob $>$ F

$\begin{array}{lrrrrr}\text { Source } & \text { DF } & \text { Sum of Squares } & \text { Mean Square } & & \\ \text { ICP Ratio Calibration Block } & 3 & 0.00094065 & 0.000314 & 2.7209 & 0.1146\end{array}$ Error 0.000115

\section{Means for Oneway Anov}

Level Number Mean Std Error Lower 95\% Upper 95\%

$\begin{array}{lllllll}1-1 & & 3 & 0.701996 & 0.00620 & 0.68770 & 0.71629 \\ 1-2 & & 3 & 0.701148 & 0.00620 & 0.68686 & 0.71544\end{array}$

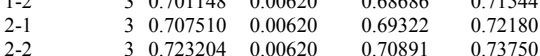

Std Error uses a pooled estimate of error variance 
Exhibit A3. PSAL Measurements by ICP Calibration Block for Samples of the

Standard Glasses by Preparation Method by Set by Analytical Plan Memo. (continued)

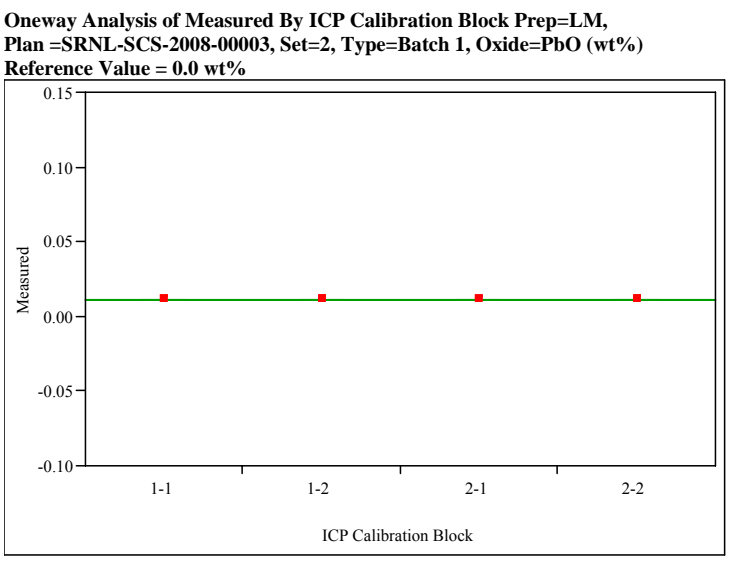

Oneway Anova
Summary of Fit

Summary of

$\begin{array}{lr}\text { Rsquare } & 0 \\ \text { Adj Rsquare } & -0.375 \\ \text { Root Mean Square Error } & 2.12 \mathrm{e}-18 \\ \text { Mean of Response } & 0.010772 \\ \text { Observations (or Sum Wgts) } & 12\end{array}$

Analysis of Variance
Source DF Sum of Squares Mean Square F Ratio Prob > F

$\begin{array}{lrrrrr}\text { Source } & \text { DF } & \text { Sum of Squares Mean Square } & \text { F Ratio } & \text { Prob }>\text { F } \\ \text { ICP Calibration Block } & 3 & 0 & 0 & 0.0000 & 1.0000\end{array}$

$\begin{array}{lrrr}\text { Error } & 8 & 3.6111 \mathrm{e}-35 & 4.514 \mathrm{e}-36 \\ \text { C. Total } & 11 & 3.611 \mathrm{e}-35 & \end{array}$

Means for Oneway Anova

Level Number $\begin{gathered}\text { Mean } \\ 1-1\end{gathered}$

$\begin{array}{lllllll}1-1 & 3 & 0.010772 & 1.227 \mathrm{e}-18 & 0.01077 & 0.01077 \\ 1-2 & & 3 & 0.010772 & 1.227 \mathrm{e}-18 & 0.01077 & 0.01077\end{array}$

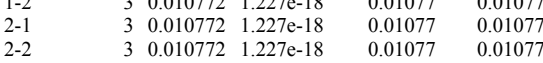

Std Error uses a pooled estimate of error variance

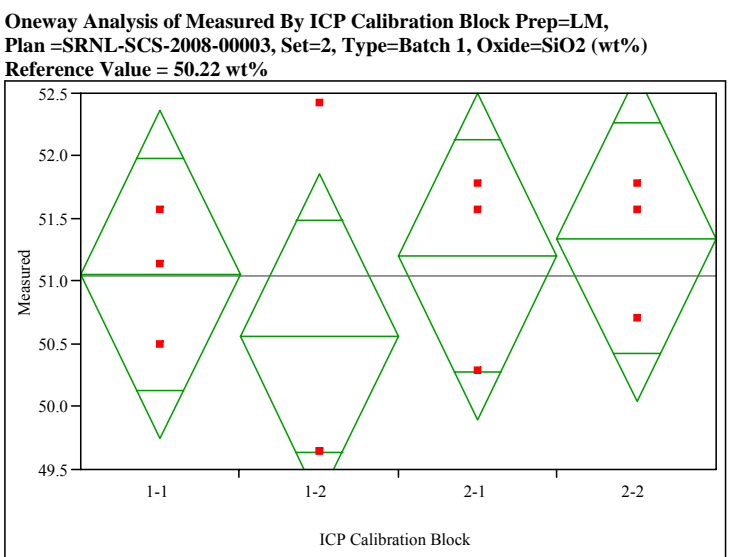

Oneway Anova
Summary of Fit

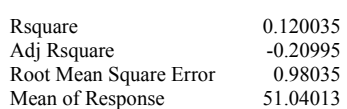
$\begin{array}{lr}\text { Mean of Response } & 51.04013 \\ \text { Observations (or Sum Wgts) } & 12\end{array}$

Analysis of Variance

Source DF Sum of Squares Mean Square F Ratio Prob $>$ F $\begin{array}{lrrrrr}\text { Source } & \text { DF } & \text { Sum of Squares } & \text { Mean Square } & \text { F Ratio } & \text { Prob }>\text { F } \\ \text { ICP Calibration Block } & 3 & 1.0488052 & 0.349602 & 0.3638 & 0.7812 \\ \text { Error } & 8 & 7.6886955 & 0.961087 & & \end{array}$ Error
C. Total 8.7375007 0.961087

Means for Oneway Anova

Level Number Mean Std Error Lower 95\% Upper 95\%

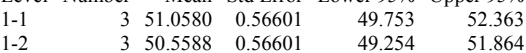

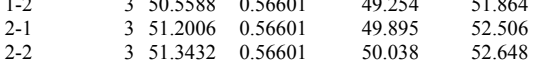

Std Error uses a pooled estimate of error variance
Oneway Analysis of Measured By ICP Calibration Block Prep=LM, Plan $=$ SRNL-SCS-2008-00003, Set $=2$, Type=Batch 1 , Oxide $=S O 4$ (wt\%) Reference Value $=0.0 \mathrm{wt} \%$

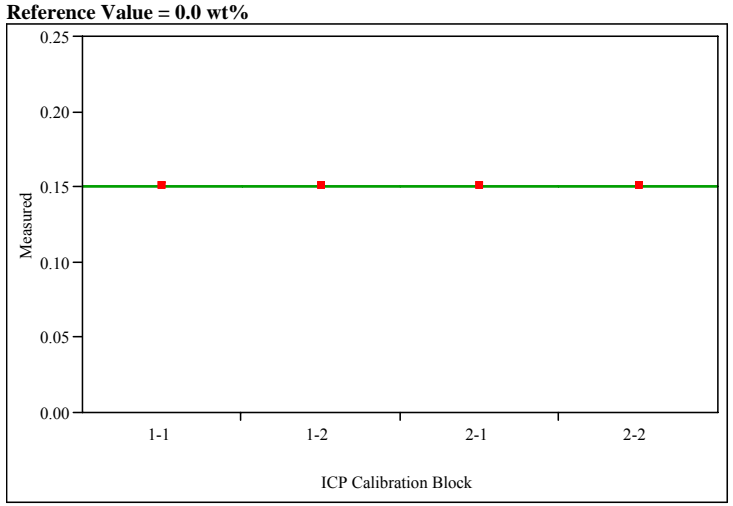

Oneway Anova

Summary of Fit

Rsquare

Root Mean Square Erro $\begin{array}{lr}\text { Mean of Response } & 0.149795 \\ \text { Observations (or Sum Wgts) } & 12\end{array}$

Analysis of Variance

Source DF Sum of Squares Mean Square F Ratio Prob $>$ F $\begin{array}{lrrr}\text { Source } & \text { DF } & \text { Sum of Squares } & \text { Mean Square } \\ \text { ICP Calibration Block } & 3 & 0 & 0 \\ \text { Error } & 8 & 0 & 0 \\ \text { C. Total } & 11 & 0 & \end{array}$ 0

Means for Oneway Anova

Level Number Mean Std Error Lower 95\% Upper 95\% $\begin{array}{rrr}1-1 & 3 & 0.149795 \\ 1-2 & 3 & 0.149795\end{array}$

$\begin{array}{llll}0 & 0.14980 & 0.14980\end{array}$

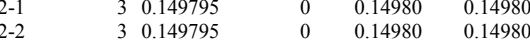

Std Error uses a pooled estimate of error variance 
Exhibit A3. PSAL Measurements by ICP Calibration Block for Samples of the

Standard Glasses by Preparation Method by Set by Analytical Plan Memo. (continued)

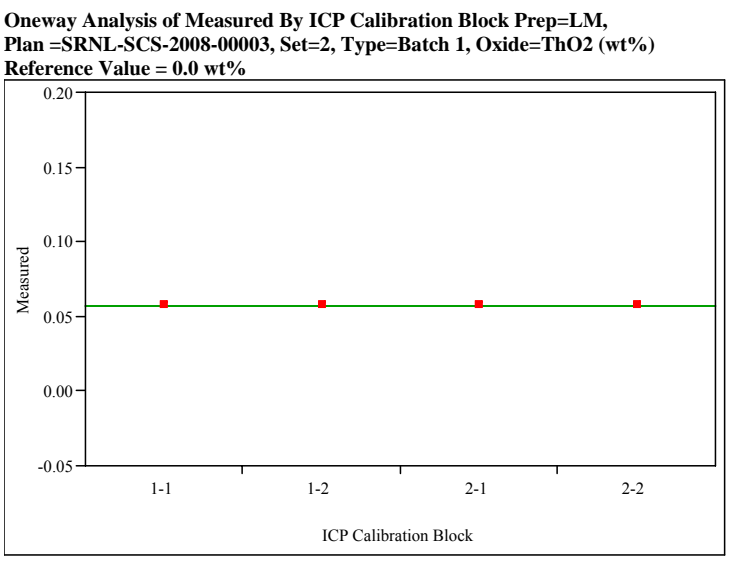

Oneway Anova

ummary of $F$

Rsquare

Root Mean Square Erro

$\begin{array}{lr}\text { Mean of Response } & 0.056895 \\ \text { Observations (or Sum Wgts) } & 12\end{array}$

Analysis of Variance
Source DF Sum of Squares Mean Square F Ratio Prob $>$ F

$\begin{array}{lcc}\text { Aource } & \text { DF } & \text { Sum of Squares } \\ \text { ICP Calibration Block Squ } & 3 & 0 \\ \text { Error } & 8 & 0\end{array}$

C. Total

8
11

0

Means for Oneway Anova

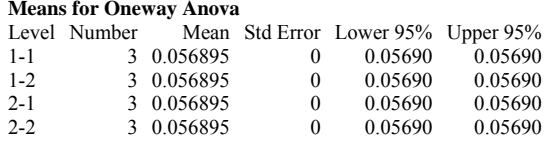

Std Error uses a pooled estimate of error variance

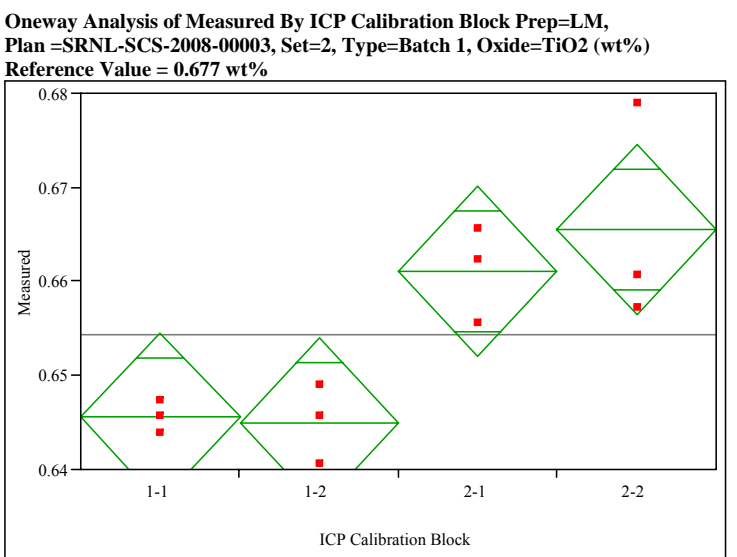

Oneway Anova
Summary of Fi

$\begin{array}{ll}\text { Rsquare } & 0.734278 \\ \text { Adj Rsquare } & 0.634632 \\ \text { Root Mean Square Error } & 0.006758 \\ \text { Mean of Response } & 0.654273\end{array}$ $\begin{array}{lr}\text { Mean of Response } & 0.654273 \\ \text { Observations (or Sum Wgts) } & 12\end{array}$

Analysis of Variance

Source DF Sum of Squares Mean Square F Ratio Prob $>F$ $\begin{array}{lrllll}\text { ICP Calibration Block } & 3 & 0.00100972 & 0.000337 & 7.3689 & 0.0109\end{array}$ Error
C. Total 0.00137511 0.000046

Means for Oneway Anova

Level Number Mean Std Error Lower 95\% Upper 95\%

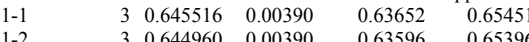

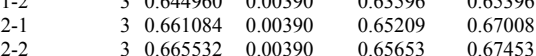

Std Error uses a pooled estimate of error variance
Oneway Analysis of Measured By ICP Calibration Block Prep=LM, Plan =SRNL-SCS-2008-00003, Set=2, Type=Batch 1, Oxide=U3O8 (wt $\%$ ) Reference Value $=0.0 \mathrm{wt} \%$

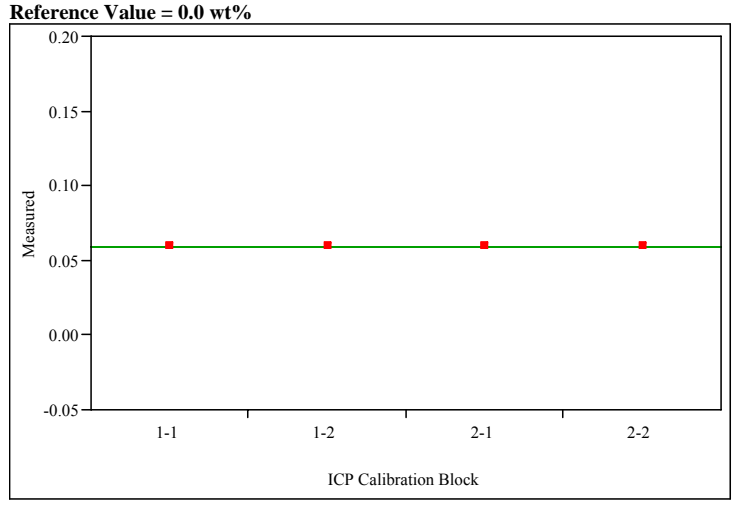

Oneway Anova

Summary of Fit

Rsquare

Root Mean Square Err

Mean of Response $\quad 0.05896$

Observations (or Sum Wgts) $\quad 12$

Analysis of Variance
Source

$\begin{array}{lcc}\text { Source } & \text { DF } & \text { Sum of Squares } \\ \text { ICP Calibration Block } & 3 & 0 \\ \text { Error } & 8 & 0\end{array}$

Error
C. Total

0
0

Means for Oneway Anova

Level Number Mean Std Error Lower 95\% Upper 95\%

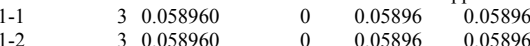

$\begin{array}{llllll}2-1 & 3 & 0.058960 & 0 & 0.05896 & 0.05896 \\ 2-2 & 3 & 0.058960 & 0 & 0.05896 & 0.05896\end{array}$

Std Error uses a pooled estimate of error variance 
Exhibit A3. PSAL Measurements by ICP Calibration Block for Samples of the

Standard Glasses by Preparation Method by Set by Analytical Plan Memo. (continued)

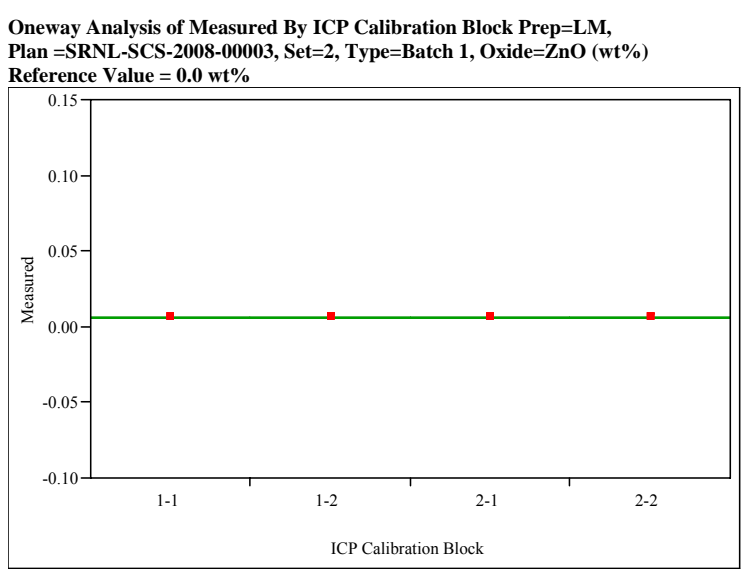

Oneway Anova
Summary of Fit

Rsquare

Adj Rsquare
Root Mean Square

Root Mean Square Error

$\begin{array}{lr}\text { Mean of Response } & 0.006224 \\ \text { Observations (or Sum Wgts) } & 12\end{array}$

Analysis of Variance

Analysis of Variance $\quad$ DF Sum of Squares Mean Square F Ratio Prob $>$ F

ICP Cal

Error
C. Total

Means for Oneway Anova
Level Number Mean Std Error Lower 95\% Upper 95\%

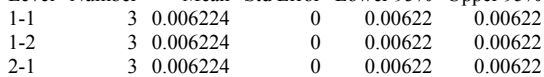

$\begin{array}{llllll}2-1 & 3 & 0.006224 & 0 & 0.00622 & 0.00622 \\ 2-2 & 3 & 0.006224 & 0 & 0.00622 & 0.00622\end{array}$

Std Error uses a pooled estimate of error variance
Oneway Analysis of Measured By ICP Calibration Block Prep=LM, Plan =SRNL-SCS-2008-00003, Set=2, Type=Batch 1, Oxide=ZrO2 $(w t \%)$

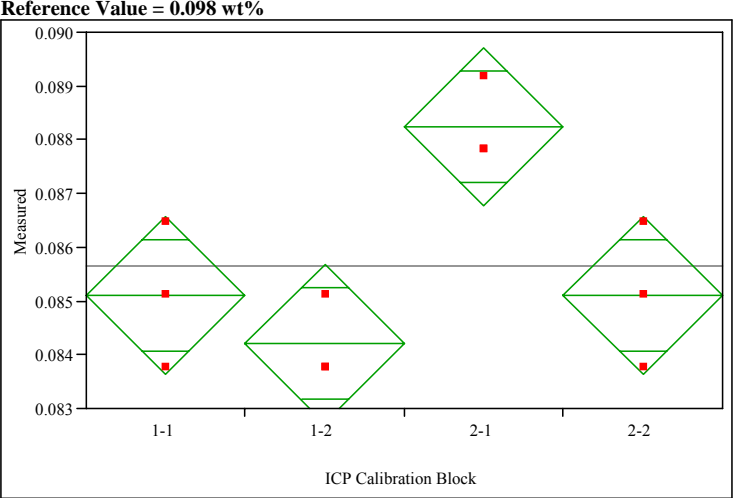

Oneway Anova
Summary of Fit

Oneway Analysis of Measured By ICP Calibration Block Prep=LM, Plan $=$ SRNL-SCS-2008-00003, Set=2, Type=Ustd, Oxide=Al2O3 $($ wt $\%$ Reference Value $=4.1 \mathrm{wt} \%$

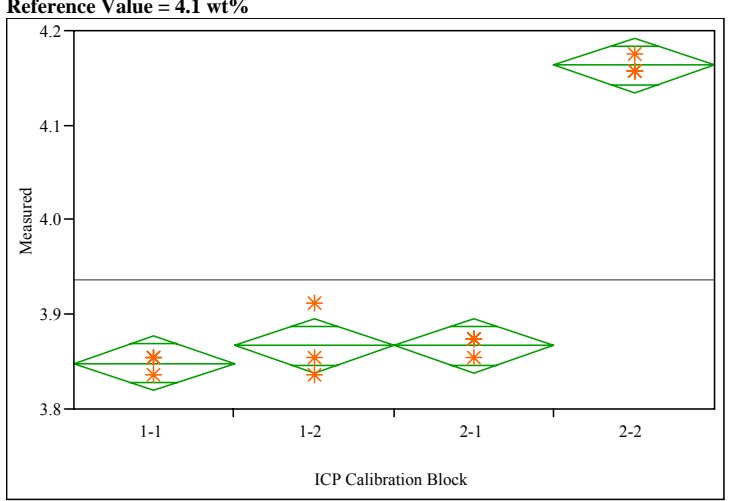

Oneway Anova
Summary of Fit

$\begin{array}{lr}\text { Rsquare } & 0.98188 \\ \text { Adj Rsquare } & 0.975085 \\ \text { Root Mean Square Error } & 0.021818 \\ \text { Mean of Response } & 3.936458 \\ \text { Observations (or Sum Wgts) } & 12\end{array}$

Analysis of Varian

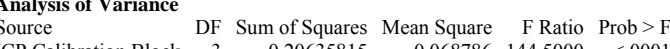

$\begin{array}{lllllll} & & & \\ \text { ICP Calibration Block } & 3 & 0.20635815 & 0.068786 & 144.5000 & <.000\end{array}$

Error
C. Total

$11 \quad 0.21016638$

.000476

Means for Oneway Anova
Level Number Mean Std Error Lower 95\% Upper 95\%

Means for Oneway Anova

$0.0002843-9.4781 \mathrm{e}-6-7.7917 \quad 0$. 0.0000381

$\begin{array}{lrrrrr}1-1 & 3 & 3.84828 & 0.01260 & 3.8192 & 3.8773 \\ 1-2 & 3 & 3.86718 & 0.01260 & 3.8381 & 3.8962 \\ 2-1 & 3 & 3.86718 & 0.01260 & 3.8381 & 3.8962 \\ 2-2 & 3 & 4.16320 & 0.01260 & 4.1342 & 4.1922\end{array}$

Std Error uses a pooled estimate of error variance 
Exhibit A3. PSAL Measurements by ICP Calibration Block for Samples of the

Standard Glasses by Preparation Method by Set by Analytical Plan Memo. (continued)

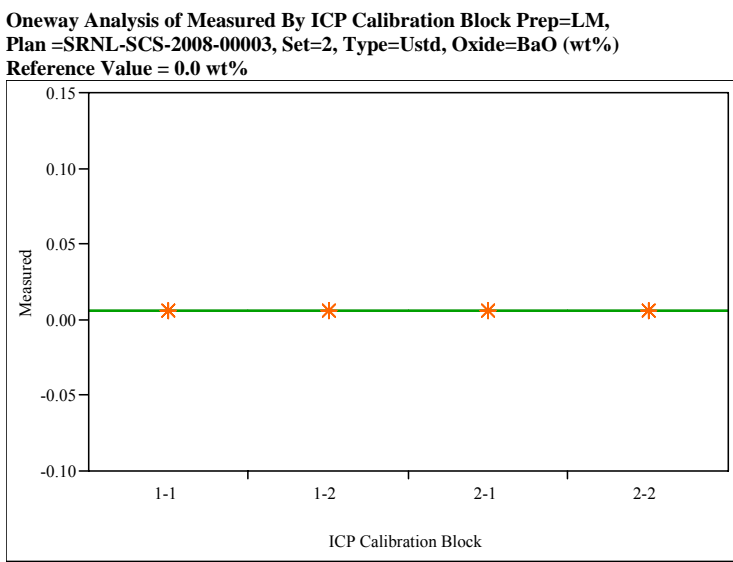

Oneway Anova
Summary of Fit

Rsquare

Adj Rsquare

$\begin{array}{ll}\text { Mean of Response } & 0.005583 \\ \text { Observations (or Sum Wgts) } & 12\end{array}$

Analysis of Varianc

Analysis of Variance DF Sum of Squares Mean Square F Ratio Prob $>$ F

ICP Cal

Error

Means for Oneway Anova

Level Number Mean Std Error Lower 95\% Upper 95\%

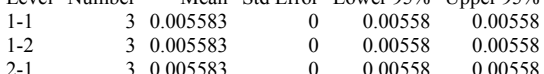

$\begin{array}{llllll}2-1 & 3 & 0.005583 & 0 & 0.00558 & 0.00558 \\ 2-2 & 3 & 0.005583 & 0 & 0.00558 & 0.00558\end{array}$

Std Error uses a pooled estimate of error variance

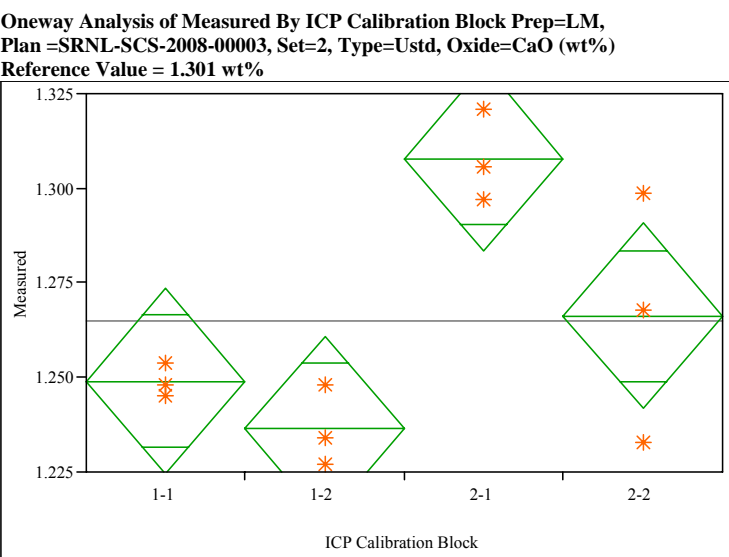

Oneway Anova
Summary of $F i t$

Rerase

Rsquare
Adj Rsquare

Mean of Response 1.264877

Observations (or Sum Wgts) $\quad 12$

Analysis of Variance

Source DF Sum of Squares Mean Square F Ratio Prob $>$ F

$\begin{array}{lllllll}\text { ICP Calibration Block } & 3 & 0.00871203 & 0.002904 & 8.5372 & 0.0071\end{array}$

Error

$\begin{array}{rr}8 & 0.00272129 \\ 11 & 0.01143332\end{array}$

0.000340

Means for Oneway Anov

Level Number Mean Std Error Lower 95\% Upper 95\%

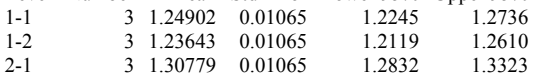

$\begin{array}{llllll}2-1 & 3 & 1.3067 & 0.01065 & 1.2832 & 1.3323 \\ 2-2 & 3 & 1.26628 & 0.01065 & 1.2417 & 1.2908\end{array}$

Std Error uses a pooled estimate of error variance
Oneway Analysis of Measured By ICP Calibration Block Prep=LM, Plan $=$ SRNL-SCS-2008-00003, Set $=2$, Type=Ustd, Oxide $=C d O(w t \%)$ Reference Value $=0.0 \mathrm{wt} \%$

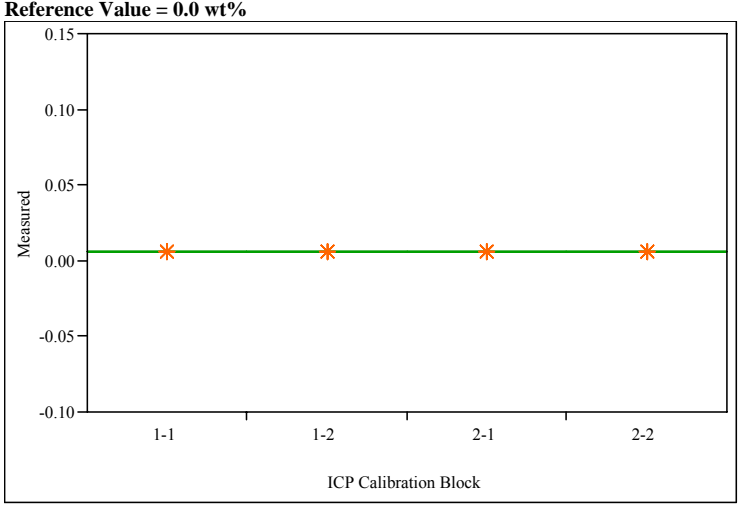

Oneway Anova
Summary of Fit

Rsquare

Adj Rsquare Mean of Response
Observations (or Sum Wgts)

Analysis of Variance

Source $\quad$ DF Sum of Squares Mean Square F Ratio Prob $>$ F

ICP Cal

Error

0

Means for Oneway Anova

Level Number Mean Std Error Lower 95\% Upper 95\%

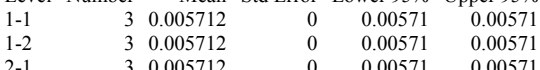

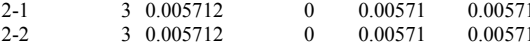

Std Error uses a pooled estimate of error variance 
Exhibit A3. PSAL Measurements by ICP Calibration Block for Samples of the

Standard Glasses by Preparation Method by Set by Analytical Plan Memo. (continued)

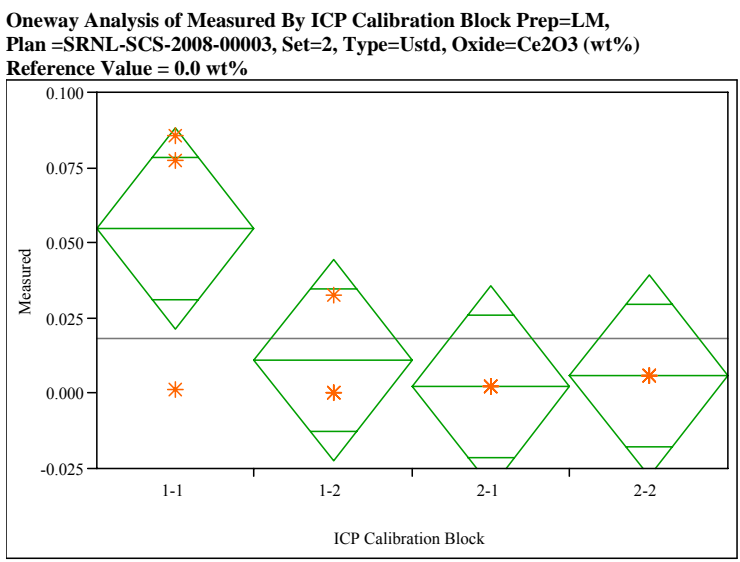

Oneway Anova
Summary of Fit

$\begin{array}{ll}\text { Rsquare } & 0.515144 \\ \text { Adj Rsquare } & 0.333322 \\ \text { Root Mean Square Error } & 0.025106 \\ \text { Mean of Response } & 0.018448\end{array}$ $\begin{array}{lr}\text { Mean of Response } & 0.018448 \\ \text { Observations (or Sum Wgts) } & 12\end{array}$

$\begin{array}{lrrrrr}\text { Analysis of Variance } & & & & & \\ \text { Source } & \text { DF } & \text { Sum of Squares } & \text { Mean Square } & \text { F Ratio } & \text { Prob }>\text { F } \\ \text { ICP Calibration Block } & 3 & 0.00535733 & 0.001786 & 2.8332 & 0.1062\end{array}$ $\begin{array}{lrrr}\text { Source } & & & \\ \text { ICP Calibration Block } & 3 & 0.00535733 & 0.001786 \\ \text { Error } & 8 & 0.00504235 & 0.00063 \\ \text { C Total } & 11 & 0.01039968 & \end{array}$

Means for Oneway Anova

Level Number Mean Std Error Lower 95\% Upper 95\%

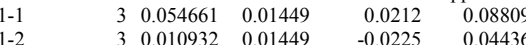

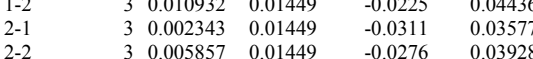

Std Error uses a pooled estimate of error variance

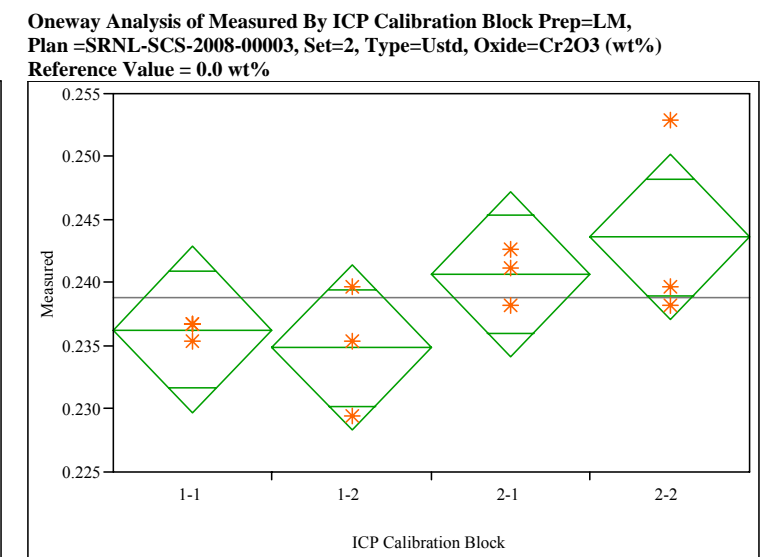

Oneway Anova
Summary of Fit

$\begin{array}{lr}\text { Rsquare } & 0.42947 \\ \text { Adj Rsquare } & 0.215522 \\ \text { Root Mean Square Error } & 0.00492 \\ \text { Mean of Response } & 0.23885 \\ \text { Observations (or Sum Wgts) } & 12\end{array}$

Analysis of Variance

Source 1 DF Sum of Squares Mean Square F Ratio Prob $>$ F

$\begin{array}{lllllll} & \text { ICP Calibration Block } & 3 & 0.00014580 & 0.000049 & 2.0074 & 0.1916\end{array}$

Error

$\begin{array}{rr}8 & 0.00019369 \\ 11 & 0.00033949\end{array}$

0.000024

Means for Oneway Anova
Level Number Mean Std Error Lower 95\% Upper 95\%

$\begin{array}{lllllll}1-1 & 3 & 0.236292 & 0.00284 & 0.22974 & 0.24284 \\ 1-2 & & 3 & 0.234830 & 0.00284 & 0.22828 & 0.24138\end{array}$

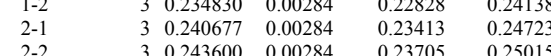

Std Error uses a pooled estimate of error variance
Oneway Analysis of Measured By ICP Calibration Block Prep=LM, Plan =SRNL-SCS-2008-00003, Set=2, Type=Ustd, Oxide=CuO (wt\%) Reference Value $=0.0 \mathrm{wt} \%$

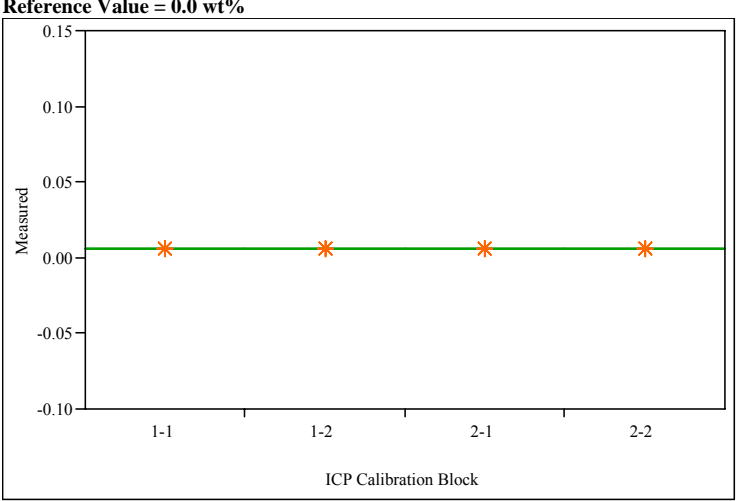

Oneway Anova
Summary of $F i t$

Rsquare

Adj Rsquare $\begin{array}{lr}\text { Mean of Response } & 0.006259 \\ \text { Observations (or Sum Wgts) } & 12\end{array}$

Analysis of Variance

Source DF Sum of Squares Mean Square F Ratio Prob $>F$

ICP Cal

Error

Means for Oneway Anova
Level Number Mean Std Error Lower 95\% Upper 95\%

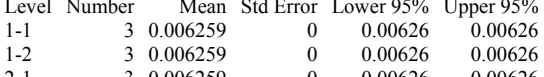

$\begin{array}{llllll}2-2 & 3 & 0.006259 & 0 & 0.00626 & 0.00626 \\ & & & & & \end{array}$

Std Error uses a pooled estimate of error variance 
Exhibit A3. PSAL Measurements by ICP Calibration Block for Samples of the

Standard Glasses by Preparation Method by Set by Analytical Plan Memo. (continued)

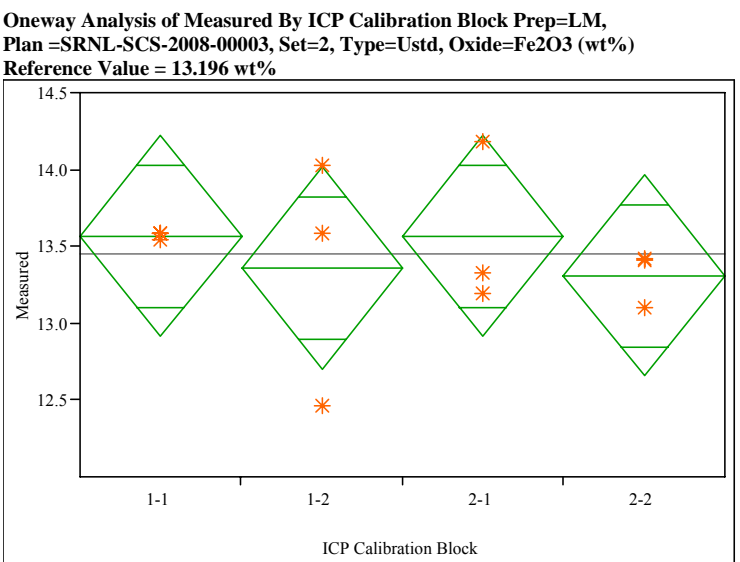

\section{Oneway Anova
Summary of Fit}

$\begin{array}{lr}\text { Rsquare } & 0.079446 \\ \text { Adj Rsquare } & -0.26576 \\ \text { Root Mean Square Error } & 0.491811 \\ \text { Mean of Response } & 13.45109 \\ \text { Observations (or Sum Wgts) } & 12\end{array}$

Analysis of Variance

$\begin{array}{lrrrrr}\text { Source } & \text { DF } & \text { Sum of Squares } & \text { Mean Square } & \text { F Ratio } & \text { Prob }>\text { F } \\ \text { ICP Calibration Block } & 3 & 0.1669982 & 0.055666 & 0.2301 & 0.8729\end{array}$

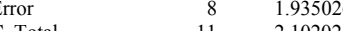

$\begin{array}{lll}0.055666 & 0.2301 & 0.8729 \\ 0.241878 & & \end{array}$

Means for Oneway Anova

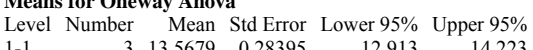

$\begin{array}{lrrrrr}1-1 & 3 & 13.5679 & 0.28395 & 12.913 & 14.223 \\ 1-2 & 3 & 13.3582 & 0.28395 & 12.703 & 14.013\end{array}$

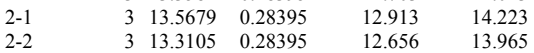

Std Error uses a pooled estimate of error variance

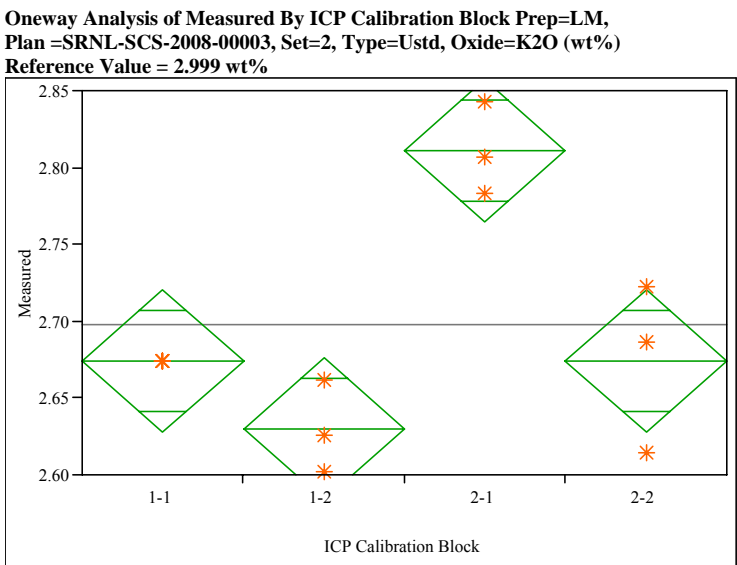

\section{Oneway Anova
Summary of Fit}

Rsquare

$\begin{array}{ll}\text { Adj Rsquare } & 0.850009 \\ 0.793763\end{array}$

Root Mean Square Error $\quad 0.034947$

Observations (or Sum Wots) 2.6973

Analysis of Variance

Source DF Sum of Squares Mean Square F Ratio Prob $>$ F

$\begin{array}{lrrrrr}\text { ICP Calibration Block } & 3 & 0.05537008 & 0.018457 & 15.1122 & 0.0012 \\ \text { Error } & 8 & 0.00977048 & 0.001221 & & \\ \text { C Total } & 11 & 0.06514055 & & & \end{array}$

$\begin{array}{rr}8 & 0.00977048 \\ 11 & 0.06514055\end{array}$

Means for Oneway Anova
Level Number Mean Std Error Lower 95\% Upper 95\%

$\begin{array}{rrrrrr}1-1 & 3 & 2.67421 & 0.02018 & 2.6277 & 2.7207\end{array}$

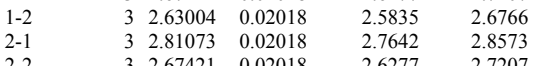

Std Error uses a pooled estimate of error variance
Oneway Analysis of Measured By ICP Calibration Block Prep=LM, Plan $=$ SRNL-SCS $-2008-00003$, Set $=2$, Type $=$ Ustd, Oxide $=L 203(w t \%)$ Reference Value $=0.0 \mathrm{wt} \%$

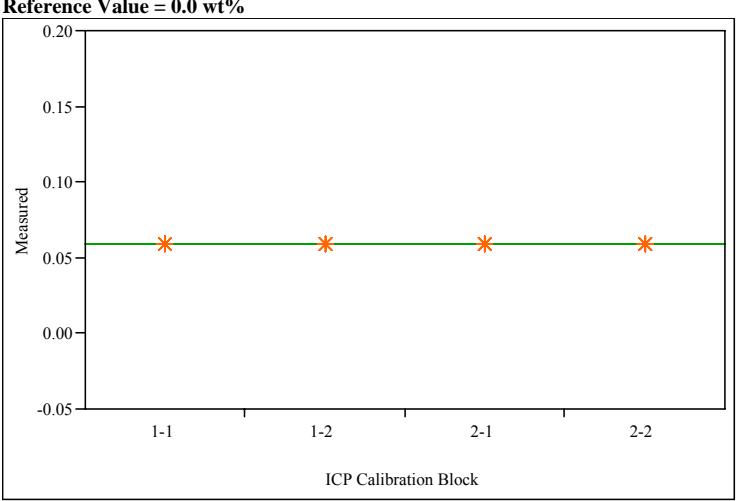

Oneway Anova
Summary of Fit

Rsquare

Adj Rsquare

Root Mean Square Erro

Observations (or Sum Wgts) $\begin{array}{r}0.05864 \\ 12\end{array}$

Analysis of Variance

Source DF Sum of Squares Mean Square F Ratio Prob $>$ F

ICP Calibration Block

C. Total

0
0

Means for Oneway Anova

Level Number Mean Std Error Lower 95\% Upper 95\%

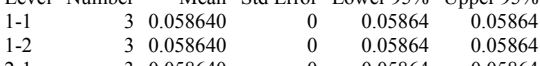

$\begin{array}{llllll}2-2 & 3 & 0.058640 & 0 & 0.05864 & 0.05864 \\ 2 & & & 0 & 0.05864 & 0.05864\end{array}$

Std Error uses a pooled estimate of error variance 
Exhibit A3. PSAL Measurements by ICP Calibration Block for Samples of the

Standard Glasses by Preparation Method by Set by Analytical Plan Memo. (continued)

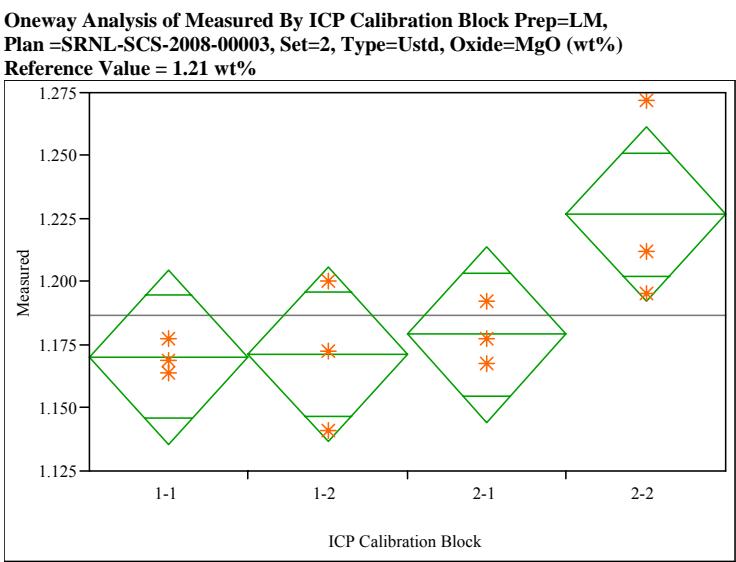

Oneway Anova

$\begin{array}{lr}\text { Rsquare } & 0.544978 \\ \text { Adj Rsquare } & 0.374344 \\ \text { Root Mean Square Error } & 0.025996 \\ \text { Mean of Response } & 1.18679 \\ \text { Observations (or Sum Wgts) } & 12\end{array}$

Analysis of Varianc

Source DF Sum of Squares Mean Square F Ratio Prob $>$ F

$\begin{array}{llllll}\text { ICP Calibration Block } & 3 & 0.00647524 & 0.002158 & 3.1939 & 0.0840\end{array}$

Error
C. Total

$\begin{array}{rr}8 & 0.00540642 \\ 11 & 0.01188166\end{array}$

Means for Oneway Anova

Level Number Mean Std Error Lower 95\% Upper 95\%

$\begin{array}{lrrrrr}1-1 & 3 & 1.17021 & 0.01501 & 1.1356 & 1.2048 \\ 1-2 & 3 & 1.17131 & 0.01501 & 1.1367 & 1.2059 \\ 2-1 & 3 & 1.17905 & 0.01501 & 1.144 & 1.2137\end{array}$

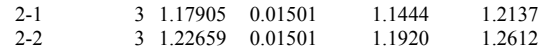

Std Error uses a pooled estimate of error variance

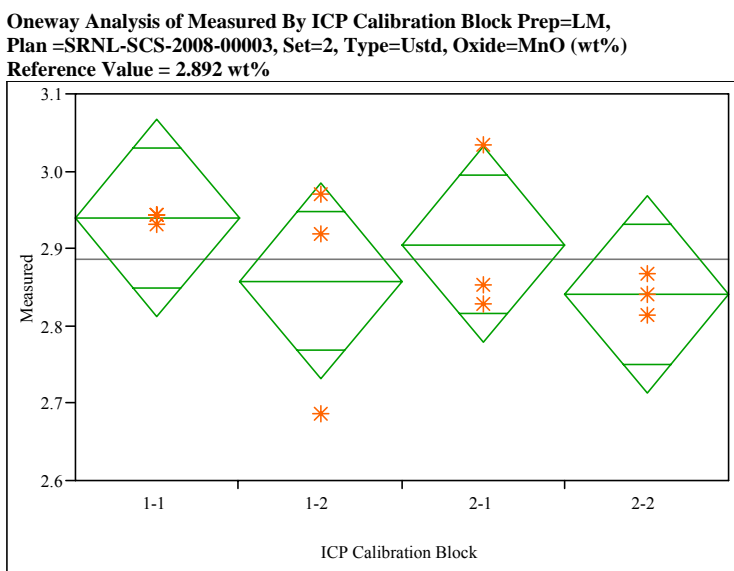

\section{Oneway Anova
Summary of Fit}

Rsquare

Adj Rsquare

Root Mean Square

Mean of Response
Observations (or Sum Wgts)

Analysis of Variance

Source DF Sum of Squares Mean Square F Ratio Prob $>$ F

$\begin{array}{llllll}\text { ICP Calibration Block } & 3 & 0.01828360 & 0.006095 & 0.6718 & 0.5930\end{array}$

Error
C. Total

$8 \quad 0.07257866$ 0.009072

Means for Oneway Anova

\begin{tabular}{lrrrrr}
\multicolumn{2}{l}{$\begin{array}{l}\text { Leans } \\
\text { Level Number }\end{array}$} & Mean & Std Error & Lower 95\% & Upper $95 \%$ \\
$1-1$ & 3 & 2.93963 & 0.05499 & 2.8128 & 3.0664 \\
$1-2$ & 3 & 2.85786 & 0.05499 & 2.7310 & 2.9847
\end{tabular}

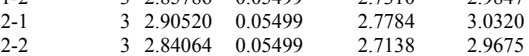

Std Error uses a pooled estimate of error variance
Oneway Analysis of Measured By ICP Calibration Block Prep=LM, Plan =SRNL-SCS-2008-00003, Set=2, Type=Ustd, Oxide $=\mathrm{Na2O}(\mathrm{wt} \%)$ Reference Value $=11.795 \mathrm{wt} \%$

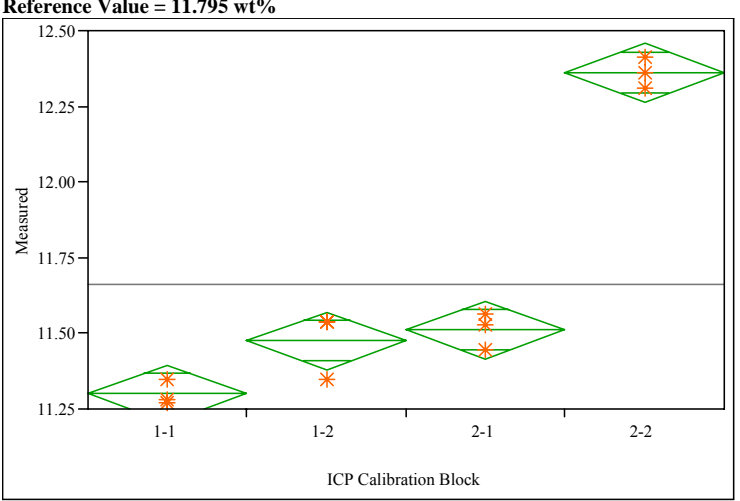

Oneway Anova
Summary of $F i t$

$\begin{array}{lr}\text { Rsquare } & 0.980223 \\ \text { Adj Rsquare } & 0.972807 \\ \text { Root Mean Square Error } & 0.071541 \\ \text { Mean of Response } & 11.66245 \\ \text { Observations (or Sum Wgts) } & 12\end{array}$

Analysis of Variance

Source $\quad$ DF Sum of Squares Mean Square $F$ Ratio Prob $>F$

$\begin{array}{lrlllll} & \text { ion Block } & 3 & 2.0294023 & 0.676467 & 132.1696 & <.000\end{array}$

C. Total

2.0703477

Means for Oneway Anova

Level Number Mean Std Error Lower 95\% Upper 95\%

$\begin{array}{lrrrrr}1-1 & 3 & 11.3007 & 0.04130 & 11.205 & 11.396 \\ 1-2 & 3 & 11.4760 & 0.04130 & 11.381 & 11.571 \\ 2-1 & 3 & 11.5119 & 0.04130 & 11.417 & 11.607\end{array}$

$\begin{array}{llllll}2-2 & 3 & 12.3612 & 0.04130 & 12.266 & 12.456\end{array}$

Std Error uses a pooled estimate of error variance 
Exhibit A3. PSAL Measurements by ICP Calibration Block for Samples of the

Standard Glasses by Preparation Method by Set by Analytical Plan Memo. (continued)

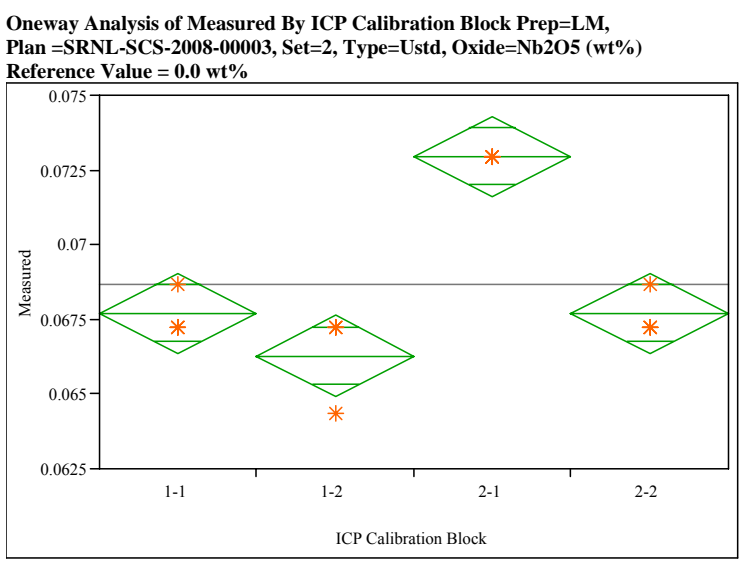

Oneway Analysis of Measured By ICP Calibration Block Prep=LM,

Plan =SRNL-SCS-2008-00003, Set=2, Type=Ustd, 0 dod

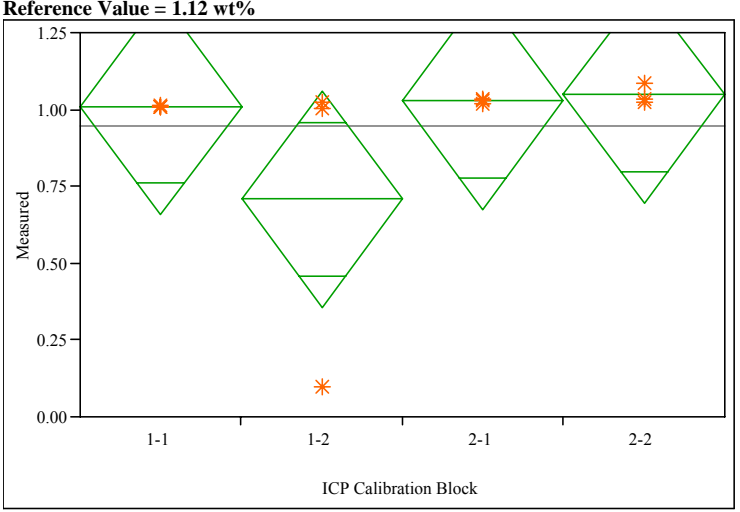

Oneway Anova
Summary of Fit

Oneway Anova
Summary of Fit

$\begin{array}{lr}\text { Rsquare } & 0.904762 \\ \text { Adj Rsquare } & 0.869048 \\ \text { Root Mean Square Error } & 0.001012 \\ \text { Mean of Response } & 0.068664 \\ \text { Observations (or Sum Wgts) } & 12\end{array}$

Analysis of Variance
Source

$\begin{array}{lrrrrr}\text { Source } & \text { DF } & \text { Sum of Squares } & \text { Mean Square } & & \\ \text { ICP Ratibration Block } & 3 & 0.00007776 & 0.000026 & 25.3333 & 0.0002\end{array}$

$\begin{array}{lrrr}\text { Error } & 8 & 0.00000819 & 1.023 \mathrm{e}-6 \\ \text { C. Total } & 11 & 0.00008595 & \end{array}$

Means for Oneway Anova

Level Number Mean Std Error Lower 95\% Upper 95\%

$\begin{array}{lllllll}1-1 & 3 & 0.067710 & 0.00058 & 0.06636 & 0.06906 \\ 1-2 & & 3 & 0.066280 & 0.00058 & 0.06493 & 0.06763 \\ 2-1 & & 3 & 0.072956 & 0.0058 & 0.0761 & 0.07430\end{array}$

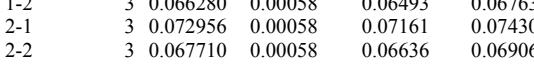

Std Error uses a pooled estimate of error variance

$\begin{array}{lrrrrr}\text { Source } & \text { DF } & \text { Sum of Squares } & \text { Mean Square } & \text { F Ratio } & \text { Prob }>F \\ \text { ICP Calibration Block } & 3 & 0.23072283 & 0.076908 & 1.0980 & 0.4045\end{array}$

Error

8
11 0.56037169 0.070046

Level Number Mean Std Error Lower 95\% Upper 95\%

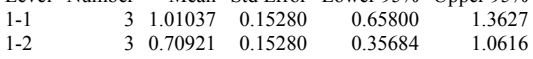

$\begin{array}{lllllll}2-1 & & 3 & 1.02691 & 0.15280 & 0.67454 & 1.3793 \\ 2-2 & & 3 & 1.04684 & 0.15280 & 0.69448 & 1.3992\end{array}$
Oneway Analysis of Measured By ICP Calibration Block Prep=LM, Plan $=$ SRNL-SCS-2008-00003, Set $=2$, Type $=$ Ustd, Oxide $=$ PbO $($ wt $\%)$ Reference Value $=0.0 \mathrm{wt} \%$

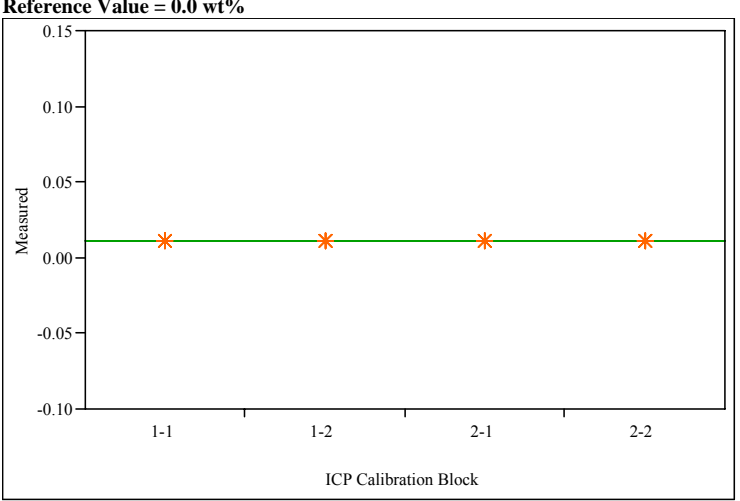

Oneway Anova
Summary of Fit

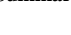

$\begin{array}{lr}\text { Rsquare } & 0 \\ \text { Adj Rsquare } & -0.375 \\ \text { Root Mean Square Error } & 2.122-18 \\ \text { Mean of Response } & 0.010772\end{array}$

Observations (or Sum Wgts) $\quad \begin{array}{r}0.010772 \\ \quad 12\end{array}$

Analysis of Variance

Source $\quad$ DF Sum of Squares Mean Square F Ratio Prob $>$ F

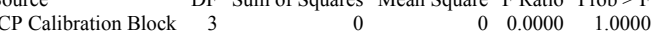

Error $\begin{array}{rr}3.6111 \mathrm{e}-35 & 4.514 \mathrm{e}-36\end{array}$

Means for Oneway Anova

Level Number Mean Std Error Lower 95\% Upper 95\%

$\begin{array}{llllll}1-1 & 3 & 0.010772 & 1.227 \mathrm{e}-18 & 0.01077 & 0.01077 \\ 1-2 & 3 & 0.010772 & 1.227 \mathrm{e}-18 & 0.01077 & 0.01077 \\ 2-1 & 3 & 0.010772 & 1.227 \mathrm{e}-18 & 0.01077 & 0.01077\end{array}$

$\begin{array}{llllll}2-2 & 3 & 0.010772 & 1.227 \mathrm{e}-18 & 0.01077 & 0.01077\end{array}$

Std Error uses a pooled estimate of error variance

Std Error uses a pooled estimate of error variance 
Exhibit A3. PSAL Measurements by ICP Calibration Block for Samples of the

Standard Glasses by Preparation Method by Set by Analytical Plan Memo. (continued)

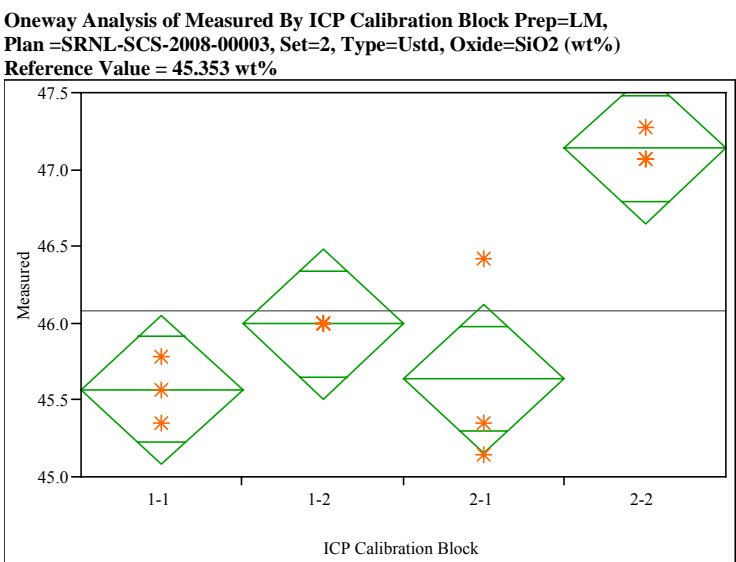

\section{Oneway Anova
Summary of Fit}

$\begin{array}{lr}\text { Rsquare } & 0.816152 \\ \text { Adj Rsquare } & 0.747209 \\ \text { Root Mean Square Error } & 0.365355 \\ \text { Mean of Response } & 46.08409 \\ \text { Observations (or Sum Wgts) } & 12\end{array}$

Analysis of Variance

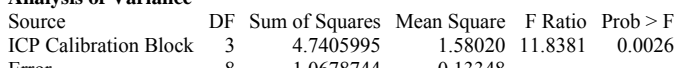

Error

$\begin{array}{lll}1.58020 & 11.8381 & 0.0026 \\ 0.13348 & \end{array}$

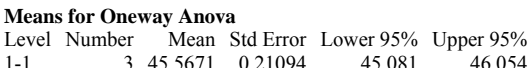

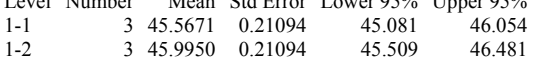

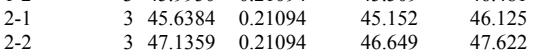

Std Error uses a pooled estimate of error variance

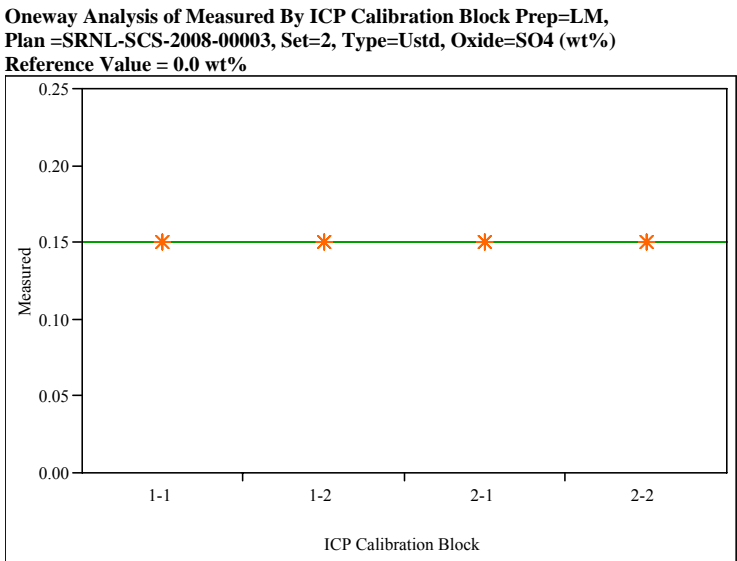

\section{Oneway Anova
Summary of Fit}

Rsquare

Adj Rsquare

Root Mean Square Error

0.149795
Observations (or Sum

Analysis of Variance

Source DF Sum of Squares Mean Square F Ratio Prob $>F$

$\begin{array}{lrrr}\text { ICP Calibration Block } & 3 & 0 & 0 \\ \text { Error } & 8 & 0 & 0\end{array}$

Ana

Level Number Mean Std Error Lower 95\% Upper 95\%

$\begin{array}{rrrrrr}1-1 & 3 & 0.149795 & 0 & 0.14980 & 0.14980 \\ 1-2 & 3 & 0.149795 & 0 & 0.14980 & 0.1480\end{array}$

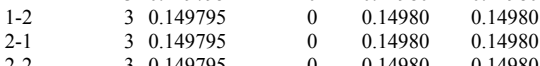

Std Error uses a pooled estimate of error variance
Oneway Analysis of Measured By ICP Calibration Block Prep=LM, Plan $=$ SRNL-SCS-2008-00003, Set $=2$, Type=Ustd, Oxide $=$ ThO2 $($ wt $\%)$ Reference Value $=0.0 \mathrm{wt} \%$

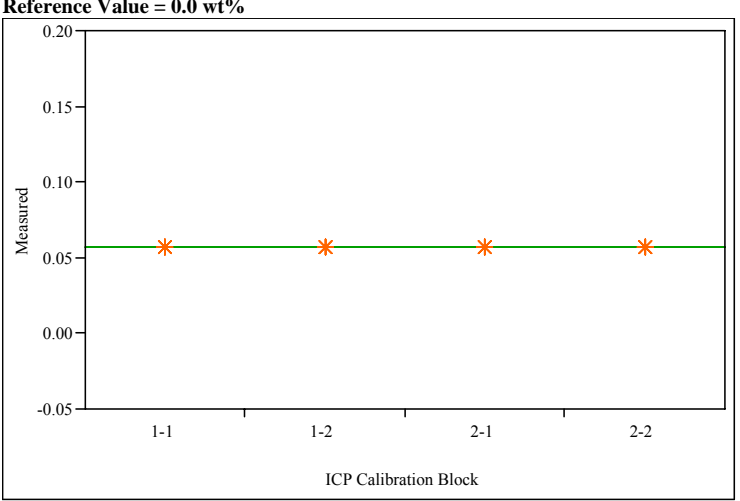

Oneway Anova
Summary of Fit

Rsquare

Adj Rsquare Mean of Response
Observations (or Sum Wgts)

Analysis of Variance

Source $\quad$ DF Sum of Squares Mean Square F Ratio Prob $>$ F

ICP Calibrat

Error
C. Total

Means for Oneway Anova

Level Number Mean Std Error Lower 95\% Upper 95\%

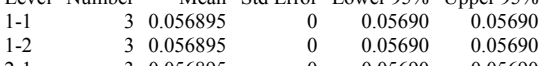

$\begin{array}{llllll}2-2 & 3 & 0.056895 & 0 & 0.05690 & 0.05690 \\ 2-2 & 3 & 0.056895 & 0 & 0.05690 & 0.05690\end{array}$

Std Error uses a pooled estimate of error variance 
Exhibit A3. PSAL Measurements by ICP Calibration Block for Samples of the

Standard Glasses by Preparation Method by Set by Analytical Plan Memo. (continued)

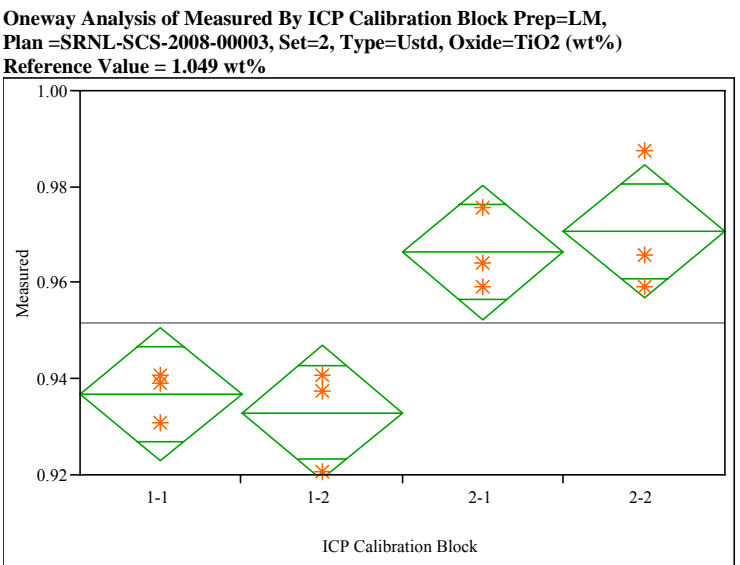

\section{Oneway Anova
Summary of Fit}

$\begin{array}{lr}\text { Rsquare } & 0.7978 \\ \text { Adj Rsquare } & 0.721975 \\ \text { Root Mean Square Error } & 0.01045 \\ \text { Mean of Response } & 0.951733 \\ \text { Observations (or Sum Wgts) } & 12\end{array}$

Analysis of Variance

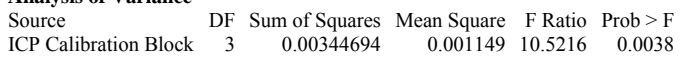

$\begin{array}{lrr}\text { Error } & 8 & 0.00087362 \\ & 11 & 0.00432056\end{array}$ $\begin{array}{lll}0.001149 & 10.5216 & 0.0038 \\ 0.000109 & \end{array}$

C. Tota 0.00432056

Means for Oneway Anova

$\begin{array}{lrrrrr}\text { Level } & \text { Number } & \text { Mean } & \text { Std Error } & \text { Lower } 95 \% & \text { Upper } 95 \% \\ 1-1 & 3 & 0.936860 & 0.00603 & 0.92295 & 0.95077\end{array}$

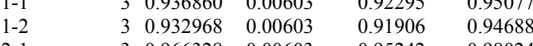

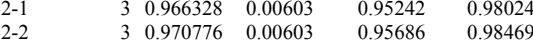

Std Error uses a pooled estimate of error variance

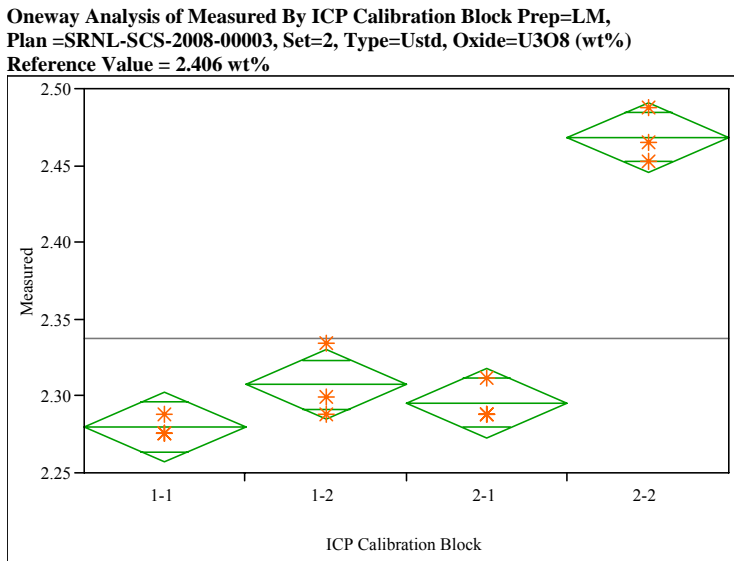

\section{Oneway Anova
Summary of Fit}

Rsquare

$\begin{array}{ll} & 0.967716 \\ \text { Adj Rsquare } & 0.955609\end{array}$

Root Mean Square Error $\quad 0.01702$

Observations (or Sum Wgts)

Analysis of Variance

$\begin{array}{lrrrrr}\text { Source } & \text { DF } & \text { Sum of Squares } & \text { Mean Square } & \text { F Ratio } & \text { Prob }>\text { F } \\ \text { ICP Calibration Block } & 3 & 0.06946769 & 0.023156 & 79.9333 & <.0001\end{array}$

Error
C. Total

$\begin{array}{rr}8 & 0.00231752 \\ 11 & 0.07178522\end{array}$

0.000290

Means for Oneway Anova

Level Number Mean Std Error Lower 95\% Upper 95\%

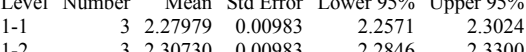

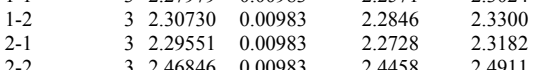

Std Error uses a pooled estimate of error variance
Oneway Analysis of Measured By ICP Calibration Block Prep=LM, Plan =SRNL-SCS-2008-00003, Set=2, Type=Ustd, Oxide=ZnO (wt\%)

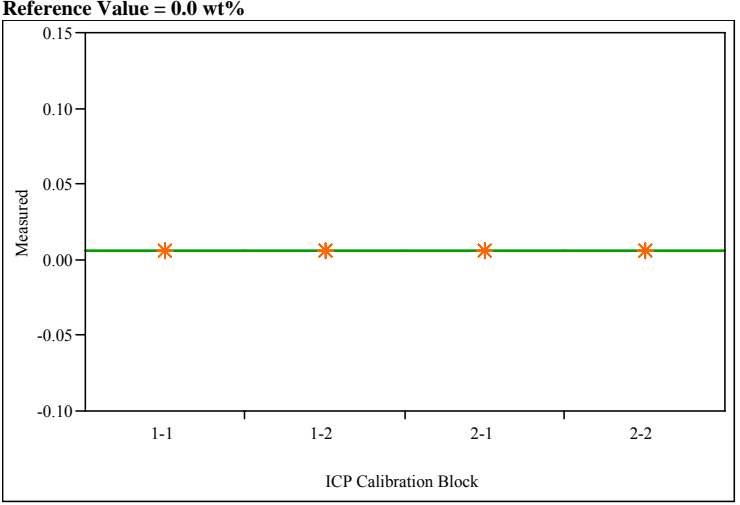

Oneway Anova
Summary of Fit

Rsquare

Adj Rsquare $\begin{array}{lr}\text { Mean of Response } & 0.006224 \\ \text { Observations (or Sum Wgts) } & 12\end{array}$

Analysis of Variance

Source DF Sum of Squares Mean Square F Ratio Prob $>$ F

ICP Calibration Block
Error
C. Total

Means for Oneway Anova

Level Number Mean Std Error Lower 95\% Upper 95\%

$\begin{array}{lrrrrr}1-1 & 3 & 0.006224 & 0 & 0.00622 & 0.00622 \\ 1-2 & 3 & 0.006224 & 0 & 0.00622 & 0.00622 \\ 2-1 & 3 & 0.00622 & 0 & 0.00622 & 0.00622\end{array}$

$\begin{array}{llllll}2-2 & 3 & 0.006224 & 0 & 0.00622 & 0.00622 \\ 2-2 & 3 & & 0 & 0.00622 & 0.00622\end{array}$

Std Error uses a pooled estimate of error variance 
Exhibit A3. PSAL Measurements by ICP Calibration Block for Samples of the

Standard Glasses by Preparation Method by Set by Analytical Plan Memo. (continued)

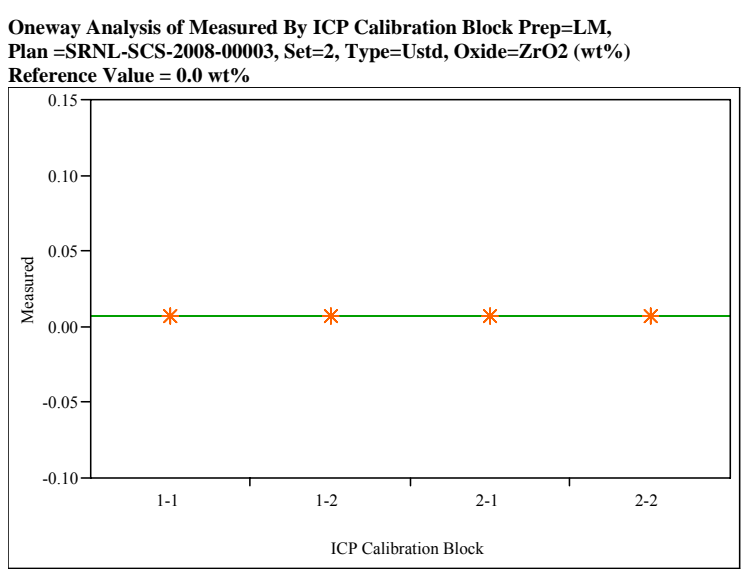

Oneway Analysis of Measured By ICP Calibration Block Prep=LM, Plan =SRNL-SCS-2008-00003, Set $=3$, Type $=$ Batch 1, Oxide $=$ Al2O3 $(\mathrm{wt} \%)$

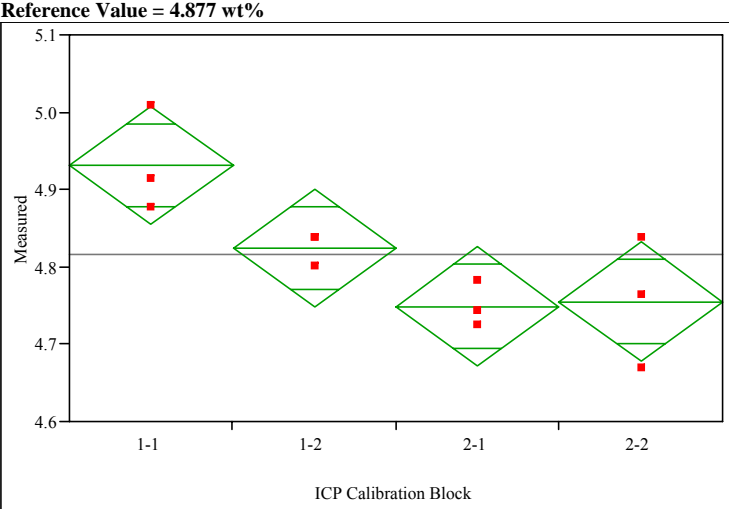

Oneway Anova
Summary of Fit

Rsquare

Adj Rsquare

$\begin{array}{lr}\text { Root Mean Square Error } & 0 \\ \text { Mean of Response } & 0.006754 \\ \text { Observations (or Sum Wgts) } & 12\end{array}$

Analysis of Variance

Analysis of Variance
Source DF Sum of Squares Mean Square F Ratio Prob $>$ F

ICP Calib

Error
C. Total

Means for Oneway Anova

Level Number Mean Std Error Lower 95\% Upper 95\%

$\begin{array}{rrrrrr}1-1 & 3 & 0.006754 & 0 & 0.00675 & 0.00675 \\ 1-2 & 3 & 0.006754 & 0 & 0.00675 & 0.00675 \\ 2-1 & 3 & 0.006754 & 0 & 0.00675 & 0.00675\end{array}$

$\begin{array}{llllll}2-1 & 3 & 0.006754 & 0 & 0.00675 & 0.00675 \\ 2-2 & 3 & 0.006754 & 0 & 0.00675 & 0.00675\end{array}$

Std Error uses a pooled estimate of error variance

\section{Oneway Anova}

Rsquare

$\begin{array}{ll}\text { Rsquare } & 0.710561 \\ \text { Adj Rsquare } & 0.602021\end{array}$

Root Mean Square Error $\quad 0.057467$

Mean of Response 4.815076

Analysis of Variance

Source DF Sum of Squares Mean Square F Ratio Prob $>F$

$\begin{array}{lrrrrr}\text { ICP Calibration Block } & 3 & 0.06485882 & 0.021620 & 6.5465 & 0.0151 \\ \text { Error } & 8 & 0.02641956 & 0.003302 & & \end{array}$

Error
C. Total

0.02641956
0.09127838

$\begin{array}{lrrrr}\text { Means for Oneway Anova } & & \\ \text { Level Number } & \text { Mean } & \text { Std Error } & \text { Lower 95\% } & \text { Upper } 95 \% \\ 1-1 & 3 & 4.93160 & 0.03318 & 4.8551 \\ 1-2 & 3 & 4.0081\end{array}$

$\begin{array}{lrrrrr}1-1 & 3 & 4.93160 & 0.03318 & 4.8551 & 5.0081 \\ 1-2 & 3 & 4.82452 & 0.03318 & 4.7480 & 4.9010\end{array}$

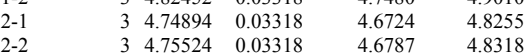

Std Error uses a pooled estimate of error variance
Oneway Analysis of Measured By ICP Calibration Block Prep=LM, Plan =SRNL-SCS-2008-00003, Set=3, Type=Batch 1 , Oxide=BaO (wto) Reference Value $=0.151 \mathrm{wt} \%$

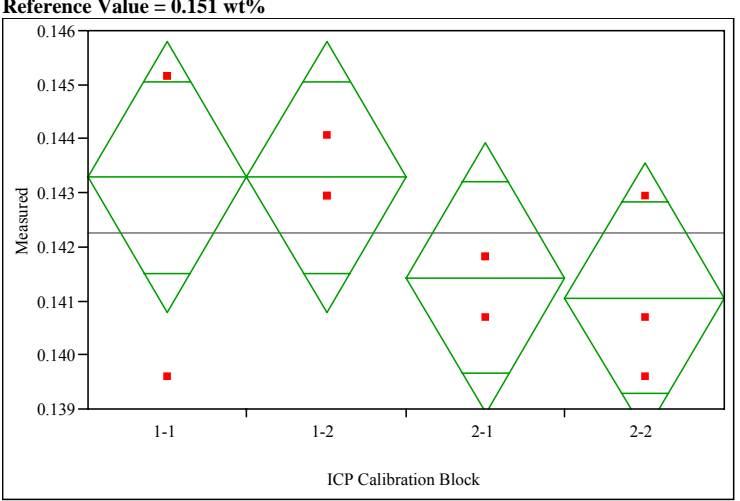

Oneway Anova
Summary of Fit

Rsquare

0.311392

Root Mean Squre Error $\quad 0.001879$

$\begin{array}{lr}\text { Mean of Response } & 0.142261 \\ \text { Observations (or Sum Wgts) } & 12\end{array}$

Analysis of Variance

Source $\quad$ DF Sum of Squares Mean Square F Ratio Prob $>F$

Error
C. Total

0.00004103

Means for Oneway Anova

Level Number Mean Std Error Lower 95\% Upper 95\%

$\begin{array}{lllllll}1-1 & & 3 & 0.143284 & 0.00109 & 0.14078 & 0.14579 \\ 1-2 & & 3 & 0.143284 & 0.00109 & 0.14078 & 0.14579\end{array}$

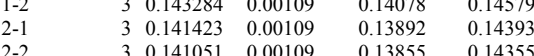

Std Error uses a pooled estimate of error variance $\begin{array}{llllll} & 3 & 0.00001278 & 4.2591 \mathrm{e}-6 & 1.2059 & 0.3681\end{array}$ 
Exhibit A3. PSAL Measurements by ICP Calibration Block for Samples of the

Standard Glasses by Preparation Method by Set by Analytical Plan Memo. (continued)

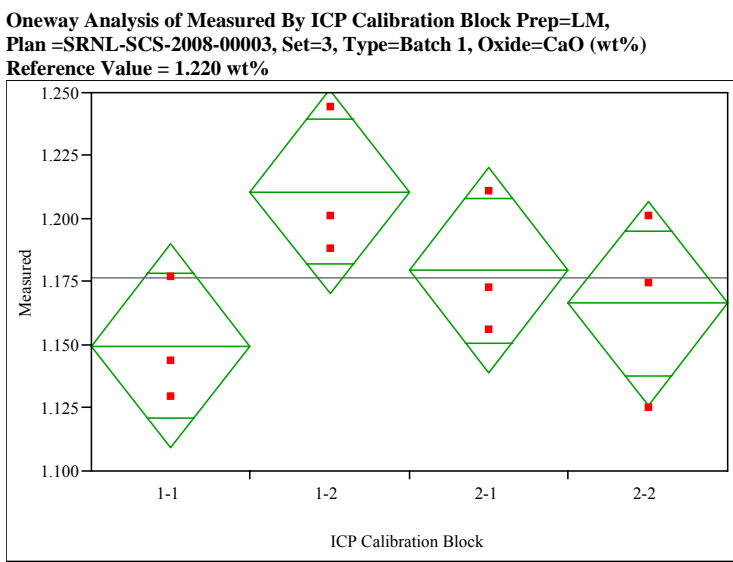

Oneway Anova
Summary of Fit

Rsquare

Adj Rsquare $\quad 0.447596$

Root Mean Square Error $\quad 0.240444$

$\begin{array}{lr}\text { Mean of Response } & 1.176611 \\ \text { Observations (or Sum Wgts) } & 12\end{array}$

Analysis of Variance

$\begin{array}{lrrrrr}\text { Source } & \text { DF } & \text { Sum of Squares } & \text { Mean Square } & \text { F Ratio } & \text { Prob }>\text { F } \\ \text { ICP Calibration Block } & 3 & 0.00601212 & 0.002004 & 2.1607 & 0.1707\end{array}$ $\begin{array}{lrrr}\text { ICP Calibration Block } & 3 & 0.00601212 & 0.002004 \\ \text { Error } & 8 & 0.00741991 & 0.000927 \\ \text { C. Total } & 11 & 0.01343203 & \end{array}$

Means for Oneway Anova

\begin{tabular}{lrrrrr}
\multicolumn{2}{l}{$\begin{array}{l}\text { Means for Oneway Anova } \\
\text { Level }\end{array}$ Number } & Mean & Std Error & Lower 95\% & Upper $95 \%$ \\
$1-1$ & 3 & 1.14968 & 0.01758 & 1.1091 & 1.1902 \\
$1-2$ & 3 & 1.21077 & 0.01758 & 1.1702 & 1.2513 \\
$2-1$ & 3 & 1.17953 & 0.01758 & 1.1390 & 1.2201 \\
$2-2$ & 3 & 1.16647 & 0.01758 & 1.1259 & 1.2070
\end{tabular}

Std Error uses a pooled estimate of error variance

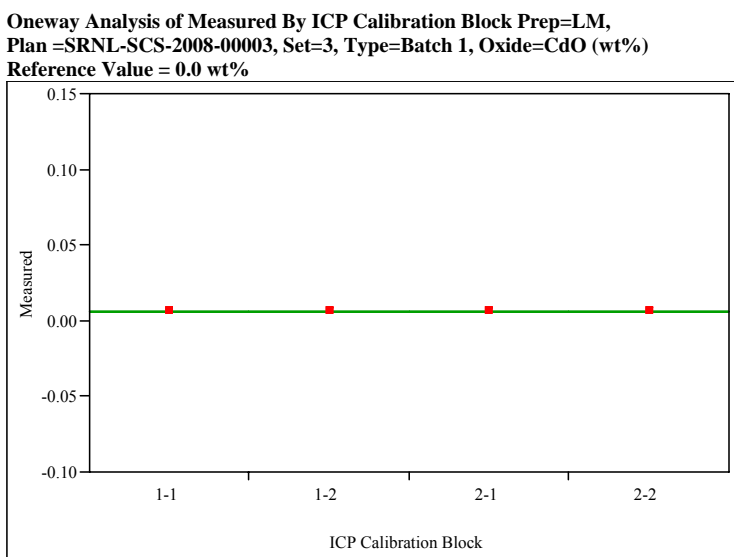

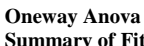

Rsquare

Adj Rsquare

Root Mean Square Erro

0.005712
Observations (or Sum Wgts)

Analysis of Variance

Source $\quad$ DF Sum of Squares Mean Square F Ratio Prob $>$ F

ICP Cal

Error

0
0

Means for Oneway Anova
$\begin{array}{llll} & \\ \text { Level Number } & \text { Mean Std Error Lower 95\% Upper 95\% }\end{array}$

$1-2$

$2-1$

Std Error uses a pooled estimate of error variance
Oneway Analysis of Measured By ICP Calibration Block Prep=LM, Plan =SRNL-SCS-2008-00003, Set=3, Type=Batch 1, Oxide=Ce2O3 (wt $\%)$ Reference Value $=0.0$ wt $\%$

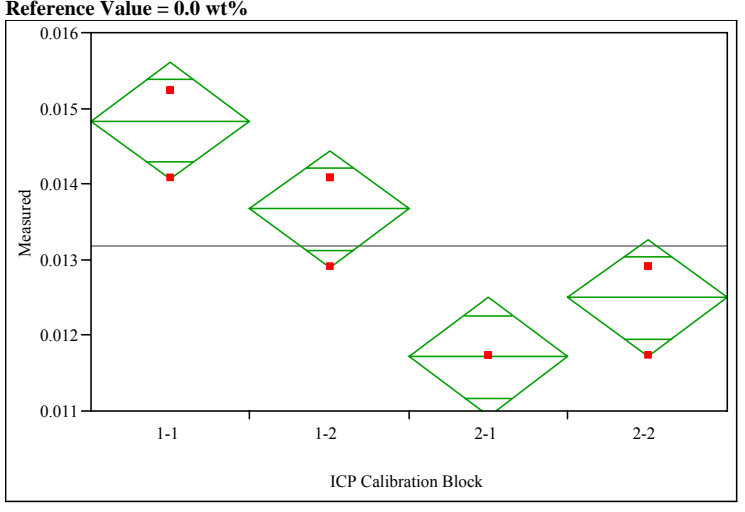

Oneway Anova
Summary of Fit

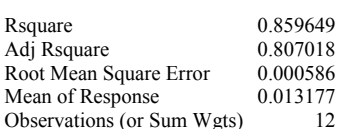

$\begin{array}{lr}\text { Mean of Response } & 0.013177 \\ \text { Observations (or Sum Wgts) } & 12\end{array}$

Analysis of Variance

Source DF Sum of Squares Mean Square F Ratio Prob $>$ F

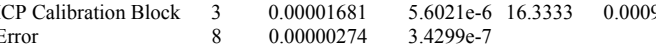

Error
C. Total

0.00001955

Means for Oneway Anova
Level Number Mean Std Error Lower 95\% Upper 95\%

$\begin{array}{lllllll}1-1 & & 3 & 0.014836 & 0.00034 & 0.01406 & 0.01562 \\ 1-2 & & 3 & 0.013665 & 0.00034 & 0.01289 & 0.01444\end{array}$

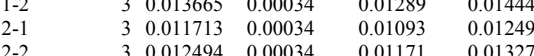

Std Error uses a pooled estimate of error variance 
Exhibit A3. PSAL Measurements by ICP Calibration Block for Samples of the

Standard Glasses by Preparation Method by Set by Analytical Plan Memo. (continued)

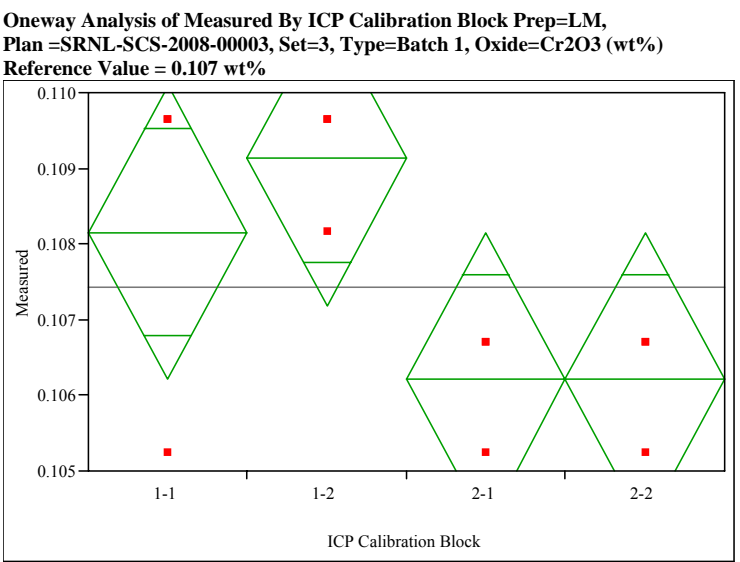

Oneway Anova
Summary of Fit

Rsquare

Adj Rsquare $\quad 0.529412$

Root Mean Square Error $\quad 0.352941$

$\begin{array}{lr}\text { Mean of Response } & 0.107428 \\ \text { Observations (or Sum Wgts) } & 12\end{array}$

Analysis of Variance

Source $\quad$ DF Sum of Squares Mean Square F Ratio Prob $>$ F

$\begin{array}{llllll}\text { ICP Calibration Block } 3 & 0.00001923 & 6.4088 \mathrm{e}-6 & 3.0000 & 0.0951\end{array}$

Error

$\begin{array}{rr}8 & 0.00001709 \\ 11 & 0.00003632\end{array}$

Means for Oneway Anova

Level Number Mean Std Error Lower 95\% Upper 95\%

$\begin{array}{lllllll}1-1 & & 3 & 0.108158 & 0.00084 & 0.10621 & 0.11010 \\ 1-2 & & 3 & 0.109133 & 0.00084 & 0.10719 & 0.11108\end{array}$

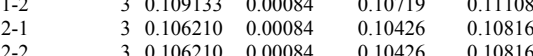

Std Error uses a pooled estimate of error variance

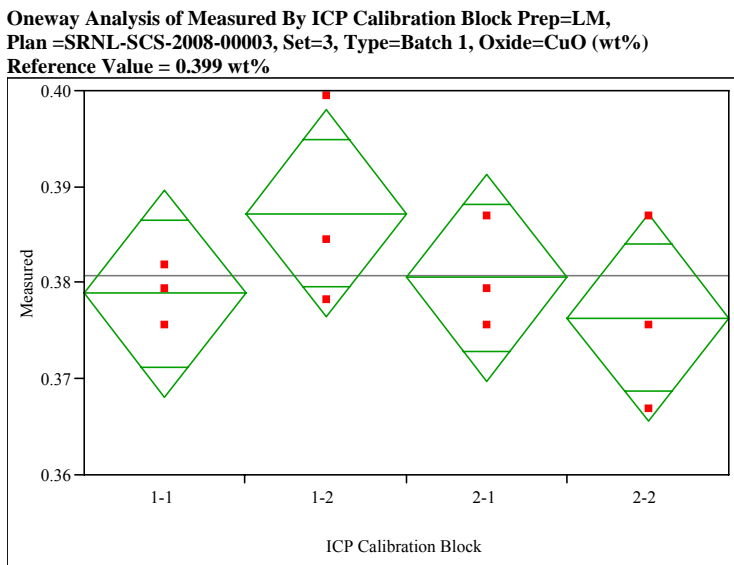

\section{Oneway Anova
Summary of Fit}

Rsquare

\begin{tabular}{ll} 
Rsquare & 0.269036 \\
Adj Rsquare & -0.00508 \\
Root Mean Square Error & 0.008113 \\
\hline
\end{tabular}

Mean of Respane Error $\quad 0.008113$

Observations (or Sum Wots)

Analysis of Variance

Source

$\begin{array}{llllll}\text { ICP Calibration Block } & 3 & 0.00019379 & 0.000065 & 0.9815 & 0.4484\end{array}$

$\begin{array}{lrr}\text { Error } & 8 & 0.00052651 \\ \text { C. Total } & 11 & 0.00072030\end{array}$ 0.000066

Means for Oneway Anova

Level Number Mean Std Error Lower 95\% Upper 95\%

$\begin{array}{lllllll}1-1 & & 3 & 0.378878 & 0.00468 & 0.36808 & 0.38968\end{array}$

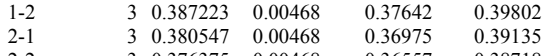

Std Error uses a pooled estimate of error variance
Oneway Analysis of Measured By ICP Calibration Block Prep=LM, Plan =SRNL-SCS-2008-00003, Set $=3$, Type=Batch 1, Oxide=Fe2O3 $($ wt $\%)$ Reference Value $=12.839 \mathrm{wt} \%$

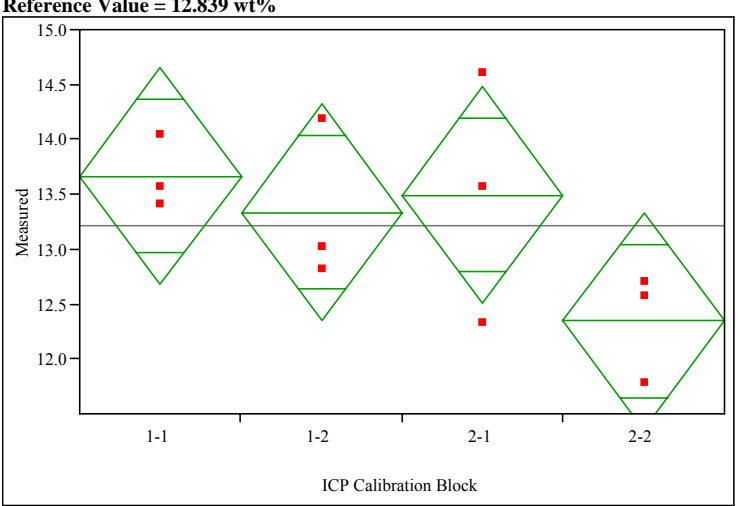

Oneway Anova
Summary of

$\begin{array}{lr}\text { Rsquare } & 0.415438 \\ \text { Adj Rsquare } & 0.196227 \\ \text { Root Mean Square Error } & 0.742011 \\ \text { Mean of Response } & 13.20924 \\ \text { Observations (or Sum Wgts) } & 12\end{array}$

Analysis of Varianc

Source DF Sum of Squares Mean Square F Ratio Prob $>$ F

$\begin{array}{llllll}\text { ICP Calibration Block } & 3 & 3.1302972 & 1.04343 & 1.8952 & 0.2089\end{array}$

$\begin{array}{lrr}\text { Error } & 8 & 4.4046382 \\ \text { C. Total } & 11 & 7.5349353\end{array}$

Means for Oneway Anova

Level Number Mean Std Error Lower 95\% Upper 95\%

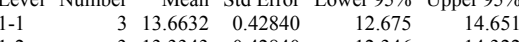

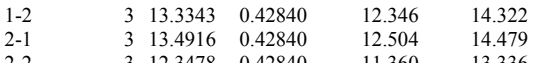

Std Error uses a pooled estimate of error variance 
Exhibit A3. PSAL Measurements by ICP Calibration Block for Samples of the

Standard Glasses by Preparation Method by Set by Analytical Plan Memo. (continued)

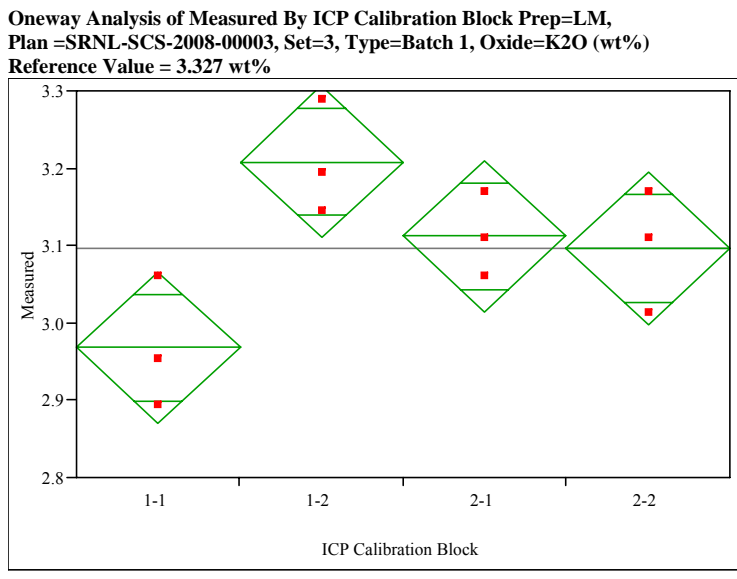

Oneway Anova
Summary of Fit

$\begin{array}{lr}\text { Rsquare } & 0.66828 \\ \text { Adj Rsquare } & 0.543886 \\ \text { Root Mean Square Error } & 0.073857 \\ \text { Mean of Response } & 3.095922 \\ \text { Observations (or Sum Wgts) } & 12\end{array}$

$\begin{array}{lrrrrr}\text { Analysis of Variance } & & & & \\ \text { Source oum } & \text { DF } & \text { Sum of Squares } & \text { Mean Square } & \text { F Ratio } & \text { Prob }>\text { F } \\ \text { ICP Calibration Block } & 3 & 0.08791532 & 0.029305 & 5.3723 & 0.0255 \\ \text { Error } & 8 & 0.04363921 & 0.005455 & & \\ \text { C. Total } & 11 & 0.13155453 & & & \end{array}$

Means for Oneway Anova

Level Number Mean Std Error Lower 95\% Upper 95\%

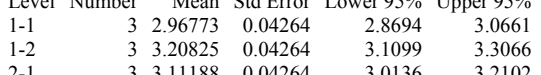

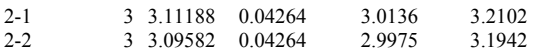

Std Error uses a pooled estimate of error variance

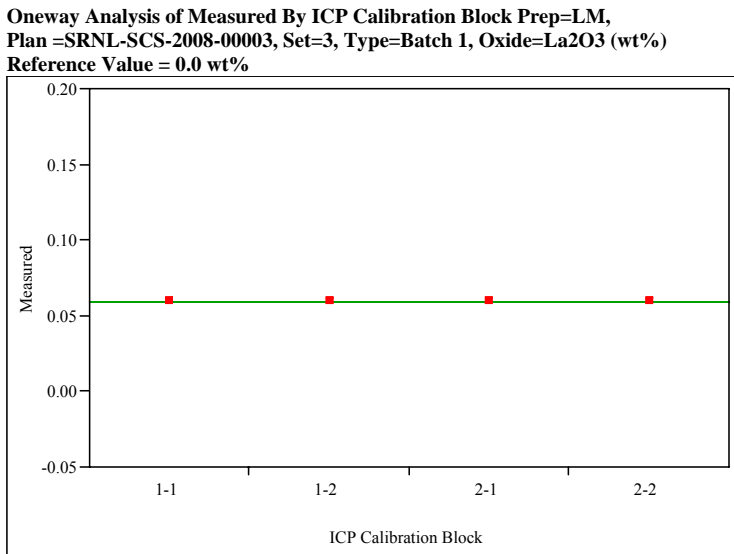

Oneway Anova
Summary of Fit

Rsquare

Adj Rsquare

Root Mean Square Eror

0.05864
Observations (or Sum Wgts)

Analysis of Variance

Source $\quad$ DF Sum of Squares Mean Square F Ratio Prob $>$ F

ICP Cal

Error
C. Total

Means for Oneway Anova

Level Number Mean Std Error Lower 95\% Upper 95\%

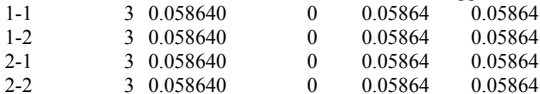

Std Error uses a pooled estimate of error variance
Oneway Analysis of Measured By ICP Calibration Block Prep=LM,

Plan =SRNL-SCS-2008-00003, Set=3, Type=Batch 1 , Oxide=MgO $\left(w_{t} \%\right)$ Reference Value $=1.419 \mathrm{wt} \%$

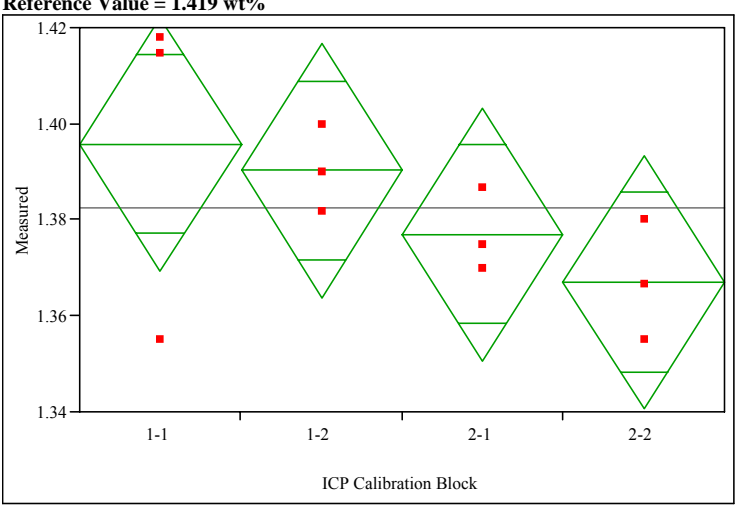

Oneway Anova
Summary of Fit

\begin{tabular}{lr} 
Rsquare & 0.326113 \\
Adj Rsquare & 0.073405 \\
Root Mean Square Error & 0.019801 \\
Mean of Response & 1.382469 \\
\hline Obserations (or Sum
\end{tabular}

Observations (or Sum

Analysis of Variance

Source $\quad$ DF Sum of Squares Mean Square F Ratio Prob $>$ F

$\begin{array}{lllllll}\text { ICP Calibration Block } & 3 & 0.00151798 & 0.000506 & 1.2905 & 0.3423\end{array}$

$\begin{array}{lrr}\text { Error } & 8 & 0.00313679 \\ \text { C. Total } & 11 & 0.00465476\end{array}$ .000392

Means for Oneway Anova

Level Number Mean Std Error Lower 95\% Upper 95\%

$\begin{array}{llllrr}1-1 & 3 & 1.39574 & 0.01143 & 1.3694 & 1.4221 \\ 1-2 & 3 & 1.39021 & 0.01143 & 1.3638 & 1.4166\end{array}$

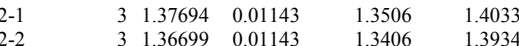

Std Error uses a pooled estimate of error variance 
Exhibit A3. PSAL Measurements by ICP Calibration Block for Samples of the

Standard Glasses by Preparation Method by Set by Analytical Plan Memo. (continued)

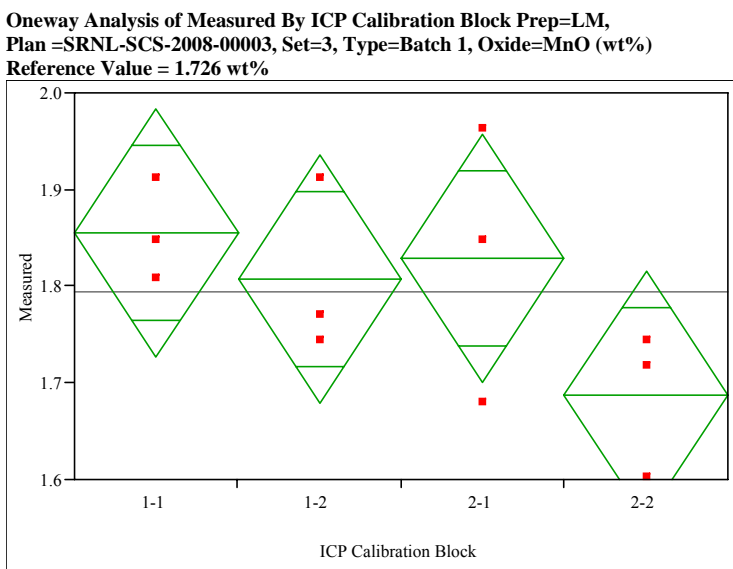

Oneway Anova

Rsquare

Adj Rsquare

Root Mean Square Error $\quad 0.096192$

$\begin{array}{lr}\text { Mean of Response } & 1.794768 \\ \text { Observations (or Sum Wgts) } & 12\end{array}$

Analysis of Variance

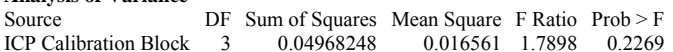

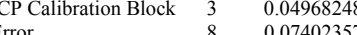

C. Total

$8 \quad 0.07402357$ 0.009253

Level Number Mean Std Error Lower 95\% Upper 95\%

$\begin{array}{lllllll}1-1 & & 3 & 1.85502 & 0.05554 & 1.7270 & 1.9831 \\ 1-2 & & 3 & 1.80768 & 0.05554 & 1.6796 & 1.9357\end{array}$

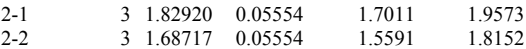

Std Error uses a pooled estimate of error variance

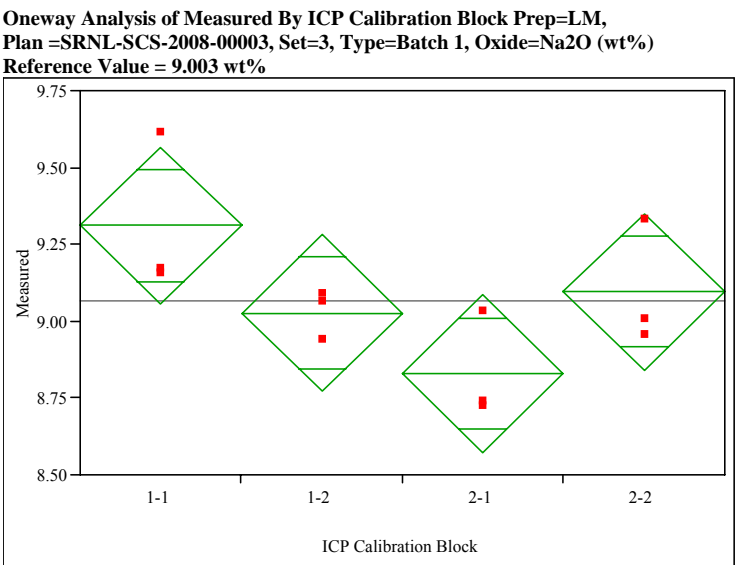

\section{Oneway Anova
Summary of Fit}

Rsquare

$\begin{array}{lr}0.546757 \\ \text { Adj Rsquare } & 0.37679\end{array}$

Root Mean Square Error $\quad 0.191468$

Observations (or Sum Wgts) $\quad 9.0653$

Analysis of Variance

Source $\quad$ DF Sum of Squares Mean Square F Ratio Prob $>F$

$\begin{array}{llllll}\text { ICP Calibration Block } & 3 & 0.35379015 & 0.117930 & 3.2169 & 0.0828\end{array}$

Error
C. Total

$\begin{array}{rr}8 & 0.29328059 \\ 11 & 0.64707073\end{array}$ 0.036660

Means for Oneway Anova

Level Number Mean Std Error Lower 95\% Upper 95\%

$\begin{array}{rrrrrr}1-1 & 3 & 9.31019 & 0.11054 & 9.0553 & 9.5651 \\ 1-2 & & 3 & 9.02711 & 0.11054 & 9.7722\end{array}$

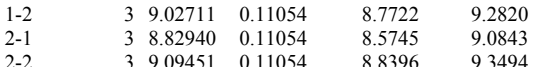

Std Error uses a pooled estimate of error variance
Oneway Analysis of Measured By ICP Calibration Block Prep=LM, Plan $=$ SRNL-SCS-2008-00003, Set=3, Type=Batch 1, Oxide $=$ Nb2O5 $($ wt $\%)$ Reference Value $=0.0 \mathrm{wt} \%$

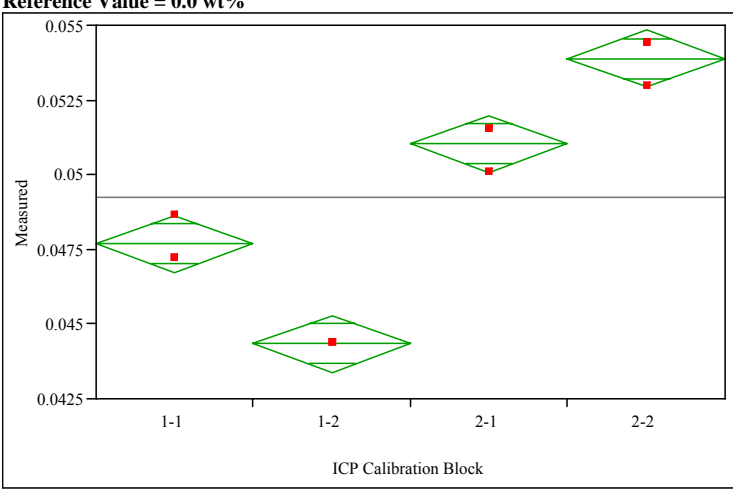

Oneway Anova

ummary of Fit

Rsquare

Root Mean Square Error $\quad 0.000715$

$\begin{array}{lr}\text { Mean of Response } & 0.049233 \\ \text { Observations (or Sum Wgts) } & 12\end{array}$

Analysis of Variance
Source

Source 1 CP Calibration Block DF S

$\begin{array}{lrrr}\text { Error } & 8 & 0.00000409 & 5.116 \mathrm{e}-7 \\ \text { C. Total } & 11 & 0.00015740 & \end{array}$

Means for Oneway Anova

Level Number Mean Std Error Lower 95\% Upper 95\%

$\begin{array}{lllllll}1-1 & 3 & 0.047683 & 0.00041 & 0.04673 & 0.0486 \\ 1-2 & 3 & 0.044346 & 0.00041 & 0.04339 & 0.04530\end{array}$

$\begin{array}{lllllll}2-1 & & 3 & 0.051021 & 0.00041 & 0.05007 & 0.05197 \\ 2-2 & & 3 & 0.053882 & 0.00041 & 0.05293 & 0.05483\end{array}$

Std Error uses a pooled estimate of error variance 
Exhibit A3. PSAL Measurements by ICP Calibration Block for Samples of the

Standard Glasses by Preparation Method by Set by Analytical Plan Memo. (continued)

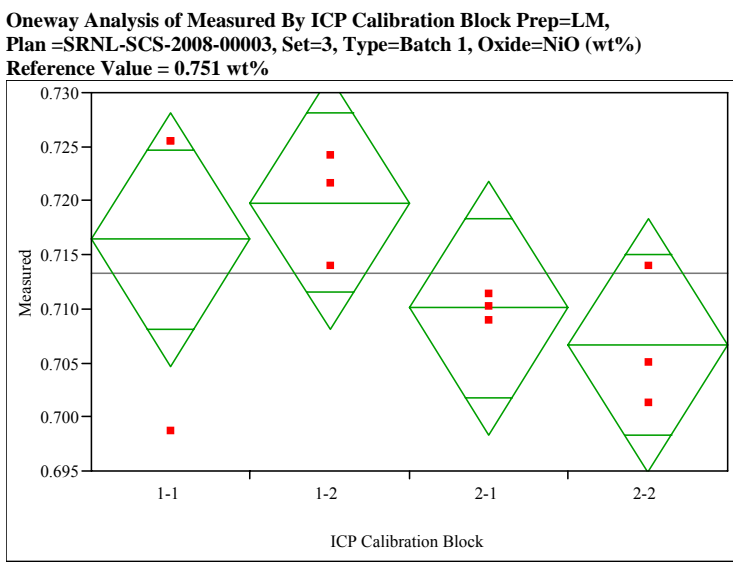

Oneway Anova
Summary of Fit

Rsquare

Adj Rsquare $\quad 0.340218$

0.0928
0.008808

$\begin{array}{lr}\text { Mean of Response } & 0.713236 \\ \text { Observations (or Sum Wgts) } & 12\end{array}$

Analysis of Variance

$\begin{array}{lrrrrr}\text { Source } & \text { DF } & \text { Sum of Squares } & \text { Mean Square } & \text { F Ratio } & \text { Prob }>\text { F } \\ \text { ICP Calibration Block } & 3 & 0.00032007 & 0.000107 & 1.3751 & 0.3185\end{array}$

$\begin{array}{lrrr}\text { ICP Calibration Block } & 3 & 0.00032007 & 0.000107 \\ \text { Error } & 8 & 0.00062071 & 0.000078 \\ \text { C. Total } & 11 & 0.00094079 & \end{array}$

Means for Oneway Anova

Level Number Mean Std Error Lower 95\% Upper 95\%

$\begin{array}{llrrrr}1-1 & 3 & 0.716418 & 0.00509 & 0.70469 & 0.72814 \\ 1-2 & 3 & 0.719811 & 0.00509 & 0.70808 & 0.73154 \\ 2-1 & 3 & 0.710055 & 0.00509 & 0.69833 & 0.72178\end{array}$

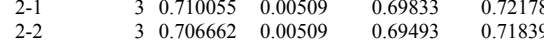

Std Error uses a pooled estimate of error variance

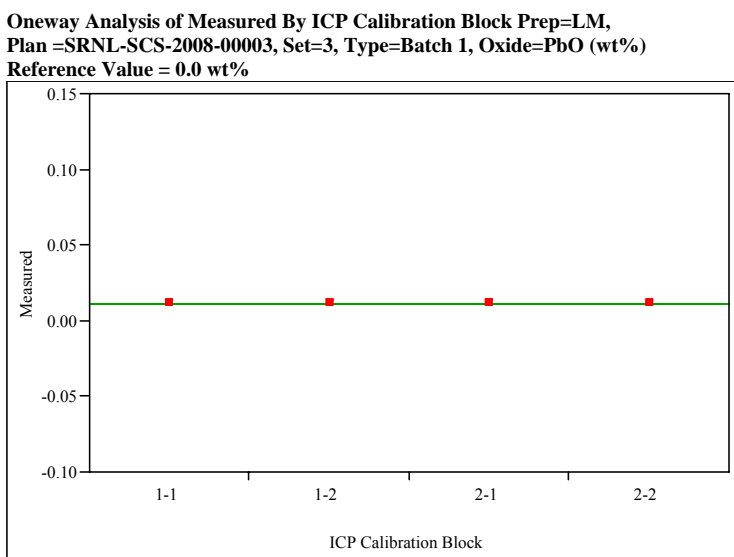

Oneway Anova
Summary of $F i t$

Rsquare

$\begin{array}{lr}\text { Rsquare } & 0 \\ \text { Adj Rsquare } & -0.375 \\ \text { Root Mean Square Error } & 2.12 \mathrm{e}-18 \\ \text { Mean of Response } & 0.010772 \\ \text { Observations (or Sum Wgts) } & 12\end{array}$

Analysis of Variance

Source DF Sum of Squares Mean Square F Ratio Prob $>F$

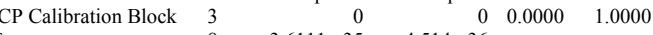

Error

$3.6111 \mathrm{e}-35$
$3.6111 \mathrm{e}-35$

$4.514 \mathrm{e}-36$

Means for Oneway Anova

Level Number Mean Std Error Lower 95\% Upper 95\%

$\begin{array}{lllllll}1-1 & 3 & 0.010772 & 1.227 \mathrm{e}-18 & 0.01077 & 0.01077 \\ 1-2 & & 3 & 0.010772 & 1.227 \mathrm{e}-18 & 0.01077 & 0.01077\end{array}$

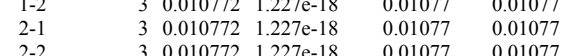

Std Error uses a pooled estimate of error variance
Oneway Analysis of Measured By ICP Calibration Block Prep=LM, Plan $=$ SRNL-SCS-2008-00003, Set=3, Type $=$ Batch 1 , Oxide $=$ SiO2 $($ wt $\%)$ Reference Value $=50.22 \mathrm{wt} \%$

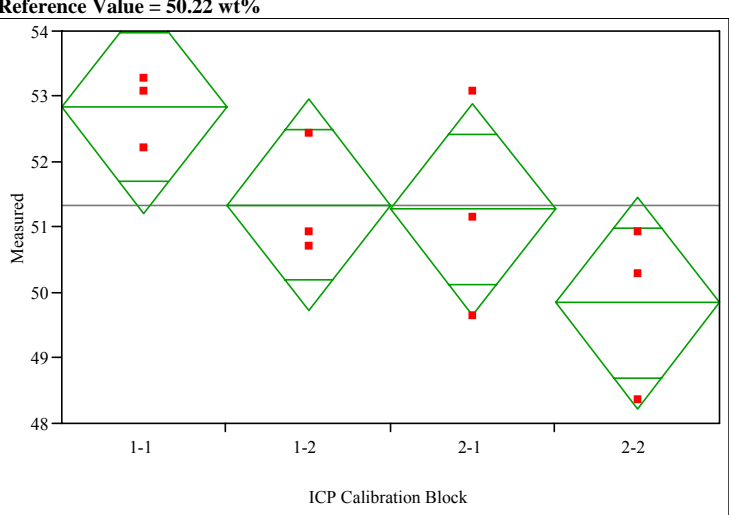

Oneway Anova

$\begin{array}{ll}\text { Rsquare } & 0.532178 \\ \text { Adj Rsquare } & 0.356745 \\ \text { Rjon } & .26457\end{array}$

Root Mean Square Error $\quad 1.216457$

$\begin{array}{lr}\text { Mean of Response } & 51.32537 \\ \text { Observations (or Sum Wgts) } & 12\end{array}$

Analysis of Variance

Source DF Sum of Squares Mean Square F Ratio Prob $>$ F

ICP Calibration Block $3 \quad 13.466659$

$\begin{array}{lrr}\text { Error } & 8 & 11.838150 \\ \text { C. Total } & 11 & 25.304809\end{array}$

$\begin{array}{lll}4.48889 & 3.0335 & 0.093\end{array}$

Means for Oneway Anova

Level Number Mean Std Error Lower 95\% Upper 95\%

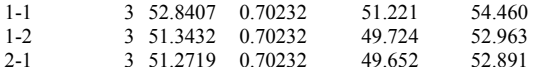

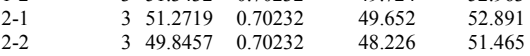

Std Error uses a pooled estimate of error variance 
Exhibit A3. PSAL Measurements by ICP Calibration Block for Samples of the

Standard Glasses by Preparation Method by Set by Analytical Plan Memo. (continued)

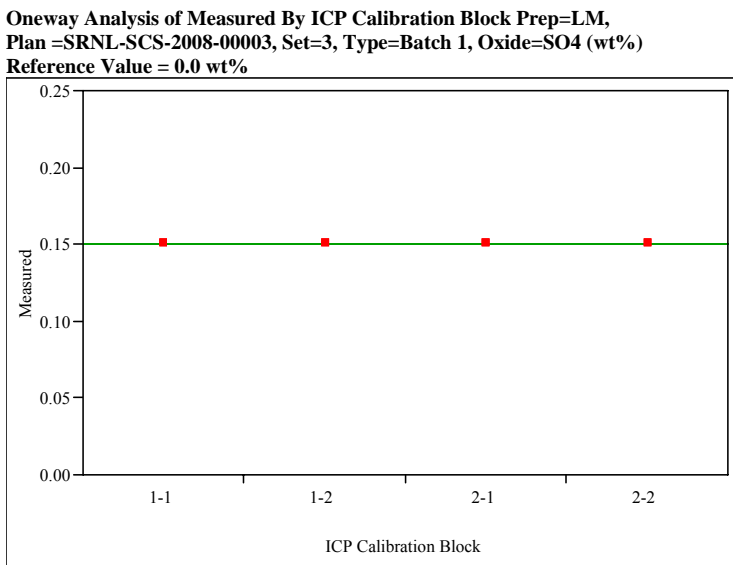

\section{Oneway Anova
Summary of Fit}

Rsquare
Adj Rsquare

$\begin{array}{lr}\text { Adj Rsquare } & \dot{0} \\ \text { Root Mean Square Error } & 0.149795 \\ \text { Mean of Response } & \text { Observations (or Sum Wgts) }\end{array}$

Analysis of Variance

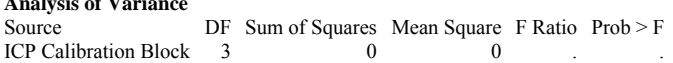

Error

$\begin{array}{rrr}3 & 0 & 0 \\ 8 & 0 & 0 \\ 11 & 0 & \end{array}$

C. Total

nova

Level Number Mean Std Error Lower 95\% Upper 95\%

$\begin{array}{rrrrrr}1-1 & 3 & 0.149795 & 0 & 0.14980 & 0.14980 \\ 1-2 & 3 & 0.149795 & 0 & 0.14980 & 0.14980\end{array}$

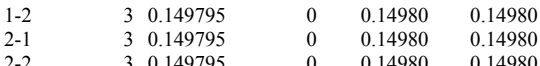

Std Error uses a pooled estimate of error variance

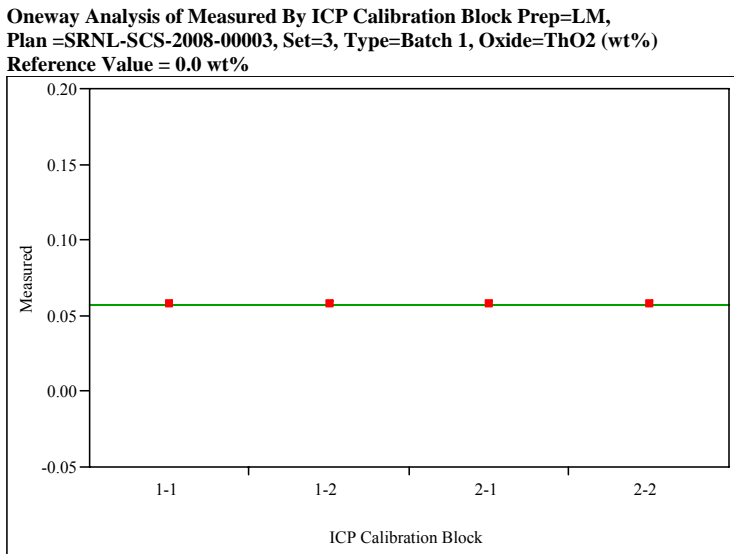

Oneway Anova

Rsquare

Adj Rsquare

$\begin{array}{lr}\text { Mean of Response } & 0.056895 \\ \text { Observations (or Sum Wgts) } & 12\end{array}$

Analysis of Variance

Source DF Sum of Squares Mean Square F Ratio Prob $>F$

ICP Cal

Error
C. Total

0
0

Means for Oneway Anova

Level Number Mean Std Error Lower 95\% Upper 95\%

$\begin{array}{lll}1-1 & 3 & 0.056895 \\ 1-2 & 3 & 0.056895 \\ 2-1 & 3 & 0.056895\end{array}$

$\begin{array}{ll}3 & 0.056895 \\ 3 & 0.056895 \\ 3 & 0.056895\end{array}$

0.05690
0.05960
0.05690
0.05690

0.05690
0.05690
0.05690

Std Error uses a pooled estimate of error variance
Oneway Analysis of Measured By ICP Calibration Block Prep=LM, Plan =SRNL-SCS-2008-00003, Set=3, Type =Batch 1, Oxide =TiO2 (wt $\%)$ Reference Value $=0.677 \mathrm{wt} \%$

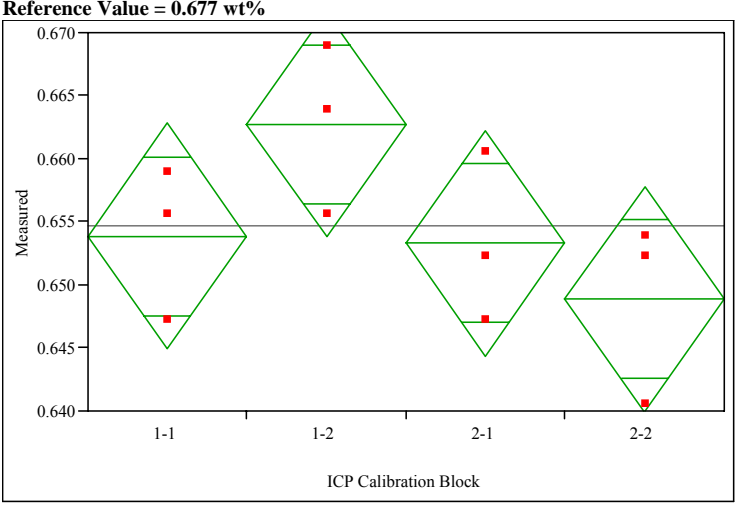

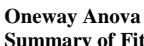

Rsquere

Rsquare
Adj Rsquare

0.458856
Error $\quad 0.255927$

Mean of Response $\quad 0.65469$

Observations (or Sum Wgts) $\quad 12$

Analysis of Variance

Source DF Sum of Squares Mean Square F Ratio Prob $>$ F

$\begin{array}{lllllll} & \text { ICP Calibration Block } & 3 & 0.00030512 & 0.000102 & 2.2612 & 0.1585\end{array}$

Error
C. Total

$11 \quad 0.00066495$

Means for Oneway Anova

Level Number Mean Std Error Lower 95\% Upper 95\%

$\begin{array}{lllllll}1-1 & & 3 & 0.653856 & 0.00387 & 0.64493 & 0.66279 \\ 1-2 & & 3 & 0.662752 & 0.00387 & 0.65382 & 0.67168 \\ 2-1 & & 3 & 0.653300 & 0.00387 & 0.6433 & 0.6623\end{array}$

$\begin{array}{llllll}2-1 & 3 & 0.653300 & 0.00387 & 0.64437 & 0.66223 \\ 2-2 & 3 & 0.648852 & 0.00387 & 0.63992 & 0.65778\end{array}$

Std Error uses a pooled estimate of error variance 
Exhibit A3. PSAL Measurements by ICP Calibration Block for Samples of the

Standard Glasses by Preparation Method by Set by Analytical Plan Memo. (continued)

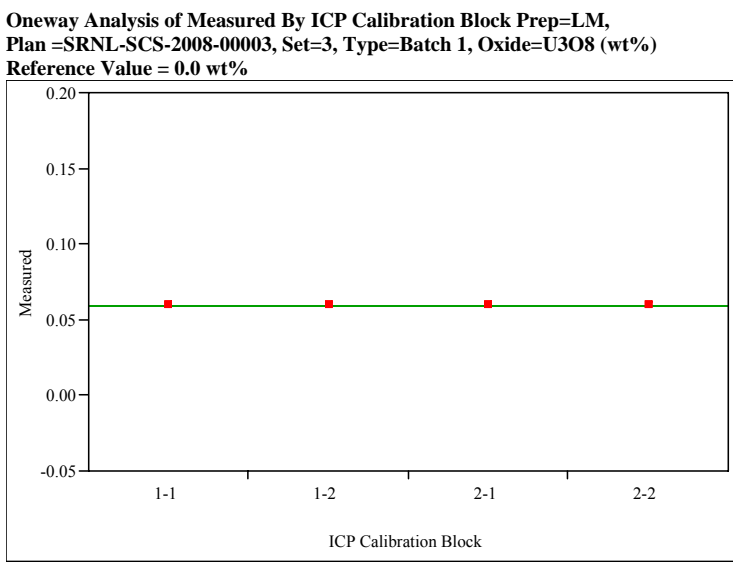

Oneway Anova
Summary of Fit

Rsquare

Adj Rsquare

Root Mean Square Erro

Observations (or Sum Wgts) $\begin{array}{r}0.05896 \\ 12\end{array}$

Analysis of Variance

Analysis of Variance $\quad$ DF Sum of Squares Mean Square F Ratio Prob $>$ F

ICP Cal

Error
C. Total

Means for Oneway Anova

Level Number Mean Std Error Lower 95\% Upper 95\%

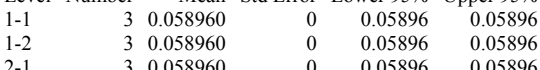

$\begin{array}{llllll}2-1 & 3 & 0.058960 & 0 & 0.05896 & 0.05896 \\ 2-2 & 3 & 0.058960 & 0 & 0.05896 & 0.05896\end{array}$

Std Error uses a pooled estimate of error variance

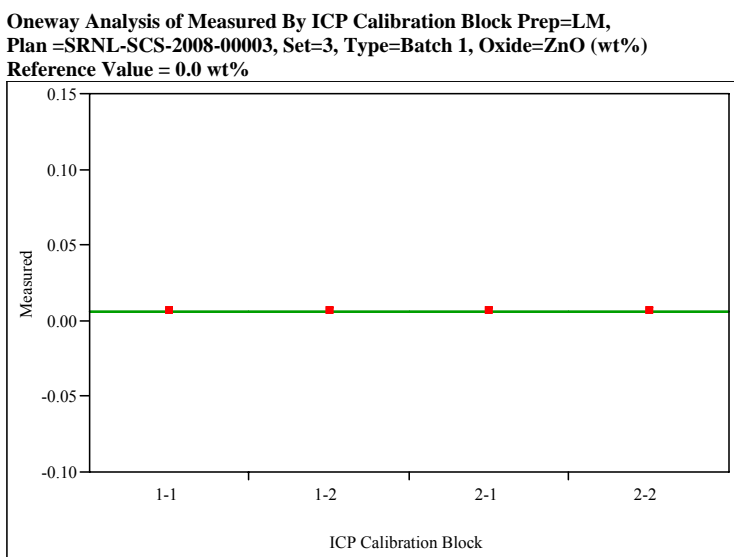

Oneway Anova

Rsquare

Adj Rsquare

Root Mean Square Erro

$\begin{array}{lr}\text { Mean of Response } & 0.006224 \\ \text { Observations (or Sum Wots) } & 12\end{array}$

Analysis of Variance

Source DF Sum of Squares Mean Square F Ratio Prob $>$ F

ICP Cal

Error
C. Total

0
0

Means for Oneway Anova
Level Number $\quad$ Mean Std Error Lower 95\% Upper 95\%

$\begin{array}{llll}1-1 & & 3 & 0.006224 \\ 1-2 & & 3 & 0.006224 \\ 2-1 & 3 & 0.006224 \\ 2-2 & & 3 & 0.00624\end{array}$

$\begin{array}{ll}3 & 0.006224 \\ 3 & 0.006224 \\ 3 & 0.006224\end{array}$

$\begin{array}{ll}0 & 0.00622 \\ 0 & 0.00622 \\ 0 & 0.00622 \\ 0 & 0.00622\end{array}$

0.00622
0.00622
0.00622

Std Error uses a pooled estimate of error variance
Oneway Analysis of Measured By ICP Calibration Block Prep=LM, Plan =SRNL-SCS-2008-00003, Set=3, Type=Batch 1, Oxide=ZrO2 (wt \%) Reference Value $=0.098 \mathrm{wt} \%$

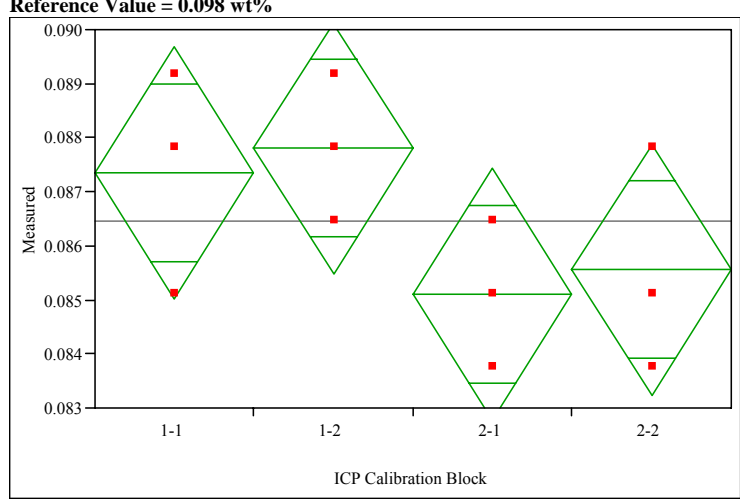

Oneway Anova
Summary of Fit

Rsquere

Rsquare
Adj Rsquare

0.393939
0.166667

Mean of Response $\quad 0.001744$

Observations (or Sum Wgts) $\quad 12$

Analysis of Variance

Source DF Sum of Squares Mean Square F Ratio Prob $>$ F

\begin{tabular}{lrrr} 
ICP Calibration Block & 3 & 0.00001581 & $5.2712 \mathrm{e}-6$ \\
Error & 8 & 0.00002433 & $3.0411 \mathrm{e}-6$ \\
C. Total & 11 & 0.00004014 & \\
\hline
\end{tabular}

Means for Oneway Anova

\begin{tabular}{lll} 
Level Number Mean Std Error Lower 95\% Upper 95\% & \\
\hline
\end{tabular}

$\begin{array}{lllllll}1-1 & 3 & 0.087352 & 0.00101 & 0.08503 & 0.08967 \\ 1-2 & & 3 & 0.087802 & 0.00101 & 0.08548 & 0.09012\end{array}$

$\begin{array}{lllllll}2-1 & & 3 & 0.087802 & 0.00101 & 0.08548 & 0.09012 \\ 2-2 & & 3 & 0.085100 & 0.00101 & 0.08278 & 0.08742 \\ & & & 0.085551 & 0.00101 & 0.08323 & 0.08787\end{array}$

Std Error uses a pooled estimate of error variance 
Exhibit A3. PSAL Measurements by ICP Calibration Block for Samples of the

Standard Glasses by Preparation Method by Set by Analytical Plan Memo. (continued)

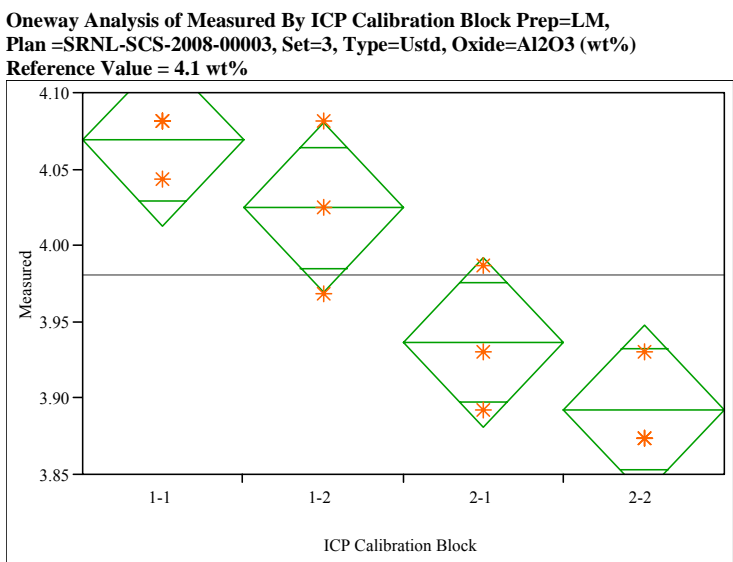

Oneway Anova
Summary of Fit

$\begin{array}{lr}\text { Rsquare } & 0.805921 \\ \text { Adj Rsquare } & 0.733141 \\ \text { Root Mean Square Error } & 0.041897 \\ \text { Mean of Response } & 3.980547 \\ \text { Observations (or Sum Wgts) } & 12\end{array}$

Analysis of Variance

Source

DF Sum of Squares Mean Square F Ratio Prob $>\mathrm{F}$

$\begin{array}{llllll}\text { ICP Calibration Block } & 3 & 0.05831343 & 0.019438 & 11.0734 & 0.0032\end{array}$

Error

$\begin{array}{rr}8 & 0.01404283 \\ 11 & 0.07235626\end{array}$

Means for Oneway Anova

$\begin{array}{lrrrrr}\text { Level } & \text { Number } & \text { Mean } & \text { Std Error } & \text { Lower 95\% } & \text { Upper } 95 \% \\ 1-1 & 3 & 4.06872 & 0.02419 & 4.0129 & 4.1245\end{array}$

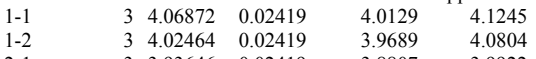

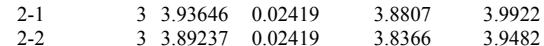

Std Error uses a pooled estimate of error variance

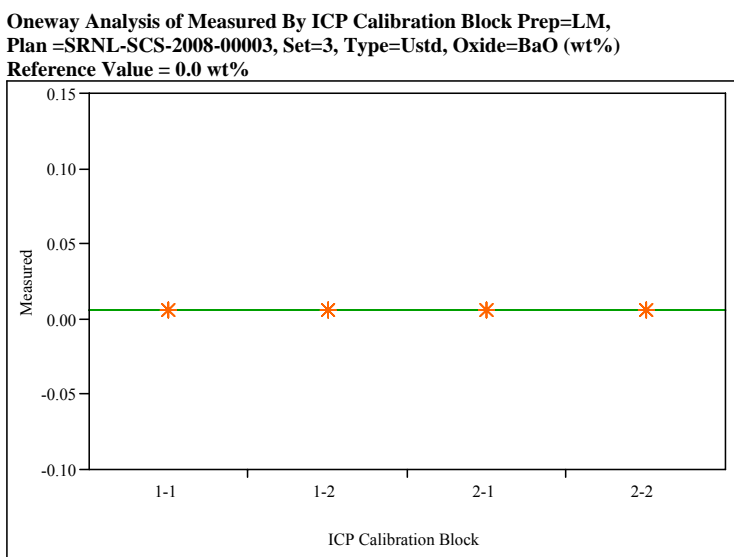

Oneway Anova
Summary of Fi

Rsquare

Adj Rsquare

Root Mean Square Erro

$\begin{array}{lr}\text { Mean of Response } & 0.005583 \\ \text { Observations (or Sum Wgts) } & 12\end{array}$

Analysis of Variance
Source

$\begin{array}{lrrr}\text { Source } & \text { DF } & \text { Sum of Squares } & \text { Mean Square } \\ \text { ICP Calibration Block } & 3 & 0 & 0 \\ \text { Error } & 8 & 0 & 0 \\ \text { C. Total } & 11 & 0 & \end{array}$

Means for Oneway Anova

Level Number Mean Std Error Lower 95\% Upper 95\%

$\begin{array}{lllllll}1-1 & & 3 & 0.005583 & 0 & 0.00558 & 0.00558 \\ 1-2 & & 3 & 0.005583 & 0 & 0.00558 & 0.00558 \\ 2-1 & & 3 & 0.05583 & 0 & 0.0058 & 0.00588\end{array}$

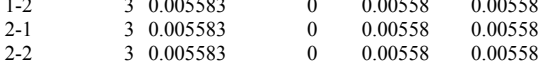

Std Error uses a pooled estimate of error variance Oneway Analysis of Measured By ICP Calibration Block Prep=LM,
Plan =SRNL-SCS-2008-00003, Set $=3$, Type=Ustd, Oxide=CaO (wt $\%$ ) Reference Value $=1.301 \mathrm{wt} \%$

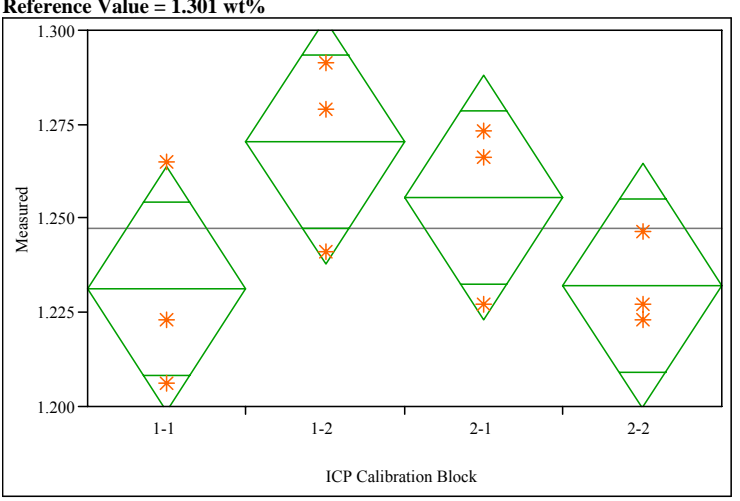

Oneway Anova

Summary of Fi

$\begin{array}{lr}\text { Rsquare } & 0.406451 \\ \text { Adj Rsquare } & 0.18387 \\ \text { Root Mean Square Error } & 0.024413 \\ \text { Mean of Response } & 1.247387 \\ \text { Observations (or Sum Wgts) } & 12\end{array}$

Analysis of Variance
Source

$\begin{array}{lrrrrr}\text { Source } & \text { DF } & \text { Sum of Squares } & \text { Mean Square } & \text { F Ratio } & \text { Prob }>\text { F } \\ \text { ICP Calibration Block } & 3 & 0.00326489 & 0.001088 & 1.8261 & 0.2205\end{array}$

$\begin{array}{lrrrr}\text { ICP Calibration Block } & 3 & 0.00326489 & 0.001088 \\ \text { Error } & 8 & 0.00476780 & 0.000596 \\ \text { C. Total } & 11 & 0.00803269 & \end{array}$

Means for Oneway Anova

Level Number Mean Std Error Lower 95\% Upper 95\%

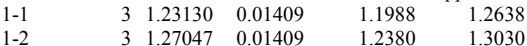

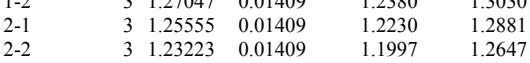

Std Error uses a pooled estimate of error variance 
Exhibit A3. PSAL Measurements by ICP Calibration Block for Samples of the

Standard Glasses by Preparation Method by Set by Analytical Plan Memo. (continued)

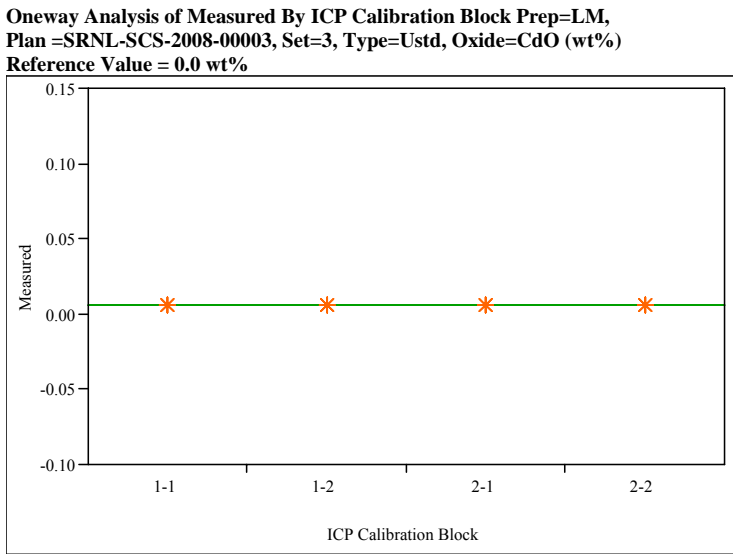

Oneway Anova
Summary of Fit

Rsquare
Adj Rsquare

Adj Rsquare

Root Mean Square Erro
Mean of Response

0.005712
Observations (or Sum Wgts)

Analysis of Variance
Source

$\begin{array}{lrrr}\text { Source } & \text { DF } & \text { Sum of Squares } & \text { Mean Square } \\ \text { ICP Calibration Block } & 3 & 0 & 0 \\ \text { Error } & 8 & 0 & 0 \\ \text { C. Total } & 11 & 0 & \end{array}$

0
0

Means for Oneway Anova

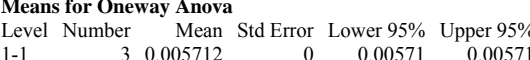

$\begin{array}{lllllll}1-1 & & 3 & 0.005712 & 0 & 0.00571 & 0.00571 \\ 1-2 & & 3 & 0.005712 & 0 & 0.00571 & 0.00571\end{array}$

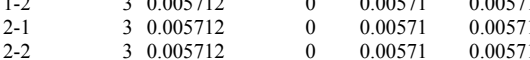

Std Error uses a pooled estimate of error variance

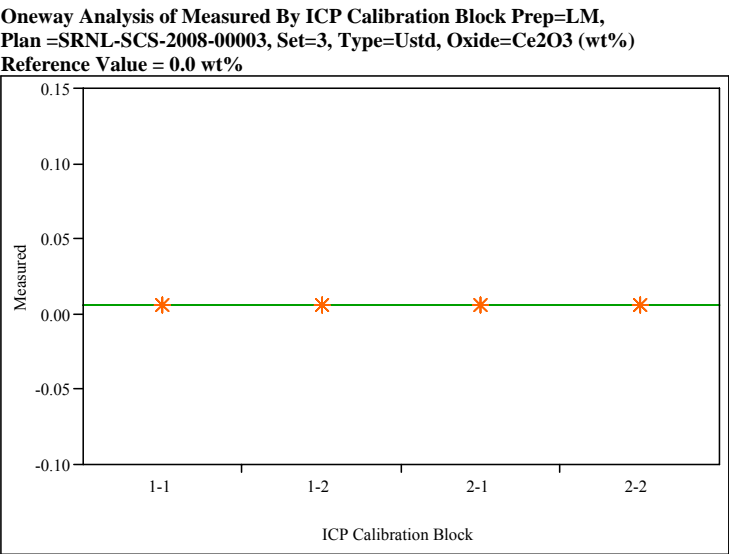

Oneway Anova

Summary of Fit

Rsquare

Root Mean Square Error

$\begin{array}{lr}\text { Mean of Response } & 0.005857 \\ \text { Observations (or Sum Wgts) } & 12\end{array}$

Analysis of Variance

Source DF Sum of Squares Mean Square F Ratio Prob $>$ F

$\begin{array}{lrrr} & & \\ \text { SF } & \text { Sum of Squares } & \text { Mean Square } \\ \text { ICP Calibration Block } & 3 & 0 & 0 \\ \text { Error } & 8 & 0 & 0 \\ \text { C. } & 11 & 0 & \end{array}$

Crror

0

Means for Oneway Anova

Level Number Mean Std Error Lower 95\% Upper 95\%

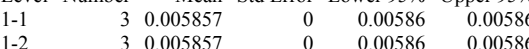

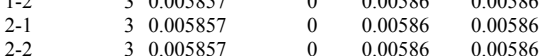

Std Error uses a pooled estimate of error variance
Oneway Analysis of Measured By ICP Calibration Block Prep=LM, Plan =SRNL-SCS-2008-00003, Set=3, Type=Ustd, Oxide=Cr203 $(w t \%)$ Reference Value $=0.0 \mathrm{wt} \%$

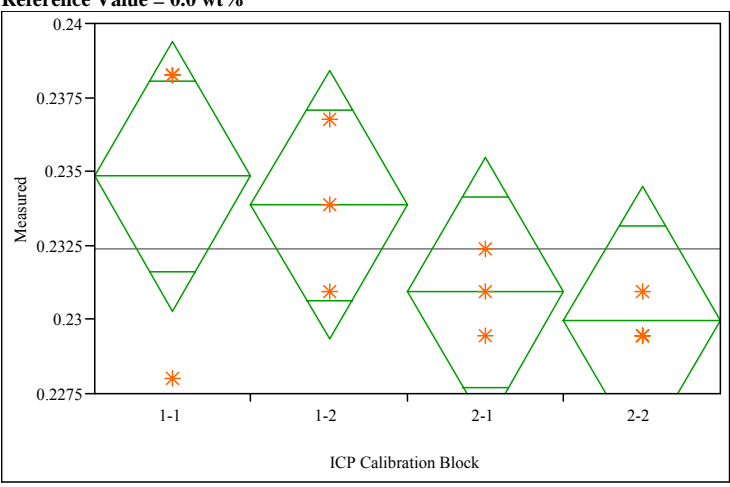

Oneway Anova
Summary of Fit

$\begin{array}{lr}\text { Rsquare } & 0.343434 \\ \text { Adj Rsquare } & 0.097222 \\ \text { Root Mean Square Error } & 0.003402 \\ \text { Mean of Response } & 0.232394 \\ \text { Observations (or Sum Wgts) } & 12\end{array}$

Analysis of Variance DF Sum of Squares Mean Square F Ratio Prob > F
Source

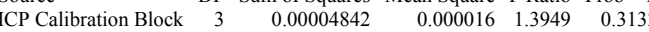

$\begin{array}{lrrr}\text { Error } & 8 & 0.00009257 & 0.000012\end{array}$

Means for Oneway Anova

Means for Oneway Anova
Level Number Mean Std Error Lower 95\% Upper 95\%

$\begin{array}{lrrrrr}\text { Level } & \text { Number } & \text { Mean } & \text { Std Error } & \text { Lower } 95 \% & \text { Upper 95\% } \\ 1-1 & 3 & 0.234830 & 0.00196 & 0.23030 & 0.23936 \\ 1-2 & 3 & 0.233856 & 0.00196 & 0.22933 & 0.23838\end{array}$

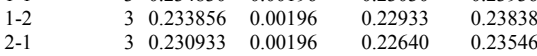

0.2344

Std Error uses a pooled estimate of error variance 
Exhibit A3. PSAL Measurements by ICP Calibration Block for Samples of the

Standard Glasses by Preparation Method by Set by Analytical Plan Memo. (continued)

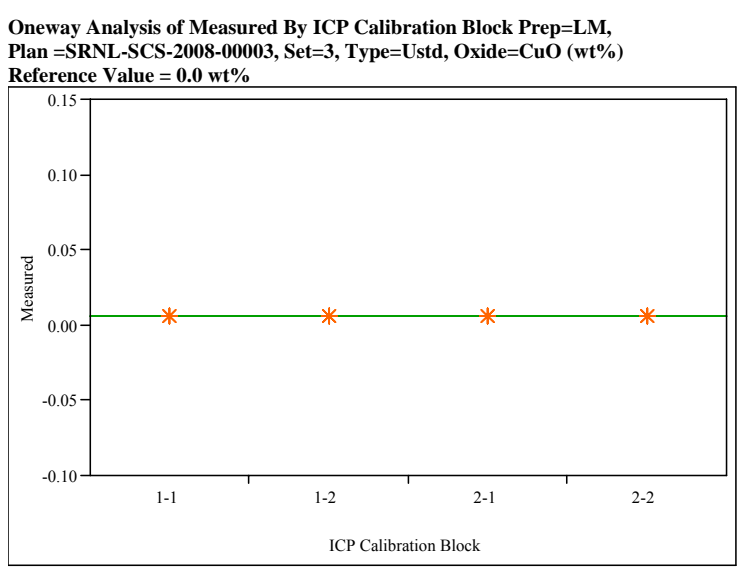

Oneway Analysis of Measured By ICP Calibration Block Prep=LM, Plan $=$ SRNL-SCS-2008-00003, Set $=3$, Type $=$ Ustd, 0 xide $=F e 203(w t \%)$

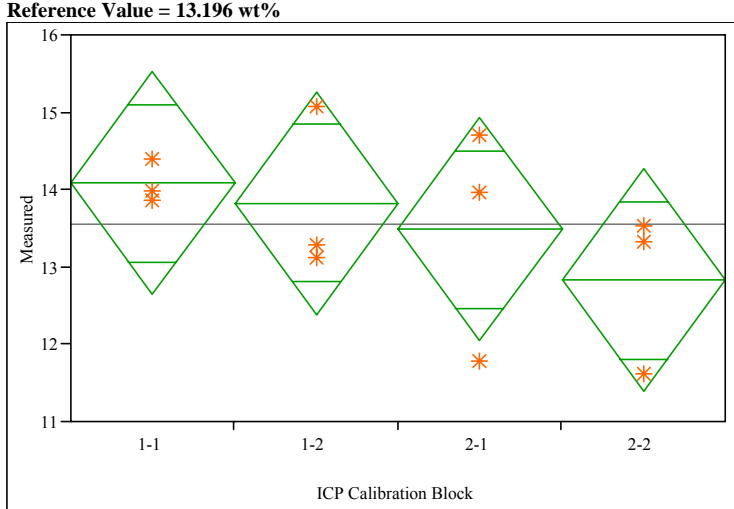

Oneway Anova
Summary of Fi

\section{Rsquare}

\section{Adj Rsquare
Root Mean S}

$\begin{array}{lr}\text { Root Mean Square Error } & 0 \\ \text { Mean of Response } & 0.006259\end{array}$

Observations (or Sum Wgts) $\quad 12$

Analysis of Variance
Source

$\begin{array}{lrrr}\text { Source } & \text { DF } & \text { Sum of Squares } & \text { Mean Square } \\ \text { ICP Calibration Block } & 3 & 0 & 0 \\ \text { Error } & 8 & 0 & 0 \\ \text { C. Total } & 11 & 0 & \end{array}$

Means for Oneway Anova
Level Number Mean Std Error Lower 95\% Upper 95\%

$\begin{array}{lrrrrr}\text { Level } & \text { Number } & \text { Mean } & \text { Std Error } & \text { Lower 95\% } & \text { Upper 95\% } \\ 1-1 & 3 & 0.006259 & 0 & 0.00626 & 0.00626 \\ 1-2 & 3 & 0.006259 & 0 & 0.00626 & 0.00626 \\ 2-1 & 3 & 0.006259 & 0 & 0.00626 & 0.00626 \\ 2-2 & 3 & 0.006259 & 0 & 0.00626 & 0.00626\end{array}$

Std Error uses a pooled estimate of error variance

\section{Oneway Anova
Summary of Fit}

Rsquare

ICP Calibration Block

-0.07252
Root Mean Square Error $\quad 1.083409$

Analysis of Variance

Source DF Sum of Squares Mean Square F Ratio Prob $>F$

$\begin{array}{lrrrrr}\text { ICP Calibration Block } & 3 & 2.648329 & 0.88278 & 0.7521 & 0.5513 \\ \text { Error } & 8 & 9.390193 & 1.17377 & & \\ \text { C. Total } & 11 & 12.038522 & & & \end{array}$

Means for Oneway Anova

$\begin{array}{llr}\text { Level Number } & \text { Mean Std Error Lower 95\% Upper 95\% } \\ 1-1 & & \end{array}$

$\begin{array}{lrrrrr}\text { Level } & \text { Number } & \text { Mean } & \text { Std Error } & \text { Lower } 95 \% & \text { Upper } 95 \% \\ 1-1 & 3 & 14.0825 & 0.62551 & 12.640 & 15.525 \\ 1-2 & 3 & 13.8252 & 0.62551 & 12.383 & 15.268\end{array}$

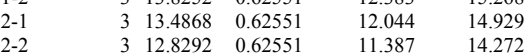

Std Error uses a pooled estimate of error variance
Oneway Analysis of Measured By ICP Calibration Block Prep=LM, Plan =SRNL-SCS-2008-00003, Set $=3$, Type=Ustd, Oxide $=K 20($ wt $\%)$ Reference Value $=2.999 \mathrm{wt} \%$

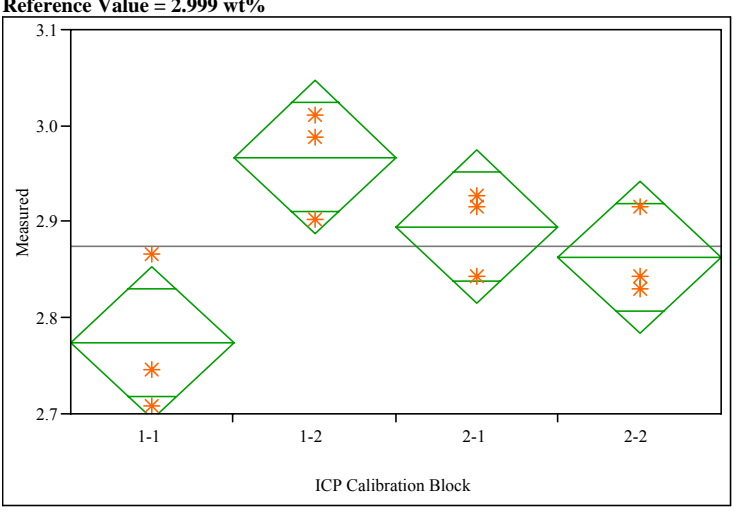

\section{Oneway Anova
Summary of Fi}

$\begin{array}{lr}\text { Rsquare } & 0.669848 \\ \text { Adj Rsquare } & 0.546041 \\ \text { Root Mean Square Error } & 0.05975 \\ \text { Mean of Response } & 2.874778 \\ \text { Observations (or Sum Wgts) } & 12\end{array}$

$\begin{array}{lr}\text { Mean of Response } & 13.55594 \\ \text { Observations (or Sum Wgts) } & 12\end{array}$
Analysis of Variance

Source $\quad$ DF Sum of Squares Mean Square F Ratio Prob $>$ F

$\begin{array}{lllllll} & \text { ICP Calibration Block } & 3 & 0.05794716 & 0.019316 & 5.4104 & 0.0251\end{array}$

$\begin{array}{lrr}\text { Error } & 8 & 0.02856075 \\ \text { C. Total } & 11 & 0.08650791\end{array}$

Means for Oneway Anova

Level Number Mean Std Error Lower 95\% Upper 95\%

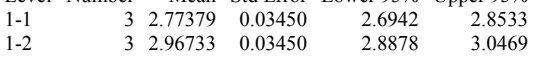

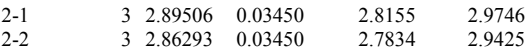

Std Error uses a pooled estimate of error variance 
Exhibit A3. PSAL Measurements by ICP Calibration Block for Samples of the

Standard Glasses by Preparation Method by Set by Analytical Plan Memo. (continued)

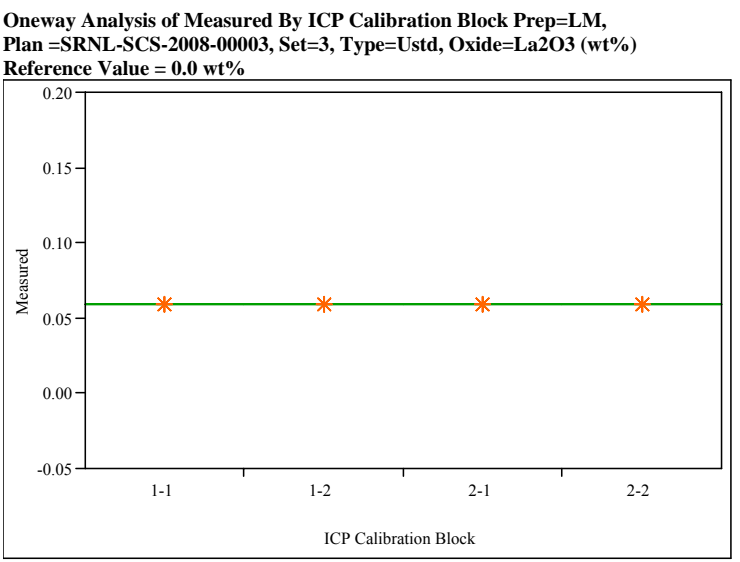

Oneway Anova
Summary of Fi

Rsquare
Adj Rsquare

Adj Rsquare

Mean of Response Error $\quad 0.05864$

Observations (or Sum Wgts) $\quad 12$

Analysis of Variance
Source

$\begin{array}{lrrr}\text { Source } & \text { DF } & \text { Sum of Squares } & \text { Mean Square } \\ \text { ICP Calibration Block } & 3 & 0 & 0 \\ \text { Error } & 8 & 0 & 0 \\ \text { C. Total } & 11 & 0 & \end{array}$

8
11

Means for Oneway Anova

$\begin{array}{lrrrrr}\text { Means for Oneway Anova } & & & \\ \text { Level } & \text { Number } & \text { Mean } & \text { Mtd Error } & \text { Lower 95\% } & \text { Upper 95\% } \\ 1-1 & 3 & 0.058640 & 0 & 0.05864 & 0.05864 \\ 1-2 & 3 & 0.058640 & 0 & 0.05864 & 0.05864 \\ 2-1 & 3 & 0.058640 & 0 & 0.05864 & 0.05864 \\ 2-2 & 3 & 0.058640 & 0 & 0.05864 & 0.05864\end{array}$

Std Error uses a pooled estimate of error variance

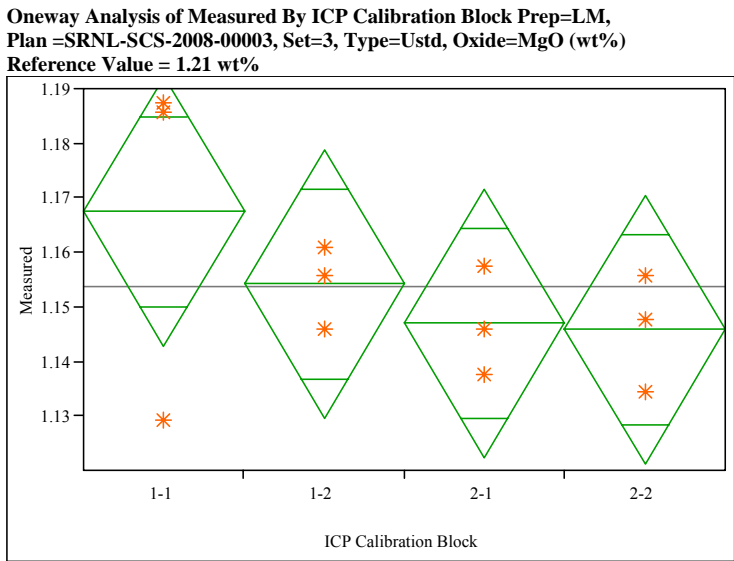

Oneway Anova
Summary of Fit

$\begin{array}{lr}\text { Rsquare } & 0.244557 \\ \text { Adj Rsquare } & -0.03873 \\ \text { Root Mean Square Error } & 0.018491 \\ \text { Mean of Response } & 1.153624 \\ \text { Observations (or Sum Wgts) } & 12\end{array}$

Analysis of Variance

Source DF Sum of Squares Mean Square F Ratio Prob $>F$

$\begin{array}{lllllll}\text { ICP Calibration Block } & 3 & 0.00088549 & 0.000295 & 0.8633 & 0.4985\end{array}$

Error

$\begin{array}{rr}8 & 0.00273529 \\ 11 & 0.00362078\end{array}$ .000342

Means for Oneway Anova

Level Number Mean Std Error Lower 95\% Upper 95\% $\begin{array}{llll}1-1 & 3 & 1.16744 & 0.01068\end{array}$

$\begin{array}{ll}1.1428 & 1.1921 \\ 1.1296 & 1.1788\end{array}$

$2-1$

Std Error uses a pooled estimate of error variance
Oneway Analysis of Measured By ICP Calibration Block Prep=LM, Plan =SRNL-SCS-2008-00003, Set=3, Type=Ustd, Oxide $=M n O(w t \%)$ Reference Value $=2.892 \mathrm{wt} \%$

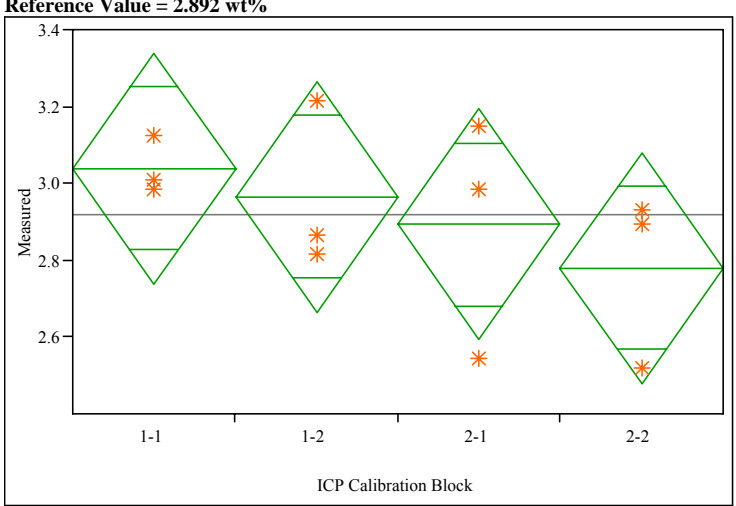

Oneway Anova

Rsquare

Adj Rsquare $\quad 0.2116$

Root Mean Square Error $\quad 0.225498$

Observations (or Sum Wgts)

Analysis of Variance

Source $\quad$ DF Sum of Squares Mean Square F Ratio Prob $>$ F

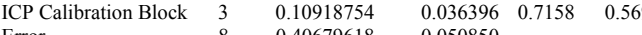

$\begin{array}{lrr}\text { Error } & 8 & 0.40679618 \\ \text { C. Total } & 11 & 0.51598371\end{array}$ .050850

Means for Oneway Anova

Level Number Mean Std Error Lower 95\% Upper 95\%

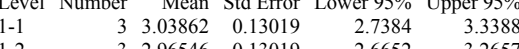

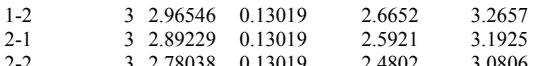

Std Error uses a pooled estimate of error variance 
Exhibit A3. PSAL Measurements by ICP Calibration Block for Samples of the

Standard Glasses by Preparation Method by Set by Analytical Plan Memo. (continued)

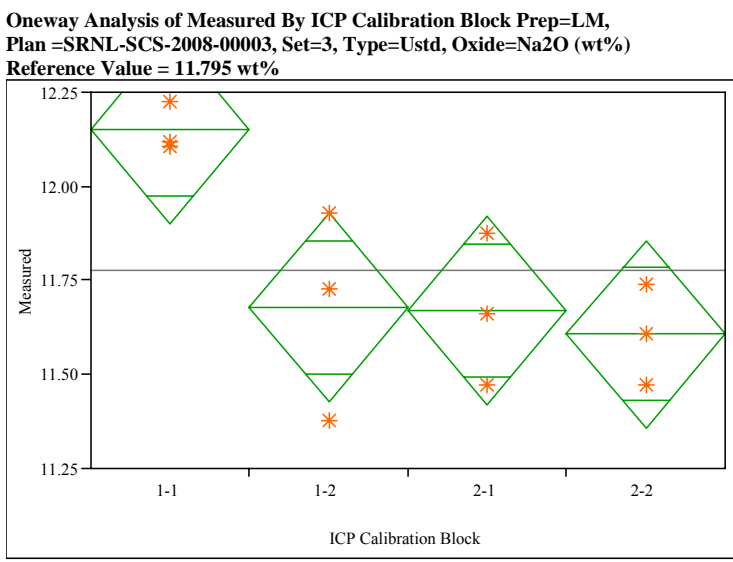

Oneway Anova
Summary of Fit

$\begin{array}{lr}\text { Rsquare } & 0.667442 \\ \text { Adj Rsquare } & 0.542733 \\ \text { Root Mean Square Error } & 0.188238 \\ \text { Mean of Response } & 11.7759\end{array}$

$\begin{array}{lr}\text { Mean of Response } & 11.7759 \\ \text { Observations (or Sum Wgts) } & 12\end{array}$

Analysis of Variance
Source

$\begin{array}{lrrrrr}\text { Source } & \text { DF } & \text { Sum of Squares } & \text { Mean Square } & \text { F Ratio } & \text { Prob }>\text { F } \\ \text { ICP Calibration Block } & 3 & 0.56892012 & 0.189640 & 5.3520 & 0.0258\end{array}$

$\begin{array}{lrrr}\text { ICP Calibration Block } & 3 & 0.56892012 & 0.189640 \\ \text { Error } & 8 & 0.28346822 & 0.035434 \\ \text { C. Total } & 11 & 0.85238834 & \end{array}$

Means for Oneway Anova

Level Number Mean Std Error Lower 95\% Upper 95\%

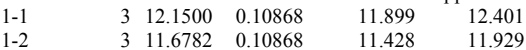

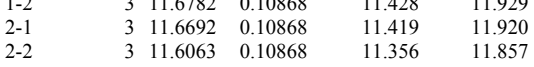

Std Error uses a pooled estimate of error variance

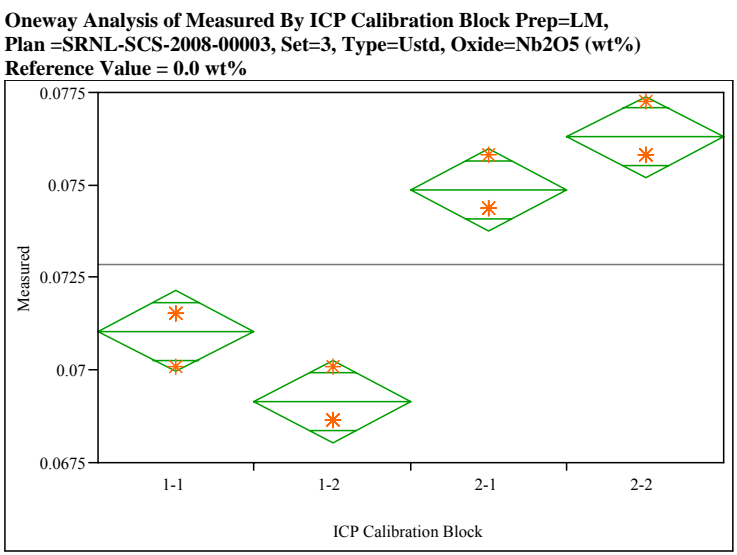

Oneway Anova

ummary of Fit

$\begin{array}{lr}\text { Rsquare } & 0.947627 \\ \text { Adj Rsquare } & 0.927987 \\ \text { Root Mean Square Error } & 0.000826 \\ \text { Mean of Response } & 0.072836 \\ \text { Observations (or Sum Wgts) } & 12\end{array}$

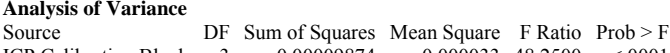

$\begin{array}{lrrrrr}\text { ICP Calibration Block } & 3 & 0.00009874 & 0.000033 & 48.2500 & <.0001\end{array}$

$\begin{array}{lrrr}\text { Error } & 8 & 0.00000546 & 6.821 \mathrm{e}-7 \\ \text { C. Total } & 11 & 0.00010419 & \end{array}$

Means for Oneway Anova

Level Number Mean Std Error Lower 95\% Upper 95\%

$\begin{array}{lllllll}1-1 & 3 & 0.071048 & 0.00048 & 0.06995 & 0.0721 \\ 1-2 & & 3 & 0.069141 & 0.00048 & 0.06804 & 0.07024\end{array}$

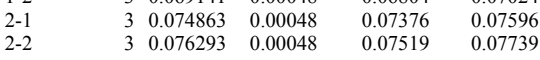

Std Error uses a pooled estimate of error variance
Oneway Analysis of Measured By ICP Calibration Block Prep=LM, Plan =SRNL-SCS-2008-00003, Set=3, Type=Ustd, Oxide=NiO $($ wto Reference Value $=1.12 \mathrm{wt} \%$

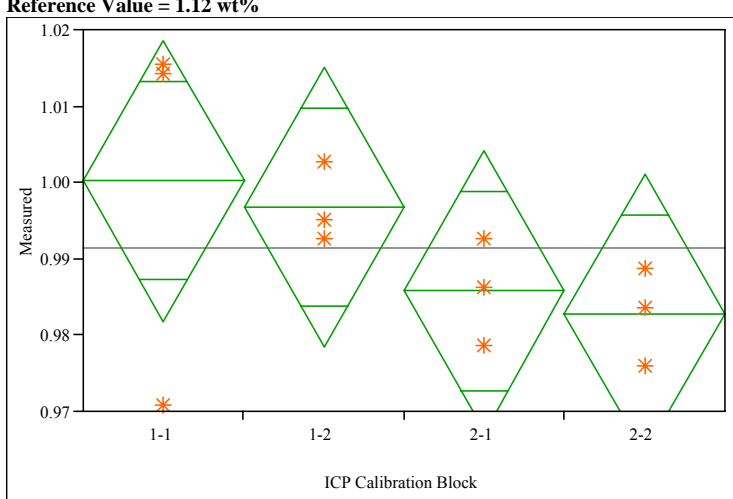

Oneway Anova

Summary of

$\begin{array}{lr}\text { Rsquare } & 0.29478 \\ \text { Adj Rsquare } & 0.030322 \\ \text { Root Mean Square Error } & 0.013794 \\ \text { Mean of Response } & 0.991384 \\ \text { Observations (or Sum Wgts) } & 12\end{array}$

Analysis of Variance

Dource Sum of Squares Mean Square F Ratio Prob $>$ F

$\begin{array}{llllll}\text { ICP Calibration Block } & 3 & 0.00063623 & 0.000212 & 1.1147 & 0.3986\end{array}$

Error

$\begin{array}{rr}8 & 0.00152210 \\ 11 & 0.00215833\end{array}$

Means for Oneway Anova

Level Number Mean Std Error Lower 95\% Upper 95\%

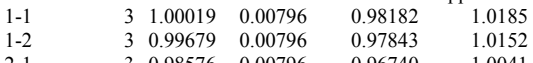

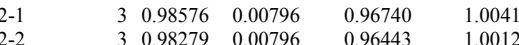

Std Error uses a pooled estimate of error variance 
Exhibit A3. PSAL Measurements by ICP Calibration Block for Samples of the

Standard Glasses by Preparation Method by Set by Analytical Plan Memo. (continued)

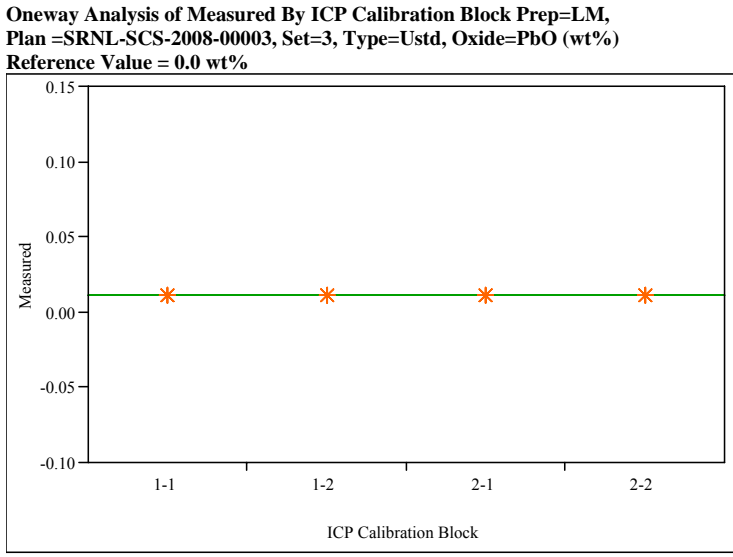

Oneway Anova
Summary of Fit

$\begin{array}{lr}\text { Rsquare } & 0 \\ \text { Adj Rsquare } & -0.375 \\ \text { Root Mean Square Error } & 2.12 \mathrm{e}-18 \\ \text { Mean of Response } & 0.010772 \\ \text { Observations (or Sum Wgts) } & 12\end{array}$

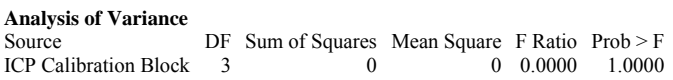

$\begin{array}{lrrr}\text { Source } & 0 & 0 \\ \text { ICP Calibration Block } & 3 & 3.6111 \mathrm{e}-35 & 4.514 \mathrm{e}-36 \\ \text { Error } & 8 & 36 \\ \text { C. Total } & 11 & 3.6111 \mathrm{e}-35 & \end{array}$

Means for Oneway Anova

Level Number Mean Std Error Lower 95\% Upper 95\%

$\begin{array}{lllllll}1-1 & & 3 & 0.010772 & 1.227 \mathrm{e}-18 & 0.01077 & 0.01077 \\ 1-2 & & 3 & 0.010772 & 1.227 \mathrm{e}-18 & 0.01077 & 0.01077\end{array}$

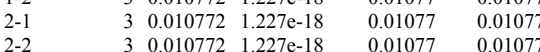

Std Error uses a pooled estimate of error variance

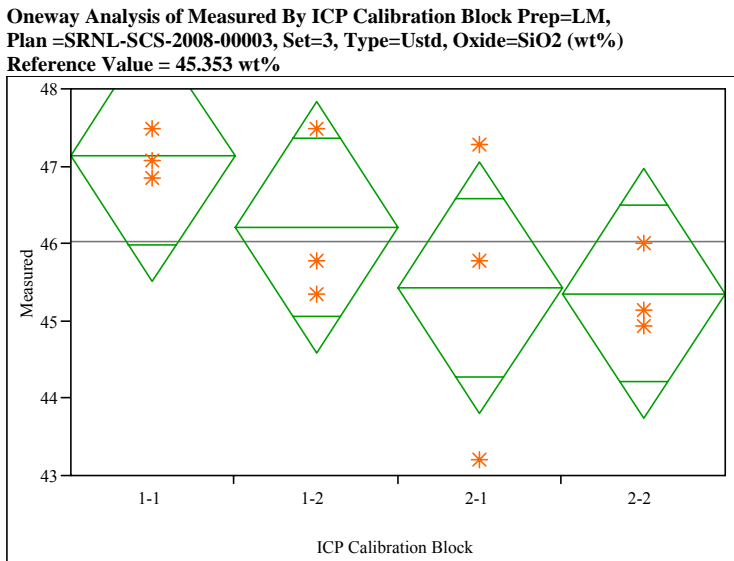

\section{Oneway Anova
Summary of Fit}

Rsquare

Rsquare
Adj Rsquare

Root Mean Square

Mean of Response

\subsection{6}

0.098778
1.218024

Analysis of Variance

$\begin{array}{lrrrrr}\text { Source } & \text { DF } & \text { Sum of Squares } & \text { Mean Square } & \text { F Ratio } & \text { Prob }>\text { F } \\ \text { ICP Calibration Block } & 3 & 6.239437 & 2.07981 & 1.4019 & 0.3114\end{array}$

C.

Error
C. Total

6.239437

18.108098 1.48358

Means for Oneway Anova

Level Number Mean Std Error Lower 95\% Upper 95\%

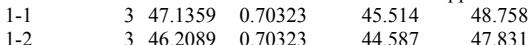

$\begin{array}{llllll}2-1 & 3 & 46.2089 & 0.70323 & 44.587 & 47.831 \\ 2 & 3 & 45.4245 & 0.70323 & 43.803 & 47.046\end{array}$

Std Error uses a pooled estimate of error variance
Oneway Analysis of Measured By ICP Calibration Block Prep=LM, Plan $=$ SRNL-SCS-2008-00003, Set $=3$, Type=Ustd, Oxide=SO4 $(\mathrm{wt} \%)$ Reference Value $=0.0 \mathrm{wt} \%$

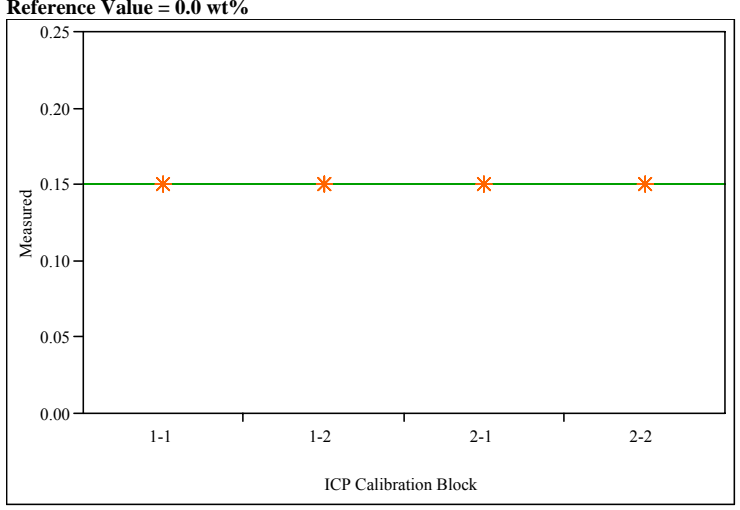

Oneway Anova

Summary of Fit

Rsquare
Adj Rsquare

Root Mean Square Error $\begin{array}{ll}\text { Mean of Response } & 0.149795 \\ \text { Observations (or Sum Wgts) }\end{array}$

Analysis of Variance

Source $\quad$ DF Sum of Squares Mean Square F Ratio Prob $>F$ $\begin{array}{lrrr}\text { ICP Calibration Block } & 3 & 0 & 0 \\ \text { Error } & 8 & 0 & 0\end{array}$

ova

Level Number Mean Std Error Lower 95\% Upper 95\%

$\begin{array}{llllllll}1-1 & & 3 & 0.149795 & & 0 & 0.14980 & 0.14980\end{array}$

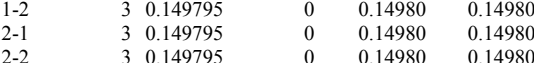

Std Error uses a pooled estimate of error variance 
Exhibit A3. PSAL Measurements by ICP Calibration Block for Samples of the

Standard Glasses by Preparation Method by Set by Analytical Plan Memo. (continued)

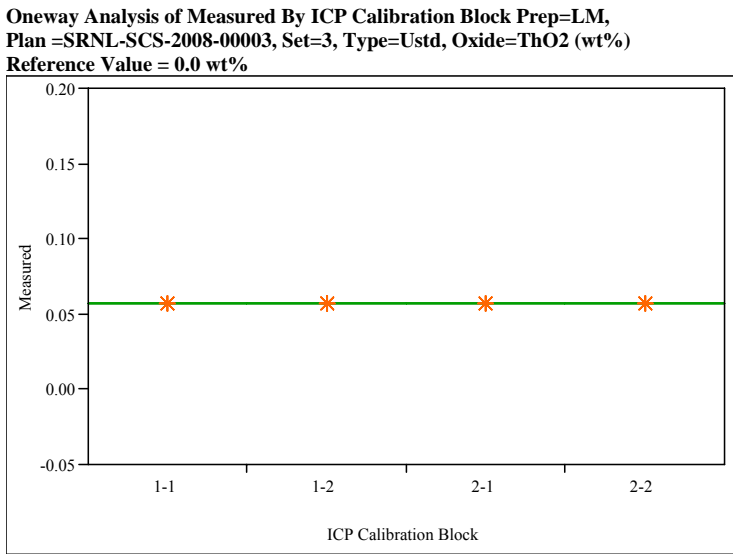

Oneway Anova
Summary of Fit

Rsquare

Adj Rsquare

Root Mean Square Erro
Mean of Response

0.056895
Observations (or Sum Wgts)

Analysis of Variance

Source DF Sum of Squares Mean Square F Ratio Prob > F

Error
C. Total

$\begin{array}{rrr}\text { DF } & \text { Sum of Squares } & \text { Mean Square } \\ 3 & 0 & 0 \\ 8 & 0 & 0 \\ 11 & 0 & \end{array}$

Means for Oneway Anova

$\begin{array}{lrrrrr}\text { Means for Oneway Anova } & & & \\ \text { Level } & \text { Number } & \text { Mean } & \text { Std Error } & \text { Lower 95\% } & \text { Upper 95\% } \\ 1-1 & 3 & 0.056895 & 0 & 0.05690 & 0.05690 \\ 1-2 & 3 & 0.056895 & 0 & 0.05690 & 0.05960 \\ 2-1 & 3 & 0.056895 & 0 & 0.05690 & 0.05690 \\ 2-2 & 3 & 0.056895 & 0 & 0.05690 & 0.05690\end{array}$

Std Error uses a pooled estimate of error variance

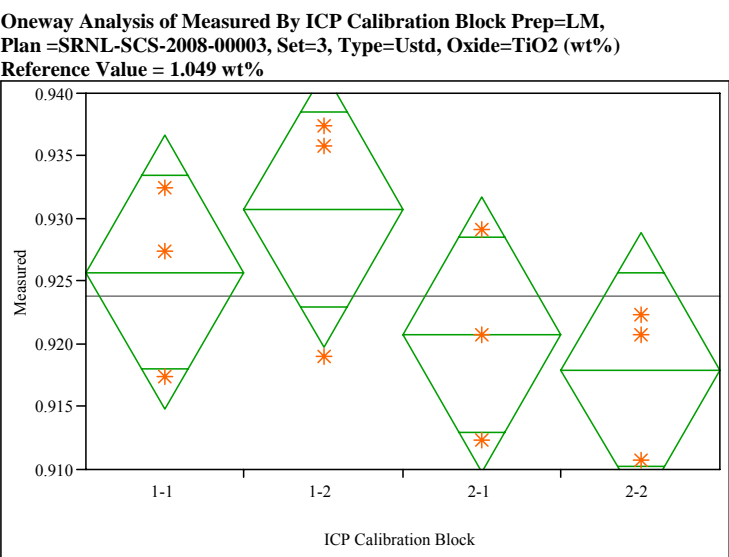

Oneway Anova
Summary of Fit

Rsquare

Root Mean Square Error $\quad 0.008228$

$\begin{array}{lr}\text { Mean of Response } & 0.923794 \\ \text { Observations (or Sum Wgts) } & 12\end{array}$

Analysis of Variance

Source DF Sum of Squares Mean Square F Ratio Prob $>$ F

$\begin{array}{llllll}\text { ICP Calibration Block } & 3 & 0.00028657 & 0.000096 & 1.4110 & 0.3090\end{array}$

Crror

0.0005416 0.000068

Means for Oneway Anova

Level Number Mean Std Error Lower 95\% Upper 95\%

$\begin{array}{lllllll}1-1 & & 3 & 0.925740 & 0.00475 & 0.91479 & 0.93669 \\ 1-2 & & 3 & 0.930744 & 0.00475 & 0.91979 & 0.94170\end{array}$

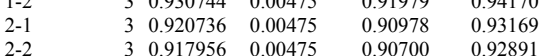

Std Error uses a pooled estimate of error variance
Oneway Analysis of Measured By ICP Calibration Block Prep=LM, Plan =SRNL-SCS-2008-00003, Set=3, Type=Ustd, Oxide=U3O8 $(\mathrm{wt} \%)$ Reference Value $=2.406 \mathrm{wt} \%$

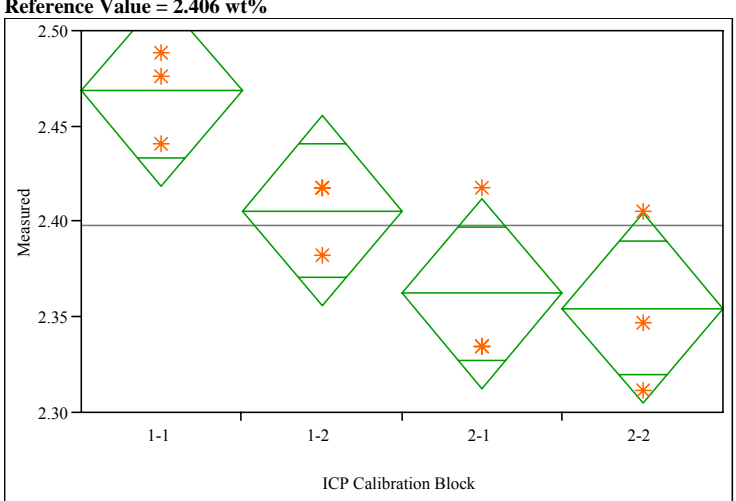

Oneway Anova

ummary of

$\begin{array}{lr}\text { Rsquare } & 0.688312 \\ \text { Adj Rsquare } & 0.571429 \\ \text { Root Mean Square Error } & 0.03729 \\ \text { Mean of Response } & 2.397707 \\ \text { Observations (or Sum Wgts) } & 12\end{array}$

Analysis of Variance
Source

$\begin{array}{llllll}\text { ICP Calibration Block } & 3 & 0.02456572 & 0.008189 & 5.8889 & 0.020\end{array}$

$\begin{array}{lrr}\text { Error } & 8 & 0.0112410 \\ \text { C. Total } & 11 & 0.03568982\end{array}$

Means for Oneway Anova

Level Number Mean Std Error Lower 95\% Upper 95\%

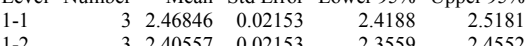

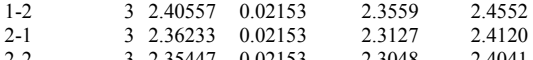

Std Error uses a pooled estimate of error variance 
Exhibit A3. PSAL Measurements by ICP Calibration Block for Samples of the

Standard Glasses by Preparation Method by Set by Analytical Plan Memo. (continued)

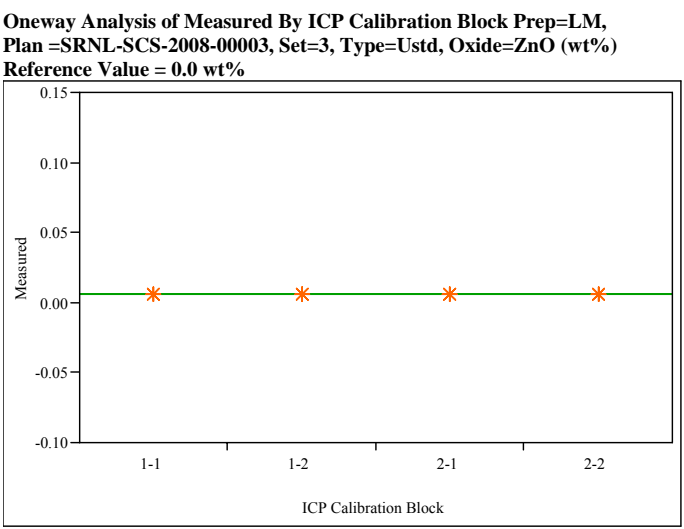

Oneway Anova

Rsquare

Root Mean Square Erro

\begin{tabular}{lr} 
Mean of Response & 0 \\
Observations (or Sum Wgts) & 0.006224 \\
\hline
\end{tabular}

Analysis of Variance
Source

$\begin{array}{lrrr}\text { Source } & & \\ \text { ICP Calibration Block } & 3 & 0 & 0 \\ \text { Error } & 8 & 0 & 0 \\ \text { C. Total } & 11 & 0 & \end{array}$

11

Means for Oneway Anova

Level Number Mean Std Error Lower 95\% Upper 95\%

$\begin{array}{rrrrrr}1-1 & 3 & 0.006224 & 0 & 0.00622 & 0.00622 \\ 1-2 & 3 & 0.006224 & 0 & 0.00622 & 0.00622\end{array}$

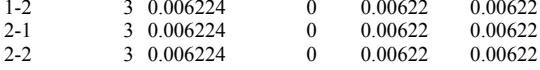

Std Error uses a pooled estimate of error variance

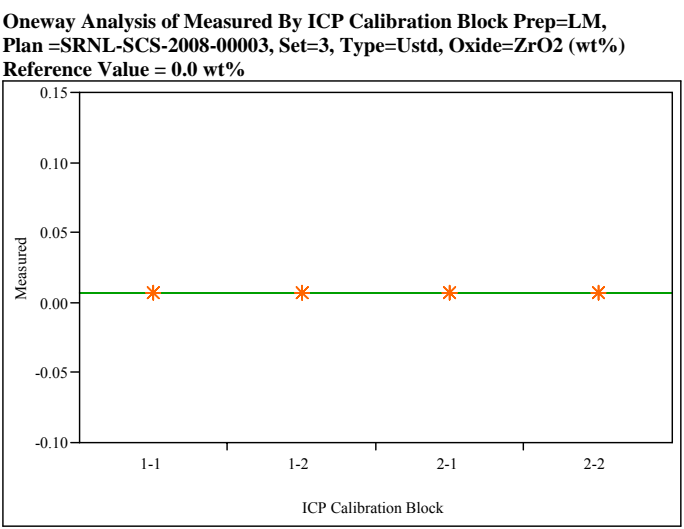

Oneway Anova

mary of Fit

Rsquare
Adj Rsquar

Root Mean Square Erro

$\begin{array}{lr}0 \\ \text { Mean of Response } & 0.006754\end{array}$

Analysis of Variance

Source of Variance DF Sum of Squares Mean Square F Ratio Prob $>$ F

$\begin{array}{lrrr}\text { Source } & & \\ \text { ICP Calibration Block } & 3 & 0 & 0 \\ \text { Error } & 8 & 0 & 0 \\ \text { C. Total } & 11 & 0 & \end{array}$

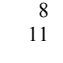

0

Means for Oneway Anova

$\begin{array}{lrrrrr}\text { Level } & \text { Number } & \text { Mean } & \text { Std Error } & \text { Lower 95\% } & \text { Upper 95\% } \\ 1-1 & 3 & 0.006754 & 0 & 0.00675 & 0.00675 \\ 1-2 & 3 & 0.006754 & 0 & 0.00675 & 0.00675 \\ 2-1 & 3 & 0.006754 & 0 & 0.00675 & 0.00675 \\ 2-2 & 3 & 0.006754 & 0 & 0.00675 & 0.00675\end{array}$

Std Error uses a pooled estimate of error variance
Oneway Analysis of Measured By ICP Calibration Block Prep=LM, Plan $=$ SRNL-SCS-2008-00043, Set $=1$, Type=Batch 1, Oxide $=$ Al2O3 $($ wt $\%)$ Reference Value $=4.877 \mathrm{wt} \%$

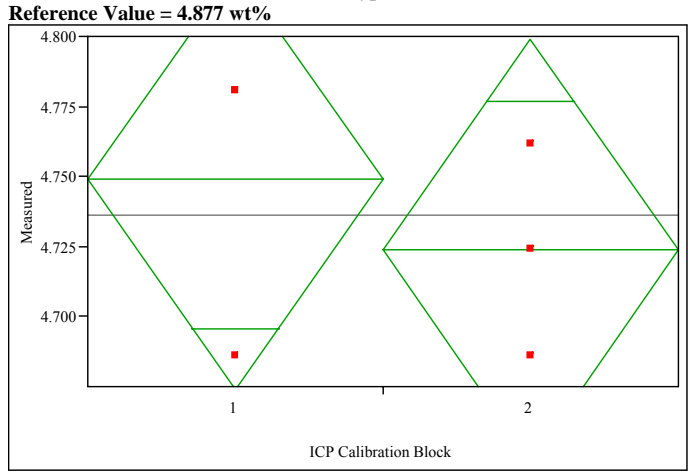

Oneway Anova

Summary of Fi

$\begin{array}{lr}\text { Rsquare } & 0.097561 \\ \text { Adj Rsquare } & -0.12805 \\ \text { Root Mean Square Error } & 0.046922 \\ \text { Mean of Response } & 4.736347 \\ \text { Observations (or Sum Wgts) } & 6\end{array}$

t Test

Assuming equal variances

Difference $\quad-0.02519$ tRatio $\quad-0.6576$

Std Err Dif 0.03831 DF

Lower CL Dif -0.13156 Prob $>|t| \quad 0.5467$

$\begin{array}{lrl}\text { Confidence } & 0.95 \text { Prob }<\mathrm{t} & 0.2734\end{array}$

\footnotetext{
Analysis of Variance

$\begin{array}{lrrrrr}\text { Source } & \text { DF } & \text { Sum of Squares } & \text { Mean Square } & \text { F Ratio } & \text { Prob }>\text { F } \\ \text { ICP Calibration Block } & 1 & 0.00095206 & 0.000952 & 0.4324 & 0.5467\end{array}$

Error
C. Total 0.00880652 0.002202

Means for Oneway Anova

Level Number Mean Std Error Lower 95\% Upper 95\%

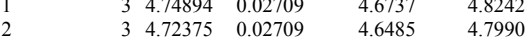

Std Error uses a pooled estimate of error variance
} 
Exhibit A3. PSAL Measurements by ICP Calibration Block for Samples of the

Standard Glasses by Preparation Method by Set by Analytical Plan Memo. (continued)

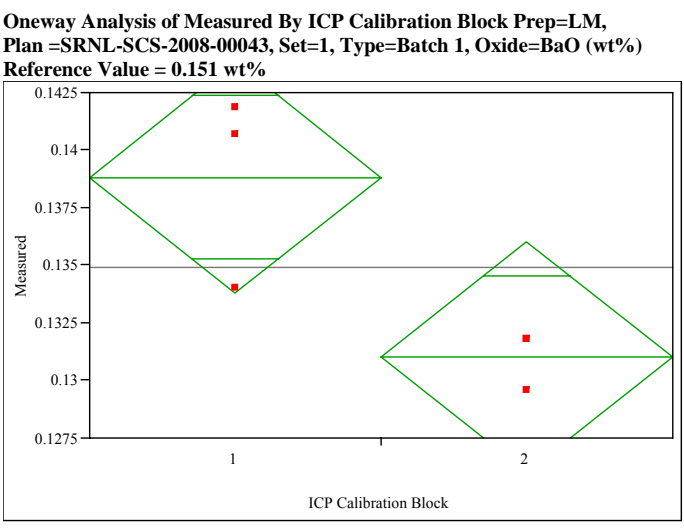

Oneway Anova

$\begin{array}{lr}\text { Rsquare } & 0.701113 \\ \text { Adj Rsquare } & 0.626391 \\ \text { Root Mean Square Error } & 0.003125 \\ \text { Mean of Response } & 0.13491 \\ \text { Observations (or Sum Wgts) } & 6\end{array}$

t Test

Assuming equal variances

Difference $\quad-0.00782$ t Ratio $\quad-3.06316$

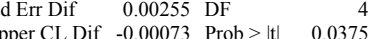

$\begin{array}{llll}\text { Upper CL Dif } & -0.00073 & \text { Prob }>|t| & 0.0375 \\ \text { Lower CL Dif }-0.01490 & \text { Prob }>t & 0.9812\end{array}$

$\begin{array}{lll}\text { Confidence } & 0.95 \text { Prob }<\mathrm{t} & 0.0188\end{array}$

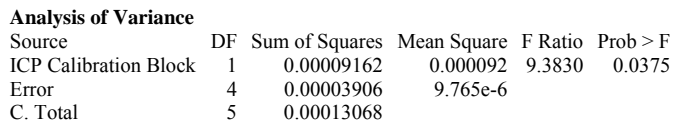

Means for Oneway Anova

Level Number Mean Std Error Lower 95\% Upper 95\%

$\begin{array}{llllll}1 & 3 & 0.138818 & 0.00180 & 0.13381 & 0.14383 \\ 2 & 3 & 0.131003 & 0.00180 & 0.12599 & 0.13601\end{array}$

Std Error uses a pooled estimate of error variance

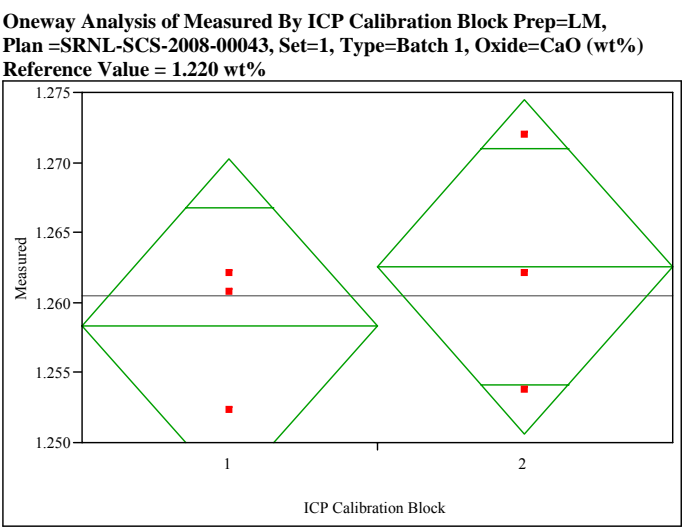

\section{Oneway Anova}

Summary of Fit

Rsquare
Adj Rsquar

Adj Rsquare $\quad-0.106439$

Root Mean Square Error $\quad 0.007448$

$\begin{array}{lr}\text { Mean of Response } & 1.260446 \\ \text { Observations (or Sum Wgts) } & 6\end{array}$

t Test
$2-1$

Assuming equal variances

Difference $\quad 0.00420$ t Ratio $\quad 0.690268$

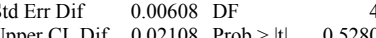

$\begin{array}{lrrr}\text { Upper CL Dif } & 0.02108 & \text { Prob }>|t| & 0.5280 \\ \text { Lower CL Dif } & -0.01269 & \text { Prob }>t & 0.2640\end{array}$

$\begin{array}{lrl}\text { Confidence } & -0.95 \text { Prob }<\mathrm{t} & 0.7360\end{array}$

Analysis of Variance
Source

$\begin{array}{lrrrrr}\text { Source } & & & & & \\ \text { ICP Calibration Block } & 1 & 0.00002643 & 0.000026 & 0.4765 & 0.5280\end{array}$

$\begin{array}{llll}\text { Error } & 4 & 0.00022188 & 0.000055 \\ \text { C. Total } & 5 & 0.00024831 & \end{array}$

Means for Oneway Anova

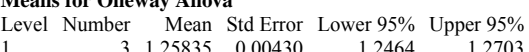

$\begin{array}{llllll}1 & 3 & 1.25835 & 0.00430 & 1.2464 & 1.2703 \\ 2 & 3 & 1.26254 & 0.00430 & 1.2506 & 1.2745\end{array}$

Std Error uses a pooled estimate of error variance
Oneway Analysis of Measured By ICP Calibration Block Prep=LM,

Plan $=$ SRNL-SCS- $2008-00043$, Set $=1$, Type $=$ Batch 1, Oxide $=$ Ce2O3 $($ wt $\%)$ Reference Value $=0.0$ wt $\%$

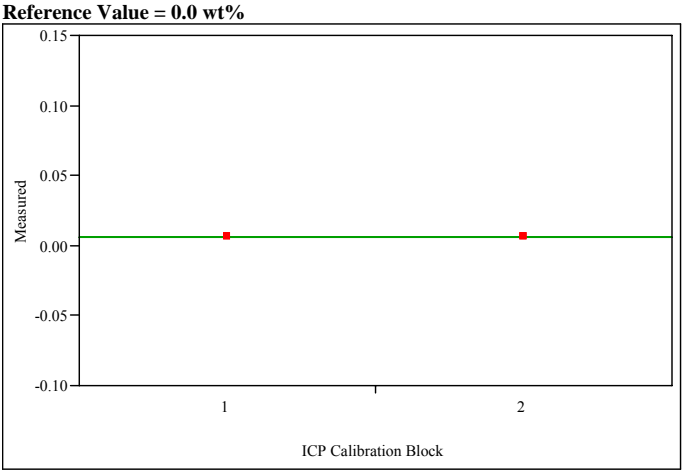

Oneway Anova

Rsquare

$\begin{array}{lr}\text { Root Mean Square Error } & 0 \\ \text { Mean of Response } & 0.005857 \\ \text { Observations (or Sum Wgts) } & 6\end{array}$

t Test

Assuming equal variances

$\begin{array}{lll}\text { Difference } & 0 & \text { t Ratio } \\ \text { Std Err Dif } & 0 & \text { DF }\end{array}$

\begin{tabular}{llll} 
Std Err Dif & 0 & DF \\
Upper CL Dif & 0 & Prob $>$ tt \\
\hline
\end{tabular}

Lower CL Dif 0 Prob $>t$

Confidence 0.95 Prob $<t$

Analysis of Variance
Source

$\begin{array}{lrrr}\text { ICP Calibration Block } & 1 & 0 & 0 \\ \text { Error } & 4 & 0 & 0\end{array}$

C. Total

Means for Oneway Anova

Level Number $\begin{array}{rrr}\text { Mean } \\ 3\end{array}$ Std Error Lower $95 \%$ Upper 95\%

$\begin{array}{llllll}2 & 3 & 0.005857 & 0 & 0.00586 & 0.00586 \\ 2 & 3 & & 0.00586\end{array}$

Std Error uses a pooled estimate of error variance 
Exhibit A3. PSAL Measurements by ICP Calibration Block for Samples of the

Standard Glasses by Preparation Method by Set by Analytical Plan Memo. (continued)

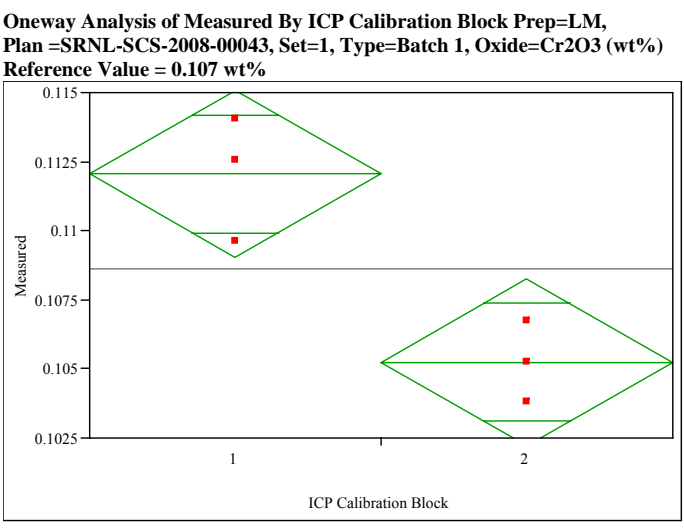

Oneway Anova

$\begin{array}{lr}\text { Rsquare } & 0.830508 \\ \text { Adj Rsquare } & 0.788136 \\ \text { Root Mean Square Error } & 0.001887 \\ \text { Mean of Response } & 0.108646 \\ \text { Observations (or Sum Wgts) } & 6\end{array}$

t Test

Assuming equal variances

$\begin{array}{llll}\text { Difference } & -0.00682 & \text { t Ratio } & -4.42719 \\ \text { Std Err Dif } & 0.00154 \quad \text { DF }\end{array}$

$\begin{array}{ll}-0.0254 \\ \text { Prob }>|t| & 0.0114\end{array}$

$\begin{array}{lll} & \\ \text { Lower CL Dif }-0.01110 & \text { Prob }>t & 0.9943\end{array}$

$\begin{array}{lll}\text { Confidence } & 0.95 \text { Prob }<\mathrm{t} \quad 0.0057\end{array}$

\footnotetext{
$\begin{array}{llll}\text { Analysis of Variance } & \\ \text { Source } & \text { DF Sum of Squares Mean Square F Ratio Prob > F }\end{array}$

$\begin{array}{lrrrrr}\text { Source Calibration Block } & 1 & 0.00006978 & 0.000070 & 19.6000 & 0.0114 \\ \text { Error } & 4 & 0.00001424 & 3.566-6 & & \end{array}$ 0.00001424

Means for Oneway Anova

Level Number Mean Std Error Lower 95\% Upper 95\%

$\begin{array}{llrrrr}1 & 3 & 0.112056 & 0.00109 & 0.10903 & 0.11508 \\ 2 & 3 & 0.105235 & 0.00109 & 0.10221 & 0.10826\end{array}$

Std Error uses a pooled estimate of error variance
}

Oneway Analysis of Measured By ICP Calibration Block Prep=LM,

Plan =SRNL-SCS-2008-00043, Set=1, Type=Batch 1, Oxide=Fe2O3 $($ wt $\%)$ Reference Value $=12.839 \mathrm{wt} \%$

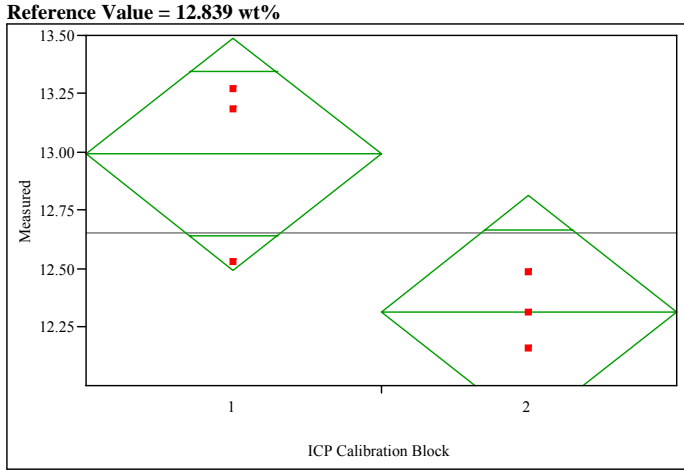

Oneway Anova

mary of Fit

Rsquare
Adj Rsquare

Root Mean Square Error $\quad 0.310226$

$\begin{array}{lr}\text { Mean of Response } & 12.65285 \\ \text { Observations (or Sum Wgts) } & 6\end{array}$

t Test

Assuming equal variances

Difference $\quad-0.6767$ t Ratio $\quad-2.67165$

Std Err Dif 0.2533 DF

Lower CL Dif -1.3800 Prob $>t-0.972$

Confidence $\quad 0.95$ Prob $<\mathrm{t} \quad 0.027$

Analysis of Variance
Source DF Sum of Squares Mean Square F Ratio Prob $>$ F

$\begin{array}{lrrrrr}\text { ICP Calibration Block } & 1 & 0.6869344 & 0.686934 & 7.1377 & 0.0557\end{array}$

$\begin{array}{lllll}\text { Error } & 4 & 0.3849613 & 0.096240\end{array}$

Means for Oneway Anova

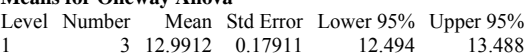

$\begin{array}{llllll}2 & 3 & 12.9912 & 0.17911 & 12.494 & 13.488 \\ 2 & 3 & 12.3145 & 0.17911 & 11.817 & 12.812\end{array}$

Std Error uses a pooled estimate of error variance
Oneway Analysis of Measured By ICP Calibration Block Prep=LM,

Plan =SRNL-SCS-2008-00043, Set=1, Type=Batch 1, Oxide=HfO2 $($ wt $\%)$ Reference Value $=0.0 \mathrm{wt} \%$

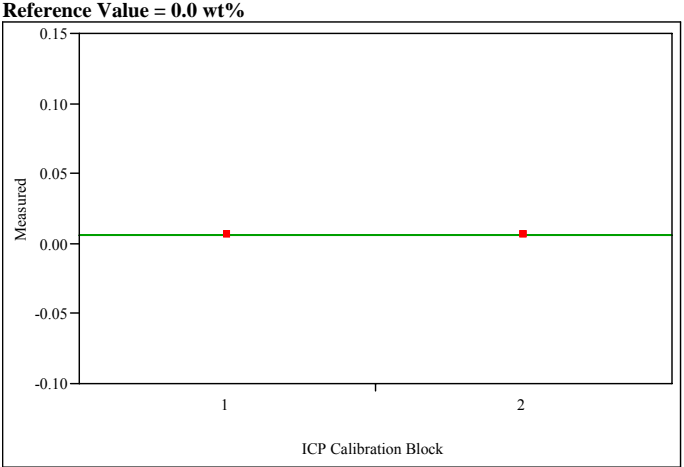

Oneway Anova

Rsquare

Root Mean Square Erro

$\begin{array}{lr}\text { Mean of Response } & 0.005897 \\ \text { Observations (or Sum Wgts) } & 6\end{array}$

t Test

Assuming equal variances

$\begin{array}{lll}\text { Difference } & 0 & \text { t Ratio } \\ \text { Std Err Dif } & 0 & \text { DF }\end{array}$

\begin{tabular}{llll} 
Std Err Dif & 0 & DF \\
Upper CL Dif & 0 & Prob $>$ tt \\
\hline
\end{tabular}

Lower CL Dif 0 Prob $>t$

Confidence 0.95 Prob $<t$

Analysis of Variance
Source

$\begin{array}{lrrr}\text { ICP Calibration Block } & 1 & 0 & 0 \\ \text { Error } & 4 & 0 & 0\end{array}$

C. Total

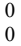

Means for Oneway Anova

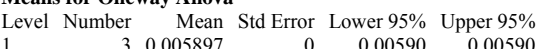

$\begin{array}{llllll}1 & 3 & 0.005897 & 0 & 0.00590 & 0.00590 \\ 2 & 3 & 0.005897 & 0 & 0.00590 & 0.00590\end{array}$

Std Error uses a pooled estimate of error variance 
Exhibit A3. PSAL Measurements by ICP Calibration Block for Samples of the

Standard Glasses by Preparation Method by Set by Analytical Plan Memo. (continued)

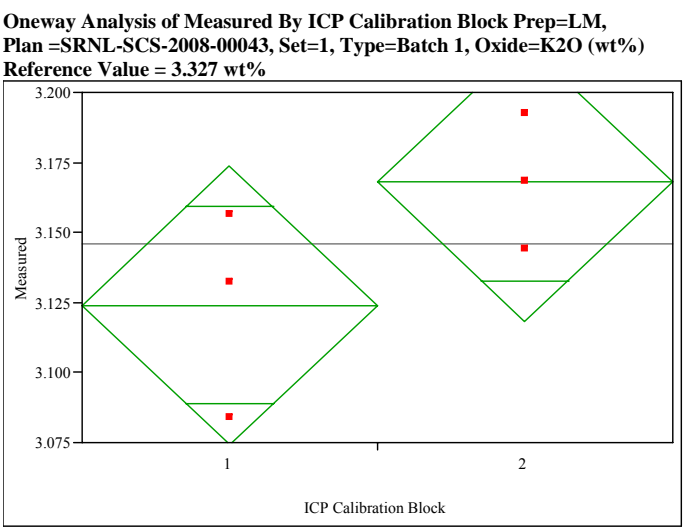

Oneway Anova

Summary of Fit

Rsquare
Adj Rsquare

\subsection{5}

$\begin{array}{ll}\text { Adj Rsquare } & 0.288256 \\ \text { Root Mean Square Error } & 0.031103\end{array}$

$\begin{array}{ll}\text { Root Mean Square Error } & 0.031103 \\ \text { Mean of Response } & 3.146014\end{array}$

Observations (or Sum Wgts)

t Test
$2-1$

Assuming equal variances

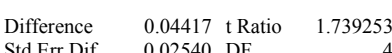

$\begin{array}{lrr}\text { Std Err Dif } & 0.02540 \text { DF } & 4 \\ \text { Upper CL Dif } & 0.11468 \text { Prob }>|t| & 0.1570\end{array}$

$\begin{array}{lrrr}\text { Upper CL Dif } & 0.11468 & \text { Prob }>|t| & 0.1570 \\ \text { Lower CL Dif } & -0.02634 & \text { Prob }>t & 0.078\end{array}$

$\begin{array}{lll}\text { Confidence } & -0.95 \text { Prob }<\mathrm{t} \quad 0.9215\end{array}$

$\begin{array}{lrrrrr}\text { Analysis of Variance } & & & & \\ \text { Source or } & \text { DF } & \text { Sum of Squares } & \text { Mean Square } & \text { F Ratio } & \text { Prob > F } \\ \text { ICP Calibration Block } & 1 & 0.00292631 & 0.002926 & 3.0250 & 0.1570 \\ \text { Error } & 4 & 0.00386950 & 0.000967 & & \\ \text { C. Total } & 5 & 0.00679580 & & & \end{array}$

Means for Oneway Anova

$\begin{array}{rrrrrr}\text { Level Number } & \text { Mean } & \text { Std Error } & \text { Lower } 95 \% & \text { Upper 95\% } \\ 1 & 3 & 3.12393 & 0.01796 & 3.0741 & 3.1738\end{array}$

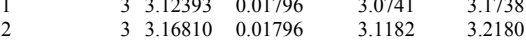

Std Error uses a pooled estimate of error variance
Oneway Analysis of Measured By ICP Calibration Block Prep=LM,
Plan =SRNL-SCS-2008-00043, Set=1, Type=Batch 1, Oxide=La2O3 (wt\%)

Reference Value $=0.0 \mathrm{wt} \%$

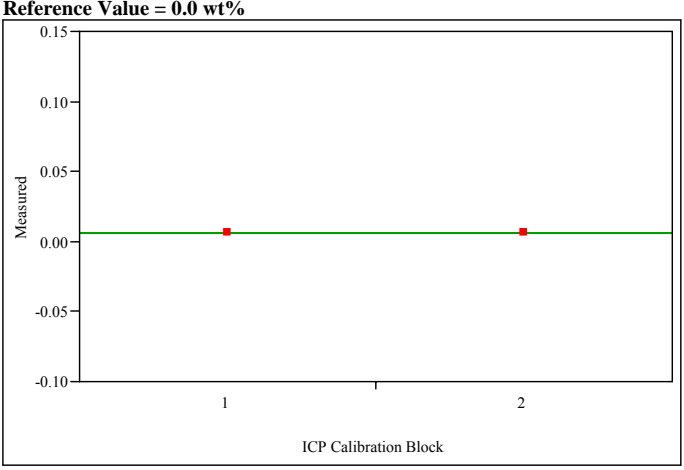

Oneway Anova

ummary of $F$

Rsquare
Adj Rsquar

Root Mean Square Erro

$\begin{array}{lr}\text { Mean of Response } & 0.005864 \\ \text { Observations (or Sum Wgts) } & 6\end{array}$

t Test

Assuming equal variances

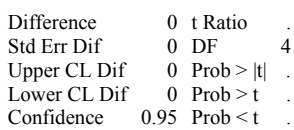

Analysis of Variance
Source DF Sum of Squares Mean Square F Ratio Prob > F

$\begin{array}{cccc}\text { Source } & & \text { Sum or Squares } & \text { Mean Square } \\ \text { ICP Calibration Block } & 1 & 0 & 0 \\ \text { Entor } & 4 & 0 & 0\end{array}$

Error

C. Total

(n)

Means for Oneway Anova

Level Number $\begin{array}{rrr}\text { Mean } \\ 1\end{array}$ Std Error Lower $95 \%$ Upper 95\%

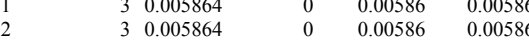

Std Error uses a pooled estimate of error variance
Oneway Analysis of Measured By ICP Calibration Block Prep=LM,

Plan $=$ SRNL-SCS-2008-00043, Set=1, Type=Batch $1,0 x i d e=M g O(w t \%)$

Reference Value $=1.419 \mathrm{wt} \%$

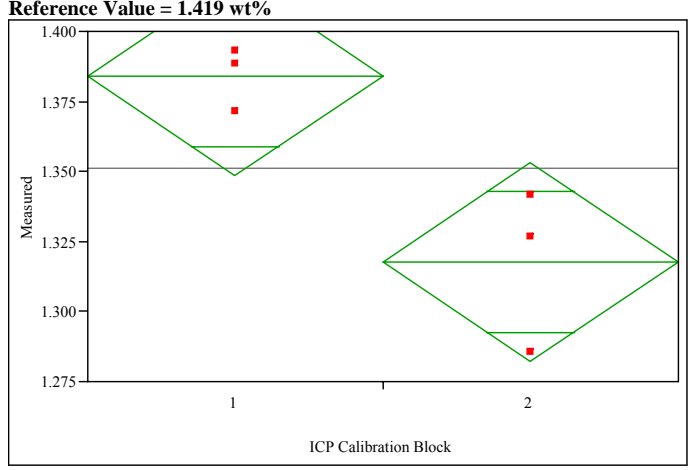

Oneway Anova

ummary of Fit

Rsquare

Root Mean Square Error $\quad 0.022145$

$\begin{array}{lr}\text { Mean of Response } & 1.350962 \\ \text { Observations (or Sum Wgts) } & 6\end{array}$

$\mathbf{t}$ Test
$2-1$

Assuming equal variances

$\begin{array}{llll}\text { Difference } & -0.06633 & t \text { Ratio } & -3.66851 \\ \text { Std Err Dif } & 0.0108 & \text { DF } & \end{array}$

$\begin{array}{llll}\text { Std Err Dif } & 0.01808 \text { DF } & 4 \\ \text { Upper CL Dif } & -0.01613 & \text { Prob }>|t| & 0.0214\end{array}$

$\begin{array}{cccc}\text { Upper CL Dif } & -0.01613 & \text { Prob }>|t| & 0.0214 \\ \text { Lower CL Dif } & -0.11653 & \text { Prob }>t & 0.9893\end{array}$

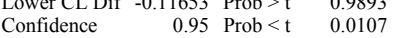

Analysis of Variance
Source

$\begin{array}{lrrrrr}\text { Source } & & & & & \\ \text { ICP Calibration Block } & 1 & 0.00659990 & 0.006600 & 13.4579 & 0.0214\end{array}$

$\begin{array}{llll}\text { Error } & 4 & 0.00196164 & 0.000490 \\ \text { C. Total } & 5 & 0.0085154 & \end{array}$

Means for Oneway Anova

Means for Oneway Anova
Level Number Mean Std Error Lower 95\% Upper 95\%

$\begin{array}{lrrrrr}\text { Level } & \text { Number } & \text { Mean } & \text { Std Error } & \text { Lower 95\% } & \text { Upper 95\% } \\ 1 & 3 & 1.38413 & 0.01279 & 1.3486 & 1.4196 \\ 2 & 3 & 1.31780 & 0.01279 & 1.2823 & 1.3533\end{array}$

Std Error uses a pooled estimate of error variance 
Exhibit A3. PSAL Measurements by ICP Calibration Block for Samples of the

Standard Glasses by Preparation Method by Set by Analytical Plan Memo. (continued)

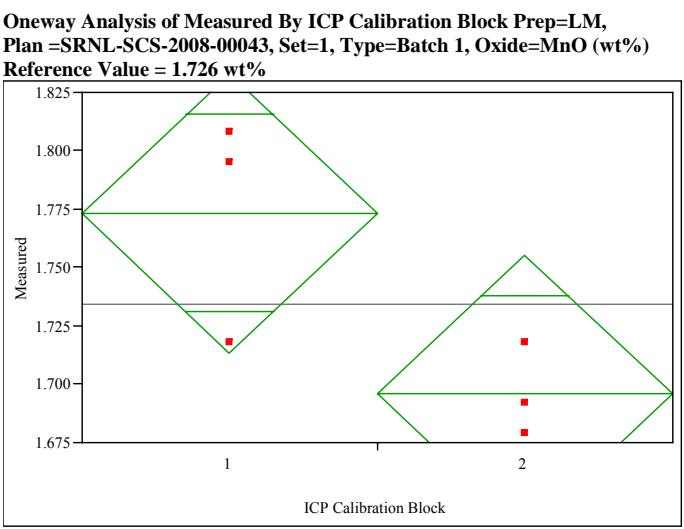

Oneway Anova

Summary of Fit

Rsquare
Adj Rsquare

Root Mean Square Error $\quad 0.037274$

$\begin{array}{lr}\text { Mean of Response } & 1.734512 \\ \text { Observations (or Sum Wgts) } & 6\end{array}$

t Test

Assuming equal variances

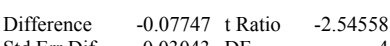

$\begin{array}{lrrr}0.03043 & \text { DF } & 4 \\ \text { Std Err Dif } & -2.5458\end{array}$

$\begin{array}{cccc}\text { Upper CL Dif } & 0.00703 & \text { Prob }>|t| & 0.0636 \\ \text { Lower CL Dif }-0.16197 & \text { Prob }>t & 0.9682\end{array}$

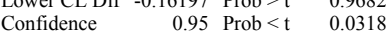

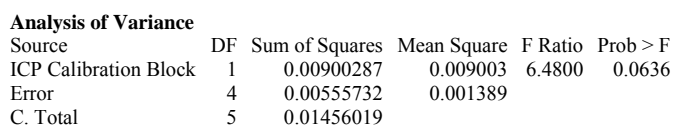

Means for Oneway Anova

\begin{tabular}{rrrrr} 
Level Number & Mean & Std Error & Lower 95\% & Upper 95\% \\
\hline & 1.77325 & 0.02152 & 1.1735 & 1.8330
\end{tabular}

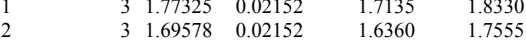

Std Error uses a pooled estimate of error variance
Oneway Analysis of Measured By ICP Calibration Block Prep=LM,

Plan =SRNL-SCS-2008-00043, Set=1, Type=Batch 1, Oxide $=$ Na2O $($ wt $\%)$

Reference Value $=9.003 \mathrm{wt} \%$

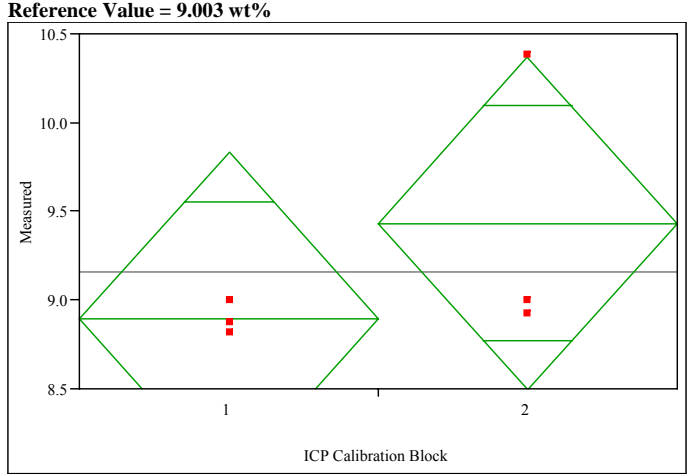

Oneway Anova

Summary of Fi

Rsquare
Adj Rsquare

Adj Rsquare

$\begin{array}{lr}\text { Mean of Response } & 9.161907 \\ \text { Observations (or Sum Wgts) } & 6\end{array}$

0.241903
0.052379
0.58531

t Test
$2-1$

Assuming equal variances

$\begin{array}{lrr}\text { Difference } & 0.5392 \text { t Ratio } & 1.129765 \\ \text { Std Err Dif } & 0.4773 \text { DF } & 4\end{array}$

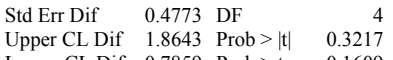
$\begin{array}{lll}\text { Confidence } & -0.95 \text { Prob }<t \quad 0.8391\end{array}$
Analysis of Variance
Source Sum of Squares Mean Square F Ratio Prob $>F$ $\begin{array}{llllll}\text { Calibration Block } & 1 & 0.4361050 & 0.436105 & 1.2764 & 0.3217 \\ & 4 & 1.3667045 & 0.341676 & & \end{array}$ C. Total 0.341676
Means for Oneway Anova
Level Number Mean Std Error Lower 95\% Upper 95\%

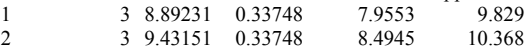

Std Error uses a pooled estimate of error variance
Oneway Analysis of Measured By ICP Calibration Block Prep=LM,

Plan =SRNL-SCS-2008-00043, Set=1, Type=Batch 1, Oxide=Nd2O3 (wt\%) Reference Value $=0.147 \mathrm{wt} \%$

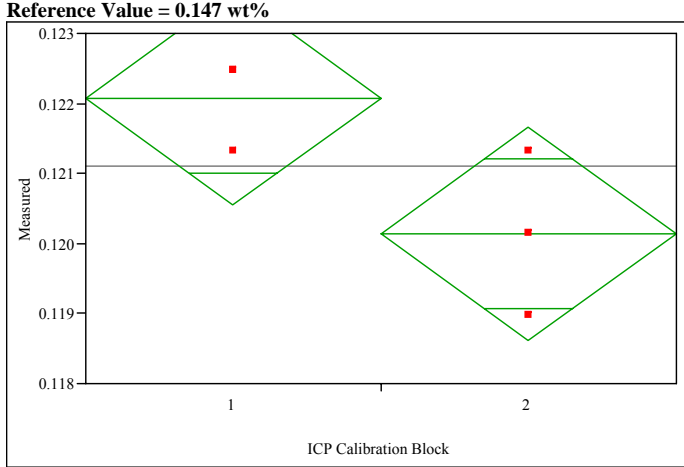

Oneway Anova

mary of $F$

Rsquare

0.609756

$\begin{array}{ll}0.512195 \\ \text { Root Mean Square Error } & 0.000952\end{array}$

Mean of Response $\quad 0.121111$

Observations (or Sum Wgts) 6

t Test

Assuming equal variances

Difference $\quad-0.00194$ t Ratio $\quad-2.5$

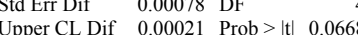

$\begin{array}{lrlll}\text { Lower CL Dif } & -0.00410 & \text { Prob }>t & 0.9666 \\ \text { Confidence } & 0.95 \text { Prob }<\text { t } & 0.033\end{array}$

Confidence $\quad 0.95$ Prob $<\mathrm{t} \quad 0.0334$

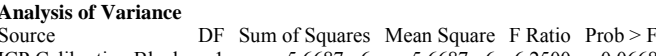

$\begin{array}{lllllll}\text { ICP Calibration Block } & 1 & 5.6687 \mathrm{e}-6 & 5.6687 \mathrm{e}-6 & 6.2500 & 0.0668\end{array}$

$\begin{array}{llll}\text { Error } & 4 & 3.62797 \mathrm{e}-6 & 9.0699 \mathrm{e}-7 \\ \text { C. Total } & 5 & 9.29667 \mathrm{e}-6 & \end{array}$

Means for Oneway Anova

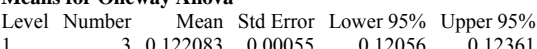

$\begin{array}{lllllll}2 & 3 & 0.120139 & 0.00055 & 0.11861 & 0.12167\end{array}$

Std Error uses a pooled estimate of error variance 
Exhibit A3. PSAL Measurements by ICP Calibration Block for Samples of the

Standard Glasses by Preparation Method by Set by Analytical Plan Memo. (continued)

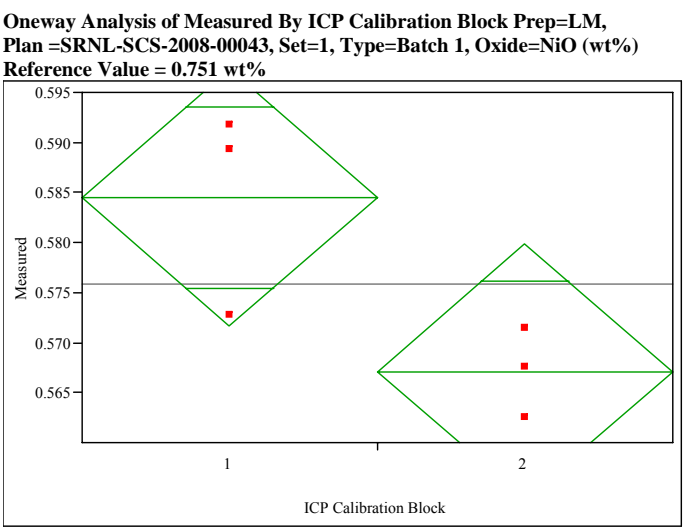

Oneway Anova

ummary of Fit

Rsquare
Adj Rsquar

Root Mean Square Error $\quad 0.00798$

$\begin{array}{lr}\text { Mean of Response } & 0.575806 \\ \text { Observations (or Sum Wgts) }\end{array}$

t Test

Assuming equal variances

$\begin{array}{llll}\text { Difference } & -0.01739 & t \text { Ratio } & -2.66887\end{array}$

$\begin{array}{lrrr}\text { Std Err Dif } & 0.00652 \text { DF } & 4 \\ \text { Upper CL Dif } & 0.00070 \text { Prob }>\text { t } 1 & 0.0559\end{array}$

$\begin{array}{lrrr}\text { Upper CL Dif } & 0.00070 & \text { Prob }>|t| & 0.0559 \\ \text { Lower CL Dif }-0.03548 & \text { Prob }>t & 0.9721\end{array}$

$\begin{array}{lrrr}\text { Confidence } & -0.95 \text { Prob }<\mathrm{t} & 0.0279\end{array}$

\begin{tabular}{lrrrrr} 
Analysis of Variance & \multicolumn{5}{l}{} \\
Source & DF & Sum of Squares & Mean Square & F Ratio & Prob $>$ F \\
ICP Calibration Block & 1 & 0.00045366 & 0.000454 & 7.1229 & 0.0559 \\
Error & 4 & 0.00025476 & 0.000064 & & \\
C. Total & 5 & 0.00070842 & & &
\end{tabular}

Means for Oneway Anova

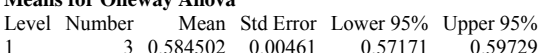

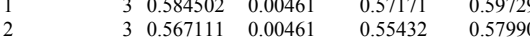

Std Error uses a pooled estimate of error variance
Oneway Analysis of Measured By ICP Calibration Block Prep=LM,
Plan =SRNL-SCS-2008-00043,

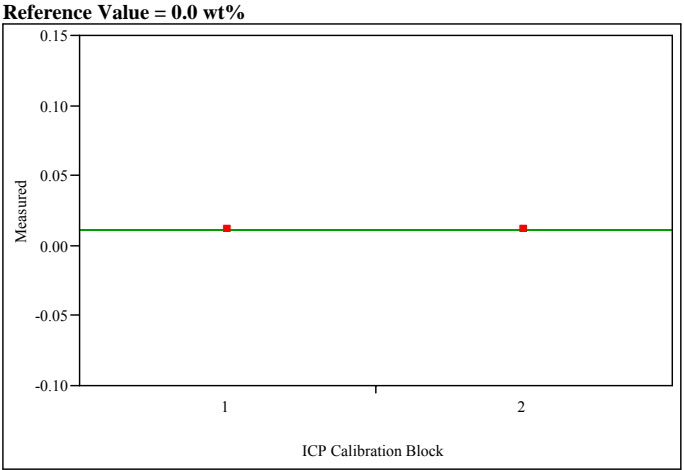

Oneway Anova

ummary of Fit

Rsquare

-0.25
Root Mean Square Error $\quad-2.120-172$

$\begin{array}{lr}2.12 \mathrm{e}-18 \\ \text { Mean of Response } & 0.010772\end{array}$

Observations (or Sum Wgt)

t Test
$2-1$

Assuming equal variances

Difference

$\begin{array}{lll} & \\ \text { Std Err Dif } & 1.735 \mathrm{e}-18 \text { DF } & 4 \\ & \end{array}$

Upper CL Dif 4.816e-18 Prob $>|t| 1.0000$

$\begin{array}{rrrr} & \\ \text { Confidence } & 0.95 & \text { Prob }<t & 0.5000\end{array}$

Analysis of Variance
Source

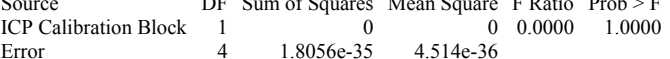

$\begin{array}{llll}\text { Error } & 4 & 1.8056 \mathrm{e}-35 & 4.514 \mathrm{e}-36 \\ \text { C. Total } & 5 & 1.8056 \mathrm{e}-35 & \end{array}$

Means for Oneway Anova

$\begin{array}{rrrrr}\text { Level Number } & \text { Mean } & \text { Std Error } & \text { Lower } 95 \% & \text { Upper 95\% } \\ 3 & 0.010772 & 1.227 \mathrm{e}-18 & 0.01077 & 0.01077\end{array}$

$\begin{array}{llllll}1 & 3 & 0.010772 & 1.227 \mathrm{e}-18 & 0.01077 & 0.0107 \\ 2 & 3 & 0.010772 & 1.227 \mathrm{e}-18 & 0.01077 & 0.01077\end{array}$

Std Error uses a pooled estimate of error variance
Oneway Analysis of Measured By ICP Calibration Block Prep=LM, Plan =SRNL-SCS-2008-00043, Set=1, Type=Batch 1, Oxide=SiO2 (wt\%) Reference Value $=50.22 \mathrm{wt} \%$

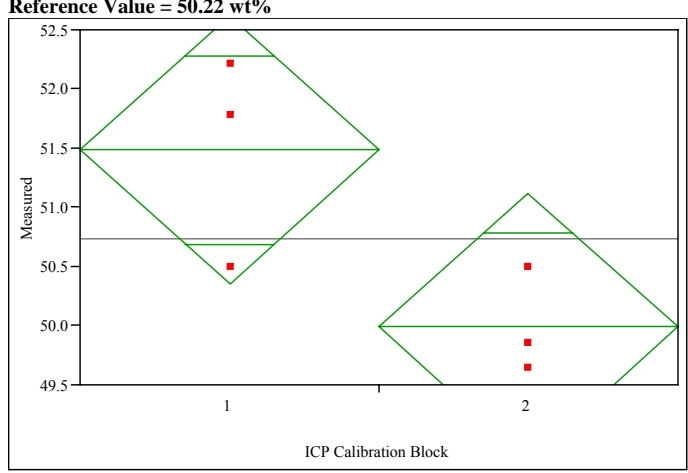

Oneway Anova

Summary of Fi

Rsquare
Adj Rsquare

0.629101

Error $\quad 0.70413$ $\begin{array}{lr}\text { Mean of Response } & 5.73707 \\ \text { Observations (or Sum Wgts) } & 6\end{array}$

t Test
$2-1$

Assuming equal variances

$\begin{array}{lrll}\text { Difference } & -1.4975 & \text { t Ratio } & -2.60473 \\ \text { Std Err Dif } & 0.5749 \text { DF } & \end{array}$ Std Err Dif $\quad 0.5749$ DF $\quad 4$ $\begin{array}{cccc}\text { Upper CL Dif } & 0.0987 & \text { Prob }>|t| & 0.0598 \\ \text { Lower CL Dif } & -3.0937 & \text { Prob }>t & 0.9701\end{array}$ Confidence $\quad 0.95$ Prob $<$ t $\quad 0.029$

\footnotetext{
Analysis of Variance

DF Sum of Squares Mean Square F Ratio Prob $>F$ $\begin{array}{llllll}\text { ICP Calibration Block } & 1 & 3.3638043 & 3.36380 & 6.7846 & 0.0598\end{array}$ C. Total 3.3638043
1.9831953
5.3469996 0.49580

Means for Oneway Anov

$\begin{array}{rrrrr}\text { Level Number } & \text { Mean } & \text { Std Error } & \text { Lower 95\% Upper 95\% } \\ 1 & 51.4858 & 0.40653 & 50.357 & 52.615\end{array}$ $\begin{array}{llllll}1 & 3 & 51.4858 & 0.40653 & 50.357 & 52.615 \\ 2 & 3 & 49.9883 & 0.40653 & 48.860 & 51.117\end{array}$
}

Std Error uses a pooled estimate of error variance 
Exhibit A3. PSAL Measurements by ICP Calibration Block for Samples of the

Standard Glasses by Preparation Method by Set by Analytical Plan Memo. (continued)

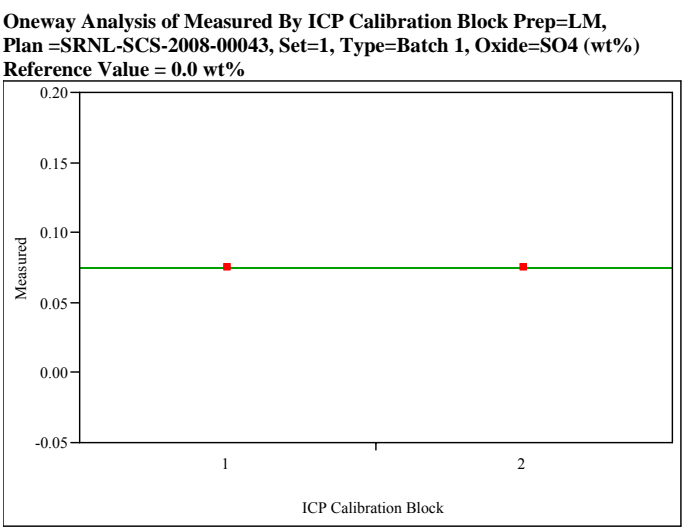

Oneway Anova

ummary of Fit

Rsquare
Adj Rsquarc

Root Mean Square Erro

$\begin{array}{lr}0.074898 \\ \text { Mean of Response } & 6 \\ \text { Observations (or Sum Wgts) } & \end{array}$

t Test

Assuming equal variances

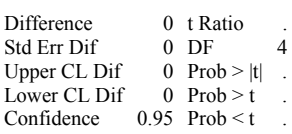

$\begin{array}{lcccc}\text { Analysis of Variance } & & & & \\ \text { Source } & \text { DF Sum of Squares } & \text { Mean Square } & \text { F Ratio Prob }>\text { F } \\ \text { ICP Calibration Block } & 1 & 0 & 0 \\ \text { Error } & 4 & 0 & 0\end{array}$

$\begin{array}{llll}\text { Error } & 4 & 0 & 0 \\ \text { C. Total } & 5 & 0 & 0\end{array}$

Means for Oneway Anova

\begin{tabular}{rrrrr} 
Level Number & Mean & Std Error & Lower 95\% & Upper 95\% \\
\hline & 0.074898 & 0 & 0.07490 & 0.07490
\end{tabular}

$\begin{array}{llllll}1 & 3 & 0.074898 & 0 & 0.07490 & 0.07490 \\ 2 & 3 & 0.074898 & 0 & 0.07490 & 0.07490\end{array}$

Std Error uses a pooled estimate of error variance

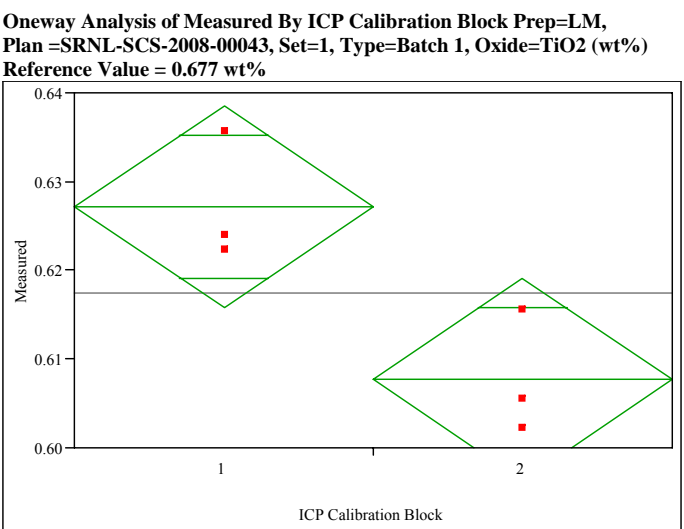

Oneway Anova
Summary of Fit

Rsquare
Adj Rsquare

$\begin{array}{ll}\text { Adj Rsquare } & 0.737508 \\ \text { Root Mean Square Error } & 0.671884 \\ 0.007109\end{array}$

Mean of Response $\quad 0.617438$

Observations (or Sum Wgts)

t Test
$2-1$

Assuming equal variances

Difference $\quad-0.01946$ t Ratio $\quad-3.35239$

Std Err Dif 0.00580 DF $\quad 4$

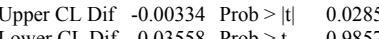

$\begin{array}{lrrr} & \text { Lowfidence } & -0.05 \text { Prob }<\mathrm{t} & 0.0143\end{array}$

$\begin{array}{lrrrrr}\text { Analysis of Variance } & & & & & \\ \text { Source } & \text { DF } & \text { Sum of Squares } & \text { Mean Square } & \text { F Ratio } & \text { Prob }>\text { F } \\ \text { ICP Calibration Block } & 1 & 0.00056804 & 0.000568 & 11.2385 & 0.0285\end{array}$

$\begin{array}{llll}\text { ICP Calibration Block } & 1 & 0.00056804 & 0.000568 \\ \text { Error } & 4 & 0.00020217 & 0.000051 \\ \text { C. Total } & 5 & 0.00077021 & \end{array}$

Means for Oneway Anova

Level Number Mean Std Error Lower 95\% Upper 95\%

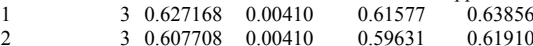

Std Error uses a pooled estimate of error variance
Oneway Analysis of Measured By ICP Calibration Block Prep=LM, Plan =SRNL-SCS-2008-00043, Set=1, Type=Batch 1, Oxide=ZnO (wt\%) Reference Value $=0.0 \mathrm{wt} \%$

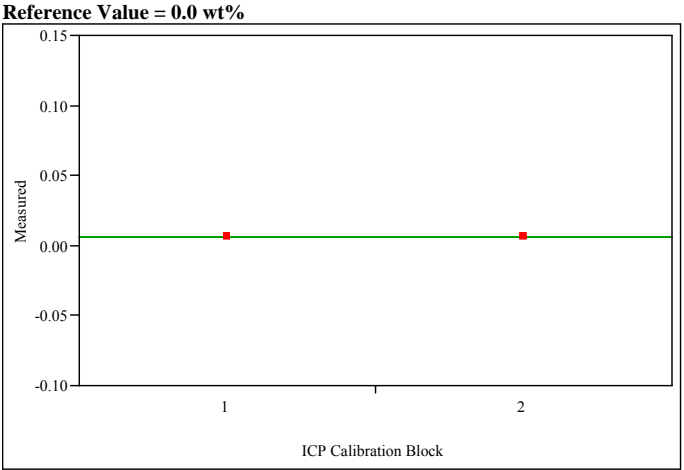

Oneway Anova

Rsquare
Adj Rsqua

Root Mean Square Erro

$\begin{array}{lr}\text { Mean of Response } & 0.006224 \\ \text { Observations (or Sum Wgts) } & 6\end{array}$

t Test

Assuming equal variances

$\begin{array}{lll}\text { Difference } & 0 & \mathrm{t} \text { Ratio } \\ \text { Std Err Dif } & 0 \mathrm{DF} \\ \text { Upper CL Dif } & 0 & \text { Prob }>|t|\end{array}$

Lower CL Dif 0 Prob $>t$

Confidence 0.95 Prob $<t$

Analysis of Variance
Source

$\begin{array}{lrrr}\text { ICP Calibration Block } & 1 & 0 & 0 \\ \text { Error } & 4 & 0 & 0\end{array}$

C. Total

Means for Oneway Anova

Level Number M Mean Std Error Lower 95\% Upper 95\%

$\begin{array}{llllll}1 & 3 & 0.006224 & 0 & 0.00622 & 0.00622 \\ 2 & 3 & 0.006224 & 0 & 0.00622 & 0.00622\end{array}$

Std Error uses a pooled estimate of error variance 
Exhibit A3. PSAL Measurements by ICP Calibration Block for Samples of the

Standard Glasses by Preparation Method by Set by Analytical Plan Memo. (continued)

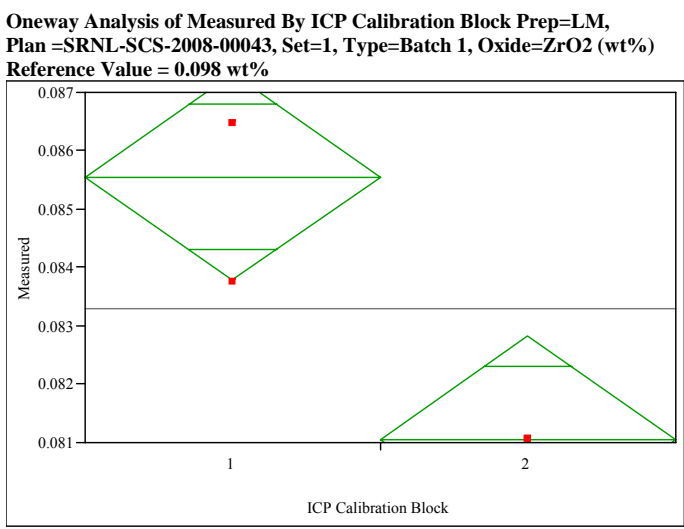

Oneway Anova

Summary of Fit

$\begin{array}{lr}\text { Rsquare } & 0.862069 \\ \text { Adj Rsquare } & 0.827586 \\ \text { Root Mean Square Error } & 0.001103 \\ \text { Mean of Response } & 0.083299 \\ \text { Observations (or Sum Wgts) } & 6\end{array}$

t Test
$2-1$

Assuming equal variances

Difference $\quad-0.00450$ tRatio

Std Err Dif 0.00090 DF

$\begin{array}{lrll}\text { Lower CL Dif } & -0.00700 & \text { Prob }>t & 0.996 \\ \text { Confidence } & 0.95 & \text { Prob }<\mathrm{t} & 0.0037\end{array}$

$\begin{array}{lccccc}\text { Analysis of Variance } & & & & & \\ \text { Source } & \text { DF } & \text { Sum of Squares } & \text { Mean Square } & \text { F Ratio } & \text { Prob }>\text { F } \\ \text { ICP Calibration Block } & 1 & 0.00003041 & 0.000030 & 25.0000 & 0.0075 \\ \text { Error } & 4 & 0.00000487 & 1.216 \mathrm{e}-6 & & \\ \text { C. Total } & 5 & 0.00003528 & & & \end{array}$

Oneway Analysis of Measured By ICP Calibration Block Prep=PF,

Plan =SRNL-SCS-2008-00003, Set=2, Type=Batch 1, Oxide $=$ B2O3 $(\mathrm{wt} \%)$ Reference Value $=7.777$ wt $\%$

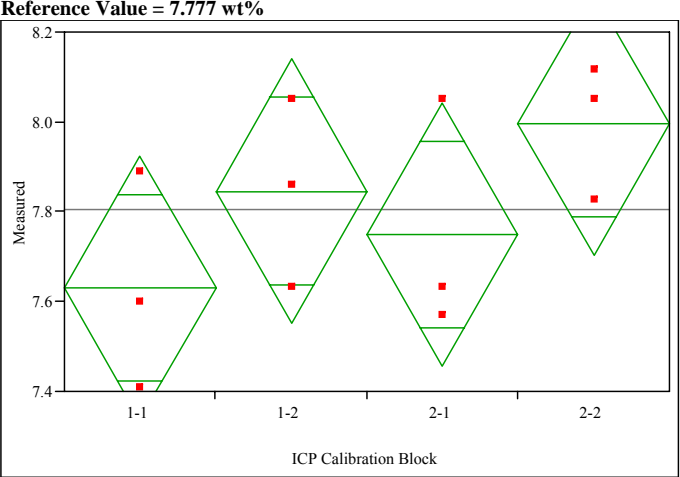

Oneway Anova

$\begin{array}{lr}\text { Rsquare } & 0.354968 \\ \text { Adj Rsquare } & 0.113081 \\ \text { Root Mean Square Error } & 0.220745 \\ \text { Mean of Response } & 7.805574 \\ \text { Observations (or Sum Wgts) } & 12\end{array}$

Analysis of Varianc

Source

$\begin{array}{lrrrrr}\text { ICP Calibration Block } & 3 & 0.21452615 & 0.071509 & 1.4675 & 0.2947 \\ \text { Error } & 8 & 0.38982763 & 0.048728 & & \\ \text { C. Total } & 11 & 0.60435378 & & & \\ \end{array}$

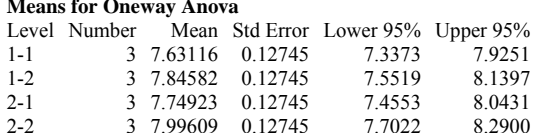

Std Error uses a pooled estimate of error variance
Oneway Analysis of Measured By ICP Calibration Block Prep=PF, Plan $=$ SRNL-SCS-2008-00003, Set=2, Type=Batch 1, Oxide=Li2O $\left({ }_{\text {wt }} \%\right)$ Reference Value $=4.429 \mathrm{wt} \%$

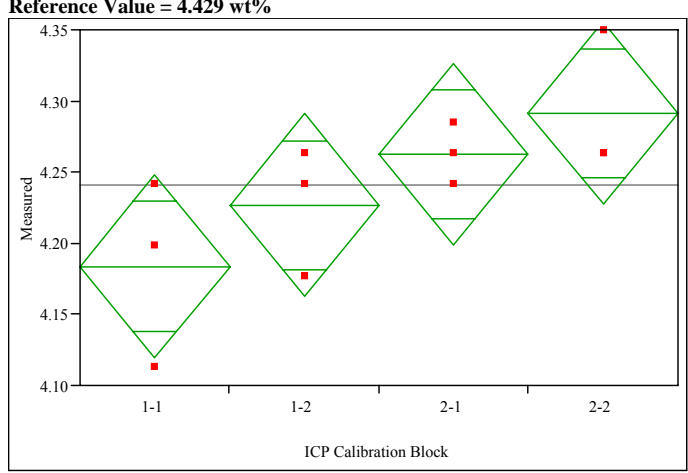

Oneway Anova
Summary of Fit

$\begin{array}{lr}\text { Rsquare } & 0.512195 \\ \text { Adj Rsquare } & 0.329268 \\ \text { Root Mean Square Error } & 0.04814 \\ \text { Mean of Response } & 4.241213 \\ \text { Observations (or Sum Wgts) } & 12\end{array}$

Analysis of Variance

Source DF Sum of Squares Mean Square F Ratio Prob $>F$ $\begin{array}{llllll}\text { CP Calibration Block } & 3 & 0.01946691 & 0.006489 & 2.8000 & 0.1086\end{array}$

C. Total

0.03800682

Means for Oneway Anova
Level Number Mean Std Error Lower 95\% Upper 95\%

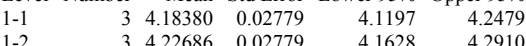

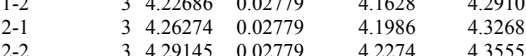

Std Error uses a pooled estimate of error variance

Means for Oneway Anova

\begin{tabular}{rrrrr} 
Level Number & Mean & Std Error & Lower 95\% & Upper 95\% \\
\hline & 0.085551 & 0.00064 & 0.08378 & 0.08732
\end{tabular}

$\begin{array}{llllll}2 & 3 & 0.085551 & 0.00064 & 0.08378 & 0.08732 \\ 2 & 3 & 0.081048 & 0.00064 & 0.07928 & 0.08282\end{array}$

Std Error uses a pooled estimate of error variance 
Exhibit A3. PSAL Measurements by ICP Calibration Block for Samples of the

Standard Glasses by Preparation Method by Set by Analytical Plan Memo. (continued)

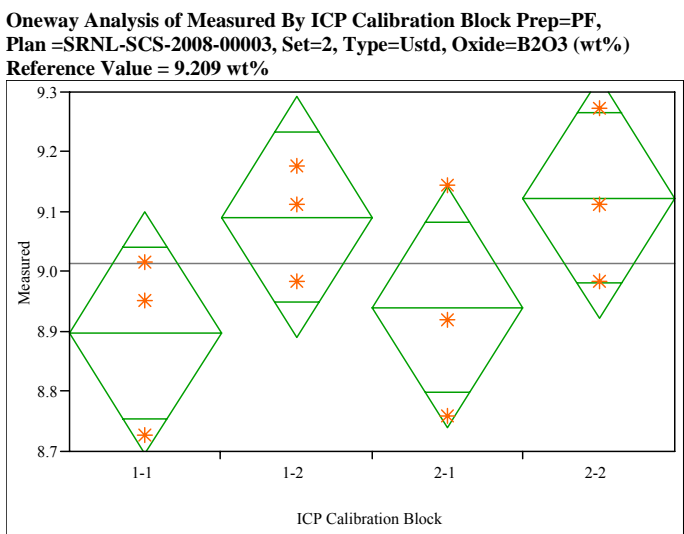

Oneway Anova
Summary of Fit

$\begin{array}{lr}\text { Rsquare } & 0.375552 \\ \text { Adj Rsquare } & 0.141384 \\ \text { Root Mean Square Error } & 0.151312 \\ \text { Mean of Response } & 9.013037 \\ \text { Observations (or Sum Wgts) } & 12\end{array}$

Analysis of Varianc

Source $\quad$ DF Sum of Squares Mean Square F Ratio Prob $>$ F

$\begin{array}{lrrrrr}\text { ICP Calibration Block } & 3 & 0.11015741 & 0.036719 & 1.6038 & 0.2634 \\ \text { Error } & 8 & 0.18316369 & 0.022895 & & \\ \text { C. Total } & 11 & 0.29332110 & & & \end{array}$

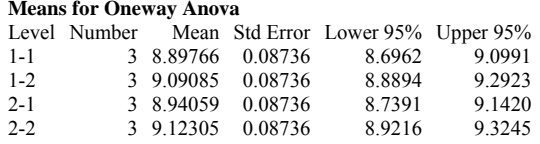

Std Error uses a pooled estimate of error variance
Oneway Analysis of Measured By ICP Calibration Block Prep=PF,

Plan =SRNL-SCS-2008-00003, Set=2, Type=Ustd, Oxide=Li2O (wt\%)

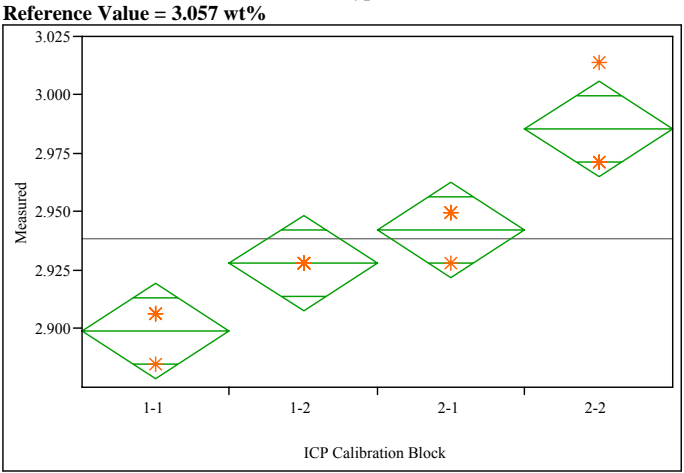

Oneway Anova

Summary of Fit

Rsquare
Adj Rsquar

Root Mean Square Error $\quad 0.810345$

Mean of Response $\quad \begin{array}{ll}0.015223 \\ 2.98708\end{array}$

Observations (or Sum Wgts) $\quad 12$

Analysis of Variance
Source DF Sum of Squares Mean Square F Ratio Prob $>$ F

$\begin{array}{lrrrrr}\text { Source } & \text { DF } & \text { Sum of Squares } & \text { Mean Square } & \text { F Ratio } & \text { Prob }>\text { F } \\ \text { ICP Calibration Block } & 3 & 0.01158745 & 0.003862 & 16.6667 & 0.0008\end{array}$ $\begin{array}{lrrr}\text { Error } & 8 & 0.00185399 & 0.000232 \\ \text { C. Total } & 11 & 0.01344144 & \end{array}$

Means for Oneway Anova

Level Number Mean Std Error Lower 95\% Upper 95\%

$\begin{array}{lllllll}1-1 & & 3 & 2.89924 & 0.00879 & 2.8790 & 2.9195 \\ 1-2 & & 3 & 2.92794 & 0.00879 & 2.9077 & 2.9482 \\ 2-1 & & 3 & 2.94230 & 0.00879 & 2.9220 & 2.9626 \\ 2-2 & & 3 & 2.9855 & 0.089 & 2.9651 & 3.0056\end{array}$

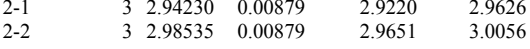

Std Error uses a pooled estimate of error variance
Oneway Analysis of Measured By ICP Calibration Block Prep=PF,

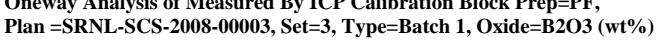
Reference Value $=7.777 \mathrm{wt} \%$

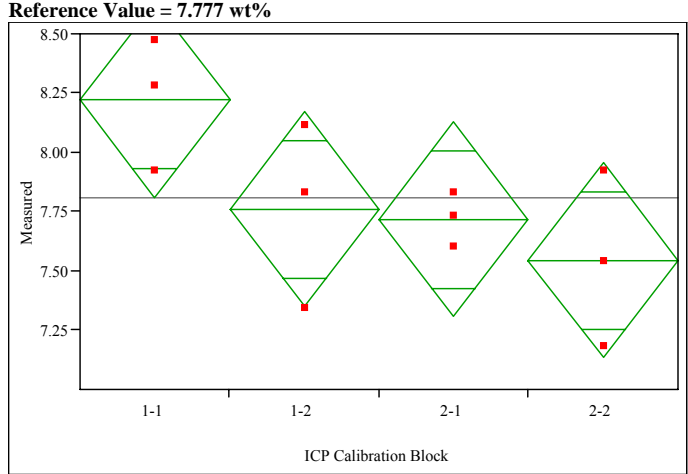

Oneway Anova

Summary of Fi

Rsquare

Adj Rsquare $\quad 0.497569$

Error $\quad 0.308002$ $\begin{array}{lr}\text { Mean of Response } & 7.810941 \\ \text { Observations (or Sum Wgts) } & 12\end{array}$

Analysis of Varian

Source DF Sum of Squares Mean Square F Ratio Prob $>$ F $\begin{array}{lrrrrr}\text { ICP Calibration Block } & 3 & 0.7515759 & 0.250525 & 2.6409 & 0.1210\end{array}$

C. Total

$\begin{array}{ll}0.7515759 & 0.250525 \\ 0.7589197 & 0.094865 \\ 1.5104957 & \end{array}$

Means for Oneway Anova
Level Number Mean Std Error Lower 95\% Upper 95\%

$\begin{array}{lllllll}1-1 & & 3 & 8.22148 & 0.17782 & 7.8114 & 8.6315 \\ 1-2 & & 3 & 7.75996 & 0.17782 & 7.3499 & 8.1700\end{array}$

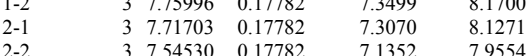

Std Error uses a pooled estimate of error variance 
Exhibit A3. PSAL Measurements by ICP Calibration Block for Samples of the

Standard Glasses by Preparation Method by Set by Analytical Plan Memo. (continued)

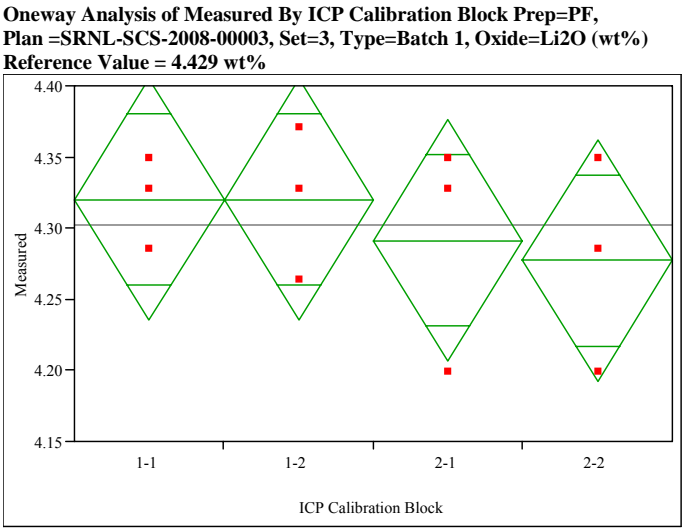

Oneway Anova
Summary of Fit

$\begin{array}{lr}\text { Rsquare } & 0.112971 \\ \text { Adj Rsquare } & -0.21967 \\ \text { Root Mean Square Error } & 0.063986 \\ \text { Mean of Response } & 4.302212 \\ \text { Observations (or Sum Wgts) } & 12\end{array}$

Analysis of Variance

Source DF Sum of Squares Mean Square F Ratio Prob $>$ F

$\begin{array}{llllll}\text { ICP Calibration Block } & 3 & 0.00417148 & 0.001390 & 0.3396 & 0.7975\end{array}$

C. Total

$\begin{array}{rrr}8 & 0.03275385 \\ 11 & 0.0369253\end{array}$

Means for Oneway Anova
Level Number Mean Std Error Lower 95\% Upper 95\%

$\begin{array}{lllllll}1-1 & & 3 & 4.32015 & 0.03694 & 4.2350 & 4.4053 \\ 1-2 & & 3 & 4.32015 & 0.03694 & 4.2350 & 4.4053\end{array}$

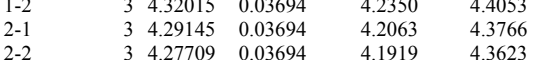

Std Error uses a pooled estimate of error variance
Oneway Analysis of Measured By ICP Calibration Block Prep=PF,
Plan =SRNL-SCS-2008-00003, Set $=3$, Type=Ustd, Oxide=B2O3 (wt $\%$ )

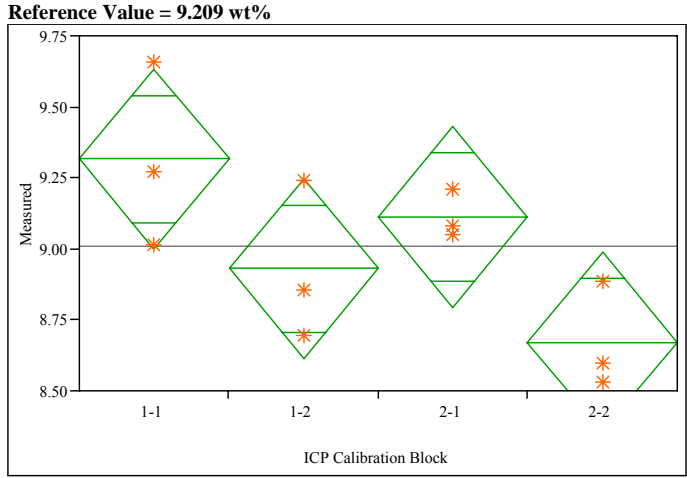

Oneway Anova

Summary of Fit

Rsquare

Adj Rsquare
Root Mean Squ

Mean of Response

Observations (or Sum Wgts)

0.597519

0.238251

9.00767
12

Analysis of Variance

Source DF Sum of Squares Mean Square F Ratio Prob $>F$

$\begin{array}{llllll}\text { ICP Calibration Block } & 3 & 0.6741633 & 0.224721 & 3.9589 & 0.0531\end{array}$

Error

0.6741633
0.4541077 0.056763

Means for Oneway Anov

Level Number Mean Std Error Lower 95\% Upper 95\%

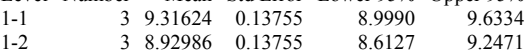

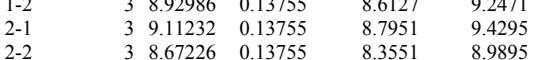

Std Error uses a pooled estimate of error variance
Oneway Analysis of Measured By ICP Calibration Block Prep $=\mathbf{P F}$, Plan =SRNL-SCS-2008-00003, Set=3, Type=Ustd, Oxide $=$ Li2 2 (wt\%) Reference Value $=3.057 \mathrm{wt} \%$

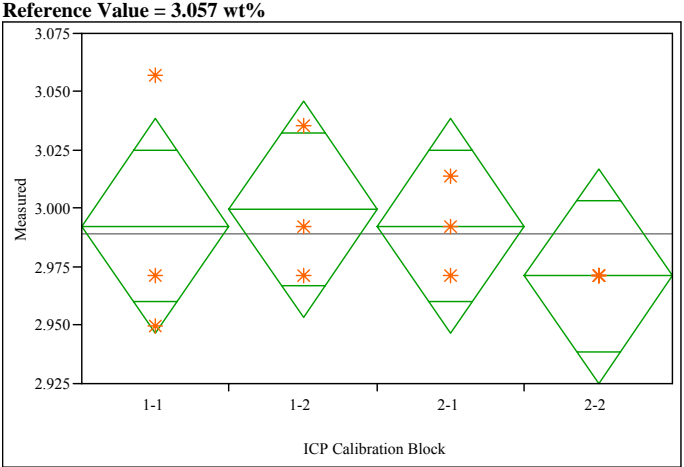

Oneway Anova

ummary of Fit

Rsquare
Adj Rsquare

Root Mean Square Error $\quad 0.034603$

$\begin{array}{lr}\text { Mean of Response } & 2.988943 \\ \text { Observations (or Sum Wgts) } & 12\end{array}$

Analysis of Variance
Source DF Sum of Squares Mean Square F Ratio Prob > F $\begin{array}{lrrrrrr} & \text { DF } & \text { Sum of Squares } & & & & \\ \text { Source } & & & & & \\ \text { ICP Calibration Block } & 3 & 0.00139049 & 0.000463 & 0.3871 & 0.7655\end{array}$ $\begin{array}{lrr}\text { Error } & 8 & 0.00957896 \\ \text { C. Total } & 11 & 0.01096945\end{array}$ .001197

Means for Oneway Anova

Level Number Mean Std Error Lower 95\% Upper 95\%

$\begin{array}{lllllll}1-1 & & 3 & 2.99253 & 0.01998 & 2.9465 & 3.0386 \\ 1-2 & & 3 & 2.99971 & 0.01998 & 2.9536 & 3.0458\end{array}$

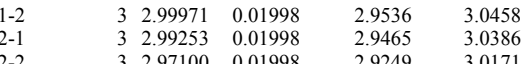

$\begin{array}{llllll}2-2 & 3 & 2.97100 & 0.01998 & 2.9249 & 3.0171\end{array}$

Std Error uses a pooled estimate of error variance 
Exhibit A3. PSAL Measurements by ICP Calibration Block for Samples of the

Standard Glasses by Preparation Method by Set by Analytical Plan Memo. (continued)

Oneway Analysis of Measured By ICP Calibration Block Prep=PF,

Plan =SRNL-SCS-2008-00043, Set $=1$, Type $=$ Batch 1, Oxide $=B 203(\mathrm{wt} \%)$

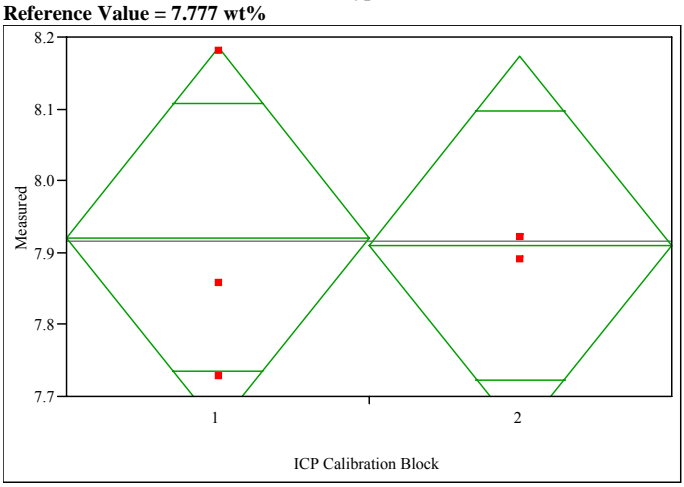

Oneway Anova
Summary of Fit

Rsquare

$\begin{array}{lr}\text { Rsquare } & 0.00159 \\ \text { Adj Rsquare } & -0.24801 \\ \text { Rot } & 0.164709\end{array}$

0.164709
7.915588

Observations (or Sum Wots)

t Test
$2-1$

Assuming equal variances

Difference $\quad-0.01073$ t Ratio $\quad-0.07981$

Std Err Dif 0.13448 DF

Upper CL Dif 0.36265 Prob $>|t| \quad 0.9402$

Lower CL Dif -0.38412 Prob $>$ t 0.5299

Analysis of Varianc

Source DF Sum of Squares Mean Square F Ratio Prob $>F$

$\begin{array}{llllll}\text { ICP Calibration Block } & 1 & 0.00017280 & 0.000173 & 0.0064 & 0.9402 \\ \text { Error } & 4 & 0.10851585 & 0.027129 & & \end{array}$

Crror

$\begin{array}{ll}4 & 0.10851585 \\ 5 & 0.10868864\end{array}$ 027129

Means for Oneway Anova

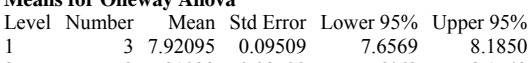

Std Error uses a pooled estimate of error variance
Oneway Analysis of Measured By ICP Calibration Block Prep=PF,

Plan =SRNL-SCS-2008-00043, Set=1, Type =Batch 1, Oxide $=$ Li2O $\left({ }^{2} \%\right)$

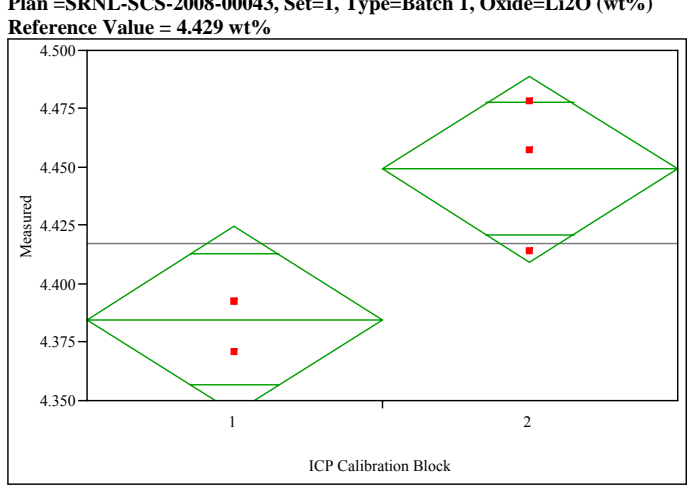

Oneway Anova

ummary of Fit

Rsquare

0.716814

$\begin{array}{lr}0.646018 \\ \text { Rdj Rsquare } & 0.02486 \\ \text { Rean Square Error } & 4.41033\end{array}$

Mean of Response $\quad 4.417033$

Observations (or Sum Wgts)

t Test
$2-1$

Assuming equal variances

Difference $\quad 0.064587$ t Ratio $\quad 3.18198$

$\begin{array}{lll}\text { Std Err Dif } & 0.020298 \text { DF } & \\ \text { Upper CL Dif } & 0.120943 \quad \text { Prob }>\text { t } & 0.0335\end{array}$

Lower CL Dif 0.008231 Prob $>t \quad 0.0163$

Confidence $\quad 0.95$ Prob $<\mathrm{t} \quad 0.9833$

$\begin{array}{lrrrrr}\text { Analysis of Variance } & & & & \\ \text { Source } & \text { DF } & \text { Sum of Squares } & \text { Mean Square } & \text { F Ratio } & \text { Prob }>\text { F } \\ \text { ICP Calibration Block } & 1 & 0.00625722 & 0.006257 & 10.1250 & 0.0335\end{array}$

$\begin{array}{llll}\text { Error } & 4 & 0.00247199 & 0.000618 \\ \text { C. Total } & 5 & 0.00872921 & \end{array}$

Means for Oneway Anova

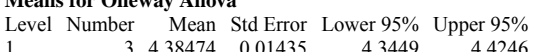

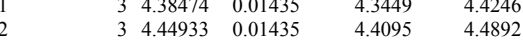

Std Error uses a pooled estimate of error variance 
Exhibit A4. Oxide Measurements by Lab ID within Glass ID Including Auxiliary Measurements for LM Preps.

Analytical Plan=SRNL-SCS-2008-00003, Prep=LM, Oxide=Al2O3 (wt\%)

Variability Chart for Measured

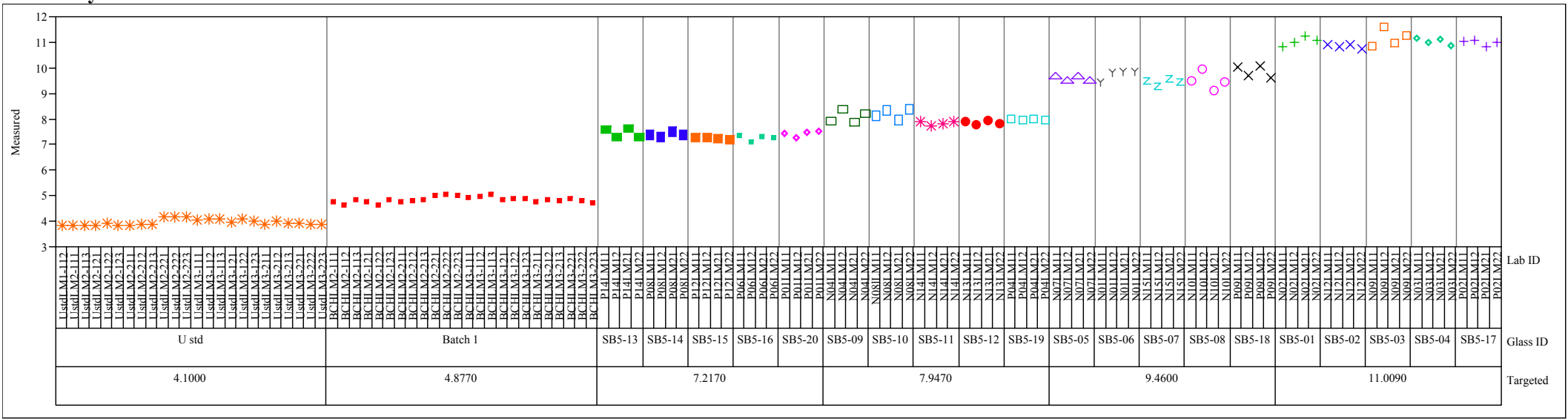

Analytical Plan =SRNL-SCS-2008-00003, Prep=LM, Oxide=Al2O3 (wt \%) Variability Chart for Measured bc

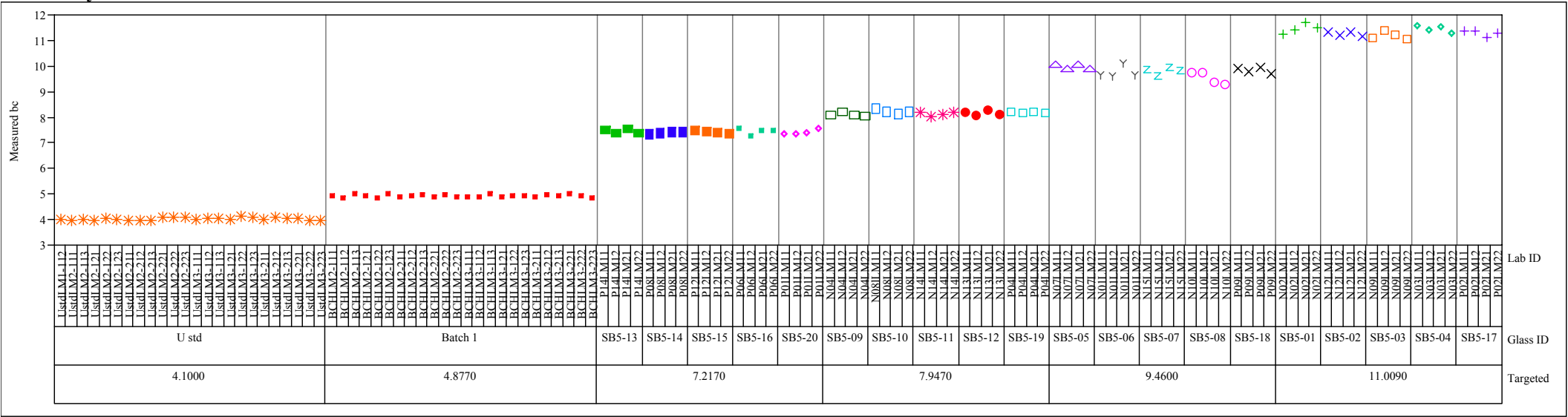


Exhibit A4. Oxide Measurements by Lab ID within Glass ID Including Auxiliary Measurements for LM Preps. (continued) Analytical Plan =SRNL-SCS-2008-00003, Prep=LM, Oxide=BaO (wt $\%)$

Variability Chart for Measured

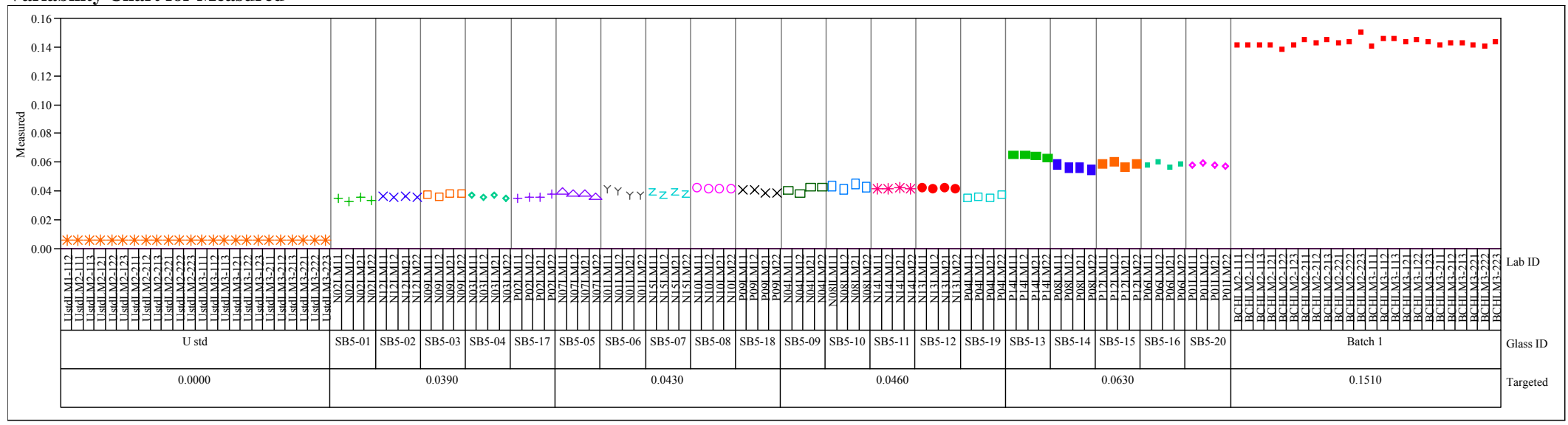

Analytical Plan =SRNL-SCS-2008-00003, Prep=LM, Oxide=BaO (wt \%)

Variability Chart for Measured bc

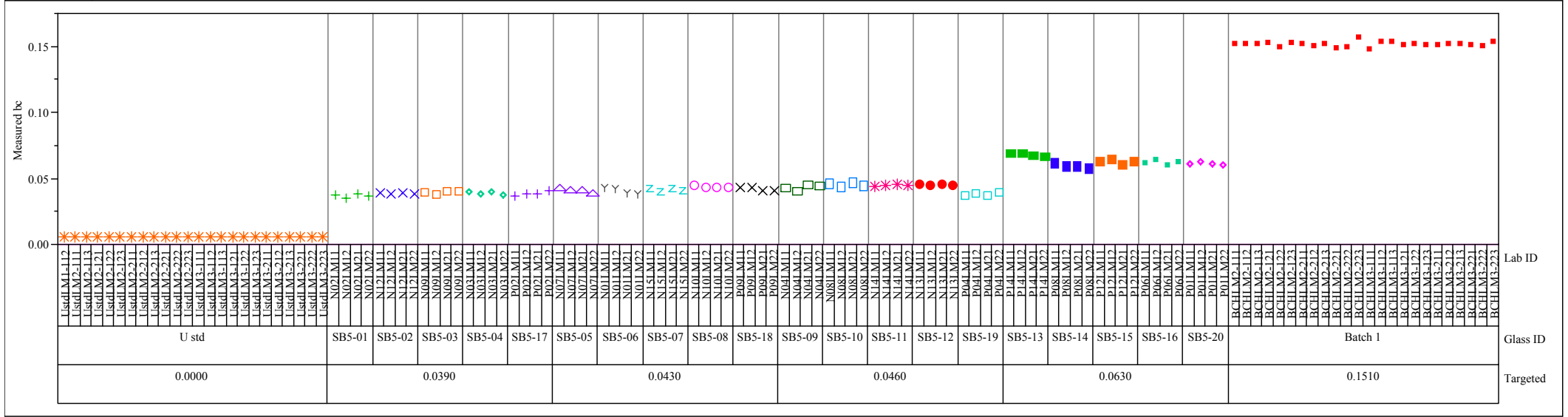


Exhibit A4. Oxide Measurements by Lab ID within Glass ID Including Auxiliary Measurements for LM Preps. (continued) Analytical Plan =SRNL-SCS-2008-00003, Prep=LM, Oxide=CaO (wt $\%)$ Variability Chart for Measured

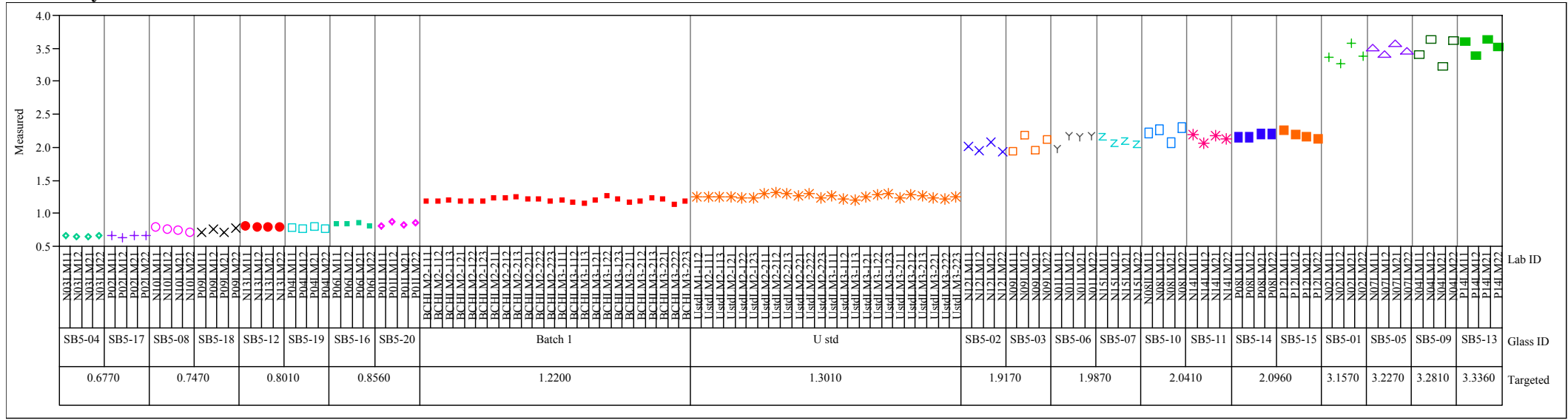

Analytical Plan $=$ SRNL-SCS-2008-00003, Prep $=$ LM, Oxide $=\mathrm{CaO}(w \mathrm{t} \%)$

Variability Chart for Measured bc

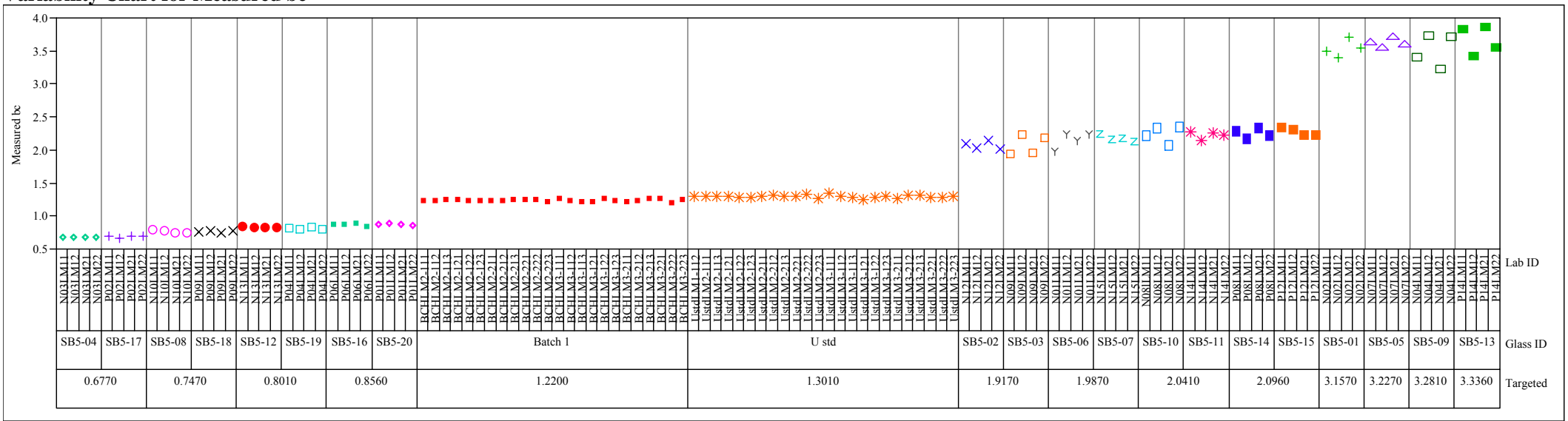


Exhibit A4. Oxide Measurements by Lab ID within Glass ID Including Auxiliary Measurements for LM Preps. (continued) Analytical Plan =SRNL-SCS-2008-00003, Prep=LM, Oxide=CdO (wt \%) Variability Chart for Measured

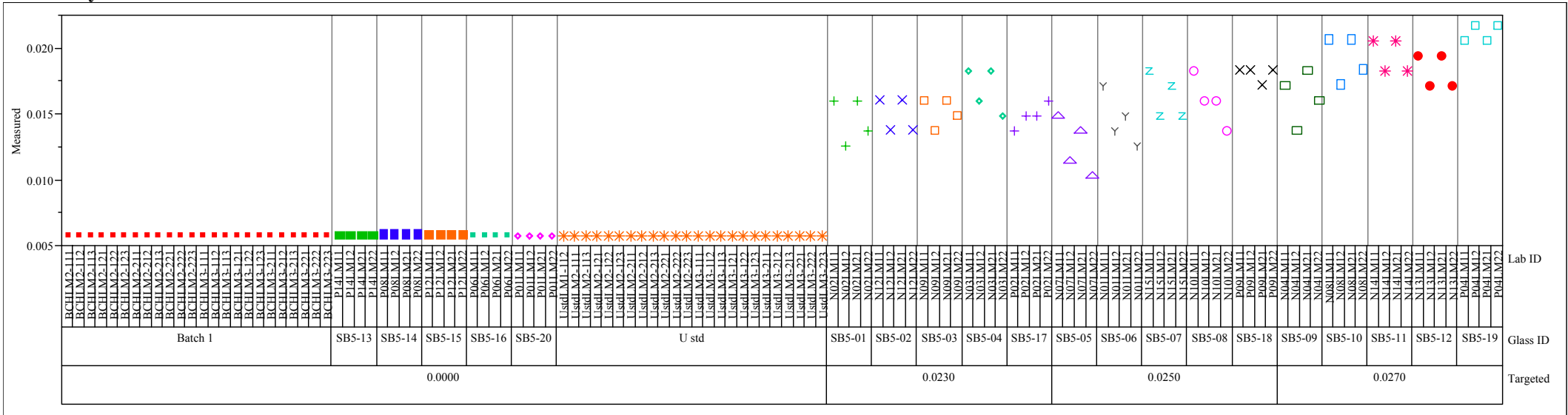

Analytical Plan =SRNL-SCS-2008-00003, Prep=LM, Oxide=CdO (wt \%) Variability Chart for Measured bc

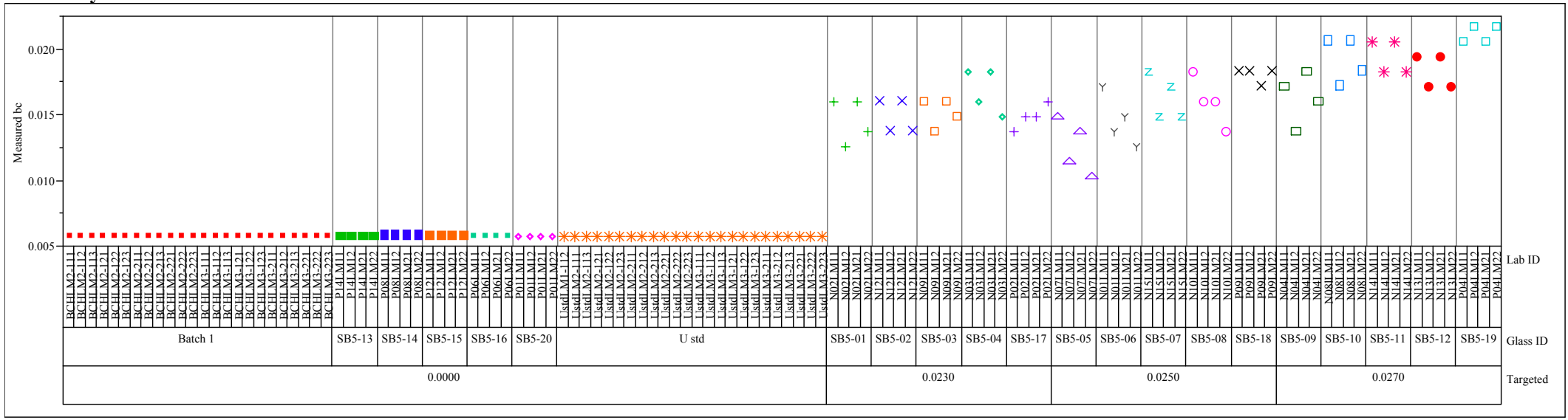


Exhibit A4. Oxide Measurements by Lab ID within Glass ID Including Auxiliary Measurements for LM Preps. (continued) Analytical Plan =SRNL-SCS-2008-00003, Prep=LM, Oxide=Ce2O3 (wt \%) Variability Chart for Measured

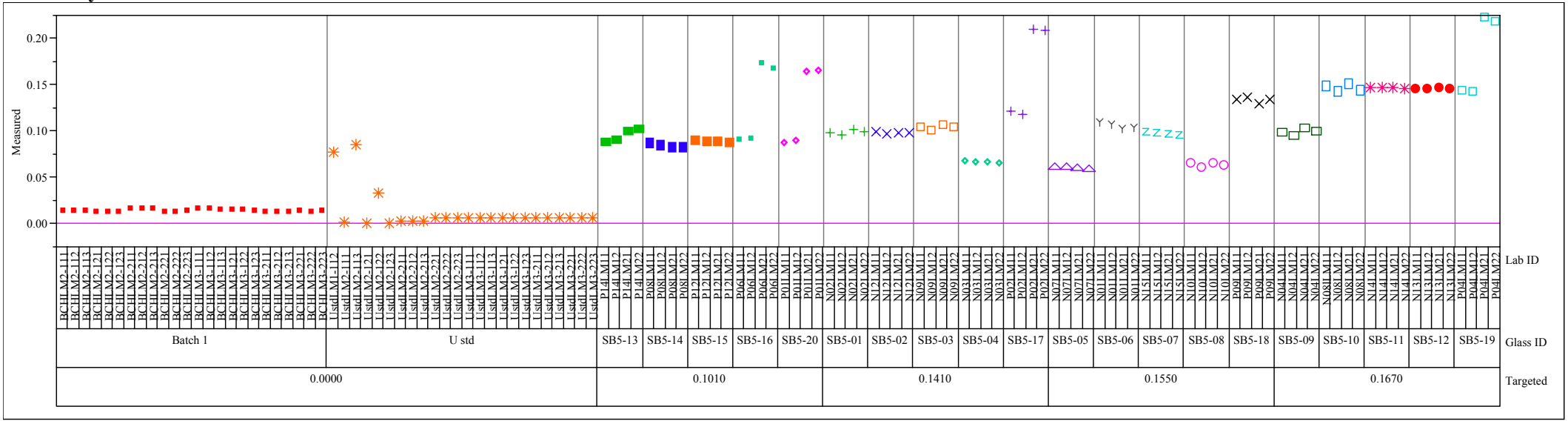

Analytical Plan =SRNL-SCS-2008-00003, Prep=LM, Oxide=Ce2O3 (wt \%) Variability Chart for Measured bc

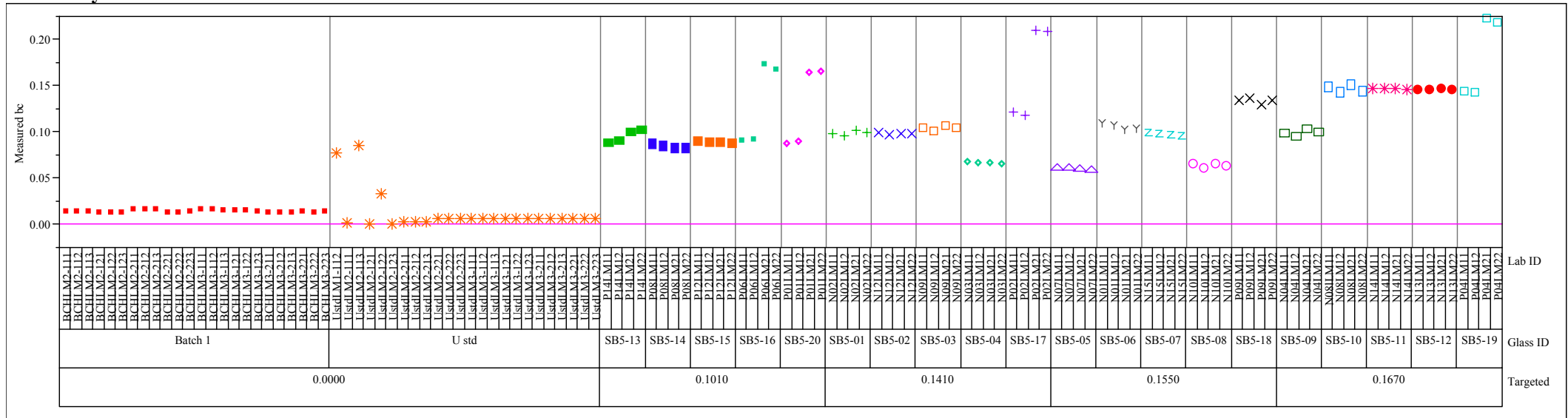


Exhibit A4. Oxide Measurements by Lab ID within Glass ID Including Auxiliary Measurements for LM Preps. (continued) Analytical Plan =SRNL-SCS-2008-00003, Prep=LM, Oxide=Cr2O3 (wt \% Variability Chart for Measured

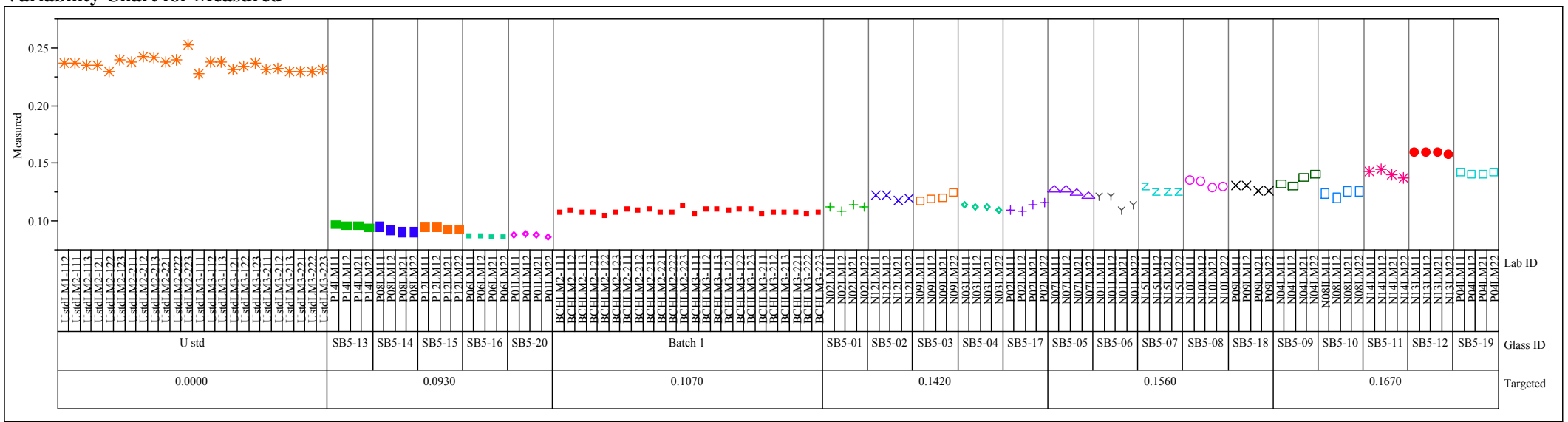

Analytical Plan =SRNL-SCS-2008-00003, Prep=LM, Oxide=Cr2O3 (wt \%) Variability Chart for Measured bc

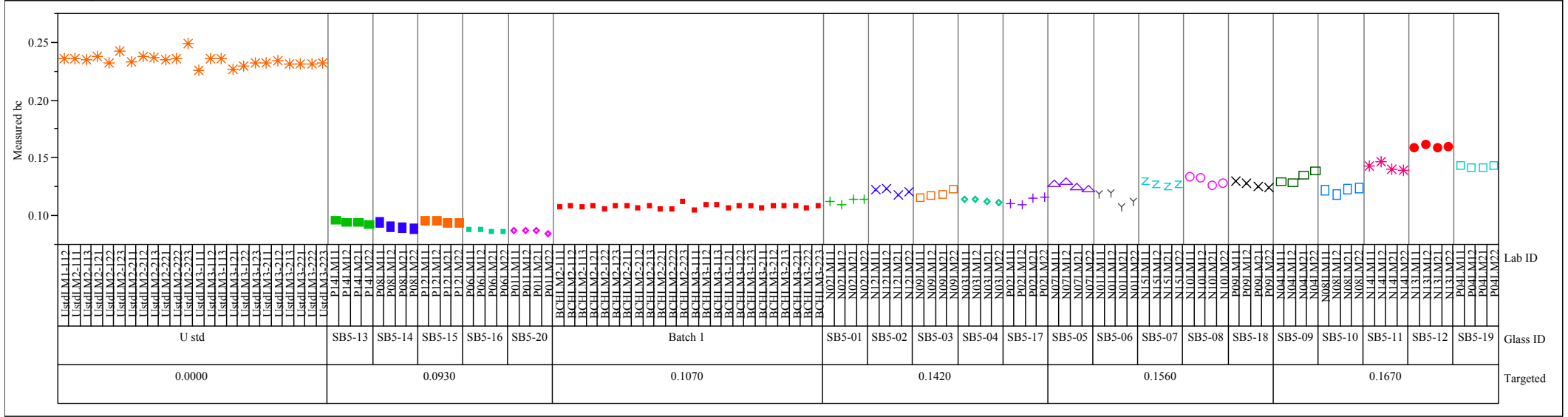


Exhibit A4. Oxide Measurements by Lab ID within Glass ID Including Auxiliary Measurements for LM Preps. (continued) Analytical Plan =SRNL-SCS-2008-00003, Prep=LM, Oxide=CuO (wt $\%)$

Variability Chart for Measured

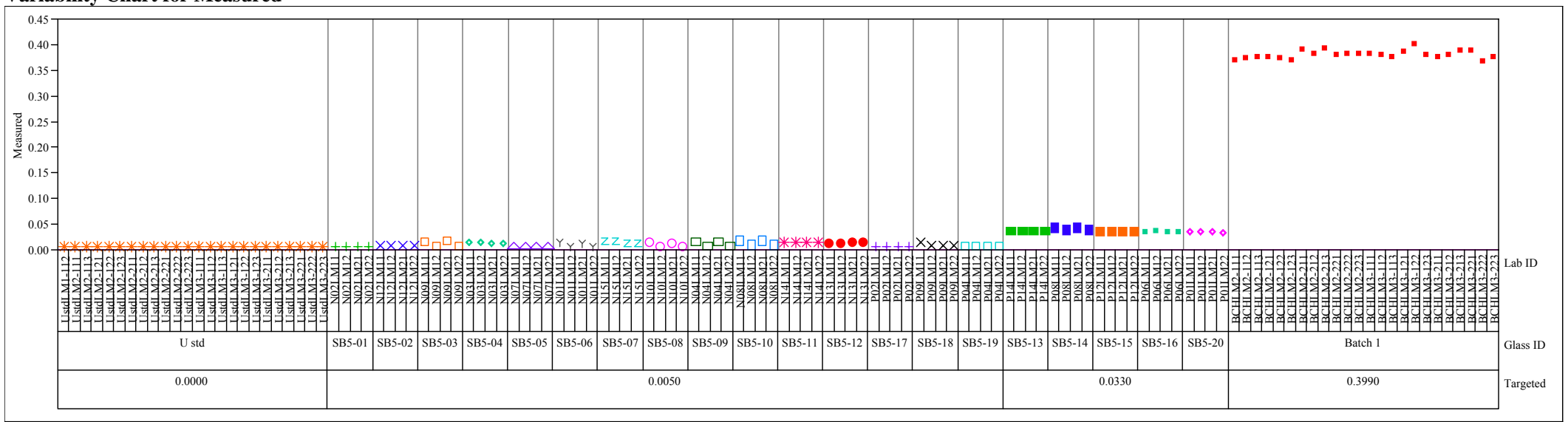

Analytical Plan =SRNL-SCS-2008-00003, Prep=LM, Oxide=CuO (wt $\%)$

Variability Chart for Measured bc

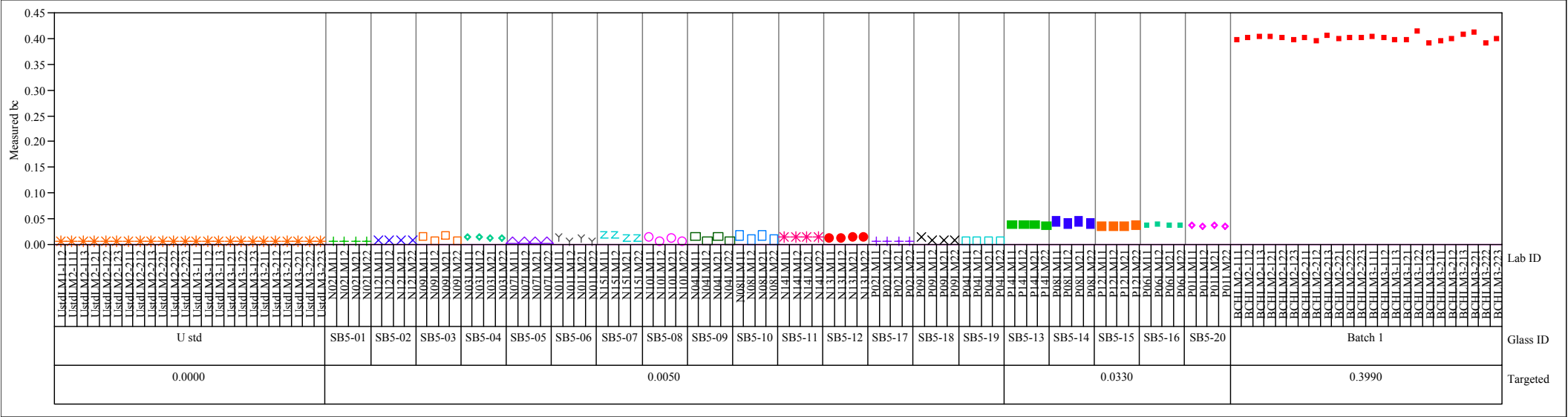


Exhibit A4. Oxide Measurements by Lab ID within Glass ID Including Auxiliary Measurements for LM Preps. (continued) Analytical Plan =SRNL-SCS-2008-00003, Prep=LM, Oxide=Fe2O3 (wt \%) Variability Chart for Measured

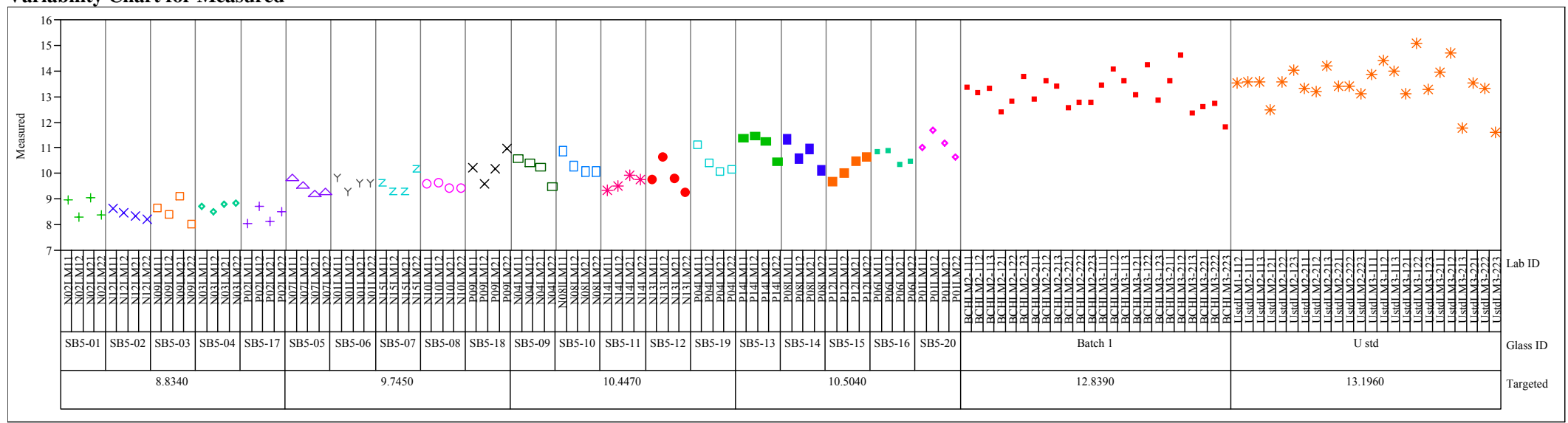

Analytical Plan =SRNL-SCS-2008-00003, Prep=LM, Oxide=Fe2O3 (wt \%) Variability Chart for Measured bc

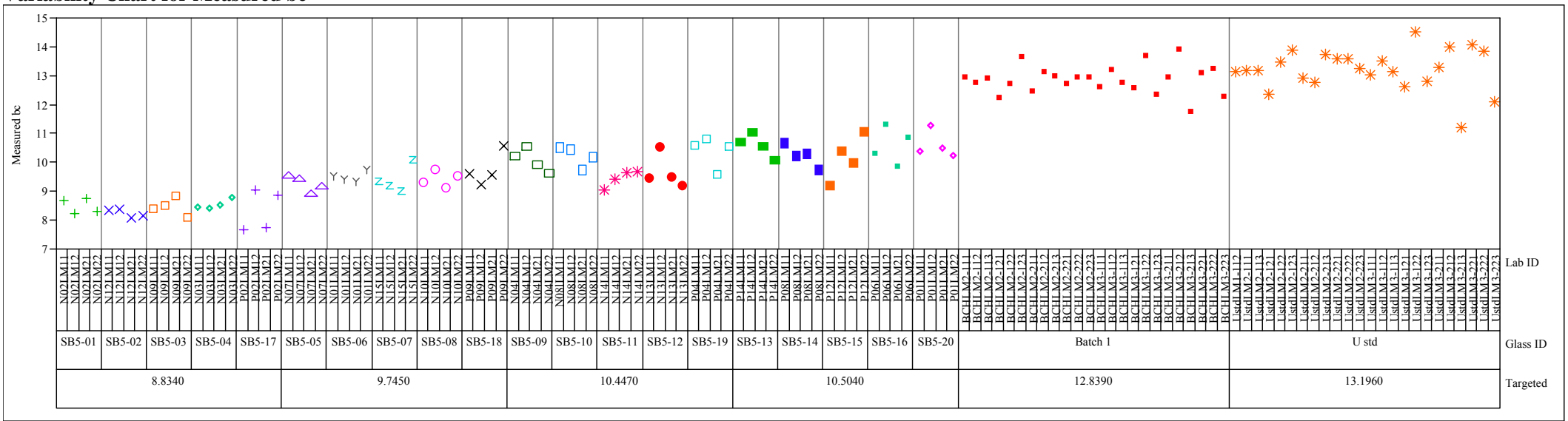


Exhibit A4. Oxide Measurements by Lab ID within Glass ID Including Auxiliary Measurements for LM Preps. (continued) Analytical Plan =SRNL-SCS-2008-00003, Prep=LM, Oxide=K2O (wt $\%)$ Variability Chart for Measured

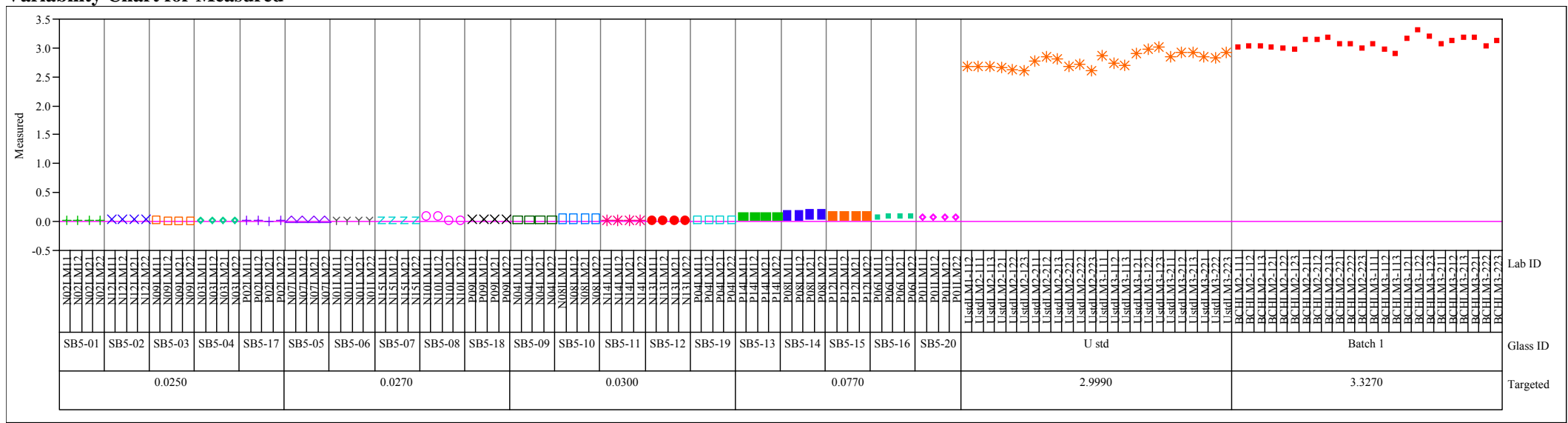

Analytical Plan =SRNL-SCS-2008-00003, Prep=LM, Oxide=K2O (wt \%)

Variability Chart for Measured bc

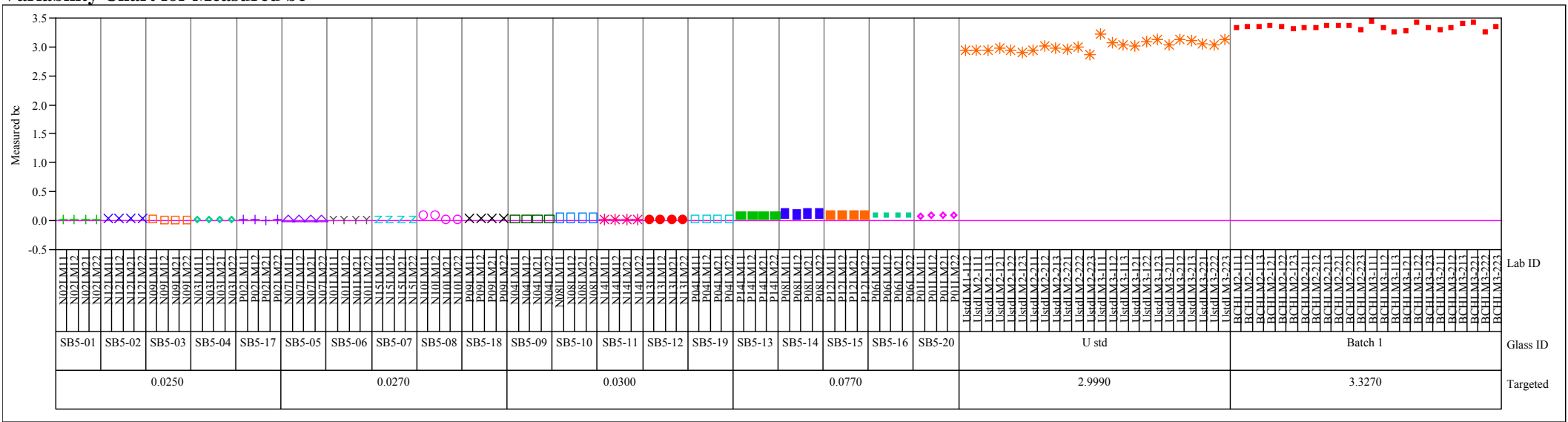


Exhibit A4. Oxide Measurements by Lab ID within Glass ID Including Auxiliary Measurements for LM Preps. (continued) Analytical Plan =SRNL-SCS-2008-00003, Prep=LM, Oxide=La2O3 (wt\%) Variability Chart for Measured

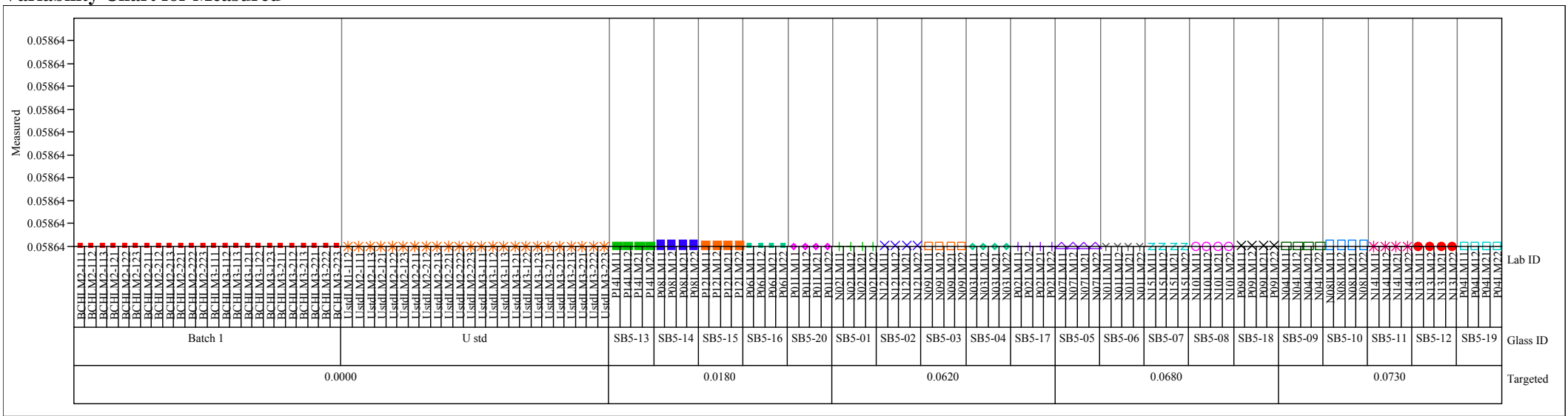

Analytical Plan =SRNL-SCS-2008-00003, Prep=LM, Oxide=La2O3 (wt \%)

Variability Chart for Measured bc

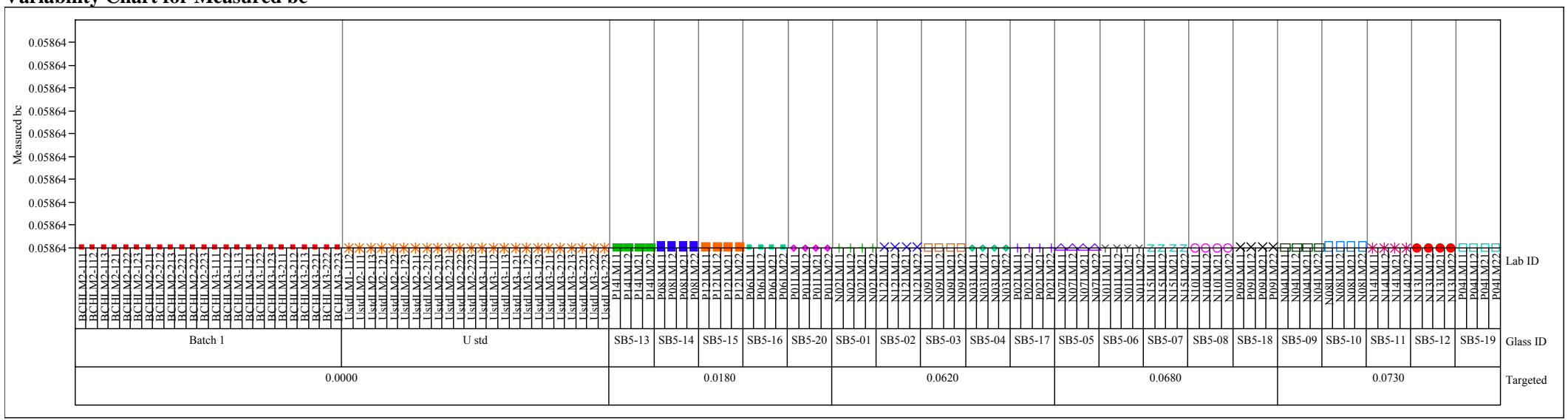


Exhibit A4. Oxide Measurements by Lab ID within Glass ID Including Auxiliary Measurements for LM Preps. (continued) Analytical Plan =SRNL-SCS-2008-00003, Prep=LM, Oxide=MgO (wt\%) Variability Chart for Measured

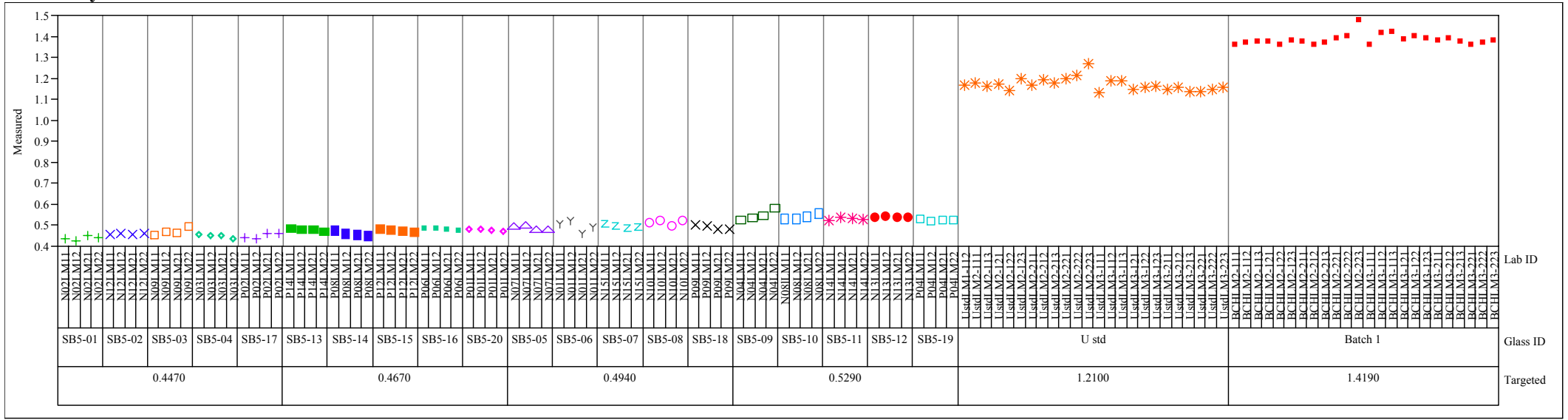

Analytical Plan $=$ SRNL-SCS-2008-00003, Prep=LM, Oxide=MgO (wt \%) Variability Chart for Measured bc

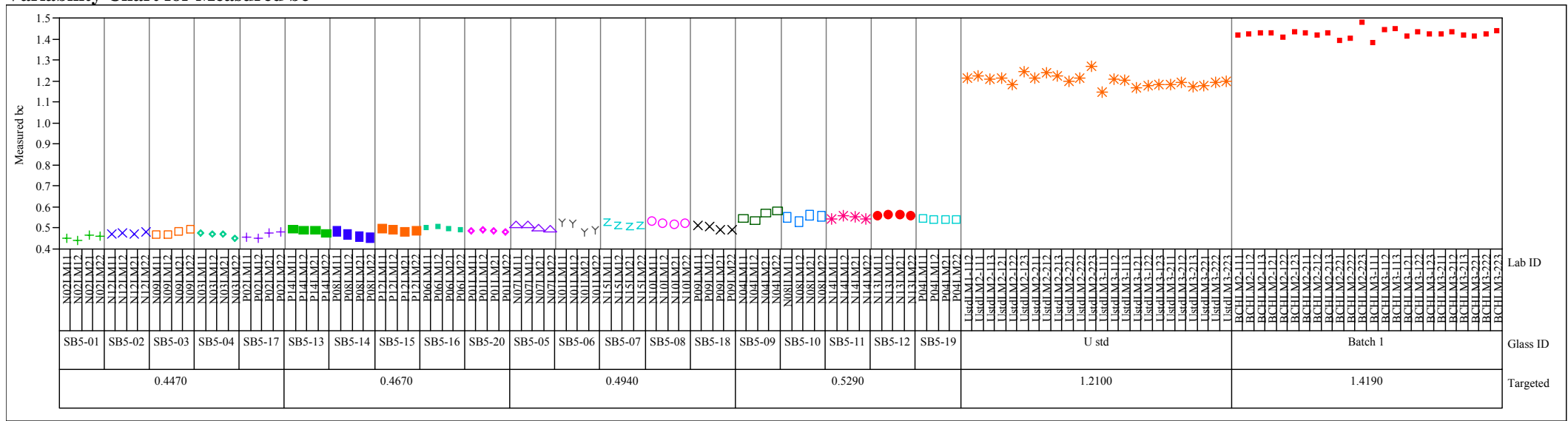


Exhibit A4. Oxide Measurements by Lab ID within Glass ID Including Auxiliary Measurements for LM Preps. (continued) Analytical Plan =SRNL-SCS-2008-00003, Prep=LM, Oxide=MnO (wt\%)

Variability Chart for Measured

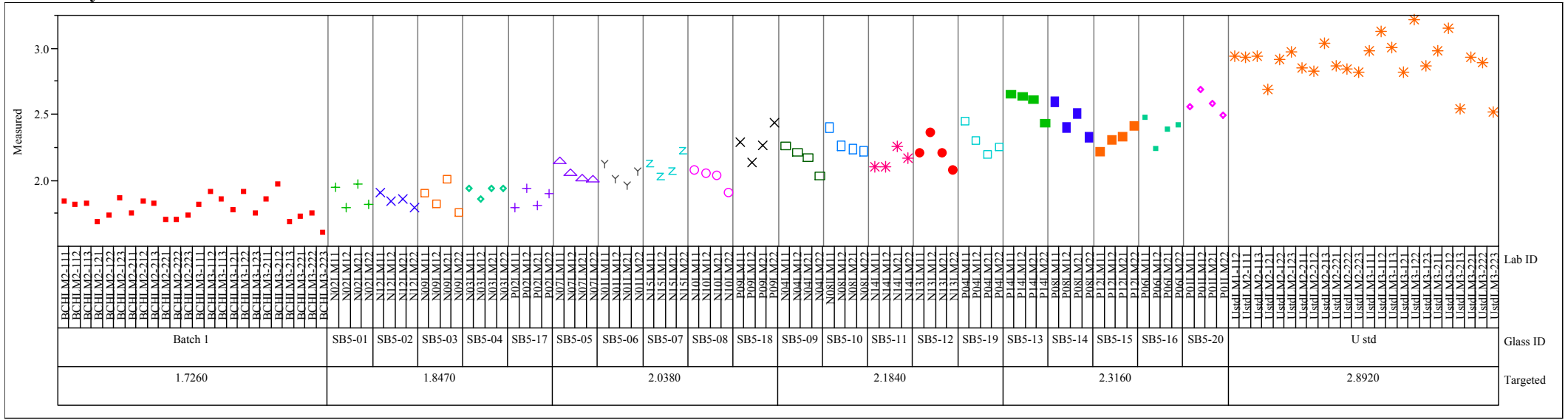

Analytical Plan =SRNL-SCS-2008-00003, Prep=LM, Oxide=MnO (wt\%)

Variability Chart for Measured bc

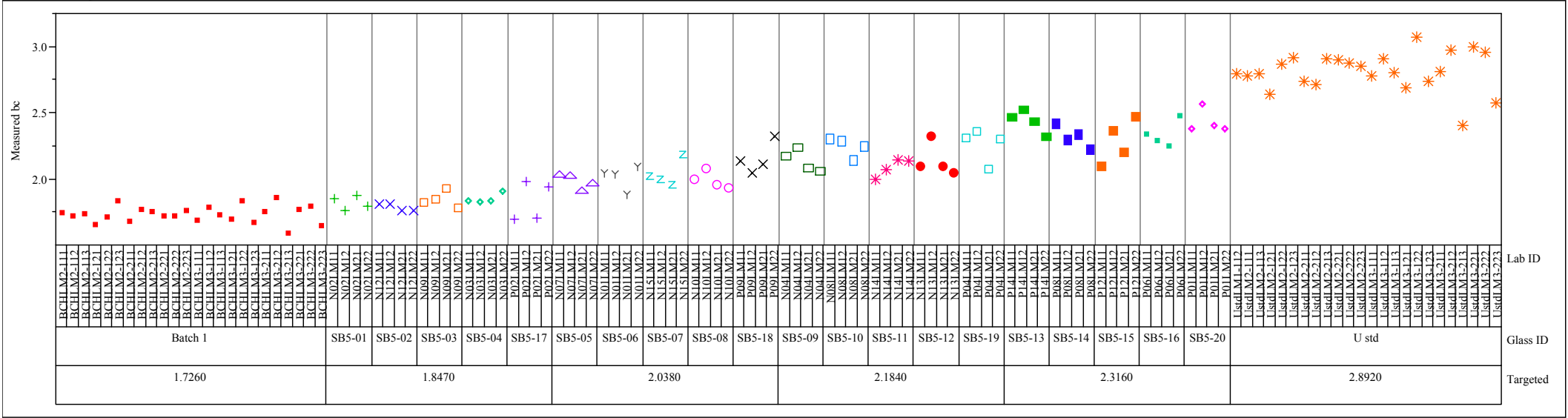


Exhibit A4. Oxide Measurements by Lab ID within Glass ID Including Auxiliary Measurements for LM Preps. (continued) Analytical Plan =SRNL-SCS-2008-00003, Prep=LM, Oxide=Na2O (wt \%)

Variability Chart for Measured

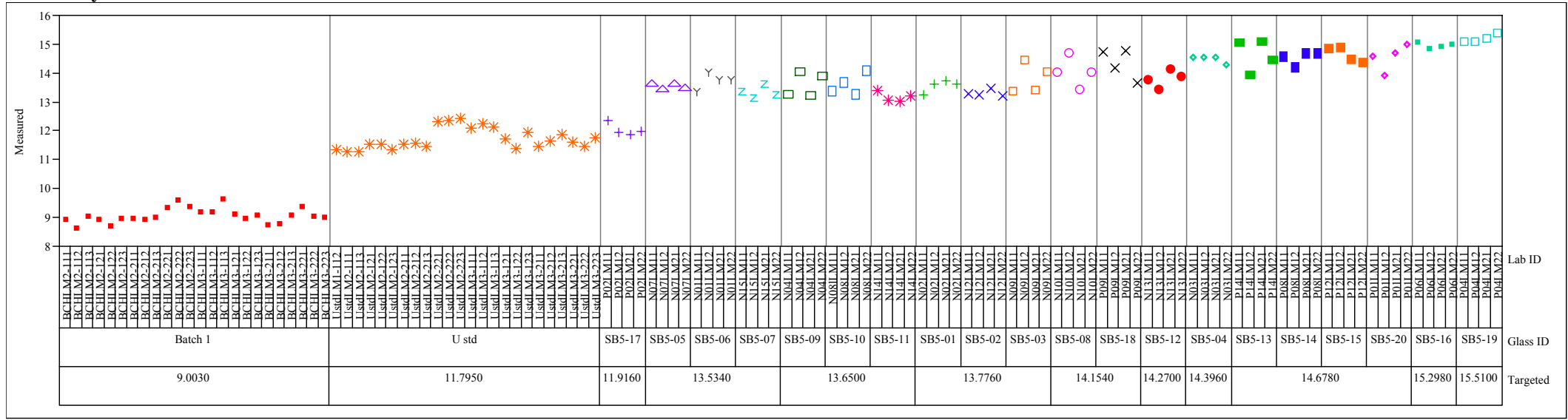

Analytical Plan $=$ SRNL-SCS-2008-00003, Prep=LM, Oxide $=$ Na2O $(w t \%)$

Variability Chart for Measured bc

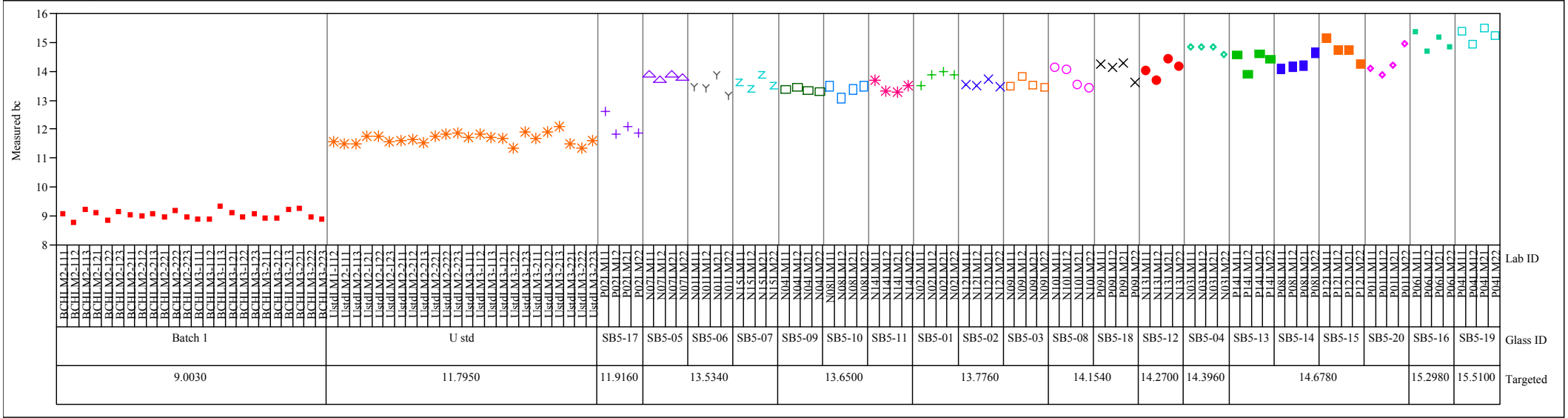


Exhibit A4. Oxide Measurements by Lab ID within Glass ID Including Auxiliary Measurements for LM Preps. (continued)

Analytical Plan =SRNL-SCS-2008-00003, Prep=LM, Oxide=Nb2O5 (wt \%)

Variability Chart for Measured

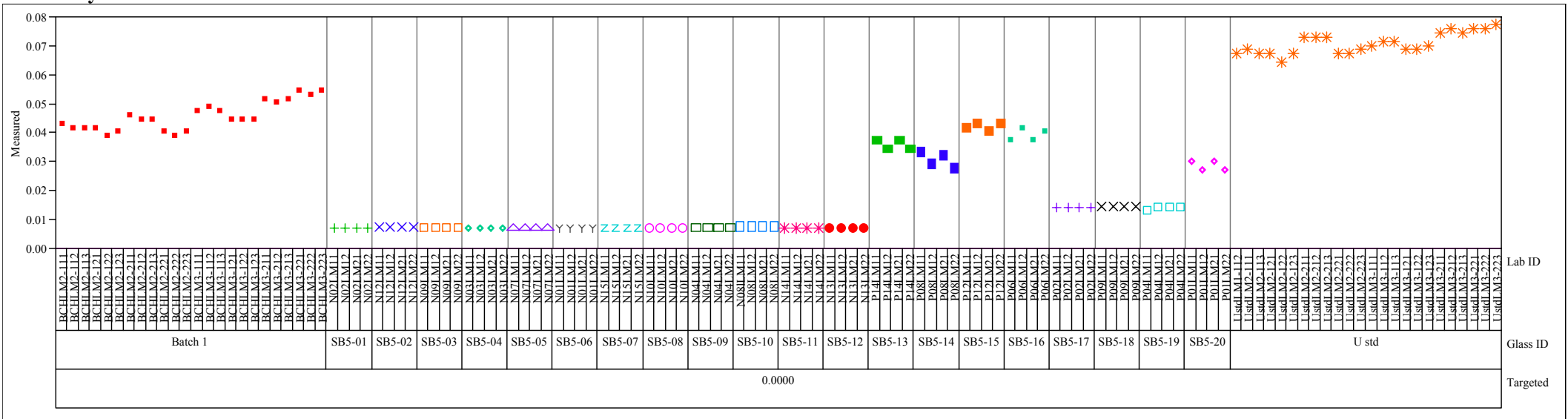

Analytical Plan =SRNL-SCS-2008-00003, Prep=LM, Oxide=Nb2O5 (wt \%)

Variability Chart for Measured bc

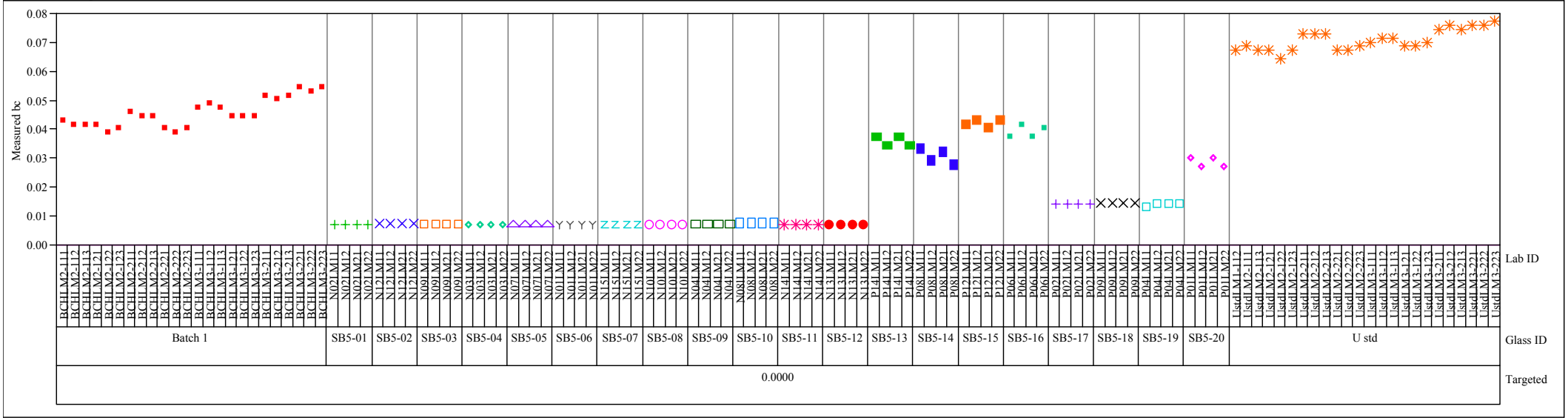


Exhibit A4. Oxide Measurements by Lab ID within Glass ID Including Auxiliary Measurements for LM Preps. (continued) Analytical Plan =SRNL-SCS-2008-00003, Prep=LM, Oxide=NiO (wt \%)

Variability Chart for Measured

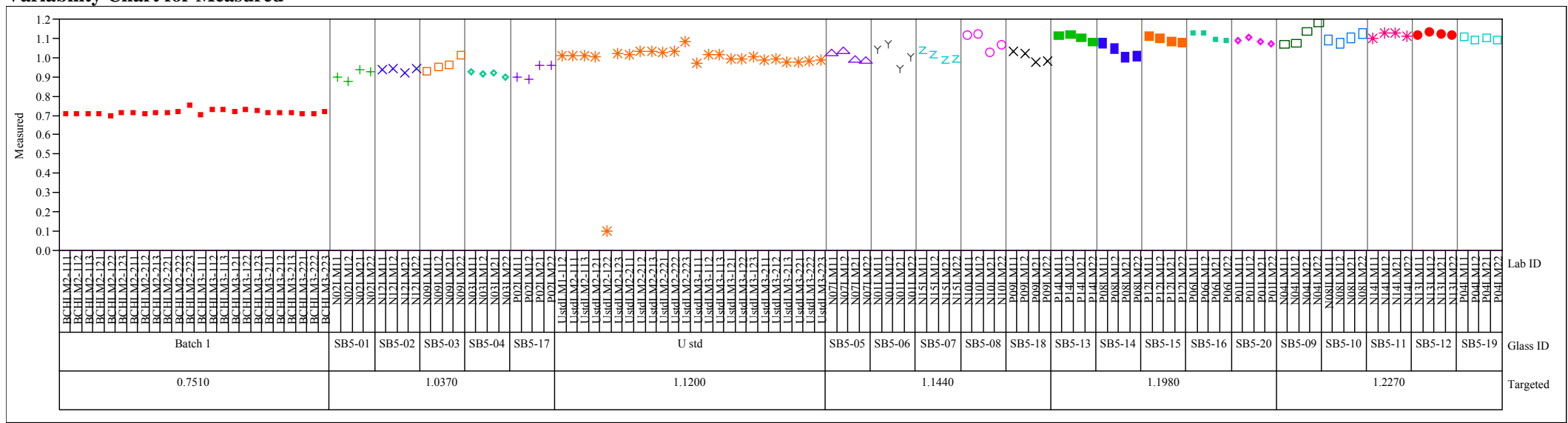

Analytical Plan $=$ SRNL-SCS-2008-00003, Prep=LM, Oxide=NiO (wt \%)

Variability Chart for Measured bc

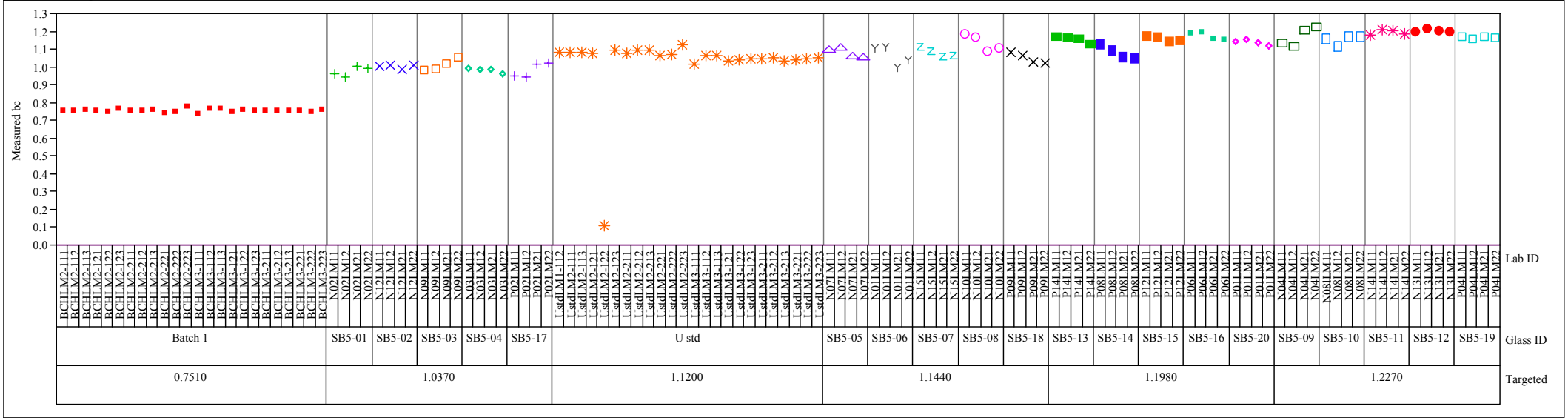


Exhibit A4. Oxide Measurements by Lab ID within Glass ID Including Auxiliary Measurements for LM Preps. (continued) Analytical Plan =SRNL-SCS-2008-00003, Prep=LM, Oxide=PbO (wt \%)

Variability Chart for Measured

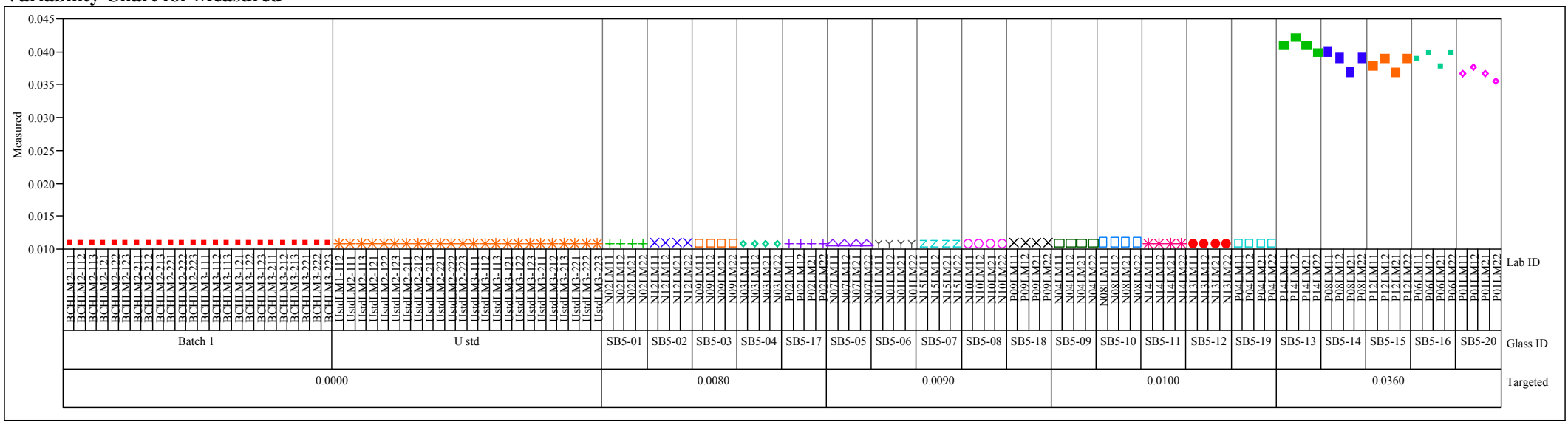

Analytical Plan =SRNL-SCS-2008-00003, Prep=LM, Oxide=PbO (wt $\%)$

Variability Chart for Measured bc

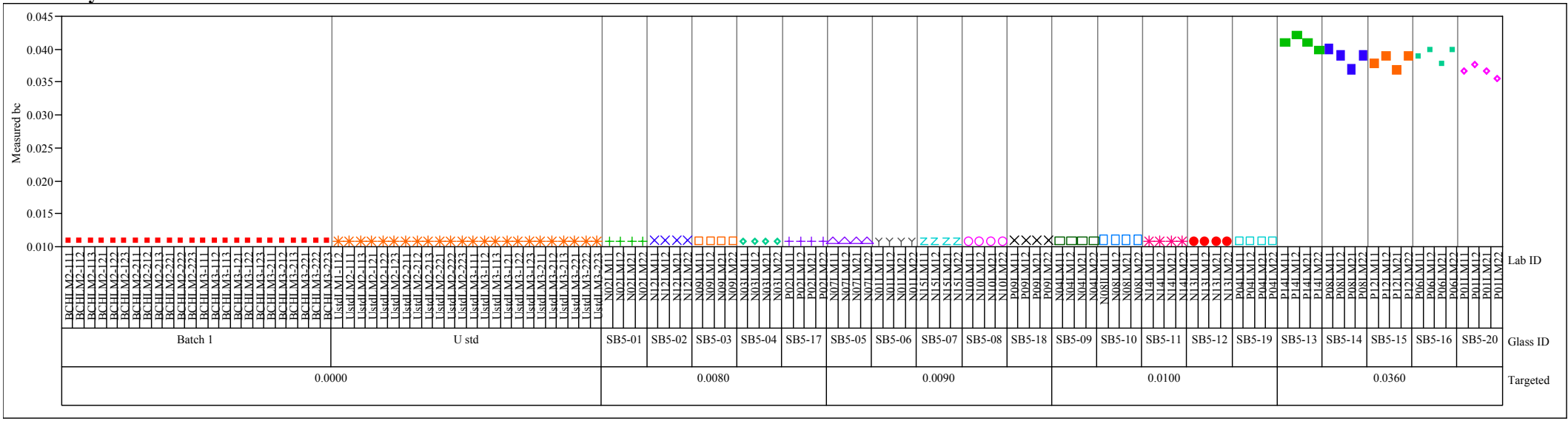


Exhibit A4. Oxide Measurements by Lab ID within Glass ID Including Auxiliary Measurements for LM Preps. (continued)

Analytical Plan =SRNL-SCS-2008-00003, Prep=LM, Oxide=SiO2 (wt \%)

Variability Chart for Measured

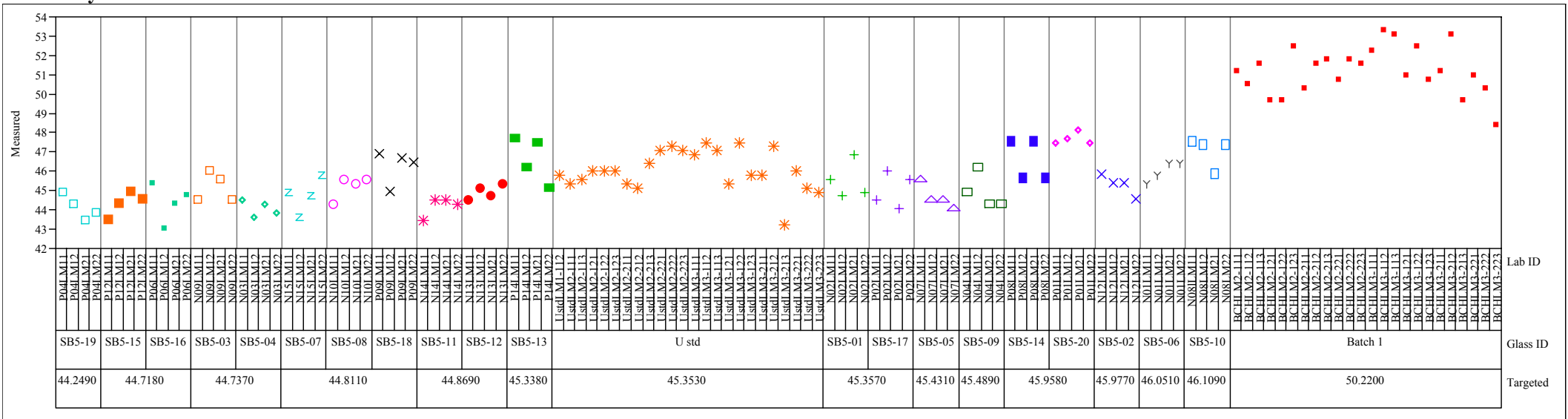

Analytical Plan =SRNL-SCS-2008-00003, Prep=LM, Oxide=SiO2 (wt \%)

Variability Chart for Measured bc

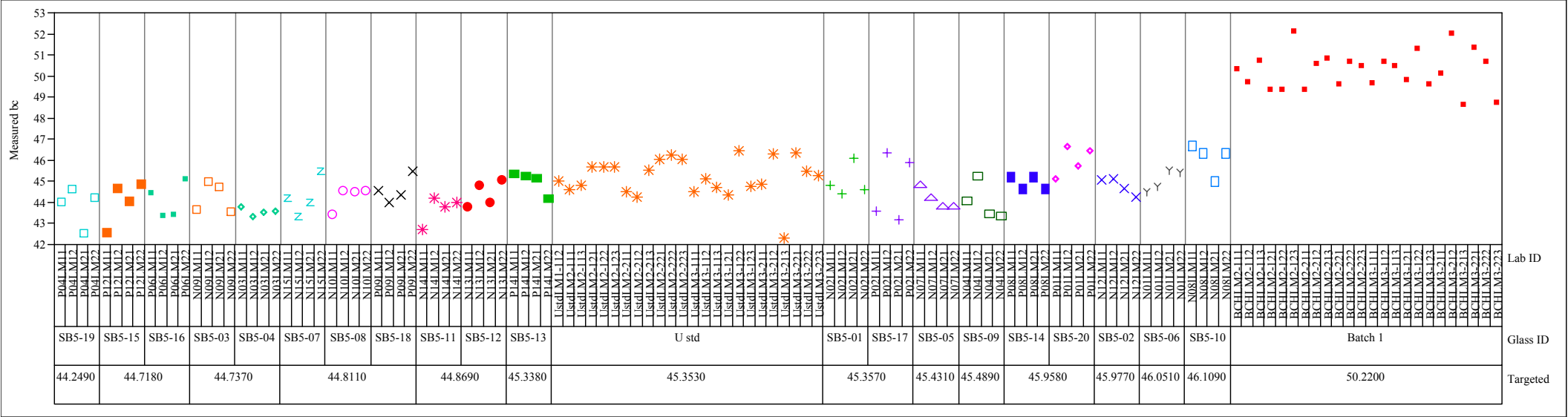


Exhibit A4. Oxide Measurements by Lab ID within Glass ID Including Auxiliary Measurements for LM Preps. (continued) Analytical Plan =SRNL-SCS-2008-00003, Prep=LM, Oxide=SO4 (wt \%)

Variability Chart for Measured

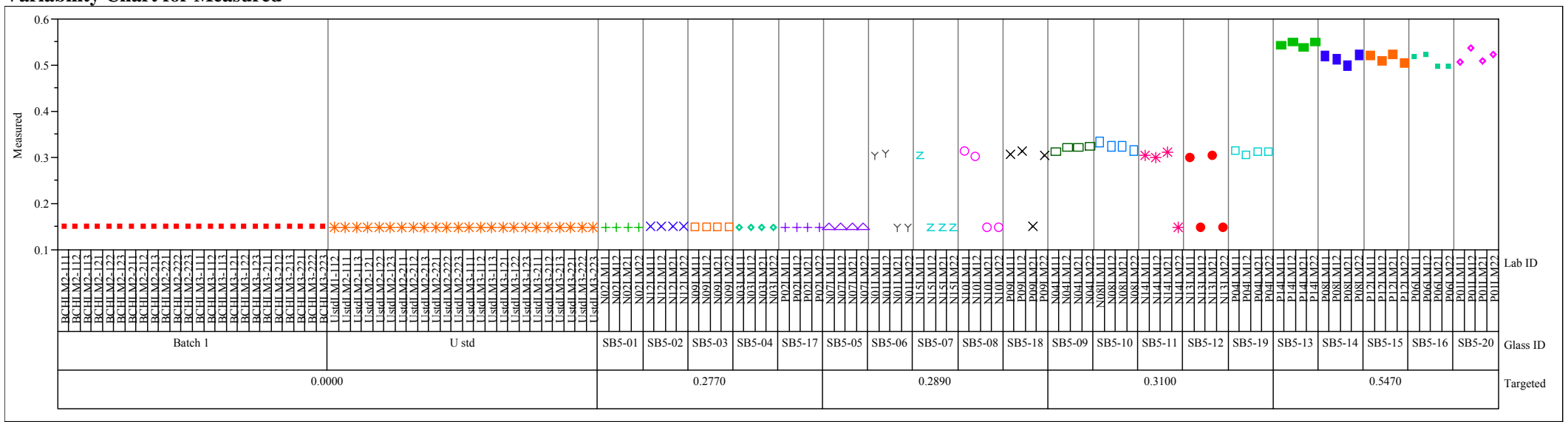

Analytical Plan =SRNL-SCS-2008-00003, Prep=LM, Oxide=SO4 (wt \%)

Variability Chart for Measured bc

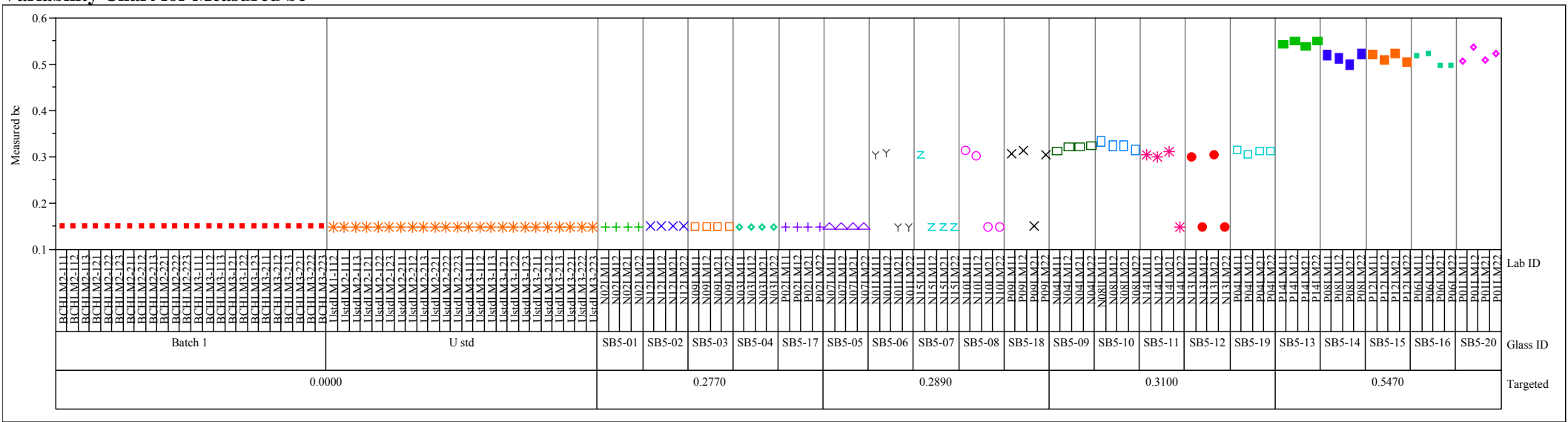


Exhibit A4. Oxide Measurements by Lab ID within Glass ID Including Auxiliary Measurements for LM Preps. (continued) Analytical Plan =SRNL-SCS-2008-00003, Prep=LM, Oxide=ThO2 (wt \%)

Variability Chart for Measured

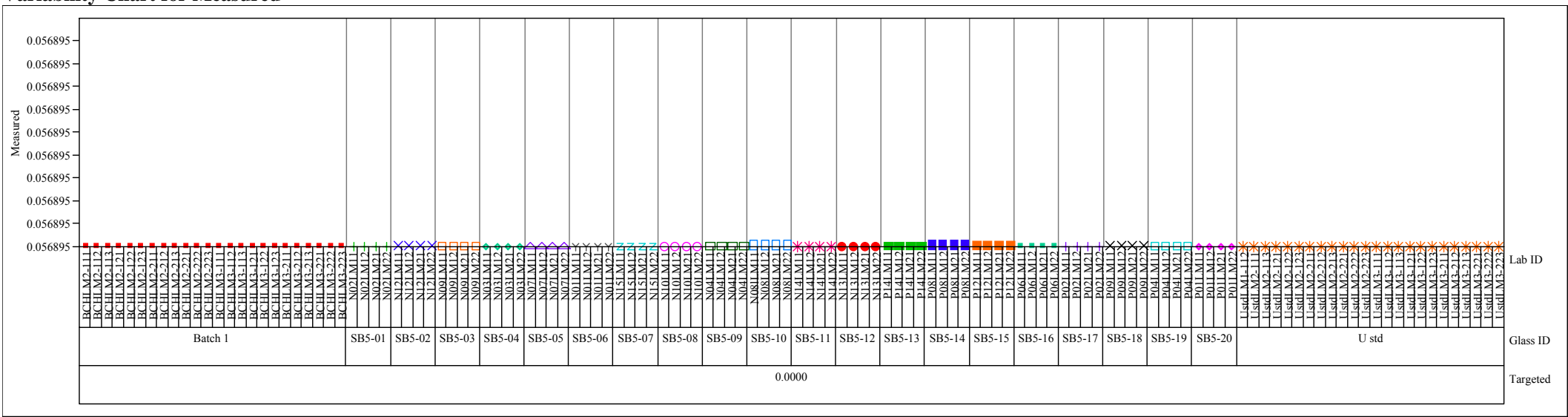

Analytical Plan =SRNL-SCS-2008-00003, Prep=LM, Oxide=ThO2 (wt \%)

Variability Chart for Measured bc

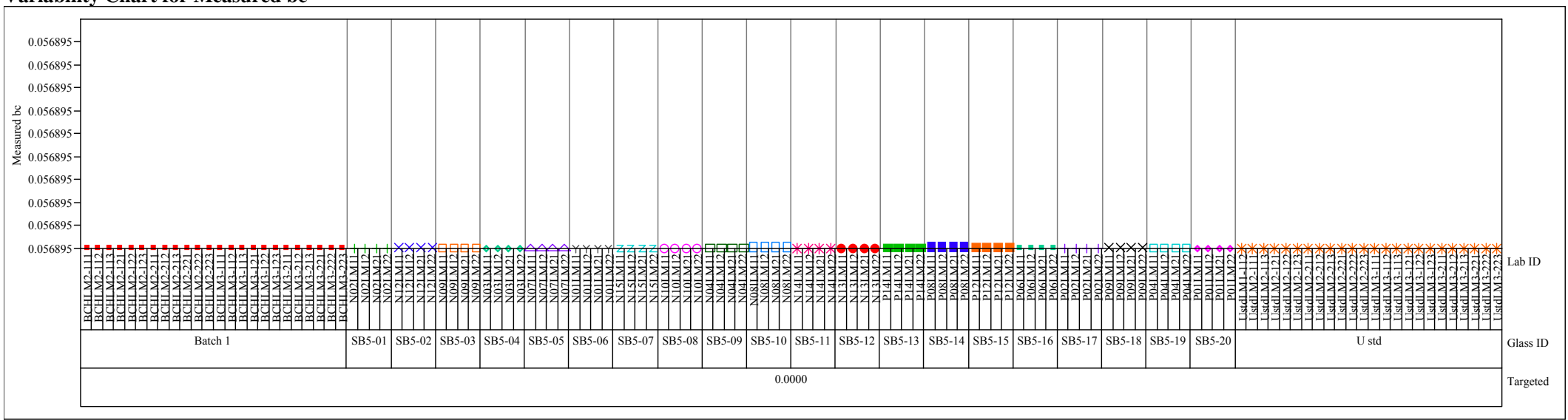


Exhibit A4. Oxide Measurements by Lab ID within Glass ID Including Auxiliary Measurements for LM Preps. (continued) Analytical Plan =SRNL-SCS-2008-00003, Prep=LM, Oxide=TiO2 (wt\%) Variability Chart for Measured

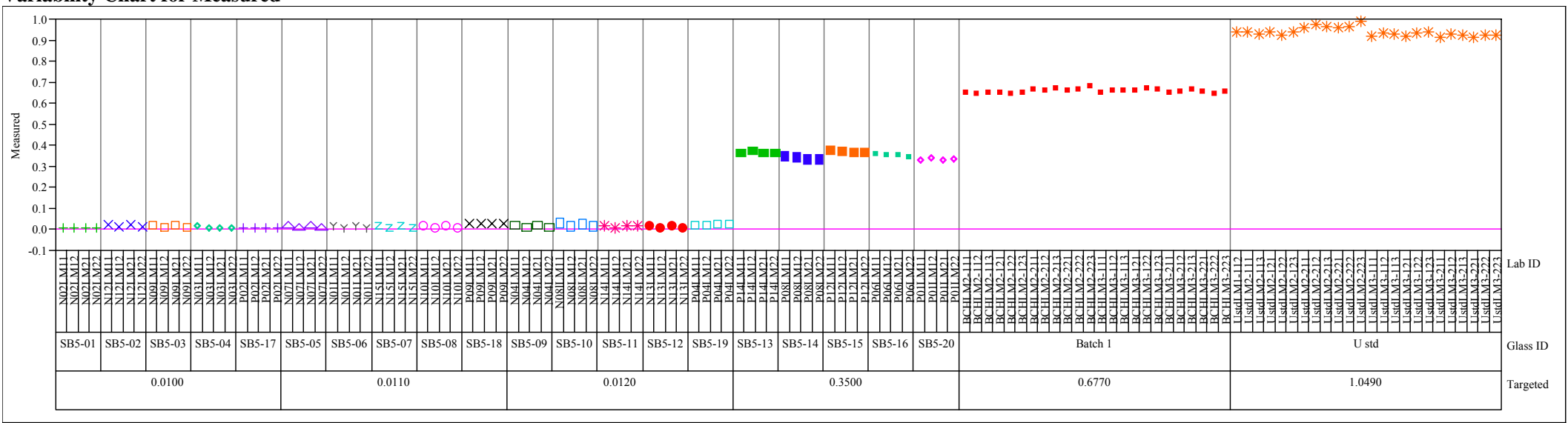

Analytical Plan =SRNL-SCS-2008-00003, Prep=LM, Oxide=TiO2 (wt \%)

Variability Chart for Measured bc

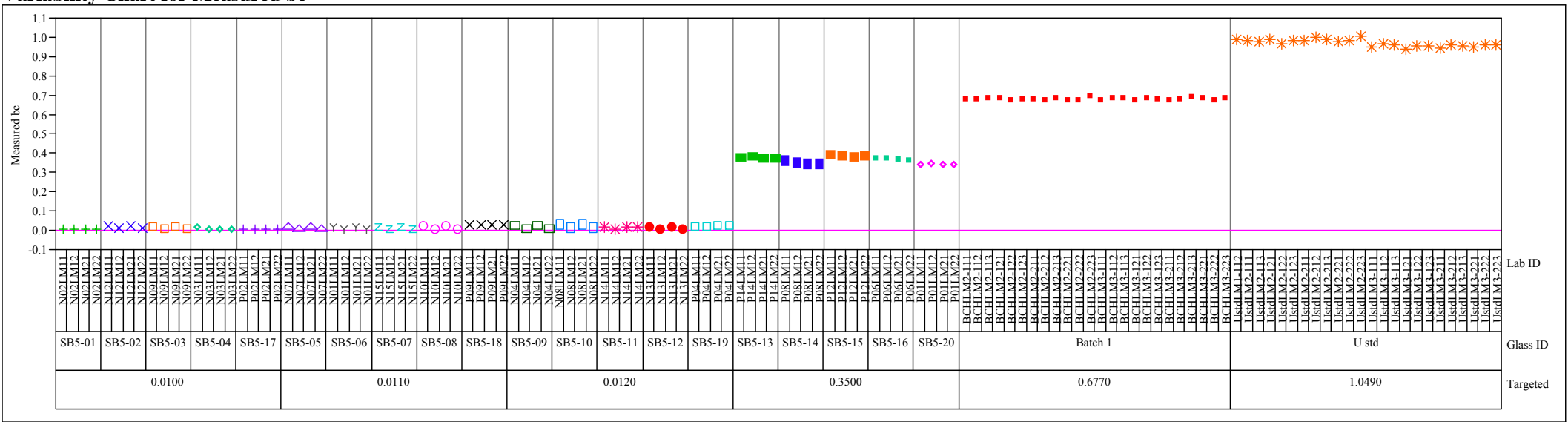


Exhibit A4. Oxide Measurements by Lab ID within Glass ID Including Auxiliary Measurements for LM Preps. (continued) Analytical Plan =SRNL-SCS-2008-00003, Prep=LM, Oxide=U3O8 (wt\%)

Variability Chart for Measured

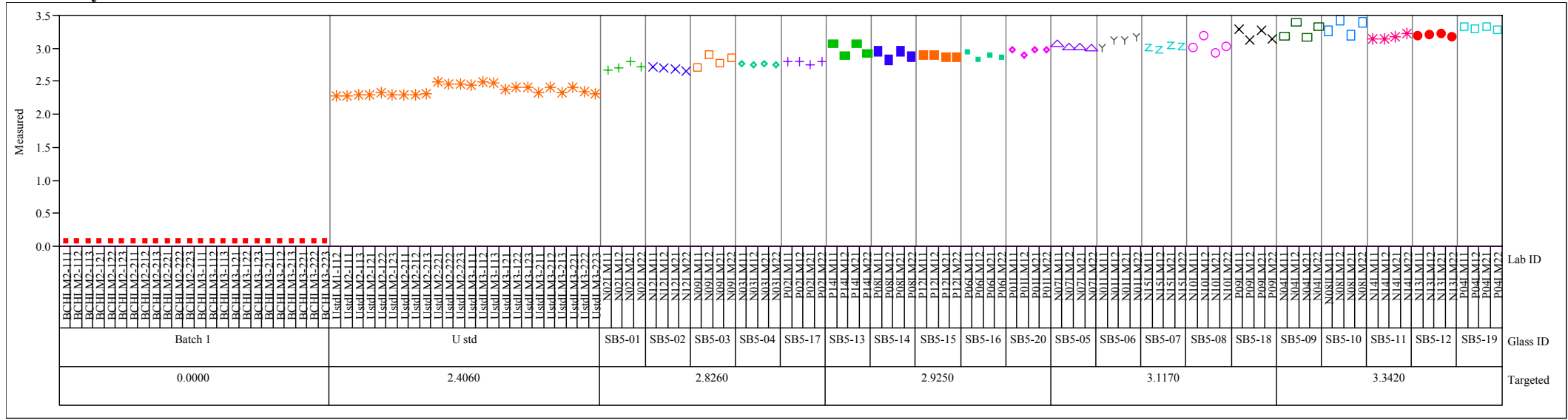

Analytical Plan =SRNL-SCS-2008-00003, Prep=LM, Oxide=U3O8 (wt \%)

Variability Chart for Measured bc

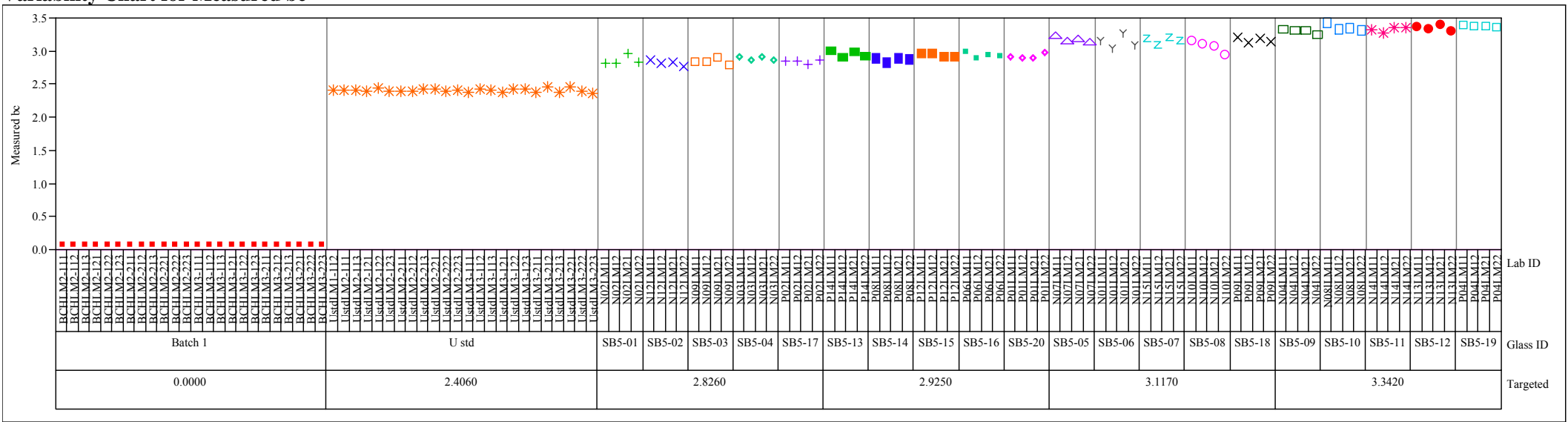


Exhibit A4. Oxide Measurements by Lab ID within Glass ID Including Auxiliary Measurements for LM Preps. (continued) Analytical Plan =SRNL-SCS-2008-00003, Prep=LM, Oxide=ZnO (wt \%

Variability Chart for Measured

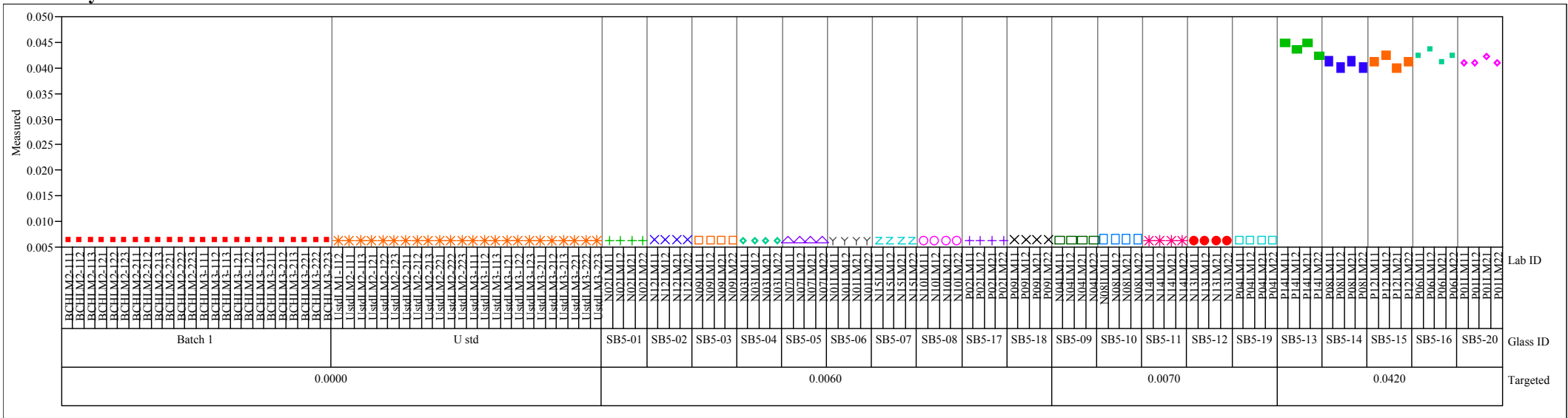

Analytical Plan =SRNL-SCS-2008-00003, Prep=LM, Oxide=ZnO (wt \%)

Variability Chart for Measured bc

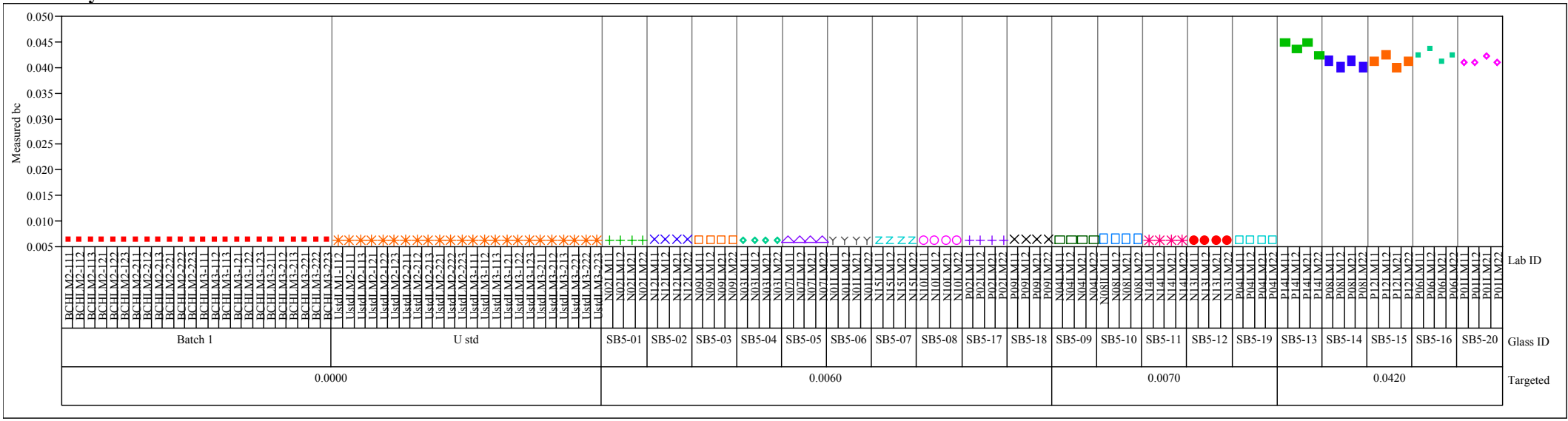


Exhibit A4. Oxide Measurements by Lab ID within Glass ID Including Auxiliary Measurements for LM Preps. (continued) Analytical Plan =SRNL-SCS-2008-00003, Prep=LM, Oxide=ZrO2 (wt \%)

Variability Chart for Measured

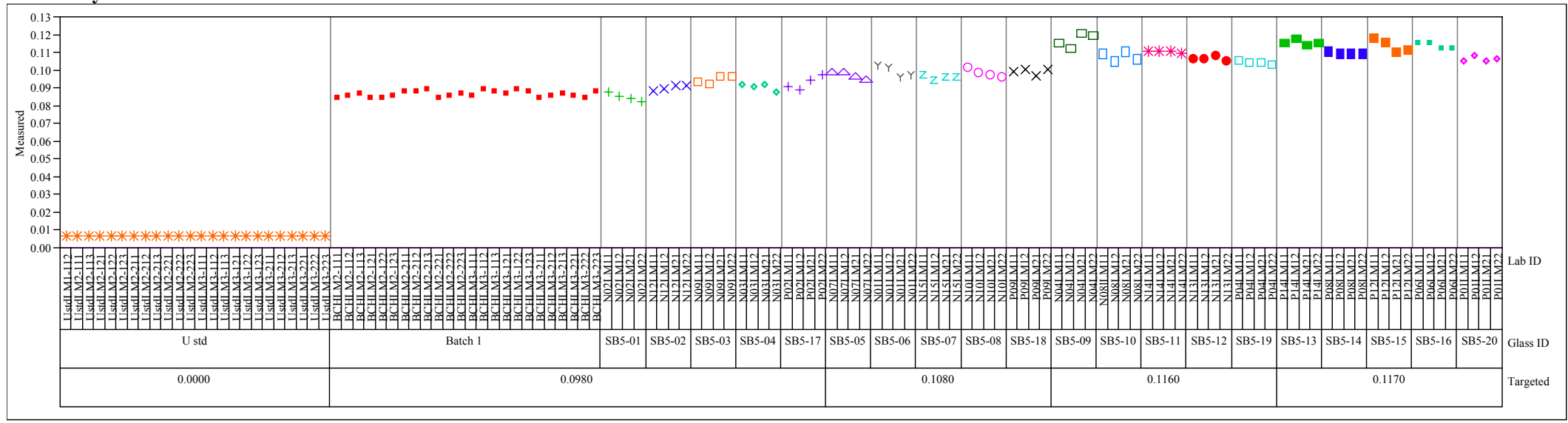

Analytical Plan =SRNL-SCS-2008-00003, Prep=LM, Oxide=ZrO2 (wt\%)

Variability Chart for Measured bc

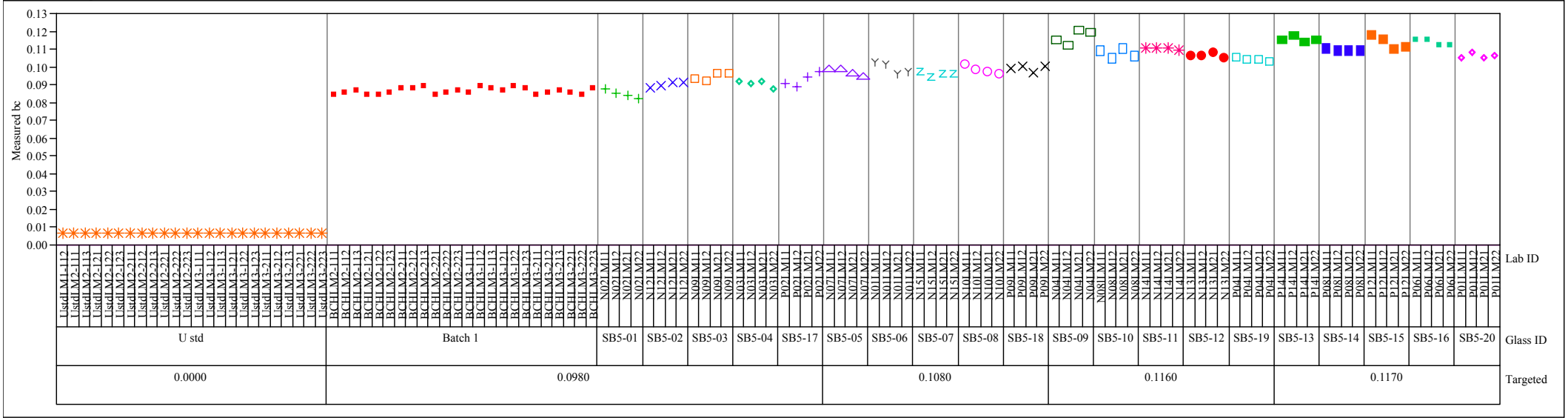


Exhibit A4. Oxide Measurements by Lab ID within Glass ID Including Auxiliary Measurements for LM Preps. (continued) Analytical Plan =SRNL-SCS-2008-00003, Prep=PF, Oxide=B2O3 (wt \%) Variability Chart for Measured

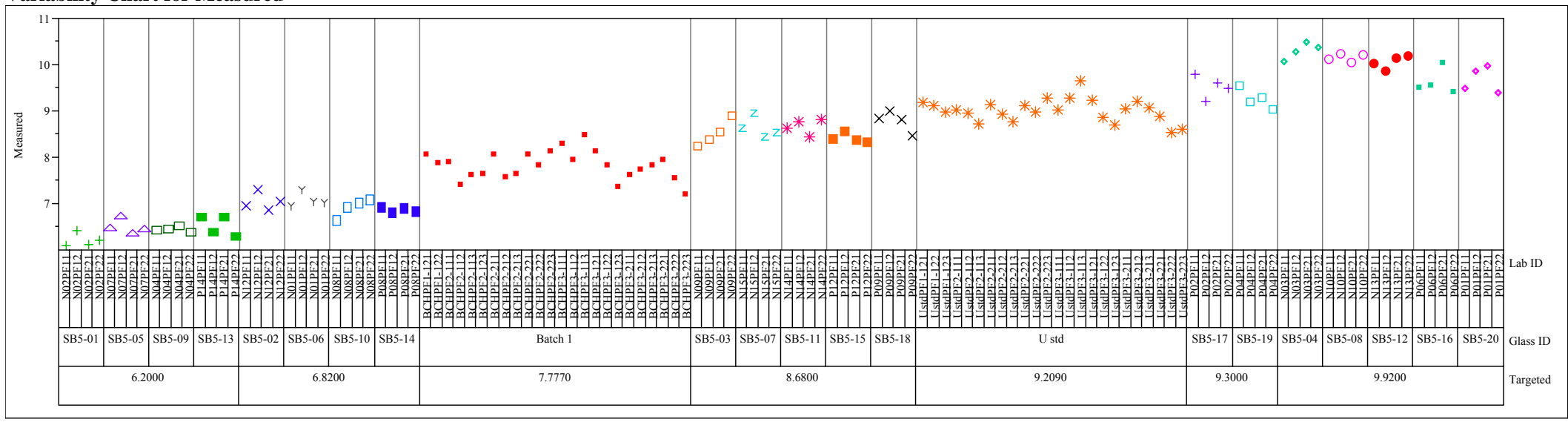

Analytical Plan =SRNL-SCS-2008-00003, Prep=PF, Oxide=B2O3 (wt \%) Variability Chart for Measured bc

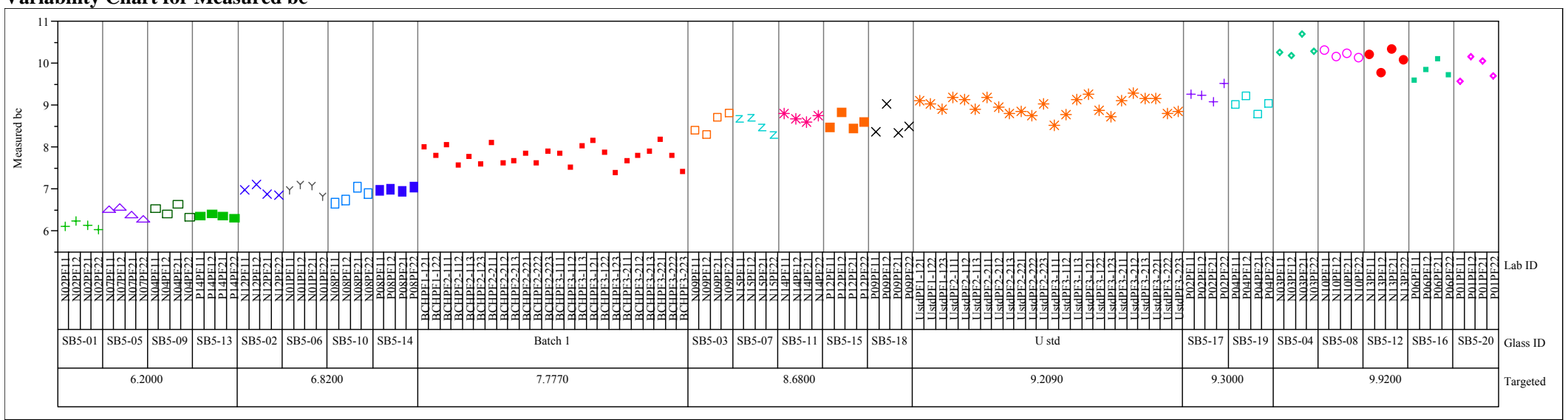


Exhibit A4. Oxide Measurements by Lab ID within Glass ID Including Auxiliary Measurements for LM Preps. (continued) Analytical Plan =SRNL-SCS-2008-00003, Prep=PF, Oxide=Li2O (wt\%) Variability Chart for Measured

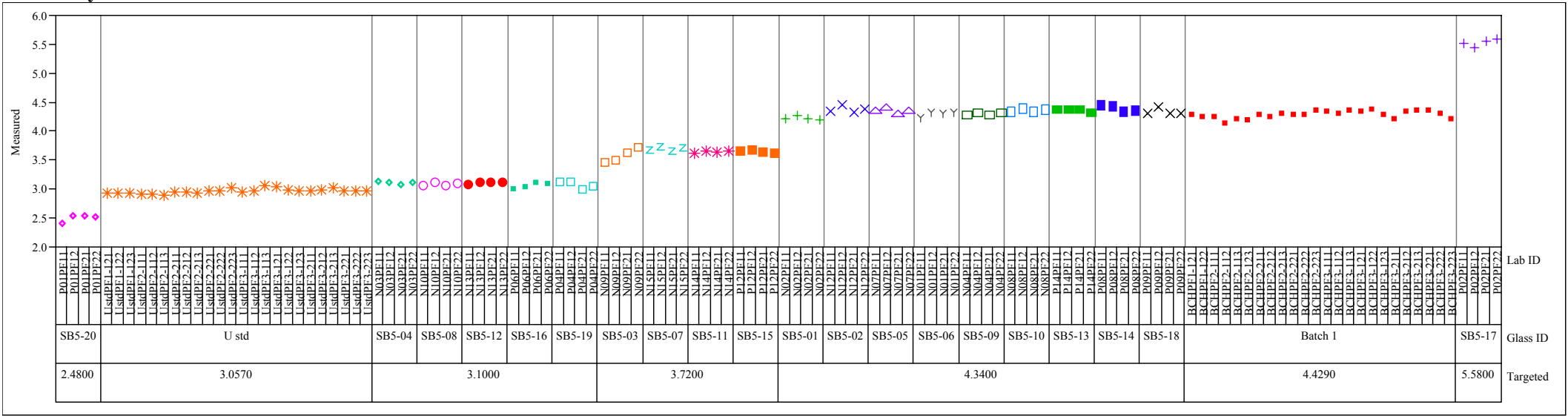

Analytical Plan =SRNL-SCS-2008-00003, Prep=PF, Oxide=Li2O (wt\%)

Variability Chart for Measured be

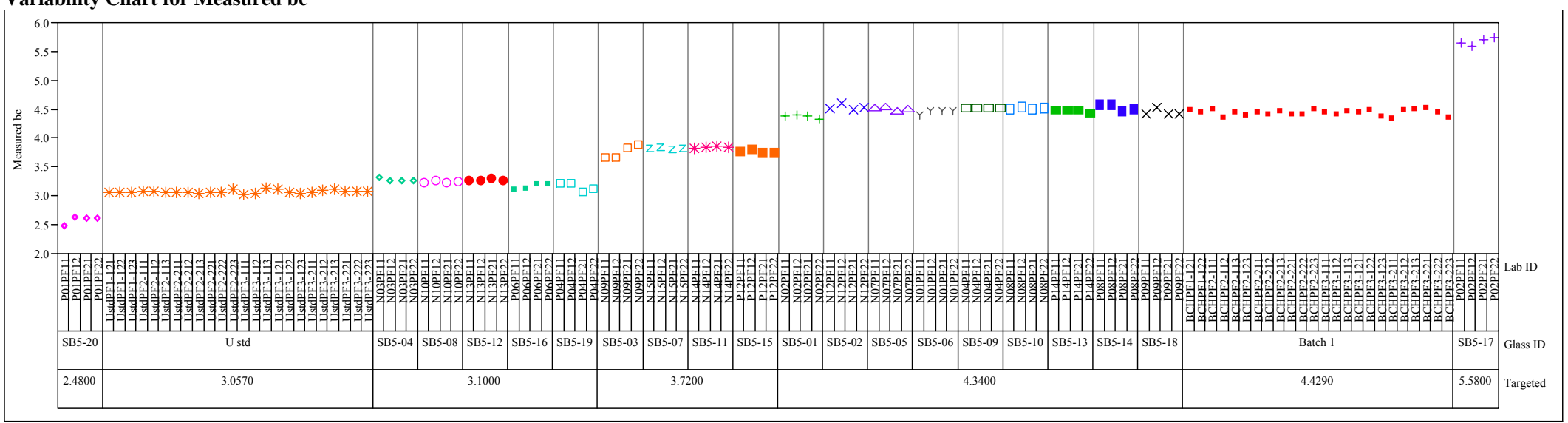


Exhibit A4. Oxide Measurements by Lab ID within Glass ID Including Auxiliary Measurements for LM Preps. (continued) Analytical Plan =SRNL-SCS-2008-00043, Prep=LM, Oxide=Al2O3 (wt \%) Variability Chart for Measured

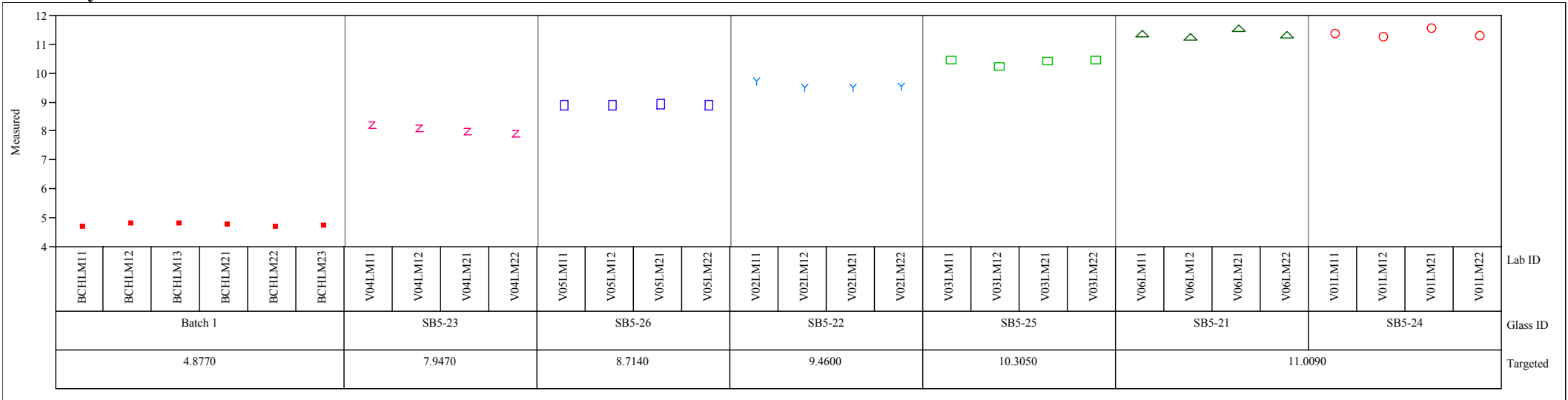

Analytical Plan =SRNL-SCS-2008-00043, Prep=LM, Oxide=Al2O3 (wt \%) Variability Chart for Measured bc

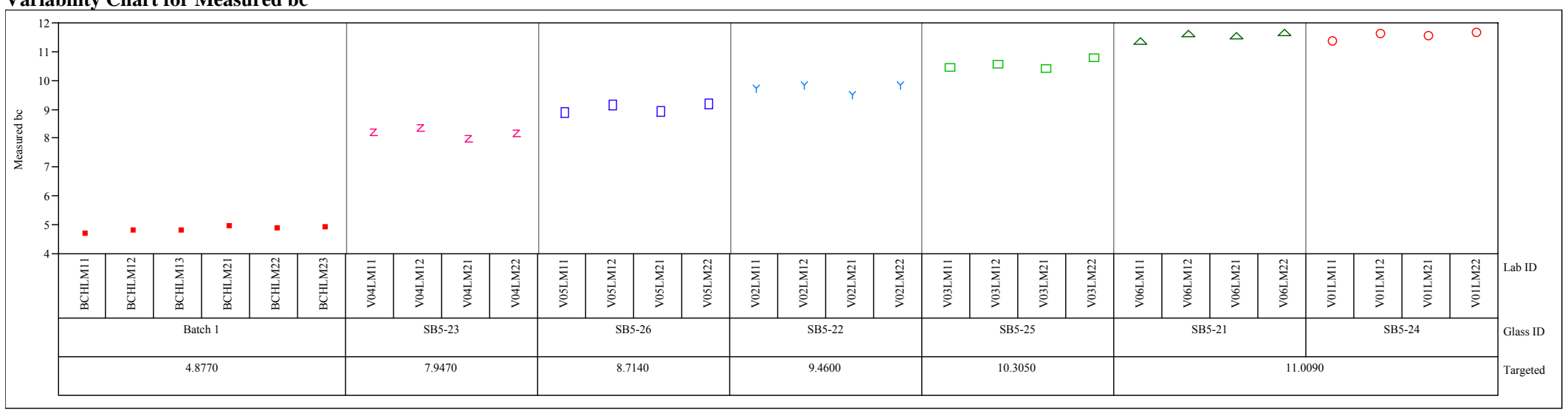


Exhibit A4. Oxide Measurements by Lab ID within Glass ID Including Auxiliary Measurements for LM Preps. (continued)

Analytical Plan =SRNL-SCS-2008-00043, Prep=LM, Oxide=BaO (wt \%)

Variability Chart for Measured

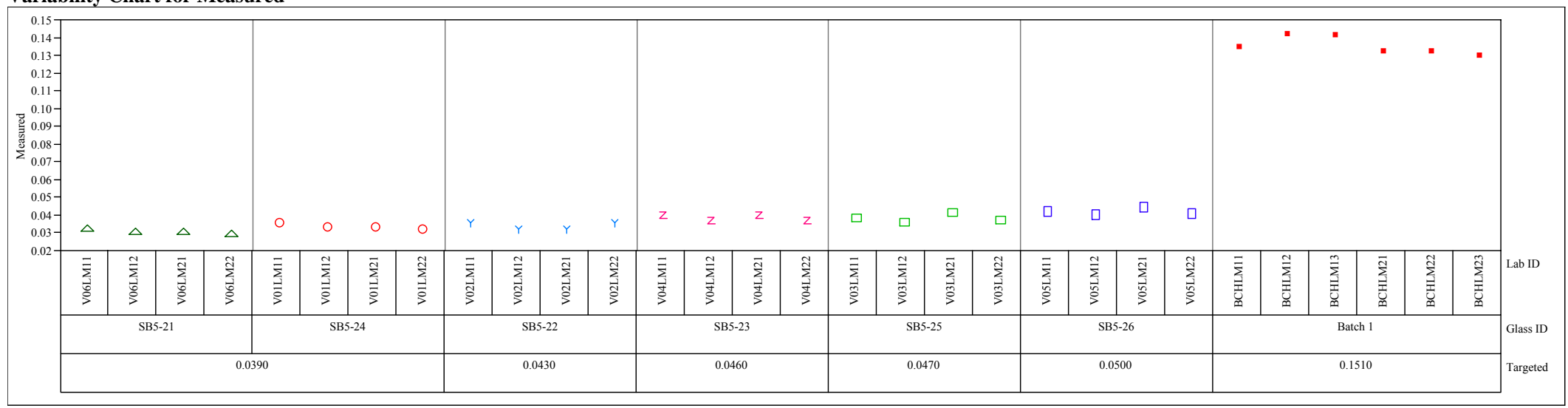

Analytical Plan $=$ SRNL-SCS-2008-00043, Prep=LM, Oxide=BaO $($ wt $\%)$

Variability Chart for Measured bc

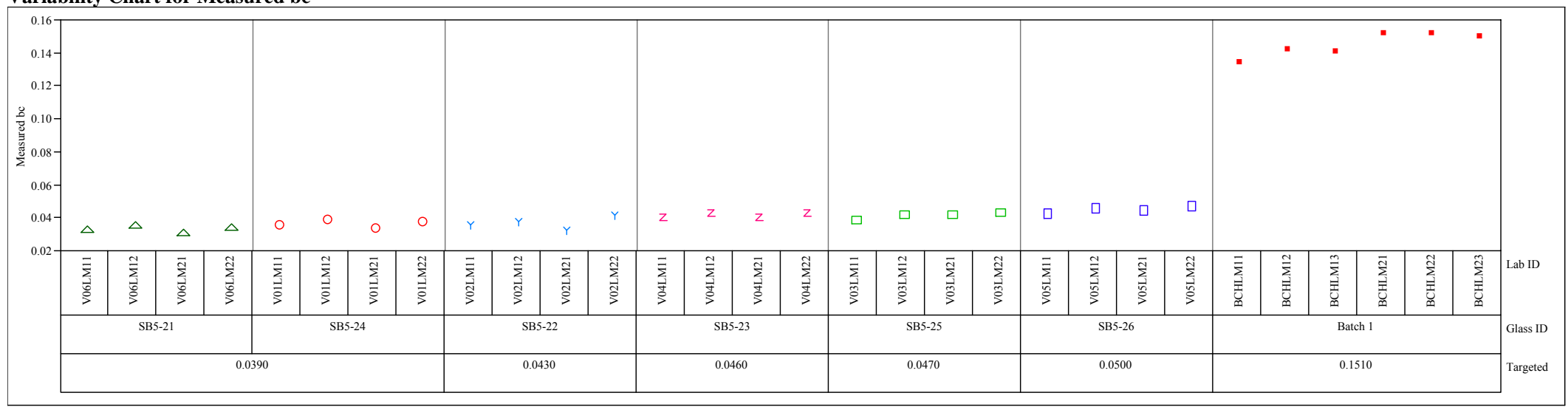


Exhibit A4. Oxide Measurements by Lab ID within Glass ID Including Auxiliary Measurements for LM Preps. (continued) Analytical Plan =SRNL-SCS-2008-00043, Prep=LM, Oxide=CaO (wt \%) Variability Chart for Measured

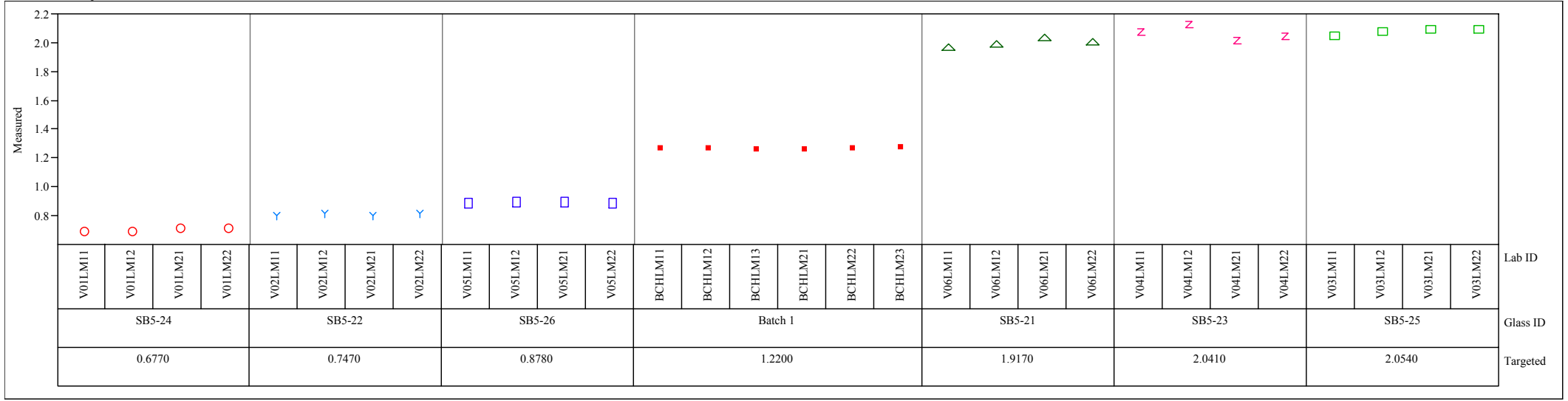

Analytical Plan $=$ SRNL-SCS-2008-00043, Prep=LM, Oxide=CaO $($ wt $\%)$

Variability Chart for Measured bc

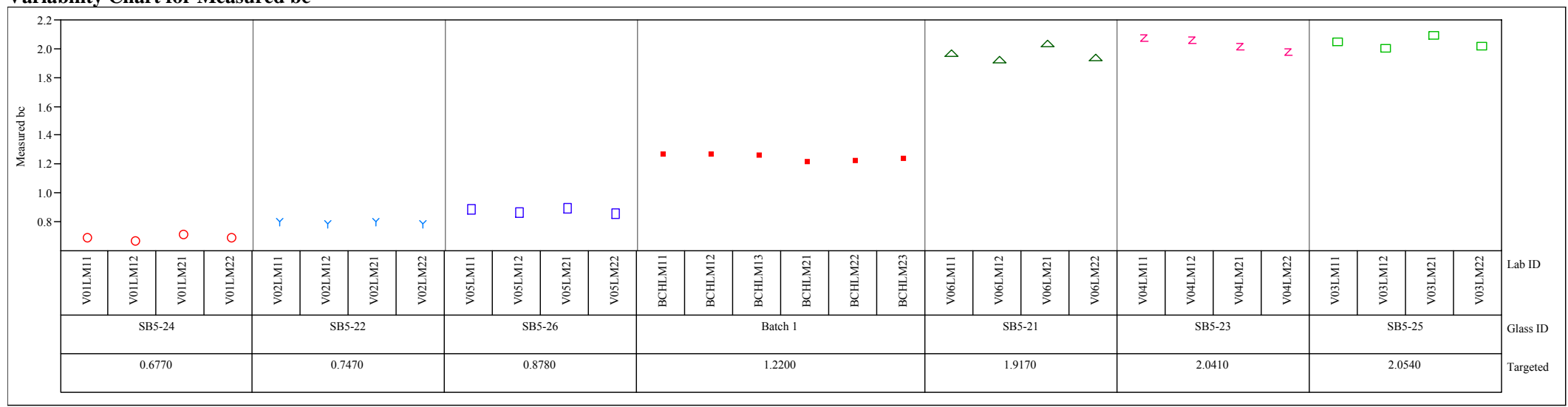


Exhibit A4. Oxide Measurements by Lab ID within Glass ID Including Auxiliary Measurements for LM Preps. (continued) Analytical Plan $=$ SRNL-SCS-2008-00043, Prep=LM, Oxide=Ce2O3 (wt $\%)$ Variability Chart for Measured

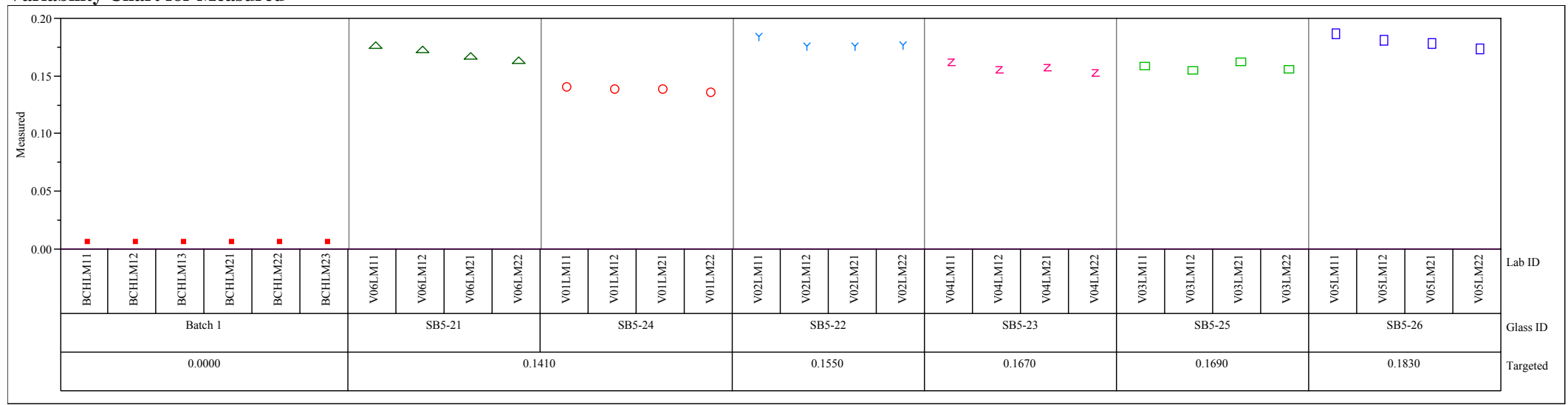

Analytical Plan =SRNL-SCS-2008-00043, Prep=LM, Oxide=Ce2O3 (wt\%)

Variability Chart for Measured bc

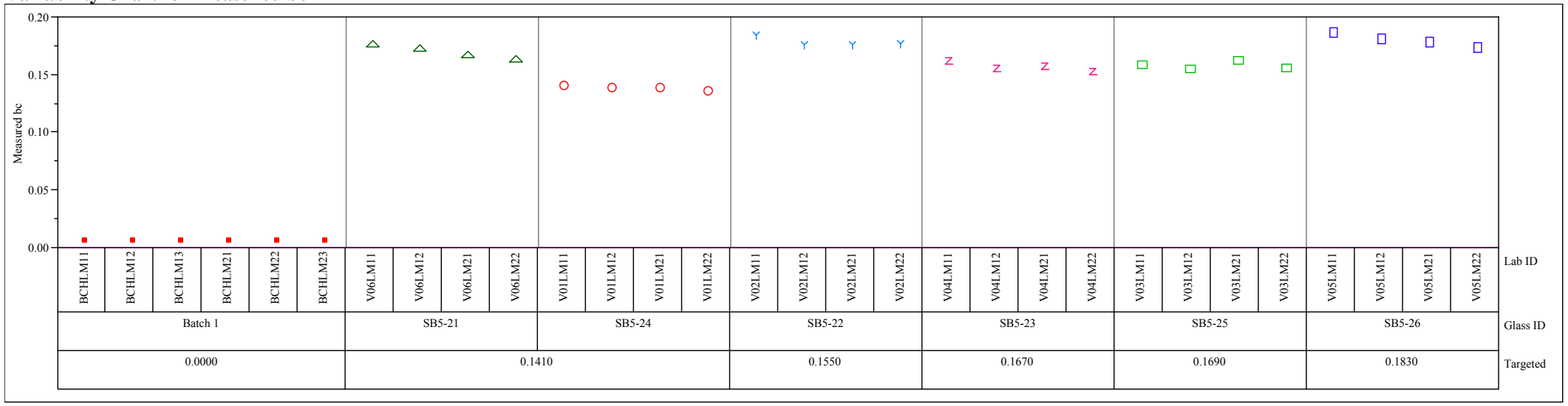


Exhibit A4. Oxide Measurements by Lab ID within Glass ID Including Auxiliary Measurements for LM Preps. (continued) Analytical Plan =SRNL-SCS-2008-00043, Prep=LM, Oxide=Cr2O3 (wt \%) Variability Chart for Measured

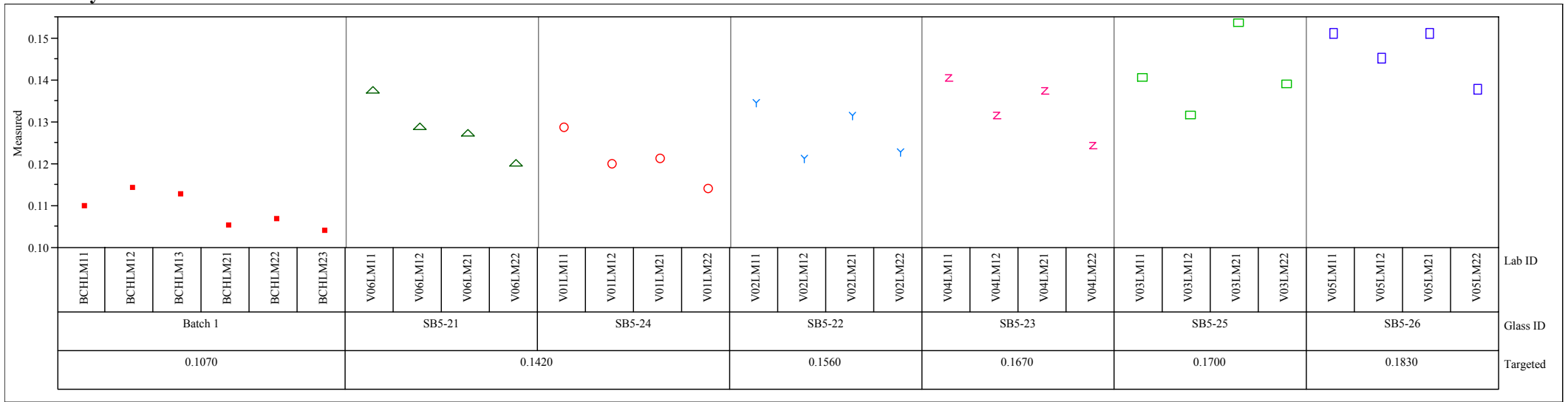

Analytical Plan =SRNL-SCS-2008-00043, Prep=LM, Oxide=Cr2O3 (wt \%) Variability Chart for Measured bc

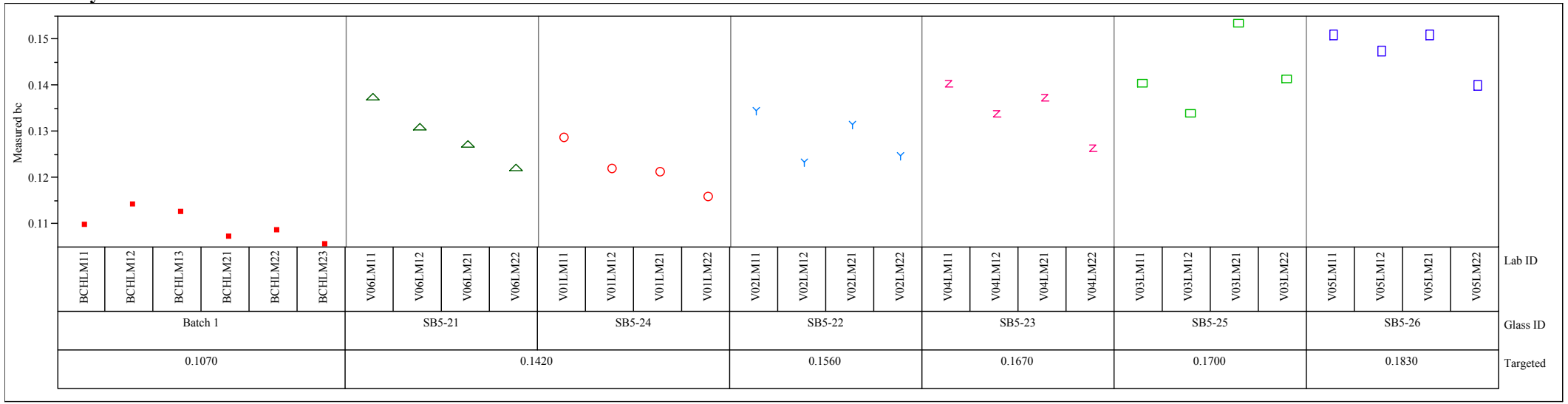


Exhibit A4. Oxide Measurements by Lab ID within Glass ID Including Auxiliary Measurements for LM Preps. (continued) Analytical Plan =SRNL-SCS-2008-00043, Prep=LM, Oxide=Fe2O3 (wt \%) Variability Chart for Measured

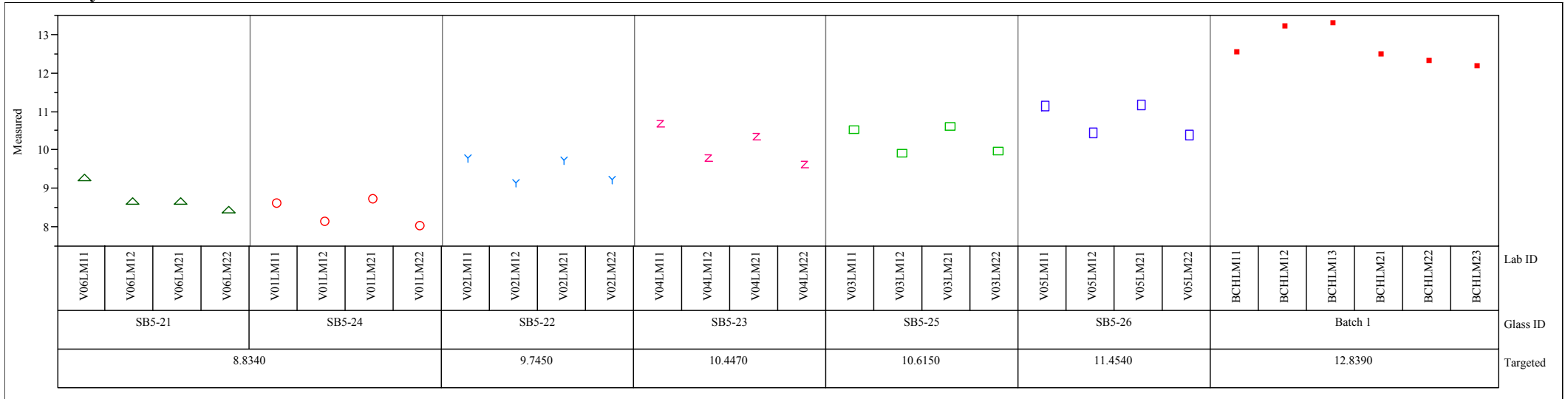

Analytical Plan =SRNL-SCS-2008-00043, Prep=LM, Oxide=Fe2O3 (wt \%) Variability Chart for Measured bc

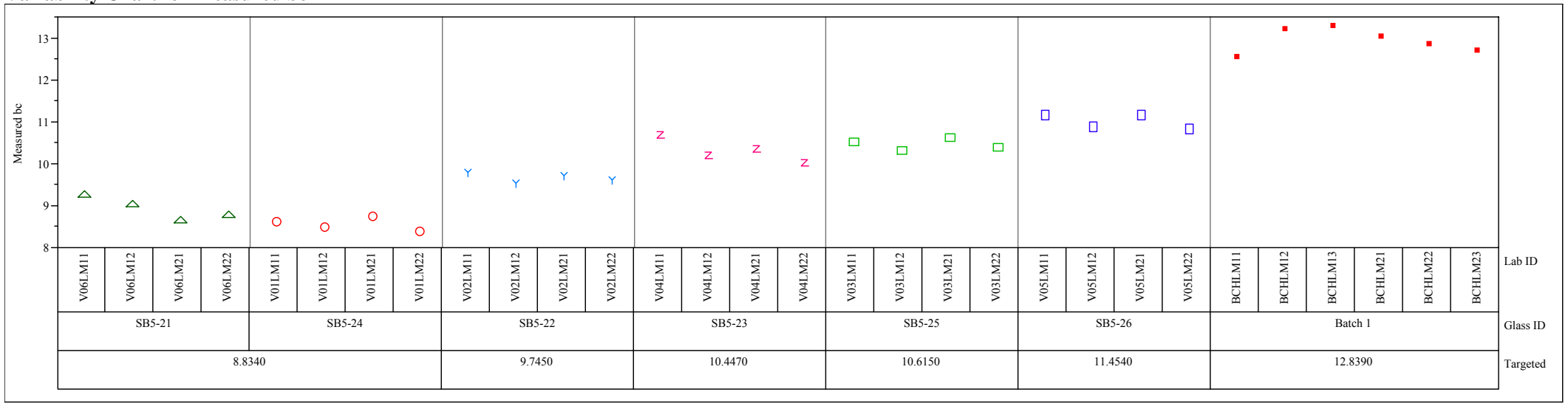


Exhibit A4. Oxide Measurements by Lab ID within Glass ID Including Auxiliary Measurements for LM Preps. (continued) Analytical Plan =SRNL-SCS-2008-00043, Prep=LM, Oxide=HfO2 (wt $\%$ )

Variability Chart for Measured

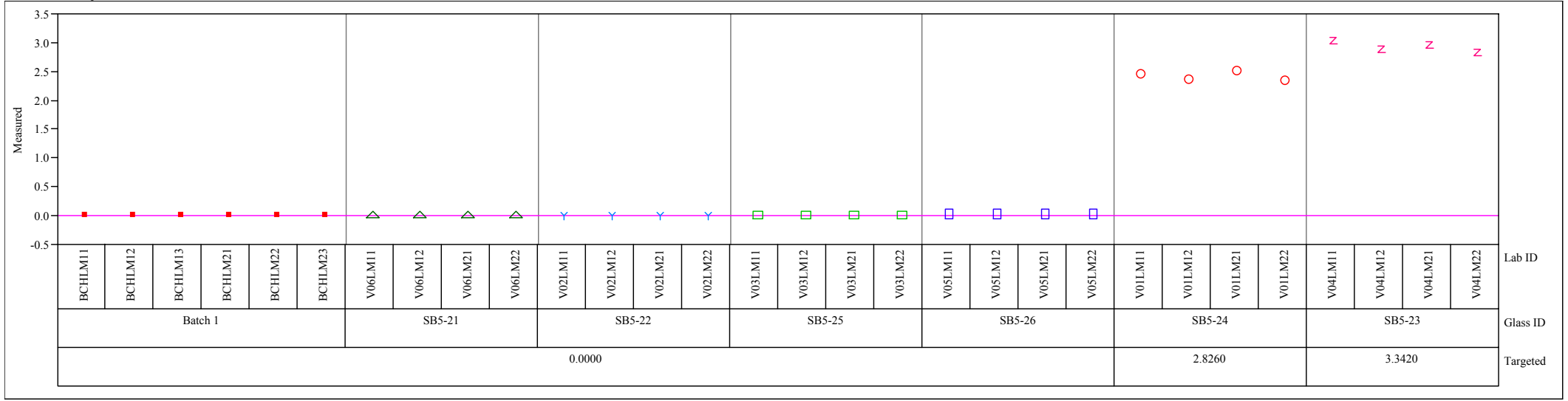

Analytical Plan =SRNL-SCS-2008-00043, Prep=LM, Oxide=HfO2 (wt\%)

Variability Chart for Measured bc

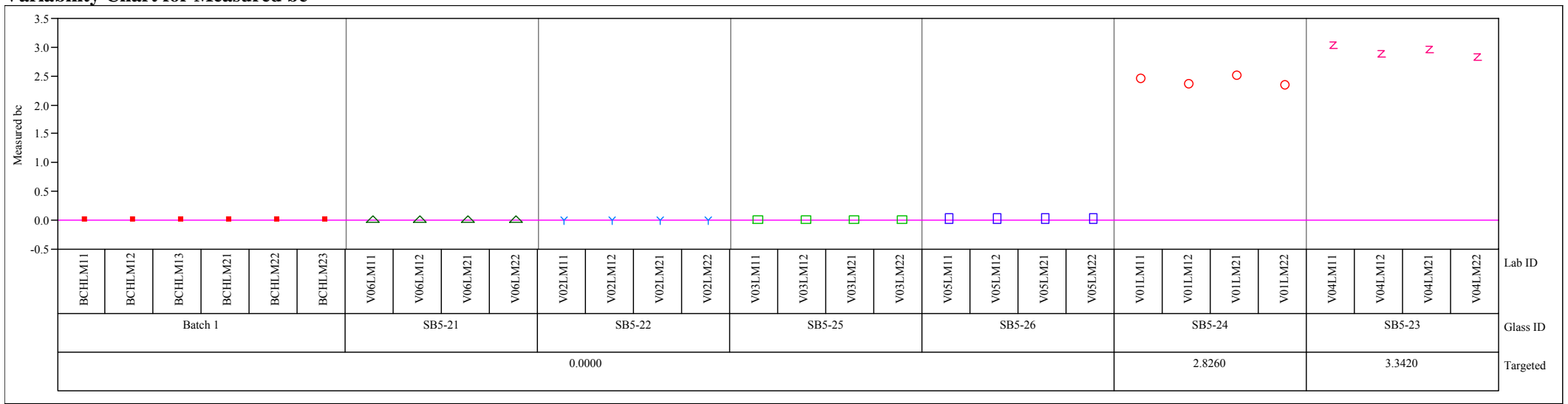


Exhibit A4. Oxide Measurements by Lab ID within Glass ID Including Auxiliary Measurements for LM Preps. (continued) Analytical Plan =SRNL-SCS-2008-00043, Prep=LM, Oxide=K2O (wt \%) Variability Chart for Measured

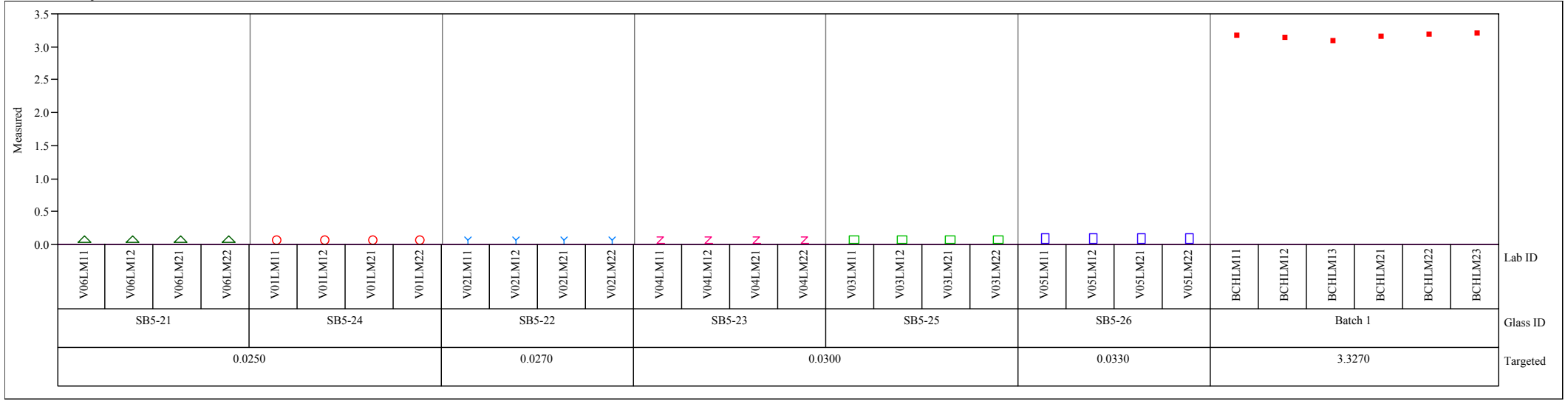

Analytical Plan =SRNL-SCS-2008-00043, Prep=LM, Oxide=K2O (wt \%)

Variability Chart for Measured bc

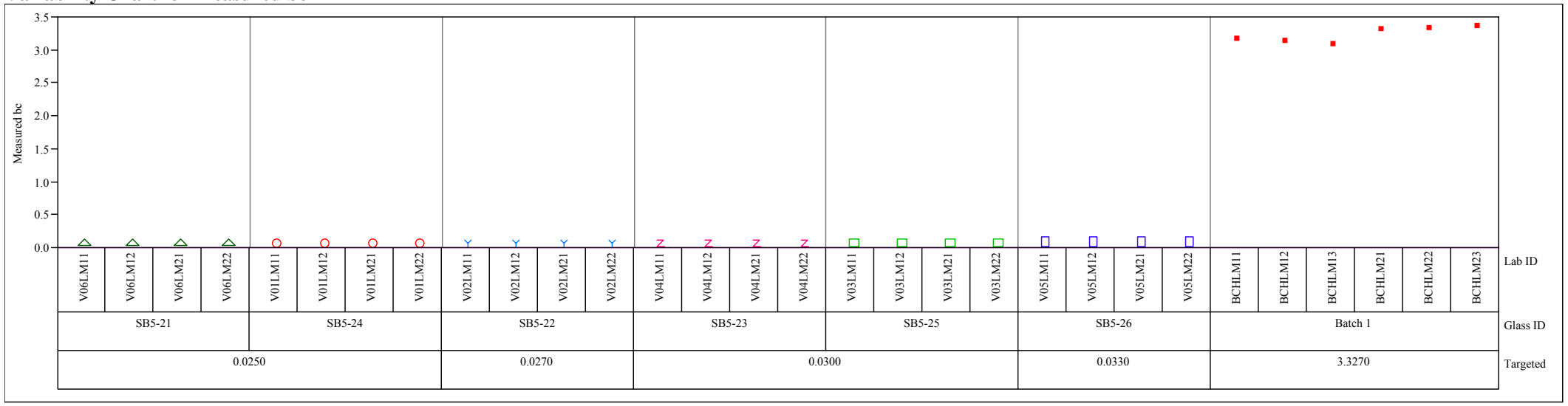


Exhibit A4. Oxide Measurements by Lab ID within Glass ID Including Auxiliary Measurements for LM Preps. (continued)

Analytical Plan =SRNL-SCS-2008-00043, Prep=LM, Oxide=La2O3 (wt \%)

Variability Chart for Measured

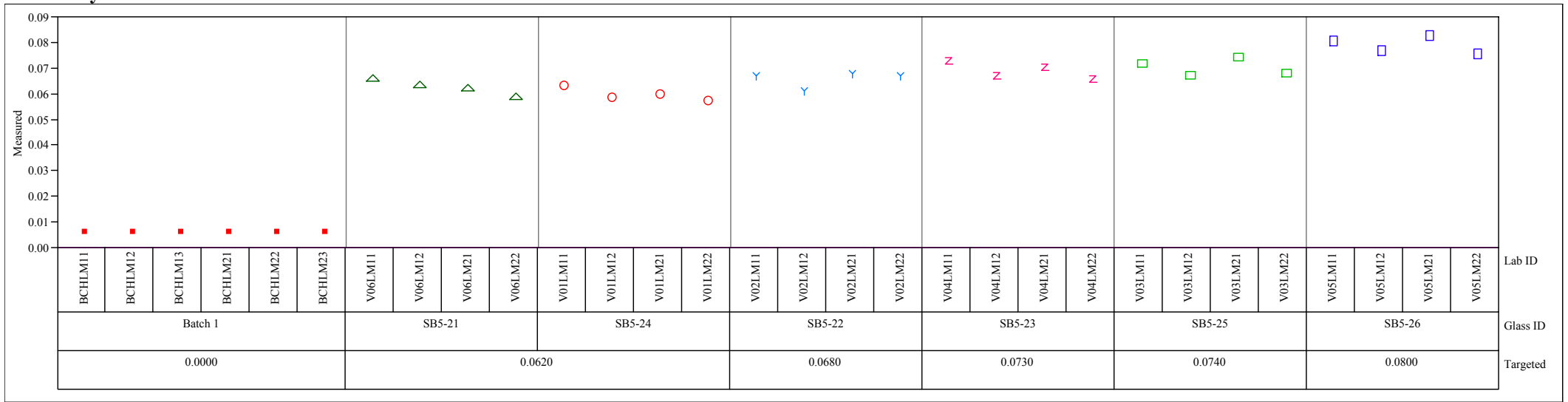

Analytical Plan =SRNL-SCS-2008-00043, Prep=LM, Oxide=La2O3 (wt\%)

Variability Chart for Measured bc

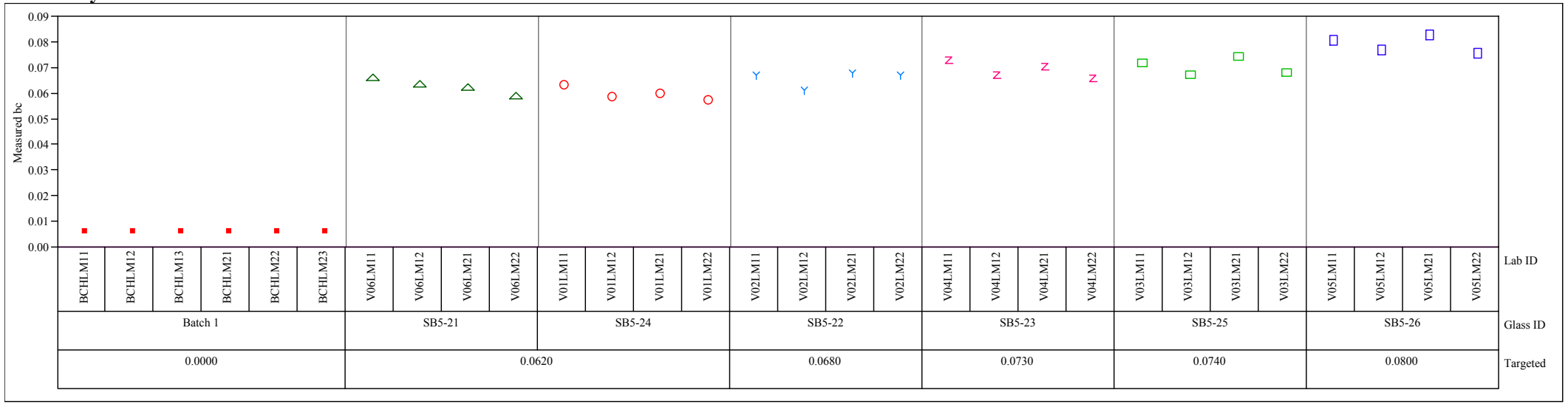


Exhibit A4. Oxide Measurements by Lab ID within Glass ID Including Auxiliary Measurements for LM Preps. (continued) Analytical Plan =SRNL-SCS-2008-00043, Prep=LM, Oxide=MgO (wt \%) Variability Chart for Measured

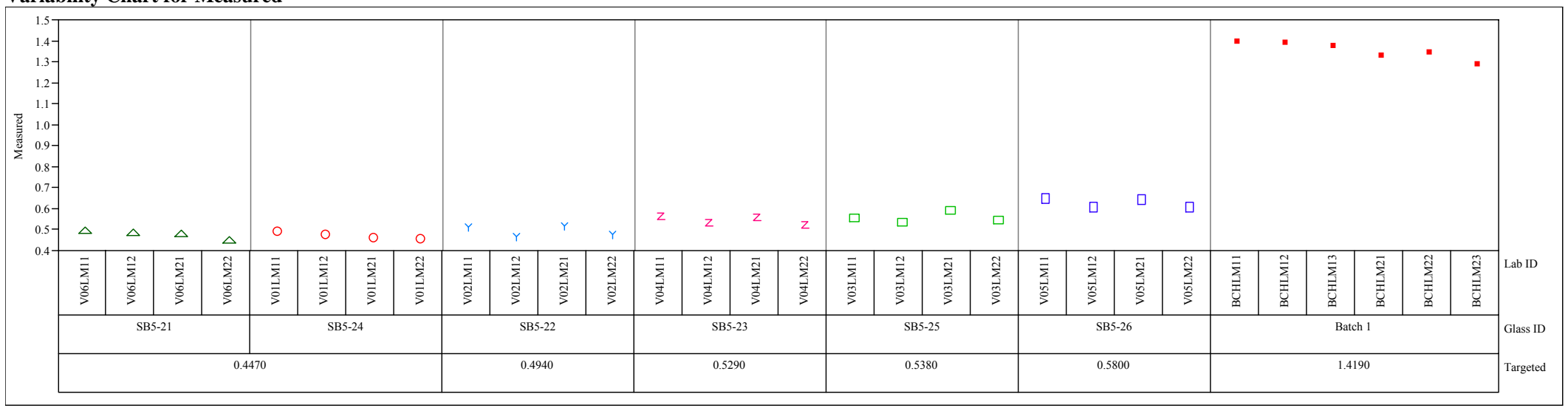

Analytical Plan =SRNL-SCS-2008-00043, Prep=LM, Oxide=MgO (wt \%)

Variability Chart for Measured bc

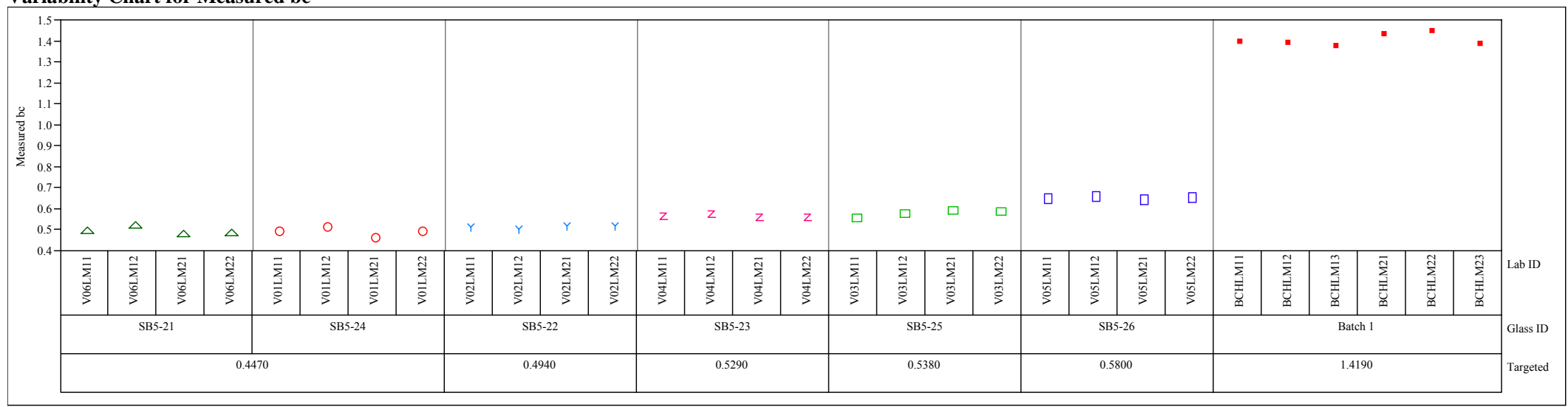


Exhibit A4. Oxide Measurements by Lab ID within Glass ID Including Auxiliary Measurements for LM Preps. (continued) Analytical Plan =SRNL-SCS-2008-00043, Prep=LM, Oxide=MnO (wt \%)

Variability Chart for Measured

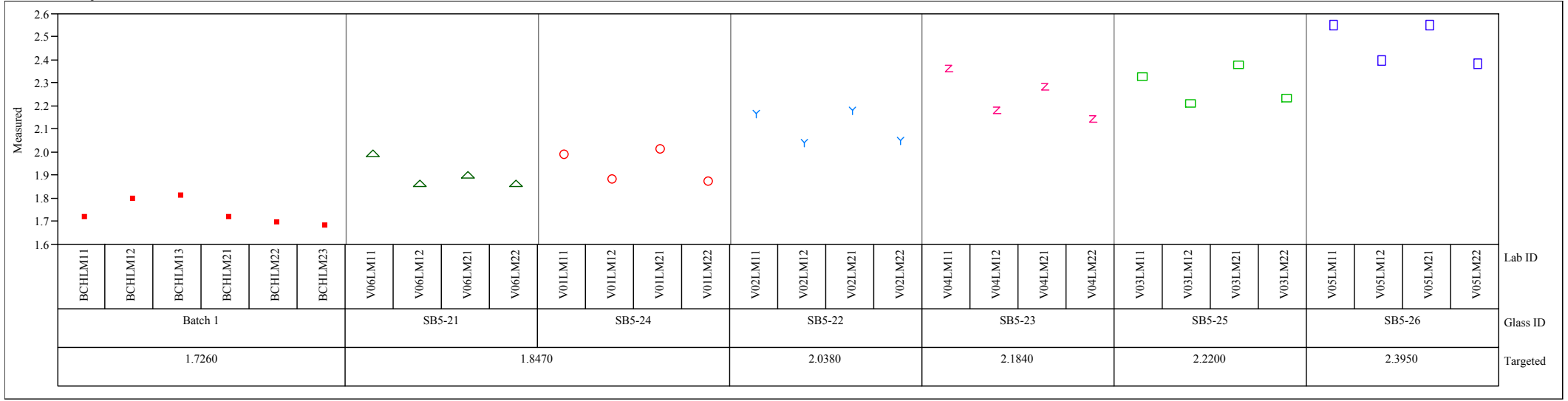

Analytical Plan =SRNL-SCS-2008-00043, Prep=LM, Oxide=MnO (wt \%)

Variability Chart for Measured bc

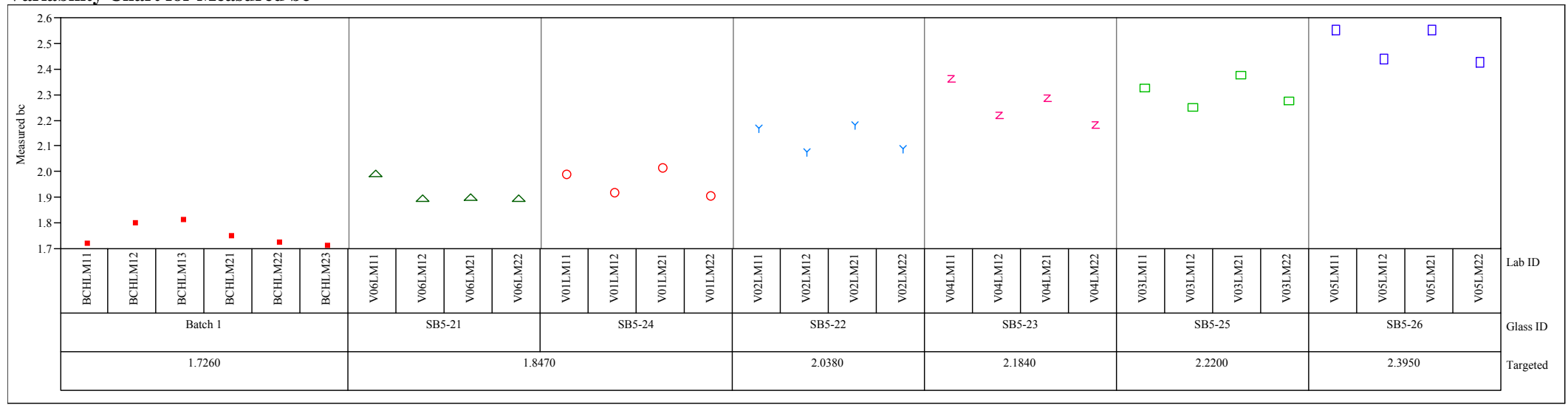


Exhibit A4. Oxide Measurements by Lab ID within Glass ID Including Auxiliary Measurements for LM Preps. (continued) Analytical Plan =SRNL-SCS-2008-00043, Prep=LM, Oxide=Na2O (wt \%)

Variability Chart for Measured

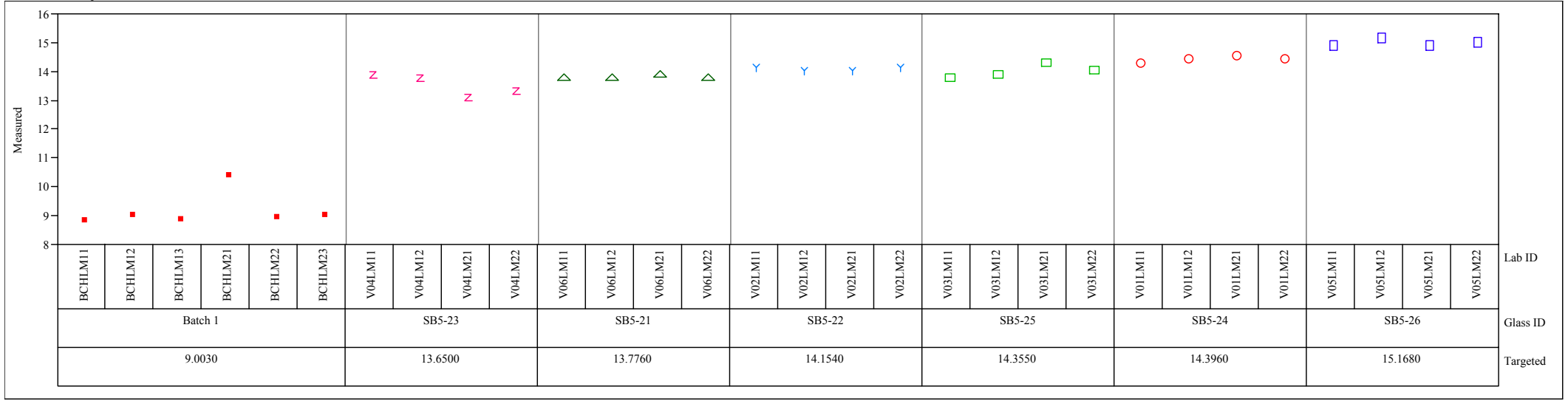

Analytical Plan =SRNL-SCS-2008-00043, Prep=LM, Oxide=Na2O (wt $\%)$

Variability Chart for Measured bc

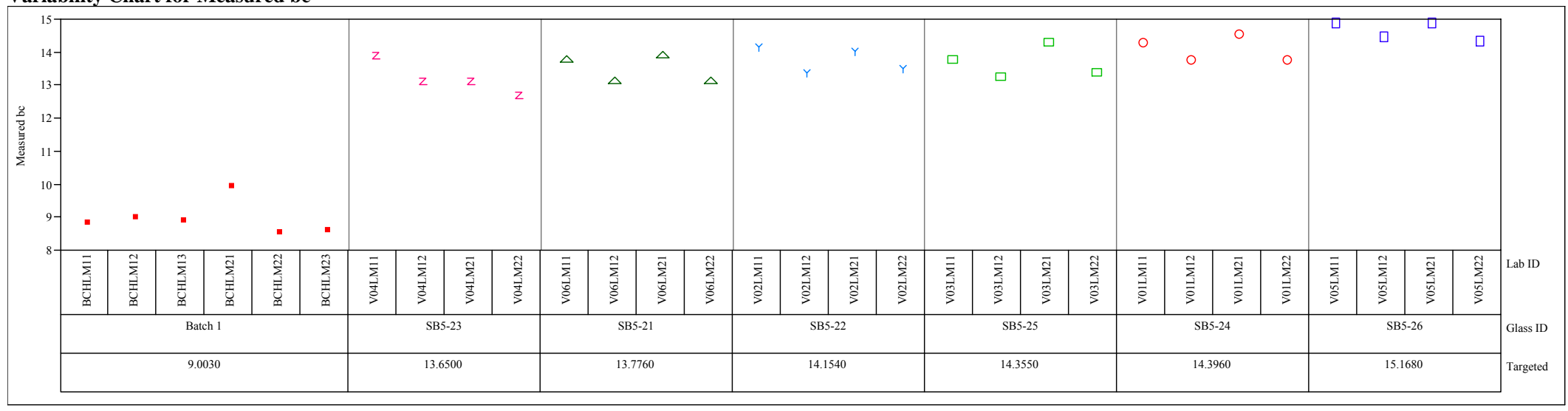


Exhibit A4. Oxide Measurements by Lab ID within Glass ID Including Auxiliary Measurements for LM Preps. (continued) Analytical Plan =SRNL-SCS-2008-00043, Prep=LM, Oxide=Nd2O3 (wt\%) Variability Chart for Measured

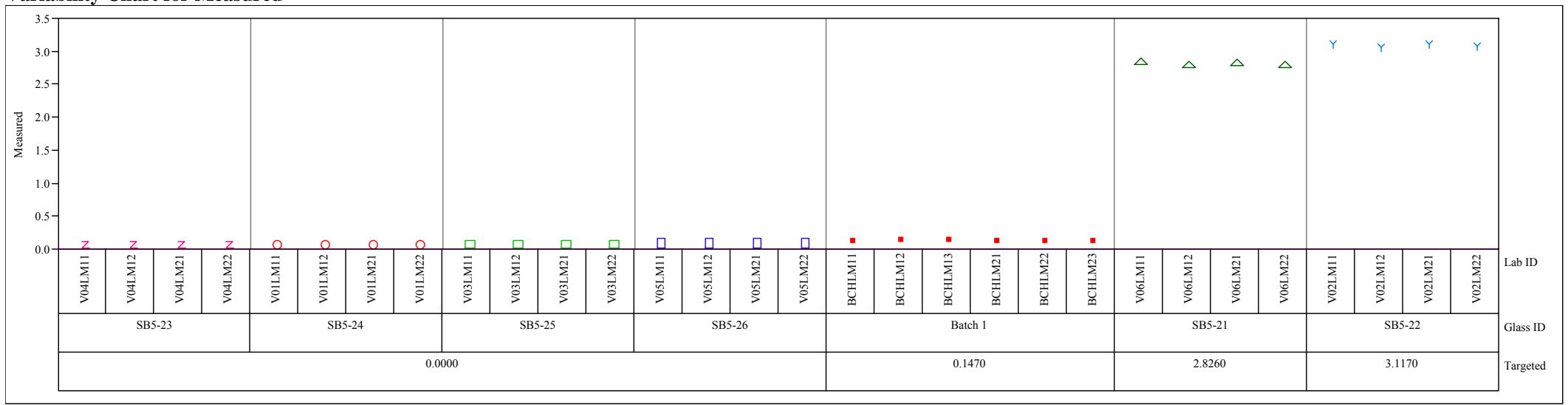

Analytical Plan =SRNL-SCS-2008-00043, Prep=LM, Oxide=Nd2O3 (wt \%)

Variability Chart for Measured bc

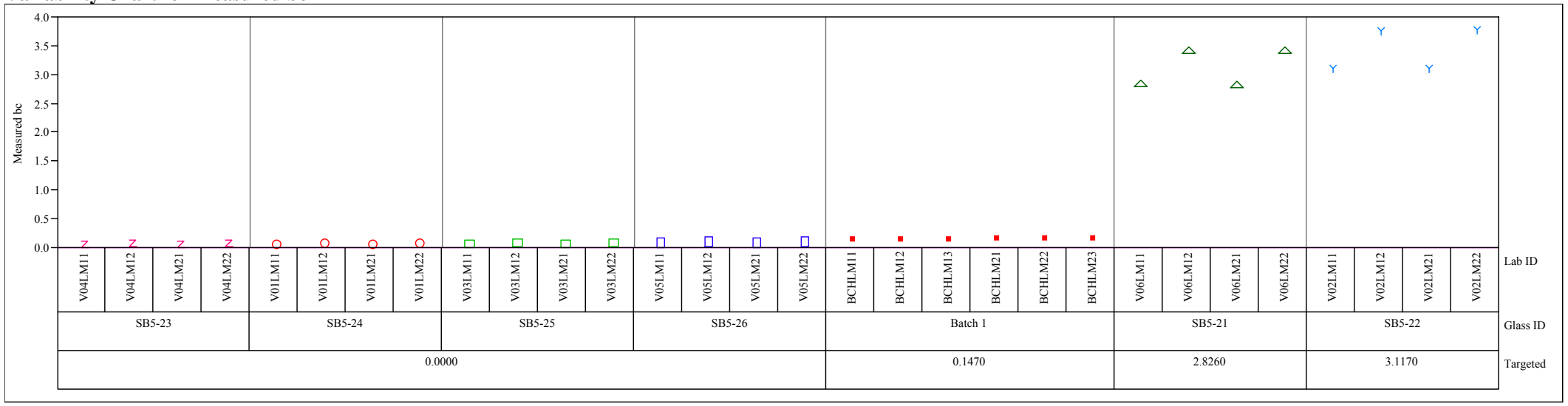


Exhibit A4. Oxide Measurements by Lab ID within Glass ID Including Auxiliary Measurements for LM Preps. (continued) Analytical Plan =SRNL-SCS-2008-00043, Prep=LM, Oxide=NiO (wt $\%)$ Variability Chart for Measured

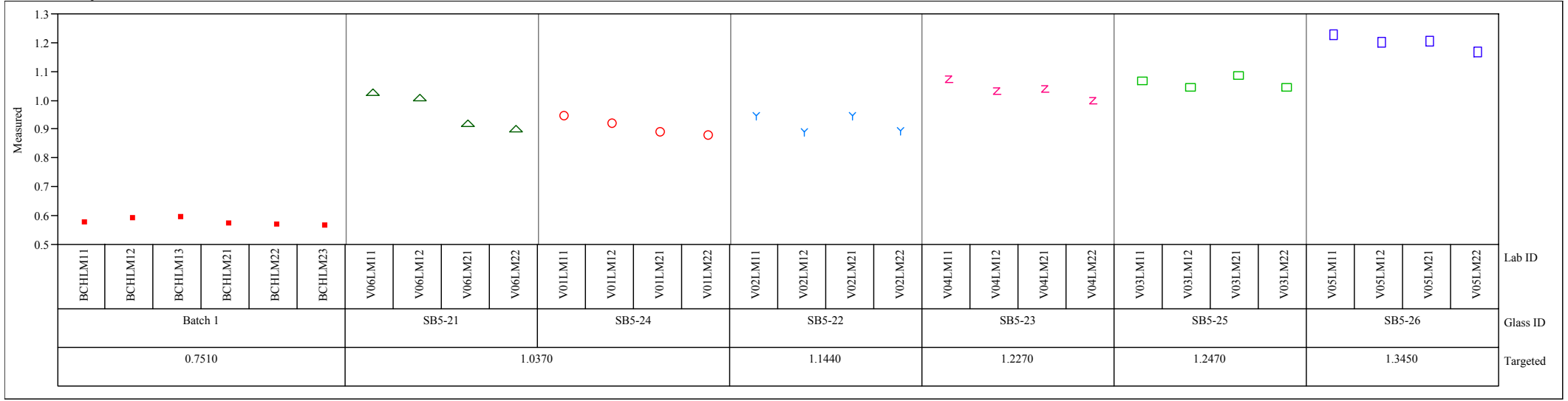

Analytical Plan =SRNL-SCS-2008-00043, Prep=LM, Oxide=NiO (wt \%)

Variability Chart for Measured bc

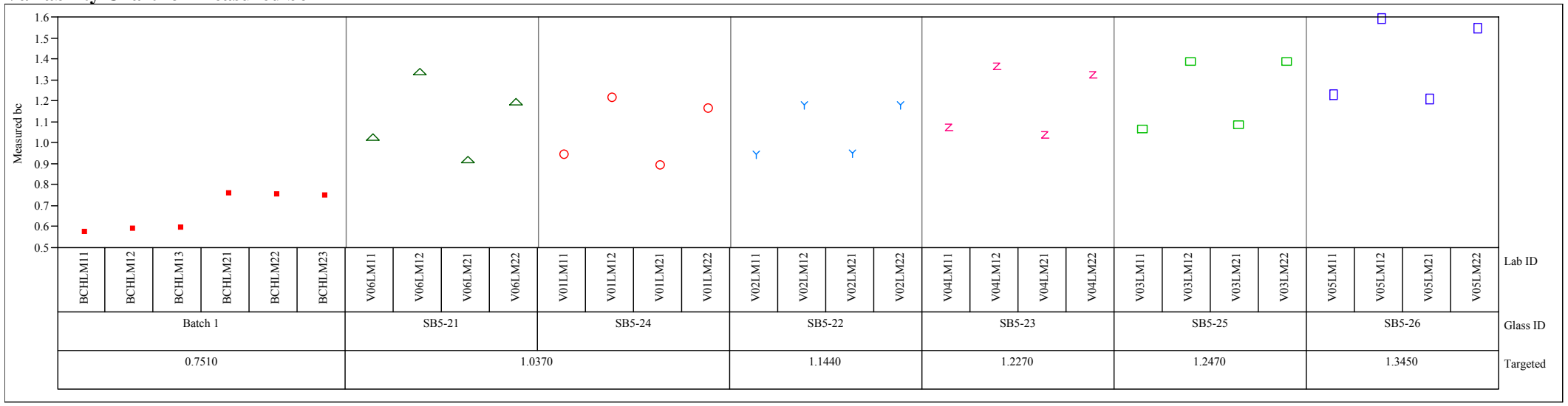


Exhibit A4. Oxide Measurements by Lab ID within Glass ID Including Auxiliary Measurements for LM Preps. (continued) Analytical Plan =SRNL-SCS-2008-00043, Prep=LM, Oxide=PbO (wt \%) Variability Chart for Measured

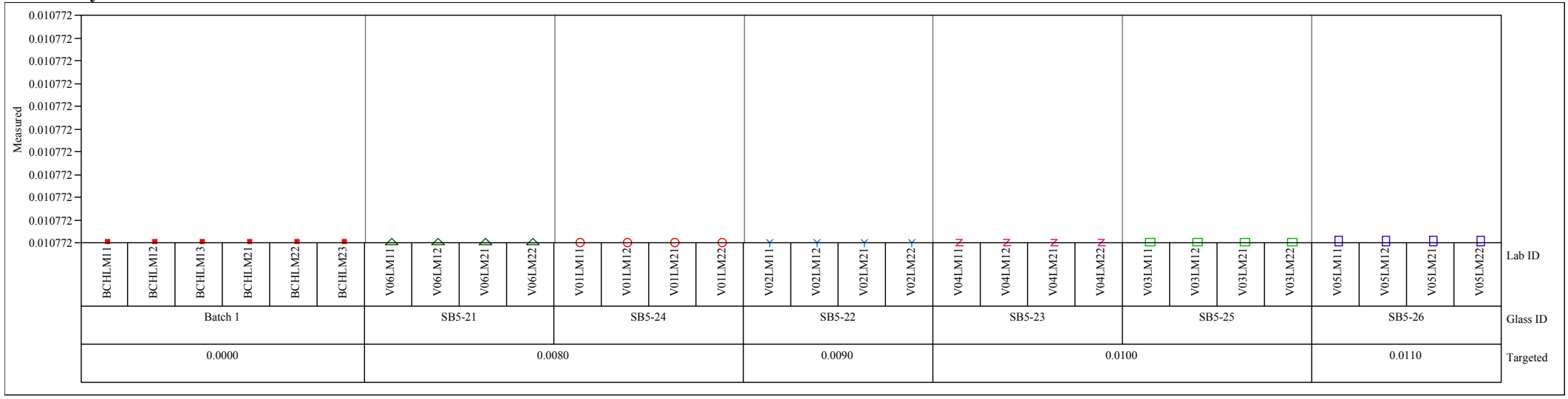

Analytical Plan =SRNL-SCS-2008-00043, Prep=LM, Oxide=PbO (wt \%) Variability Chart for Measured bc

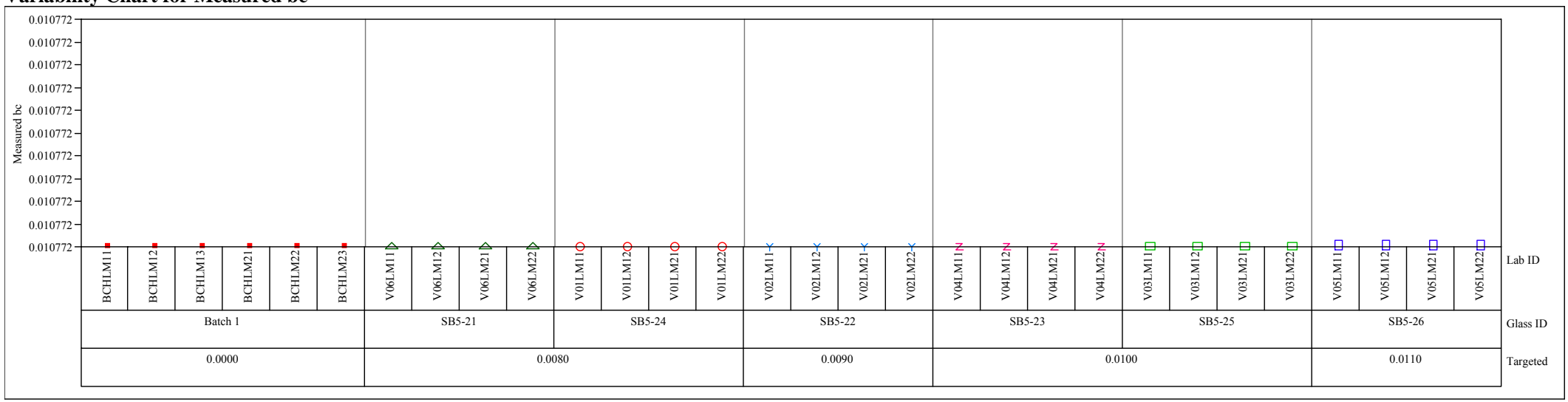


Exhibit A4. Oxide Measurements by Lab ID within Glass ID Including Auxiliary Measurements for LM Preps. (continued) Analytical Plan =SRNL-SCS-2008-00043, Prep=LM, Oxide=SiO2 (wt \%) Variability Chart for Measured

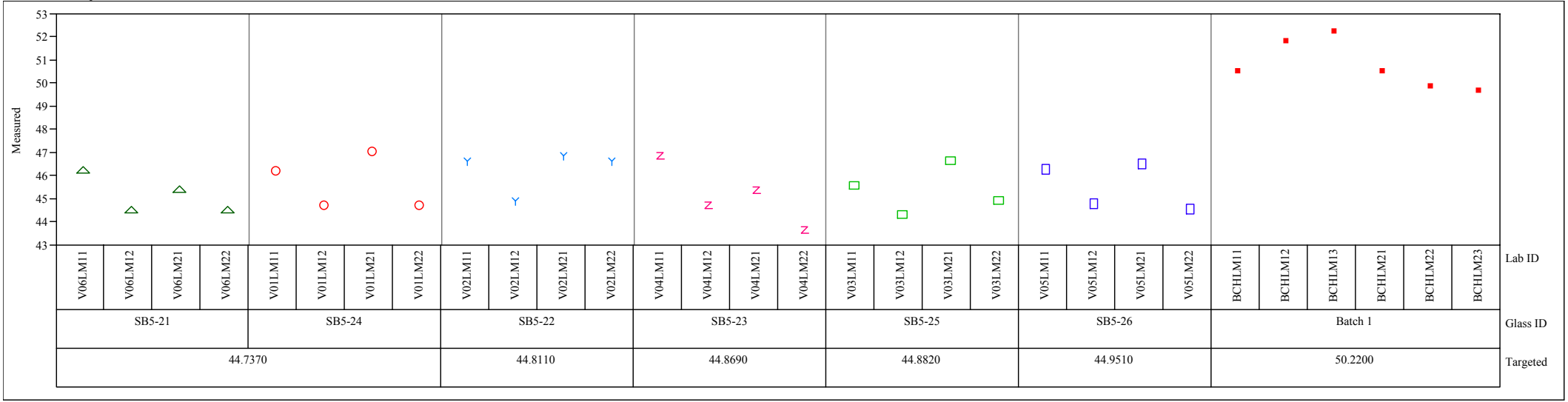

Analytical Plan =SRNL-SCS-2008-00043, Prep=LM, Oxide=SiO2 (wt \%)

Variability Chart for Measured bc

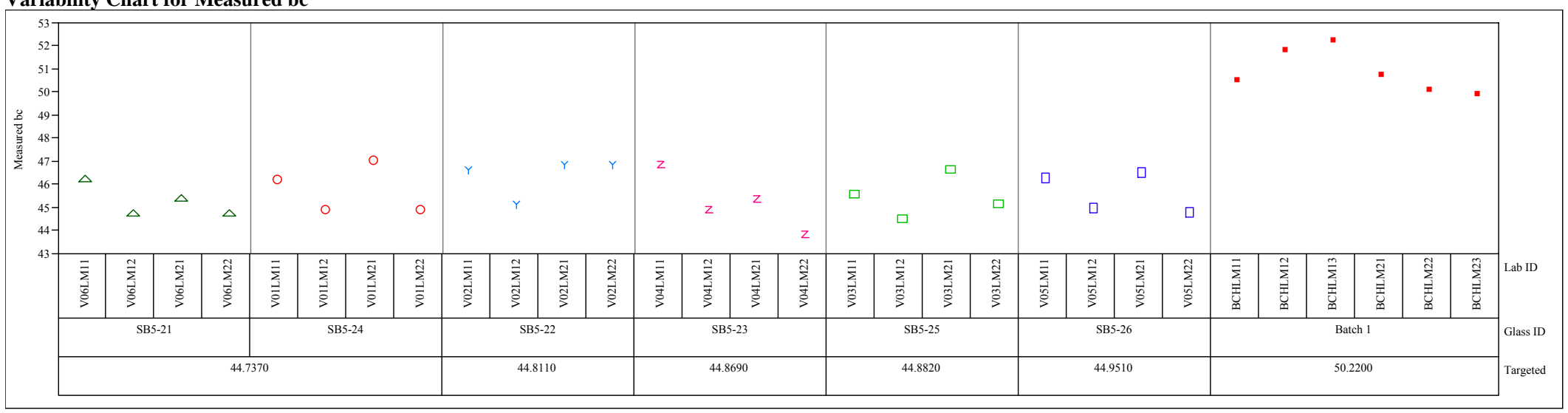


Exhibit A4. Oxide Measurements by Lab ID within Glass ID Including Auxiliary Measurements for LM Preps. (continued) Analytical Plan =SRNL-SCS-2008-00043, Prep=LM, Oxide=SO4 (wt $\%)$ Variability Chart for Measured

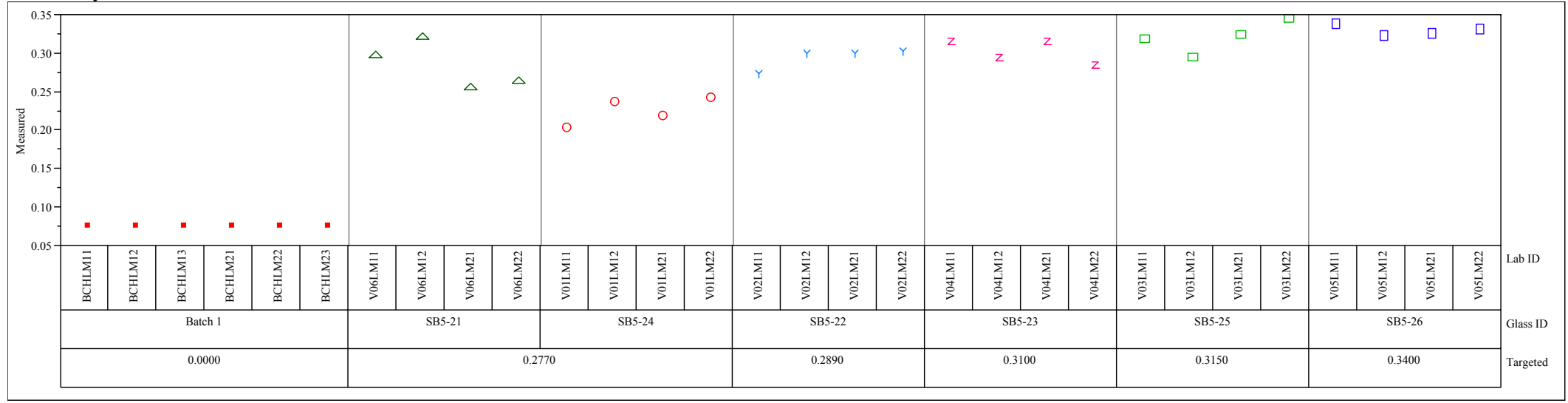

Analytical Plan =SRNL-SCS-2008-00043, Prep=LM, Oxide=SO4 (wt\%)

Variability Chart for Measured bc

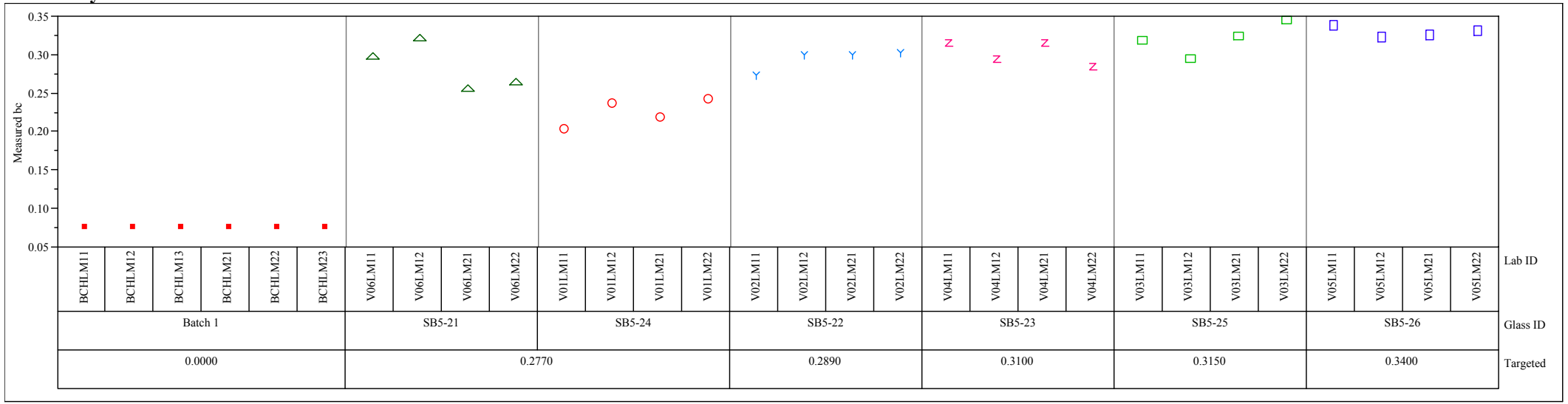


Exhibit A4. Oxide Measurements by Lab ID within Glass ID Including Auxiliary Measurements for LM Preps. (continued) Analytical Plan =SRNL-SCS-2008-00043, Prep=LM, Oxide=TiO2 (wt \%) Variability Chart for Measured

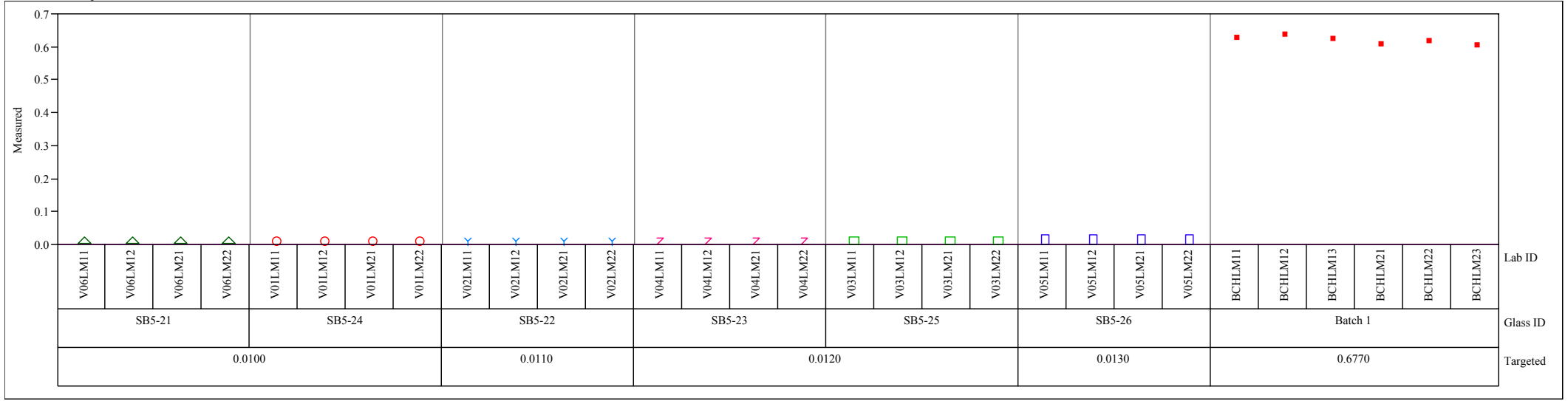

Analytical Plan =SRNL-SCS-2008-00043, Prep=LM, Oxide=TiO2 (wt \%)

Variability Chart for Measured bc

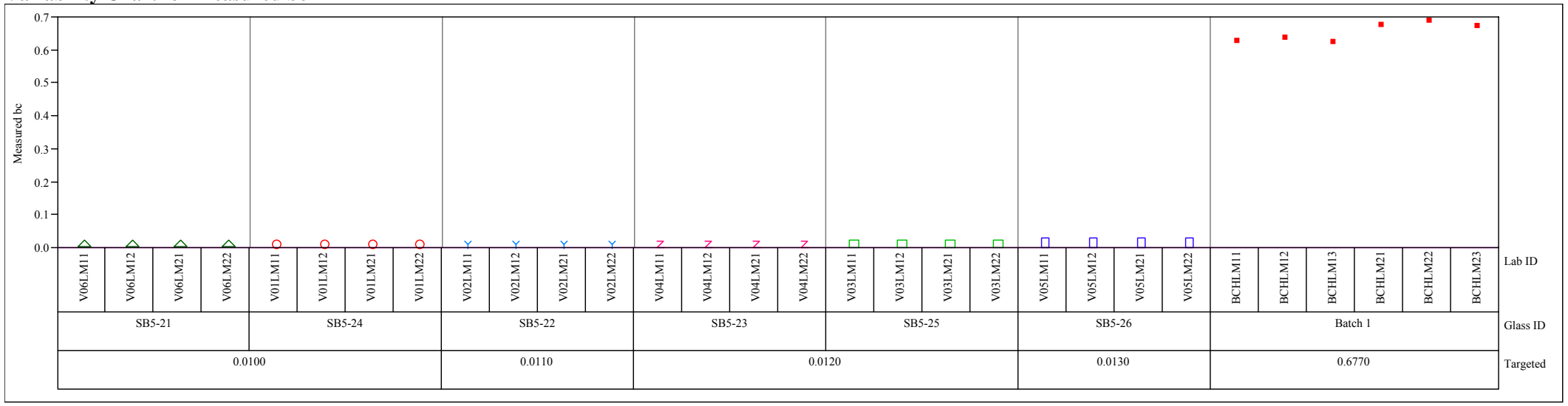


Exhibit A4. Oxide Measurements by Lab ID within Glass ID Including Auxiliary Measurements for LM Preps. (continued)

Analytical Plan =SRNL-SCS-2008-00043, Prep=LM, Oxide=ZnO (wt\%)

Variability Chart for Measured

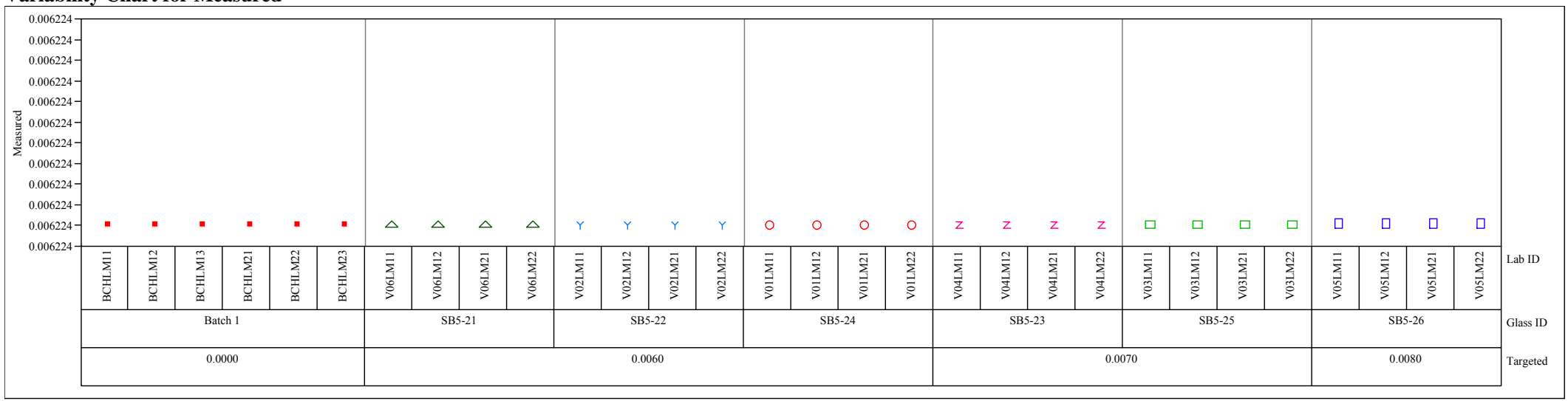

Analytical Plan $=$ SRNL-SCS-2008-00043, Prep $=$ LM, Oxide $=$ ZnO $(w t \%)$

Variability Chart for Measured bc

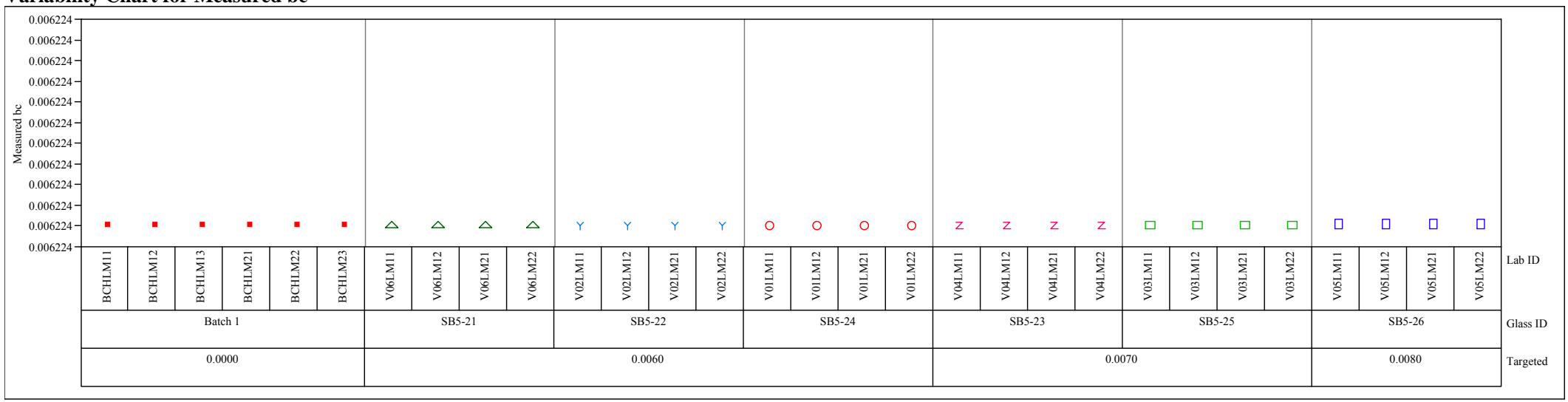


Exhibit A4. Oxide Measurements by Lab ID within Glass ID Including Auxiliary Measurements for LM Preps. (continued) Analytical Plan =SRNL-SCS-2008-00043, Prep=LM, Oxide=ZrO2 (wt\%) Variability Chart for Measured

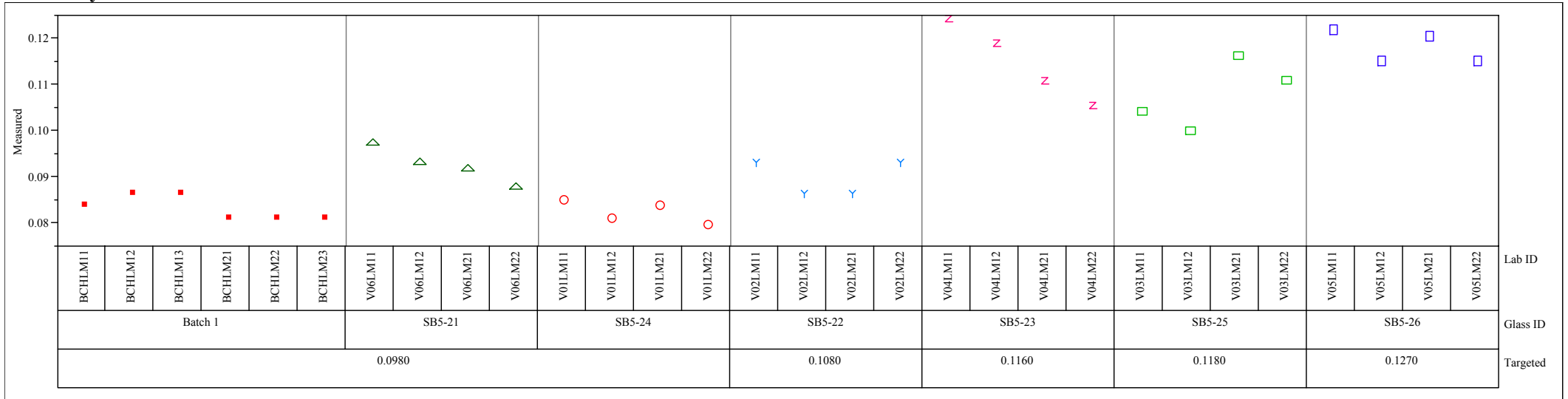

Analytical Plan =SRNL-SCS-2008-00043, Prep=LM, Oxide=ZrO2 (wt \%) Variability Chart for Measured bc

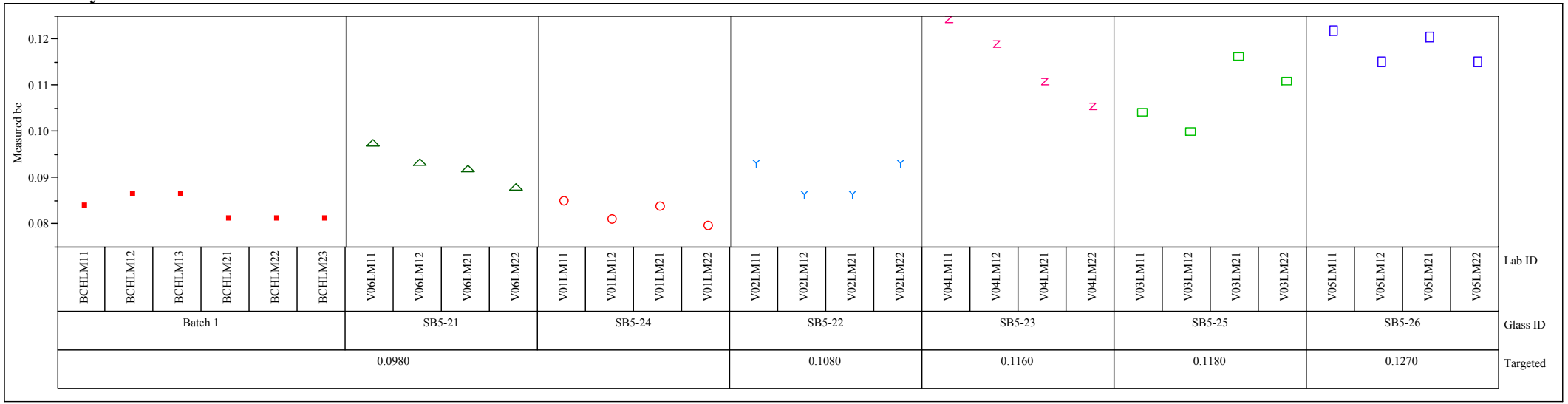


Exhibit A4. Oxide Measurements by Lab ID within Glass ID Including Auxiliary Measurements for LM Preps. (continued) Analytical Plan =SRNL-SCS-2008-00043, Prep=PF, Oxide=B2O3 (wt \%) Variability Chart for Measured

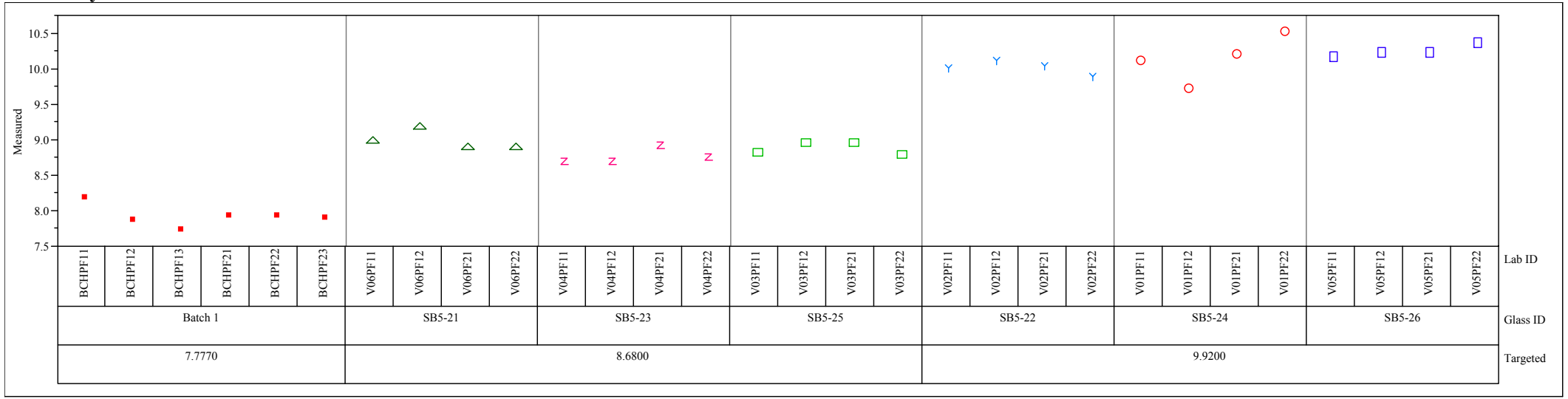

Analytical Plan =SRNL-SCS-2008-00043, Prep=PF, Oxide=B2O3 (wt $\%)$

Variability Chart for Measured bc

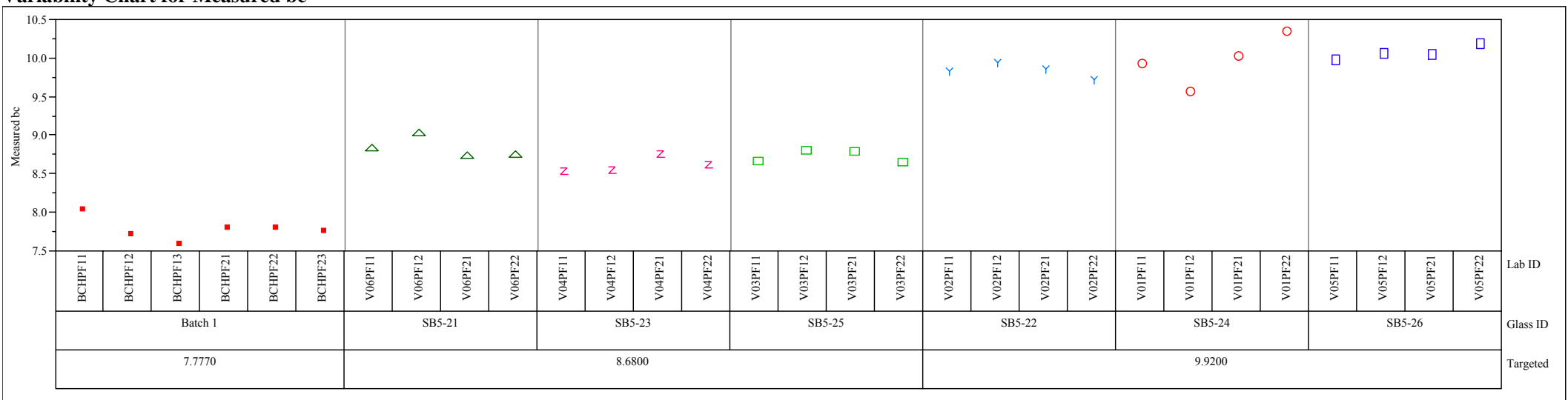


Exhibit A4. Oxide Measurements by Lab ID within Glass ID Including Auxiliary Measurements for LM Preps. (continued) Analytical Plan =SRNL-SCS-2008-00043, Prep=PF, Oxide=Li2O (wt\%) Variability Chart for Measured

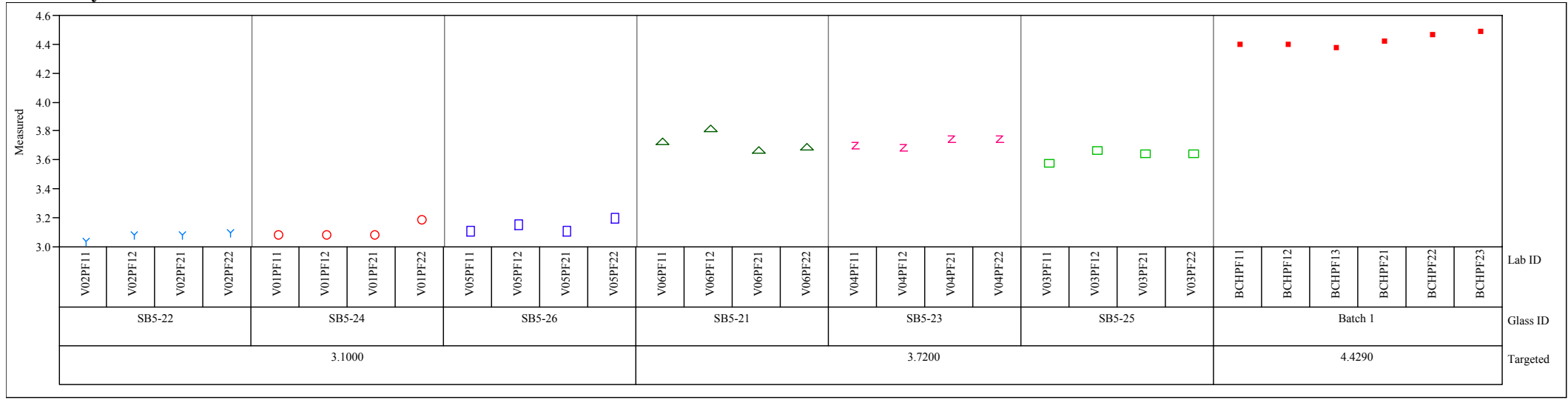

Analytical Plan =SRNL-SCS-2008-00043, Prep=PF, Oxide=Li2O (wt \%)

Variability Chart for Measured bc

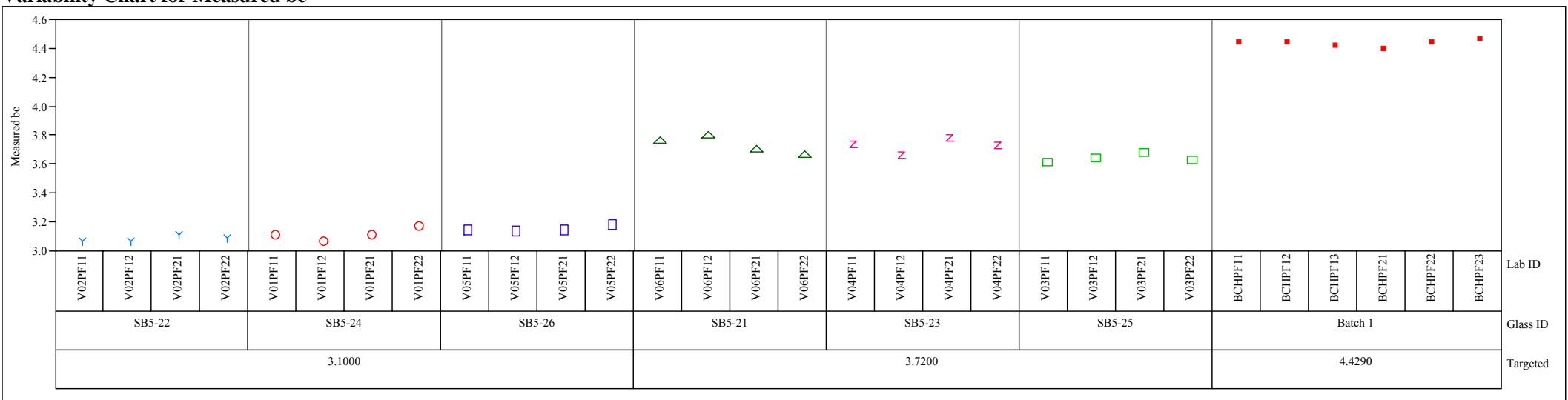


Exhibit A5. Measured, Measured bc, and Targeted Concentrations by Oxide by Glass ID for each Memo and Analytical Set.
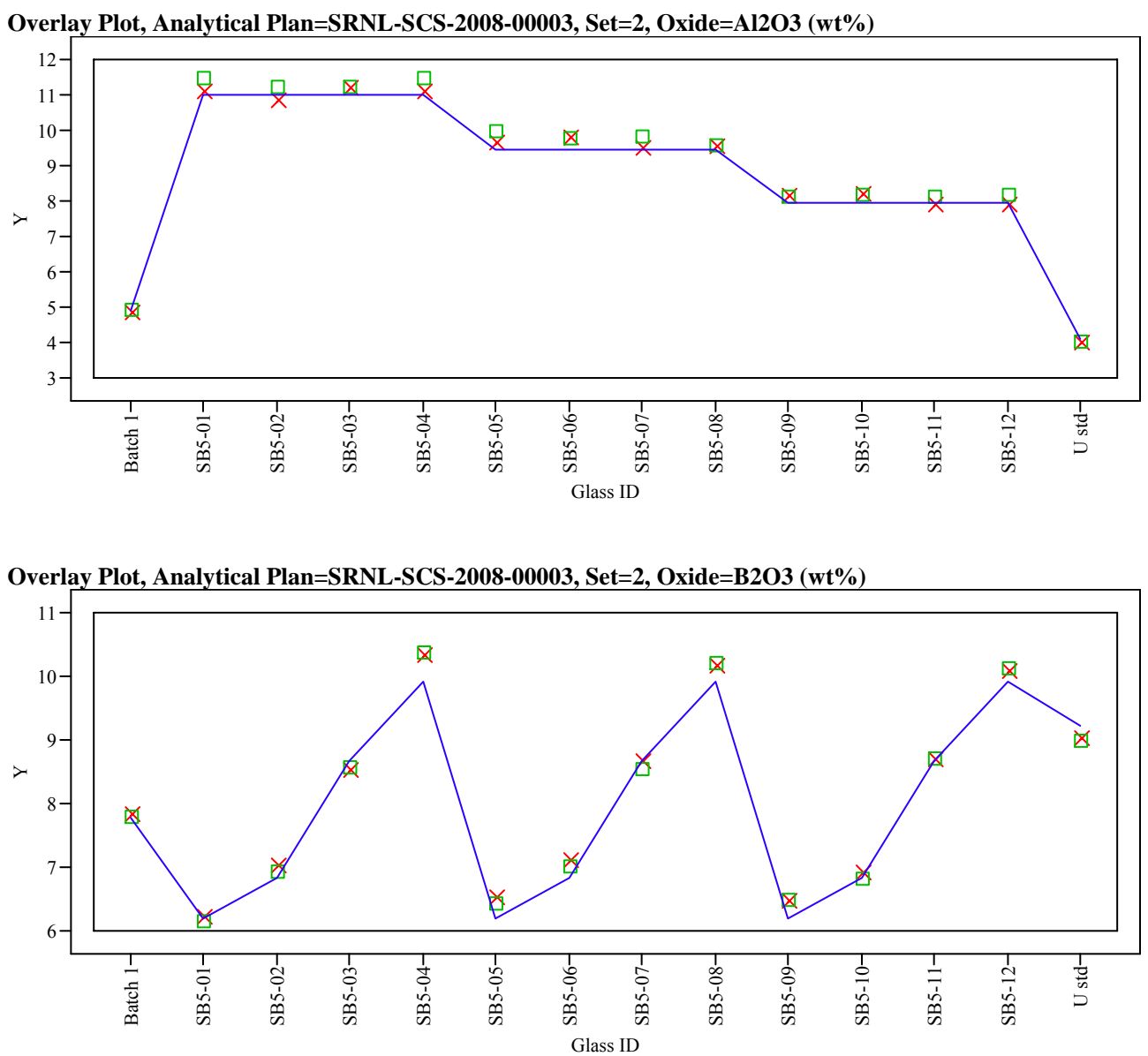

Overlay Plot, Analytical Plan=SRNL-SCS-2008-00003, Set=2, Oxide=BaO (wt \%)

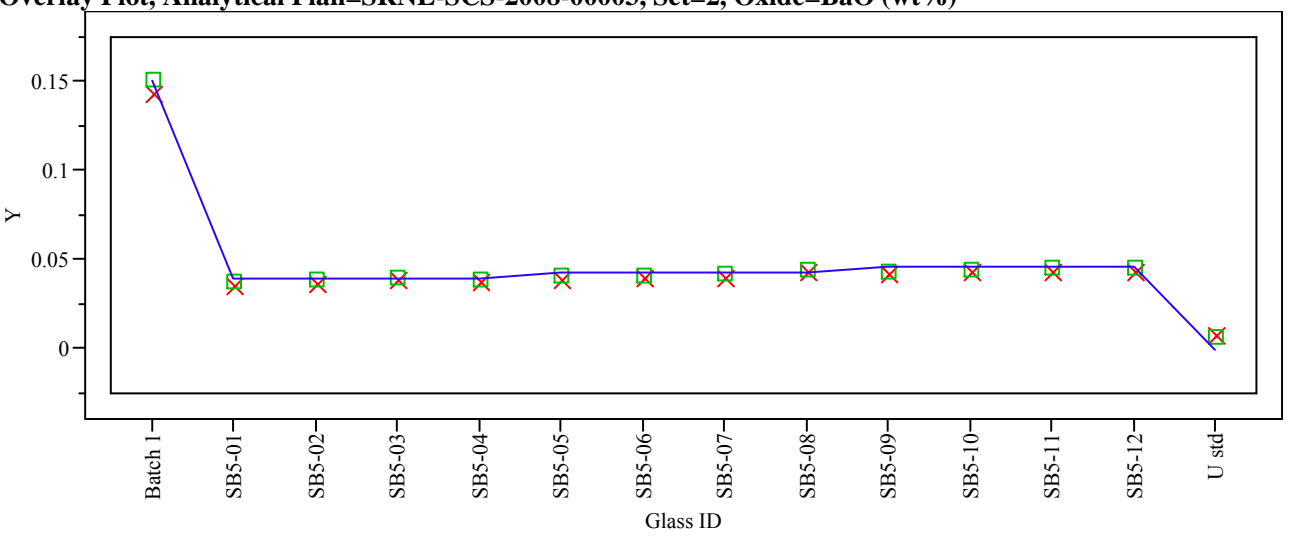

Y $\times$ Measured

- Measured bc Targeted 
Exhibit A5. Measured, Measured bc, and Targeted Concentrations by Oxide by Glass ID for each Memo and Analytical Set. (continued)

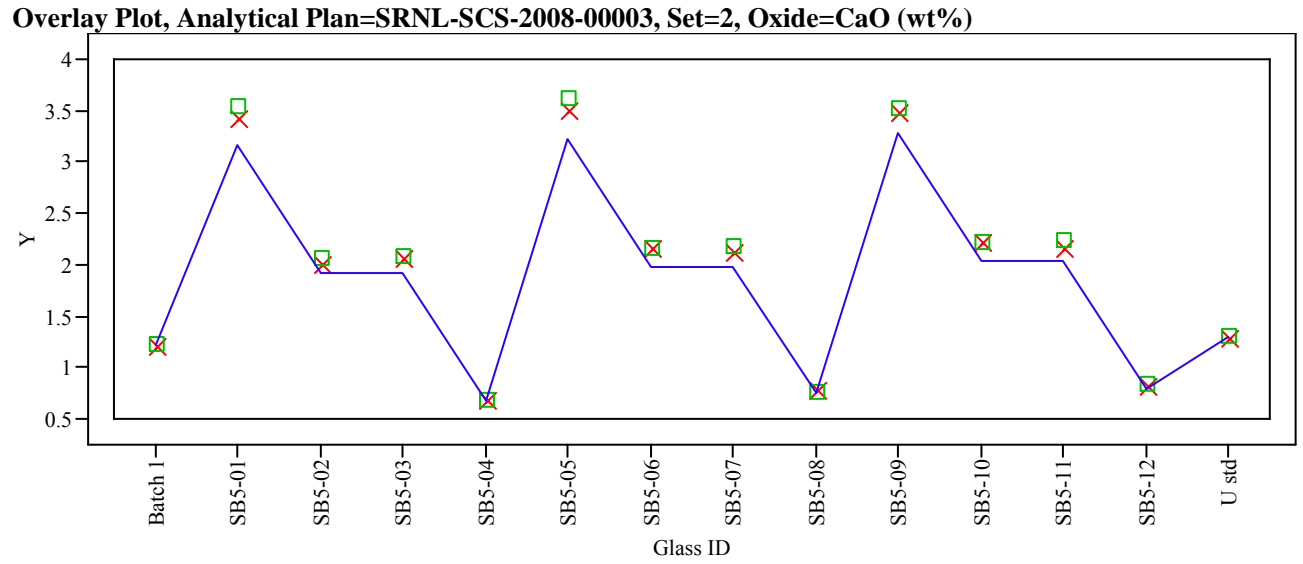

Overlay Plot, Analytical Plan=SRNL-SCS-2008-00003, Set=2, Oxide=CdO (wt \%)

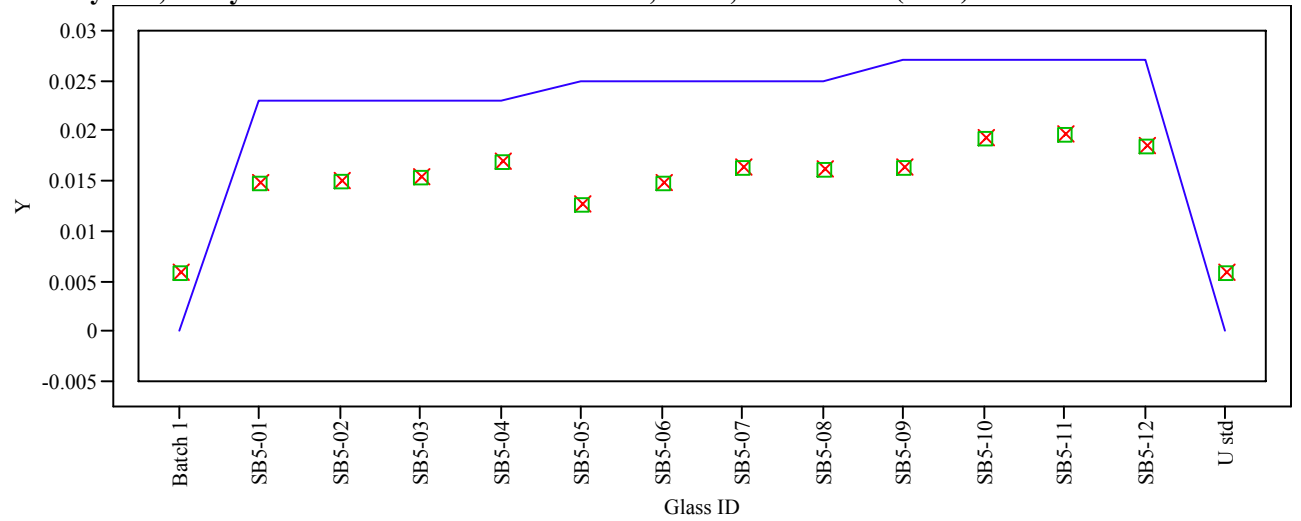

Overlay Plot, Analytical Plan=SRNL-SCS-2008-00003, Set=2, Oxide=Ce2O3 (wt\%)

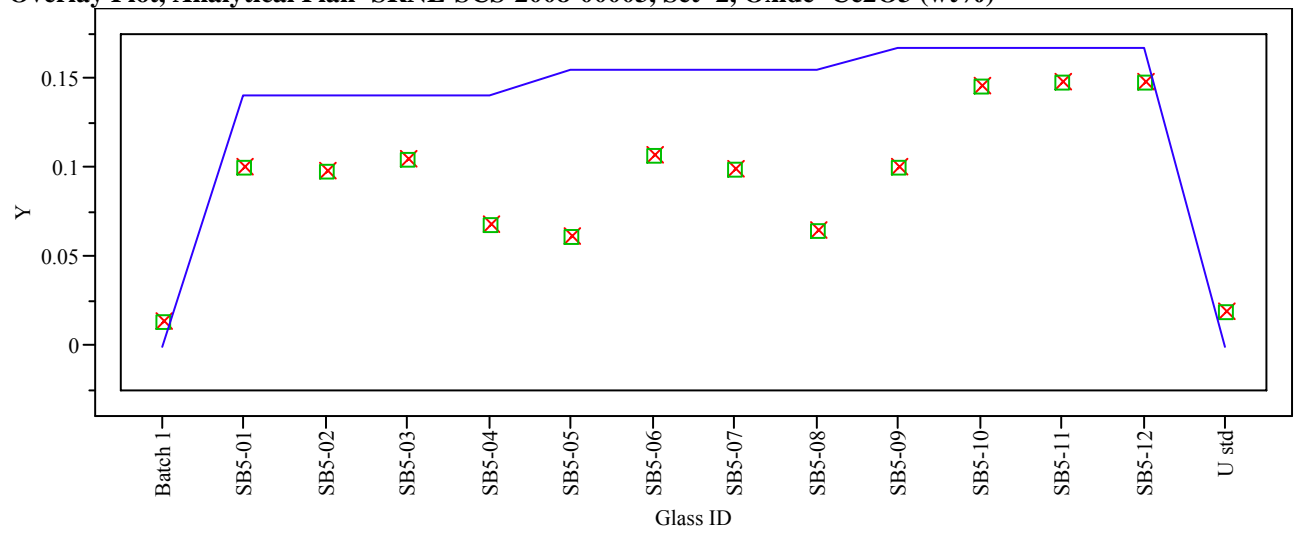

$\mathrm{Y} \times$ Measured $\square$ Measured bc Targeted 
Exhibit A5. Measured, Measured bc, and Targeted Concentrations by Oxide by Glass ID for each Memo and Analytical Set. (continued)
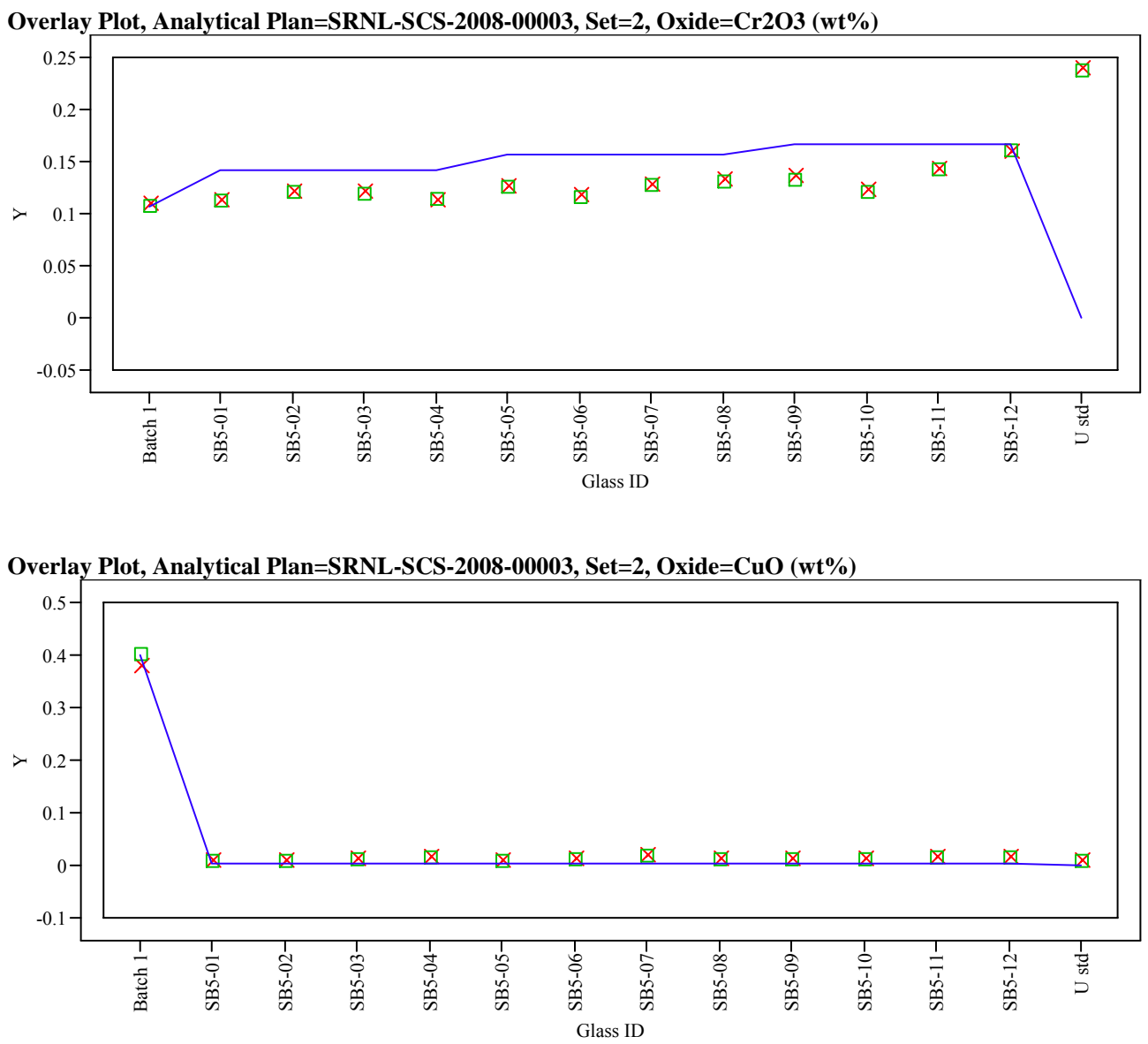

Overlay Plot, Analytical Plan=SRNL-SCS-2008-00003, Set=2, Oxide=Fe2O3 (wt\%)

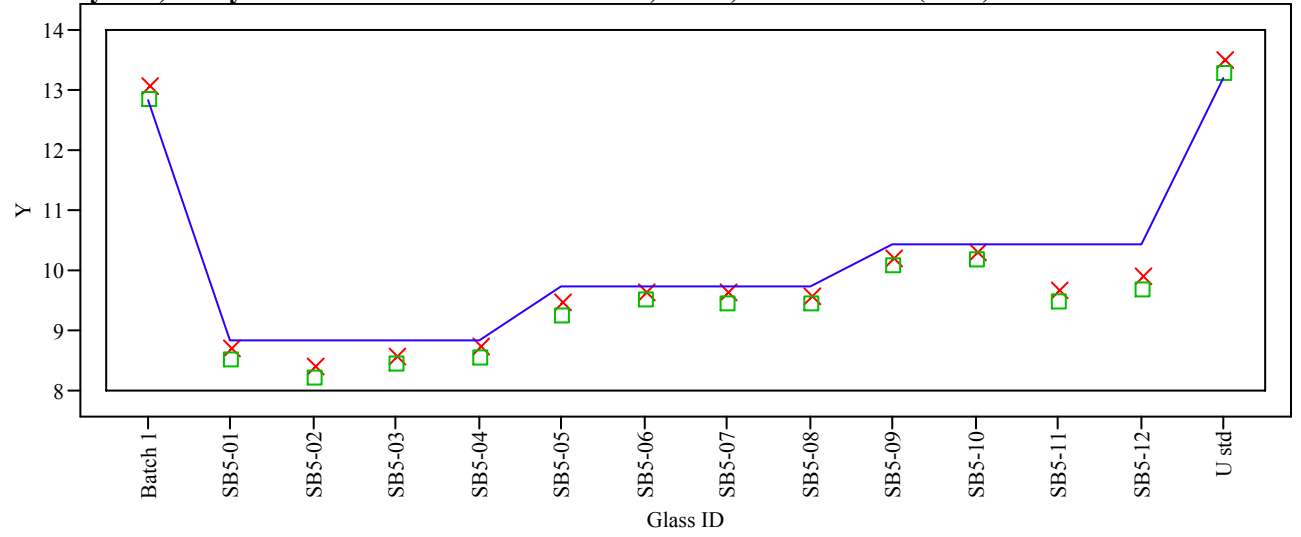

Y $\times$ Measured $\square$ Measured bc $\quad$ Targeted 
Exhibit A5. Measured, Measured bc, and Targeted Concentrations by Oxide by Glass ID for each Memo and Analytical Set. (continued)

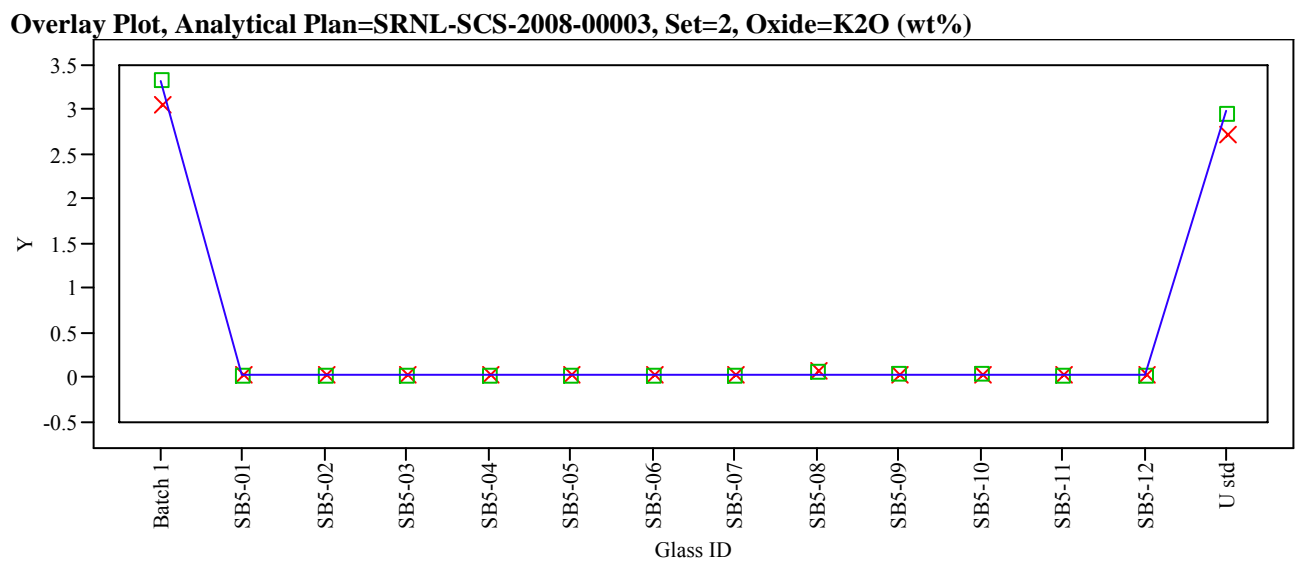

Overlay Plot, Analytical Plan=SRNL-SCS-2008-00003, Set=2, Oxide=La2O3 (wt\%)

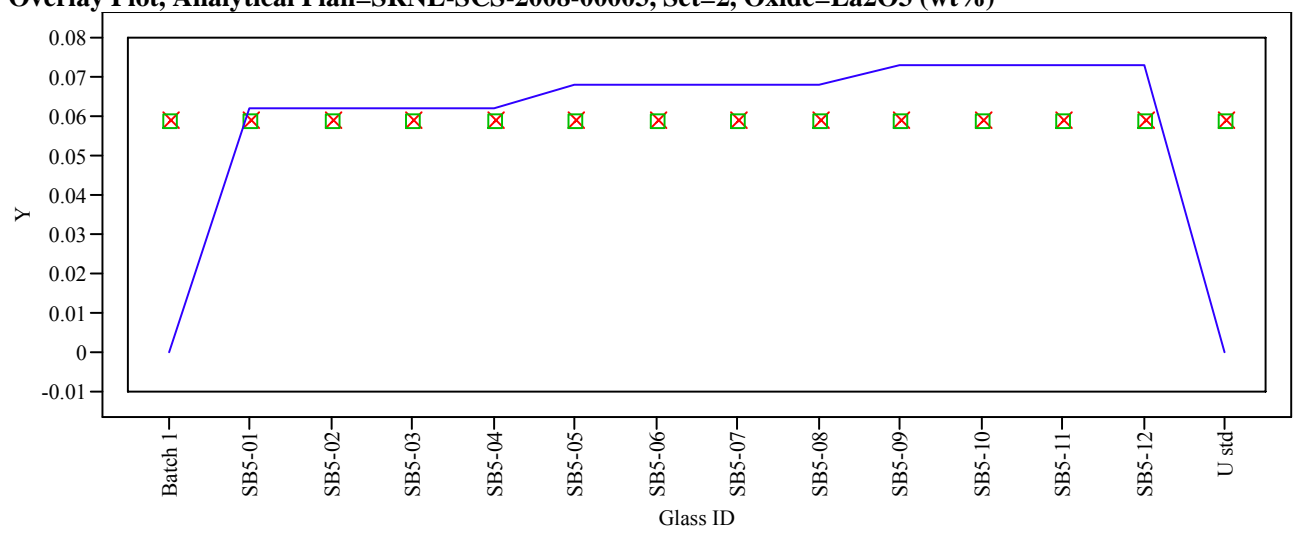

Overlay Plot, Analytical Plan=SRNL-SCS-2008-00003, Set=2, Oxide=Li2O (wt \%)

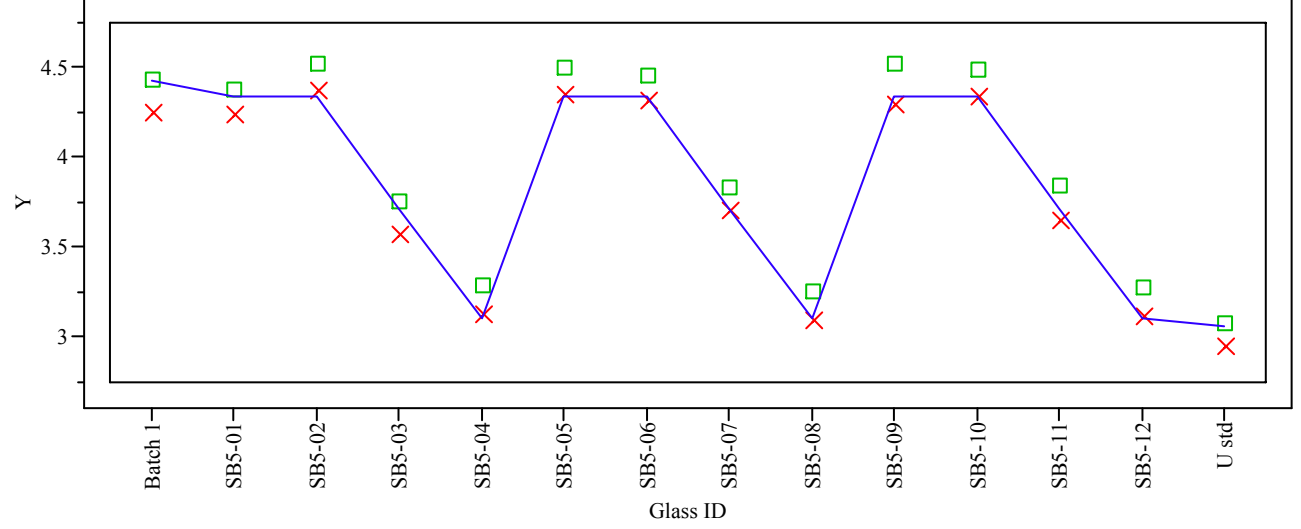

$\mathrm{Y} \times$ Measured $\square$ Measured bc Targeted 
Exhibit A5. Measured, Measured bc, and Targeted Concentrations by Oxide by Glass ID for each Memo and Analytical Set. (continued)
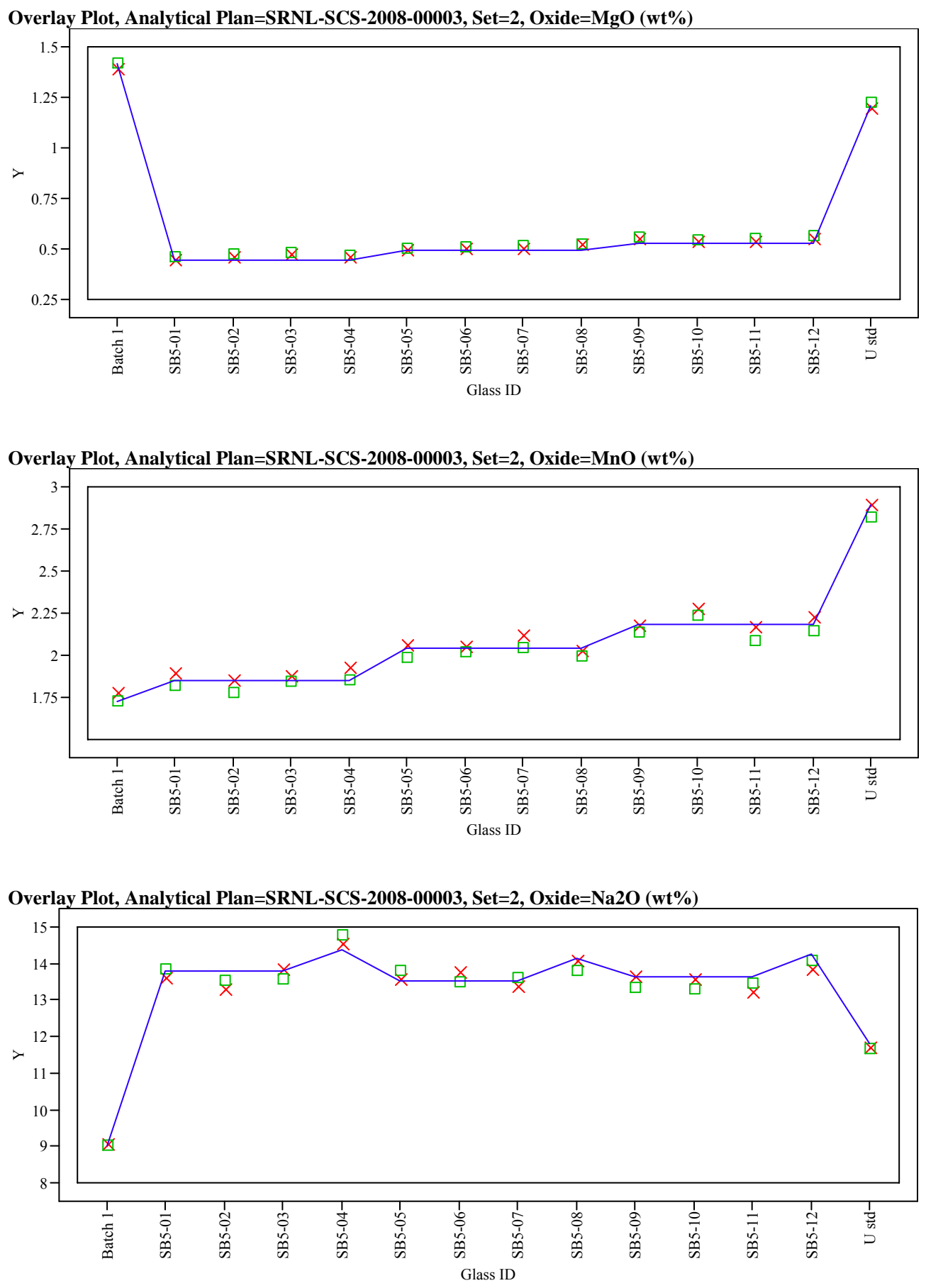

$\mathrm{Y} \times$ Measured $\quad$ Measured bc Targeted 
Exhibit A5. Measured, Measured bc, and Targeted Concentrations by Oxide by Glass ID for each Memo and Analytical Set. (continued)
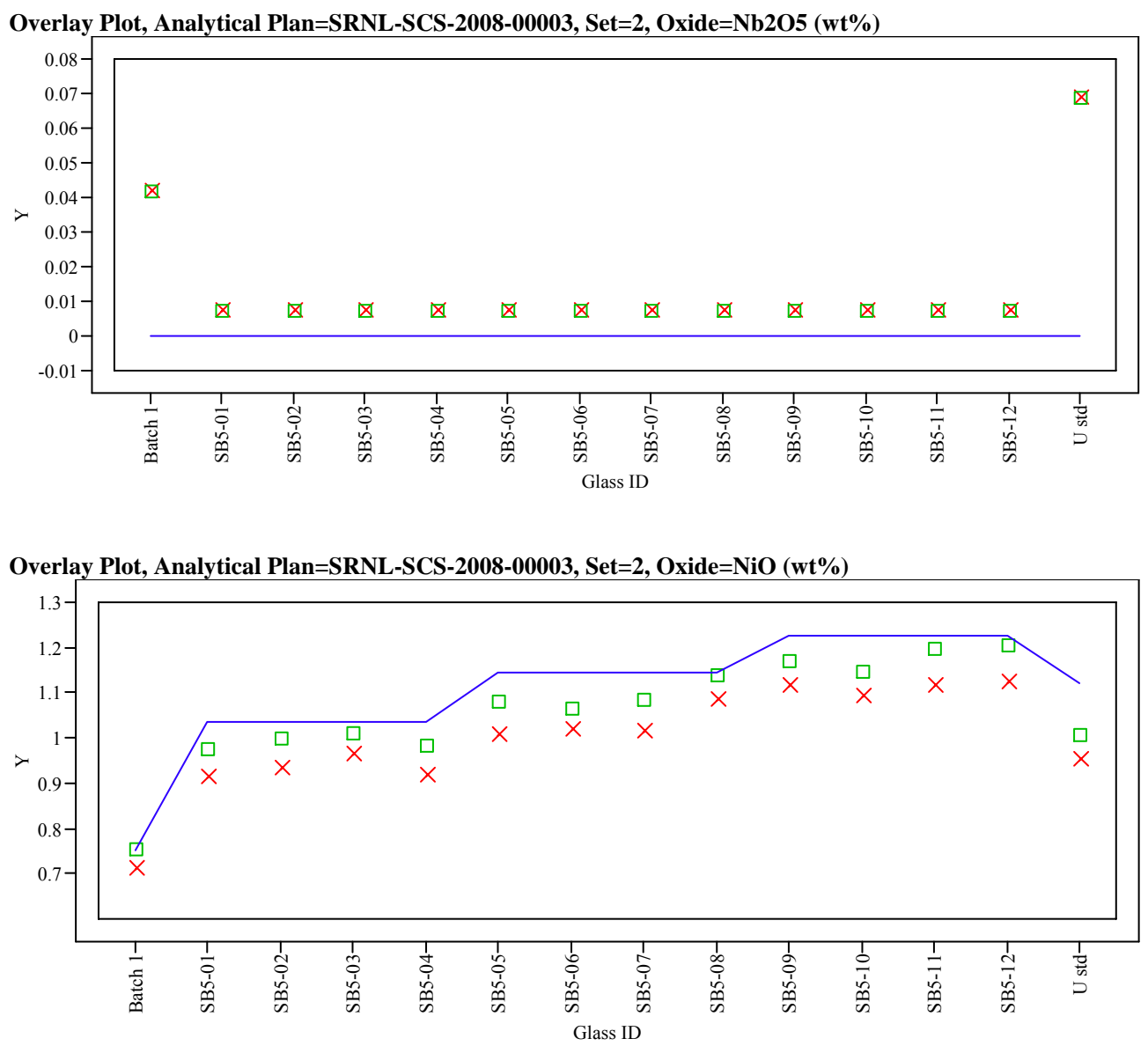

Overlay Plot, Analytical Plan=SRNL-SCS-2008-00003, Set=2, Oxide=PbO (wt\%)

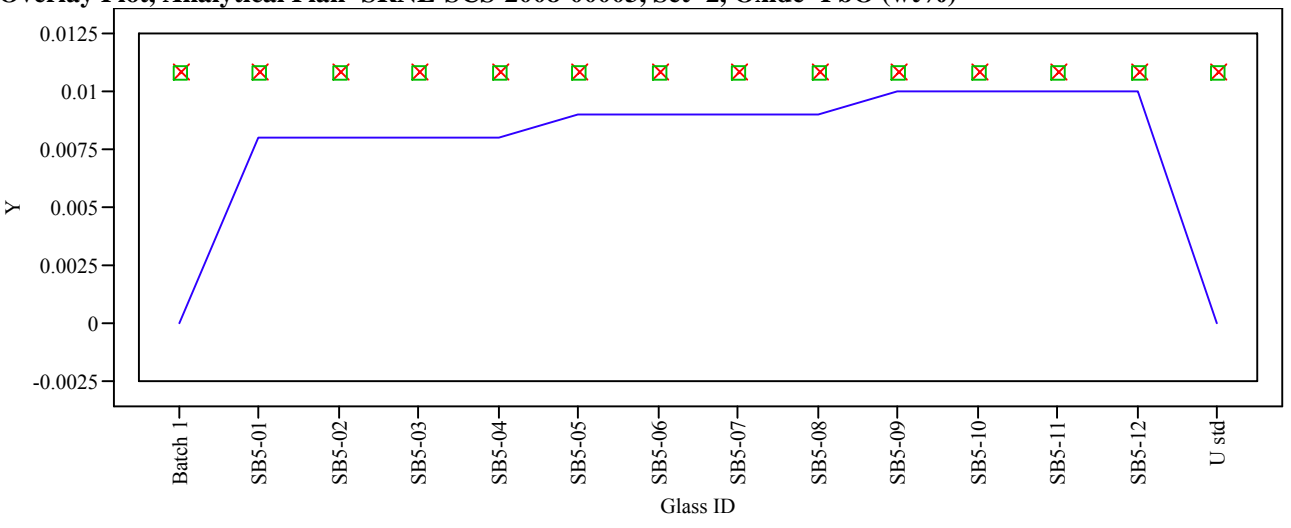

$\mathrm{Y} \times$ Measured $\square$ Measured bc $\quad$ Targeted 
Exhibit A5. Measured, Measured bc, and Targeted Concentrations by Oxide by Glass ID for each Memo and Analytical Set. (continued)

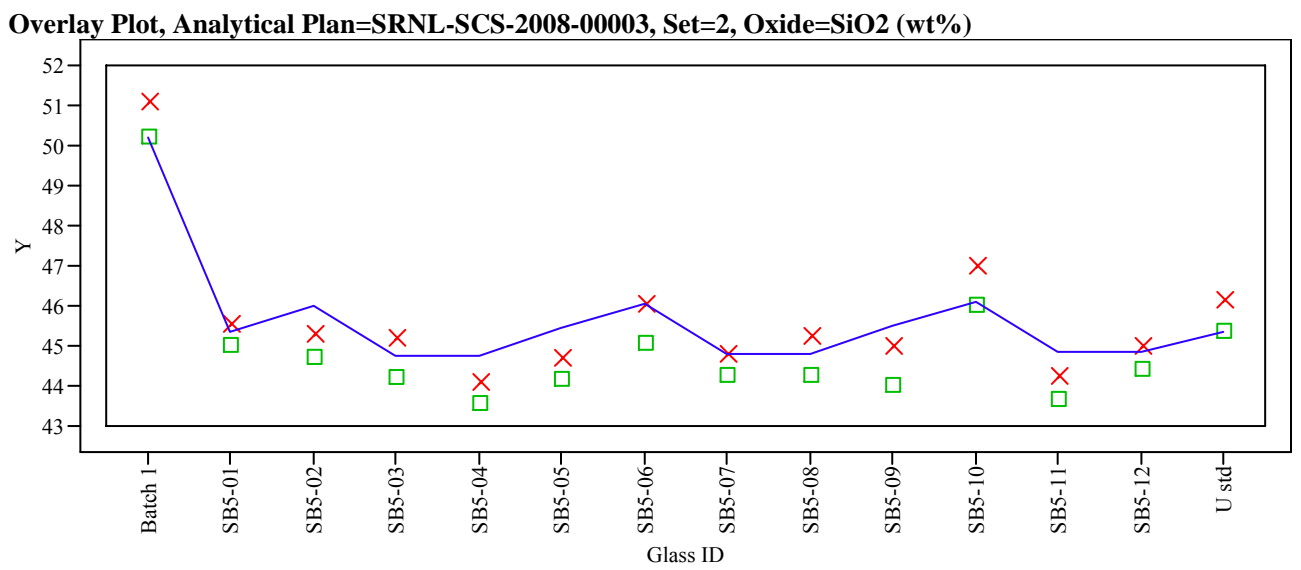

Overlay Plot, Analytical Plan=SRNL-SCS-2008-00003, Set=2, Oxide=SO4 (wt\%)

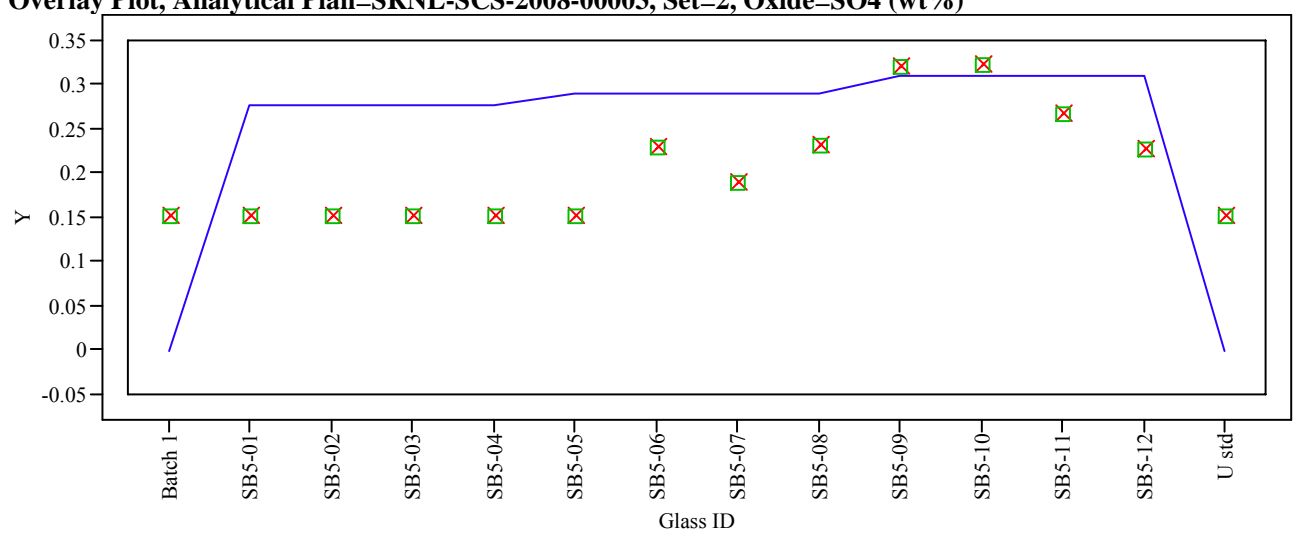

Overlay Plot, Analytical Plan=SRNL-SCS-2008-00003, Set=2, Oxide=ThO2 (wt \%)

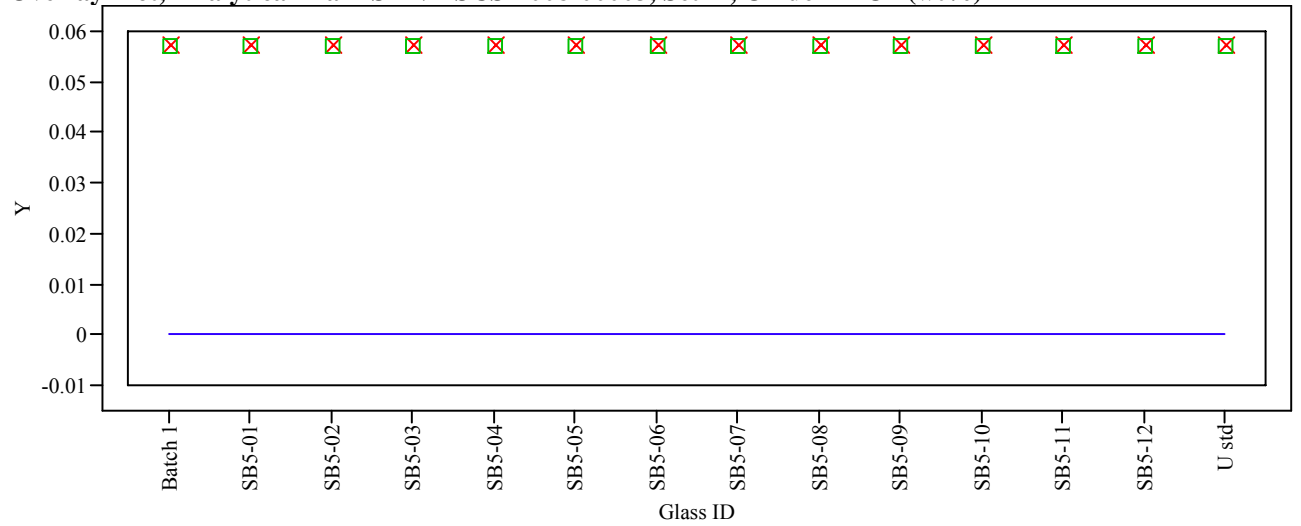

$\mathrm{Y} \times$ Measured $\square$ Measured bc $\quad$ Targeted 
Exhibit A5. Measured, Measured bc, and Targeted Concentrations by Oxide by Glass ID for each Memo and Analytical Set. (continued)
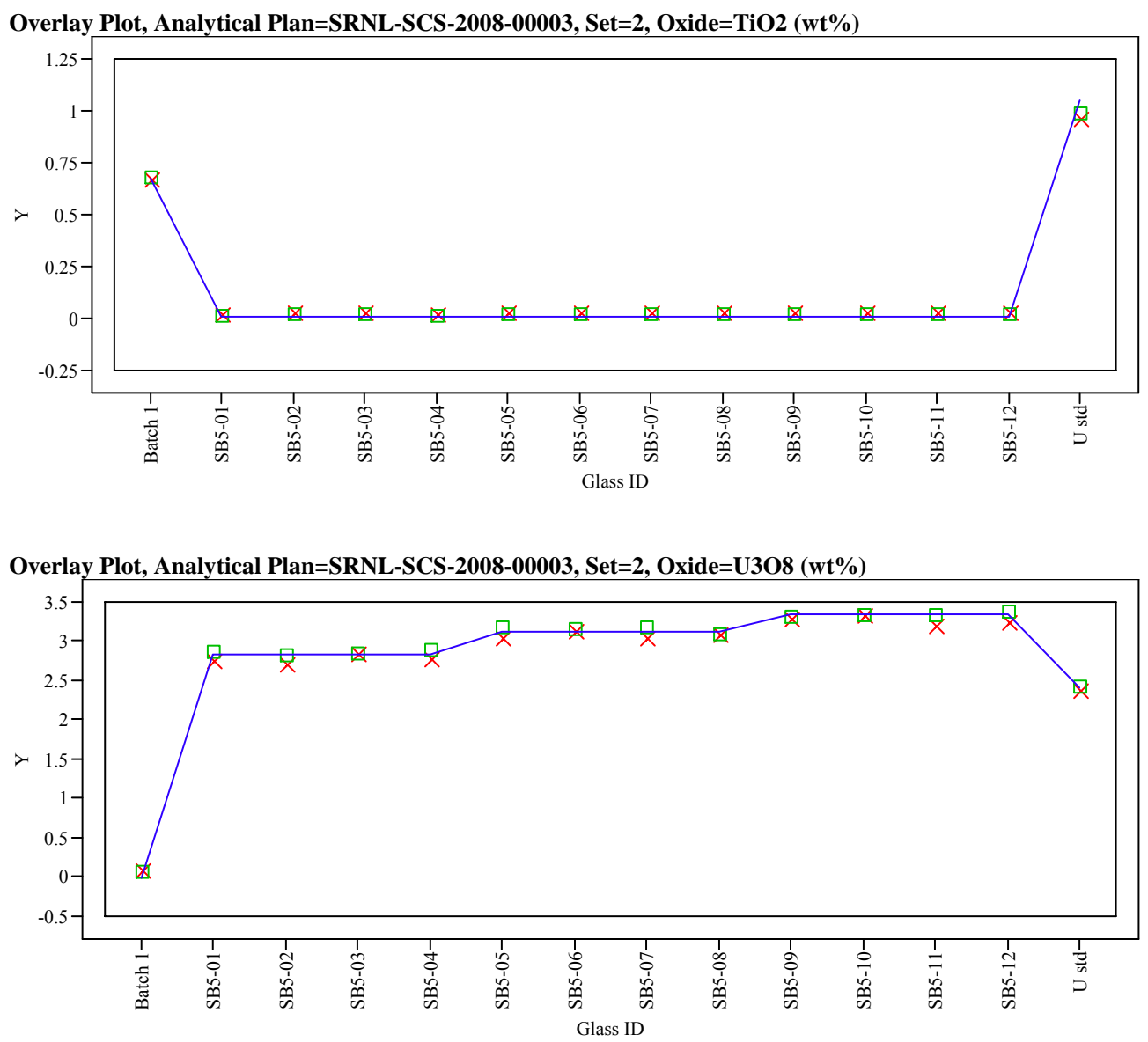

Overlay Plot, Analytical Plan=SRNL-SCS-2008-00003, Set $=2$, Oxide $=$ ZnO (wt \%)

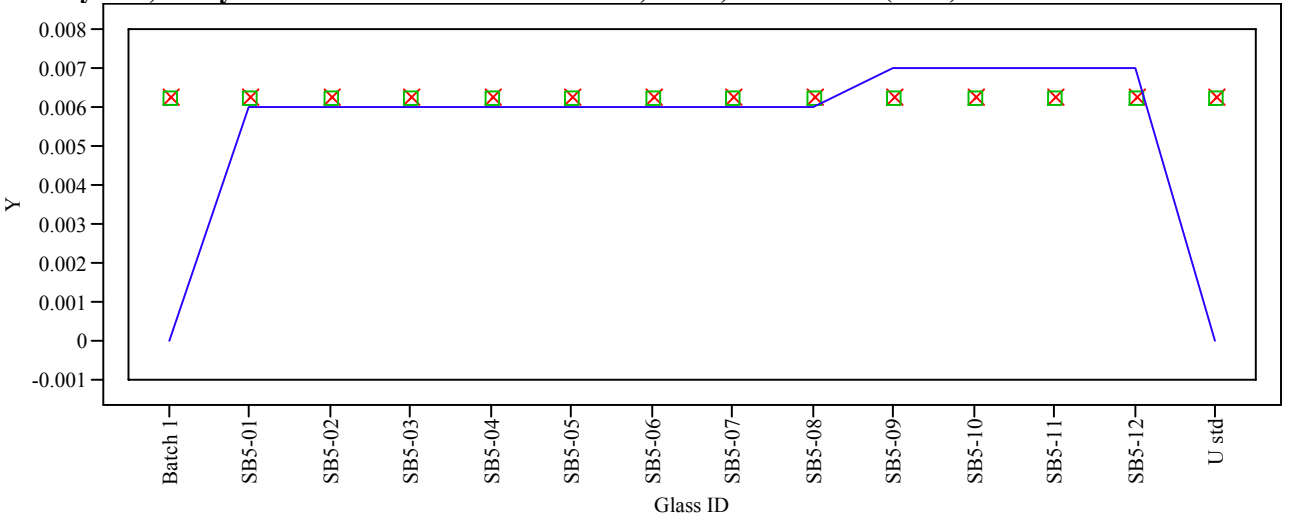

$\mathrm{Y} \times$ Measured $\square$ Measured bc Targeted 
Exhibit A5. Measured, Measured bc, and Targeted Concentrations by Oxide by Glass ID for each Memo and Analytical Set. (continued)

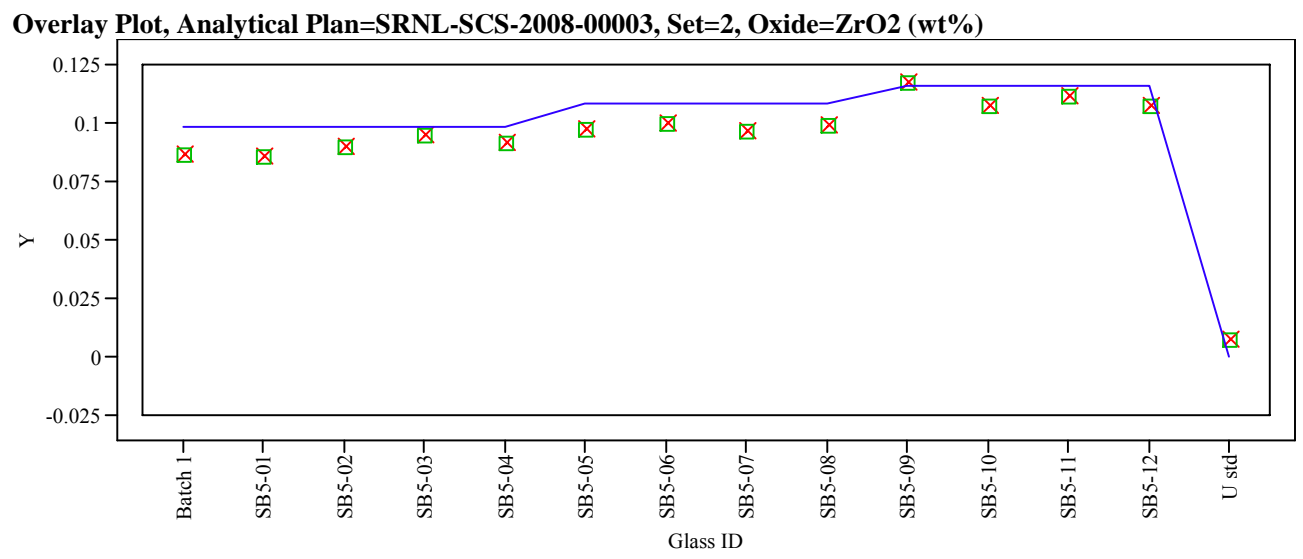

Overlay Plot, Analytical Plan=SRNL-SCS-2008-00003, Set=2, Oxide $=$ Sum of Oxides

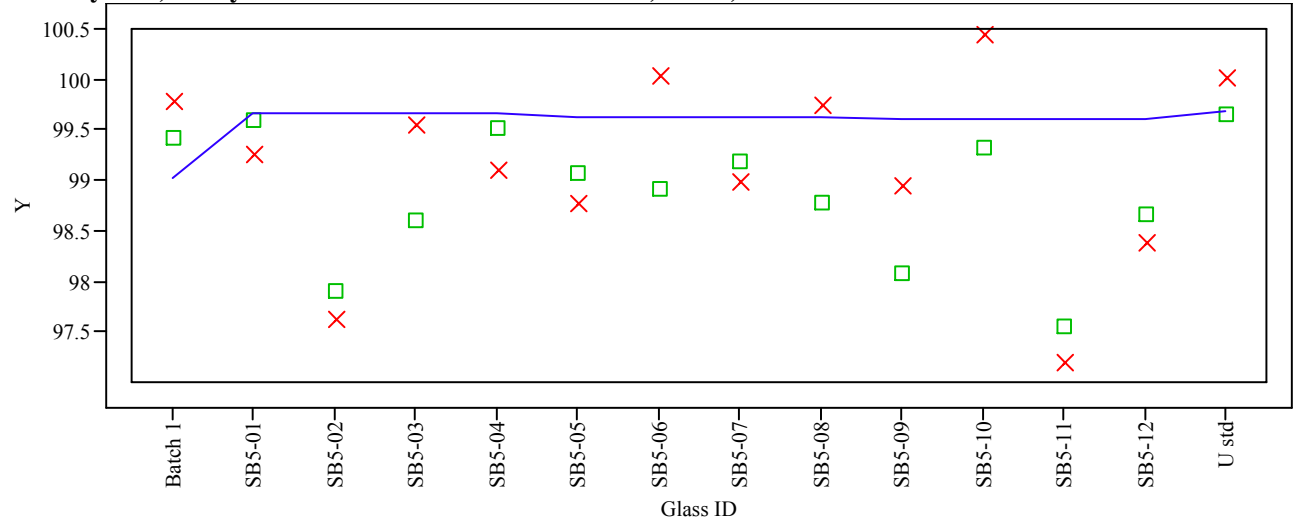

Overlay Plot, Analytical Plan=SRNL-SCS-2008-00003, Set=3, Oxide=Al2O3 (wt\%)

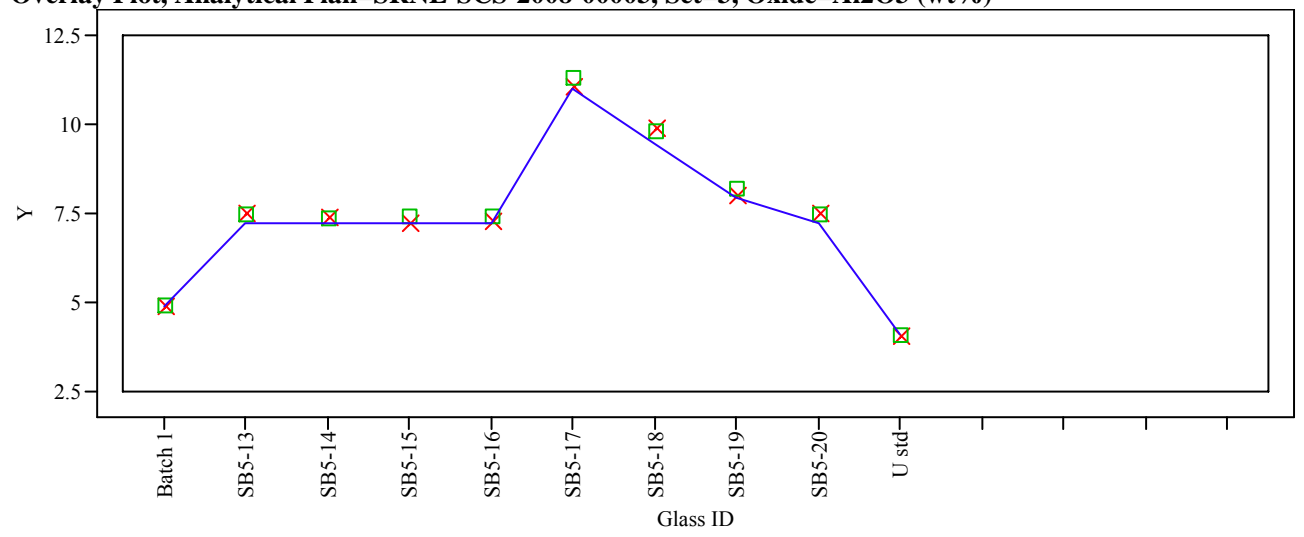

$\mathrm{Y} \times$ Measured $\square$ Measured bc $\quad$ Targeted 
Exhibit A5. Measured, Measured bc, and Targeted Concentrations by Oxide by Glass ID for each Memo and Analytical Set. (continued)

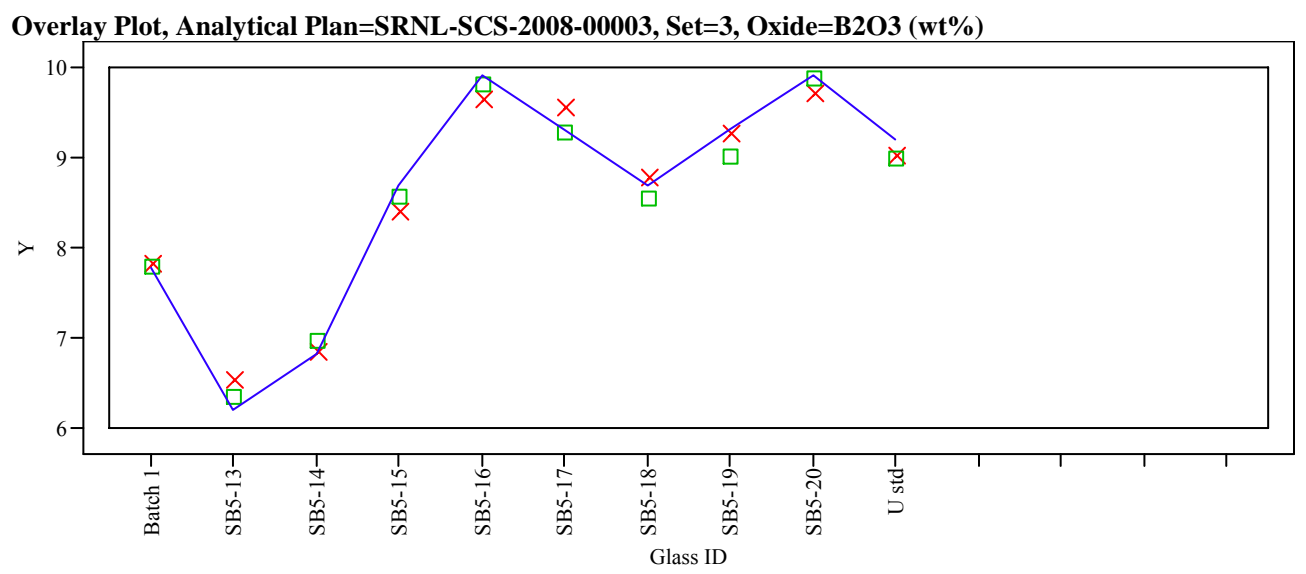

Overlay Plot, Analytical Plan=SRNL-SCS-2008-00003, Set=3, Oxide=BaO (wt\%)

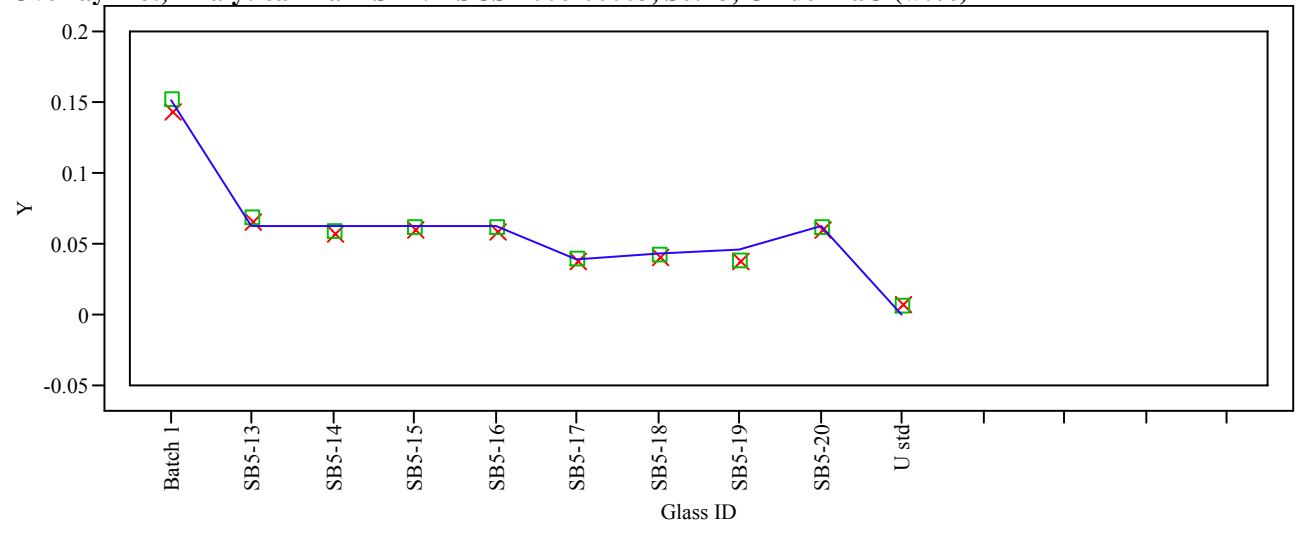

Overlay Plot, Analytical Plan=SRNL-SCS-2008-00003, Set=3, Oxide=CaO (wt \%)

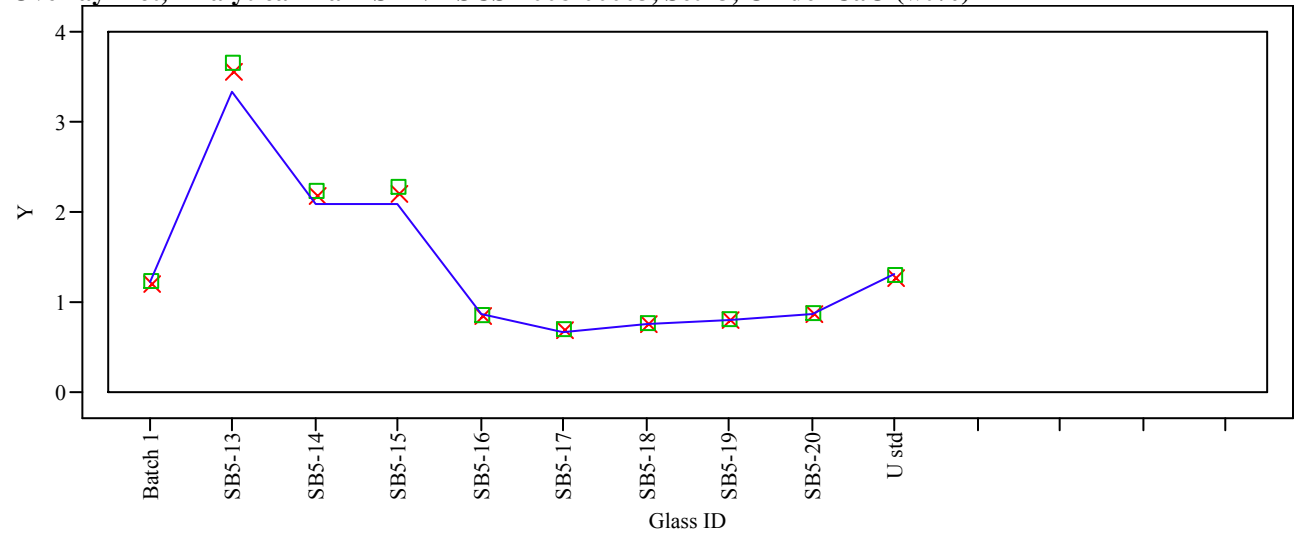

$\mathrm{Y} \times$ Measured $\square$ Measured bc $\quad$ Targeted 
SRNS-STI-2008-00060

Revision 0

Appendix A

Exhibit A5. Measured, Measured bc, and Targeted Concentrations by Oxide by Glass ID for each Memo and Analytical Set. (continued)
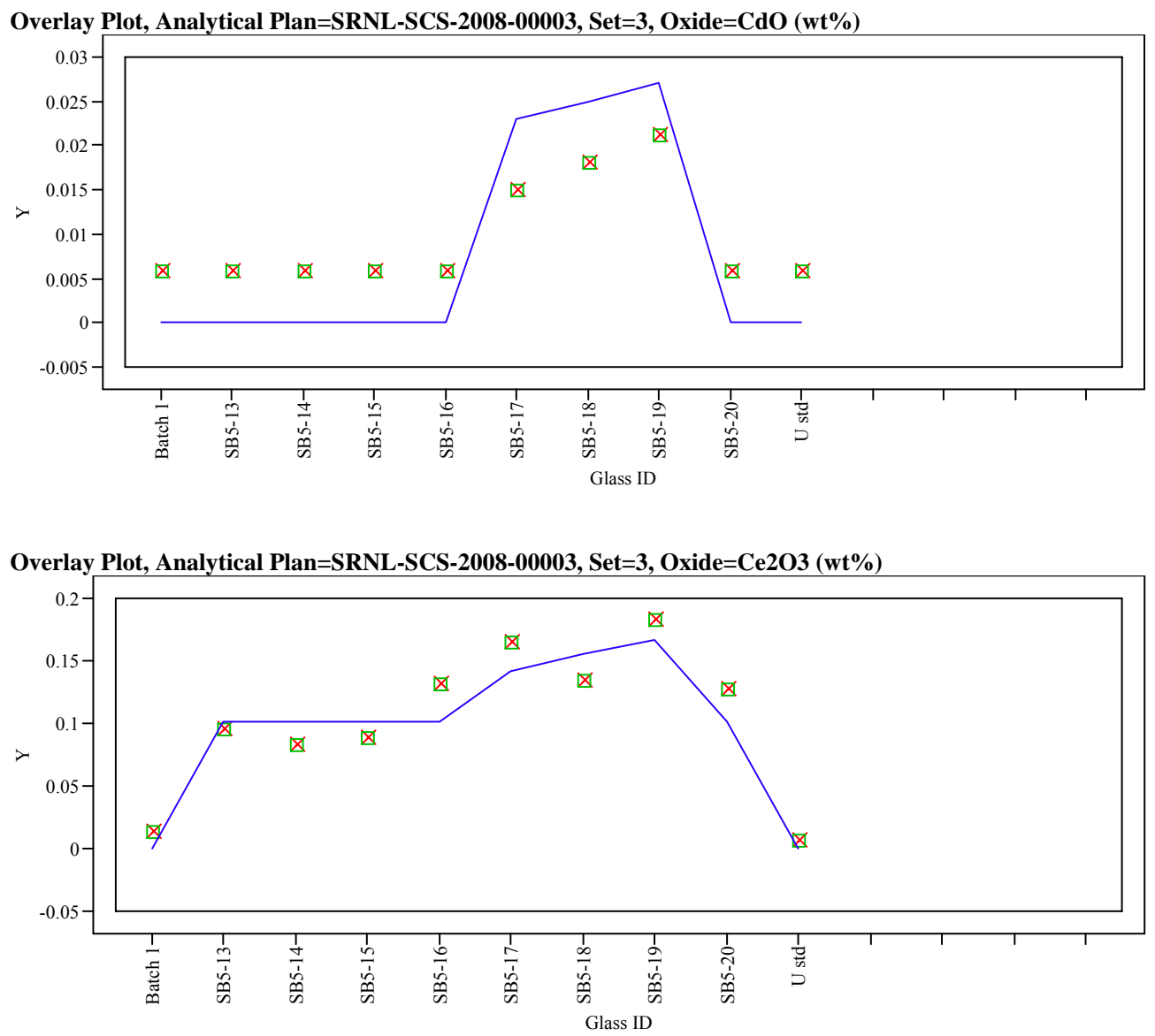

Overlay Plot, Analytical Plan=SRNL-SCS-2008-00003, Set=3, Oxide=Cr2O3 (wt\%)

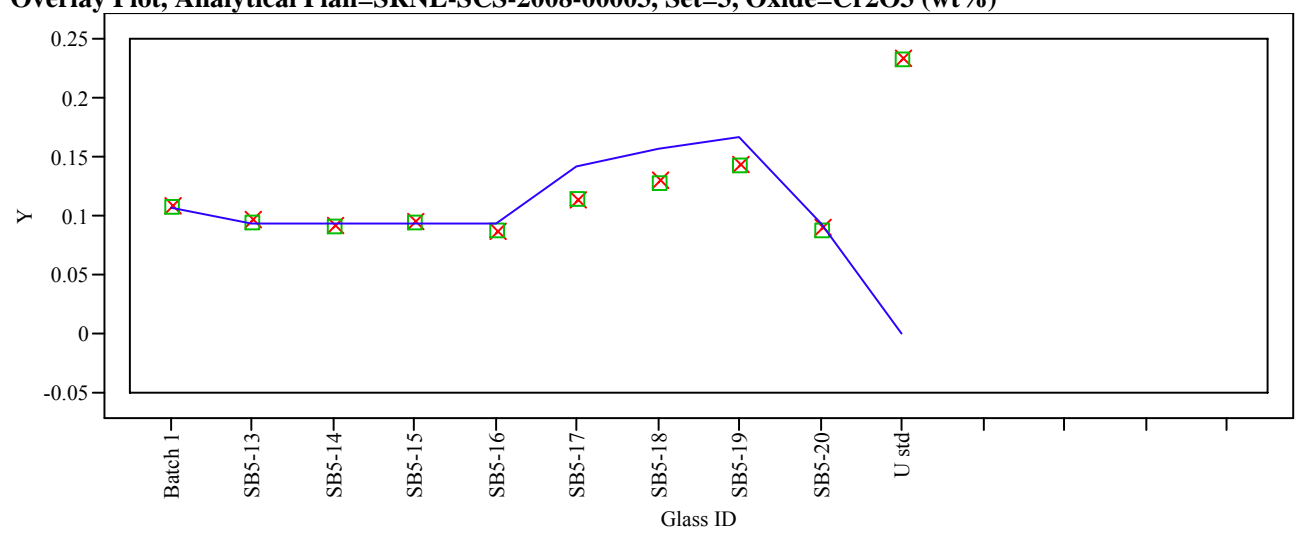

Y $\times$ Measured $\square$ Measured bc - Targeted 
SRNS-STI-2008-00060

Revision 0

Appendix A

Exhibit A5. Measured, Measured bc, and Targeted Concentrations by Oxide by Glass ID for each Memo and Analytical Set. (continued)

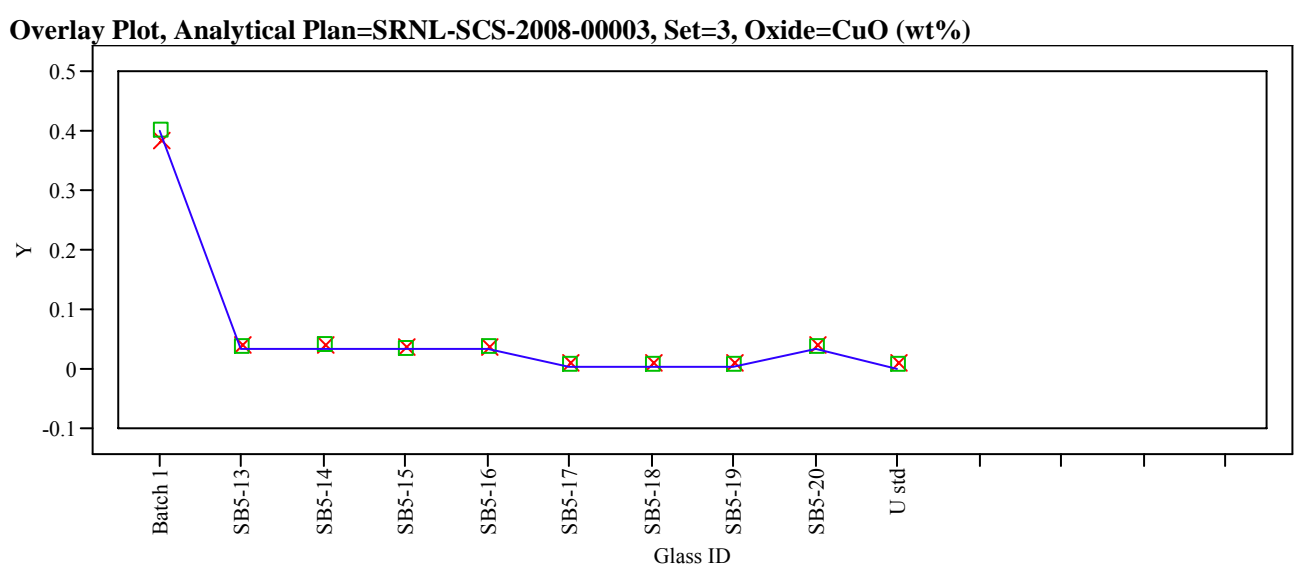

Overlay Plot, Analytical Plan=SRNL-SCS-2008-00003, Set=3, Oxide=Fe2O3 (wt\%)

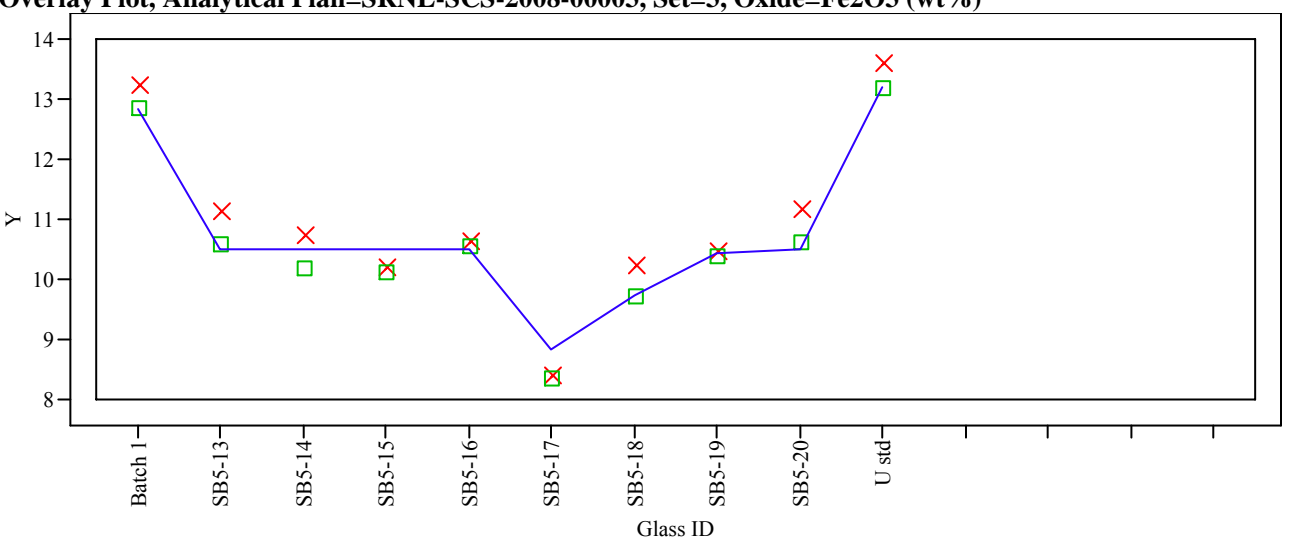

Overlay Plot, Analytical Plan=SRNL-SCS-2008-00003, Set=3, Oxide=K2O (wt\%)

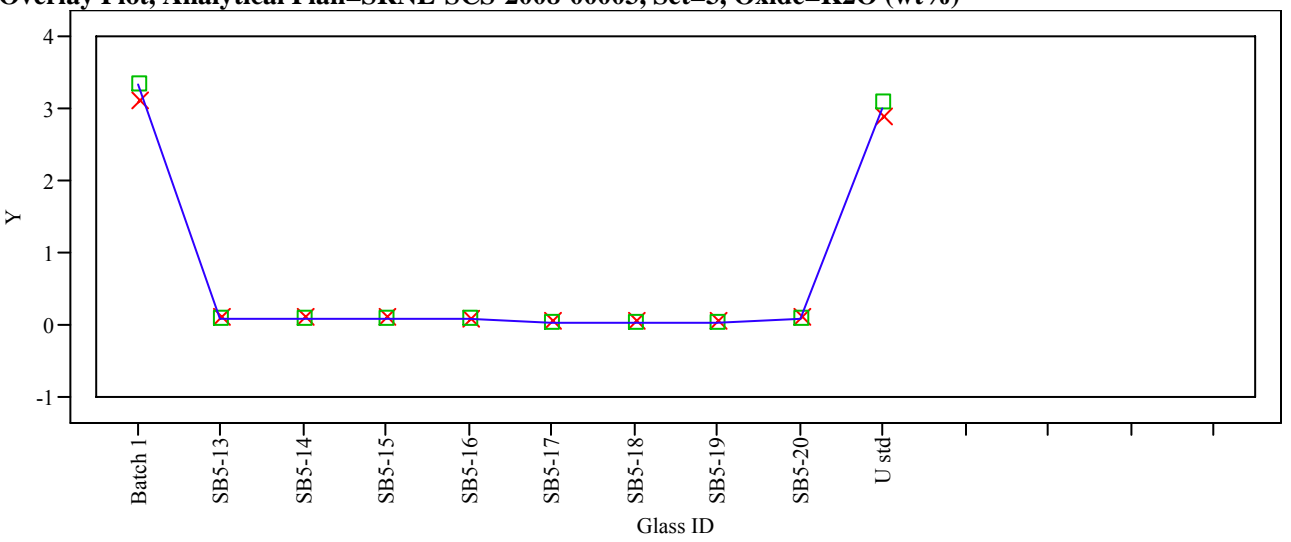

$\mathrm{Y} \times$ Measured $\quad$ Measured bc Targeted 
Exhibit A5. Measured, Measured bc, and Targeted Concentrations by Oxide by Glass ID for each Memo and Analytical Set. (continued)
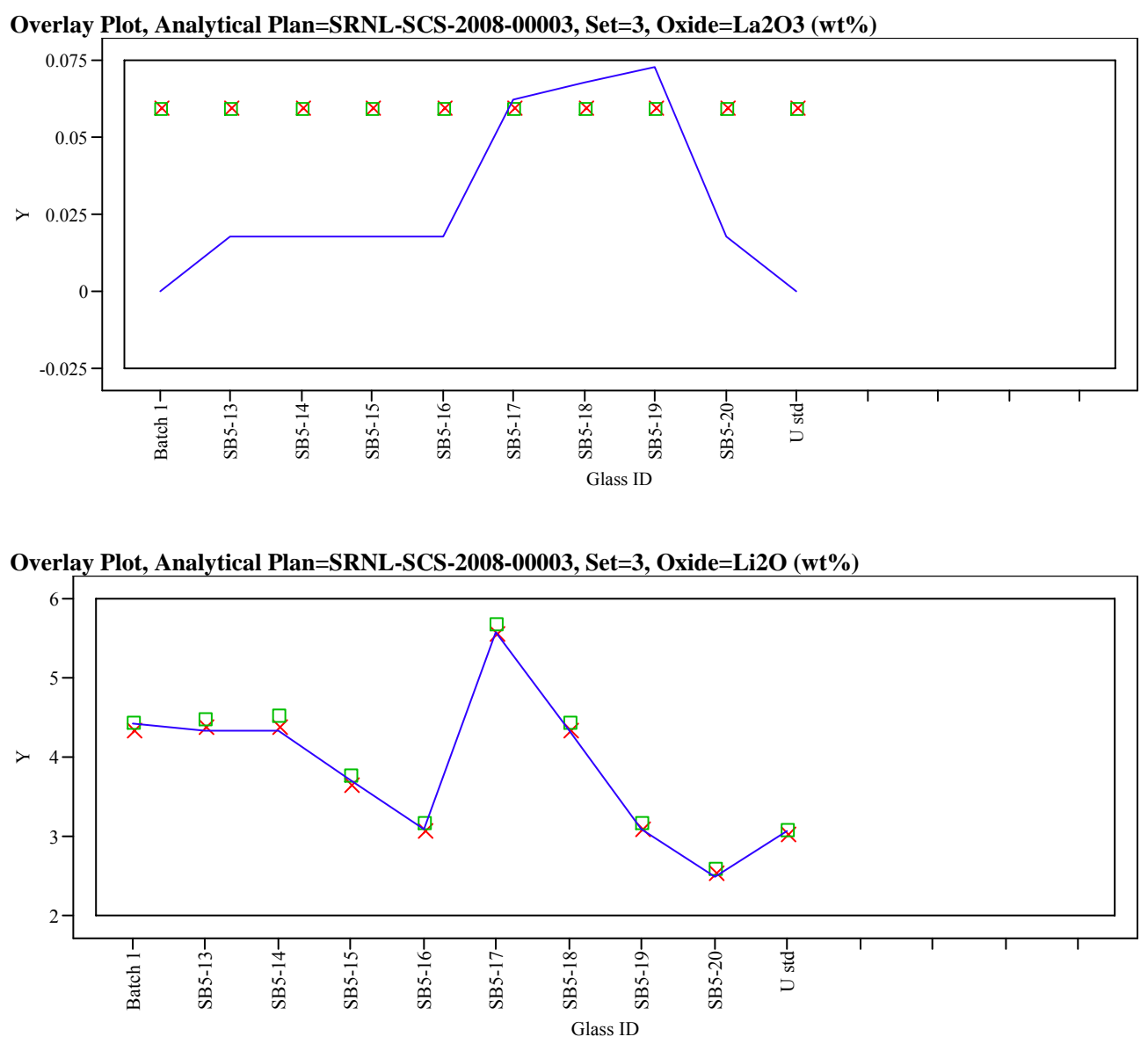

Overlay Plot, Analytical Plan=SRNL-SCS-2008-00003, Set=3, Oxide=MgO (wt\%)

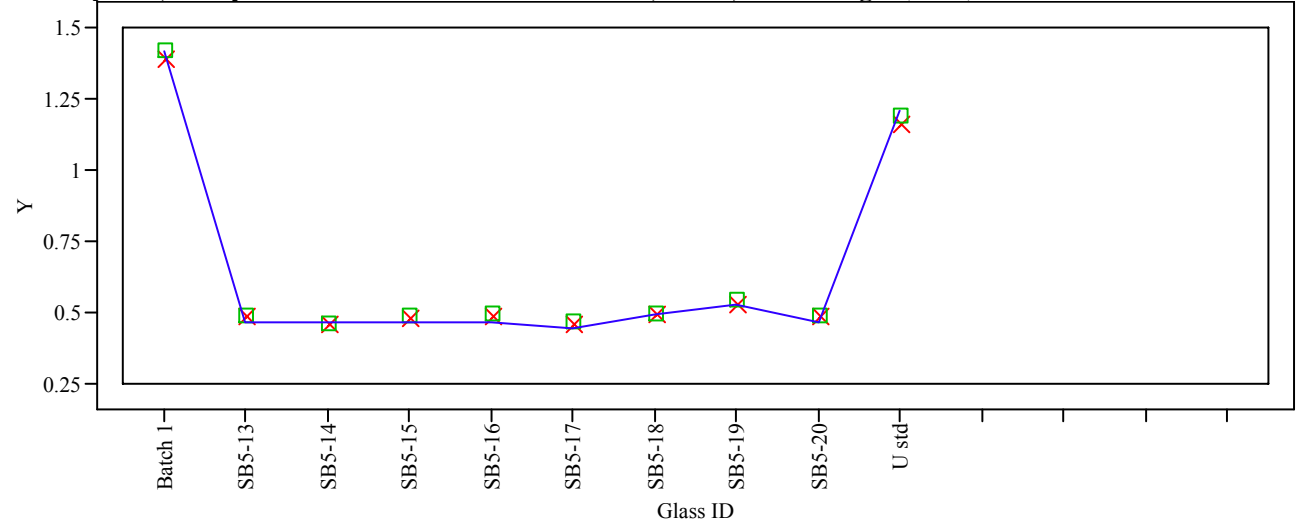

$\mathrm{Y} \times$ Measured $\quad$ Measured bc Targeted 
Exhibit A5. Measured, Measured bc, and Targeted Concentrations by Oxide by Glass ID for each Memo and Analytical Set. (continued)
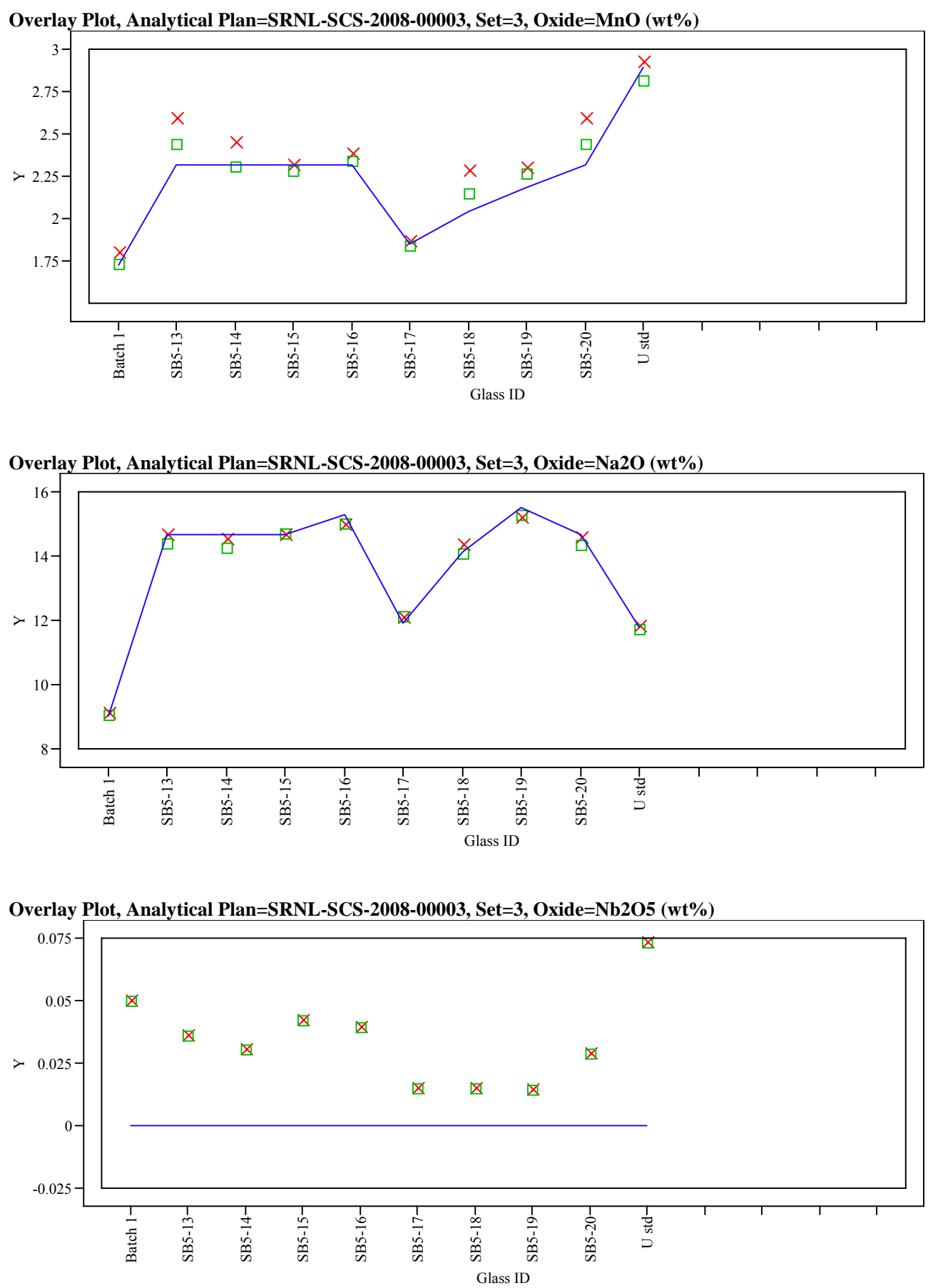

$\mathrm{Y} \times$ Measured $\quad$ Measured bc $\quad$ Targeted 
Exhibit A5. Measured, Measured bc, and Targeted Concentrations by Oxide by Glass ID for each Memo and Analytical Set. (continued)
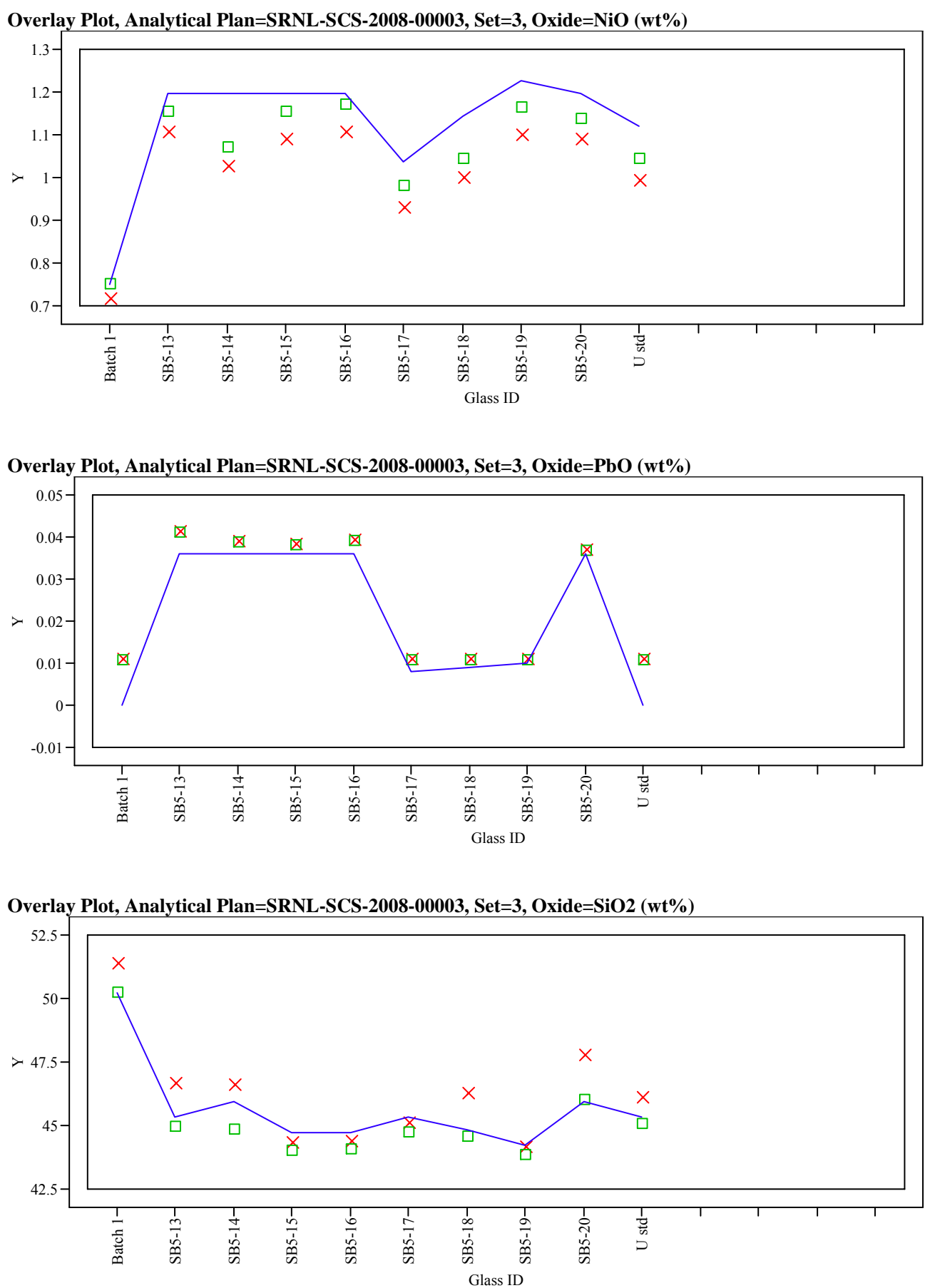

$\mathrm{Y} \times$ Measured $\square$ Measured bc $\quad$ Targeted 
Exhibit A5. Measured, Measured bc, and Targeted Concentrations by Oxide by Glass ID for each Memo and Analytical Set. (continued)
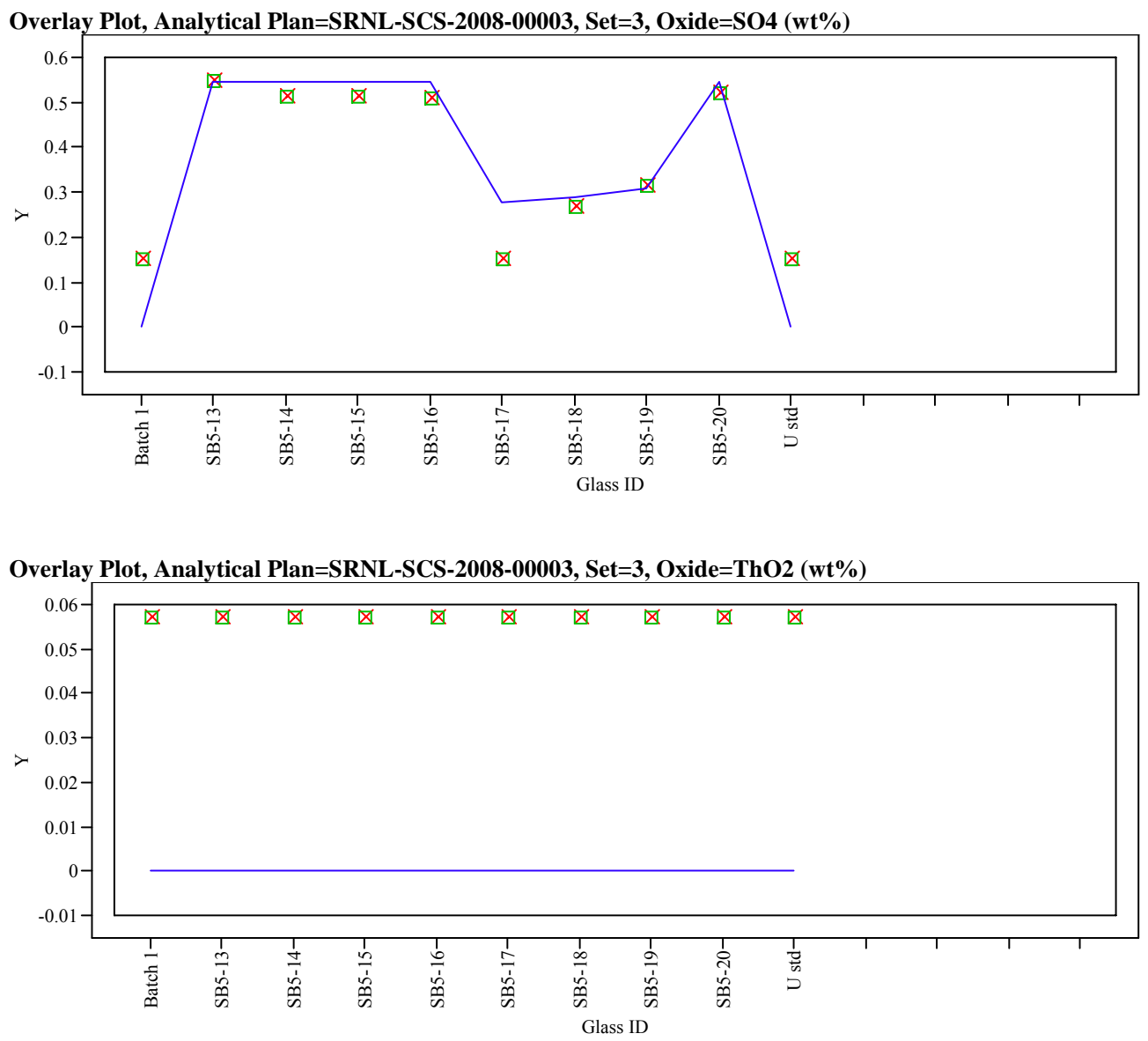

Overlay Plot, Analytical Plan=SRNL-SCS-2008-00003, Set=3, Oxide=TiO2 (wt\%)

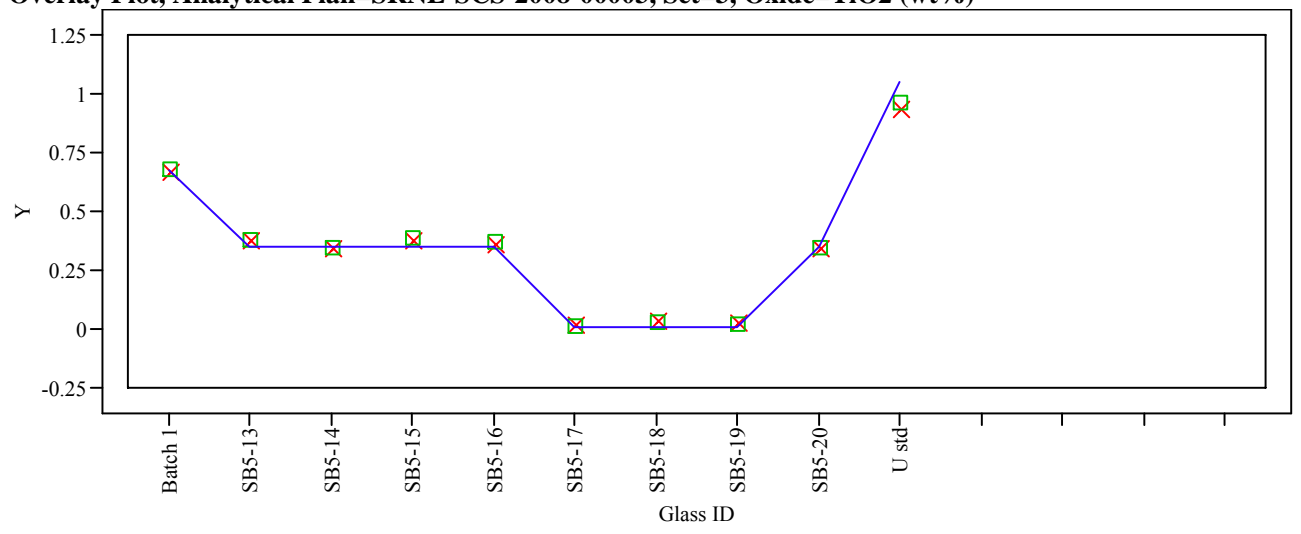

$\mathrm{Y} \times$ Measured $\square$ Measured bc $\quad$ Targeted 
Exhibit A5. Measured, Measured bc, and Targeted Concentrations by Oxide by Glass ID for each Memo and Analytical Set. (continued)
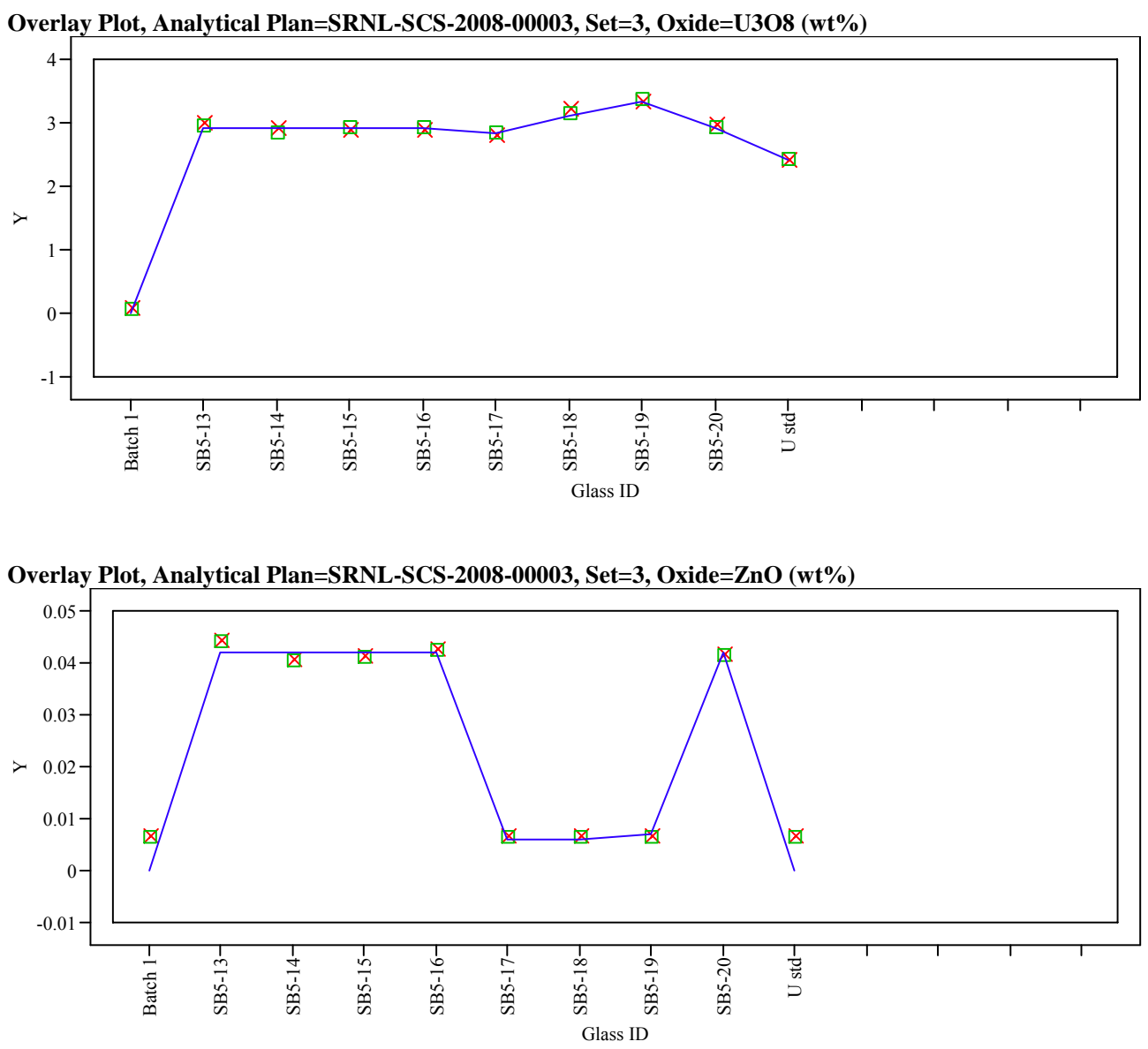

Overlay Plot, Analytical Plan=SRNL-SCS-2008-00003, Set=3, Oxide=ZrO2 (wt \%)

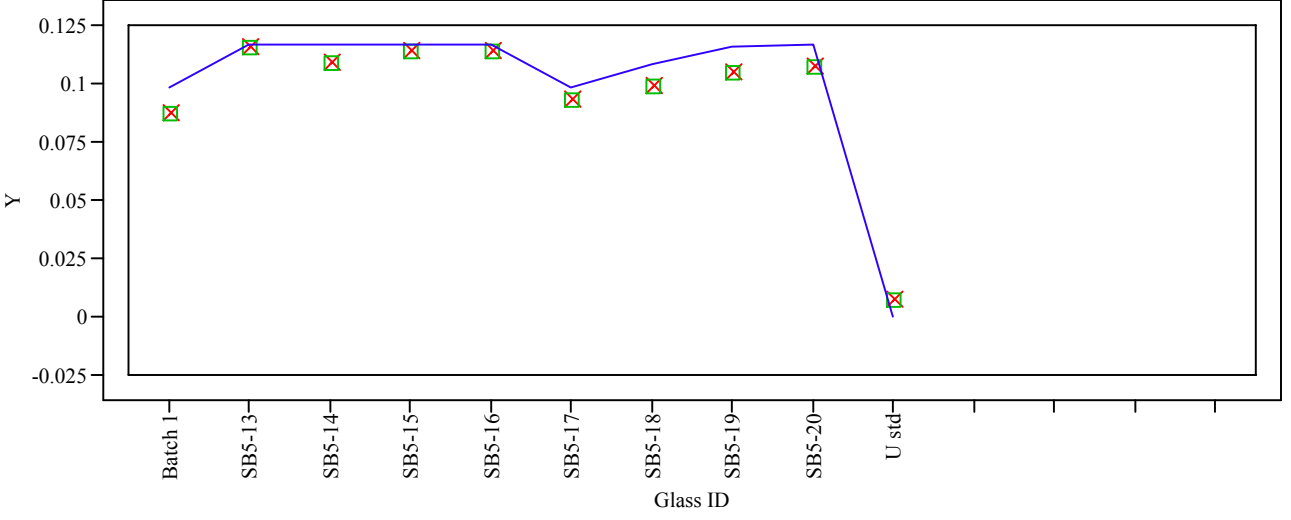

Y $\times$ Measured $\quad$ Measured bc - Targeted 
Exhibit A5. Measured, Measured bc, and Targeted Concentrations by Oxide by Glass ID for each Memo and Analytical Set. (continued)

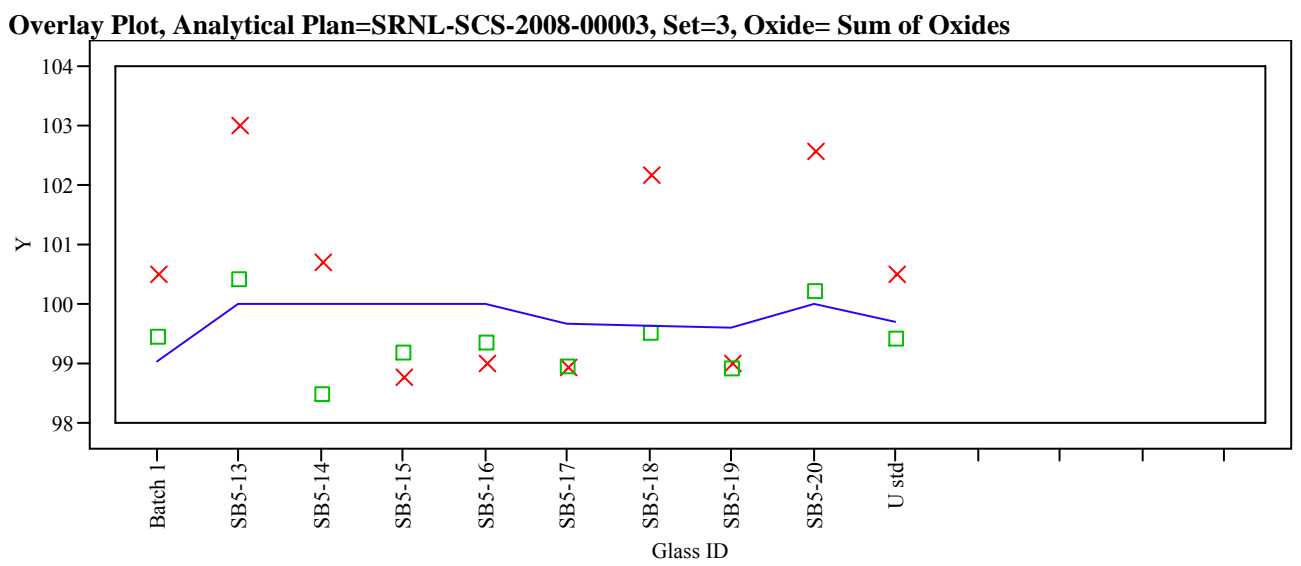

Overlay Plot, Analytical Plan=SRNL-SCS-2008-00043, Set=1, Oxide=Al2O3 (wt\%)

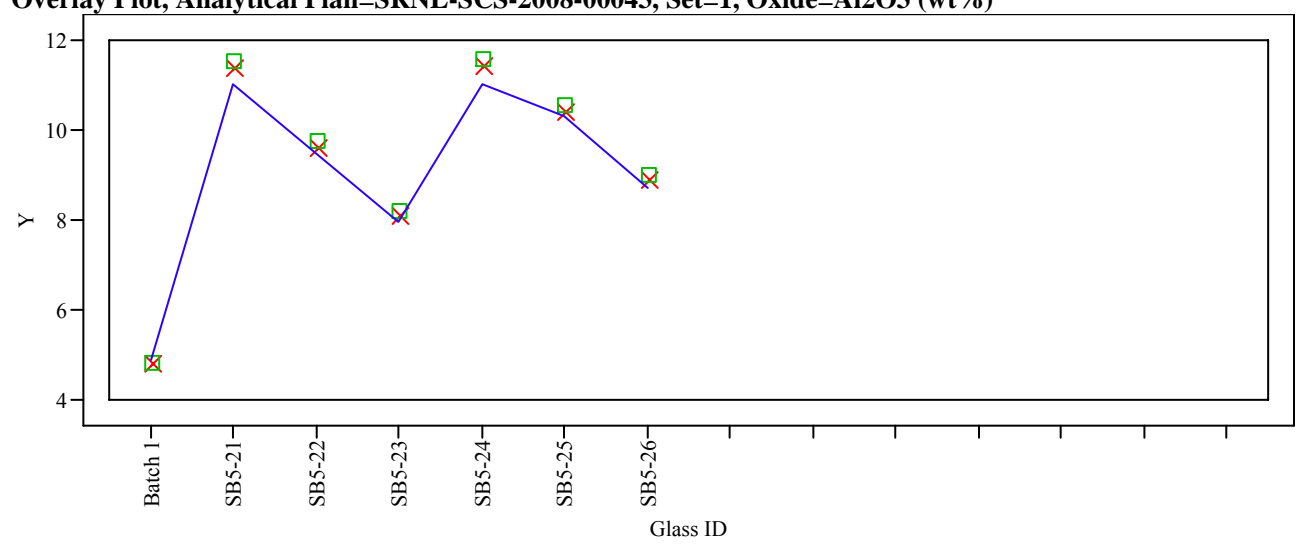

Overlay Plot, Analytical Plan=SRNL-SCS-2008-00043, Set=1, Oxide=B2O3 (wt\%)

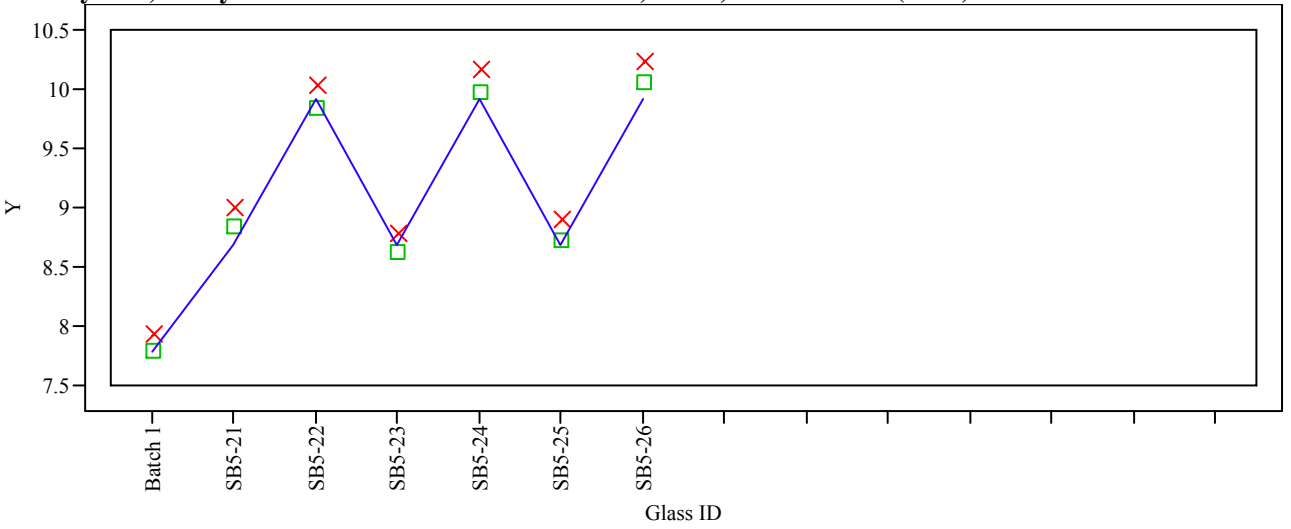

$\mathrm{Y} \times$ Measured $\square$ Measured bc $\quad$ Targeted 
SRNS-STI-2008-00060

Revision 0

Appendix A

Exhibit A5. Measured, Measured bc, and Targeted Concentrations by Oxide by Glass ID for each Memo and Analytical Set. (continued)

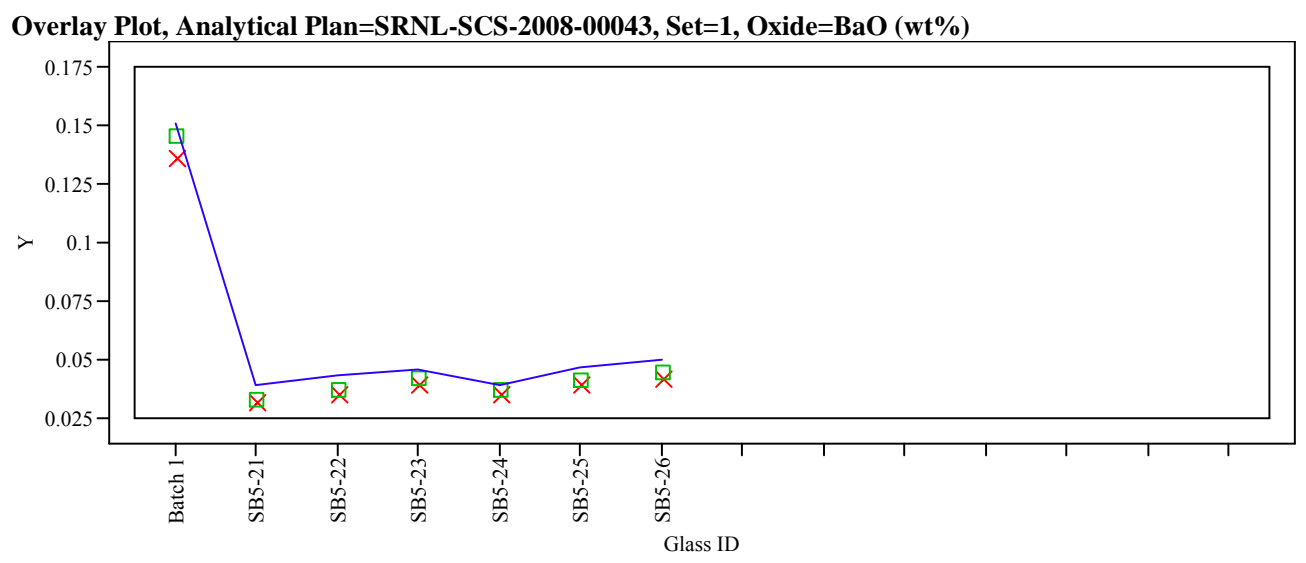

Overlay Plot, Analytical Plan=SRNL-SCS-2008-00043, Set=1, Oxide=CaO (wt\%)

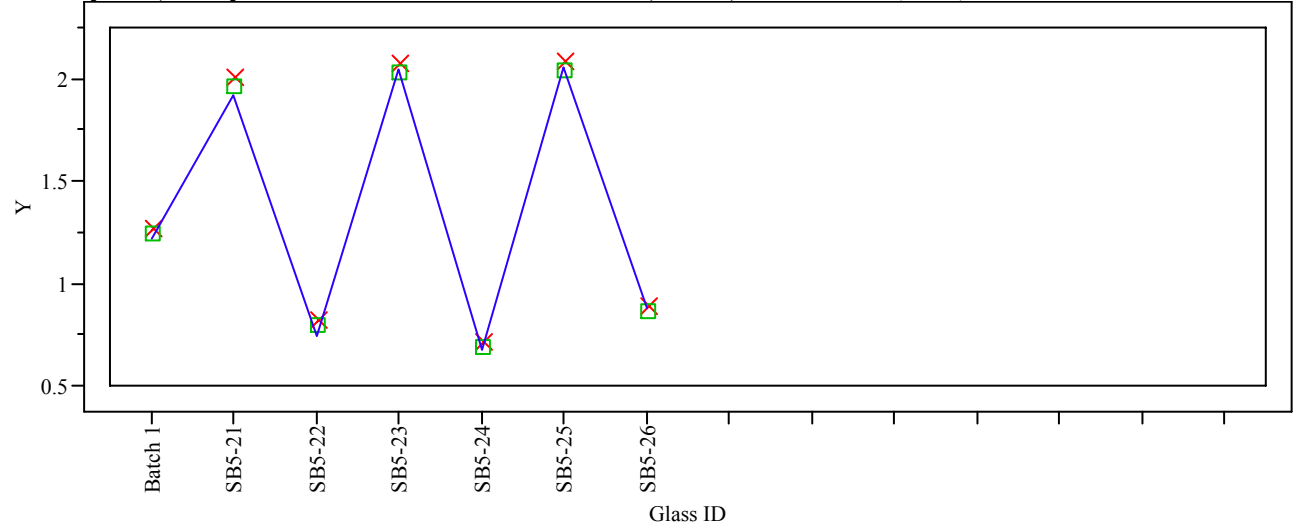

Overlay Plot, Analytical Plan=SRNL-SCS-2008-00043, Set=1, Oxide=Ce2O3 (wt\%)

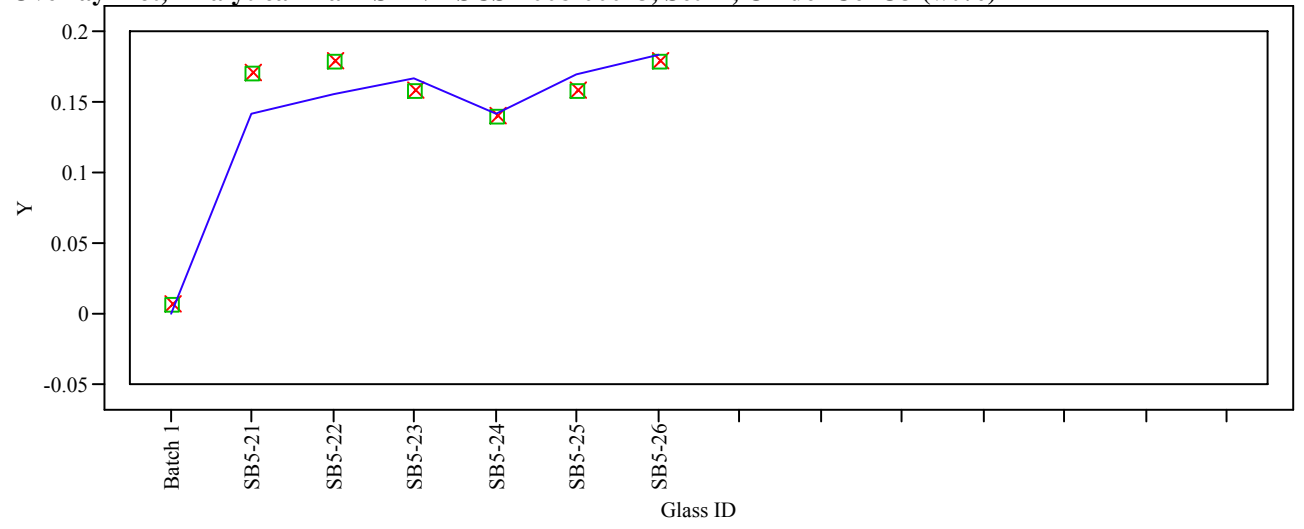

$\mathrm{Y} \times$ Measured $\square$ Measured bc Targeted 
SRNS-STI-2008-00060

Revision 0

Appendix A

Exhibit A5. Measured, Measured bc, and Targeted Concentrations by Oxide by Glass ID for each Memo and Analytical Set. (continued)
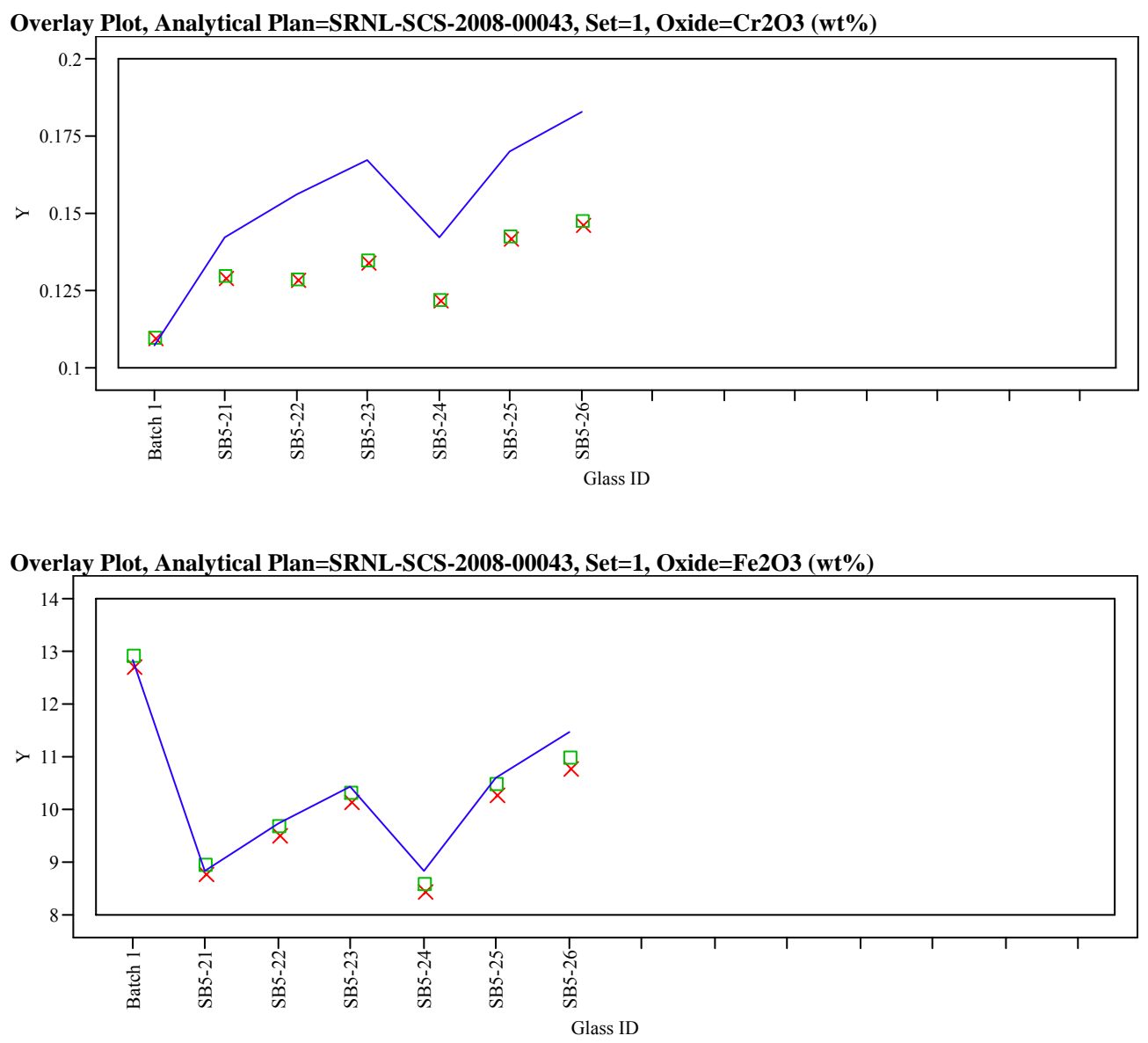

Overlay Plot, Analytical Plan=SRNL-SCS-2008-00043, Set=1, Oxide=HfO2 (wt\%)

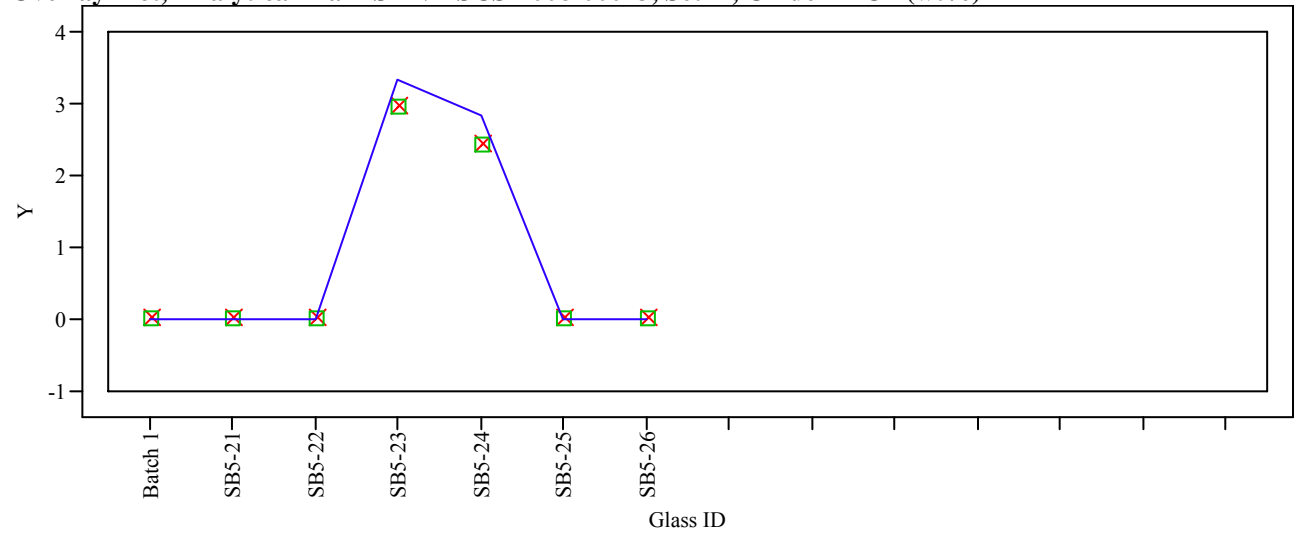

$\mathrm{Y} \times$ Measured $\square$ Measured bc Targeted 
SRNS-STI-2008-00060

Revision 0

Appendix A

Exhibit A5. Measured, Measured bc, and Targeted Concentrations by Oxide by Glass ID for each Memo and Analytical Set. (continued)

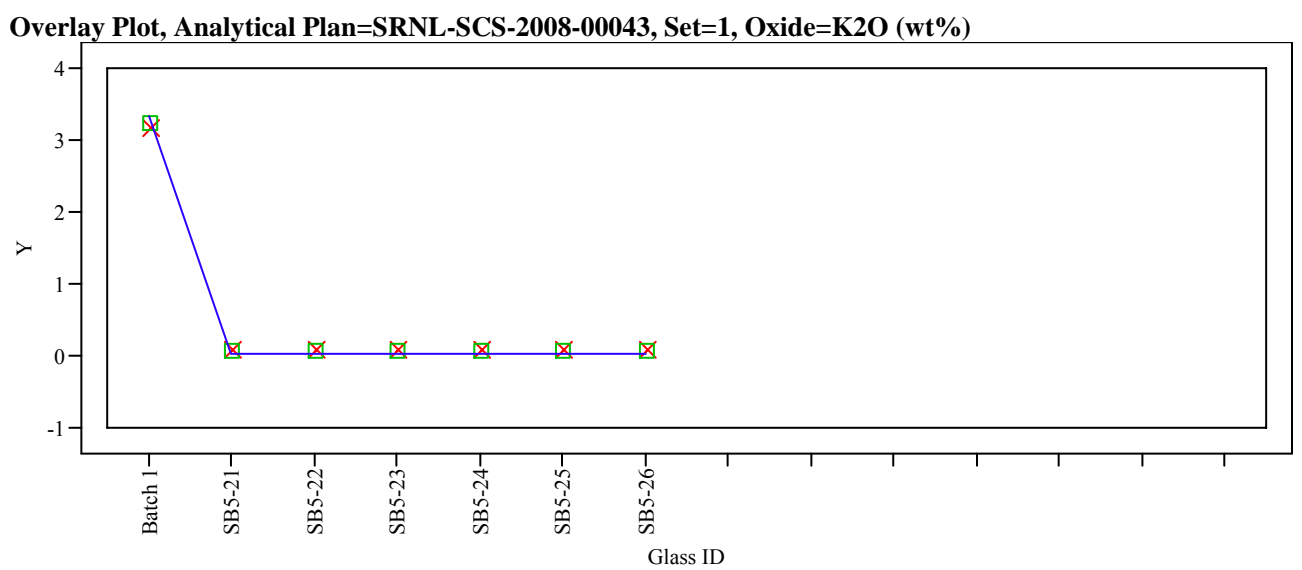

Overlay Plot, Analytical Plan=SRNL-SCS-2008-00043, Set=1, Oxide=La2O3 (wt\%)

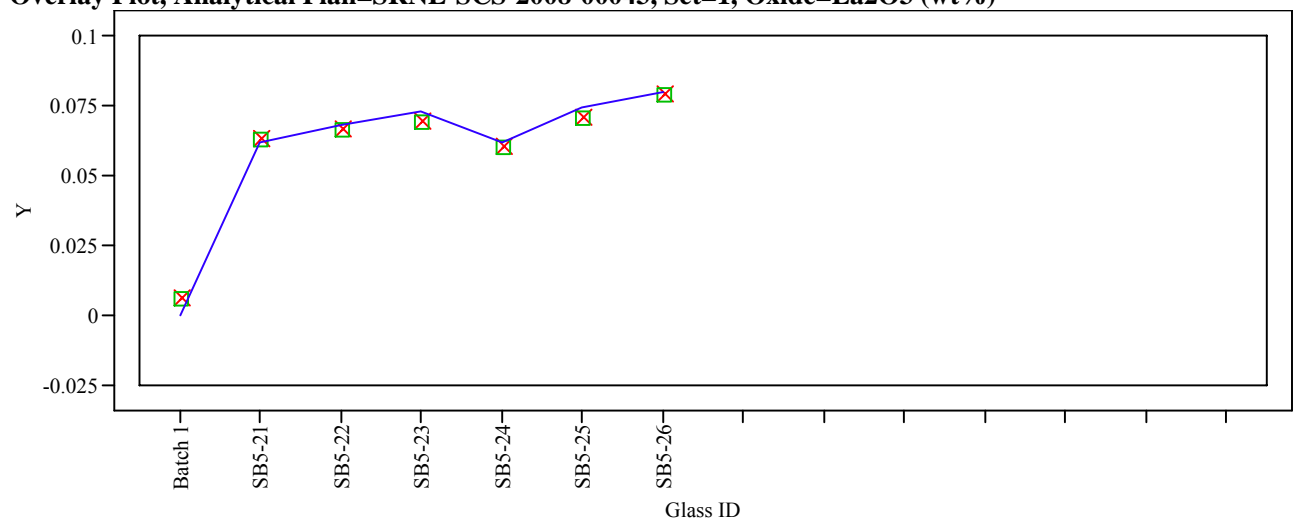

Overlay Plot, Analytical Plan=SRNL-SCS-2008-00043, Set=1, Oxide=Li2O (wt\%)

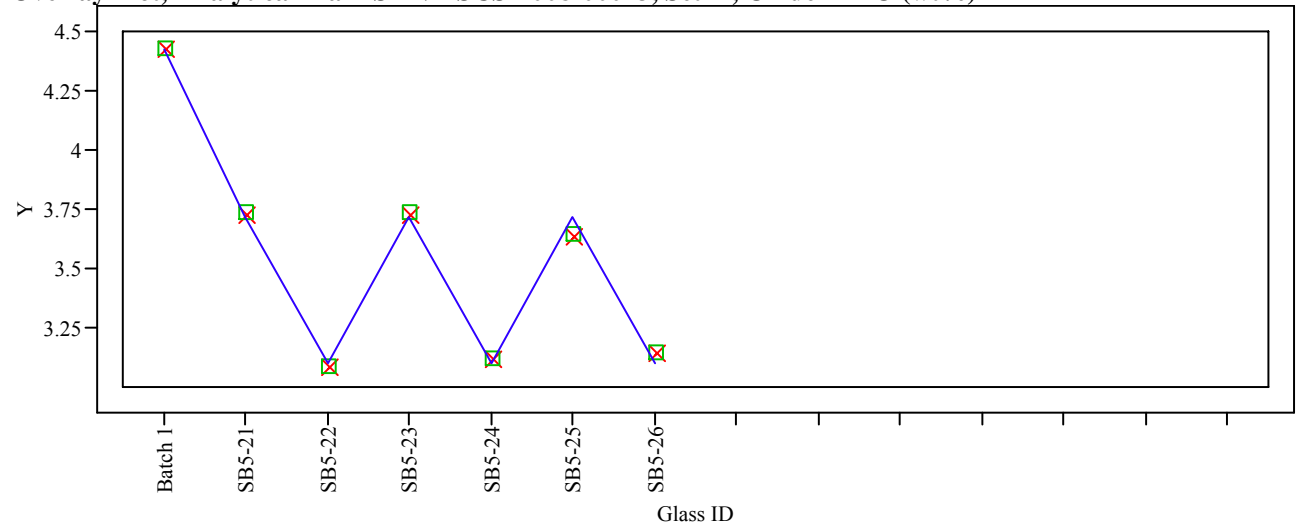

$\mathrm{Y} \times$ Measured $\square$ Measured bc Targeted 
SRNS-STI-2008-00060

Revision 0

Appendix A

Exhibit A5. Measured, Measured bc, and Targeted Concentrations by Oxide by Glass ID for each Memo and Analytical Set. (continued)
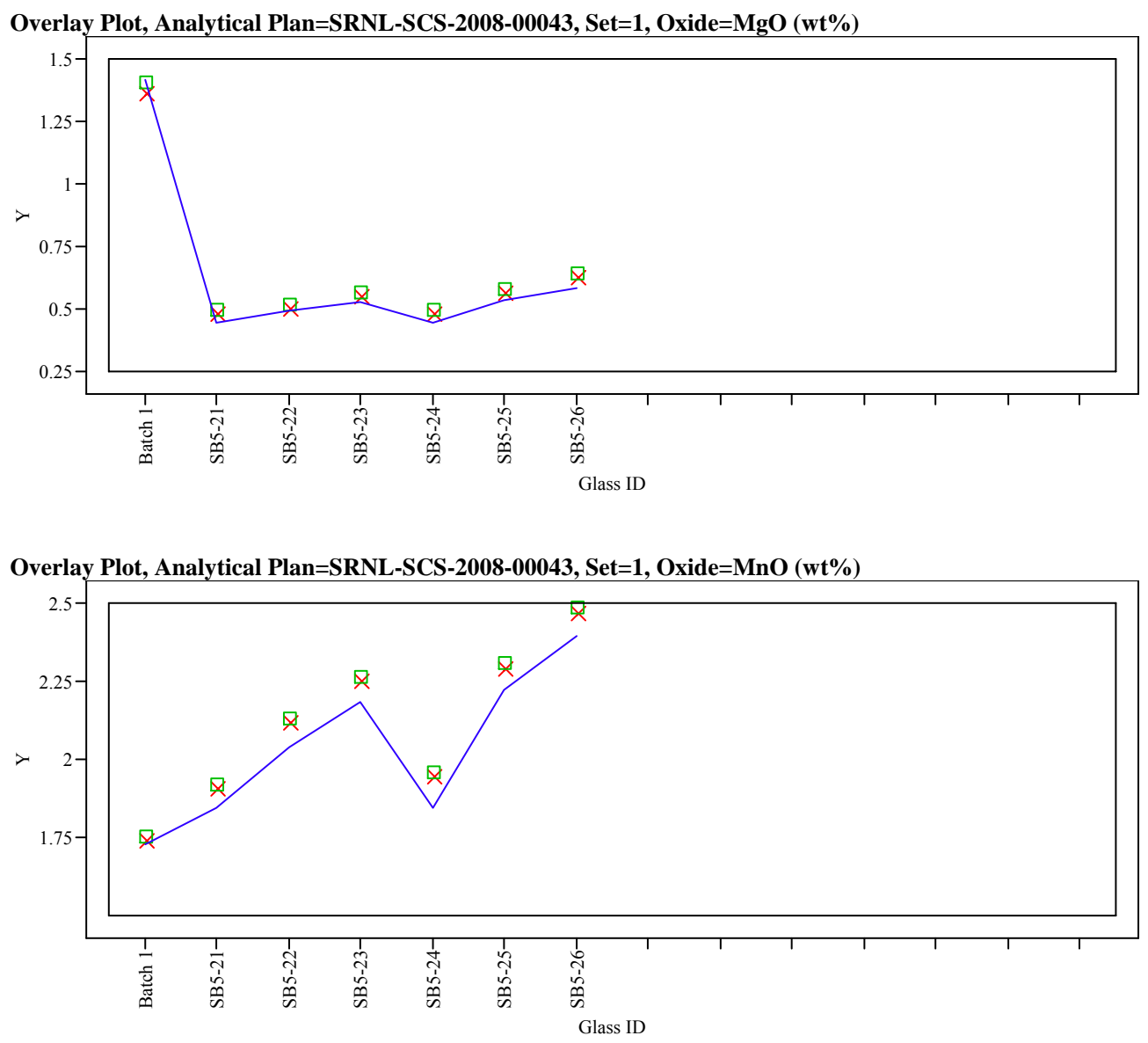

Overlay Plot, Analytical Plan=SRNL-SCS-2008-00043, Set=1, Oxide=Na2O (wt\%)

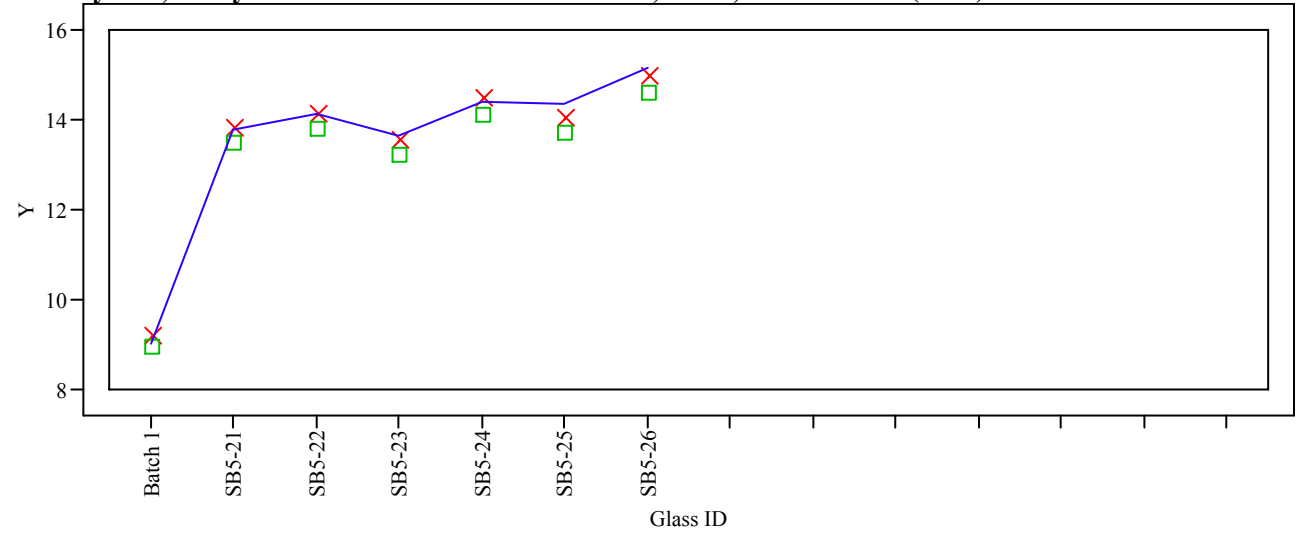

$\mathrm{Y} \times$ Measured $\quad$ Measured bc - Targeted 
SRNS-STI-2008-00060

Revision 0

Appendix A

Exhibit A5. Measured, Measured bc, and Targeted Concentrations by Oxide by Glass ID for each Memo and Analytical Set. (continued)
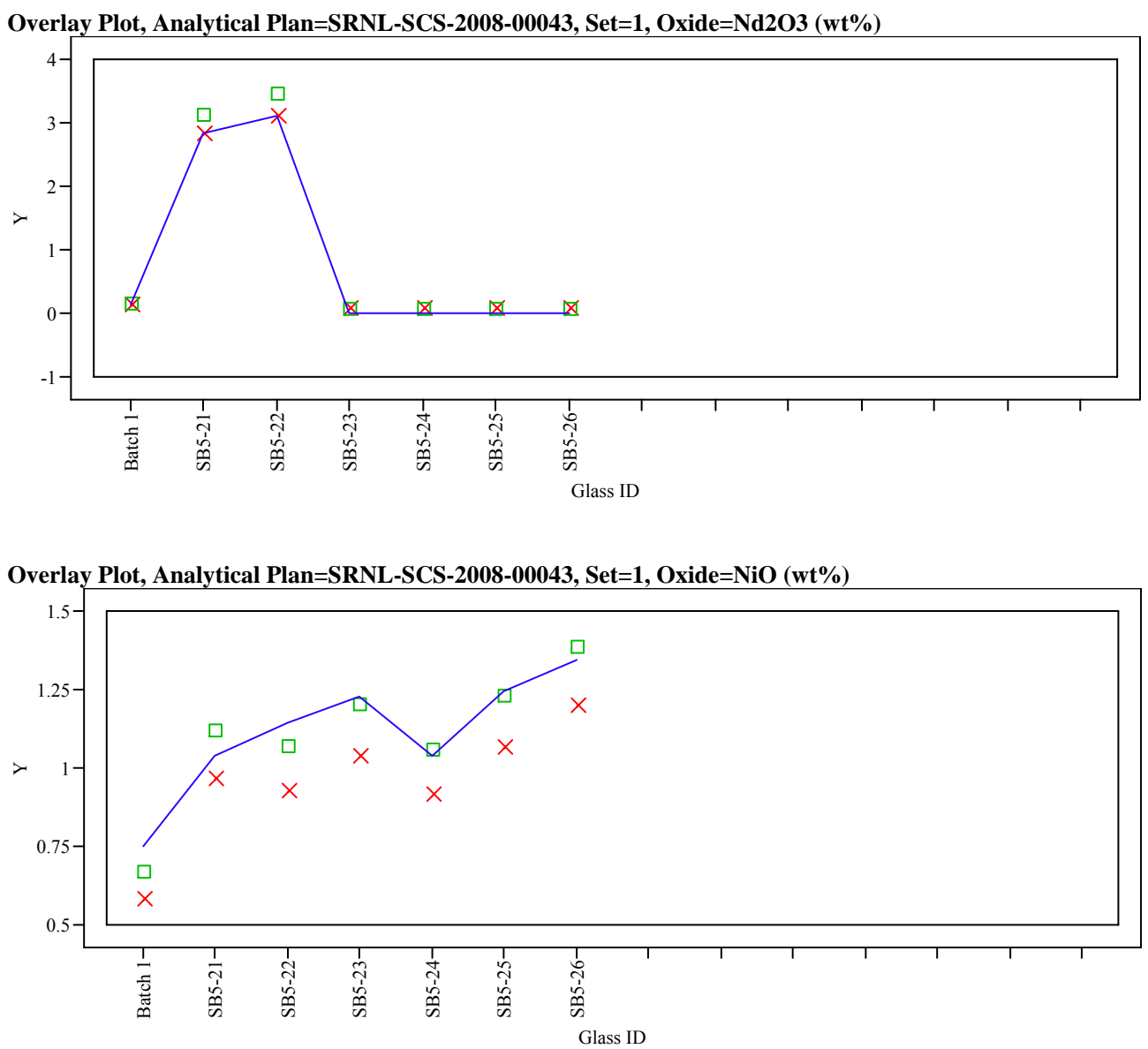

Overlay Plot, Analytical Plan=SRNL-SCS-2008-00043, Set=1, Oxide=PbO (wt\%)

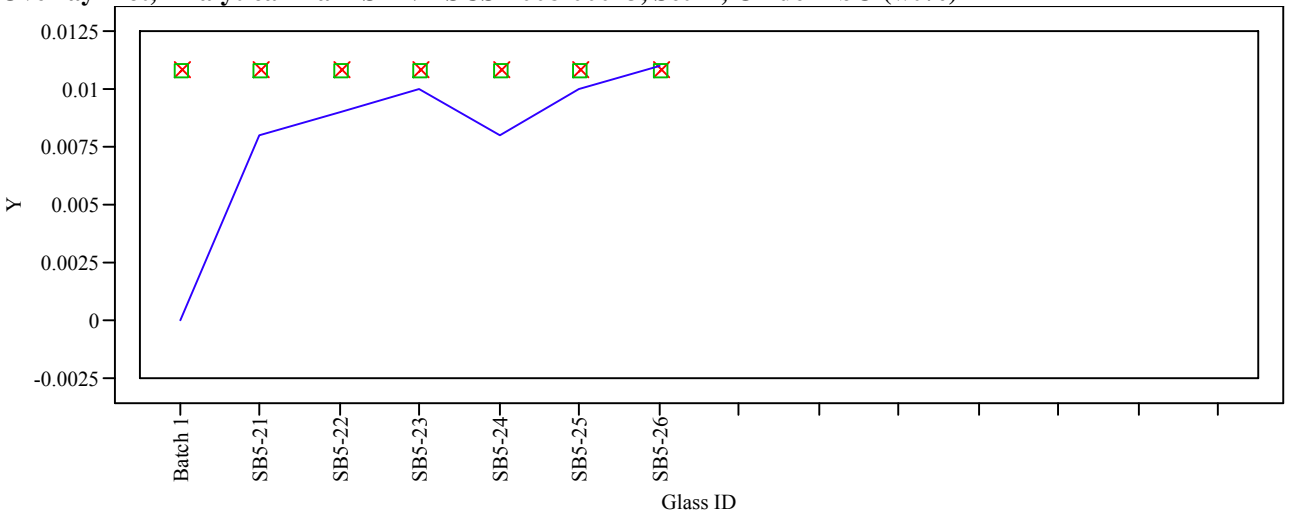

$\mathrm{Y} \times$ Measured $\square$ Measured bc Targeted 
Exhibit A5. Measured, Measured bc, and Targeted Concentrations by Oxide by Glass ID for each Memo and Analytical Set. (continued)
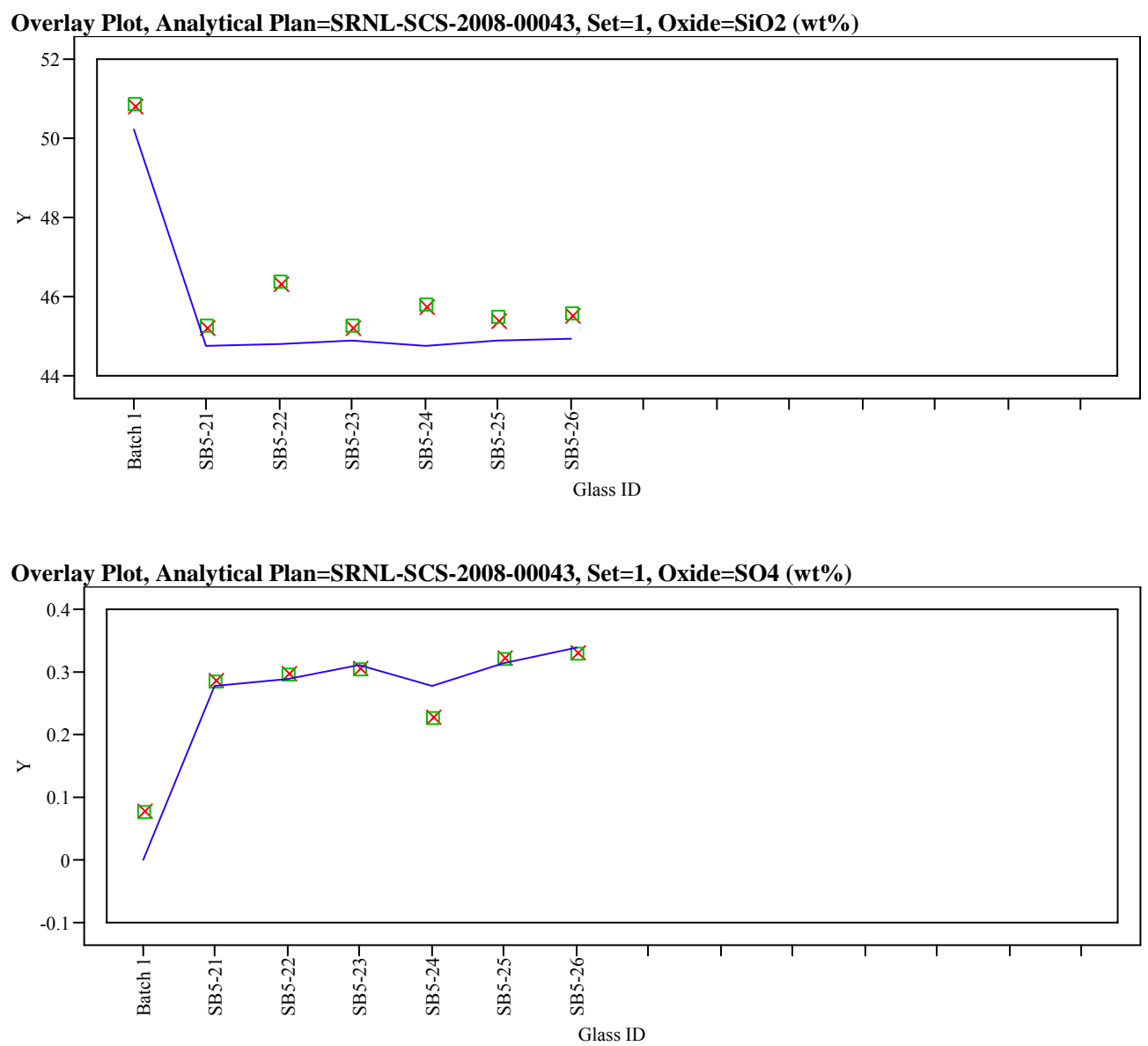

Overlay Plot, Analytical Plan=SRNL-SCS-2008-00043, Set=1, Oxide=TiO2 (wt\%)

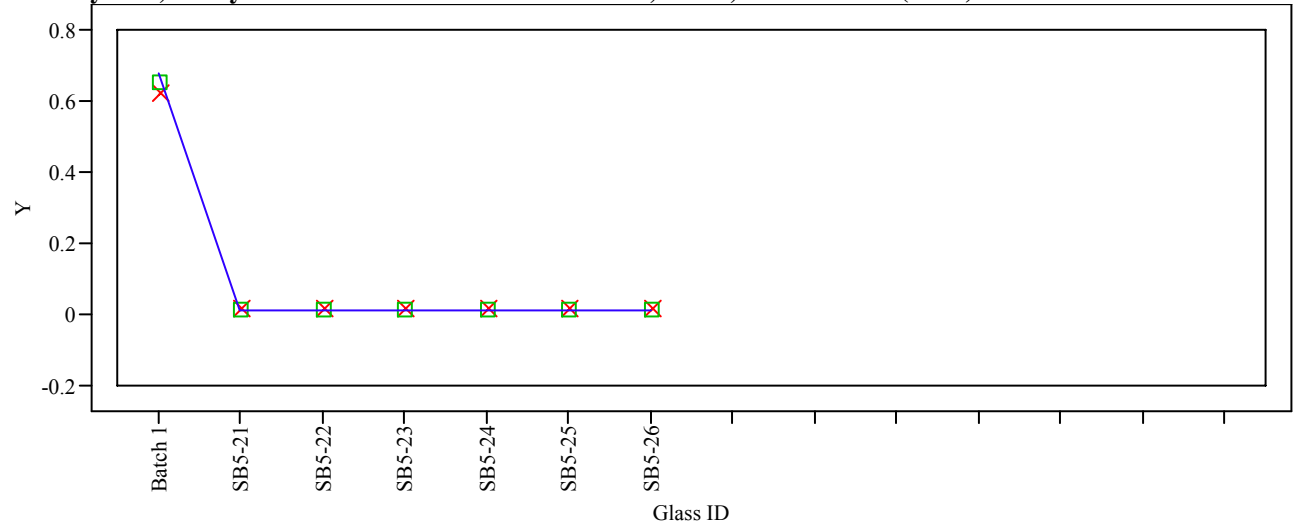

$\mathrm{Y} \times$ Measured $\square$ Measured bc $\quad$ Targeted 
SRNS-STI-2008-00060

Revision 0

Appendix A

Exhibit A5. Measured, Measured bc, and Targeted Concentrations by Oxide by Glass ID for each Memo and Analytical Set. (continued)
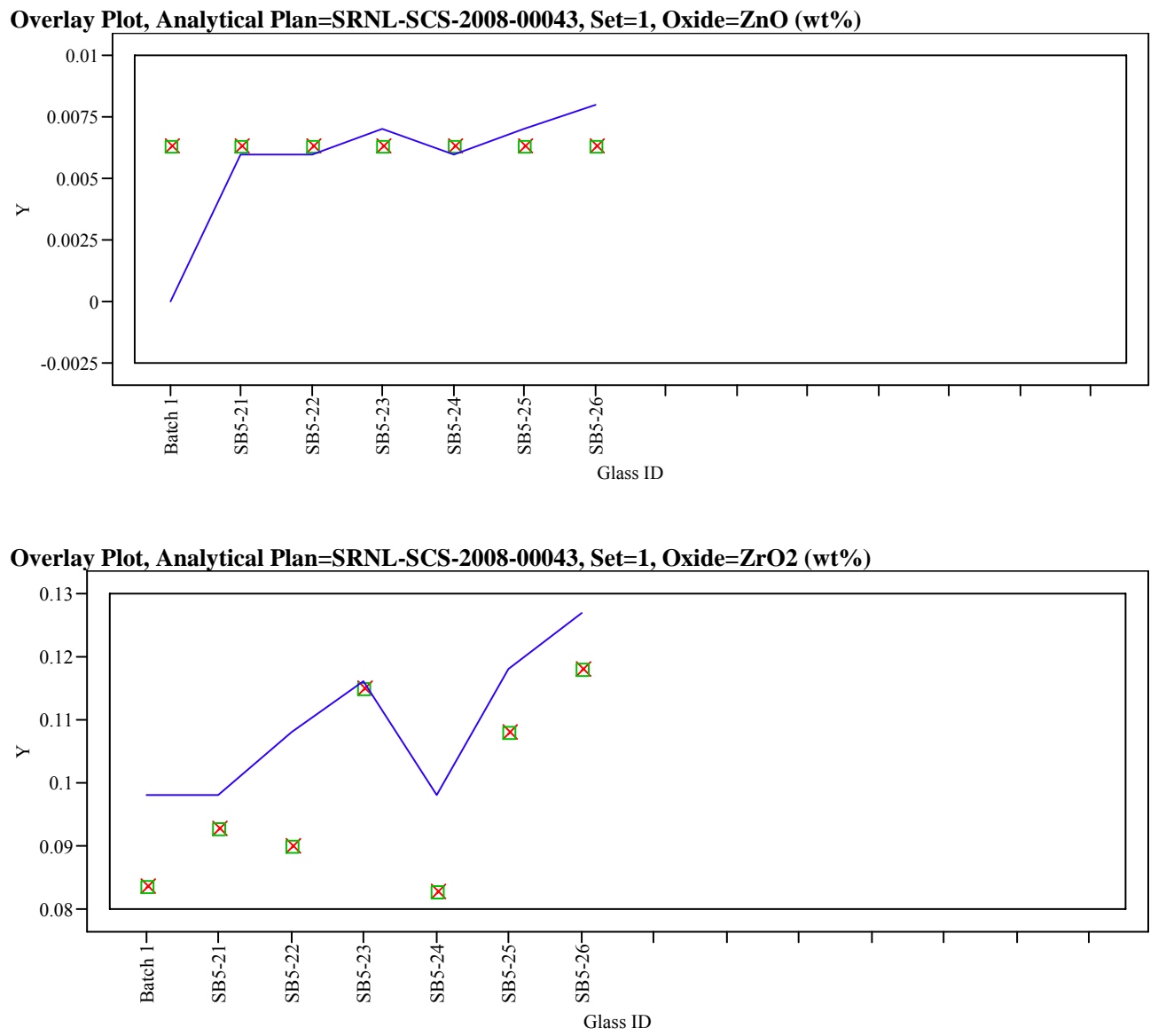

Overlay Plot, Analytical Plan=SRNL-SCS-2008-00043, Set=1, Oxide=Sum of Oxides

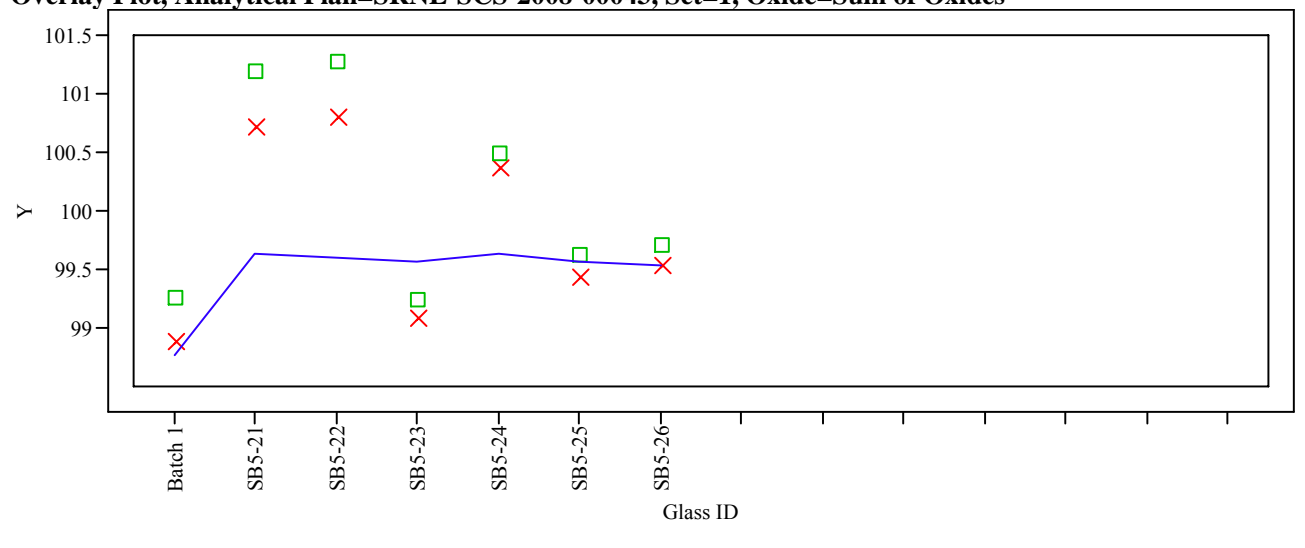

$\mathrm{Y} \times$ Measured $\square$ Measured bc $\quad$ Targeted 
SRNS-STI-2008-00060

Revision 0

\section{Appendix B}

Tables and Exhibits Supporting the Analysis of the PCT Results 
Table B1. PSAL Measurements of the PCT Solutions (in ppm) for the Study Glasses As-Received (ar) and After Appropriate Adjustments.

\begin{tabular}{|c|c|c|c|c|c|c|c|c|c|c|c|c|c|c|}
\hline $\begin{array}{c}\text { Analytical Plan } \\
\text { Memo }\end{array}$ & Set & Glass ID & $\begin{array}{c}\text { Heat } \\
\text { Treament }\end{array}$ & Block & Sequence & Lab ID & B (ar) & Li (ar) & Na (ar) & $\mathrm{Si}$ (ar) & B & $\mathbf{L i}$ & $\mathrm{Na}$ & Si \\
\hline SRNL-SCS-2008-00005 & $\mathrm{r}$ & Soln Std & ref & 1 & 1 & STD-21-1 & 20.5 & 9.89 & 79.3 & 50.7 & 20.5 & 9.89 & 79.3 & 50.7 \\
\hline SRNL-SCS-2008-00005 & $\mathrm{r}$ & EA & ref & 1 & 2 & $\mathrm{R} 62$ & 36.5 & 11 & 98.2 & 53.3 & 608.33455 & 183.3337 & 1636.6699 & 888.33511 \\
\hline SRNL-SCS-2008-00005 & $\mathrm{r}$ & SB5-17 & quenched & 1 & 3 & $\mathrm{R} 55$ & 11.9 & 11.3 & 34.1 & 59.4 & 19.83373 & 18.83371 & 56.83447 & 99.00198 \\
\hline SRNL-SCS-2008-00005 & $\mathrm{r}$ & SB4VAR13 & $\mathrm{ccc}$ & 1 & 4 & R09 & 23.8 & 17.9 & 65.9 & 71.6 & 39.66746 & 29.83393 & 109.83553 & 119.33572 \\
\hline SRNL-SCS-2008-00005 & $\mathrm{r}$ & SB5-18 & $\mathrm{ccc}$ & 1 & 5 & $\mathrm{R} 38$ & 11.7 & 8.53 & 45.8 & 58 & 19.50039 & 14.216951 & 76.33486 & 96.6686 \\
\hline SRNL-SCS-2008-00005 & $\mathrm{r}$ & SB4VAR33 & quenched & 1 & 6 & $\mathrm{R} 77$ & 12.5 & 10.3 & 44.9 & 57.5 & 20.83375 & 17.16701 & 74.83483 & 95.83525 \\
\hline SRNL-SCS-2008-00005 & $\mathrm{r}$ & SB4VAR11 & $\mathrm{ccc}$ & 1 & 7 & $\mathrm{R} 06$ & 12.2 & 10.6 & 35.2 & 61.1 & 20.33374 & 17.66702 & 58.66784 & 101.83537 \\
\hline SRNL-SCS-2008-00005 & $\mathrm{r}$ & SB5-14 & $\mathrm{ccc}$ & 1 & 8 & $\mathrm{R} 49$ & 11.1 & 10.5 & 60.5 & 68.5 & 18.50037 & 17.50035 & 100.83535 & 114.16895 \\
\hline SRNL-SCS-2008-00005 & $\mathrm{r}$ & SB5-17 & $\mathrm{ccc}$ & 1 & 9 & $\mathrm{R} 45$ & 11.3 & 10.5 & 32 & 58.4 & 18.83371 & 17.50035 & 53.3344 & 97.33528 \\
\hline SRNL-SCS-2008-00005 & $\mathrm{r}$ & SB4VAR33 & $\mathrm{ccc}$ & 1 & 10 & R84 & 13.1 & 10.7 & 43.6 & 59.1 & 21.83377 & 17.83369 & 72.66812 & 98.50197 \\
\hline SRNL-SCS-2008-00005 & $\mathrm{r}$ & SB5-20 & $\mathrm{ccc}$ & 1 & 11 & $\mathrm{R} 26$ & 11.7 & 4.55 & 39 & 49.9 & 19.50039 & 7.583485 & 65.0013 & 83.16833 \\
\hline SRNL-SCS-2008-00005 & $\mathrm{r}$ & SB4VAR32 & quenched & 1 & 12 & $\mathrm{R} 52$ & 12.3 & 10.4 & 37.7 & 59.9 & 20.50041 & 17.33368 & 62.83459 & 99.83533 \\
\hline SRNL-SCS-2008-00005 & $\mathrm{r}$ & SB5-16 & quenched & 1 & 13 & R68 & 15 & 6.42 & 55.3 & 56.2 & 25.0005 & 10.700214 & 92.16851 & 93.66854 \\
\hline SRNL-SCS-2008-00005 & $\mathrm{r}$ & ARM-1 & ref & 1 & 14 & $\mathrm{R} 17$ & 10.2 & 8.03 & 21.1 & 36 & 17.00034 & 13.383601 & 35.16737 & 60.0012 \\
\hline SRNL-SCS-2008-00005 & $\mathrm{r}$ & SB5-15 & quenched & 1 & 15 & R69 & 13.3 & 8.12 & 58.9 & 59.2 & 22.16711 & 13.533604 & 98.16863 & 98.66864 \\
\hline SRNL-SCS-2008-00005 & $\mathrm{r}$ & Soln Std & ref & 1 & 16 & STD-21-2 & 19.8 & 9.69 & 79.1 & 49.4 & 19.8 & 9.69 & 79.1 & 49.4 \\
\hline SRNL-SCS-2008-00005 & $\mathrm{r}$ & SB5-16 & $\mathrm{ccc}$ & 1 & 17 & R61 & 13.8 & 5.94 & 48.2 & 54.4 & 23.00046 & 9.900198 & 80.33494 & 90.66848 \\
\hline SRNL-SCS-2008-00005 & $\mathrm{r}$ & SB4VAR12 & quenched & 1 & 18 & $\mathrm{R} 85$ & 12.9 & 10.5 & 41.5 & 59.1 & 21.50043 & 17.50035 & 69.16805 & 98.50197 \\
\hline SRNL-SCS-2008-00005 & $\mathrm{r}$ & SB5-15 & $\mathrm{ccc}$ & 1 & 19 & $\mathrm{R} 78$ & 12.8 & 7.93 & 51.6 & 57.3 & 21.33376 & 13.216931 & 86.00172 & 95.50191 \\
\hline SRNL-SCS-2008-00005 & $\mathrm{r}$ & SB4VAR13 & quenched & 1 & 20 & R57 & 13.3 & 10.5 & 48.7 & 56.6 & 22.16711 & 17.50035 & 81.16829 & 94.33522 \\
\hline SRNL-SCS-2008-00005 & $\mathrm{r}$ & SB5-20 & quenched & 1 & 21 & $\mathrm{R} 31$ & 11.8 & 4.44 & 41.1 & 48.3 & 19.66706 & 7.400148 & 68.50137 & 80.50161 \\
\hline SRNL-SCS-2008-00005 & $\mathrm{r}$ & SB4VAR31 & quenched & 1 & 22 & $\mathrm{R} 30$ & 12.4 & 10.9 & 34.3 & 61.2 & 20.66708 & 18.16703 & 57.16781 & 102.00204 \\
\hline SRNL-SCS-2008-00005 & $\mathrm{r}$ & blank & ref & 1 & 23 & R08 & $<1.00$ & $<1.00$ & $<1.00$ & 0.116 & 0.83335 & 0.83335 & 0.83335 & 0.1933372 \\
\hline SRNL-SCS-2008-00005 & $\mathrm{r}$ & SB4VAR12 & $\mathrm{ccc}$ & 1 & 24 & $\mathrm{R} 46$ & 12.2 & 10.3 & 46.2 & 57.7 & 20.33374 & 17.16701 & 77.00154 & 96.16859 \\
\hline SRNL-SCS-2008-00005 & $\mathrm{r}$ & SB5-18 & quenched & 1 & 25 & $\mathrm{R} 48$ & 11.7 & 8.35 & 46.7 & 57.4 & 19.50039 & 13.916945 & 77.83489 & 95.66858 \\
\hline SRNL-SCS-2008-00005 & $\mathrm{r}$ & SB4VAR11 & quenched & 1 & 26 & $\mathrm{R} 01$ & 12.7 & 10.7 & 36.9 & 62 & 21.16709 & 17.83369 & 61.50123 & 103.3354 \\
\hline SRNL-SCS-2008-00005 & $\mathrm{r}$ & SB4VAR31 & $\mathrm{ccc}$ & 1 & 27 & R59 & 12.4 & 11.1 & 34.3 & 62.2 & 20.66708 & 18.50037 & 57.16781 & 103.66874 \\
\hline
\end{tabular}


Table B1. PSAL Measurements of the PCT Solutions (in ppm) for the Study Glasses As-Received (ar) and After Appropriate Adjustments. (continued)

\begin{tabular}{|c|c|c|c|c|c|c|c|c|c|c|c|c|c|c|}
\hline $\begin{array}{c}\text { Analytical Plan } \\
\text { Memo }\end{array}$ & Set & Glass ID & $\begin{array}{c}\text { Heat } \\
\text { Treament }\end{array}$ & Block & Sequence & Lab ID & B (ar) & Li (ar) & Na (ar) & Si (ar) & B & $\mathbf{L i}$ & $\mathrm{Na}$ & Si \\
\hline SRNL-SCS-2008-00005 & $\mathrm{r}$ & SB5-19 & quenched & 1 & 28 & $\mathrm{R} 65$ & 12.3 & 5.63 & 51.5 & 52.8 & 20.50041 & 9.383521 & 85.83505 & 88.00176 \\
\hline SRNL-SCS-2008-00005 & $\mathrm{r}$ & SB4VAR32 & $\mathrm{ccc}$ & 1 & 29 & $\mathrm{R} 02$ & 12 & 10.4 & 38.4 & 58.4 & 20.0004 & 17.33368 & 64.00128 & 97.33528 \\
\hline SRNL-SCS-2008-00005 & $\mathrm{r}$ & SB5-19 & $\mathrm{ccc}$ & 1 & 30 & $\mathrm{R} 37$ & 11.9 & 5.66 & 49.7 & 51.8 & 19.83373 & 9.433522 & 82.83499 & 86.33506 \\
\hline SRNL-SCS-2008-00005 & $\mathrm{r}$ & SB5-14 & quenched & 1 & 31 & $\mathrm{R} 53$ & 11.1 & 10.2 & 64.4 & 67.8 & 18.50037 & 17.00034 & 107.33548 & 113.00226 \\
\hline SRNL-SCS-2008-00005 & $\mathrm{r}$ & Soln Std & ref & 1 & 32 & STD-21-3 & 20.1 & 9.82 & 79.4 & 49.9 & 20.1 & 9.82 & 79.4 & 49.9 \\
\hline SRNL-SCS-2008-00005 & $\mathrm{r}$ & Soln Std & ref & 2 & 1 & STD-22-1 & 20.1 & 9.63 & 78.7 & 50.8 & 20.1 & 9.63 & 78.7 & 50.8 \\
\hline SRNL-SCS-2008-00005 & $\mathrm{r}$ & SB5-17 & $\mathrm{ccc}$ & 2 & 2 & $\mathrm{R} 83$ & 10.8 & 10.4 & 31.5 & 58.1 & 18.00036 & 17.33368 & 52.50105 & 96.83527 \\
\hline SRNL-SCS-2008-00005 & $\mathrm{r}$ & SB4VAR11 & $\mathrm{ccc}$ & 2 & 3 & $\mathrm{R} 03$ & 11.4 & 10 & 34.1 & 59.3 & 19.00038 & 16.667 & 56.83447 & 98.83531 \\
\hline SRNL-SCS-2008-00005 & $\mathrm{r}$ & SB5-15 & quenched & 2 & 4 & $\mathrm{R} 20$ & 12.3 & 7.6 & 56.8 & 56.8 & 20.50041 & 12.66692 & 94.66856 & 94.66856 \\
\hline SRNL-SCS-2008-00005 & $\mathrm{r}$ & SB5-14 & quenched & 2 & 5 & R54 & 10.1 & 9.53 & 61.3 & 65 & 16.83367 & 15.883651 & 102.16871 & 108.3355 \\
\hline SRNL-SCS-2008-00005 & $\mathrm{r}$ & SB4VAR11 & quenched & 2 & 6 & $\mathrm{R} 13$ & 11 & 9.73 & 35.4 & 57.9 & 18.3337 & 16.216991 & 59.00118 & 96.50193 \\
\hline SRNL-SCS-2008-00005 & $\mathrm{r}$ & SB5-15 & $\mathrm{ccc}$ & 2 & 7 & $\mathrm{R} 80$ & 11.4 & 7.42 & 52.8 & 55.1 & 19.00038 & 12.366914 & 88.00176 & 91.83517 \\
\hline SRNL-SCS-2008-00005 & $\mathrm{r}$ & SB4VAR13 & $\mathrm{ccc}$ & 2 & 8 & $\mathrm{R} 47$ & 21.5 & 16.5 & 62.3 & 65.8 & 35.83405 & 27.50055 & 103.83541 & 109.66886 \\
\hline SRNL-SCS-2008-00005 & $\mathrm{r}$ & SB4VAR12 & quenched & 2 & 9 & $\mathrm{R} 82$ & 11.7 & 10 & 41.3 & 58.1 & 19.50039 & 16.667 & 68.83471 & 96.83527 \\
\hline SRNL-SCS-2008-00005 & $\mathrm{r}$ & SB5-18 & quenched & 2 & 10 & R66 & 10.4 & 7.51 & 44.6 & 54.6 & 17.33368 & 12.516917 & 74.33482 & 91.00182 \\
\hline SRNL-SCS-2008-00005 & $\mathrm{r}$ & SB4VAR12 & $\mathrm{ccc}$ & 2 & 11 & $\mathrm{R} 32$ & 11.7 & 10.1 & 39.7 & 59 & 19.50039 & 16.83367 & 66.16799 & 98.3353 \\
\hline SRNL-SCS-2008-00005 & $\mathrm{r}$ & SB4VAR13 & quenched & 2 & 12 & $\mathrm{R} 40$ & 12.2 & 10 & 48.9 & 56.2 & 20.33374 & 16.667 & 81.50163 & 93.66854 \\
\hline SRNL-SCS-2008-00005 & $\mathrm{r}$ & SB4VAR33 & $\mathrm{ccc}$ & 2 & 13 & $\mathrm{R} 74$ & 12 & 10.2 & 53.4 & 56.9 & 20.0004 & 17.00034 & 89.00178 & 94.83523 \\
\hline SRNL-SCS-2008-00005 & $\mathrm{r}$ & SB5-19 & $\mathrm{ccc}$ & 2 & 14 & $\mathrm{R} 50$ & 11.2 & 5.38 & 48.9 & 52.3 & 18.66704 & 8.966846 & 81.50163 & 87.16841 \\
\hline SRNL-SCS-2008-00005 & $\mathrm{r}$ & SB5-17 & quenched & 2 & 15 & $\mathrm{R} 27$ & 11.1 & 11 & 34.3 & 60.2 & 18.50037 & 18.3337 & 57.16781 & 100.33534 \\
\hline SRNL-SCS-2008-00005 & $\mathrm{r}$ & Soln Std & ref & 2 & 16 & STD-22-2 & 19.6 & 9.64 & 80.1 & 50.9 & 19.6 & 9.64 & 80.1 & 50.9 \\
\hline SRNL-SCS-2008-00005 & $\mathrm{r}$ & SB5-14 & $\mathrm{ccc}$ & 2 & 17 & $\mathrm{R} 81$ & 10.4 & 10.1 & 60.5 & 68.1 & 17.33368 & 16.83367 & 100.83535 & 113.50227 \\
\hline SRNL-SCS-2008-00005 & $\mathrm{r}$ & SB4VAR33 & quenched & 2 & 18 & $\mathrm{R} 42$ & 12.2 & 10.1 & 44.7 & 58.1 & 20.33374 & 16.83367 & 74.50149 & 96.83527 \\
\hline SRNL-SCS-2008-00005 & $\mathrm{r}$ & ARM-1 & ref & 2 & 19 & $\mathrm{R} 25$ & 9.64 & 7.65 & 20.5 & 35.6 & 16.066988 & 12.750255 & 34.16735 & 59.33452 \\
\hline SRNL-SCS-2008-00005 & $\mathrm{r}$ & SB5-16 & $\mathrm{ccc}$ & 2 & 20 & $\mathrm{R} 35$ & 12.4 & 5.52 & 47.9 & 52.6 & 20.66708 & 9.200184 & 79.83493 & 87.66842 \\
\hline SRNL-SCS-2008-00005 & $\mathrm{r}$ & SB5-19 & quenched & 2 & 21 & $\mathrm{R} 41$ & 12.2 & 5.6 & 52.9 & 53.6 & 20.33374 & 9.33352 & 88.16843 & 89.33512 \\
\hline SRNL-SCS-2008-00005 & $\mathrm{r}$ & SB5-20 & $\mathrm{ccc}$ & 2 & 22 & $\mathrm{R} 19$ & 11.1 & 4.34 & 40.6 & 49.6 & 18.50037 & 7.233478 & 67.66802 & 82.66832 \\
\hline SRNL-SCS-2008-00005 & $\mathrm{r}$ & EA & ref & 2 & 23 & $\mathrm{R} 51$ & 36.6 & 10.7 & 98.6 & 53.8 & 610.00122 & 178.33369 & 1643.3366 & 896.66846 \\
\hline
\end{tabular}


Table B1. PSAL Measurements of the PCT Solutions (in ppm) for the Study Glasses As-Received (ar) and After Appropriate Adjustments. (continued)

\begin{tabular}{|c|c|c|c|c|c|c|c|c|c|c|c|c|c|c|}
\hline $\begin{array}{c}\text { Analytical Plan } \\
\text { Memo } \\
\end{array}$ & Set & Glass ID & $\begin{array}{c}\text { Heat } \\
\text { Treament } \\
\end{array}$ & Block & Sequence & Lab ID & B (ar) & $\mathrm{Li}(\mathrm{ar})$ & Na (ar) & Si (ar) & B & $\mathbf{L i}$ & $\mathrm{Na}$ & Si \\
\hline SRNL-SCS-2008-00005 & $\mathrm{r}$ & SB4VAR32 & quenched & 2 & 24 & $\mathrm{R} 36$ & 11.9 & 10.2 & 38.8 & 60.9 & 19.83373 & 17.00034 & 64.66796 & 101.50203 \\
\hline SRNL-SCS-2008-00005 & $\mathrm{r}$ & SB5-20 & quenched & 2 & 25 & R11 & 11.7 & 4.3 & 41.1 & 51.1 & 19.50039 & 7.16681 & 68.50137 & 85.16837 \\
\hline SRNL-SCS-2008-00005 & $\mathrm{r}$ & SB4VAR32 & $\operatorname{ccc}$ & 2 & 26 & $\mathrm{R} 34$ & 12.5 & 10.5 & 37.8 & 62.7 & 20.83375 & 17.50035 & 63.00126 & 104.50209 \\
\hline SRNL-SCS-2008-00005 & $\mathrm{r}$ & SB4VAR31 & quenched & 2 & 27 & $\mathrm{R} 24$ & 12.3 & 10.8 & 34.1 & 63.4 & 20.50041 & 18.00036 & 56.83447 & 105.66878 \\
\hline SRNL-SCS-2008-00005 & $\mathrm{r}$ & SB5-18 & $\mathrm{ccc}$ & 2 & 28 & $\mathrm{R} 12$ & 11.5 & 8.4 & 46.1 & 59.9 & 19.16705 & 14.00028 & 76.83487 & 99.83533 \\
\hline SRNL-SCS-2008-00005 & $\mathrm{r}$ & SB4VAR31 & $\mathrm{ccc}$ & 2 & 29 & $\mathrm{R} 72$ & 11.7 & 10.4 & 34.1 & 62.4 & 19.50039 & 17.33368 & 56.83447 & 104.00208 \\
\hline SRNL-SCS-2008-00005 & $\mathrm{r}$ & SB5-16 & quenched & 2 & 30 & $\mathrm{R} 70$ & 14.8 & 6.11 & 55.1 & 57.2 & 24.66716 & 10.183537 & 91.83517 & 95.33524 \\
\hline SRNL-SCS-2008-00005 & $\mathrm{r}$ & Soln Std & ref & 2 & 31 & STD-22-3 & 21.5 & 10.1 & 81.5 & 54.3 & 21.5 & 10.1 & 81.5 & 54.3 \\
\hline SRNL-SCS-2008-00005 & $\mathrm{r}$ & Soln Std & ref & 3 & 1 & STD-23-1 & 20.8 & 9.7 & 79.1 & 49.9 & 20.8 & 9.7 & 79.1 & 49.9 \\
\hline SRNL-SCS-2008-00005 & $\mathrm{r}$ & SB4VAR32 & ccc & 3 & 2 & $\mathrm{R} 73$ & 12.5 & 10.7 & 40.2 & 60.4 & 20.83375 & 17.83369 & 67.00134 & 100.66868 \\
\hline SRNL-SCS-2008-00005 & $\mathrm{r}$ & SB5-17 & quenched & 3 & 3 & R60 & 11.1 & 10.1 & 31.8 & 54.8 & 18.50037 & 16.83367 & 53.00106 & 91.33516 \\
\hline SRNL-SCS-2008-00005 & $\mathrm{r}$ & SB4VAR12 & $\mathrm{ccc}$ & 3 & 4 & $\mathrm{R} 23$ & 11.9 & 10 & 40.1 & 56.6 & 19.83373 & 16.667 & 66.83467 & 94.33522 \\
\hline SRNL-SCS-2008-00005 & $\mathrm{r}$ & SB4VAR11 & $\mathrm{ccc}$ & 3 & 5 & $\mathrm{R} 15$ & 11.8 & 10 & 34.2 & 59.2 & 19.66706 & 16.667 & 57.00114 & 98.66864 \\
\hline SRNL-SCS-2008-00005 & $\mathrm{r}$ & SB4VAR31 & $\mathrm{ccc}$ & 3 & 6 & $\mathrm{R} 58$ & 11.9 & 10.3 & 32.7 & 60.5 & 19.83373 & 17.16701 & 54.50109 & 100.83535 \\
\hline SRNL-SCS-2008-00005 & $\mathrm{r}$ & SB5-19 & $\mathrm{ccc}$ & 3 & 7 & $\mathrm{R} 76$ & 12.5 & 5.46 & 48.4 & 54.5 & 20.83375 & 9.100182 & 80.66828 & 90.83515 \\
\hline SRNL-SCS-2008-00005 & $\mathrm{r}$ & SB5-14 & quenched & 3 & 8 & $\mathrm{R} 79$ & 11.5 & 10 & 63.7 & 70.2 & 19.16705 & 16.667 & 106.16879 & 117.00234 \\
\hline SRNL-SCS-2008-00005 & $\mathrm{r}$ & SB4VAR33 & $\mathrm{ccc}$ & 3 & 9 & $\mathrm{R} 63$ & 13.7 & 10.8 & 45.1 & 60.6 & 22.83379 & 18.00036 & 75.16817 & 101.00202 \\
\hline SRNL-SCS-2008-00005 & $\mathrm{r}$ & SB4VAR32 & quenched & 3 & 10 & R67 & 12.5 & 10.1 & 37.5 & 59.4 & 20.83375 & 16.83367 & 62.50125 & 99.00198 \\
\hline SRNL-SCS-2008-00005 & $\mathrm{r}$ & EA & ref & 3 & 11 & R64 & 36.9 & 10.7 & 91.3 & 55 & 615.00123 & 178.33369 & 1521.6697 & 916.6685 \\
\hline SRNL-SCS-2008-00005 & $\mathrm{r}$ & SB5-20 & $\mathrm{ccc}$ & 3 & 12 & R22 & 12.6 & 4.39 & 40.2 & 51.4 & 21.00042 & 7.316813 & 67.00134 & 85.66838 \\
\hline SRNL-SCS-2008-00005 & $\mathrm{r}$ & SB4VAR12 & quenched & 3 & 13 & R44 & 13.6 & 10.6 & 43.9 & 63.3 & 22.66712 & 17.66702 & 73.16813 & 105.50211 \\
\hline SRNL-SCS-2008-00005 & $\mathrm{r}$ & SB5-17 & $\mathrm{ccc}$ & 3 & 14 & R07 & 12.6 & 11.2 & 33 & 64.2 & 21.00042 & 18.66704 & 55.0011 & 107.00214 \\
\hline SRNL-SCS-2008-00005 & $\mathrm{r}$ & SB4VAR13 & $\mathrm{ccc}$ & 3 & 15 & $\mathrm{R} 28$ & 25 & 18.1 & 64.6 & 73.7 & 41.6675 & 30.16727 & 107.66882 & 122.83579 \\
\hline SRNL-SCS-2008-00005 & $\mathrm{r}$ & Soln Std & ref & 3 & 16 & STD-23-2 & 22 & 10.1 & 80 & 54.6 & 22 & 10.1 & 80 & 54.6 \\
\hline SRNL-SCS-2008-00005 & $\mathrm{r}$ & SB4VAR11 & quenched & 3 & 17 & $\mathrm{R} 33$ & 12.8 & 10.4 & 37.1 & 58.9 & 21.33376 & 17.33368 & 61.83457 & 98.16863 \\
\hline SRNL-SCS-2008-00005 & $\mathrm{r}$ & SB4VAR31 & quenched & 3 & 18 & R04 & 14.2 & 11.2 & 36.4 & 67.2 & 23.66714 & 18.66704 & 60.66788 & 112.00224 \\
\hline SRNL-SCS-2008-00005 & $\mathrm{r}$ & SB5-19 & quenched & 3 & 19 & $\mathrm{R} 86$ & 14 & 6 & 56 & 57.2 & 23.3338 & 10.0002 & 93.3352 & 95.33524 \\
\hline SRNL-SCS-2008-00005 & $\mathrm{r}$ & SB4VAR13 & quenched & 3 & 20 & $\mathrm{R} 10$ & 12.3 & 10.4 & 50.9 & 52.6 & 20.50041 & 17.33368 & 84.83503 & 87.66842 \\
\hline
\end{tabular}


Table B1. PSAL Measurements of the PCT Solutions (in ppm) for the Study Glasses

As-Received (ar) and After Appropriate Adjustments. (continued)

\begin{tabular}{|c|c|c|c|c|c|c|c|c|c|c|c|c|c|c|}
\hline $\begin{array}{c}\text { Analytical Plan } \\
\text { Memo } \\
\end{array}$ & Set & Glass ID & $\begin{array}{c}\text { Heat } \\
\text { Treament } \\
\end{array}$ & Block & Sequence & Lab ID & B (ar) & Li (ar) & Na (ar) & Si (ar) & B & $\mathbf{L i}$ & $\mathrm{Na}$ & Si \\
\hline SRNL-SCS-2008-00005 & $\mathrm{r}$ & SB5-15 & $\mathrm{ccc}$ & 3 & 21 & $\mathrm{R} 16$ & 11.4 & 7.68 & 55.1 & 51.2 & 19.00038 & 12.800256 & 91.83517 & 85.33504 \\
\hline SRNL-SCS-2008-00005 & $\mathrm{r}$ & SB5-14 & $\mathrm{ccc}$ & 3 & 22 & $\mathrm{R} 71$ & 9.94 & 10.2 & 62.5 & 60.3 & 16.566998 & 17.00034 & 104.16875 & 100.50201 \\
\hline SRNL-SCS-2008-00005 & $\mathrm{r}$ & SB5-20 & quenched & 3 & 23 & $\mathrm{R} 56$ & 11.3 & 4.29 & 42.3 & 46.7 & 18.83371 & 7.150143 & 70.50141 & 77.83489 \\
\hline SRNL-SCS-2008-00005 & $\mathrm{r}$ & SB5-16 & quenched & 3 & 24 & $\mathrm{R} 75$ & 13.8 & 6.17 & 58 & 53.3 & 23.00046 & 10.283539 & 96.6686 & 88.83511 \\
\hline SRNL-SCS-2008-00005 & $\mathrm{r}$ & blank & ref & 3 & 25 & $\mathrm{R} 39$ & $<1.00$ & $<1.00$ & $<1.00$ & 0.456 & 0.83335 & 0.83335 & 0.83335 & 0.7600152 \\
\hline SRNL-SCS-2008-00005 & $\mathrm{r}$ & SB5-18 & quenched & 3 & 26 & $\mathrm{R} 21$ & 10.9 & 8.22 & 49.7 & 53.5 & 18.16703 & 13.700274 & 82.83499 & 89.16845 \\
\hline SRNL-SCS-2008-00005 & $\mathrm{r}$ & SB4VAR33 & quenched & 3 & 27 & $\mathrm{R} 18$ & 12.3 & 10.7 & 48.4 & 57 & 20.50041 & 17.83369 & 80.66828 & 95.0019 \\
\hline SRNL-SCS-2008-00005 & $\mathrm{r}$ & ARM-1 & ref & 3 & 28 & $\mathrm{R} 29$ & 10.1 & 8.25 & 23.2 & 35 & 16.83367 & 13.750275 & 38.66744 & 58.3345 \\
\hline SRNL-SCS-2008-00005 & $\mathrm{r}$ & SB5-16 & $\mathrm{ccc}$ & 3 & 29 & $\mathrm{R} 14$ & 13.7 & 6.3 & 55.1 & 53.2 & 22.83379 & 10.50021 & 91.83517 & 88.66844 \\
\hline SRNL-SCS-2008-00005 & $\mathrm{r}$ & SB5-18 & $\mathrm{ccc}$ & 3 & 30 & $\mathrm{R} 43$ & 10.8 & 8.28 & 48.5 & 54.2 & 18.00036 & 13.800276 & 80.83495 & 90.33514 \\
\hline SRNL-SCS-2008-00005 & $\mathrm{r}$ & SB5-15 & quenched & 3 & 31 & R05 & 14.4 & 8.23 & 58.7 & 61.6 & 24.00048 & 13.716941 & 97.83529 & 102.66872 \\
\hline SRNL-SCS-2008-00005 & $\mathrm{r}$ & Soln Std & ref & 3 & 32 & STD-23-3 & 19.7 & 10.2 & 83.1 & 50 & 19.7 & 10.2 & 83.1 & 50 \\
\hline SRNL-SCS-2008-00005 & $\mathrm{s}$ & Soln Std & ref & 1 & 1 & STD-31-1 & 19.3 & 9.53 & 80.7 & 47.8 & 19.3 & 9.53 & 80.7 & 47.8 \\
\hline SRNL-SCS-2008-00005 & $\mathrm{s}$ & SB5-11 & $\mathrm{ccc}$ & 1 & 2 & S84 & 12.2 & 7.65 & 47.2 & 56.5 & 20.33374 & 12.750255 & 78.66824 & 94.16855 \\
\hline SRNL-SCS-2008-00005 & $\mathrm{s}$ & SB5-04 & quenched & 1 & 3 & S59 & 11.4 & 5.12 & 41.4 & 49.4 & 19.00038 & 8.533504 & 69.00138 & 82.33498 \\
\hline SRNL-SCS-2008-00005 & $\mathrm{s}$ & SB5-01 & quenched & 1 & 4 & S19 & 6.19 & 7.26 & 43.6 & 43.3 & 10.316873 & 12.100242 & 72.66812 & 72.16811 \\
\hline SRNL-SCS-2008-00005 & $\mathrm{s}$ & SB5-12 & quenched & 1 & 5 & $\mathrm{~S} 82$ & 12.3 & 5.77 & 46.7 & 52.1 & 20.50041 & 9.616859 & 77.83489 & 86.83507 \\
\hline SRNL-SCS-2008-00005 & $\mathrm{s}$ & SB5-05 & quenched & 1 & 6 & S07 & 7.43 & 8.54 & 50.4 & 49.3 & 12.383581 & 14.233618 & 84.00168 & 82.16831 \\
\hline SRNL-SCS-2008-00005 & $\mathrm{s}$ & SB5-07 & $\mathrm{ccc}$ & 1 & 7 & $\mathrm{~S} 74$ & 9.49 & 6.46 & 39.4 & 47.4 & 15.816983 & 10.766882 & 65.66798 & 79.00158 \\
\hline SRNL-SCS-2008-00005 & $\mathrm{s}$ & SB5-08 & quenched & 1 & 8 & $\mathrm{~S} 83$ & 11.7 & 5.38 & 40.7 & 51.4 & 19.50039 & 8.966846 & 67.83469 & 85.66838 \\
\hline SRNL-SCS-2008-00005 & $\mathrm{s}$ & SB5-13 & quenched & 1 & 9 & $\mathrm{~S} 60$ & 10.8 & 11.2 & 72.7 & 68.5 & 18.00036 & 18.66704 & 121.16909 & 114.16895 \\
\hline SRNL-SCS-2008-00005 & $\mathrm{s}$ & EA & ref & 1 & 10 & S48 & 35.9 & 10.9 & 101.2 & 53 & 598.33453 & 181.66703 & 1686.67 & 883.3351 \\
\hline SRNL-SCS-2008-00005 & $\mathrm{s}$ & SB5-11 & quenched & 1 & 11 & S61 & 11.7 & 7.33 & 47.5 & 53.6 & 19.50039 & 12.216911 & 79.16825 & 89.33512 \\
\hline SRNL-SCS-2008-00005 & $\mathrm{s}$ & SB5-08 & $\mathrm{ccc}$ & 1 & 12 & S23 & 11.6 & 5.18 & 36.6 & 50.1 & 19.33372 & 8.633506 & 61.00122 & 83.50167 \\
\hline SRNL-SCS-2008-00005 & $\mathrm{s}$ & SB5-13 & $\mathrm{ccc}$ & 1 & 13 & S67 & 10.7 & 11.4 & 70.6 & 68.4 & 17.83369 & 19.00038 & 117.66902 & 114.00228 \\
\hline SRNL-SCS-2008-00005 & $\mathrm{s}$ & ARM-1 & ref & 1 & 14 & S86 & 10.5 & 8.12 & 23.2 & 37.2 & 17.50035 & 13.533604 & 38.66744 & 62.00124 \\
\hline SRNL-SCS-2008-00005 & $\mathrm{s}$ & SB5-02 & $\mathrm{ccc}$ & 1 & 15 & S66 & 7.14 & 7.38 & 39.4 & 49.9 & 11.900238 & 12.300246 & 65.66798 & 83.16833 \\
\hline SRNL-SCS-2008-00005 & $\mathrm{s}$ & Soln Std & ref & 1 & 16 & STD-31-2 & 20 & 9.71 & 79.4 & 51.5 & 20 & 9.71 & 79.4 & 51.5 \\
\hline
\end{tabular}


Table B1. PSAL Measurements of the PCT Solutions (in ppm) for the Study Glasses

As-Received (ar) and After Appropriate Adjustments. (continued)

\begin{tabular}{|c|c|c|c|c|c|c|c|c|c|c|c|c|c|c|}
\hline $\begin{array}{c}\text { Analytical Plan } \\
\text { Memo }\end{array}$ & Set & Glass ID & $\begin{array}{c}\text { Heat } \\
\text { Treament }\end{array}$ & Block & Sequence & Lab ID & B (ar) & Li (ar) & $\mathrm{Na}(\mathrm{ar})$ & Si (ar) & B & $\mathbf{L i}$ & $\mathrm{Na}$ & $\mathbf{S i}$ \\
\hline SRNL-SCS-2008-00005 & $\mathrm{s}$ & SB5-02 & quenched & 1 & 17 & S24 & 8.07 & 7.58 & 44 & 52.4 & 13.450269 & 12.633586 & 73.3348 & 87.33508 \\
\hline SRNL-SCS-2008-00005 & $\mathrm{s}$ & SB5-03 & quenched & 1 & 18 & S63 & 9.37 & 6.24 & 40 & 47.4 & 15.616979 & 10.400208 & 66.668 & 79.00158 \\
\hline SRNL-SCS-2008-00005 & $\mathrm{s}$ & SB5-09 & $\mathrm{ccc}$ & 1 & 19 & S29 & 9.41 & 10.2 & 57 & 59.9 & 15.683647 & 17.00034 & 95.0019 & 99.83533 \\
\hline SRNL-SCS-2008-00005 & $\mathrm{s}$ & SB5-01 & $\mathrm{ccc}$ & 1 & 20 & $\mathrm{~S} 73$ & 6.64 & 7.67 & 41.8 & 45.8 & 11.066888 & 12.783589 & 69.66806 & 76.33486 \\
\hline SRNL-SCS-2008-00005 & $\mathrm{s}$ & blank & ref & 1 & 21 & S49 & $<1.00$ & $<1.00$ & $<1.00$ & $<0.100$ & 0.83335 & 0.83335 & 0.83335 & 0.083335 \\
\hline SRNL-SCS-2008-00005 & $\mathrm{s}$ & SB5-10 & $\mathrm{ccc}$ & 1 & 22 & S76 & 10 & 9.39 & 50.9 & 63.3 & 16.667 & 15.650313 & 84.83503 & 105.50211 \\
\hline SRNL-SCS-2008-00005 & $\mathrm{s}$ & SB5-06 & $\mathrm{ccc}$ & 1 & 23 & S01 & 9.79 & 6.38 & 38 & 48.9 & 16.316993 & 10.633546 & 63.3346 & 81.50163 \\
\hline SRNL-SCS-2008-00005 & $\mathrm{s}$ & SB5-07 & quenched & 1 & 24 & S65 & 11.6 & 7.01 & 44.7 & 53.6 & 19.33372 & 11.683567 & 74.50149 & 89.33512 \\
\hline SRNL-SCS-2008-00005 & $\mathrm{s}$ & SB5-04 & $\mathrm{ccc}$ & 1 & 25 & S39 & 11.4 & 5.22 & 37.4 & 52 & 19.00038 & 8.700174 & 62.33458 & 86.6684 \\
\hline SRNL-SCS-2008-00005 & $\mathrm{s}$ & SB5-05 & $\mathrm{ccc}$ & 1 & 26 & S09 & 8.57 & 9.09 & 48.4 & 53 & 14.283619 & 15.150303 & 80.66828 & 88.3351 \\
\hline SRNL-SCS-2008-00005 & $\mathrm{s}$ & SB5-03 & $\mathrm{ccc}$ & 1 & 27 & $\mathrm{~S} 78$ & 11.3 & 9.62 & 48.6 & 59 & 18.83371 & 16.033654 & 81.00162 & 98.3353 \\
\hline SRNL-SCS-2008-00005 & $\mathrm{s}$ & SB5-06 & quenched & 1 & 28 & $\mathrm{~S} 16$ & 9.03 & 8.5 & 55.6 & 55.6 & 15.050301 & 14.16695 & 92.66852 & 92.66852 \\
\hline SRNL-SCS-2008-00005 & $\mathrm{s}$ & SB5-10 & quenched & 1 & 29 & $\mathrm{~S} 38$ & 14.8 & 9.88 & 63.4 & 66.7 & 24.66716 & 16.466996 & 105.66878 & 111.16889 \\
\hline SRNL-SCS-2008-00005 & $\mathrm{s}$ & SB5-09 & quenched & 1 & 30 & S04 & 10.6 & 11 & 72 & 67.1 & 17.66702 & 18.3337 & 120.0024 & 111.83557 \\
\hline SRNL-SCS-2008-00005 & $\mathrm{s}$ & SB5-12 & $\mathrm{ccc}$ & 1 & 31 & S57 & 12.1 & 5.83 & 49.7 & 56.6 & 20.16707 & 9.716861 & 82.83499 & 94.33522 \\
\hline SRNL-SCS-2008-00005 & $\mathrm{s}$ & Soln Std & ref & 1 & 32 & STD-31-3 & 19.8 & 10.1 & 79.5 & 53.5 & 19.8 & 10.1 & 79.5 & 53.5 \\
\hline SRNL-SCS-2008-00005 & $\mathrm{s}$ & Soln Std & ref & 2 & 1 & STD-32-1 & 20 & 9.7 & 79.9 & 50.3 & 20 & 9.7 & 79.9 & 50.3 \\
\hline SRNL-SCS-2008-00005 & $\mathrm{s}$ & SB5-11 & $\mathrm{ccc}$ & 2 & 2 & $\mathrm{~S} 15$ & 11.6 & 7.61 & 45.3 & 53.8 & 19.33372 & 12.683587 & 75.50151 & 89.66846 \\
\hline SRNL-SCS-2008-00005 & $\mathrm{s}$ & SB5-02 & $\mathrm{ccc}$ & 2 & 3 & S33 & 6.92 & 7.38 & 38.7 & 48.1 & 11.533564 & 12.300246 & 64.50129 & 80.16827 \\
\hline SRNL-SCS-2008-00005 & $\mathrm{s}$ & SB5-10 & $\mathrm{ccc}$ & 2 & 4 & S05 & 9.16 & 9.37 & 50.1 & 59.6 & 15.266972 & 15.616979 & 83.50167 & 99.33532 \\
\hline SRNL-SCS-2008-00005 & $\mathrm{s}$ & EA & ref & 2 & 5 & S54 & 35.8 & 11.2 & 100 & 53.4 & 596.66786 & 186.66704 & 1666.67 & 890.00178 \\
\hline SRNL-SCS-2008-00005 & $\mathrm{s}$ & SB5-04 & $\mathrm{ccc}$ & 2 & 6 & S56 & 10.2 & 5.07 & 35.9 & 47.3 & 17.00034 & 8.450169 & 59.83453 & 78.83491 \\
\hline SRNL-SCS-2008-00005 & $\mathrm{s}$ & SB5-13 & $\mathrm{ccc}$ & 2 & 7 & $\mathrm{~S} 10$ & 10.1 & 11.1 & 69.2 & 64 & 16.83367 & 18.50037 & 115.33564 & 106.6688 \\
\hline SRNL-SCS-2008-00005 & $\mathrm{s}$ & SB5-06 & quenched & 2 & 8 & $\mathrm{~S} 02$ & 7.87 & 8.04 & 44.7 & 51.4 & 13.116929 & 13.400268 & 74.50149 & 85.66838 \\
\hline SRNL-SCS-2008-00005 & $\mathrm{s}$ & SB5-05 & quenched & 2 & 9 & S26 & 7.18 & 8.51 & 49.6 & 48.2 & 11.966906 & 14.183617 & 82.66832 & 80.33494 \\
\hline SRNL-SCS-2008-00005 & $\mathrm{s}$ & SB5-12 & $\mathrm{ccc}$ & 2 & 10 & S47 & 11.4 & 5.6 & 42.6 & 49.9 & 19.00038 & 9.33352 & 71.00142 & 83.16833 \\
\hline SRNL-SCS-2008-00005 & $\mathrm{s}$ & ARM-1 & ref & 2 & 11 & S58 & 9.79 & 7.98 & 21.8 & 35.2 & 16.316993 & 13.300266 & 36.33406 & 58.66784 \\
\hline SRNL-SCS-2008-00005 & $\mathrm{s}$ & SB5-01 & quenched & 2 & 12 & S79 & 5.95 & 7.37 & 42.5 & 42.6 & 9.916865 & 12.283579 & 70.83475 & 71.00142 \\
\hline
\end{tabular}


Table B1. PSAL Measurements of the PCT Solutions (in ppm) for the Study Glasses

As-Received (ar) and After Appropriate Adjustments. (continued)

\begin{tabular}{|c|c|c|c|c|c|c|c|c|c|c|c|c|c|c|}
\hline $\begin{array}{c}\text { Analytical Plan } \\
\text { Memo }\end{array}$ & Set & Glass ID & $\begin{array}{c}\text { Heat } \\
\text { Treament }\end{array}$ & Block & Sequence & Lab ID & B (ar) & Li (ar) & $\mathrm{Na}(\mathrm{ar})$ & Si (ar) & B & $\mathbf{L i}$ & $\mathrm{Na}$ & $\mathbf{S i}$ \\
\hline SRNL-SCS-2008-00005 & $\mathrm{s}$ & SB5-13 & quenched & 2 & 13 & S64 & 10.6 & 11.6 & 75.2 & 67.2 & 17.66702 & 19.33372 & 125.33584 & 112.00224 \\
\hline SRNL-SCS-2008-00005 & $\mathrm{s}$ & SB5-12 & quenched & 2 & 14 & $\mathrm{~S} 18$ & 12.4 & 5.88 & 45.3 & 52.2 & 20.66708 & 9.800196 & 75.50151 & 87.00174 \\
\hline SRNL-SCS-2008-00005 & $\mathrm{s}$ & SB5-07 & $\mathrm{ccc}$ & 2 & 15 & S36 & 9.68 & 6.86 & 40.9 & 48.5 & 16.133656 & 11.433562 & 68.16803 & 80.83495 \\
\hline SRNL-SCS-2008-00005 & $\mathrm{s}$ & Soln Std & ref & 2 & 16 & STD-32-2 & 18.9 & 9.55 & 80.2 & 48.6 & 18.9 & 9.55 & 80.2 & 48.6 \\
\hline SRNL-SCS-2008-00005 & $\mathrm{s}$ & SB5-08 & $\mathrm{ccc}$ & 2 & 17 & S27 & 10.5 & 5.12 & 37.4 & 45.7 & 17.50035 & 8.533504 & 62.33458 & 76.16819 \\
\hline SRNL-SCS-2008-00005 & $\mathrm{s}$ & SB5-09 & $\mathrm{ccc}$ & 2 & 18 & $\mathrm{~S} 80$ & 8.7 & 9.91 & 55.1 & 55.8 & 14.50029 & 16.516997 & 91.83517 & 93.00186 \\
\hline SRNL-SCS-2008-00005 & $\mathrm{s}$ & SB5-04 & quenched & 2 & 19 & $\mathrm{~S} 30$ & 10.8 & 5.21 & 40.3 & 47.6 & 18.00036 & 8.683507 & 67.16801 & 79.33492 \\
\hline SRNL-SCS-2008-00005 & $\mathrm{s}$ & SB5-01 & $\mathrm{ccc}$ & 2 & 20 & S69 & 5.56 & 7.18 & 38.9 & 40.4 & 9.266852 & 11.966906 & 64.83463 & 67.33468 \\
\hline SRNL-SCS-2008-00005 & $\mathrm{s}$ & SB5-03 & $\mathrm{ccc}$ & 2 & 21 & $\mathrm{~S} 21$ & 8.11 & 8.49 & 45.1 & 54.4 & 13.516937 & 14.150283 & 75.16817 & 90.66848 \\
\hline SRNL-SCS-2008-00005 & $\mathrm{s}$ & SB5-02 & quenched & 2 & 22 & S41 & 6.41 & 7.02 & 38.8 & 45.3 & 10.683547 & 11.700234 & 64.66796 & 75.50151 \\
\hline SRNL-SCS-2008-00005 & $\mathrm{s}$ & SB5-09 & quenched & 2 & 23 & $\mathrm{~S} 20$ & 8.88 & 10 & 57.9 & 56.9 & 14.800296 & 16.667 & 96.50193 & 94.83523 \\
\hline SRNL-SCS-2008-00005 & $\mathrm{s}$ & SB5-03 & quenched & 2 & 24 & S62 & 8.48 & 6.25 & 38.9 & 44.8 & 14.133616 & 10.416875 & 64.83463 & 74.66816 \\
\hline SRNL-SCS-2008-00005 & $\mathrm{s}$ & SB5-10 & quenched & 2 & 25 & S31 & 8.54 & 8.92 & 51.3 & 56.5 & 14.233618 & 14.866964 & 85.50171 & 94.16855 \\
\hline SRNL-SCS-2008-00005 & $\mathrm{s}$ & SB5-06 & $\mathrm{ccc}$ & 2 & 26 & S77 & 7.7 & 5.9 & 37.2 & 42.1 & 12.83359 & 9.83353 & 62.00124 & 70.16807 \\
\hline SRNL-SCS-2008-00005 & $\mathrm{s}$ & SB5-11 & quenched & 2 & 27 & $\mathrm{~S} 70$ & 10.3 & 7.14 & 45.5 & 50.1 & 17.16701 & 11.900238 & 75.83485 & 83.50167 \\
\hline SRNL-SCS-2008-00005 & $\mathrm{s}$ & SB5-05 & $\mathrm{ccc}$ & 2 & 28 & S37 & 6.99 & 8.66 & 47.8 & 47.5 & 11.650233 & 14.433622 & 79.66826 & 79.16825 \\
\hline SRNL-SCS-2008-00005 & $\mathrm{s}$ & SB5-08 & quenched & 2 & 29 & S43 & 10.5 & 5.25 & 39.3 & 47.5 & 17.50035 & 8.750175 & 65.50131 & 79.16825 \\
\hline SRNL-SCS-2008-00005 & $\mathrm{s}$ & SB5-07 & quenched & 2 & 30 & $\mathrm{~S} 13$ & 9.36 & 6.63 & 41.8 & 46.5 & 15.600312 & 11.050221 & 69.66806 & 77.50155 \\
\hline SRNL-SCS-2008-00005 & $\mathrm{s}$ & Soln Std & ref & 2 & 31 & STD-32-2 & 18.2 & 9.45 & 80.4 & 47.2 & 18.2 & 9.45 & 80.4 & 47.2 \\
\hline SRNL-SCS-2008-00005 & $\mathrm{s}$ & Soln Std & ref & 3 & 1 & STD-33-1 & 19.8 & 9.64 & 81.7 & 49.8 & 19.8 & 9.64 & 81.7 & 49.8 \\
\hline SRNL-SCS-2008-00005 & $\mathrm{s}$ & SB5-10 & $\mathrm{ccc}$ & 3 & 2 & S44 & 9.19 & 9.18 & 49.3 & 59.3 & 15.316973 & 15.300306 & 82.16831 & 98.83531 \\
\hline SRNL-SCS-2008-00005 & $\mathrm{s}$ & SB5-02 & $\mathrm{ccc}$ & 3 & 3 & S81 & 6.85 & 7.42 & 38.8 & 49.4 & 11.416895 & 12.366914 & 64.66796 & 82.33498 \\
\hline SRNL-SCS-2008-00005 & $\mathrm{s}$ & SB5-06 & $\mathrm{ccc}$ & 3 & 4 & S35 & 8.45 & 6.19 & 36.5 & 45.5 & 14.083615 & 10.316873 & 60.83455 & 75.83485 \\
\hline SRNL-SCS-2008-00005 & $\mathrm{s}$ & SB5-06 & quenched & 3 & 5 & S51 & 8.19 & 8.33 & 45.8 & 54.9 & 13.650273 & 13.883611 & 76.33486 & 91.50183 \\
\hline SRNL-SCS-2008-00005 & $\mathrm{s}$ & blank & ref & 3 & 6 & S42 & $<1.00$ & $<1.00$ & $<1.00$ & $<0.100$ & 0.83335 & 0.83335 & 0.83335 & 0.083335 \\
\hline SRNL-SCS-2008-00005 & $\mathrm{s}$ & SB5-04 & $\mathrm{ccc}$ & 3 & 7 & $\mathrm{~S} 12$ & 10.1 & 5.21 & 36.8 & 49.4 & 16.83367 & 8.683507 & 61.33456 & 82.33498 \\
\hline SRNL-SCS-2008-00005 & $\mathrm{s}$ & SB5-13 & quenched & 3 & 8 & $\mathrm{~S} 72$ & 11 & 12 & 77 & 70.6 & 18.3337 & 20.0004 & 128.3359 & 117.66902 \\
\hline SRNL-SCS-2008-00005 & $\mathrm{s}$ & SB5-09 & $\mathrm{ccc}$ & 3 & 9 & S46 & 8.65 & 10.1 & 55 & 57.9 & 14.416955 & 16.83367 & 91.6685 & 96.50193 \\
\hline
\end{tabular}


Table B1. PSAL Measurements of the PCT Solutions (in ppm) for the Study Glasses

As-Received (ar) and After Appropriate Adjustments. (continued)

\begin{tabular}{|c|c|c|c|c|c|c|c|c|c|c|c|c|c|c|}
\hline $\begin{array}{c}\text { Analytical Plan } \\
\text { Memo }\end{array}$ & Set & Glass ID & $\begin{array}{c}\text { Heat } \\
\text { Treament }\end{array}$ & Block & Sequence & Lab ID & B (ar) & Li (ar) & $\mathrm{Na}(\mathrm{ar})$ & Si (ar) & B & $\mathbf{L i}$ & $\mathrm{Na}$ & $\mathbf{S i}$ \\
\hline SRNL-SCS-2008-00005 & $\mathrm{s}$ & SB5-05 & quenched & 3 & 10 & $\mathrm{~S} 28$ & 7.25 & 8.6 & 48.8 & 49.4 & 12.083575 & 14.33362 & 81.33496 & 82.33498 \\
\hline SRNL-SCS-2008-00005 & $\mathrm{s}$ & SB5-12 & quenched & 3 & 11 & $\mathrm{~S} 40$ & 11.9 & 5.7 & 44.6 & 51.2 & 19.83373 & 9.50019 & 74.33482 & 85.33504 \\
\hline SRNL-SCS-2008-00005 & $\mathrm{s}$ & SB5-04 & quenched & 3 & 12 & $\mathrm{~S} 03$ & 10.5 & 5.08 & 39.6 & 47.6 & 17.50035 & 8.466836 & 66.00132 & 79.33492 \\
\hline SRNL-SCS-2008-00005 & $\mathrm{s}$ & SB5-01 & quenched & 3 & 13 & S50 & 5.88 & 7.46 & 42.8 & 44.2 & 9.800196 & 12.433582 & 71.33476 & 73.66814 \\
\hline SRNL-SCS-2008-00005 & $\mathrm{s}$ & SB5-11 & $\mathrm{ccc}$ & 3 & 14 & S06 & 11 & 7.51 & 44.7 & 54.1 & 18.3337 & 12.516917 & 74.50149 & 90.16847 \\
\hline SRNL-SCS-2008-00005 & $\mathrm{s}$ & SB5-03 & $\mathrm{ccc}$ & 3 & 15 & S22 & 8 & 8.38 & 43.6 & 54.7 & 13.3336 & 13.966946 & 72.66812 & 91.16849 \\
\hline SRNL-SCS-2008-00005 & $\mathrm{s}$ & Soln Std & ref & 3 & 16 & STD-33-2 & 19 & 9.61 & 80.2 & 49.6 & 19 & 9.61 & 80.2 & 49.6 \\
\hline SRNL-SCS-2008-00005 & $\mathrm{s}$ & SB5-12 & $\mathrm{ccc}$ & 3 & 17 & S68 & 12.2 & 5.86 & 43.1 & 53.5 & 20.33374 & 9.766862 & 71.83477 & 89.16845 \\
\hline SRNL-SCS-2008-00005 & $\mathrm{s}$ & SB5-03 & quenched & 3 & 18 & $\mathrm{~S} 71$ & 8.34 & 6.1 & 37.5 & 44 & 13.900278 & 10.16687 & 62.50125 & 73.3348 \\
\hline SRNL-SCS-2008-00005 & $\mathrm{s}$ & SB5-10 & quenched & 3 & 19 & S25 & 8.69 & 9.07 & 51.4 & 58.7 & 14.483623 & 15.116969 & 85.66838 & 97.83529 \\
\hline SRNL-SCS-2008-00005 & $\mathrm{s}$ & SB5-01 & $\mathrm{ccc}$ & 3 & 20 & S52 & 5.65 & 7.43 & 40 & 42.6 & 9.416855 & 12.383581 & 66.668 & 71.00142 \\
\hline SRNL-SCS-2008-00005 & $\mathrm{s}$ & SB5-08 & $\mathrm{ccc}$ & 3 & 21 & $\mathrm{~S} 11$ & 10.8 & 5.38 & 37.9 & 49.4 & 18.00036 & 8.966846 & 63.16793 & 82.33498 \\
\hline SRNL-SCS-2008-00005 & $\mathrm{s}$ & SB5-02 & quenched & 3 & 22 & $\mathrm{~S} 32$ & 6.42 & 7.05 & 39.7 & 48.2 & 10.700214 & 11.750235 & 66.16799 & 80.33494 \\
\hline SRNL-SCS-2008-00005 & $\mathrm{s}$ & SB5-05 & $\mathrm{ccc}$ & 3 & 23 & $\mathrm{~S} 45$ & 6.99 & 8.65 & 46.9 & 49.2 & 11.650233 & 14.416955 & 78.16823 & 82.00164 \\
\hline SRNL-SCS-2008-00005 & $\mathrm{s}$ & EA & ref & 3 & 24 & $\mathrm{~S} 55$ & 35.2 & 10.9 & 99 & 52 & 586.66784 & 181.66703 & 1650.0033 & 866.6684 \\
\hline SRNL-SCS-2008-00005 & $\mathrm{s}$ & SB5-09 & quenched & 3 & 25 & S08 & 8.67 & 9.81 & 58 & 55.5 & 14.450289 & 16.350327 & 96.6686 & 92.50185 \\
\hline SRNL-SCS-2008-00005 & $\mathrm{s}$ & SB5-07 & $\mathrm{ccc}$ & 3 & 26 & S17 & 8.77 & 6.38 & 38.6 & 45 & 14.616959 & 10.633546 & 64.33462 & 75.0015 \\
\hline SRNL-SCS-2008-00005 & $\mathrm{s}$ & SB5-08 & quenched & 3 & 27 & S34 & 10.4 & 5.12 & 37.4 & 47.1 & 17.33368 & 8.533504 & 62.33458 & 78.50157 \\
\hline SRNL-SCS-2008-00005 & $\mathrm{s}$ & ARM-1 & ref & 3 & 28 & S85 & 8.9 & 7.5 & 19.8 & 34.3 & 14.83363 & 12.50025 & 33.00066 & 57.16781 \\
\hline SRNL-SCS-2008-00005 & $\mathrm{s}$ & SB5-13 & $\mathrm{ccc}$ & 3 & 29 & $\mathrm{~S} 14$ & 10.2 & 11.6 & 71.3 & 68.3 & 17.00034 & 19.33372 & 118.83571 & 113.83561 \\
\hline SRNL-SCS-2008-00005 & $\mathrm{s}$ & SB5-11 & quenched & 3 & 30 & $\mathrm{~S} 75$ & 10.7 & 7.34 & 46.3 & 52.2 & 17.83369 & 12.233578 & 77.16821 & 87.00174 \\
\hline SRNL-SCS-2008-00005 & $\mathrm{s}$ & SB5-07 & quenched & 3 & 31 & S53 & 9.37 & 6.57 & 40.7 & 47 & 15.616979 & 10.950219 & 67.83469 & 78.3349 \\
\hline SRNL-SCS-2008-00005 & $\mathrm{s}$ & Soln Std & ref & 3 & 32 & STD-33-3 & 19.2 & 9.75 & 81.1 & 50.3 & 19.2 & 9.75 & 81.1 & 50.3 \\
\hline SRNL-SCS-2008-00042 & $\mathrm{u}$ & Soln Std & ref & 1 & 1 & STD-11 & 20.2 & 10 & 83.1 & 51.3 & 20.2 & 10 & 83.1 & 51.3 \\
\hline SRNL-SCS-2008-00042 & $\mathrm{u}$ & SB5-21 & quenched & 1 & 2 & $\mathrm{U} 43$ & 8.69 & 6.35 & 43 & 41.1 & 14.483623 & 10.583545 & 71.6681 & 68.50137 \\
\hline SRNL-SCS-2008-00042 & $\mathrm{u}$ & EA & ref & 1 & 3 & U02 & 37.2 & 11.46 & 103 & 54.2 & 620.00124 & 191.00038 & 1716.6701 & 903.33514 \\
\hline SRNL-SCS-2008-00042 & $\mathrm{u}$ & SB5-24 & quenched & 1 & 4 & U14 & 7.54 & 4.31 & 31.2 & 36.6 & 12.566918 & 7.183477 & 52.00104 & 61.00122 \\
\hline SRNL-SCS-2008-00042 & $\mathrm{u}$ & SB5-22 & $\mathrm{ccc}$ & 1 & 5 & U36 & 9.87 & 5.28 & 38.2 & 42.2 & 16.450329 & 8.800176 & 63.66794 & 70.33474 \\
\hline
\end{tabular}


Table B1. PSAL Measurements of the PCT Solutions (in ppm) for the Study Glasses

As-Received (ar) and After Appropriate Adjustments. (continued)

\begin{tabular}{|c|c|c|c|c|c|c|c|c|c|c|c|c|c|c|}
\hline $\begin{array}{c}\text { Analytical Plan } \\
\text { Memo } \\
\end{array}$ & Set & Glass ID & $\begin{array}{c}\text { Heat } \\
\text { Treament } \\
\end{array}$ & Block & Sequence & Lab ID & B (ar) & Li (ar) & $\mathrm{Na}(\mathrm{ar})$ & Si (ar) & B & $\mathbf{L i}$ & $\mathrm{Na}$ & Si \\
\hline SRNL-SCS-2008-00042 & $\mathrm{u}$ & SB5-23 & quenched & 1 & 6 & U07 & 8.21 & 6.24 & 39.1 & 39.8 & 13.683607 & 10.400208 & 65.16797 & 66.33466 \\
\hline SRNL-SCS-2008-00042 & $\mathrm{u}$ & SB5-26 & quenched & 1 & 7 & $\mathrm{U} 27$ & 10.4 & 5.04 & 45 & 47.5 & 17.33368 & 8.400168 & 75.0015 & 79.16825 \\
\hline SRNL-SCS-2008-00042 & $\mathrm{u}$ & SB5-24 & $\mathrm{ccc}$ & 1 & 8 & $\mathrm{U} 30$ & 6.98 & 4.32 & 29.2 & 36.7 & 11.633566 & 7.200144 & 48.66764 & 61.16789 \\
\hline SRNL-SCS-2008-00042 & $\mathrm{u}$ & Soln Std & ref & 1 & 9 & STD-11 & 18.8 & 9.87 & 82.4 & 50 & 18.8 & 9.87 & 82.4 & 50 \\
\hline SRNL-SCS-2008-00042 & $\mathrm{u}$ & SB5-26 & $\mathrm{ccc}$ & 1 & 10 & $\mathrm{U} 26$ & 10.8 & 5.32 & 43 & 49.2 & 18.00036 & 8.866844 & 71.6681 & 82.00164 \\
\hline SRNL-SCS-2008-00042 & $\mathrm{u}$ & blank & ref & 1 & 11 & $\mathrm{U} 01$ & $<0.100$ & $<1.00$ & $<0.100$ & $<0.100$ & 0.083335 & 0.83335 & 0.083335 & 0.083335 \\
\hline SRNL-SCS-2008-00042 & $\mathrm{u}$ & ARM-1 & ref & 1 & 12 & U09 & 11.5 & 9.35 & 25.5 & 40.5 & 19.16705 & 15.583645 & 42.50085 & 67.50135 \\
\hline SRNL-SCS-2008-00042 & $\mathrm{u}$ & SB5-25 & $\mathrm{ccc}$ & 1 & 13 & $\mathrm{U} 23$ & 8.43 & 6.52 & 42.8 & 46 & 14.050281 & 10.866884 & 71.33476 & 76.6682 \\
\hline SRNL-SCS-2008-00042 & $\mathrm{u}$ & SB5-21 & $\mathrm{ccc}$ & 1 & 14 & $\mathrm{U} 22$ & 8.67 & 6.55 & 39.7 & 41.2 & 14.450289 & 10.916885 & 66.16799 & 68.66804 \\
\hline SRNL-SCS-2008-00042 & $\mathrm{u}$ & SB5-25 & quenched & 1 & 15 & $\mathrm{U} 42$ & 8.54 & 6.45 & 44.5 & 46.5 & 14.233618 & 10.750215 & 74.16815 & 77.50155 \\
\hline SRNL-SCS-2008-00042 & $\mathrm{u}$ & SB5-22 & quenched & 1 & 16 & U19 & 10.1 & 5.42 & 41.7 & 44.1 & 16.83367 & 9.033514 & 69.50139 & 73.50147 \\
\hline SRNL-SCS-2008-00042 & $\mathrm{u}$ & SB5-23 & $\mathrm{ccc}$ & 1 & 17 & U06 & 8.23 & 6.4 & 38 & 40.2 & 13.716941 & 10.66688 & 63.3346 & 67.00134 \\
\hline SRNL-SCS-2008-00042 & $\mathrm{u}$ & Soln Std & ref & 1 & 18 & STD-13 & 19.1 & 10.1 & 84.3 & 50.9 & 19.1 & 10.1 & 84.3 & 50.9 \\
\hline SRNL-SCS-2008-00042 & $\mathrm{u}$ & Soln Std & ref & 2 & 1 & STD-21 & 21.7 & 10.2 & 82.5 & 54 & 21.7 & 10.2 & 82.5 & 54 \\
\hline SRNL-SCS-2008-00042 & $\mathrm{u}$ & SB5-21 & quenched & 2 & 2 & U39 & 10.4 & 6.79 & 43.3 & 47 & 17.33368 & 11.316893 & 72.16811 & 78.3349 \\
\hline SRNL-SCS-2008-00042 & $\mathrm{u}$ & SB5-26 & quenched & 2 & 3 & $\mathrm{U} 21$ & 12.1 & 5.16 & 45.2 & 51.9 & 20.16707 & 8.600172 & 75.33484 & 86.50173 \\
\hline SRNL-SCS-2008-00042 & $\mathrm{u}$ & EA & ref & 2 & 4 & $\mathrm{U} 31$ & 41.8 & 12.1 & 105 & 59.9 & 696.66806 & 201.66707 & 1750.0035 & 998.33533 \\
\hline SRNL-SCS-2008-00042 & $\mathrm{u}$ & SB5-24 & $\mathrm{ccc}$ & 2 & 5 & $\mathrm{U} 13$ & 8.37 & 4.29 & 29.4 & 39.6 & 13.950279 & 7.150143 & 49.00098 & 66.00132 \\
\hline SRNL-SCS-2008-00042 & $\mathrm{u}$ & SB5-24 & quenched & 2 & 6 & $\mathrm{U} 15$ & 8.56 & 4.36 & 31.2 & 40.1 & 14.266952 & 7.266812 & 52.00104 & 66.83467 \\
\hline SRNL-SCS-2008-00042 & $\mathrm{u}$ & SB5-22 & quenched & 2 & 7 & $\mathrm{U} 12$ & 11.3 & 5.22 & 38.8 & 45.5 & 18.83371 & 8.700174 & 64.66796 & 75.83485 \\
\hline SRNL-SCS-2008-00042 & $\mathrm{u}$ & SB5-25 & $\mathrm{ccc}$ & 2 & 8 & U16 & 9.23 & 6.19 & 40 & 46.3 & 15.383641 & 10.316873 & 66.668 & 77.16821 \\
\hline SRNL-SCS-2008-00042 & $\mathrm{u}$ & Soln Std & ref & 2 & 9 & STD-22 & 20.5 & 9.94 & 83 & 52.2 & 20.5 & 9.94 & 83 & 52.2 \\
\hline SRNL-SCS-2008-00042 & $\mathrm{u}$ & SB5-25 & quenched & 2 & 10 & $\mathrm{U} 28$ & 10.4 & 6.21 & 42.9 & 47.9 & 17.33368 & 10.350207 & 71.50143 & 79.83493 \\
\hline SRNL-SCS-2008-00042 & $\mathrm{u}$ & SB5-26 & $\mathrm{ccc}$ & 2 & 11 & $\mathrm{U} 40$ & 12.3 & 5.43 & 44.4 & 52.5 & 20.50041 & 9.050181 & 74.00148 & 87.50175 \\
\hline SRNL-SCS-2008-00042 & $\mathrm{u}$ & ARM-1 & ref & 2 & 12 & $\mathrm{U} 20$ & 13.7 & 9.71 & 25 & 44.4 & 22.83379 & 16.183657 & 41.6675 & 74.00148 \\
\hline SRNL-SCS-2008-00042 & $\mathrm{u}$ & SB5-22 & $\mathrm{ccc}$ & 2 & 13 & $\mathrm{U} 35$ & 11.7 & 5.37 & 37.5 & 46.5 & 19.50039 & 8.950179 & 62.50125 & 77.50155 \\
\hline SRNL-SCS-2008-00042 & $\mathrm{u}$ & SB5-23 & quenched & 2 & 14 & $\mathrm{U} 33$ & 9.73 & 6.37 & 38.9 & 43.8 & 16.216991 & 10.616879 & 64.83463 & 73.00146 \\
\hline SRNL-SCS-2008-00042 & $\mathrm{u}$ & SB5-21 & $\mathrm{ccc}$ & 2 & 15 & U11 & 10.2 & 6.64 & 39.9 & 45 & 17.00034 & 11.066888 & 66.50133 & 75.0015 \\
\hline
\end{tabular}


Table B1. PSAL Measurements of the PCT Solutions (in ppm) for the Study Glasses

As-Received (ar) and After Appropriate Adjustments. (continued)

\begin{tabular}{|c|c|c|c|c|c|c|c|c|c|c|c|c|c|c|}
\hline $\begin{array}{c}\text { Analytical Plan } \\
\text { Memo } \\
\end{array}$ & Set & Glass ID & $\begin{array}{c}\text { Heat } \\
\text { Treament } \\
\end{array}$ & Block & Sequence & Lab ID & B (ar) & Li (ar) & Na (ar) & $\mathrm{Si}$ (ar) & B & $\mathbf{L i}$ & $\mathrm{Na}$ & Si \\
\hline SRNL-SCS-2008-00042 & $\mathrm{u}$ & SB5-23 & $\mathrm{ccc}$ & 2 & 16 & $\mathrm{U} 32$ & 10.1 & 6.76 & 38.3 & 45.9 & 16.83367 & 11.266892 & 63.83461 & 76.50153 \\
\hline SRNL-SCS-2008-00042 & $\mathrm{u}$ & Soln Std & ref & 2 & 17 & STD-23 & 21.8 & 10.3 & 83.1 & 54.9 & 21.8 & 10.3 & 83.1 & 54.9 \\
\hline SRNL-SCS-2008-00042 & $\mathrm{u}$ & Soln Std & ref & 3 & 1 & STD-31 & 20.7 & 10 & 83 & 51.7 & 20.7 & 10 & 83 & 51.7 \\
\hline SRNL-SCS-2008-00042 & $\mathrm{u}$ & SB5-24 & $\mathrm{ccc}$ & 3 & 2 & $\mathrm{U} 41$ & 7.87 & 4.31 & 29.8 & 38 & 13.116929 & 7.183477 & 49.66766 & 63.3346 \\
\hline SRNL-SCS-2008-00042 & $\mathrm{u}$ & SB5-22 & quenched & 3 & 3 & $\mathrm{U} 18$ & 10.5 & 5.28 & 41 & 44.3 & 17.50035 & 8.800176 & 68.3347 & 73.83481 \\
\hline SRNL-SCS-2008-00042 & $\mathrm{u}$ & SB5-23 & quenched & 3 & 4 & U08 & 8.7 & 6.28 & 39.7 & 40.8 & 14.50029 & 10.466876 & 66.16799 & 68.00136 \\
\hline SRNL-SCS-2008-00042 & $\mathrm{u}$ & SB5-22 & $\mathrm{ccc}$ & 3 & 5 & $\mathrm{U} 05$ & 11 & 5.65 & 41.1 & 47 & 18.3337 & 9.416855 & 68.50137 & 78.3349 \\
\hline SRNL-SCS-2008-00042 & $\mathrm{u}$ & blank & ref & 3 & 6 & $\mathrm{U} 29$ & $<0.100$ & $<1.00$ & $<0.100$ & $<0.100$ & 0.083335 & 0.83335 & 0.083335 & 0.083335 \\
\hline SRNL-SCS-2008-00042 & $\mathrm{u}$ & SB5-26 & $\mathrm{ccc}$ & 3 & 7 & U03 & 11 & 5.42 & 44 & 51.3 & 18.3337 & 9.033514 & 73.3348 & 85.50171 \\
\hline SRNL-SCS-2008-00042 & $\mathrm{u}$ & SB5-25 & $\mathrm{ccc}$ & 3 & 8 & U17 & 8.49 & 6.19 & 40.3 & 45.3 & 14.150283 & 10.316873 & 67.16801 & 75.50151 \\
\hline SRNL-SCS-2008-00042 & $\mathrm{u}$ & Soln Std & ref & 3 & 9 & STD-32 & 19.7 & 9.98 & 82.7 & 51.6 & 19.7 & 9.98 & 82.7 & 51.6 \\
\hline SRNL-SCS-2008-00042 & $\mathrm{u}$ & ARM-1 & ref & 3 & 10 & $\mathrm{U} 24$ & 12.6 & 9.38 & 25.7 & 41 & 21.00042 & 15.633646 & 42.83419 & 68.3347 \\
\hline SRNL-SCS-2008-00042 & $\mathrm{u}$ & SB5-21 & $\mathrm{ccc}$ & 3 & 11 & $\mathrm{U} 38$ & 9.48 & 6.6 & 39.8 & 42.7 & 15.800316 & 11.00022 & 66.33466 & 71.16809 \\
\hline SRNL-SCS-2008-00042 & $\mathrm{u}$ & SB5-25 & quenched & 3 & 12 & $\mathrm{U} 10$ & 9.39 & 6.55 & 44.6 & 48.6 & 15.650313 & 10.916885 & 74.33482 & 81.00162 \\
\hline SRNL-SCS-2008-00042 & $\mathrm{u}$ & EA & ref & 3 & 13 & $\mathrm{U} 25$ & 38.4 & 11.8 & 107 & 56.8 & 640.00128 & 196.66706 & 1783.3369 & 946.66856 \\
\hline SRNL-SCS-2008-00042 & $\mathrm{u}$ & SB5-26 & quenched & 3 & 14 & $\mathrm{U} 34$ & 11.03 & 5.06 & 46.3 & 47.9 & 18.383701 & 8.433502 & 77.16821 & 79.83493 \\
\hline SRNL-SCS-2008-00042 & $\mathrm{u}$ & SB5-21 & quenched & 3 & 15 & U04 & 9.43 & 6.62 & 43.9 & 43.3 & 15.716981 & 11.033554 & 73.16813 & 72.16811 \\
\hline SRNL-SCS-2008-00042 & $\mathrm{u}$ & SB5-23 & $\mathrm{ccc}$ & 3 & 16 & $\mathrm{U} 44$ & 8.89 & 6.67 & 40.1 & 42.9 & 14.816963 & 11.116889 & 66.83467 & 71.50143 \\
\hline SRNL-SCS-2008-00042 & $\mathrm{u}$ & SB5-24 & quenched & 3 & 17 & U37 & 8.01 & 4.62 & 34.4 & 39.4 & 13.350267 & 7.700154 & 57.33448 & 65.66798 \\
\hline SRNL-SCS-2008-00042 & $\mathrm{u}$ & Soln Std & ref & 3 & 18 & STD-33 & 19.6 & 10.1 & 84.6 & 51.5 & 19.6 & 10.1 & 84.6 & 51.5 \\
\hline
\end{tabular}


Exhibit B1. Laboratory PCT Measurements in Analytical Sequence by Block for Each Set and Analytical Plan Memo.

Memo=SRNL-SCS-2008-00005, Set $=r$

Chart for log[B ppm]

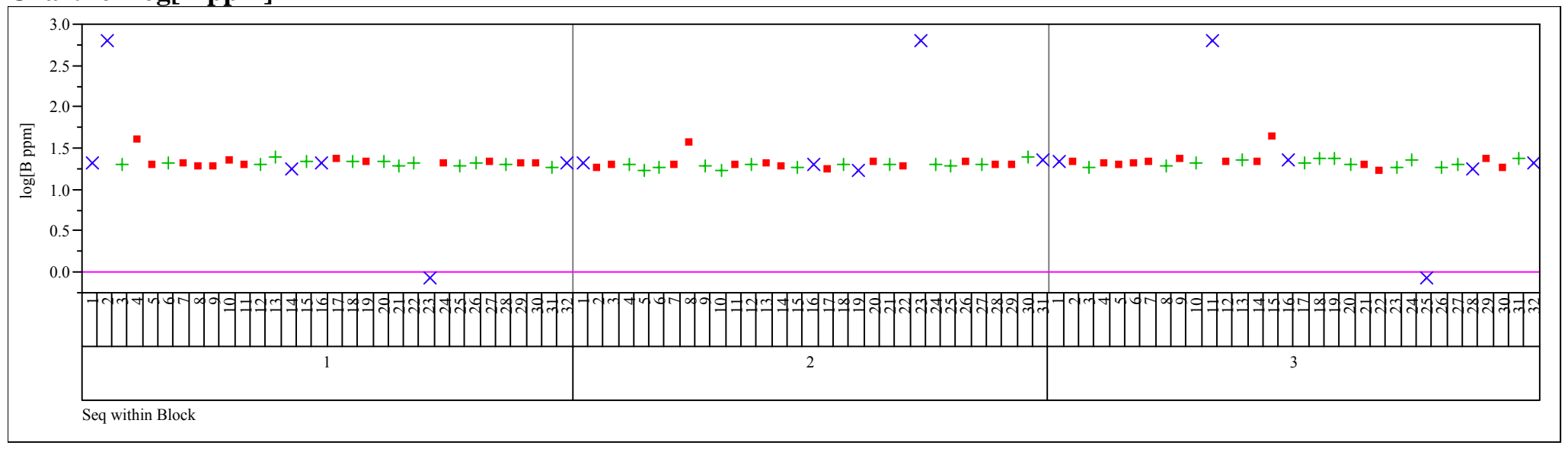

\section{Memo=SRNL-SCS-2008-00005, Set $=r$}

Chart for $\log [\mathrm{Li}$ ppm]

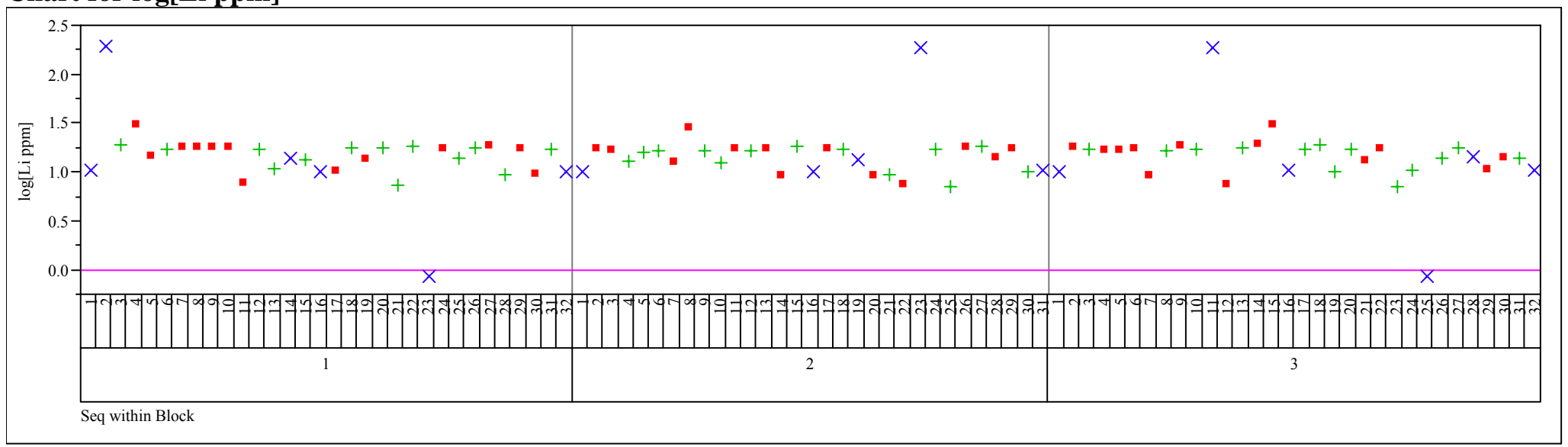


Exhibit B1. Laboratory PCT Measurements in Analytical Sequence by Block for Each Set and Analytical Plan Memo. (continued)

Memo=SRNL-SCS-2008-00005, Set $=r$

Chart for log[Na ppm]

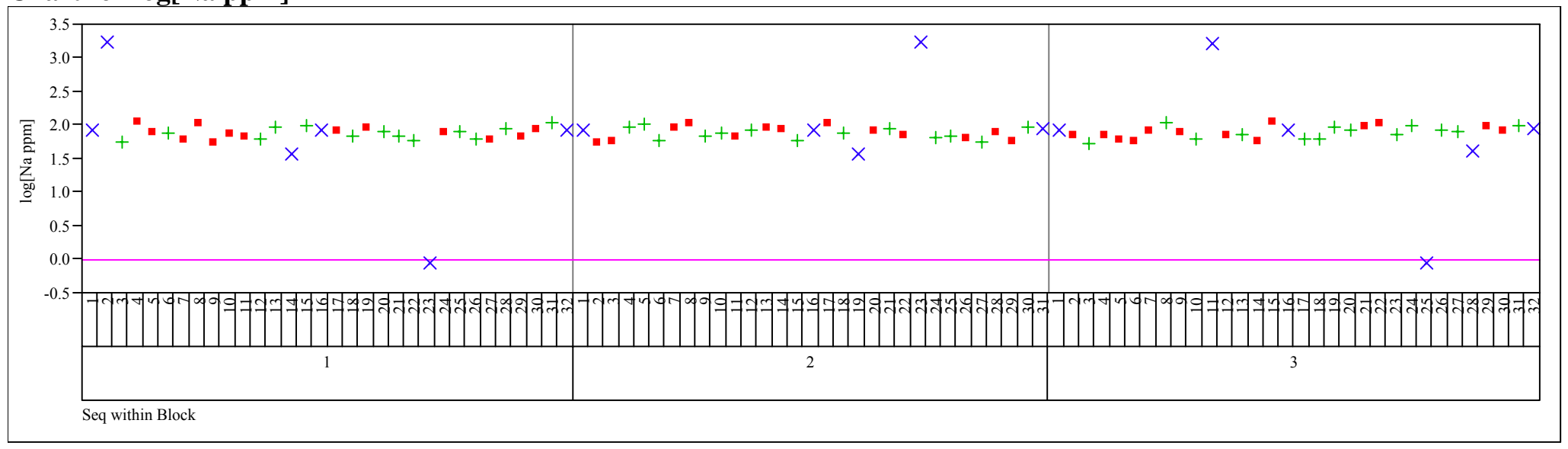

\section{Memo=SRNL-SCS-2008-00005, Set $=r$}

Chart for $\log [\mathrm{Si}$ ppm]

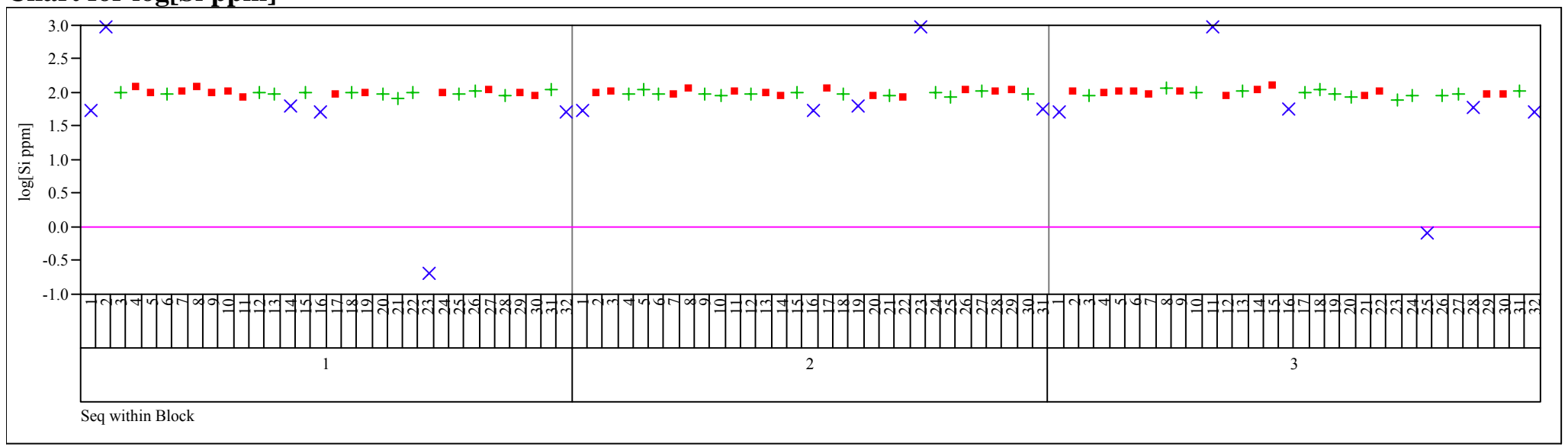


Exhibit B1. Laboratory PCT Measurements in Analytical Sequence by Block for Each Set and Analytical Plan Memo. (continued)

Memo=SRNL-SCS-2008-00005, Set=s

Chart for $\log [\mathrm{B}$ ppm]

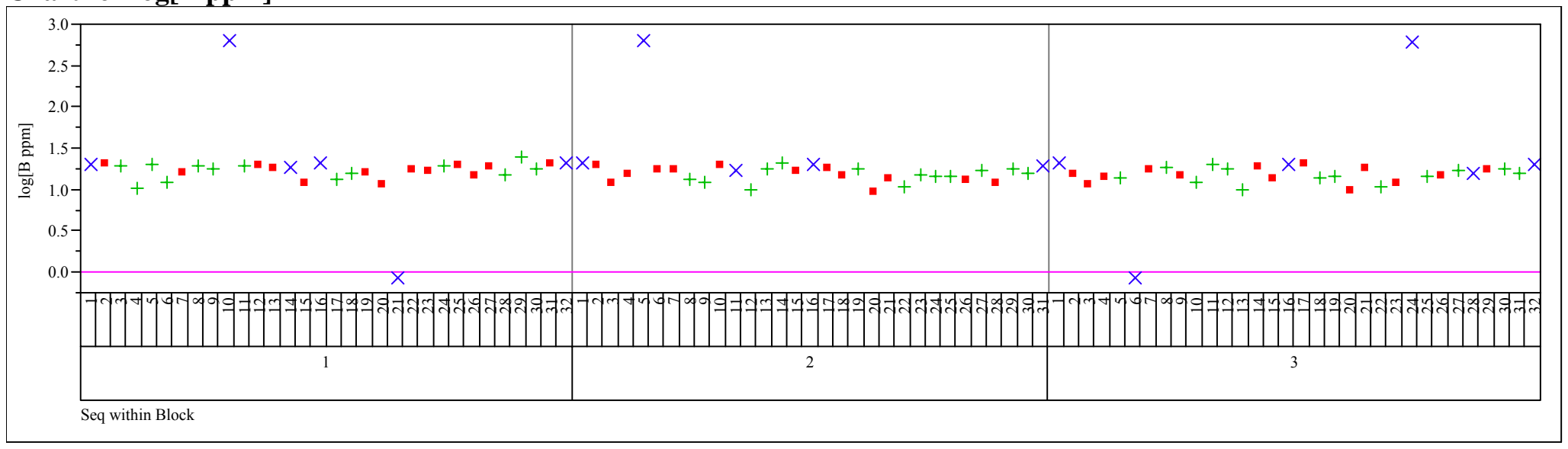

\section{Memo=SRNL-SCS-2008-00005, Set $=$ s}

Chart for $\log [\mathrm{Li}$ ppm]

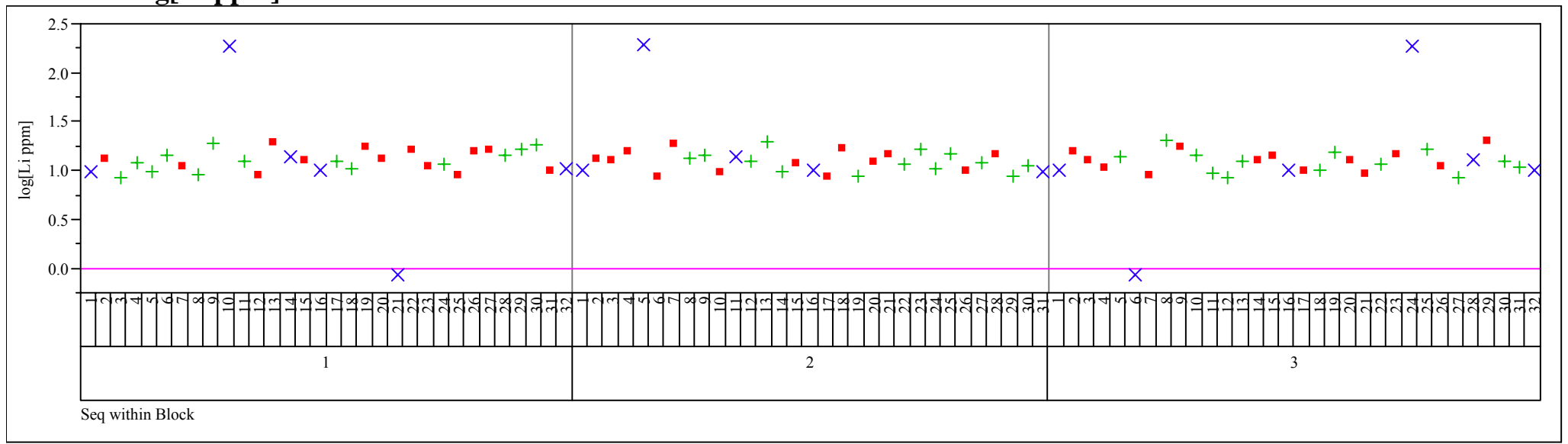


Exhibit B1. Laboratory PCT Measurements in Analytical Sequence by Block for Each Set and Analytical Plan Memo. (continued)

Memo=SRNL-SCS-2008-00005, Set=s

Chart for log[Na ppm]

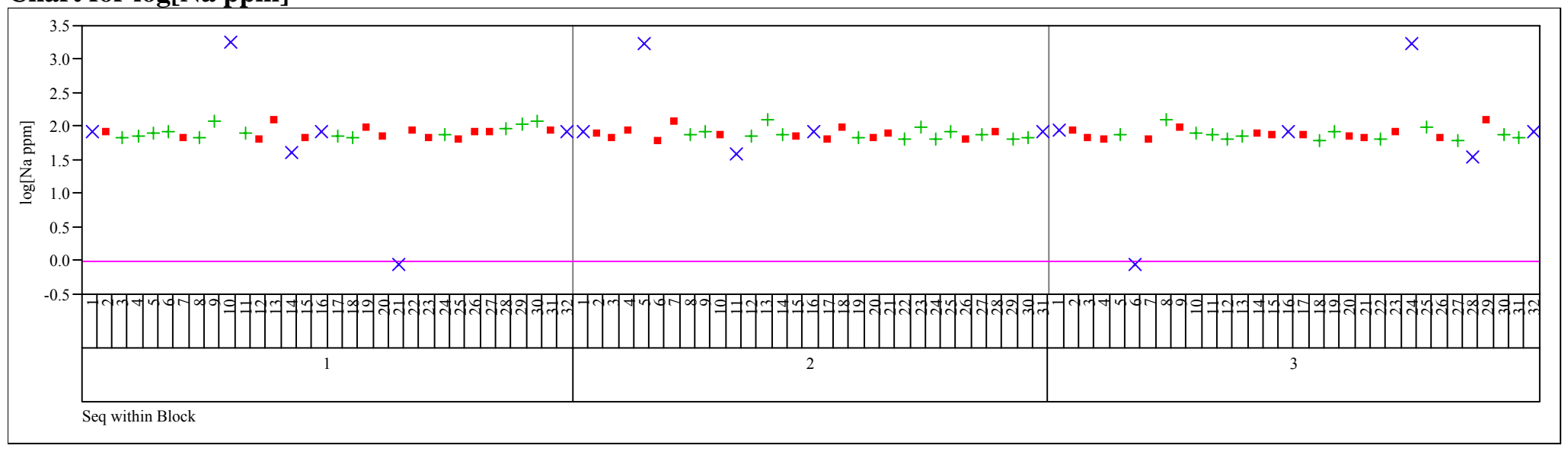

\section{Memo=SRNL-SCS-2008-00005, Set $=$ s}

Chart for $\log [\mathrm{Si}$ ppm]

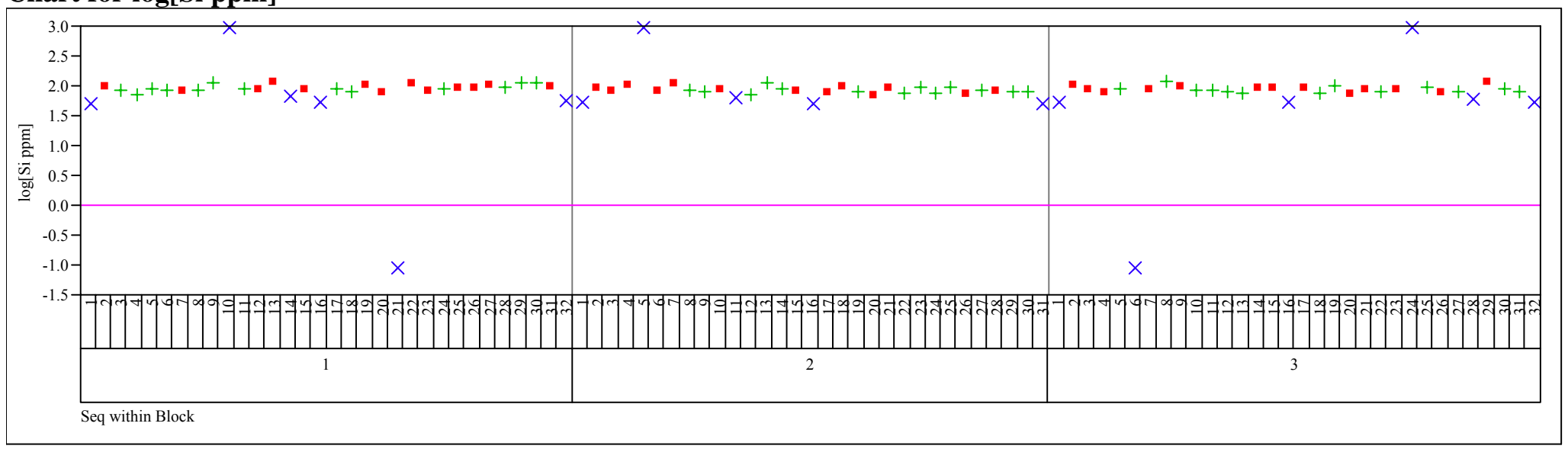


Exhibit B1. Laboratory PCT Measurements in Analytical Sequence by Block for Each Set and Analytical Plan Memo. (continued)

Memo=SRNL-SCS-2008-00042, Set $=u$

Chart for $\log [\mathrm{B}$ ppm]

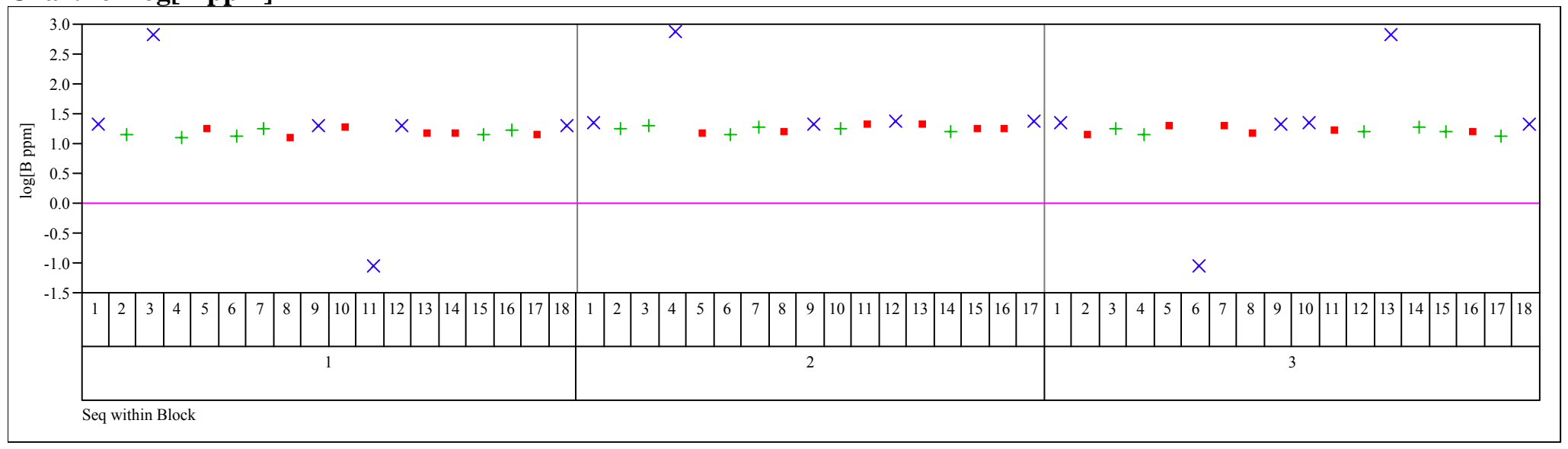

Memo=SRNL-SCS-2008-00042, Set $=\mathbf{u}$

Chart for $\log [\mathrm{Li}$ ppm]

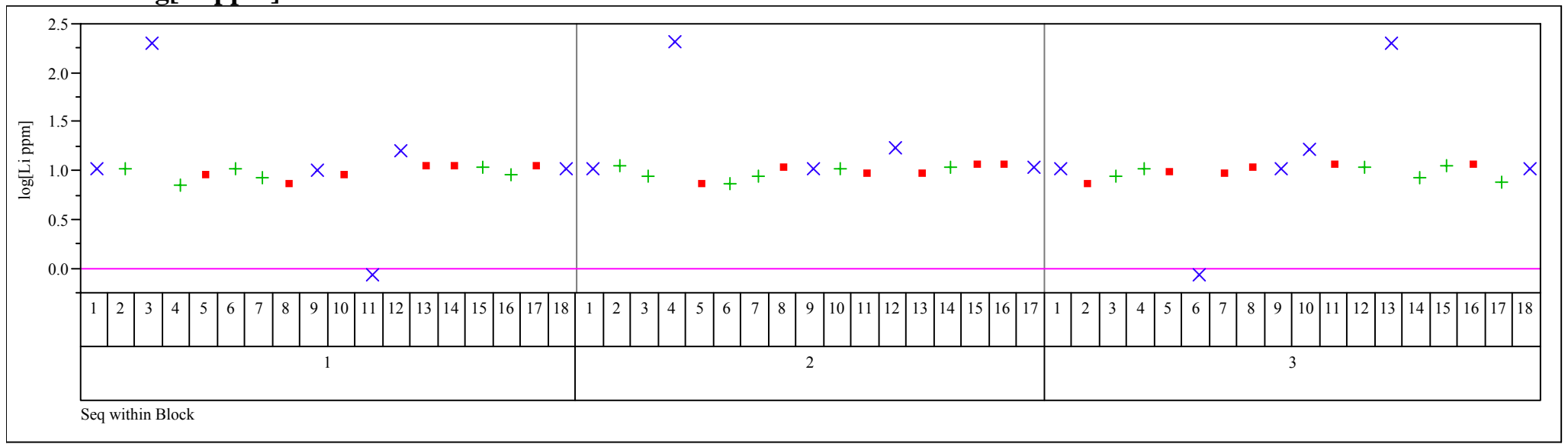


Exhibit B1. Laboratory PCT Measurements in Analytical Sequence by Block for Each Set and Analytical Plan Memo. (continued)

Memo=SRNL-SCS-2008-00042, Set $=u$

Chart for log[Na ppm]

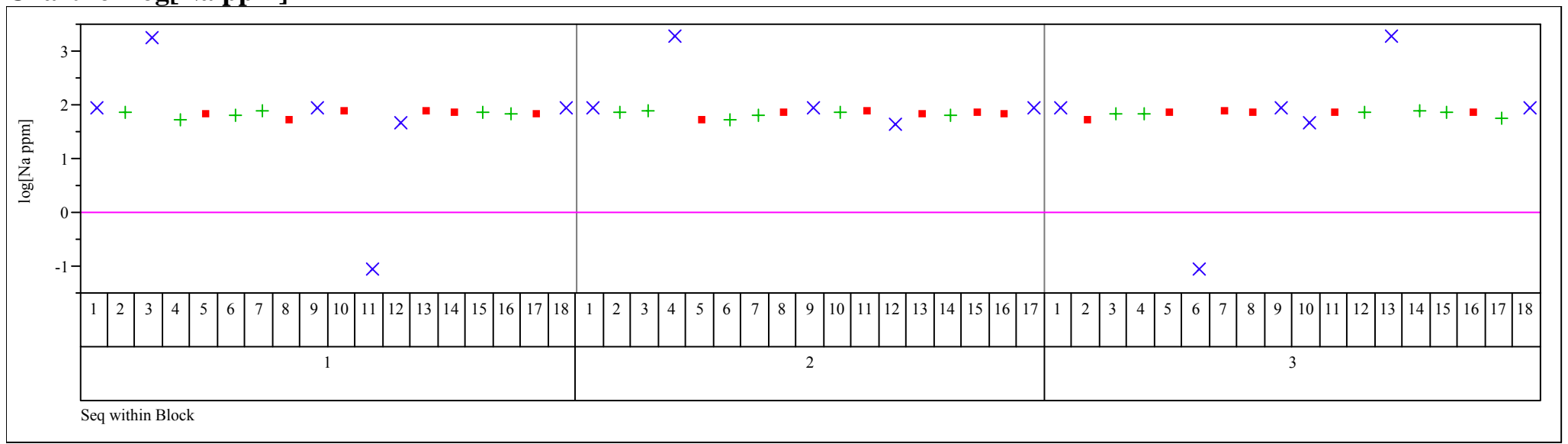

\section{Memo=SRNL-SCS-2008-00042, Set $=u$}

Chart for $\log [\mathrm{Si}$ ppm]

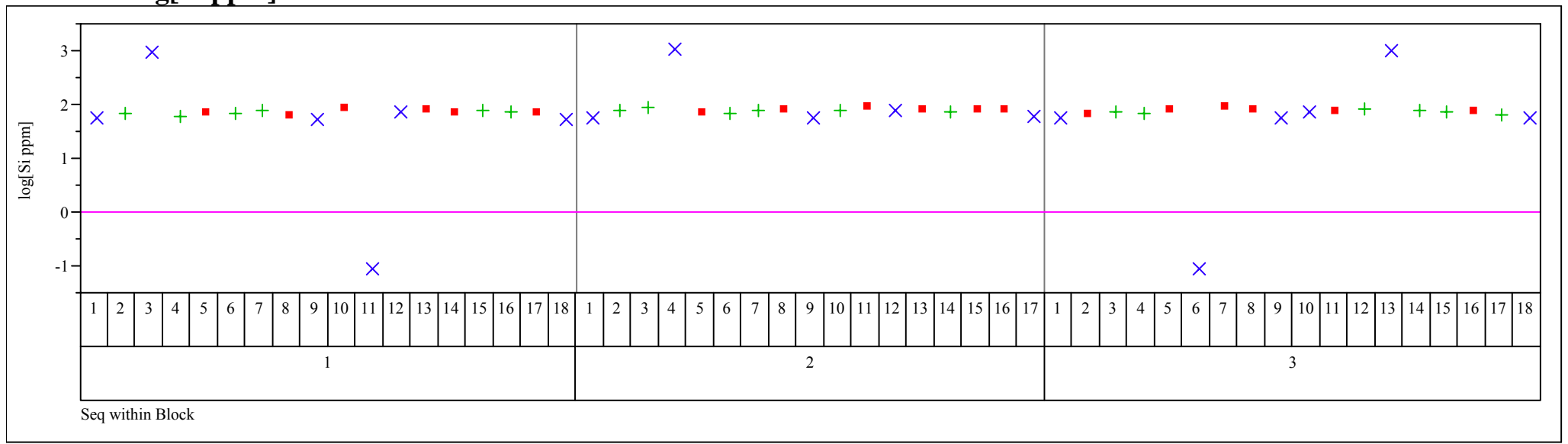




\section{Exhibit B2. Measurements of the Multi-Element Solution Standard by ICP Block.}

Oneway Analysis of Si (ppm) By Block

Memo=SRNL-SCS-2008-00005, Set $=r$

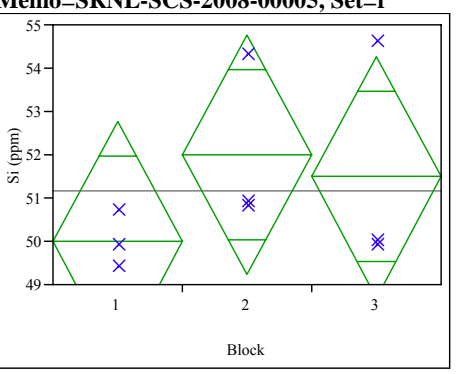

Oneway Anova

Summary of Fit

$\begin{array}{lr}\text { Rsquare } & 0.218708 \\ \text { Adj Rsquare } & -0.04172 \\ \text { Root Mean Square Error } & 1.967232 \\ \text { Mean of Response } & 51.16667 \\ \text { Observations (or Sum Wgts) } & 9\end{array}$

Observations (or Sum Wgts) 9

Analysis of Variance

Source DF Sum of Squares Mean Square F Ratio Prob $>$ F

$\begin{array}{llllll}\text { Block } & 2 & 6.500000 & 3.25000 & 0.8398 & 0.4769\end{array}$

$\begin{array}{llll}\text { Error } & 6 & 23.220000 & 3.87000\end{array}$

C. Total $8 \quad 29.720000$

Means for Oneway Anova

Level Number Mean Std Error Lower 95\% Upper 95\%

$\begin{array}{llllll}1 & 3 & 50.0000 & 1.1358 & 47.221 & 52.779\end{array}$

$\begin{array}{llllll}2 & 3 & 52.0000 & 1.1358 & 49.221 & 54.779\end{array}$

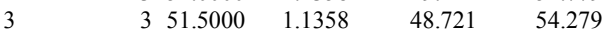

Std Error uses a pooled estimate of error variance

Oneway Analysis of Na (ppm) By Block

Memo=SRNL-SCS-2008-00005, Set $=r$

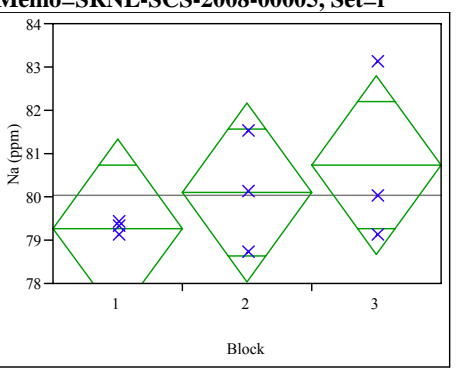

Oneway Anova

Summary of Fit

$\begin{array}{lr}\text { Rsquare } & 0.202663 \\ \text { Adj Rsquare } & -0.06312 \\ \text { Root Mean Square Error } & 1.459071 \\ \text { Mean of Response } & 80.03333 \\ \text { Observations (or Sum Wgts) } & 9\end{array}$

Analysis of Variance

Source DF Sum of Squares Mean Square F Ratio Prob > F

$\begin{array}{llllll}\text { Block } & 2 & 3.246667 & 1.62333 & 0.7625 & 0.5069\end{array}$

$\begin{array}{llrr}\text { Error } & 6 & 12.773333 & 2.12889\end{array}$

$\begin{array}{lll}\text { C. Total } & 8 & 16.020000\end{array}$

Means for Oneway Anova

Level Number Mean Std Error Lower 95\% Upper 95\%

$\begin{array}{llllll}1 & 3 & 79.2667 & 0.84240 & 77.205 & 81.328\end{array}$

$\begin{array}{llllll}2 & 3 & 80.1000 & 0.84240 & 78.039 & 82.16\end{array}$

$\begin{array}{llllll}3 & 3 & 80.7333 & 0.84240 & 78.672 & 82.795\end{array}$

Std Error uses a pooled estimate of error variance
Oneway Analysis of $\mathrm{Li}(\mathrm{ppm})$ By Block

Memo=SRNL-SCS-2008-00005, Set $=r$

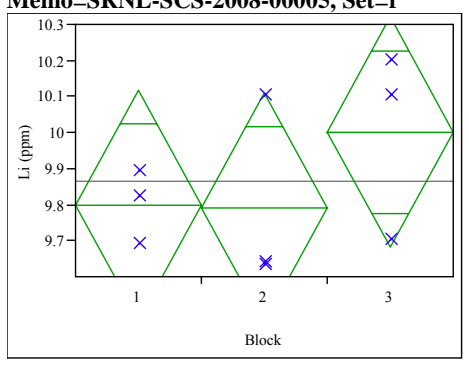

Oneway Anova

Summary of Fit

Rsquare

0.216452

Adj Rsquare

$-0.04473$

Root Mean Square Error $\quad 0.225389$

Mean of Response $\quad 9.863333$

Observations (or Sum Wgts) 9

Analysis of Variance

Source DF Sum of Squares Mean Square F Ratio Prob $>$ F

$\begin{array}{lrrrrr}\text { Block } & 2 & 0.08420000 & 0.042100 & 0.8287 & 0.4811\end{array}$

$\begin{array}{llll}\text { Error } & 6 & 0.30480000 & 0.050800\end{array}$

C. Total $8 \quad 0.38900000$

Means for Oneway Anova

Level Number Mean Std Error Lower 95\% Upper 95\%

$\begin{array}{lllrrr}1 & 3 & 9.8000 & 0.13013 & 9.4816 & 10.118 \\ 2 & 3 & 9.7900 & 0.13013 & 9.4716 & 10.108\end{array}$

$\begin{array}{llllll}3 & 3 & 10.0000 & 0.13013 & 9.6816 & 10.318\end{array}$

Std Error uses a pooled estimate of error variance

Oneway Analysis of B (ppm) By Block Memo=SRNL-SCS-2008-00005, Set $=r$

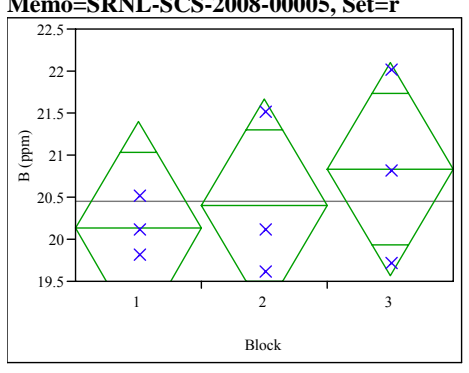

Oneway Anova

Summary of Fit

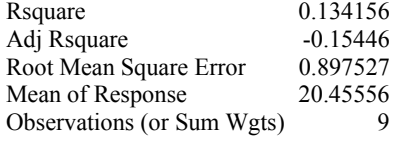

Analysis of Variance

Source DF Sum of Squares Mean Square F Ratio Prob $>$ F

$\begin{array}{llllll}\text { Block } & 2 & 0.7488889 & 0.374444 & 0.4648 & 0.6491\end{array}$

$\begin{array}{llll}\text { Error } & 6 & 4.8333333 & 0.805556\end{array}$

C. Total $8 \quad 5.5822222$

Means for Oneway Anova

Level Number Mean Std Error Lower 95\% Upper 95\%

$\begin{array}{llllll}1 & 3 & 20.1333 & 0.51819 & 18.865 & 21.401 \\ 2 & 3 & 20.4000 & 0.51819 & 19.132 & 21.668\end{array}$

$\begin{array}{llllll}2 & 3 & 20.4000 & 0.51819 & 19.132 & 21.668 \\ 3 & 3 & 20.8333 & 0.51819 & 19.565 & 22.101\end{array}$

Std Error uses a pooled estimate of error variance 
Exhibit B2. Measurements of the Multi-Element Solution Standard by ICP Block. (cont'd)

Oneway Analysis of Si (ppm) By Block

Memo=SRNL-SCS-2008-00005, Set $=$ s

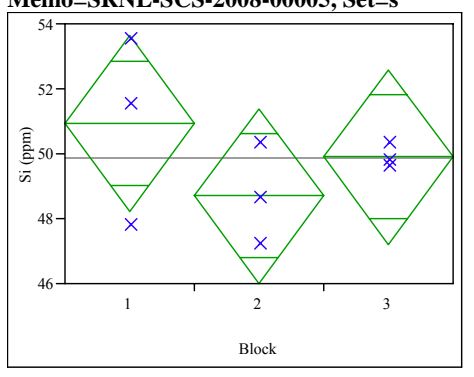

Oneway Anova

Summary of Fit

$\begin{array}{lr}\text { Rsquare } & 0.255802 \\ \text { Adj Rsquare } & 0.007735 \\ \text { Root Mean Square Error } & 1.906422 \\ \text { Mean of Response } & 49.84444 \\ \text { Observations (or Sum Wgts) } & 9\end{array}$

Observations (or Sum Wgts) 9

Analysis of Variance

Source DF Sum of Squares Mean Square F Ratio Prob $>$ F

$\begin{array}{llllll}\text { Block } & 2 & 7.495556 & 3.74778 & 1.0312 & 0.4122\end{array}$

$\begin{array}{llll}\text { Error } & 6 & 21.806667 & 3.63444\end{array}$

C. Total $8 \quad 29.302222$

Means for Oneway Anova

Level Number Mean Std Error Lower 95\% Upper 95\%

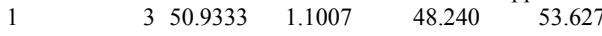

$\begin{array}{llllll}2 & 3 & 48.7000 & 1.1007 & 46.007 & 51.393\end{array}$

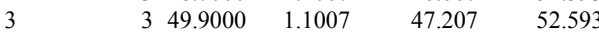

Std Error uses a pooled estimate of error variance

Oneway Analysis of Na (ppm) By Block

Memo=SRNL-SCS-2008-00005, Set $=$ s

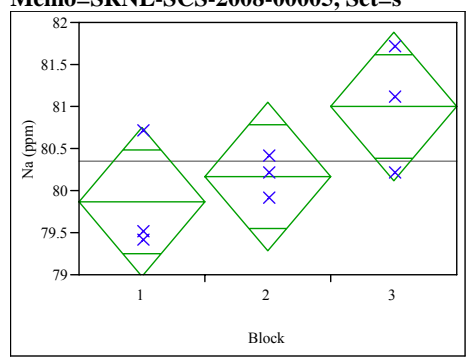

Oneway Anova

Summary of Fit

Rsquare

0.47211

Adj Rsquare

0.296146

Root Mean Square Error $\quad 0.62093$

Mean of Response $\quad 80.34444$

Observations (or Sum Wgts) 9

Analysis of Variance

Source DF Sum of Squares Mean Square F Ratio Prob $>$ F

$\begin{array}{llllll}\text { Block } & 2 & 2.0688889 & 1.03444 & 2.6830 & 0.1471\end{array}$

$\begin{array}{llll}\text { Error } & 6 & 2.3133333 & 0.38556\end{array}$

C. Total $8 \quad 4.3822222$

Means for Oneway Anova

Level Number Mean Std Error Lower 95\% Upper 95\%

$\begin{array}{lllllr}1 & 3 & 79.8667 & 0.35849 & 78.989 & 80.744\end{array}$

$\begin{array}{llllll}2 & 3 & 80.1667 & 0.35849 & 79.289 & 81.044\end{array}$

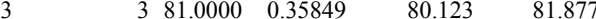

Std Error uses a pooled estimate of error variance
Oneway Analysis of Li (ppm) By Block

Memo=SRNL-SCS-2008-00005, Set $=$ s

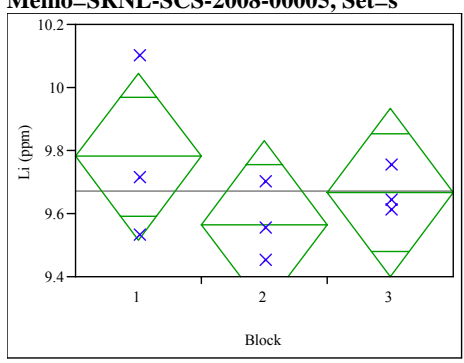

Oneway Anova

Summary of Fit

Rsquare

0.243528

Adj Rsquare

$-0.00863$

Root Mean Square Error $\quad 0.188119$

Mean of Response $\quad 9.671111$

Observations (or Sum Wgts) 9

Analysis of Variance

Source DF Sum of Squares Mean Square F Ratio Prob $>$ F

$\begin{array}{lrrrrr}\text { Block } & 2 & 0.06835556 & 0.034178 & 0.9658 & 0.4329\end{array}$

$\begin{array}{llll}\text { Error } & 6 & 0.21233333 & 0.035389\end{array}$

C. Total $8 \quad 0.28068889$

Means for Oneway Anova

Level Number Mean Std Error Lower 95\% Upper 95\%

$\begin{array}{lrrrrr}1 & 3 & 9.78000 & 0.10861 & 9.5142 & 10.046 \\ 2 & 3 & 9.56667 & 0.10861 & 9.3009 & 9.832\end{array}$

$\begin{array}{lllll}3 & 3 & 9.66667 & 0.10861 & 9.4009\end{array}$

Std Error uses a pooled estimate of error variance

Oneway Analysis of B (ppm) By Block Memo=SRNL-SCS-2008-00005, Set $=$ s

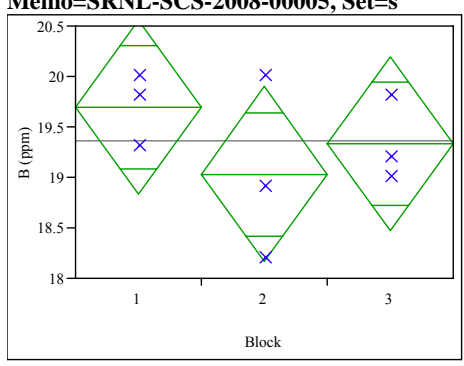

Oneway Anova

Summary of Fit

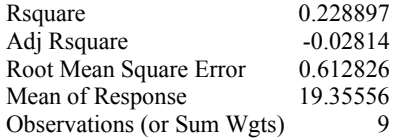

Observations (or Sum Wgts) 19.35556

Analysis of Variance

Source DF Sum of Squares Mean Square F Ratio Prob $>$ F

$\begin{array}{llllll}\text { Block } & 2 & 0.6688889 & 0.334444 & 0.8905 & 0.4585\end{array}$

$\begin{array}{llll}\text { Error } & 6 & 2.2533333 & 0.375556\end{array}$

C. Total $8 \quad 2.9222222$

Means for Oneway Anova

Level Number Mean Std Error Lower 95\% Upper 95\%

$\begin{array}{llllll}1 & 3 & 19.7000 & 0.35382 & 18.834 & 20.566 \\ 2 & 3 & 19.0333 & 0.35382 & 18.168 & 19.899\end{array}$

$\begin{array}{llllll}2 & 3 & 19.0333 & 0.35382 & 18.168 & 19.899 \\ 3 & 3 & 19.3333 & 0.35382 & 18.468 & 20.199\end{array}$

Std Error uses a pooled estimate of error variance 
Exhibit B2. Measurements of the Multi-Element Solution Standard by ICP Block. (cont'd)

Oneway Analysis of Si (ppm) By Block

Memo=SRNL-SCS-2008-00042, Set $=u$

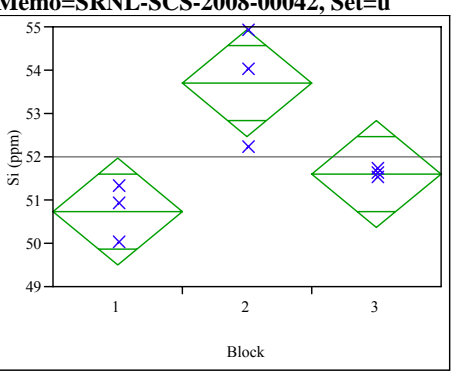

Oneway Anova

Summary of Fit

$\begin{array}{lr}\text { Rsquare } & 0.748689 \\ \text { Adj Rsquare } & 0.664919 \\ \text { Root Mean Square Error } & 0.883805 \\ \text { Mean of Response } & 52.01111 \\ \text { Observations (or Sum Wgts) } & 9\end{array}$

Observations (or Sum Wgts) 9

Analysis of Variance

Source DF Sum of Squares Mean Square F Ratio Prob $>$ F

$\begin{array}{llllll}\text { Block } & 2 & 13.962222 & 6.98111 & 8.9374 & 0.0159\end{array}$

$\begin{array}{llrr}\text { Error } & 6 & 4.686667 & 0.78111\end{array}$

C. Total $8 \quad 18.648889$

Means for Oneway Anova

Level Number Mean Std Error Lower 95\% Upper 95\%

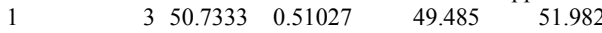

$\begin{array}{llllll}2 & 3 & 53.7000 & 0.51027 & 52.451 & 54.949\end{array}$

$\begin{array}{llllll}3 & 3 & 51.6000 & 0.51027 & 50.351 & 52.849\end{array}$

Std Error uses a pooled estimate of error variance

Oneway Analysis of Na (ppm) By Block

Memo=SRNL-SCS-2008-00042, Set $=u$

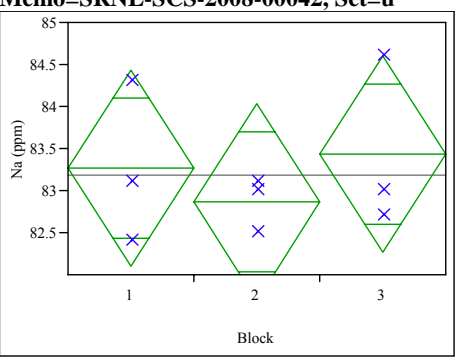

Oneway Anova

Summary of Fit

Rsquare $\quad 0.109465$

Adj Rsquare $\quad-0.18738$

Root Mean Square Error $\quad 0.830662$

Mean of Response 83.18889

Observations (or Sum Wgts) 9

Analysis of Variance

Source DF Sum of Squares Mean Square F Ratio Prob $>$ F

$\begin{array}{llllll}\text { Block } & 2 & 0.5088889 & 0.254444 & 0.3688 & 0.7062\end{array}$

Error $\quad 6 \quad 4.1400000$

C. Total $8 \quad 4.6488889$

Means for Oneway Anova

Level Number Mean Std Error Lower 95\% Upper 95\%

$\begin{array}{lllrrr}1 & 3 & 83.2667 & 0.47958 & 82.093 & 84.440\end{array}$

$\begin{array}{llllll}2 & 3 & 82.8667 & 0.47958 & 81.693 & 84.040\end{array}$

$\begin{array}{llllll}3 & 3 & 83.4333 & 0.47958 & 82.260 & 84.607\end{array}$

Std Error uses a pooled estimate of error variance
Oneway Analysis of Li (ppm) By Block

Memo=SRNL-SCS-2008-00042, Set $=u$

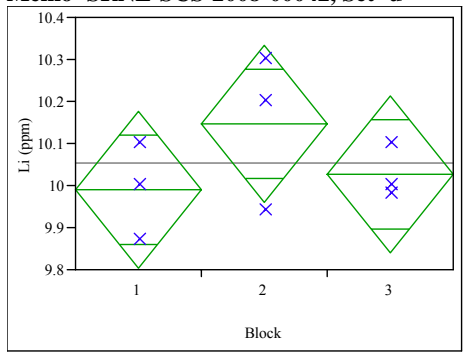

Oneway Anova

Summary of Fit

Rsquare

0.279353

Adj Rsquare

0.039137

Root Mean Square Error $\quad 0.131614$

Mean of Response $\quad 10.05444$

Observations (or Sum Wgts) 9

Analysis of Variance

Source DF Sum of Squares Mean Square F Ratio Prob $>$ F

$\begin{array}{llllll}\text { Block } & 2 & 0.04028889 & 0.020144 & 1.1629 & 0.3743\end{array}$

$\begin{array}{llll}\text { Error } & 6 & 0.10393333 & 0.017322\end{array}$

C. Total $8 \quad 0.14422222$

Means for Oneway Anova

Level Number Mean Std Error Lower 95\% Upper 95\%

$\begin{array}{lrrrrr}1 & 3 & 9.9900 & 0.07599 & 9.8041 & 10.176 \\ 2 & 3 & 10.1467 & 0.07599 & 9.9607 & 10.333\end{array}$

$\begin{array}{llllll}3 & 3 & 10.0267 & 0.07599 & 9.8407 & 10.213\end{array}$

Std Error uses a pooled estimate of error variance

Oneway Analysis of B (ppm) By Block Memo=SRNL-SCS-2008-00042, Set $=u$

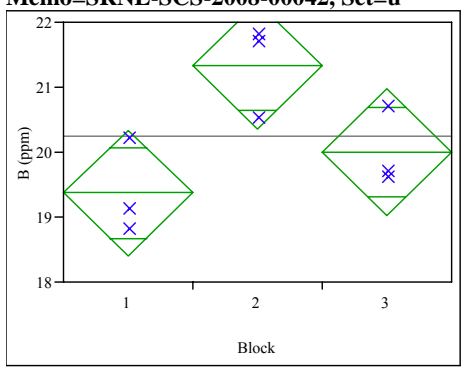

Oneway Anova

Summary of Fit

$\begin{array}{lr}\text { Rsquare } & 0.677877 \\ \text { Adj Rsquare } & 0.570503 \\ \text { Root Mean Square Error } & 0.692018 \\ \text { Mean of Response } & 20.23333 \\ \text { Observations (or Sum Wgts) } & 9\end{array}$

Analysis of Variance

Source DF Sum of Squares Mean Square F Ratio Prob $>$ F $\begin{array}{llllll}\text { Block } & 2 & 6.0466667 & 3.02333 & 6.3132 & 0.0334\end{array}$ $\begin{array}{llll}\text { Error } & 6 & 2.8733333 & 0.47889\end{array}$

C. Total $8 \quad 8.9200000$

Means for Oneway Anova

Level Number Mean Std Error Lower 95\% Upper 95\%

$\begin{array}{lllllr}1 & 3 & 19.3667 & 0.39954 & 18.389 & 20.344 \\ 2 & 3 & 21.3333 & 0.39954 & 20.356 & 22.311\end{array}$

$\begin{array}{llllll}3 & 3 & 20.0000 & 0.39954 & 19.022 & 20.978\end{array}$

Std Error uses a pooled estimate of error variance 
Exhibit B3. Laboratory PCT Measurements by Glass Identifier for Study Glasses and Standards.

Memo=SRNL-SCS-2008-00005, Set $=\mathbf{r} \quad$ Chart for B (ppm)

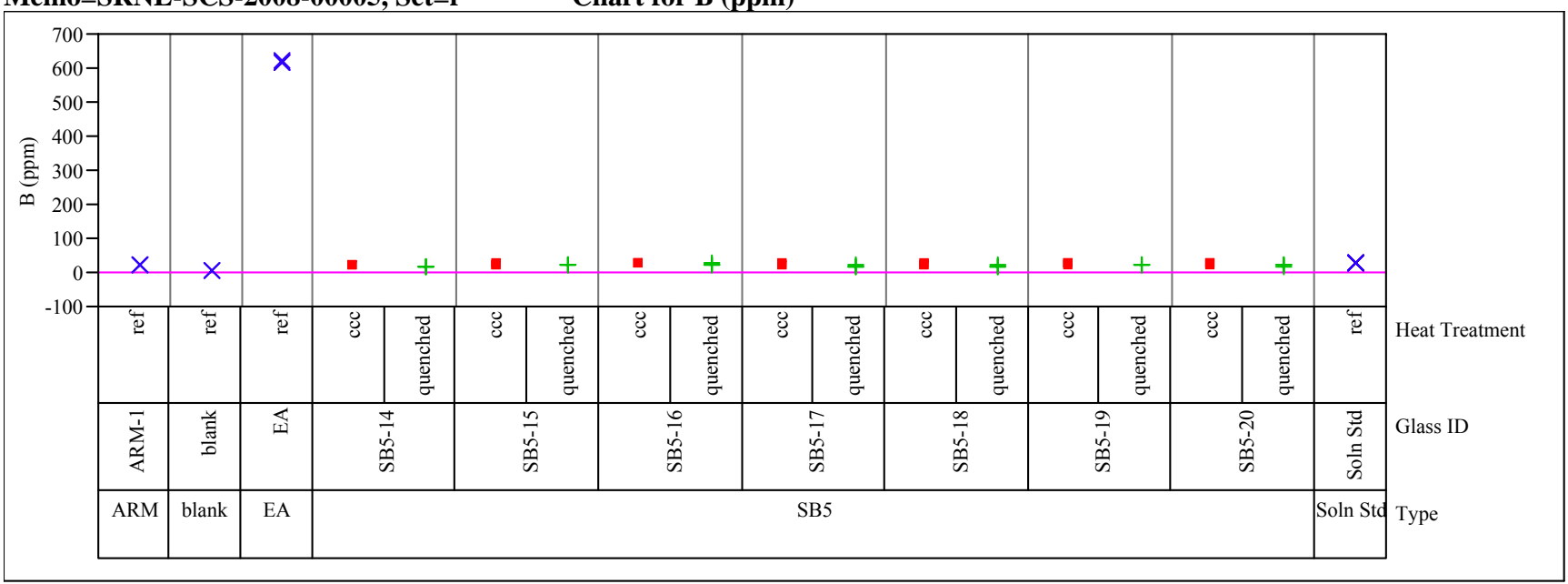

Memo=SRNL-SCS-2008-00005, Set=r Chart for Li (ppm)

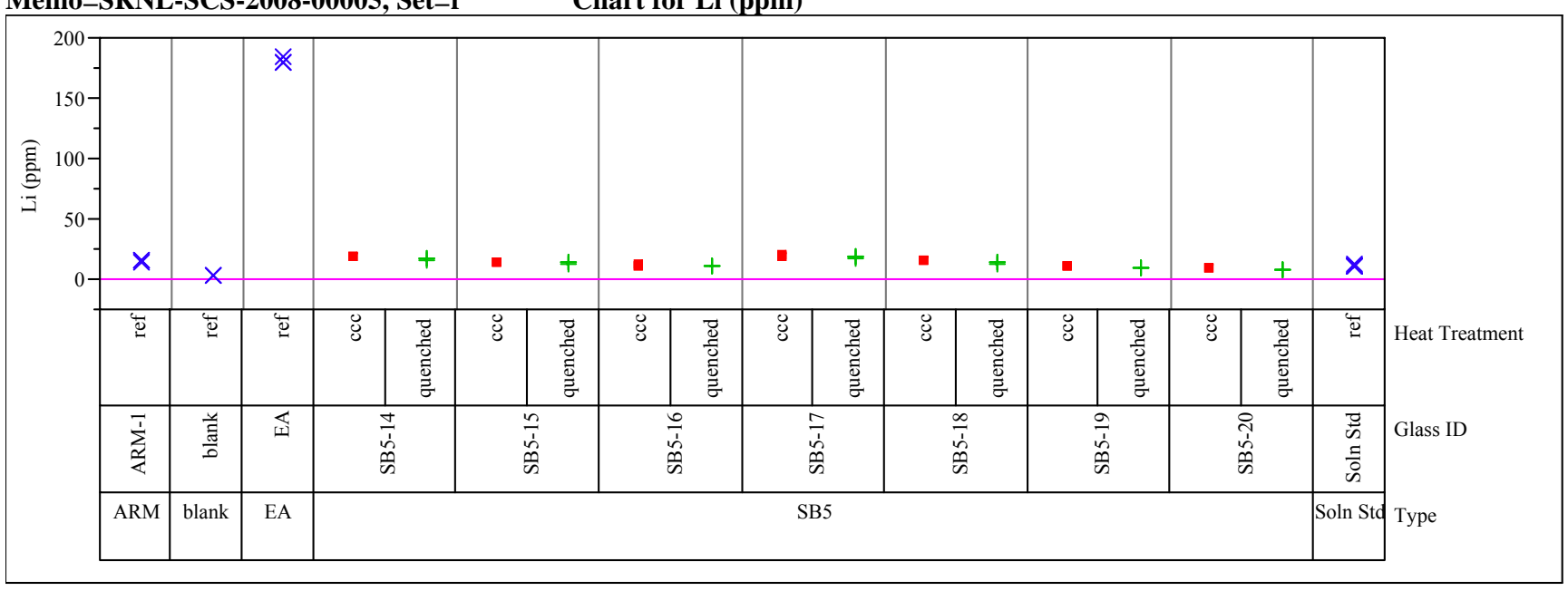


Exhibit B3. Laboratory PCT Measurements by Glass Identifier for Study Glasses and Standards. (continued)

Memo=SRNL-SCS-2008-00005, Set $=r \quad$ Chart for Na $(\mathbf{p p m})$

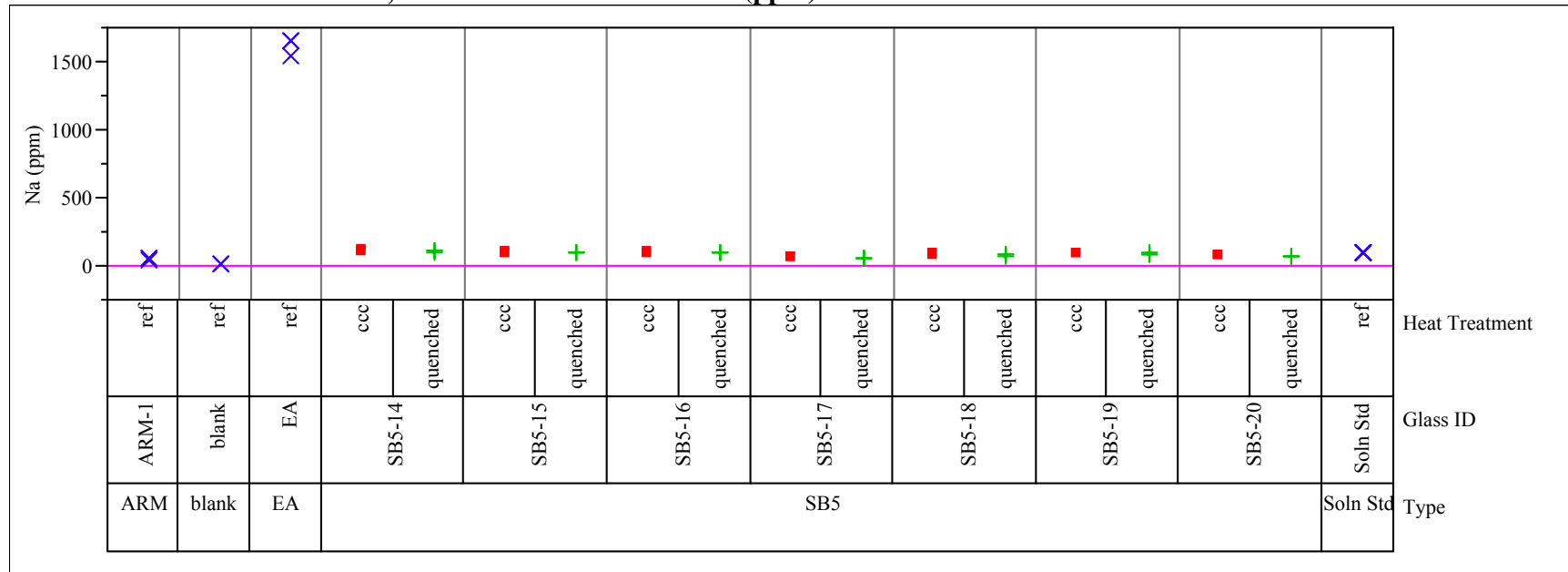

Memo=SRNL-SCS-2008-00005, Set $=r \quad$ Chart for $\mathrm{Si}(\mathrm{ppm})$

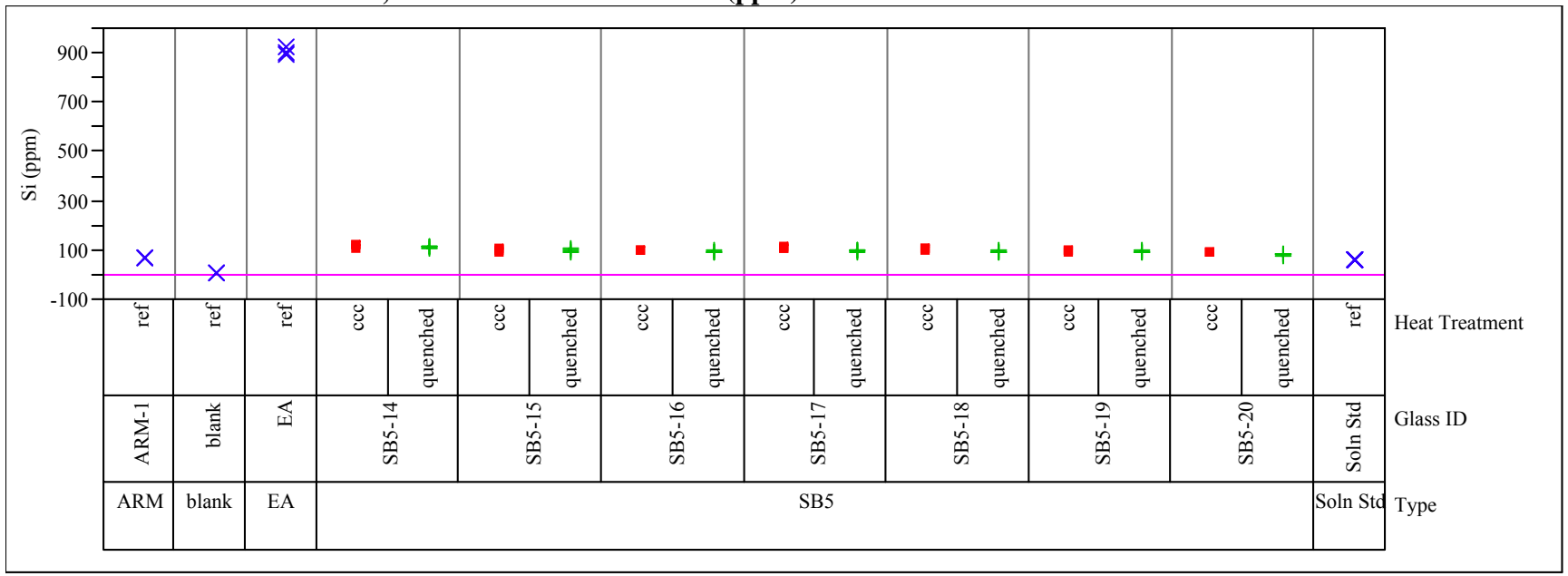


Exhibit B3. Laboratory PCT Measurements by Glass Identifier for Study Glasses and Standards. (continued)

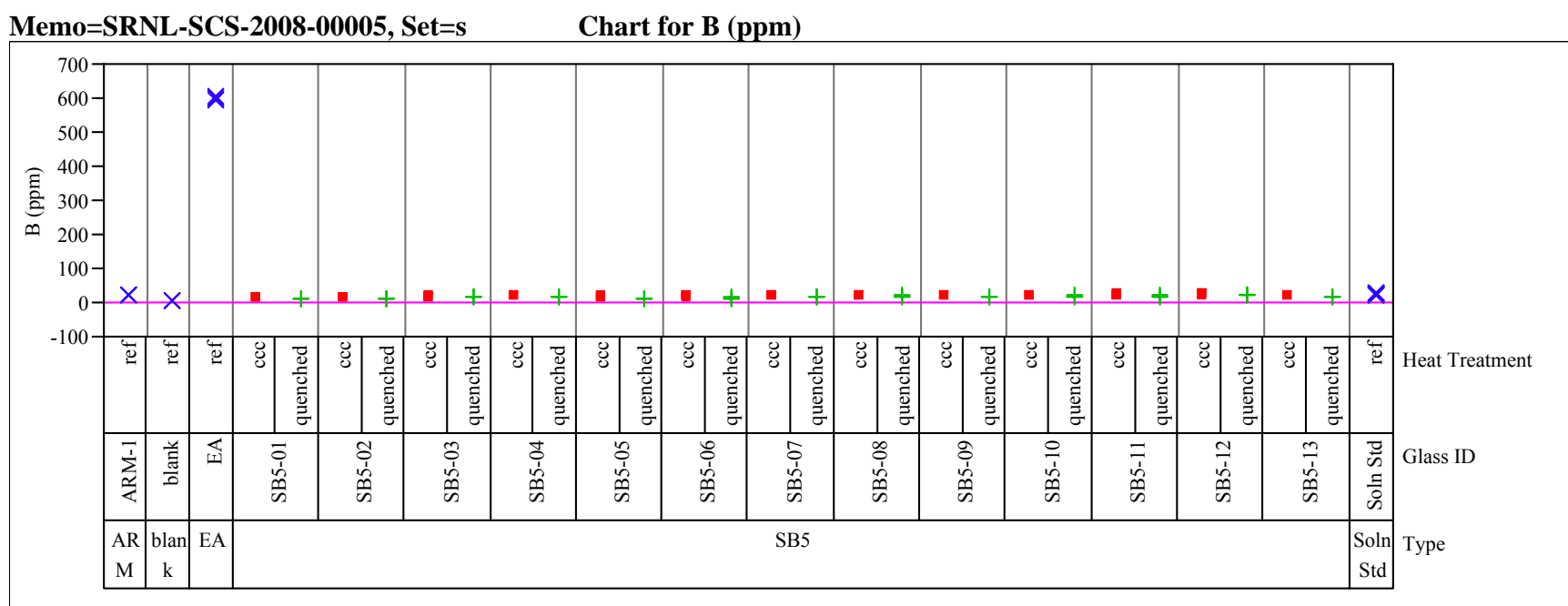

Memo=SRNL-SCS-2008-00005, Set=s Chart for Li (ppm)

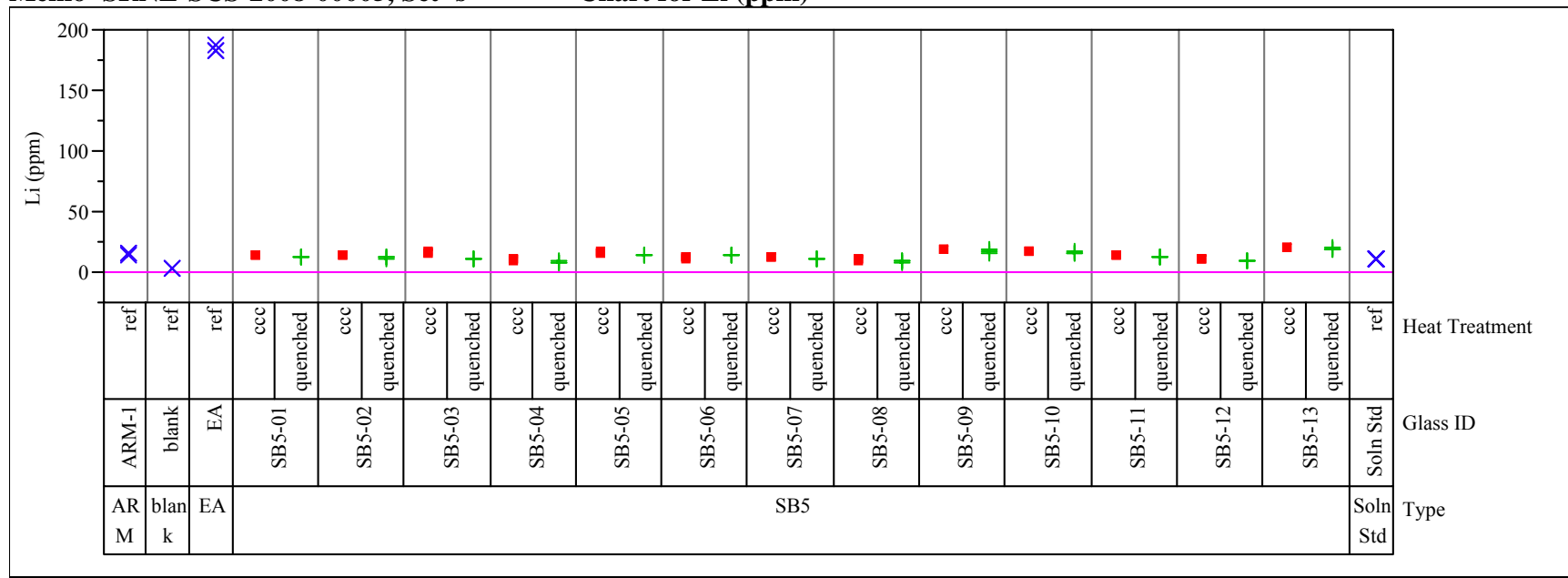


Exhibit B3. Laboratory PCT Measurements by Glass Identifier for Study Glasses and Standards. (continued)

Memo=SRNL-SCS-2008-00005, Set=s Chart for Na (ppm)

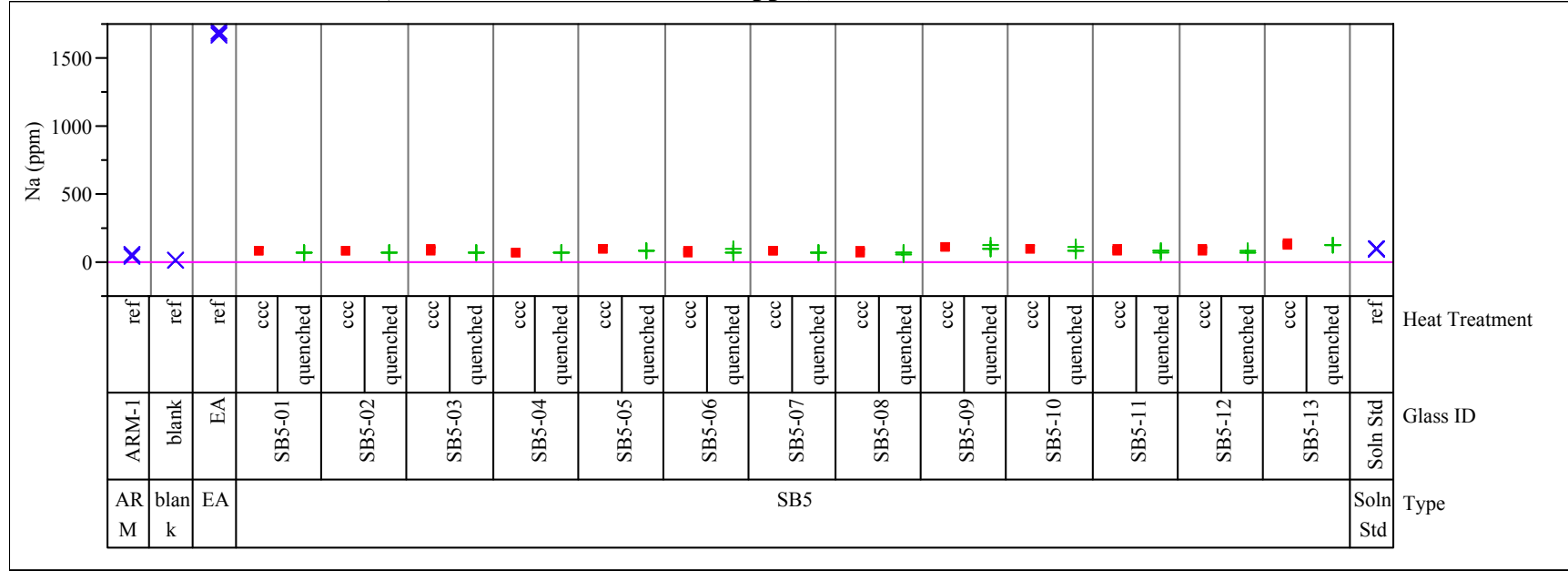

Memo=SRNL-SCS-2008-00005, Set=s Chart for Si (ppm)

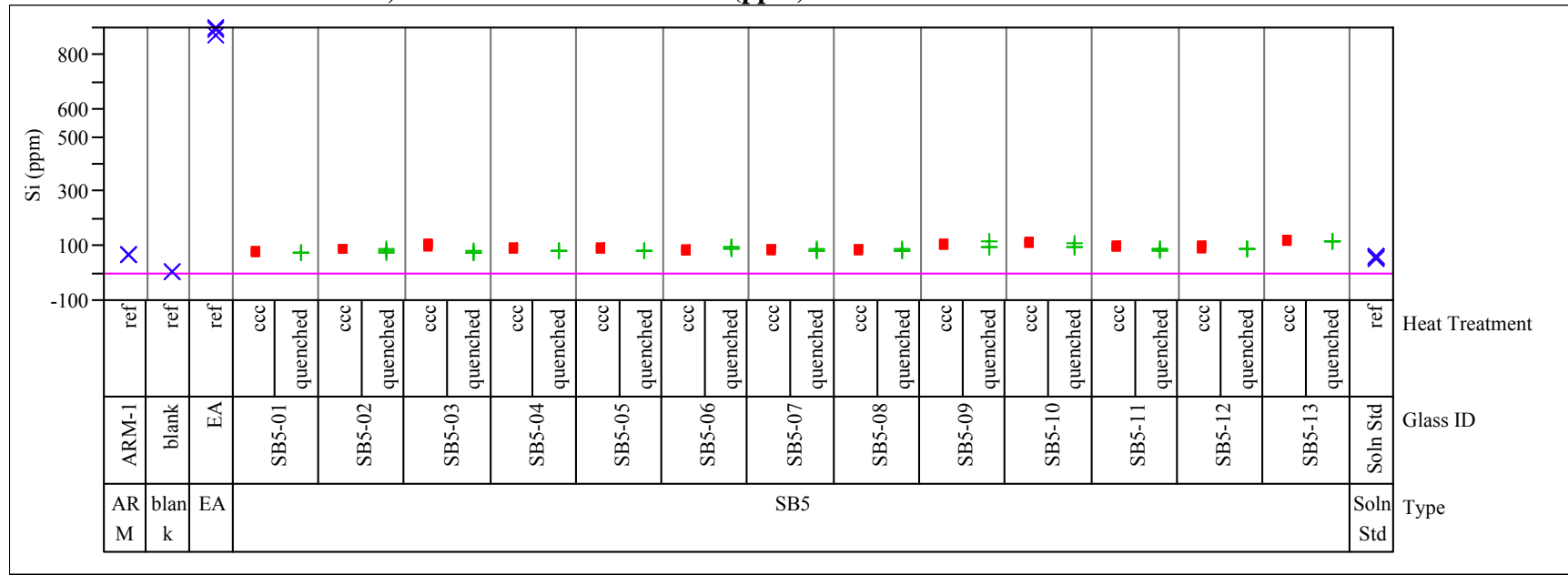


Exhibit B3. Laboratory PCT Measurements by Glass Identifier for Study Glasses and Standards. (continued)

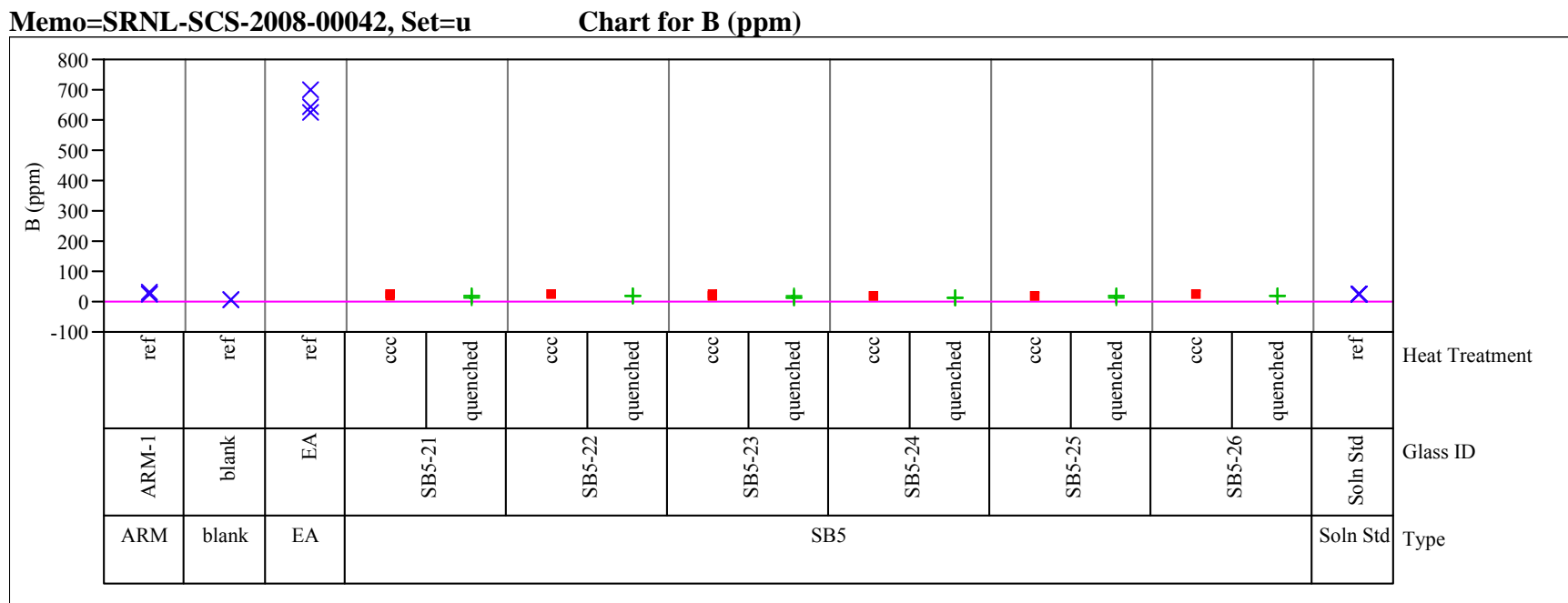

Memo=SRNL-SCS-2008-00042, Set=u Chart for Li (ppm)

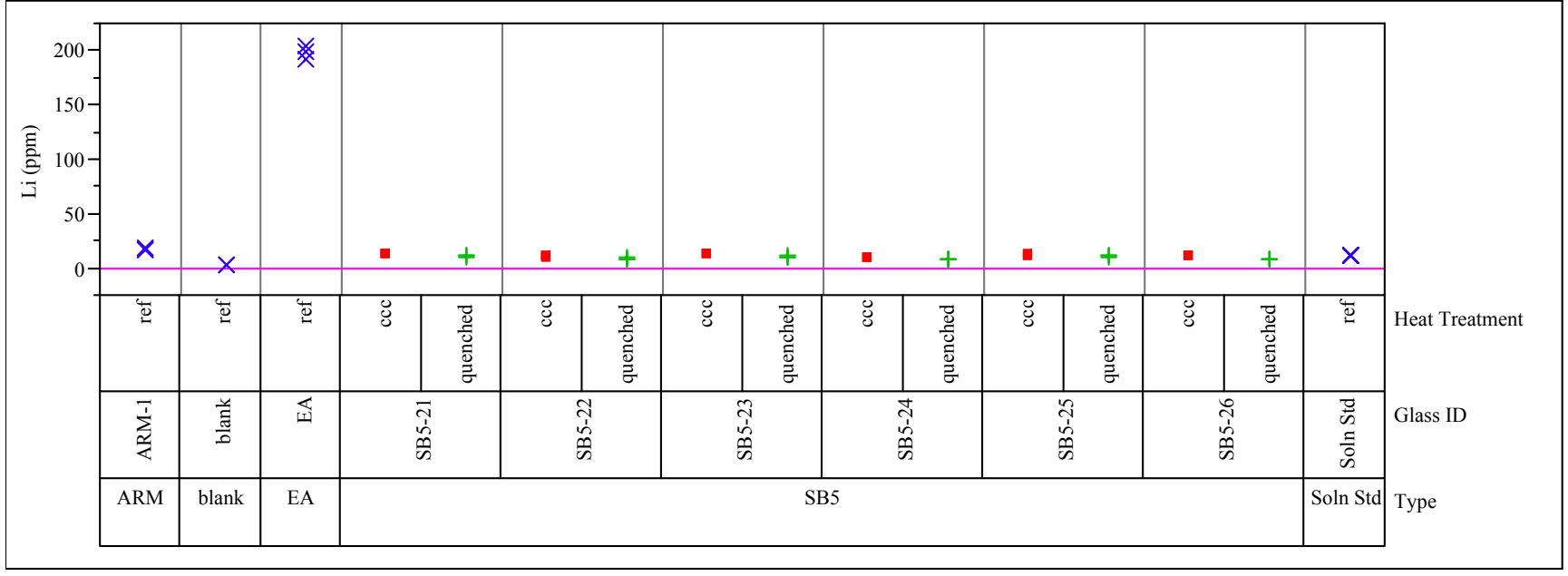


Exhibit B3. Laboratory PCT Measurements by Glass Identifier for Study Glasses and Standards. (continued)

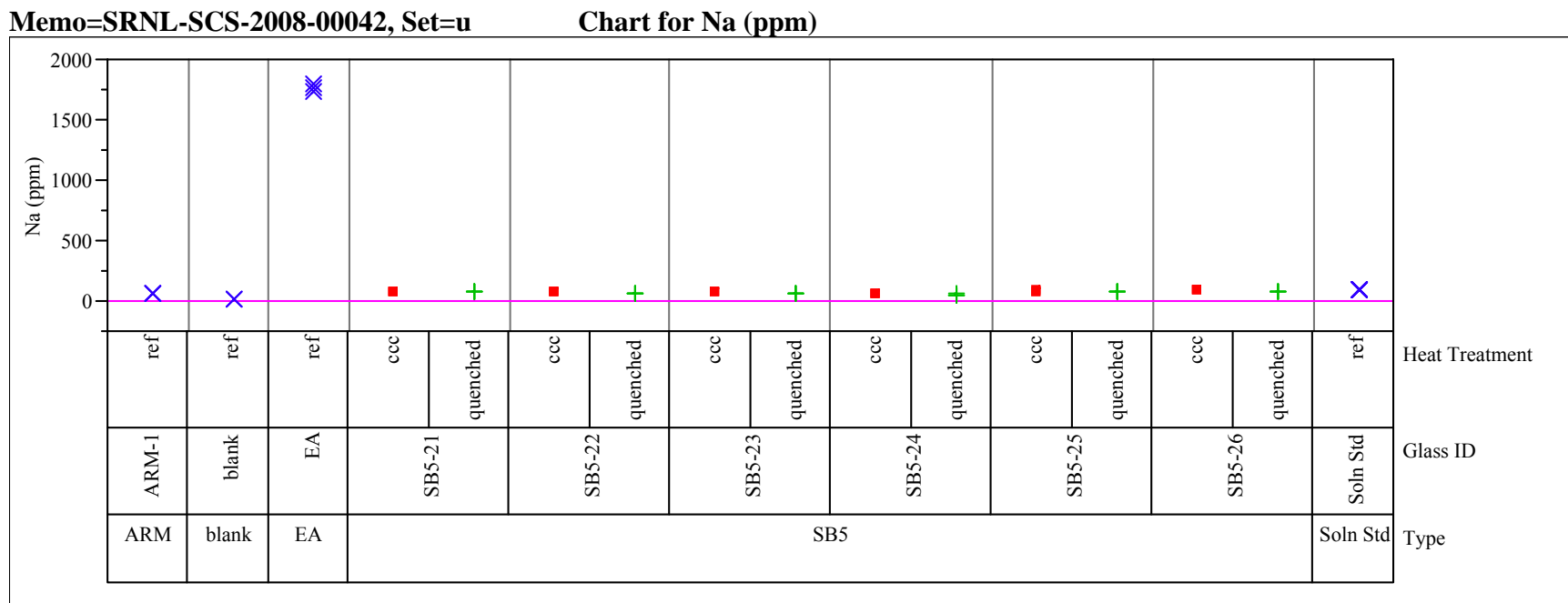

Memo=SRNL-SCS-2008-00042, Set=u Chart for Si (ppm)

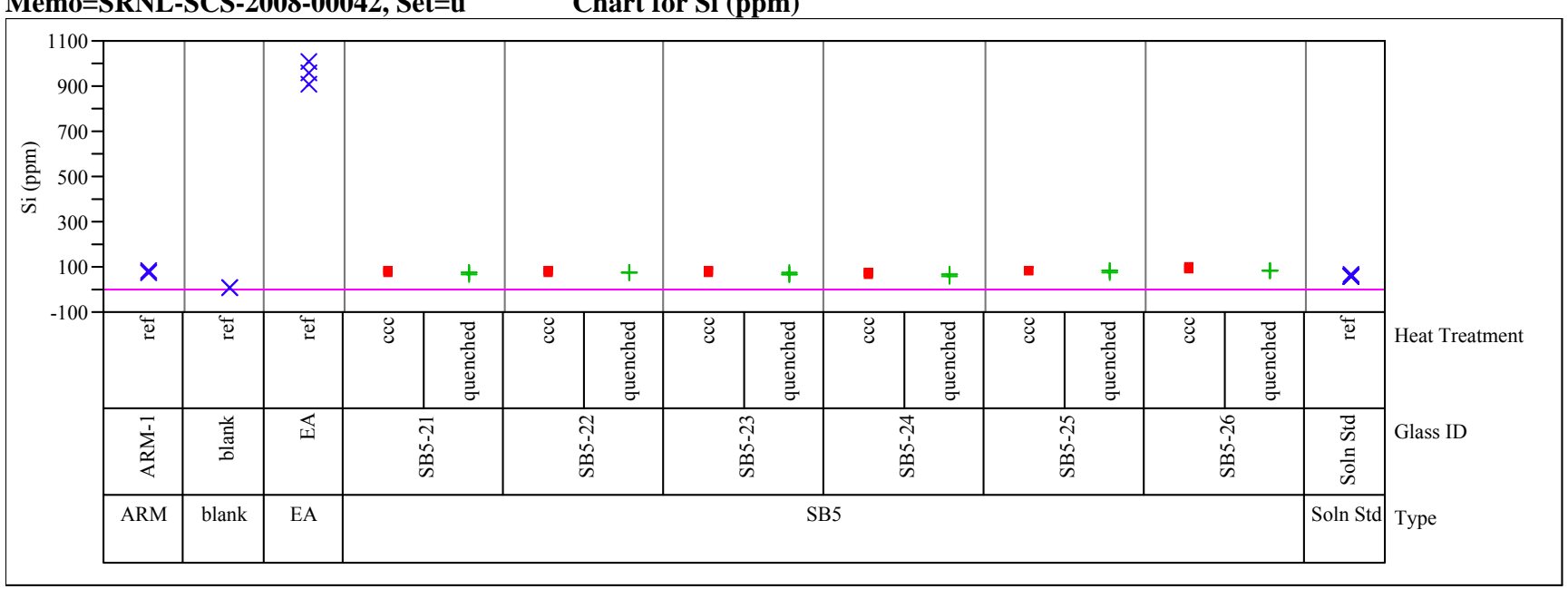




\section{Exhibit B4. Laboratory PCT Measurements by Glass Identifier for Study Glasses.}

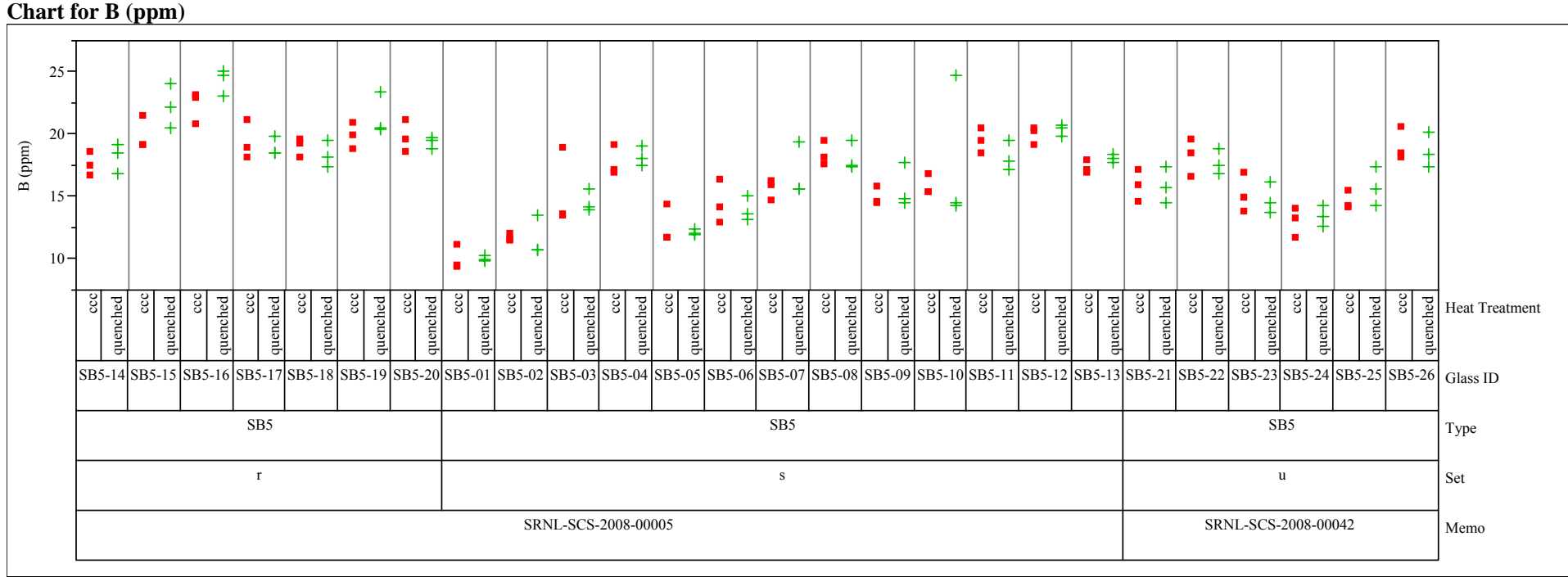

\section{Chart for Li (ppm)}

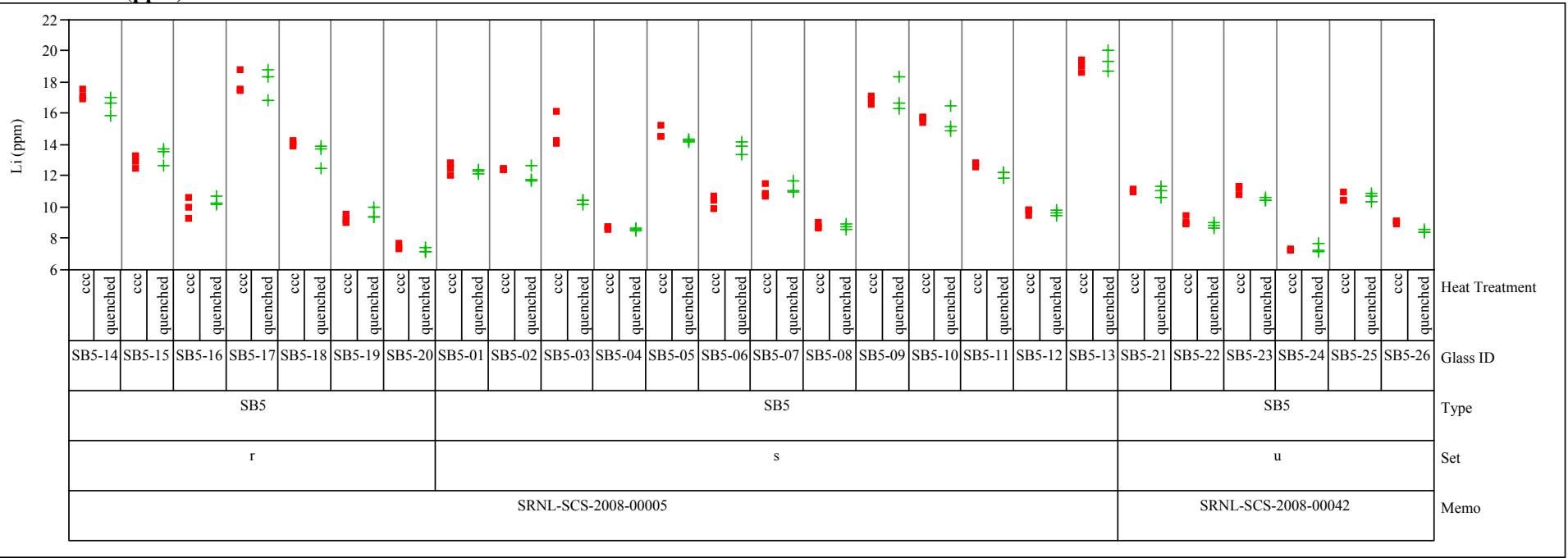


Exhibit B4. Laboratory PCT Measurements by Glass Identifier for Study Glasses. (continued)
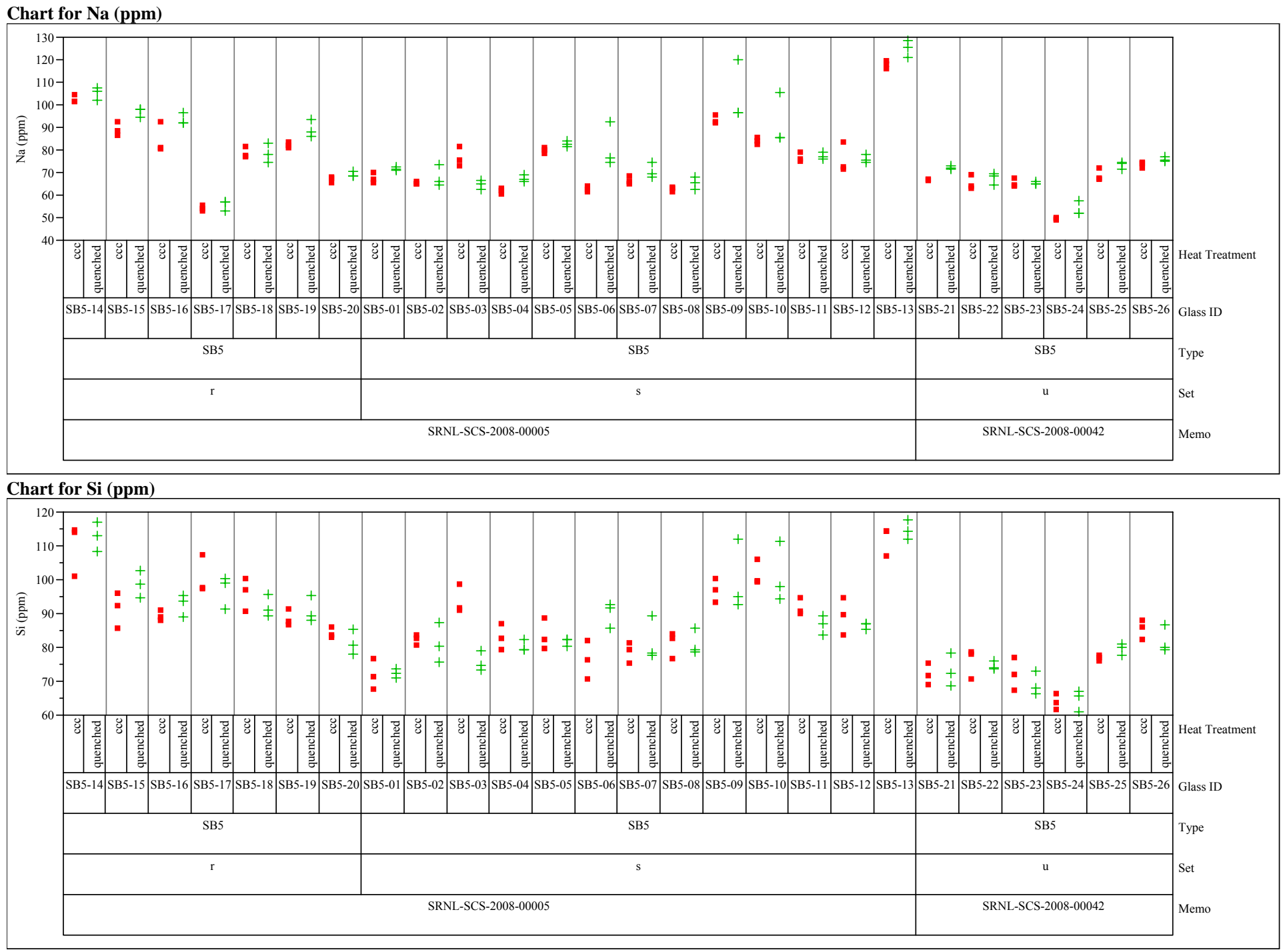


\section{Exhibit B5. Correlations and Scatter Plots of Normalized PCTs Over All Compositional Views and Heat Treatments.}

Multivariate, Analytical Plan=SRNL-SCS-2008-00005, Set $=r$

\section{Correlations}

$\begin{array}{lrrrr} & \log \mathrm{NL}[\mathrm{B}(\mathrm{g} / \mathrm{L})] & \log \mathrm{NL}[\mathrm{Li}(\mathrm{g} / \mathrm{L})] & \log \mathrm{NL}[\mathrm{Na}(\mathrm{g} / \mathrm{L})] & \log \mathrm{NL}[\mathrm{Si}(\mathrm{g} / \mathrm{L})] \\ \log \mathrm{NL}[\mathrm{B}(\mathrm{g} / \mathrm{L})] & 1.0000 & 0.9914 & 0.9871 & 0.9793 \\ \log \mathrm{NL}[\mathrm{Li}(\mathrm{g} / \mathrm{L})] & 0.9914 & 1.0000 & 0.9779 & 0.9811 \\ \log \mathrm{NL}[\mathrm{Na}(\mathrm{g} / \mathrm{L})] & 0.9871 & 0.9779 & 1.0000 & 0.9642 \\ \log \mathrm{NL}[\mathrm{Si}(\mathrm{g} / \mathrm{L})] & 0.9793 & 0.9811 & 0.9642 & 1.0000\end{array}$

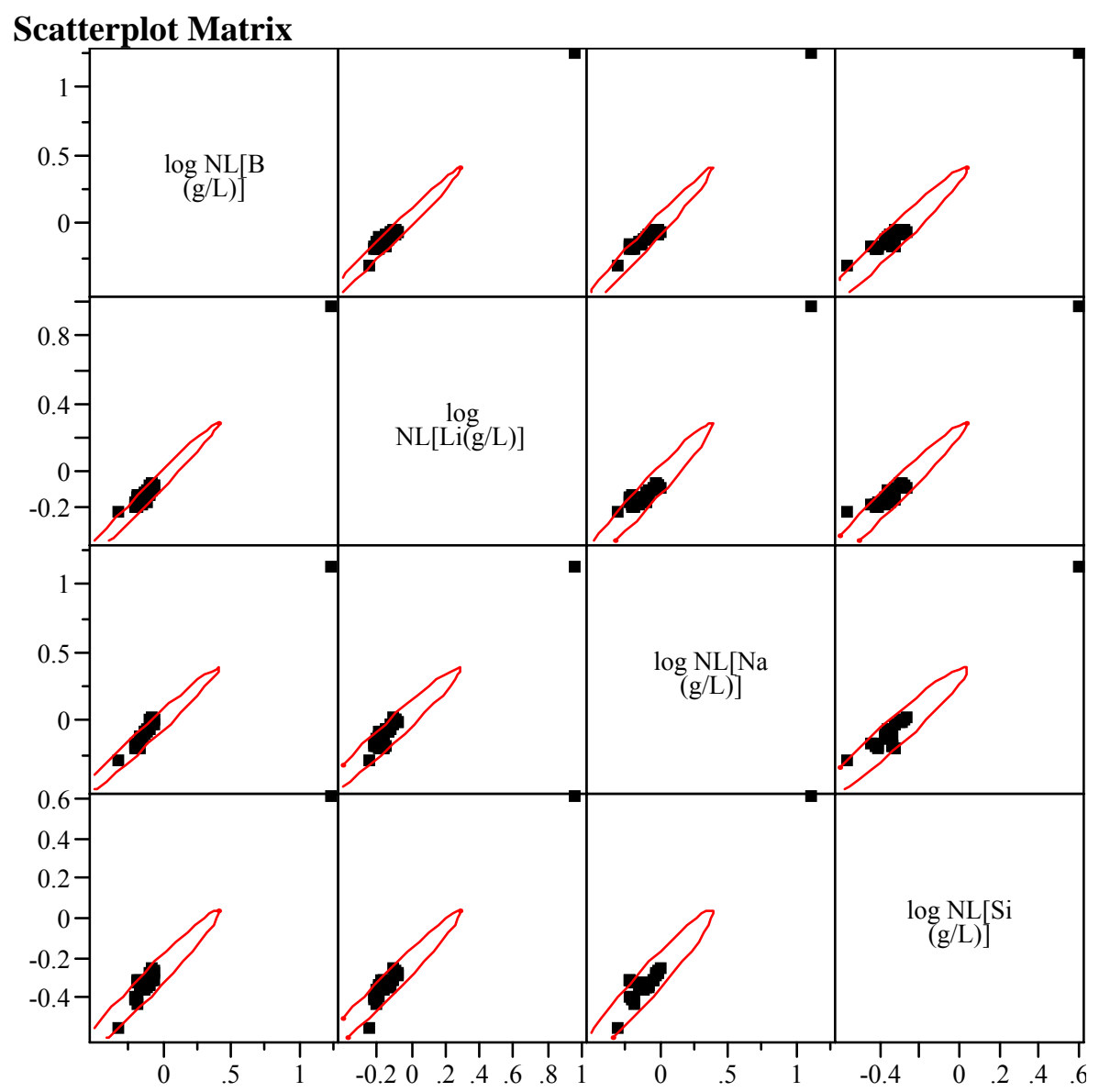


Exhibit B5. Correlations and Scatter Plots of Normalized PCTs Over All Compositional Views and Heat Treatments. (continued)

Multivariate, Analytical Plan=SRNL-SCS-2008-00005, Set $=\mathbf{s}$

\section{Correlations}

$\begin{array}{lrrrr} & \log \mathrm{NL}[\mathrm{B}(\mathrm{g} / \mathrm{L})] & \log \mathrm{NL}[\mathrm{Li}(\mathrm{g} / \mathrm{L})] & \log \mathrm{NL}[\mathrm{Na}(\mathrm{g} / \mathrm{L})] & \log \mathrm{NL}[\mathrm{Si}(\mathrm{g} / \mathrm{L})] \\ \log \mathrm{NL}[\mathrm{B}(\mathrm{g} / \mathrm{L})] & 1.0000 & 0.9502 & 0.9669 & 0.9756 \\ \log \mathrm{NL}[\mathrm{Li}(\mathrm{g} / \mathrm{L})] & 0.9502 & 1.0000 & 0.9763 & 0.9680 \\ \log \mathrm{NL}[\mathrm{Na}(\mathrm{g} / \mathrm{L})] & 0.9669 & 0.9763 & 1.0000 & 0.9634 \\ \log \mathrm{NL}[\mathrm{Si}(\mathrm{g} / \mathrm{L})] & 0.9756 & 0.9680 & 0.9634 & 1.0000\end{array}$

\section{Scatterplot Matrix}

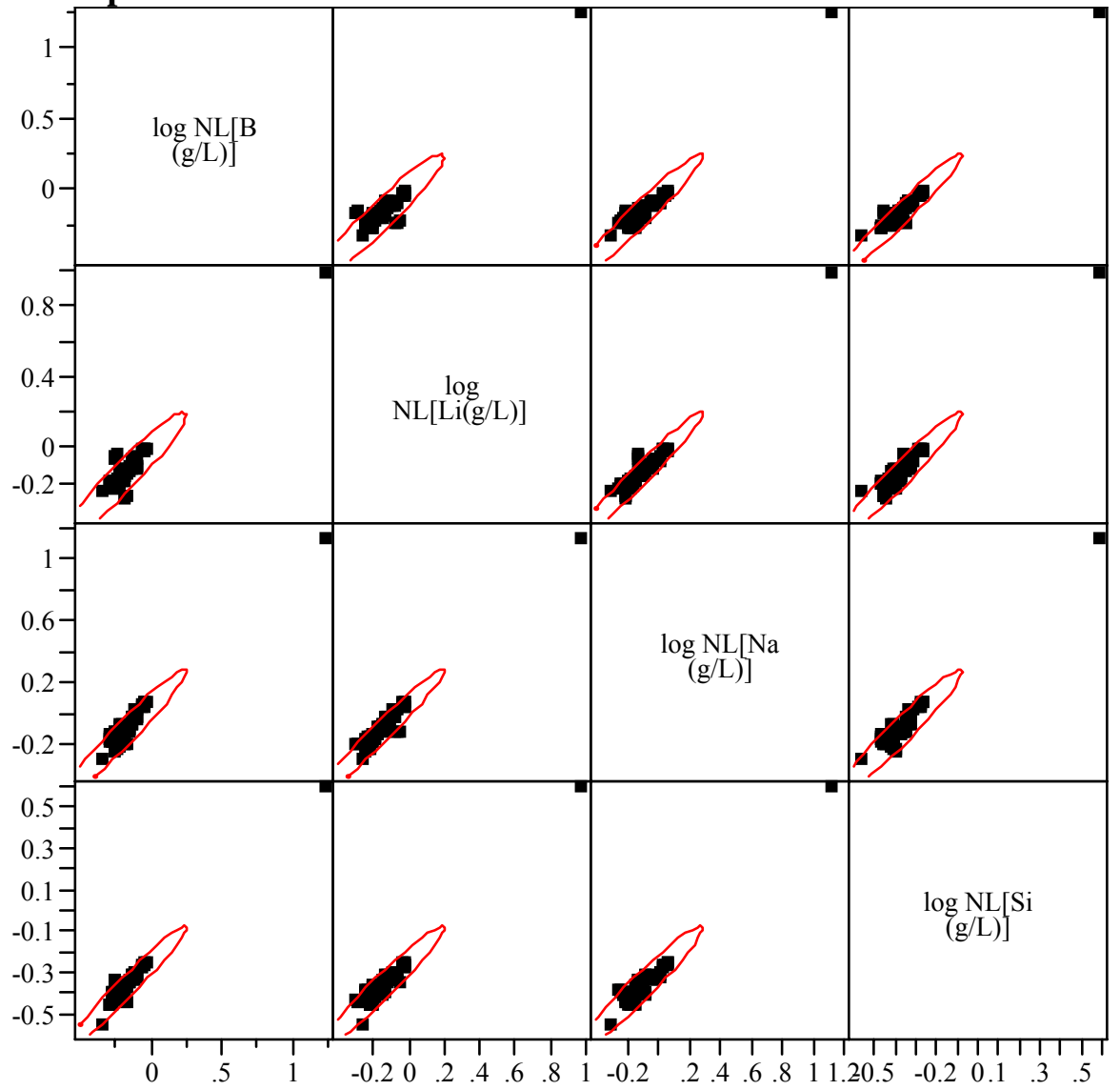


Exhibit B5. Correlations and Scatter Plots of Normalized PCTs Over All Compositional Views and Heat Treatments. (continued)

\section{Multivariate, Analytical Plan=SRNL-SCS-2008-00042, Set $=\mathbf{u}$}

\section{Correlations}

$\begin{array}{lrrrr} & \log \mathrm{NL}[\mathrm{B}(\mathrm{g} / \mathrm{L})] & \log \mathrm{NL}[\mathrm{Li}(\mathrm{g} / \mathrm{L})] & \log \mathrm{NL}[\mathrm{Na}(\mathrm{g} / \mathrm{L})] & \log \mathrm{NL}[\mathrm{Si}(\mathrm{g} / \mathrm{L})] \\ \log \mathrm{NL}[\mathrm{B}(\mathrm{g} / \mathrm{L})] & 1.0000 & 0.9962 & 0.9944 & 0.9916 \\ \log \mathrm{NL}[\mathrm{Li}(\mathrm{g} / \mathrm{L})] & 0.9962 & 1.0000 & 0.9917 & 0.9847 \\ \log \mathrm{NL}[\mathrm{Na}(\mathrm{g} / \mathrm{L})] & 0.9944 & 0.9917 & 1.0000 & 0.9877 \\ \log \mathrm{NL}[\mathrm{Si}(\mathrm{g} / \mathrm{L})] & 0.9916 & 0.9847 & 0.9877 & 1.0000\end{array}$

\section{Scatterplot Matrix}

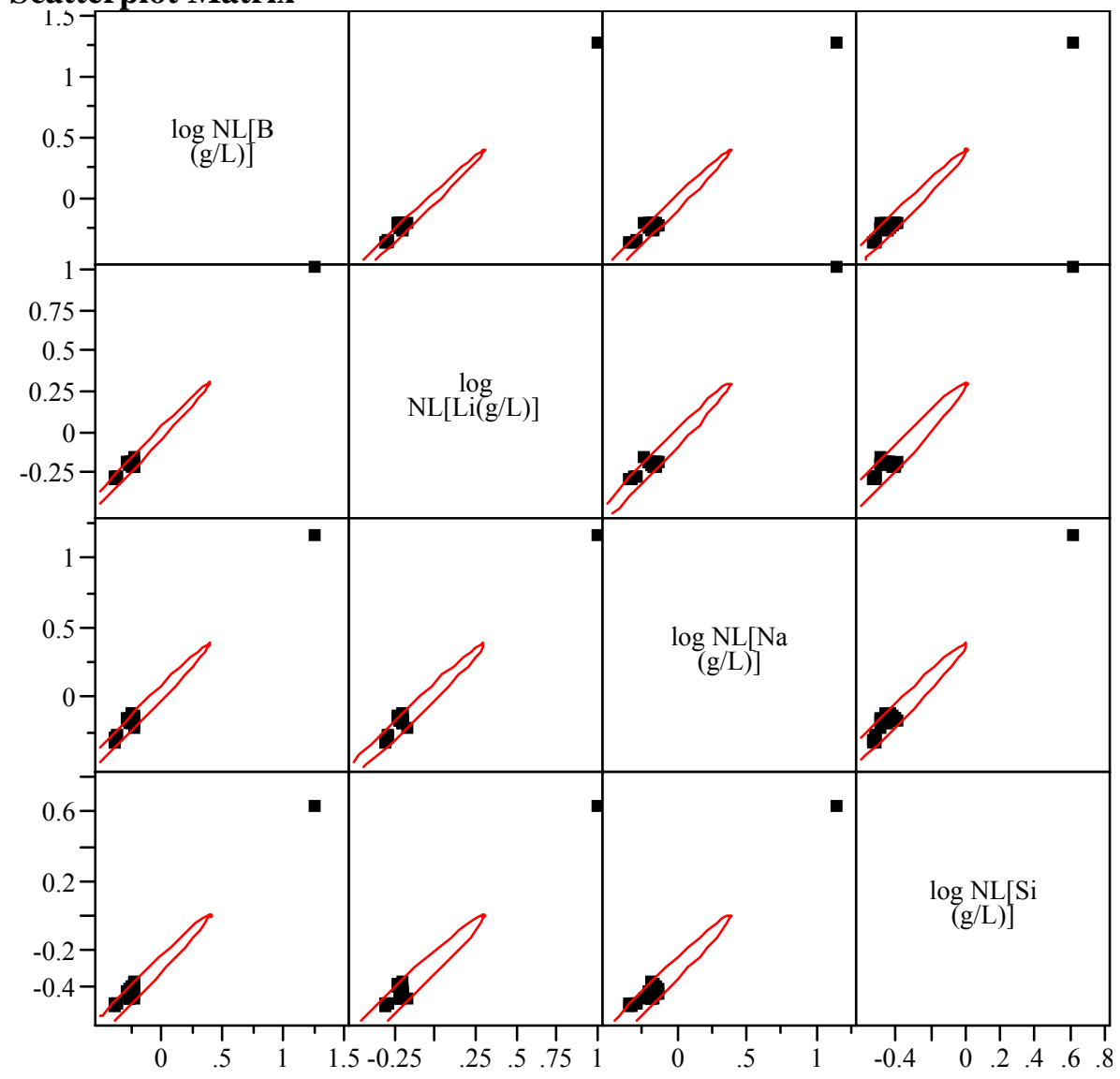




\section{Exhibit B6. Effects of Heat Treatment on PCT Response of Study Glasses.}

Oneway Analysis of log[Si ppm] By Heat Treatment Glass ID=SB5-01

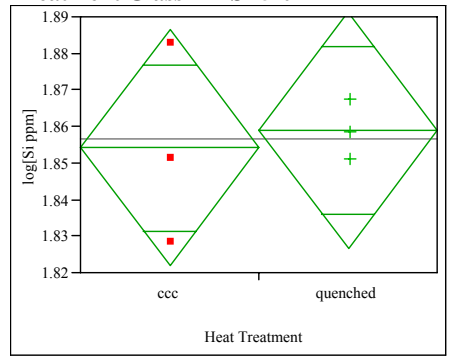

Difference $\quad 0.00489$ t Ratio $\quad 0.297014$ Std Err Dif 0.01646 DF

Upper CL Dif 0.05058 Prob $>|t| \quad 0.7812$

Lower CL Dif -0.04080 Prob $>t \quad 0.3906$

$\begin{array}{lll}\text { Confidence } & 0.95 \text { Prob }<\mathrm{t} \quad 0.6094\end{array}$

Oneway Analysis of log[Na ppm] By Heat

Treatment Glass ID=SB5-01

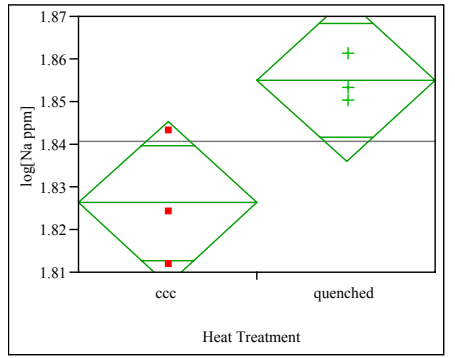

Difference $\quad 0.028711$ t Ratio 2.968021 Std Err Dif $\quad 0.009673$ DF

Upper CL Dif 0.055569 Prob $>|t| \quad 0.0412$

Lower CL Dif 0.001853 Prob $>$ t 0.0206

Confidence $\quad 0.95$ Prob $<\mathrm{t} \quad 0.9794$

Oneway Analysis of $\log [\mathrm{Li}$ ppm $]$ By Heat Treatment Glass ID=SB5-01

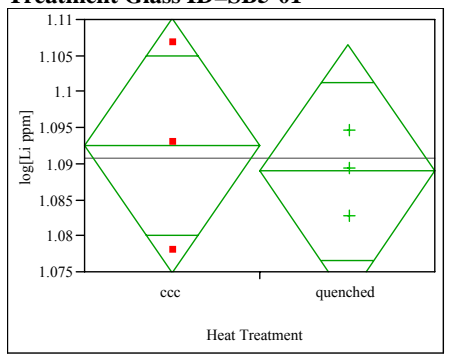

Difference $\quad-0.00359$ t Ratio $\quad-0.40075$ Std Err Dif 0.00895 DF

Upper CL Dif 0.02127 Prob $>|t| \quad 0.7091$

Lower CL Dif -0.02845 Prob $>\mathrm{t} \quad 0.6455$

$\begin{array}{lll}\text { Confidence } & 0.95 \text { Prob }<\mathrm{t} \quad 0.3545\end{array}$
Oneway Analysis of $\log [B \mathrm{ppm}]$ By Heat Treatment Glass ID=SB5-01

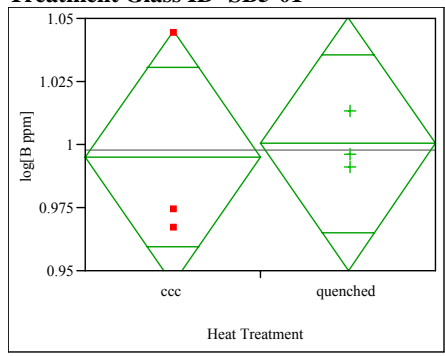

Difference $\quad 0.00543$ t Ratio 0.212775 Std Err Dif $\quad 0.02553$ DF

Upper CL Dif 0.07630 Prob $>|\mathrm{t}| \quad 0.8419$

Lower CL Dif -0.06544 Prob $>\mathrm{t} \quad 0.4210$

$\begin{array}{lll}\text { Confidence } & 0.95 \text { Prob }<\mathrm{t} \quad 0.5790\end{array}$

Oneway Analysis of $\log [\mathrm{Si}$ ppm] By Heat Treatment Glass ID=SB5-02

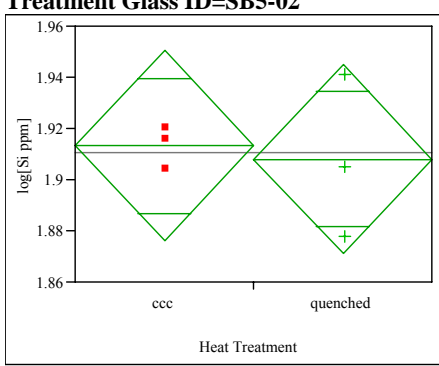

Difference $\quad-0.00517$ t Ratio $\quad-0.27289$ Std Err Dif $\quad 0.01893$ DF

Upper CL Dif 0.04739 Prob $>|t| \quad 0.7984$

Lower CL Dif -0.05772 Prob $>t \quad 0.6008$

Confidence $\quad 0.95$ Prob $<\mathrm{t} \quad 0.3992$

Oneway Analysis of log[Na ppm] By Heat Treatment Glass ID=SB5-02

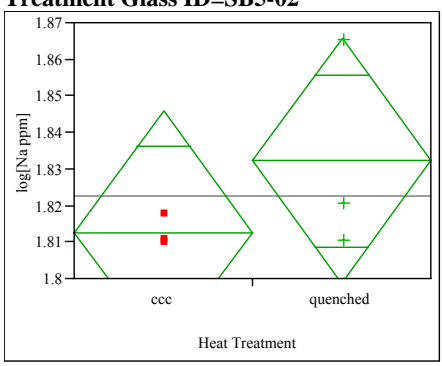

Difference $\quad 0.01968$ t Ratio 1.159619 Std Err Dif $\quad 0.01697$ DF

Upper CL Dif 0.06679 Prob $>|t| \quad 0.3107$ Lower CL Dif -0.02744 Prob $>\mathrm{t} \quad 0.1554$

Confidence $\quad 0.95$ Prob $<\mathrm{t} \quad 0.8446$
Oneway Analysis of $\log [\mathrm{Li}$ ppm] By Heat Treatment Glass ID=SB5-02

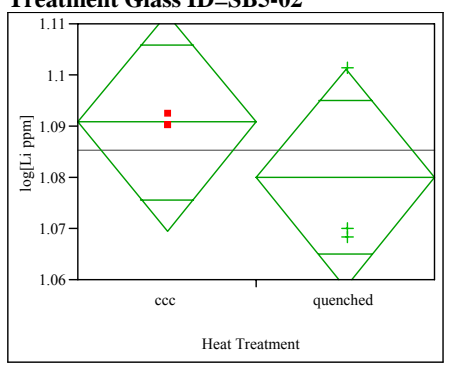

Difference $\quad-0.01077$ t Ratio $\quad-0.99356$ Std Err Dif $\quad 0.01084$ DF 4 Upper CL Dif 0.01933 Prob $>|\mathrm{t}| \quad 0.3767$ Lower CL Dif -0.04088 Prob $>t \quad 0.8117$ Confidence $\quad 0.95$ Prob $<\mathrm{t} \quad 0.1883$

Oneway Analysis of $\log [\mathrm{B}$ ppm] By Heat Treatment Glass ID=SB5-02

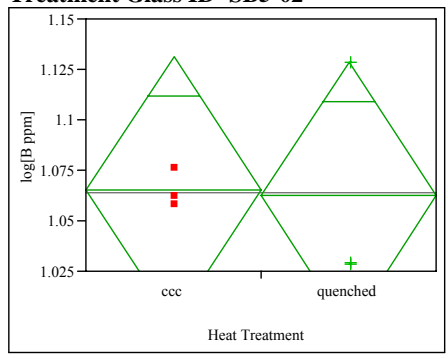

Difference $\quad-0.00274$ t Ratio $\quad-0.08147$ Std Err Dif $\quad 0.03367$ DF 4 Upper CL Dif 0.09073 Prob $>|t| \quad 0.9390$ Lower CL Dif -0.09621 Prob $>t \quad 0.5305$ Confidence $\quad 0.95$ Prob $<\mathrm{t} \quad 0.4695$

Oneway Analysis of $\log [\mathrm{Si}$ ppm] By Heat Treatment Glass ID=SB5-03

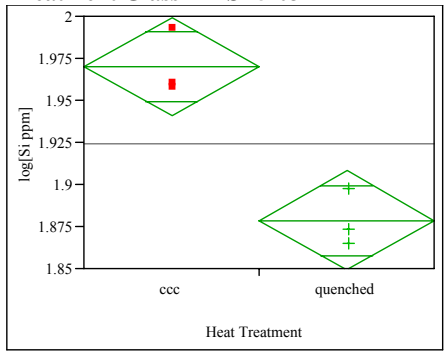

Difference $\quad-0.09131$ t Ratio -6.0986 Std Err Dif $\quad 0.01497$ DF Upper CL Dif -0.04974 Prob $>|t| 0.0037$ Lower CL Dif -0.13288 Prob $>\mathrm{t} \quad 0.9982$ Confidence $\quad 0.95$ Prob $<\mathrm{t} \quad 0.0018$ 
Exhibit B6. Effects of Heat Treatment on PCT Response of Study Glasses. (continued)

Oneway Analysis of log[Na ppm] By Heat Treatment Glass ID=SB5-03

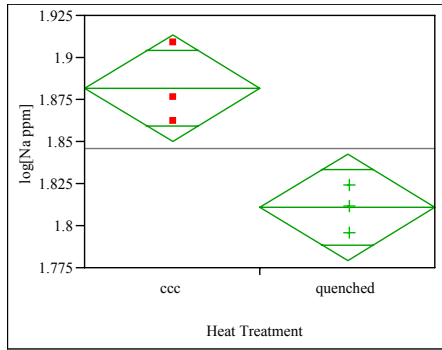

Difference $\quad-0.07142$ t Ratio $\quad-4.4301$ Std Err Dif $\quad 0.01612$ DF

Upper CL Dif -0.02666 Prob > $|\mathrm{t}| \quad 0.0114$ Lower CL Dif -0.11618 Prob $>t \quad 0.9943$ Confidence $\quad 0.95$ Prob $<\mathrm{t} \quad 0.0057$

Oneway Analysis of $\log [\mathrm{Li} \mathrm{ppm}]$ By Heat Treatment Glass ID=SB5-03

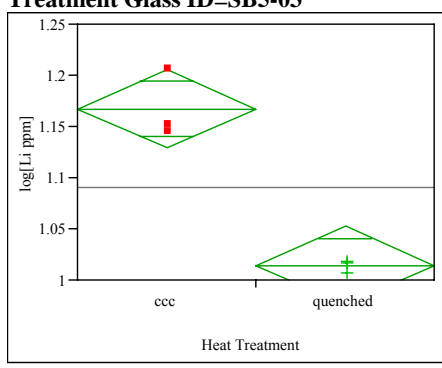

Difference $\quad-0.15298$ t Ratio $\quad-7.88358$ Std Err Dif $\quad 0.01940$ DF

Upper CL Dif -0.09910 Prob $>|t| \quad 0.0014$

Lower CL Dif -0.20685 Prob $>t \quad 0.9993$

Confidence $\quad 0.95$ Prob $<\mathrm{t} \quad 0.0007$

Oneway Analysis of $\log [\mathrm{B}$ ppm] By Heat Treatment Glass ID=SB5-03

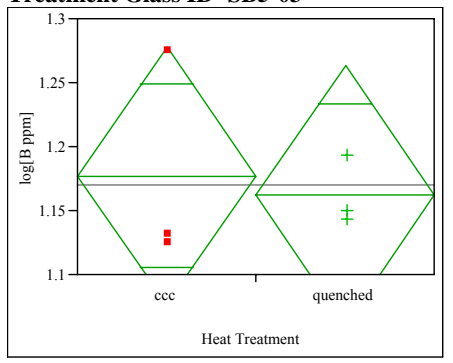

Difference -0.01463 t Ratio -0.28397 Std Err Dif $\quad 0.05152$ DF

Upper CL Dif 0.12841 Prob $>|t| \quad 0.7905$

Lower CL Dif -0.15766 Prob $>t \quad 0.6047$

Confidence $\quad 0.95$ Prob $<\mathrm{t} \quad 0.3953$
Oneway Analysis of $\log [\mathrm{Si}$ ppm] By Heat Treatment Glass ID=SB5-04

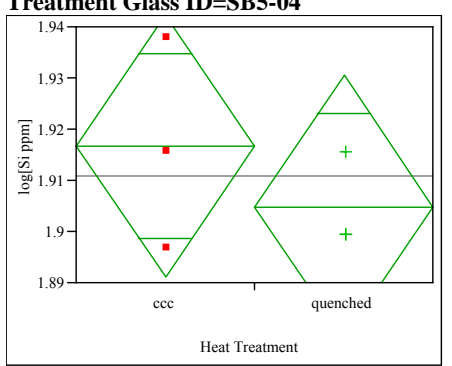

Difference $\quad-0.01188$ t Ratio $\quad-0.91075$ Std Err Dif $\quad 0.01305$ DF

Upper CL Dif 0.02434 Prob $>|t| \quad 0.4139$

Lower CL Dif -0.04811 Prob $>\mathrm{t} \quad 0.7930$

Confidence $\quad 0.95$ Prob $<\mathrm{t} \quad 0.2070$

Oneway Analysis of log[Na ppm] By Heat Treatment Glass ID=SB5-04

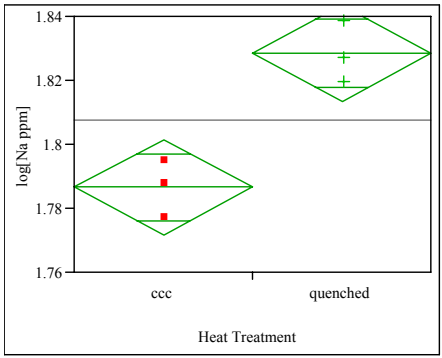

Difference $\quad 0.042062$ t Ratio 5.511507 Std Err Dif $\quad 0.007632$ DF

Upper CL Dif 0.063251 Prob $>|t| \quad 0.0053$ Lower CL Dif 0.020873 Prob $>$ t 0.0026

Confidence $\quad 0.95$ Prob $<\mathrm{t} \quad 0.9974$

Oneway Analysis of $\log [\mathrm{Li}$ ppm] By Heat Treatment Glass ID=SB5-04

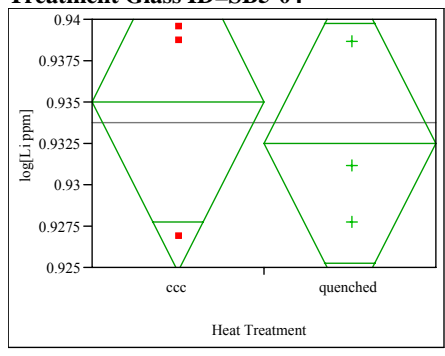

Difference $\quad-0.00251$ t Ratio -0.48188 Std Err Dif $\quad 0.00522$ DF

Upper CL Dif 0.01198 Prob $>|t| \quad 0.6551$

Lower CL Dif -0.01701 Prob $>t \quad 0.6725$

$\begin{array}{lll}\text { Confidence } & 0.95 \text { Prob }<\mathrm{t} \quad 0.3275\end{array}$
Oneway Analysis of $\log [\mathrm{B}$ ppm] By Heat Treatment Glass ID=SB5-04

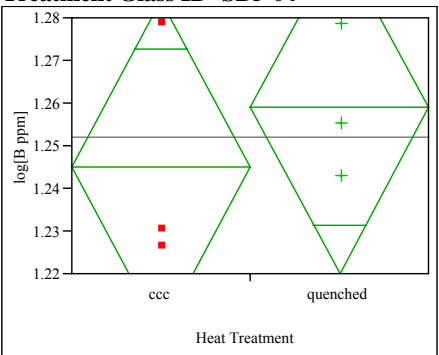

Difference $\quad 0.01390$ t Ratio 0.700064 Std Err Dif $\quad 0.01985$ DF

Upper CL Dif 0.06901 Prob $>|t| \quad 0.5225$

Lower CL Dif -0.04122 Prob $>t \quad 0.2612$

Confidence $\quad 0.95$ Prob $<\mathrm{t} \quad 0.7388$

Oneway Analysis of $\log [\mathrm{Si}$ ppm] By Heat Treatment Glass ID=SB5-05

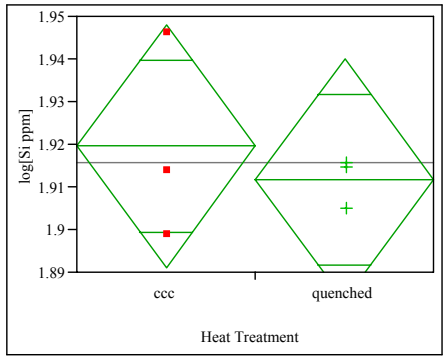

Difference $\quad-0.00777$ t Ratio $\quad-0.53825$ Std Err Dif $\quad 0.01444$ DF 4 Upper CL Dif 0.03231 Prob $>|t| \quad 0.6190$ Lower CL Dif -0.04786 Prob $>t \quad 0.6905$ Confidence $\quad 0.95$ Prob $<\mathrm{t} \quad 0.3095$

Oneway Analysis of log[Na ppm] By Heat Treatment Glass ID=SB5-05

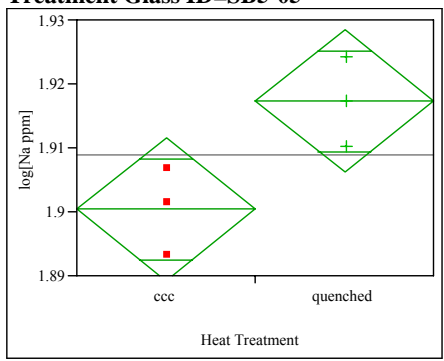

Difference $\quad 0.016962$ t Ratio 2.99099 Std Err Dif $\quad 0.005671$ DF 4 Upper CL Dif 0.032707 Prob $>|t| \quad 0.0403$

Lower CL Dif 0.001217 Prob $>t \quad 0.0201$ Confidence $\quad 0.95$ Prob $<\mathrm{t} \quad 0.9799$ 
Exhibit B6. Effects of Heat Treatment on PCT Response of Study Glasses. (continued)

Oneway Analysis of $\log [\mathrm{Li}$ ppm] By Heat Treatment Glass ID=SB5-05

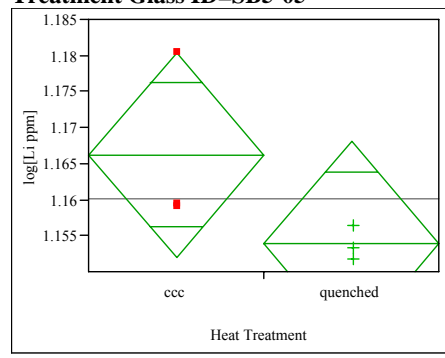

Difference $\quad-0.01240$ t Ratio $\quad-1.71651$ Std Err Dif 0.00723 DF

Upper CL Dif 0.00766 Prob $>|t| \quad 0.1612$

Lower CL Dif -0.03247 Prob $>t \quad 0.9194$

$\begin{array}{lr}\text { Confidence } & 0.95 \text { Prob }<\mathrm{t} \quad 0.0806\end{array}$

Oneway Analysis of $\log [\mathrm{B}$ ppm $]$ By Heat Treatment Glass ID=SB5-05

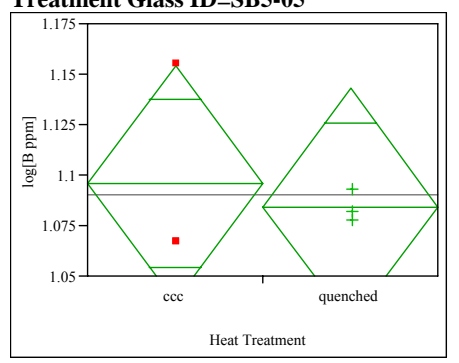

Difference $\quad-0.01149$ t Ratio $\quad-0.38533$ Std Err Dif $\quad 0.02983$ DF

Upper CL Dif 0.07133 Prob $>|\mathrm{t}| \quad 0.7196$ Lower CL Dif -0.09432 Prob $>t \quad 0.6402$ $\begin{array}{lll}\text { Confidence } & 0.95 \text { Prob }<\mathrm{t} & 0.3598\end{array}$

Oneway Analysis of log[Si ppm] By Heat Treatment Glass ID=SB5-06

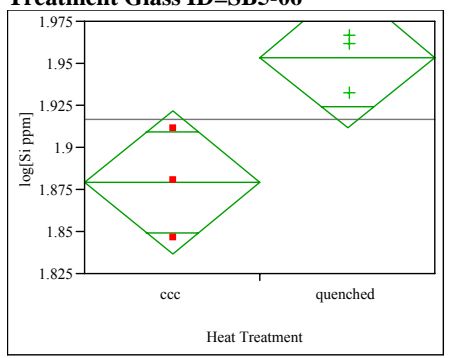

Difference $\quad 0.074669$ t Ratio 3.465181 Std Err Dif 0.021548 DF

Upper CL Dif 0.134497 Prob $>|\mathrm{t}| \quad 0.0257$

Lower CL Dif 0.014841 Prob $>$ t 0.0128

$\begin{array}{lll}\text { Confidence } & 0.95 \text { Prob }<\mathrm{t} & 0.9872\end{array}$
Oneway Analysis of $\log [\mathrm{Na}$ ppm] By Hea Treatment Glass ID=SB5-06

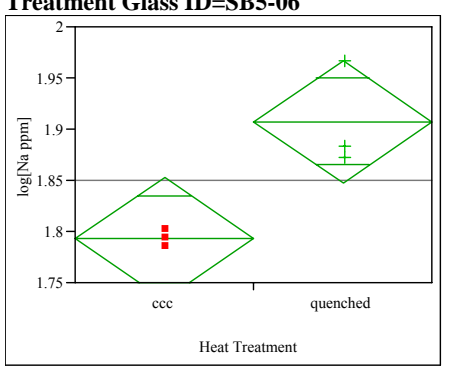

Difference $\quad 0.114543$ t Ratio 3.766948 Std Err Dif $\quad 0.030407$ DF

Upper CL Dif 0.198967 Prob $>|t| \quad 0.0197$

Lower CL Dif 0.030119 Prob $>\mathrm{t} \quad 0.0098$

Confidence $\quad 0.95$ Prob $<\mathrm{t} \quad 0.9902$

Oneway Analysis of $\log [\mathrm{Li}$ ppm] By Heat Treatment Glass ID=SB5-06

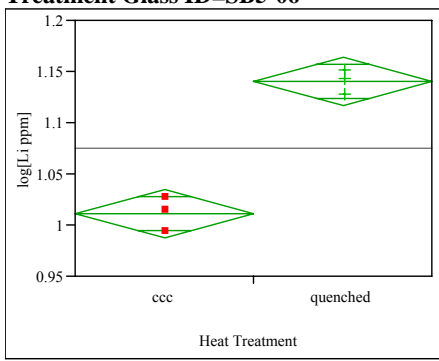

Difference $\quad 0.129319$ t Ratio 10.64161 Std Err Dif 0.012152 DF

Upper CL Dif 0.163059 Prob $>|t| \quad 0.0004$ Lower CL Dif 0.095579 Prob $>$ t 0.0002

Confidence $\quad 0.95$ Prob $<\mathrm{t} \quad 0.9998$

Oneway Analysis of $\log [\mathrm{B}$ ppm] By Heat Treatment Glass ID=SB5-06

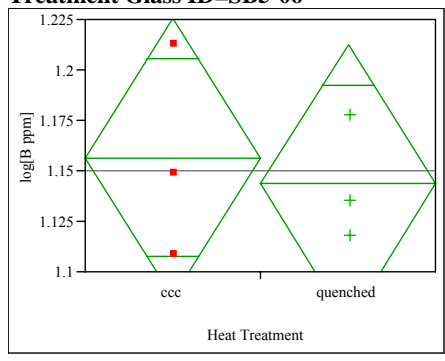

Difference $\quad-0.01306$ t Ratio $\quad-0.37145$ Std Err Dif $\quad 0.03516$ DF

Upper CL Dif 0.08457 Prob $>|t| \quad 0.7291$

Lower CL Dif -0.11069 Prob $>t \quad 0.6354$

Confidence $\quad 0.95$ Prob $<\mathrm{t} \quad 0.3646$
Oneway Analysis of $\log [\mathrm{Si}$ ppm] By Heat Treatment Glass ID=SB5-07

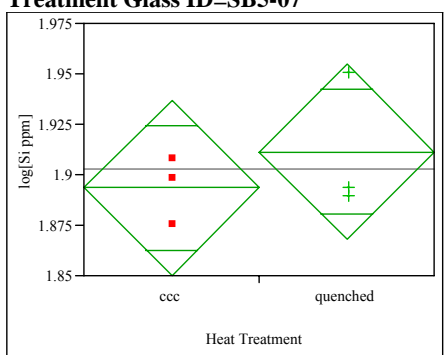

Difference $\quad 0.01799$ t Ratio $\quad 0.815997$ Std Err Dif $\quad 0.02205$ DF

Upper CL Dif 0.07922 Prob $>|t| \quad 0.4603$

Lower CL Dif -0.04323 Prob $>t \quad 0.2302$

$\begin{array}{lll}\text { Confidence } & 0.95 \text { Prob }<\mathrm{t} & 0.7698\end{array}$

Oneway Analysis of log[Na ppm] By Heat Treatment Glass ID=SB5-07

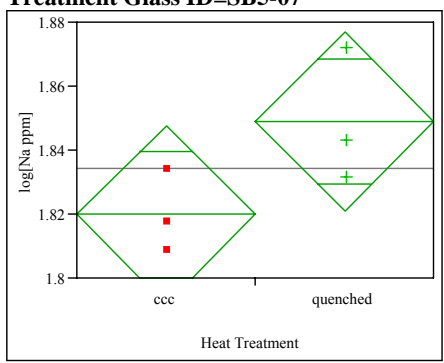

Difference $\quad 0.02909$ t Ratio 2.052782 Std Err Dif $\quad 0.01417$ DF

Upper CL Dif 0.06844 Prob $>|t| \quad 0.1093$

Lower CL Dif -0.01026 Prob $>t \quad 0.0547$

Confidence $\quad 0.95$ Prob $<\mathrm{t} \quad 0.9453$

Oneway Analysis of $\log [\mathrm{Li}$ ppm] By Heat Treatment Glass ID $=$ SB5-07

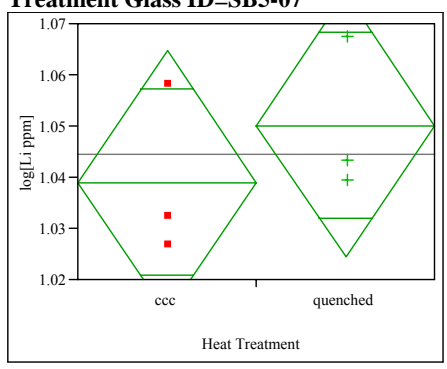

Difference $\quad 0.01114$ t Ratio $\quad 0.849332$ Std Err Dif $\quad 0.01312$ DF 4

Upper CL Dif 0.04756 Prob $>|t| \quad 0.4435$

Lower CL Dif -0.02528 Prob $>t \quad 0.2218$

Confidence $\quad 0.95$ Prob $<\mathrm{t} \quad 0.7782$ 
Exhibit B6. Effects of Heat Treatment on PCT Response of Study Glasses. (continued)

Oneway Analysis of log[B ppm] By Heat Treatment Glass ID=SB5-07

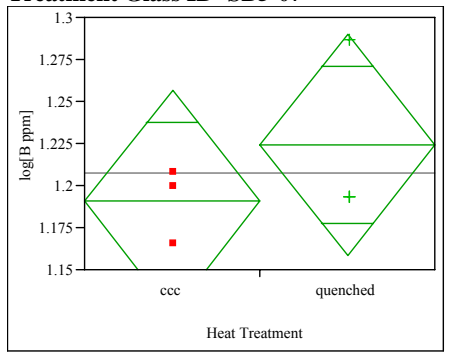

Difference $\quad 0.03378$ t Ratio 1.004164 Std Err Dif 0.03364 DF

Upper CL Dif 0.12717 Prob $>|t| \quad 0.3721$

Lower CL Dif -0.05961 Prob $>$ t 0.1861

$\begin{array}{lll}\text { Confidence } & 0.95 \text { Prob }<\mathrm{t} \quad 0.8139\end{array}$

Oneway Analysis of $\log [\mathrm{Si}$ ppm $]$ By Heat Treatment Glass ID=SB5-08

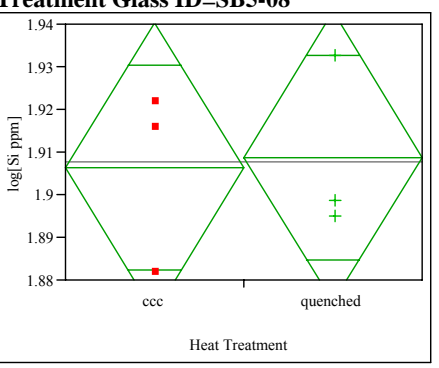

Difference $\quad 0.00240$ t Ratio 0.138479 Std Err Dif 0.01732 DF

Upper CL Dif 0.05050 Prob $>|t| \quad 0.8966$

Lower CL Dif -0.04570 Prob $>t \quad 0.4483$

$\begin{array}{lll}\text { Confidence } & 0.95 \text { Prob }<\mathrm{t} & 0.5517\end{array}$

Oneway Analysis of log[Na ppm] By Heat Treatment Glass ID=SB5-08

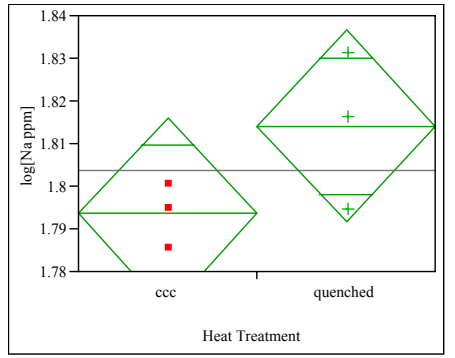

Difference $\quad 0.02062$ t Ratio 1.788163 Std Err Dif 0.01153 DF

Upper CL Dif 0.05264 Prob $>|t| \quad 0.1483$

Lower CL Dif -0.01140 Prob $>t \quad 0.0741$

Confidence $\quad 0.95$ Prob $<\mathrm{t} \quad 0.9259$
Oneway Analysis of $\log [\mathrm{Li}$ ppm] By Heat Treatment Glass ID=SB5-08

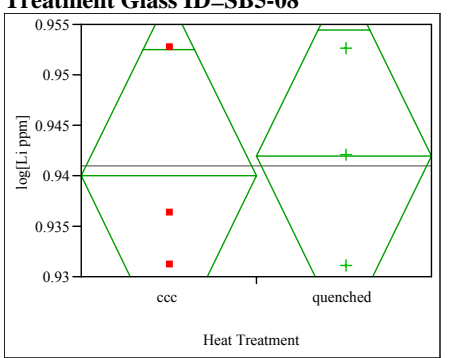

Difference $\quad 0.00194$ t Ratio 0.21626 Std Err Dif 0.00899 DF

Upper CL Dif 0.02689 Prob $>|\mathrm{t}| \quad 0.8394$ Lower CL Dif -0.02300 Prob $>t \quad 0.4197$

$\begin{array}{lll}\text { Confidence } & 0.95 \text { Prob }<\mathrm{t} \quad 0.5803\end{array}$

Oneway Analysis of $\log [B$ ppm] By Heat Treatment Glass ID=SB5-08

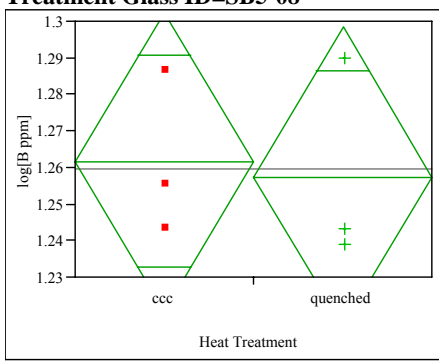

Difference $\quad-0.00422$ t Ratio -0.20241 Std Err Dif $\quad 0.02085$ DF

Upper CL Dif 0.05368 Prob $>|t| \quad 0.8495$

Lower CL Dif -0.06212 Prob $>t \quad 0.5753$

Confidence $\quad 0.95$ Prob $<\mathrm{t} \quad 0.4247$

Oneway Analysis of log[Si ppm] By Heat Treatment Glass ID=SB5-09

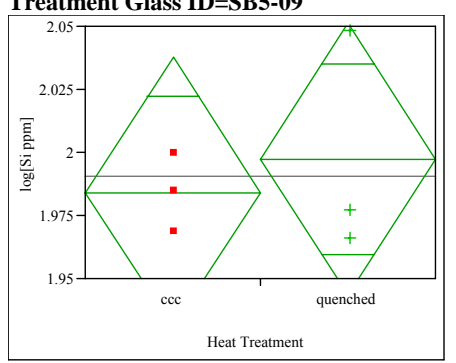

Difference $\quad 0.01313$ t Ratio 0.480079 Std Err Dif $\quad 0.02735$ DF

Upper CL Dif 0.08906 Prob $>|t| \quad 0.6562$

Lower CL Dif -0.06280 Prob $>t \quad 0.3281$

$\begin{array}{lrl}\text { Confidence } & 0.95 \text { Prob }<\mathrm{t} & 0.6719\end{array}$
Oneway Analysis of log[Na ppm] By Heat Treatment Glass ID=SB5-09

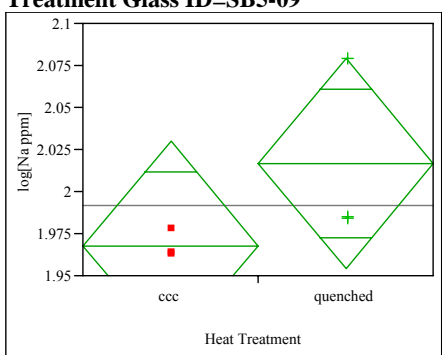

Difference $\quad 0.04868$ t Ratio $\quad 1.529507$ Std Err Dif 0.03183 DF 4 Upper CL Dif 0.13706 Prob $>|\mathrm{t}| \quad 0.2009$ Lower CL Dif -0.03969 Prob $>\mathrm{t} \quad 0.1004$ $\begin{array}{lll}\text { Confidence } & 0.95 \text { Prob }<\mathrm{t} \quad 0.8996\end{array}$

Oneway Analysis of $\log [\mathrm{Li}$ ppm] By Heat Treatment Glass ID=SB5-09

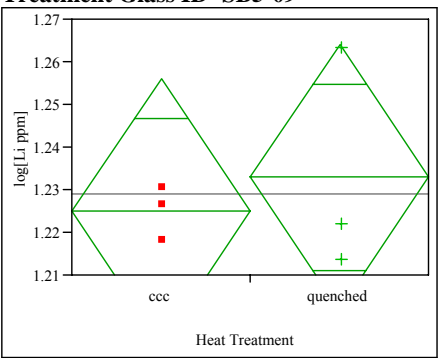

Difference $\quad 0.00802$ t Ratio 0.507454 Std Err Dif $\quad 0.01581$ DF

Upper CL Dif 0.05191 Prob $>|t| \quad 0.6385$

Lower CL Dif -0.03587 Prob $>t \quad 0.3193$

Confidence $\quad 0.95$ Prob $<\mathrm{t} \quad 0.6807$

Oneway Analysis of $\log [\mathrm{B}$ ppm] By Heat Treatment Glass ID=SB5-09

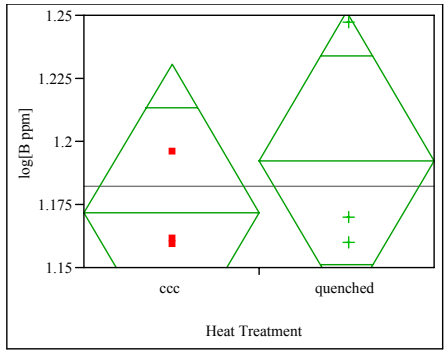

Difference $\quad 0.02054$ t Ratio 0.68577 Std Err Dif 0.02995 DF Upper CL Dif 0.10369 Prob $>|t| \quad 0.5305$ Lower CL Dif -0.06261 Prob $>t \quad 0.2653$ Confidence $\quad 0.95$ Prob $<\mathrm{t} \quad 0.7347$ 
Exhibit B6. Effects of Heat Treatment on PCT Response of Study Glasses. (continued)

Oneway Analysis of log[Si ppm] By Heat Treatment Glass ID=SB5-10

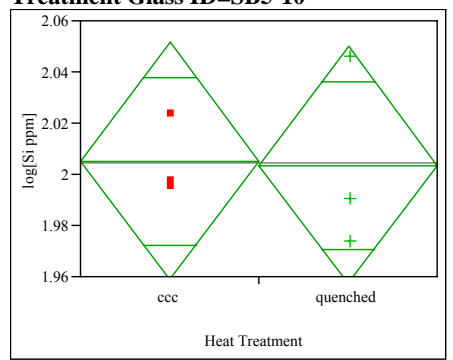

Difference $\quad-0.00163$ t Ratio $\quad-0.06904$ Std Err Dif 0.02362 DF

Upper CL Dif 0.06395 Prob $>|t| \quad 0.9483$

Lower CL Dif -0.06721 Prob $>$ t 0.5259

Confidence $\quad 0.95$ Prob $<\mathrm{t} \quad 0.4741$

Oneway Analysis of log[Na ppm] By Heat Treatment Glass ID=SB5-10

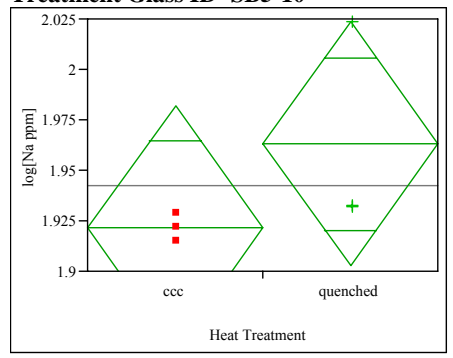

Difference $\quad 0.04126$ t Ratio $\quad 1.340392$ Std Err Dif $\quad 0.03078$ DF

Upper CL Dif 0.12671 Prob $>|t| \quad 0.2512$

Lower CL Dif -0.04420 Prob $>t \quad 0.1256$

Confidence $\quad 0.95$ Prob $<\mathrm{t} \quad 0.8744$

Oneway Analysis of $\log [\mathrm{Li}$ ppm $]$ By Heat Treatment Glass ID=SB5-10

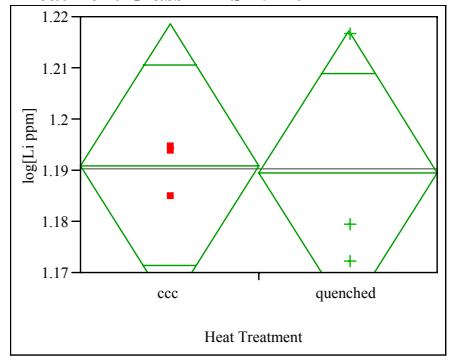

Difference $\quad-0.00151$ t Ratio $\quad-0.10681$ Std Err Dif $\quad 0.01410$ DF

Upper CL Dif 0.03765 Prob $>|t| \quad 0.9201$ Lower CL Dif -0.04066 Prob $>t \quad 0.5400$

Confidence $\quad 0.95$ Prob $<\mathrm{t} \quad 0.4600$
Oneway Analysis of $\log [\mathrm{B}$ ppm] By Heat Treatment Glass ID=SB5-10

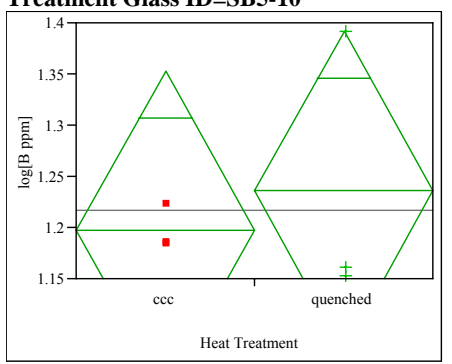

Difference $\quad 0.03851$ t Ratio 0.485266 Std Err Dif $\quad 0.07936$ DF

Upper CL Dif 0.25884 Prob $>|t| \quad 0.6529$

Lower CL Dif -0.18182 Prob $>\mathrm{t} \quad 0.3264$

Confidence $\quad 0.95$ Prob $<\mathrm{t} \quad 0.6736$

Oneway Analysis of $\log [\mathrm{Si}$ ppm] By Heat Treatment Glass ID=SB5-11

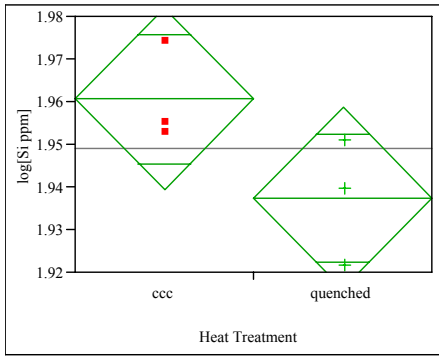

Difference $\quad-0.02312$ t Ratio $\quad-2.12839$ Std Err Dif $\quad 0.01086$ DF

Upper CL Dif 0.00704 Prob $>|t| \quad 0.1004$ Lower CL Dif -0.05328 Prob $>t \quad 0.9498$

Confidence $\quad 0.95$ Prob $<\mathrm{t} \quad 0.0502$

Oneway Analysis of log[Na ppm] By Heat Treatment Glass ID=SB5-11

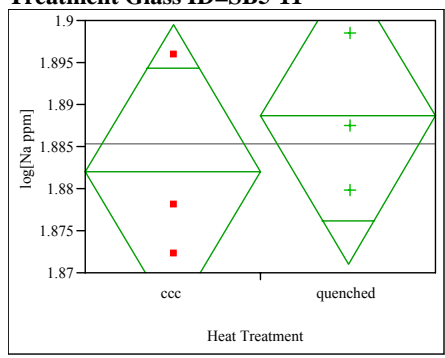

Difference $\quad 0.00665$ t Ratio 0.742969 Std Err Dif $\quad 0.00895$ DF

Upper CL Dif 0.03148 Prob $>|t| \quad 0.4988$ Lower CL Dif -0.01819 Prob $>t \quad 0.2494$

Confidence $\quad 0.95$ Prob $<\mathrm{t} \quad 0.7506$
Oneway Analysis of $\log [\mathrm{Li}$ ppm] By Heat Treatment Glass ID=SB5-11

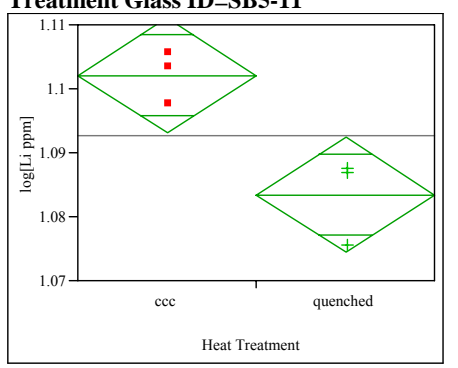

Difference $\quad-0.01873$ t Ratio $\quad-4.09291$ Std Err Dif $\quad 0.00458$ DF 4 Upper CL Dif -0.00602 Prob $>|t| \quad 0.0149$ Lower CL Dif -0.03143 Prob $>t \quad 0.9925$ Confidence $\quad 0.95$ Prob $<\mathrm{t} \quad 0.0075$

Oneway Analysis of $\log [\mathrm{B}$ ppm] By Heat Treatment Glass ID=SB5-11

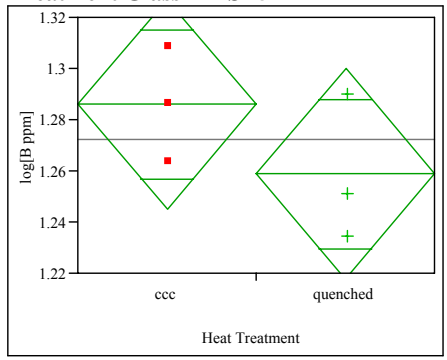

Difference $\quad-0.02727$ t Ratio $\quad-1.30352$ Std Err Dif $\quad 0.02092$ DF

Upper CL Dif 0.03081 Prob $>|t| \quad 0.2624$ Lower CL Dif -0.08535 Prob $>t \quad 0.8688$ Confidence $\quad 0.95$ Prob $<\mathrm{t} \quad 0.1312$

Oneway Analysis of $\log [\mathrm{Si}$ ppm] By Heat Treatment Glass ID=SB5-12

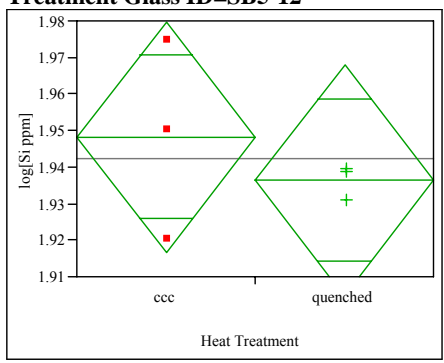

Difference $\quad-0.01183$ t Ratio $\quad-0.73719$ Std Err Dif $\quad 0.01605$ DF 4 Upper CL Dif 0.03273 Prob $>|t| \quad 0.5019$ Lower CL Dif -0.05639 Prob $>t \quad 0.7490$ Confidence $\quad 0.95$ Prob $<\mathrm{t} \quad 0.2510$ 
Exhibit B6. Effects of Heat Treatment on PCT Response of Study Glasses. (continued)

Oneway Analysis of log[Na ppm] By Heat Treatment Glass ID=SB5-12

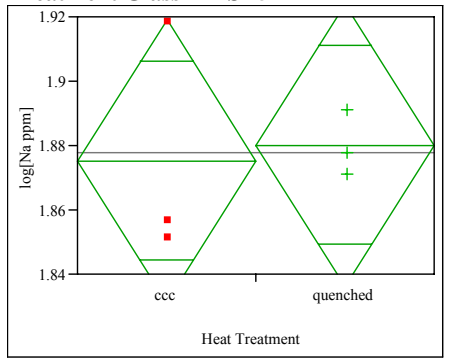

Difference $\quad 0.00484$ t Ratio $\quad 0.216779$ Std Err Dif $\quad 0.02231$ DF

Upper CL Dif 0.06677 Prob $>|t| \quad 0.8390$ Lower CL Dif -0.05710 Prob $>\mathrm{t} \quad 0.4195$

Confidence $\quad 0.95$ Prob $<\mathrm{t} \quad 0.5805$

Oneway Analysis of $\log [\mathrm{Li}$ ppm] By Heat

Treatment Glass ID=SB5-12

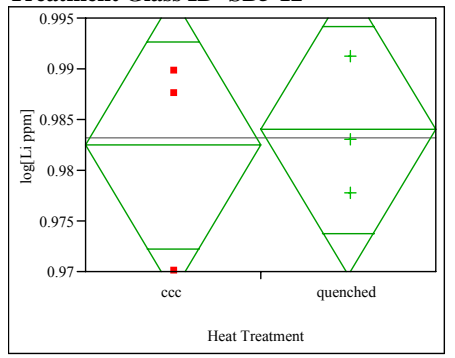

Difference $\quad 0.00156$ t Ratio $\quad 0.211498$ Std Err Dif 0.00737 DF

Upper CL Dif 0.02201 Prob $>|t| \quad 0.8428$

Lower CL Dif -0.01889 Prob $>$ t $\quad 0.4214$

Confidence $\quad 0.95$ Prob $<\mathrm{t} \quad 0.5786$

Oneway Analysis of $\log [\mathrm{B}$ ppm] By Heat Treatment Glass ID=SB5-12

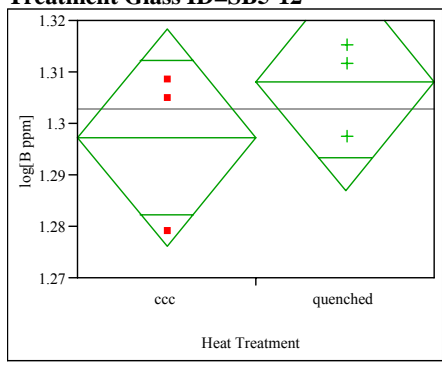

Difference $\quad 0.01094$ t Ratio $\quad 1.015819$ Std Err Dif $\quad 0.01077$ DF Upper CL Dif 0.04085 Prob $>|t| \quad 0.3672$

Lower CL Dif -0.01896 Prob $>t \quad 0.1836$ Confidence $\quad 0.95$ Prob $<\mathrm{t} \quad 0.8164$
Oneway Analysis of $\log [\mathrm{Si}$ ppm] By Heat Treatment Glass ID=SB5-13

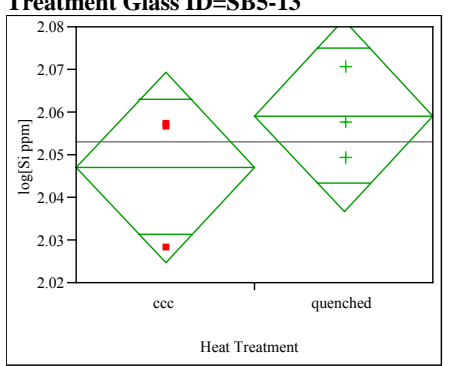

Difference $\quad 0.01207$ t Ratio $\quad 1.06025$ Std Err Dif $\quad 0.01138$ DF

Upper CL Dif 0.04367 Prob $>|t| 0.3488$

Lower CL Dif -0.01954 Prob $>t \quad 0.1744$

Confidence $\quad 0.95$ Prob $<\mathrm{t} \quad 0.8256$

Oneway Analysis of log[Na ppm] By Heat Treatment Glass ID=SB5-13

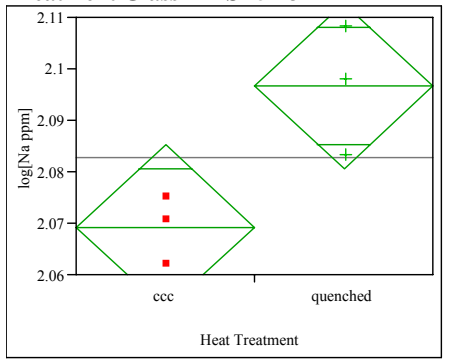

Difference $\quad 0.027414$ t Ratio 3.348419 Std Err Dif 0.008187 DF

Upper CL Dif 0.050146 Prob $>|t| \quad 0.0286$ Lower CL Dif 0.004683 Prob $>t \quad 0.0143$

$\begin{array}{lll}\text { Confidence } & 0.95 \text { Prob }<\mathrm{t} \quad 0.9857\end{array}$

Oneway Analysis of $\log [\mathrm{Li}$ ppm] By Heat Treatment Glass ID=SB5-13

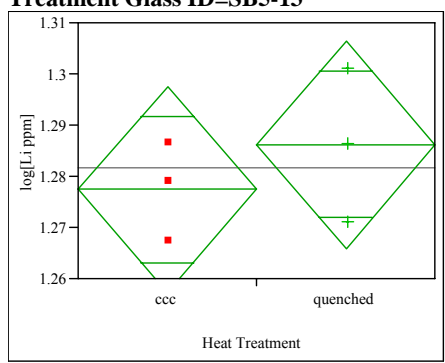

Difference $\quad 0.00872$ t Ratio 0.848185 Std Err Dif $\quad 0.01029$ DF

Upper CL Dif 0.03728 Prob $>|t| \quad 0.4441$

Lower CL Dif -0.01983 Prob $>t \quad 0.2221$

$\begin{array}{lll}\text { Confidence } & 0.95 \text { Prob }<\mathrm{t} & 0.7779\end{array}$
Oneway Analysis of $\log [\mathrm{B}$ ppm] By Heat Treatment Glass ID=SB5-13

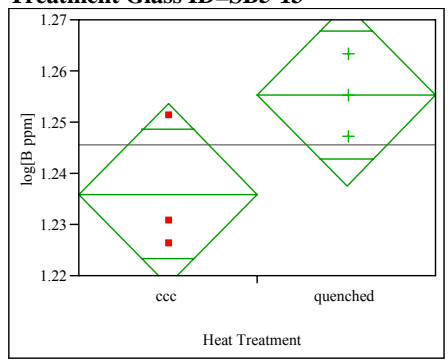

Difference $\quad 0.01927$ t Ratio 2.135096 Std Err Dif 0.00903 DF

Upper CL Dif 0.04433 Prob $>|t| \quad 0.0996$

Lower CL Dif -0.00579 Prob $>t \quad 0.0498$

Confidence $\quad 0.95$ Prob $<\mathrm{t} \quad 0.9502$

Oneway Analysis of $\log [\mathrm{Si}$ ppm] By Heat Treatment Glass ID=SB5-14

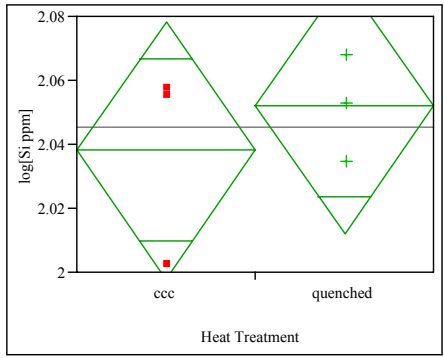

Difference $\quad 0.01378$ t Ratio 0.672843 Std Err Dif $\quad 0.02047$ DF

Upper CL Dif 0.07062 Prob $>|t| \quad 0.5379$ Lower CL Dif -0.04307 Prob $>t \quad 0.2690$

Confidence $\quad 0.95$ Prob $<t \quad 0.7310$

Oneway Analysis of $\log [\mathrm{Na} \mathrm{ppm}]$ By Heat Treatment Glass ID=SB5-14

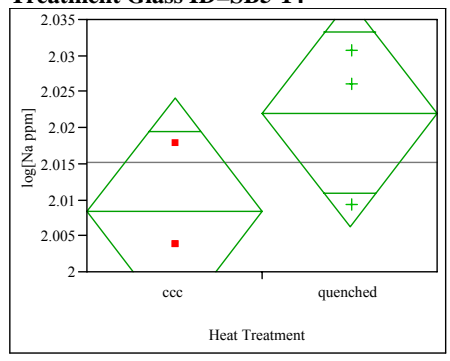

Difference $\quad 0.01370$ t Ratio $\quad 1.707279$ Std Err Dif $\quad 0.00802$ DF 4

Upper CL Dif 0.03598 Prob $>|t| \quad 0.1630$ Lower CL Dif -0.00858 Prob $>t \quad 0.0815$

Confidence $\quad 0.95$ Prob $<\mathrm{t} \quad 0.9185$ 
Exhibit B6. Effects of Heat Treatment on PCT Response of Study Glasses. (continued)

Oneway Analysis of $\log [\mathrm{Li}$ ppm] By Heat Treatment Glass ID=SB5-14

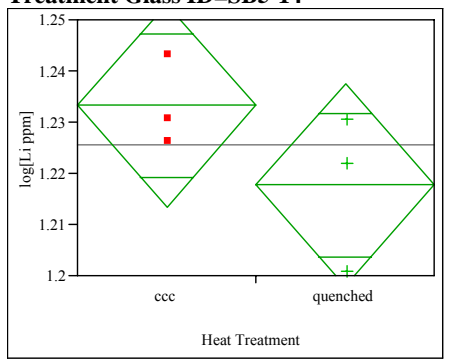

Difference $\quad-0.01547$ t Ratio $\quad-1.52907$ Std Err Dif $\quad 0.01012$ DF

Upper CL Dif 0.01262 Prob $>|t| \quad 0.2010$

Lower CL Dif -0.04357 Prob $>\mathrm{t} \quad 0.8995$

Confidence $\quad 0.95$ Prob $<\mathrm{t} \quad 0.1005$

Oneway Analysis of $\log [\mathrm{B}$ ppm] By Heat

Treatment Glass ID=SB5-14

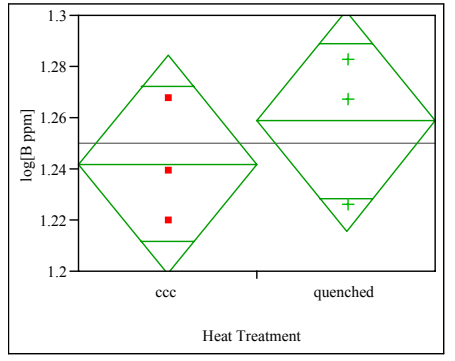

Difference $\quad 0.01687$ t Ratio $\quad 0.772532$

Std Err Dif $\quad 0.02183$ DF

Upper CL Dif 0.07748 Prob $>|t|$

Lower CL Dif -0.04375 Prob $>t \quad 0.2415$

Confidence $\quad 0.95$ Prob $<\mathrm{t} \quad 0.7585$

Oneway Analysis of $\log [\mathrm{Si}$ ppm] By Heat Treatment Glass ID=SB5-15

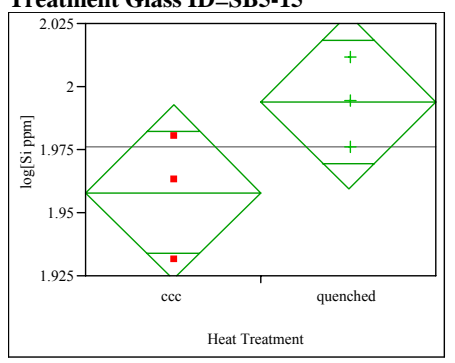

Difference $\quad 0.03589$ t Ratio 2.042626 Std Err Dif $\quad 0.01757$ DF

Upper CL Dif 0.08468 Prob $>|t| \quad 0.1106$

Lower CL Dif -0.01289 Prob $>t \quad 0.0553$

Confidence $\quad 0.95$ Prob $<\mathrm{t} \quad 0.9447$
Oneway Analysis of log[Na ppm] By Heat Treatment Glass ID=SB5-15

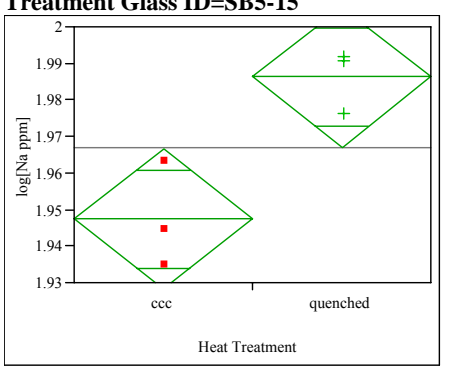

Difference $\quad 0.038889$ t Ratio 3.989999 Std Err Dif 0.009747 DF

Upper CL Dif 0.065950 Prob $>|t| \quad 0.0163$

Lower CL Dif 0.011828 Prob $>t \quad 0.008$

Confidence $\quad 0.95$ Prob $<\mathrm{t} \quad 0.9919$

Oneway Analysis of $\log [\mathrm{Li}$ ppm] By Heat Treatment Glass ID=SB5-15

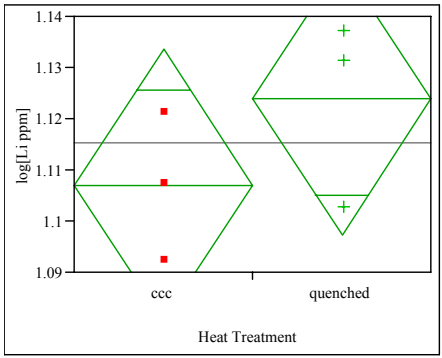

Difference $\quad 0.01691$ t Ratio $\quad 1.247557$ Std Err Dif $\quad 0.01355$ DF

Upper CL Dif 0.05454 Prob $>|t| \quad 0.2802$ Lower CL Dif -0.02072 Prob $>t \quad 0.1401$

Confidence $\quad 0.95$ Prob $<\mathrm{t} \quad 0.8599$

Oneway Analysis of $\log [B$ ppm] By Heat Treatment Glass ID=SB5-15

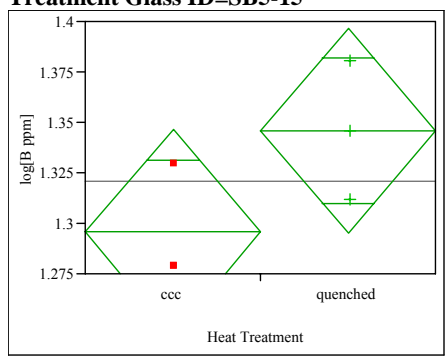

Difference $\quad 0.05037$ t Ratio 1.943333 Std Err Dif 0.02592 DF

Upper CL Dif 0.12233 Prob $>|t| \quad 0.1239$ Lower CL Dif -0.02159 Prob $>t \quad 0.0620$

Confidence $\quad 0.95$ Prob $<\mathrm{t} \quad 0.9380$
Oneway Analysis of $\log [\mathrm{Si}$ ppm] By Heat Treatment Glass ID=SB5-16

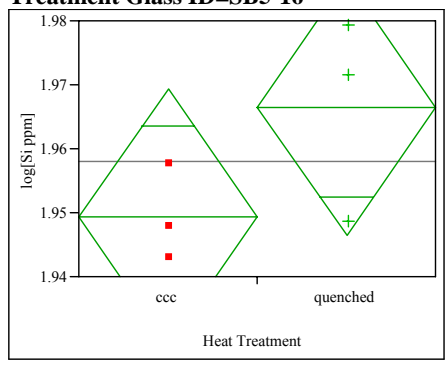

Difference $\quad 0.01712$ t Ratio 1.684124 Std Err Dif $\quad 0.01017$ DF

Upper CL Dif 0.04535 Prob $>|t| \quad 0.1674$

Lower CL Dif -0.01110 Prob $>t \quad 0.0837$

Confidence $\quad 0.95$ Prob $<\mathrm{t} \quad 0.9163$

Oneway Analysis of log[Na ppm] By Heat Treatment Glass ID=SB5-16

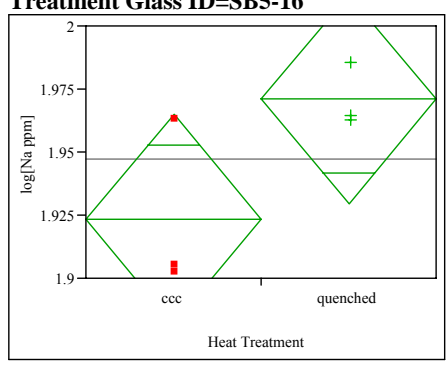

Difference $\quad 0.04759$ t Ratio 2.256074 Std Err Dif $\quad 0.02109$ DF

Upper CL Dif 0.10616 Prob $>|t| 0.0871$

Lower CL Dif -0.01098 Prob $>t \quad 0.0435$

Confidence $\quad 0.95$ Prob $<\mathrm{t} \quad 0.9565$

Oneway Analysis of $\log [\mathrm{Li}$ ppm] By Heat Treatment Glass ID=SB5-16

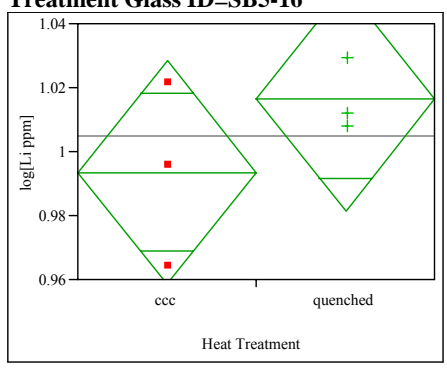

Difference $\quad 0.02293$ t Ratio $\quad 1.284185$ Std Err Dif $\quad 0.01786$ DF

Upper CL Dif 0.07251 Prob $>|t| \quad 0.2684$ Lower CL Dif -0.02665 Prob $>t \quad 0.1342$

Confidence $\quad 0.95$ Prob $<\mathrm{t} \quad 0.8658$ 
Exhibit B6. Effects of Heat Treatment on PCT Response of Study Glasses. (continued)

Oneway Analysis of log[B ppm] By Heat Treatment Glass ID=SB5-16

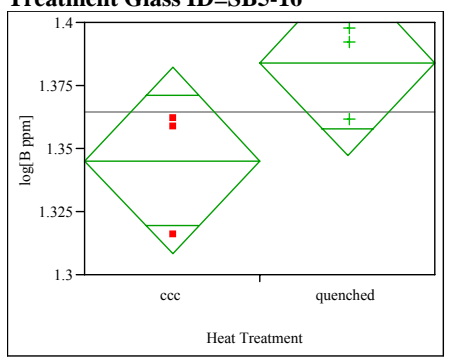

Difference $\quad 0.03874$ t Ratio 2.068693 Std Err Dif 0.01873 DF

Upper CL Dif 0.09073 Prob $>|t| \quad 0.1074$ Lower CL Dif -0.01325 Prob $>\mathrm{t} \quad 0.0537$ $\begin{array}{lr}\text { Confidence } & 0.95 \text { Prob }<\mathrm{t} \quad 0.9463\end{array}$

Oneway Analysis of log[Si ppm] By Heat Treatment Glass ID=SB5-17

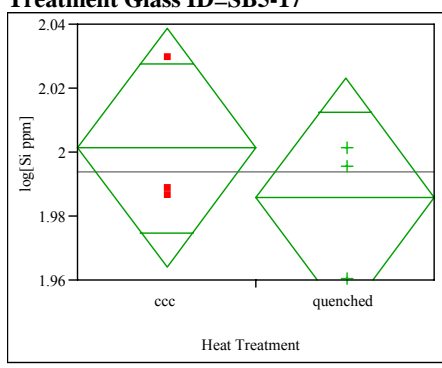

Difference $\quad-0.01532$ t Ratio $\quad-0.80613$ Std Err Dif $\quad 0.01900$ DF

Upper CL Dif 0.03745 Prob $>|t| \quad 0.4654$

Lower CL Dif -0.06809 Prob $>t \quad 0.7673$

Confidence $\quad 0.95$ Prob $<\mathrm{t} \quad 0.2327$

Oneway Analysis of log[Na ppm] By Heat Treatment Glass ID=SB5-17

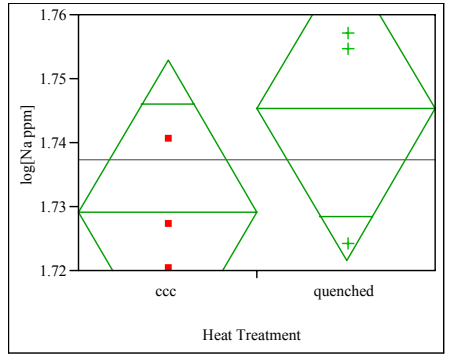

Difference $\quad 0.01617$ t Ratio 1.334955 Std Err Dif $\quad 0.01211$ DF

Upper CL Dif 0.04979 Prob $>|t| \quad 0.2528$

Lower CL Dif -0.01746 Prob $>t \quad 0.1264$

Confidence $\quad 0.95$ Prob $<\mathrm{t} \quad 0.8736$
Oneway Analysis of $\log [\mathrm{Li} \mathrm{ppm}]$ By Heat Treatment Glass ID=SB5-17

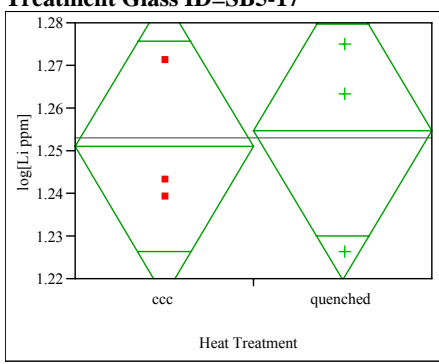

Difference $\quad 0.00378$ t Ratio 0.212141 Std Err Dif $\quad 0.01784$ DF

Upper CL Dif 0.05331 Prob $>|\mathrm{t}| \quad 0.8424$

Lower CL Dif -0.04574 Prob $>t \quad 0.4212$

Confidence $\quad 0.95$ Prob $<\mathrm{t} \quad 0.5788$

Oneway Analysis of $\log [\mathrm{B}$ ppm] By Heat Treatment Glass ID=SB5-17

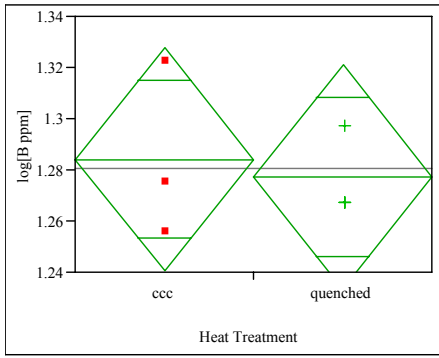

Difference $\quad-0.00689$ t Ratio $\quad-0.30945$ Std Err Dif 0.02228 DF

Upper CL Dif 0.05495 Prob $>|t| \quad 0.7724$ Lower CL Dif -0.06874 Prob $>t \quad 0.6138$

Confidence $\quad 0.95$ Prob $<\mathrm{t} \quad 0.3862$

Oneway Analysis of $\log [\mathrm{Si}$ ppm] By Heat Treatment Glass ID=SB5-18

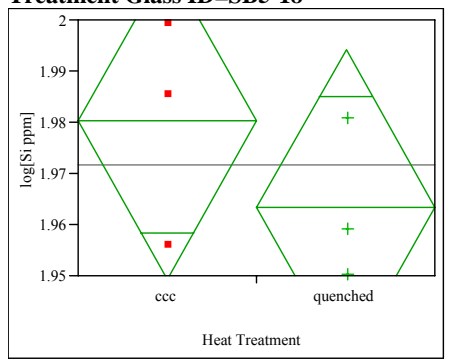

Difference $\quad-0.01680$ t Ratio $\quad-1.0706$ Std Err Dif $\quad 0.01569$ DF 4 Upper CL Dif 0.02677 Prob $>|t| \quad 0.3446$ Lower CL Dif -0.06036 Prob $>t \quad 0.8277$ Confidence $\quad 0.95$ Prob $<\mathrm{t} \quad 0.1723$
Oneway Analysis of log[Na ppm] By Heat Treatment Glass ID=SB5-18

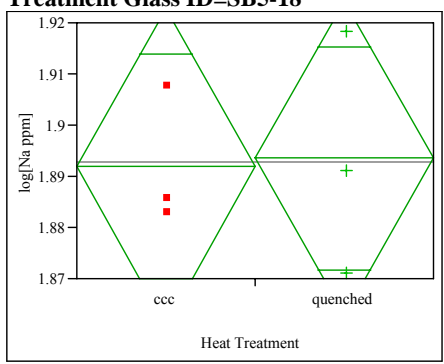

Difference $\quad 0.00157$ t Ratio 0.099594 Std Err Dif $\quad 0.01573$ DF

Upper CL Dif 0.04524 Prob $>|t| \quad 0.9255$

Lower CL Dif -0.04211 Prob $>t \quad 0.4627$

Confidence $\quad 0.95$ Prob $<\mathrm{t} \quad 0.5373$

Oneway Analysis of $\log [\mathrm{Li}$ ppm] By Heat Treatment Glass ID=SB5-18

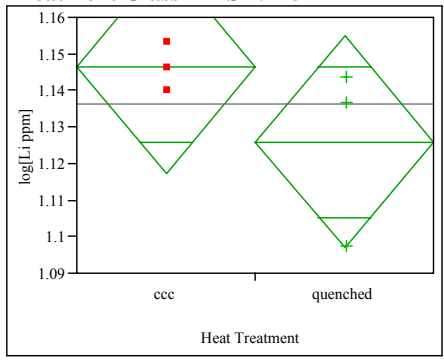

Difference $\quad-0.02035$ t Ratio $\quad-1.37287$ Std Err Dif $\quad 0.01483$ DF 4 Upper CL Dif 0.02081 Prob $>|t| \quad 0.2417$ Lower CL Dif -0.06152 Prob $>t \quad 0.8791$ Confidence $\quad 0.95$ Prob $<\mathrm{t} \quad 0.1209$

Oneway Analysis of $\log [\mathrm{B}$ ppm] By Heat Treatment Glass ID=SB5-18

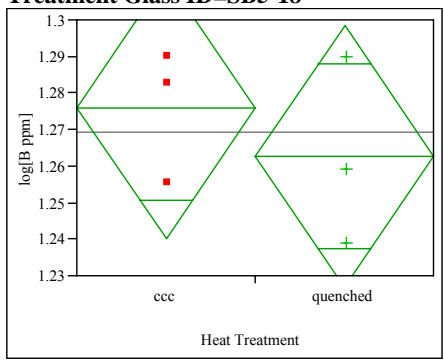

Difference $\quad-0.01322$ t Ratio $\quad-0.72491$ Std Err Dif $\quad 0.01824$ DF 4 Upper CL Dif 0.03741 Prob $>|t| \quad 0.5086$ Lower CL Dif -0.06386 Prob $>t \quad 0.7457$ Confidence $\quad 0.95$ Prob $<\mathrm{t} \quad 0.2543$ 
Exhibit B6. Effects of Heat Treatment on PCT Response of Study Glasses. (continued)

Oneway Analysis of log[Si ppm] By Heat Treatment Glass ID=SB5-19

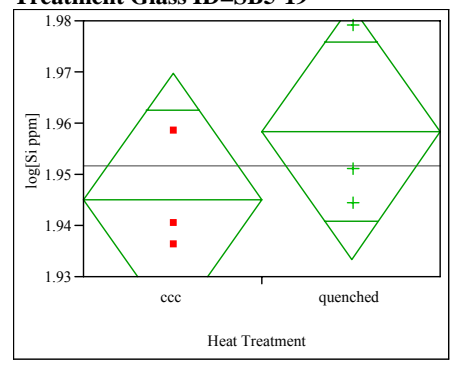

Difference $\quad 0.01332$ t Ratio $\quad 1.054568$ Std Err Dif 0.01263 DF

Upper CL Dif 0.04840 Prob $>|t| \quad 0.3511$

Lower CL Dif -0.02175 Prob $>t \quad 0.1756$

Confidence $\quad 0.95$ Prob $<\mathrm{t} \quad 0.8244$

Oneway Analysis of log[Na ppm] By Heat Treatment Glass ID=SB5-19

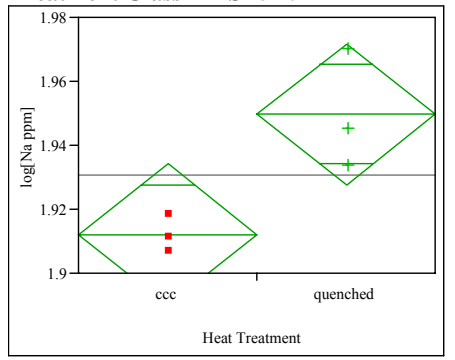

Difference $\quad 0.037647$ t Ratio $\quad 3.350126$ Std Err Dif $\quad 0.011237$ DF

Upper CL Dif 0.068847 Prob $>|t| \quad 0.0286$

Lower CL Dif 0.006447 Prob $>$ t 0.0143

Confidence $\quad 0.95$ Prob $<\mathrm{t} \quad 0.9857$

Oneway Analysis of $\log [\mathrm{Li}$ ppm] By Heat Treatment Glass ID=SB5-19

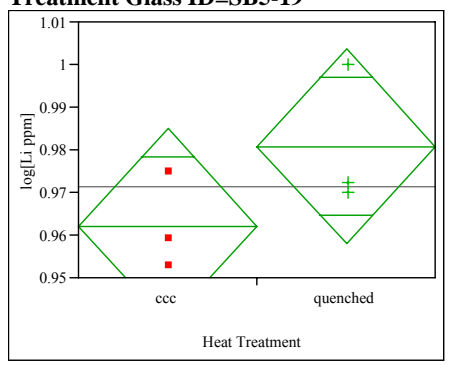

Difference $\quad 0.01869$ t Ratio 1.605544 Std Err Dif $\quad 0.01164$ DF Upper CL Dif 0.05100 Prob $>|t| \quad 0.1836$

Lower CL Dif -0.01363 Prob $>t \quad 0.0918$ Confidence $\quad 0.95$ Prob $<\mathrm{t} \quad 0.9082$
Oneway Analysis of $\log [B \mathrm{ppm}]$ By Heat Treatment Glass ID=SB5-19

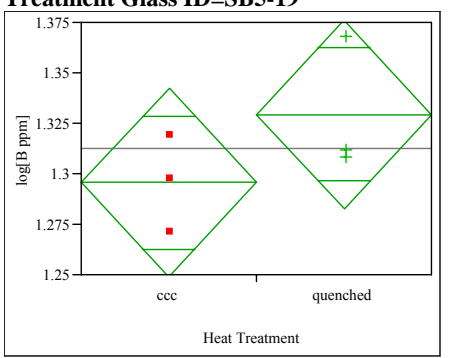

Difference $\quad 0.03357$ t Ratio 1.412416 Std Err Dif $\quad 0.02377$ DF

Upper CL Dif 0.09957 Prob > $|\mathrm{t}| \quad 0.2307$ Lower CL Dif -0.03242 Prob $>t \quad 0.1153$

$\begin{array}{lll}\text { Confidence } & 0.95 \text { Prob }<\mathrm{t} \quad 0.8847\end{array}$

Oneway Analysis of $\log [\mathrm{Si}$ ppm] By Heat Treatment Glass ID $=$ SB5-20

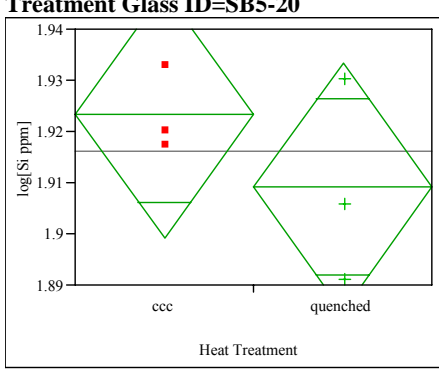

Difference $\quad-0.01429$ t Ratio $\quad-1.155$ Std Err Dif $\quad 0.01237$ DF

Upper CL Dif 0.02006 Prob $>|t| 0.3124$

Lower CL Dif -0.04863 Prob $>t \quad 0.8438$

Confidence $\quad 0.95$ Prob $<\mathrm{t} \quad 0.1562$

Oneway Analysis of log[Na ppm] By Heat Treatment Glass ID=SB5-20

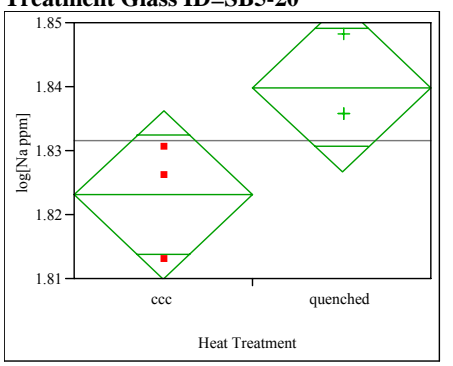

Difference $\quad 0.01674$ t Ratio 2.496298 Std Err Dif $\quad 0.00670$ DF

Upper CL Dif 0.03535 Prob $>|t| \quad 0.0670$ Lower CL Dif -0.00188 Prob $>t \quad 0.0335$

Confidence $\quad 0.95$ Prob $<\mathrm{t} \quad 0.9665$
Oneway Analysis of $\log [\mathrm{Li}$ ppm] By Heat Treatment Glass ID $=$ SB5-20

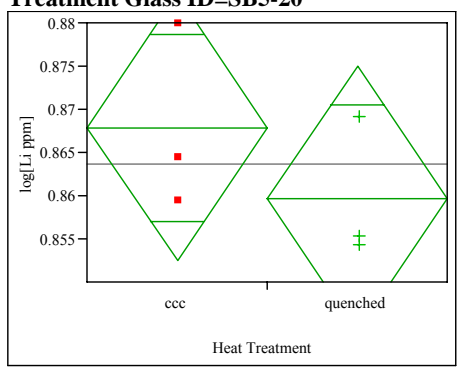

Difference $\quad-0.00822$ t Ratio $\quad-1.04899$ Std Err Dif $\quad 0.00784$ DF

Upper CL Dif 0.01353 Prob $>|t| \quad 0.3534$ Lower CL Dif -0.02997 Prob $>t \quad 0.8233$

Confidence $\quad 0.95$ Prob $<\mathrm{t} \quad 0.1767$

Oneway Analysis of $\log [\mathrm{B}$ ppm] By Heat Treatment Glass ID=SB5-20

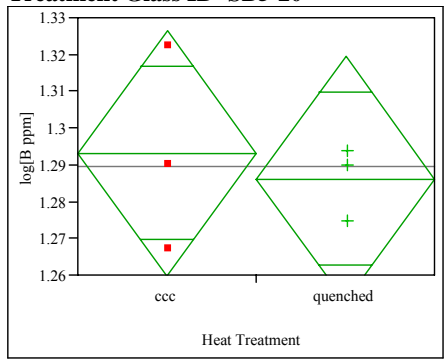

Difference $\quad-0.00691$ t Ratio $\quad-0.40723$ Std Err Dif $\quad 0.01697$ DF 4 Upper CL Dif 0.04021 Prob $>|\mathrm{t}| \quad 0.7047$ Lower CL Dif -0.05403 Prob $>\mathrm{t} \quad 0.6477$ Confidence $\quad 0.95$ Prob $<\mathrm{t} \quad 0.3523$

Oneway Analysis of $\log [\mathrm{Si}$ ppm] By Heat Treatment Glass ID=SB5-21

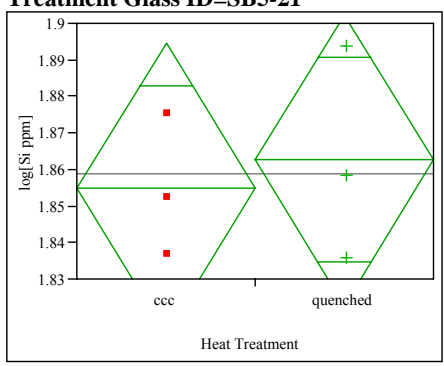

Difference $\quad 0.00796$ t Ratio $\quad 0.392667$ Std Err Dif $\quad 0.02028$ DF 4 Upper CL Dif 0.06427 Prob $>|\mathrm{t}| \quad 0.7146$ Lower CL Dif -0.04834 Prob $>\mathrm{t} \quad 0.3573$ $\begin{array}{lll}\text { Confidence } & 0.95 \text { Prob }<\mathrm{t} \quad 0.6427\end{array}$ 
Exhibit B6. Effects of Heat Treatment on PCT Response of Study Glasses. (continued)

Oneway Analysis of log[Na ppm] By Heat Treatment Glass ID=SB5-21

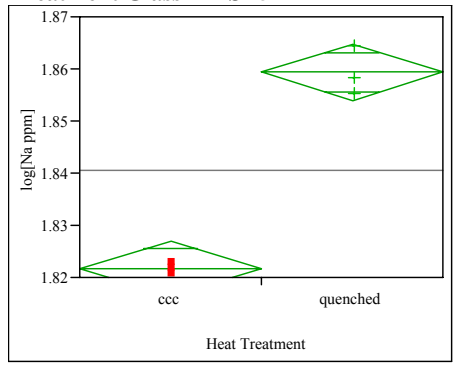

Difference $\quad 0.037591$ t Ratio 13.83394 Std Err Dif $\quad 0.002717$ DF

Upper CL Dif 0.045136 Prob $>|t| \quad 0.0002$ Lower CL Dif 0.030047 Prob $>t \quad<.0001$

Confidence $\quad 0.95$ Prob $<\mathrm{t} \quad 0.9999$

Oneway Analysis of $\log [\mathrm{Li}$ ppm] By Heat Treatment Glass ID=SB5-21

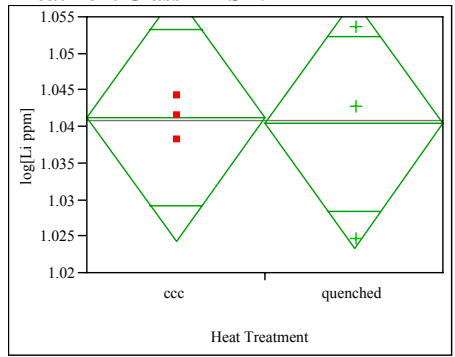

Difference $\quad-0.00082$ t Ratio -0.09445 Std Err Dif 0.00865 DF

Upper CL Dif 0.02321 Prob $>|t| 0.9293$

Lower CL Dif -0.02484 Prob $>\mathrm{t} \quad 0.5354$

Confidence $\quad 0.95$ Prob $<\mathrm{t} \quad 0.4646$

Oneway Analysis of $\log [\mathrm{B}$ ppm] By Heat Treatment Glass ID=SB5-21

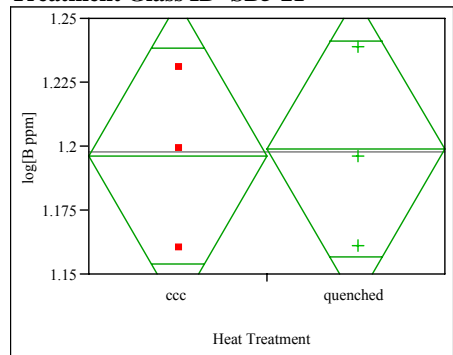

Difference $\quad 0.00238$ t Ratio 0.078221 Std Err Dif 0.03041 DF

Upper CL Dif 0.08682 Prob $>|\mathrm{t}| \quad 0.9414$

Lower CL Dif -0.08207 Prob $>t \quad 0.4707$

$\begin{array}{lrl}\text { Confidence } & 0.95 \text { Prob }<\mathrm{t} & 0.5293\end{array}$
Oneway Analysis of $\log [\mathrm{Si}$ ppm] By Heat Treatment Glass ID=SB5-22

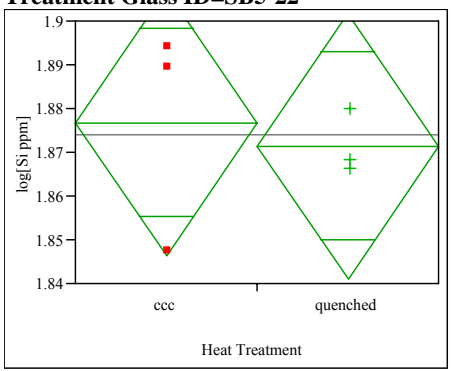

Difference $\quad-0.00534$ t Ratio $\quad-0.34491$ Std Err Dif $\quad 0.01547$ DF

Upper CL Dif 0.03762 Prob $>|t| \quad 0.7475$

Lower CL Dif -0.04829 Prob $>t \quad 0.6262$

Confidence $\quad 0.95$ Prob $<\mathrm{t} \quad 0.3738$

Oneway Analysis of $\log [\mathrm{Na} \mathrm{ppm}]$ By Heat Treatment Glass ID=SB5-22

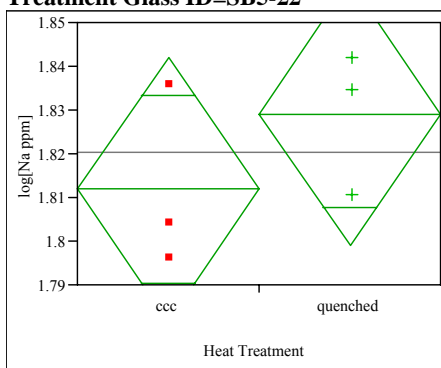

Difference $\quad 0.01727$ t Ratio $\quad 1.12179$ Std Err Dif $\quad 0.01540$ DF

Upper CL Dif 0.06002 Prob $>|t| 0.3247$

Lower CL Dif -0.02548 Prob $>t \quad 0.1624$

Confidence $\quad 0.95$ Prob $<\mathrm{t} \quad 0.8376$

Oneway Analysis of $\log [\mathrm{Li}$ ppm] By Heat

Treatment Glass ID=SB5-22

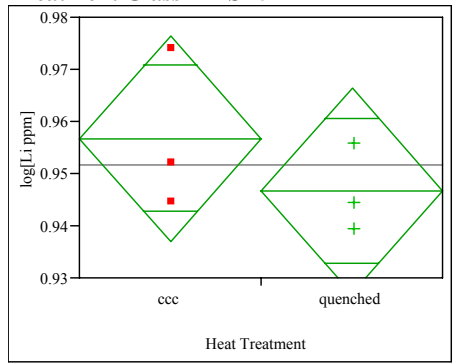

Difference $\quad-0.01012$ t Ratio $\quad-1.00432$ Std Err Dif $\quad 0.01007$ DF

Upper CL Dif 0.01785 Prob $>|\mathrm{t}| \quad 0.3721$

Lower CL Dif -0.03809 Prob $>\mathrm{t} \quad 0.8140$ $\begin{array}{lll}\text { Confidence } & 0.95 \text { Prob }<\mathrm{t} & 0.1860\end{array}$
Oneway Analysis of $\log [\mathrm{B}$ ppm] By Heat Treatment Glass ID=SB5-22

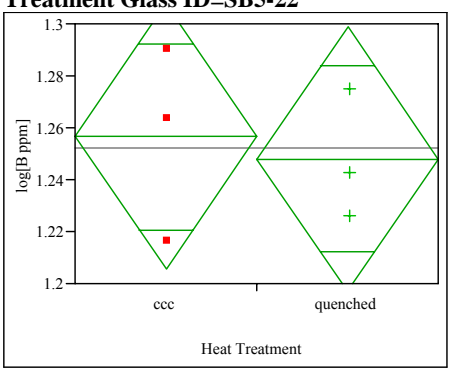

Difference $\quad-0.00844$ t Ratio $\quad-0.32577$ Std Err Dif $\quad 0.02589$ DF 4

Upper CL Dif 0.06346 Prob $>|t| \quad 0.7609$ Lower CL Dif -0.08033 Prob $>t \quad 0.6195$

Confidence $\quad 0.95$ Prob $<\mathrm{t} \quad 0.3805$

Oneway Analysis of $\log [\mathrm{Si}$ ppm] By Heat Treatment Glass ID=SB5-23

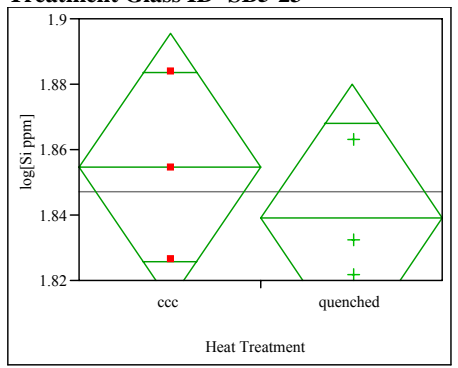

Difference $\quad-0.01549$ t Ratio $\quad-0.74567$ Std Err Dif $\quad 0.02078$ DF 4

Upper CL Dif 0.04219 Prob $>|t| 0.4973$ Lower CL Dif -0.07318 Prob $>t \quad 0.7513$ $\begin{array}{lll}\text { Confidence } & 0.95 \text { Prob }<\mathrm{t} \quad 0.2487\end{array}$

Oneway Analysis of $\log [\mathrm{Na} \mathrm{ppm}] \mathrm{By}$ Heat Treatment Glass ID=SB5-23

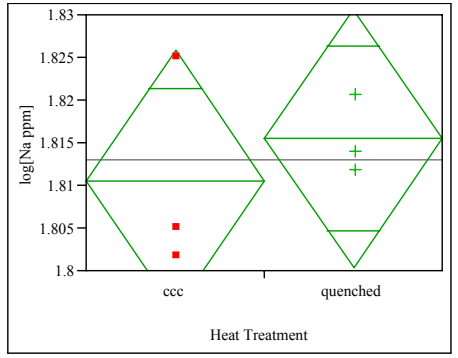

Difference $\quad 0.00493$ t Ratio 0.635854 Std Err Dif $\quad 0.00775$ DF

Upper CL Dif 0.02646 Prob $>|t| \quad 0.5594$ Lower CL Dif -0.01660 Prob $>t \quad 0.2797$ Confidence $\quad 0.95$ Prob $<\mathrm{t} \quad 0.7203$ 
Exhibit B6. Effects of Heat Treatment on PCT Response of Study Glasses. (continued)

Oneway Analysis of $\log [\mathrm{Li}$ ppm] By Heat Treatment Glass ID=SB5-23

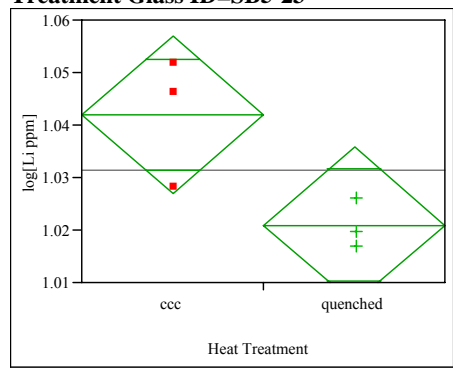

Difference $\quad-0.02099$ t Ratio $\quad-2.75229$ Std Err Dif 0.00763 DF

Upper CL Dif 0.00018 Prob $>|t| \quad 0.0513$

Lower CL Dif -0.04216 Prob $>t \quad 0.9744$

Confidence $\quad 0.95$ Prob $<t \quad 0.0256$

Oneway Analysis of $\log [\mathrm{B}$ ppm] By Heat Treatment Glass ID=SB5-23

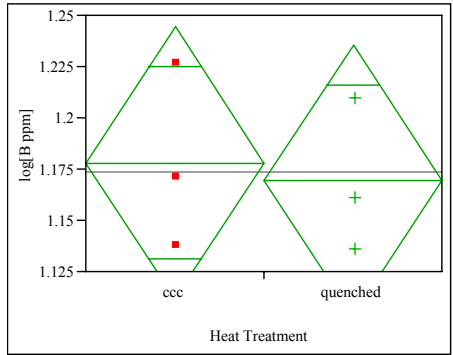

Difference $\quad-0.00888$ t Ratio $\quad-0.26296$ Std Err Dif 0.03378 DF

Upper CL Dif 0.08490 Prob $>|t| \quad 0.8056$

Lower CL Dif -0.10267 Prob $>t \quad 0.5972$

Confidence $\quad 0.95$ Prob $<\mathrm{t} \quad 0.4028$

Oneway Analysis of log[Si ppm] By Heat Treatment Glass ID=SB5-24

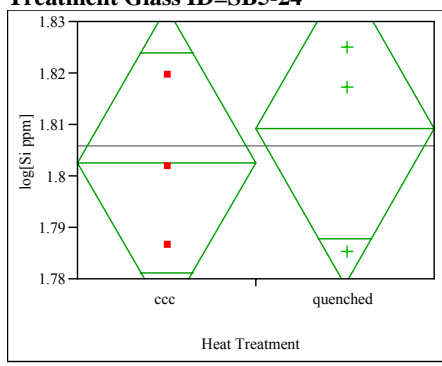

Difference $\quad 0.00666$ t Ratio 0.430985 Std Err Dif $\quad 0.01545$ DF

Upper CL Dif 0.04956 Prob $>|t| \quad 0.6887$

Lower CL Dif -0.03624 Prob $>t \quad 0.3443$

$\begin{array}{lll}\text { Confidence } & 0.95 \text { Prob }<\mathrm{t} & 0.6557\end{array}$
Oneway Analysis of $\log [\mathrm{Na}$ ppm] By Hea Treatment Glass ID=SB5-24

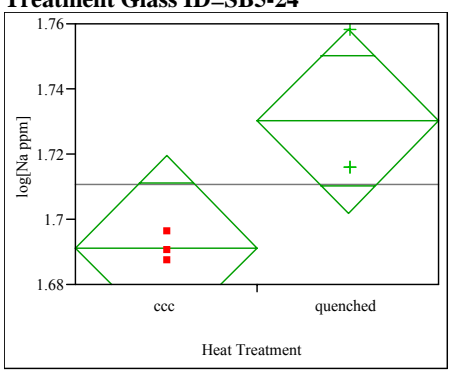

Difference $\quad 0.03897$ t Ratio $\quad 2.71198$ Std Err Dif $0.01437 \mathrm{DF}$

Upper CL Dif 0.07887 Prob $>|t| \quad 0.0534$ Lower CL Dif -0.00093 Prob $>t \quad 0.0267$

Confidence $\quad 0.95$ Prob $<\mathrm{t} \quad 0.9733$

Oneway Analysis of $\log [\mathrm{Li}$ ppm] By Heat Treatment Glass ID=SB5-24

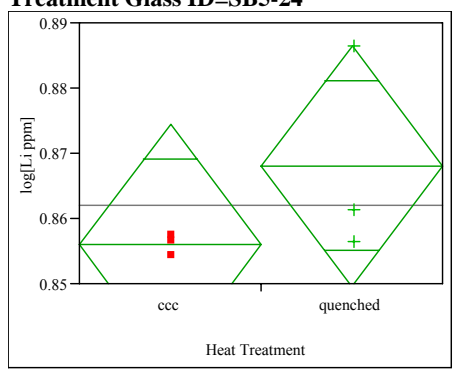

Difference $\quad 0.01206$ t Ratio $\quad 1.286655$ Std Err Dif 0.00938 DF

Upper CL Dif 0.03809 Prob $>|\mathrm{t}| \quad 0.2676$ Lower CL Dif -0.01397 Prob $>\mathrm{t} \quad 0.1338$ $\begin{array}{lll}\text { Confidence } & 0.95 \text { Prob }<\mathrm{t} \quad 0.8662\end{array}$

Oneway Analysis of $\log [\mathrm{B}$ ppm] By Heat Treatment Glass ID=SB5-24

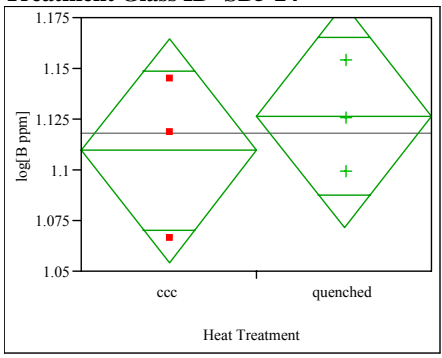

Difference $\quad 0.01697$ t Ratio $\quad 0.604115$ Std Err Dif $\quad 0.02810$ DF

Upper CL Dif 0.09498 Prob $>|\mathrm{t}| \quad 0.5784$

Lower CL Dif -0.06104 Prob $>\mathrm{t} \quad 0.2892$

$\begin{array}{lll}\text { Confidence } & 0.95 \text { Prob }<\mathrm{t} & 0.7108\end{array}$
Oneway Analysis of $\log [\mathrm{Si}$ ppm] By Heat Treatment Glass ID=SB5-25

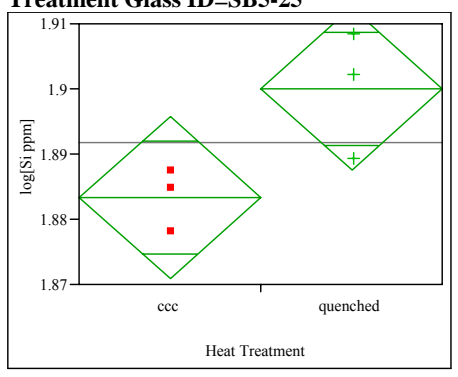

Difference $\quad 0.01666$ t Ratio 2.64211 Std Err Dif $\quad 0.00631$ DF Upper CL Dif 0.03417 Prob $>|t| \quad 0.0575$ Lower CL Dif -0.00085 Prob $>t \quad 0.0287$ Confidence $\quad 0.95$ Prob $<\mathrm{t} \quad 0.9713$

Oneway Analysis of log[Na ppm] By Heat Treatment Glass ID=SB5-25

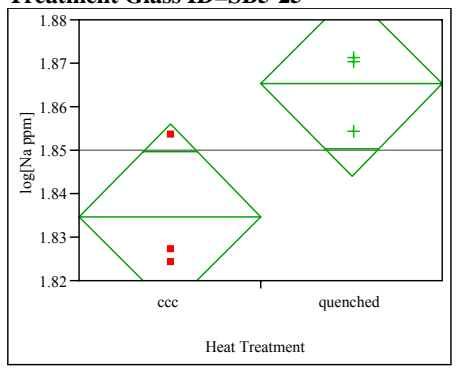

Difference $\quad 0.030448$ t Ratio 2.821694 Std Err Dif $\quad 0.010791$ DF

Upper CL Dif 0.060407 Prob $>|t| \quad 0.0477$ Lower CL Dif 0.000488 Prob $>$ t 0.0239 $\begin{array}{lll}\text { Confidence } & 0.95 \text { Prob }<\mathrm{t} & 0.9761\end{array}$

Oneway Analysis of $\log [\mathrm{Li}$ ppm] By Heat Treatment Glass ID=SB5-25

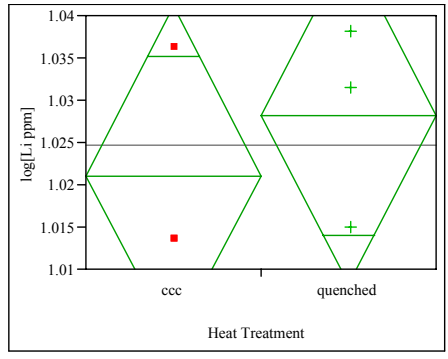

Difference $\quad 0.00709$ t Ratio $\quad 0.695513$ Std Err Dif 0.01019 DF 4

Upper CL Dif 0.03538 Prob $>|t| \quad 0.5250$

Lower CL Dif -0.02121 Prob $>t \quad 0.2625$

Confidence $\quad 0.95$ Prob $<\mathrm{t} \quad 0.7375$ 
Exhibit B6. Effects of Heat Treatment on PCT Response of Study Glasses. (continued)

Oneway Analysis of log[B ppm] By Heat Treatment Glass ID=SB5-25

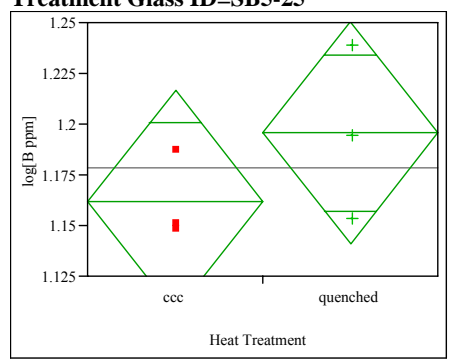

Difference $\quad 0.03374$ t Ratio $\quad 1.215605$ Std Err Dif $\quad 0.02776$ DF

Upper CL Dif 0.11080 Prob $>|t| \quad 0.2910$ Lower CL Dif -0.04332 Prob $>\mathrm{t} \quad 0.1455$

Confidence $\quad 0.95$ Prob $<\mathrm{t} \quad 0.8545$

Oneway Analysis of $\log [\mathrm{Si}$ ppm $]$ By Heat Treatment Glass ID=SB5-26

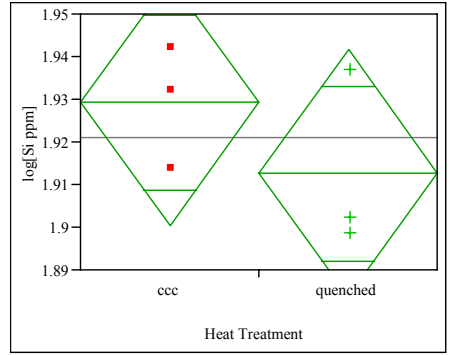

Difference $\quad-0.01668$ t Ratio $\quad-1.12868$ Std Err Dif 0.01478 DF

Upper CL Dif 0.02435 Prob $>|t| \quad 0.3221$

Lower CL Dif -0.05772 Prob $>t \quad 0.8389$

Confidence $\quad 0.95$ Prob $<\mathrm{t} \quad 0.1611$

Oneway Analysis of log[Na ppm] By Heat

Treatment Glass ID=SB5-26

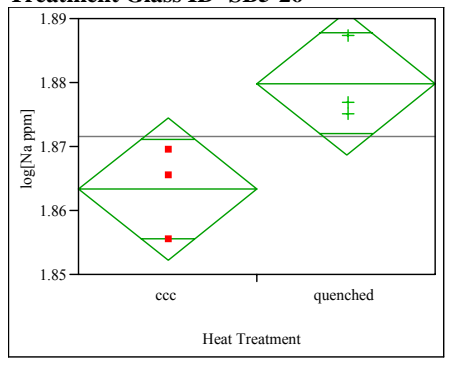

Difference $\quad 0.016543$ t Ratio 2.928212 Std Err Dif 0.005649 DF

Upper CL Dif 0.032228 Prob $>|t| \quad 0.0429$

Lower CL Dif 0.000857 Prob $>t \quad 0.0214$

Confidence $\quad 0.95$ Prob $<\mathrm{t} \quad 0.9786$
Oneway Analysis of $\log [\mathrm{Li}$ ppm] By Heat Treatment Glass ID=SB5-26

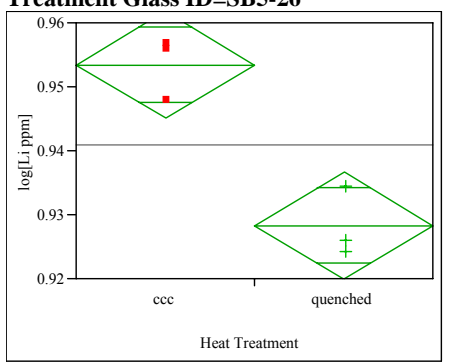

Difference $\quad-0.02516$ t Ratio $\quad-5.92408$ Std Err Dif $\quad 0.00425$ DF

Upper CL Dif -0.01337 Prob $>|t| \quad 0.0041$

Lower CL Dif -0.03695 Prob $>t \quad 0.9980$

Confidence $\quad 0.95$ Prob $<\mathrm{t} \quad 0.0020$

Oneway Analysis of $\log [\mathrm{B}$ ppm] By Heat Treatment Glass ID=SB5-26

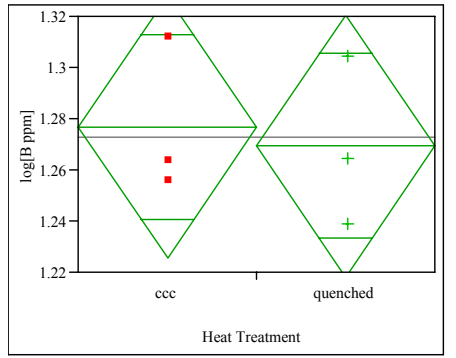

Difference $\quad-0.00744$ t Ratio $\quad-0.28588$ Std Err Dif 0.02603 DF

Upper CL Dif 0.06484 Prob $>|t| \quad 0.7892$

Lower CL Dif -0.07972 Prob $>t \quad 0.6054$

Confidence $\quad 0.95$ Prob $<\mathrm{t} \quad 0.3946$ 
Exhibit B7. Effects of Heat Treatment for Study Glasses by Compositional View.

Comp View=Measured

Chart for NL B(g/L)

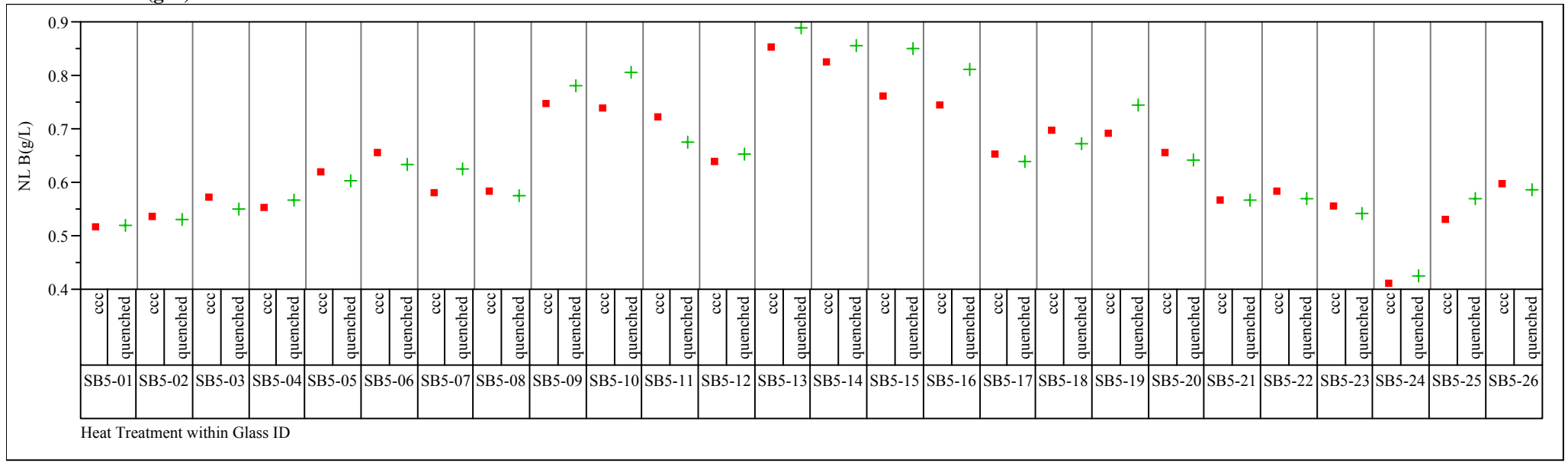

Comp View=Measured

Chart for NL Li (g/L)

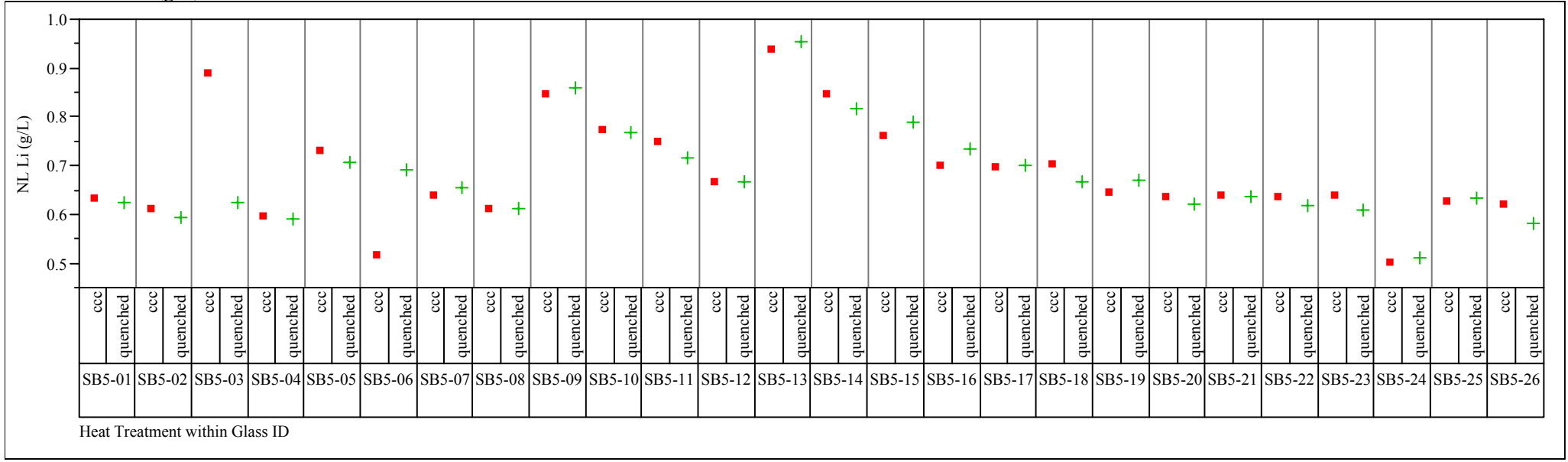


Exhibit B7. Effects of Heat Treatment for Study Glasses by Compositional View. (continued)

Comp View=Measured

Chart for NL Na (g/L)

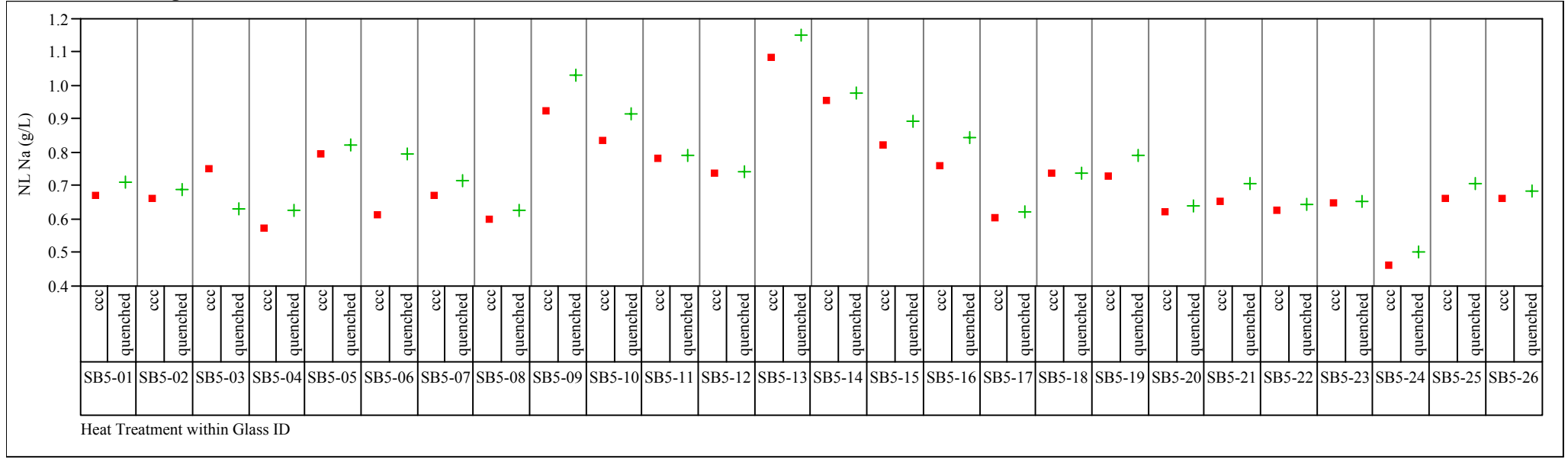

Comp View=Measured

Chart for NL Si (g/L)

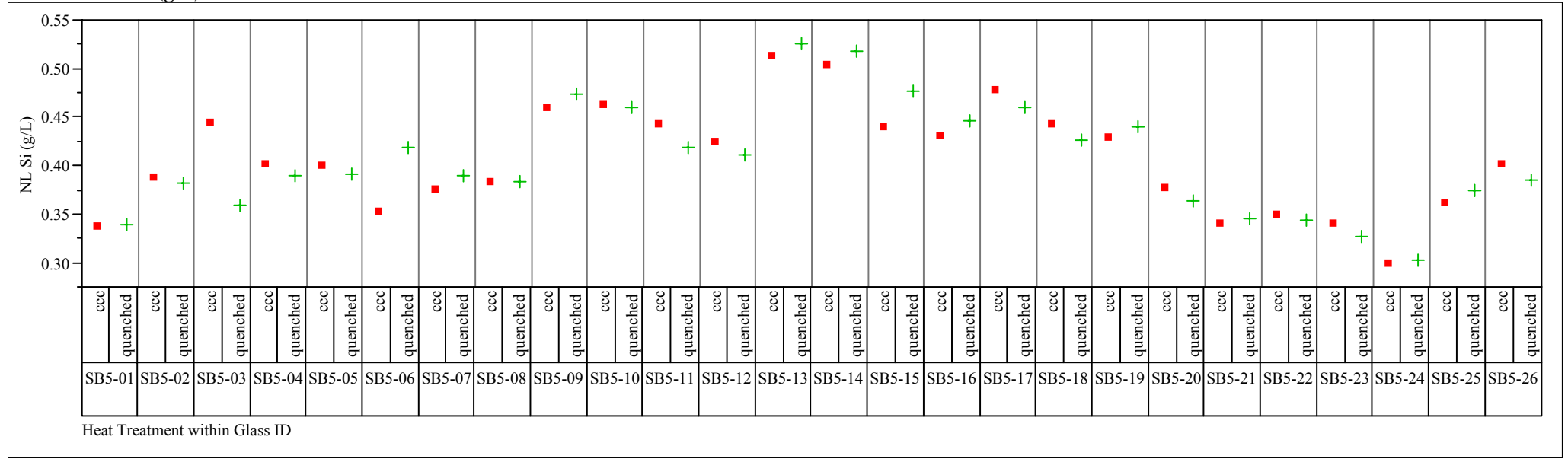


Exhibit B7. Effects of Heat Treatment for Study Glasses by Compositional View. (continued)

Comp View=Measured bc

Chart for NL B(g/L)

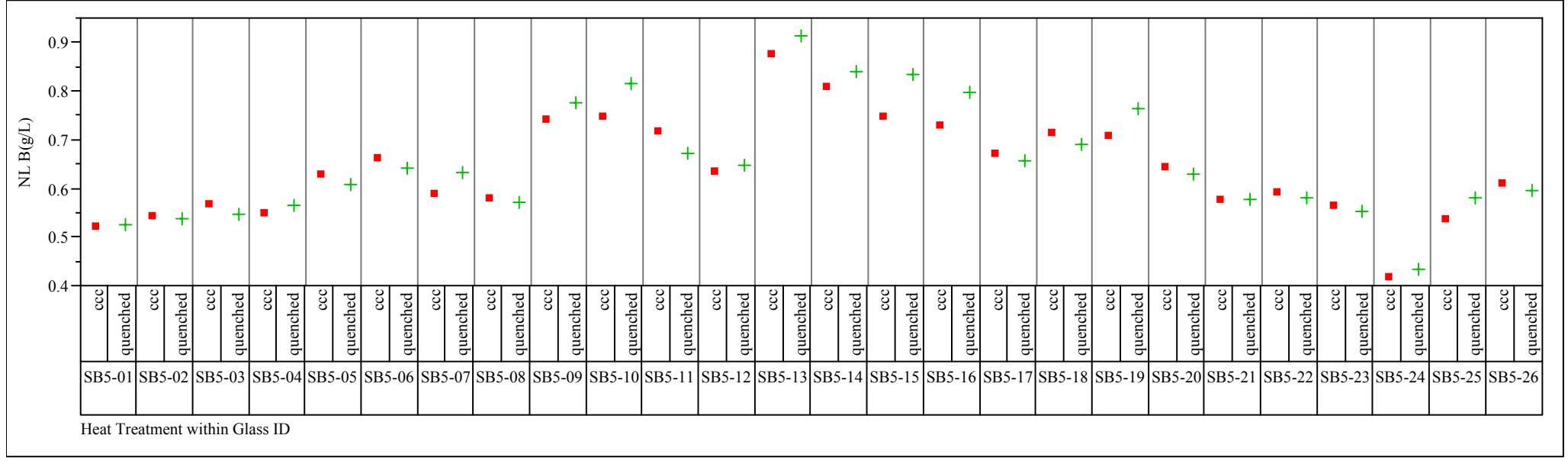

Comp View=Measured bc

Chart for NL Li (g/L)

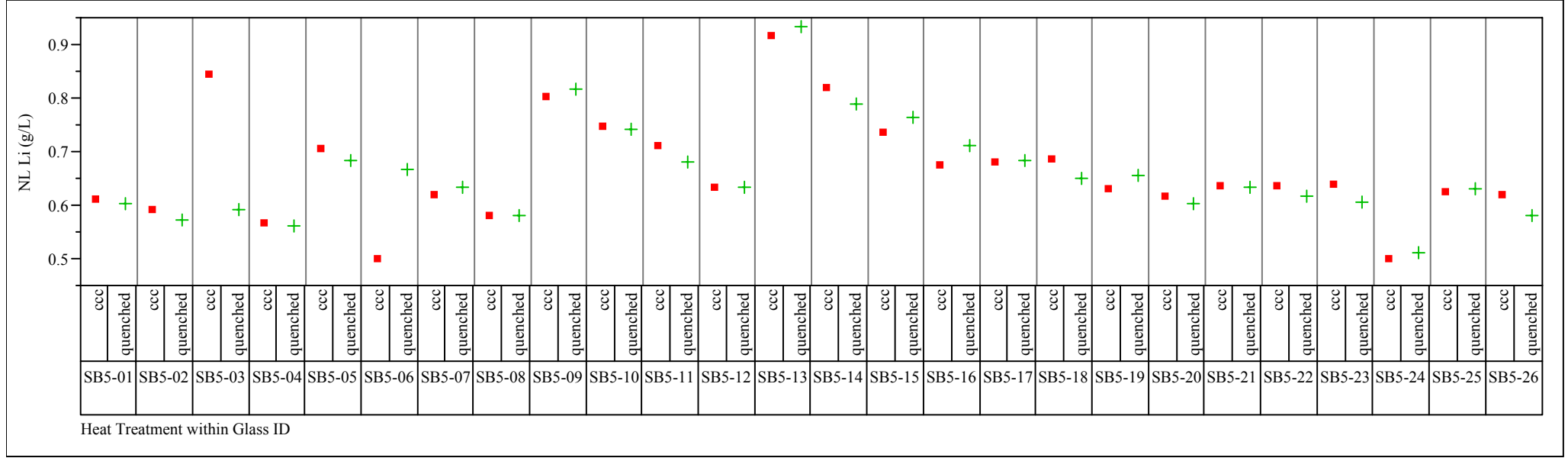


Exhibit B7. Effects of Heat Treatment for Study Glasses by Compositional View. (continued)

Comp View=Measured bc

Chart for NL Na (g/L)

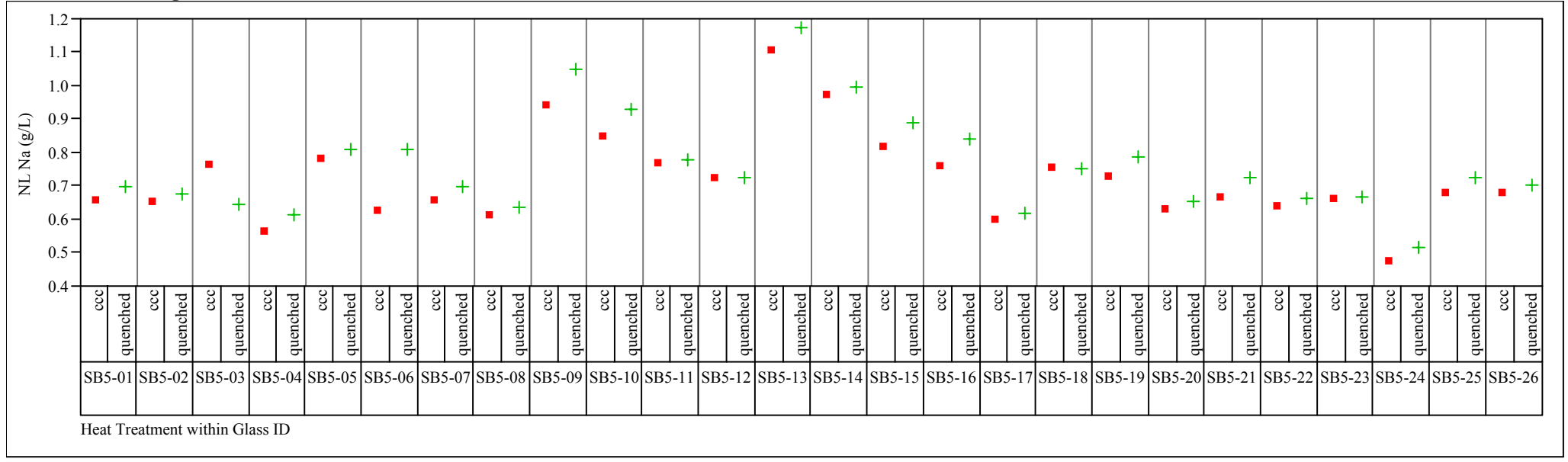

Comp View=Measured bc

Chart for NL Si $(g / L)$

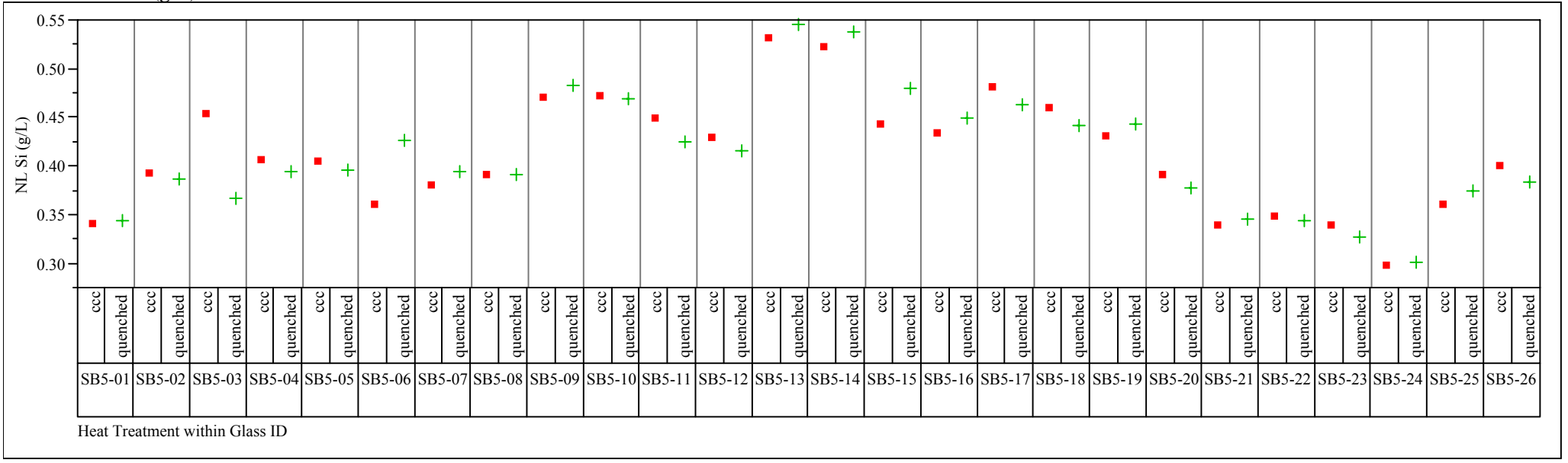


Exhibit B7. Effects of Heat Treatment for Study Glasses by Compositional View. (continued)

Comp View=Targeted

Chart for NL B(g/L)

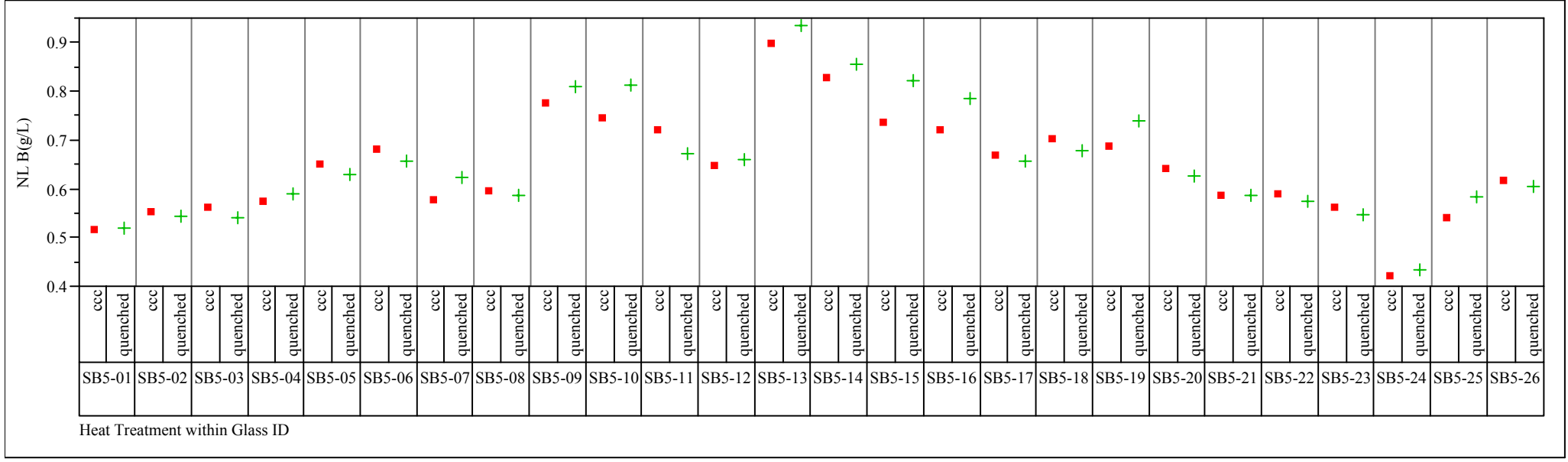

Comp View=Targeted

Chart for NL Li (g/L)

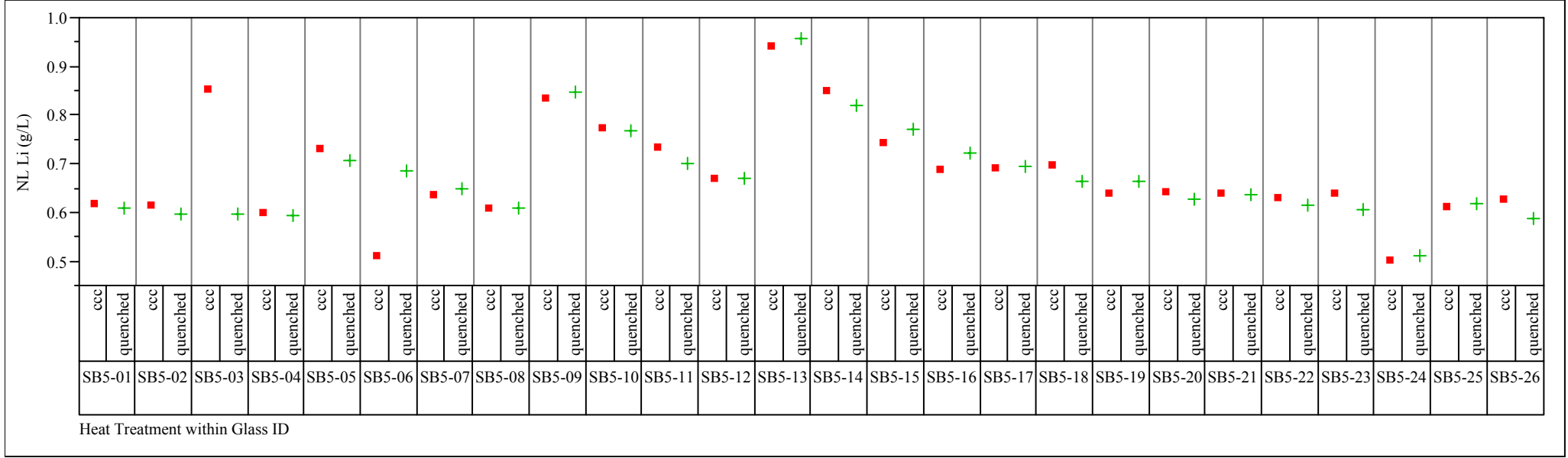


Exhibit B7. Effects of Heat Treatment for Study Glasses by Compositional View. (continued)

Comp View=Targeted

Chart for NL Na (g/L)

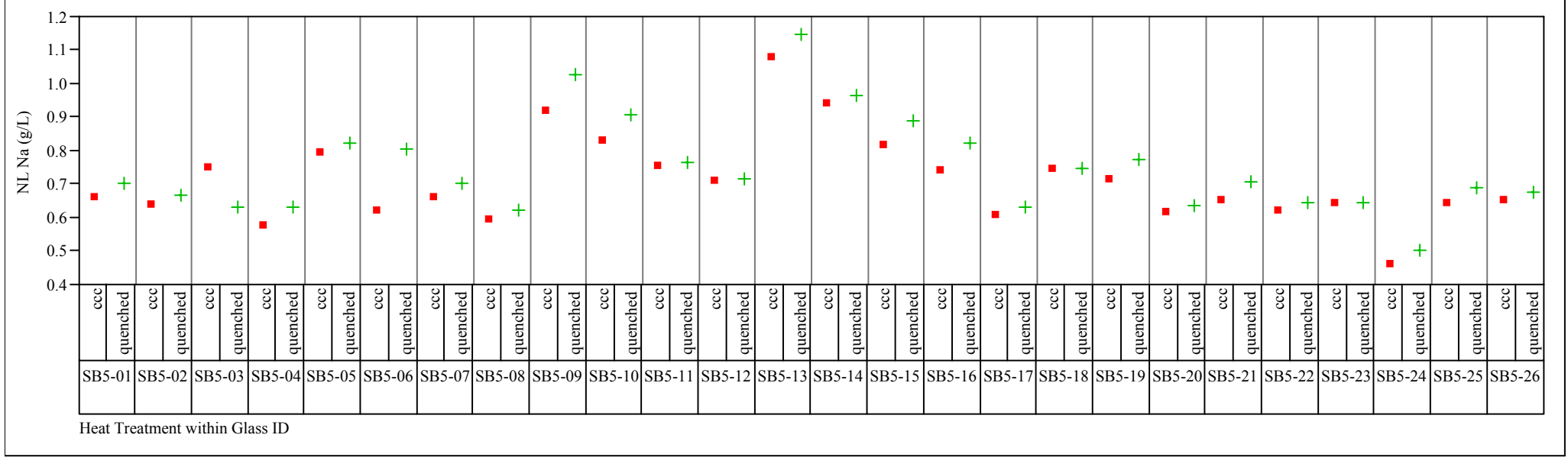

Comp View=Targeted

Chart for NL Si (g/L)

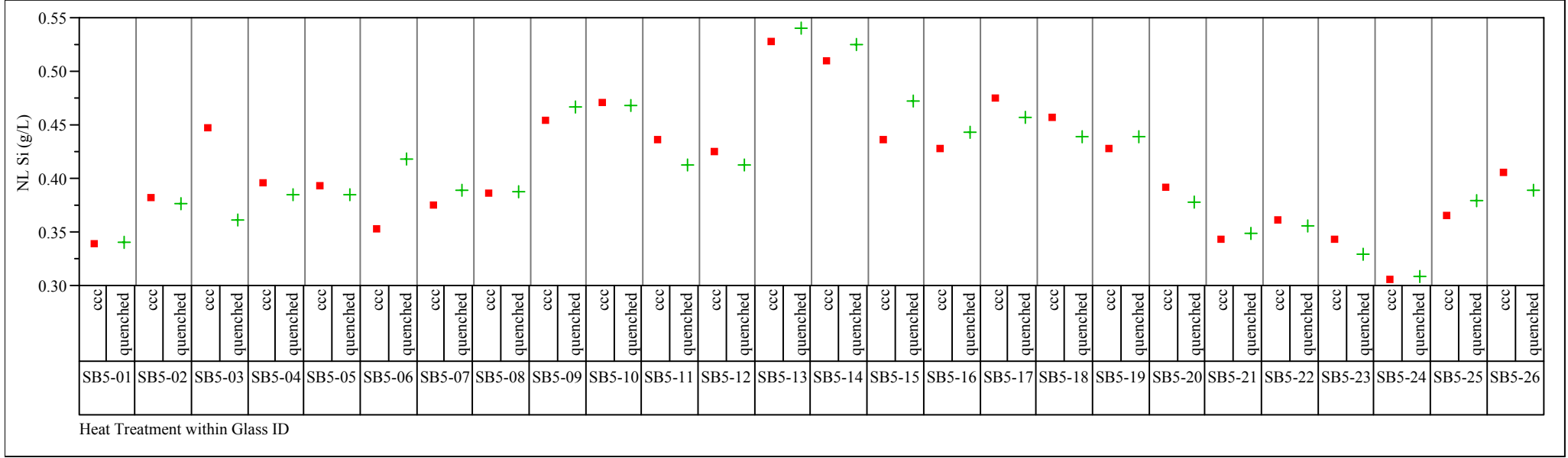


Exhibit B8. Comparisons Between the Potential Surrogate Pairs of Glasses. (continued) Variability Chart for B (ppm)

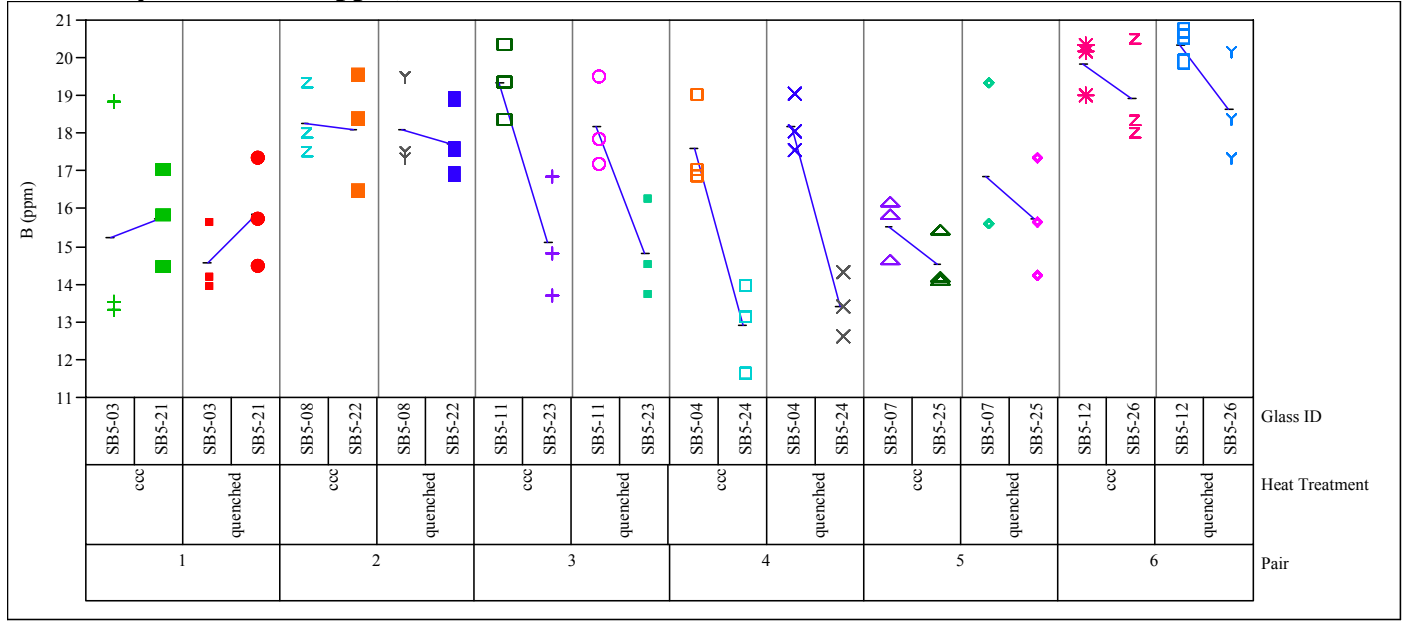

Variability Chart for Li (ppm)

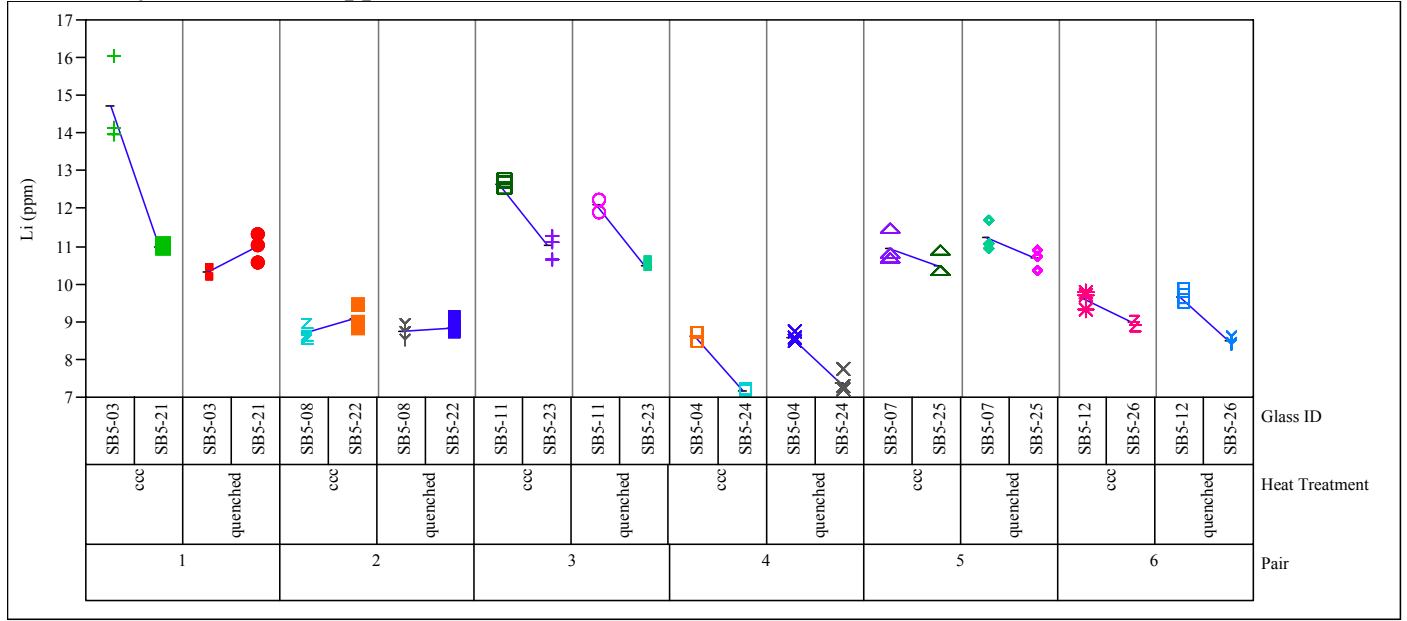

Variability Chart for Na (ppm)

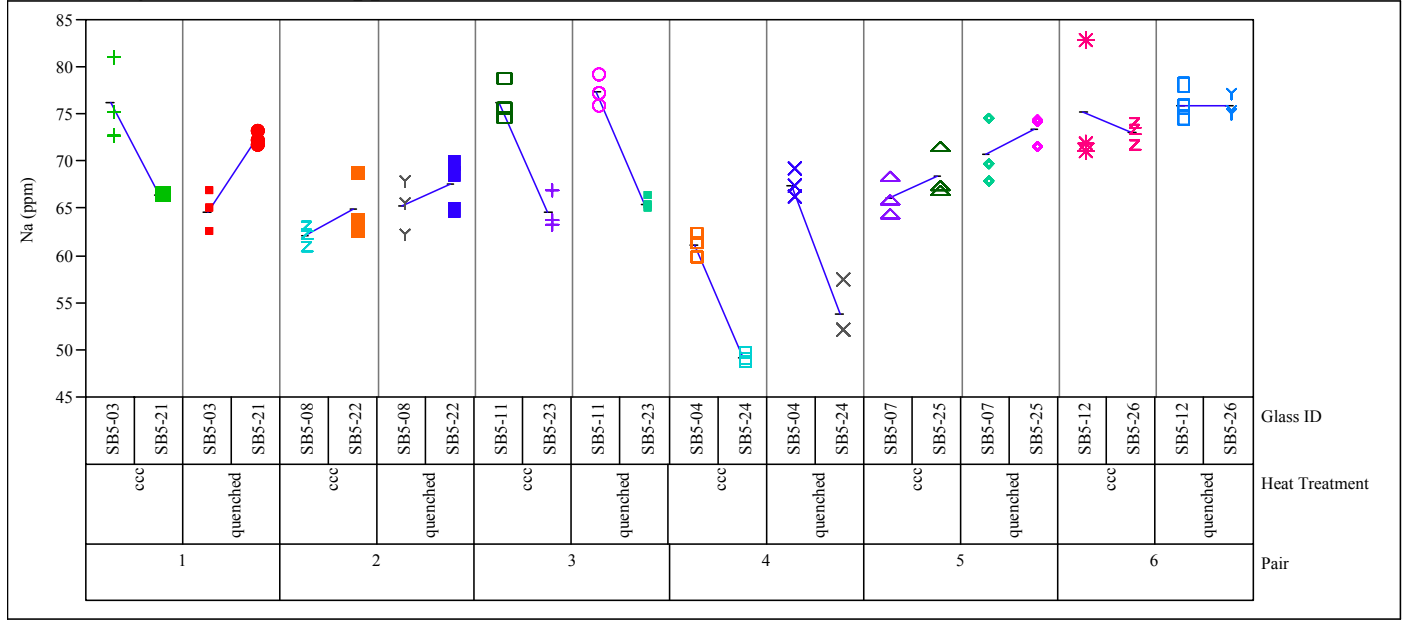


Exhibit B8. Comparisons Between the Potential Surrogate Pairs of Glasses. (continued)

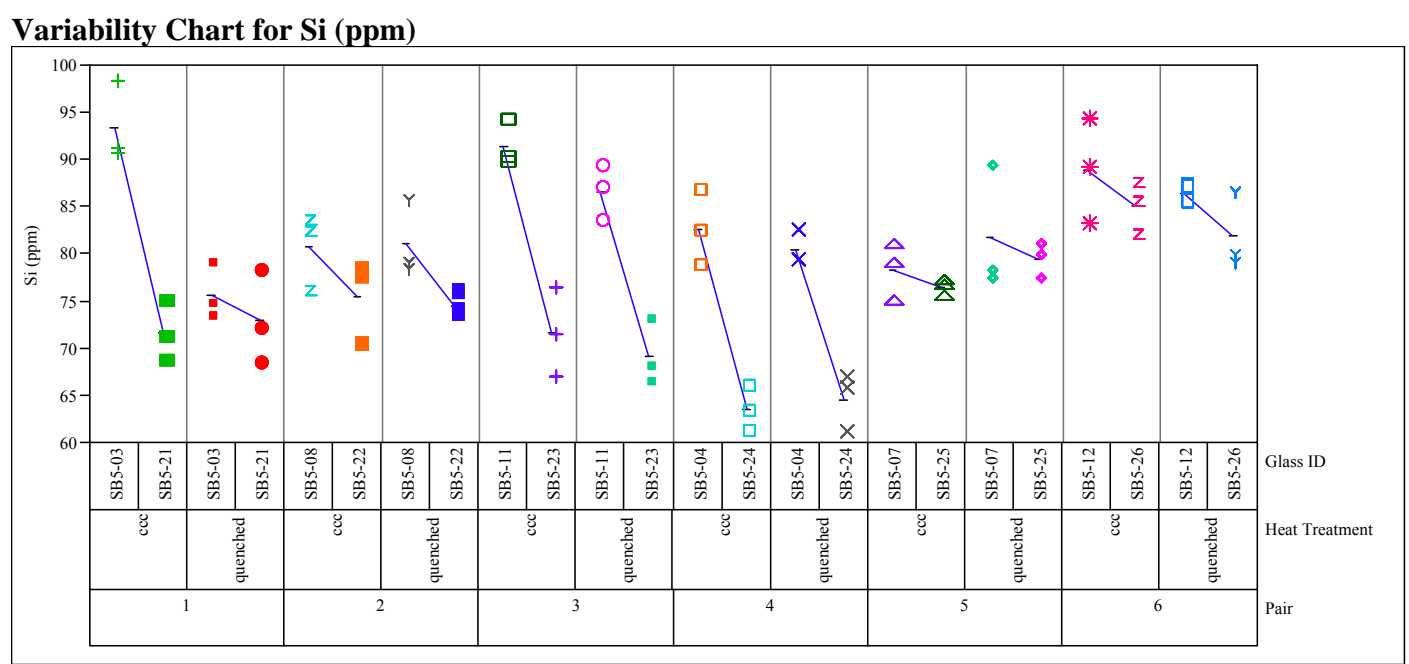

Variability Chart for $\log [\mathrm{B}$ ppm]

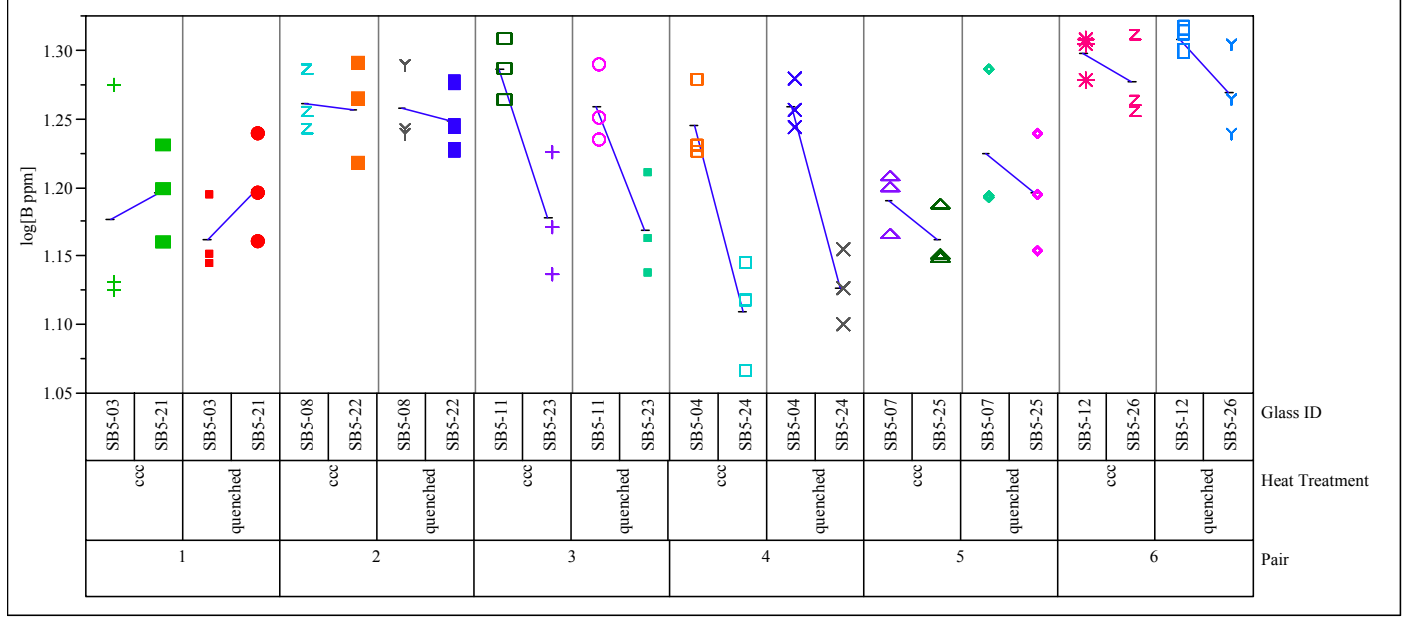

Variability Chart for log[Li ppm]

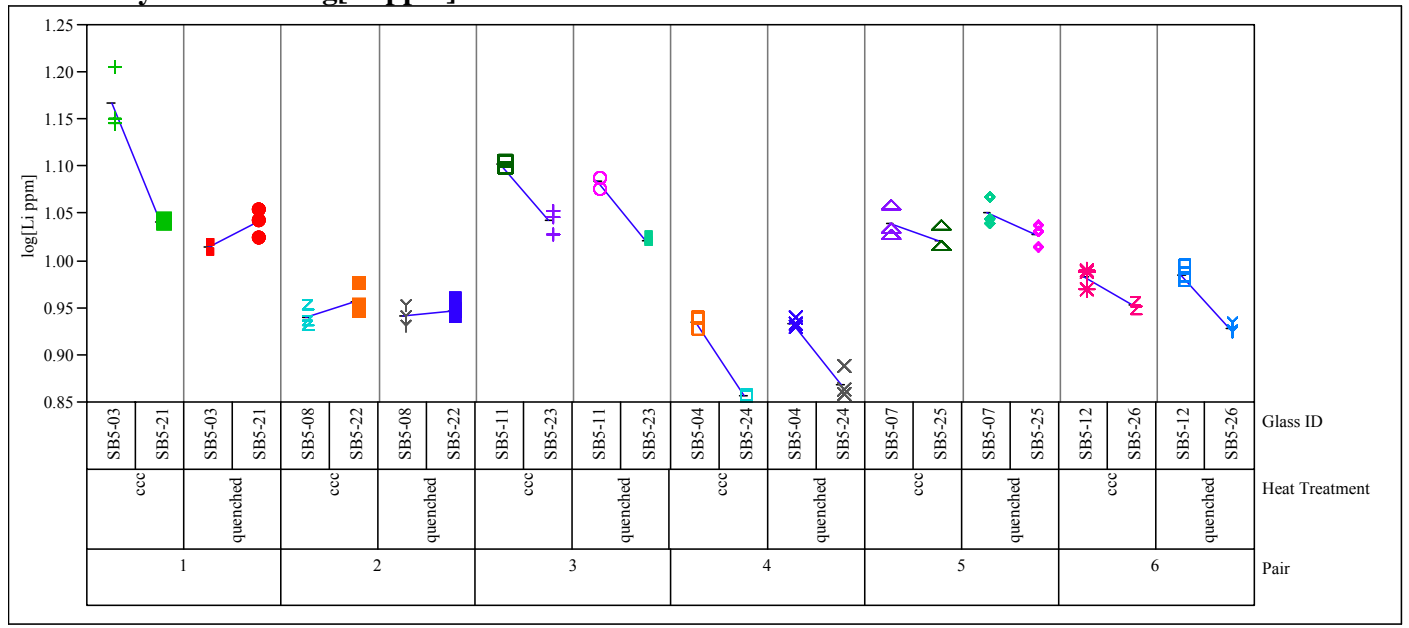


Exhibit B8. Comparisons Between the Potential Surrogate Pairs of Glasses. (continued)

Variability Chart for log[Na ppm]

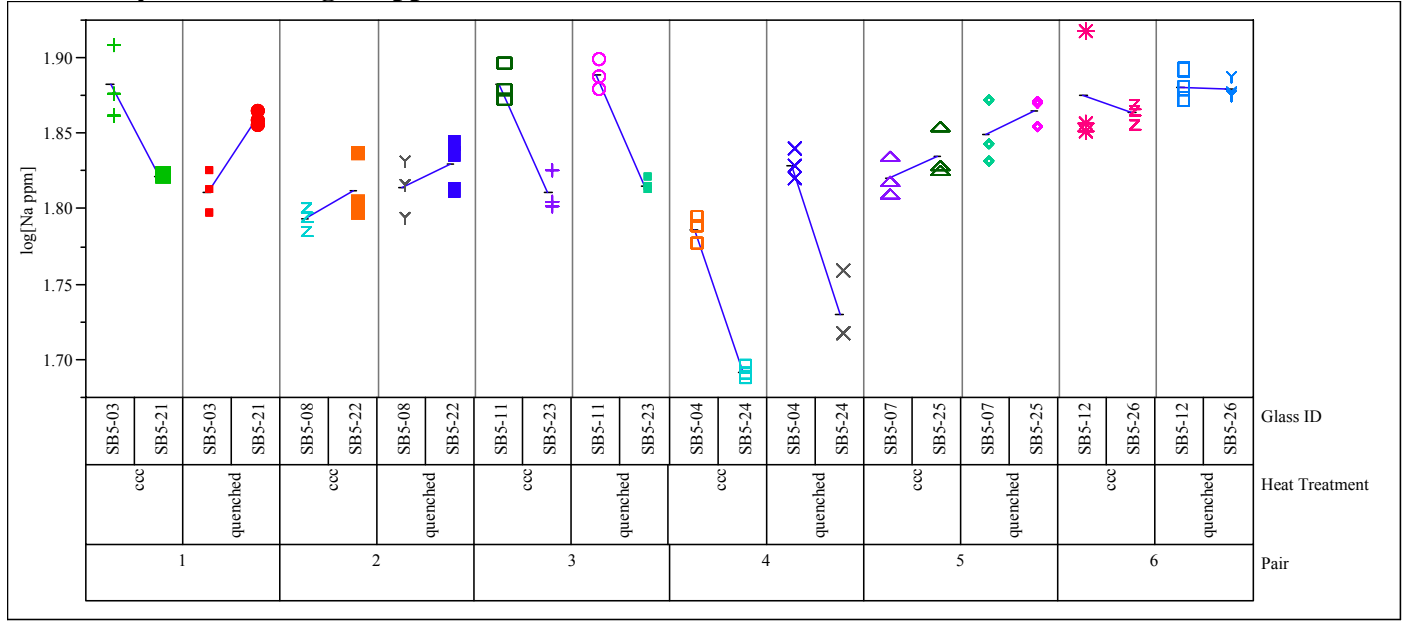

Variability Chart for log[Si ppm]

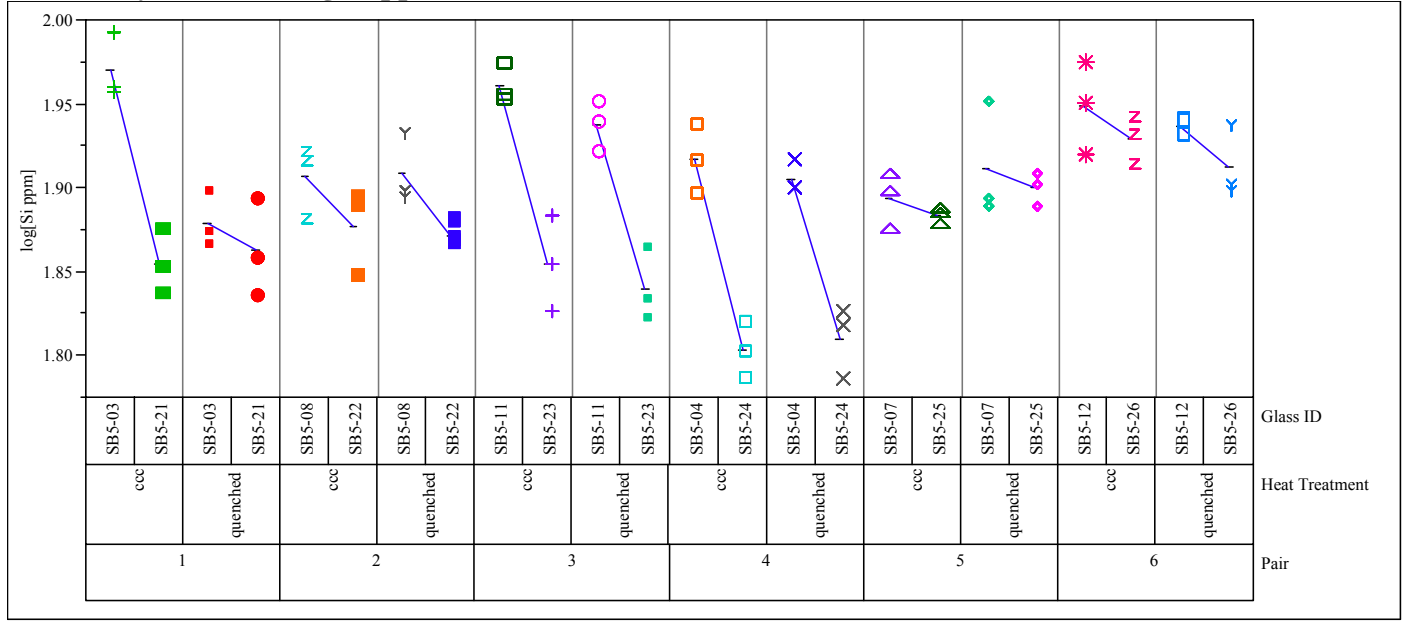

Variability Chart for $\log$ NL[B (g/L)]

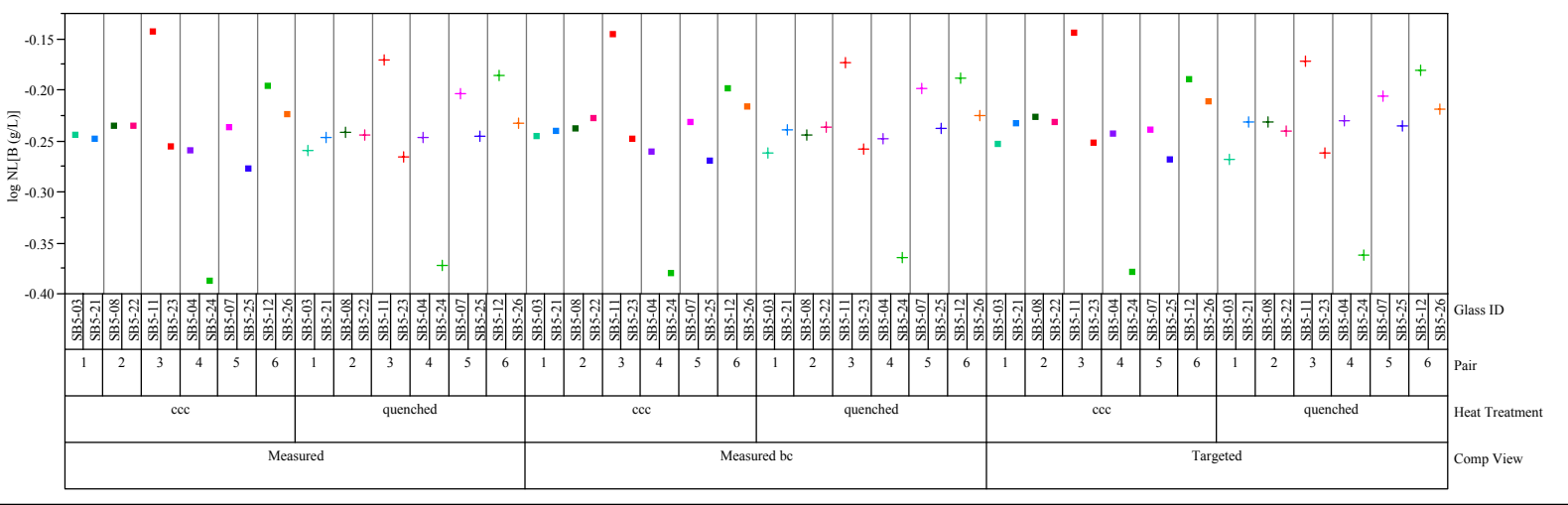


Exhibit B8. Comparisons Between the Potential Surrogate Pairs of Glasses. (continued)

Variability Chart for log NL[Li(g/L)]

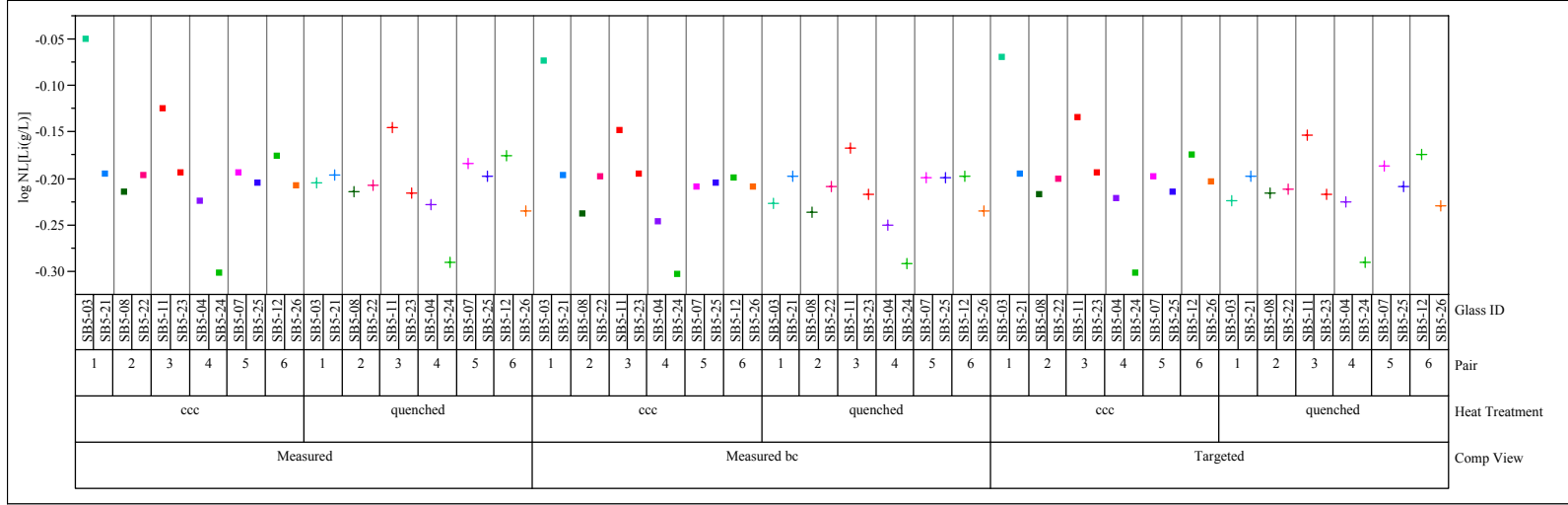

Variability Chart for log NL[Na (g/L)]

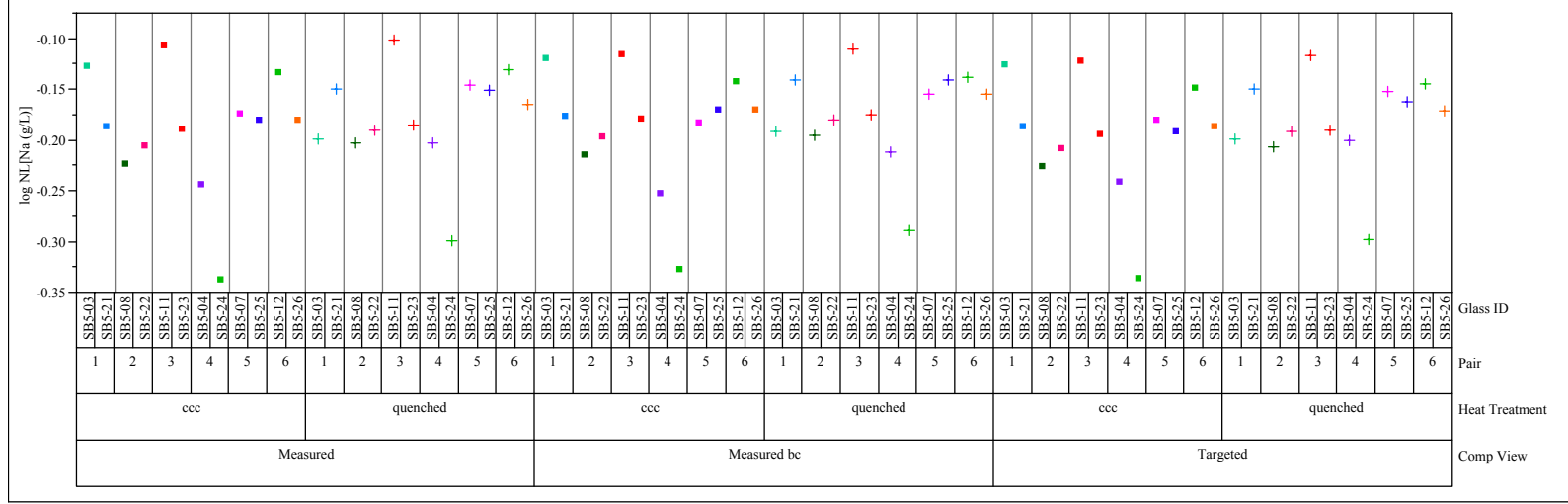

Variability Chart for $\log$ NL[Si (g/L)]

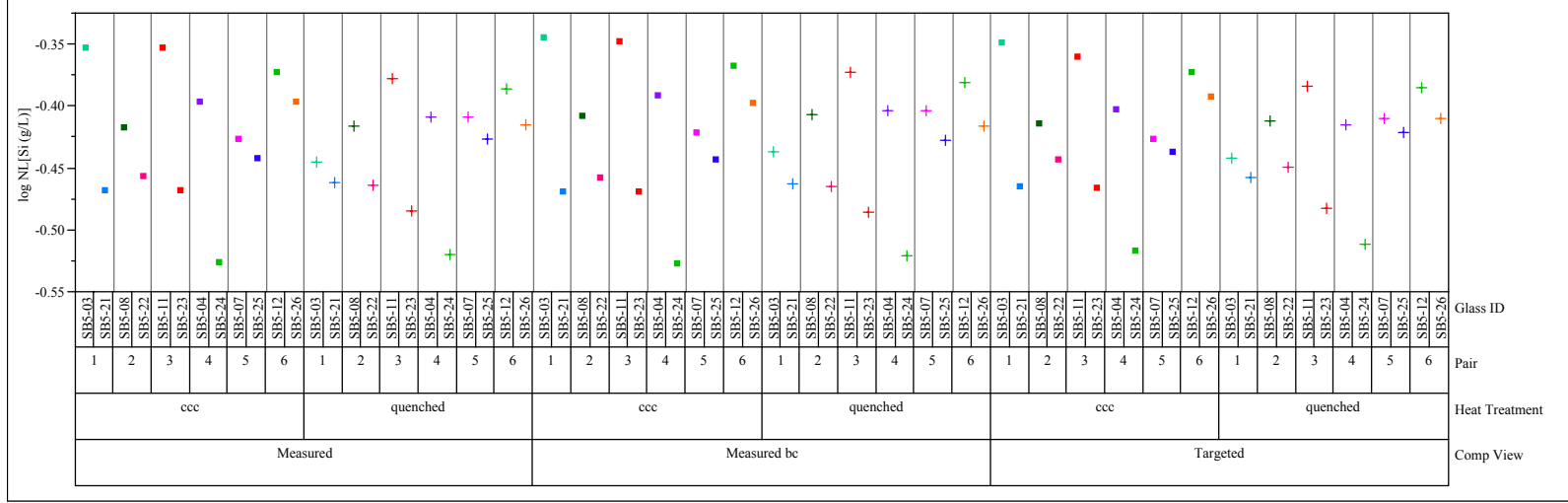


Exhibit B9. del $G p\left(\Delta G_{p}\right)$ Predictions versus Common Logarithm Normalized Leachate (log NL[.]) for B, Li, Na, and Si Over All Compositional Views and Heat Treatments for Study Glasses SB5-01 through SB5-20.

\begin{tabular}{|c|c|c|}
\hline & & Glass Standard or Heat Treatment-Compositional View \\
\hline $\mathbf{z}$ & 1 & ARM \\
\hline$\diamond$ & 2 & EA \\
\hline 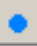 & 3 & measured bc-ccc \\
\hline $\mathbf{a}$ & 4 & measured bc-quenched \\
\hline 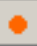 & 5 & measured-cce \\
\hline 口 & 6 & measured-quenched \\
\hline 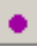 & 7 & targeted-ccc \\
\hline $\mathbf{a}$ & 8 & targeted-quenched \\
\hline
\end{tabular}

Bivariate Fit of log NL[B (g/L)] By del Gp

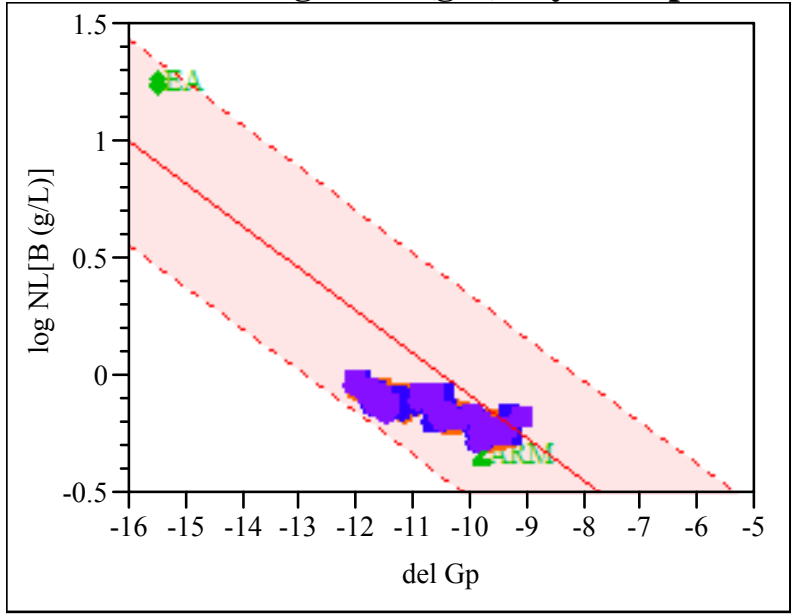

Bivariate Fit of log NL[Li (g/L)] By del Gp

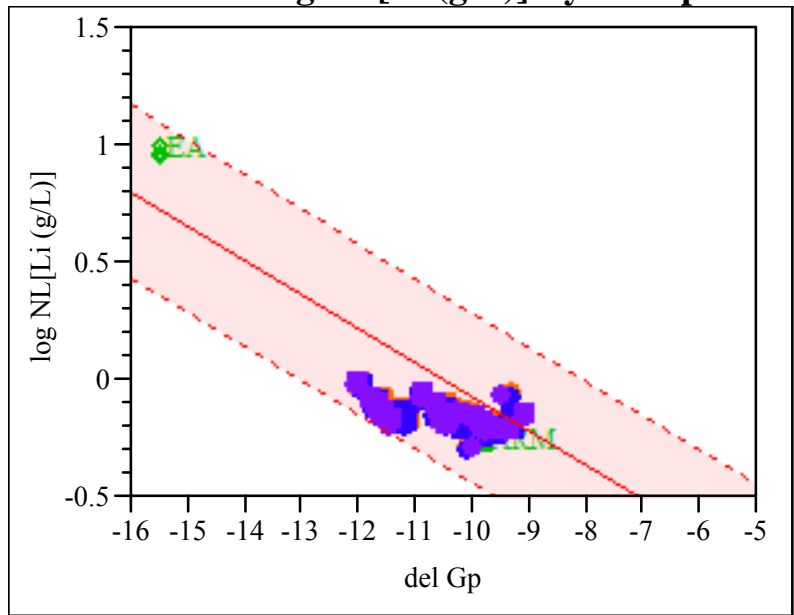

Bivariate Fit of log NL[Na (g/L)] By del Gp

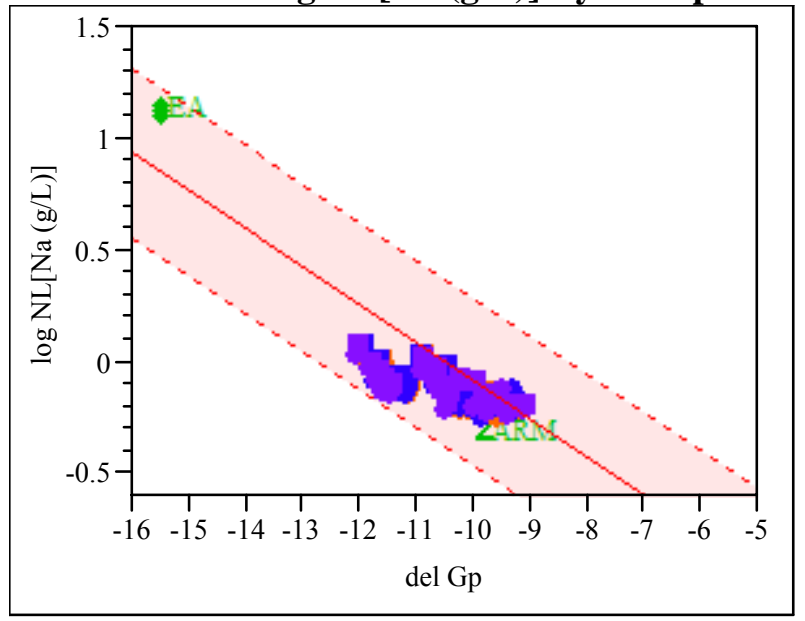

Bivariate Fit of log NL[Si (g/L)] By del Gp

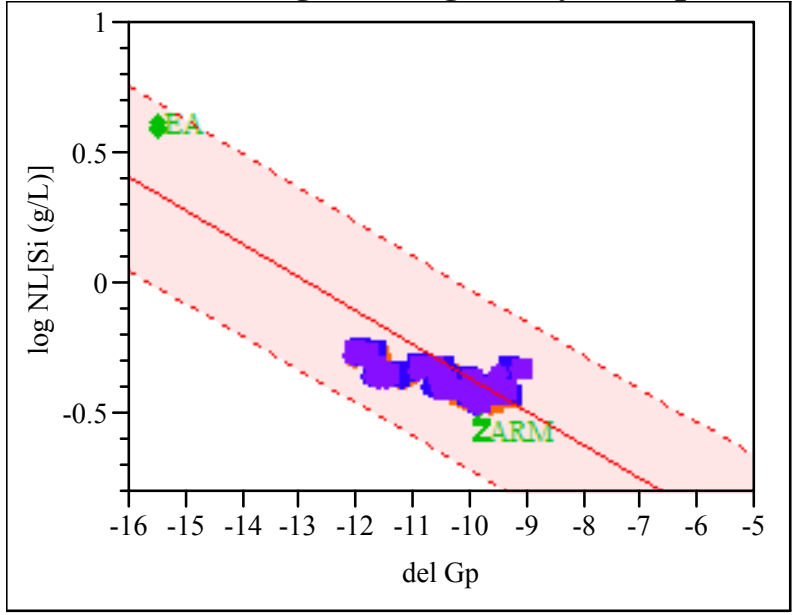


Exhibit B10. del Gp $\left(\Delta G_{p}\right)$ Predictions versus Common Logarithm Normalized Leachate $(\log \mathrm{NL}[]$.$) for B, Li, Na, and Si Over All Compositional Views$ for Quenched Versions of Study Glasses SB5-01 through SB5-20.

\begin{tabular}{|c|c|c|}
\hline & & Glass Standard or Heat Treatment-Compositional View \\
\hline$z$ & 1 & ARM \\
\hline$\diamond$ & 2 & EA \\
\hline - & 3 & measured bc-ccc \\
\hline $\mathbf{0}$ & 4 & measured bc-quenched \\
\hline 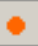 & 5 & measured-cce \\
\hline 口 & 6 & measured-quenched \\
\hline$\bullet$ & 7 & targeted-ccc \\
\hline $\mathbf{a}$ & 8 & targeted-quenched \\
\hline
\end{tabular}

Bivariate Fit of $\log$ NL[B (g/L)] By del Gp

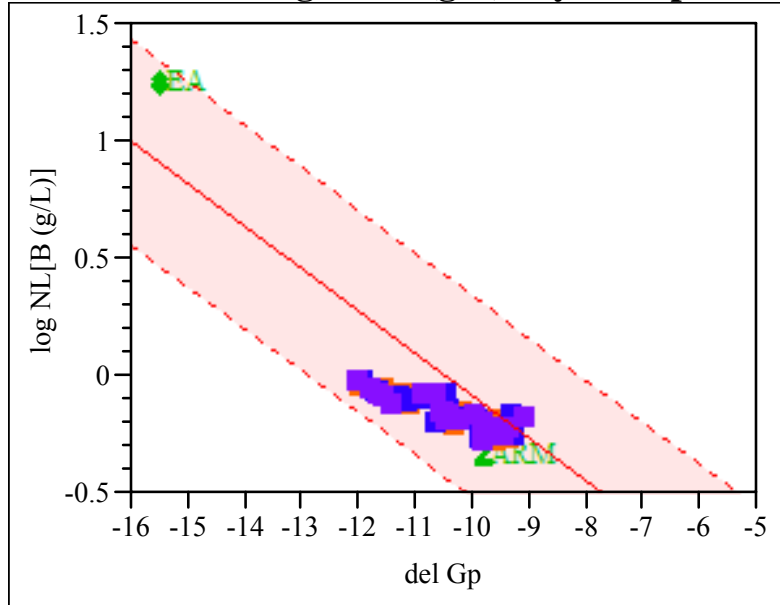

Bivariate Fit of $\log$ NL[Li (g/L)] By del Gp

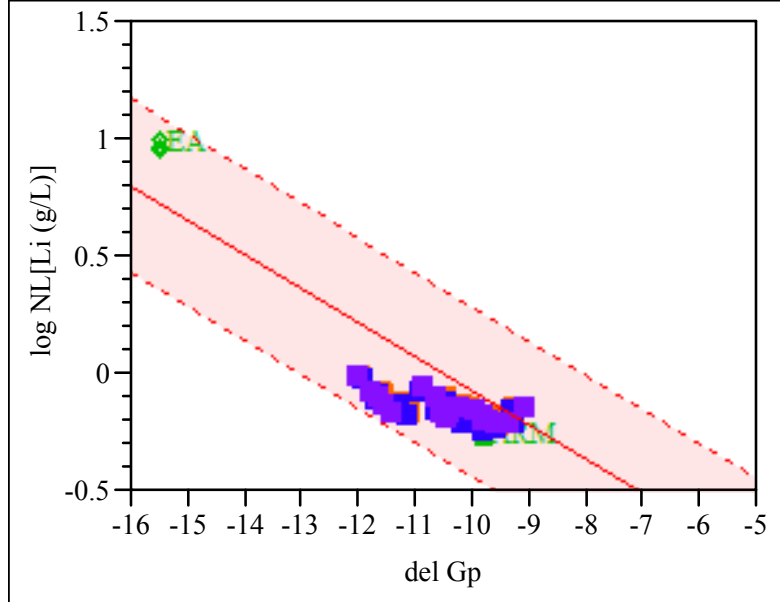

Bivariate Fit of log NL[Na (g/L)] By del Gp

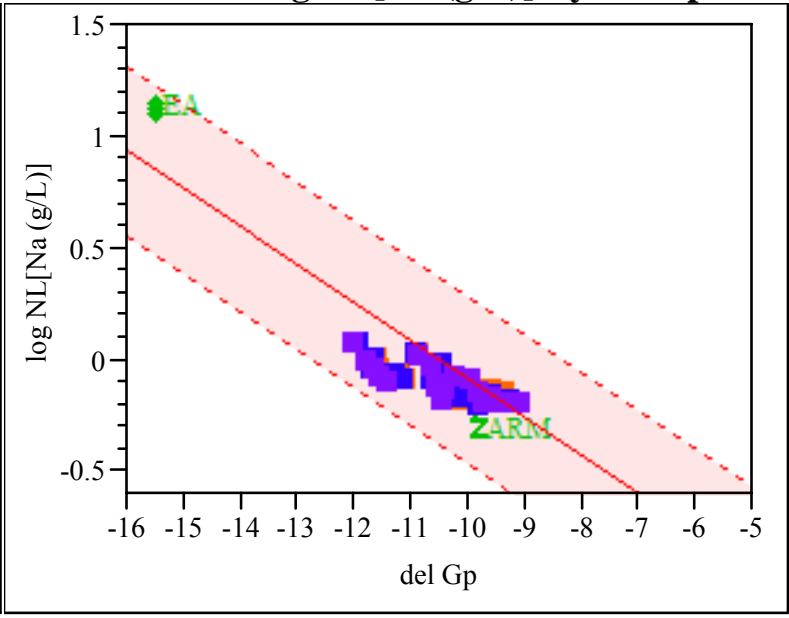

Bivariate Fit of $\log$ NL[Si (g/L)] By del Gp

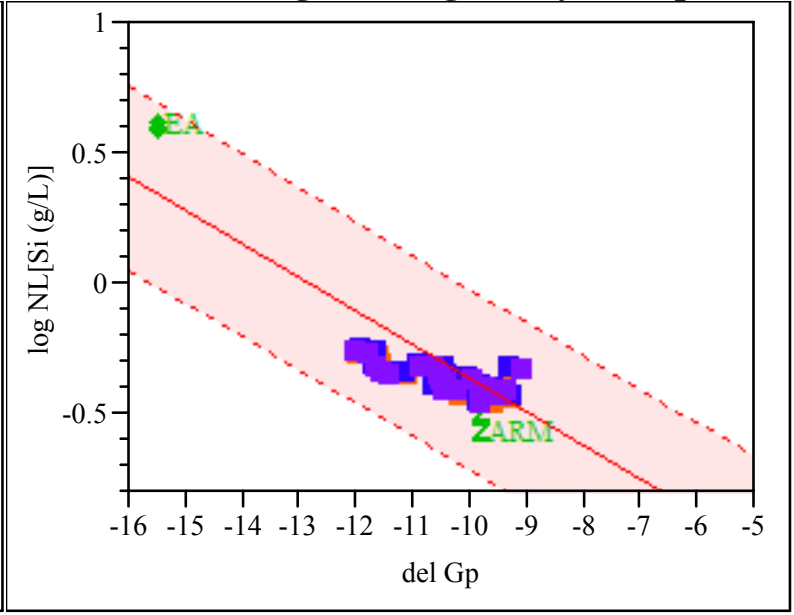


Exhibit B11. del Gp $\left(\Delta G_{p}\right)$ Predictions versus Common Logarithm Normalized Leachate (log NL[.]) for B, Li, Na, and Si Over All Compositional Views

for CCC Versions of Study Glasses SB5-01 through SB5-20.

\begin{tabular}{|c|c|c|}
\hline & & Glass Standard or Heat Treatment-Compositional View \\
\hline $\mathbf{z}$ & 1 & ARM \\
\hline$\diamond$ & 2 & EA \\
\hline$\bullet$ & 3 & measured bc-cce \\
\hline - & 4 & measured bc-quenched \\
\hline$\bullet$ & 5 & measured-cce \\
\hline 물 & 6 & measured-quenched \\
\hline$\bullet$ & 7 & targeted-ccc \\
\hline घ & 8 & targeted-quenched \\
\hline
\end{tabular}

Bivariate Fit of log NL[B (g/L)] By del Gp

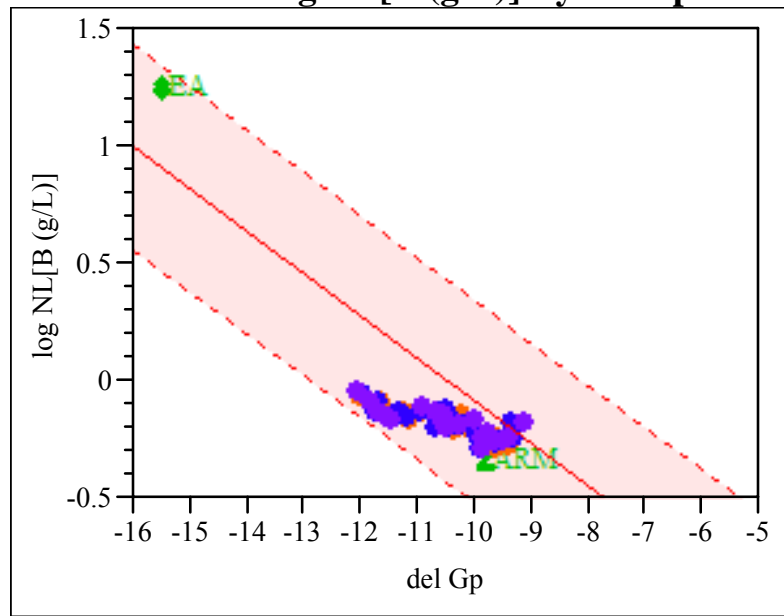

Bivariate Fit of log NL[Li (g/L)] By del Gp

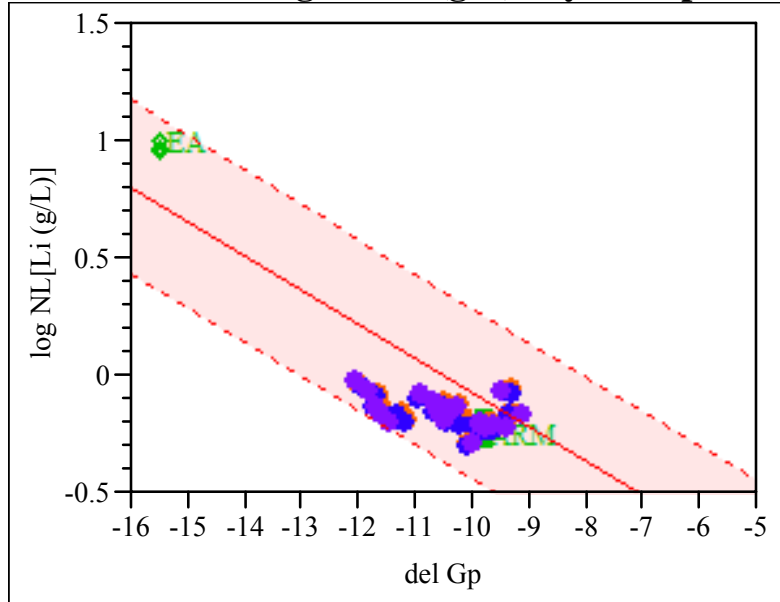

Bivariate Fit of log NL[Na (g/L)] By del Gp

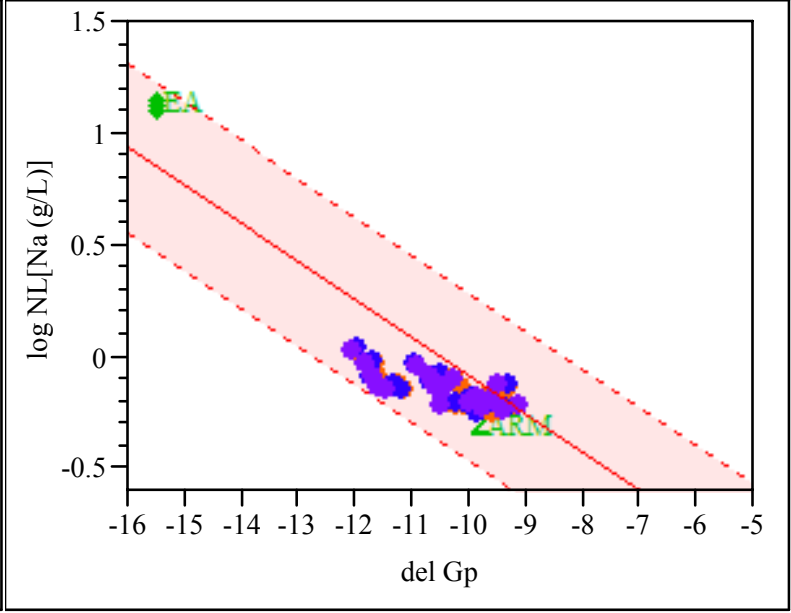

Bivariate Fit of log NL[Si (g/L)] By del Gp

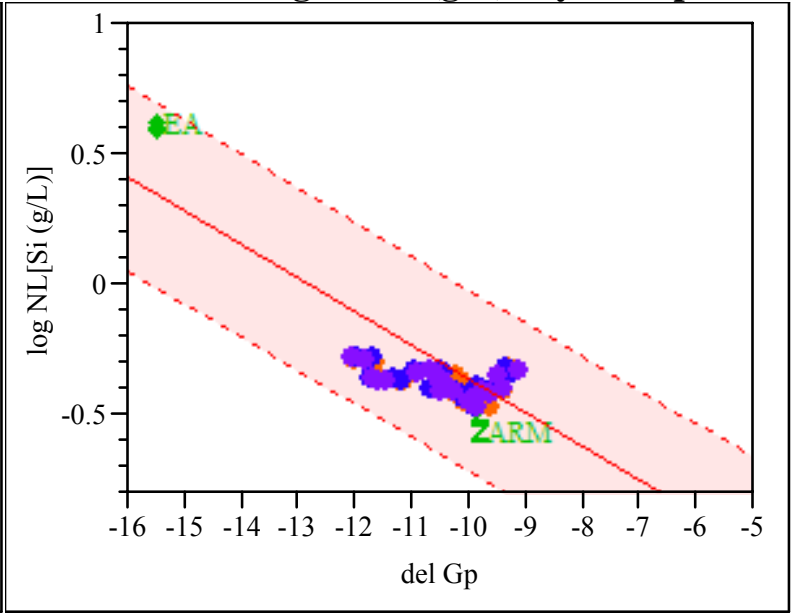




\section{Distribution:}

C.J. Bannochie, 773-42A

D.D. Larsen, 766-H

A.B. Barnes, 999-W

D.J. McCabe, 773-42A

D.R. Best, 786-1A

R.T. McNew, 704-27S

J.M. Bricker, 704-27S

D.H. Miller, 999-W

D.A. Crowley, 773-43A

T.A. Nance, 773-42A

B.A. Davis, 704-27S

J.D. Newell, 999-W

T.B. Edwards, 999-W

J.E. Occhipinti, 704-S

T.L. Fellinger, 704-26S

D.K. Peeler, 999-W

S. D. Fink, 773-A

F.C. Raszewski, 999-W

K.M. Fox, 999-W

J.W. Ray, 704-S

C.W. Gardner, 773-A

B.J. Giddings, 786-5A

I.A. Reamer, 999-1W

J.M. Gillam, 766-H

H.B. Shah, 766-H

D.C. Sherburne, 704-S

J.C. Griffin, 773-A

B.A. Hamm, 766-H

M.E. Smith, 999-W

M.E. Stone, 999-W

C.C. Herman, 999-W

J. Stuberfield, 766-H

J.F. Iaukea, 704-30S

R.J. Workman, 999-1 W

T.M. Jones, 999-W

A.L. Youchak-Billings, 999-W 